\title{
Meso- and Macro-Scale Facies and Chemostratigraphic Analysis of Middle Devonian Marcellus Shale in Northern West Virginia, USA
}

Thomas J Paronish

Follow this and additional works at: https://researchrepository.wvu.edu/etd

\section{Recommended Citation}

Paronish, Thomas J, "Meso- and Macro-Scale Facies and Chemostratigraphic Analysis of Middle Devonian Marcellus Shale in Northern West Virginia, USA" (2018). Graduate Theses, Dissertations, and Problem Reports. 7225.

https://researchrepository.wvu.edu/etd/7225

This Thesis is protected by copyright and/or related rights. It has been brought to you by the The Research Repository @ WVU with permission from the rights-holder(s). You are free to use this Thesis in any way that is permitted by the copyright and related rights legislation that applies to your use. For other uses you must obtain permission from the rights-holder(s) directly, unless additional rights are indicated by a Creative Commons license in the record and/ or on the work itself. This Thesis has been accepted for inclusion in WVU Graduate Theses, Dissertations, and Problem Reports collection by an authorized administrator of The Research Repository @ WVU. For more information, please contact researchrepository@mail.wvu.edu. 
Meso- and Macro-Scale Facies and Chemostratigraphic Analysis of Middle Devonian Marcellus Shale in Northern West Virginia, USA

Thomas J. Paronish

Thesis submitted to the

Eberly College of Arts and Sciences

at West Virginia University

in partial fulfillment of the requirements for the degree of

Master of Science in

Geology

Timothy R. Carr, Ph.D., Chair

Shikha Sharma, Ph.D.

Amy Weislogel, Ph.D.

Department of Geology and Geography

Morgantown, West Virginia

2018

Keywords: Marcellus Shale, Appalachian Basin, Chemostratigraphy, Lithofacies, Petrophysics

Copyright@ 2018 Thomas J. Paronish 


\section{ABSTRACT \\ Meso- and Macro-Scale Facies and Chemostratigraphic Analysis of Middle Devonian Marcellus Shale in Northern West Virginia, USA}

\section{Thomas J Paronish}

The Marcellus Shale Energy and Environmental Laboratory (MSEEL), consists of four producing horizontal wells, two vertical pilot wells and a vertical microseismic observation well. To gain a detailed understanding of the Middle Devonian Marcellus Shale and surrounding shale and limestone intervals, I focused on the two vertical pilot wells (MIP-3H and MIP-4H). Understanding the vertical and lateral distribution of the shale lithofacies and changes in chemostratigraphy are critical to understanding the impact of depositional and diagenetic environments on hydrocarbon generation and production in shale gas reservoirs. Integrated geological and petrophysical characterization of the Marcellus and adjacent Onondaga Limestone through Mahantango Formation used available core and well log data.

Macro-scale lithofacies were determined through a combination of core and CT-scan descriptions. Meso-scale shale lithofacies, based on mineralogy and total organic content (TOC), used a combination of triple combo and advanced logging tools, which were calibrated to core data (XRD and source-rock pyrolysis). Chemostratigraphic analysis utilized X-ray fluorescence (XRF) to determine the major and trace-element trends associated within the Devonian Marcellus-Mahantango interval. Integration of these three approaches were used to develop a depositional model.

The Devonian Marcellus-Mahantango interval is composed of six shale lithofacies both at the meso- and macro-scale. Petrophysical analysis shows that three well developed organic mudstone facies are present in the Marcellus interval. Chemostratigraphic (trace element concentrations) and petrophysical data (spectral gamma derived uranium content) indicate the highly organic-rich mudstone (TOC > 6.5 weight percent) facies in the lowest part of the Marcellus Shale compared to overlying units was deposited in a highly anoxic environment with decreased detrital influence indicated by silicon, aluminum, and titanium trends. The anoxic conditions and decreased detrital input allowed for preservation of organic matter in the lowest part of the Marcellus Shale. 


\section{Acknowledgements}

I would like to express my gratitude to my advisor, Dr. Timothy Carr for supporting this thesis. Thank you for accepting me as your student, providing financial assistance, guidance, and for your boundless patience in reviewing my thesis. The skills I have learned from you have made me both a better geologist and individual.

I would like to thank the faculty and staff in the Department of Geology and Geography for aiding in this process. Specifically I would like to thank Dr. Shikha Sharma and Dr. Amy Wieslogel for being on my committee. Thank you both for the time and insights for my research.

I would like to thank the Department of Energy for funding this project and the scores of individuals and groups associated with the Marcellus Shale Energy and Environmental Laboratory (MSEEL). Specifically I would like to thank Northeast Natural Energy for providing the $\log$ and core data for this study. I would like to express my gratitude to the National Energy Technology Laboratory, specifically, Dustin Crandall, Johnathan Moore, Sarah Brown, and Chloe Wonnel. Thank you for providing the pyrolysis data, XRF data sets, and medical CTscans, as well as, your guidance and support in acquiring the core description.

I would like to express my appreciation to my friends and colleagues within the department. Thank you for your suggestions and motivation during this process. Brittany Hupp, thank you for your help in providing the XRD data for this study. Payam Kavousi, Shuvajit Bhattacharya, Keithan Martin, and Liaosha Song, thank you all for friendship, guidance, and for your willingness to take the time and share your skills and knowledge. 
Lastly and most importantly, I would like to thank my family and friends for their constant support, encouragement, and patience during this process. Special thanks to my parents and siblings without your love and support this journey would not have been possible.

Thomas Paronish

April 19, 2018 


\section{Table of Contents}

Abstract ii

Acknowledgements $\quad$ iii

Table of Contents $\quad$ V

List of Figures vii

List of Tables $\quad$ xiii

Chapter 1, Introduction 1

1.1 Geologic Setting 3

$\begin{array}{ll}\text { 1.2 Previous Studies } & 6\end{array}$

$\begin{array}{ll}\text { Lithofacies } & 6\end{array}$

$\begin{array}{ll}\text { XRF/Chemostratigraphy } & 7\end{array}$

1.3 Study Area $\quad 7$

$\begin{array}{ll}\text { Chapter 2, Lithofacies } & 7\end{array}$

$\begin{array}{ll}\text { 2.1 Macrofacies } & 7\end{array}$

$\begin{array}{ll}\text { Core description } & 7\end{array}$

$\begin{array}{ll}\text { Ct-scan description } & 16\end{array}$

2.2 Mesofacies 39

Total Organic Carbon Evaluation $\quad 39$

$\begin{array}{ll}\text { Mineralogy } & 46\end{array}$

$\begin{array}{ll}\text { XRD } & 46\end{array}$

Petrophysical Mineralogy 48

Meso-facies classification $\quad 55$

$\begin{array}{lr}\text { Chapter 3, Chemostratigraphy } & 59\end{array}$ 
3.1 X-ray Fluorescence $\quad 59$

$\begin{array}{ll}\text { 3.2 Paleo Proxies } & 69\end{array}$

$\begin{array}{ll}\text { Detrital } & 70\end{array}$

$\begin{array}{ll}\text { Redox Condition } & 71\end{array}$

$\begin{array}{ll}\text { Paleo-Production } & 72\end{array}$

$\begin{array}{ll}3.3 \text { Pulse Neutron Spectroscopy } & 76\end{array}$

Chapter 4, Discussion and Conclusions $\quad 81$

Chapter 5, References Cited $\quad 83$

Appendix A: Source rock Pyrolysis data 86

$\begin{array}{lr}\text { Appendix B: X-Ray Diffraction data } & 87\end{array}$

$\begin{array}{ll}\text { RIR Mineralogy } & 87\end{array}$

$\begin{array}{ll}\text { XRF-XRD Mineralogy } & 89\end{array}$

Appendix C: Shale Lithofacies PETRA Advanced Transform file 90

Appendix D: XRF Data Sets 92

Hamilton Suite $\quad 93$

MSCL Mining-plus Suite 106

$\begin{array}{ll}\text { MSCL Soil Suite } & 160\end{array}$

MSCL Mining Suite $\quad 264$ 


\section{Chapter 1}

\section{LIST OF FIGURES}

Figure 1-1: The MSEEL study area is located in the central portion of the Marcellus Play in Northern West Virginia (USA). The site consists of 3 vertical pilot holes (MIPSW, MIP3H, MIP4H), and 4 lateral wells (MIP3H, MIP4H, MIP5H and MIP6H). The wells in blue indicate well drilled in 2011 and in the red are the wells drilled in 2015 and associated with the Marcellus Shale Energy and Environmental Laboratory (MSEEL) project. This proposed study will focus on the MIP3H and MIP4H vertical pilots. Modified from MSEEL.org.

Figure 1-2: This study focuses on the Middle Devonian Mahantango/Marcellus formations within the Appalachian basin

Figure 1-3: System tracts and boundaries defined by Lash and Engelder (2011) on left compared to the MIP3H well on right. Dark gray intervals represent the transgressive systems tracts (TST) and light gray represents the regressive systems tracts (RST), separated by max regressive surface (MRS) represented by the thick line, and maximum flooding surface (MFS) by the thinner line. Log tracts displayed are gamma ray in API units (GR) and Density (grams/cm3). For simplicity, the stratigraphy is divided into the upper, middle and lower members defined by the GR log trends capturing the three peaks separated by thin limestone intervals.

Figure 1-4: Middle Devonian paleogeography, red circle indicates the position of the Appalachian basin with the Arcadian mountains to the south and the Cincinnati arch to the north, The yellow star indicates our study location, and the white lines indicate the approximate orientation of the paleo-equator and $30^{\circ} \mathrm{S}$ latitude according to Witzke and Heckel (1988), (Modified from Blakey, 2010).

Figure 1-5: Workflow for classifying shale lithofacies (Modified from Wang 2012)

\section{Chapter 2}

Figure 2-1: Upper Marcellus lithology summary, Track 1: lithofacies, Track 2: pyrite density, Track 3: fracture density, Track 4- lithology summary

Figure 2-.2: Middle Marcellus lithology summary, Track 1: lithofacies, Track 2: pyrite density, Track 3: fracture density, Track 4- lithology summary

Figure 2-3: Lower Marcellus lithology summary, Track 1: lithofacies, Track 2: pyrite density, Track 3: fracture density, Track 4- lithology summary

Figure 2-4: Marco-facies, Track 1: gamma ray, Track 2: Marco-scale lithofacies (colors described by key to the left of the figure), and pictures to the right of the figure indicates how each lithofacies appears in core.

Figure 2-5: denotes the orientation (YZ-plane in blue and XZ-plane in red) of the medical CT scan images displayed in figures 5-12. 
Figure 2-6: 2D isolated planes ( $\mathrm{YZ}$ on left and $\mathrm{XZ}$ on right) through the vertical center of the medical CT scans of the MSEEL MIP 3H core from 7445' - 7454'. See Table 2.1 for key.

Figure 2-7: 2D isolated planes (YZ on left and $\mathrm{XZ}$ on right) through the vertical center of the medical CT scans of the MSEEL MIP 3H core from 7454' - 7460'

Figure 2-8: 2D isolated planes ( $\mathrm{YZ}$ on left and $\mathrm{XZ}$ on right) through the vertical center of the medical CT scans of the MSEEL MIP 3H core from 7460' - 7467'

Figure 2-9: 2D isolated planes (YZ on left and $\mathrm{XZ}$ on right) through the vertical center of the medical CT scans of the MSEEL MIP 3H core from $7467-7473$ '.....

Figure 2-10: 2D isolated planes (YZ on left and $\mathrm{XZ}$ on right) through the vertical center of the medical CT scans of the MSEEL MIP 3H core from $7473-7481$ '.

Figure 2-11: 2D isolated planes ( $\mathrm{YZ}$ on left and $\mathrm{XZ}$ on right) through the vertical center of the medical CT scans of the MSEEL MIP 3H core from $7482-7485$ '......

Figure 2-12: $2 \mathrm{D}$ isolated planes ( $\mathrm{YZ}$ on left and $\mathrm{XZ}$ on right) through the vertical center of the medical CT scans of the MSEEL MIP 3H core from $7488-7495$

Figure 2-13: $2 \mathrm{D}$ isolated planes ( $\mathrm{YZ}$ on left and $\mathrm{XZ}$ on right) through the vertical center of the medical CT scans of the MSEEL MIP 3H core from 7495 - 7501

Figure 2-14: 2D isolated planes ( $\mathrm{YZ}$ on left and $\mathrm{XZ}$ on right) through the vertical center of the medical CT scans of the MSEEL MIP 3H core from $7501-7510^{\prime}$......

Figure 2-15: 2D isolated planes (YZ on left and $\mathrm{XZ}$ on right) through the vertical center of the medical CT scans of the MSEEL MIP 3H core from 7510 - 7516'.

Figure 2-16: 2D isolated planes (YZ on left and $\mathrm{XZ}$ on right) through the vertical center of the medical CT scans of the MSEEL MIP 3H core from $7516-7525^{\prime}$......

Figure 2-17: 2D isolated planes (YZ on left and $\mathrm{XZ}$ on right) through the vertical center of the medical CT scans of the MSEEL MIP 3H core from 7525 - 7529'...

Figure 2-18: 2D isolated planes (YZ on left and $\mathrm{XZ}$ on right) through the vertical center of the medical CT scans of the MSEEL MIP 3H core from 7529 - 7538'......

Figure 2-19: 2D isolated planes (YZ on left and $\mathrm{XZ}$ on right) through the vertical center of the medical CT scans of the MSEEL MIP 3H core from 7538 - 7544'......

Figure 2-20: 2D isolated planes (YZ on left and $\mathrm{XZ}$ on right) through the vertical center of the medical CT scans of the MSEEL MIP 3H core from $7544-7553$ '.

Figure 2-21: 2D isolated planes (YZ on left and $\mathrm{XZ}$ on right) through the vertical center of the medical CT scans of the MSEEL MIP 3H core from 7553 - 7557'......

Figure 2-22: This figure shows the results of the pyrite density and fracture density analysis from the medical CT scans. A.(on the right) denotes pyrite density at .5' increments with the blue 
representing the $\mathrm{YZ}$ slice and red representing the $\mathrm{XZ}$ slice. (B.)(on the left) shows fracture density within a $.5 \mathrm{ft}$. window, blue represents the $\mathrm{YZ}$ slice and red represents the $\mathrm{XZ}$ slice. 36

Figure 2-23: Shown in the plot to the left is an example of how CT-scan imagery was used to aid the macro-facies description. Average grayscales from each slice $(.5 \mathrm{~mm})$ are plotted with depth. Grayscales generally range from about 1600 to 2200, high values relate to denser minerals and lower values have less dense minerals. Low gray scale values $(<1600)$ are associated with missing material, either due to a fracture or missing core. High gray scale values $(>2400)$ are associated with nodules and other high density minerals.

Figure 2-24: Cross-plot of Schmokers where RHOB and Core TOC, the equation is the linear relationship between the two TOC measurements.

Figure 2-25: Crossplot of TOC and DLogR, the red line denotes the linear fit from Petra used to determine the approximate LOM.

Figure 2-26: cross-plot of Passey's calculated TOC and Core TOC, the equation is the linear relationship between the two TOC measurements.

Figure 2-27: Crossplot of gamma ray and core TOC, the green line represents the linear fit for the equation at the top of the crossplot.

Figure 2-28: Crossplot of uranium and core TOC, the green line represents the linear fit for the equation at the top of the crossplot.

Figure 2-29: the log plot above shows the relationship between the Core TOC (blue dots) and log derived TOC's. Track 1- Gamma Ray (0-250 API displayed, colored from 0-600 API); Track 2DTCO (Compressional Sonic) in red $(\mu \mathrm{s} / \mathrm{ft})$ and deep resistivity in black with orange in fill $>200$ ohm-m. Leftward separation between DTCO and RT represents the amount or organic matter present as determined by Passey's Method. Track 3- bulk density (grams/cc), Track 4- TOC determined by SRA from core shifted to log depth (blue dots) TOC_URAN (Uranium derived TOC) in purple, Passey_Sonic_TOC is TOC derived using Passey's method in green, TOC_Schmoker's method is TOC derived from the modified Schmoker's equation in orange, and GR-TOC is TOC derived from gamma ray in black.

Figure 2-30: The figure above displays the mineralogy for the MIP-3H well. A. Represents the mineralogy from the RIR method. B. Represents the XRF-XRD mineralogy model, both from Hupp and Donovan, 2018.

Figure 2-31: This figure displays the mineralogy from ECS logs in MIP-3H (A) and MIP-4H (C) compared to the XRF-XRD derived mineralogy, from Hupp and Donovan (2018). Key is on right of figure: Clay mineral (gray), QFM (yellow), carbonate (blue), high density minerals (pyrite in gold; barite in pink, siderite in dark red).

Figure 2-32: This figure shows the distribution of NPhi vs normalized Gamma Ray for MIP-3H (blue), MIP-4H (green) and MIP-SW (red). In the crossplot on the left we can see that NPhi in MIP-4H is higher overall with the same distribution with like GR values. The right crossplot show the results after normalization is complete. 
Figure 2-33: Lambda-Mu crossplot with mineralogy ternary plot overlay (from Perez-Altamar \& Marfurt, 2014). Formations for both MIP3H and MIP4H listed with their associated color on the left. The Marcellus in green in the formation of interest and is represented as an siliceous/argillaceous shale reservoir.

Figure 2-34: This figure represents the comparison between the three mineralogy suites in the MIP-3H well: the XRF-XRD points (blue), ECS log (red), Modeled mineralogy (yellow). 55

Figure 2-35: Key for macro-facies: Warm colors are organic-rich facies $(>6.5 \%)$ and cool colors are organic-lean $(<6.5 \%)$ or 'gray' modified after Bhattacharya and Carr, 2016

Figure 2-36: Quartz-Clay-Carbonate Ternary diagrams with z-axis showing Shale lithofacies for each mineralogy model: A. XRF-XRD, B. PNS MIP-3H, C. PNS MIP-4H, D. Modeled MIP-3H, E. Modeled MIP-4H

Figure 2-37: This cross-section between MIP-3H and MIP-4H compares the shale lithofacies models generated for each well. Tract 1: Gamma Ray (0-200 API with color display 0 - 300 API); Tract 2: lithofacies from PNS and TOC_URAN, discrete points shale lithofacies from XRD and TOC-URANIUM (MIP-3H only); Tract 3:PNS mineralogy, quartz (yellow), Calcite (blue), Clays (gray), discrete points XRD mineralogy, quartz (yellow), calcite (blue), clays (gray); Tract 4: lithofacies from Modeled mineralogy and TOC_URAN, discrete points shale lithofacies from XRD and TOC-URANIUM (MIP-3H only), Tract 5: modeled mineralogy, quartz (yellow), Calcite (blue), Clays (gray), discrete points XRD mineralogy, quartz (yellow), calcite (blue), clays (gray); Tract 6:Macro-facies (core scale facies) key in Figure 2-2.

\section{Chapter 3}

Figure 3-1: the Multi Sensor Core Logger (MSCL) allows us to continuously run petrophysical measurements on whole core: (A) natural gamma detector; (B) X-Ray Fluorescence Spectrometry; (C) Magnetic Susceptibility Loop Sensor; (D) Magnetic Susceptibility Point Sensor; (E) Non-Contacting Electrical Resistivity Sensor; (F) P-Wave Velocity Transducers, (G) Gamma Density Source.

Figure 3-2: Four suites of XRF data obtained from the MIP 3H well using the Multi Sensor Core Logger (MSCL) and traditional XRF analysis showing elements that can be identified with each suite are highlighted. Green represents elements quantified with lower errors and orange represents elements with higher error. Parameters of each suite are outlined.

Figure 3-3: The six crossplots above represent the standards ran in the Hamilton Suite. Hamilton values plotted along the $\mathrm{x}$-axis and the standards values are measured along the $\mathrm{y}$-axis. All elements are displayed in the $\mathrm{x}$-plots above.

Figure 3-4: Correlation between MSCL and Hamilton suites. Correlations are displayed with bars between -1 and 1 , where, negative values are red and positive values are blue.

Figure 3-5: This correlation matrix shows the relationship between elements, the bolded results are the correlations of like elements between the Mining and Hamilton Suites. The Color scale is dark blue with a strong positive correlation and dark red with a strong negative correlation both values get lighter as they approach zero. 
Figure 3-6: This correlation matrix shows the relationship between elements, the bolded results are the correlations of like elements between the Mining and Hamilton Suites. The Color scale is dark blue with a strong positive correlation and dark red with a strong negative correlation both values get lighter as they approach zero.

Figure 3-7: This correlation matrix shows the relationship between elements. The Color scale is dark blue with a strong positive correlation and dark red with a strong negative correlation both values get lighter as they approach zero.

Figure 3-8: This correlation matrix shows the relationship between elements, the bolded results are the correlations of like elements between the Mining and Hamilton Suites. The Color scale is dark blue with a strong positive correlation and dark

Figure 3-9: This figure ties chemical proxies to their importance in the paleo-environment. Green denotes paleo-redox proxies, red denotes paleo-production proxies, and orange denotes the detrital proxies. Modified from Sageman et al. 2003.

Figure 3-10: Well and core data from the MIP 3H. XRF data is from MSCL and Hamilton Lab analyses showing trends in elements used to determine detrital influence. Column 1 - Gamma ray log, Column 2 - Core Facies legend at base of figure, Column 3 - Aluminum (Al) from Hamilton (green), MSCL Mining Suite (black), MSCL Mining-Plus Suite (blue); Column 4 Silicon (Si) from Hamilton (green), MSCL Mining Suite (black), MSCL Mining-Plus Suite (blue); Column 5 - Calcium (Ca) from Hamilton (green), MSCL Soil Suite (red), MSCL MiningPlus Suite (blue); Column 6 -- Zircon (Zr) from Hamilton (green), MSCL Soil Suite (red), MSCL Mining-Plus Suite (blue); Column 7 - Ratio of Silicon to Aluminum (Si/Al) from Hamilton (green), MSCL Mining Suite (black), MSCL Mining-Plus Suite (blue); Column 8 Ratio of Titanium to Aluminum (Si/Al) from Hamilton (green) and MSCL Mining-Plus Suite (blue), Column 9 - Total organic carbon from core (blue) and total organic carbon from uranium (red)

Figure 3-11: Well and core data from the MIP 3H. XRF data is from MSCL and Hamilton Lab analyses showing trends in elements used to determine paleo-redox conditions. Column $1-$ Gamma ray log, Column 2 - Core Facies legend at base of figure, Column 3 - total organic carbon from core (blue) and total organic carbon from uranium (red), Column 4 - Manganese (Mn) from Hamilton Suite (green), MSCL Soil Suite (red), and MSCL Mining-Plus Suite (Blue); Column 5 - Molybdenum (Mo) from Hamilton Suite (green) and MSCL Mining-Plus Suite (blue), Column 6 - Sulfur (S) from Hamilton Suite (green), MSCL Mining Suite (black), and MSCL Mining-Plus Suite (blue); Column 7 - Iron (Fe) from Hamilton Suite (green), MSCL Mining Suite (black), and MSCL Mining-Plus Suite (blue); Column 8 - Spectral Gamma Ray logs: uranium (U) (ppm) (green), thorium (Th) (ppm) (red), and Potassium (K) (\%) (blue); Column 9 - ratio of Thorium to Uranium $(\mathrm{Th} / \mathrm{U})$ values less than 3 are shaded yellow and represent a fixed uranium suggesting anoxic conditions throughout the Marcellus.

Figure 3-12: Well and core data from the MIP 3H. XRF data is from MSCL and Hamilton Lab analyses showing trends in elements used to determine paleo-redox conditions. Column $1-$ Gamma ray log, Column 2 - Core Facies legend at base of figure, Column 3 - total organic carbon from core (blue) and total organic carbon from uranium (red), Column 4 - Yttrium (Y) from MSCL Soil Suite (red) and Phosphorous (P) from Hamilton Suite (green), Column 5 - 
Nickel (Ni) from Hamilton Suite (green), MSCL Soil Suite (red), and MSCL Mining-Plus Suite (blue); Column 6 - Vanadium (V) from Hamilton Suite, Column 7 - Zinc from Hamilton Suite (green) and MSCL Mining-Plus Suite (blue).

Figure 3-13: PNS derived elements, green are all elements capture through the lithoscanner tool and orange elements are the elements captured through the SpectroLith $\log$.

Figure 3-14: Correlation coefficients between the PNS log and Hamilton/Mining-plus suites for the MIP-3H well, Correlations are displayed with bars between -1 and 1 , where, negative values are red and positive values are blue.

Figure 3-15: Cross-section of MIP3H and MIP4H: Tract 1: Gamma Ray (0-200 API with color display 0 - 300 API); Tract 2: PNS Titanium (0-0.02), Aluminum (0-0.15), Silicon (0-0.5); Tract 3: PNS Calcium (0-.3); Tract 4: PNS Iron and Sulfur (0-.1), Tract 5: Ratio of Silicon to Aluminum (Si/Al) (0-20); Tract 6: Ratio of Titanium to Aluminum (Ti/Al)(0-0.5)

Figure 3-16: This figure shows concretions precipitated given bottom water conditions. The red box indicates the regime of the MIP-3H and MIP-4H (after Clark and Mosier 1989).

Figure 3-17: Cross-section of MIP3H and MIP4H: Tract 1: Gamma Ray (0-200 API with color display 0 - 300 API); Tract 2: Deep lateral array resistivity (ohmm) orange shading $(>100$ ohmm); Tract 3: neutron porosity (blue) density porosity (red); Tract 4: Neutron-Density separation (NEU_DEN_SEP)(red), Photoelectric log (PEFZ)(black/wide), Redox condition (black, extra wide) larger bracket indicates anoxic to dysoxic zone, smaller bracket (euxinic/anoxic conditions), Tract 5: Gamma Ray TOC (black line/orange shading) and effective porosity (dark blue). Red shading across logs indicates regions of high density minerals (High PE, strong positive NDS, decrease in RT), Blue shaded regions relate to carbonate intervals (NDS approaching 0, moderate PE, high RT)

\section{Appendix B}

Figure B-1: Visual display of RIR XRD mineralogy from Table B-2 


\section{LIST OF TABLES}

\section{Chapter 2}

Table 2-1: This table denotes the designated markers for the CT-Scan descriptions from figure 26 to $2-21$

Table 2-2: Log properties matrix for mineralogy solution, Top is from the MIP-3H solution and the bottom matrix is for the MIP-4H solution. Lambda values are shifted down 1 to $3 \mathrm{GPa} . \ldots \ldots 4$

\section{Appendices}

\section{Appendix A}

Table A-1: Pyrolysis Data from NETL Source rock analyzer, 7445 to 7544' (Core depth) _......... 86

Table A-2: Pyrolysis Data from NETL Source rock analyzer, 7547 to 7557 ' (Core depth) _......... 87.

\section{Appendix B}

Table B-1: RIR XRD, Depth in red indicates points in the Mahantango and purple points indicate points in the Marcellus 88

Table B-2: XRF-XRD Mineralogy from Hupp and Donovan, 2018

\section{Appendix D}

Table D-1: Oxide to Element conversions 93

Table D-2: Standards measured as unknowns Hamilton suite

Table D-3: Actual values of standards, \# indicates data derived from Jochum et al., 2015

Geostandards and Geoanalytical Research 40, 333-350

Table D-4: Hamilton XRF Suite. 96

Table D-5: MSCL Mining-Plus Suite 106

Table D-6: MSCL Soil Suite 160

Table D-7: MSCL Mining Suite 264 


\section{CHAPTER 1, INTRODUCTION}

The Middle Devonian Marcellus Shale is one of the most prolific unconventional shale plays in the United States with an estimated technically recoverable resource of 148.7 trillion cubic feet (Tcf) of natural gas and an additional 5.7 billion barrels (Bbbl) of natural gas liquids over a lateral extent of 20,757 square miles (53,760 sq. km) in the Appalachian basin (US EIA, 2015). Given the potential of the Marcellus as a long-term major producer of gas and gas liquids, it is important to better understand and utilize best practices to identify and produce economically and in a manner safest to the environment. The Marcellus Shale Energy and Environmental Laboratory (MSEEL) project was founded on these foundations in order to pursue an increased understanding of the Marcellus Shale by utilizing new technologies to optimize production, while reducing the environmental impacts.

The MSEEL project is located in the Morgantown Industrial Park, Monongalia County, northeastern West Virginia. The project is a collaboration of the Department of Energy's National Energy Technology Laboratory (NETL), West Virginia University (WVU), Northeast Natural Energy (NNE), and the Ohio State University. The study wells in the area were initially drilled in the summer of 2011 with two Marcellus gas wells; the MIP4H pilot and lateral well, and MIP6H lateral well (Figure 1.1, blue). The MSEEL project drilled three additional wells in the fall of 2015: the MIP3H, which includes a cored and logged pilot well and subsequent lateral production well; the MIPSW a microseismic observation well; and MIP5H, which consists of only the lateral production well (Figure 1-1, red). 


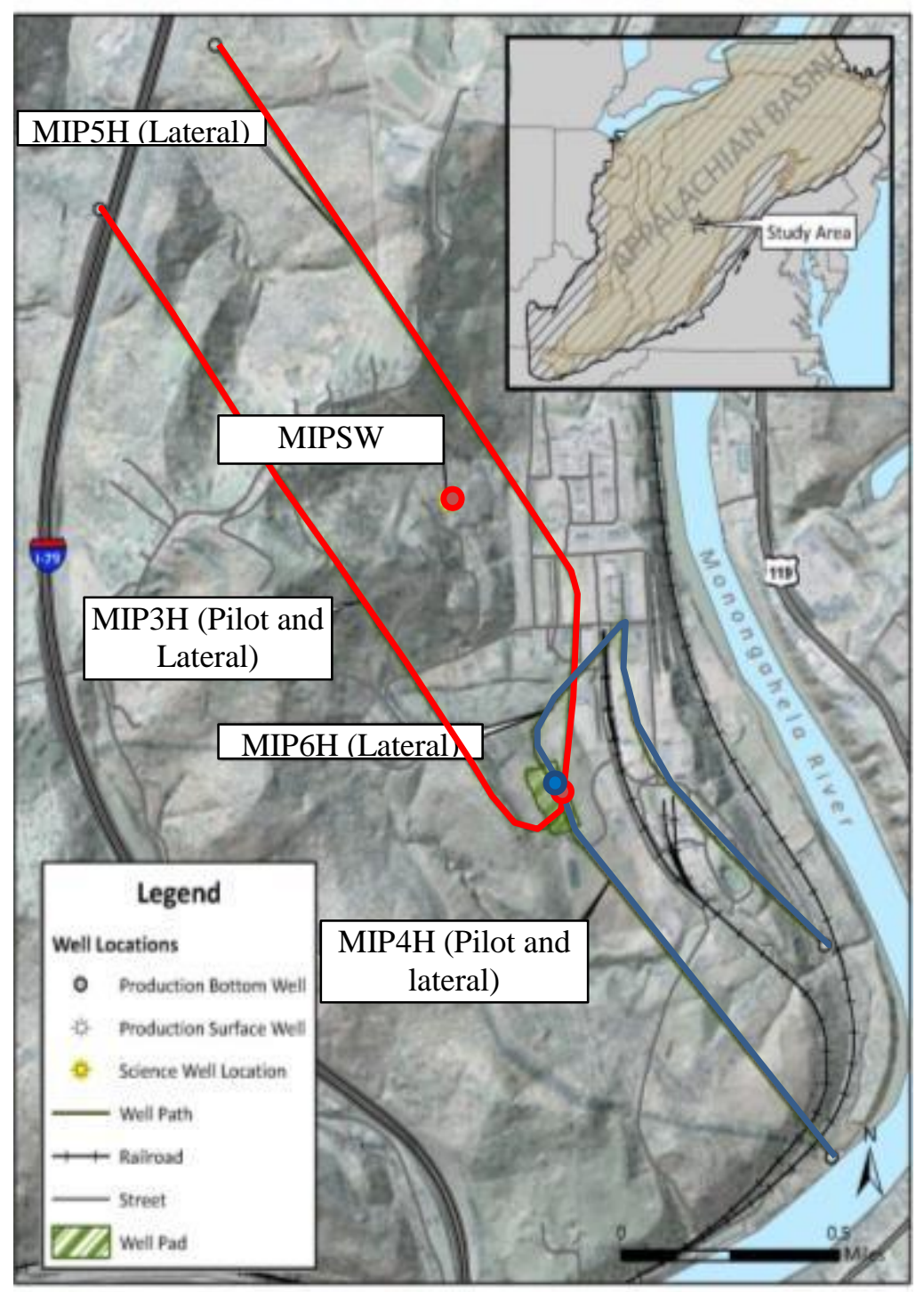

Figure 1-1: The MSEEL study area is located in the central portion of the Marcellus Play in northern West Virginia (USA). The site consists of 3 vertical pilot holes (MIPSW, MIP3H, MIP4H), and 4 lateral wells (MIP3H, MIP4H, MIP5H and MIP6H). The wells in blue indicate well drilled in 2011 and in the red are the wells drilled in 2015 and associated with the Marcellus Shale Energy and Environmental Laboratory (MSEEL) project. This proposed study will focus on the MIP3H and MIP4H vertical pilots. Modified from MSEEL.org 


\subsection{Geological Background}

The Marcellus Shale (or often referred to as the Marcellus Formation) is a middle Devonian (Eifelian to Givetian) unit at the base of the Hamilton Group (Lash \& Engelder, 2011) (Figure 12). Formally the Marcellus is divided into three members; the Union Springs Member, Cherry Valley Member, and Oatka Creek Member, which represent the division between two third-order transgressive-regressive sequences (Figure 1.3) (Lash and Engelder, 2011). Overall the sequence stratigraphy shows a coarsening upward trend due to the ongoing Arcadian orogeny (Lash and Engelder, 2011). For simplicity, we divide the Marcellus into the upper, middle and lower Marcellus units in the study area, which encompass the three gamma ray peaks separated by lower gamma-ray, carbonate intervals (Figure 1-3). Although formally called the Marcellus Shale most organic-rich shale reservoirs are not made of shale but are better referred to as organic-rich mudstone.

The Arcadian orogeny accommodates oblique collision between the Avalonian terrain and the Laurentian terrain (Ettensohn, 1985). Due to this oblique collision, closure is to the east and southern part of the basin due to the Acadian mountains geometry and closure to the north and west by the Cincinnati arch (Williams \& Hatcher, 1982; and Ettensohn 1985). The Acadian orogeny created major subsidence in the eastern portion of the basin, just west of the Acadian mountains, and to the west the Cincinnati arch fore-bulge closing off the basin (Brett \& Baird, 1996). Due to this enclosure, along with eustatic sea-level rise, shallow shelf carbonate deposition ended and allowed for the deposition of the organic-rich shale units of the Catskill delta (Lash \& Engelder, 2011). Due to the large amount of accommodation space in the east, deposition of the shale units is thicker in eastern portion of the basin and thin toward the western portion of the basin. The paleogeography position of the basin differed greatly from our current 
latitude; studies show that the basin was oriented east-west at approximately 30 to 35 degrees south of the Devonian equator (Witzke \& Heckel, 1988) (Figure 1-4). The Appalachian basin sat within a subtropical zone resulting in seasonal variation between dry conditions and stormy conditions due to the basin's position within the subtropical trade winds and its close proximity to the horse latitudes (Witzke and Heckel, 1988). This paleogeography plays an important role in understanding the mode and direction of sediment transport within the Appalachian basin during the Middle Devonian.

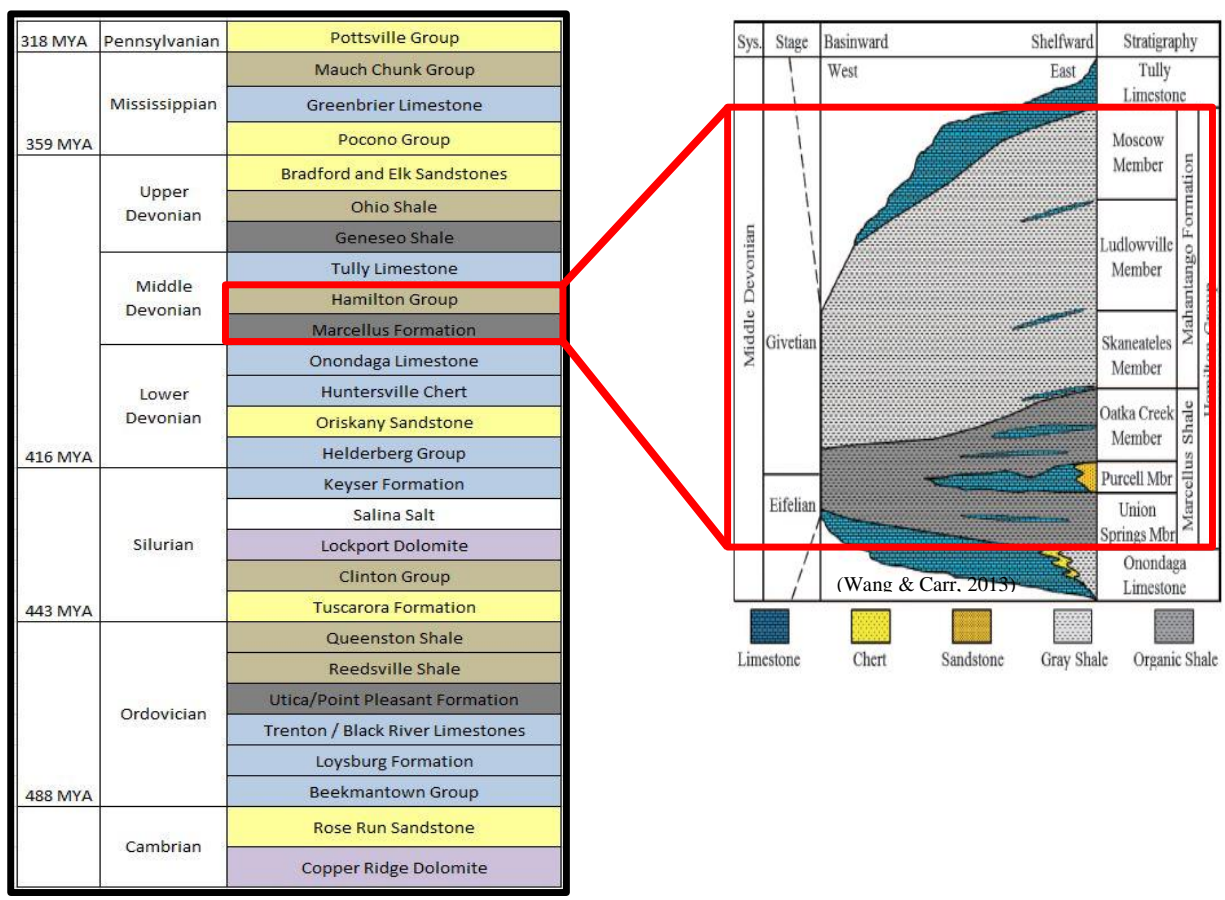

Figure 1-2: This study focuses on the Middle Devonian Mahantango/Marcellus formations within the Appalachian basin. 


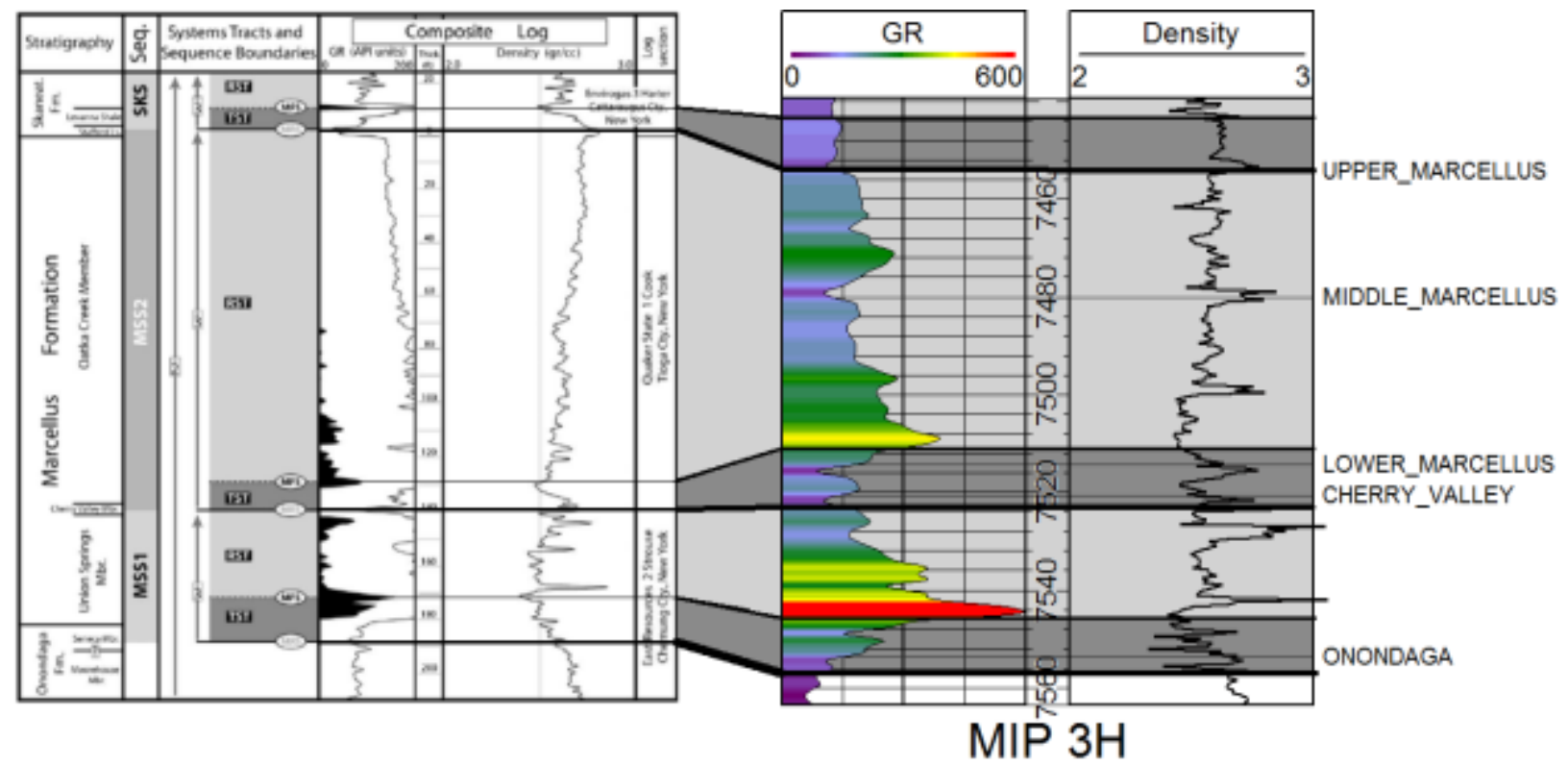

Figure 1-3: System tracts and boundaries defined by Lash and Engelder (2011) on left compared to the MIP3H well on right. Dark gray intervals represent the transgressive systems tracts (TST) and light gray represents the regressive systems tracts (RST), separated by max regressive surface (MRS) represented by the thick line, and maximum flooding surface (MFS) by the thinner line. Log tracts displayed are gamma ray in API units (GR) and Density $\left(\mathrm{grams} / \mathrm{cm}^{3}\right)$. For simplicity, the stratigraphy is divided into the upper, middle and lower members defined by the GR log trends capturing the three peaks separated by thin limestone intervals.

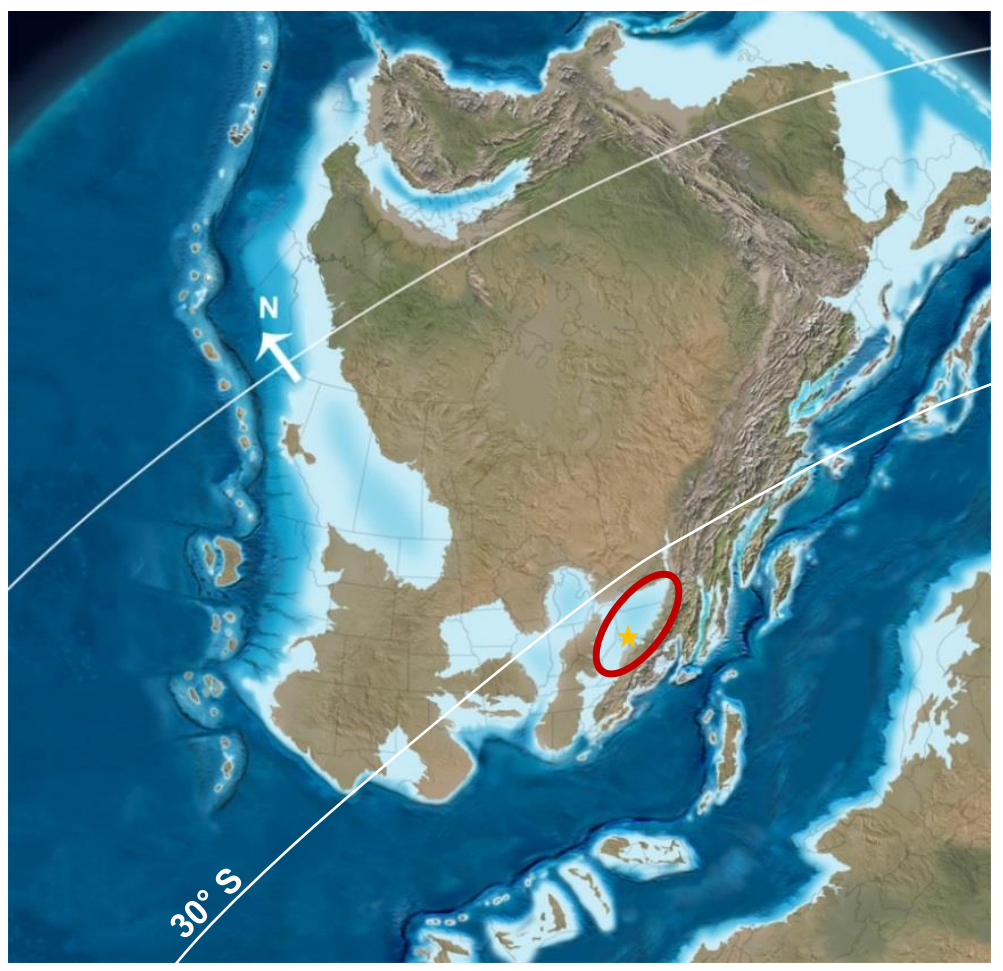

Figure 1-4: Middle Devonian paleogeography, red circle indicates the position of the Appalachian basin with the Arcadian mountains to the south and the Cincinnati arch to the north, The yellow star indicates our study location, and the white lines indicate the approximate orientation of the paleo-equator and $30^{\circ} \mathrm{S}$ latitude according to Witzke and Heckel (1988), (Modified from Blakey, 2010). 


\subsection{Previous Works}

Lithofacies:

Lithofacies can be described visually, through core description, and quantitatively through petrophysical analysis. Lithofacies classification has typically been used in clastic and carbonate dominated environments and has more recently been incorporated into documenting heterogeneity in shale facies (Wang and Carr, 2013; Bhattacharya and Carr 2016). Wang and Carr (2013), developed a multiscale facies model based on total organic carbon (TOC) and mineralogy. They defined seven shale lithofacies, which include; organic mudstone, organic siliceous, organic mixed, gray (organic-lean) mudstone, gray siliceous shale, gray mixed shale and carbonate facies (Figure 1-5). Bhattacharya and Carr (2016) utilizing a similar workflow found the model can be utilized in other basins to analyze the distribution of facies (e.g., Bakken Shale of the Williston basin).

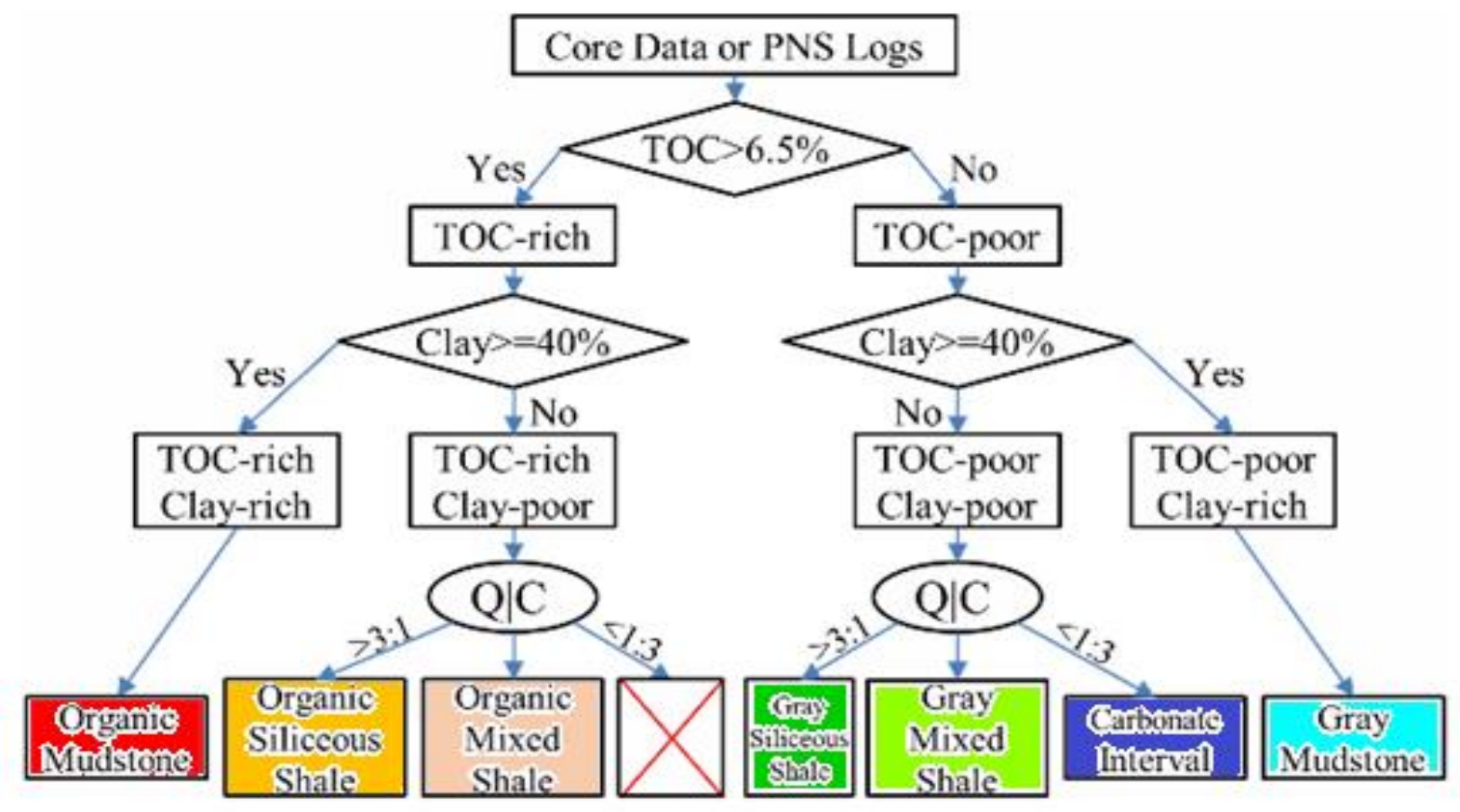

Figure 1-5: Workflow for classifying shale lithofacies (Modified from Wang 2012) 
$\mathrm{XRF} /$ chemostratigraphy:

X-ray fluorescence (XRF) has played an important role in understanding the changes in relative abundances of major elemental concentrations in rocks at short intervals at detection limits of parts per million (ppm) (Potts \& Webb, 1992). XRF has been used as a tool to understand the relative distribution of major elements and more recently has allowed inference of stratigraphic and paleoenvironmental changes based on chemical signatures (Potts \& Webb, 1992; and Sageman et al., 2003). In organic-rich mudstone facies, chemostratigraphy contributes to understanding the interaction between production, decomposition, and dilution in the accumulation of strata (Sageman et al., 2003). These chemical proxies are inferred by the chemical relations observed in modern depositional environments. For instance, chemostratigraphy is a useful to in understanding changes in redox environments based on trace elements (Algeo and Maynard, 2004).

\subsection{Study Area:}

In the study area, located in northeastern West Virginia, the Marcellus interval is about 100 feet $(30 \mathrm{~m})$ thick and defined by three distinct high gamma peaks separated by thin carbonate intervals. For simplicity, the unit was separated into the informal upper, middle and lower Marcellus (Figure 1-1).

\section{CHAPTER 2: LITHOFACIES}

\subsection{Macro facies}

Core Description:

The MIP-3H well core is 112 feet (33m) thick, ranging from 7445 to 7557 feet (2269.24 to 2303.37 m). This depth interval encompasses the strata from the Onondaga Limestone upwards 
to the base of the Mahantango. The core was described extensively in two approaches; first, through visual description from the whole rock core keeping to methods established by Lazar et al. (2015), and second, through CT-scan description looking specifically for pyrite distribution and structural changes in the core. Following the core description, the core was illustrated in a graphical log.

The method established by Lazar et al. (2015) focuses on describing fine-grained sedimentary rocks using three major components texture, bedding, and composition. The first pass of our core description focused on determining the "texture" and "composition" of the core. Focusing on the amount of silt quartz grains present in the mudstone and separating them into course, medium and fine, in descending size of silt. Additionally, the composition of the mudstone was determined based on the amount of silt, carbonate and clay present to categorize them into siliceous, calcareous, and argillaceous (Lazar et al., 2015).

The second pass on the core focused on the description of sedimentary and structural features present in the core. This includes fracture type and intensity, nodules and concretions, bedding and other fabric, fossils, and bioturbation. Bedding and fabric focused on the laminae geometry, continuity, and shape. Overall the fabric in the Marcellus lacked laminations and was either massive or finely laminated. Most laminations in the MIP-3H core were observed in the scattered calcareous intervals of the Marcellus, and moderately in the Mahantango Formation. Nodules, concretions, and fractures were distributed throughout the core, but increase in intensity within the middle and lower Marcellus. Cross-cutting relationships from the nodules and fractures help to determine the relative time of features during and after deposition. Bioturbation and fossils were most intense and abundant in the limestone and calcareous intervals. 
The Marcellus Shale, in our study is a heterogeneous mudstone with varying degrees of carbonate, clay, organics and silt material. For simplicity, we divide the Marcellus into, upper, middle, and lower units which are further divided by 1 to $3 \mathrm{ft}(.3$ to $1 \mathrm{~m})$ packages of calcareous marl/limestone. Figures 2-1-2-3, show a visual summary of the core description.

The lower Marcellus is relatively more heterogeneous compared to the middle and upper Marcellus. The lower Marcellus includes all the observed lithofacies and has relatively equal distribution of each lithofacies. The base of the lower Marcellus and contact with the underlying Onondaga Limestone is a gradation contact between black to dark gray organic shale to gray to light gray calcareous marl to wackestone. Within the transition are two bentonite ash layers (7552.8 to 7552.9 and 7555.1 to 7555.3 ), both ash layers have mica present and are much coarser compared to the surrounding strata. We consider the lower ash to be the Tiago K-bentonite and place the Onondaga-Marcellus contact there. Above this contact, the lower Marcellus is composed of two black to dark-brown organic-rich mudstone facies. Also, these intervals are pyrite-rich, siliceous, and are relatively massive. They have almost no silt present and lack flocculate layers. There are prominent pyrite bands and nodules, as well as, an increase in open fracture density (Figure 2-22). Separating these two black shale intervals is a gradational transition from black fine-grained massive mudstone into coarser dark-gray mudstone and then into a light-gray marl. This transition shows an increase in the amount of silt-sized grains, crossbedding, and bioturbation until terminating in a sharp contact with black to dark gray mediumgrained mudstone. From 7529.45 to 7531.2 , there is an abundance of calcite nodules, with pyritization. This zone likely represents the boundary between anoxic and oxic water conditions that favor this growth of nodules (Maynard, 2014). Above this zone, we return to a dark-gray mudstone coarsening upward to the Cherry Valley Limestone. 
The Cherry Valley Limestone in the MIP-3H well is about $2.25 \mathrm{ft}(.68 \mathrm{~m})$ thick and is composed of a bioturbated, fossiliferous wackestone.

The Middle Marcellus is composed dominantly of dark-gray to gray, medium mudstone. The mudstone in the middle Marcellus consists of planar laminations, but there is a $2.7 \mathrm{ft}$ interval of massive black organic-rich mudstone at the base of the middle Marcellus unit. There are some small interbedded calcareous shale intervals within the middle Marcellus, but they are less frequent compared to the number in the upper Marcellus. Pyrite nodules decrease with an increase in depth. The middle Marcellus has an abundance of calcite filled fractures (749). The fractures are relatively late stage feature with horizontal fractures forming followed by the vertical fractures. This is based on cross-cutting relations. The number of fractures observed in core is much higher than observed with the image logs (Yixuan Zhi, pers. comm.).

The upper Marcellus is like the lower and middle units; it is dominantly comprised of dark-gray mudstone. However, the upper unit has an increase in silt and clay. There is an overall increase in the frequency of interbedded flocculate intervals (1-2mm thick, light gray clay). There is relatively less pyrite in this interval and the pyrite present is on the size-order of millimeter to sub-millimeter. The upper Marcellus has a smaller frequency of calcite filled fractures. The zones where horizontal fractures dominate the fracture density increases, unlike in the middle and lower units, where vertical and sub-vertical orientations dominate.

The Mahantango Formation and Marcellus Shale form a sharp contact between a large calcite concretion that is heavily burrowed and fossiliferous. Above the concretion is a fining upward sequence from a coarse, cross-bedded calcareous shale to a planar-laminated, medium-grained, 
dark-gray mudstone. The Mahantango has a very sparse distribution of both fractures and pyrite nodules.

Macro-Facies (core-scale facies) are based on a combination of core and CT scan descriptions. I identified 6 facies (limestone, light-gray shale, mixed dark-gray shale, dark-gray shale, black shale, k-bentonite) and one sub-facies (dark gray shale with nodules). The facies are:

- Limestone made up of light-gray color, heavy bioturbation, generally massive (some minor lamination) with an abundance of fossils;

- Light-gray shale made up of highly laminated (strongly cross-bedded) with flocculate zones, and are typically carbonate-rich;

- Mixed dark-gray shale made up of light gray to gray laminations (cross-bedding) on the scale of millimeter to submillimeter.

- Dark-gray shale characterized by minor laminations, minor pyrite;

- Dark-gray shale with pyrite-replaced calcite nodules and a high frequency of large calcite nodules; and

- Black shale: black shale with very minor lamination, abundance of pyrite; and KBentonite made up of gray/grayish yellow, platy structure, mica-rich.

Figure 2-4, provides an example of each facies observed in the core. 


\section{Core Lithofacies Key}

Limestone

Mixed Dark-Gray Shale

K-Bentonite

Dark-Gray Shale w/Nodules

\section{Light-Gray Shale \\ Dark-Gray Shale \\ Black Shale}

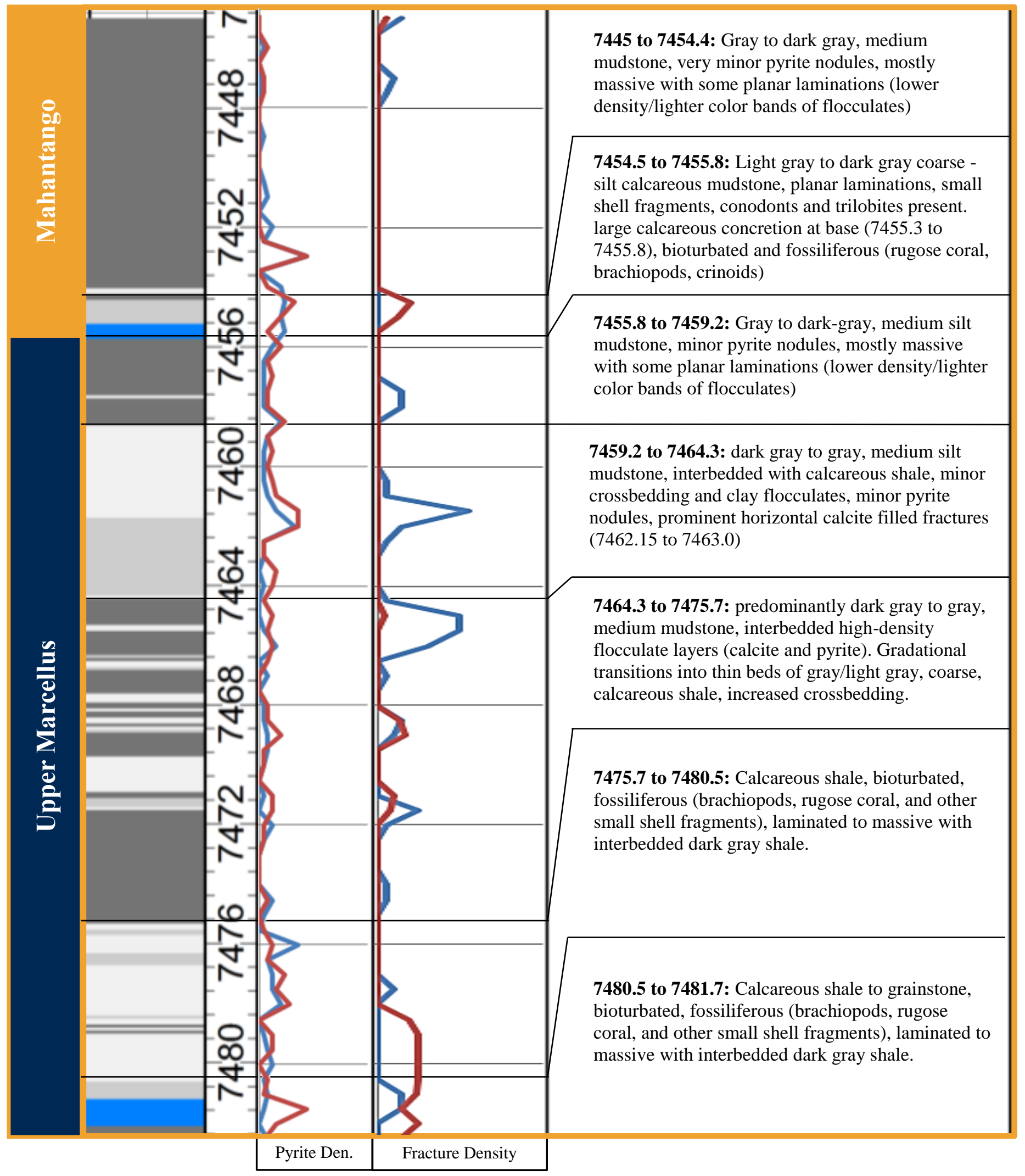

Figure 2-1: Upper Marcellus lithology summary, Track 1: lithofacies, Track 2: pyrite density, Track 3: fracture density, Track 4- lithology summary 


\section{Core Lithofacies Key}

Limestone

\section{Mixed Dark-Gray Shale}

K-Bentonite

\section{Dark-Gray Shale w/Nodules}

Light-Gray Shale

Dark-Gray Shale

Black Shale

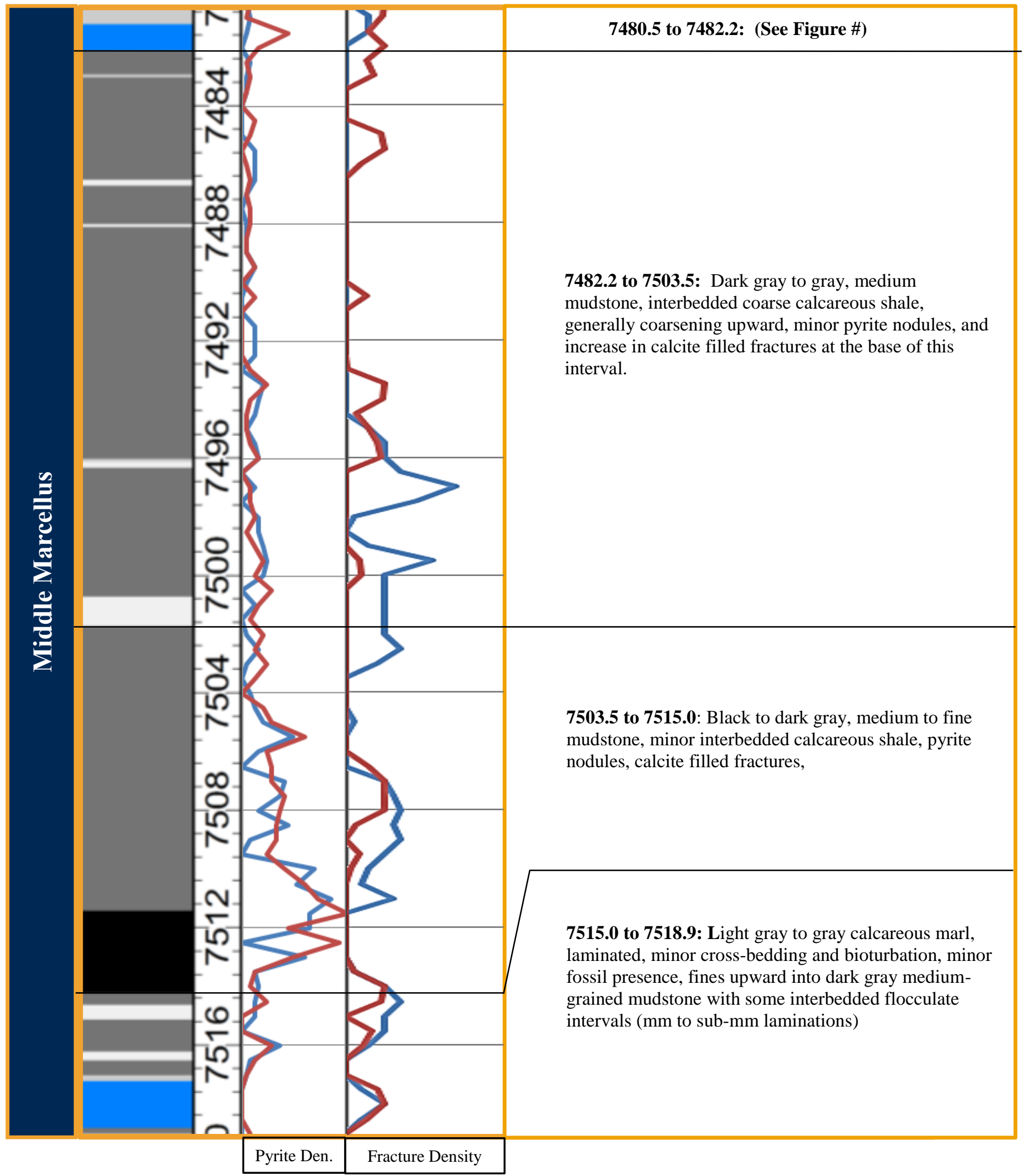

Figure 2-2: Middle Marcellus lithology summary, Track 1: lithofacies, Track 2: pyrite density, Track 3: fracture density, Track 4- 


\section{Core Lithofacies Key}

Limestone

Mixed Dark-Gray Shale

K-Bentonite

\section{Dark-Gray Shale w/Nodules}

\section{Light-Gray Shale \\ Dark-Gray Shale \\ Black Shale}

늘 7518.9 to $7523.55:$ Dark gray to gray, medium mudstone, interbedded calcareous shale, platy clay flocculates

7523.55 to 7525.8: Light gray to gray calcareous shale to wackestone, bioturbated, fossiliferous, platy flocculates coarsening upward.

7525.8 to 7529.45: Dark gray to gray, medium mudstone, interbedded calcareous shale, crossbedding, small platy shell fragments, open fractures present at base of interval.

7529.45 to 7533.95: Dark gray to gray, medium mudstone, thin interbedded layer of calcareous shale, abundant calcite nodules with some pyritization present from 7529.45 to 7531.2

7533.95 to 7540: Black to dark brown, fine mudstone, siliceous, pyrite interbedded in bands, organic-rich, vertical to sub-vertical fractures and a mineralized shear fractures (7539.5 to 7540 ).

7540 to 7545.65: Dark gray to gray, medium mudstone, thin interbedded layer of calcareous shale/wackestone, calcareous shale intervals dominated by clay flocculates coarsening upward in 1 to 2 " packages

7545.65 to 7549.6: Black to dark brown, fine mudstone, siliceous, pyrite interbedded in bands, organic-rich

7549.6 to 7552.9: Calcareous shale/wackestone, calcareous shale intervals dominated by clay flocculate coarsening upward in 1 to 2" packages between medium mudstone and course calcareous shale. Wackestone at base, fossiliferous, bioturbated, minor fracturing at base from overpressure.

7552.9 to 7557: Calcareous shale with interbedded layers of black to dark gray shale. Two K-bentonite ash layers at 7552.8-7552.9 and 7555.1 to 7555.3

Fracture Density

Figure 2-3: Lower Marcellus lithology summary, Track 1: lithofacies, Track 2: pyrite density, Track 3: fracture density, Track 4- lithology summary 


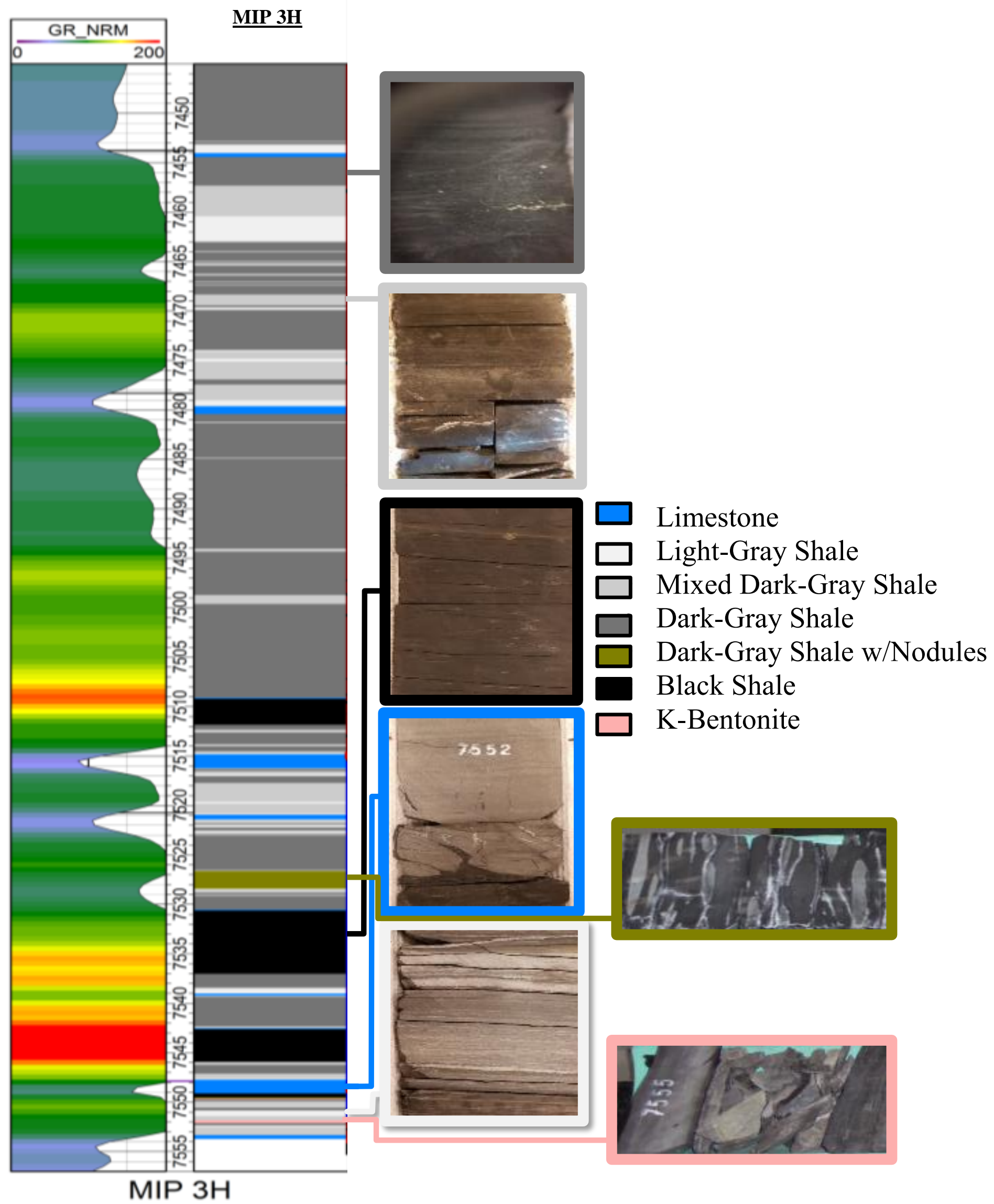

Figure 2-4: Marco-facies, Track 1: gamma ray, Track 2: Marco-scale lithofacies (colors described by key to the left of the figure), and pictures to the right of the figure indicates how each lithofacies appears in core. 


\section{CT-Scans:}

The entire MIP-3H core was scanned using a medical Toshiba ${ }^{\circledR}$ Aquilion RXL $^{\mathrm{TM}}$ Multi-slice Helical Computed Tomography Scanner (medical CT). The medical CT scanner produces images at high speeds at millimeter-scale resolution, with a voxel resolution of $0.43 \mathrm{~mm} \times 0.43$ $\mathrm{mm}$ in the XY plane and $0.50 \mathrm{~mm}$ in the $\mathrm{z}$-plane. The images from the medical CT scans used a voltage of $135 \mathrm{kV}$ and at $200 \mathrm{~mA}$ using a helical detector rotation/acquisition over a $220 \mathrm{~mm}$ collection diameter (Crandall et al., 2017). A 3D volume and a 2D cross-section through the middle of the core was processed from the acquired stacks at NETL using ImageJ (Figure 2-5).

The variation in the grayscale of the $\mathrm{CT}$ images is directly proportional to the changes in attenuation and the density of the scanned rock. Lower density regions (e.g. clays and gas-filled fractures) are represented by dark grayscales, and higher density regions (e.g. carbonate and pyrite) are represented by lighter grayscales. Utilizing this relationship, the CT-scans are used to aid in finding structural changes in the core, including bedding changes (planar vs. crossbedding), fractures (type and intensity), and pyrite nodule distribution.

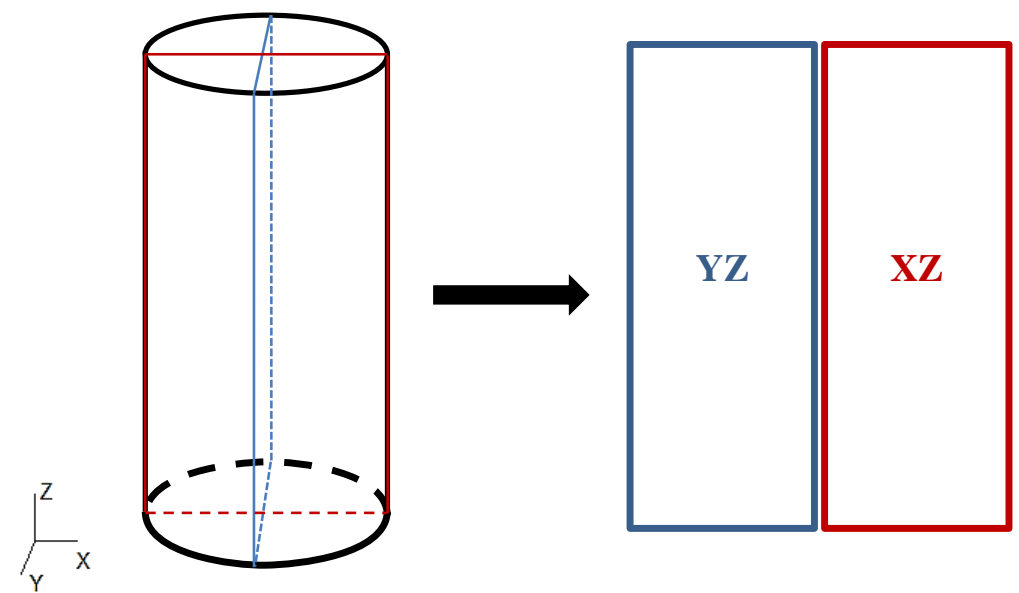

Figure 2-5: denotes the orientation (YZ-plane in blue and XZ-plane in red) of the medical CT scan images displayed in figures 5-12. 
Figures 2-6 to 2-21 display the results of the medical CT scanner in a ' $\mathrm{XZ}$ ' and 'YZ' isolated plane views. To the right of each CT image pair is a scale, where each individual bar is 1 in (2.54 $\mathrm{cm})$. Depths displayed at the base of the images; represent the upper depth of the image. Grayscale is shifted image-by-image to best represent the structures present in the $\mathrm{XZ}$ images and the original grayscale was maintained in the $\mathrm{YZ}$ images.

CT-images were scaled (to left of each slice, each bar is 1 inch $(2.54 \mathrm{~cm})$ ) and structural features were annotated in Weatherford@ PreView. Pyrite nodule, concretions, and fractures (open and filled) were digitized and depth indexed for each CT-image. Following digitization, features and their associated depths were exported and converted into pyrite nodule intensity and P20 fracture intensity; both intensities were taken at a half-foot increment (Figure 2-22). Additionally, CTimages were used to denote bedding structures and lamination styles within the resolution of the medical CT-scan (.5 mm z-axis and $.43 \mathrm{~mm}$ in $\mathrm{x}$ - and $\mathrm{y}$-axes $)$.

Pyrite nodules were picked from the CT-scan slices by visually picking out "white-spots" in the images. The "white-spots" are a result of the high attenuation when the x-rays hit the highdensity pyrite nodules $(5.01 \mathrm{grams} / \mathrm{cc})$. Due to the resolution of the medical CT-scans, any pyrite nodules that are smaller than $.5 \mathrm{~mm}$ may not be imaged. The overall density of the pyrite nodules is consistent between the $\mathrm{XZ}$ and $\mathrm{YZ}$ planes (Figure 2-22). This suggests that there is little preferential orientation of the pyrite nodules. This is observed more clearly in larger pyrite nodules not compromised by excessively high attenuation values (e.g. the base of 7473 ' and $\left.\sim 7498^{\prime}\right)$. These nodules form in a pancake-like shape, where the primary stress on the nodule is vertical due to burial and smaller variations in stress in the horizontal directions. The vertical density increases with depth peaking at the base of the middle Marcellus. The Mahantango has a sparse distribution of pyrite compared to the Marcellus, as a whole (Figure 2-22). 
Fractures picked visually from the CT-scan slices by white linear features, denoting calcite filled fractures (2.71 grams/cc), and dark lineations denoting gas filled fractures (or open fractures). There are limitations to this method due to the resolution of the medical CT-scans; it is possible to misrepresent the length of the fractures or miss them entirely, if the aperture decreases to less than $0.5 \mathrm{~mm}$ in horizontal fractures or $0.47 \mathrm{~mm}$ in vertical fractures. Comparing the results of this analysis between the $\mathrm{XZ}$ and $\mathrm{YZ}$ slices, there is separation between the curves, suggesting that fractures are preferentially distributed. The CT-scans are not consistently oriented, so the variation between the $\mathrm{XZ}$ and $\mathrm{YZ}$ cannot be determined. There is variation between the slice with high fracture density, however the majority of the fractures appear to be perpendicular to the $\mathrm{YZ}$ slice and parallel to the $\mathrm{XZ}$ direction.

Laminations were difficult to distinguish due to the termination in cross-laminations occurring below the resolution of the medical CT-scans. We were able to denote intervals with soft sediment deformation using the CT-scans to aid in the core description. Soft sediment deformation occurs along intervals where we have observed higher density objects, such as nodules or carbonate intervals that were deposited or formed during the time of deposition. An example of this can be seen at $\sim 7471.5$ '. 


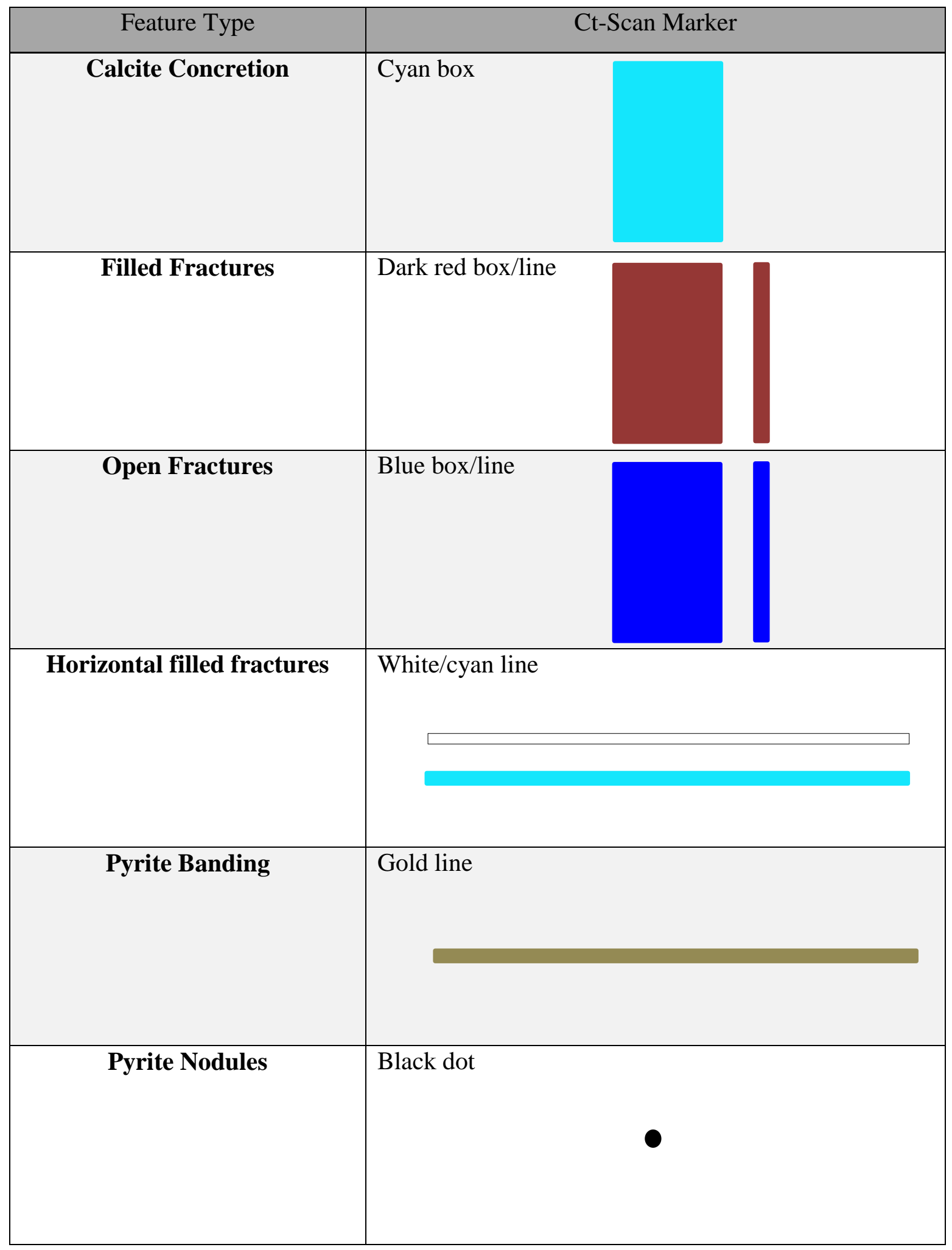

Table 2-1: This table denotes the designated markers for the CT-Scan descriptions from Figure 2-6 to 2.21 


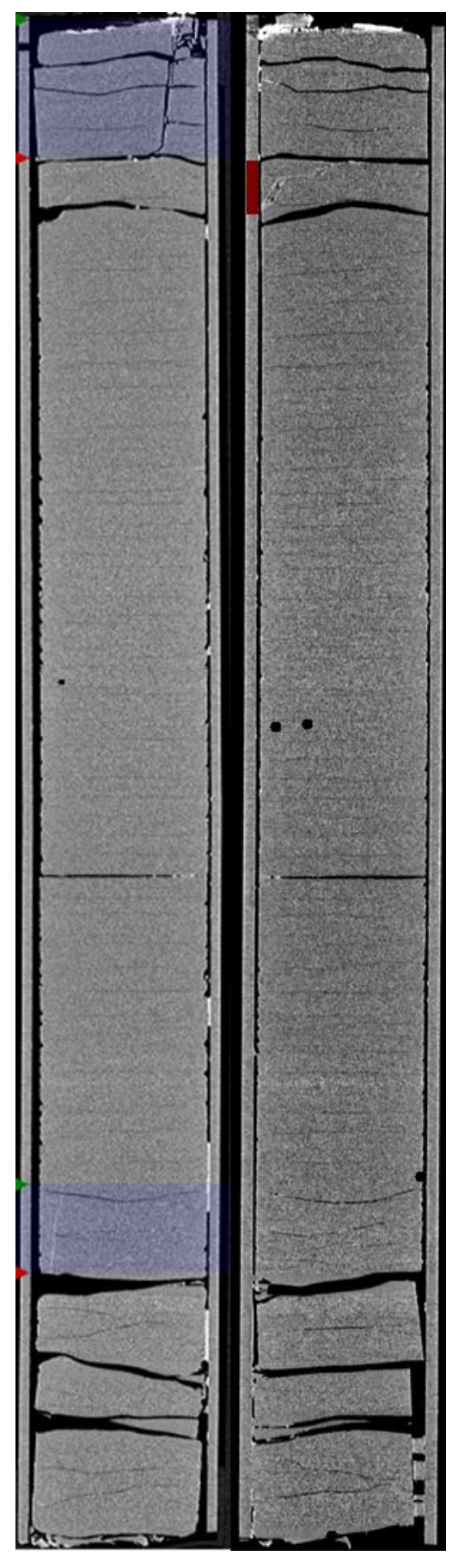

$7445^{\prime}$

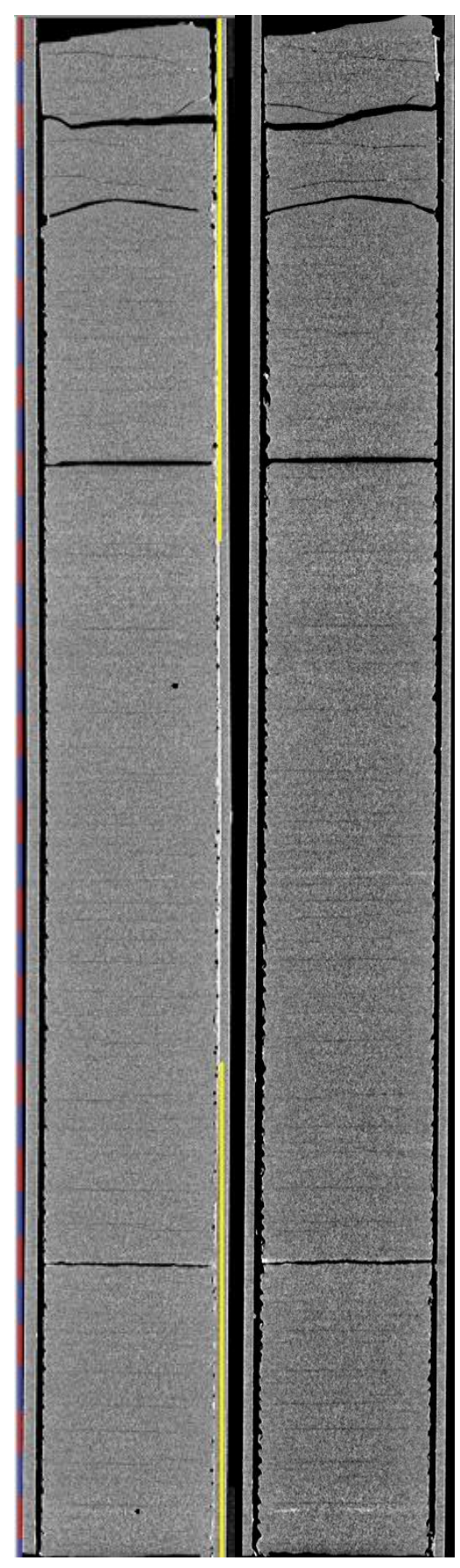

7448'

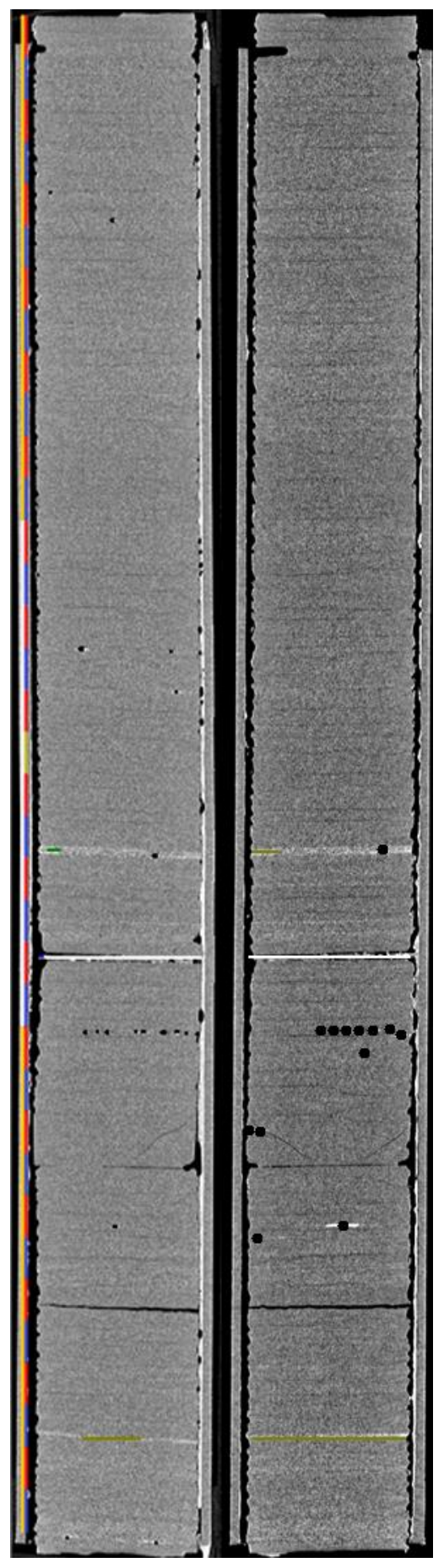

7451'

Figure 2-6: 2D isolated planes ( $\mathrm{YZ}$ on left and $\mathrm{XZ}$ on right) through the vertical center of the medical $\mathrm{CT}$ scans of the MSEEL MIP 3H core from 7445' - 7454'. See Table 2.1 for key. 


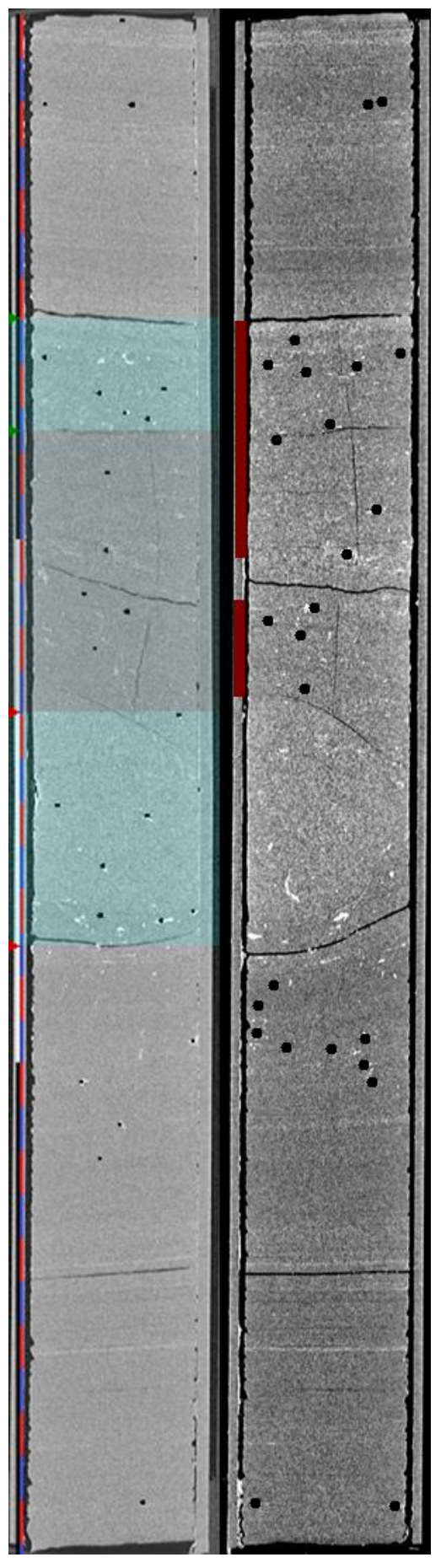

$7454^{\prime}$

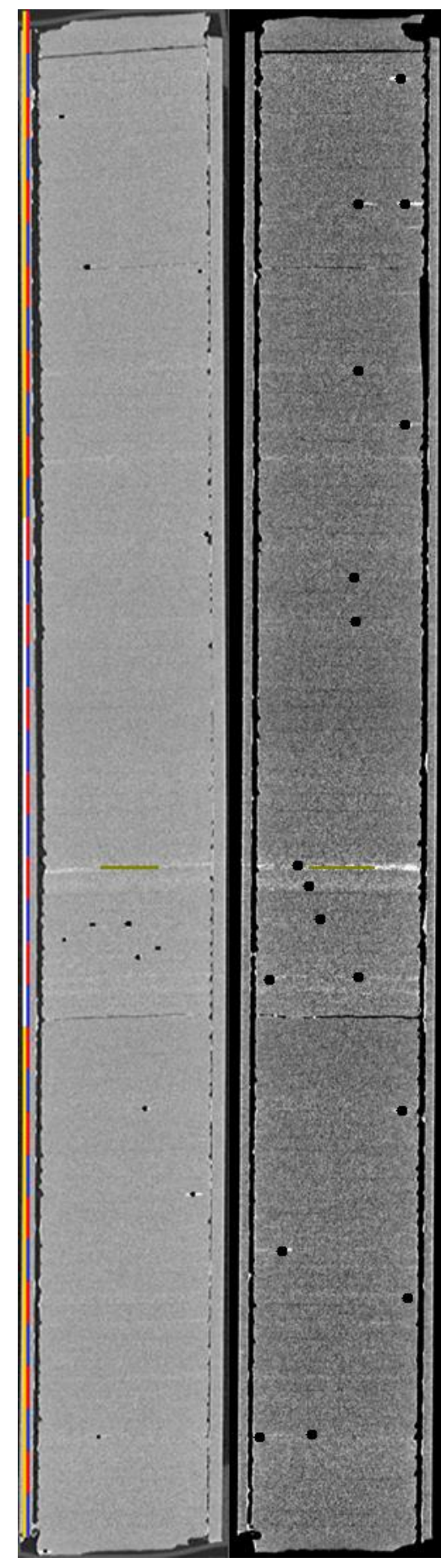

$7457^{\prime}$

Figure 2-7: 2D isolated planes ( $\mathrm{YZ}$ on left and $\mathrm{XZ}$ on right) through the vertical center of the medical $\mathrm{CT}$ scans of the MSEEL MIP 3H core from 7454' - 7460' 

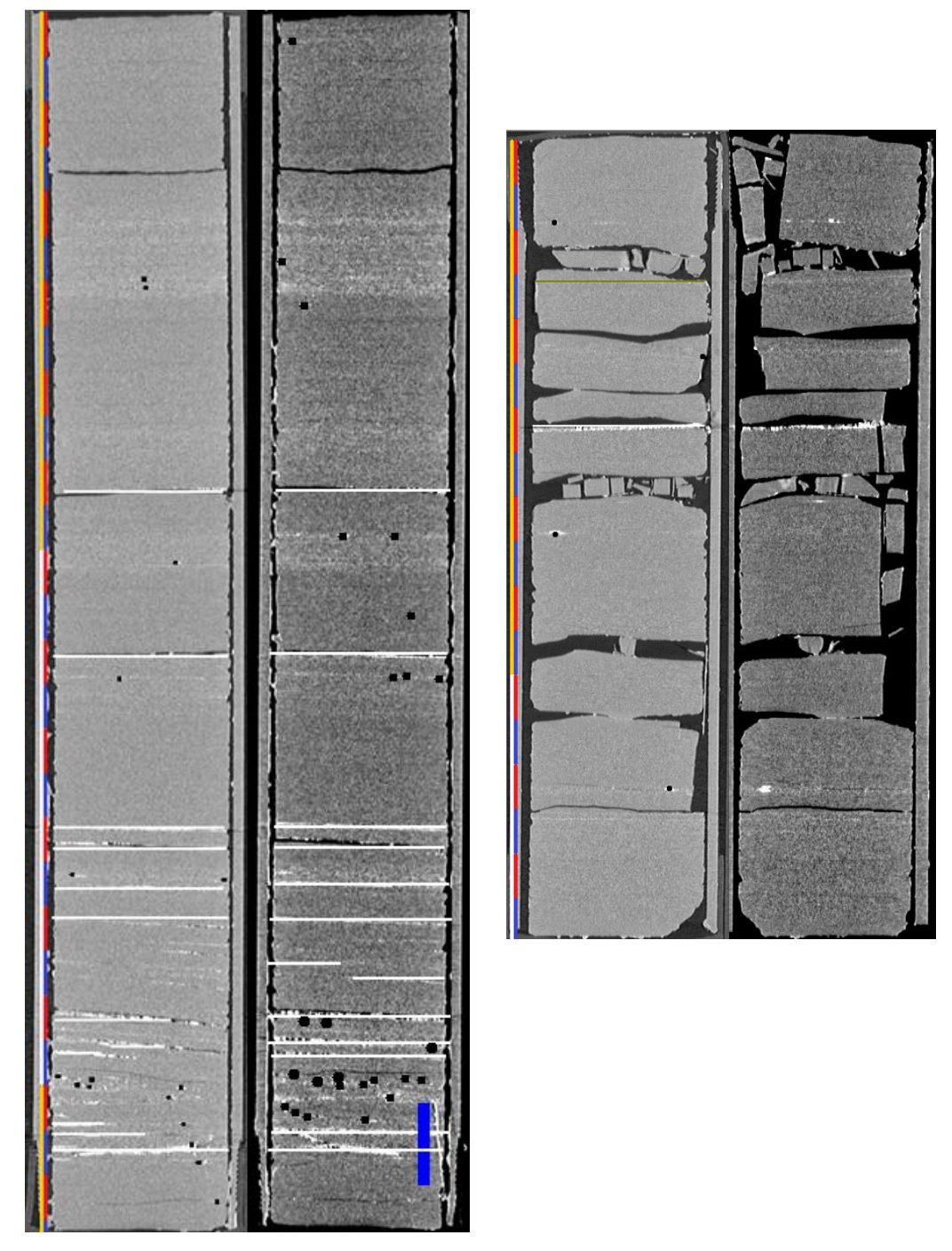

7460

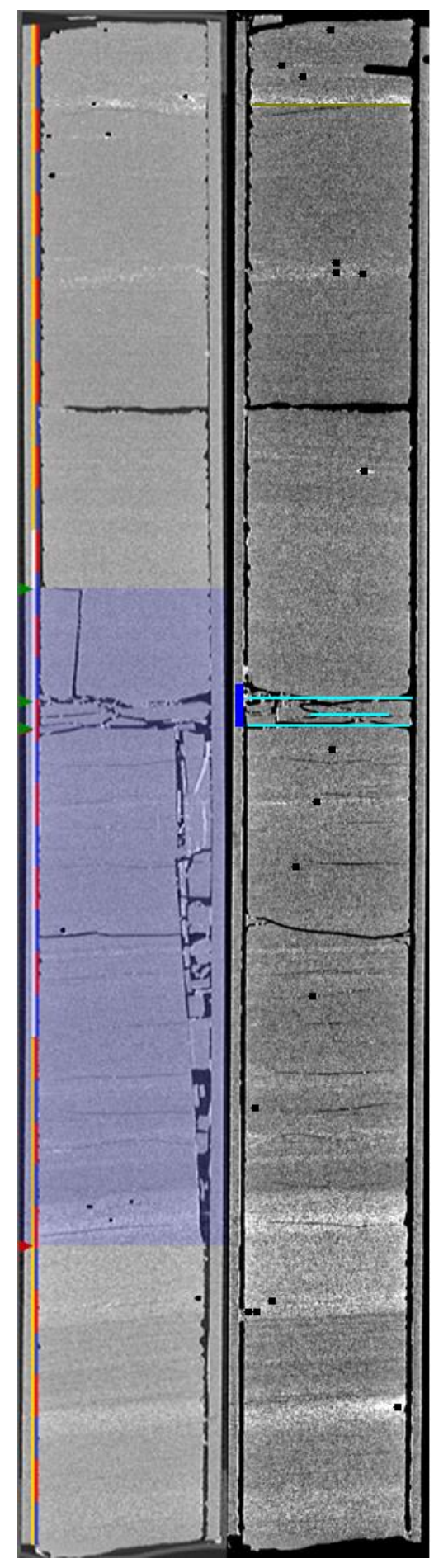

7464'

Figure 2-8: 2D isolated planes ( $\mathrm{YZ}$ on left and $\mathrm{XZ}$ on right) through the vertical center of the medical CT scans of the MSEEL MIP 3H core from 7460' - 7467' 


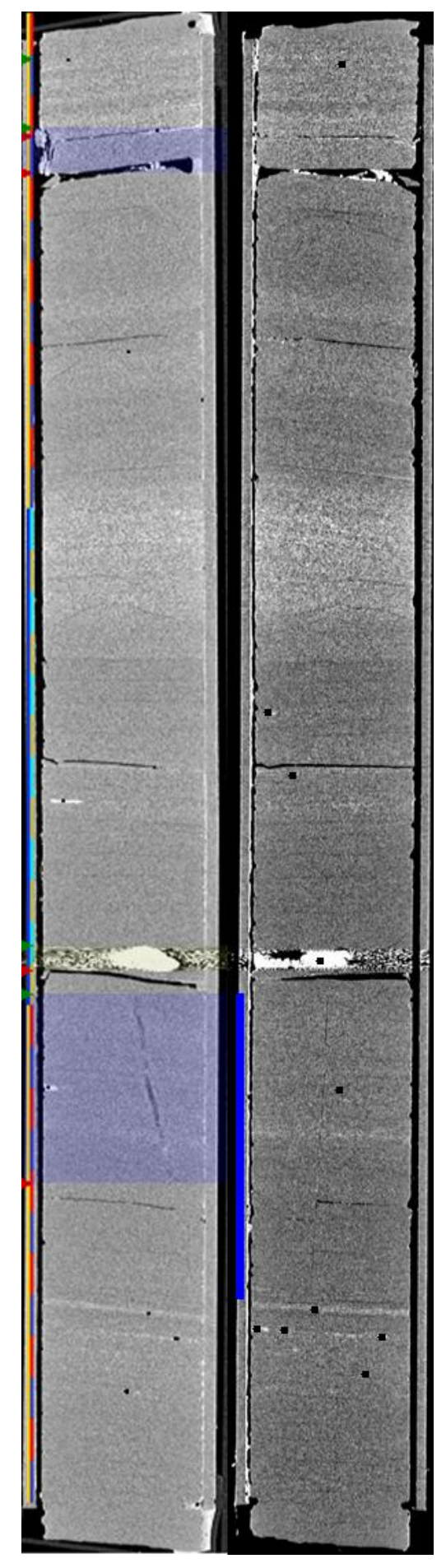

$7467^{\prime}$

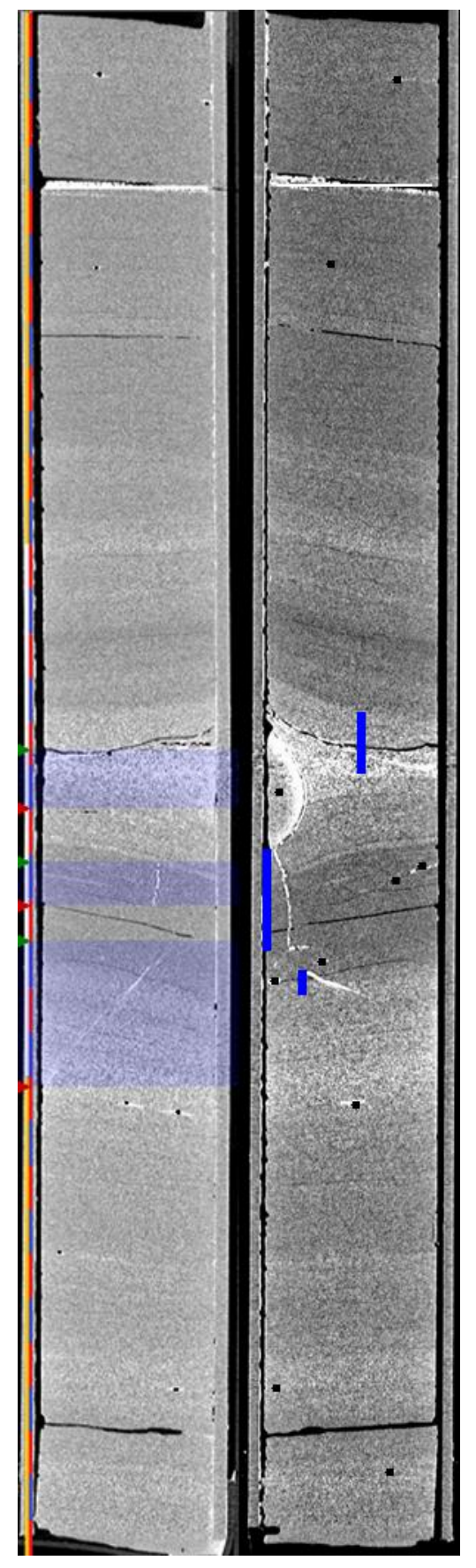

7470'

Figure 2-9: 2D isolated planes (YZ on left and $\mathrm{XZ}$ on right) through the vertical center of the medical CT scans of the MSEEL MIP 3H core from 7467 - 7473' 


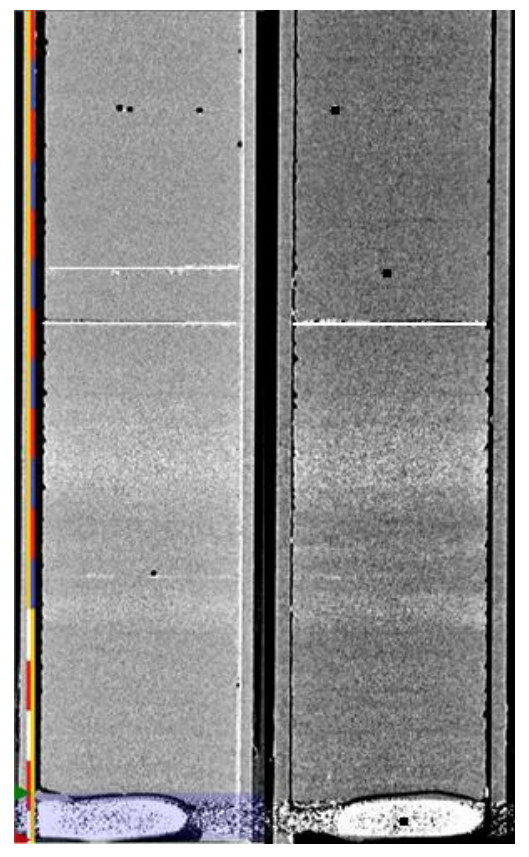

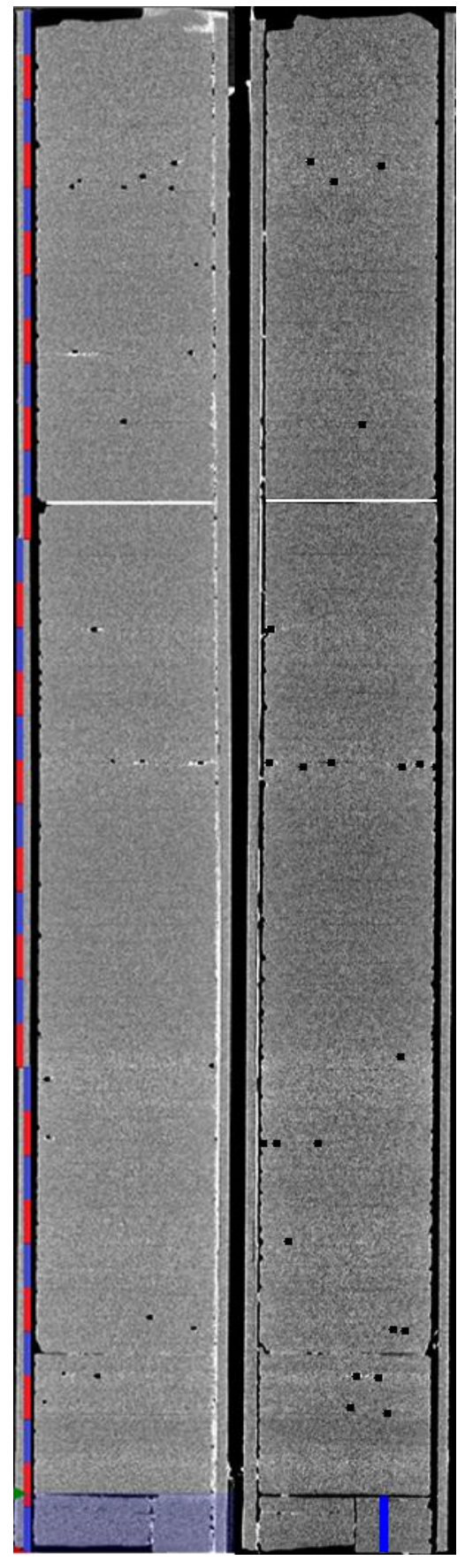

7476'

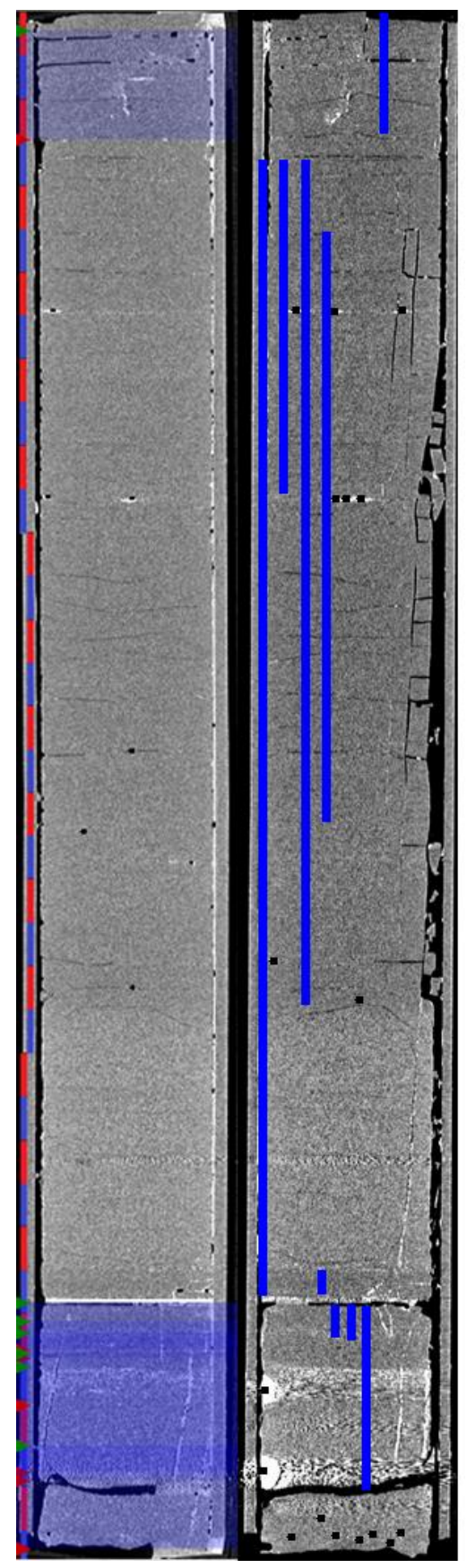

7479'

Figure 2-10: 2D isolated planes ( $\mathrm{YZ}$ on left and $\mathrm{XZ}$ on right) through the vertical center of the medical CT scans of the MSEEL MIP 3H core from 7473 - 7481' 


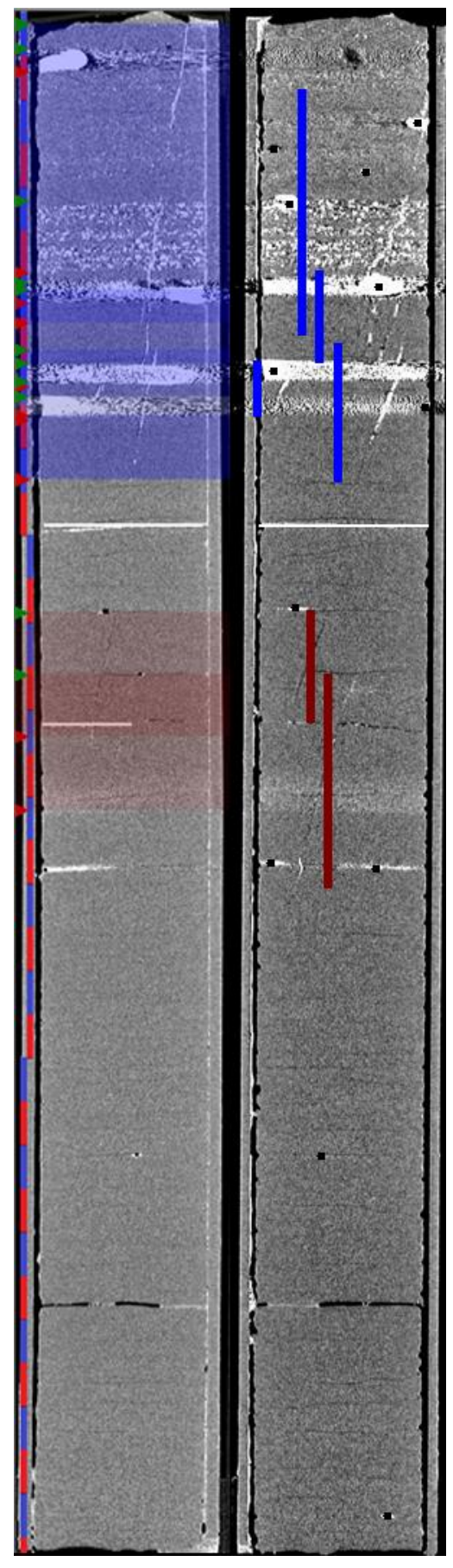

$7482^{\prime}$

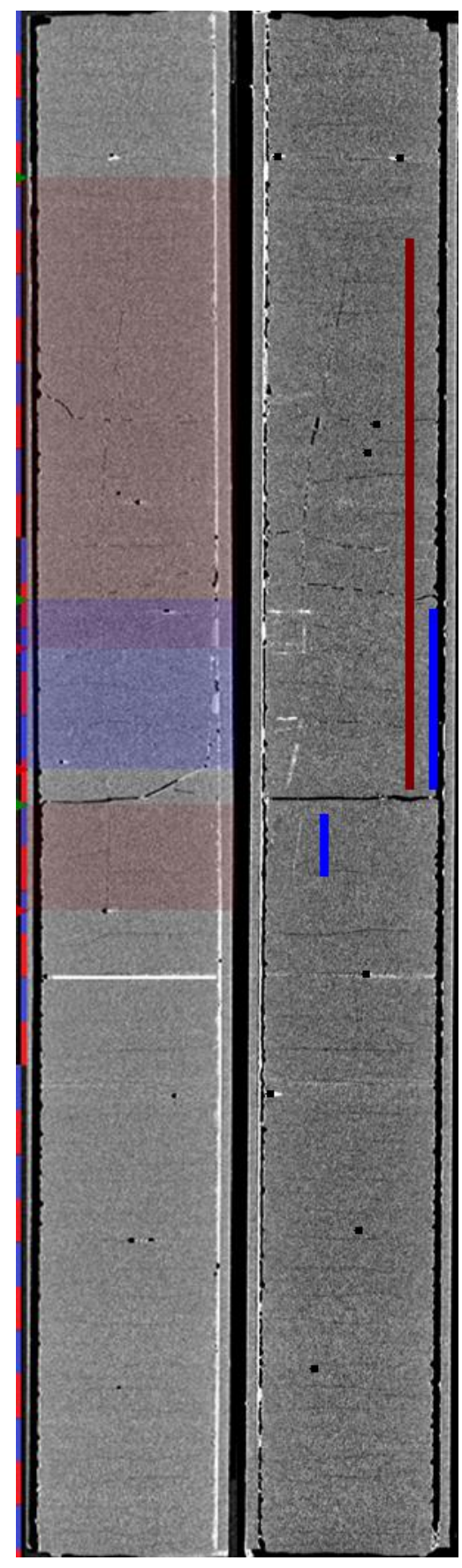

7485

Figure 2-11: 2D isolated planes (YZ on left and $\mathrm{XZ}$ on right) through the vertical center of the medical CT scans of the MSEEL MIP 3H core from 7482 - 7485' 


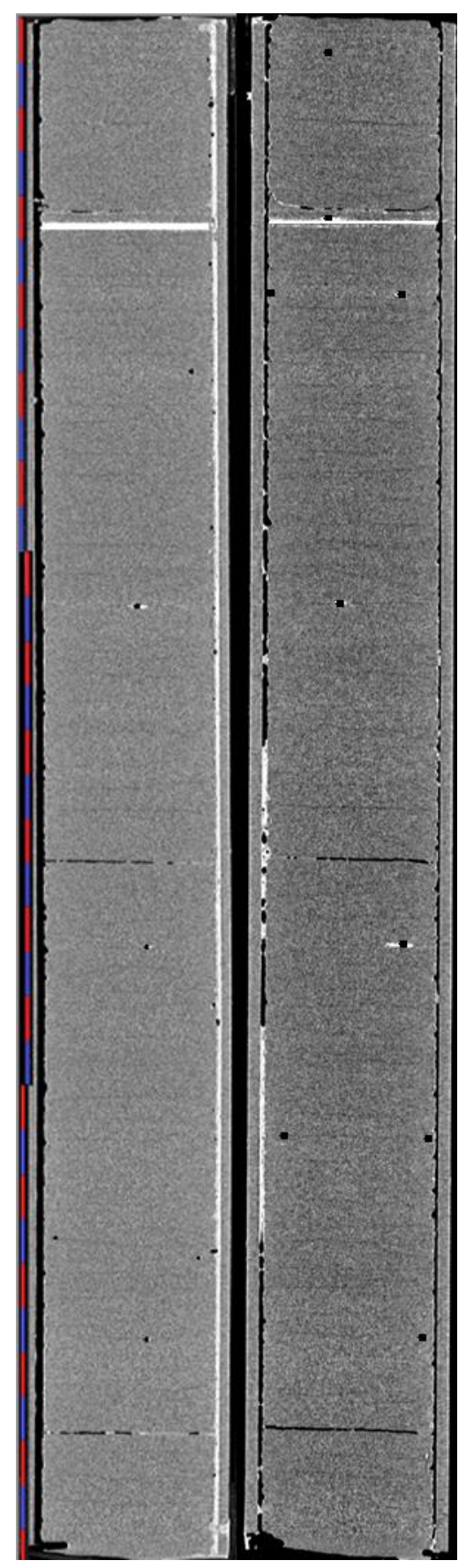

$7488^{\prime}$

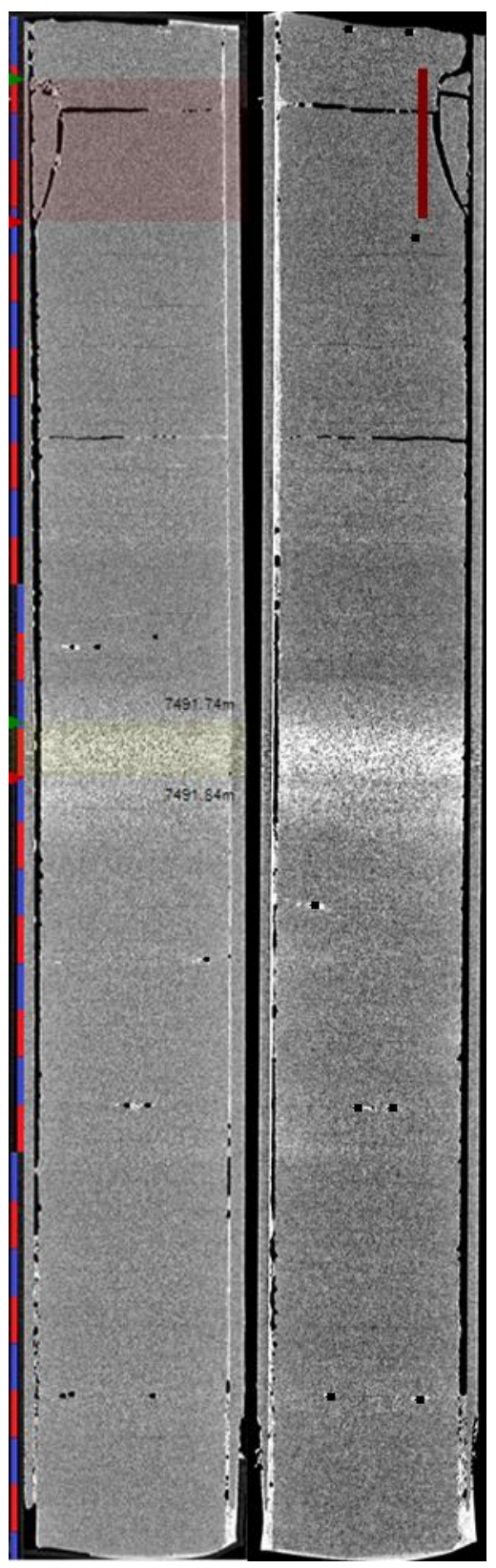

7491'

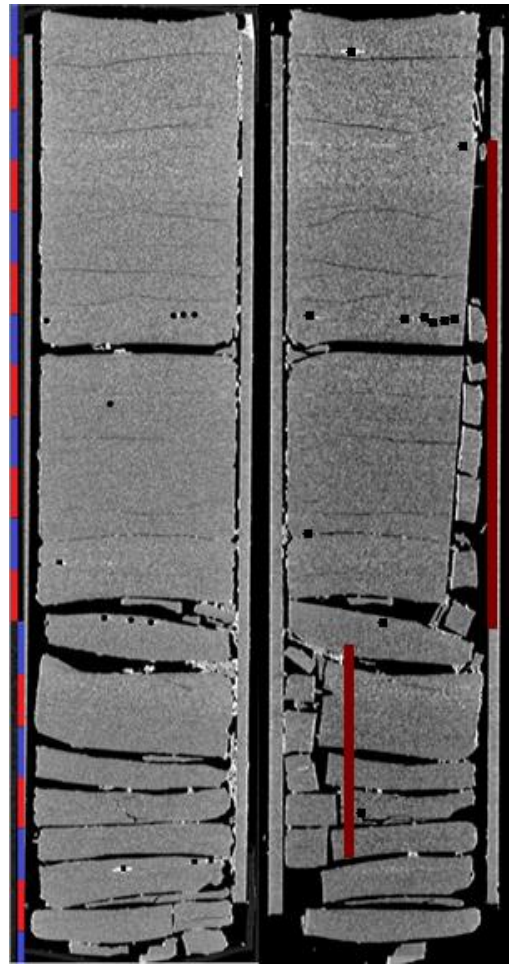

7493.25'

Figure 2-12: 2D isolated planes ( $\mathrm{YZ}$ on left and $\mathrm{XZ}$ on right) through the vertical center of the medical CT scans of the MSEEL MIP 3H core from $7488-7495$ 


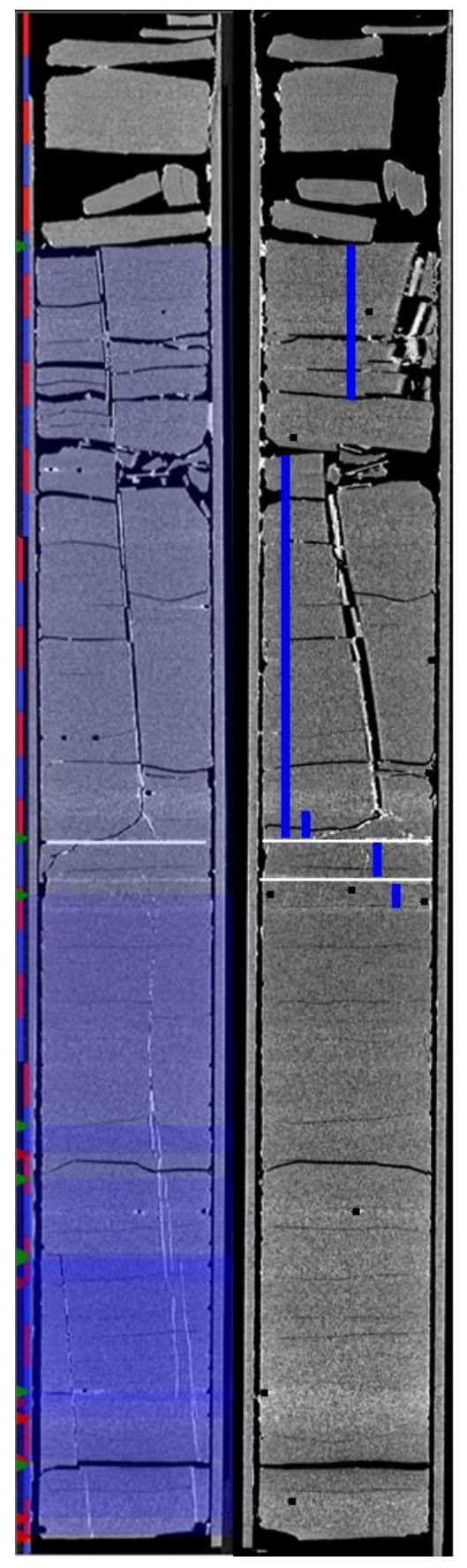

7495

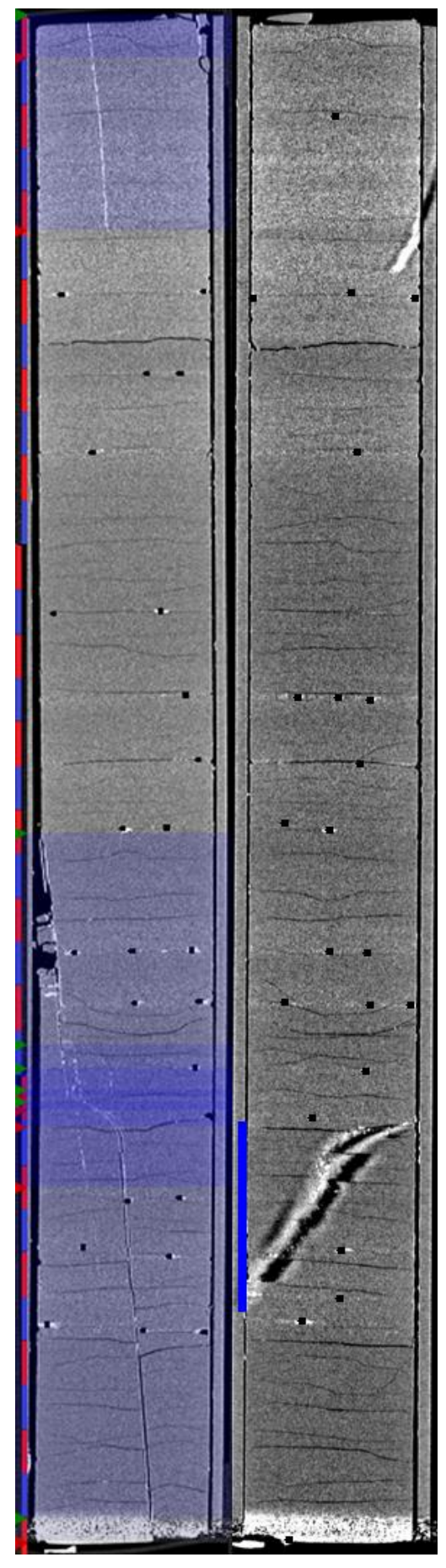

7498,

Figure 2-13: 2D isolated planes ( $\mathrm{YZ}$ on left and $\mathrm{XZ}$ on right) through the vertical center of the medical CT scans of the MSEEL MIP 3H core from 7495 - 7501 


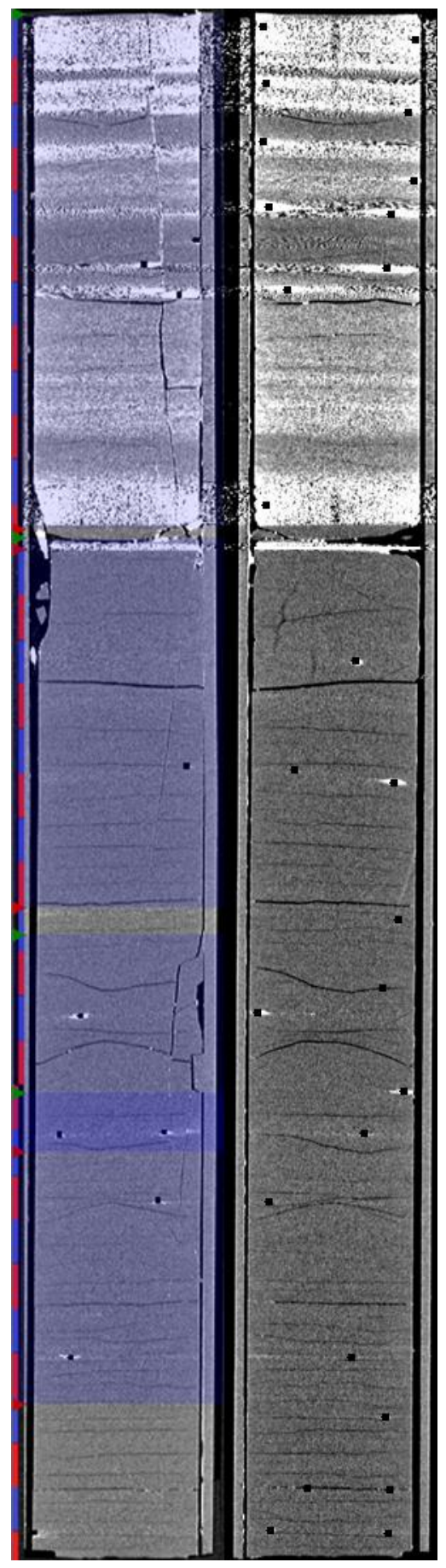

$7501^{\prime}$

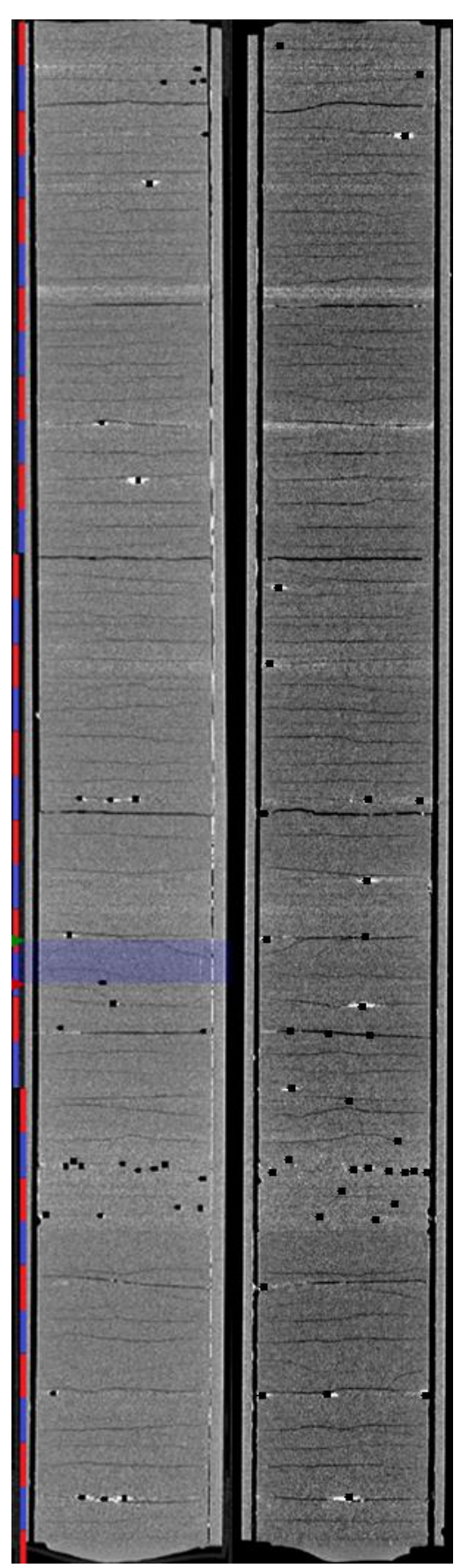

7504'

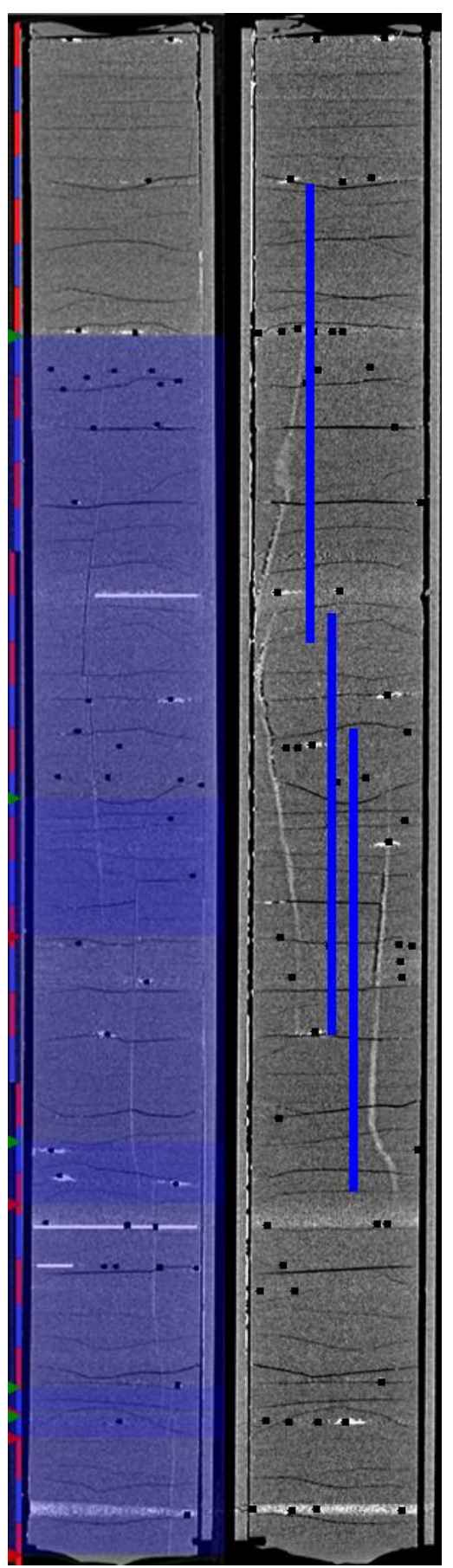

7507'

Figure 2-14: 2D isolated planes ( $Y Z$ on left and $X Z$ on right) through the vertical center of the medical CT scans of the MSEEL MIP 3H core from 7501 - 7510' 


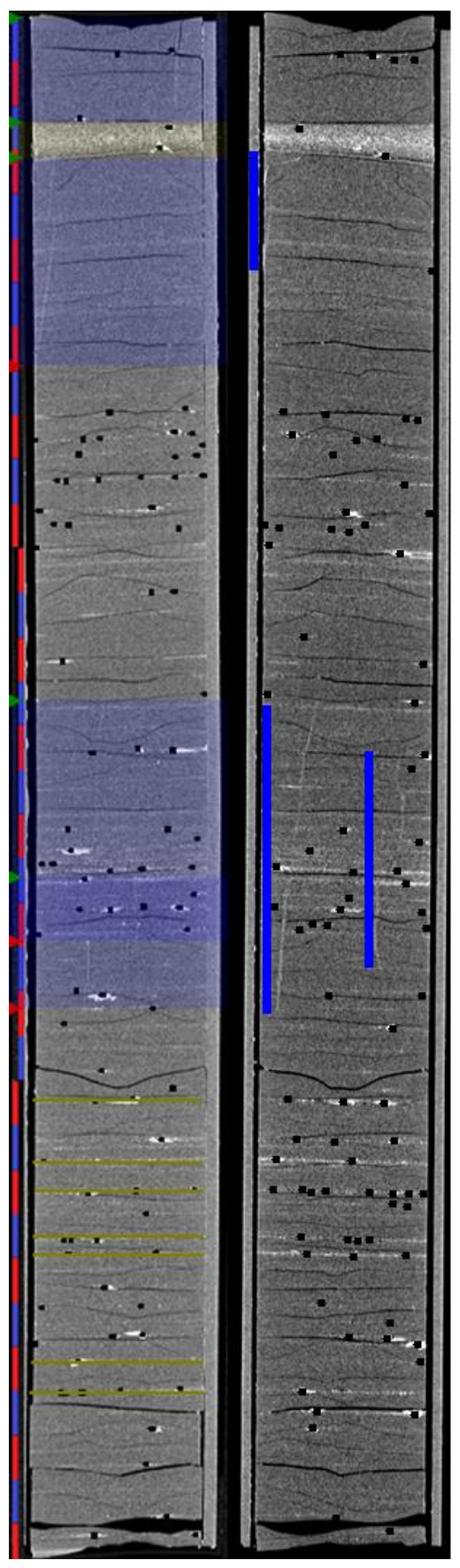

$7510^{\prime}$

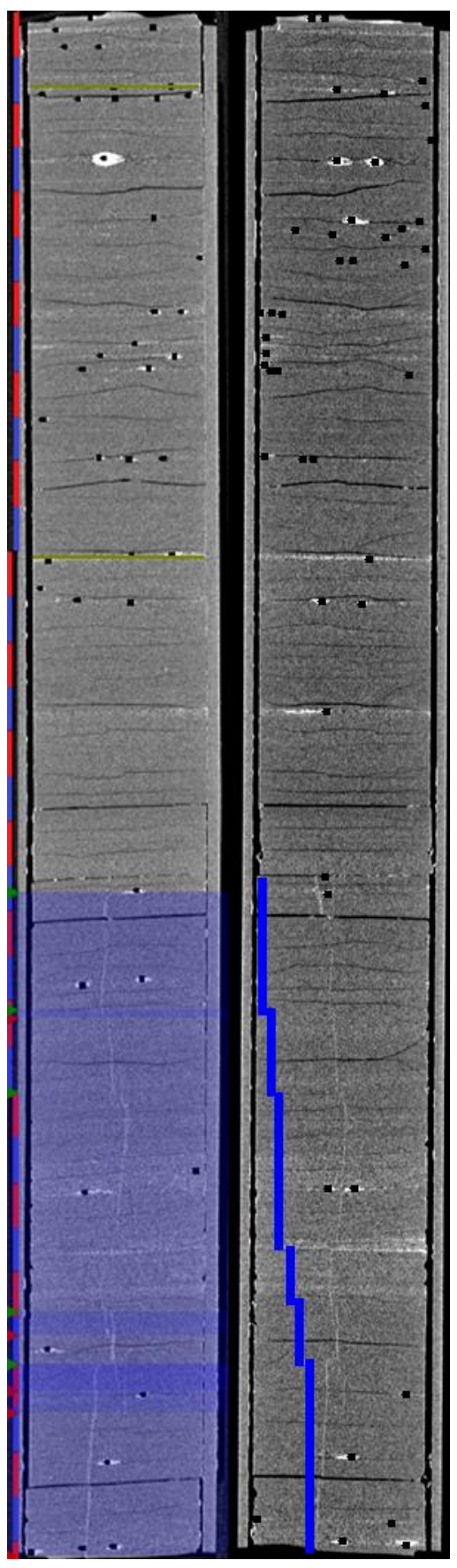

7513'

Figure 2-15: 2D isolated planes ( $\mathrm{YZ}$ on left and $\mathrm{XZ}$ on right) through the vertical center of the medical CT scans of the MSEEL MIP 3H core from 7510 - 7516' 


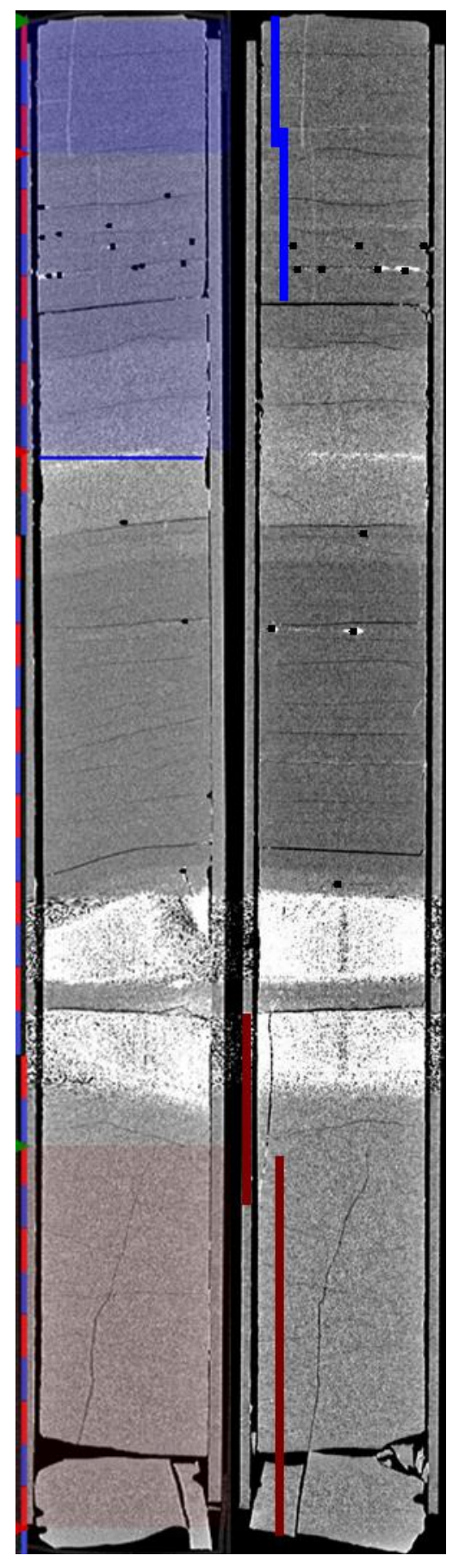

7516

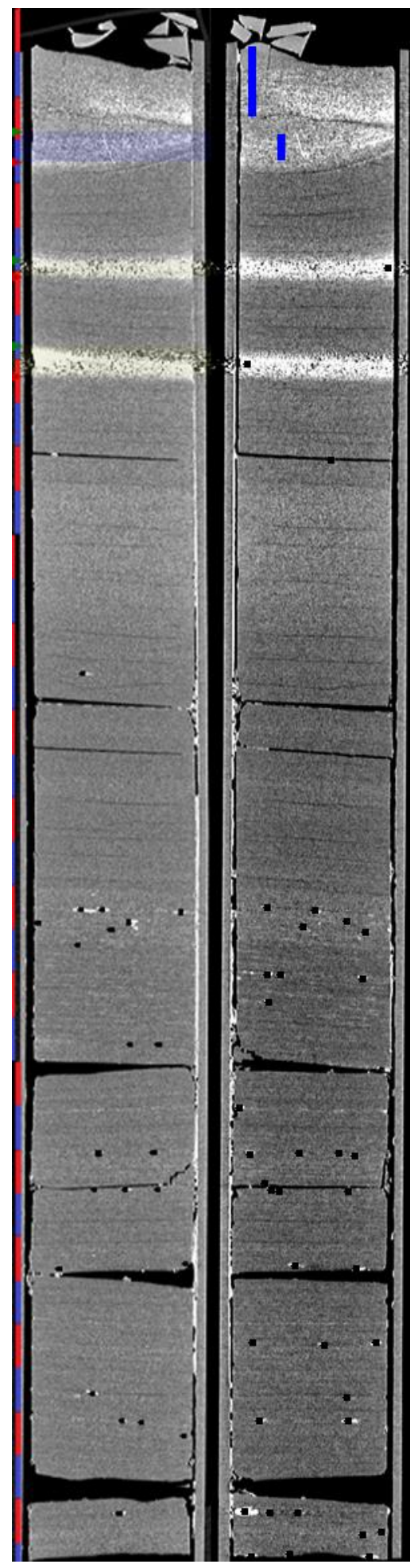

7519'

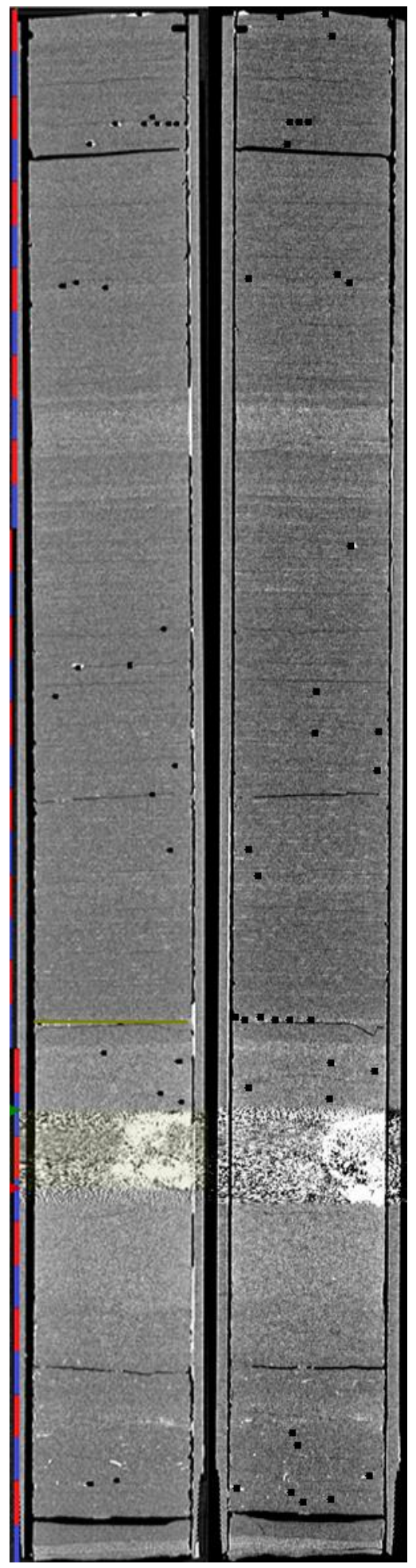

7522,

Figure 2-16: 2D isolated planes ( $\mathrm{YZ}$ on left and $\mathrm{XZ}$ on right) through the vertical center of the medical CT scans of the MSEEL MIP 3H core from 7516 - 7525' 

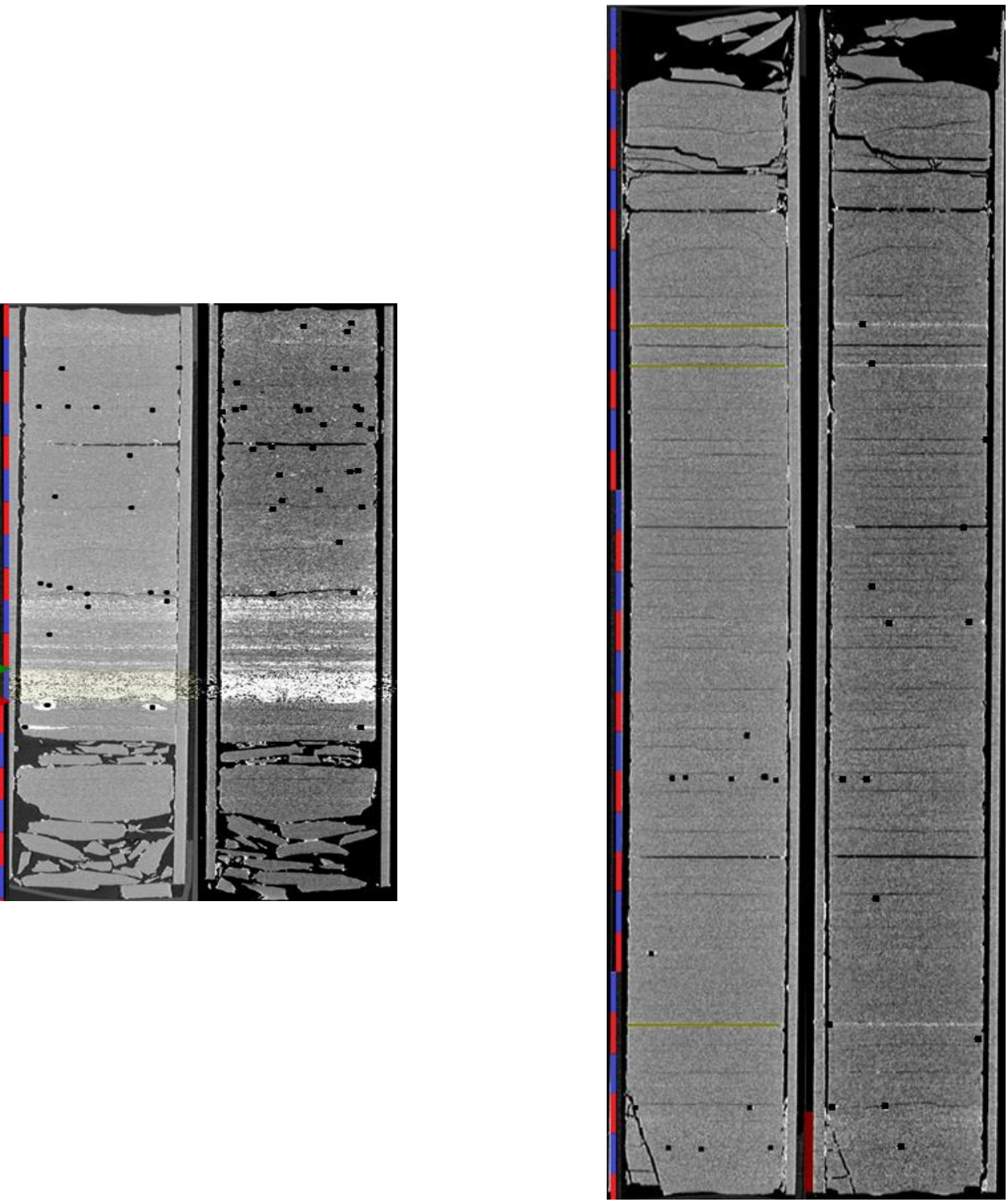

$7525^{\prime}$

7526.5

Figure 2-17: 2D isolated planes ( $Y Z$ on left and $X Z$ on right) through the vertical center of the medical CT scans of the MSEEL MIP 3H core from 7525 - 7529' 


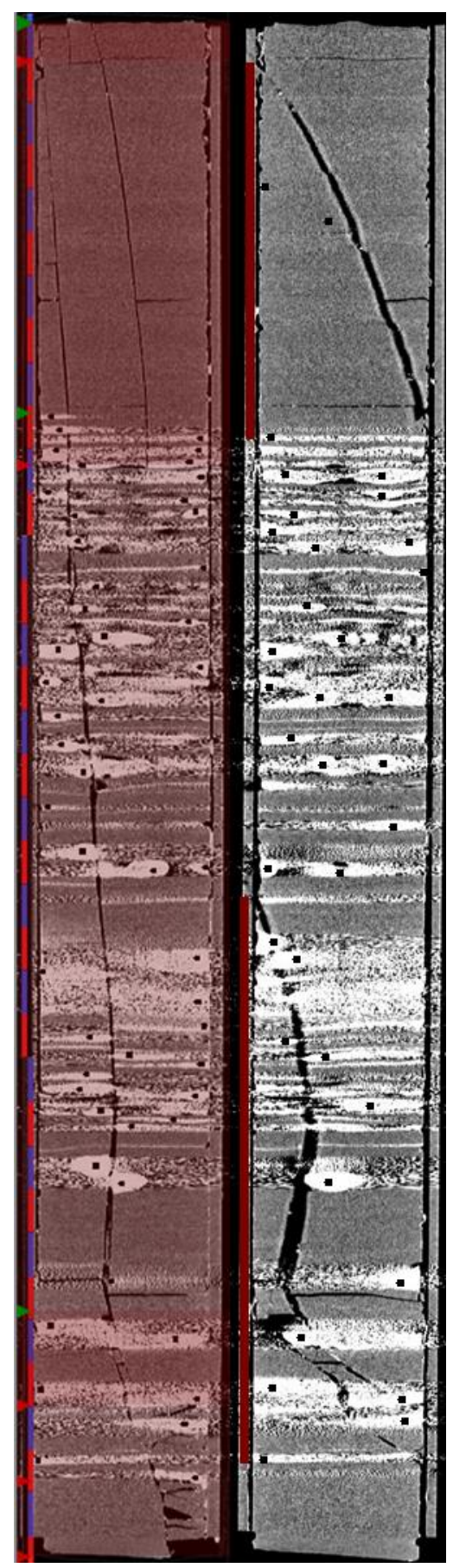

$7529^{\prime}$

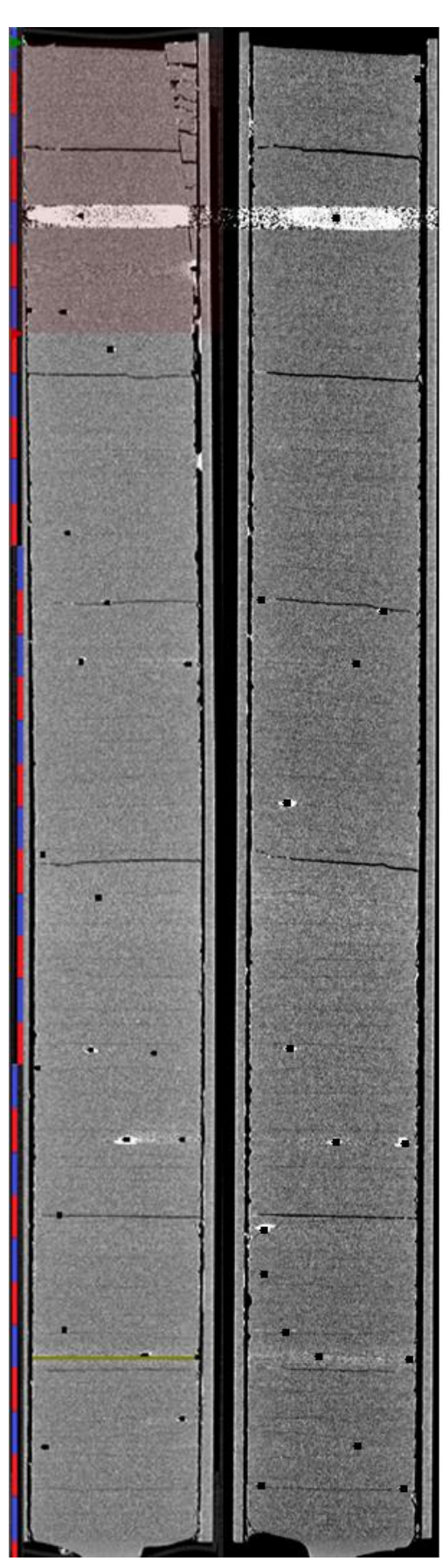

7532'

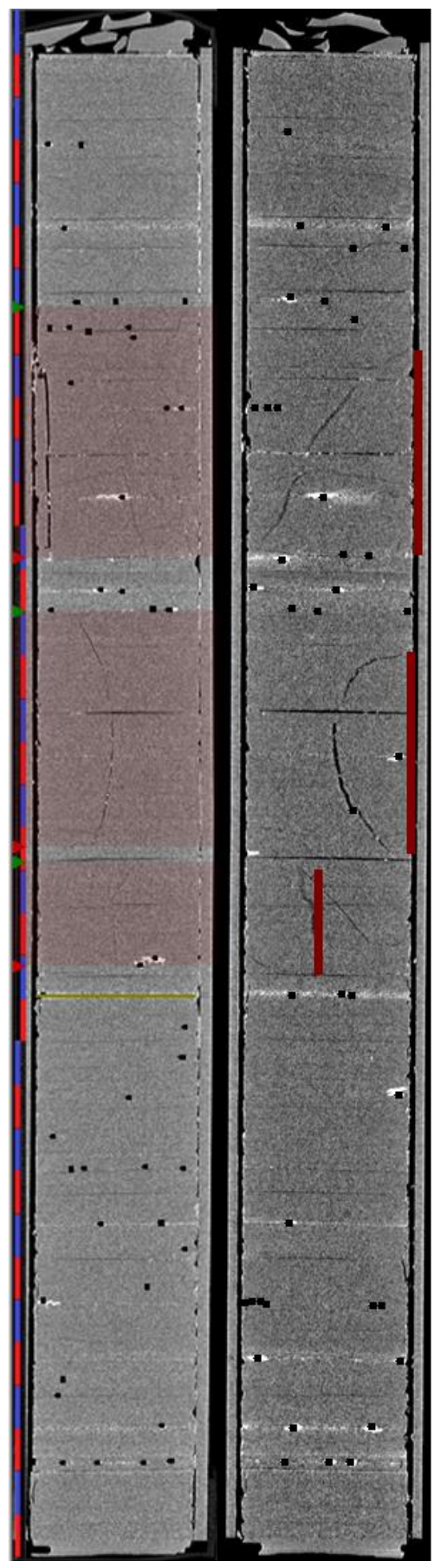

7535'

Figure 2-18: 2D isolated planes ( $\mathrm{YZ}$ on left and $\mathrm{XZ}$ on right) through the vertical center of the medical CT scans of the MSEEL MIP 3H core from 7529 - 7538' 


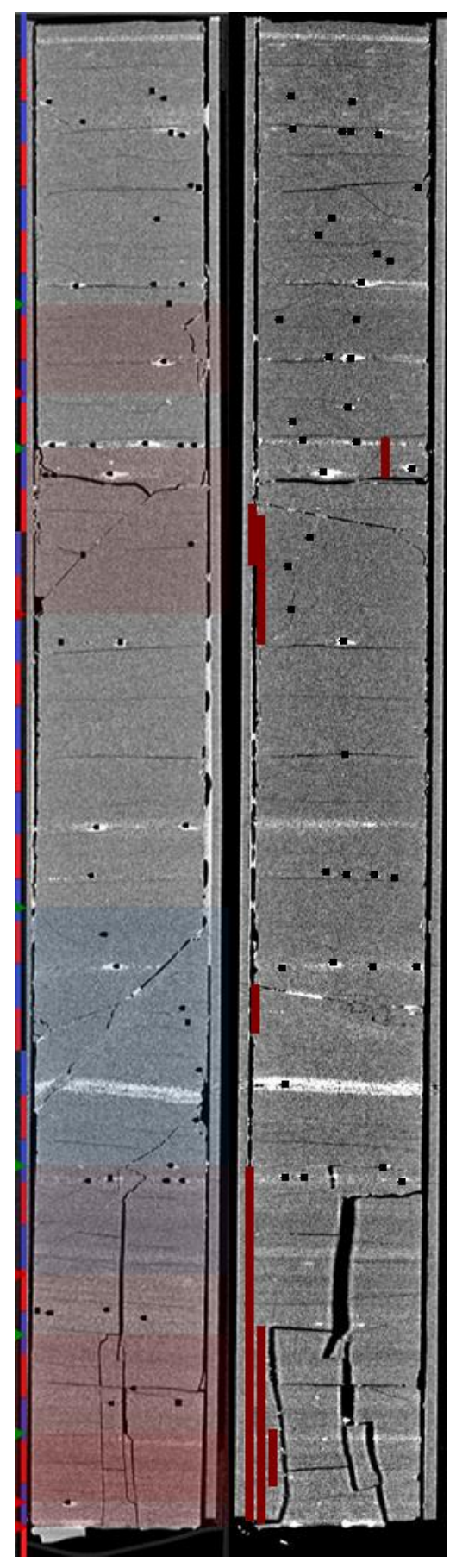

$7538^{\prime}$

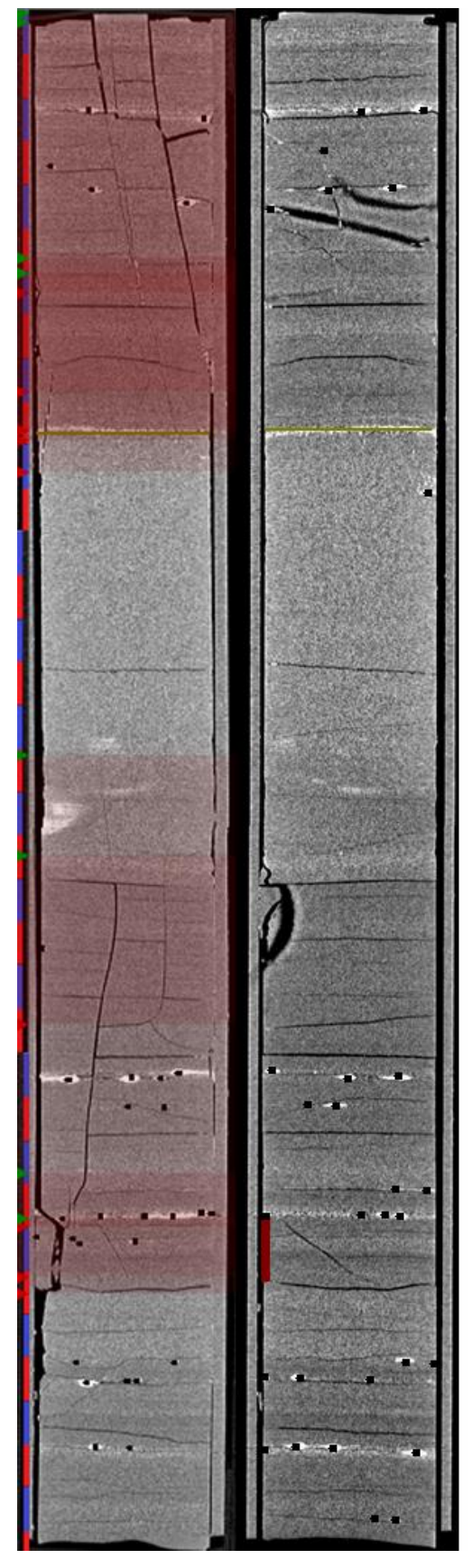

7541'

Figure 2-19: 2D isolated planes ( $\mathrm{YZ}$ on left and $\mathrm{XZ}$ on right) through the vertical center of the medical CT scans of the MSEEL MIP 3H core from 7538 - 7544' 


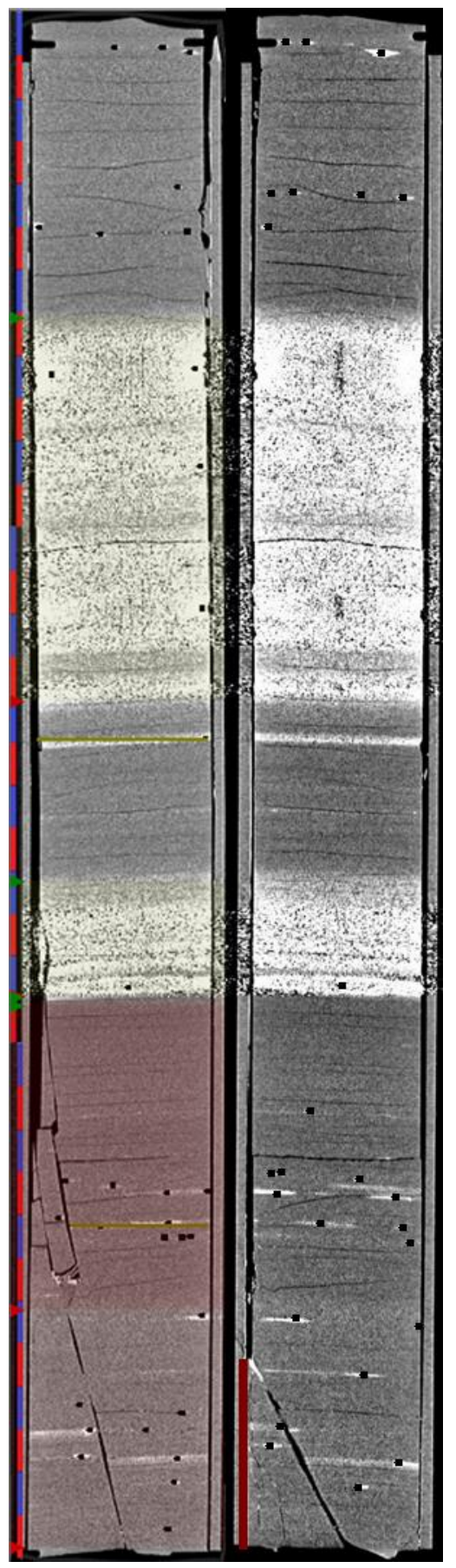

$7544^{\prime}$

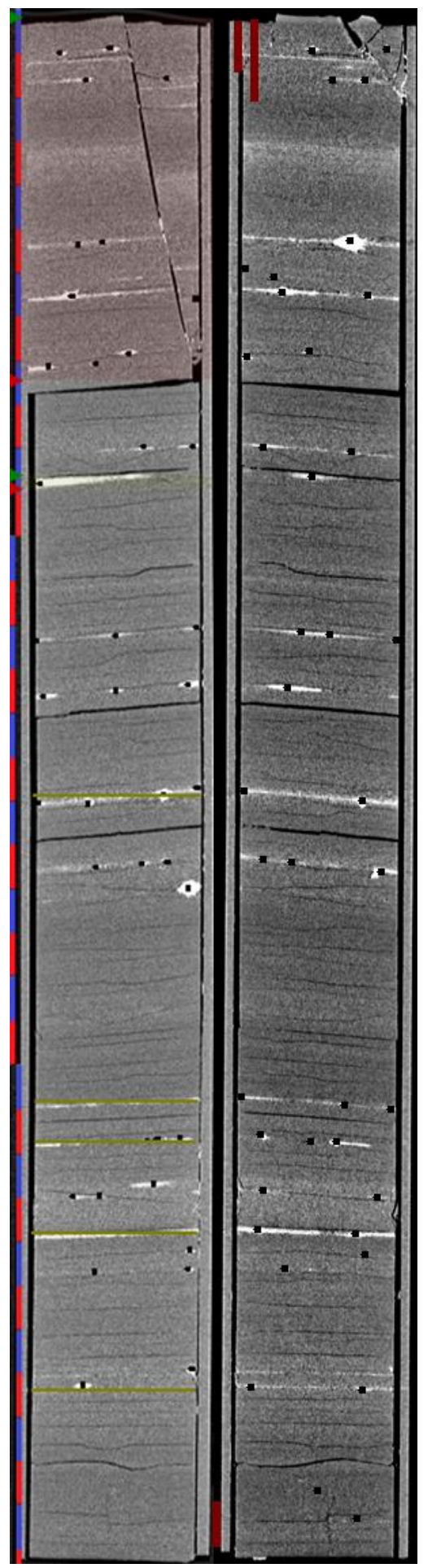

7547'

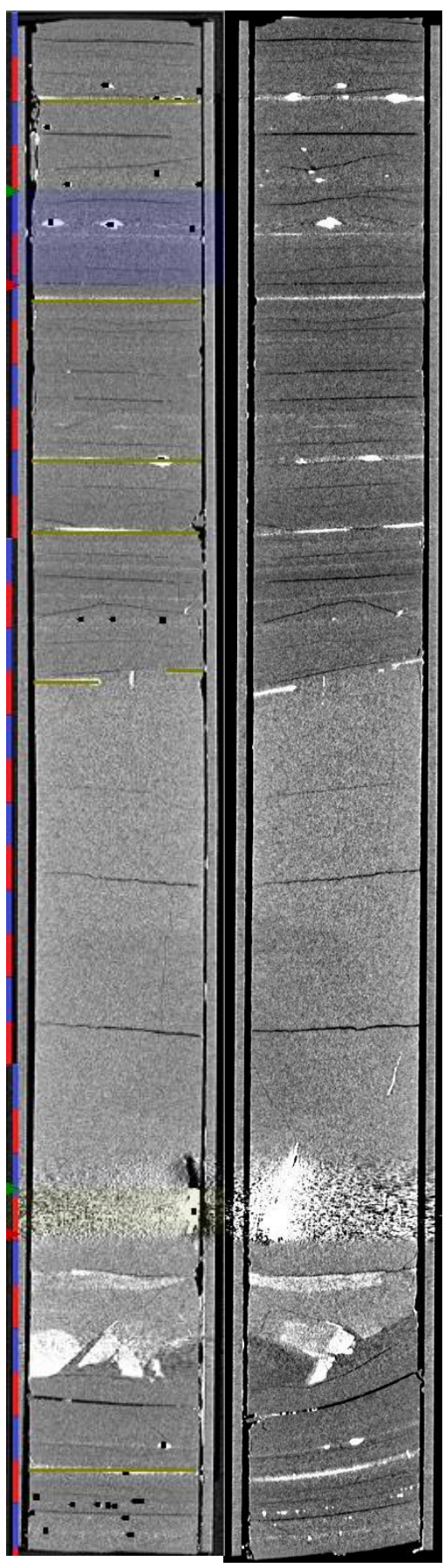

7550'

Figure 2-20: 2D isolated planes ( $\mathrm{YZ}$ on left and $\mathrm{XZ}$ on right) through the vertical center of the medical CT scans of the MSEEL MIP 3H core from $7544-7553$, 


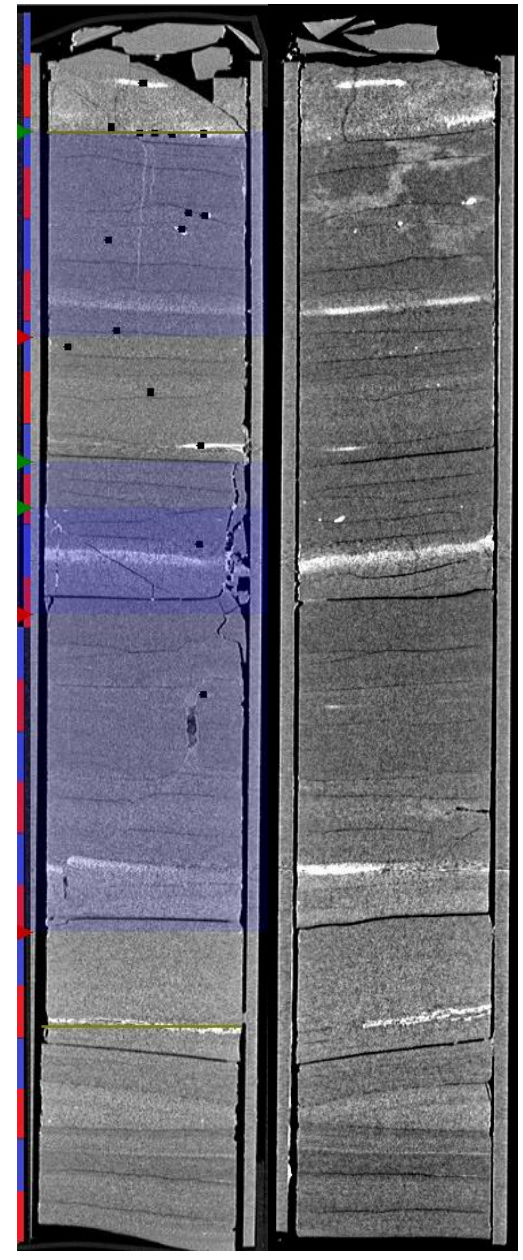

$7553^{\prime}$
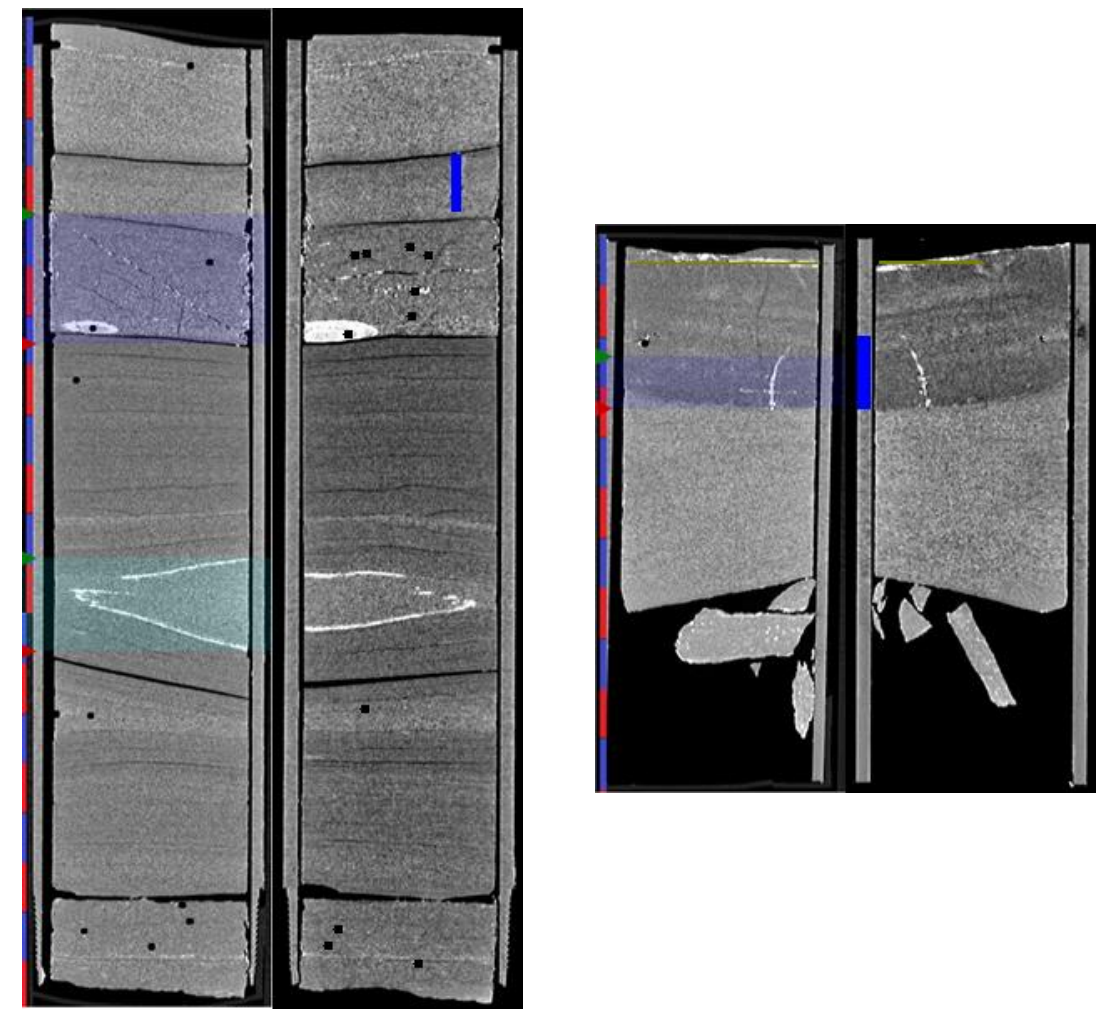

7555

Figure 2-21: 2D isolated planes ( $\mathrm{YZ}$ on left and $\mathrm{XZ}$ on right) through the vertical center of the medical $\mathrm{CT}$ scans of the MSEEL MIP 3H core from $7553-7557$ ' 
A. Pyrite density

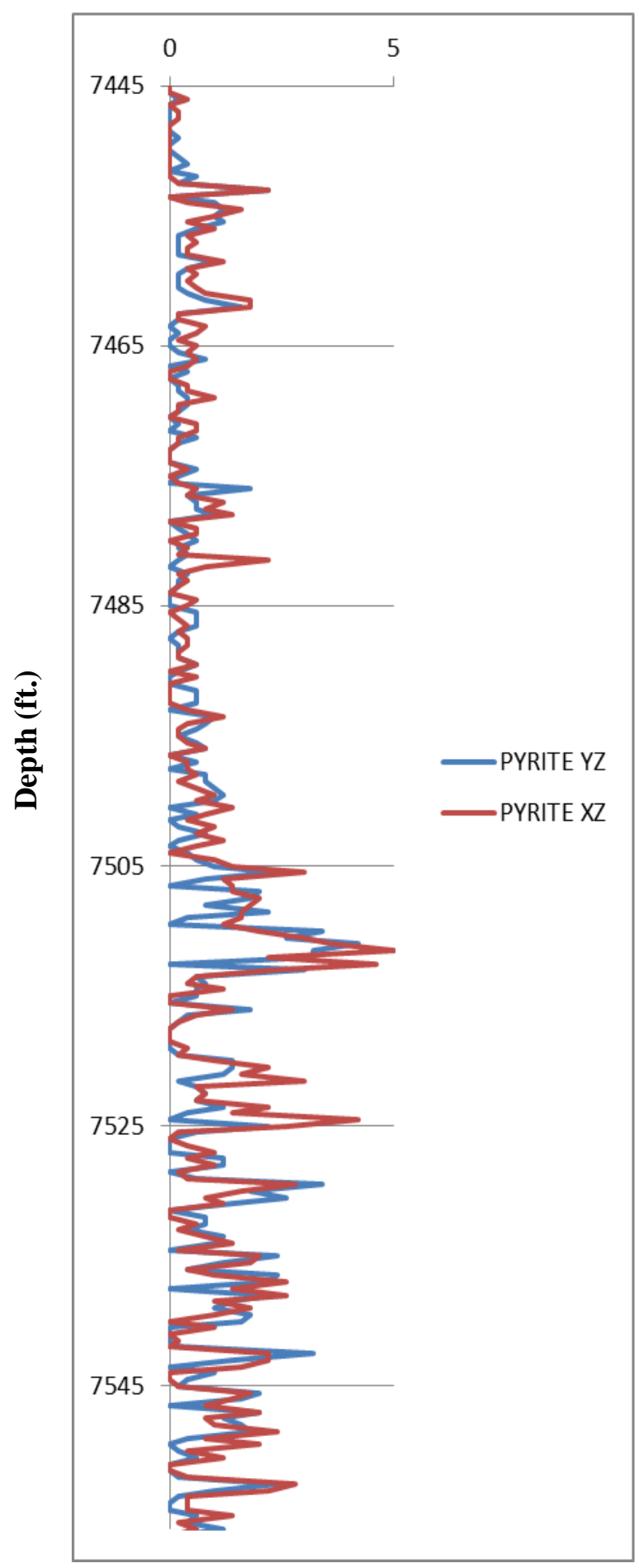

B. Fracture density (P20)

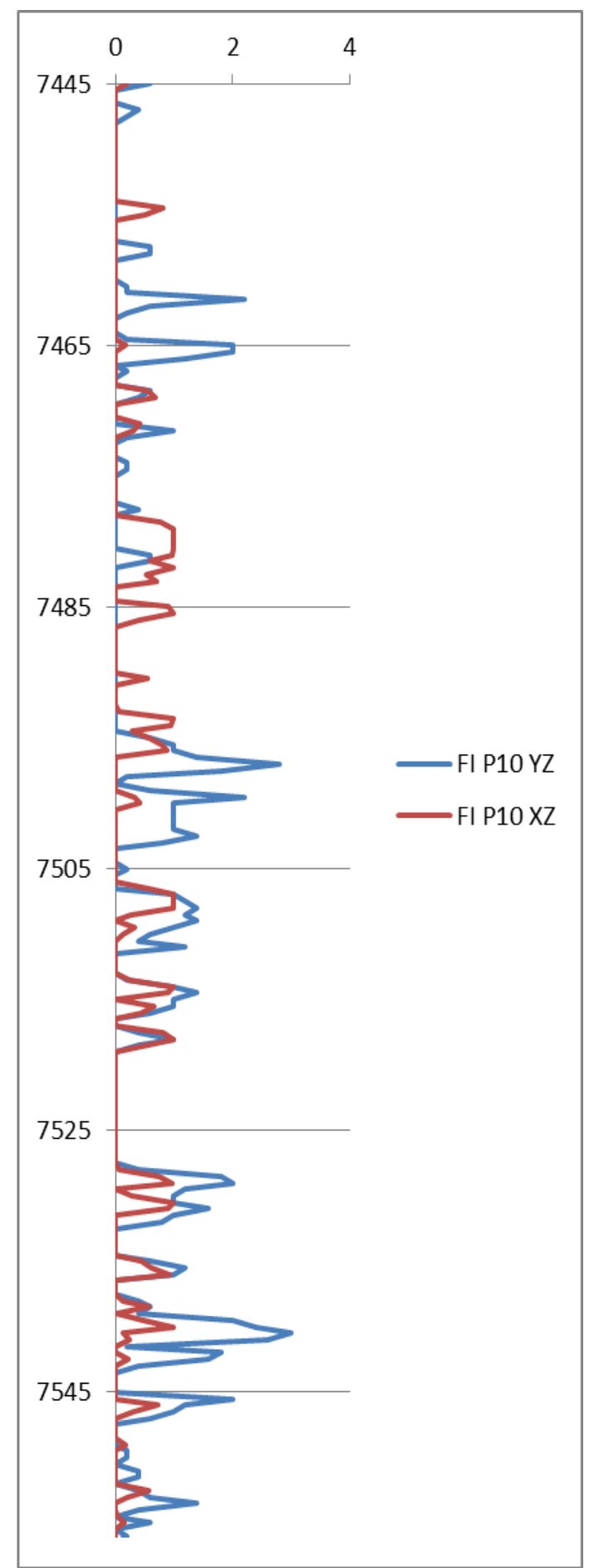

Figure 2-22: This figure shows the results of the pyrite density and fracture density analysis from the medical CT scans. A.(on the right) denotes pyrite density at .5' increments with the blue representing the $Y Z$ slice and red representing the $\mathrm{XZ}$ slice. (B.)(on the left) shows fracture density within a $.5 \mathrm{ft}$. window, blue represents the $\mathrm{YZ}$ slice and red represents the XZ slice. 
In addition to structural descriptions the medical CT-scans can aid in the breakout of the macrofacies. Figure 2-23, shows average grayscale versus depth compared with our macro-facies. Grayscales generally range from about 1600 to 2200, high values relate to denser minerals and lower values have less dense minerals. Low gray-scale values $(<1600)$ are associated with missing material, either due to a fracture or missing core. High gray-scale values (>2400) are associated with nodules and other high-density minerals. The lower Marcellus has an overall lower grayscale value (1700 on average) compared to the upper portion of the middle Marcellus and the upper Marcellus (1900 on average). We also see a slight increase in the frequency of carbonate intervals in the lower Marcellus. This is particularly clear when looking at the Marcellus-Onondaga contact, where there is a high frequency change from high to low grayscale values. In addition to facies information the average gray scale can be used to pick of rubble zones and high-density minerals. These zones sit distinctly below and above the average grayscale trends, respectively. 


\section{Average grayscale from CT-Scan images}

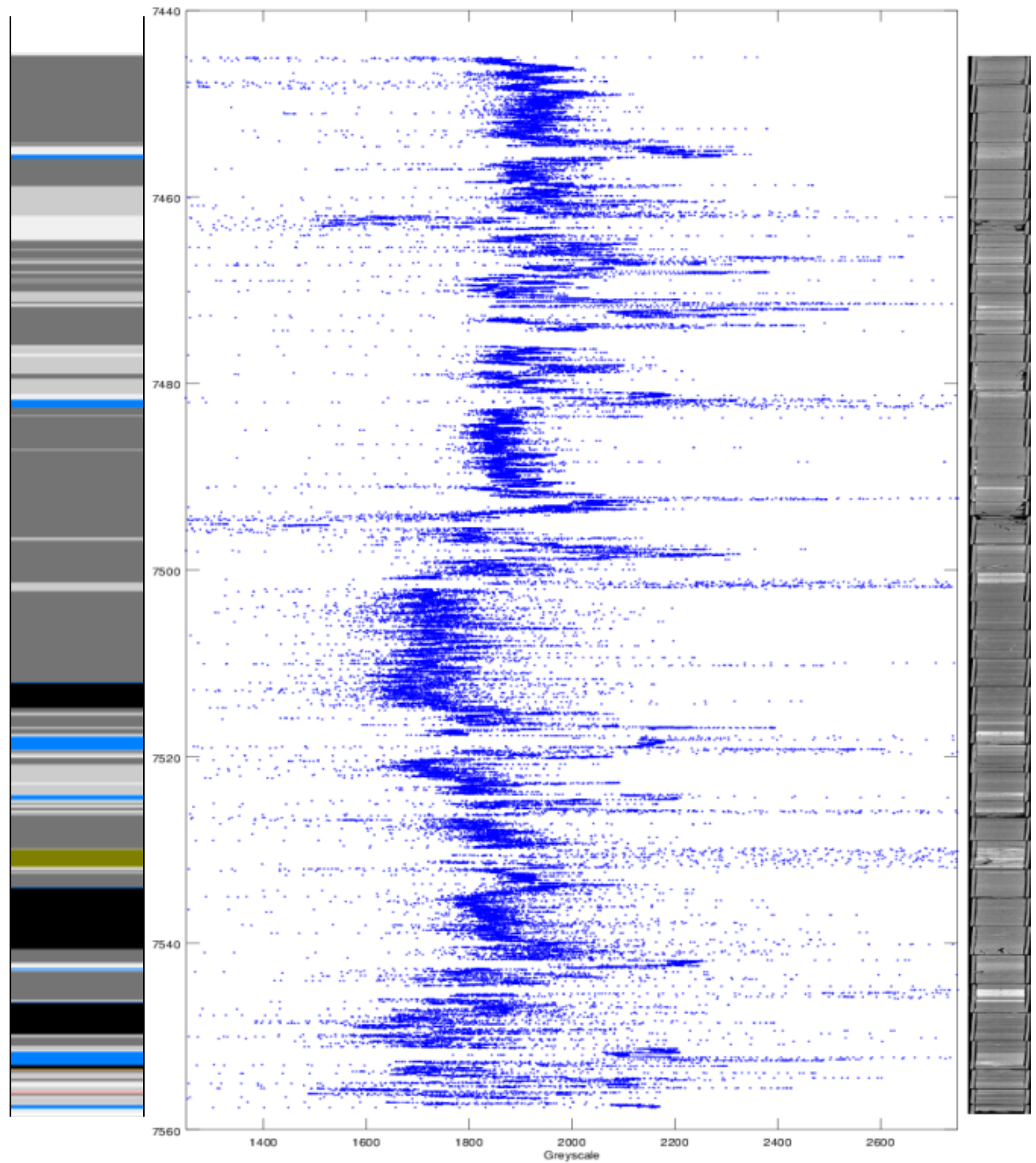

Figure 2-23: Shown in the plot to the left is an example of how CT-scan imagery was used to aid the macro-facies description. Average grayscales from each slice $(.5 \mathrm{~mm})$ plotted with depth. Grayscales generally range from about 1600 to 2200 , high values relate to denser minerals and lower values have less dense minerals. Low gray scale values $(<1600)$ are associated with missing material, either due to a fracture or missing core. High gray scale values $(>2400)$ are associated with nodules and other high-density minerals. 


\subsection{Meso-Facies}

Meso-scale (core-scale) lithofacies analysis is derived from the properties that represent the lithology. For mudrock and shale reservoirs, lithofacies are often differentiated by total organic content and mineralogy (Wang 2012, Wang and Carr 2013, Bhattacharya and Carr 2016). In this section, we investigate organic matter prediction, mineralogy prediction, and meso-facies prediction between MIP-3H and MIP-4H.

\section{Organic Matter Evaluation:}

Total organic carbon (TOC) was determined from the MIP3H core using the source rock analyzer (SRA) at National Energy Technology Laboratory (Chloe Wonnel pers. comm.). The TOC core data were used to calibrate and confirm TOC derived from logs. There were a total 47 TOC samples from the MIP3H well taken from roughly every $3 \mathrm{ft}(1 \mathrm{~m})$ with 5 repeat samples to confirm accuracy. TOC ranges from $2.6 \mathrm{wt} \%$ to $10.9 \mathrm{wt} \%$ through the Marcellus. TOC values generally decrease from the lower Marcellus to upper with three peaks at the base of each of the high gamma-ray intervals. Specific values for SRA derived TOC are in Appendix A.

\section{Predicting TOC from Log Data:}

Numerous methods exist to determine TOC from common well logs. In this study Schmoker's Method, Passey's Method, Gamma Ray derived TOC, and Uranium derived TOC were used to derive TOC from well logs and were compared with the SRA TOC to identify the best method.

Schmoker's Method uses the inverse relationship between density and organic content, mainly through increasing relatively low-density kerogen content (1.1 to 1.8 grams/cc) compared to more typical quartz and carbonate densities (2.64 to 2.71 grams/cc) decreasing the observed density to determine the TOC (Schmoker, 1981). Figure 2-24, shows the relationship between 
density and core TOC. There is a moderate correlation between core TOC and density with an $\mathrm{r}^{2}$ of .558. The equation tracks the core TOC closely in the upper and middle Marcellus, however it under-predicts TOC in the lower Marcellus. This is likely due to the increase in the higher density pyrite through this zone (5.01 grams/cc).

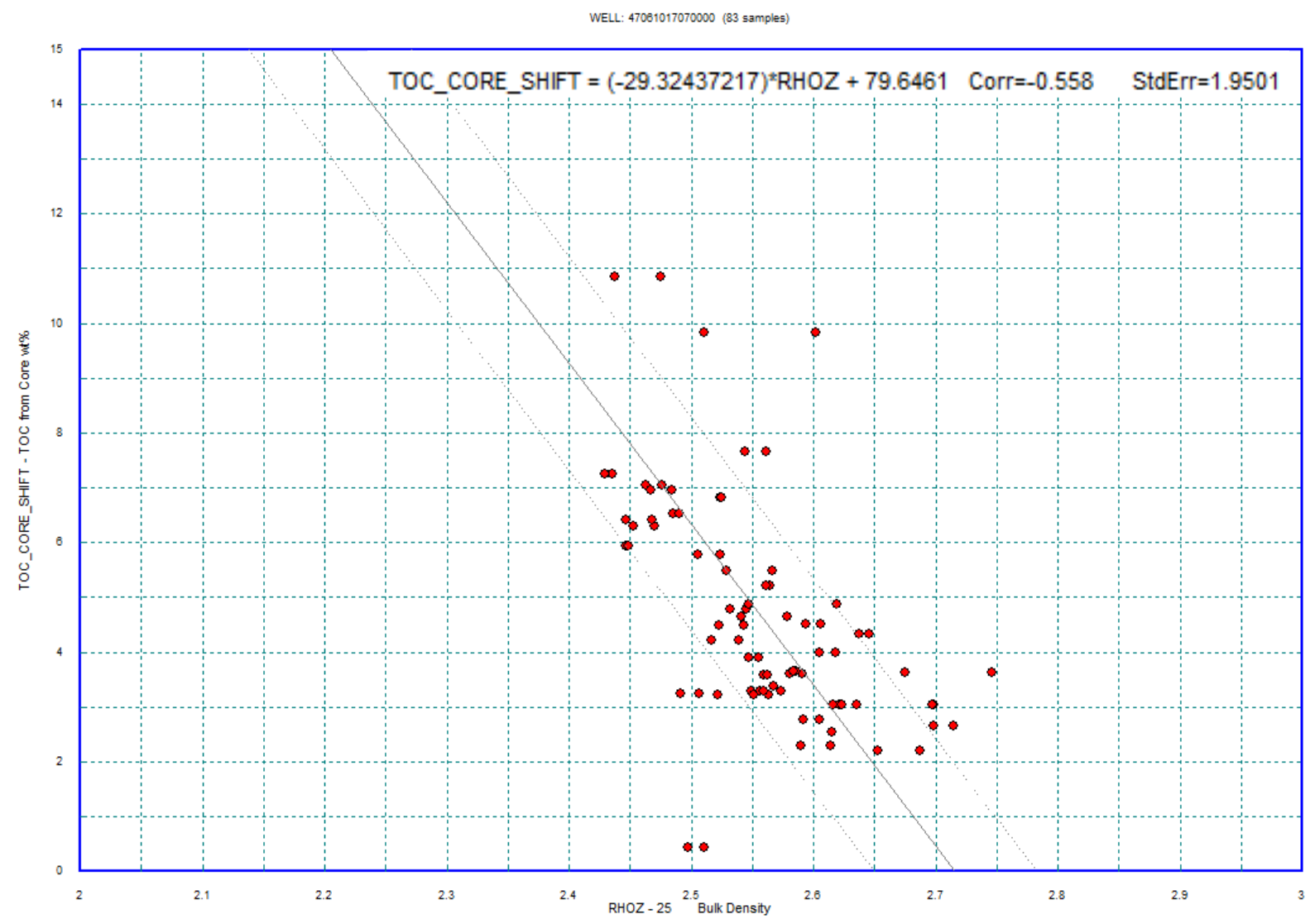

Figure 2-24: Cross-plot of Schmoker's method where RHOB and Core TOC, the equation is the linear relationship between the two TOC measurements.

Passey's Method utilizes the relationship between sonic transit time (DT) and resistivity (R) to determine the amount of kerogen in source rocks (Passey, et al., 1990). The Passey's Equation is a two-part equation:

(1) $\mathrm{DLogR}=\log _{10}\left(\mathrm{R} / \mathrm{R}_{\text {baseline }}\right)+0.02 *\left(\mathrm{DT}-\mathrm{DT}_{\text {baseline }}\right)$ 
Where, $\mathrm{R}$ is resistivity, DT is the measured transit time in $\mu \mathrm{s} / \mathrm{ft}, \mathrm{R}_{\text {baseline }}$ and $\mathrm{DT}$ baseline are corresponding values baselined in non-source, clay-rich rocks. In our calculation, we use a resistivity baseline of $72 \mathrm{ohm}-\mathrm{m}$ and DT baseline of $81 \mu \mathrm{s} / \mathrm{ft}$, taken from the Mahantango at 7380 $\mathrm{ft}(2249.43 \mathrm{~m})$.

(2) $\mathrm{TOC}=(\mathrm{DLogR}) * 10^{(2.297-01688 * \mathrm{LOM})}$

Where, LOM is maturity from $5-12,7$ being the onset of maturity for oil-prone reservoirs and 12 being over mature. In our calculation, we use an LOM of 8.5 determined through a x-plot of TOC and DLogR (Figure 2-25).

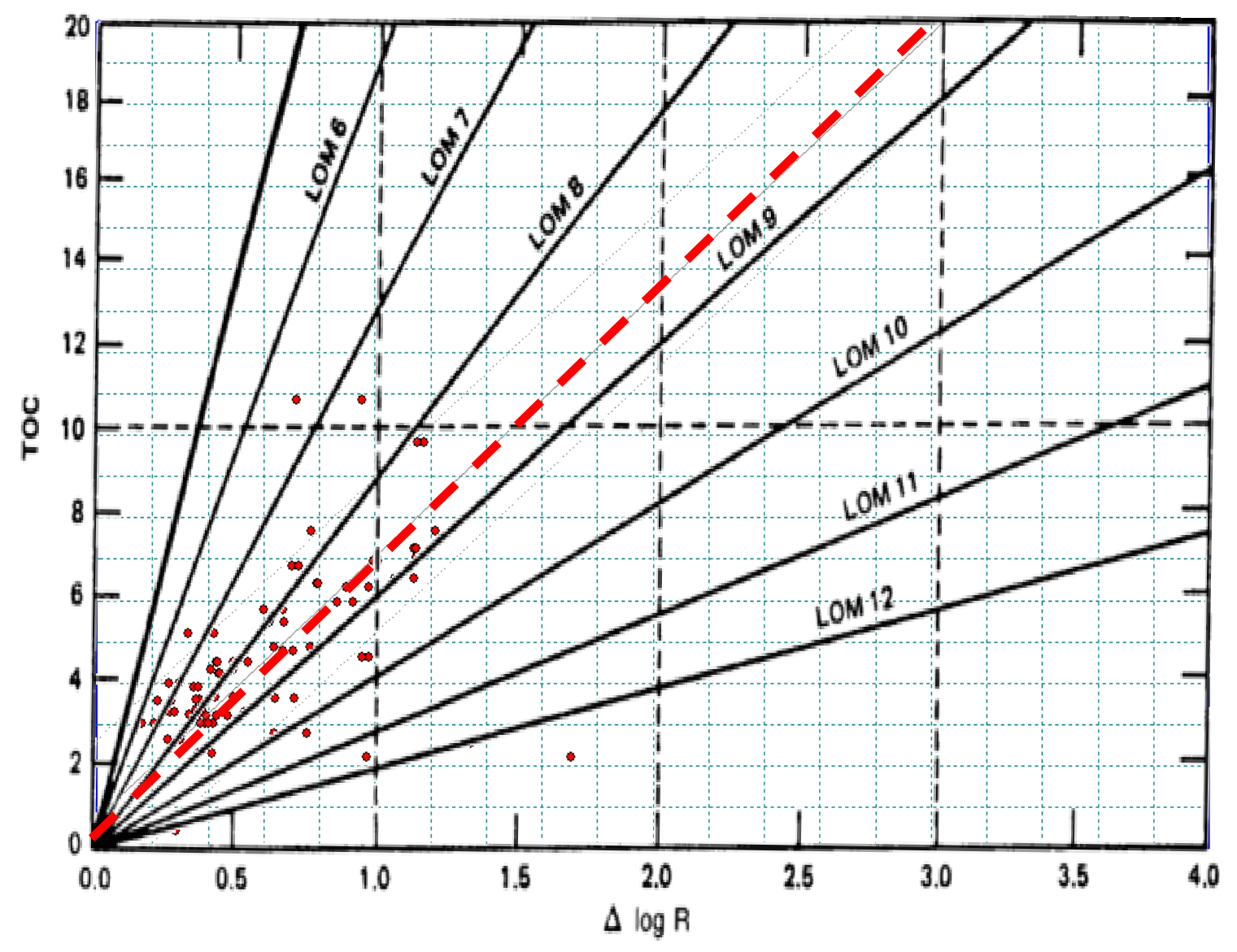

Figure 2-25: Crossplot of TOC and DLogR, the red line denotes the linear fit from Petra used to determine the approximate LOM. 
TOC determined using Passey's method has an $\mathrm{r}^{2}$ of .585. The log curve has a strong fit in the upper and middle Marcellus, however, it breaks down when predicting TOC across the carbonate intervals (overpredicting TOC) and in the most organic portion of the lower Marcellus (under-predicting TOC) (Figure

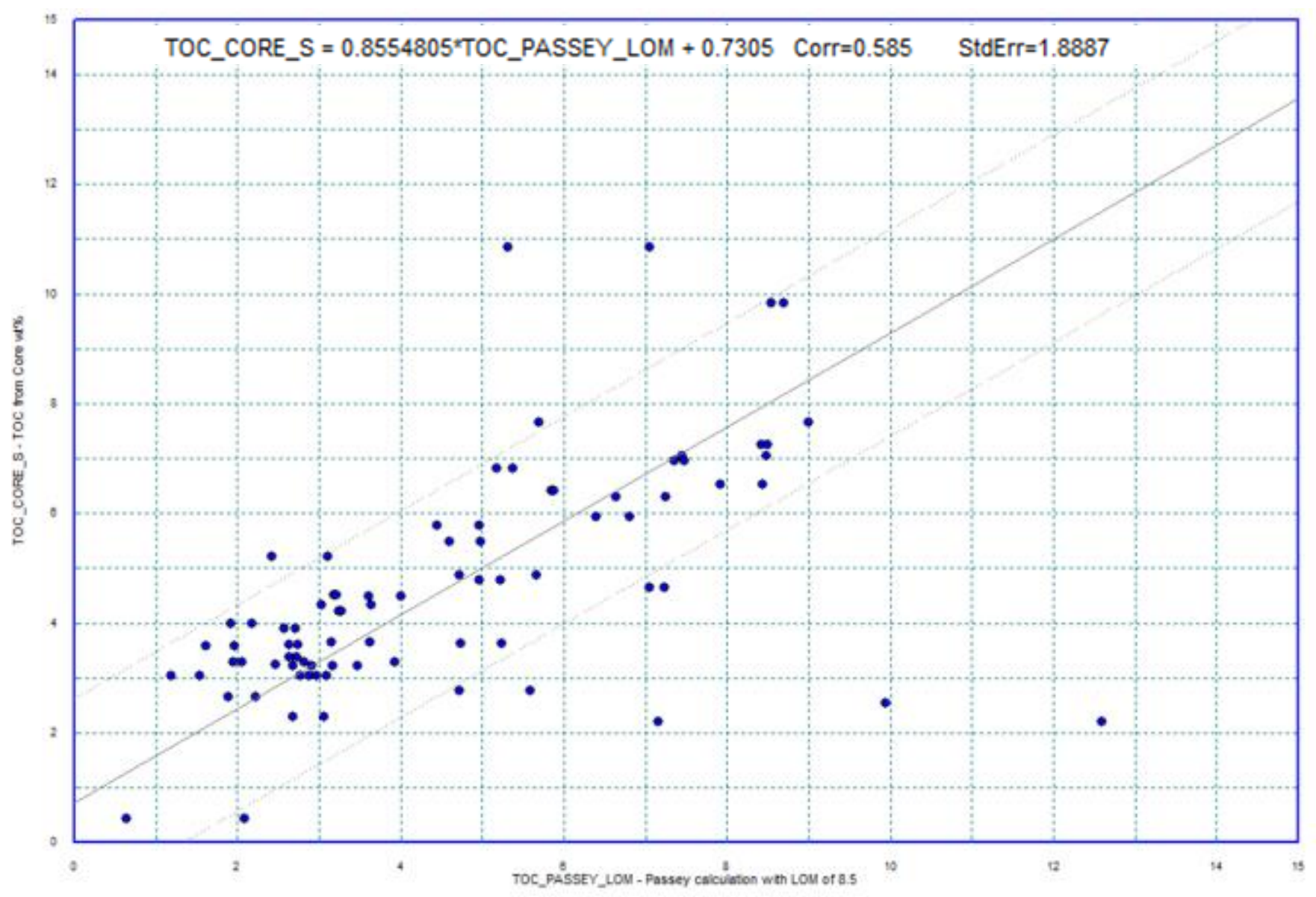

Figure 2-26: cross-plot of Passey's calculated TOC and Core TOC, the equation is the linear relationship between the two TOC measurements.

2-26).

Gamma ray and uranium derived correlations utilize the linear relationship between TOC and uranium via uranyl carbonate complexes, which occur in oxygen limited zones (Algeo \& Maynard, 2004). Figure 2-27, shows the linear relationship between core TOC and gamma ray and Figure 2-28 shows the linear relationship between uranium and core TOC. TOC correlations have $\mathrm{r}^{2}$ values great than 0.75 , TOC from gamma ray has a correlation of .77 and TOC from 
uranium has an $r^{2}$ of .81 . Both gamma ray and uranium derived TOC track the core derived TOC closely (Figure 2-28). Both slightly over predict TOC at the gamma ray peaks, however uranium derived TOC is closer to the core TOC values at these points ( $\pm 2 \mathrm{wt} \%)$.

All methods track the core derived TOC from the source rock analyzer and provide higher depth resolution while sacrificing some accuracy. However, given uncertainties due to core-log depth shifts and the differences in sample resolution ( 0.25 feet for logs and 3 feet for core) results are interpreted as good to excellent (Figure 2-29). 


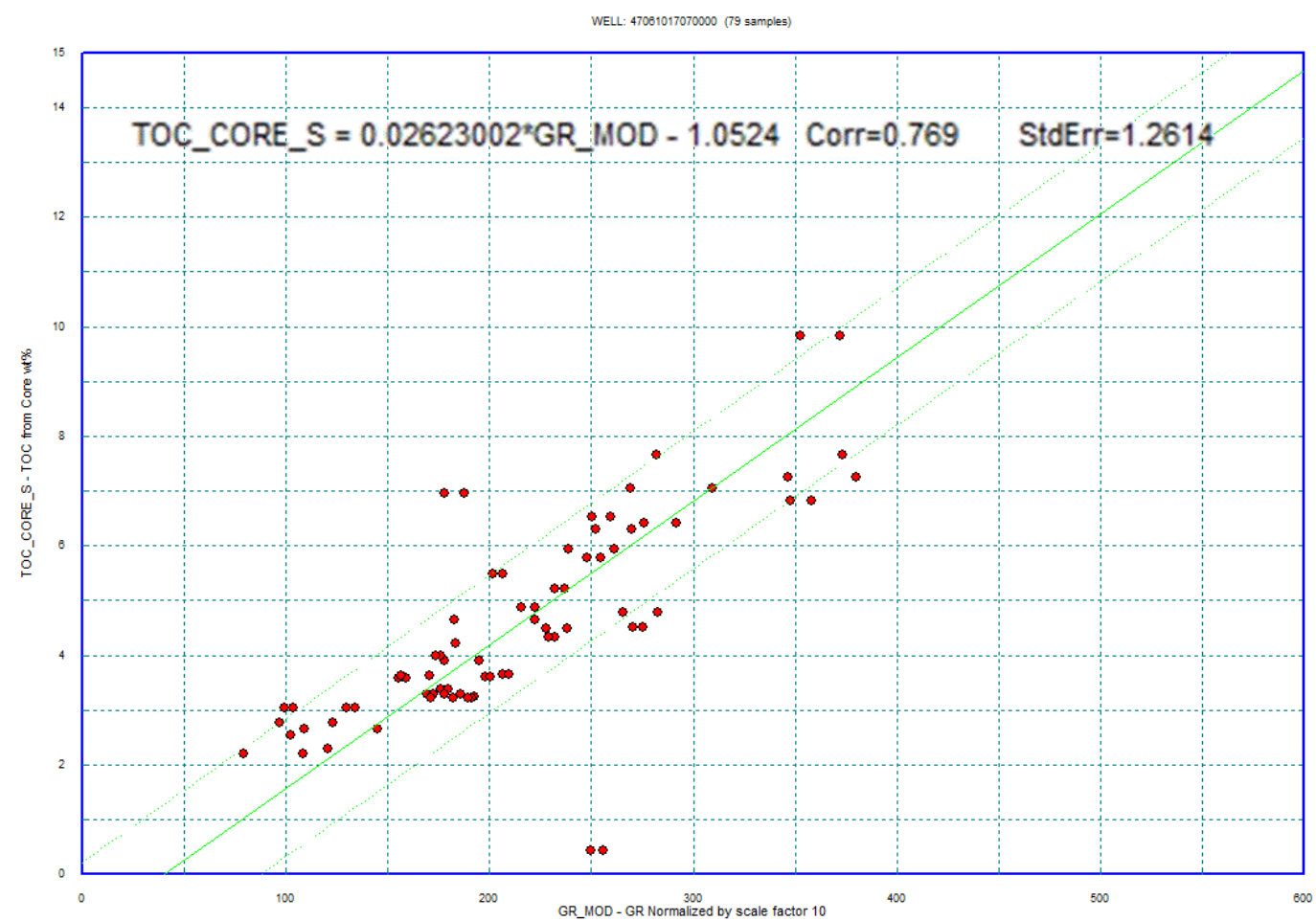

Figure 2-27: Crossplot of gamma ray and core TOC, the green line represents the linear fit for the equation at the top of the crossplot.

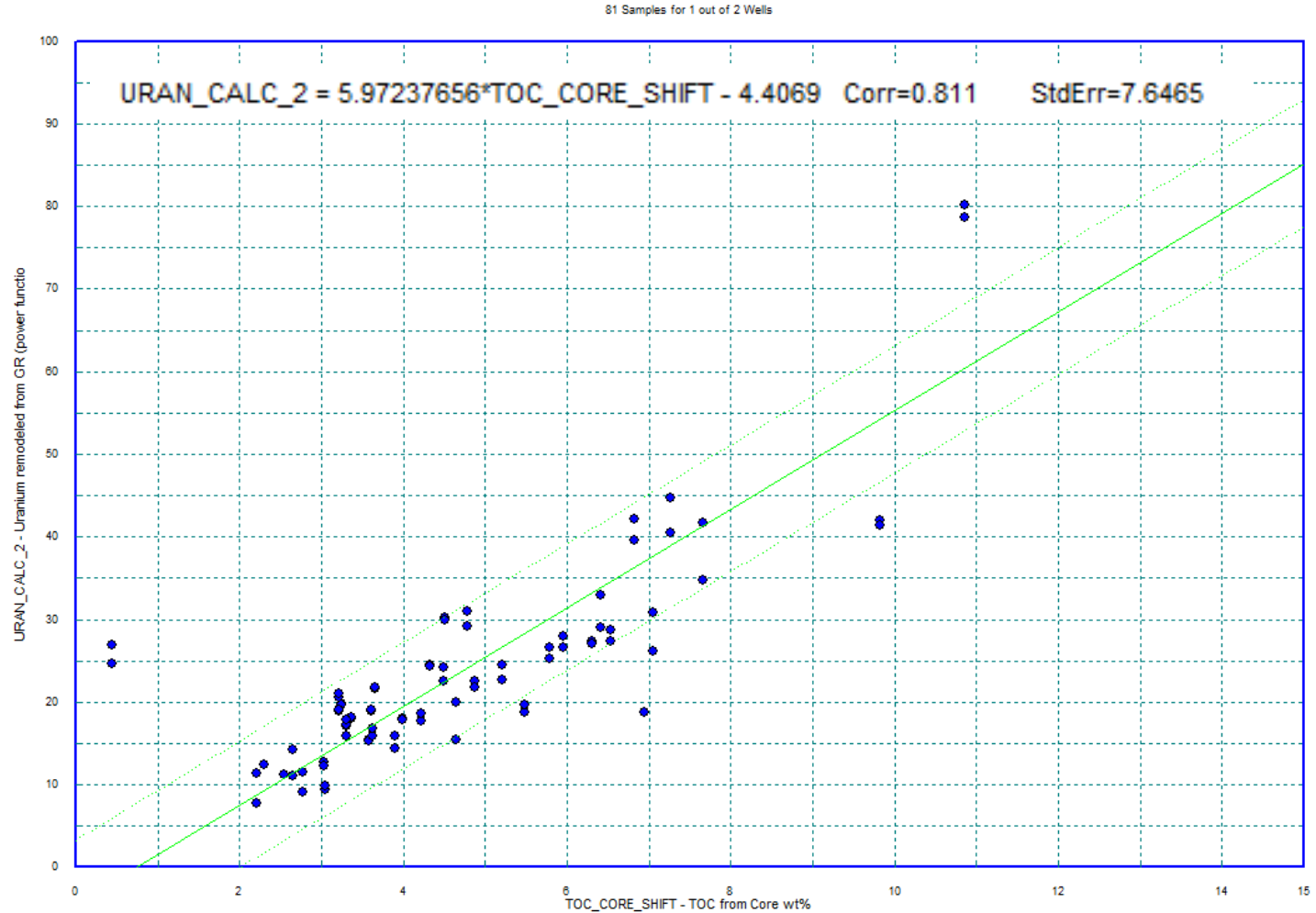

Figure 2-28: Crossplot of uranium and core TOC, the green line represents the linear fit for the equation at the top of the crossplot. 


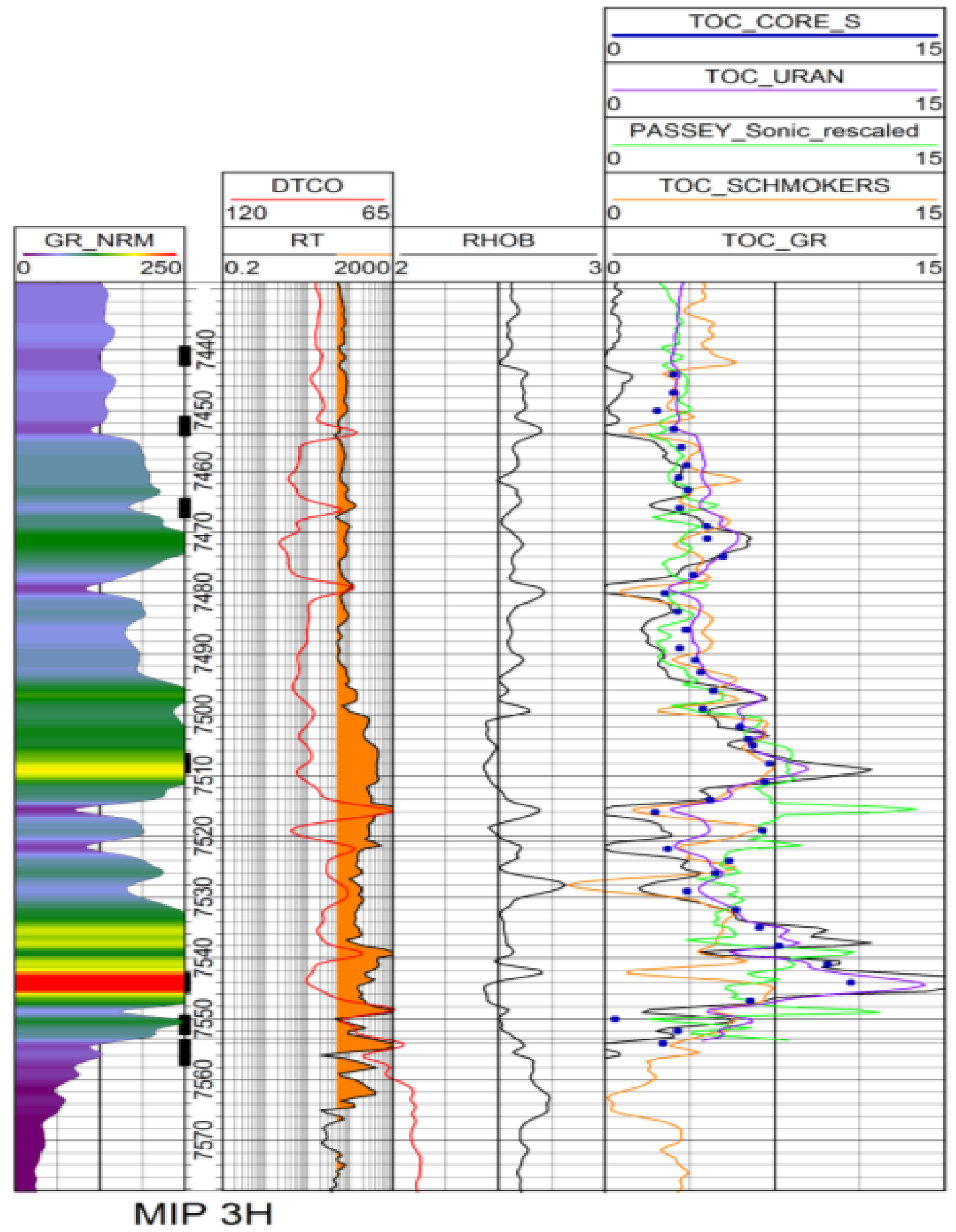

Figure 2-29: the log plot above shows the relationship between the Core TOC (blue dots) and log derived TOC's. Track 1Gamma Ray (0-250 API displayed, colored from 0-600 API); Track 2- DTCO (Compressional Sonic) in red ( $\mu \mathrm{s} / \mathrm{ft})$ and deep resistivity in black with orange in fill $>200 \mathrm{ohm}-\mathrm{m}$. Leftward separation between DTCO and RT represents the amount or organic matter present as determined by Passey's Method. Track 3- bulk density (grams/cc), Track 4- TOC determined by SRA from core shifted to log depth (blue dots) TOC_URAN (Uranium derived TOC) in purple, Passey's_Sonic_TOC is TOC derived using Passey's method in green, TOC_Schmoker's method is TOC derived from the modified Schmoker's equation in orange, and GR_TOC is TOC derived from gamma ray in black. 
Mineralogy:

Mineralogy was determined in this well using a combination of core and log derived techniques. Core derived techniques included semi-quantitative x-ray diffraction (XRD) and integration of XRF-XRD method to quantify the data (Hupp and Donovan, 2018). Additionally, a discrete multi-mineral model was developed to compare facies change between the MIP-3H and MIP-4H.

XRD:

Core derived mineralogy for this study was provided by Brittany Hupp. Numerous samples (55) were taken between $7455^{\prime}$ and 7556.2 at intervals of 1.7 feet on average. Samples were from whole-core using a horizontal side-wall mini corer providing 25 -mm-diameter side-wall plugs at 1.5 to $6 \mathrm{~cm}$ lengths. Samples were segmented to $\sim 1 \mathrm{~cm}$ and pulverized for 4 to 6 minutes using a Spex ${ }^{\odot}$ Model 5100 steel shatterbox, allowing for a $65 \%$ minimum of the grains to be smaller than $100 \mu \mathrm{m}$. The pulverized samples were then hydraulically press into Chemplex ${ }^{\mathrm{TM}}$ pellets for XRD and WDXRF (Hupp and Donovan, 2018).

XRD was run on the Chemplex pellets using a PANalytical X'Pert Pro ${ }^{\text {TM }}$ X-ray diffractometer with a $\mathrm{CuK}_{\alpha}$ source at $2 \theta$ angles from $5^{\circ}$ to $75^{\circ}$ at a step time of 12 degrees per second. X-rays were focused using $20 \mathrm{~mm}$ silt on an Xcellerator ${ }^{\mathrm{TM}}$ detector. Mineral phases were qualitatively identified using PDF2 reference library (ICDD, 2004) and the PANalytical X'pert HighScore

Plus $^{\circledR}$. Percentage breakouts were semi-quantitatively determined using reference intensity ratio (RIR) matrix flushing method (Chung, 1974a, 19754b) from the mineral phases chosen (albite, barite, calcite, dolomite, muscovite+illite, pyrite, quartz). RIR phases were consistent for all samples and no amorphous phases were identified aside from organic matter, allowing concentrations to be based on the total inorganic fraction of the sample (Hupp and Donovan, 2018). 
Following the RIR calculations, the XRD data taken for semi- quantitative to quantitative by using the stoichiometric relationships for each phase and the elemental oxides from the WDXRF (Hupp and Donovan, 2018). The XRF-XRD integrated mineralogy was used for this study (Appendix B).

The results of the XRF-XRD mineralogy limited the overall clay volume and more evenly distributed the smaller mineral fractions (chlorite, dolomite, albite, barite, pyrite by utilizing a cluster analysis to determine the fractionation of silicate and aluminum phases (Hupp and Donovan, 2018) (figure 2-30).

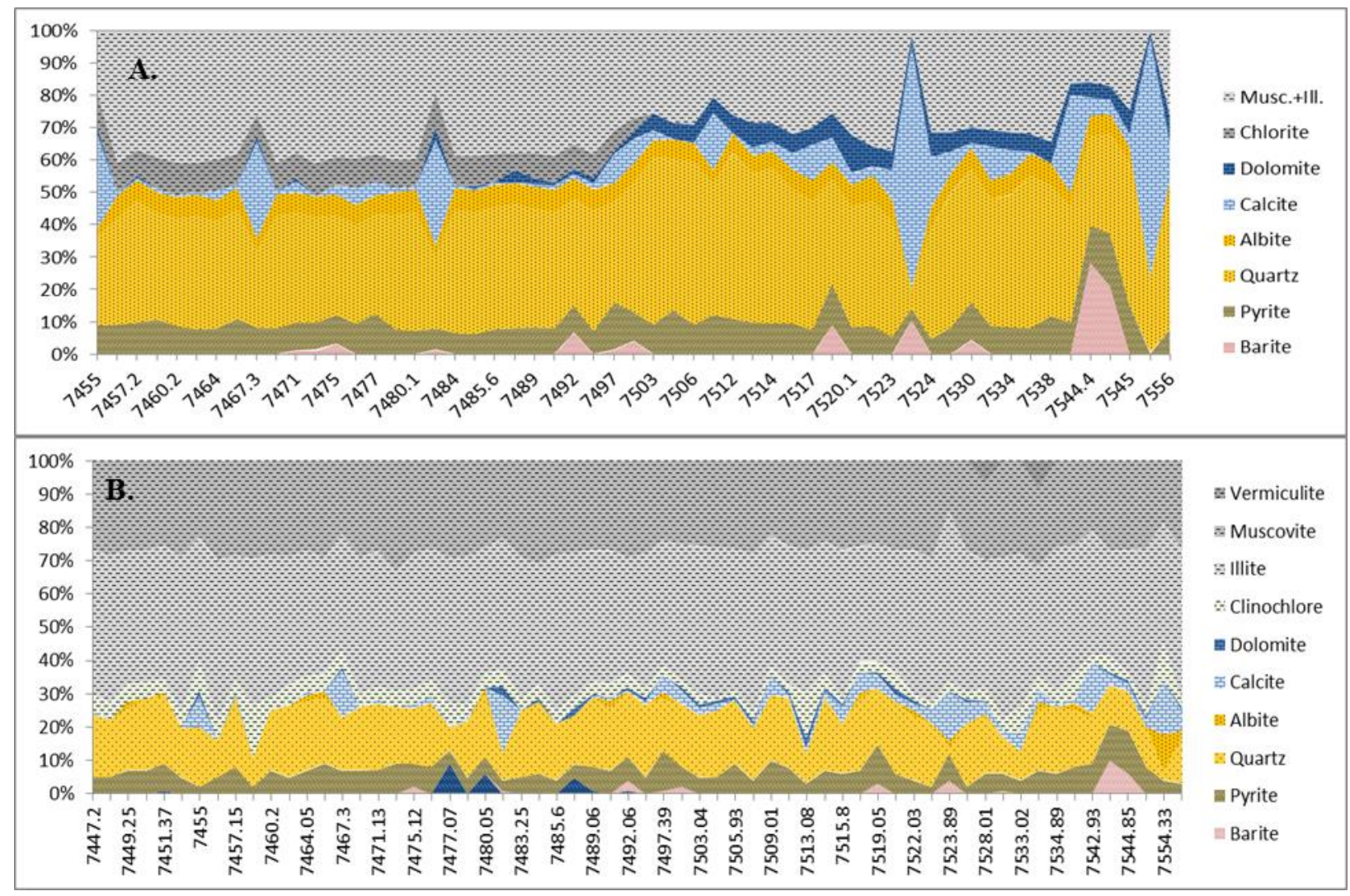

Figure 2-30: The figure above displays the mineralogy for the MIP-3H well. A. Represents the mineralogy from the RIR method. B. Represents the XRF-XRD mineralogy model, both from Hupp and Donovan, 2018. 


\section{Petrophysical Mineralogy:}

Petrophysical mineralogy for this study is generated through elemental capture spectroscopy (ECS) logs in MIP-4H and MIP-3H. Additional, we derived 3-mineral deterministic lithology solution and calibrated the model using the core-derived XRD data.

\section{ECS:}

Elemental capture spectroscopy utilizes an americium beryllium $(\mathrm{AmBe})$ neutron source with a bismuth germinate $(\mathrm{BGO})$ detector to measure the relative elemental proportions based on a spectrum of gamma-ray values. ECS tools capture elemental distributions for the formation, and deterministically modeled to derive a lithology solution (Hertzog et al., 1989). MIP-4H was equipped with Schlumberger's SpectroLith log. The SpectroLith log provides, elemental concentrations for $\mathrm{Si}, \mathrm{Al}, \mathrm{Ca}, \mathrm{Fe}, \mathrm{S}, \mathrm{Ti}$, and $\mathrm{Gd}$, which are used to derive total carbonate, total clay, anhydrite + gypsum $(\mathrm{S}$ and $\mathrm{Ca})$, quartz + feldspar + mica (QFM), pyrite, siderite (high deviations in Fe), Coal, and salt. The SpectroLith log has the sensitivity to resolve C, oxides, Na, $\mathrm{Mg}$, and Mn, which allows differentiation between clay types and carbonate type.

The MIP-3H well utilizes Schlumberger's Lithoscanner ECS logging suite. The Lithoscanner $\log$ suite provides Al, Ba, C, Ca, Cl, Cu, Fe, Gd, H, K, Mg, Mn, Na, Ni, O, S, Si, and Ti (Aboud et al., 2014). Due to the tools ability to resolve the lighter elements $(\mathrm{C}, \mathrm{Cl}, \mathrm{Mg}, \mathrm{Mn}, \mathrm{O}$, and $\mathrm{H})$ MIP-3H provided with mineralogy solutions for anhydrite, illite, chlorite, calcite, dolomite, pyrite, siderite, quartz, barite and kerogen. To keep consistent between wells, we consolidate these minerals into clays (illite, chlorite) and carbonate (calcite, dolomite).

The Lithoscanner mineralogy has moderate correlations with carbonate and clay XRD values $\left(\mathrm{r}^{2}\right.$ $=.56$ and .575 , respectively) and poor correlation with QFM. The ECS model has a more 
variable distribution of the mineral components compared to the XRF-XRD data. ECS quartz, in the MIP-3H well, is generally under predicted (20 to $60 \%$ ECS vs. 40 to $65 \%$ XRF-XRD) (Figure 2-31a, 2-31b). ECS clay generally decreases with depth reflecting core trend and trends from the XRD models. This can also be seen in the MIP-4H ECS mineralogy; however, it is less pronounced (Figure 2-31c). Carbonate content follows the trend of the XRD data, we see an overall increase with depth, however the peaks of calcite and magnitude of the carbonate content do not correlate well. The distribution of carbonate in the MIP-4H well not evenly distributed compared to the MIP-3H and in some instances calcite approaches $0 \%$. In the areas, where the carbonate cuts out there is an increase in siderite content. The SpectroLith ECS log bins carbonate minerals into siderite where the iron-content is excessively high. There was no siderite present, suggesting this is likely carbonate not siderite. Barite and pyrite distributions are similar between the ECS and XRD suites, peaks align and are generally between $0-15 \%$. Barite content over the zone from 7544' to 7545' is about half as much in the MIP-3H ECS logs as it is in the XRD-XRD model. Pyrite content in the MIP-4H well is generally lower than the MIP-3H well; however the distribution increases across the high gamma-ray peaks and transition intervals separating anoxic/euxinic zones from dysoxic zones (discussed in Chapter 3). 


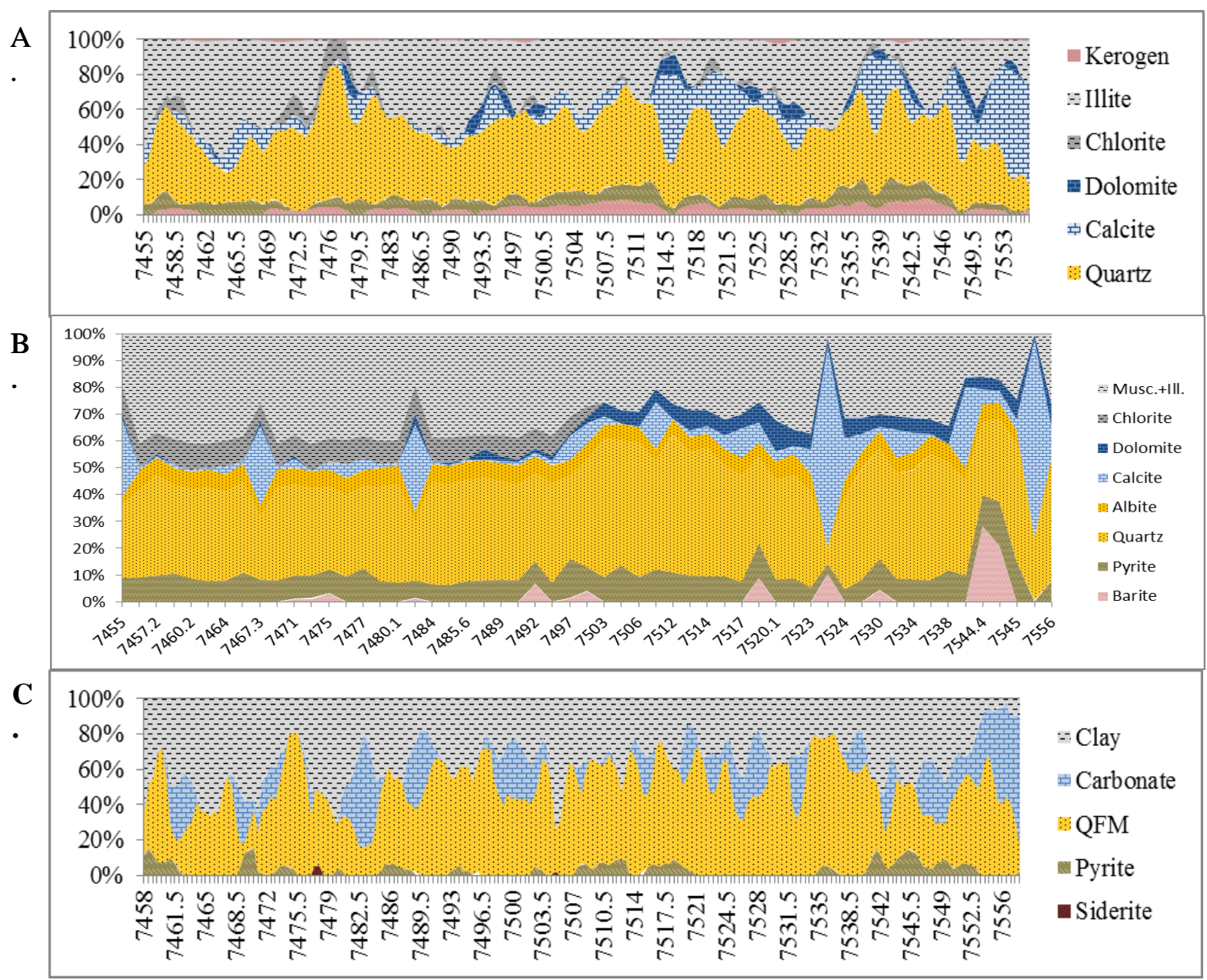

Figure 2-31: This figure displays the mineralogy from ECS logs in MIP-3H (A) and MIP-4H (C) compared to the XRFXRD derived mineralogy, from Hupp and Donovan (2018). Key is on right of figure: Clay mineral (gray), QFM (yellow), carbonate (blue), high density minerals (pyrite in gold; barite in pink, siderite in dark red).

\section{Modeled Mineralogy:}

Petrophysical analysis and log preparation:

Prior to the prediction of mineralogy, a full petrophysical analysis was applied to the MIP-3H and MIP-4H wells. Logs were first normalized between the MIP-3H and MIP-4H wells, starting with gamma-ray. This was accomplished using a stretch-and-pull method between the 'grayshale' average in the Mahantango and the average API across the Onondaga limestone:

(3) GR_NRM = GRMIN + (GRMAX - GRMIN) * (GR - GRLOW) / (GRHIGH - GRLOW $)$ 
Where, GRMIN and GRMAX are the values to normalized to, in this case MIP-3H, and

GRLOW and GRHIGH are the values corresponding to Min and Max in the well to be modified, MIP-4H. In the MIP-3H well GRMIN and GRMAX were determined to be 30 API and 140 API, respectively; and GRLOW and GRHIGH were determined to be 144 and 30 API in MIP-4H.

With gamma ray corrected, we can quickly compare distributions between wells and correct the logs for separation between one another by picking the max and min values for each well to normalize the well. Figure 2-32, shows an example of how this was undertaken for the neutron porosity $\log$.
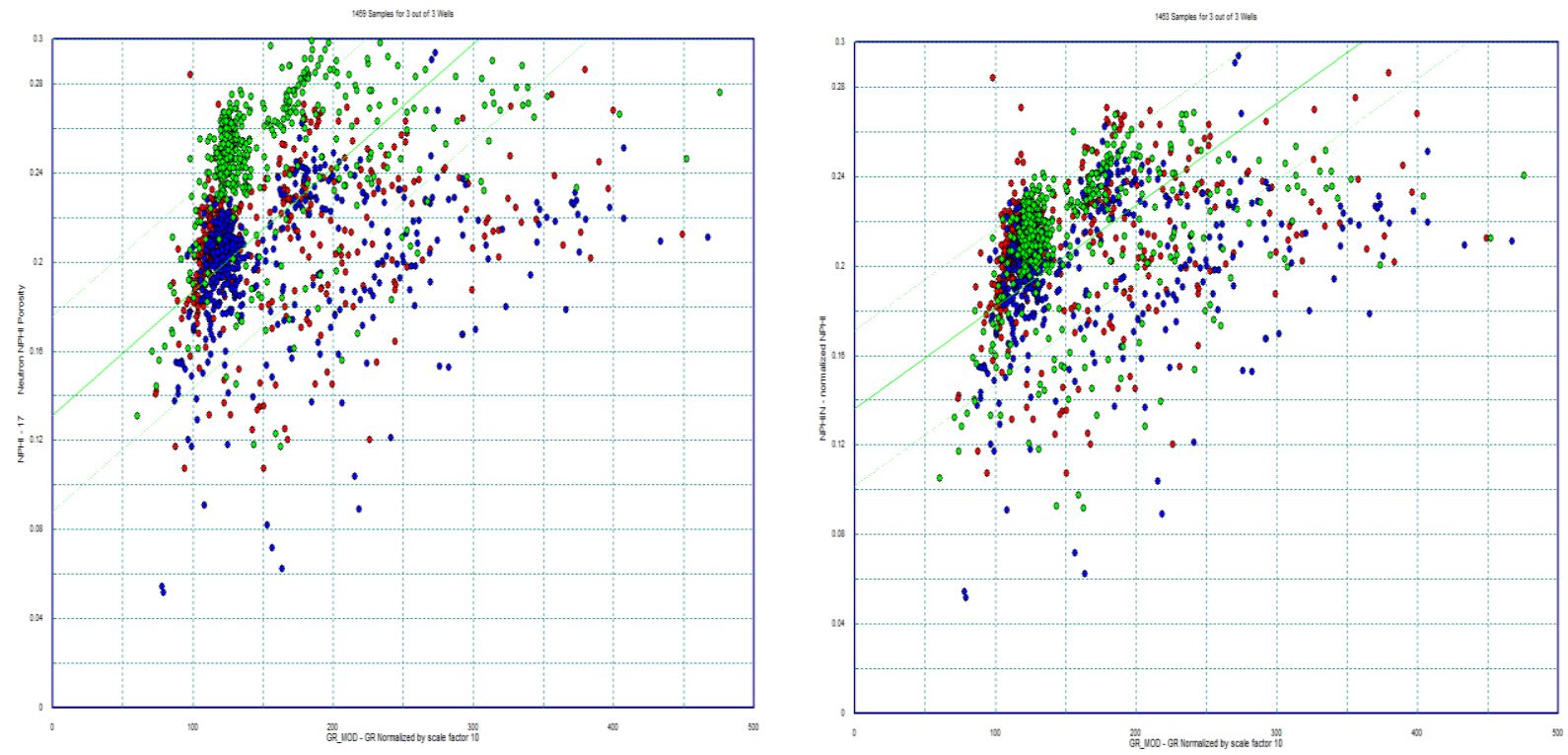

Figure 2-32: This figure shows the distribution of NPhi vs normalized Gamma Ray for MIP-3H (blue), MIP-4H (green) and MIP-SW (red). In the crossplot on the left we can see that NPhi in MIP-4H is higher overall with the same distribution with like GR values. The right crossplot show the results after normalization is complete.

Porosity corrections:

Following the normalization of the log suites, we corrected the neutron and density porosities for clay and kerogen volumes.

(4) DPHIc $=$ PHID $-\left(\right.$ Vsh $^{*}$ DphiSH $)-\left(\right.$ Vkero $^{*}$ DphiK $)$ 
(5) NPHIc $=$ PHIN $-\left(\right.$ Vsh ${ }^{*}$ NphiSH $)-\left(\right.$ Vkero ${ }^{*}$ NphiK $)$

Kerogen volume was calculated from uranium using a regional formula developed by Wang, 2012:

(6) $V K E R O U=-.00381+0.00350 * U R A N$

We used neutron porosity to calculate for volume clay and corrected using a series of crossplots for kerogen (volume clay neutron vs. kerogen from uranium) and fluids (volume clay neutron uranium corrected vs XRD clay (muscovite+illite and chlorite).

(7) $V C L N=\left(\frac{N P H I}{\text { Nclay }} * \frac{\text { Nphi-Nclean }}{\text { Nclay }- \text { Nclean }}\right)^{\cdot 5}$

(8) VCLN $=$ VCLNclay + VCLNkerogen + VCLNFluid

(9) $V C L N U=0.277-0.784 * V C L N-(V K E R O U-1.017)$

(10) $V C L N U F=1.10718^{*} V C L N U-0.2839$

Shear Moduli (or Mu) and Lame's first parameter (or lambda) are two elastic moduli, which represent rigidity and incompressibility, respectively. These equations derived using $\mathrm{p}$-and swave velocities and bulk density. Lame's parameters use these equations:

(11) Lambda ( $\lambda): \quad \lambda=\rho\left(V_{p}^{2}-2 V_{s}^{2}\right)$

(12) $\mathrm{Mu}(\mu): \quad \mu=\rho V_{s}^{2}$

Typically, these moduli derived from seismic data from p- and s-wave impedance. Therefore, throughout these plots are often referred to as Lambda-rho and Mu-rho crossplots. These elastic moduli when cross-plotted provide a multipurpose solution which can help us to determine brittle intervals, organic-rich intervals, correlate with Poisson's ratio and Young's Moduli and can be used to determine lithology (Alzate and Devegowda, 2013; Weicht, 2015). Lambda is more sensitive to pore fluids than the overall matrix and $\mathrm{Mu}$ is related to the matrix connectivity 
alone (Alzate, 2012). Lame's parameters are also directly related to Poisson's ratio and Young's moduli. The relationship between Poisson's ratio has a linear trend from the y-axis to the $x$-axis due to the influence by the where perfectly compressible $(v=0)$ objects have a lambda of 0 , and incompressible objects have lambda near infinity (Pollard and Fletcher, 2010). The Young's modulus relationship increases with increasing Mu values (Alzate, 2012; Weicht, 2015). These relationships classify intervals into different zone of TOC richness, brittleness, and mineralogy. Figure 2-33, shows the relationship between Lame's parameters and mineralogy with log data from the Geneseo Shale to the Huntersville Chert (Figure 2-33).

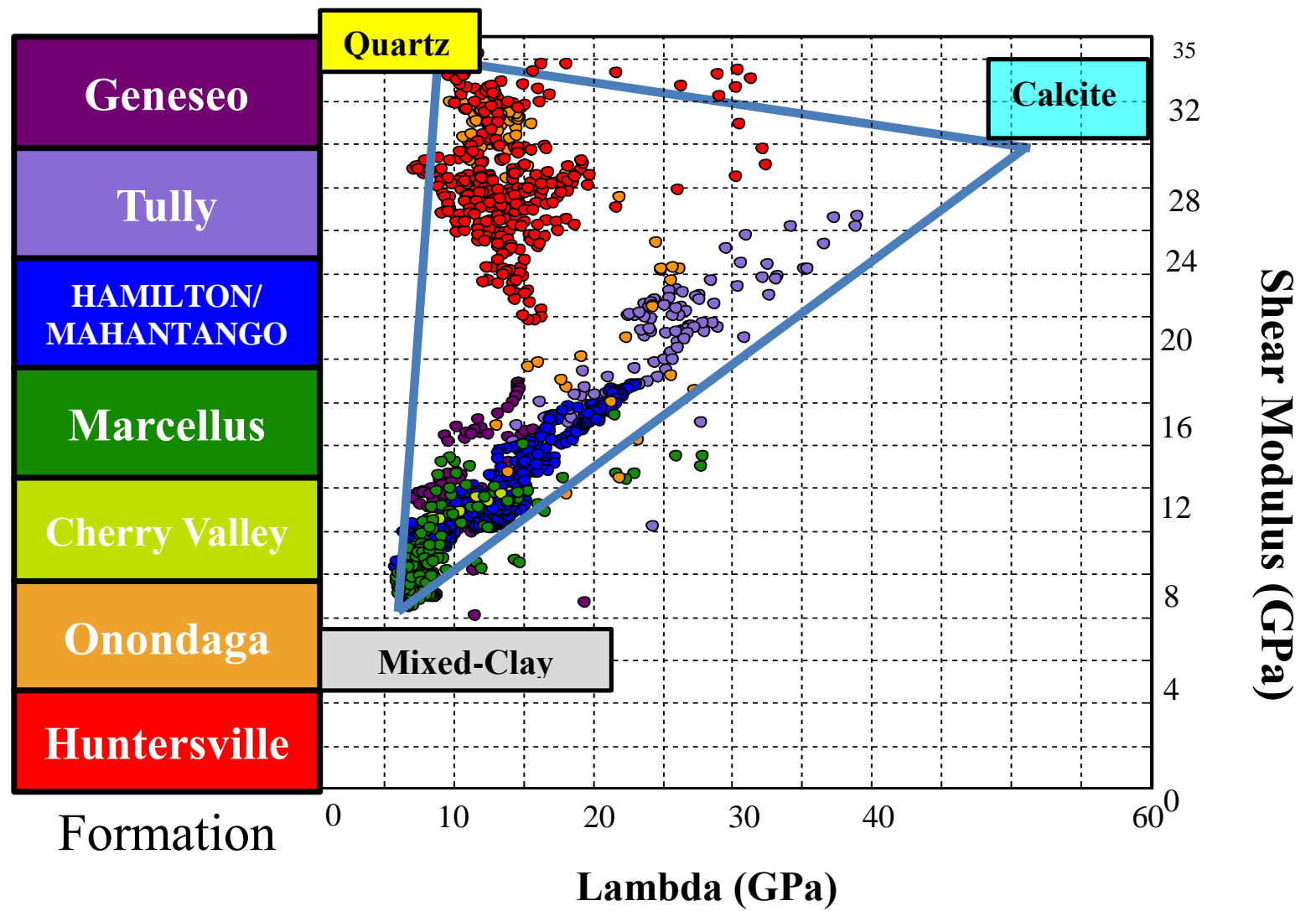

Figure 2-33: Lambda-Mu crossplot with mineralogy ternary plot overlay (from Perez-Altamar \& Marfurt, 2014). Formations for both MIP3H and MIP4H listed with their associated color on the left. The Marcellus in green in the formation of interest and is represented as a siliceous/argillaceous shale reservoir. 
Petrophysical Mineralogy Model:

Mineralogy was modeled using a reverse deterministic 3-mineral model (clay, quartz, and carbonate) for the MIP-3H and MIP-4H wells. This matrix algebra solution is determining the original mineralogy given log responses and a matrix of log properties (Doveton, 1994). Fitting the model to the core mineralogy is a time intensive process due to the fact the solution is relatively simple but over a dynamic, heterogeneous reservoir. The model calculated uses bulk density from corrected density porosity, corrected neutron porosity, lambda, and a unity function (Table 2-2). The photoelectric log, corrected to UMAA, was initially used. However due to degree of high density minerals present the solution could not produce an accurate solution without utilizing constants outside of geologic reality. Lambda, due to the high spatial separation between data points provides a strong correlation between the XRD and model.

\begin{tabular}{|c|c|c|c|c|}
\hline C: MIP-3H & Quartz & CALCITE & CLAY & POROSITY \\
\hline PHIN & -5 & 0 & 25 & 100 \\
\hline DENS & 2.65 & 2.71 & 2.85 & 1 \\
\hline Lambda & 5 & 28 & 8 & 0.01 \\
\hline UNITY & 1 & 1 & 1 & 1 \\
\hline C: MIP-4H & Quartz & CALCITE & CLAY & POROSITY \\
\hline PHIN & -5 & 0 & 25 & 100 \\
\hline DENS & 2.65 & 2.71 & 2.85 & 1 \\
\hline Lambda & 4 & 25 & 7.5 & 0.01 \\
\hline UNITY & 1 & 1 & 1 & 1 \\
\hline
\end{tabular}

Table 2-2: Log properties matrix for mineralogy solution, Top is from the MIP-3H solution and the bottom matrix is for the MIP-4H solution. Lambda values are shifted down 1 to 3 GPa.

Comparing the Mineralogy model with the XRD data we find a relatively close fit with moderate correlations for all minerals $(\mathrm{r}=.48)$. Ternary comparison between the ECS, XRF-XRD, and modeled mineralogy shows a relatively close match between the modeled and core-derived 
mineralogy (Figure 2-34). Similar to the XRF-XRD data, the modeled mineralogy has slightly lower clay content than the ECS log.

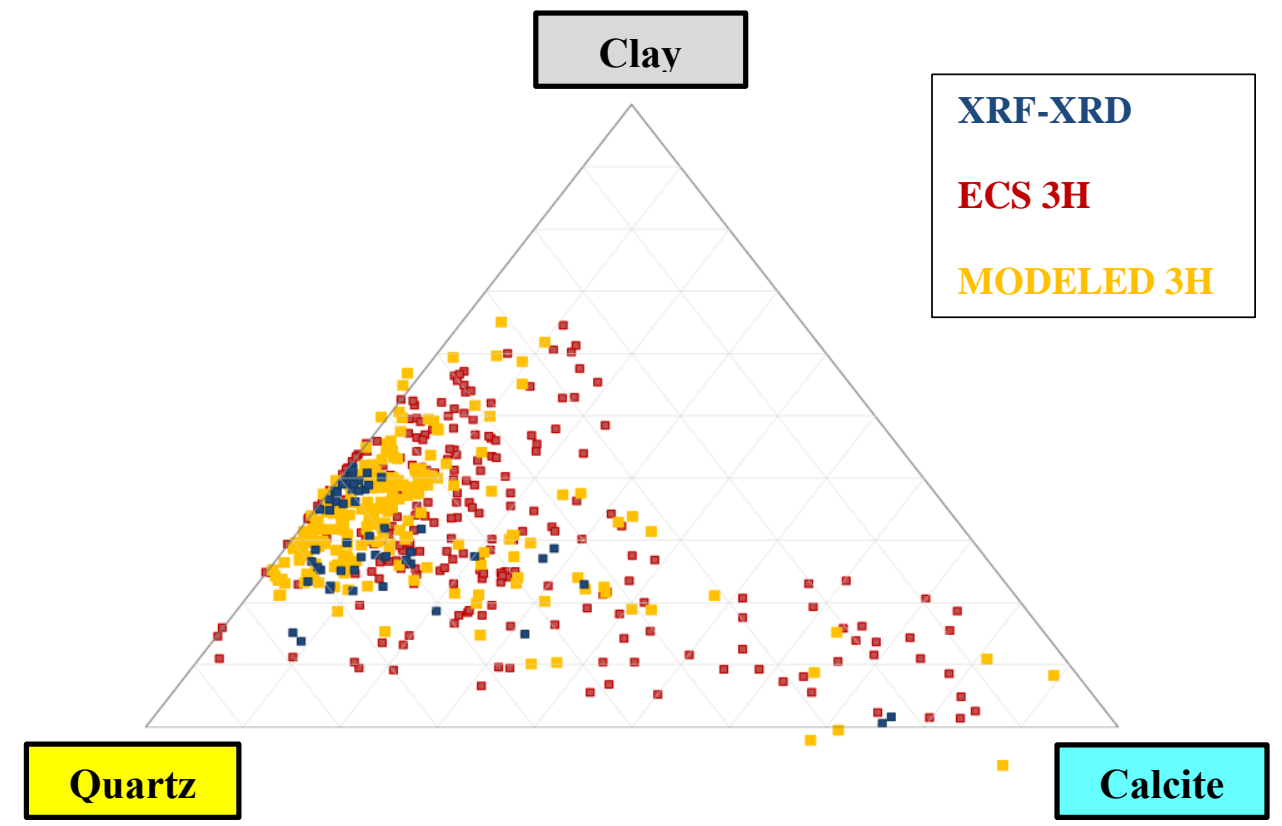

Figure 2-34: The comparison between the three mineralogy suites in the MIP-3H well: the XRF-XRD points (blue), ECS $\log$ (red), Modeled mineralogy (yellow).

\section{Meso-facies calculation:}

Mesofacies were calculated using distinct cut-offs modified from Bhattacharya and Carr, 2015 (Figure 2-35). To quickly calculate the facies cutoffs a PETRA Advanced transforms file was constuctred (Appendix C). Three sets of shale lithofacies were derived for the MIP-3H well (XRD, PNS, and Modeled) and two sets for the MIP-4H well (PNS and Modeled). Ternary plot displays show each facies set mineralogical distribution (Figure 2-36). Organic-rich facies (6.5 wt $\%$ cutoff) generally remains in facies below the $30 \%$ clay content and entirely so in facies with less than $40 \%$ clay content. Both the modeled and XRD lithofacies show generally more narrow distributions compared to the PNS logs. Organic-facies in the MIP-4H PNS model occur in mostly mixed facies with some siliceous organic facies present. Organic-facies in the 
modeled-mineralogy lithofacies solution occurs mostly in the siliceous-rich organic facies with some facies occuring within the mixed and clay-rich facies.

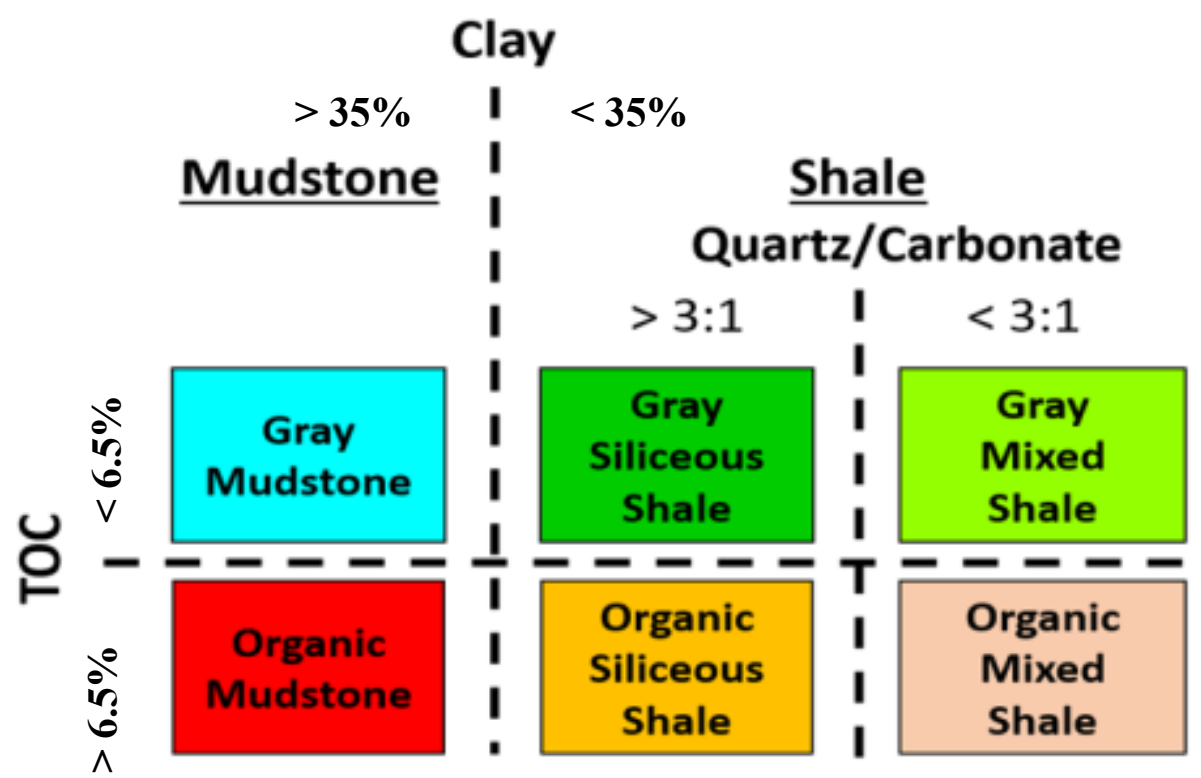

Figure 2-35: Key for macro-facies: warm colors are organic-rich facies $(>6.5 \%)$ and cool colors are organic-lean $(<6.5 \%)$ or 'gray' modified after Bhattacharya and Carr, 2016

Figure 2-37, shows a comparison between MIP-3h and MIP-4H wells, in both well tracts 2 and 3 are from the ECS logs and tracts 4 and 5 are from the modeled mineralogy. The discrete point data is form the XRF-XRD data. All models show that there is an increase in mudstone facies in the upper and upper portion of the middle Marcellus compared to the lower Marcellus. The high gamma peaks correlate with siliceous facies and organic facies are primarily siliceous. The only instance of the organic mudstone occurs in two one-foot facies from 7538 to 7542 in MIP-4H well. Low gamma values relate to mixed shale facies and approaches limestone (at 7515, MIP3H). The MIP-4H well facies are similar to the MIP-3H with a slight increase in the total clay and carbonate and slightly low TOC values, compared to the MIP-3H well. Due to the lower TOC values in the MIP-4H well the thickness of the modelled organic-rich facies is smaller. 


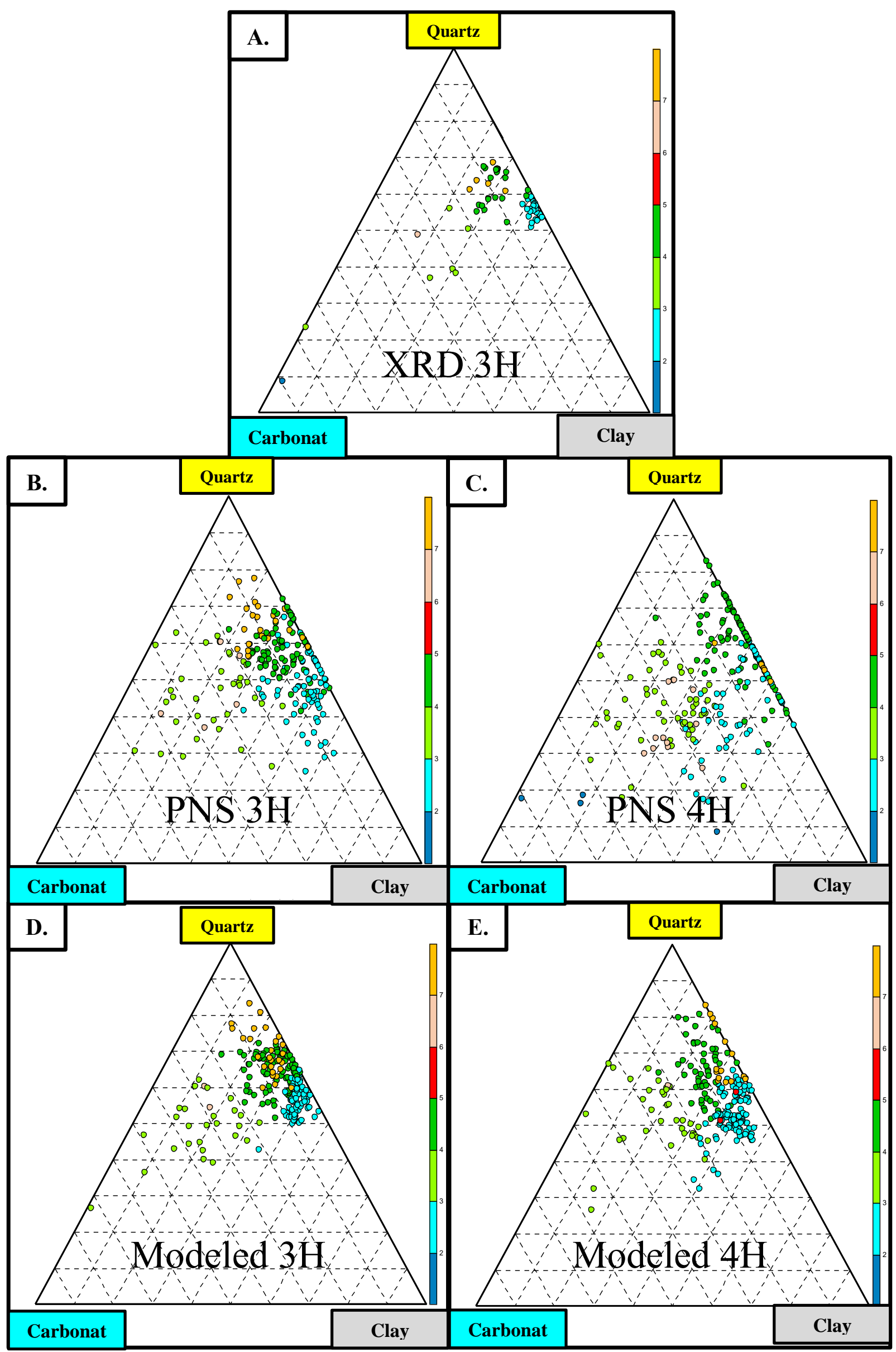

Figure 2-36: Quartz-Clay-Carbonate Ternary diagrams with z-axis showing Shale lithofacies for each mineralogy model: A. XRF-XRD, B. PNS MIP-3H, C. PNS MIP-4H, D. Modeled MIP-3H, E. Modeled MIP-4H 

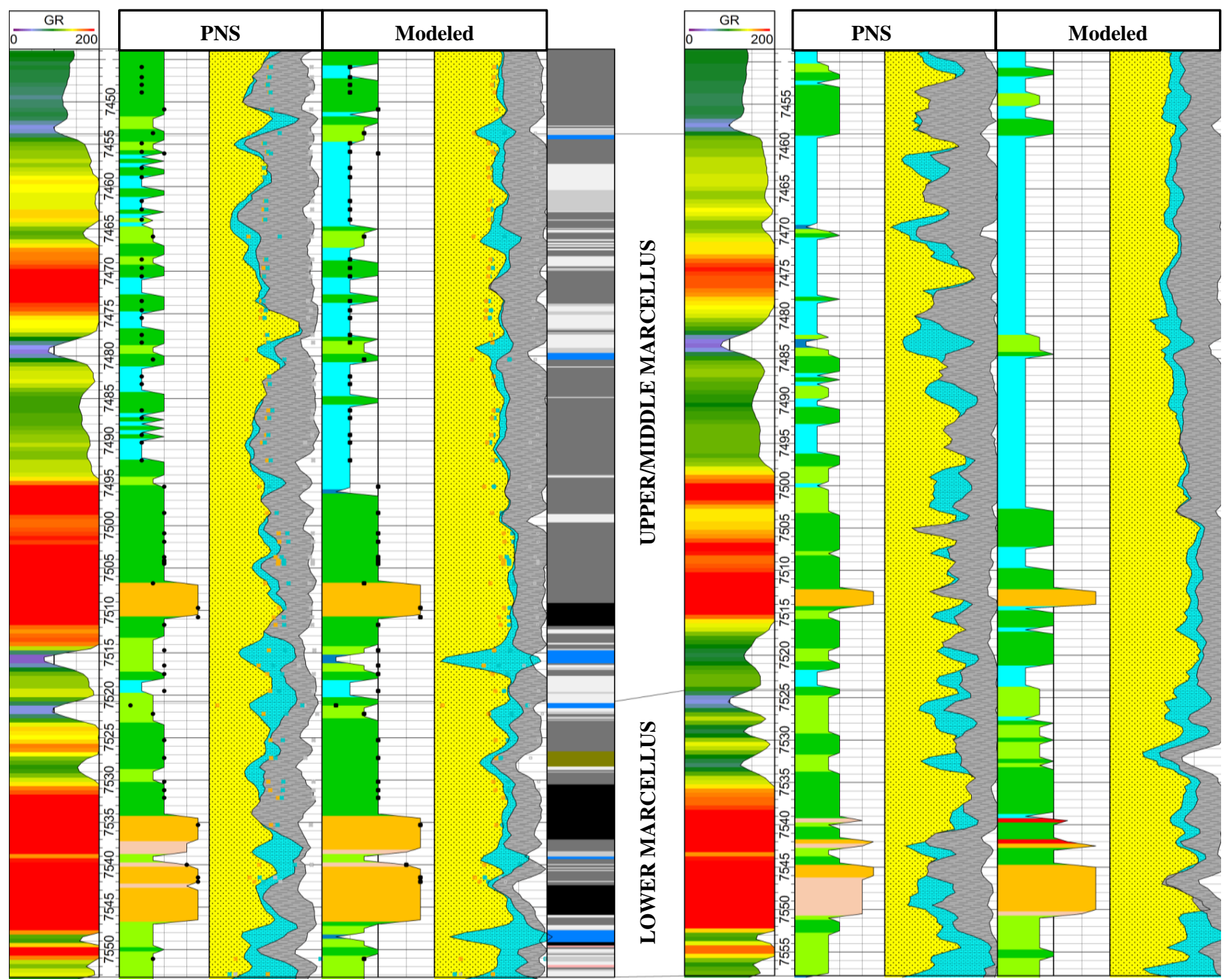

MIP $3 \mathrm{H}$

MIP $4 \mathrm{H}$

Figure 2-37: This cross-section between MIP-3H and MIP-4H compares the shale lithofacies models generated for each well. Tract 1: Gamma Ray (0-200 API with color display 0 - 300 API); Tract 2: lithofacies from PNS and TOC_URAN, discrete points shale lithofacies from XRD and TOC-URANIUM (MIP-3H only); Tract 3:PNS mineralogy, quartz (yellow), calcite (blue), clays (gray), discrete points XRD mineralogy, quartz (yellow), calcite (blue), clays (gray); Tract 4: lithofacies from Modeled mineralogy and TOC_URAN, discrete points shale lithofacies from XRD and TOC-URANIUM (MIP-3H only), Tract 5: modeled mineralogy, quartz (yellow), calcite (blue), Clays (gray), discrete points XRD mineralogy, quartz (yellow), calcite (blue), clays (gray);, Tract 6:Macro-facies (core scale facies) key in Figure 2-2. 


\section{CHAPTER 3, CHEMOSTRATIGRAPHY}

\subsection{X-ray Fluorescence (XRF):}

XRF measures elemental proportions in a sample by measuring the energy return after an atom is disrupted by a source X-ray proton. Four different XRF 'suites' were used in analysis of the core from the MIP-3H well to determine the elemental concentration of a larger number of elements and to confirm the reliability of each suite when compared to other methods of analysis.

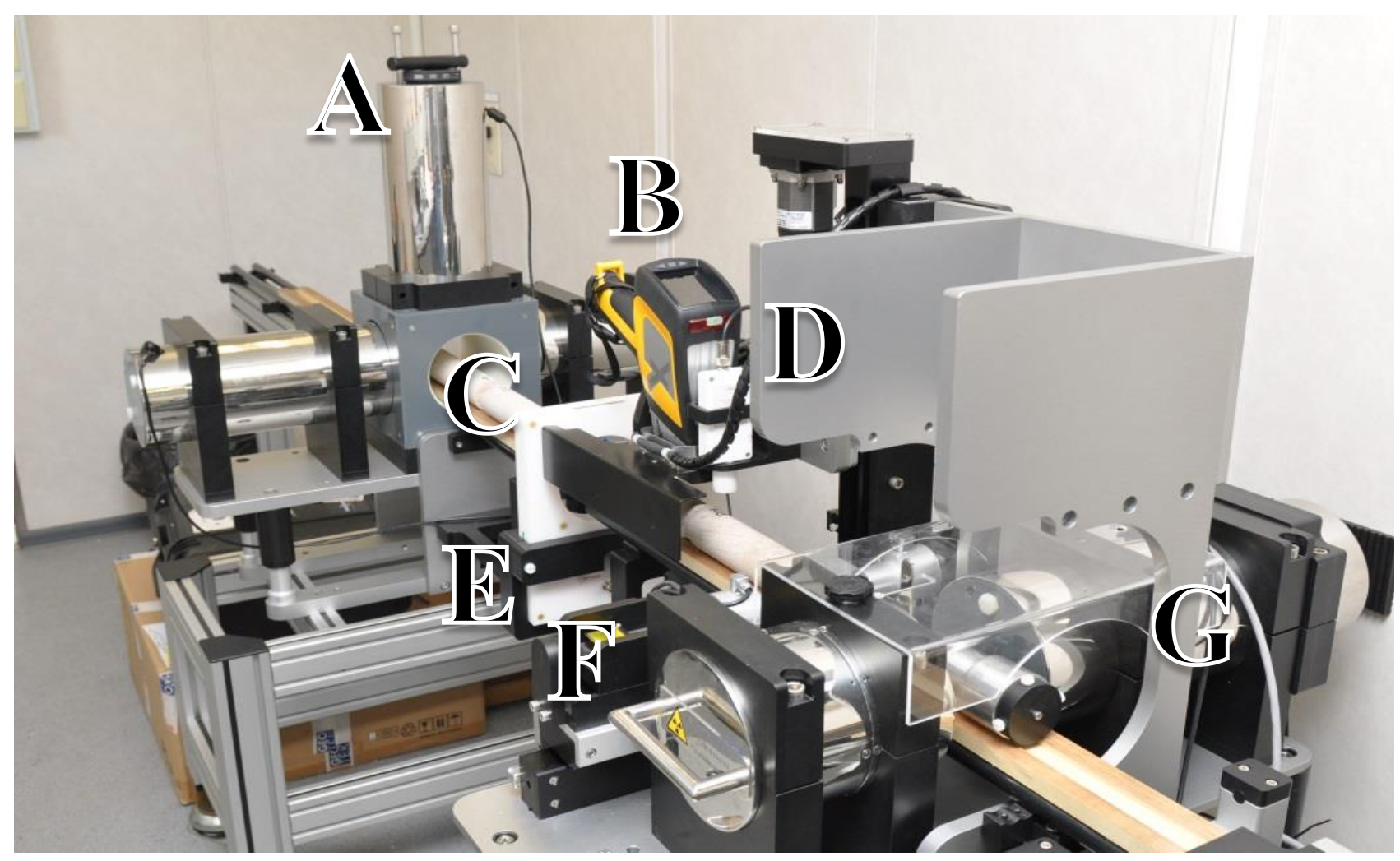

Figure 3-1: the Multi Sensor Core Logger (MSCL) allows us to continuously run petrophysical measurements on whole core: (A) natural gamma detector; (B) X-Ray Fluorescence Spectrometry; (C) Magnetic Susceptibility Loop Sensor; (D) Magnetic Susceptibility Point Sensor; (E) Non-Contacting Electrical Resistivity Sensor; (F) P-Wave Velocity Transducers, (G) Gamma Density Source.

The Multi Sensor Core Logger (MSCL) measures petrophysical parameters on whole core (Figure 3-1). For this study, the MSCL analyzed X-Ray fluorescence (XRF), magnetic susceptibility, p-wave velocity, and gamma ray density. The MSCL provides continuous 
measurements at an end user provided interval to allow for unbiased sampling. We ran the $1 / 3^{\text {rd }}$ core piece from the MIP-3H well through the MSCL using a sample increment of $2 \mathrm{~cm}$. The average length of each core piece was 3 feet $(0.9144 \mathrm{~m})$ which required a total of 41 core boats to sample the entire core $\left(7445^{\prime}\right.$ to $\left.7557^{\prime}\right)$. Three suites of XRF samples were obtained using the Multi Sensor Core Logger at NETL include a Mining and Mining Suite Plus at a longer exposure time for a limited portion of the core. These MSCL suites were subsequently compared to a limited number of samples run by an outside lab using traditional XRF techniques (Figure 3.2). Appendix D, includes data for all XRF Suites.

The Mining Suite measures the light elements $(\mathrm{Mg}$ to $\mathrm{Pb})$. We used an exposure time of 20 seconds at $2 \mathrm{~cm}$ resolution through the whole core length (7444 to $\left.7555^{\prime}\right)$. The Soil Suite best measures some major elements $(\mathrm{K}, \mathrm{Ca}, \mathrm{S}, \mathrm{P}, \mathrm{Cl}, \mathrm{Ti}, \mathrm{Cr}, \mathrm{Mn}, \mathrm{Fe}, \mathrm{Ni}, \mathrm{Cu})$ and trace elements $(\mathrm{Zn}$, $\mathrm{Hg}$, As, Pb, Bi, Se, Rb, U, Sr, Zr, Mo, Ag, Cd, Sn, Sb, and Ba). Again, an exposure time of 20 seconds at $2 \mathrm{~cm}$ resolution for the entire length of the core was used (7444' to $\left.7555^{\prime}\right)$. The Soil Suite utilizes a dual beam at two energy levels to better resolve the trace elements and major elements separately giving a total exposure time of 40 seconds. The Mining-Plus Suite works in a similar fashion to the Soil Suite, the tool measures the heavy and transition metals, then changes to a high energy to measure the major elements. It was determined following the analysis of the Soil Suite samples that the short exposure time significantly limited the accuracy of the trace element determination. Therefore, the exposure time of the Mining-Plus Suite was increased to 60 secs per beam, while keeping the $2 \mathrm{~cm}$ resolution. Due to time constraints, we limited our analysis to 38 feet through the lower Marcellus in the MIP3H core (7517 to 7555'). The lower Marcellus was selected due to the heterogeneity of facies that are represented and the rapid changes in TOC through this interval. 


\section{MSCL Mining Suite}

- $20 \mathrm{sec}$ exposure time

- $2 \mathrm{~cm}$ resolution (7444 to $7555^{\prime}$ )

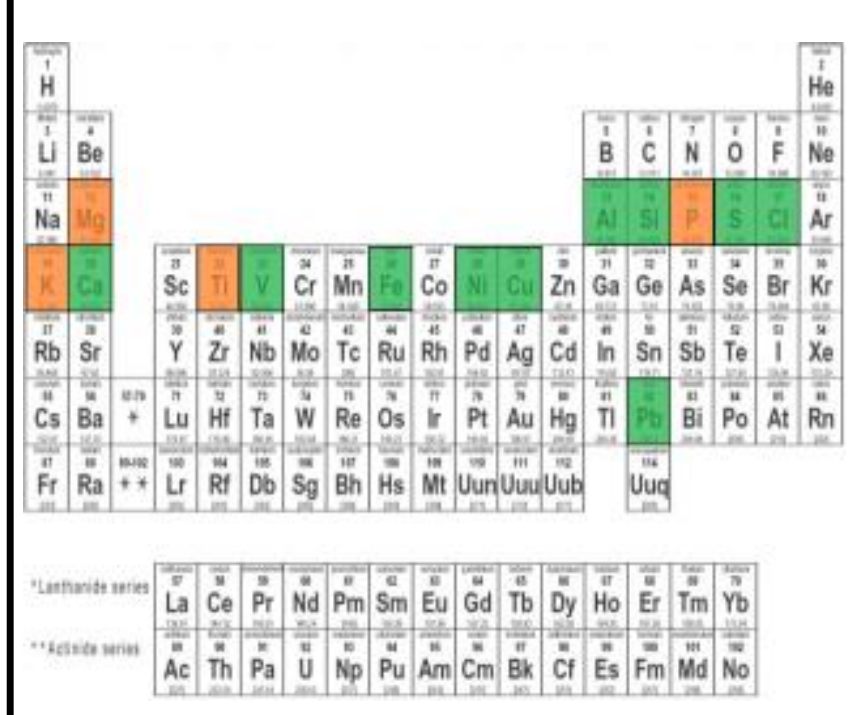

\section{MSCL Mining-Plus Suite}

- $120 \mathrm{sec}$ exposure time

- (Dual Beam 60 per beam)

- 2 cm resolution (7515 to $7555^{\prime}$ )
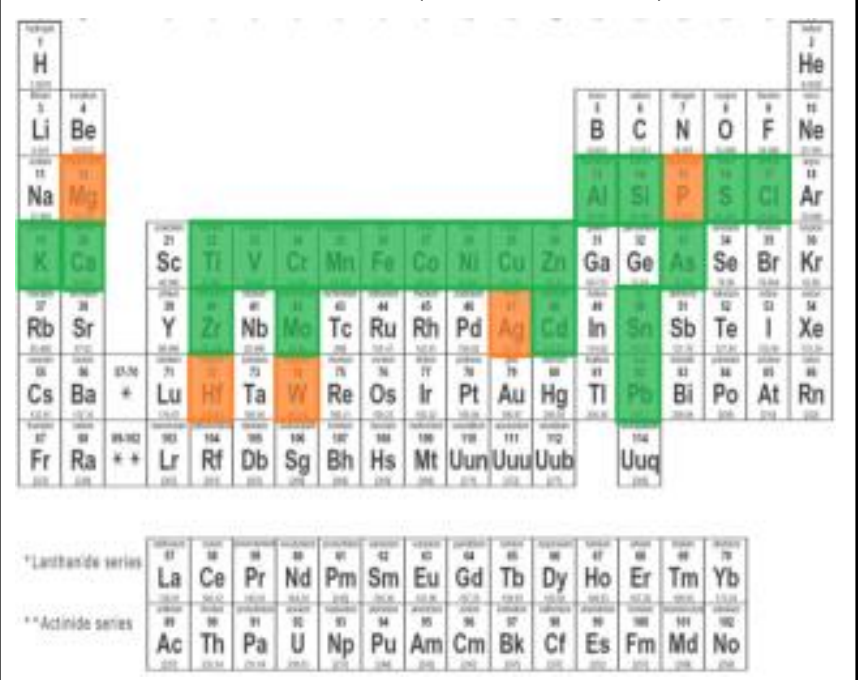

\section{MSCL Soil Suite}

- $40 \mathrm{sec}$ exposure time

- (Dual Beam 20 per beam)

- $2 \mathrm{~cm}$ resolution (7444 to 7555 ')

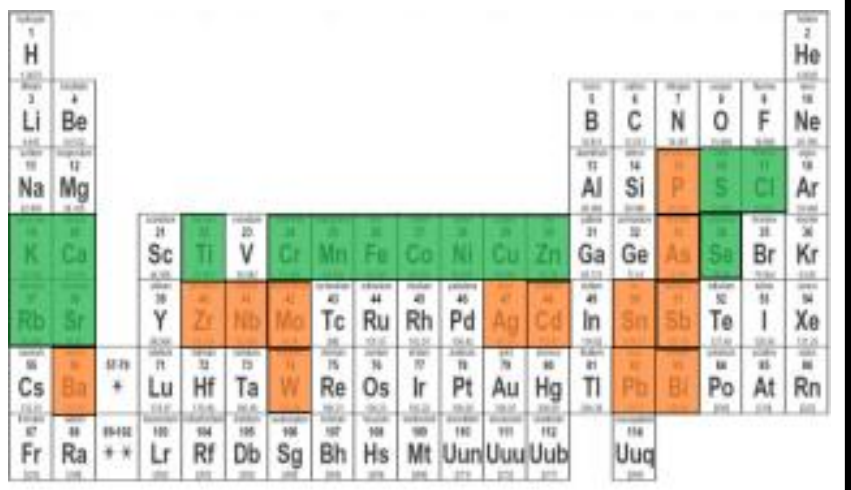

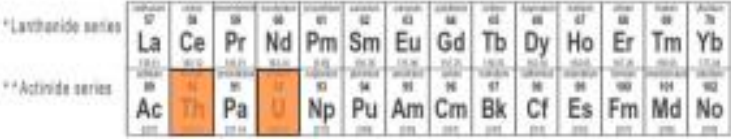

\section{Hamilton Suite}

- Loss on Ignition at $900^{\circ} \mathrm{C}$

- Higher precision but lower resolution.

- $1-3 \mathrm{ft}$ resolution (7444 to $7555^{\prime}$ )

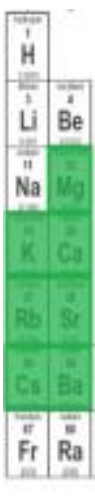

$$
\text { in }
$$

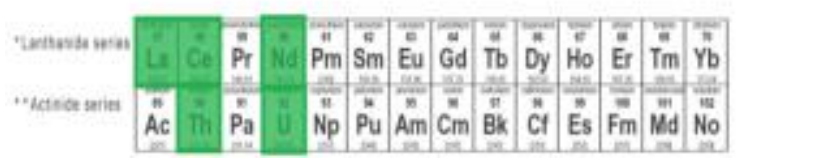

Figure 3-2: Four suites of XRF data obtained from the MIP 3H well using the Multi Sensor Core Logger (MSCL) and traditional XRF analysis showing elements that are identified with each suite are highlighted. Green represents elements quantified with lower errors and orange represents elements with higher error. Parameters of each suite are outlined. 
Finally, a limited set of powdered samples was run using traditional methods by Hamilton University using a Thermo ARL Preform'X spectrometer, about 2 hours of exposure time (Hupp 2017). The Hamilton Suite includes major elements, transition metals, and trace elements. The long exposure time allows for high accuracy and precision but given expenses and available samples limited vertical resolution and possible introduction of sampling biases (Hupp 2017).

Figure 3-3, shows the Hamilton measured data verse the laboratory standards all displayed results have an $r^{2}$ of 0.99 . Therefore, the Hamilton XRF provides a method to evaluate the reliability of the XRF data obtained from the Multi Sensor Core Logger (MSCL).

\section{Hamilton Suite vs Standards}

BCR-2

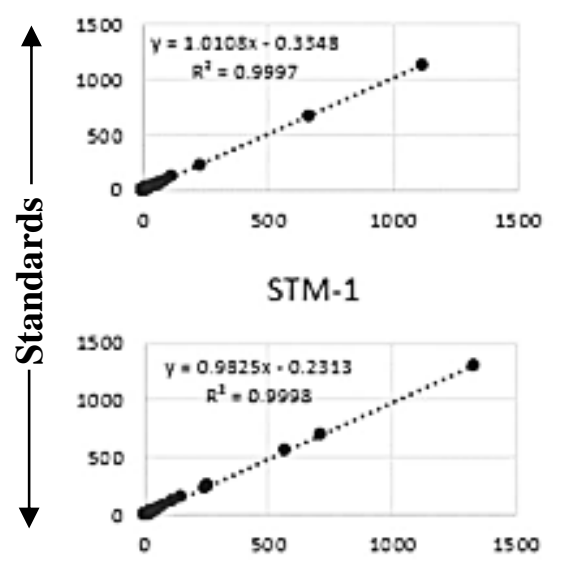

AGV-2

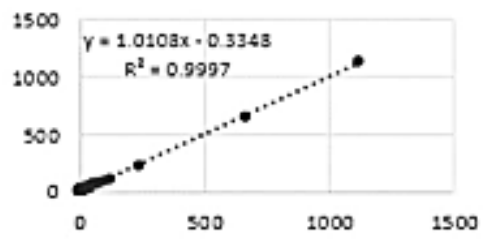

W-2

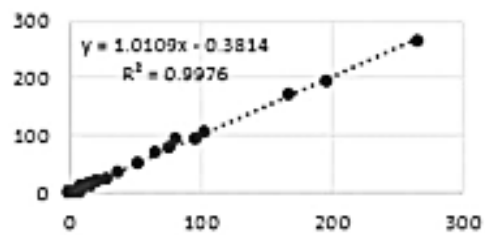

Hamilton Suite
G-2
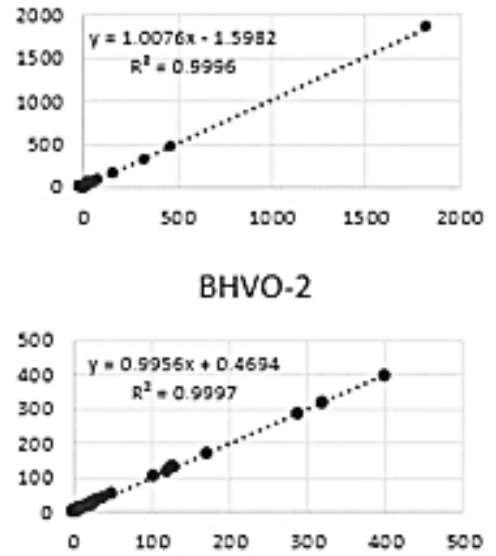

$\quad 200 \quad 300 \quad 400 \quad 500$

Figure 3-3: The six crossplots above represent the standards ran in the Hamilton Suite. Hamilton values plotted along the $x$-axis and the standards values are measured along the $y$-axis. All elements are displayed in the $x$-plots above.

To evaluate the reliability of the MSCL data, we converted the oxides in the Hamilton Suite to their elemental state and compared all like elements between each MSCL suite (Figure 3-4). The mining-plus suite compared to the Hamilton shows a strong correlation with the major elements $(\mathrm{Si}, \mathrm{Al}, \mathrm{Ca}, \mathrm{Fe})$ and some of the trace elements $(\mathrm{Ni}, \mathrm{Mo}, \mathrm{Zn}, \mathrm{Cu})$. The correlations between 
Hamilton and Mining-plus were poor for phosphorous, magnesium, potassium, sulfur and titanium. The poor correlation for sulfur is due to loss on fusion of the powdered samples in the Hamilton suites (Hupp and Donovan, 2018). The other low correlations are likely due to limits of detection for the Mining-plus suite. The Soil and Mining suites both have generally lower correlation coefficients overall. The Soil Suite has moderate correlations between some major and transition elements $(\mathrm{Fe}, \mathrm{K}, \mathrm{Ni}$, and $\mathrm{Ti})$. The Mining Suite has low correlations between all elements. These lower correlations in the mining and Soil suites are likely a result of lower runtimes to resolve the element proportions.

Figures 3-5 to 3-8 show correlations between elements within each XRF suite. This allows a better understanding of the general relationships and functions of each element and what proxies can be used to understand the paleo-environment. We find strong positive correlations between $\mathrm{Si}, \mathrm{Cr}, \mathrm{K}, \mathrm{Ti}, \mathrm{Al}, \mathrm{Hf}, \mathrm{Ti}$ and $\mathrm{Zr}$ and a strong negative correlation between these element and $\mathrm{Ca}$. These elements all have strong affinity for silicates and are strongly related to sedimentation trends (Sagemen 2003). We also find strong to moderate positive correlations between $\mathrm{Mo}, \mathrm{Fe}$, $\mathrm{Cu}, \mathrm{Ni}, \mathrm{Pb}, \mathrm{S}, \mathrm{Zn}, \mathrm{As}$, and $\mathrm{V}$; and a strong to moderate negative correlation to Mn. These elements generally have a strong affinity for sulfides and/or native iron, as such, these elements follow redox trends. These correlation plots also show a problem with the hhXRF tool, specifically in the Mining and Mining-plus suites where elements with high errors (e.g. Ti, V) can show a false correlation. Generally, a strong correlation between vanadium and titanium would occur among mafic-rich rocks (Shervais, 1982). This doesn't match the depositional environment and does not match the correlation coefficient in the Hamilton suite. 


\begin{tabular}{|c|c|}
\hline Mining-Plus vs Hamilton & Correlation \\
\hline $\mathrm{Al}$ & 0.93 \\
\hline As & 0.78 \\
\hline $\mathrm{Ca}$ & 0.99 \\
\hline $\mathrm{Cl}$ & 0.29 \\
\hline $\mathrm{Cr}$ & 0.72 \\
\hline $\mathrm{Cu}$ & 0.94 \\
\hline $\mathrm{Fe}$ & 0.64 \\
\hline $\mathrm{Hf}$ & 0.30 \\
\hline $\mathrm{K}$ & 0.38 \\
\hline $\mathrm{Mg}$ & nd \\
\hline $\mathrm{Mn}$ & 0.26 \\
\hline Mo & 0.98 \\
\hline $\mathrm{Ni}$ & 0.95 \\
\hline $\mathrm{Pb}$ & 0.41 \\
\hline $\mathrm{P}$ & 0.15 \\
\hline $\mathrm{Si}$ & 0.80 \\
\hline $\mathrm{S}$ & -0.01 \\
\hline $\mathrm{Ti}$ & -0.37 \\
\hline $\mathrm{V}$ & 0.11 \\
\hline $\mathrm{Zn}$ & 0.96 \\
\hline $\mathrm{Zr}$ & -0.03 \\
\hline Soil vs. Hamilton & Correlation \\
\hline As & 0.38 \\
\hline $\mathrm{Ba}$ & -0.06 \\
\hline $\mathrm{Ca}$ & 0.25 \\
\hline $\mathrm{Cl}$ & 0.09 \\
\hline $\mathrm{Cr}$ & -0.03 \\
\hline $\mathrm{Cu}$ & 0.10 \\
\hline $\mathrm{Fe}$ & 0.66 \\
\hline $\mathrm{K}$ & 0.67 \\
\hline $\mathrm{Mn}$ & 0.59 \\
\hline Mo & -0.12 \\
\hline $\mathrm{Nb}$ & -0.31 \\
\hline $\mathrm{Ni}$ & 0.60 \\
\hline $\mathrm{Pb}$ & 0.21 \\
\hline $\mathrm{P}$ & -0.17 \\
\hline $\mathrm{Rb}$ & -0.35 \\
\hline $\mathrm{S}$ & -0.01 \\
\hline $\mathrm{Sr}$ & 0.05 \\
\hline Th & -0.28 \\
\hline $\mathrm{Ti}$ & 0.50 \\
\hline$U$ & 0.09 \\
\hline $\mathrm{Y}$ & -0.22 \\
\hline $\mathrm{Zn}$ & -0.06 \\
\hline $\mathrm{Zr}$ & 0.09 \\
\hline Mining vs. Hamilton & Correlation \\
\hline $\mathrm{Al}$ & 0.26 \\
\hline $\mathrm{Ca}$ & 0.25 \\
\hline $\mathrm{Cl}$ & -0.16 \\
\hline $\mathrm{Cu}$ & 0.27 \\
\hline $\mathrm{Fe}$ & 0.32 \\
\hline $\mathrm{K}$ & 0.38 \\
\hline $\mathrm{Mg}$ & 0.14 \\
\hline $\mathrm{Ni}$ & 0.28 \\
\hline $\mathrm{Pb}$ & 0.09 \\
\hline $\mathrm{P}$ & nd \\
\hline $\mathrm{Si}$ & 0.16 \\
\hline $\mathrm{S}$ & -0.02 \\
\hline $\mathrm{Ti}$ & -0.03 \\
\hline $\mathrm{V}$ & 0.45 \\
\hline
\end{tabular}

Figure3-4: Correlation between MSCL and Hamilton suites. Correlations displayed with bars between -1 and 1, where, negative values are red and positive values are blue. 


\section{MSCL Mining}

\begin{tabular}{|c|c|c|c|c|c|c|c|c|c|c|c|c|c|c|}
\hline & Al-Mining & Ca-Mining & Cl-Mining & Cu-Mining $F$ & Fe-Mining & K-Mining & Mg-Mining & Ni-Mining F & $P b$-Mining & P-Mining & Si-Mining & S-Mining & Ti-Mining & V-Mining \\
\hline Al-Mining & 1.00 & & & & & & & & & & & & & \\
\hline Ca-Mining & -0.36 & 1.00 & & & & & & & & & & & & \\
\hline Cl-Mining & -0.71 & -0.27 & 1.00 & & & & & & & & & & & \\
\hline Cu-Mining & 0.79 & -0.28 & -0.71 & 1.00 & & & & & & & & & & \\
\hline Fe-Mining & 0.71 & -0.37 & -0.49 & 0.68 & 1.00 & & & & & & & & & \\
\hline \begin{tabular}{|l|} 
K-Mining \\
\end{tabular} & 0.77 & -0.21 & -0.45 & 0.45 & 0.46 & 1.00 & & & & & & & & \\
\hline Mg-Mining & 0.20 & -0.10 & -0.03 & 0.09 & 0.14 & 0.30 & 1.00 & & & & & & & \\
\hline Ni-Mining & 0.79 & -0.28 & -0.71 & 1.00 & 0.68 & 0.44 & 0.09 & 1.00 & & & & & & \\
\hline Pb-Mining & 0.80 & -0.27 & -0.72 & 1.00 & 0.66 & 0.45 & 0.08 & 1.00 & 1.00 & & & & & \\
\hline P-Mining & -- & -- & -- & -- & -- & -- & -- & -- & -- & 1.00 & & & & \\
\hline Si-Mining & 0.90 & -0.28 & -0.82 & 0.84 & 0.64 & 0.50 & 0.09 & 0.84 & 0.85 & -- & 1.00 & & & \\
\hline S-Mining & 0.55 & -0.14 & -0.62 & 0.70 & 0.76 & 0.19 & -0.03 & 0.71 & 0.71 & -- & 0.71 & 1.00 & & \\
\hline Ti-Mining & 0.20 & -0.10 & -0.20 & 0.51 & 0.24 & 0.08 & 0.06 & 0.50 & 0.52 & -- & 0.30 & 0.56 & 1.00 & \\
\hline V-Mining & 0.42 & -0.15 & -0.45 & 0.81 & 0.41 & 0.16 & 0.05 & 0.81 & 0.81 & -- & 0.56 & 0.69 & 0.89 & 1.00 \\
\hline
\end{tabular}

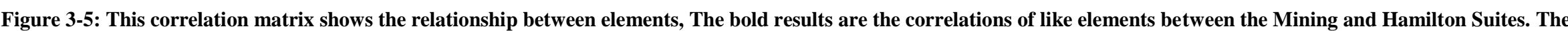
Color scale is dark blue with a strong positive correlation and dark red with a strong negative correlation both values get lighter as they approach zero. 


\section{MSCL Soil}

\begin{tabular}{|c|c|c|c|c|c|c|c|c|c|c|c|c|c|c|c|c|c|c|c|c|c|c|c|}
\hline & As-SOIL & Ba-SOIL & Ca-SOIL & Cl-SOIL & Cr-SOIL & Cu-SOIL & $\mathrm{Fe}$-SOIL & K-SOIL & Mn-SOIL & Mo-SOIL & Nb-SOIL & Ni-SOIL & $P b-S O I L$ & P-SOIL & $R b$-SOIL & S-SOIL & Sr-SOIL & Th-SOIL & Ti-SOIL & U-SOIL & Y-SOIL & Zn-SOIL & Zr-SOIL \\
\hline As-SOIL & 1.00 & & & & & & & & & & & & & & & & & & & & & & \\
\hline Ba-SOIL & 0.62 & 1.00 & & & & & & & & & & & & & & & & & & & & & \\
\hline Ca-SOIL & -0.07 & -0.04 & 1.00 & & & & & & & & & & & & & & & & & & & & \\
\hline Cl-SOIL & 0.10 & 0.33 & -0.12 & 1.00 & & & & & & & & & & & & & & & & & & & \\
\hline Cr-SOIL & -0.02 & -0.10 & -0.50 & -0.16 & 1.00 & & & & & & & & & & & & & & & & & & \\
\hline Cu-SOIL & -0.07 & 0.02 & -0.03 & 0.05 & -0.06 & 1.00 & & & & & & & & & & & & & & & & & \\
\hline Fe-SOIL & 0.04 & -0.02 & -0.58 & -0.07 & 0.59 & 0.01 & 1.00 & & & & & & & & & & & & & & & & \\
\hline K-SOIL & -0.32 & -0.16 & -0.50 & -0.37 & 0.43 & -0.01 & 0.65 & 1.00 & & & & & & & & & & & & & & & \\
\hline \begin{tabular}{|l|} 
Mn-SOIL \\
\end{tabular} & -0.42 & -0.12 & 0.27 & -0.17 & 0.04 & 0.03 & 0.12 & 0.26 & 1.00 & & & & & & & & & & & & & & \\
\hline Mo-SOIL & 0.40 & 0.60 & $\begin{array}{ll}0.02 \\
\end{array}$ & 0.27 & 0.15 & -0.12 & $\begin{array}{l}-0.02 \\
\end{array}$ & -0.35 & -0.11 & 1.00 & & & & & & & & & & & & & \\
\hline Nb-SOIL & 0.31 & -0.20 & -0.19 & -0.09 & 0.07 & -0.05 & 0.11 & -0.12 & -0.48 & -0.29 & 1.00 & & & & & & & & & & & & \\
\hline Ni-SOIL & 0.69 & 0.33 & -0.32 & 0.06 & 0.31 & 0.02 & 0.35 & -0.02 & -0.44 & 0.22 & 0.55 & 1.00 & & & & & & & & & & & \\
\hline $\mathrm{Pb}$-SOIL & 0.30 & 0.44 & -0.45 & 0.24 & 0.02 & 0.09 & 0.19 & 0.22 & -0.31 & 0.09 & 0.14 & 0.34 & 1.00 & & & & & & & & & & \\
\hline P-SOIL & 0.12 & 0.05 & $\begin{array}{ll}0.16 \\
\end{array}$ & -0.21 & 0.15 & -0.13 & 0.12 & 0.06 & -0.03 & -0.10 & 0.13 & 0.24 & 0.07 & 1.00 & & & & & & & & & \\
\hline Rb-SOIL & -0.04 & \begin{tabular}{|c|}
-0.10 \\
\end{tabular} & 0.11 & $\begin{array}{l}-0.02 \\
\end{array}$ & 0.55 & -0.05 & 0.01 & -0.32 & 0.01 & 0.46 & -0.02 & 0.09 & -0.33 & 0.12 & 1.00 & & & & & & & & \\
\hline S-SOIL & 0.53 & 0.14 & -0.28 & 0.08 & 0.52 & -0.06 & 0.61 & 0.01 & -0.30 & 0.25 & 0.37 & 0.66 & 0.09 & 0.30 & 0.37 & 1.00 & & & & & & & \\
\hline Sr-SOIL & 0.59 & 0.79 & 0.19 & 0.16 & -0.16 & 0.04 & -0.14 & -0.29 & 0.02 & 0.50 & -0.16 & 0.25 & 0.28 & 0.19 & 0.04 & 0.15 & 1.00 & & & & & & \\
\hline Th-SOIL & 0.51 & -0.01 & -0.17 & 0.05 & 0.09 & 0.08 & 0.12 & -0.27 & -0.49 & -0.06 & 0.69 & 0.60 & 0.20 & 0.12 & 0.05 & 0.58 & 0.13 & 1.00 & & & & & \\
\hline Ti-SOIL & -0.28 & -0.08 & -0.28 & -0.14 & -0.10 & -0.01 & 0.30 & 0.61 & 0.33 & -0.23 & -0.30 & -0.23 & 0.13 & -0.15 & -0.54 & -0.35 & -0.18 & -0.40 & 1.00 & & & & \\
\hline U-SOIL & 0.27 & 0.37 & 0.33 & 0.39 & -0.63 & 0.06 & -0.58 & -0.45 & -0.25 & 0.03 & 0.02 & 0.03 & 0.27 & -0.05 & -0.34 & -0.23 & 0.31 & 0.04 & -0.14 & 1.00 & & & \\
\hline Y-SOIL & -0.36 & -0.44 & -0.44 & -0.30 & 0.41 & -0.04 & 0.33 & 0.69 & 0.10 & -0.53 & 0.00 & -0.09 & 0.13 & 0.10 & -0.09 & -0.07 & -0.46 & -0.23 & 0.37 & -0.27 & 1.00 & & \\
\hline Zn-SOIL & 0.39 & 0.75 & -0.11 & 0.37 & 0.02 & -0.04 & 0.09 & -0.10 & -0.16 & 0.40 & -0.09 & 0.34 & 0.32 & 0.04 & -0.01 & 0.20 & 0.39 & 0.04 & -0.08 & 0.21 & -0.32 & 1.00 & \\
\hline Zr-SOIL & 0.56 & 0.96 & \begin{tabular}{l|l}
-0.16 \\
\end{tabular} & 0.32 & -0.03 & -0.02 & 0.08 & 0.03 & -0.09 & 0.53 & -0.25 & 0.32 & 0.49 & 0.04 & -0.19 & 0.12 & 0.71 & -0.09 & 0.06 & $\begin{array}{ll}0.32 \\
\end{array}$ & -0.26 & 0.68 & 1.00 \\
\hline
\end{tabular}

Figure 3-6: This correlation matrix shows the relationship between elements. The bold results are the correlations of like elements between the Mining and Hamilton Suites. The Color scale is dark blue with a strong positive correlation and dark red with a strong negative correlation both values get lighter as they approach zero. 


\section{MSCL Mining-plus}

\begin{tabular}{|c|c|c|c|c|c|c|c|c|c|c|c|c|c|c|c|c|c|c|c|c|c|}
\hline & $A l-M P$ & As-MP & Ca-MP & CIMP & Cr-MP & Cu-MP & $\mathrm{Fe}-\mathrm{MP}$ & $H f-M P$ & $K-M P$ & $M g-M P$ & $M n-M P$ & Mo-MP & Ni-MP & $P b-M P$ & $P-M P$ & Si-MP & $S-M P$ & Ti-MP & $V-M P$ & $Z n-M P$ & Zr-MP \\
\hline Al-MP & 1.00 & & & & & & & & & & & & & & & & & & & & \\
\hline As-MP & 0.00 & 1.00 & & & & & & & & & & & & & & & & & & & \\
\hline Ca-MP & -0.78 & -0.52 & 1.00 & & & & & & & & & & & & & & & & & & \\
\hline Cl-MP & -0.02 & 0.48 & -0.16 & 1.00 & & & & & & & & & & & & & & & & & \\
\hline Cr-MP & 0.63 & -0.32 & -0.27 & 0.03 & 1.00 & & & & & & & & & & & & & & & & \\
\hline Cu-MP & 0.32 & 0.72 & -0.65 & 0.15 & 0.15 & 1.00 & & & & & & & & & & & & & & & \\
\hline Fe-MP & 0.09 & 0.83 & -0.55 & 0.45 & -0.37 & 0.44 & 1.00 & & & & & & & & & & & & & & \\
\hline Hf-MP & 0.47 & 0.01 & -0.54 & -0.40 & -0.11 & 0.17 & 0.09 & 1.00 & & & & & & & & & & & & & \\
\hline K-MP & 0.56 & -0.23 & -0.33 & -0.36 & 0.48 & 0.29 & -0.23 & 0.35 & 1.00 & & & & & & & & & & & & \\
\hline Mg-MP & -- & -- & -- & -- & -- & -- & -- & -- & -- & 1.00 & & & & & & & & & & & \\
\hline Mn-MP & -0.32 & 0.22 & 0.02 & -0.18 & -0.63 & 0.14 & 0.11 & 0.43 & 0.19 & -- & 1.00 & & & & & & & & & & \\
\hline Mo-MP & 0.02 & 0.79 & -0.41 & 0.36 & 0.04 & 0.71 & 0.56 & -0.29 & -0.25 & -- & -0.13 & 1.00 & & & & & & & & & \\
\hline Ni-MP & 0.08 & 0.88 & -0.57 & 0.18 & -0.16 & 0.85 & 0.62 & 0.04 & -0.11 & -- & 0.16 & 0.86 & 1.00 & & & & & & & & \\
\hline Pb-MP & 0.42 & 0.86 & -0.81 & 0.32 & -0.04 & 0.86 & 0.72 & 0.29 & 0.08 & -- & 0.17 & 0.74 & 0.86 & 1.00 & & & & & & & \\
\hline P-MP & 0.23 & -0.35 & -0.04 & -0.10 & 0.32 & -0.15 & -0.26 & 0.06 & 0.44 & -- & -0.08 & -0.32 & -0.28 & -0.27 & 1.00 & & & & & & \\
\hline Si-MP & 0.82 & 0.16 & -0.78 & -0.10 & 0.52 & 0.44 & 0.06 & 0.53 & 0.33 & -- & -0.26 & 0.19 & 0.33 & 0.49 & 0.16 & 1.00 & & & & & \\
\hline S-MP & -0.11 & 0.72 & -0.45 & 0.21 & -0.64 & 0.32 & 0.85 & 0.35 & -0.33 & -- & 0.44 & 0.39 & 0.56 & 0.60 & -0.31 & -0.02 & 1.00 & & & & \\
\hline Ti-MP & -0.30 & 0.16 & 0.03 & -0.21 & -0.61 & 0.13 & 0.10 & 0.42 & 0.26 & -- & 0.98 & -0.19 & 0.10 & 0.14 & -0.10 & -0.33 & 0.41 & 1.00 & & & \\
\hline V-MP & -0.39 & 0.22 & 0.09 & -0.17 & -0.69 & 0.10 & 0.13 & 0.37 & 0.13 & -- & 0.99 & -0.12 & 0.14 & 0.14 & -0.11 & -0.36 & 0.46 & 0.98 & 1.00 & & \\
\hline Zn-MP & -0.07 & 0.74 & -0.19 & 0.69 & 0.04 & 0.50 & 0.54 & -0.46 & -0.20 & -- & -0.19 & 0.68 & 0.56 & 0.49 & -0.10 & -0.01 & 0.21 & -0.23 & -0.17 & 1.00 & \\
\hline Zr-MP & 0.09 & 0.17 & -0.28 & -0.24 & -0.33 & 0.30 & 0.09 & 0.64 & 0.52 & -- & 0.90 & -0.17 & 0.16 & 0.30 & 0.14 & 0.05 & 0.36 & 0.90 & 0.86 & -0.25 & 1.00 \\
\hline
\end{tabular}

Figure 3-7: This correlation matrix shows the relationship between elements. The Color scale is dark blue with a strong positive correlation and dark red with a strong negative correlation both values get lighter as they approach zero. 


\section{Hamilton Suite}

\begin{tabular}{|c|c|c|c|c|c|c|c|c|c|c|c|c|c|c|c|c|c|c|c|c|c|}
\hline & Al & $A s>=$ & $\mathrm{Ca}$ & $C l>=$ & $\mathrm{Cr}$ & $\mathrm{Cu}$ & $\mathrm{Fe}$ & $H f$ & $K$ & $M g$ & $M n$ & Mo & $\mathrm{Ni}$ & $P b>=$ & $P$ & $\mathrm{Si}$ & $S>=$ & $T i$ & $V$ & $Z n$ & $\mathrm{Zr}$ \\
\hline Al & 1.00 & & & & & & & & & & & & & & & & & & & & \\
\hline As $>=$ & 0.15 & 1.00 & & & & & & & & & & & & & & & & & & & \\
\hline $\mathrm{Ca}$ & -0.78 & -0.58 & 1.00 & & & & & & & & & & & & & & & & & & \\
\hline$C \mid>=$ & 0.16 & 0.25 & -0.12 & 1.00 & & & & & & & & & & & & & & & & & \\
\hline $\mathrm{Cr}$ & 0.92 & 0.22 & -0.70 & 0.17 & 1.00 & & & & & & & & & & & & & & & & \\
\hline $\mathrm{Cu}$ & 0.41 & 0.72 & -0.69 & -0.03 & 0.57 & 1.00 & & & & & & & & & & & & & & & \\
\hline $\mathrm{Fe}$ & 0.19 & 0.90 & -0.64 & 0.26 & 0.21 & 0.69 & 1.00 & & & & & & & & & & & & & & \\
\hline $\mathrm{Hf}$ & 0.75 & 0.08 & -0.48 & -0.01 & 0.78 & 0.36 & 0.02 & 1.00 & & & & & & & & & & & & & \\
\hline$K$ & 1.00 & 0.16 & -0.79 & 0.14 & 0.92 & 0.42 & 0.21 & 0.74 & 1.00 & & & & & & & & & & & & \\
\hline $\mathrm{Mg}$ & 0.65 & 0.22 & -0.51 & 0.22 & 0.80 & 0.55 & 0.23 & 0.74 & 0.64 & 1.00 & & & & & & & & & & & \\
\hline $\mathrm{Mn}$ & -0.02 & -0.49 & 0.36 & -0.04 & -0.05 & -0.22 & -0.46 & 0.33 & -0.06 & 0.19 & 1.00 & & & & & & & & & & \\
\hline Mo & 0.19 & 0.63 & -0.47 & 0.11 & 0.36 & 0.67 & 0.61 & -0.11 & 0.22 & 0.32 & -0.67 & 1.00 & & & & & & & & & \\
\hline $\mathrm{Ni}$ & 0.20 & 0.74 & -0.58 & 0.08 & 0.35 & 0.81 & 0.75 & -0.05 & 0.23 & 0.28 & -0.59 & 0.93 & 1.00 & & & & & & & & \\
\hline $\mathrm{Pb}>=$ & 0.58 & 0.16 & -0.50 & -0.16 & 0.66 & 0.48 & 0.29 & 0.26 & 0.60 & 0.44 & -0.45 & 0.59 & 0.50 & 1.00 & & & & & & & \\
\hline P & -0.48 & -0.47 & 0.58 & -0.12 & -0.39 & -0.54 & -0.66 & -0.28 & -0.49 & -0.31 & -0.03 & -0.37 & $\begin{array}{l}-0.52 \\
\end{array}$ & -0.33 & 1.00 & & & & & & \\
\hline $\mathrm{Si}$ & 0.92 & 0.27 & -0.86 & 0.11 & 0.85 & 0.45 & 0.32 & 0.57 & 0.94 & 0.55 & -0.35 & 0.39 & 0.38 & 0.70 & -0.45 & 1.00 & & & & & \\
\hline$S\rangle=$ & 0.33 & 0.20 & -0.35 & -0.46 & 0.32 & 0.46 & 0.07 & 0.38 & 0.33 & 0.28 & 0.20 & 0.07 & 0.15 & 0.24 & -0.29 & 0.33 & 1.00 & & & & \\
\hline $\mathrm{Ti}$ & 0.99 & 0.06 & -0.71 & 0.12 & 0.92 & 0.37 & 0.10 & 0.81 & 0.98 & 0.68 & 0.09 & 0.08 & 0.09 & 0.56 & -0.41 & 0.88 & 0.35 & 1.00 & & & \\
\hline $\mathrm{V}$ & 0.27 & 0.82 & -0.68 & 0.17 & 0.40 & 0.86 & 0.78 & 0.06 & 0.30 & 0.36 & -0.53 & 0.88 & 0.96 & 0.41 & -0.53 & 0.42 & 0.22 & 0.17 & 1.00 & & \\
\hline $2 n$ & 0.09 & 0.36 & -0.17 & 0.04 & 0.32 & 0.46 & 0.24 & 0.10 & 0.11 & 0.29 & -0.37 & 0.68 & 0.65 & 0.31 & -0.16 & 0.20 & 0.12 & 0.02 & 0.61 & 1.00 & \\
\hline $2 r$ & 0.93 & 0.09 & -0.64 & 0.11 & 0.96 & 0.50 & 0.10 & 0.86 & 0.91 & 0.78 & 0.15 & 0.13 & 0.15 & 0.57 & -0.36 & 0.80 & 0.39 & 0.96 & 0.22 & 0.14 & 1.00 \\
\hline
\end{tabular}

Figure 3-8: This correlation matrix shows the relationship between elements. The bold results are the correlations of like elements between the Mining and Hamilton Suites. The Color scale is dark blue with a strong positive correlation and dark 


\subsection{Paleo Proxies:}

Chemostratigraphy is the study of the chemical variations within sedimentary sequences to determine stratigraphic relationships. Chemostratigraphy is particularly important in intervals that visually appear homogenous and lack abundant environment of deposition indicators such as the Marcellus Shale. Sageman et al. 2003, provides a framework to understand organic matter preservation in clay-rich intervals using three processes: dilution, or the amount of sediment input; decomposition, or the paleo-redox conditions; and production both in terms of nutrient supply and primary production. Using elemental enrichments and ratios, we can resolve changes in paleo-redox environments, sedimentation, and paleo-production (Figure 3-9).

Chemical proxies for sedimentation primarily focus on the change in detrital (fluvial and eolian) and skeletal (benthic and pelagic) delivery. Detrital delivery is primarily resolved using the ratio of $\mathrm{Ti}$ and $\mathrm{Si}$ to $\mathrm{Al}$ and elemental enrichments in $\mathrm{Zr}$. Skeletal influx is resolved by increase s in Ca. Chemical proxies for redox conditions primarily focus on elemental enrichment and they dominate electron acceptor. For example, Manganese is primarily associated with $\mathrm{O}_{2}$, therefore increases in $\mathrm{Mn}$ indicate oxic redox conditions. One also uses $\mathrm{Fe}, \mathrm{S}$, and Mo to determine anoxic to euxinic (sulfate reducing anoxic condition). Mo and Fe (from pyrite) accumulate during intervals of sulfate reduction.

Chemical proxies for paleo-production can be separated into two categories: nutrient supply and bio-productivity. Nutrient supply is determined through enrichments in $\mathrm{Ni}, \mathrm{Zn}$, and V. Bioproductivity is determined using $\mathrm{P}, \mathrm{Y}$ enrichment, and the TOC. 


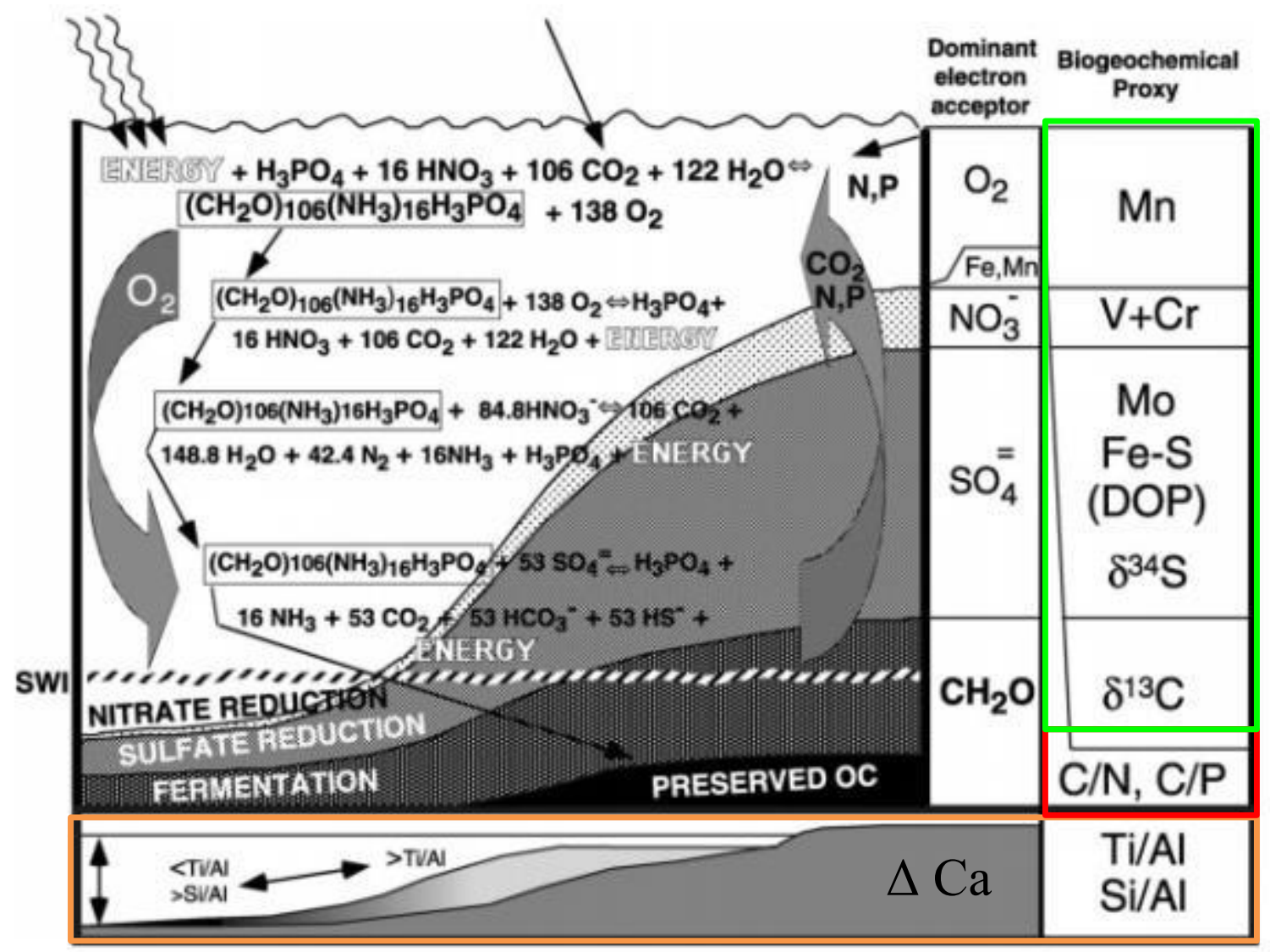

Figure 3.9: This figure ties chemical proxies to their importance in the paleo-environment. Green denotes paleo-redox proxies, red denotes paleo-production proxies, and orange denotes the detrital proxies. Modified from Sageman et al. 2003.

Detrital:

XRF results of the core from the MIP-3H well show an increase from the Onondaga below 7549 feet through the Marcellus in the amount of detrital influence as evidenced by a generally persistent decrease in the $\mathrm{Si} / \mathrm{Al}$ ratio from greater than 10 to less than 5 (Figure 3-10). In the lower part of the Marcellus there is a less than 1-meter interval at 7539 feet picked up by the MSCL with a ratio of greater than 20, indicating very low detrital influence. The interval also has a high $\mathrm{Ca}$ percentage. Similar elevated $\mathrm{Si} / \mathrm{Al}$ peaks and associated Ca peaks are picked up through the lower Marcellus (e.g., 7516 feet). There is a persistent increase in $\mathrm{Zr}$ enrichment in 
the Marcellus upwards from less than 100ppm to more than 150ppm also indicating and increase in detrital influence. The most volatile changes in detrital or skeletal influence in the well occur within the thin intervals of carbonate shown by the $\mathrm{Ca}$ (Figure 3-10). Note the variation in absolute concentration of $\mathrm{Al}$ and $\mathrm{Si}$ between the MSCL and Hamilton XRF is the result of report as oxides versus elemental concentrations.

The TOC determined from core and spectral gamma ray logs (Uranium) shows that changes in detrital input as indicated by the $\mathrm{Si} / \mathrm{Al}$ ratio and $\mathrm{Zr}$ enrichment do have influence on organic matter.

Redox:

In the MIP 3H core, maganeese (Mn) shows a persistent increase with scattered peaks through the Marcellus Shale from less than 100ppm to more than 200ppm. Throughout there are scattered peaks in Mn approaching 400ppm which are associated with peaks in Ca (Figure 3-11). The molybdenum (Mo) concentration in the lower Marcellus decreases rapidily above and below the interval and gradually decreases from the lower marcellus to the Mahantango. A similar upwards decreasing trend with significant volatility is obseserved in both the sulfur/iron ratio (S/Fe), also represented as degree of pyritization (DOP). the degree of pyritization trend decreases from the lower marcellus to the Mahantago. This trend relates closely to the pyrite density curve established from the CT-scan image analysis. The S/Fe show an irregular periodicity of 5 to 10 feet. The Thorium (Th) and Potassium (K) decrease with depth and are lowest in the organic peak of the lower Marcellus. Uranium (U) increases in concentration with depth and peaks in the middle and lower Marcellus. The Th/U ratio shows a presistent increase through the Marcellus Shale from 0.1 to about 2. The ratio remains below 2 suggesting the the uranium is fixed throughout the deposition of the Marcellus Shale. Additionally relating this 
trend to oxygen content we see an increase in oxygen from the lower Marcellus to the Mahantango with decreases in oxygen content at the TOC peaks in the middle and low Marcellus.

Paleo-production:

Yttrium (Y) and phosphorous (P) show only a small decrease upwards through the Marcellus (Figure 3-12). Nickel (Ni), zinc (Zn) and vandadium (V) show a significant decrease upwards from the lower part of the Marcellus that correlates with the decrease in TOC. This suggest that there is better preservation of organic matter in the lower Marcellus compared to the rest of the unit, and is consistent with the euxinic redox conditions and the decreased in detrital influence in the Lower Marcellus. 


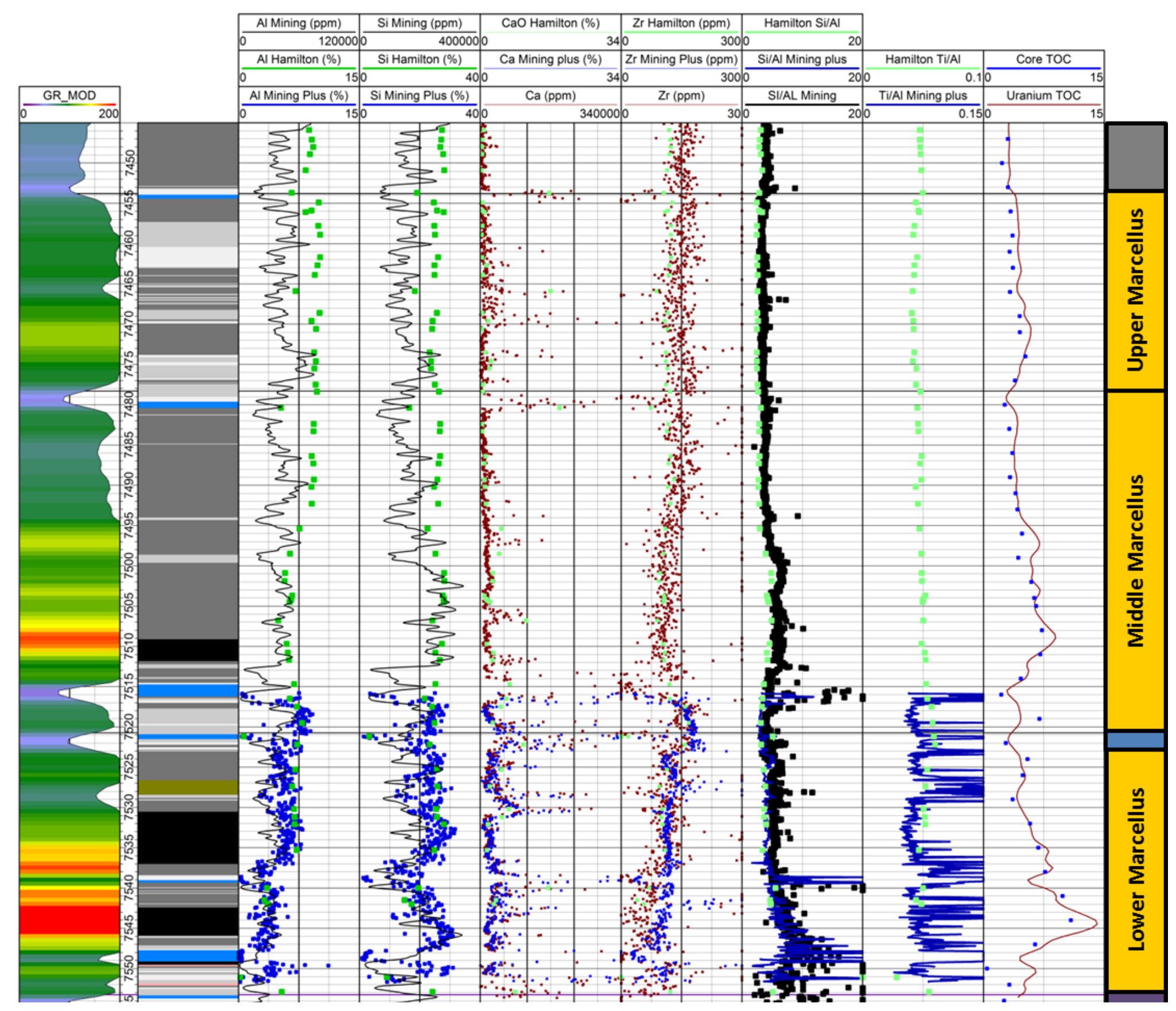

Figure 3.10: Well and core data from the MIP 3H. XRF data is from MSCL and Hamilton Lab analyses showing trends in elements used to determine detrital influence. Column 1 - Gamma ray log, Column 2 - Core Facies legend at base of figure, Column 3 - aluminum (Al) from Hamilton (green), MSCL Mining Suite (black), MSCL Mining-Plus Suite (blue); Column 4 - silicon (Si) from Hamilton (green), MSCL Mining Suite (black), MSCL Mining-Plus Suite (blue); Column 5 calcium (Ca) from Hamilton (green), MSCL Soil Suite (red), MSCL Mining-Plus Suite (blue); Column 6 -- zircon (Zr) from Hamilton (green), MSCL Soil Suite (red), MSCL Mining-Plus Suite (blue); Column 7 - Ratio of silicon to aluminum (Si/Al) from Hamilton (green), MSCL Mining Suite (black), MSCL Mining-Plus Suite (blue): Column 8 - Ratio of titanium to aluminum (Si/Al) from Hamilton (green) and MSCL Mining-Plus Suite (blue). Column 9 - Total organic carbon from 


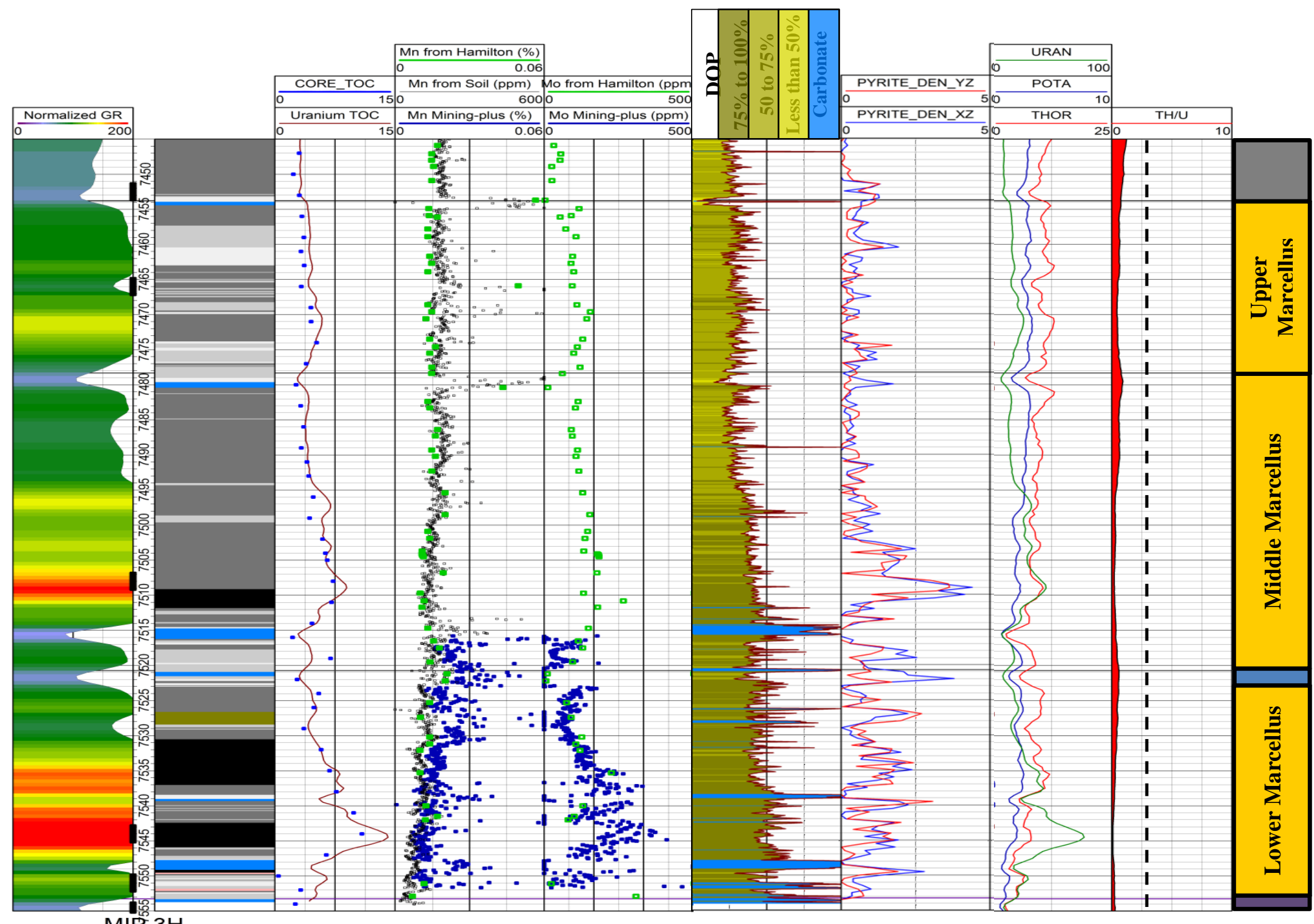

$\mathrm{MIP} 3 \mathrm{H}$

Figure 3.11: Well and core data from the MIP 3H. XRF data is from MSCL and Hamilton Lab analyses showing trends in elements used to determine paleoredox conditions. Column 1 - Gamma ray log, Column 2 - Core Facies legend at base of figure, Column 3 - total organic carbon from core (blue) and total organic carbon from uranium (red), Column 4 - manganese (Mn) from Hamilton Suite (green), MSCL Soil Suite (red), and MSCL Mining-Plus Suite (Blue); Column 5 - molybdenum (Mo) from Hamilton Suite (green) and MSCL Mining-Plus Suite (blue), Column 6 -S/Fe ratio from Soil Suite (red) shading behing the curve denote the degree of pyritization (DOP) (100 to 75\%, dark yellow, 75 to $50 \%$,gold-yellow, less than 50, yellow, limestone, blue); Column 7 - pyrite density from medical CT-scan (red YZ plan, blue XZ plan); Column 8 - Spectral Gamma Ray logs: uranium (U) (ppm) (green), thorium (Th) (ppm) (red), and potassium (K) (\%) (blue); Column 9 - ratio of thorium to uranium $(\mathrm{Th} / \mathrm{U})$ values less than 2 are shaded red and represent a fixed uranium suggesting anoxic conditions throughout the Marcellus. 


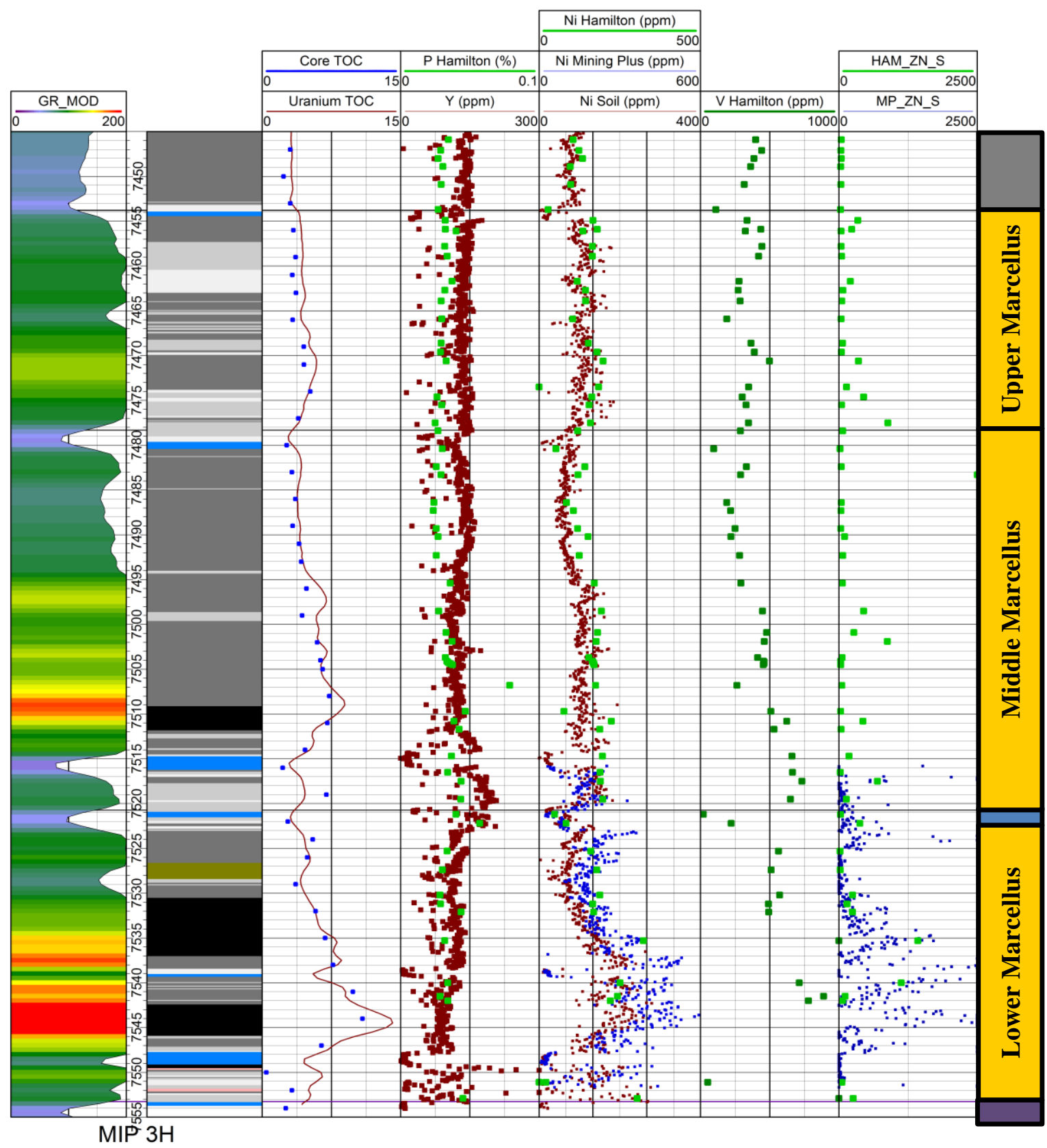

Figure 3-12: Well and core data from the MIP 3H. XRF data is from MSCL and Hamilton Lab analyses showing trends in elements used to determine paleo-redox conditions. Column 1 - Gamma ray log, Column 2 - Core Facies legend at base of figure, Column 3 - total organic carbon from core (blue) and total organic carbon from uranium (red), Column 4 - yttrium (Y) from MSCL Soil Suite (red) and phosphorous (P) from Hamilton Suite (green), Column 5 - nickel (Ni) from Hamilton Suite (green), MSCL Soil Suite (red), and MSCL Mining-Plus Suite (blue); Column 6 - vanadium (V) from Hamilton Suite, Column 7 - zinc from Hamilton Suite (green) and MSCL Mining-Plus Suite (blue). 


\section{Pulse Neutron Spectroscopy (PNS) logs}

To compare the chemostratigraphy between the MIP-3H and MIP-4H wells, we examine chemical proxies between each well using the Pulse Neutron Spectroscopy (PNS) logs. The PNS log emits high energy neutrons into the formation and detects the returning neutron energy by a 254-channel gamma-ray detector. The returned spectrum includes both elastic/capture $(\mathrm{C}, \mathrm{O}, \mathrm{Ca}$, $\mathrm{Al}, \mathrm{Mg}, \mathrm{Si}, \mathrm{S}$, and $\mathrm{Fe}$ ) and inelastic components $(\mathrm{H}, \mathrm{Na}, \mathrm{Cl}, \mathrm{K}, \mathrm{Ti}, \mathrm{Cr}, \mathrm{Ni}, \mathrm{Ba}, \mathrm{Gd}, \mathrm{Ca}, \mathrm{Al}, \mathrm{Mg}$, $\mathrm{Si}, \mathrm{S}$, and $\mathrm{Fe}$ ) (Schlumberger, 2006). The PNS in MIP-3H returns mostly major elements and Gadolinium and the PNS Suite in MIP-4H returns only major elements (Figure 3-13).

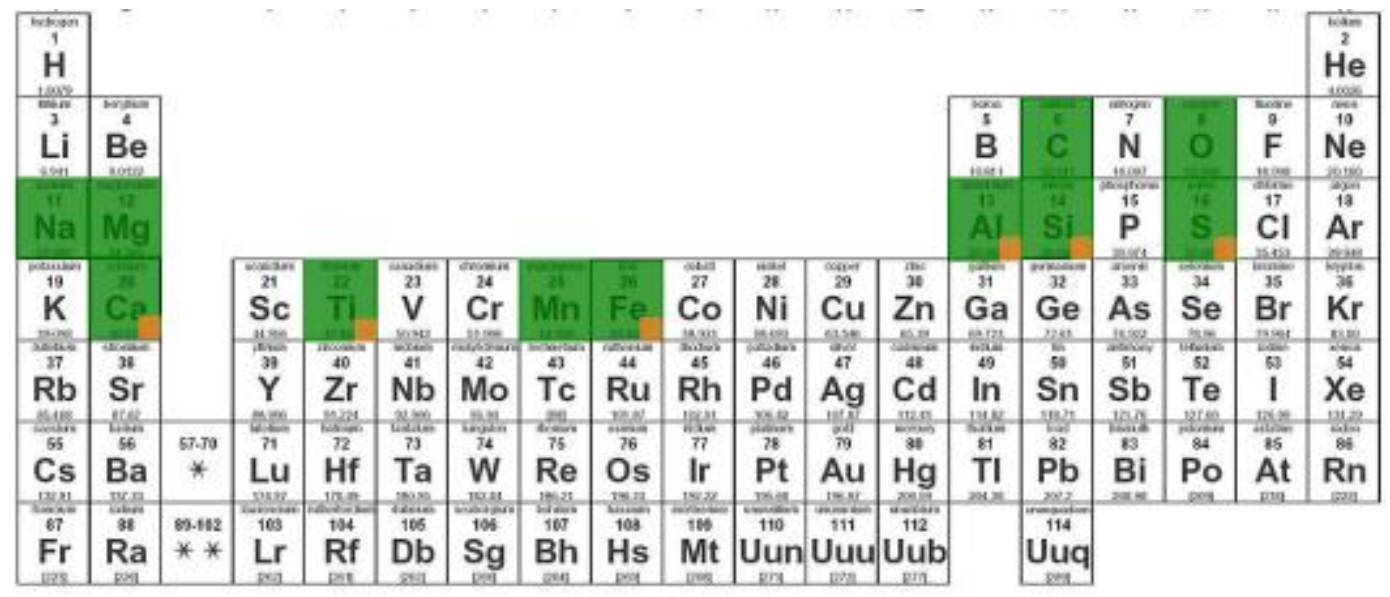

\begin{tabular}{|c|c|c|c|c|c|c|c|c|c|c|c|c|c|c|}
\hline - Lanthanide series & ${ }^{5 r}$ & $\mathrm{Ce}_{\mathrm{si}}$ & Pr & Nd & $\mathrm{Pm}^{\star 1}$ & $\mathrm{Sm}$ & Eu & $\mathrm{Gd}$ & $\begin{array}{l}\text { sis } \\
\text { Tb }\end{array}$ & Dy & $\begin{array}{l}\text { if } \\
\text { Ho }\end{array}$ & $\mathrm{Er}$ & $\mathrm{Tm}$ & $\begin{array}{l}\mathrm{Yb} \\
\mathrm{Y} b\end{array}$ \\
\hline Actinide series & $\mathrm{Ac}^{10}$ & $\begin{array}{l}\text { Then } \\
\text { Th }\end{array}$ & $\frac{n+m}{P_{a}^{n}}$ & U & $\begin{array}{l}{ }^{31} \\
\mathrm{~Np}\end{array}$ & $\mathrm{Pu}$ & Am & $\stackrel{50}{\mathrm{Cm}}$ & Bk & Cf & $\mathrm{Es}^{n}$ & $\mathrm{Fm}$ & Md & No \\
\hline
\end{tabular}

Figure 3-13: PNS derived elements, green are all elements capture through the lithoscanner tool and orange elements are the elements captured through the SpectroLith log

The PNS elements have strong/moderate correlations with $\mathrm{Al}, \mathrm{Ca}, \mathrm{Fe}, \mathrm{K}, \mathrm{S}, \mathrm{Si}$, and Ti and poor correlations with $\mathrm{Mg}$ and $\mathrm{Mn}$ (Figure 3-14). Given the strong correlations between the XRF and PNS logs, we are confident in the ability to use the PNS logs in MIP-4H. From the elementals present, we can use the PNS logs to distinguish differences in detrital input throughout the wells. 
However, to understand paleo-redox conditions, we need to infer based on petrophysical logs (Density and Photoelectric specifically).

\begin{tabular}{|l|l|l|}
\hline \multicolumn{3}{|c|}{ Mining-plus vs. PNS } \\
\hline $\mathrm{Al}$ & 0.31 & \\
\hline $\mathrm{Ca}$ & 0.60 & \\
\hline $\mathrm{Fe}$ & 0.73 & \\
\hline $\mathrm{K}$ & 0.18 & \\
\hline $\mathrm{Mn}$ & -0.09 & \\
\hline $\mathrm{S}$ & 0.54 & \\
\hline $\mathrm{Si}$ & 0.63 & \\
\hline $\mathrm{Ti}$ & 0.03 & \\
\hline & Hamilton vs. & PNS \\
\hline $\mathrm{Al}$ & 0.59 & \\
\hline $\mathrm{Ca}$ & 0.56 & \\
\hline $\mathrm{Fe}$ & 0.47 & \\
\hline $\mathrm{K}$ & 0.50 & \\
\hline $\mathrm{Mg}$ & -0.01 & \\
\hline $\mathrm{Mn}$ & -0.17 & \\
\hline $\mathrm{Na}$ & 0.15 & \\
\hline $\mathrm{Si}$ & 0.30 & \\
\hline $\mathrm{Ti}$ & 0.54 \\
\hline
\end{tabular}

Figure 3-14: Correlation coefficients between the PNS log and Hamilton/Mining-plus suites for the MIP-3H well, correlations are displayed with bars between $\mathbf{- 1}$ and 1 , where, negative values are red and positive values are blue.

The PNS elemental suite show similar trends to the XRF data detrital influence decreases with depth. Al and Ti concentrations decrease with depth with an increase in the Si/Al ratio. Si remains consistent throughout both wells suggesting that biogenic silica is likely present. Ca aligns with the low gamma peaks and increases in frequency and concentration in the lower Marcellus. Fe remains relatively consistent through both wells with a slight decrease in concentration in the MIP-4H well. Sulfur slightly increases with depth and Fe-S separation decreases with depth with some negative separation in the lower middle Marcellus and lower Marcellus. These zones align with the high gamma peaks and are likely attributed to anoxic to euxinic redox conditions (Figure 3-15). 


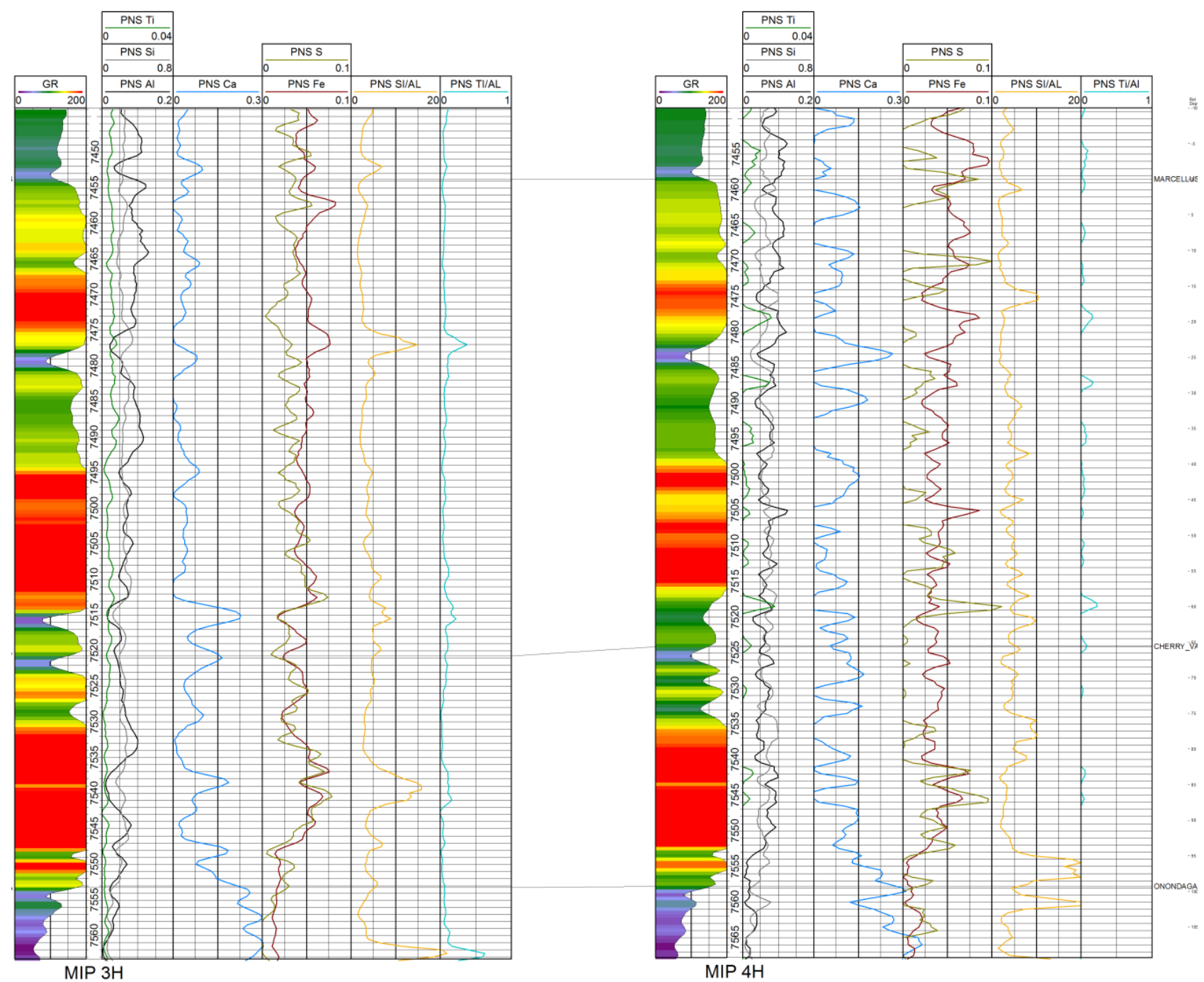

Figure 3-15: Cross-section of MIP3H and MIP4H: Tract 1: Gamma Ray (0-200 API with color display 0 - 300 API); Tract 2: PNS titanium (0-0.02), aluminum (0-0.15), Silicon (0-0.5); Tract 3: PNS calcium (0-.3); Tract 4: PNS iron and xulfur (0-.1), Tract 5: Ratio of silicon to aluminum (Si/Al) (0-20); Tract 6: Ratio of titanium to aluminum $(\mathrm{Ti} / \mathrm{Al})(0-0.5)$

Due to the limitations of the PNS elemental data to distinguish redox zones, we use the petrophysical logs, specifically looking for zones of high density minerals and carbonate zones. High density pyrite nodules and calcite concretions occur across transitions from anoxic/euxinic to dysoxic/oxic bottom water conditions (Clark and Mosier, 1989) (Figure 3-16). 


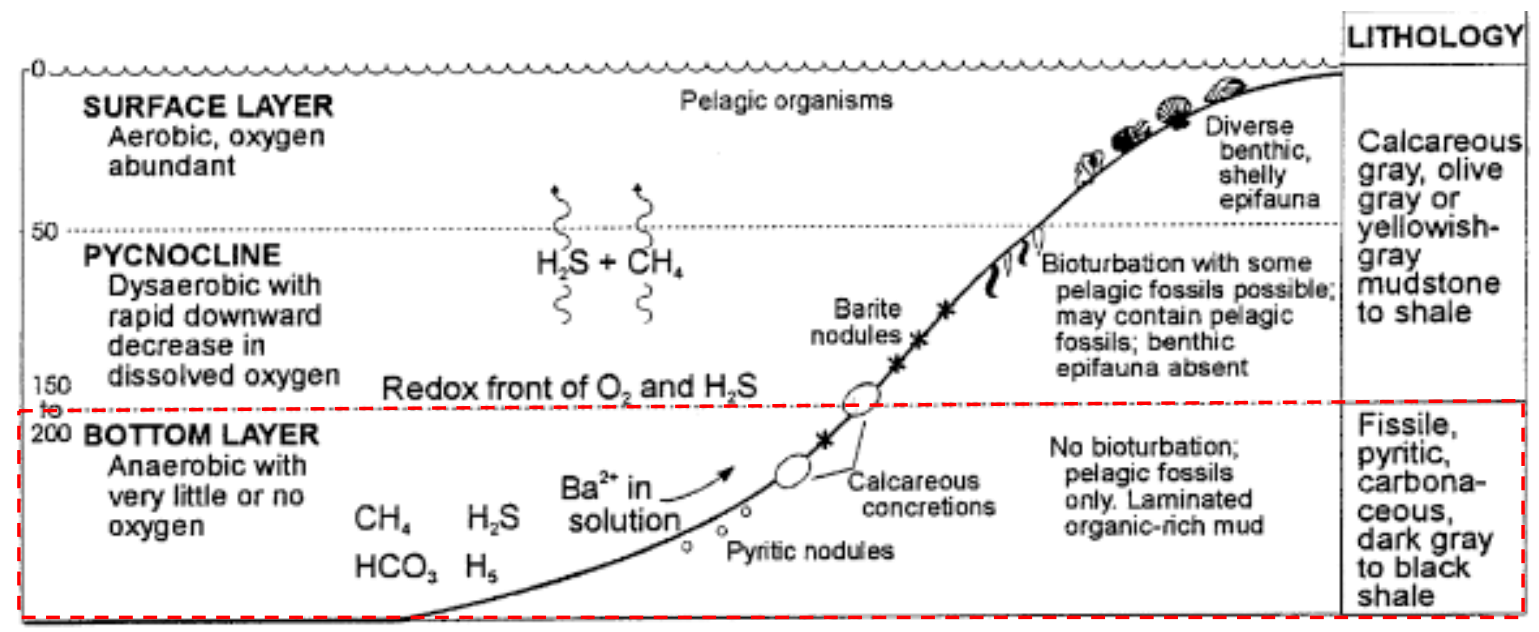

Figure 3-16: This figure shows concretions precipitated given bottom water conditions. The red box indicates the regime of the MIP-3H and MIP-4H (after Clark and Mosier 1989).

Marking out these zones we can redox zones that have approached euxinic redox conditions.

Both MIP-3H and MIP-4H wells have similar distributions of high density and carbonate zones with one exception in the transition between the middle and lower Marcellus through the Cherry Valley zone. MIP-4H has a small (2 foot) carbonate facies which pinches out in MIP-3H. The lower Marcellus has four redox transition zones, the bottom three are ranging from euxinic to anoxic and the top zone never reaches euxinic conditions (lacks high density mineral zone at transition). Seven zones were chosen from the middle Marcellus to the Mahantango. We find four zones that reach euxinic bottom water conditions, form the Middle Marcellus to the base of the Lower Marcellus and three zones that are anoxic to dysoxic in the upper portion of the upper Marcellus to the Marcellus-Mahantango contact. (Figure 3-17). 


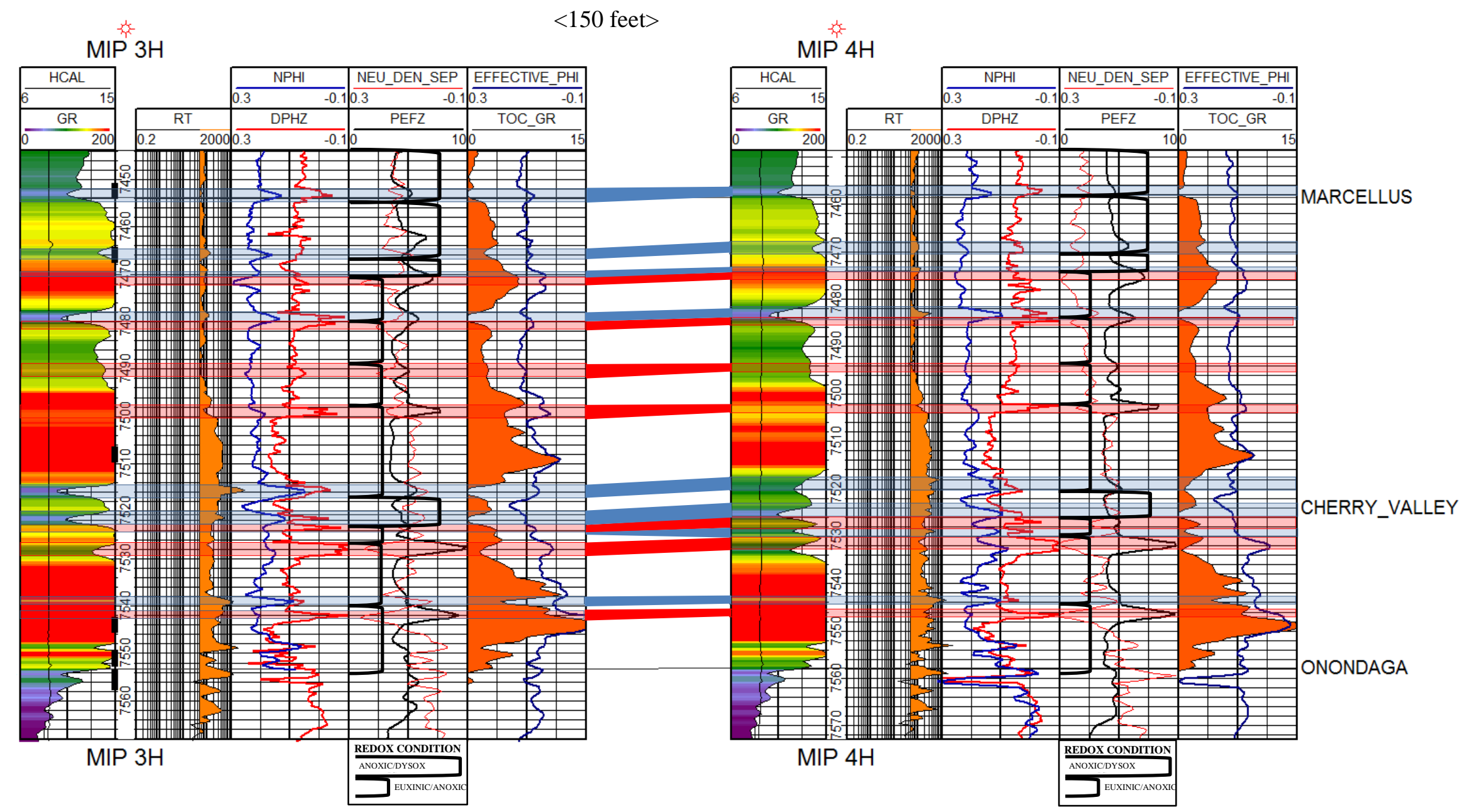

Figure 3-17: Cross-section of MIP3H and MIP4H: Tract 1: Gamma Ray (0-200 API with color display 0 - 300 API); Tract 2: Deep lateral array resistivity (ohm-m) orange shading (>100 ohm-m); Tract 3: neutron porosity (blue) density porosity (red); Tract 4: Neutron-Density separation (NEU_DEN_SEP)(red), Photoelectric log (PEFZ)(black/wide), Redox condition (black, extra wide) larger bracket indicates anoxic to dysoxic zone, smaller bracket (euxinic/anoxic conditions), Tract 5: Gamma Ray TOC (black line/orange shading) and effective porosity (dark blue). Red shading across logs indicates regions of high density minerals (High PE, strong positive NDS, decrease in RT), Blue shaded regions relate to carbonate intervals (NDS approaching 0, moderate PE, high RT) 


\section{CHAPTER 4: CONCLUSIONS}

Marcellus Shale has been divided into 6 different meso-facies and 5 different macro-facies units, such as Organic Siliceous Shale, Organic Mudstone, Organic Mixed Shale, Gray Siliceous Shale, Gray Mixed Shale and Gray Mudstone (Carbonate interlayers are present sometimes (in Mahantango) in the meso-facies; and Limestone, Light-Gray Shale, Mixed Dark-Gray Shale, Dark-Gray Shale, and Black Shale (K-Bentonite found in Onondaga Limestone) in the macrofacies.

MIP-3H contains 5 meso-scale facies (lacking organic mudstone) and MIP-4H includes all 6 facies. We find that silica content increases with TOC in the high GR peaks in both the MIP-3H and MIP-4H wells. Lower TOC values in MIP-4H and slightly high clay content are attributed to thinner organic-rich facies in the well compared to MIP-3H. In the transition between middle Marcellus and lower Marcellus, there are more thinly layers carbonate intervals in MIP-4H.

XRF data from the hhXRF from NETL is limited by the total exposure time and detection limits. We find that a minimum exposure time of 60 seconds is optimal for the best results, however the hhXRF cannot differentiate phosphorous or magnesium regardless of runtime due to the close proximity of the detection limit ( 2 and $1.2 \%$, respectively). The limitation of the hhXRF tool below and nearing the detection limits could also be a reason for the low correlation between the MSCL mining plus and Hamilton suites for titanium, considering there is lower detrital input in the lower Marcellus.

XRF data shows the Marcellus Shale in the MIP3H well ranges from dysoxic to anoxic throughout the well, increase in molybdenum and uranium trends in the Lower Marcellus suggests anoxic/euxinic conditions. Using zones of high density minerals at the contact of 
dysoxic/oxic zones (via carbonate intervals), we were able to distinguish redox zones in both the MIP-3H and MIP-4H that relate to the XRF data. From this analysis, we find 10 redox transition zones in the Marcellus Shale, suggesting rapid changes in redox environment. The three-high gamma-ray peaks fall within euxinic to anoxic zones. From the Cherry Valley Limestone to the base of the middle Marcellus, there is a zone of anoxic/dysoxic bottom water conditions. Additionally, this is seen from the top of the high gamma-ray peak in the upper Marcellus through the Marcellus-Mahantango contact.

Production increases with depth, with peak production in the Lower Marcellus, as seen through a decrease in phosphorous and yttrium and an increase in nickel, zinc, and vanadium. This increase in production likely aids in the higher TOC values present in the lower Marcellus.

Additionally, the increase in TOC is aided by an overall decrease in the amount of detrital influence. This is show through the XRF and PNS elemental data. Aluminum and titanium values decrease with depth and $\mathrm{Si} / \mathrm{Al}$ ratio increase. The increase in $\mathrm{Si} / \mathrm{Al}$ also suggest that silica content in the lower Marcellus is likely biogenic. This trend is mirrored by the overall decrease in clay mineralogy and mudstone facies and an increase in quartz and carbonate content and their associated facies in at meso- and macro-facies scale. 


\section{REFERENCES CITED}

Alzate, J.H. (2012). Integration of surface seismic, microseismic, and production logs for shale gas characterization: Methodology and field application (master's thesis). Retrieved from Mewbourne School of Petroleum and Geological Engineering.

Alzate, J. H., and Devegowda, D (2013). Integration of surface seismic, microseismic, and production logs for shale gas characterization: Methodology and field application. Interpretation, 1(2), SB37-SB49 pp.

Aboud, M., Badry, R., Grau, J., Herron, S., Hamichi, F., Horkowitz, J., Hemingway, J., MacDonald, R., Saldungaray, P., Stachiw, D., \& Stoller, C. (2014). High-definition spectroscopy_determining mineralogic complexity. Oilfield Review, 26(1), 34-50.

Algeo, T. J., \& Maynard, J. B. (2004). Trace-element behavior and redox facies in core shales of Upper Pennsylvanian Kansas-type cyclothems. Chemical Geology, 206(3), 289-318.

Brett, C. E., \& Baird, G. C. (1996). Middle Devonian sedimentary cycles and sequences in the northern Appalachian Basin. Special Papers-Geological Society Of America, 213-242.

Bhattacharya, S., \& Carr, T. R. (2016). Integrated Petrofacies Characterization and Interpretation of Depositional Environment of the Bakken Shale in the Williston Basin, North America. Petrophysics, 57(02), 96-111.

Chung, F.H. (1975a). Quantitative interpretation of x-ray diffraction patterns of mixtures. III. Simultaneous determination of a set of reference intensities. Journal of Applied Crystallography, 8, 17-19

Chung, F.H. (1975b). Quantitative interpretation of x-ray diffraction patterns of mixtures. I. Matrix-flushing method for quantitative multicomponent analysis. Journal of Applied Crystallography, 7, 519-525

Clark, S. H., \& Mosier, E. L. (1989). Barite nodules in Devonian shale and mudstone of western Virginia.

Crain, E. R., \& Holgate, D. (2014). A 12-Step Program to Reduce Uncertainty in Kerogen-Rich Reservoirs. GeoConvention: Focus. Canadian Society of Petroleum Geologists, Calgary, $1-11$.

Crandall, D., Moore, J., Brown, S.,\& Paronish, T. (2017). CT Scanning and Geophysical Logging of Core from the Marcellus Shale Energy and Environment Laboratory; NETLTRS- X-2017; EPAct Technical Report Series.U.S. Department of Energy, National Energy Technology Laboratory: Morgantown, WV, 2017, 1-41.

Doveton, J. H. (1994). Geologic Log Analysis Using Computer Methods (1994).

Ettensohn, F. R. (1985). Controls on development of Catskill Delta complex basinfacies. Geological Society of America Special Papers, 201, 65-78. 
Hertzog, R., Colson, L., Seeman, O., O'Brien, M., Scott, H., McKeon, D, Wraight, P., Grau, J., Ellis, D., Schweitzer, J., \& Herron, M. (1989). Geochemical logging with spectrometry tools. SPE Formation Evaluation, 4(02), 153-162.

Hupp, B. N. (2017). Provenance of the Hamilton Group: A Study of Source-to-Sink Relationships within the Middle Devonian Central Appalachian Basin. West Virginia (master's thesis). Retrieved from Eberly College of Arts and Sciences at West Virginia University. Order No. 10608023.

Hupp, B. N., \& Donovan J. J. (2017). Quantitative mineralogy of the Marcellus Shale, Appalachian Basin, USA, based on XRD-XRF. Chemical Geology. Manuscript submitted for publication.

International Center for Diffraction Data (ICDD), 2004, PDF-2 diffraction database.

Lash, G. G., \& Engelder, T. (2011). Thickness trends and sequence stratigraphy of the Middle Devonian Marcellus Formation, Appalachian Basin: Implications for Acadian foreland basin evolution. AAPG bulletin, 95(1), 61-103.

Lazar, O. R., Bohacs, K. M., Macquaker, J. H., Schieber, J., \& Demko, T. M. (2015). Capturing Key Attributes of Fine-Grained Sedimentary Rocks In Outcrops, Cores, and Thin Sections: Nomenclature and Description Guidelines. Journal of Sedimentary Research, 85(3), 230-246.

Mavko, G., Mukerji, T., \& Dvorkin, J. (2009). The Rock Physics Handbook: Tools for Seismic Analysis of Porous Media. Cambridge: Cambridge University Press.

Maynard, B. J. (2014). Manganiferous Sediments, Rocks, and Ores. In: Holland H.D. and Turekian K.K. (eds.) Treatise on Geochemistry, Second Edition, 9, 327-349. Oxford: Elsevier.

Passey, Q. R., Creaney, S., Kulla, J. B., Moretti, F. J., \& Stroud, J. D. (1990). A practical model for organic richness from porosity and resistivity logs. AAPG bulletin, 74(12), 17771794.

Potts, P. J., \& Webb, P. C. (1992). X-ray fluorescence spectrometry. Journal of Geochemical Exploration, 44(1-3), 251-296.

Pollard, D. D., \& Fletcher, R. C. (2005). Fundamentals of structural geology. Cambridge University Press.

Perez Altamar, R., \& Marfurt, K. (2014). Mineralogy-based brittleness prediction from surface seismic data: Application to the Barnett Shale. Interpretation, 2(4), T255-T271.

Sageman, B. B., Murphy, A. E., Werne, J. P., Ver Straeten, C. A., Hollander, D. J., \& Lyons, T. W. (2003). A tale of shales: the relative roles of production, decomposition, and dilution in the accumulation of organic-rich strata, Middle-Upper Devonian, Appalachian basin. Chemical Geology, 195(1), 229-273. 
Schlumberger (2006). ECS Elemental Capture Spectroscopy Sonde [Brochure]. N.P.: Schlumberger.

Schmoker, J. W. (1981). Determination of organic-matter content of Appalachian Devonian shales from gamma-ray logs. AAPG Bulletin, 65(7), 1285-1298.

Shervais, J. W. (1982). Ti-V plots and the petrogenesis of modern and ophiolitic lavas. Earth and Planetary Science Letters, 59(1), 101-118.

Wang, G. (2012). Black Shale Lithofacies Prediction and Distribution Pattern Analysis of Middle Devonian Marcellus Shale in the Appalachian Basin, Northeastern U.S.A. West Virginia University, Morgantown, unpublished $\mathrm{PhD}$ dissertation.

Wang, G., \& Carr, T. R. (2013). Organic-rich Marcellus Shale lithofacies modeling and distribution pattern analysis in the Appalachian Basin. AAPG bulletin, 97(12), 21732205.

Williams, H., \& Hatcher, R. D. (1982). Suspect terranes and accretionary history of the Appalachian orogen. Geology, 10(10), 530-536.

Witzke, B. J., \& Heckel, P. H. (1988). Paleoclimatic indicators and inferred Devonian paleolatitudes of Euramerica. Canadian Society of Petroleum Geologist Memoir, 14(1), 49-63.

Weicht, D. (2015). 3D Seismic, Mechanical Stratigraphy, and Petrophysical Analysis of the Marcellus Shale in Taylor County, West Virginia (master's thesis). Retrieved from Eberly College of Arts and Sciences at West Virginia University. (UMI Number: 1588158)

US EIA (2015). Oil and Gas Supply Module. Assumptions to Annual Energy Outlook 2015. 128146. 


\section{Appendix A: Source rock Pyrolysis data}

\begin{tabular}{|c|c|c|c|c|c|c|c|c|c|c|c|}
\hline ID & Depth & TOC & vTPH(S1) & pTPH(S2) & S3 & cTemp(Tmax) & $\mathrm{HI}$ & OI & PI & S1/TOC & tTemp \\
\hline MIP 3H - 01 & 7445 & 3.04 & 0.06 & 0.12 & 0.20 & 306.8 & 4 & 7 & 0.35 & 0.02 & 345.8 \\
\hline MIP 3H - 02 & 7448 & 3.07 & 0.08 & 0.13 & 0.21 & 323.3 & 4 & 7 & 0.38 & 0.03 & 362.3 \\
\hline MIP $3 \mathrm{H}-03$ & 7451 & 2.27 & 0.28 & 0.27 & 0.47 & 318.0 & 12 & 21 & 0.51 & 0.12 & 357.0 \\
\hline MIP $3 \mathrm{H}-04$ & 7454 & 3.01 & 0.06 & 0.05 & 0.24 & 307.4 & 2 & 8 & 0.51 & 0.02 & 346.4 \\
\hline MIP $3 \mathrm{H}-05$ & 7457 & 3.34 & 0.11 & 0.16 & 0.34 & 288.5 & 5 & 10 & 0.41 & 0.03 & 327.5 \\
\hline MIP $3 \mathrm{H}-06$ & 7460 & 3.62 & 0.21 & 0.30 & 1.34 & 320.5 & 8 & 37 & 0.41 & 0.06 & 359.5 \\
\hline MIP 3H - 07 & 7462 & 3.22 & 0.07 & 0.15 & 0.23 & 322.2 & 5 & 7 & 0.32 & 0.02 & 361.2 \\
\hline MIP $3 \mathrm{H}-08$ & 7464 & 3.66 & 0.05 & 0.15 & 0.26 & 543.4 & 4 & 7 & 0.25 & 0.01 & 582.4 \\
\hline MIP 3H - 09 & 7467 & 3.19 & 0.14 & 0.12 & 0.24 & 317.8 & 4 & 8 & 0.54 & 0.04 & 356.8 \\
\hline MIP $3 \mathrm{H}-10$ & 7470 & 4.45 & 0.12 & 0.22 & 0.40 & 310.2 & 5 & 9 & 0.36 & 0.03 & 349.2 \\
\hline MIP 3H - 11 & 7473 & 4.54 & 0.07 & 0.20 & 0.20 & 360.7 & 4 & 4 & 0.27 & 0.02 & 399.7 \\
\hline MIP $3 \mathrm{H}-12$ & 7476 & 5.16 & 0.04 & 0.16 & 0.20 & 566.1 & 3 & 4 & 0.21 & 0.01 & 605.1 \\
\hline MIP $3 \mathrm{H}-13$ & 7479 & 3.73 & 0.06 & 0.12 & 0.14 & 567.0 & 3 & 4 & 0.32 & 0.02 & 606.0 \\
\hline MIP $3 \mathrm{H}-14$ & 7482 & 2.60 & 0.16 & 0.29 & 0.42 & 325.0 & 11 & 16 & 0.35 & 0.06 & 364.0 \\
\hline MIP $3 \mathrm{H}-15$ & 7485 & 3.26 & 0.04 & 0.11 & 0.13 & 566.2 & 3 & 4 & 0.24 & 0.01 & 605.2 \\
\hline MIP $3 \mathrm{H}-16$ & 7488 & 3.60 & 0.11 & 0.19 & 0.35 & 315.4 & 5 & 10 & 0.38 & 0.03 & 354.4 \\
\hline 3H-7488_16 & 7488 & 3.19 & 0.11 & 0.14 & 0.31 & 314.8 & 4 & 10 & 0.45 & 0.04 & 353.8 \\
\hline MIP $3 \mathrm{H}-17$ & 7491 & 3.31 & 0.12 & 0.17 & 0.37 & 314.1 & 5 & 11 & 0.42 & 0.04 & 353.1 \\
\hline MIP $3 \mathrm{H}-18$ & 7493 & 4.01 & 0.17 & 0.28 & 0.34 & 316.1 & 7 & 9 & 0.37 & 0.04 & 355.1 \\
\hline MIP $3 \mathrm{H}-19$ & 7495 & 4.23 & 0.06 & 0.20 & 0.31 & 342.2 & 5 & 7 & 0.24 & 0.01 & 381.2 \\
\hline MIP 3H - 20 & 7498 & 4.78 & 0.09 & 0.18 & 0.24 & 566.1 & 4 & 5 & 0.33 & 0.02 & 605.1 \\
\hline MIP 3H - 21 & 7501 & 4.30 & 0.05 & 0.14 & 0.31 & 562.8 & 3 & 7 & 0.29 & 0.01 & 601.8 \\
\hline MIP 3H - 22 & 7504 & 5.92 & 0.13 & 0.28 & 0.26 & 563.4 & 5 & 4 & 0.32 & 0.02 & 602.4 \\
\hline MIP $3 \mathrm{H}-23$ & 7507 & 6.34 & 0.30 & 0.36 & 0.31 & 296.8 & 6 & 5 & 0.46 & 0.05 & 335.8 \\
\hline MIP $3 \mathrm{H}-24$ & 7510 & 7.21 & 0.09 & 0.27 & 0.31 & 563.2 & 4 & 4 & 0.26 & 0.01 & 602.2 \\
\hline MIP $3 \mathrm{H}-25$ & 7513 & 7.18 & 0.12 & 0.26 & 0.20 & 563.3 & 4 & 3 & 0.33 & 0.02 & 602.3 \\
\hline MIP $3 \mathrm{H}-26$ & 7516 & 5.07 & 0.11 & 0.18 & 0.33 & 562.7 & 3 & 6 & 0.39 & 0.02 & 601.7 \\
\hline MIP 3H - 27 & 7519 & 1.80 & 0.10 & 0.10 & 0.35 & 316.8 & 6 & 19 & 0.49 & 0.05 & 355.8 \\
\hline MIP $3 \mathrm{H}-28$ & 7522 & 7.25 & 0.19 & 0.29 & 0.43 & 564.4 & 4 & 6 & 0.40 & 0.03 & 603.4 \\
\hline MIP 3H - 29 & 7525 & 2.72 & 0.06 & 0.07 & 0.31 & 561.9 & 3 & 12 & 0.45 & 0.02 & 600.9 \\
\hline MIP $3 \mathrm{H}-30$ & 7527 & 5.55 & 0.22 & 0.41 & 0.31 & 391.6 & 7 & 6 & 0.34 & 0.04 & 430.6 \\
\hline MIP 3H - 31 & 7529 & 4.76 & 0.08 & 0.12 & 0.37 & 558.6 & 3 & 8 & 0.39 & 0.02 & 597.6 \\
\hline MIP $3 \mathrm{H}-32$ & 7532 & 3.53 & 0.09 & 0.11 & 0.44 & 563.7 & 3 & 13 & 0.44 & 0.03 & 602.7 \\
\hline MIP 3H - 33 & 7535 & 5.87 & 0.14 & 0.16 & 0.28 & 565.4 & 3 & 5 & 0.47 & 0.02 & 604.4 \\
\hline MIP 3H - 34 & 7538 & 6.86 & 0.14 & 0.24 & 0.33 & 560.7 & 3 & 5 & 0.37 & 0.02 & 599.7 \\
\hline 3Н-7538_34 & 7538 & 6.54 & 0.18 & 0.30 & 0.33 & 564.7 & 5 & 5 & 0.38 & 0.03 & 603.7 \\
\hline 3H-7541_35 & 7541 & 7.70 & 0.13 & 0.29 & 0.38 & 547.6 & 4 & 5 & 0.32 & 0.02 & 586.6 \\
\hline MIP $3 \mathrm{H}-36$ & 7544 & 9.88 & 0.12 & 0.36 & 0.40 & 562.9 & 4 & 4 & 0.25 & 0.01 & 601.9 \\
\hline 3H-7544_36 & 7544 & 9.20 & 0.17 & 0.45 & 0.33 & 562.3 & 5 & 4 & 0.28 & 0.02 & 601.3 \\
\hline
\end{tabular}

Table A-1: Pyrolysis Data from NETL Source rock analyzer, 7445 to 7544' (Core depth). 


\begin{tabular}{|l|c|c|c|c|c|c|c|c|c|c|c|}
\hline ID & Depth & TOC & vTPH(S1) & pTPH(S2) & S3 & cTemp(Tmax) & HI & OI & PI & S1/TOC & tTemp \\
\hline MIP 3H - 37 & 7547 & 10.91 & 0.12 & 0.52 & 0.46 & 562.7 & 5 & 4 & 0.18 & 0.01 & 601.7 \\
\hline 3H-7547_37 & 7547 & 10.13 & 0.15 & 0.60 & 0.39 & 564.6 & 6 & 4 & 0.20 & 0.01 & 603.6 \\
\hline MIP 3H - 38 & 7550 & 7.00 & 0.19 & 0.29 & 0.20 & 564.4 & 4 & 3 & 0.39 & 0.03 & 603.4 \\
\hline MIP 3H - 39 & 7553 & 0.44 & 0.06 & 0.09 & 0.07 & 358.9 & 21 & 15 & 0.41 & 0.14 & 397.9 \\
\hline MIP 3H - 40 & 7555 & 3.17 & 0.06 & 0.07 & 0.37 & 563.4 & 2 & 12 & 0.45 & 0.02 & 602.4 \\
\hline 3H-7555_40 & 7555 & 2.88 & 0.07 & 0.08 & 0.36 & 566.1 & 3 & 12 & 0.45 & 0.02 & 605.1 \\
\hline MIP 3H - 41 & 7557 & 2.55 & 0.07 & 0.11 & 0.24 & 337.1 & 4 & 9 & 0.38 & 0.03 & 376.1 \\
\hline 3H-7557_41 & 7557 & 2.37 & 0.07 & 0.14 & 0.21 & 354.5 & 6 & 9 & 0.35 & 0.03 & 393.5 \\
\hline
\end{tabular}

Table A-2: Pyrolysis Data from NETL Source rock analyzer, 7547 to 7557' (Core depth).

\section{Appendix B: X-Ray Diffraction data}

Two series of XRD data were used in this study from Hupp, 2017. The RIR XRD data set (Table B-1).and the quantitative XRF-XRD set (Table B-2).

\section{RIR X-ray Diffraction}

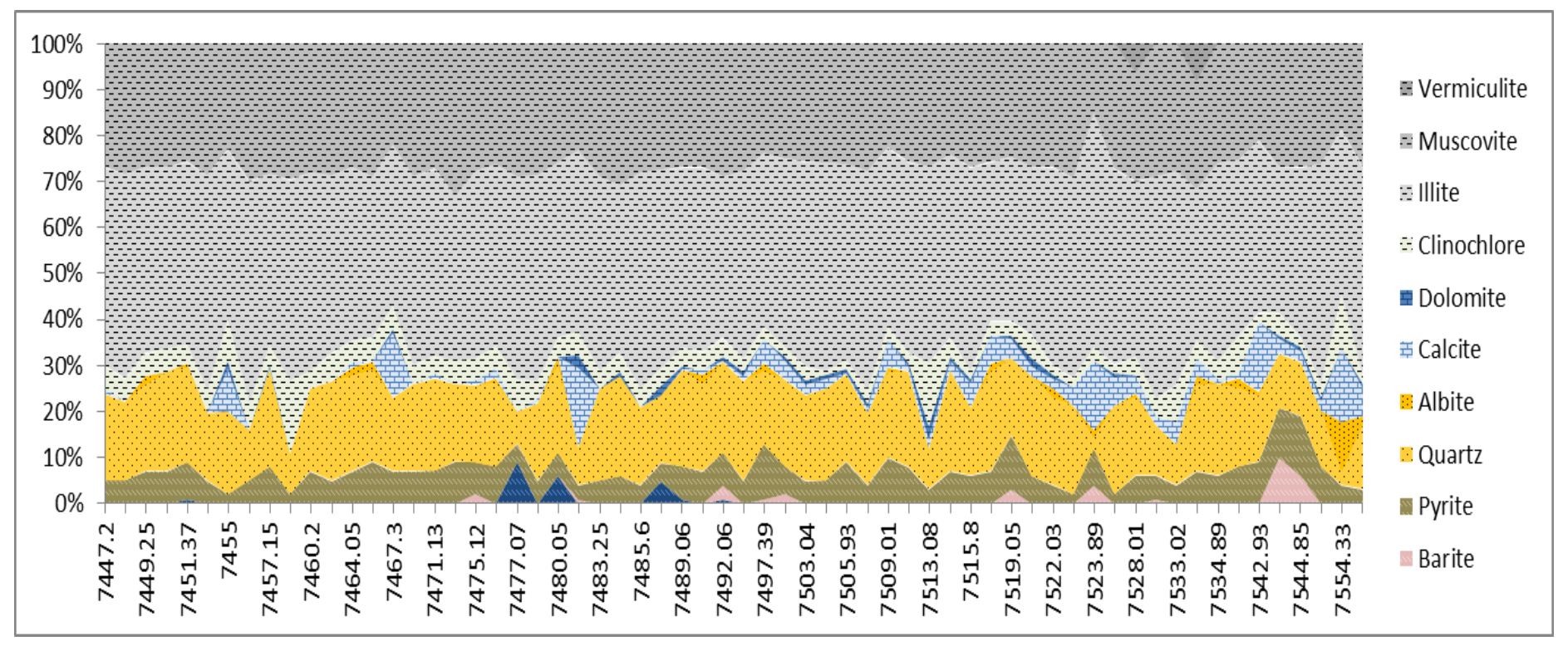

Figure B-1: Visual display of RIR XRD mineralogy from Table B-2 


\begin{tabular}{|c|c|c|c|c|c|c|c|c|c|c|c|c|c|}
\hline Depth & Quartz & Illite & Muscovite & Clinochlor Vermiculite & e Pyrite & Covellite & Albite & Calcite & Dolomite & Barite & Zeolite & Quartz & Illite+Musc \\
\hline 7447.2 & 19 & 43 & 27 & 5 & 5 & 0 & 0 & 1 & 0 & 0 & 0 & 19 & 70 \\
\hline 7448.35 & 17 & 44 & 28 & 5 & 5 & 0 & 0 & 0 & 0 & 0 & 0 & 17 & 72 \\
\hline 7449.25 & 19 & 41 & 27 & 5 & 7 & 0 & 2 & 0 & 0 & 0 & 0 & 19 & 68 \\
\hline 7450.2 & 22 & 40 & 27 & 5 & 7 & 0 & 0 & 0 & 0 & 0 & 0 & 22 & 67 \\
\hline 7451.37 & 21 & 40 & 26 & 4 & 8 & 1 & 1 & 0 & 0 & 0 & 0 & 21 & 66 \\
\hline 7452.22 & 15 & 47 & 29 & 5 & 5 & 0 & 0 & 0 & 0 & 0 & 0 & 15 & 76 \\
\hline 7455 & 18 & 37 & 23 & 9 & 2 & 0 & 0 & 9 & 2 & 0 & 0 & 18 & 60 \\
\hline 7456.16 & 11 & 48 & 30 & 6 & 5 & 0 & 0 & 0 & 0 & 0 & 0 & 11 & 78 \\
\hline 7457.15 & 21 & 37 & 28 & 4 & 8 & 0 & 0 & 0 & 1 & 0 & 0 & 21 & 65 \\
\hline 7459.13 & 9 & 44 & 30 & 16 & 2 & 0 & 0 & 0 & 0 & 0 & 0 & 9 & 74 \\
\hline 7460.2 & 18 & 43 & 28 & 4 & 7 & 0 & 0 & 0 & 0 & 0 & 0 & 18 & 71 \\
\hline 7463.1 & 22 & 39 & 29 & 6 & 5 & 0 & 0 & 0 & 0 & 0 & 0 & 22 & 68 \\
\hline 7464.05 & 21 & 38 & 27 & 5 & 7 & 0 & 2 & 1 & 0 & 0 & 0 & 21 & 65 \\
\hline 7465.3 & 20 & 35 & 29 & 5 & 9 & 0 & 2 & 0 & 0 & 0 & 0 & 20 & 64 \\
\hline 7467.3 & 16 & 35 & 22 & 5 & 7 & 0 & 0 & 14 & 1 & 0 & 0 & 16 & 57 \\
\hline 7470.05 & 19 & 41 & 29 & 4 & 7 & 0 & 0 & 0 & 0 & 0 & 0 & 19 & 70 \\
\hline 7471.13 & 20 & 40 & 27 & 4 & 7 & 0 & 0 & 1 & 0 & 0 & 0 & 20 & 67 \\
\hline 7472.09 & 17 & 36 & 33 & 5 & 9 & 0 & 0 & 0 & 0 & 0 & 0 & 17 & 69 \\
\hline 7475.12 & 17 & 41 & 28 & 5 & 7 & 0 & 0 & 1 & 0 & 2 & 0 & 17 & 69 \\
\hline 7476.15 & 18 & 39 & 26 & 5 & 8 & 0 & 1 & 2 & 0 & 0 & 0 & 18 & 65 \\
\hline 7477.07 & 7 & 44 & 29 & 7 & 4 & 9 & 0 & 0 & 0 & 0 & 0 & 7 & 73 \\
\hline 7479.05 & 17 & 45 & 29 & 4 & 5 & 0 & 0 & 1 & 0 & 0 & 0 & 17 & 74 \\
\hline 7480.05 & 19 & 38 & 26 & 4 & 5 & 6 & 2 & 0 & 0 & 0 & 0 & 19 & 64 \\
\hline 7482.12 & 8 & 39 & 23 & 5 & 3 & 0 & 0 & 17 & 3 & 1 & 0 & 8 & 62 \\
\hline 7483.25 & 20 & 42 & 29 & 4 & 5 & 0 & 0 & 0 & 0 & 0 & 0 & 20 & 71 \\
\hline 7485.03 & 22 & 37 & 31 & 4 & 6 & 0 & 0 & 0 & 1 & 0 & 0 & 22 & 68 \\
\hline 7485.6 & 17 & 47 & 28 & 4 & 4 & 0 & 0 & 0 & 0 & 0 & 0 & 17 & 75 \\
\hline 7488.15 & 15 & 44 & 28 & 4 & 4 & 5 & 0 & 0 & 2 & 0 & 0 & 15 & 72 \\
\hline 7489.06 & 21 & 39 & 27 & 4 & 7 & 1 & 0 & 0 & 1 & 0 & 0 & 21 & 66 \\
\hline 7491.22 & 19 & 40 & 27 & 4 & 7 & 0 & 2 & 1 & 0 & 0 & 0 & 19 & 67 \\
\hline 7492.06 & 20 & 35 & 29 & 4 & 7 & 1 & 0 & 0 & 1 & 3 & 0 & 20 & 64 \\
\hline 7494.2 & 22 & 41 & 28 & 3 & 5 & 0 & 0 & 1 & 1 & 0 & 0 & 22 & 69 \\
\hline 7497.39 & 16 & 38 & 24 & 3 & 12 & 0 & 2 & 5 & 0 & 1 & 0 & 16 & 62 \\
\hline 7500.6 & 19 & 41 & 25 & 2 & 6 & 0 & 0 & 4 & 1 & 2 & 0 & 19 & 66 \\
\hline 7503.04 & 19 & 45 & 26 & 3 & 5 & 0 & 0 & 2 & 1 & 0 & 0 & 19 & 71 \\
\hline 7503.98 & 20 & 45 & 26 & 1 & 5 & 0 & 0 & 2 & 1 & 0 & 0 & 20 & 71 \\
\hline 7505.93 & 19 & 42 & 26 & 2 & 9 & 0 & 0 & 0 & 1 & 0 & 0 & 19 & 68 \\
\hline 7506.99 & 16 & 48 & 28 & 3 & 4 & 0 & 0 & 1 & 1 & 0 & 0 & 16 & 76 \\
\hline 7509.01 & 19 & 39 & 23 & 3 & 10 & 0 & 1 & 6 & 0 & 0 & 0 & 19 & 62 \\
\hline 7512.01 & 21 & 42 & 26 & 2 & 8 & 0 & 0 & 1 & 1 & 0 & 0 & 21 & 68 \\
\hline 7513.08 & 9 & 43 & 27 & 14 & 3 & 0 & 0 & 2 & 3 & 0 & 0 & 9 & 70 \\
\hline 7514.1 & 22 & 40 & 24 & 4 & 7 & 0 & 0 & 2 & 1 & 0 & 0 & 22 & 64 \\
\hline 7515.8 & 15 & 44 & 27 & 2 & 6 & 0 & 0 & 5 & 1 & 0 & 0 & 15 & 71 \\
\hline 7517.23 & 22 & 35 & 26 & 3 & 7 & 0 & 2 & 6 & 0 & 0 & 0 & 22 & 61 \\
\hline 7519.05 & 17 & 36 & 25 & 3 & 12 & 0 & 0 & 4 & 1 & 3 & 0 & 17 & 61 \\
\hline 7520.13 & 22 & 37 & 27 & 5 & 6 & 0 & 0 & 2 & 2 & 0 & 0 & 22 & 64 \\
\hline 7522.03 & 19 & 43 & 27 & 2 & 4 & 0 & 2 & 2 & 1 & 0 & 0 & 19 & 70 \\
\hline 7523.03 & 19 & 43 & 29 & 2 & 2 & 0 & 0 & 4 & 0 & 0 & 0 & 19 & 72 \\
\hline 7523.89 & 2 & 51 & 15 & 3 & 8 & 0 & 2 & 15 & 0 & 4 & 0 & 2 & 66 \\
\hline 7524.9 & 19 & 42 & 27 & 2 & 2 & 0 & 0 & 6 & 1 & 0 & 0 & 19 & 69 \\
\hline 7528.01 & 17 & 38 & 24 & 4 & 6 & 0 & 1 & 4 & 0 & 0 & 0 & 17 & 62 \\
\hline 7530.05 & 11 & 48 & 29 & 4 & 5 & 0 & 0 & 2 & 0 & 1 & 0 & 11 & 77 \\
\hline 7533.02 & 9 & 47 & 28 & 8 & 4 & 0 & 0 & 5 & 0 & 0 & 0 & 9 & 75 \\
\hline 7534.04 & 19 & 33 & 24 & 4 & 7 & 0 & 2 & 4 & 0 & 0 & 0 & 19 & 57 \\
\hline 7534.89 & 20 & 43 & 26 & 4 & 6 & 0 & 0 & 1 & 0 & 0 & 0 & 20 & 69 \\
\hline 7538.15 & 17 & 38 & 25 & 8 & 8 & 0 & 2 & 1 & 0 & 0 & 0 & 17 & 63 \\
\hline 7542.93 & 14 & 37 & 21 & 2 & 9 & 0 & 1 & 15 & 0 & 0 & 0 & 14 & 58 \\
\hline 7544.37 & 12 & 32 & 27 & 5 & 11 & 0 & 0 & 3 & 1 & 10 & 0 & 12 & 59 \\
\hline 7544.85 & 12 & 37 & 27 & 2 & 13 & 0 & 0 & 2 & 1 & 6 & 0 & 12 & 64 \\
\hline 7545.77 & 12 & 47 & 26 & 3 & 8 & 0 & 0 & 2 & 1 & 0 & 0 & 12 & 73 \\
\hline 7554.33 & 3 & 36 & 19 & 12 & 4 & 0 & 11 & 16 & 0 & 0 & 0 & 3 & 55 \\
\hline 7556.15 & 14 & 45 & 27 & 2 & 3 & 0 & 2 & 6 & 1 & 0 & 0 & 14 & 72 \\
\hline
\end{tabular}

Table B-1: RIR XRD, Depth in red indicates points in the Mahantango and purple points indicate points in the Marcellus. 
XRF-XRD Mineralogy:

\begin{tabular}{|c|c|c|c|c|c|c|c|c|c|}
\hline Sample & Quartz & Musc.+Ill. & Chlorite & Pyrite & Albite & Calcite & Dolomite & Barite & Normalization \\
\hline 7455 & 27.2 & 19.6 & 10.66 & 8.66 & 2.94 & 28.7 & 2.01 & 0.19 & 0.939 \\
\hline 7456 & 33 & 40.8 & 9.3 & 8.82 & 6.79 & 0.97 & 0 & 0.3 & 0.987 \\
\hline 7457.2 & 37.4 & 36.8 & 8.04 & 9.37 & 6.55 & 0.67 & 0.76 & 0.38 & 0.98 \\
\hline 7459 & 32.9 & 39.2 & 9.73 & 10.37 & 6.43 & 1.01 & 0 & 0.3 & 0.954 \\
\hline 7460.2 & 33.3 & 40.8 & 10.01 & 8.46 & 6.42 & 0.66 & 0 & 0.28 & 0.965 \\
\hline 7463 & 34.8 & 40.4 & 9.53 & 7.22 & 6.63 & 0.84 & 0 & 0.58 & 0.987 \\
\hline 7464 & 33.2 & 40 & 9.54 & 7.7 & 6.39 & 2.94 & 0 & 0.3 & 0.99 \\
\hline 7465 & 33.7 & 38.2 & 9.14 & 10.14 & 6.77 & 1.27 & 0 & 0.73 & 0.976 \\
\hline 7467.3 & 23.4 & 25.9 & 6.75 & 8.03 & 4.04 & 29.9 & 1.68 & 0.25 & 0.945 \\
\hline 7470.1 & 34.6 & 40.7 & 9.15 & 7.69 & 6.68 & 0.95 & 0 & 0.27 & 0.966 \\
\hline 7471 & 33.8 & 37.9 & 7.93 & 8.6 & 6.21 & 3.46 & 0.81 & 1.31 & 0.984 \\
\hline 7472 & 32.5 & 41 & 9.35 & 8.25 & 6.61 & 0.84 & 0 & 1.44 & 0.983 \\
\hline 7475 & 30.8 & 39 & 8.9 & 8.9 & 6.44 & 2.65 & 0 & 3.24 & 0.985 \\
\hline 7476 & 30.6 & 39.7 & 8.89 & 9.16 & 6.19 & 5.16 & 0 & 0.29 & 0.98 \\
\hline 7477 & 30.9 & 38.1 & 8.58 & 12.06 & 5.8 & 4.27 & 0 & 0.25 & 0.97 \\
\hline 7479 & 35.1 & 40.2 & 8.51 & 7.59 & 6.84 & 1.49 & 0 & 0.27 & 0.988 \\
\hline 7480.1 & 36.6 & 39.6 & 8.49 & 6.88 & 6.87 & 1.2 & 0 & 0.35 & 0.989 \\
\hline 7482.1 & 21.8 & 19.2 & 10.85 & 6.41 & 3.68 & 32.4 & 4.09 & 1.63 & 0.783 \\
\hline 7484 & 38.1 & 38.8 & 8.93 & 6.21 & 6.79 & 0.89 & 0 & 0.27 & 0.984 \\
\hline 7485 & 37.5 & 38.6 & 9.32 & 5.85 & 6.89 & 0.57 & 0.94 & 0.27 & 0.978 \\
\hline 7485.6 & 37.8 & 38 & 8.85 & 7.49 & 6.62 & 0.94 & 0 & 0.28 & 0.977 \\
\hline 7488 & 38.4 & 37.7 & 5.38 & 7.65 & 6.41 & 0.49 & 3.7 & 0.28 & 0.983 \\
\hline 7489 & 36.7 & 37.9 & 7.83 & 8.06 & 6.55 & 1.02 & 1.68 & 0.26 & 0.981 \\
\hline 7491.2 & 35.6 & 38.6 & 8.38 & 7.81 & 6.92 & 1.46 & 0.85 & 0.3 & 0.981 \\
\hline 7492 & 32.8 & 35.3 & 7.39 & 8.41 & 6.4 & 1.33 & 1.6 & 6.73 & 0.938 \\
\hline 7494 & 37.1 & 38 & 7.41 & 6.95 & 6.67 & 1.78 & 1.87 & 0.25 & 0.985 \\
\hline 7497 & 31.1 & 30.9 & 6.2 & 14.3 & 5.7 & 9 & 1.12 & 1.63 & 0.935 \\
\hline 7500.6 & 39.9 & 26.9 & 4.3 & 8.81 & 6.02 & 7.49 & 2.34 & 4.27 & 0.961 \\
\hline 7503 & 51.7 & 25.4 & 0 & 8.87 & 5.19 & 3.19 & 5.48 & 0.18 & 0.99 \\
\hline 7505 & 46.9 & 28.3 & 0 & 13.45 & 5.91 & 0 & 5.21 & 0.2 & 0.961 \\
\hline 7506 & 50.2 & 29.1 & 0 & 9.03 & 5.86 & 0.07 & 5.52 & 0.19 & 0.986 \\
\hline 7509 & 40.5 & 20.4 & 0 & 11.93 & 4.36 & 17.4 & 5.25 & 0.19 & 0.933 \\
\hline 7512 & 51.1 & 26.2 & 0 & 10.81 & 6 & 0.31 & 5.4 & 0.21 & 0.952 \\
\hline 7513 & 45.9 & 28.5 & 0 & 9.67 & 5.59 & 2.59 & 7.58 & 0.2 & 0.991 \\
\hline 7514 & 47.9 & 28.4 & 0 & 9.43 & 5.34 & 2.88 & 5.78 & 0.19 & 0.995 \\
\hline 7515 & 41.3 & 31.8 & 0 & 9.38 & 5.91 & 5.24 & 6.07 & 0.2 & 0.971 \\
\hline 7517 & 39.9 & 30 & 0 & 7.33 & 6.44 & 11 & 5.23 & 0.22 & 0.971 \\
\hline 7519 & 32.1 & 25.6 & 0 & 12.81 & 5.45 & 7.37 & 7.62 & 8.97 & 0.884 \\
\hline 7520.1 & 37.4 & 32.4 & 0 & 8.02 & 6.7 & 3.56 & 11.74 & 0.23 & 0.971 \\
\hline
\end{tabular}




\begin{tabular}{|r|r|r|r|r|r|r|r|r|r|}
\hline 7522 & 38.5 & 35.8 & 0 & 8.45 & 7.89 & 2.86 & 6.26 & 0.29 & 0.998 \\
\hline 7523 & 35.6 & 37.3 & 0 & 5.19 & 6.75 & 8.97 & 5.79 & 0.32 & 0.969 \\
\hline 7523.9 & 5.3 & 1.6 & 0 & 3.81 & 0.9 & 75.3 & 2.72 & 10.34 & 0.854 \\
\hline 7524 & 34.2 & 31.5 & 0 & 4.41 & 6.43 & 15.8 & 7.37 & 0.31 & 0.975 \\
\hline 7528 & 41.4 & 31.4 & 0 & 8.17 & 6.09 & 6.94 & 5.76 & 0.2 & 0.929 \\
\hline 7530 & 41.3 & 29.9 & 0 & 11.56 & 6.28 & 1.81 & 4.69 & 4.53 & 0.979 \\
\hline 7533 & 39.4 & 30.7 & 0 & 8.24 & 6.03 & 10.4 & 5.04 & 0.23 & 0.982 \\
\hline 7534 & 40.8 & 31.5 & 0 & 8.13 & 6.56 & 7.37 & 5.37 & 0.29 & 0.995 \\
\hline 7534.9 & 47.2 & 32 & 0 & 7.94 & 6.58 & 0.93 & 5.18 & 0.19 & 0.993 \\
\hline 7538 & 40.2 & 34.3 & 0 & 11.5 & 7.06 & 0 & 6.66 & 0.22 & 0.94 \\
\hline 7542.9 & 35.4 & 16 & 0 & 9.64 & 4.77 & 30.4 & 3.64 & 0.18 & 0.933 \\
\hline 7544.4 & 27.7 & 15.9 & 0 & 11.36 & 6.21 & 5.95 & 4.61 & 28.28 & 0.955 \\
\hline 7544.9 & 30.7 & 17.1 & 0 & 16.5 & 6.14 & 4.71 & 3.93 & 20.94 & 0.95 \\
\hline 7545 & 44 & 24.4 & 0 & 14.73 & 5.05 & 3.96 & 7.65 & 0.22 & 0.977 \\
\hline 7554.3 & 23.2 & 0 & 0 & 0.6 & 0 & 74.1 & 1.99 & 0.06 & 1.036 \\
\hline 7556 & 40.4 & 26.2 & 0 & 7.4 & 5.57 & 12 & 8.26 & 0.19 & 0.951 \\
\hline
\end{tabular}

Table B-2: XRF-XRD Mineralogy from Hupp and Donovan, 2018

\section{Appendix C: Shale Lithofacies PETRA Advanced Transform file}

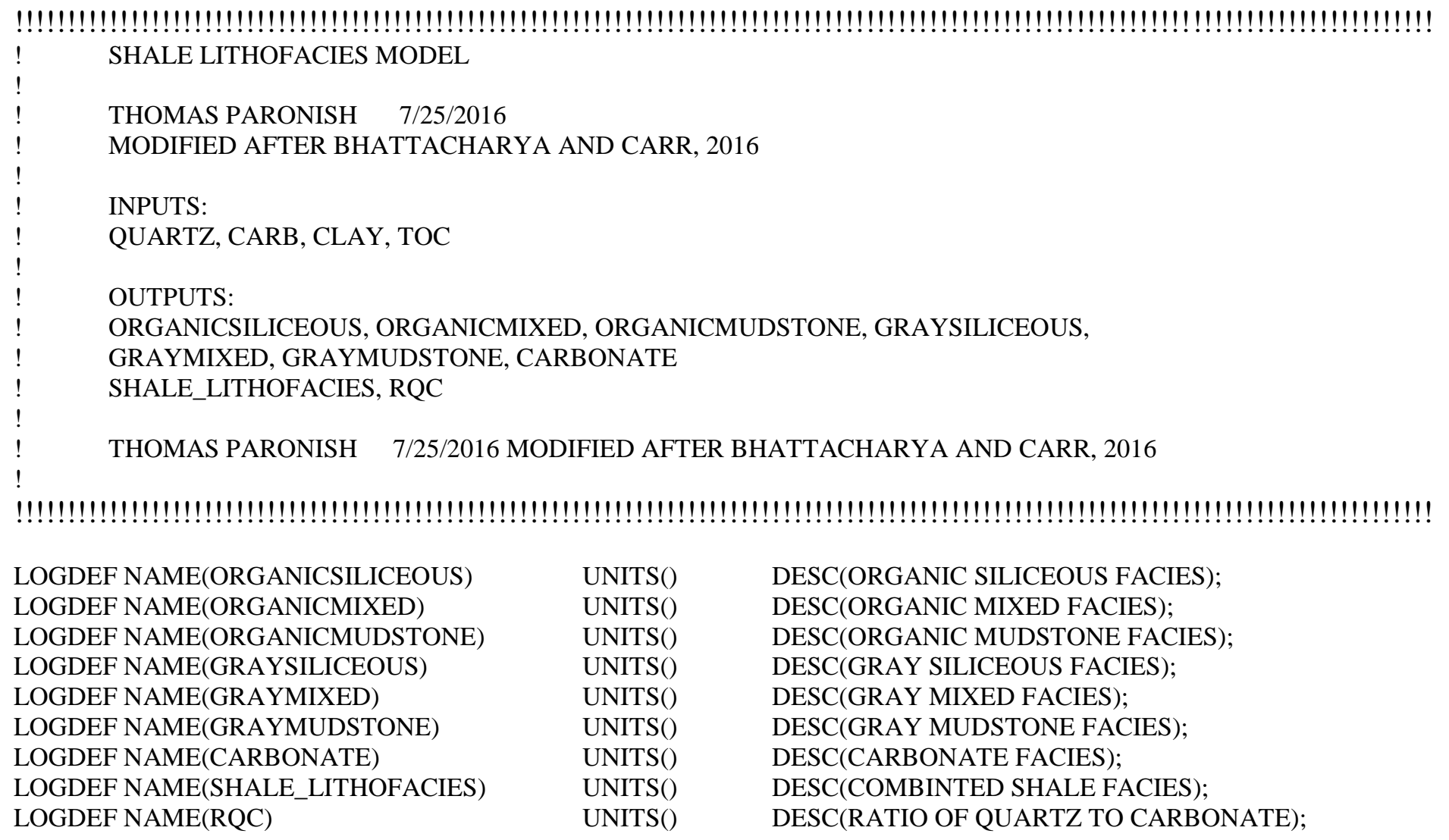


!!!!!!!!!!!!!!!!!!!!!!!!!!!!!!!!!!!!!!!!!!!!!!!!!!!!!!!!!!!!!!!!!!! ! INPUT LOGS USED IN THE MODEL

!!!!!!!!!!!!!!!!!!!!!!!!!!!!!!!!!!!!!!!!!!!!!!!!!!!!!!!!!!!!!!!

$\begin{array}{lll}\text { LOG } & \text { QUARTZ } & \text { IN; } \\ \text { LOG } & \text { CARB } & \text { IN; } \\ \text { LOG } & \text { CLAY } & \text { IN; } \\ \text { LOG } & \text { TOC } & \text { IN; }\end{array}$

!!!!!!!!!!!!!!!!!!!!!!!!!!!!!!!!!!!!!!!!!!!!!!!!!!!!!!!!!!!!!!! ! OUTPUT LOG USED IN THE MODEL

!!!!!!!!!!!!!!!!!!!!!!!!!!!!!!!!!!!!!!!!!!!!!!!!!!!!!!!!!!!!!!!!!!!

$\begin{array}{lll}\text { LOG } & \text { ORGANICSILICEOUS } & \text { OUT; } \\ \text { LOG } & \text { ORGANICMIXED } & \text { OUT; } \\ \text { LOG } & \text { ORGANICMUDSTONE } & \text { OUT; } \\ \text { LOG } & \text { GRAYSILICEOUS } & \text { OUT; } \\ \text { LOG } & \text { GRAYMUDSTONE } & \text { OUT; } \\ \text { LOG } & \text { GRAYMIXED } & \text { OUT; } \\ \text { LOG } & \text { CARBONATE } & \text { OUT; } \\ \text { LOG } & \text { SHALE_LITHOFACIES } & \text { OUT; } \\ \text { LOG } & \text { RQC } & \text { OUT; }\end{array}$

!!!!!!!!!!!!!!!!!!!!!!!!!!!!!!!!!!!!!!!!!!!!!!!!!!!!!!!!!!!!!!!!!!

! CONSTANTS USED IN THE MODEL

!!!!!!!!!!!!!!!!!!!!!!!!!!!!!!!!!!!!!!!!!!!!!!!!!!!!!!!!!!!!!!!!!

CONST TOC_CUTOFF 6.5;

CONST CLAY_CUTOFF $\quad 0.35$;

CONST RQC_SILICEOUS 3.0;

CONST RQC_MIXED_LS 0.3 ;

CONST NULL;

!!!!!!!!!!!!!!!!!!!!!!!!!!!!!!!!!!!!!!!!!!!!!!!!!!!!!!!!!!!!!!!!!

!!!!!!!!!!!!!!!!!!!!!!!!!!!!!!!!!!!!!!!!!!!!!!!!!!!!!!!!!!!!!!!!

!!

!! BEGIN MODEL EXECUTION SECTION

!!

!!!!!!!!!!!!!!!!!!!!!!!!!!!!!!!!!!!!!!!!!!!!!!!!!!!!!!!!!!!!!!!!!!!!

!!!!!!!!!!!!!!!!!!!!!!!!!!!!!!!!!!!!!!!!!!!!!!!!!!!!!!!!!!!!!!!!!!!

!!!!!!!!!!!!!!!!!!!!!!!!!!!!!!!!!!!!!!!!!!!!!!!!!!!!!!!!!!!!!!!!!!!!

!! INIT OUTPUTS TO NULL

!!!!!!!!!!!!!!!!!!!!!!!!!!!!!!!!!!!!!!!!!!!!!!!!!!!!!!!!!!!!!!!!!

ORGANICSILICEOUS = NULL;

ORGANICMIXED = NULL;

ORGANICMUDSTONE = NULL;

GRAYSILICEOUS = NULL;

GRAYMIXED = NULL;

GRAYMUDSTONE = NULL;

CARBONATE = NULL;

SHALE_LITHOFACIES = NULL; 
RQC NULL;

IF (CARB .NE. NULL) THEN BEGIN; ～～～CHECKS FOR NULL VALUES IN CARBONATE MINERALOGY !!!!!!!!!!!!!!!!!!!!!!!!!!!!!!!!!!

! CALCULATE RATIO OF QUARTZ TO CARBONATE (RQC)

!!!!!!!!!!!!!!!!!!!!!!!!!!!!!!!!!!

IF (CARB .EQ. 0) THEN BEGIN;

$\mathrm{RQC}=3.0$;

END;

ELSE BEGIN;

END;

$\mathrm{RQC}=((\mathrm{QUARTZ}) /(\mathrm{CARB})) ;$

!!!!!!!!!!!!!!!!!!!!!!!!!!!!!!!!!!!!

! LITHOFACIES LOGIC STATEMENTS

!!!!!!!!!!!!!!!!!!!!!!!!!!!!!!!!!!

! TEST FOR ORGANIC OR GRAY

IF (TOC .GE. TOC_CUTOFF) THEN GOTO ORGANIC;

ELSE GOTO GRAY;

ORGANIC:

IF (CLAY .GE. CLAY_CUTOFF) THEN BEGIN;

SHALE_LITHOFACIES $=5$;

GOTO DONE;

END;

ELSE GOTO OSILICIEOUSTEST;

OSILICIEOUSTEST:

IF (RQC .GE. RQC_SILICEOUS) THEN BEGIN; SHALE_LITHOFACIES $=7$;

GOTO DONE;

END;

ELSE BEGIN;

SHALE_LITHOFACIES $=6$;

GOTO DONE;

END;

GRAY:

IF (CLAY .GE. CLAY_CUTOFF) THEN BEGIN;

SHALE_LITHOFACIES $=2$;

GOTO DONE;

END;

ELSE GOTO GRSILICIEOUSTEST;

GRSILICIEOUSTEST:

IF (RQC .GE. RQC_SILICEOUS) THEN BEGIN;

GOTO DONE;

SHALE_LITHOFACIES $=4$;

END;

ELSE GOTO MIXEDTEST;

MIXEDTEST:

IF (RQC .GE. RQC_MIXED_LS) THEN BEGIN;

SHALE_LITHOFACIES $=3$;

GOTO DONE; 
END;

ELSE BEGIN;

SHALE_LITHOFACIES $=1$;

GOTO DONE;

END;

END;

ELSE BEGIN;

SHALE_LITHOFACIES = NULL;

END;

DONE:

ENDMOD;

!!!!!!!!!!!!!!!!!!!!!!!!!!!!!!!!!!!!!!!!!!!!!!!!!!!!!!!!!!!!!!!!!!!!!!!!!!!!!!!!!!!!!!!!!!!!!!!!!!!!!!!!!!!!!!!!!!!!!!!!!!!!!!!!!!!!!!!

\section{Appendix D: XRF Data Sets}

\section{Hamilton Suite:}

The Hamilton suite was run using WDXRF methods. Through this method the Hamilton suite outputs both elemental and oxides. To compare to the MSCL hhXRF suites we corrected oxides using variables from Table D-1.

\begin{tabular}{|l|r|c|}
\hline Oxide & Mol. Wt. & Factor \\
\hline $\mathrm{Al}_{2} \mathrm{O}_{3}$ & 101.9613 & 0.529251 \\
\hline $\mathrm{CaO}$ & 56.0794 & 0.714701 \\
\hline $\mathrm{FeO}$ & 172.1188 & 0.777311 \\
\hline $\mathrm{K}_{2} \mathrm{O}$ & 94.1954 & 0.830147 \\
\hline $\mathrm{MgO}$ & 40.3044 & 0.603036 \\
\hline $\mathrm{MnO}$ & 70.9374 & 0.774457 \\
\hline $\mathrm{Na}_{2} \mathrm{O}$ & 61.97894 & 0.741857 \\
\hline $\mathrm{P}_{2} \mathrm{O}_{5}$ & 141.9445 & 0.436421 \\
\hline $\mathrm{SO}_{3}$ & 80.0582 & 0.400459 \\
\hline $\mathrm{SiO}_{2}$ & 60.0848 & 0.467439 \\
\hline $\mathrm{TiO}_{2}$ & 79.8988 & 0.599508 \\
\hline
\end{tabular}

Table D-1: Oxide to Element conversions 


\begin{tabular}{|c|c|c|c|c|c|c|c|c|}
\hline \multicolumn{9}{|c|}{ CRMs run as unknowns } \\
\hline Sample & $\mathrm{SiO2}$ & TiO2 & $\mathrm{Al2O3}$ & $\mathrm{FeO}^{*}$ & MnO & $\mathrm{MgO}$ & $\mathrm{CaO}$ & $\mathrm{Na2O}$ \\
\hline AGV-2 & 58.97 & 1.044 & 16.82 & 6.12 & 0.098 & 1.77 & 5.13 & 4.16 \\
\hline BCR-2 & 54.07 & 2.280 & 13.49 & 12.41 & 0.197 & 3.63 & 7.17 & 3.18 \\
\hline BHVO-2 & 49.45 & 2.757 & 13.56 & 11.09 & 0.168 & 7.25 & 11.34 & 2.23 \\
\hline $\mathrm{G}-2$ & 68.68 & 0.492 & 15.14 & 2.44 & 0.033 & 0.76 & 1.91 & 4.08 \\
\hline SCo-1 & 62.50 & 0.605 & 13.43 & 4.69 & 0.051 & 2.63 & 2.57 & 0.93 \\
\hline STM-1 & 59.10 & 0.138 & 18.40 & 4.76 & 0.217 & 0.12 & 1.18 & 8.81 \\
\hline W-2 & 52.55 & 1.071 & 15.40 & 9.53 & 0.167 & 6.40 & 10.88 & 2.23 \\
\hline Sample & $\mathrm{K} 2 \mathrm{O}$ & P2O5 & $\mathrm{Cl}>=$ & SO3 >= & $\mathrm{Br}>=$ & As $>=$ & $\mathrm{Ni}$ & $\mathrm{Cr}$ \\
\hline AGV-2 & 2.90 & 0.474 & 0.01 & 0.00 & 1 & 0 & 19 & 14 \\
\hline BCR-2 & 1.79 & 0.360 & 0.02 & 0.05 & 1 & 3 & 12 & 17 \\
\hline BHVO-2 & 0.52 & 0.271 & 0.04 & 0.01 & 1 & 1 & 119 & 289 \\
\hline G-2 & 4.47 & 0.133 & 0.02 & 0.02 & 0 & 4 & 1 & 7 \\
\hline SCo-1 & 2.79 & 0.207 & 0.03 & 0.18 & 0 & 14 & 29 & 73 \\
\hline STM-1 & 4.24 & 0.159 & 0.05 & 0.00 & 2 & 4 & 5 & 4 \\
\hline W-2 & 0.63 & 0.125 & 0.02 & 0.03 & 0 & 0 & 66 & 81 \\
\hline Sample & $\mathbf{V}$ & Sc & $\mathrm{Cu}$ & $Z n$ & $\mathrm{Ga}$ & $\mathrm{Ba}$ & $\mathbf{R b}$ & Cs \\
\hline AGV-2 & 115 & 14 & 49 & 87 & 20 & 1115 & 67 & 0 \\
\hline BCR-2 & 414 & 33 & 17 & 131 & 21 & 679 & 46 & 2 \\
\hline BHVO-2 & 318 & 30 & 129 & 102 & 22 & 125 & 10 & 0 \\
\hline $\mathrm{G}-2$ & 34 & 4 & 11 & 84 & 23 & 1843 & 167 & 0 \\
\hline SCo-1 & 137 & 12 & 29 & 107 & 16 & 588 & 116 & 8 \\
\hline STM-1 & 3 & 1 & 6 & 245 & 35 & 572 & 113 & 3 \\
\hline W-2 & 266 & 37 & 104 & 76 & 17 & 168 & 21 & 2 \\
\hline Sample & $\mathrm{Sr}$ & $\bar{Y}$ & $\mathrm{Zr}$ & $\mathrm{Hf}$ & $\mathrm{Nb}$ & Mo & La & $\mathrm{Ce}$ \\
\hline AGV-2 & 665 & 21 & 232 & 6 & 13 & 0 & 39 & 67 \\
\hline BCR-2 & 344 & 37 & 190 & 6 & 14 & 257 & 20 & 52 \\
\hline BHVO-2 & 398 & 27 & 172 & 3 & 18 & 3 & 10 & 38 \\
\hline G-2 & 472 & 10 & 339 & 8 & 12 & 1 & 88 & 167 \\
\hline SCo-1 & 172 & 23 & 178 & 5 & 12 & 0 & 28 & 60 \\
\hline STM-1 & 710 & 46 & 1333 & 32 & 258 & 8 & 151 & 257 \\
\hline W-2 & 197 & 23 & 97 & 2 & 8 & 0 & 8 & 29 \\
\hline Sample & $\mathrm{Nd}$ & Th & $\mathbf{U}$ & $\mathrm{Pb}>=$ & 900 C LOI & sumMaj & sumAll & \\
\hline AGV-2 & 27 & 5 & 2 & 13 & 1.33 & 98.81 & 99.07 & \\
\hline BCR-2 & 27 & 6 & 2 & 11 & 1.09 & 99.65 & 99.95 & \\
\hline BHVO-2 & 23 & 0 & 0 & 2 & 0.58 & 99.22 & 99.44 & \\
\hline $\mathrm{G}-2$ & 52 & 25 & 1 & 30 & 0.53 & 98.66 & 99.04 & \\
\hline SCo-1 & 29 & 9 & 2 & 31 & 8.34 & 98.75 & 99.13 & \\
\hline STM-1 & 78 & 31 & 7 & 18 & 1.48 & 98.61 & 99.04 & \\
\hline W-2 & 17 & 4 & 3 & 8 & 0.59 & 99.58 & 99.75 & \\
\hline
\end{tabular}

Table D-2: Standards measured as unknowns Hamilton suite 


\begin{tabular}{|c|c|c|c|c|c|c|c|c|}
\hline \multicolumn{9}{|c|}{ CRMs values } \\
\hline Sample\# & SiO2\# & TiO2\# & Al203\# & $\mathrm{FeO}^{*} \#$ & MnO\# & MgO\# & $\mathrm{CaO \#}$ & Na2O\# \\
\hline AGV-2\# & 59.14 & 1.051 & 17.03 & 6.10 & 0.100 & 1.80 & 5.15 & 4.20 \\
\hline BCR-2\# & 54.00 & 2.265 & 13.48 & 12.39 & 0.197 & 3.60 & 7.11 & 3.12 \\
\hline BHVO-2\# & 49.60 & 2.731 & 13.44 & 11.15 & 0.169 & 7.26 & 11.40 & 2.22 \\
\hline G-2\# & 68.74 & 0.480 & 15.31 & 2.38 & 0.031 & 0.75 & 1.91 & 4.05 \\
\hline SCo-1 & 62.78 & 0.628 & 13.67 & 4.62 & 0.053 & 2.72 & 2.62 & 0.90 \\
\hline STM-1 & 59.65 & 0.135 & 18.39 & 4.70 & 0.220 & 0.10 & 1.09 & 8.94 \\
\hline W-2\# & 52.57 & 1.064 & 15.38 & 9.72 & 0.166 & 6.43 & 10.91 & 2.20 \\
\hline Sample\# & K2O\# & P2O5\# & $\mathrm{Cl}>=\#$ & SO3 >=\# & $\mathrm{Br}>=\#$ & As >=\# & $\mathrm{Ni \#}$ & Cr\# \\
\hline AGV-2\# & 2.90 & 0.483 & 0.01 & 0.01 & & 1 & 19 & 16 \\
\hline BCR-2\# & 1.77 & 0.359 & 0.01 & 0.04 & & 1 & 13 & 16 \\
\hline BHVO-2\# & 0.51 & 0.269 & 0.02 & 0.04 & 0 & 1 & 120 & 287 \\
\hline G-2\# & 4.50 & 0.129 & 0.01 & 0.03 & & 0 & 4 & 8 \\
\hline SCo-1 & 2.77 & 0.206 & 0.01 & 0.16 & 1 & 12 & 27 & 68 \\
\hline STM-1 & 4.28 & 0.158 & 0.05 & 0.01 & 2 & 5 & 3 & 4 \\
\hline W-2\# & 0.62 & 0.136 & 0.02 & 0.02 & & 1 & 72 & 92 \\
\hline Sample\# & V\# & Sc\# & Cu\# & Zn\# & Ga\# & Ba\# & Rb\# & Cs\# \\
\hline AGV-2\# & 119 & 13 & 52 & 87 & 20 & 1134 & 68 & 1 \\
\hline BCR-2\# & 418 & 34 & 20 & 130 & 22 & 684 & 46 & 1 \\
\hline BHVO-2\# & 318 & 32 & 129 & 104 & 21 & 131 & 9 & 0 \\
\hline G-2\# & 35 & 4 & 11 & 84 & 23 & 1860 & 169 & 1 \\
\hline SCo-1 & 131 & 12 & 29 & 100 & 15 & 570 & 110 & 8 \\
\hline STM-1 & 9 & 1 & 5 & 235 & 36 & 560 & 118 & 2 \\
\hline W-2\# & 266 & 36 & 106 & 78 & 18 & 173 & 20 & 1 \\
\hline Sample\# & Sr\# & Y\# & Zr\# & $\mathrm{Hf \#}$ & $\mathrm{Nb \#}$ & Mo\# & La\# & Ce\# \\
\hline AGV-2\# & 660 & 19 & 233 & 5 & 14 & 2 & 38 & 69 \\
\hline BCR-2\# & 337 & 36 & 187 & 5 & 12 & 251 & 25 & 53 \\
\hline BHVO-2\# & 394 & 26 & 171 & 5 & 18 & 4 & 15 & 38 \\
\hline G-2\# & 475 & 10 & 319 & 8 & 12 & 0 & 88 & 161 \\
\hline SCo-1 & 170 & 26 & 169 & 5 & 11 & 1 & 29 & 57 \\
\hline STM-1 & 700 & 46 & 1308 & 29 & 249 & 5 & 151 & 259 \\
\hline W-2\# & 195 & 22 & 93 & 2 & 8 & 1 & 11 & 23 \\
\hline Sample\# & Nd\# & Th\# & U\# & $\mathrm{Pb}>=$ & 900 C LOI\# & sumMaj\# & sumAll\# & \\
\hline AGV-2\# & 31 & 6 & 2 & & 1.33 & 99.29 & 99.57 & \\
\hline BCR-2\# & 28 & 6 & 2 & & 1.09 & 99.39 & 99.68 & \\
\hline BHVO-2\# & 24 & 1 & 0 & & 0.58 & 99.33 & 99.57 & \\
\hline G-2\# & 54 & 25 & 2 & & 0.53 & 98.81 & 99.18 & \\
\hline SCo-1 & 26 & 9 & 3 & & 8.34 & 99.30 & 99.63 & \\
\hline STM-1 & 79 & 29 & 9 & & 1.48 & 99.14 & 99.58 & \\
\hline W-2\# & 13 & 2 & 1 & & 0.59 & 99.79 & 99.95 & \\
\hline
\end{tabular}

Table D-3: Actual values of standards, \# indicates data derived from Jochum et al 2015 Geostandards and Geoanalytical Research 40, 333-350 
Table D-4, Hamilton XRF Suite:

\begin{tabular}{|c|c|c|c|c|c|c|c|c|c|c|}
\hline Sample & Time/Date & SiO2 (\%) & Si (\%) & TiO2 (\%) & $\mathrm{Ti}(\%)$ & Al2O3 (\%) & Al (\%) & $\mathrm{FeO}^{*}(\%)$ & Fe (\%) & MnO (\%) \\
\hline 7447.20 & $17: 45$ 6-3-17 & 58.54 & 27.37 & 0.69 & 0.41 & 16.52 & 8.74 & 5.86 & 4.56 & 0.02 \\
\hline 7448.35 & 20:21 6-3-17 & 57.98 & 27.10 & 0.72 & 0.43 & 17.18 & 9.09 & 5.71 & 4.44 & 0.02 \\
\hline 7449.25 & 22:57 6-3-17 & 57.34 & 26.80 & 0.74 & 0.44 & 17.50 & 9.26 & 6.66 & 5.18 & 0.02 \\
\hline 7450.20 & 1:33 7-3-17 & 59.40 & 27.77 & 0.71 & 0.42 & 16.73 & 8.85 & 5.84 & 4.54 & 0.02 \\
\hline 7452.22 & $6: 457-3-17$ & 60.10 & 28.09 & 0.67 & 0.40 & 15.72 & 8.32 & 6.03 & 4.69 & 0.02 \\
\hline 7455.00 & 11:35 7-3-17 & 40.83 & 19.08 & 0.55 & 0.33 & 12.41 & 6.57 & 6.82 & 5.30 & 0.07 \\
\hline 7456.16 & 14:11 7-3-17 & 53.01 & 24.78 & 0.73 & 0.44 & 18.86 & 9.98 & 6.03 & 4.69 & 0.02 \\
\hline 7457.15 & 16:47 7-3-17 & 55.14 & 25.78 & 0.70 & 0.42 & 17.03 & 9.01 & 6.18 & 4.81 & 0.02 \\
\hline 7457.37 & 4:09 7-3-17 & 59.77 & 27.94 & 0.65 & 0.39 & 15.68 & 8.30 & 6.89 & 5.36 & 0.02 \\
\hline 7459.13 & $20: 43$ 15-12-16 & 53.09 & 24.81 & 0.71 & 0.42 & 18.71 & 9.90 & 7.05 & 5.48 & 0.02 \\
\hline 7460.20 & $22: 5315-12-16$ & 53.53 & 25.02 & 0.71 & 0.42 & 19.12 & 10.12 & 5.97 & 4.64 & 0.02 \\
\hline 7463.10 & 19:23 7-3-17 & 55.76 & 26.06 & 0.76 & 0.46 & 19.12 & 10.12 & 5.33 & 4.15 & 0.02 \\
\hline 7464.05 & 21:59 7-3-17 & 53.12 & 24.83 & 0.70 & 0.42 & 18.50 & 9.79 & 5.52 & 4.29 & 0.02 \\
\hline 7465.30 & 0:35 8-3-17 & 53.21 & 24.87 & 0.67 & 0.40 & 17.88 & 9.46 & 6.81 & 5.29 & 0.02 \\
\hline 7467.30 & 3:118-3-17 & 39.31 & 18.37 & 0.49 & 0.29 & 13.40 & 7.09 & 5.75 & 4.47 & 0.06 \\
\hline 7470.05 & 1:03 16-12-16 & 54.87 & 25.65 & 0.68 & 0.41 & 18.99 & 10.05 & 5.46 & 4.25 & 0.02 \\
\hline 7471.13 & 5:47 8-3-17 & 51.90 & 24.26 & 0.63 & 0.38 & 17.11 & 9.06 & 5.73 & 4.45 & 0.02 \\
\hline 7472.09 & 8:23 8-3-17 & 51.59 & 24.12 & 0.68 & 0.41 & 18.17 & 9.62 & 5.62 & 4.37 & 0.02 \\
\hline 7475.12 & 10:59 8-3-17 & 49.81 & 23.28 & 0.67 & 0.40 & 17.74 & 9.39 & 5.99 & 4.66 & 0.02 \\
\hline 7476.15 & 13:35 8-3-17 & 50.75 & 23.72 & 0.67 & 0.40 & 18.13 & 9.59 & 6.25 & 4.86 & 0.02 \\
\hline 7477.07 & 16:11 8-3-17 & 50.69 & 23.69 & 0.70 & 0.42 & 17.78 & 9.41 & 7.90 & 6.14 & 0.02 \\
\hline 7479.05 & 18:47 8-3-17 & 53.43 & 24.98 & 0.70 & 0.42 & 18.07 & 9.57 & 5.15 & 4.01 & 0.02 \\
\hline 7480.05 & 21:23 8-3-17 & 56.48 & 26.40 & 0.78 & 0.47 & 18.40 & 9.74 & 4.95 & 3.85 & 0.02 \\
\hline 7482.12 & $3: 13$ 16-12-16 & 35.33 & 16.51 & 0.40 & 0.24 & 9.95 & 5.27 & 5.44 & 4.23 & 0.06 \\
\hline 7484.11 & 23:59 8-3-17 & 57.21 & 26.74 & 0.71 & 0.43 & 17.63 & 9.33 & 4.62 & 3.59 & 0.02 \\
\hline 7485.03 & 2:35 9-3-17 & 56.92 & 26.61 & 0.72 & 0.43 & 17.56 & 9.29 & 4.49 & 3.49 & 0.02 \\
\hline 7485.60 & 5:10 9-3-17 & 57.79 & 27.01 & 0.72 & 0.43 & 17.63 & 9.33 & 5.43 & 4.22 & 0.02 \\
\hline 7488.15 & 7:46 9-3-17 & 57.15 & 26.71 & 0.74 & 0.44 & 17.16 & 9.08 & 5.01 & 3.89 & 0.02 \\
\hline 7489.06 & 10:22 9-3-17 & 56.22 & 26.28 & 0.74 & 0.44 & 17.52 & 9.27 & 5.57 & 4.33 & 0.02 \\
\hline 7491.22 & 12:58 9-3-17 & 55.26 & 25.83 & 0.76 & 0.45 & 17.67 & 9.35 & 5.45 & 4.24 & 0.02 \\
\hline 7492.06 & $5: 2216-12-16$ & 52.84 & 24.70 & 0.66 & 0.40 & 17.08 & 9.04 & 5.87 & 4.56 & 0.02 \\
\hline 7494.20 & 15:34 9-3-17 & 55.85 & 26.11 & 0.70 & 0.42 & 17.20 & 9.10 & 4.83 & 3.76 & 0.02 \\
\hline 7497.39 & 18:10 9-3-17 & 48.22 & 22.54 & 0.59 & 0.36 & 14.31 & 7.57 & 9.00 & 7.00 & 0.03 \\
\hline
\end{tabular}




\begin{tabular}{|c|c|c|c|c|c|c|c|c|c|c|}
\hline Sample & Time/Date & $\mathrm{SiO2}(\%)$ & Si (\%) & TiO2 (\%) & $\mathrm{Ti}(\%)$ & Al2O3 (\%) & Al (\%) & $\mathrm{FeO}^{*}(\%)$ & $\mathrm{Fe}(\%)$ & MnO (\%) \\
\hline 7500.60 & 20:46 9-3-17 & 53.90 & 25.19 & 0.52 & 0.31 & 12.07 & 6.39 & 5.55 & 4.32 & 0.03 \\
\hline 7503.04 & 23:22 9-3-17 & 60.00 & 28.05 & 0.47 & 0.28 & 10.90 & 5.77 & 4.74 & 3.68 & 0.02 \\
\hline 7505.93 & 1:58 10-3-17 & 59.08 & 27.62 & 0.57 & 0.34 & 12.59 & 6.66 & 7.41 & 5.76 & 0.01 \\
\hline 7506.99 & 4:34 10-3-17 & 59.95 & 28.02 & 0.54 & 0.33 & 12.32 & 6.52 & 4.77 & 3.71 & 0.01 \\
\hline 7509.01 & 7:10 10-3-17 & 51.75 & 24.19 & 0.41 & 0.25 & 9.30 & 4.92 & 6.97 & 5.41 & 0.03 \\
\hline 7513.08 & 9:46 10-3-17 & 53.85 & 25.17 & 0.52 & 0.31 & 11.53 & 6.10 & 4.94 & 3.84 & 0.02 \\
\hline 7514.10 & $12: 22$ 10-3-17 & 56.98 & 26.63 & 0.55 & 0.33 & 11.84 & 6.27 & 4.95 & 3.85 & 0.01 \\
\hline 7515.80 & $14: 5810-3-17$ & 54.51 & 25.48 & 0.64 & 0.39 & 13.59 & 7.19 & 5.07 & 3.94 & 0.02 \\
\hline 7517.23 & 22:09 11-3-17 & 53.86 & 25.18 & 0.60 & 0.36 & 13.02 & 6.89 & 4.05 & 3.15 & 0.02 \\
\hline 7519.05 & $11: 5216-12-16$ & 46.23 & 21.61 & 0.57 & 0.34 & 12.01 & 6.36 & 7.40 & 5.75 & 0.02 \\
\hline 7524.90 & $4: 2112-3-17$ & 49.83 & 23.29 & 0.73 & 0.44 & 13.75 & 7.28 & 2.46 & 1.91 & 0.03 \\
\hline 7528.01 & $14: 0216-12-16$ & 54.91 & 25.67 & 0.63 & 0.38 & 13.32 & 7.05 & 4.45 & 3.46 & 0.02 \\
\hline 7530.15 & $6: 3411-3-17$ & 55.29 & 25.84 & 0.58 & 0.35 & 12.58 & 6.66 & 6.42 & 4.99 & 0.01 \\
\hline 7533.02 & 9:09 11-3-17 & 54.36 & 25.41 & 0.58 & 0.35 & 13.00 & 6.88 & 4.62 & 3.59 & 0.02 \\
\hline 7534.04 & $11: 4511-3-17$ & 54.88 & 25.65 & 0.60 & 0.36 & 13.12 & 6.95 & 4.45 & 3.46 & 0.02 \\
\hline 7534.89 & $14: 2111-3-17$ & 59.84 & 27.97 & 0.60 & 0.36 & 13.14 & 6.96 & 4.26 & 3.31 & 0.01 \\
\hline 7538.15 & $16: 12$ 16-12-16 & 53.01 & 24.78 & 0.56 & 0.33 & 13.62 & 7.21 & 5.96 & 4.63 & 0.01 \\
\hline 7542.93 & $16: 5711-3-17$ & 41.87 & 19.57 & 0.29 & 0.18 & 6.65 & 3.52 & 5.24 & 4.07 & 0.02 \\
\hline 7544.37 & $19: 3311-3-17$ & 32.00 & 14.96 & 0.23 & 0.14 & 6.02 & 3.18 & 5.52 & 4.29 & 0.02 \\
\hline 7544.85 & 6:57 12-3-17 & 36.60 & 17.11 & 0.29 & 0.17 & 6.75 & 3.57 & 8.42 & 6.55 & 0.02 \\
\hline
\end{tabular}




\begin{tabular}{|c|c|c|c|c|c|c|c|c|c|c|c|c|}
\hline Sample & Mn (\%) & MgO (\%) & Mg (\%) & $\mathrm{CaO}(\%)$ & $\mathrm{Ca}(\%)$ & Na2O (\%) & $\mathrm{Na}(\%)$ & K2O (\%) & K (\%) & P2O5 (\%) & P (\%) & SO3 >= (\%) \\
\hline 7447.20 & 0.02 & 1.40 & 0.84 & 0.86 & 0.62 & 0.67 & 0.49 & 3.97 & 3.30 & 0.08 & 0.07 & 0.45 \\
\hline 7448.35 & 0.01 & 1.38 & 0.83 & 0.42 & 0.30 & 0.63 & 0.28 & 4.13 & 3.43 & 0.07 & 0.05 & 0.27 \\
\hline 7449.25 & 0.01 & 1.41 & 0.85 & 0.36 & 0.26 & 0.71 & 0.29 & 4.20 & 3.49 & 0.06 & 0.05 & 0.34 \\
\hline 7450.20 & 0.02 & 1.34 & 0.81 & 0.43 & 0.31 & 0.70 & 0.33 & 4.02 & 3.34 & 0.07 & 0.06 & 0.31 \\
\hline 7452.22 & 0.01 & 1.28 & 0.77 & 0.30 & 0.21 & 0.69 & 0.41 & 3.78 & 3.14 & 0.07 & 0.06 & 0.27 \\
\hline 7455.00 & 0.06 & 2.23 & 1.34 & 16.68 & 11.92 & 0.35 & 0.23 & 2.43 & 2.01 & 0.06 & 0.05 & 1.77 \\
\hline 7456.16 & 0.01 & 1.40 & 0.85 & 0.49 & 0.35 & 0.72 & 0.00 & 4.54 & 3.77 & 0.07 & 0.06 & 0.36 \\
\hline 7457.15 & 0.01 & 1.37 & 0.82 & 0.55 & 0.39 & 0.70 & 0.00 & 4.12 & 3.42 & 0.07 & 0.06 & 0.30 \\
\hline 7457.37 & 0.02 & 1.36 & 0.82 & 0.98 & 0.70 & 0.68 & 0.00 & 3.84 & 3.19 & 0.09 & 0.08 & 0.56 \\
\hline 7459.13 & 0.01 & 1.49 & 0.90 & 0.52 & 0.37 & 0.69 & 0.00 & 4.44 & 3.68 & 0.07 & 0.06 & 0.14 \\
\hline 7460.20 & 0.01 & 1.52 & 0.92 & 0.33 & 0.24 & 0.68 & 0.00 & 4.56 & 3.79 & 0.08 & \begin{tabular}{|l|}
0.06 \\
\end{tabular} & 0.42 \\
\hline 7463.10 & 0.01 & 1.47 & 0.89 & 0.43 & 0.31 & 0.72 & 0.00 & 4.60 & 3.82 & 0.08 & \begin{tabular}{|l|}
0.07 \\
\end{tabular} & 0.33 \\
\hline 7464.05 & 0.02 & 1.45 & 0.88 & 1.49 & 1.07 & 0.68 & 0.00 & 4.49 & 3.73 & 0.07 & \begin{tabular}{|l|}
0.06 \\
\end{tabular} & 0.76 \\
\hline 7465.30 & 0.01 & 1.40 & 0.84 & 0.65 & 0.46 & 0.73 & 0.00 & 4.31 & 3.57 & 0.07 & 0.06 & 0.44 \\
\hline 7467.30 & 0.05 & 1.48 & 0.89 & 16.98 & 12.14 & 0.47 & 0.00 & 3.16 & 2.62 & 0.07 & 0.06 & 3.10 \\
\hline 7470.05 & 0.01 & 1.40 & 0.84 & 0.48 & 0.35 & 0.72 & 0.00 & 4.57 & 3.80 & 0.07 & 0.06 & 0.22 \\
\hline 7471.13 & 0.02 & 1.36 & 0.82 & 1.97 & 1.41 & 0.66 & 0.00 & 4.22 & 3.50 & 0.07 & 0.06 & 1.25 \\
\hline 7472.09 & 0.01 & 1.38 & 0.83 & 0.42 & 0.30 & 0.69 & 0.00 & 4.47 & 3.71 & 0.07 & 0.06 & 0.50 \\
\hline 7475.12 & 0.01 & 1.34 & 0.81 & 1.33 & 0.95 & 0.68 & 0.00 & 4.32 & 3.59 & 0.30 & 0.25 & 1.07 \\
\hline 7476.15 & 0.02 & 1.36 & 0.82 & 2.64 & 1.89 & 0.67 & 0.00 & 4.48 & 3.72 & 0.06 & 0.05 & 1.50 \\
\hline 7477.07 & 0.01 & 1.33 & 0.80 & 2.21 & 1.58 & 0.63 & 0.00 & 4.36 & 3.62 & 0.07 & 0.06 & 1.28 \\
\hline 7479.05 & 0.01 & 1.26 & 0.76 & 0.73 & 0.52 & 0.71 & 0.00 & 4.38 & 3.64 & 0.06 & 0.05 & 0.43 \\
\hline 7480.05 & 0.01 & 1.30 & 0.78 & 0.62 & 0.44 & 0.74 & 0.00 & 4.47 & 3.71 & 0.06 & 0.05 & 0.35 \\
\hline 7482.12 & 0.04 & 1.80 & 1.08 & 19.15 & 13.68 & 0.43 & 0.00 & 2.34 & 1.95 & 0.07 & 0.06 & 0.94 \\
\hline 7484.11 & 0.01 & 1.36 & 0.82 & 0.45 & 0.32 & 0.73 & 0.00 & 4.36 & 3.62 & 0.06 & 0.05 & 0.25 \\
\hline 7485.03 & 0.01 & 1.42 & 0.86 & 0.55 & 0.39 & 0.74 & 0.00 & 4.35 & 3.61 & 0.07 & 0.06 & 0.30 \\
\hline 7485.60 & 0.01 & 1.38 & 0.83 & 0.49 & 0.35 & 0.73 & 0.00 & 4.37 & 3.63 & 0.06 & 0.05 & 0.22 \\
\hline 7488.15 & 0.02 & 1.58 & 0.96 & 1.30 & 0.93 & 0.70 & 0.00 & 4.32 & 3.58 & 0.06 & 0.05 & 0.83 \\
\hline 7489.06 & 0.02 & 1.55 & 0.94 & 1.00 & 0.72 & 0.71 & 0.00 & 4.33 & 3.60 & 0.05 & 0.05 & 0.51 \\
\hline 7491.22 & 0.02 & 1.45 & 0.88 & 0.98 & 0.70 & 0.75 & 0.00 & 4.37 & 3.62 & 0.06 & \begin{tabular}{|l|}
0.05 \\
\end{tabular} & 0.37 \\
\hline 7492.06 & 0.02 & 1.51 & 0.91 & 1.17 & 0.84 & 0.72 & 0.00 & 4.16 & 3.45 & 0.06 & 0.05 & 0.35 \\
\hline 7494.20 & 0.01 & 1.51 & 0.91 & 1.43 & 1.02 & 0.72 & 0.00 & 4.29 & 3.56 & 0.06 & 0.05 & 0.53 \\
\hline 7497.39 & 0.02 & 1.22 & 0.73 & 5.09 & 3.64 & 0.64 & 0.00 & 3.62 & 3.00 & 0.08 & 0.07 & 2.67 \\
\hline
\end{tabular}




\begin{tabular}{|c|c|c|c|c|c|c|c|c|c|c|c|c|}
\hline Sample & Mn (\%) & MgO (\%) & Mg (\%) & $\mathrm{CaO}(\%)$ & $\mathrm{Ca}(\%)$ & Na2O (\%) & $\mathrm{Na}(\%)$ & K2O (\%) & K (\%) & P2O5 (\%) & P (\%) & SO3 >= (\%) \\
\hline 7500.60 & 0.02 & 1.15 & 0.69 & 4.59 & 3.28 & 0.66 & 0.00 & 3.11 & 2.58 & 0.06 & 0.05 & 2.84 \\
\hline 7503.04 & 0.01 & 1.07 & 0.64 & 3.08 & 2.20 & 0.55 & 0.00 & 2.80 & 2.32 & 0.08 & 0.06 & 1.20 \\
\hline 7505.93 & 0.01 & 1.05 & 0.63 & 1.00 & 0.71 & 0.64 & 0.00 & 3.22 & 2.67 & 0.07 & 0.06 & 0.45 \\
\hline 7506.99 & 0.01 & 1.06 & 0.64 & 1.51 & 1.08 & 0.61 & 0.00 & 3.18 & 2.64 & 0.08 & 0.06 & 0.83 \\
\hline 7509.01 & 0.02 & 1.12 & 0.68 & 11.04 & 7.89 & 0.50 & 0.00 & 2.46 & 2.04 & 0.18 & 0.15 & 4.70 \\
\hline 7513.08 & 0.01 & 1.41 & 0.85 & 3.20 & 2.29 & 0.56 & 0.00 & 3.01 & 2.50 & 0.09 & 0.07 & 1.48 \\
\hline 7514.10 & 0.01 & 1.11 & 0.67 & 2.96 & 2.11 & 0.55 & 0.00 & 3.08 & 2.56 & 0.10 & 0.08 & 0.95 \\
\hline 7515.80 & 0.01 & 1.20 & 0.72 & 4.32 & 3.09 & 0.63 & 0.00 & 3.55 & 2.95 & 0.11 & 0.09 & 2.19 \\
\hline 7517.23 & 0.01 & 1.05 & 0.64 & 7.12 & 5.09 & 0.70 & 0.00 & 3.42 & 2.84 & 0.08 & 0.07 & 3.11 \\
\hline 7519.05 & 0.02 & 1.61 & 0.97 & 6.21 & 4.44 & 0.62 & 0.00 & 3.06 & 2.54 & 0.08 & 0.06 & 0.64 \\
\hline 7524.90 & 0.02 & 1.50 & 0.91 & 10.34 & 7.39 & 0.71 & 0.00 & 3.63 & 3.01 & 0.13 & 0.11 & 2.02 \\
\hline 7528.01 & 0.01 & 1.14 & 0.69 & 5.12 & 3.66 & 0.65 & 0.00 & 3.53 & 2.93 & 0.08 & 0.06 & 0.34 \\
\hline 7530.15 & 0.01 & 0.95 & 0.57 & 2.26 & 1.62 & 0.69 & 0.00 & 3.42 & 2.84 & 0.07 & 0.06 & 1.01 \\
\hline 7533.02 & 0.01 & 1.03 & 0.62 & 6.88 & 4.92 & 0.67 & 0.00 & 3.56 & 2.95 & 0.07 & \begin{tabular}{|l|}
0.05 \\
\end{tabular} & 2.00 \\
\hline 7534.04 & 0.01 & 1.07 & 0.65 & 5.26 & 3.76 & 0.71 & 0.00 & 3.55 & 2.95 & 0.07 & 0.05 & 1.08 \\
\hline 7534.89 & 0.01 & 1.02 & 0.61 & 1.88 & 1.34 & 0.70 & 0.00 & 3.55 & 2.94 & 0.10 & 0.08 & 0.60 \\
\hline 7538.15 & 0.01 & 1.26 & 0.76 & 1.65 & 1.18 & 0.72 & 0.00 & 3.68 & 3.05 & 0.07 & 0.06 & 0.54 \\
\hline 7542.93 & 0.01 & 0.72 & 0.44 & 16.44 & 11.75 & 0.51 & 0.00 & 1.80 & 1.49 & 0.08 & 0.06 & 2.06 \\
\hline 7544.37 & 0.02 & 0.82 & 0.49 & 3.84 & 2.75 & 0.59 & 0.00 & 1.60 & 1.33 & 0.07 & 0.05 & 1.85 \\
\hline 7544.85 & 0.01 & 0.73 & 0.44 & 3.27 & 2.34 & 0.62 & 0.00 & 1.80 & 1.50 & 0.08 & 0.07 & 1.60 \\
\hline
\end{tabular}




\begin{tabular}{|c|c|c|c|c|c|c|c|c|}
\hline Sample & $S>=(\%)$ & $\mathrm{Cl}>=(\%)$ & $\mathrm{Br}>=(\mathrm{ppm})$ & As $>=(p p m)$ & $\mathrm{Ni}(\mathrm{ppm})$ & $\operatorname{Cr}(p p m)$ & V (ppm) & Sc (ppm) \\
\hline 7447.20 & 0.18 & 0.00 & 1 & 22 & 105 & 104 & 398 & 16 \\
\hline 7448.35 & 0.11 & 0.00 & 2 & 24 & 124 & 105 & 441 & 18 \\
\hline 7449.25 & 0.14 & 0.00 & 0 & 31 & 135 & 102 & 385 & 15 \\
\hline 7450.20 & 0.12 & 0.00 & 1 & 21 & 96 & 100 & 361 & 17 \\
\hline 7452.22 & 0.11 & 0.00 & 0 & 23 & 98 & 87 & 315 & 17 \\
\hline 7455.00 & 0.71 & 0.01 & 3 & 6 & 28 & 54 & 111 & 15 \\
\hline 7456.16 & 0.14 & 0.00 & 0 & 23 & 170 & 94 & 343 & 21 \\
\hline 7457.15 & 0.12 & 0.00 & 1 & 24 & 179 & 96 & 436 & 19 \\
\hline 7457.37 & 0.22 & 0.00 & 1 & 24 & 135 & 89 & 322 & 18 \\
\hline 7459.13 & 0.05 & 0.01 & 1 & 42 & 165 & 119 & 444 & 19 \\
\hline 7460.20 & 0.17 & 0.01 & 2 & 32 & 165 & 103 & 421 & 20 \\
\hline 7463.10 & 0.13 & 0.00 & 4 & 16 & 118 & 92 & 277 & 19 \\
\hline 7464.05 & 0.30 & 0.00 & 0 & 14 & 144 & 90 & 272 & 20 \\
\hline 7465.30 & 0.18 & 0.00 & 2 & 25 & 144 & 96 & 286 & 15 \\
\hline 7467.30 & 1.24 & 0.01 & 2 & 21 & 104 & 62 & 189 & 15 \\
\hline 7470.05 & 0.09 & 0.02 & 1 & 20 & 153 & 100 & 365 & 18 \\
\hline 7471.13 & 0.50 & 0.00 & 0 & 27 & 180 & 89 & 389 & 18 \\
\hline 7472.09 & 0.20 & 0.00 & 1 & 21 & 198 & 94 & 499 & 19 \\
\hline 7475.12 & 0.43 & 0.00 & 0 & 28 & 184 & 85 & 346 & 17 \\
\hline 7476.15 & 0.60 & 0.00 & 2 & 25 & 163 & 88 & 301 & 22 \\
\hline 7477.07 & 0.51 & 0.00 & 0 & 42 & 155 & 88 & 329 & 23 \\
\hline 7479.05 & 0.17 & 0.00 & 3 & 14 & 160 & 100 & 347 & 20 \\
\hline 7480.05 & 0.14 & 0.00 & 2 & 17 & 118 & 98 & 285 & 19 \\
\hline 7482.12 & 0.38 & 0.07 & 0 & 19 & 52 & 52 & 94 & 7 \\
\hline 7484.11 & 0.10 & 0.00 & 2 & 11 & 142 & 85 & 332 & 19 \\
\hline 7485.03 & 0.12 & 0.00 & 1 & 16 & 121 & 89 & 291 & 19 \\
\hline 7485.60 & 0.09 & 0.00 & 0 & 14 & 113 & 85 & 254 & 18 \\
\hline 7488.15 & 0.33 & 0.00 & 1 & 13 & 85 & 84 & 189 & 19 \\
\hline 7489.06 & 0.20 & 0.00 & 1 & 18 & 106 & 92 & 217 & 18 \\
\hline 7491.22 & 0.15 & 0.00 & 0 & 19 & 120 & 93 & 250 & 20 \\
\hline 7492.06 & 0.14 & 0.03 & 0 & 23 & 152 & 89 & 220 & 19 \\
\hline 7494.20 & 0.21 & 0.00 & 3 & 20 & 124 & 94 & 282 & 19 \\
\hline 7497.39 & 1.07 & 0.00 & 1 & 53 & 171 & 84 & 291 & 20 \\
\hline
\end{tabular}




\begin{tabular}{|c|c|c|c|c|c|c|c|c|}
\hline Sample & $S>=(\%)$ & $\mathrm{Cl}>=(\%)$ & $\mathrm{Br}>=(\mathrm{ppm})$ & As >= (ppm) & $\mathrm{Ni}$ (ppm) & $\mathrm{Cr}$ (ppm) & V (ppm) & Sc (ppm) \\
\hline 7500.60 & 1.14 & 0.00 & 0 & 20 & 193 & 79 & 448 & 17 \\
\hline 7503.04 & 0.48 & 0.00 & 0 & 23 & 181 & 81 & 477 & 16 \\
\hline 7503.98 & 0.11 & 0.04 & 4 & 22 & 177 & 73 & 461 & 14 \\
\hline 7505.93 & 0.18 & 0.00 & 4 & 31 & 154 & 80 & 412 & 15 \\
\hline 7506.99 & 0.33 & 0.00 & 3 & 23 & 166 & 78 & 459 & 14 \\
\hline 7509.01 & 1.88 & 0.00 & 1 & 35 & 175 & 56 & 261 & 17 \\
\hline 7512.01 & 0.07 & 0.03 & 4 & 18 & 77 & 81 & 507 & 15 \\
\hline 7513.08 & 0.59 & 0.00 & 1 & 29 & 224 & 81 & 622 & 19 \\
\hline 7514.10 & 0.38 & 0.00 & 3 & 32 & 188 & 81 & 529 & 17 \\
\hline 7515.80 & 0.88 & 0.00 & 2 & 28 & 207 & 85 & 472 & 18 \\
\hline 7517.23 & 1.24 & 0.00 & 2 & 28 & 196 & 91 & 661 & 20 \\
\hline 7519.05 & 0.26 & 0.06 & 1 & 35 & 189 & 99 & 665 & 15 \\
\hline 7520.13 & 0.90 & 0.00 & 2 & 26 & 190 & 130 & 732 & 23 \\
\hline 7522.05 & 0.45 & 0.00 & 1 & 21 & 199 & 123 & 656 & 21 \\
\hline 7523.03 & 0.78 & 0.00 & 3 & 14 & 120 & 121 & 404 & 22 \\
\hline 7523.89 & 0.04 & 0.00 & 0 & 2 & 46 & 0 & 17 & 0 \\
\hline 7524.90 & 0.81 & 0.00 & 0 & 8 & 84 & 107 & 224 & 19 \\
\hline 7528.01 & 0.14 & 0.06 & 3 & 22 & 160 & 93 & 563 & 16 \\
\hline 7530.15 & 0.40 & 0.00 & 0 & 24 & 179 & 72 & 510 & 14 \\
\hline 7533.02 & 0.80 & 0.00 & 3 & 25 & 188 & 88 & 571 & 18 \\
\hline 7534.04 & 0.43 & 0.00 & 2 & 16 & 166 & 78 & 491 & 17 \\
\hline 7534.89 & 0.24 & 0.00 & 1 & 15 & 169 & 90 & 492 & 17 \\
\hline 7538.15 & 0.22 & 0.04 & 3 & 41 & 323 & 108 & 1106 & 17 \\
\hline 7542.93 & 0.83 & 0.00 & 1 & 26 & 251 & 61 & 713 & 14 \\
\hline 7544.37 & 0.74 & 0.00 & 0 & 25 & 243 & 23 & 889 & 8 \\
\hline 7544.85 & 0.64 & 0.00 & 0 & 58 & 220 & 40 & 777 & 7 \\
\hline 7545.77 & 0.42 & 0.00 & 1 & 41 & 466 & 102 & 1274 & 13 \\
\hline 7554.33 & 0.07 & 0.00 & 1 & 4 & 21 & 0 & 52 & 0 \\
\hline 7556.15 & 0.94 & 0.00 & 1 & 32 & 304 & 89 & 1083 & 14 \\
\hline
\end{tabular}




\begin{tabular}{|c|c|c|c|c|c|c|c|c|}
\hline Sample & Cu (ppm) & Zn (ppm) & Ga (ppm) & $\mathrm{Ba}$ (ppm) & Rb (ppm) & Cs (ppm) & Sr (ppm) & $Y(p p m)$ \\
\hline 7447.20 & 93 & 41 & 21 & 1496 & 187 & 10 & 124 & 36 \\
\hline 7448.35 & 97 & 36 & 22 & 1172 & 197 & 17 & 130 & 31 \\
\hline 7449.25 & 105 & 41 & 23 & 1382 & 202 & 11 & 124 & 32 \\
\hline 7450.20 & 97 & 33 & 22 & 1278 & 193 & 16 & 119 & 31 \\
\hline 7452.22 & 86 & 35 & 22 & 1185 & 177 & 10 & 111 & 27 \\
\hline 7455.00 & 22 & 29 & 17 & 907 & 105 & 6 & 266 & 76 \\
\hline 7456.16 & 110 & 348 & 26 & 1676 & 219 & 16 & 134 & 29 \\
\hline 7457.15 & 125 & 224 & 23 & 2138 & 200 & 16 & 135 & 32 \\
\hline 7457.37 & 113 & 36 & 20 & 1097 & 181 & 11 & 124 & 32 \\
\hline 7459.13 & 132 & 39 & 25 & 1722 & 222 & 18 & 128 & 33.3 \\
\hline 7460.20 & 115 & 48 & 27 & 1588 & 228 & 16 & 136 & 36.6 \\
\hline 7463.10 & 92 & 207 & 27 & 3292 & 225 & 16 & 161 & 38 \\
\hline 7464.05 & 144 & 71 & 25 & 1656 & 217 & 14 & 151 & 35 \\
\hline 7465.30 & 165 & 52 & 24 & 4270 & 206 & 15 & 161 & 30 \\
\hline 7467.30 & 124 & 39 & 20 & 1310 & 145 & 6 & 240 & 123 \\
\hline 7470.05 & 86 & 64 & 27 & 1520 & 229 & 15 & 127 & 25.9 \\
\hline 7471.13 & 134 & 44 & 23 & 7634 & 200 & 13 & 229 & 32 \\
\hline 7472.09 & 142 & 350 & 26 & 8395 & 217 & 18 & 221 & 30 \\
\hline 7475.12 & 151 & 139 & 26 & 19000 & 204 & 12 & 386 & 63 \\
\hline 7476.15 & 157 & 447 & 24 & 1599 & 215 & 15 & 140 & 35 \\
\hline 7477.07 & 180 & 46 & 25 & 1362 & 208 & 14 & 162 & 33 \\
\hline 7479.05 & 130 & 893 & 25 & 1497 & 210 & 16 & 106 & 23 \\
\hline 7480.05 & 98 & 62 & 25 & 1988 & 214 & 17 & 121 & 26 \\
\hline 7482.12 & 83 & 20 & 13 & 11800 & 103 & 3 & 420 & 62.4 \\
\hline 7484.11 & 105 & 37 & 25 & 1508 & 206 & 13 & 118 & 28 \\
\hline 7485.03 & 91 & 2965 & 24 & 1502 & 202 & 15 & 117 & 31 \\
\hline 7485.60 & 79 & 75 & 22 & 1587 & 207 & 13 & 118 & 29 \\
\hline 7488.15 & 79 & 43 & 24 & 1560 & 199 & 13 & 118 & 31 \\
\hline 7489.06 & 68 & 37 & 22 & 1430 & 198 & 13 & 122 & 32 \\
\hline 7491.22 & 83 & 34 & 24 & 1669 & 198 & 12 & 130 & 35 \\
\hline 7492.06 & 99 & 102 & 26 & 41600 & 196 & 11 & 605 & 34.9 \\
\hline 7494.20 & 103 & 71 & 24 & 1379 & 196 & 14 & 126 & 35 \\
\hline 7497.39 & 140 & 62 & 20 & 10000 & 162 & 12 & 281 & 43 \\
\hline
\end{tabular}




\begin{tabular}{|c|c|c|c|c|c|c|c|c|}
\hline Sample & Cu (ppm) & Zn (ppm) & Ga (ppm) & $\mathrm{Ba}$ (ppm) & Rb (ppm) & Cs (ppm) & Sr (ppm) & Y (ppm) \\
\hline 7500.60 & 143 & 446 & 16 & 25700 & 136 & 11 & 435 & 38 \\
\hline 7503.04 & 137 & 270 & 16 & 909 & 126 & 11 & 132 & 39 \\
\hline 7503.98 & 134 & 877 & 16 & 1110 & 123 & 10 & 125 & 38.1 \\
\hline 7505.93 & 134 & 55 & 17 & 1108 & 146 & 14 & 112 & 39 \\
\hline 7506.99 & 139 & 31 & 18 & 1025 & 143 & 12 & 118 & 40 \\
\hline 7509.01 & 148 & 50 & 14 & 1000 & 107 & 7 & 192 & 108 \\
\hline 7512.01 & 51 & 31 & 16 & 1170 & 127 & 7 & 122 & 45.0 \\
\hline 7513.08 & 142 & 436 & 16 & 1069 & 132 & 10 & 137 & 48 \\
\hline 7514.10 & 147 & 35 & 17 & 995 & 135 & 9 & 146 & 50 \\
\hline 7515.80 & 136 & 27 & 20 & 1048 & 155 & 10 & 171 & 33 \\
\hline 7517.23 & 154 & 183 & 19 & 1085 & 139 & 12 & 257 & 37 \\
\hline 7519.05 & 153 & 26 & 22 & 59000 & 131 & 7 & 751 & 28.5 \\
\hline 7520.13 & 187 & 696 & 20 & 1248 & 151 & 12 & 146 & 31 \\
\hline 7522.05 & 197 & 135 & 20 & 1590 & 159 & 11 & 147 & 31 \\
\hline 7523.03 & 130 & 130 & 21 & 1760 & 171 & 10 & 211 & 37 \\
\hline 7523.89 & 35 & 29 & nd & 70200 & 9 & nd & 1064 & 21 \\
\hline 7524.90 & 113 & 378 & 19 & 1568 & 141 & 11 & 282 & 46 \\
\hline 7528.01 & 88 & 22 & 18 & 1098 & 151 & 6 & 164 & 37.2 \\
\hline 7530.15 & 103 & 25 & 19 & 26800 & 143 & 7 & 464 & 25 \\
\hline 7533.02 & 107 & 247 & 19 & 1149 & 148 & 10 & 207 & 38 \\
\hline 7534.04 & 99 & 146 & 19 & 1531 & 149 & 11 & 182 & 33 \\
\hline 7534.89 & 102 & 243 & 18 & 1022 & 148 & 12 & 128 & 32 \\
\hline 7538.15 & 161 & 1435 & 20 & 1253 & 156 & 13 & 124 & 38.0 \\
\hline 7542.93 & 151 & 1129 & 10 & 804 & 68 & 5 & 332 & 84 \\
\hline 7544.37 & 166 & 121 & nd & 172000 & 58 & nd & 2224 & 29 \\
\hline 7544.85 & 173 & 57 & nd & 128000 & 68 & nd & 1676 & 37 \\
\hline 7545.77 & 231 & 986 & 16 & 1193 & 99 & 5 & 158 & 46 \\
\hline 7554.33 & 6 & 53 & 0 & 102 & 3 & 0 & 261 & 54 \\
\hline 7556.15 & 224 & 257 & 16 & 930 & 116 & 9 & 248 & 31 \\
\hline
\end{tabular}




\begin{tabular}{|c|c|c|c|c|c|c|c|c|c|}
\hline Sample & $\mathrm{Zr}$ (ppm) & Hf (ppm) & $\mathrm{Nb}$ (ppm) & Mo (ppm) & Ce (ppm) & Nd (ppm) & Th (ppm) & U (ppm) & $\mathrm{Pb}>=(\mathrm{ppm})$ \\
\hline 7447.20 & 119 & 2 & 11 & 32 & 77 & 36 & 11 & 8 & 31 \\
\hline 7448.35 & 122 & 5 & 11 & 56 & 75 & 31 & 12 & 9 & 29 \\
\hline 7449.25 & 124 & 4 & 12 & 53 & 71 & 32 & 12 & 8 & 33 \\
\hline 7450.20 & 120 & 3 & 12 & 25 & 71 & 36 & 11 & 6 & 32 \\
\hline 7452.22 & 114 & 4 & 10 & 26 & 71 & 27 & 13 & 7 & 33 \\
\hline 7455.00 & 106 & 3 & 11 & 3 & 94 & 47 & 7 & 4 & 7 \\
\hline 7456.16 & 127 & 4 & 13 & 119 & 65 & 30 & 12 & 15 & 26 \\
\hline 7457.15 & 116 & 4 & 9 & 89 & 69 & 30 & 13 & 13 & 36 \\
\hline 7457.37 & 109 & 4 & 11 & 53 & 68 & 32 & 11 & 7 & 33 \\
\hline 7459.13 & 124 & 4.4 & 11.0 & 72 & 72 & 36 & 12 & 17 & 24 \\
\hline 7460.20 & 124 & 4.3 & 11.6 & 107 & 78 & 37 & 11 & 19 & 26 \\
\hline 7463.10 & 124 & 5 & 12 & 95 & 76 & 41 & 13 & 16 & 30 \\
\hline 7464.05 & 119 & 3 & 11 & 90 & 83 & 36 & 13 & 16 & 32 \\
\hline 7465.30 & 118 & 3 & 12 & 99 & 69 & 30 & 11 & 20 & 33 \\
\hline 7467.30 & 88 & 2 & 9 & 94 & 97 & 57 & 9 & 23 & 19 \\
\hline 7470.05 & 116 & 3.4 & 10.7 & 110 & 64 & 31 & 11 & 21 & 23 \\
\hline 7471.13 & 109 & 1 & 9 & 155 & 71 & 34 & 12 & 17 & 31 \\
\hline 7472.09 & 115 & 3 & 11 & 143 & 79 & 34 & 13 & 24 & 31 \\
\hline 7475.12 & 111 & 3 & 11 & 129 & 92 & 57 & 11 & 30 & 33 \\
\hline 7476.15 & 111 & 2 & 10 & 111 & 74 & 40 & 11 & 22 & 32 \\
\hline 7477.07 & 115 & 4 & 12 & 99 & 88 & 36 & 13 & 19 & 35 \\
\hline 7479.05 & 111 & 4 & 11 & 121 & 68 & 30 & 12 & 15 & 29 \\
\hline 7480.05 & 122 & 5 & 12 & 59 & 66 & 26 & 11 & 11 & 27 \\
\hline 7482.12 & 74 & 3.2 & 7.0 & 12 & 52 & 29 & 5 & 7 & 18 \\
\hline 7484.11 & 117 & 4 & 11 & 114 & 67 & 29 & 14 & 15 & 27 \\
\hline 7485.03 & 114 & 4 & 10 & 105 & 68 & 33 & 10 & 15 & 26 \\
\hline 7485.60 & 116 & 4 & 11 & 97 & 68 & 34 & 13 & 16 & 28 \\
\hline 7488.15 & 123 & 3 & 11 & 91 & 72 & 36 & 12 & 10 & 23 \\
\hline 7489.06 & 118 & 4 & 10 & 94 & 76 & 31 & 13 & 11 & 27 \\
\hline 7491.22 & 131 & 5 & 12 & 112 & 78 & 34 & 10 & 14 & 24 \\
\hline 7492.06 & 116 & 2.8 & 12.4 & 107 & 64 & 29 & 11 & 16 & 19 \\
\hline 7494.20 & 123 & 5 & 12 & 116 & 65 & 30 & 11 & 14 & 27 \\
\hline 7497.39 & 110 & 4 & 11 & 129 & 68 & 40 & 9 & 28 & 37 \\
\hline
\end{tabular}




\begin{tabular}{|c|c|c|c|c|c|c|c|c|c|}
\hline Sample & $\mathrm{Zr}$ (ppm) & Hf (ppm) & $\mathrm{Nb}$ (ppm) & Mo (ppm) & Ce (ppm) & Nd (ppm) & Th (ppm) & U (ppm) & $\mathrm{Pb}>=(p p m)$ \\
\hline 7500.60 & 105 & 4 & 9 & 153 & 73 & 29 & 9 & 24 & 33 \\
\hline 7503.04 & 97 & 2 & 8 & 146 & 55 & 33 & 9 & 24 & 26 \\
\hline 7503.98 & 94 & 3.5 & 7.5 & 137 & 52 & 31 & 7 & 23 & 7 \\
\hline 7505.93 & 107 & 3 & 9 & 133 & 65 & 34 & 9 & 23 & 34 \\
\hline 7506.99 & 107 & 3 & 9 & 181 & 62 & 35 & 10 & 21 & 32 \\
\hline 7509.01 & 90 & 3 & 7 & 177 & 79 & 61 & 6 & 47 & 36 \\
\hline 7512.01 & 104 & 3.7 & 9.2 & 132 & 59 & 36 & 9 & 47 & 5 \\
\hline 7513.08 & 108 & 3 & 8 & 266 & 56 & 36 & 9 & 46 & 26 \\
\hline 7514.10 & 107 & 3 & 9 & 179 & 64 & 40 & 10 & 31 & 34 \\
\hline 7515.80 & 123 & 4 & 11 & 181 & 57 & 30 & 11 & 25 & 33 \\
\hline 7517.23 & 120 & 3 & 10 & 148 & 63 & 31 & 8 & 14 & 30 \\
\hline 7519.05 & 121 & 2.3 & 11.8 & 114 & 45 & 21 & 5 & 15 & 20 \\
\hline 7520.13 & 159 & 6 & 12 & 129 & 55 & 29 & 9 & 17 & 29 \\
\hline 7522.05 & 168 & 4 & 12 & 97 & 74 & 34 & 11 & 18 & 36 \\
\hline 7523.03 & 153 & 4 & 13 & 21 & 83 & 40 & 11 & 10 & 18 \\
\hline 7523.89 & 16 & 1 & 5 & 10 & nd & nd & 0 & 1 & nd \\
\hline 7524.90 & 158 & 5 & 14 & 6 & 81 & 40 & 12 & 5 & 11 \\
\hline 7528.01 & 114 & 3.4 & 9.4 & 75 & 63 & 31 & 9 & 16 & 12 \\
\hline 7530.15 & 95 & 3 & 10 & 91 & 65 & 21 & 8 & 18 & 37 \\
\hline 7533.02 & 103 & 3 & 8 & 126 & 61 & 27 & 9 & 16 & 27 \\
\hline 7534.04 & 100 & 3 & 10 & 106 & 57 & 28 & 8 & 18 & 24 \\
\hline 7534.89 & 102 & 3 & 9 & 120 & 54 & 28 & 10 & 28 & 28 \\
\hline 7538.15 & 110 & 3.3 & 8.8 & 225 & 52 & 31 & 9 & 60 & 17 \\
\hline 7542.93 & 70 & 2 & 7 & 130 & 64 & 43 & 4 & 44 & 26 \\
\hline 7544.37 & 40 & 1 & 11 & 101 & nd & nd & 8 & 28 & nd \\
\hline 7544.85 & 55 & 3 & 9 & 80 & nd & nd & 6 & 33 & nd \\
\hline 7545.77 & 93 & 2 & 5 & 317 & 46 & 32 & 7 & 71 & 41 \\
\hline 7554.33 & 1 & 0 & 1 & 24 & 32 & 26 & 1 & 7 & 2 \\
\hline 7556.15 & 122 & 3 & 8 & 308 & 29 & 16 & 7 & 32 & 34 \\
\hline
\end{tabular}


Table D-5, MSCL Mining-plus Suite:

\begin{tabular}{|c|c|c|c|c|c|c|c|c|c|c|c|c|}
\hline Depth & XRF & XRF Live Time & $\mathrm{Ti}$ & $\mathrm{V}$ & $\mathrm{Cr}$ & $\mathrm{Mn}$ & $\mathrm{Fe}$ & Co & $\mathrm{Ni}$ & $\mathrm{Cu}$ & $\mathrm{Zn}$ & As \\
\hline & Total Counts & secs & $\mathrm{ppm}$ & $\mathrm{ppm}$ & $\mathrm{ppm}$ & $\mathrm{ppm}$ & $\mathrm{ppm}$ & $\mathrm{ppm}$ & $\mathrm{ppm}$ & $\mathrm{ppm}$ & $\mathrm{ppm}$ & $\mathrm{ppm}$ \\
\hline 7517.07 & 1285030 & 48.00 & 4304.74 & 1226.93 & 260.47 & 233.45 & 37595.11 & 145.45 & 251.59 & 269.78 & 1515.15 & 32.00 \\
\hline 7517.13 & 1281743 & 48.00 & 4165.22 & 1233.02 & 157.19 & 207.37 & 29043.50 & 116.24 & 212.00 & 188.75 & 420.58 & 30.00 \\
\hline 7517.20 & 1445098 & 47.00 & 3854.24 & 1066.77 & 263.02 & 199.13 & 28824.21 & 109.37 & 216.01 & 195.95 & 242.56 & 26.57 \\
\hline 7517.26 & 1339818 & 48.00 & 3834.02 & 1130.28 & 230.30 & 203.99 & 31031.38 & 98.38 & 209.44 & 194.02 & 131.82 & 29.17 \\
\hline 7517.33 & 1123231 & 50.00 & 4213.73 & 1055.57 & 254.53 & 192.50 & 30253.96 & 98.17 & 206.38 & 207.03 & 48.16 & 26.01 \\
\hline 7517.39 & 2134367 & 38.00 & 1114.44 & 444.85 & 0.00 & 445.50 & 7582.51 & 0.00 & 33.39 & 45.85 & 0.00 & 0.00 \\
\hline 7517.46 & 2200254 & 37.00 & 6073.60 & 3858.89 & 0.00 & 692.95 & 4140.97 & 0.00 & 0.00 & 0.00 & 0.00 & 0.00 \\
\hline 7517.52 & 2133698 & 38.00 & 1582.34 & 861.32 & 0.00 & 404.60 & 4044.26 & 0.00 & 27.58 & 0.00 & 0.00 & 0.00 \\
\hline 7517.59 & 2055572 & 39.00 & 4101.69 & 2214.45 & 0.00 & 662.37 & 9855.62 & 0.00 & 43.13 & 49.24 & 0.00 & 0.00 \\
\hline 7517.66 & 1721921 & 43.00 & 12847.06 & 7674.25 & 0.00 & 871.03 & 11362.99 & 0.00 & 60.22 & 42.66 & 0.00 & 0.00 \\
\hline 7517.72 & 2245382 & 36.00 & 42508.37 & 26101.65 & 0.00 & 2043.41 & 17533.79 & 0.00 & 55.38 & 63.07 & 0.00 & 0.00 \\
\hline 7517.79 & 2232828 & 36.00 & 59483.02 & 37714.52 & 0.00 & 2590.21 & 12049.97 & 0.00 & 61.31 & 62.30 & 0.00 & 0.00 \\
\hline 7517.85 & 2267388 & 35.00 & 1973.78 & 783.90 & 0.00 & 515.51 & 4771.54 & 0.00 & 40.52 & 41.55 & 0.00 & 0.00 \\
\hline 7517.92 & 1340565 & 48.00 & 2368.88 & 832.10 & 194.61 & 567.68 & 10906.93 & 0.00 & 73.91 & 0.00 & 0.00 & 0.00 \\
\hline 7517.98 & 1970704 & 40.00 & 1034.49 & 459.27 & 121.31 & 477.37 & 4286.33 & 0.00 & 30.54 & 0.00 & 0.00 & 0.00 \\
\hline 7518.05 & 2057128 & 39.00 & 1966.08 & 357.15 & 161.49 & 437.39 & 3243.54 & 0.00 & 31.98 & 0.00 & 0.00 & 0.00 \\
\hline 7518.12 & 2106156 & 38.00 & 788.29 & 215.40 & 174.88 & 408.06 & 4393.79 & 0.00 & 29.28 & 0.00 & 0.00 & 0.00 \\
\hline 7518.18 & 2049743 & 39.00 & 747.99 & 280.97 & 0.00 & 509.23 & 5044.78 & 0.00 & 30.51 & 0.00 & 0.00 & 0.00 \\
\hline 7518.25 & 1668617 & 44.00 & 675.51 & 339.77 & 167.50 & 497.55 & 5433.41 & 0.00 & 44.20 & 0.00 & 0.00 & 0.00 \\
\hline 7518.31 & 1644446 & 44.00 & 808.00 & 333.26 & 0.00 & 567.06 & 4828.75 & 0.00 & 36.14 & 0.00 & 0.00 & 0.00 \\
\hline 7518.38 & 1626289 & 44.00 & 675.76 & 304.30 & 0.00 & 625.71 & 5025.15 & 0.00 & 32.85 & 0.00 & 0.00 & 0.00 \\
\hline 7518.44 & 1604762 & 45.00 & 955.15 & 311.95 & 0.00 & 683.44 & 6206.01 & 0.00 & 33.67 & 0.00 & 0.00 & 0.00 \\
\hline 7518.51 & 1467920 & 46.00 & 1119.79 & 435.52 & 0.00 & 656.25 & 5522.34 & 0.00 & 51.53 & 0.00 & 0.00 & 0.00 \\
\hline 7518.57 & 2163442 & 37.00 & 847.32 & 323.54 & 108.50 & 680.90 & 5809.95 & 0.00 & 54.38 & 0.00 & 0.00 & 0.00 \\
\hline 7518.64 & 2120365 & 38.00 & 917.69 & 458.30 & 130.58 & 576.84 & 6236.13 & 0.00 & 37.56 & 46.84 & 0.00 & 0.00 \\
\hline 7518.71 & 1952572 & 40.00 & 1384.33 & 672.31 & 0.00 & 477.77 & 4574.11 & 0.00 & 48.62 & 45.23 & 0.00 & 0.00 \\
\hline 7518.77 & 1978439 & 40.00 & 4876.08 & 2959.10 & 0.00 & 541.82 & 7114.98 & 0.00 & 53.08 & 46.82 & 0.00 & 0.00 \\
\hline 7518.84 & 1917661 & 41.00 & 4548.55 & 2536.55 & 0.00 & 436.59 & 7727.09 & 0.00 & 56.24 & 65.61 & 0.00 & 0.00 \\
\hline 7518.90 & 1782762 & 43.00 & 5316.31 & 2792.44 & 0.00 & 494.83 & 10949.71 & 0.00 & 87.70 & 89.65 & 0.00 & 0.00 \\
\hline 7518.97 & 1418590 & 47.00 & 4608.68 & 1100.12 & 250.70 & 223.94 & 29880.61 & 0.00 & 244.57 & 238.81 & 0.00 & 24.43 \\
\hline 7519.03 & 1056806 & 51.00 & 4921.46 & 1430.76 & 196.32 & 232.17 & 29385.39 & 85.26 & 184.81 & 215.91 & 0.00 & 25.90 \\
\hline 7519.10 & 1296713 & 48.00 & 18131.93 & 9346.28 & 0.00 & 935.95 & 44506.56 & 0.00 & 223.55 & 227.77 & 0.00 & 29.57 \\
\hline
\end{tabular}




\begin{tabular}{|c|c|c|c|c|c|c|c|c|c|c|c|c|}
\hline Depth & $\mathrm{XRF}$ & XRF Live Time & $\mathrm{Ti}$ & $\mathrm{V}$ & $\mathrm{Cr}$ & $\mathrm{Mn}$ & $\mathrm{Fe}$ & $\mathrm{Co}$ & $\mathrm{Ni}$ & $\mathrm{Cu}$ & $\mathrm{Zn}$ & As \\
\hline & Total Counts & secs & $\mathrm{ppm}$ & ppm & ppm & $\mathrm{ppm}$ & $\mathrm{ppm}$ & $\mathrm{ppm}$ & $\mathrm{ppm}$ & $\mathrm{ppm}$ & $\mathrm{ppm}$ & $\mathrm{ppm}$ \\
\hline 7519.17 & 1347911 & 48.00 & 4274.88 & 1381.00 & 243.67 & 236.53 & 26301.23 & 0.00 & 208.50 & 197.25 & 0.00 & 28.62 \\
\hline 7519.23 & 1185508 & 49.00 & 3953.39 & 1213.92 & 286.11 & 214.98 & 26728.88 & 92.26 & 204.26 & 211.50 & 0.00 & 22.94 \\
\hline 7519.30 & 1590471 & 45.00 & 19699.63 & 10921.87 & 0.00 & 1086.73 & 57720.67 & 0.00 & 204.98 & 218.11 & 0.00 & 47.04 \\
\hline 7519.36 & 1528581 & 46.00 & 3075.33 & 998.98 & 231.13 & 317.60 & 21143.49 & 0.00 & 175.62 & 157.96 & 0.00 & 22.26 \\
\hline 7519.43 & 1249264 & 49.00 & 3680.09 & 1097.90 & 199.87 & 251.58 & 28255.01 & 90.17 & 185.95 & 190.60 & 44.38 & 26.71 \\
\hline 7519.49 & 1371788 & 47.00 & 3252.44 & 1121.99 & 240.87 & 329.35 & 22290.21 & 0.00 & 146.09 & 172.05 & 71.21 & 24.91 \\
\hline 7519.56 & 1953982 & 40.00 & 1963.35 & 723.93 & 109.16 & 367.87 & 15926.25 & 0.00 & 99.88 & 125.20 & 619.04 & 14.82 \\
\hline 7519.62 & 1876197 & 41.00 & 1677.33 & 633.10 & 111.46 & 342.97 & 11385.77 & 0.00 & 72.68 & 90.04 & 1508.14 & 0.00 \\
\hline 7519.69 & 1760645 & 43.00 & 1495.38 & 619.43 & 167.16 & 349.30 & 12356.89 & 0.00 & 100.93 & 115.69 & 3508.33 & 0.00 \\
\hline 7519.76 & 1572498 & 45.00 & 2900.22 & 893.41 & 226.39 & 331.94 & 16959.27 & 0.00 & 129.98 & 142.59 & 4644.38 & 17.13 \\
\hline 7519.82 & 1258867 & 49.00 & 3407.98 & 1222.62 & 222.22 & 240.12 & 23414.62 & 0.00 & 185.67 & 219.85 & 2921.05 & 19.26 \\
\hline 7519.89 & 1031755 & 51.00 & 3930.60 & 1037.54 & 191.44 & 177.94 & 26665.18 & 72.76 & 195.53 & 264.99 & 7526.90 & 25.95 \\
\hline 7519.95 & 1027093 & 51.00 & 3818.16 & 1056.52 & 184.41 & 165.45 & 29133.32 & 82.51 & 182.92 & 221.67 & 1082.73 & 24.76 \\
\hline 7520.02 & 1075664 & 50.00 & 4201.86 & 1054.40 & 217.07 & 169.92 & 27807.72 & 71.74 & 194.88 & 208.66 & 448.27 & 25.15 \\
\hline 7520.08 & 1131334 & 50.00 & 4609.54 & 1169.51 & 225.84 & 210.40 & 25075.06 & 78.72 & 196.08 & 203.73 & 983.19 & 17.35 \\
\hline 7520.15 & 1195299 & 49.00 & 4443.24 & 1207.91 & 261.84 & 277.30 & 30808.21 & 0.00 & 213.98 & 255.60 & 738.72 & 33.19 \\
\hline 7520.22 & 1056097 & 51.00 & 4644.20 & 1090.94 & 240.07 & 228.98 & 31924.63 & 98.07 & 235.01 & 254.76 & 436.28 & 33.29 \\
\hline 7520.28 & 992632 & 51.00 & 5337.68 & 1213.54 & 244.28 & 165.09 & 26730.11 & 0.00 & 149.69 & 170.69 & 0.00 & 26.01 \\
\hline 7520.35 & 1066127 & 50.00 & 4510.69 & 1023.40 & 287.13 & 172.16 & 35014.41 & 93.45 & 165.01 & 195.12 & 0.00 & 28.20 \\
\hline 7520.41 & 1113749 & 50.00 & 4896.36 & 1114.09 & 299.77 & 226.56 & 42462.59 & 92.01 & 168.39 & 234.35 & 53.56 & 37.71 \\
\hline 7520.48 & 1169875 & 49.00 & 4823.19 & 984.70 & 273.94 & 253.85 & 39407.53 & 86.28 & 168.40 & 228.26 & 0.00 & 37.05 \\
\hline 7520.54 & 1147691 & 50.00 & 4587.32 & 900.87 & 327.99 & 281.91 & 39896.27 & 101.53 & 194.16 & 262.36 & 0.00 & 28.78 \\
\hline 7520.61 & 1030686 & 51.00 & 4998.76 & 1019.52 & 293.70 & 260.50 & 29069.34 & 0.00 & 171.37 & 218.70 & 0.00 & 20.33 \\
\hline 7520.67 & 969778 & 51.00 & 4780.39 & 962.01 & 257.06 & 214.90 & 37911.26 & 108.17 & 167.75 & 251.56 & 0.00 & 28.78 \\
\hline 7520.74 & 1039994 & 51.00 & 5282.30 & 955.84 & 349.83 & 166.91 & 25410.72 & 0.00 & 139.14 & 212.09 & 0.00 & 16.84 \\
\hline 7520.81 & 1107018 & 50.00 & 4845.04 & 1013.80 & 242.93 & 189.98 & 28807.37 & 76.62 & 160.56 & 223.30 & 0.00 & 22.27 \\
\hline 7520.87 & 1129854 & 50.00 & 4862.54 & 926.40 & 291.21 & 227.23 & 41518.28 & 147.97 & 138.96 & 262.95 & 37.80 & 20.75 \\
\hline 7520.94 & 1052013 & 51.00 & 5141.46 & 991.89 & 294.68 & 210.98 & 33955.58 & 81.38 & 163.24 & 241.61 & 0.00 & 18.02 \\
\hline 7521.00 & 967594 & 51.00 & 5057.79 & 1053.73 & 349.70 & 225.32 & 32241.45 & 0.00 & 184.00 & 262.05 & 0.00 & 17.81 \\
\hline 7521.07 & 1134376 & 50.00 & 5022.66 & 1027.18 & 326.26 & 211.31 & 28436.73 & 0.00 & 142.90 & 222.03 & 33.60 & 16.15 \\
\hline 7521.13 & 1171116 & 49.00 & 5005.08 & 804.27 & 299.65 & 234.19 & 38985.38 & 105.64 & 146.05 & 244.86 & 29.93 & 19.73 \\
\hline 7521.20 & 1196560 & 49.00 & 4628.42 & 899.23 & 298.21 & 224.12 & 44611.23 & 146.08 & 148.17 & 285.11 & 149.26 & 23.02 \\
\hline
\end{tabular}




\begin{tabular}{|c|c|c|c|c|c|c|c|c|c|c|c|c|}
\hline Depth & $\mathrm{XRF}$ & XRF Live Time & $\mathrm{Ti}$ & $\mathrm{V}$ & $\mathrm{Cr}$ & $\mathrm{Mn}$ & $\mathrm{Fe}$ & $\mathrm{Co}$ & $\mathrm{Ni}$ & $\mathrm{Cu}$ & $\mathrm{Zn}$ & As \\
\hline & Total Counts & secs & $\mathrm{ppm}$ & ppm & ppm & $\mathrm{ppm}$ & $\mathrm{ppm}$ & $\mathrm{ppm}$ & $\mathrm{ppm}$ & $\mathrm{ppm}$ & $\mathrm{ppm}$ & $\mathrm{ppm}$ \\
\hline 7521.26 & 1007590 & 51.00 & 4904.68 & 786.97 & 335.46 & 240.33 & 31775.43 & 86.73 & 142.99 & 223.77 & 31.69 & 17.32 \\
\hline 7521.33 & 1182746 & 49.00 & 3914.18 & 762.59 & 146.59 & 216.36 & 64142.17 & 165.13 & 138.10 & 278.81 & 84.68 & 34.50 \\
\hline 7521.39 & 1038470 & 51.00 & 4773.76 & 865.32 & 280.90 & 239.13 & 29875.33 & 78.31 & 144.59 & 206.67 & 34.12 & 16.07 \\
\hline 7521.46 & 1102153 & 50.00 & 4859.50 & 989.12 & 303.25 & 223.33 & 33059.85 & 114.99 & 125.99 & 247.01 & 38.95 & 0.00 \\
\hline 7521.52 & 1256802 & 49.00 & 4795.64 & 895.19 & 307.89 & 211.74 & 45852.86 & 149.07 & 130.94 & 271.51 & 90.58 & 22.94 \\
\hline 7521.59 & 1193412 & 49.00 & 4527.94 & 873.80 & 243.74 & 225.65 & 41938.45 & 115.73 & 159.48 & 285.71 & 98.08 & 21.49 \\
\hline 7521.66 & 1014089 & 51.00 & 5015.69 & 966.71 & 289.00 & 231.51 & 28478.13 & 95.00 & 159.87 & 222.81 & 34.19 & 0.00 \\
\hline 7521.72 & 1091419 & 50.00 & 5243.87 & 1092.48 & 265.88 & 233.61 & 30257.92 & 0.00 & 199.09 & 226.48 & 34.38 & 21.50 \\
\hline 7521.79 & 1096462 & 50.00 & 4657.85 & 991.74 & 247.77 & 199.41 & 41376.38 & 173.48 & 211.94 & 236.76 & 37.30 & 29.20 \\
\hline 7521.85 & 1187084 & 49.00 & 4330.43 & 1072.14 & 265.38 & 211.20 & 45673.15 & 131.48 & 227.32 & 289.13 & 55.77 & 32.07 \\
\hline 7521.92 & 1193293 & 49.00 & 4821.06 & 1159.74 & 251.50 & 199.92 & 38163.31 & 113.48 & 205.06 & 226.47 & 68.33 & 27.52 \\
\hline 7521.98 & 1138335 & 50.00 & 4554.67 & 1176.15 & 241.09 & 223.09 & 38875.24 & 123.44 & 251.23 & 301.48 & 355.74 & 25.89 \\
\hline 7522.05 & 1091127 & 50.00 & 19279.61 & 757.17 & 283.58 & 198.80 & 29117.91 & 0.00 & 206.44 & 294.11 & 77.89 & 19.07 \\
\hline 7522.12 & 1052438 & 51.00 & 5041.86 & 1036.83 & 235.80 & 197.05 & 36758.33 & 0.00 & 189.35 & 207.71 & 42.79 & 25.99 \\
\hline 7522.18 & 1132548 & 50.00 & 5077.74 & 1172.80 & 216.28 & 259.49 & 31732.52 & 81.48 & 215.40 & 222.93 & 92.51 & 25.99 \\
\hline 7522.25 & 1216157 & 49.00 & 4758.12 & 1170.35 & 243.40 & 280.93 & 35036.74 & 88.47 & 235.69 & 271.06 & 43.47 & 26.42 \\
\hline 7522.31 & 1404019 & 47.00 & 4628.37 & 1148.01 & 265.84 & 271.91 & 42328.03 & 90.84 & 330.50 & 304.87 & 56.33 & 24.37 \\
\hline 7522.38 & 1951533 & 40.00 & 2144.69 & 733.83 & 190.63 & 484.98 & 14038.17 & 0.00 & 89.53 & 101.84 & 0.00 & 0.00 \\
\hline 7522.44 & 1525066 & 46.00 & 4106.03 & 975.21 & 233.65 & 341.90 & 32893.95 & 0.00 & 144.72 & 281.71 & 0.00 & 0.00 \\
\hline 7522.51 & 1402856 & 47.00 & 4083.35 & 1052.44 & 216.94 & 310.71 & 24122.46 & 85.36 & 151.19 & 189.16 & 0.00 & 0.00 \\
\hline 7522.57 & 1161288 & 50.00 & 4998.58 & 1144.13 & 268.06 & 242.83 & 23027.00 & 78.76 & 150.67 & 165.20 & 0.00 & 0.00 \\
\hline 7522.64 & 1366972 & 47.00 & 4880.45 & 1135.15 & 274.19 & 256.32 & 27062.09 & 0.00 & 169.58 & 199.86 & 0.00 & 17.32 \\
\hline 7522.71 & 1290507 & 48.00 & 4796.40 & 1184.19 & 218.47 & 337.15 & 35187.71 & 102.73 & 230.86 & 297.13 & 56.35 & 18.97 \\
\hline 7522.77 & 1148069 & 50.00 & 4693.98 & 1188.41 & 268.66 & 252.15 & 29780.47 & 89.60 & 219.36 & 321.68 & 936.34 & 19.65 \\
\hline 7522.84 & 1113971 & 50.00 & 5037.08 & 1157.98 & 263.46 & 292.77 & 26146.05 & 77.57 & 167.20 & 223.85 & 68.70 & 16.19 \\
\hline 7522.90 & 1059302 & 51.00 & 5242.71 & 1355.37 & 272.46 & 257.29 & 24222.17 & 0.00 & 185.78 & 253.18 & 123.77 & 19.24 \\
\hline 7522.97 & 1112731 & 50.00 & 5239.57 & 1188.37 & 202.91 & 285.58 & 21496.79 & 0.00 & 162.85 & 198.60 & 263.76 & 14.25 \\
\hline 7523.03 & 1218752 & 49.00 & 13735.56 & 1044.94 & 241.71 & 306.61 & 28362.90 & 0.00 & 141.96 & 166.88 & 61.23 & 16.21 \\
\hline 7523.10 & 1149113 & 50.00 & 5094.00 & 1021.34 & 222.77 & 288.22 & 21684.39 & 65.62 & 129.60 & 138.16 & 46.57 & 0.00 \\
\hline 7523.17 & 1221323 & 49.00 & 5389.01 & 1086.89 & 300.76 & 271.48 & 18627.63 & 0.00 & 122.16 & 147.50 & 45.35 & 13.36 \\
\hline 7523.23 & 1227373 & 49.00 & 5262.94 & 835.74 & 272.13 & 254.69 & 15695.36 & 0.00 & 128.15 & 147.71 & 36.61 & 0.00 \\
\hline 7523.30 & 1374354 & 47.00 & 4713.75 & 807.41 & 261.21 & 346.94 & 31825.27 & 0.00 & 168.96 & 187.14 & 203.13 & 0.00 \\
\hline
\end{tabular}




\begin{tabular}{|c|c|c|c|c|c|c|c|c|c|c|c|c|}
\hline Depth & $\mathrm{XRF}$ & XRF Live Time & $\mathrm{Ti}$ & $\mathrm{V}$ & $\mathrm{Cr}$ & $\mathrm{Mn}$ & $\mathrm{Fe}$ & $\mathrm{Co}$ & $\mathrm{Ni}$ & $\mathrm{Cu}$ & $\mathrm{Zn}$ & As \\
\hline & Total Counts & secs & $\mathrm{ppm}$ & ppm & $\mathrm{ppm}$ & $\mathrm{ppm}$ & $\mathrm{ppm}$ & $\mathrm{ppm}$ & $\mathrm{ppm}$ & $\mathrm{ppm}$ & $\mathrm{ppm}$ & $\mathrm{ppm}$ \\
\hline 7523.36 & 1141981 & 50.00 & 5383.61 & 924.82 & 233.67 & 331.32 & 20596.06 & 0.00 & 154.81 & 155.72 & 42.73 & 0.00 \\
\hline 7523.43 & 1053591 & 51.00 & 6017.80 & 1112.61 & 326.05 & 248.98 & 14225.79 & 0.00 & 109.40 & 112.43 & 64.03 & 0.00 \\
\hline 7523.49 & 1033383 & 51.00 & 5644.55 & 818.59 & 240.91 & 252.98 & 15708.90 & 0.00 & 113.22 & 79.79 & 29.19 & 0.00 \\
\hline 7523.56 & 1132478 & 50.00 & 5240.71 & 785.05 & 272.53 & 253.04 & 13327.80 & 0.00 & 95.68 & 81.97 & 42.16 & 0.00 \\
\hline 7523.62 & 1474030 & 46.00 & 4391.41 & 756.13 & 162.37 & 381.32 & 13704.88 & 0.00 & 75.76 & 66.82 & 306.87 & 0.00 \\
\hline 7523.69 & 1875581 & 41.00 & 2393.43 & 420.52 & 158.52 & 621.49 & 11170.06 & 0.00 & 40.72 & 0.00 & 0.00 & 0.00 \\
\hline 7523.76 & 1896705 & 41.00 & 1414.40 & 225.30 & 115.16 & 674.19 & 11259.66 & 0.00 & 41.65 & 0.00 & 77.41 & 0.00 \\
\hline 7523.82 & 1883790 & 41.00 & 17961.36 & 11082.94 & 0.00 & 798.25 & 6838.88 & 0.00 & 29.39 & 0.00 & 0.00 & 0.00 \\
\hline 7523.89 & 1971907 & 40.00 & 34147.58 & 20927.67 & 0.00 & 1386.94 & 10725.98 & 0.00 & 24.97 & 0.00 & 0.00 & 0.00 \\
\hline 7523.95 & 2023916 & 39.00 & 3132.44 & 1525.30 & 0.00 & 382.16 & 16446.13 & 0.00 & 27.20 & 59.33 & 0.00 & 0.00 \\
\hline 7524.02 & 2046707 & 39.00 & 33669.94 & 0.00 & 235.06 & 332.63 & 4702.69 & 0.00 & 22.32 & 56.39 & 0.00 & 0.00 \\
\hline 7524.08 & 2033768 & 39.00 & 843.13 & 240.93 & 148.62 & 424.99 & 5922.02 & 0.00 & 29.63 & 0.00 & 0.00 & 0.00 \\
\hline 7524.15 & 1802650 & 42.00 & 1961.41 & 457.57 & 142.25 & 496.05 & 7976.31 & 0.00 & 40.83 & 50.65 & 0.00 & 0.00 \\
\hline 7524.22 & 1684136 & 44.00 & 2567.05 & 493.19 & 210.90 & 468.88 & 10532.07 & 0.00 & 75.32 & 80.33 & 549.58 & 0.00 \\
\hline 7524.28 & 1198367 & 49.00 & 4464.48 & 700.80 & 165.49 & 320.93 & 15269.35 & 0.00 & 88.38 & 127.11 & 289.59 & 0.00 \\
\hline 7524.35 & 1484359 & 46.00 & 4040.96 & 640.84 & 220.83 & 330.60 & 14170.75 & 0.00 & 78.84 & 77.04 & 213.24 & 0.00 \\
\hline 7524.41 & 1246603 & 49.00 & 4551.36 & 785.32 & 208.92 & 308.88 & 17175.68 & 0.00 & 91.30 & 102.84 & 100.69 & 0.00 \\
\hline 7524.48 & 1074734 & 50.00 & 5318.06 & 896.19 & 264.25 & 243.77 & 14046.45 & 0.00 & 85.74 & 105.73 & 62.11 & 0.00 \\
\hline 7524.54 & 1049602 & 51.00 & 4849.95 & 788.75 & 161.28 & 256.12 & 15150.18 & 0.00 & 91.37 & 121.68 & 216.99 & 0.00 \\
\hline 7524.61 & 1109083 & 50.00 & 5221.27 & 721.27 & 179.06 & 277.52 & 15334.08 & 0.00 & 86.47 & 113.34 & 173.59 & 0.00 \\
\hline 7524.67 & 1349396 & 48.00 & 4455.48 & 775.39 & 266.01 & 368.02 & 17941.95 & 0.00 & 80.80 & 136.72 & 446.43 & 0.00 \\
\hline 7524.74 & 1384906 & 47.00 & 3994.47 & 605.52 & 219.88 & 372.01 & 11378.21 & 61.18 & 69.19 & 112.86 & 815.31 & 0.00 \\
\hline 7524.81 & 1501193 & 46.00 & 4093.89 & 630.80 & 189.94 & 388.52 & 14209.67 & 0.00 & 83.84 & 91.82 & 90.99 & 0.00 \\
\hline 7524.87 & 1301222 & 48.00 & 4619.98 & 617.30 & 232.39 & 339.84 & 13923.31 & 0.00 & 89.38 & 90.22 & 82.71 & 0.00 \\
\hline 7524.94 & 1052051 & 51.00 & 5049.57 & 624.82 & 284.21 & 248.95 & 13754.36 & 0.00 & 93.36 & 134.40 & 115.30 & 0.00 \\
\hline 7525.00 & 13998 & 51.55 & 5270.27 & 1088.41 & 232.05 & 250.94 & 23771.21 & 0.00 & 112.98 & 132.63 & 236.56 & 0.00 \\
\hline 7525.07 & 15811 & 50.43 & 5244.80 & 693.29 & 230.23 & 227.28 & 15577.22 & 0.00 & 86.75 & 139.88 & 92.53 & 0.00 \\
\hline 7525.13 & 16265 & 50.27 & 5269.80 & 832.19 & 340.99 & 263.58 & 15028.12 & 57.98 & 84.92 & 125.50 & 122.30 & 0.00 \\
\hline 7525.20 & 16166 & 50.34 & 5359.78 & 743.16 & 297.88 & 275.25 & 15954.75 & 67.11 & 77.16 & 103.11 & 60.63 & 0.00 \\
\hline 7525.26 & 15250 & 50.66 & 5704.76 & 901.72 & 201.22 & 308.14 & 19645.66 & 0.00 & 105.59 & 105.44 & 60.67 & 0.00 \\
\hline 7525.33 & 17514 & 48.24 & 4210.76 & 607.33 & 161.16 & 298.91 & 16469.25 & 70.15 & 75.45 & 85.57 & 68.06 & 0.00 \\
\hline 7525.39 & 17679 & 48.11 & 4076.11 & 590.82 & 216.34 & 309.78 & 21300.33 & 0.00 & 96.88 & 90.73 & 74.28 & 0.00 \\
\hline
\end{tabular}




\begin{tabular}{|c|c|c|c|c|c|c|c|c|c|c|c|c|}
\hline Depth & $\mathrm{XRF}$ & XRF Live Time & $\mathrm{Ti}$ & $\mathrm{V}$ & $\mathrm{Cr}$ & $\mathrm{Mn}$ & $\mathrm{Fe}$ & $\mathrm{Co}$ & $\mathrm{Ni}$ & $\mathrm{Cu}$ & $\mathrm{Zn}$ & As \\
\hline & Total Counts & secs & $\mathrm{ppm}$ & ppm & ppm & $\mathrm{ppm}$ & $\mathrm{ppm}$ & $\mathrm{ppm}$ & $\mathrm{ppm}$ & $\mathrm{ppm}$ & ppm & $\mathrm{ppm}$ \\
\hline 7525.46 & 19138 & 46.53 & 3684.86 & 937.47 & 236.72 & 348.21 & 29850.79 & 0.00 & 113.74 & 123.66 & 925.85 & 20.66 \\
\hline 7525.52 & 22895 & 43.21 & 8617.94 & 4297.46 & 0.00 & 758.61 & 19823.96 & 0.00 & 86.01 & 95.28 & 160.53 & 0.00 \\
\hline 7525.59 & 16993 & 49.28 & 6729.37 & 2244.10 & 271.23 & 323.64 & 32884.51 & 0.00 & 180.07 & 264.52 & 161.78 & 0.00 \\
\hline 7525.66 & 15995 & 50.03 & 6247.92 & 1883.58 & 292.49 & 317.49 & 22347.76 & 0.00 & 146.01 & 177.71 & 0.00 & 0.00 \\
\hline 7525.72 & 18305 & 46.79 & 22307.10 & 13026.58 & 0.00 & 1166.31 & 16020.43 & 0.00 & 95.60 & 81.41 & 0.00 & 0.00 \\
\hline 7525.79 & 16937 & 48.93 & 4000.99 & 1384.42 & 139.78 & 231.68 & 50305.39 & 0.00 & 297.27 & 228.26 & 58.75 & 69.35 \\
\hline 7525.85 & 16952 & 49.07 & 3879.14 & 1307.15 & 183.16 & 178.75 & 55538.80 & 111.43 & 353.90 & 257.57 & 60.85 & 70.02 \\
\hline 7525.92 & 19975 & 47.53 & 4056.25 & 1319.78 & 229.15 & 207.80 & 46684.99 & 108.84 & 342.22 & 248.15 & 246.53 & 49.21 \\
\hline 7525.98 & 19930 & 47.63 & 4344.99 & 1415.50 & 246.86 & 127.55 & 51332.70 & 128.91 & 360.58 & 246.03 & 517.57 & 63.88 \\
\hline 7526.05 & 17310 & 49.82 & 11639.06 & 1379.62 & 203.94 & 185.74 & 34933.66 & 80.35 & 321.74 & 203.14 & 961.43 & 38.77 \\
\hline 7526.12 & 14535 & 51.04 & 4074.56 & 1615.59 & 218.91 & 161.11 & 36529.15 & 99.81 & 306.15 & 205.43 & 513.34 & 40.36 \\
\hline 7526.18 & 19012 & 48.88 & 3637.03 & 1456.24 & 208.08 & 161.98 & 36483.36 & 0.00 & 278.87 & 189.27 & 761.78 & 39.48 \\
\hline 7526.25 & 15677 & 50.58 & 3431.53 & 1296.50 & 158.91 & 125.97 & 34509.31 & 94.68 & 240.69 & 175.97 & 698.82 & 36.28 \\
\hline 7526.31 & 15340 & 50.63 & 3425.07 & 1164.52 & 166.74 & 168.48 & 36433.51 & 0.00 & 264.91 & 182.76 & 2662.99 & 42.94 \\
\hline 7526.38 & 16988 & 49.53 & 3327.20 & 1212.04 & 154.21 & 181.43 & 31007.54 & 98.04 & 234.90 & 178.36 & 1531.75 & 33.71 \\
\hline 7526.44 & 17453 & 49.21 & 3086.91 & 1046.89 & 0.00 & 199.07 & 32622.35 & 80.98 & 271.84 & 153.96 & 304.72 & 33.27 \\
\hline 7526.51 & 16928 & 49.86 & 3770.97 & 1057.78 & 169.94 & 178.53 & 34362.52 & 79.73 & 267.98 & 166.84 & 267.39 & 32.53 \\
\hline 7526.57 & 16015 & 50.39 & 3563.14 & 1122.02 & 169.25 & 157.66 & 32678.84 & 0.00 & 244.01 & 131.91 & 257.64 & 32.10 \\
\hline 7526.64 & 15493 & 50.56 & 3420.37 & 1173.80 & 220.03 & 192.01 & 33484.22 & 95.60 & 204.36 & 164.65 & 345.88 & 38.15 \\
\hline 7526.71 & 15226 & 50.70 & 3020.57 & 1181.28 & 138.76 & 164.89 & 31440.05 & 101.10 & 207.38 & 150.71 & 323.34 & 37.40 \\
\hline 7526.77 & 17765 & 49.78 & 3083.96 & 945.18 & 219.99 & 155.89 & 32368.98 & 87.47 & 207.26 & 147.01 & 20460.84 & 40.27 \\
\hline 7526.84 & 16665 & 50.05 & 3379.70 & 1046.22 & 154.24 & 200.35 & 29882.81 & 85.20 & 217.71 & 159.39 & 1650.71 & 35.27 \\
\hline 7526.90 & 15646 & 50.76 & 3344.79 & 1133.84 & 168.07 & 190.67 & 29333.99 & 76.10 & 206.57 & 137.31 & 1798.88 & 29.01 \\
\hline 7527.07 & 1061278 & 51.00 & 3154.90 & 845.14 & 200.12 & 244.58 & 28092.74 & 98.18 & 175.13 & 122.40 & 249.50 & 28.11 \\
\hline 7527.13 & 996807 & 51.00 & 3468.63 & 968.52 & 235.25 & 176.26 & 32305.62 & 138.16 & 222.55 & 146.18 & 255.78 & 34.58 \\
\hline 7527.20 & 1000165 & 51.00 & 3784.19 & 1041.67 & 179.99 & 178.55 & 31919.71 & 108.76 & 238.17 & 151.01 & 460.67 & 29.85 \\
\hline 7527.26 & 1018320 & 51.00 & 3649.06 & 985.37 & 136.18 & 184.16 & 34906.98 & 110.55 & 232.84 & 185.56 & 362.89 & 30.10 \\
\hline 7527.33 & 1021399 & 51.00 & 3544.56 & 948.28 & 189.35 & 186.05 & 35642.96 & 150.92 & 203.51 & 149.88 & 148.43 & 31.38 \\
\hline 7527.39 & 988503 & 51.00 & 3580.79 & 791.32 & 172.55 & 203.35 & 34532.80 & 126.08 & 213.39 & 123.95 & 267.44 & 30.55 \\
\hline 7527.46 & 1083259 & 50.00 & 4166.67 & 1085.18 & 181.48 & 257.45 & 33957.26 & 128.99 & 209.14 & 146.18 & 219.68 & 31.87 \\
\hline 7527.52 & 1065827 & 50.00 & 4243.06 & 1002.26 & 156.13 & 181.80 & 35416.53 & 0.00 & 220.93 & 110.72 & 156.82 & 27.84 \\
\hline 7527.59 & 1281905 & 48.00 & 3321.08 & 732.00 & 203.26 & 273.42 & 24054.09 & 88.69 & 130.82 & 76.90 & 112.28 & 20.70 \\
\hline
\end{tabular}




\begin{tabular}{|c|c|c|c|c|c|c|c|c|c|c|c|c|}
\hline Depth & $\mathrm{XRF}$ & XRF Live Time & $\mathrm{Ti}$ & $\mathrm{V}$ & $\mathrm{Cr}$ & $\mathrm{Mn}$ & $\mathrm{Fe}$ & $\mathrm{Co}$ & $\mathrm{Ni}$ & $\mathrm{Cu}$ & $\mathrm{Zn}$ & As \\
\hline & Total Counts & secs & $\mathrm{ppm}$ & ppm & $\mathrm{ppm}$ & $\mathrm{ppm}$ & $\mathrm{ppm}$ & $\mathrm{ppm}$ & $\mathrm{ppm}$ & $\mathrm{ppm}$ & $\mathrm{ppm}$ & $\mathrm{ppm}$ \\
\hline 7527.66 & 1359983 & 47.00 & 2900.08 & 825.60 & 244.43 & 286.22 & 21275.13 & 96.10 & 130.23 & 72.60 & 167.95 & 19.59 \\
\hline 7527.72 & 1215486 & 49.00 & 3985.48 & 958.21 & 151.43 & 251.76 & 28435.94 & 106.33 & 170.04 & 98.06 & 330.81 & 23.76 \\
\hline 7527.79 & 1411965 & 47.00 & 3052.35 & 782.68 & 206.50 & 328.38 & 24195.68 & 75.30 & 174.84 & 113.98 & 302.47 & 20.81 \\
\hline 7527.85 & 1194067 & 49.00 & 3999.69 & 1062.98 & 183.76 & 234.09 & 29538.59 & 112.92 & 164.57 & 117.56 & 442.58 & 23.38 \\
\hline 7527.92 & 1381205 & 47.00 & 3863.72 & 907.43 & 137.96 & 324.07 & 31164.29 & 104.76 & 179.27 & 117.49 & 38.63 & 25.50 \\
\hline 7527.98 & 1225072 & 49.00 & 4278.37 & 1066.91 & 190.63 & 254.27 & 32657.79 & 116.99 & 192.32 & 119.81 & 39.88 & 28.75 \\
\hline 7528.05 & 1198329 & 49.00 & 16405.21 & 710.86 & 211.09 & 182.54 & 35465.46 & 123.28 & 191.76 & 126.06 & 40.25 & 31.10 \\
\hline 7528.12 & 1112014 & 50.00 & 4598.63 & 994.00 & 168.69 & 237.15 & 33332.62 & 102.10 & 198.49 & 106.00 & 0.00 & 30.02 \\
\hline 7528.18 & 1110067 & 50.00 & 4219.69 & 1020.42 & 231.95 & 189.93 & 33381.37 & 112.01 & 216.35 & 118.22 & 0.00 & 29.06 \\
\hline 7528.25 & 1172911 & 49.00 & 3967.48 & 930.57 & 179.63 & 128.24 & 40359.17 & 127.46 & 278.79 & 198.49 & 0.00 & 52.30 \\
\hline 7528.31 & 1092925 & 50.00 & 4322.82 & 920.40 & 141.95 & 175.73 & 45643.49 & 174.91 & 295.68 & 210.74 & 33.45 & 58.01 \\
\hline 7528.38 & 1251460 & 49.00 & 4170.21 & 918.95 & 172.45 & 139.09 & 46912.99 & 134.71 & 280.52 & 197.08 & 40.98 & 62.58 \\
\hline 7528.44 & 1158589 & 50.00 & 4282.22 & 799.63 & 149.39 & 134.12 & 54031.44 & 180.82 & 200.15 & 160.90 & 0.00 & 65.27 \\
\hline 7528.51 & 1020996 & 51.00 & 4339.74 & 928.88 & 175.43 & 180.15 & 37812.48 & 116.86 & 197.76 & 151.29 & 0.00 & 46.21 \\
\hline 7528.57 & 1174871 & 49.00 & 3775.84 & 826.97 & 148.93 & 125.56 & 41647.58 & 118.68 & 244.93 & 140.14 & 0.00 & 53.46 \\
\hline 7528.64 & 1080763 & 50.00 & 3850.73 & 912.12 & 212.40 & 190.31 & 41168.59 & 146.85 & 217.94 & 143.40 & 37.62 & 44.46 \\
\hline 7528.71 & 1037005 & 51.00 & 3743.12 & 897.20 & 108.22 & 203.54 & 36454.92 & 117.13 & 201.06 & 135.82 & 0.00 & 38.41 \\
\hline 7528.77 & 906159 & 52.00 & 4076.68 & 759.87 & 167.49 & 239.05 & 37582.74 & 129.42 & 218.49 & 125.41 & 0.00 & 26.22 \\
\hline 7528.84 & 907332 & 52.00 & 3929.26 & 744.72 & 189.07 & 147.41 & 51815.18 & 133.39 & 197.72 & 126.58 & 0.00 & 50.32 \\
\hline 7528.90 & 805834 & 53.00 & 3764.09 & 934.97 & 0.00 & 215.66 & 37490.88 & 140.86 & 167.82 & 131.74 & 0.00 & 33.91 \\
\hline 7528.97 & 634535 & 55.00 & 4088.53 & 1007.00 & 146.44 & 160.25 & 37595.31 & 127.45 & 221.83 & 124.65 & 0.00 & 32.76 \\
\hline 7529.03 & 818138 & 53.00 & 22675.60 & 3962.58 & 0.00 & 398.61 & 38829.45 & 151.53 & 222.23 & 139.14 & 61.89 & 32.46 \\
\hline 7529.10 & 834764 & 53.00 & 6004.00 & 2202.08 & 0.00 & 264.33 & 42300.49 & 145.09 & 267.99 & 165.86 & 0.00 & 38.88 \\
\hline 7529.17 & 949304 & 52.00 & 7744.15 & 2910.45 & 0.00 & 284.68 & 48529.46 & 180.00 & 302.73 & 189.77 & 221.18 & 40.28 \\
\hline 7529.23 & 1105726 & 50.00 & 4132.86 & 875.52 & 144.11 & 162.22 & 42909.00 & 124.96 & 243.25 & 177.57 & 0.00 & 33.83 \\
\hline 7529.30 & 1098136 & 50.00 & 4016.85 & 1059.56 & 143.56 & 163.21 & 45347.91 & 184.38 & 268.73 & 168.99 & 55.73 & 44.09 \\
\hline 7529.36 & 1012739 & 51.00 & 4556.61 & 1054.70 & 138.86 & 229.94 & 36884.25 & 132.37 & 221.83 & 118.97 & 35.87 & 32.59 \\
\hline 7529.43 & 1244719 & 49.00 & 50494.42 & 28970.64 & 0.00 & 2083.54 & 54543.65 & 145.15 & 205.20 & 117.40 & 0.00 & 27.77 \\
\hline 7529.49 & 1529438 & 46.00 & 149695.94 & 91185.03 & 0.00 & 8248.47 & 27953.25 & 132.64 & 108.22 & 111.39 & 0.00 & 0.00 \\
\hline 7529.56 & 1227483 & 49.00 & 8255.06 & 3150.10 & 0.00 & 287.10 & 34067.93 & 140.42 & 194.98 & 124.70 & 0.00 & 30.31 \\
\hline 7529.62 & 1398568 & 47.00 & 80341.91 & 48320.07 & 0.00 & 3495.00 & 26098.19 & 0.00 & 135.08 & 88.86 & 77.48 & 0.00 \\
\hline 7529.69 & 1203838 & 49.00 & 4289.08 & 1172.10 & 184.85 & 283.22 & 25517.87 & 0.00 & 147.46 & 103.19 & 0.00 & 21.49 \\
\hline
\end{tabular}




\begin{tabular}{|c|c|c|c|c|c|c|c|c|c|c|c|c|}
\hline Depth & $\mathrm{XRF}$ & XRF Live Time & $\mathrm{Ti}$ & $\mathrm{V}$ & $\mathrm{Cr}$ & $\mathrm{Mn}$ & $\mathrm{Fe}$ & $\mathrm{Co}$ & $\mathrm{Ni}$ & $\mathrm{Cu}$ & $\mathrm{Zn}$ & As \\
\hline & Total Counts & secs & $\mathrm{ppm}$ & ppm & $\mathrm{ppm}$ & $\mathrm{ppm}$ & $\mathrm{ppm}$ & $\mathrm{ppm}$ & $\mathrm{ppm}$ & $\mathrm{ppm}$ & $\mathrm{ppm}$ & $\mathrm{ppm}$ \\
\hline 7529.76 & 1119307 & 50.00 & 13656.34 & 6901.41 & 0.00 & 598.80 & 30023.61 & 119.58 & 174.61 & 128.11 & 0.00 & 20.06 \\
\hline 7529.82 & 1051841 & 51.00 & 4913.07 & 1389.42 & 147.64 & 210.67 & 31255.24 & 122.60 & 180.60 & 131.60 & 30.97 & 21.21 \\
\hline 7529.89 & 1022736 & 51.00 & 6473.05 & 2543.23 & 0.00 & 264.18 & 32924.13 & 108.02 & 203.26 & 144.52 & 0.00 & 24.24 \\
\hline 7529.95 & 1049273 & 51.00 & 7804.86 & 3057.66 & 0.00 & 258.06 & 33285.99 & 96.20 & 196.55 & 137.07 & 36.10 & 23.84 \\
\hline 7530.02 & 922379 & 52.00 & 7658.88 & 2628.40 & 181.25 & 304.98 & 34588.84 & 93.23 & 217.02 & 150.62 & 0.00 & 20.29 \\
\hline 7530.08 & 973431 & 51.00 & 41450.76 & 23668.03 & 0.00 & 1694.94 & 42412.49 & 0.00 & 148.93 & 121.87 & 58.46 & 0.00 \\
\hline 7530.15 & 1518060 & 46.00 & 18907.33 & 10457.62 & 0.00 & 752.60 & 90286.33 & 159.71 & 220.99 & 131.97 & 0.00 & 45.58 \\
\hline 7530.22 & 1169140 & 49.00 & 5064.87 & 1694.65 & 177.65 & 222.66 & 34453.65 & 112.14 & 197.45 & 151.70 & 0.00 & 21.04 \\
\hline 7530.28 & 1226637 & 49.00 & 30833.29 & 17700.79 & 0.00 & 1354.39 & 33538.38 & 121.37 & 190.09 & 116.68 & 35.79 & 25.77 \\
\hline 7530.35 & 1109800 & 50.00 & 7515.72 & 3222.42 & 0.00 & 348.48 & 29131.55 & 78.62 & 182.32 & 147.91 & 0.00 & 19.08 \\
\hline 7530.41 & 1372476 & 47.00 & 7532.74 & 3134.87 & 254.32 & 398.96 & 32512.35 & 96.78 & 229.68 & 143.09 & 0.00 & 26.00 \\
\hline 7530.48 & 1076086 & 50.00 & 12218.28 & 5795.71 & 0.00 & 703.18 & 29111.79 & 0.00 & 183.53 & 131.50 & 0.00 & 19.86 \\
\hline 7530.54 & 1237850 & 49.00 & 28938.64 & 16828.87 & 0.00 & 1572.51 & 25031.29 & 0.00 & 151.21 & 122.74 & 0.00 & 0.00 \\
\hline 7530.61 & 1172556 & 49.00 & 6775.75 & 2559.66 & 181.04 & 349.50 & 31456.03 & 87.26 & 175.96 & 115.06 & 0.00 & 26.74 \\
\hline 7530.67 & 1162842 & 50.00 & 7614.33 & 3219.61 & 0.00 & 362.83 & 30943.57 & 98.78 & 177.62 & 134.81 & 0.00 & 24.40 \\
\hline 7530.74 & 1227613 & 49.00 & 107280.82 & 64635.92 & 0.00 & 6041.70 & 73971.83 & 0.00 & 133.63 & 122.29 & 115.50 & 0.00 \\
\hline 7530.81 & 1072276 & 50.00 & 18474.79 & 9995.95 & 0.00 & 688.41 & 29730.29 & 96.27 & 185.29 & 113.64 & 0.00 & 21.27 \\
\hline 7530.87 & 1003383 & 51.00 & 4757.00 & 1690.59 & 0.00 & 242.04 & 28336.99 & 104.70 & 169.14 & 104.08 & 0.00 & 19.16 \\
\hline 7530.94 & 1195174 & 49.00 & 5059.29 & 1796.43 & 0.00 & 210.72 & 28974.14 & 102.68 & 137.66 & 118.38 & 0.00 & 24.76 \\
\hline 7531.00 & 782239 & 53.00 & 14814.16 & 7369.20 & 0.00 & 702.75 & 32268.56 & 0.00 & 185.39 & 130.34 & 0.00 & 30.47 \\
\hline 7531.07 & 1066665 & 50.00 & 5561.88 & 1974.80 & 158.62 & 307.27 & 28814.82 & 82.37 & 154.48 & 124.24 & 39.05 & 22.48 \\
\hline 7531.13 & 963302 & 51.00 & 28155.76 & 16699.96 & 0.00 & 1173.24 & 33260.22 & 0.00 & 139.23 & 135.75 & 54.87 & 25.18 \\
\hline 7531.20 & 959728 & 52.00 & 17699.58 & 9733.56 & 0.00 & 729.90 & 21695.81 & 0.00 & 132.24 & 88.81 & 386.85 & 23.89 \\
\hline 7531.26 & 1604393 & 45.00 & 91766.94 & 56122.02 & 0.00 & 4567.29 & 53288.41 & 0.00 & 112.30 & 84.12 & 136.98 & 0.00 \\
\hline 7531.33 & 1334137 & 48.00 & 5497.12 & 2234.61 & 0.00 & 442.09 & 16205.76 & 0.00 & 108.52 & 68.35 & 137.69 & 0.00 \\
\hline 7531.39 & 1286681 & 48.00 & 18505.43 & 10600.01 & 0.00 & 934.82 & 18583.20 & 0.00 & 115.11 & 64.55 & 133.21 & 0.00 \\
\hline 7531.46 & 1759884 & 43.00 & 2451.05 & 564.29 & 137.39 & 343.01 & 15454.53 & 0.00 & 94.37 & 64.52 & 0.00 & 0.00 \\
\hline 7531.52 & 1638709 & 44.00 & 2280.57 & 695.23 & 155.03 & 324.14 & 17268.26 & 0.00 & 103.61 & 65.52 & 0.00 & 0.00 \\
\hline 7531.59 & 1268276 & 48.00 & 3135.28 & 794.48 & 168.07 & 251.94 & 22616.25 & 0.00 & 138.57 & 107.75 & 0.00 & 23.43 \\
\hline 7531.66 & 1419938 & 47.00 & 3777.13 & 775.19 & 175.84 & 209.29 & 27789.93 & 89.12 & 169.08 & 116.21 & 0.00 & 18.00 \\
\hline 7531.72 & 1359999 & 47.00 & 3627.03 & 814.11 & 179.72 & 205.60 & 29669.54 & 136.69 & 167.79 & 119.00 & 0.00 & 26.06 \\
\hline 7531.79 & 1369553 & 47.00 & 4051.38 & 830.95 & 133.36 & 240.92 & 29712.08 & 0.00 & 179.12 & 128.00 & 39.36 & 20.54 \\
\hline
\end{tabular}




\begin{tabular}{|c|c|c|c|c|c|c|c|c|c|c|c|c|}
\hline Depth & $\mathrm{XRF}$ & XRF Live Time & $\mathrm{Ti}$ & $\mathrm{V}$ & $\mathrm{Cr}$ & $\mathrm{Mn}$ & $\mathrm{Fe}$ & $\mathrm{Co}$ & $\mathrm{Ni}$ & $\mathrm{Cu}$ & $\mathrm{Zn}$ & As \\
\hline & Total Counts & secs & $\mathrm{ppm}$ & ppm & $\mathrm{ppm}$ & $\mathrm{ppm}$ & $\mathrm{ppm}$ & $\mathrm{ppm}$ & $\mathrm{ppm}$ & $\mathrm{ppm}$ & $\mathrm{ppm}$ & $\mathrm{ppm}$ \\
\hline 7531.85 & 1237600 & 49.00 & 4015.68 & 732.14 & 209.22 & 218.52 & 30211.66 & 84.87 & 179.93 & 116.42 & 0.00 & 24.52 \\
\hline 7531.92 & 1458154 & 46.00 & 31232.37 & 17756.21 & 0.00 & 1237.79 & 71705.24 & 0.00 & 150.99 & 105.48 & 73.07 & 29.45 \\
\hline 7531.98 & 1299333 & 48.00 & 13174.22 & 680.32 & 152.09 & 226.98 & 29102.99 & 108.50 & 166.42 & 121.00 & 35.93 & 21.34 \\
\hline 7532.05 & 1199907 & 49.00 & 3784.52 & 845.07 & 156.44 & 206.44 & 29272.40 & 108.61 & 173.47 & 124.70 & 0.00 & 24.87 \\
\hline 7532.12 & 1302051 & 48.00 & 3944.72 & 853.27 & 182.82 & 246.55 & 30184.59 & 0.00 & 164.44 & 116.79 & 0.00 & 22.35 \\
\hline 7532.18 & 1314292 & 48.00 & 3950.62 & 841.95 & 186.24 & 257.62 & 28643.68 & 83.40 & 162.31 & 119.53 & 0.00 & 23.26 \\
\hline 7532.25 & 1175983 & 49.00 & 3890.92 & 912.08 & 212.69 & 213.54 & 30295.61 & 118.38 & 182.16 & 112.67 & 46.06 & 23.62 \\
\hline 7532.31 & 1459721 & 46.00 & 3947.76 & 812.22 & 198.04 & 250.91 & 31129.95 & 98.35 & 164.82 & 125.14 & 0.00 & 24.28 \\
\hline 7532.38 & 1303700 & 48.00 & 4007.51 & 871.29 & 215.15 & 270.85 & 29928.27 & 110.56 & 179.76 & 141.81 & 0.00 & 21.37 \\
\hline 7532.44 & 1373057 & 47.00 & 3260.31 & 745.77 & 176.78 & 236.07 & 28697.14 & 95.89 & 161.94 & 115.19 & 0.00 & 26.68 \\
\hline 7532.51 & 1265418 & 49.00 & 3476.26 & 917.84 & 203.52 & 265.39 & 28323.64 & 0.00 & 172.63 & 112.83 & 0.00 & 22.81 \\
\hline 7532.57 & 1238775 & 49.00 & 3345.37 & 874.83 & 165.63 & 271.05 & 30945.54 & 86.46 & 178.39 & 123.05 & 0.00 & 26.02 \\
\hline 7532.64 & 1284449 & 48.00 & 3285.18 & 947.72 & 148.52 & 261.71 & 27586.08 & 82.68 & 189.49 & 133.94 & 0.00 & 23.71 \\
\hline 7532.71 & 1123205 & 50.00 & 3883.27 & 1105.90 & 183.69 & 224.40 & 31372.89 & 128.56 & 183.56 & 141.53 & 0.00 & 24.36 \\
\hline 7532.77 & 1393862 & 47.00 & 3463.80 & 958.29 & 167.50 & 266.72 & 31725.21 & 89.29 & 190.20 & 142.66 & 32.40 & 23.52 \\
\hline 7532.84 & 1396285 & 47.00 & 3654.47 & 918.38 & 181.91 & 241.66 & 31139.79 & 0.00 & 163.66 & 114.53 & 0.00 & 26.03 \\
\hline 7532.90 & 1362860 & 47.00 & 3662.20 & 989.45 & 203.42 & 218.16 & 30931.01 & 103.63 & 201.41 & 127.37 & 66.19 & 29.36 \\
\hline 7532.97 & 1201510 & 49.00 & 5305.81 & 927.35 & 194.55 & 174.26 & 32683.12 & 113.06 & 200.80 & 135.88 & 376.04 & 25.44 \\
\hline 7533.03 & 1184678 & 49.00 & 3694.90 & 951.33 & 171.21 & 226.76 & 33008.79 & 102.32 & 213.83 & 151.32 & 298.64 & 25.96 \\
\hline 7533.10 & 1307410 & 48.00 & 3390.84 & 966.90 & 133.98 & 283.98 & 33019.19 & 115.13 & 187.80 & 154.10 & 704.57 & 26.32 \\
\hline 7533.17 & 1416140 & 47.00 & 3140.62 & 778.29 & 196.22 & 240.28 & 26398.22 & 0.00 & 163.82 & 106.92 & 60.28 & 21.45 \\
\hline 7533.23 & 1733533 & 43.00 & 2351.45 & 668.21 & 165.01 & 341.87 & 22317.62 & 77.19 & 125.64 & 83.82 & 92.46 & 15.73 \\
\hline 7533.30 & 1719751 & 43.00 & 2230.28 & 646.49 & 144.19 & 359.05 & 23028.29 & 85.84 & 113.86 & 91.49 & 105.76 & 18.68 \\
\hline 7533.36 & 1562526 & 45.00 & 2597.23 & 756.75 & 206.07 & 276.56 & 23726.94 & 0.00 & 144.11 & 89.97 & 314.49 & 17.21 \\
\hline 7533.43 & 1532782 & 46.00 & 2601.19 & 723.30 & 150.67 & 350.27 & 22777.97 & 0.00 & 155.89 & 101.06 & 202.32 & 0.00 \\
\hline 7533.49 & 1472085 & 46.00 & 3380.70 & 754.26 & 184.23 & 305.27 & 25945.08 & 92.61 & 158.49 & 134.36 & 100.78 & 19.46 \\
\hline 7533.56 & 1212987 & 49.00 & 3412.45 & 826.40 & 199.99 & 228.29 & 30394.95 & 91.44 & 168.92 & 126.02 & 197.64 & 22.87 \\
\hline 7533.62 & 1331464 & 48.00 & 3555.31 & 800.92 & 156.31 & 268.02 & 28005.64 & 0.00 & 165.28 & 102.85 & 179.54 & 21.45 \\
\hline 7533.69 & 1349908 & 48.00 & 3666.65 & 811.66 & 174.46 & 249.21 & 28156.99 & 90.90 & 161.38 & 99.90 & 110.57 & 20.62 \\
\hline 7533.76 & 1219281 & 49.00 & 3610.59 & 869.20 & 162.83 & 244.47 & 34096.25 & 115.41 & 159.51 & 113.61 & 0.00 & 26.85 \\
\hline 7533.82 & 1427189 & 47.00 & 3886.07 & 941.19 & 174.91 & 212.41 & 32046.41 & 94.57 & 193.83 & 147.49 & 138.86 & 27.67 \\
\hline 7533.89 & 1317628 & 48.00 & 3690.00 & 1022.44 & 235.84 & 221.61 & 31904.19 & 90.87 & 192.34 & 147.28 & 182.73 & 23.91 \\
\hline
\end{tabular}




\begin{tabular}{|c|c|c|c|c|c|c|c|c|c|c|c|c|}
\hline Depth & $\mathrm{XRF}$ & XRF Live Time & $\mathrm{Ti}$ & $\mathrm{V}$ & $\mathrm{Cr}$ & $\mathrm{Mn}$ & $\mathrm{Fe}$ & $\mathrm{Co}$ & $\mathrm{Ni}$ & $\mathrm{Cu}$ & $\mathrm{Zn}$ & As \\
\hline & Total Counts & secs & $\mathrm{ppm}$ & $\mathrm{ppm}$ & ppm & $\mathrm{ppm}$ & $\mathrm{ppm}$ & $\mathrm{ppm}$ & $\mathrm{ppm}$ & $\mathrm{ppm}$ & $\mathrm{ppm}$ & $\mathrm{ppm}$ \\
\hline 7533.95 & 1425530 & 47.00 & 3919.42 & 1001.47 & 141.26 & 303.48 & 29571.51 & 88.26 & 185.46 & 116.46 & 123.74 & 26.49 \\
\hline 7534.02 & 1238300 & 49.00 & 3856.54 & 843.41 & 196.44 & 177.15 & 30932.00 & 104.20 & 177.42 & 124.99 & 140.99 & 26.42 \\
\hline 7534.08 & 1154375 & 50.00 & 3833.27 & 923.79 & 172.93 & 182.94 & 32506.26 & 101.21 & 207.54 & 142.09 & 113.72 & 28.49 \\
\hline 7534.15 & 1026478 & 51.00 & 3768.10 & 876.82 & 157.25 & 207.13 & 34904.65 & 100.26 & 198.98 & 143.20 & 0.00 & 24.35 \\
\hline 7534.22 & 960489 & 51.00 & 3837.02 & 887.14 & 204.52 & 186.37 & 35983.71 & 139.74 & 183.16 & 148.00 & 0.00 & 28.77 \\
\hline 7534.28 & 1194802 & 49.00 & 3946.42 & 1015.44 & 132.05 & 224.64 & 36598.49 & 112.73 & 213.47 & 179.11 & 29.15 & 30.66 \\
\hline 7534.35 & 1116069 & 50.00 & 3735.91 & 1002.31 & 118.29 & 170.69 & 36636.47 & 94.90 & 230.00 & 165.80 & 152.42 & 32.21 \\
\hline 7534.41 & 998520 & 51.00 & 3858.42 & 952.09 & 169.05 & 174.02 & 36532.89 & 86.50 & 254.52 & 209.20 & 352.84 & 25.56 \\
\hline 7534.48 & 1231396 & 49.00 & 4253.21 & 981.54 & 165.93 & 156.28 & 41429.62 & 105.09 & 247.91 & 165.72 & 1241.68 & 30.91 \\
\hline 7534.54 & 1119915 & 50.00 & 4038.41 & 922.91 & 166.54 & 167.79 & 35922.97 & 98.49 & 215.66 & 150.93 & 245.22 & 28.09 \\
\hline 7534.61 & 972578 & 51.00 & 3375.58 & 967.25 & 144.66 & 182.52 & 35741.24 & 86.47 & 219.64 & 175.41 & 1147.40 & 26.60 \\
\hline 7534.67 & 1201501 & 49.00 & 3775.29 & 1072.94 & 154.55 & 163.68 & 34629.91 & 114.05 & 251.01 & 199.29 & 485.75 & 33.69 \\
\hline 7534.74 & 1026263 & 51.00 & 4014.10 & 851.91 & 208.00 & 156.00 & 33999.89 & 125.84 & 175.88 & 144.27 & 98.57 & 29.37 \\
\hline 7534.81 & 1109833 & 50.00 & 3963.92 & 748.14 & 156.01 & 142.57 & 32745.48 & 88.64 & 168.87 & 140.76 & 92.62 & 27.85 \\
\hline 7534.87 & 1270692 & 48.00 & 3463.43 & 857.54 & 165.79 & 156.48 & 29985.13 & 86.36 & 209.70 & 140.72 & 140.46 & 24.12 \\
\hline 7535.07 & 1229111 & 49.00 & 3571.53 & 1005.61 & 154.08 & 183.81 & 36075.17 & 103.23 & 254.96 & 196.61 & 856.83 & 33.37 \\
\hline 7535.13 & 1308648 & 48.00 & 3439.95 & 1077.51 & 204.55 & 133.06 & 38405.93 & 82.58 & 268.49 & 208.61 & 427.56 & 35.43 \\
\hline 7535.20 & 1151888 & 50.00 & 3903.19 & 995.20 & 188.78 & 174.16 & 36337.63 & 126.92 & 226.75 & 196.74 & 248.66 & 33.68 \\
\hline 7535.26 & 687619 & 54.00 & 3449.89 & 823.35 & 193.11 & 142.87 & 32633.46 & 111.60 & 181.49 & 161.05 & 510.43 & 33.54 \\
\hline 7535.33 & 1009590 & 51.00 & 4224.09 & 751.88 & 237.24 & 178.41 & 32887.11 & 92.99 & 208.47 & 138.93 & 199.23 & 29.01 \\
\hline 7535.39 & 1022079 & 51.00 & 3722.53 & 835.53 & 182.61 & 170.26 & 31482.68 & 89.14 & 190.03 & 138.13 & 215.90 & 31.30 \\
\hline 7535.46 & 922666 & 52.00 & 3619.75 & 869.31 & 188.94 & 146.17 & 30528.16 & 91.00 & 194.29 & 145.41 & 112.54 & 26.11 \\
\hline 7535.52 & 1203502 & 49.00 & 3815.23 & 895.16 & 185.82 & 165.85 & 33303.96 & 121.28 & 190.02 & 149.20 & 77.71 & 30.12 \\
\hline 7535.59 & 1218909 & 49.00 & 3994.49 & 964.89 & 119.64 & 152.93 & 35939.47 & 111.33 & 222.19 & 174.65 & 342.46 & 26.83 \\
\hline 7535.66 & 1234984 & 49.00 & 3406.90 & 926.43 & 163.04 & 157.03 & 37214.38 & 100.58 & 223.80 & 188.53 & 156.19 & 26.69 \\
\hline 7535.72 & 1201984 & 49.00 & 3509.11 & 898.55 & 151.66 & 130.52 & 47548.36 & 164.99 & 195.44 & 190.53 & 175.92 & 37.10 \\
\hline 7535.79 & 1126574 & 50.00 & 3145.99 & 957.04 & 144.38 & 130.26 & 38739.48 & 101.84 & 254.34 & 193.64 & 467.97 & 31.87 \\
\hline 7535.85 & 1154499 & 50.00 & 3342.23 & 995.13 & 133.10 & 177.49 & 33363.60 & 106.74 & 239.57 & 186.54 & 367.88 & 32.05 \\
\hline 7535.92 & 1048264 & 51.00 & 3434.79 & 916.47 & 183.64 & 198.93 & 35050.76 & 111.30 & 222.65 & 188.74 & 323.26 & 32.10 \\
\hline 7535.98 & 1246849 & 49.00 & 3631.32 & 1025.41 & 155.42 & 195.42 & 32204.84 & 93.88 & 173.51 & 157.39 & 229.72 & 27.05 \\
\hline 7536.05 & 1146873 & 50.00 & 3698.39 & 977.28 & 149.45 & 178.40 & 30526.86 & 101.38 & 197.51 & 149.43 & 415.47 & 26.47 \\
\hline 7536.12 & 1085922 & 50.00 & 3548.52 & 884.52 & 212.59 & 164.14 & 34675.78 & 118.17 & 211.83 & 177.88 & 891.25 & 32.16 \\
\hline
\end{tabular}




\begin{tabular}{|c|c|c|c|c|c|c|c|c|c|c|c|c|}
\hline Depth & $\mathrm{XRF}$ & XRF Live Time & $\mathrm{Ti}$ & $\mathrm{V}$ & $\mathrm{Cr}$ & $\mathrm{Mn}$ & $\mathrm{Fe}$ & $\mathrm{Co}$ & $\mathrm{Ni}$ & $\mathrm{Cu}$ & $\mathrm{Zn}$ & As \\
\hline & Total Counts & secs & $\mathrm{ppm}$ & $\mathrm{ppm}$ & ppm & $\mathrm{ppm}$ & $\mathrm{ppm}$ & $\mathrm{ppm}$ & $\mathrm{ppm}$ & $\mathrm{ppm}$ & $\mathrm{ppm}$ & $\mathrm{ppm}$ \\
\hline 7536.18 & 1043985 & 51.00 & 3333.53 & 938.09 & 118.40 & 138.52 & 33162.70 & 88.67 & 209.83 & 146.52 & 489.09 & 33.40 \\
\hline 7536.25 & 1115786 & 50.00 & 3729.63 & 964.25 & 163.11 & 152.15 & 35175.66 & 91.04 & 213.16 & 153.33 & 814.57 & 31.57 \\
\hline 7536.31 & 1105804 & 50.00 & 3204.66 & 857.11 & 227.65 & 168.72 & 31017.02 & 106.91 & 186.31 & 147.93 & 271.18 & 29.82 \\
\hline 7536.38 & 1108861 & 50.00 & 3664.14 & 1018.98 & 157.41 & 166.99 & 32537.79 & 109.14 & 201.63 & 169.66 & 223.32 & 30.67 \\
\hline 7536.44 & 800209 & 53.00 & 3093.66 & 821.07 & 220.50 & 151.45 & 29294.00 & 0.00 & 189.94 & 142.19 & 199.13 & 29.25 \\
\hline 7536.51 & 1256477 & 49.00 & 3370.82 & 838.69 & 166.91 & 194.43 & 41387.54 & 160.10 & 172.60 & 153.47 & 257.33 & 31.84 \\
\hline 7536.57 & 1231900 & 49.00 & 3580.36 & 892.05 & 233.34 & 176.41 & 32708.14 & 110.57 & 189.32 & 155.59 & 343.32 & 31.70 \\
\hline 7536.64 & 1254893 & 49.00 & 3450.07 & 896.12 & 278.48 & 165.35 & 32532.27 & 88.82 & 213.36 & 166.04 & 185.06 & 29.19 \\
\hline 7536.71 & 1132940 & 50.00 & 3399.13 & 1025.31 & 156.61 & 164.97 & 36218.65 & 101.08 & 240.35 & 163.39 & 358.41 & 39.39 \\
\hline 7536.77 & 1173745 & 49.00 & 3518.32 & 929.51 & 111.73 & 192.26 & 36108.28 & 142.26 & 223.79 & 180.20 & 772.82 & 37.28 \\
\hline 7536.84 & 1155135 & 50.00 & 3388.20 & 981.79 & 175.60 & 160.67 & 37778.99 & 134.29 & 207.27 & 175.05 & 611.76 & 32.39 \\
\hline 7536.90 & 1100748 & 50.00 & 3406.03 & 927.91 & 226.70 & 178.91 & 35056.88 & 107.24 & 208.71 & 184.85 & 612.02 & 34.98 \\
\hline 7536.97 & 770038 & 53.00 & 3722.93 & 1091.28 & 159.63 & 193.23 & 38483.81 & 0.00 & 230.52 & 192.83 & 1399.10 & 35.82 \\
\hline 7537.07 & 996926 & 51.00 & 7119.46 & 935.90 & 231.95 & 187.67 & 38084.86 & 139.01 & 238.07 & 215.00 & 585.29 & 38.37 \\
\hline 7537.13 & 1033836 & 51.00 & 4496.44 & 1315.50 & 278.81 & 198.50 & 42244.80 & 97.26 & 287.13 & 230.14 & 412.19 & 48.59 \\
\hline 7537.20 & 1210089 & 49.00 & 3773.61 & 1178.74 & 160.85 & 190.35 & 34638.59 & 106.34 & 199.89 & 191.06 & 408.29 & 40.64 \\
\hline 7537.26 & 1119116 & 50.00 & 3650.62 & 1014.71 & 201.30 & 171.55 & 42868.61 & 138.26 & 178.40 & 176.16 & 328.48 & 42.15 \\
\hline 7537.33 & 1115254 & 50.00 & 4048.74 & 1100.37 & 156.38 & 200.99 & 36232.72 & 147.70 & 203.83 & 213.41 & 282.59 & 38.84 \\
\hline 7537.39 & 1099514 & 50.00 & 3667.99 & 963.00 & 187.58 & 167.40 & 43396.68 & 142.47 & 240.07 & 203.61 & 520.03 & 45.07 \\
\hline 7537.46 & 1385127 & 47.00 & 3776.42 & 1024.00 & 183.91 & 196.89 & 52290.19 & 158.39 & 218.20 & 218.48 & 226.63 & 38.58 \\
\hline 7537.52 & 1221509 & 49.00 & 3688.74 & 1061.48 & 192.13 & 183.00 & 39738.30 & 86.92 & 239.19 & 201.27 & 307.10 & 40.66 \\
\hline 7537.59 & 1177529 & 49.00 & 4095.13 & 1260.52 & 204.92 & 181.61 & 40976.45 & 129.77 & 252.66 & 189.31 & 1500.26 & 52.20 \\
\hline 7537.66 & 1241446 & 49.00 & 3496.60 & 1194.59 & 220.36 & 164.02 & 57172.50 & 179.46 & 327.10 & 219.27 & 1804.64 & 78.02 \\
\hline 7537.72 & 1161889 & 50.00 & 3356.51 & 1195.60 & 184.20 & 150.55 & 36900.10 & 105.11 & 339.43 & 253.09 & 889.08 & 96.53 \\
\hline 7537.79 & 1052051 & 51.00 & 3744.82 & 1335.37 & 212.45 & 133.19 & 35626.66 & 114.00 & 347.26 & 224.82 & 891.53 & 76.87 \\
\hline 7537.85 & 1309952 & 48.00 & 3590.25 & 1316.05 & 200.99 & 120.27 & 47984.31 & 194.69 & 292.30 & 224.20 & 797.32 & 89.51 \\
\hline 7537.92 & 1138486 & 50.00 & 3566.07 & 1498.60 & 168.70 & 151.33 & 37218.37 & 136.55 & 355.40 & 260.60 & 1593.26 & 64.97 \\
\hline 7537.98 & 1070860 & 50.00 & 4140.30 & 1713.71 & 193.02 & 156.82 & 39906.52 & 118.03 & 371.24 & 257.96 & 964.25 & 65.32 \\
\hline 7538.05 & 1254251 & 49.00 & 10046.31 & 1360.30 & 216.98 & 151.45 & 48189.89 & 180.96 & 370.04 & 273.52 & 1191.79 & 55.31 \\
\hline 7538.12 & 1196104 & 49.00 & 3809.10 & 1564.39 & 169.84 & 156.38 & 40888.28 & 124.40 & 389.35 & 262.42 & 1165.16 & 48.19 \\
\hline 7538.18 & 1272223 & 48.00 & 2857.08 & 1307.48 & 138.10 & 98.94 & 83841.98 & 317.47 & 328.31 & 274.65 & 2192.26 & 84.86 \\
\hline 7538.25 & 1013408 & 51.00 & 3618.18 & 1490.45 & 189.02 & 135.30 & 37710.40 & 125.40 & 365.66 & 221.19 & 908.36 & 50.99 \\
\hline
\end{tabular}




\begin{tabular}{|c|c|c|c|c|c|c|c|c|c|c|c|c|}
\hline Depth & $\mathrm{XRF}$ & XRF Live Time & $\mathrm{Ti}$ & $\mathrm{V}$ & $\mathrm{Cr}$ & $\mathrm{Mn}$ & $\mathrm{Fe}$ & $\mathrm{Co}$ & $\mathrm{Ni}$ & $\mathrm{Cu}$ & $\mathrm{Zn}$ & As \\
\hline & Total Counts & secs & $\mathrm{ppm}$ & ppm & $\mathrm{ppm}$ & $\mathrm{ppm}$ & ppm & $\mathrm{ppm}$ & $\mathrm{ppm}$ & $\mathrm{ppm}$ & $\mathrm{ppm}$ & $\mathrm{ppm}$ \\
\hline 7538.31 & 1039533 & 51.00 & 3474.48 & 1406.03 & 132.04 & 155.20 & 37334.43 & 103.52 & 364.14 & 227.79 & 1077.23 & 42.70 \\
\hline 7538.38 & 1194477 & 49.00 & 3354.26 & 1242.41 & 178.89 & 170.70 & 37524.02 & 151.25 & 360.52 & 235.99 & 760.08 & 39.17 \\
\hline 7538.44 & 982156 & 51.00 & 3718.32 & 1363.32 & 129.01 & 155.36 & 40759.35 & 99.74 & 364.47 & 251.38 & 787.82 & 47.38 \\
\hline 7538.51 & 1337667 & 48.00 & 3286.89 & 1267.34 & 126.01 & 139.28 & 47019.77 & 180.03 & 310.64 & 246.76 & 490.00 & 44.36 \\
\hline 7538.57 & 916694 & 52.00 & 3696.99 & 1508.01 & 203.84 & 157.00 & 45468.09 & 140.19 & 335.00 & 286.76 & 1243.68 & 52.66 \\
\hline 7538.64 & 1071533 & 50.00 & 3719.66 & 1360.34 & 184.26 & 157.47 & 42063.24 & 142.14 & 347.01 & 263.10 & 918.86 & 46.85 \\
\hline 7538.71 & 1119030 & 50.00 & 4027.16 & 1367.30 & 214.25 & 169.08 & 37885.73 & 129.01 & 307.90 & 225.11 & 756.84 & 41.76 \\
\hline 7538.77 & 916950 & 52.00 & 3920.39 & 1073.47 & 226.46 & 136.52 & 34791.84 & 103.35 & 255.84 & 182.40 & 380.81 & 42.31 \\
\hline 7538.84 & 825469 & 53.00 & 3583.58 & 1101.17 & 118.28 & 161.93 & 33926.51 & 0.00 & 225.36 & 178.25 & 541.01 & 42.13 \\
\hline 7538.90 & 1052485 & 51.00 & 3689.67 & 1148.36 & 238.91 & 155.56 & 38301.28 & 118.06 & 238.57 & 189.66 & 514.74 & 47.18 \\
\hline 7538.97 & 1116674 & 50.00 & 3923.12 & 1130.43 & 218.41 & 147.76 & 37733.04 & 120.01 & 247.39 & 188.34 & 391.86 & 47.42 \\
\hline 7539.03 & 1199442 & 49.00 & 12198.48 & 1035.58 & 190.36 & 147.88 & 37461.39 & 108.72 & 246.05 & 197.25 & 885.30 & 48.15 \\
\hline 7539.10 & 1050111 & 51.00 & 3607.09 & 1196.39 & 149.56 & 131.21 & 41253.81 & 106.10 & 269.72 & 186.79 & 768.46 & 51.57 \\
\hline 7539.17 & 1413399 & 47.00 & 3686.73 & 1157.48 & 214.28 & 131.37 & 36173.90 & 98.76 & 317.61 & 214.14 & 510.80 & 45.53 \\
\hline 7539.23 & 1333970 & 48.00 & 3619.61 & 1279.16 & 152.92 & 109.31 & 39302.40 & 150.91 & 239.83 & 188.04 & 421.29 & 45.30 \\
\hline 7539.30 & 1097954 & 50.00 & 3876.94 & 1149.38 & 204.06 & 154.40 & 36756.04 & 103.06 & 255.66 & 211.05 & 379.39 & 50.61 \\
\hline 7539.36 & 1050107 & 51.00 & 3632.69 & 1132.36 & 208.54 & 141.15 & 40334.34 & 145.16 & 260.45 & 200.91 & 495.03 & 50.57 \\
\hline 7539.43 & 1293609 & 48.00 & 3846.53 & 1189.35 & 186.87 & 168.27 & 38663.12 & 142.83 & 262.89 & 198.59 & 1467.04 & 51.65 \\
\hline 7539.49 & 1433555 & 47.00 & 2980.34 & 998.96 & 287.08 & 175.86 & 37395.19 & 100.67 & 242.64 & 219.71 & 0.00 & 45.72 \\
\hline 7539.56 & 1254365 & 49.00 & 3453.90 & 916.66 & 181.59 & 169.43 & 42520.80 & 112.96 & 242.21 & 237.20 & 63.95 & 60.45 \\
\hline 7539.62 & 1135777 & 50.00 & 25545.59 & 15042.91 & 0.00 & 1237.11 & 29416.20 & 0.00 & 184.22 & 148.15 & 202.25 & 33.95 \\
\hline 7539.69 & 1059321 & 51.00 & 2622.21 & 895.04 & 162.67 & 185.35 & 33140.87 & 0.00 & 277.77 & 189.95 & 0.00 & 40.81 \\
\hline 7539.76 & 1357384 & 48.00 & 4770.53 & 1848.84 & 0.00 & 332.50 & 109590.84 & 381.74 & 0.00 & 63.63 & 0.00 & 130.26 \\
\hline 7539.82 & 1559205 & 45.00 & 3569.45 & 1196.51 & 165.13 & 166.47 & 52295.64 & 173.28 & 357.29 & 267.42 & 46.00 & 67.86 \\
\hline 7539.89 & 1253541 & 49.00 & 3690.88 & 1147.31 & 183.25 & 120.52 & 49976.85 & 135.17 & 341.87 & 255.12 & 44.92 & 64.18 \\
\hline 7539.95 & 1711047 & 43.00 & 2709.96 & 1005.82 & 0.00 & 128.39 & 112354.35 & 252.52 & 337.30 & 288.32 & 80.32 & 191.67 \\
\hline 7540.02 & 609810 & 55.00 & 4270.43 & 1603.61 & 232.12 & 186.62 & 51014.86 & 0.00 & 343.62 & 287.11 & 418.86 & 80.63 \\
\hline 7540.07 & 863287 & 53.00 & 3018.89 & 1597.45 & 190.89 & 199.70 & 54845.04 & 128.46 & 498.81 & 346.54 & 560.59 & 77.31 \\
\hline 7540.13 & 872577 & 52.00 & 3418.69 & 2054.20 & 175.19 & 157.51 & 54645.92 & 191.48 & 526.23 & 303.28 & 262.23 & 82.78 \\
\hline 7540.20 & 856395 & 53.00 & 3501.58 & 1915.46 & 0.00 & 113.35 & 55086.64 & 148.67 & 447.47 & 302.85 & 2689.55 & 105.36 \\
\hline 7540.26 & 826528 & 53.00 & 2874.01 & 1888.55 & 195.09 & 115.75 & 57112.65 & 144.29 & 470.76 & 307.41 & 955.28 & 105.80 \\
\hline 7540.33 & 667904 & 54.00 & 3513.08 & 2050.19 & 0.00 & 172.91 & 80628.46 & 0.00 & 411.22 & 356.35 & 314.96 & 122.25 \\
\hline
\end{tabular}




\begin{tabular}{|c|c|c|c|c|c|c|c|c|c|c|c|c|}
\hline Depth & $\mathrm{XRF}$ & XRF Live Time & $\mathrm{Ti}$ & $\mathrm{V}$ & $\mathrm{Cr}$ & $\mathrm{Mn}$ & $\mathrm{Fe}$ & $\mathrm{Co}$ & $\mathrm{Ni}$ & $\mathrm{Cu}$ & $\mathrm{Zn}$ & As \\
\hline & Total Counts & secs & $\mathrm{ppm}$ & $\mathrm{ppm}$ & ppm & $\mathrm{ppm}$ & $\mathrm{ppm}$ & $\mathrm{ppm}$ & $\mathrm{ppm}$ & $\mathrm{ppm}$ & $\mathrm{ppm}$ & $\mathrm{ppm}$ \\
\hline 7540.39 & 590748 & 55.00 & 4823.96 & 2732.41 & 0.00 & 228.89 & 62494.76 & 0.00 & 453.47 & 317.67 & 216.89 & 133.49 \\
\hline 7540.46 & 1419341 & 47.00 & 3602.12 & 2099.96 & 254.71 & 153.34 & 74195.90 & 162.55 & 528.04 & 358.22 & 120.66 & 131.42 \\
\hline 7540.52 & 1177092 & 49.00 & 2393.18 & 1490.61 & 160.88 & 103.48 & 68655.06 & 172.20 & 502.51 & 312.04 & 199.27 & 109.36 \\
\hline 7540.59 & 1127870 & 50.00 & 2331.00 & 1248.59 & 216.19 & 90.97 & 82906.86 & 248.28 & 335.37 & 301.30 & 170.28 & 118.17 \\
\hline 7540.66 & 1049870 & 51.00 & 2329.99 & 1201.19 & 157.56 & 169.77 & 78180.99 & 203.74 & 509.43 & 275.71 & 113.57 & 125.16 \\
\hline 7540.72 & 941900 & 52.00 & 2806.19 & 1537.02 & 271.20 & 99.30 & 66444.65 & 191.29 & 484.52 & 304.13 & 437.63 & 100.51 \\
\hline 7540.79 & 893806 & 52.00 & 2792.49 & 1273.72 & 193.85 & 80.81 & 65582.83 & 187.96 & 516.59 & 333.63 & 224.30 & 87.47 \\
\hline 7540.85 & 1066397 & 50.00 & 2598.98 & 1512.07 & 152.52 & 144.86 & 59661.27 & 146.88 & 383.79 & 289.04 & 981.88 & 86.38 \\
\hline 7540.92 & 1093060 & 50.00 & 3702.59 & 2001.38 & 167.53 & 189.31 & 61699.88 & 146.69 & 487.33 & 335.51 & 993.37 & 76.03 \\
\hline 7540.98 & 1003018 & 51.00 & 16043.02 & 1365.27 & 219.65 & 101.65 & 66438.71 & 0.00 & 488.66 & 352.62 & 2518.50 & 113.66 \\
\hline 7541.05 & 857311 & 53.00 & 2875.55 & 1470.56 & 0.00 & 104.25 & 53378.94 & 152.15 & 426.46 & 325.39 & 1785.32 & 77.74 \\
\hline 7541.12 & 763310 & 53.00 & 2852.31 & 1601.81 & 246.31 & 133.29 & 60851.90 & 0.00 & 457.41 & 334.92 & 2057.57 & 81.71 \\
\hline 7541.18 & 1441947 & 47.00 & 2730.65 & 1349.12 & 0.00 & 155.19 & 60473.07 & 127.91 & 472.93 & 284.75 & 2363.72 & 80.35 \\
\hline 7541.25 & 1214706 & 49.00 & 2692.87 & 1296.16 & 184.82 & 195.00 & 47292.05 & 112.21 & 380.53 & 310.58 & 4304.78 & 69.38 \\
\hline 7541.31 & 1248468 & 49.00 & 2561.55 & 1309.81 & 120.59 & 171.57 & 38532.40 & 91.96 & 318.15 & 273.45 & 1490.47 & 65.52 \\
\hline 7541.38 & 1095081 & 50.00 & 2533.57 & 1003.17 & 212.42 & 173.57 & 36410.92 & 112.55 & 346.23 & 298.44 & 1855.20 & 53.90 \\
\hline 7541.44 & 1374051 & 47.00 & 2381.42 & 972.38 & 149.29 & 236.15 & 40583.64 & 0.00 & 171.19 & 279.42 & 488.66 & 39.60 \\
\hline 7541.51 & 2043872 & 39.00 & 1178.35 & 517.71 & 0.00 & 327.25 & 4401.75 & 0.00 & 26.68 & 0.00 & 0.00 & 0.00 \\
\hline 7541.57 & 2081646 & 38.00 & 1556.51 & 801.19 & 0.00 & 390.94 & 2243.96 & 0.00 & 24.10 & 0.00 & 0.00 & 0.00 \\
\hline 7541.64 & 2106754 & 38.00 & 956.63 & 542.05 & 0.00 & 260.51 & 1790.17 & 31.46 & 18.71 & 0.00 & 0.00 & 0.00 \\
\hline 7541.71 & 2091045 & 38.00 & 704.40 & 413.12 & 0.00 & 291.85 & 2710.99 & 0.00 & 38.82 & 0.00 & 0.00 & 0.00 \\
\hline 7541.77 & 2099394 & 38.00 & 923.06 & 409.00 & 116.81 & 255.10 & 4006.73 & 0.00 & 23.37 & 0.00 & 0.00 & 0.00 \\
\hline 7541.84 & 2075631 & 39.00 & 895.80 & 355.14 & 0.00 & 277.82 & 4925.31 & 0.00 & 31.69 & 0.00 & 0.00 & 0.00 \\
\hline 7541.90 & 2151859 & 37.00 & 884.11 & 321.16 & 0.00 & 314.46 & 5331.62 & 0.00 & 32.00 & 44.72 & 0.00 & 0.00 \\
\hline 7541.97 & 2286215 & 35.00 & 1183.15 & 621.10 & 0.00 & 312.94 & 5259.34 & 0.00 & 39.69 & 47.19 & 0.00 & 0.00 \\
\hline 7542.03 & 2141958 & 38.00 & 2959.82 & 495.57 & 195.50 & 339.56 & 6176.83 & 0.00 & 32.90 & 60.10 & 0.00 & 0.00 \\
\hline 7542.10 & 1857107 & 42.00 & 535.88 & 396.30 & 108.91 & 260.13 & 15107.06 & 0.00 & 36.00 & 40.61 & 0.00 & 0.00 \\
\hline 7542.17 & 1566075 & 45.00 & 612.76 & 255.03 & 0.00 & 155.40 & 24737.69 & 0.00 & 44.22 & 58.42 & 0.00 & 15.16 \\
\hline 7542.23 & 1005886 & 51.00 & 735.29 & 421.86 & 0.00 & 161.93 & 12473.10 & 0.00 & 64.86 & 84.28 & 42.48 & 0.00 \\
\hline 7542.30 & 1132235 & 50.00 & 1179.51 & 593.95 & 180.45 & 198.89 & 31492.83 & 0.00 & 112.25 & 105.14 & 154.90 & 26.20 \\
\hline 7542.36 & 988289 & 51.00 & 2908.51 & 1096.77 & 204.33 & 207.64 & 50948.60 & 122.56 & 322.12 & 305.06 & 478.59 & 53.20 \\
\hline 7542.43 & 1096115 & 50.00 & 2817.86 & 1287.00 & 181.77 & 117.51 & 49285.74 & 184.43 & 467.79 & 329.44 & 1379.37 & 71.82 \\
\hline
\end{tabular}




\begin{tabular}{|c|c|c|c|c|c|c|c|c|c|c|c|c|}
\hline Depth & $\mathrm{XRF}$ & XRF Live Time & $\mathrm{Ti}$ & $\mathrm{V}$ & $\mathrm{Cr}$ & $\mathrm{Mn}$ & $\mathrm{Fe}$ & $\mathrm{Co}$ & $\mathrm{Ni}$ & $\mathrm{Cu}$ & $\mathrm{Zn}$ & As \\
\hline & Total Counts & secs & $\mathrm{ppm}$ & ppm & ppm & $\mathrm{ppm}$ & ppm & $\mathrm{ppm}$ & $\mathrm{ppm}$ & $\mathrm{ppm}$ & $\mathrm{ppm}$ & $\mathrm{ppm}$ \\
\hline 7542.49 & 929795 & 52.00 & 2460.61 & 1261.69 & 188.92 & 159.31 & 47314.37 & 0.00 & 468.49 & 314.04 & 2158.01 & 58.16 \\
\hline 7542.56 & 1090256 & 50.00 & 2693.12 & 1390.95 & 211.96 & 156.19 & 44440.33 & 150.41 & 400.34 & 311.63 & 1493.30 & 59.03 \\
\hline 7542.62 & 832141 & 53.00 & 3019.26 & 1321.33 & 252.69 & 109.82 & 43096.92 & 0.00 & 469.93 & 325.72 & 1725.74 & 54.19 \\
\hline 7542.69 & 887855 & 52.00 & 2552.95 & 1322.94 & 134.73 & 88.34 & 46550.90 & 0.00 & 391.95 & 311.83 & 1929.48 & 62.05 \\
\hline 7542.76 & 1149389 & 50.00 & 1426.00 & 701.98 & 0.00 & 0.00 & 134551.73 & 212.61 & 288.82 & 256.43 & 1525.48 & 202.72 \\
\hline 7542.82 & 828156 & 53.00 & 2587.69 & 1319.64 & 200.15 & 128.89 & 52656.74 & 152.82 & 425.84 & 328.92 & 1263.65 & 72.86 \\
\hline 7542.89 & 1167461 & 49.00 & 2934.53 & 1486.27 & 208.36 & 143.68 & 46664.71 & 0.00 & 412.06 & 293.71 & 1365.89 & 64.34 \\
\hline 7542.95 & 1678256 & 44.00 & 1782.49 & 922.74 & 108.51 & 185.86 & 36489.22 & 102.11 & 237.75 & 178.89 & 1001.84 & 43.03 \\
\hline 7543.07 & 1347867 & 48.00 & 2995.40 & 1532.49 & 191.41 & 132.94 & 56067.90 & 154.97 & 454.74 & 399.68 & 1204.00 & 86.47 \\
\hline 7543.13 & 1265572 & 48.00 & 3040.29 & 1590.89 & 148.27 & 130.03 & 54504.80 & 170.44 & 509.37 & 348.93 & 715.65 & 66.16 \\
\hline 7543.20 & 1586545 & 45.00 & 1765.03 & 1278.09 & 188.47 & 243.17 & 26701.83 & 0.00 & 277.04 & 207.96 & 642.17 & 29.11 \\
\hline 7543.26 & 1628903 & 44.00 & 2933.06 & 1403.16 & 175.65 & 218.91 & 51585.04 & 137.65 & 376.62 & 319.83 & 1117.81 & 73.43 \\
\hline 7543.33 & 1445655 & 47.00 & 2575.09 & 1346.43 & 0.00 & 143.18 & 62168.63 & 164.33 & 337.30 & 293.45 & 1743.91 & 96.91 \\
\hline 7543.39 & 1328659 & 48.00 & 2833.35 & 1270.12 & 193.16 & 144.67 & 62075.98 & 156.84 & 466.14 & 383.28 & 549.90 & 74.14 \\
\hline 7543.46 & 1213694 & 49.00 & 2919.69 & 1656.22 & 257.03 & 134.50 & 45761.23 & 115.13 & 384.03 & 308.12 & 382.12 & 67.52 \\
\hline 7543.52 & 1570048 & 45.00 & 2541.20 & 1252.90 & 133.95 & 89.95 & 90016.29 & 277.88 & 354.98 & 328.61 & 343.80 & 87.33 \\
\hline 7543.59 & 1268282 & 48.00 & 2916.89 & 1406.19 & 207.22 & 153.82 & 38593.38 & 101.31 & 319.43 & 285.67 & 638.25 & 66.84 \\
\hline 7543.66 & 1249256 & 49.00 & 2444.49 & 1374.84 & 192.07 & 125.43 & 69808.60 & 149.49 & 411.88 & 382.42 & 1148.40 & 106.94 \\
\hline 7543.72 & 1359511 & 47.00 & 2279.05 & 1562.18 & 221.47 & 127.09 & 52640.18 & 146.33 & 459.51 & 363.69 & 595.38 & 84.05 \\
\hline 7543.79 & 1439176 & 47.00 & 2464.68 & 1535.42 & 214.03 & 167.11 & 60488.93 & 174.88 & 468.16 & 370.08 & 936.11 & 97.03 \\
\hline 7543.85 & 1388138 & 47.00 & 2565.30 & 1558.75 & 203.98 & 140.59 & 56772.09 & 163.17 & 429.87 & 378.18 & 1255.38 & 86.65 \\
\hline 7543.92 & 1346719 & 48.00 & 2713.30 & 1605.38 & 242.15 & 141.24 & 54028.48 & 142.07 & 431.64 & 370.41 & 1566.89 & 81.65 \\
\hline 7543.98 & 1311102 & 48.00 & 2177.56 & 1405.29 & 259.23 & 184.74 & 48718.25 & 122.89 & 309.11 & 277.88 & 497.11 & 71.81 \\
\hline 7544.05 & 1322055 & 48.00 & 11787.12 & 1395.72 & 177.39 & 140.34 & 63122.13 & 193.36 & 413.28 & 375.17 & 637.17 & 103.16 \\
\hline 7544.12 & 1110677 & 50.00 & 2369.60 & 1643.14 & 132.69 & 110.99 & 55628.28 & 155.94 & 387.64 & 308.31 & 958.44 & 76.11 \\
\hline 7544.18 & 1206231 & 49.00 & 2289.33 & 1547.96 & 189.83 & 113.84 & 55757.88 & 166.29 & 447.38 & 342.40 & 529.42 & 76.81 \\
\hline 7544.25 & 999797 & 51.00 & 2139.15 & 1547.91 & 212.67 & 145.79 & 42738.42 & 120.41 & 378.82 & 296.76 & 787.72 & 63.22 \\
\hline 7544.31 & 1143314 & 50.00 & 12016.33 & 7507.12 & 210.83 & 742.74 & 41179.31 & 116.24 & 344.88 & 260.52 & 517.19 & 59.97 \\
\hline 7544.38 & 1328139 & 48.00 & 92808.01 & 57767.70 & 0.00 & 5278.28 & 47409.88 & 0.00 & 301.97 & 266.54 & 168.17 & 56.84 \\
\hline 7544.44 & 1014131 & 51.00 & 60829.18 & 38231.81 & 0.00 & 3545.70 & 50664.98 & 0.00 & 307.11 & 257.53 & 0.00 & 59.97 \\
\hline 7544.51 & 1049612 & 51.00 & 20019.98 & 11959.39 & 0.00 & 1143.58 & 41712.52 & 0.00 & 351.03 & 293.47 & 316.51 & 63.10 \\
\hline 7544.57 & 1147855 & 50.00 & 34763.14 & 21430.19 & 0.00 & 2001.41 & 44363.83 & 0.00 & 369.52 & 285.09 & 938.32 & 57.77 \\
\hline
\end{tabular}




\begin{tabular}{|c|c|c|c|c|c|c|c|c|c|c|c|c|}
\hline Depth & $\mathrm{XRF}$ & XRF Live Time & $\mathrm{Ti}$ & $\mathrm{V}$ & $\mathrm{Cr}$ & $\mathrm{Mn}$ & $\mathrm{Fe}$ & $\mathrm{Co}$ & $\mathrm{Ni}$ & $\mathrm{Cu}$ & $\mathrm{Zn}$ & As \\
\hline & Total Counts & secs & $\mathrm{ppm}$ & ppm & $\mathrm{ppm}$ & $\mathrm{ppm}$ & $\mathrm{ppm}$ & $\mathrm{ppm}$ & $\mathrm{ppm}$ & $\mathrm{ppm}$ & $\mathrm{ppm}$ & $\mathrm{ppm}$ \\
\hline 7544.64 & 1034307 & 51.00 & 22464.98 & 14106.32 & 0.00 & 1347.76 & 31285.68 & 0.00 & 318.90 & 253.11 & 525.67 & 47.70 \\
\hline 7544.71 & 953864 & 52.00 & 14536.20 & 8588.08 & 0.00 & 824.44 & 35263.09 & 0.00 & 319.65 & 307.46 & 452.35 & 48.60 \\
\hline 7544.77 & 1324891 & 48.00 & 37447.83 & 22789.78 & 0.00 & 2089.48 & 41656.09 & 0.00 & 425.12 & 306.74 & 336.90 & 53.15 \\
\hline 7544.84 & 1292709 & 48.00 & 30144.82 & 18431.43 & 0.00 & 1770.71 & 37806.05 & 0.00 & 356.69 & 275.94 & 83.87 & 48.29 \\
\hline 7544.90 & 1383066 & 47.00 & 47226.73 & 29344.66 & 0.00 & 2663.83 & 43878.77 & 0.00 & 278.75 & 240.50 & 56.89 & 58.89 \\
\hline 7544.97 & 1155465 & 50.00 & 18401.71 & 10863.00 & 0.00 & 1044.70 & 29531.11 & 0.00 & 299.85 & 273.75 & 40.89 & 39.74 \\
\hline 7545.07 & 1162680 & 50.00 & 15334.57 & 8658.37 & 0.00 & 899.18 & 29274.75 & 0.00 & 310.01 & 265.09 & 135.31 & 45.94 \\
\hline 7545.13 & 1328734 & 48.00 & 5956.78 & 3240.98 & 147.72 & 363.84 & 49623.94 & 112.83 & 480.33 & 348.48 & 209.24 & 50.77 \\
\hline 7545.20 & 1314727 & 48.00 & 2850.22 & 1597.28 & 186.45 & 102.25 & 54040.04 & 97.12 & 495.62 & 376.81 & 873.23 & 59.09 \\
\hline 7545.26 & 1269144 & 48.00 & 3110.74 & 1442.85 & 220.82 & 142.84 & 53958.84 & 103.88 & 483.02 & 326.37 & 704.85 & 57.09 \\
\hline 7545.33 & 1239891 & 49.00 & 2659.20 & 1494.11 & 145.33 & 147.65 & 55484.71 & 131.45 & 463.96 & 310.12 & 506.03 & 60.96 \\
\hline 7545.39 & 1328363 & 48.00 & 2988.74 & 1365.17 & 173.01 & 196.56 & 46748.54 & 105.79 & 453.90 & 305.04 & 344.36 & 42.24 \\
\hline 7545.46 & 1606307 & 45.00 & 11327.85 & 6672.35 & 255.40 & 778.61 & 19656.42 & 0.00 & 206.57 & 166.46 & 44.19 & 22.39 \\
\hline 7545.52 & 1615556 & 45.00 & 22994.72 & 14489.07 & 0.00 & 1411.55 & 43327.16 & 0.00 & 223.05 & 198.59 & 0.00 & 48.61 \\
\hline 7545.59 & 1515843 & 46.00 & 30279.15 & 18628.20 & 0.00 & 1810.50 & 55271.80 & 135.44 & 375.36 & 287.78 & 0.00 & 61.61 \\
\hline 7545.66 & 1513086 & 46.00 & 36619.16 & 22930.65 & 0.00 & 1655.48 & 54334.88 & 0.00 & 396.36 & 302.59 & 50.24 & 47.70 \\
\hline 7545.72 & 1147382 & 50.00 & 2659.40 & 1453.72 & 260.04 & 111.77 & 48040.96 & 131.77 & 516.61 & 357.35 & 81.81 & 59.28 \\
\hline 7545.79 & 1184826 & 49.00 & 2628.58 & 1766.51 & 196.13 & 96.51 & 54931.34 & 142.12 & 515.51 & 364.28 & 1159.06 & 70.51 \\
\hline 7545.85 & 1205756 & 49.00 & 2559.92 & 1837.78 & 155.99 & 100.83 & 58820.78 & 183.98 & 516.42 & 359.29 & 1299.50 & 74.88 \\
\hline 7545.92 & 1292056 & 48.00 & 2434.95 & 1505.66 & 211.84 & 98.24 & 54031.05 & 173.43 & 510.84 & 344.55 & 1340.56 & 65.84 \\
\hline 7545.98 & 1206450 & 49.00 & 2547.11 & 1525.26 & 133.06 & 128.71 & 51942.54 & 105.00 & 444.08 & 334.40 & 801.97 & 73.60 \\
\hline 7546.05 & 1459850 & 46.00 & 7071.30 & 1231.30 & 0.00 & 74.07 & 124457.40 & 274.69 & 356.06 & 387.82 & 293.44 & 141.80 \\
\hline 7546.12 & 1212101 & 49.00 & 2659.88 & 1481.15 & 136.97 & 93.32 & 59318.44 & 147.07 & 429.91 & 355.66 & 720.77 & 84.67 \\
\hline 7546.18 & 1178478 & 49.00 & 2245.26 & 1467.99 & 168.80 & 90.30 & 47703.60 & 0.00 & 484.49 & 334.48 & 1273.93 & 54.98 \\
\hline 7546.25 & 1435079 & 47.00 & 2288.72 & 1404.53 & 180.08 & 152.45 & 42791.03 & 96.70 & 456.61 & 287.06 & 1089.15 & 52.98 \\
\hline 7546.31 & 1512816 & 46.00 & 2227.33 & 1420.32 & 216.38 & 220.66 & 43739.36 & 121.54 & 420.08 & 282.87 & 2082.23 & 64.61 \\
\hline 7546.38 & 1253405 & 49.00 & 2034.69 & 1346.98 & 188.63 & 123.41 & 40204.25 & 0.00 & 450.90 & 268.13 & 1642.43 & 46.31 \\
\hline 7546.44 & 965822 & 52.00 & 1920.25 & 1134.31 & 157.98 & 181.11 & 32763.76 & 0.00 & 359.81 & 280.34 & 1520.94 & 54.01 \\
\hline 7546.51 & 679663 & 54.00 & 2131.05 & 1136.95 & 173.54 & 116.14 & 45446.18 & 0.00 & 391.23 & 240.80 & 828.14 & 51.18 \\
\hline 7546.57 & 997494 & 51.00 & 2051.33 & 1416.35 & 156.97 & 163.31 & 48868.17 & 0.00 & 460.28 & 359.76 & 1254.77 & 80.85 \\
\hline 7546.64 & 906226 & 52.00 & 2232.20 & 1511.52 & 0.00 & 109.94 & 57845.06 & 161.98 & 491.74 & 395.29 & 1742.29 & 100.13 \\
\hline 7546.71 & 726527 & 54.00 & 5959.59 & 3445.88 & 206.19 & 284.96 & 40953.77 & 0.00 & 473.10 & 308.70 & 1863.87 & 56.25 \\
\hline
\end{tabular}




\begin{tabular}{|c|c|c|c|c|c|c|c|c|c|c|c|c|}
\hline Depth & $\mathrm{XRF}$ & XRF Live Time & $\mathrm{Ti}$ & $\mathrm{V}$ & $\mathrm{Cr}$ & $\mathrm{Mn}$ & $\mathrm{Fe}$ & $\mathrm{Co}$ & $\mathrm{Ni}$ & $\mathrm{Cu}$ & $\mathrm{Zn}$ & As \\
\hline & Total Counts & secs & $\mathrm{ppm}$ & $\mathrm{ppm}$ & $\mathrm{ppm}$ & $\mathrm{ppm}$ & $\mathrm{ppm}$ & $\mathrm{ppm}$ & $\mathrm{ppm}$ & $\mathrm{ppm}$ & ppm & $\mathrm{ppm}$ \\
\hline 7546.77 & 1360060 & 47.00 & 2774.52 & 1898.73 & 204.83 & 95.52 & 53267.83 & 171.47 & 594.91 & 409.70 & 3583.92 & 80.01 \\
\hline 7546.84 & 516474 & 56.00 & 4682.38 & 3041.37 & 0.00 & 252.29 & 34342.64 & 0.00 & 396.38 & 247.98 & 2385.40 & 49.69 \\
\hline 7546.90 & 732319 & 54.00 & 3313.49 & 2159.03 & 0.00 & 166.77 & 56776.45 & 0.00 & 391.06 & 273.89 & 1481.15 & 72.53 \\
\hline 7546.97 & 1299521 & 48.00 & 5836.44 & 1540.35 & 244.54 & 77.59 & 68461.41 & 139.32 & 587.49 & 415.09 & 31063.29 & 95.80 \\
\hline 7547.03 & 1247702 & 49.00 & 2575.07 & 1745.79 & 148.17 & 124.36 & 50086.80 & 0.00 & 512.16 & 355.80 & 6832.81 & 73.52 \\
\hline 7547.10 & 1302702 & 48.00 & 2321.25 & 1519.91 & 131.56 & 134.04 & 44694.86 & 159.89 & 493.28 & 339.63 & 1475.54 & 64.90 \\
\hline 7547.17 & 1153968 & 50.00 & 3166.91 & 1818.84 & 0.00 & 178.85 & 47378.21 & 120.55 & 418.78 & 294.19 & 469.50 & 62.76 \\
\hline 7547.23 & 1271966 & 48.00 & 2475.59 & 1368.44 & 202.34 & 108.07 & 49449.79 & 106.28 & 416.57 & 317.75 & 1636.81 & 82.16 \\
\hline 7547.30 & 1040701 & 51.00 & 2430.27 & 1667.75 & 139.80 & 145.58 & 37883.04 & 110.09 & 470.58 & 282.00 & 1278.00 & 47.85 \\
\hline 7547.36 & 1116173 & 50.00 & 2794.73 & 1716.25 & 120.14 & 113.83 & 55366.60 & 151.98 & 545.29 & 436.58 & 8089.06 & 80.82 \\
\hline 7547.43 & 1013180 & 51.00 & 2575.70 & 1691.30 & 0.00 & 76.18 & 47818.93 & 111.65 & 439.27 & 349.29 & 2195.33 & 84.41 \\
\hline 7547.49 & 1179981 & 49.00 & 2426.75 & 1528.00 & 158.42 & 93.13 & 40153.39 & 154.38 & 485.02 & 371.03 & 2266.89 & 56.38 \\
\hline 7547.56 & 1300297 & 48.00 & 2360.20 & 1496.52 & 148.77 & 82.68 & 72212.19 & 144.89 & 389.68 & 388.47 & 7965.40 & 120.68 \\
\hline 7547.62 & 1102143 & 50.00 & 2447.18 & 1434.62 & 163.42 & 102.21 & 40257.64 & 105.24 & 352.56 & 335.82 & 1762.29 & 68.18 \\
\hline 7547.69 & 1064228 & 51.00 & 2467.14 & 1565.18 & 0.00 & 120.65 & 37888.90 & 91.19 & 358.00 & 299.35 & 1688.59 & 61.51 \\
\hline 7547.76 & 1050840 & 51.00 & 2312.57 & 1581.01 & 162.73 & 110.50 & 37214.01 & 97.78 & 363.96 & 295.69 & 4900.99 & 65.08 \\
\hline 7547.82 & 972278 & 51.00 & 2421.05 & 1478.87 & 177.92 & 116.99 & 38674.63 & 93.08 & 346.13 & 296.39 & 14609.85 & 79.75 \\
\hline 7547.89 & 1006482 & 51.00 & 2551.18 & 1436.75 & 157.27 & 109.73 & 39007.53 & 105.93 & 331.85 & 245.70 & 751.81 & 67.96 \\
\hline 7547.95 & 1081292 & 50.00 & 2309.76 & 1498.21 & 180.51 & 96.19 & 41207.06 & 90.55 & 461.66 & 328.00 & 1345.54 & 56.04 \\
\hline 7548.02 & 1302183 & 48.00 & 5633.65 & 1315.88 & 0.00 & 102.44 & 60461.91 & 134.59 & 489.83 & 363.62 & 1210.36 & 79.94 \\
\hline 7548.08 & 1251679 & 49.00 & 2463.79 & 1383.75 & 188.46 & 106.66 & 43275.78 & 131.53 & 416.01 & 328.66 & 3260.26 & 60.64 \\
\hline 7548.15 & 1209721 & 49.00 & 2820.61 & 1576.10 & 224.70 & 132.99 & 47570.19 & 104.37 & 511.34 & 433.11 & 1392.05 & 63.30 \\
\hline 7548.22 & 1175075 & 49.00 & 2967.35 & 1343.20 & 143.70 & 68.49 & 49962.07 & 120.21 & 364.96 & 333.93 & 218.50 & 76.85 \\
\hline 7548.28 & 1117606 & 50.00 & 2606.85 & 1227.55 & 170.29 & 115.51 & 41810.56 & 115.50 & 399.64 & 336.30 & 312.09 & 54.01 \\
\hline 7548.35 & 1217156 & 49.00 & 2765.14 & 1510.09 & 147.26 & 102.47 & 61588.76 & 119.89 & 493.82 & 397.07 & 174.40 & 74.77 \\
\hline 7548.41 & 1111289 & 50.00 & 2336.28 & 1290.11 & 140.54 & 96.84 & 45863.27 & 119.91 & 369.95 & 308.03 & 106.13 & 66.11 \\
\hline 7548.48 & 1061402 & 51.00 & 1966.71 & 1287.06 & 0.00 & 78.64 & 32786.11 & 83.36 & 382.50 & 278.01 & 278.42 & 42.60 \\
\hline 7548.54 & 1130860 & 50.00 & 1988.23 & 1038.74 & 115.91 & 111.05 & 37050.87 & 80.77 & 386.98 & 292.47 & 385.78 & 46.13 \\
\hline 7548.61 & 1103148 & 50.00 & 2025.07 & 1064.77 & 96.68 & 93.76 & 36268.92 & 116.98 & 337.24 & 261.01 & 838.96 & 51.65 \\
\hline 7548.67 & 1156713 & 50.00 & 2473.31 & 1202.14 & 107.83 & 115.13 & 37483.88 & 0.00 & 324.89 & 270.82 & 670.93 & 47.87 \\
\hline 7548.74 & 1085827 & 50.00 & 1907.36 & 1024.63 & 169.61 & 78.57 & 26098.13 & 88.40 & 275.52 & 193.52 & 269.30 & 31.28 \\
\hline 7548.81 & 1115009 & 50.00 & 1938.78 & 1212.72 & 188.90 & 85.52 & 27752.38 & 95.27 & 310.12 & 228.61 & 89.15 & 34.69 \\
\hline
\end{tabular}




\begin{tabular}{|c|c|c|c|c|c|c|c|c|c|c|c|c|}
\hline Depth & $\mathrm{XRF}$ & XRF Live Time & $\mathrm{Ti}$ & $\mathrm{V}$ & $\mathrm{Cr}$ & $\mathrm{Mn}$ & $\mathrm{Fe}$ & $\mathrm{Co}$ & $\mathrm{Ni}$ & $\mathrm{Cu}$ & $\mathrm{Zn}$ & As \\
\hline & Total Counts & secs & $\mathrm{ppm}$ & $\mathrm{ppm}$ & $\mathrm{ppm}$ & $\mathrm{ppm}$ & $\mathrm{ppm}$ & $\mathrm{ppm}$ & $\mathrm{ppm}$ & $\mathrm{ppm}$ & $\mathrm{ppm}$ & $\mathrm{ppm}$ \\
\hline 7548.87 & 1063933 & 51.00 & 2401.82 & 1114.76 & 161.94 & 108.85 & 39608.61 & 93.51 & 307.03 & 257.99 & 0.00 & 55.81 \\
\hline 7548.94 & 964379 & 52.00 & 2464.36 & 995.68 & 205.41 & 115.96 & 35774.56 & 0.00 & 272.19 & 227.78 & 0.00 & 50.94 \\
\hline 7549.07 & 745269 & 54.00 & 3026.96 & 1308.13 & 181.74 & 181.48 & 28957.88 & 123.10 & 232.36 & 211.12 & 338.20 & 39.12 \\
\hline 7549.13 & 1067278 & 50.00 & 2343.08 & 886.85 & 189.65 & 179.17 & 25562.68 & 92.44 & 208.63 & 147.03 & 0.00 & 32.01 \\
\hline 7549.20 & 1280979 & 48.00 & 2398.29 & 1018.89 & 136.47 & 117.47 & 44733.54 & 133.63 & 283.86 & 258.18 & 0.00 & 58.82 \\
\hline 7549.26 & 1233309 & 49.00 & 2890.43 & 1184.98 & 131.05 & 145.18 & 37917.63 & 119.85 & 271.56 & 252.94 & 28.39 & 53.31 \\
\hline 7549.33 & 1132234 & 50.00 & 2805.18 & 1063.89 & 184.09 & 102.83 & 33495.14 & 122.83 & 303.74 & 256.42 & 31.71 & 44.83 \\
\hline 7549.39 & 1256909 & 49.00 & 2585.98 & 981.08 & 187.52 & 95.22 & 42271.28 & 139.23 & 284.46 & 267.33 & 39.20 & 50.98 \\
\hline 7549.46 & 1362557 & 47.00 & 2429.26 & 789.94 & 184.05 & 110.72 & 60769.59 & 188.21 & 187.85 & 210.77 & 0.00 & 69.93 \\
\hline 7549.52 & 1112889 & 50.00 & 2568.81 & 837.47 & 160.42 & 136.36 & 30422.72 & 108.76 & 226.13 & 185.55 & 0.00 & 39.57 \\
\hline 7549.59 & 805186 & 53.00 & 1725.95 & 773.26 & 0.00 & 141.19 & 19532.96 & 77.61 & 242.82 & 148.49 & 112.56 & 17.43 \\
\hline 7549.66 & 964705 & 52.00 & 1459.49 & 702.55 & 166.08 & 86.24 & 23530.09 & 85.10 & 212.95 & 167.39 & 90.74 & 30.68 \\
\hline 7549.72 & 938155 & 52.00 & 1812.64 & 938.36 & 105.18 & 131.17 & 39661.72 & 121.96 & 342.88 & 185.35 & 112.36 & 45.16 \\
\hline 7549.79 & 846631 & 53.00 & 2528.27 & 940.67 & 114.65 & 86.01 & 29138.92 & 0.00 & 265.01 & 190.25 & 69.28 & 34.54 \\
\hline 7549.85 & 1085507 & 50.00 & 2482.64 & 989.26 & 139.88 & 121.23 & 30545.69 & 99.47 & 346.01 & 225.80 & 521.75 & 34.63 \\
\hline 7549.92 & 1068436 & 50.00 & 2475.29 & 980.64 & 152.39 & 126.19 & 33819.44 & 131.48 & 290.54 & 224.10 & 241.54 & 43.18 \\
\hline 7549.98 & 970551 & 51.00 & 2478.43 & 1037.00 & 162.28 & 114.74 & 26191.90 & 83.40 & 210.87 & 211.17 & 182.41 & 40.56 \\
\hline 7550.05 & 1258257 & 49.00 & 6791.56 & 924.58 & 190.16 & 107.09 & 28945.84 & 115.29 & 297.30 & 233.46 & 724.66 & 40.31 \\
\hline 7550.12 & 1342770 & 48.00 & 2221.73 & 1092.64 & 174.73 & 174.83 & 59273.37 & 139.14 & 395.11 & 304.32 & 1358.50 & 87.07 \\
\hline 7550.18 & 1160449 & 50.00 & 2441.60 & 1286.12 & 157.71 & 132.12 & 38540.37 & 151.82 & 332.43 & 270.23 & 411.95 & 54.14 \\
\hline 7550.25 & 1045810 & 51.00 & 2508.42 & 1028.80 & 138.48 & 126.52 & 32391.51 & 87.35 & 233.80 & 238.03 & 280.17 & 48.23 \\
\hline 7550.31 & 842355 & 53.00 & 2249.53 & 1049.73 & 129.23 & 99.67 & 19910.54 & 90.67 & 205.01 & 159.26 & 262.34 & 31.16 \\
\hline 7550.38 & 1029461 & 51.00 & 2227.87 & 1067.03 & 133.97 & 102.38 & 36583.28 & 103.01 & 311.05 & 273.60 & 516.16 & 41.31 \\
\hline 7550.44 & 950209 & 52.00 & 1949.52 & 1035.12 & 102.24 & 125.65 & 23144.74 & 71.77 & 180.12 & 151.25 & 3613.50 & 37.07 \\
\hline 7550.51 & 910897 & 52.00 & 2393.47 & 1105.89 & 153.81 & 92.55 & 24681.68 & 89.38 & 217.24 & 175.43 & 226.27 & 29.57 \\
\hline 7550.57 & 1280874 & 48.00 & 3188.53 & 1531.70 & 198.08 & 144.97 & 45148.71 & 167.61 & 431.20 & 319.14 & 6102.09 & 59.87 \\
\hline 7550.64 & 1162093 & 50.00 & 2758.84 & 1119.27 & 119.37 & 90.84 & 35336.38 & 112.30 & 211.87 & 218.99 & 233.62 & 42.60 \\
\hline 7550.71 & 1100324 & 50.00 & 2356.07 & 989.04 & 164.71 & 129.59 & 24836.45 & 104.68 & 231.93 & 181.52 & 310.00 & 34.85 \\
\hline 7550.77 & 913551 & 52.00 & 1998.34 & 1150.45 & 106.80 & 63.75 & 25485.31 & 88.92 & 252.10 & 193.62 & 641.21 & 32.00 \\
\hline 7550.84 & 768168 & 53.00 & 2162.54 & 1142.04 & 131.88 & 88.75 & 29528.83 & 120.37 & 239.82 & 195.09 & 316.13 & 35.65 \\
\hline 7550.90 & 1047071 & 51.00 & 2164.27 & 1140.09 & 155.95 & 125.79 & 29650.42 & 98.93 & 334.57 & 230.74 & 978.93 & 34.46 \\
\hline 7550.97 & 1115511 & 50.00 & 2871.91 & 1221.52 & 168.96 & 116.63 & 35179.43 & 0.00 & 369.20 & 259.46 & 13384.98 & 47.08 \\
\hline
\end{tabular}




\begin{tabular}{|c|c|c|c|c|c|c|c|c|c|c|c|c|}
\hline Depth & $\mathrm{XRF}$ & XRF Live Time & $\mathrm{Ti}$ & $\mathrm{V}$ & $\mathrm{Cr}$ & $\mathrm{Mn}$ & $\mathrm{Fe}$ & $\mathrm{Co}$ & $\mathrm{Ni}$ & $\mathrm{Cu}$ & $\mathrm{Zn}$ & As \\
\hline & Total Counts & secs & $\mathrm{ppm}$ & $\mathrm{ppm}$ & $\mathrm{ppm}$ & $\mathrm{ppm}$ & $\mathrm{ppm}$ & $\mathrm{ppm}$ & $\mathrm{ppm}$ & $\mathrm{ppm}$ & $\mathrm{ppm}$ & $\mathrm{ppm}$ \\
\hline 7551.03 & 1042194 & 51.00 & 1473.29 & 450.53 & 0.00 & 170.95 & 17475.24 & 0.00 & 74.91 & 60.71 & 870.05 & 0.00 \\
\hline 7551.10 & 1444019 & 47.00 & 807.02 & 390.75 & 0.00 & 248.06 & 2288.22 & 0.00 & 47.07 & 0.00 & 117.70 & 0.00 \\
\hline 7551.17 & 2232312 & 36.00 & 779.15 & 250.66 & 109.04 & 275.99 & 2911.38 & 0.00 & 37.82 & 52.37 & 80.83 & 0.00 \\
\hline 7551.23 & 2267609 & 35.00 & 761.26 & 327.33 & 119.35 & 245.54 & 3092.49 & 0.00 & 33.82 & 0.00 & 0.00 & 0.00 \\
\hline 7551.30 & 2082659 & 38.00 & 559.62 & 324.10 & 134.95 & 276.10 & 3987.52 & 0.00 & 39.85 & 0.00 & 0.00 & 0.00 \\
\hline 7551.36 & 2001981 & 40.00 & 847.04 & 462.48 & 0.00 & 212.34 & 3291.45 & 0.00 & 31.14 & 0.00 & 0.00 & 0.00 \\
\hline 7551.43 & 2132020 & 38.00 & 779.73 & 422.03 & 137.88 & 238.18 & 2668.88 & 0.00 & 34.91 & 0.00 & 0.00 & 0.00 \\
\hline 7551.49 & 2244638 & 36.00 & 786.47 & 352.90 & 134.18 & 288.11 & 1659.47 & 0.00 & 21.36 & 0.00 & 0.00 & 0.00 \\
\hline 7551.56 & 2210967 & 36.00 & 1301.69 & 533.51 & 0.00 & 293.29 & 1711.57 & 0.00 & 29.32 & 0.00 & 0.00 & 0.00 \\
\hline 7551.62 & 2325363 & 34.00 & 987.28 & 544.36 & 111.46 & 291.13 & 1713.58 & 0.00 & 21.80 & 0.00 & 0.00 & 0.00 \\
\hline 7551.69 & 2187388 & 37.00 & 1013.21 & 577.44 & 0.00 & 272.20 & 1441.73 & 0.00 & 31.77 & 91.50 & 43.11 & 0.00 \\
\hline 7551.76 & 1950249 & 40.00 & 793.19 & 392.61 & 153.32 & 273.50 & 1044.38 & 0.00 & 25.79 & 0.00 & 0.00 & 0.00 \\
\hline 7551.82 & 2194980 & 37.00 & 813.78 & 362.46 & 167.70 & 236.01 & 968.24 & 0.00 & 35.02 & 0.00 & 0.00 & 0.00 \\
\hline 7551.89 & 2118622 & 38.00 & 644.50 & 277.11 & 0.00 & 307.59 & 640.24 & 0.00 & 22.85 & 0.00 & 0.00 & 0.00 \\
\hline 7551.95 & 2064445 & 39.00 & 711.05 & 431.91 & 0.00 & 241.21 & 1315.37 & 0.00 & 0.00 & 0.00 & 0.00 & 0.00 \\
\hline 7552.02 & 2027627 & 39.00 & 9592.61 & 495.43 & 0.00 & 262.54 & 1232.18 & 0.00 & 30.00 & 0.00 & 0.00 & 0.00 \\
\hline 7552.08 & 2027456 & 39.00 & 4226.90 & 2406.60 & 0.00 & 442.27 & 836.86 & 0.00 & 20.95 & 0.00 & 0.00 & 0.00 \\
\hline 7552.15 & 1930324 & 41.00 & 1367.65 & 890.56 & 0.00 & 290.78 & 22255.13 & 0.00 & 0.00 & 0.00 & 0.00 & 0.00 \\
\hline 7552.22 & 1717619 & 43.00 & 1305.19 & 753.19 & 0.00 & 211.71 & 9003.89 & 0.00 & 37.58 & 86.14 & 38.47 & 0.00 \\
\hline 7552.28 & 2254848 & 36.00 & 553.53 & 324.53 & 0.00 & 253.67 & 14104.83 & 0.00 & 19.85 & 45.87 & 0.00 & 0.00 \\
\hline 7552.35 & 2055337 & 39.00 & 742.58 & 449.82 & 0.00 & 224.66 & 44665.39 & 131.60 & 68.16 & 84.70 & 0.00 & 23.16 \\
\hline 7552.41 & 1060758 & 51.00 & 2944.37 & 1124.20 & 164.50 & 111.76 & 34325.83 & 140.96 & 264.00 & 295.51 & 0.00 & 45.72 \\
\hline 7552.48 & 1060499 & 51.00 & 3210.45 & 1643.12 & 126.63 & 126.34 & 34634.46 & 100.82 & 460.98 & 293.55 & 42.36 & 43.03 \\
\hline 7552.54 & 1278680 & 48.00 & 2813.37 & 1164.56 & 160.23 & 90.36 & 36486.31 & 139.08 & 352.08 & 324.91 & 0.00 & 46.85 \\
\hline 7552.61 & 1133008 & 50.00 & 3110.29 & 1348.46 & 0.00 & 109.93 & 39283.49 & 118.27 & 354.06 & 289.05 & 0.00 & 44.85 \\
\hline 7552.67 & 1115254 & 50.00 & 2563.10 & 909.92 & 0.00 & 100.40 & 43220.52 & 110.99 & 290.23 & 243.40 & 0.00 & 52.94 \\
\hline 7552.74 & 1382409 & 47.00 & 4008.04 & 1499.78 & 159.60 & 215.64 & 32362.91 & 112.31 & 118.20 & 146.45 & 0.00 & 50.27 \\
\hline 7552.81 & 672248 & 54.00 & 6008.26 & 988.08 & 0.00 & 196.54 & 23760.74 & 90.54 & 0.00 & 0.00 & 0.00 & 59.85 \\
\hline 7552.87 & 1023555 & 51.00 & 11273.15 & 1479.87 & 156.94 & 368.46 & 37904.92 & 150.52 & 19.95 & 74.90 & 0.00 & 76.92 \\
\hline 7552.94 & 753236 & 53.00 & 2046.37 & 836.57 & 128.59 & 108.90 & 23405.29 & 0.00 & 237.58 & 183.34 & 0.00 & 31.54 \\
\hline 7553.07 & 1058519 & 51.00 & 2315.87 & 1177.36 & 120.34 & 94.93 & 31741.80 & 108.58 & 285.13 & 229.60 & 0.00 & 41.81 \\
\hline 7553.13 & 1246195 & 49.00 & 2839.58 & 1165.26 & 199.56 & 128.18 & 33838.25 & 94.59 & 303.56 & 289.30 & 0.00 & 50.46 \\
\hline
\end{tabular}




\begin{tabular}{|c|c|c|c|c|c|c|c|c|c|c|c|c|}
\hline Depth & $\mathrm{XRF}$ & XRF Live Time & $\mathrm{Ti}$ & $\mathrm{V}$ & $\mathrm{Cr}$ & $\mathrm{Mn}$ & $\mathrm{Fe}$ & $\mathrm{Co}$ & $\mathrm{Ni}$ & $\mathrm{Cu}$ & $\mathrm{Zn}$ & As \\
\hline & Total Counts & secs & $\mathrm{ppm}$ & $\mathrm{ppm}$ & $\mathrm{ppm}$ & $\mathrm{ppm}$ & $\mathrm{ppm}$ & $\mathrm{ppm}$ & $\mathrm{ppm}$ & $\mathrm{ppm}$ & $\mathrm{ppm}$ & $\mathrm{ppm}$ \\
\hline 7553.20 & 1089428 & 50.00 & 2365.02 & 1024.62 & 116.68 & 96.31 & 39798.64 & 178.29 & 281.42 & 347.16 & 49.20 & 45.07 \\
\hline 7553.26 & 889368 & 52.00 & 2624.12 & 1042.11 & 194.39 & 129.12 & 24378.55 & 81.69 & 172.21 & 177.82 & 0.00 & 38.19 \\
\hline 7553.33 & 1182239 & 49.00 & 2829.39 & 1109.84 & 213.24 & 96.86 & 28280.42 & 121.85 & 266.95 & 241.54 & 25.81 & 30.02 \\
\hline 7553.39 & 1281977 & 48.00 & 2893.87 & 1152.40 & 183.87 & 78.37 & 44214.02 & 166.39 & 429.01 & 308.96 & 39.72 & 48.88 \\
\hline 7553.46 & 1276875 & 48.00 & 2573.51 & 1029.90 & 174.95 & 78.75 & 32651.53 & 128.82 & 211.62 & 290.72 & 0.00 & 42.62 \\
\hline 7553.52 & 1199557 & 49.00 & 2841.38 & 1119.55 & 168.25 & 118.12 & 27029.53 & 102.80 & 323.62 & 266.18 & 0.00 & 34.04 \\
\hline 7553.59 & 1146892 & 50.00 & 2657.30 & 1260.82 & 165.45 & 134.72 & 26451.67 & 112.34 & 259.82 & 231.70 & 33.63 & 35.92 \\
\hline 7553.66 & 718183 & 54.00 & 2868.64 & 1090.87 & 0.00 & 151.53 & 23349.70 & 114.88 & 22.81 & 51.38 & 0.00 & 49.71 \\
\hline 7553.72 & 1080697 & 50.00 & 2797.34 & 941.04 & 192.86 & 105.36 & 19594.61 & 82.68 & 196.23 & 176.93 & 0.00 & 29.59 \\
\hline 7553.79 & 1046807 & 51.00 & 2835.72 & 1175.06 & 205.95 & 105.06 & 25430.23 & 75.47 & 302.22 & 231.40 & 109.60 & 33.35 \\
\hline 7553.85 & 1181333 & 49.00 & 2929.97 & 1302.03 & 115.71 & 117.61 & 37283.33 & 136.01 & 351.89 & 314.17 & 79.67 & 58.30 \\
\hline 7553.92 & 994082 & 51.00 & 2176.19 & 777.88 & 131.21 & 135.34 & 17614.06 & 0.00 & 151.37 & 152.86 & 0.00 & 28.27 \\
\hline 7553.98 & 996554 & 51.00 & 3204.21 & 1099.04 & 182.74 & 142.08 & 27061.37 & 105.29 & 177.94 & 195.34 & 34.23 & 39.69 \\
\hline 7554.05 & 1158533 & 50.00 & 3170.70 & 997.44 & 167.70 & 140.74 & 26287.27 & 82.69 & 254.21 & 230.50 & 129.32 & 36.10 \\
\hline 7554.12 & 1353012 & 48.00 & 3357.10 & 878.41 & 164.06 & 115.81 & 29842.05 & 87.79 & 232.92 & 241.66 & 0.00 & 39.01 \\
\hline 7554.18 & 2202958 & 37.00 & 1344.27 & 761.52 & 0.00 & 549.39 & 9782.04 & 0.00 & 29.65 & 0.00 & 0.00 & 0.00 \\
\hline 7554.25 & 2047940 & 39.00 & 1419.54 & 596.01 & 0.00 & 407.28 & 7291.94 & 0.00 & 69.39 & 74.63 & 0.00 & 0.00 \\
\hline 7554.31 & 1924669 & 41.00 & 909.15 & 295.21 & 182.85 & 161.84 & 5675.51 & 0.00 & 74.45 & 75.24 & 0.00 & 0.00 \\
\hline 7554.38 & 2092658 & 38.00 & 375.55 & 323.88 & 0.00 & 326.40 & 1560.57 & 0.00 & 30.42 & 37.41 & 129.67 & 0.00 \\
\hline 7554.44 & 1537503 & 46.00 & 1479.96 & 609.68 & 0.00 & 233.48 & 7281.53 & 0.00 & 71.33 & 51.21 & 0.00 & 0.00 \\
\hline 7554.51 & 1521051 & 46.00 & 4068.40 & 943.37 & 167.79 & 130.86 & 35389.72 & 130.65 & 276.88 & 322.35 & 72.11 & 47.61 \\
\hline 7554.57 & 2117703 & 38.00 & 1337.46 & 696.42 & 170.79 & 219.92 & 4268.75 & 0.00 & 70.60 & 0.00 & 0.00 & 0.00 \\
\hline 7554.64 & 1556898 & 45.00 & 2828.94 & 1068.12 & 157.31 & 133.39 & 22245.88 & 90.85 & 306.10 & 312.51 & 2140.56 & 35.48 \\
\hline 7554.71 & 1226495 & 49.00 & 2584.18 & 1192.01 & 168.36 & 117.89 & 27667.37 & 0.00 & 423.10 & 478.76 & 3069.61 & 44.02 \\
\hline 7554.77 & 1046676 & 51.00 & 3221.15 & 1680.75 & 220.56 & 123.56 & 25465.68 & 103.47 & 413.87 & 530.55 & 3955.29 & 42.74 \\
\hline 7554.84 & 2095933 & 38.00 & 793.09 & 549.63 & 125.99 & 305.67 & 6756.80 & 0.00 & 75.63 & 58.06 & 9061.43 & 15.03 \\
\hline 7554.90 & 1973090 & 40.00 & 568.13 & 341.96 & 0.00 & 318.25 & 3660.04 & 0.00 & 53.89 & 0.00 & 0.00 & 0.00 \\
\hline 7554.97 & 1859281 & 42.00 & 756.54 & 334.00 & 0.00 & 345.84 & 3707.06 & 0.00 & 57.18 & 41.60 & 0.00 & 0.00 \\
\hline
\end{tabular}




\begin{tabular}{|c|c|c|c|c|c|c|c|c|c|c|c|c|c|c|}
\hline Depth & $\mathrm{Zr}$ & Mo & $\mathrm{Ag}$ & $\mathrm{Cd}$ & $\mathrm{Sn}$ & $\mathrm{Sb}$ & $\mathrm{Hf}$ & $\mathrm{W}$ & $\mathrm{Pb}$ & $\mathrm{Bi}$ & LE & $\mathrm{Mg}$ & $\mathrm{Al}$ & $\mathrm{Si}$ \\
\hline & ppm & ppm & ppm & $\mathrm{ppm}$ & $\mathrm{ppm}$ & $\mathrm{ppm}$ & $\mathrm{ppm}$ & $\mathrm{ppm}$ & $\mathrm{ppm}$ & ppm & $\mathrm{ppm}$ & $\mathrm{ppm}$ & ppm & ppm \\
\hline 7517.07 & 162.19 & 177.75 & 0.00 & 239.53 & 89.85 & 122.24 & 0.00 & 55.86 & 40.84 & 27.54 & 538265.06 & 0.00 & 75034.45 & 250036.70 \\
\hline 7517.13 & 148.78 & 116.44 & 0.00 & 239.53 & 95.04 & 123.69 & 45.79 & 0.00 & 30.95 & 23.26 & 546059.41 & 0.00 & 64463.51 & 248154.39 \\
\hline 7517.20 & 143.84 & 133.73 & 0.00 & 235.21 & 84.93 & 118.18 & 32.41 & 24.79 & 34.57 & 24.27 & 558969.59 & 0.00 & 59182.88 & 218718.85 \\
\hline 7517.26 & 144.44 & 116.99 & 0.00 & 236.59 & 87.23 & 125.67 & 37.23 & 0.00 & 32.22 & 20.26 & 581498.62 & 0.00 & 60875.43 & 212989.86 \\
\hline 7517.33 & 145.93 & 125.98 & 0.00 & 230.34 & 89.68 & 123.99 & 0.00 & 0.00 & 38.95 & 23.50 & 570876.83 & 0.00 & 74691.11 & 233292.03 \\
\hline 7517.39 & 42.00 & 0.00 & 0.00 & 243.91 & 97.84 & 129.24 & 0.00 & 0.00 & 0.00 & 0.00 & 555683.23 & 0.00 & 6802.95 & 78720.63 \\
\hline 7517.46 & 104.73 & 0.00 & 0.00 & 270.80 & 104.41 & 128.07 & 0.00 & 0.00 & 7.66 & 0.00 & 536434.48 & 0.00 & 4720.81 & 57641.71 \\
\hline 7517.52 & 41.76 & 0.00 & 0.00 & 267.67 & 96.93 & 121.20 & 0.00 & 0.00 & 0.00 & 0.00 & 563357.83 & 0.00 & 4026.66 & 58102.90 \\
\hline 7517.59 & 69.31 & 0.00 & 0.00 & 264.16 & 95.57 & 115.90 & 0.00 & 29.33 & 0.00 & 0.00 & 528199.53 & 0.00 & 7855.13 & 137800.95 \\
\hline 7517.66 & 84.81 & 0.00 & 0.00 & 271.36 & 108.21 & 127.64 & 0.00 & 28.31 & 0.00 & 0.00 & 487294.37 & 0.00 & 14407.67 & 198147.64 \\
\hline 7517.72 & 144.26 & 0.00 & 0.00 & 267.13 & 109.14 & 125.17 & 0.00 & 48.08 & 11.68 & 0.00 & 419480.95 & 0.00 & 8372.03 & 50745.54 \\
\hline 7517.79 & 168.76 & 14.20 & 0.00 & 255.14 & 104.38 & 120.32 & 0.00 & 42.84 & 19.88 & 0.00 & 399571.75 & 0.00 & 9758.52 & 46535.72 \\
\hline 7517.85 & 32.31 & 0.00 & 0.00 & 257.33 & 93.93 & 124.13 & 0.00 & 0.00 & 0.00 & 0.00 & 557453.91 & 0.00 & 6817.71 & 38785.30 \\
\hline 7517.92 & 41.16 & 0.00 & 0.00 & 273.98 & 106.94 & 138.24 & 0.00 & 0.00 & 0.00 & 0.00 & 590429.06 & 0.00 & 10849.29 & 41724.61 \\
\hline 7517.98 & 27.60 & 0.00 & 0.00 & 262.06 & 102.45 & 132.87 & 0.00 & 0.00 & 0.00 & 0.00 & 591529.25 & 0.00 & 6543.75 & 32265.42 \\
\hline 7518.05 & 28.85 & 0.00 & 0.00 & 256.28 & 94.00 & 125.62 & 0.00 & 0.00 & 0.00 & 0.00 & 600452.96 & 0.00 & 6324.77 & 27774.22 \\
\hline 7518.12 & 29.53 & 0.00 & 0.00 & 249.65 & 97.97 & 123.70 & 0.00 & 0.00 & 10.03 & 0.00 & 580615.17 & 0.00 & 3746.60 & 27389.68 \\
\hline 7518.18 & 27.89 & 0.00 & 0.00 & 249.62 & 100.82 & 125.84 & 0.00 & 0.00 & 0.00 & 0.00 & 593552.14 & 0.00 & 4051.61 & 31906.81 \\
\hline 7518.25 & 29.61 & 0.00 & 0.00 & 261.98 & 94.78 & 126.69 & 0.00 & 0.00 & 0.00 & 0.00 & 620915.74 & 0.00 & 3429.37 & 30463.51 \\
\hline 7518.31 & 31.63 & 0.00 & 0.00 & 264.50 & 92.48 & 135.97 & 0.00 & 0.00 & 0.00 & 0.00 & 623971.74 & 0.00 & 3215.47 & 27131.67 \\
\hline 7518.38 & 32.20 & 0.00 & 0.00 & 252.00 & 101.15 & 133.53 & 0.00 & 0.00 & 0.00 & 0.00 & 622682.76 & 0.00 & 4532.24 & 32636.72 \\
\hline 7518.44 & 30.36 & 0.00 & 0.00 & 257.86 & 97.35 & 133.82 & 0.00 & 0.00 & 0.00 & 0.00 & 619743.31 & 0.00 & 4511.57 & 38200.51 \\
\hline 7518.51 & 37.52 & 0.00 & 0.00 & 270.88 & 109.70 & 126.48 & 0.00 & 0.00 & 0.00 & 0.00 & 636214.23 & 0.00 & 3985.46 & 36154.83 \\
\hline 7518.57 & 37.51 & 0.00 & 0.00 & 287.83 & 101.35 & 132.11 & 0.00 & 0.00 & 0.00 & 0.00 & 557046.81 & 0.00 & 8979.32 & 57291.69 \\
\hline 7518.64 & 34.05 & 9.90 & 0.00 & 271.46 & 90.06 & 125.61 & 0.00 & 0.00 & 7.52 & 0.00 & 563426.17 & 0.00 & 7716.01 & 55869.39 \\
\hline 7518.71 & 35.26 & 0.00 & 0.00 & 254.64 & 102.23 & 127.71 & 0.00 & 0.00 & 0.00 & 0.00 & 564570.63 & 0.00 & 7534.60 & 61045.99 \\
\hline 7518.77 & 67.50 & 0.00 & 0.00 & 239.11 & 94.09 & 126.80 & 0.00 & 0.00 & 9.69 & 0.00 & 554158.17 & 0.00 & 6322.21 & 84266.12 \\
\hline 7518.84 & 67.58 & 19.16 & 0.00 & 248.98 & 90.18 & 120.94 & 0.00 & 0.00 & 0.00 & 0.00 & 551368.99 & 0.00 & 10177.17 & 112784.55 \\
\hline 7518.90 & 82.11 & 44.32 & 0.00 & 247.64 & 91.95 & 126.63 & 0.00 & 0.00 & 11.74 & 0.00 & 561573.92 & 0.00 & 19253.44 & 116820.91 \\
\hline 7518.97 & 164.69 & 139.80 & 0.00 & 237.52 & 97.61 & 128.56 & 35.44 & 22.80 & 36.59 & 20.92 & 531614.85 & 0.00 & 76846.68 & 226512.16 \\
\hline 7519.03 & 151.13 & 113.96 & 0.00 & 267.61 & 102.65 & 132.20 & 43.36 & 28.72 & 37.31 & 25.30 & 558663.64 & 0.00 & 71578.43 & 234175.36 \\
\hline 7519.10 & 216.68 & 107.80 & 0.00 & 240.30 & 90.38 & 106.85 & 48.70 & 0.00 & 44.50 & 26.38 & 491507.62 & 0.00 & 69462.13 & 240151.15 \\
\hline
\end{tabular}




\begin{tabular}{|c|c|c|c|c|c|c|c|c|c|c|c|c|c|c|}
\hline Depth & $\mathrm{Zr}$ & Mo & $\mathrm{Ag}$ & $\mathrm{Cd}$ & $\mathrm{Sn}$ & $\mathrm{Sb}$ & $\mathrm{Hf}$ & $\mathrm{W}$ & $\mathrm{Pb}$ & $\mathrm{Bi}$ & LE & $\mathrm{Mg}$ & $\mathrm{Al}$ & $\mathrm{Si}$ \\
\hline & ppm & ppm & $\mathrm{ppm}$ & $\mathrm{ppm}$ & $\mathrm{ppm}$ & $\mathrm{ppm}$ & $\mathrm{ppm}$ & $\mathrm{ppm}$ & $\mathrm{ppm}$ & ppm & $\mathrm{ppm}$ & $\mathrm{ppm}$ & ppm & ppm \\
\hline 7519.17 & 152.22 & 103.14 & 0.00 & 243.68 & 90.90 & 125.32 & 37.14 & 25.12 & 27.26 & 24.56 & 578718.72 & 0.00 & 62279.44 & 211417.94 \\
\hline 7519.23 & 146.70 & 122.98 & 0.00 & 265.19 & 99.95 & 132.64 & 40.53 & 0.00 & 34.52 & 20.85 & 555470.77 & 0.00 & 67860.46 & 241129.25 \\
\hline 7519.30 & 196.11 & 97.93 & 0.00 & 241.51 & 89.41 & 114.63 & 0.00 & 0.00 & 38.46 & 21.60 & 459400.63 & 0.00 & 52873.73 & 208777.91 \\
\hline 7519.36 & 127.77 & 76.13 & 0.00 & 243.99 & 91.85 & 125.32 & 40.43 & 29.53 & 24.71 & 14.88 & 555455.03 & 0.00 & 47077.35 & 194757.60 \\
\hline 7519.43 & 144.64 & 103.83 & 0.00 & 265.61 & 96.91 & 129.05 & 56.50 & 0.00 & 31.53 & 21.57 & 572764.14 & 0.00 & 57457.24 & 216475.49 \\
\hline 7519.49 & 128.42 & 85.96 & 0.00 & 266.52 & 100.18 & 141.09 & 0.00 & 30.83 & 23.56 & 21.92 & 551270.87 & 0.00 & 54057.84 & 188929.33 \\
\hline 7519.56 & 84.51 & 50.57 & 0.00 & 256.09 & 95.41 & 129.27 & 0.00 & 40.90 & 13.89 & 0.00 & 535786.09 & 0.00 & 30016.02 & 127350.14 \\
\hline 7519.62 & 64.49 & 32.01 & 0.00 & 275.63 & 88.20 & 127.19 & 0.00 & 56.26 & 11.36 & 0.00 & 559668.01 & 0.00 & 16413.59 & 119138.30 \\
\hline 7519.69 & 83.21 & 47.78 & 0.00 & 298.40 & 94.49 & 122.98 & 0.00 & 80.53 & 13.55 & 0.00 & 569131.71 & 0.00 & 22095.47 & 119761.81 \\
\hline 7519.76 & 123.42 & 79.56 & 0.00 & 313.51 & 90.63 & 133.44 & 0.00 & 143.46 & 21.11 & 0.00 & 580567.52 & 0.00 & 34432.14 & 143619.30 \\
\hline 7519.82 & 140.32 & 110.69 & 0.00 & 296.43 & 95.02 & 122.49 & 49.60 & 90.03 & 30.76 & 21.28 & 581017.26 & 0.00 & 50647.95 & 208854.18 \\
\hline 7519.89 & 141.92 & 123.10 & 0.00 & 349.28 & 91.13 & 128.49 & 49.26 & 187.88 & 34.09 & 22.33 & 583441.97 & 0.00 & 58836.63 & 253471.23 \\
\hline 7519.95 & 135.35 & 104.13 & 0.00 & 250.09 & 95.62 & 123.20 & 39.39 & 39.82 & 38.84 & 23.69 & 580346.78 & 0.00 & 63353.88 & 269867.48 \\
\hline 7520.02 & 139.36 & 81.45 & 0.00 & 263.51 & 95.56 & 125.72 & 40.75 & 34.72 & 38.39 & 20.44 & 550503.23 & 0.00 & 69048.41 & 300479.77 \\
\hline 7520.08 & 155.32 & 73.78 & 0.00 & 243.72 & 96.12 & 120.02 & 42.51 & 55.50 & 35.05 & 22.60 & 554488.70 & 0.00 & 76472.70 & 274325.02 \\
\hline 7520.15 & 159.84 & 116.59 & 0.00 & 250.30 & 93.43 & 122.71 & 43.96 & 45.21 & 32.13 & 25.93 & 561272.15 & 0.00 & 72325.45 & 246358.83 \\
\hline 7520.22 & 181.79 & 113.09 & 0.00 & 265.47 & 93.92 & 120.05 & 58.11 & 0.00 & 35.91 & 28.78 & 568892.86 & 0.00 & 76732.66 & 257973.93 \\
\hline 7520.28 & 153.31 & 37.82 & 0.00 & 238.28 & 92.95 & 122.74 & 46.05 & 0.00 & 26.73 & 24.67 & 581406.20 & 0.00 & 76086.05 & 256057.22 \\
\hline 7520.35 & 158.26 & 47.23 & 0.00 & 229.34 & 89.78 & 118.75 & 38.73 & 25.58 & 29.97 & 28.56 & 554160.74 & 0.00 & 77636.21 & 269998.84 \\
\hline 7520.41 & 155.01 & 35.04 & 0.00 & 254.01 & 87.15 & 120.95 & 36.28 & 0.00 & 25.35 & 26.32 & 549936.25 & 0.00 & 80090.14 & 250920.37 \\
\hline 7520.48 & 161.60 & 31.47 & 0.00 & 202.09 & 83.20 & 120.40 & 39.68 & 25.48 & 22.53 & 29.09 & 541576.96 & 0.00 & 85158.98 & 257898.66 \\
\hline 7520.54 & 161.91 & 31.80 & 0.00 & 238.58 & 85.74 & 122.95 & 43.39 & 0.00 & 27.84 & 24.64 & 547936.61 & 0.00 & 84298.01 & 258680.33 \\
\hline 7520.61 & 166.79 & 19.77 & 0.00 & 226.20 & 83.53 & 121.53 & 51.73 & 0.00 & 16.19 & 27.08 & 578477.54 & 0.00 & 79911.40 & 250552.61 \\
\hline 7520.67 & 153.83 & 32.25 & 0.00 & 241.48 & 89.17 & 112.29 & 42.55 & 26.68 & 32.01 & 27.58 & 577849.16 & 0.00 & 75561.19 & 240237.49 \\
\hline 7520.74 & 177.84 & 19.29 & 0.00 & 237.77 & 94.40 & 120.38 & 48.35 & 0.00 & 23.81 & 29.54 & 553664.23 & 0.00 & 89762.06 & 278683.88 \\
\hline 7520.81 & 166.41 & 44.06 & 0.00 & 245.15 & 89.98 & 112.85 & 39.05 & 0.00 & 28.26 & 29.03 & 567548.77 & 0.00 & 78211.96 & 245706.98 \\
\hline 7520.87 & 165.94 & 34.68 & 0.00 & 237.46 & 94.08 & 118.05 & 38.97 & 0.00 & 22.49 & 25.70 & 566022.34 & 0.00 & 73402.99 & 228466.42 \\
\hline 7520.94 & 166.36 & 32.39 & 0.00 & 237.46 & 91.67 & 121.43 & 45.51 & 0.00 & 22.73 & 30.81 & 578877.84 & 0.00 & 73947.96 & 235338.66 \\
\hline 7521.00 & 165.00 & 28.43 & 0.00 & 223.23 & 80.58 & 114.35 & 0.00 & 0.00 & 23.38 & 28.66 & 589203.75 & 0.00 & 76225.29 & 238207.84 \\
\hline 7521.07 & 163.01 & 25.91 & 0.00 & 245.65 & 89.90 & 118.87 & 53.24 & 21.66 & 21.10 & 28.74 & 536160.91 & 0.00 & 93468.76 & 282442.32 \\
\hline 7521.13 & 163.40 & 28.35 & 0.00 & 240.02 & 92.98 & 121.57 & 49.58 & 0.00 & 24.50 & 29.20 & 529707.45 & 0.00 & 88690.82 & 275913.82 \\
\hline 7521.20 & 156.66 & 38.24 & 0.00 & 237.11 & 92.77 & 120.14 & 39.77 & 0.00 & 28.91 & 24.13 & 529251.38 & 0.00 & 87777.50 & 262196.95 \\
\hline
\end{tabular}




\begin{tabular}{|c|c|c|c|c|c|c|c|c|c|c|c|c|c|c|}
\hline Depth & $\mathrm{Zr}$ & Mo & $\mathrm{Ag}$ & $\mathrm{Cd}$ & $\mathrm{Sn}$ & $\mathrm{Sb}$ & Hf & $\mathrm{W}$ & $\mathrm{Pb}$ & $\mathrm{Bi}$ & LE & $\mathrm{Mg}$ & $\mathrm{Al}$ & $\mathrm{Si}$ \\
\hline & ppm & ppm & ppm & $\mathrm{ppm}$ & $\mathrm{ppm}$ & $\mathrm{ppm}$ & ppm & $\mathrm{ppm}$ & $\mathrm{ppm}$ & ppm & $\mathrm{ppm}$ & $\mathrm{ppm}$ & ppm & ppm \\
\hline 7521.26 & 163.07 & 27.09 & 0.00 & 224.20 & 92.06 & 124.34 & 40.44 & 0.00 & 23.77 & 26.57 & 583564.25 & 0.00 & 77980.94 & 242601.41 \\
\hline 7521.33 & 159.52 & 30.18 & 0.00 & 219.96 & 80.62 & 116.47 & 52.32 & 0.00 & 30.91 & 24.04 & 559828.57 & 0.00 & 70814.49 & 206034.50 \\
\hline 7521.39 & 159.91 & 29.01 & 0.00 & 248.46 & 86.66 & 123.20 & 41.30 & 0.00 & 23.23 & 26.67 & 573676.99 & 0.00 & 81270.99 & 247545.54 \\
\hline 7521.46 & 166.05 & 31.22 & 0.00 & 251.17 & 97.88 & 127.97 & 36.77 & 26.58 & 26.16 & 27.01 & 566359.45 & 0.00 & 83096.75 & 245642.76 \\
\hline 7521.52 & 166.47 & 34.28 & 0.00 & 252.67 & 93.41 & 121.42 & 49.10 & 0.00 & 28.68 & 28.10 & 536480.98 & 0.00 & 82542.28 & 239812.05 \\
\hline 7521.59 & 162.36 & 47.89 & 0.00 & 257.03 & 87.45 & 124.38 & 44.04 & 0.00 & 27.83 & 24.74 & 554572.69 & 0.00 & 78380.66 & 229913.82 \\
\hline 7521.66 & 161.23 & 42.15 & 0.00 & 218.33 & 87.92 & 126.51 & 47.04 & 24.47 & 28.05 & 25.67 & 591727.61 & 0.00 & 74226.57 & 230566.41 \\
\hline 7521.72 & 169.61 & 57.47 & 0.00 & 243.48 & 90.78 & 124.52 & 39.77 & 0.00 & 25.16 & 24.46 & 578758.88 & 0.00 & 78173.26 & 240342.31 \\
\hline 7521.79 & 166.19 & 70.77 & 0.00 & 221.36 & 88.06 & 115.18 & 43.78 & 24.47 & 40.35 & 24.77 & 568743.53 & 0.00 & 74281.11 & 229279.81 \\
\hline 7521.85 & 180.10 & 97.00 & 0.00 & 248.07 & 92.72 & 131.66 & 51.69 & 25.62 & 36.02 & 26.65 & 533262.03 & 0.00 & 83666.55 & 250300.88 \\
\hline 7521.92 & 178.77 & 78.66 & 0.00 & 260.57 & 95.17 & 128.66 & 47.65 & 23.42 & 41.72 & 27.92 & 529058.65 & 0.00 & 86822.67 & 271219.16 \\
\hline 7521.98 & 176.98 & 107.99 & 0.00 & 232.69 & 90.35 & 120.58 & 0.00 & 37.81 & 42.74 & 23.76 & 572988.70 & 0.00 & 71964.74 & 226701.37 \\
\hline 7522.05 & 170.78 & 75.75 & 0.00 & 230.49 & 94.04 & 121.88 & 47.10 & 0.00 & 33.60 & 24.11 & 613355.96 & 0.00 & 67789.45 & 208466.53 \\
\hline 7522.12 & 172.07 & 63.68 & 0.00 & 265.84 & 98.94 & 126.40 & 45.48 & 28.44 & 33.90 & 25.81 & 570375.21 & 0.00 & 75956.86 & 236019.64 \\
\hline 7522.18 & 178.57 & 89.98 & 0.00 & 243.21 & 95.06 & 123.48 & 43.49 & 26.22 & 30.11 & 27.96 & 571537.05 & 0.00 & 74797.80 & 231518.73 \\
\hline 7522.25 & 177.87 & 92.06 & 0.00 & 245.08 & 93.67 & 123.11 & 42.90 & 26.91 & 34.37 & 24.57 & 564458.04 & 0.00 & 74890.00 & 223285.36 \\
\hline 7522.31 & 183.61 & 184.46 & 0.00 & 270.45 & 101.54 & 120.62 & 45.65 & 26.16 & 44.87 & 24.76 & 521295.76 & 0.00 & 82250.74 & 238241.79 \\
\hline 7522.38 & 116.26 & 24.72 & 0.00 & 246.27 & 97.80 & 125.85 & 0.00 & 32.96 & 17.48 & 0.00 & 547005.03 & 0.00 & 30238.32 & 115766.59 \\
\hline 7522.44 & 169.80 & 46.25 & 0.00 & 241.54 & 94.96 & 122.38 & 46.60 & 0.00 & 35.52 & 24.59 & 555406.69 & 0.00 & 66347.66 & 164555.20 \\
\hline 7522.51 & 174.71 & 33.78 & 0.00 & 223.47 & 95.44 & 118.60 & 0.00 & 0.00 & 28.13 & 20.86 & 573154.61 & 0.00 & 59222.91 & 180859.88 \\
\hline 7522.57 & 174.63 & 23.10 & 0.00 & 234.92 & 94.16 & 131.38 & 40.57 & 27.17 & 22.95 & 23.08 & 558370.69 & 0.00 & 76466.29 & 230855.98 \\
\hline 7522.64 & 181.90 & 30.23 & 0.00 & 253.66 & 87.77 & 115.20 & 40.49 & 24.50 & 26.87 & 29.92 & 538185.79 & 0.00 & 76309.25 & 234495.43 \\
\hline 7522.71 & 178.65 & 53.97 & 0.00 & 241.88 & 91.62 & 123.56 & 0.00 & 0.00 & 41.33 & 25.81 & 549725.88 & 0.00 & 76043.42 & 227775.90 \\
\hline 7522.77 & 187.75 & 59.05 & 0.00 & 245.93 & 92.63 & 125.83 & 0.00 & 53.71 & 36.39 & 26.13 & 571467.81 & 0.00 & 77604.74 & 229964.86 \\
\hline 7522.84 & 184.50 & 35.86 & 0.00 & 249.86 & 94.07 & 124.23 & 45.06 & 0.00 & 25.28 & 28.16 & 578845.59 & 0.00 & 74374.97 & 227143.69 \\
\hline 7522.90 & 186.04 & 38.21 & 0.00 & 255.23 & 92.78 & 120.99 & 47.54 & 0.00 & 23.69 & 29.07 & 586930.95 & 0.00 & 76717.70 & 227845.06 \\
\hline 7522.97 & 174.17 & 13.97 & 0.00 & 230.63 & 88.58 & 122.92 & 44.88 & 0.00 & 16.69 & 25.59 & 563373.79 & 0.00 & 82505.35 & 246509.53 \\
\hline 7523.03 & 165.84 & 0.00 & 0.00 & 260.06 & 96.39 & 127.19 & 36.72 & 32.45 & 24.76 & 27.14 & 579542.50 & 0.00 & 78629.06 & 222294.21 \\
\hline 7523.10 & 172.52 & 0.00 & 0.00 & 239.50 & 92.15 & 121.91 & 0.00 & 0.00 & 17.77 & 26.49 & 562804.46 & 0.00 & 82578.51 & 244396.86 \\
\hline 7523.17 & 174.93 & 0.00 & 0.00 & 240.09 & 93.75 & 118.63 & 38.92 & 0.00 & 10.33 & 29.16 & 533097.25 & 0.00 & 89618.96 & 266834.54 \\
\hline 7523.23 & 187.29 & 0.00 & 0.00 & 245.82 & 89.14 & 125.65 & 34.22 & 0.00 & 12.12 & 29.22 & 550731.91 & 0.00 & 81745.76 & 249464.63 \\
\hline 7523.30 & 176.35 & 0.00 & 0.00 & 229.98 & 88.82 & 127.72 & 36.06 & 26.80 & 21.12 & 27.22 & 550133.20 & 0.00 & 69147.84 & 210298.45 \\
\hline
\end{tabular}




\begin{tabular}{|c|c|c|c|c|c|c|c|c|c|c|c|c|c|c|}
\hline Depth & $\mathrm{Zr}$ & Mo & $\mathrm{Ag}$ & $\mathrm{Cd}$ & $\mathrm{Sn}$ & $\mathrm{Sb}$ & $\mathrm{Hf}$ & $\mathrm{W}$ & $\mathrm{Pb}$ & $\mathrm{Bi}$ & LE & $\mathrm{Mg}$ & $\mathrm{Al}$ & $\mathrm{Si}$ \\
\hline & $\mathrm{ppm}$ & ppm & ppm & $\mathrm{ppm}$ & $\mathrm{ppm}$ & $\mathrm{ppm}$ & $\mathrm{ppm}$ & $\mathrm{ppm}$ & $\mathrm{ppm}$ & ppm & $\mathrm{ppm}$ & $\mathrm{ppm}$ & ppm & ppm \\
\hline 7523.36 & 177.33 & 0.00 & 0.00 & 244.11 & 92.30 & 124.13 & 46.91 & 0.00 & 19.57 & 26.60 & 572913.44 & 0.00 & 77016.91 & 231371.12 \\
\hline 7523.43 & 183.00 & 0.00 & 0.00 & 251.31 & 96.26 & 131.57 & 39.20 & 24.84 & 11.18 & 24.92 & 580790.47 & 0.00 & 80185.17 & 239220.30 \\
\hline 7523.49 & 171.89 & 0.00 & 0.00 & 248.95 & 92.33 & 120.51 & 34.11 & 22.45 & 14.55 & 29.46 & 571960.02 & 0.00 & 84288.36 & 251253.49 \\
\hline 7523.56 & 174.49 & 0.00 & 0.00 & 246.70 & 97.01 & 126.49 & 33.65 & 26.60 & 11.22 & 29.37 & 578492.97 & 0.00 & 74997.51 & 229273.14 \\
\hline 7523.62 & 180.60 & 0.00 & 0.00 & 240.13 & 91.89 & 117.73 & 0.00 & 27.90 & 9.76 & 21.37 & 568429.07 & 0.00 & 54053.51 & 190423.16 \\
\hline 7523.69 & 171.54 & 0.00 & 0.00 & 259.34 & 101.19 & 137.53 & 0.00 & 0.00 & 8.37 & 16.33 & 579984.55 & 0.00 & 20535.61 & 98484.92 \\
\hline 7523.76 & 118.43 & 0.00 & 0.00 & 269.63 & 94.27 & 136.55 & 0.00 & 0.00 & 0.00 & 0.00 & 594733.66 & 0.00 & 9742.72 & 56963.75 \\
\hline 7523.82 & 95.61 & 0.00 & 0.00 & 274.17 & 104.01 & 127.54 & 0.00 & 0.00 & 0.00 & 0.00 & 581552.84 & 0.00 & 4409.95 & 18703.25 \\
\hline 7523.89 & 130.54 & 0.00 & 0.00 & 249.28 & 103.38 & 124.71 & 0.00 & 0.00 & 0.00 & 0.00 & 537547.74 & 0.00 & 4437.57 & 18637.56 \\
\hline 7523.95 & 55.11 & 0.00 & 0.00 & 271.11 & 99.01 & 131.83 & 0.00 & 0.00 & 8.63 & 0.00 & 584728.46 & 0.00 & 8338.25 & 26857.76 \\
\hline 7524.02 & 41.58 & 0.00 & 0.00 & 236.51 & 95.08 & 116.74 & 0.00 & 0.00 & 0.00 & 0.00 & 587822.07 & 0.00 & 14824.49 & 33332.81 \\
\hline 7524.08 & 59.80 & 0.00 & 0.00 & 242.69 & 91.28 & 128.51 & 0.00 & 0.00 & 0.00 & 0.00 & 591687.11 & 0.00 & 8890.94 & 37227.12 \\
\hline 7524.15 & 117.34 & 0.00 & 0.00 & 236.96 & 100.85 & 125.61 & 0.00 & 0.00 & 0.00 & 0.00 & 595222.60 & 0.00 & 23219.78 & 94550.48 \\
\hline 7524.22 & 160.56 & 0.00 & 0.00 & 242.58 & 101.38 & 130.90 & 0.00 & 0.00 & 10.92 & 0.00 & 591368.94 & 0.00 & 30023.40 & 117767.21 \\
\hline 7524.28 & 190.24 & 0.00 & 0.00 & 279.02 & 99.70 & 136.21 & 40.20 & 0.00 & 0.00 & 22.03 & 573290.19 & 0.00 & 58798.05 & 199334.95 \\
\hline 7524.35 & 195.81 & 0.00 & 0.00 & 259.41 & 100.39 & 117.93 & 0.00 & 29.55 & 6.17 & 20.56 & 568122.30 & 0.00 & 54050.69 & 190642.96 \\
\hline 7524.41 & 181.72 & 0.00 & 0.00 & 257.22 & 97.60 & 124.49 & 37.91 & 30.33 & 11.03 & 22.16 & 574140.51 & 0.00 & 64530.76 & 214618.33 \\
\hline 7524.48 & 185.94 & 0.00 & 0.00 & 260.71 & 101.08 & 123.51 & 0.00 & 0.00 & 13.17 & 25.58 & 591063.42 & 0.00 & 71899.13 & 224737.66 \\
\hline 7524.54 & 175.14 & 0.00 & 0.00 & 261.38 & 96.74 & 128.79 & 39.68 & 0.00 & 12.27 & 23.19 & 594583.83 & 0.00 & 68873.89 & 218041.75 \\
\hline 7524.61 & 179.50 & 0.00 & 0.00 & 246.28 & 102.07 & 131.49 & 48.87 & 0.00 & 8.90 & 26.64 & 580119.80 & 0.00 & 73509.92 & 227276.22 \\
\hline 7524.67 & 204.66 & 0.00 & 0.00 & 263.79 & 100.79 & 122.20 & 0.00 & 0.00 & 11.09 & 19.63 & 610751.49 & 0.00 & 49969.23 & 184315.10 \\
\hline 7524.74 & 199.82 & 0.00 & 0.00 & 293.61 & 103.63 & 131.55 & 0.00 & 39.88 & 8.58 & 18.20 & 594289.94 & 0.00 & 41172.18 & 164029.37 \\
\hline 7524.81 & 169.74 & 0.00 & 0.00 & 238.03 & 98.15 & 121.37 & 33.90 & 0.00 & 0.00 & 22.21 & 573522.02 & 0.00 & 49948.69 & 179774.08 \\
\hline 7524.87 & 171.66 & 0.00 & 0.00 & 252.62 & 89.96 & 120.38 & 38.18 & 26.22 & 8.59 & 23.31 & 571910.87 & 0.00 & 62724.65 & 207781.00 \\
\hline 7524.94 & 198.55 & 0.00 & 0.00 & 261.99 & 98.58 & 128.38 & 40.24 & 0.00 & 14.24 & 27.25 & 586041.20 & 0.00 & 70510.48 & 235914.40 \\
\hline 7525.00 & 173.94 & 0.00 & 0.00 & 273.92 & 104.24 & 136.77 & 50.21 & 0.00 & 14.40 & 23.03 & 595892.82 & 0.00 & 68905.37 & 223830.85 \\
\hline 7525.07 & 175.51 & 0.00 & 0.00 & 254.46 & 97.93 & 123.96 & 43.58 & 0.00 & 10.16 & 22.69 & 572793.75 & 0.00 & 73878.56 & 241413.02 \\
\hline 7525.13 & 182.61 & 0.00 & 0.00 & 254.76 & 98.11 & 130.15 & 38.97 & 23.88 & 6.13 & 25.92 & 569524.10 & 0.00 & 78771.60 & 245426.42 \\
\hline 7525.20 & 178.66 & 0.00 & 0.00 & 262.91 & 99.71 & 130.37 & 40.04 & 28.85 & 16.59 & 24.77 & 570092.17 & 0.00 & 75734.67 & 246500.06 \\
\hline 7525.26 & 178.67 & 0.00 & 0.00 & 229.14 & 93.63 & 123.11 & 35.71 & 0.00 & 14.57 & 25.95 & 592699.87 & 0.00 & 66592.14 & 219871.86 \\
\hline 7525.33 & 198.61 & 0.00 & 0.00 & 244.46 & 95.56 & 133.17 & 0.00 & 0.00 & 13.17 & 19.90 & 599294.62 & 0.00 & 45142.79 & 177911.80 \\
\hline 7525.39 & 209.34 & 0.00 & 0.00 & 266.71 & 93.52 & 137.13 & 0.00 & 0.00 & 12.08 & 18.86 & 593780.44 & 0.00 & 43111.51 & 184057.27 \\
\hline
\end{tabular}




\begin{tabular}{|c|c|c|c|c|c|c|c|c|c|c|c|c|c|c|}
\hline Depth & $\mathrm{Zr}$ & Mo & $\mathrm{Ag}$ & $\mathrm{Cd}$ & $\mathrm{Sn}$ & $\mathrm{Sb}$ & $\mathrm{Hf}$ & $\mathrm{W}$ & $\mathrm{Pb}$ & $\mathrm{Bi}$ & LE & $\mathrm{Mg}$ & $\mathrm{Al}$ & $\mathrm{Si}$ \\
\hline & $\mathrm{ppm}$ & ppm & ppm & $\mathrm{ppm}$ & $\mathrm{ppm}$ & $\mathrm{ppm}$ & $\mathrm{ppm}$ & $\mathrm{ppm}$ & $\mathrm{ppm}$ & ppm & $\mathrm{ppm}$ & $\mathrm{ppm}$ & ppm & ppm \\
\hline 7525.46 & 201.60 & 0.00 & 0.00 & 248.07 & 95.96 & 124.21 & 0.00 & 44.95 & 16.98 & 19.70 & 575752.70 & 0.00 & 34454.57 & 149648.80 \\
\hline 7525.52 & 171.14 & 0.00 & 0.00 & 253.53 & 96.93 & 125.03 & 0.00 & 29.94 & 9.29 & 16.22 & 534162.15 & 0.00 & 40578.59 & 142309.20 \\
\hline 7525.59 & 187.36 & 37.57 & 0.00 & 250.97 & 92.75 & 122.92 & 0.00 & 0.00 & 29.38 & 26.57 & 563672.41 & 0.00 & 70921.25 & 214434.69 \\
\hline 7525.66 & 210.01 & 15.03 & 0.00 & 243.14 & 92.92 & 121.18 & 44.22 & 28.35 & 15.98 & 30.49 & 575967.92 & 0.00 & 75772.72 & 215836.03 \\
\hline 7525.72 & 271.00 & 0.00 & 0.00 & 241.84 & 108.18 & 124.55 & 0.00 & 36.71 & 13.66 & 29.13 & 528063.10 & 0.00 & 41705.53 & 170352.94 \\
\hline 7525.79 & 135.51 & 140.28 & 0.00 & 222.77 & 98.84 & 125.70 & 43.98 & 0.00 & 42.44 & 24.00 & 577956.87 & 0.00 & 55607.30 & 184485.11 \\
\hline 7525.85 & 128.71 & 148.85 & 0.00 & 226.64 & 96.04 & 129.14 & 0.00 & 29.18 & 43.49 & 21.57 & 572298.64 & 0.00 & 59070.06 & 194710.19 \\
\hline 7525.92 & 134.06 & 163.02 & 0.00 & 257.23 & 93.43 & 123.34 & 43.39 & 0.00 & 52.31 & 24.74 & 543733.69 & 0.00 & 66171.99 & 218114.03 \\
\hline 7525.98 & 134.39 & 177.42 & 0.00 & 262.19 & 97.75 & 123.11 & 45.43 & 31.58 & 40.33 & 26.29 & 519609.03 & 0.00 & 72923.37 & 252155.03 \\
\hline 7526.05 & 140.57 & 148.01 & 0.00 & 268.48 & 99.45 & 127.11 & 43.59 & 37.36 & 41.20 & 24.85 & 588533.18 & 0.00 & 63526.99 & 242948.20 \\
\hline 7526.12 & 132.90 & 155.76 & 0.00 & 238.39 & 93.41 & 130.60 & 43.54 & 0.00 & 40.62 & 26.76 & 659276.09 & 0.00 & 44939.94 & 184088.68 \\
\hline 7526.18 & 124.47 & 107.28 & 0.00 & 238.46 & 92.38 & 126.48 & 37.13 & 30.16 & 37.51 & 26.13 & 533553.54 & 0.00 & 65732.64 & 285888.87 \\
\hline 7526.25 & 111.82 & 97.83 & 0.00 & 232.20 & 93.10 & 135.46 & 0.00 & 0.00 & 30.70 & 22.09 & 588353.98 & 0.00 & 49924.69 & 251418.62 \\
\hline 7526.31 & 106.10 & 108.53 & 0.00 & 279.93 & 95.65 & 126.94 & 40.61 & 89.34 & 33.93 & 20.92 & 615009.59 & 0.00 & 42292.71 & 228035.25 \\
\hline 7526.38 & 107.65 & 100.44 & 0.00 & 267.40 & 93.36 & 130.61 & 0.00 & 65.44 & 30.42 & 19.37 & 594917.94 & 0.00 & 44179.52 & 231112.67 \\
\hline 7526.44 & 106.42 & 117.90 & 0.00 & 247.08 & 96.03 & 135.65 & 0.00 & 0.00 & 25.04 & 18.76 & 590055.70 & 0.00 & 49394.49 & 232722.58 \\
\hline 7526.51 & 119.03 & 110.35 & 0.00 & 259.38 & 94.27 & 131.17 & 0.00 & 0.00 & 30.91 & 22.90 & 567199.87 & 0.00 & 54054.26 & 261988.97 \\
\hline 7526.57 & 117.89 & 96.07 & 0.00 & 245.59 & 97.12 & 123.26 & 0.00 & 28.87 & 27.96 & 21.89 & 577403.99 & 0.00 & 52072.93 & 264977.77 \\
\hline 7526.64 & 113.55 & 89.78 & 0.00 & 236.96 & 98.16 & 124.48 & 0.00 & 0.00 & 31.10 & 20.53 & 592762.75 & 0.00 & 49635.56 & 234625.87 \\
\hline 7526.71 & 103.24 & 86.68 & 0.00 & 241.65 & 96.12 & 124.85 & 36.99 & 0.00 & 27.39 & 23.02 & 598448.12 & 0.00 & 43717.04 & 236185.66 \\
\hline 7526.77 & 115.42 & 122.20 & 0.00 & 464.41 & 96.05 & 127.33 & 0.00 & 533.70 & 27.08 & 22.08 & 558960.44 & 0.00 & 51267.63 & 251787.18 \\
\hline 7526.84 & 113.24 & 113.18 & 0.00 & 269.64 & 94.99 & 127.25 & 35.14 & 47.80 & 23.27 & 23.12 & 562213.47 & 0.00 & 59188.88 & 269731.25 \\
\hline 7526.90 & 104.68 & 97.38 & 0.00 & 263.60 & 91.64 & 123.19 & 0.00 & 50.76 & 25.71 & 19.82 & 587509.49 & 0.00 & 47763.06 & 258876.27 \\
\hline 7527.07 & 100.54 & 66.88 & 0.00 & 242.26 & 93.15 & 126.75 & 40.60 & 0.00 & 27.80 & 19.86 & 576547.67 & 0.00 & 48865.42 & 265695.60 \\
\hline 7527.13 & 110.06 & 97.31 & 0.00 & 235.65 & 92.32 & 130.43 & 40.49 & 0.00 & 21.50 & 23.66 & 580970.57 & 0.00 & 56600.57 & 273382.91 \\
\hline 7527.20 & 121.21 & 98.15 & 0.00 & 240.64 & 97.74 & 132.63 & 35.12 & 0.00 & 28.19 & 21.94 & 576448.59 & 0.00 & 57348.24 & 271419.07 \\
\hline 7527.26 & 122.00 & 122.97 & 0.00 & 246.33 & 89.89 & 125.40 & 0.00 & 0.00 & 31.55 & 26.37 & 590814.92 & 0.00 & 54034.21 & 241942.01 \\
\hline 7527.33 & 111.17 & 90.77 & 0.00 & 229.85 & 90.78 & 123.33 & 0.00 & 0.00 & 29.86 & 17.79 & 578346.80 & 0.00 & 54619.71 & 257680.81 \\
\hline 7527.39 & 112.91 & 79.98 & 0.00 & 224.03 & 90.30 & 127.55 & 37.75 & 0.00 & 30.23 & 19.35 & 600098.09 & 0.00 & 50020.31 & 234625.89 \\
\hline 7527.46 & 131.91 & 80.25 & 0.00 & 232.00 & 93.80 & 127.29 & 42.16 & 30.24 & 27.95 & 26.76 & 579596.50 & 0.00 & 58903.72 & 233232.13 \\
\hline 7527.52 & 137.78 & 86.74 & 0.00 & 237.38 & 94.17 & 127.85 & 48.15 & 0.00 & 35.42 & 22.67 & 577170.30 & 0.00 & 60816.99 & 240432.19 \\
\hline 7527.59 & 112.06 & 51.40 & 0.00 & 240.75 & 94.02 & 127.48 & 42.64 & 26.54 & 19.04 & 17.64 & 559336.46 & 0.00 & 49154.68 & 232073.23 \\
\hline
\end{tabular}




\begin{tabular}{|c|c|c|c|c|c|c|c|c|c|c|c|c|c|c|}
\hline Depth & $\mathrm{Zr}$ & Mo & $\mathrm{Ag}$ & $\mathrm{Cd}$ & $\mathrm{Sn}$ & $\mathrm{Sb}$ & $\mathrm{Hf}$ & $\mathrm{W}$ & $\mathrm{Pb}$ & $\mathrm{Bi}$ & LE & $\mathrm{Mg}$ & $\mathrm{Al}$ & $\mathrm{Si}$ \\
\hline & ppm & ppm & $\mathrm{ppm}$ & $\mathrm{ppm}$ & $\mathrm{ppm}$ & $\mathrm{ppm}$ & $\mathrm{ppm}$ & $\mathrm{ppm}$ & $\mathrm{ppm}$ & ppm & $\mathrm{ppm}$ & $\mathrm{ppm}$ & ppm & ppm \\
\hline 7527.66 & 100.97 & 52.99 & 0.00 & 247.56 & 97.61 & 128.15 & 33.53 & 0.00 & 16.60 & 20.76 & 565006.05 & 0.00 & 43625.05 & 212364.75 \\
\hline 7527.72 & 116.36 & 61.47 & 0.00 & 249.70 & 100.84 & 128.13 & 37.12 & 0.00 & 24.90 & 22.39 & 550446.86 & 0.00 & 58846.86 & 259703.25 \\
\hline 7527.79 & 102.19 & 66.77 & 0.00 & 270.18 & 95.85 & 129.51 & 0.00 & 0.00 & 19.26 & 16.42 & 546492.99 & 0.00 & 49522.78 & 220853.11 \\
\hline 7527.85 & 117.29 & 59.59 & 0.00 & 261.83 & 98.68 & 129.52 & 42.15 & 0.00 & 24.51 & 21.02 & 563012.10 & 0.00 & 61121.27 & 235070.96 \\
\hline 7527.92 & 125.49 & 65.99 & 0.00 & 261.31 & 103.40 & 126.98 & 47.98 & 25.64 & 25.80 & 29.12 & 512277.83 & 0.00 & 69281.22 & 269037.57 \\
\hline 7527.98 & 129.32 & 65.38 & 0.00 & 238.96 & 97.95 & 122.14 & 40.80 & 0.00 & 27.21 & 23.85 & 542685.78 & 0.00 & 66914.37 & 258459.15 \\
\hline 7528.05 & 136.56 & 68.56 & 0.00 & 239.39 & 94.32 & 120.49 & 35.09 & 25.66 & 29.85 & 25.94 & 583446.06 & 0.00 & 63238.45 & 220134.71 \\
\hline 7528.12 & 131.48 & 66.98 & 0.00 & 245.68 & 97.02 & 132.86 & 45.84 & 0.00 & 26.94 & 20.45 & 557784.23 & 0.00 & 69301.12 & 257262.51 \\
\hline 7528.18 & 128.80 & 65.85 & 0.00 & 235.11 & 94.35 & 129.52 & 42.07 & 30.09 & 30.92 & 22.17 & 561833.57 & 0.00 & 65823.75 & 257924.41 \\
\hline 7528.25 & 125.05 & 97.82 & 0.00 & 237.52 & 91.09 & 119.28 & 35.43 & 0.00 & 26.45 & 27.06 & 558321.92 & 0.00 & 65790.67 & 246134.36 \\
\hline 7528.31 & 137.80 & 129.49 & 0.00 & 245.76 & 91.80 & 124.34 & 40.10 & 0.00 & 35.93 & 26.51 & 591159.10 & 0.00 & 55177.99 & 209462.13 \\
\hline 7528.38 & 133.41 & 146.97 & 0.00 & 242.38 & 99.04 & 129.18 & 39.80 & 0.00 & 31.67 & 26.86 & 541322.76 & 0.00 & 69986.68 & 243763.76 \\
\hline 7528.44 & 130.89 & 105.32 & 0.00 & 240.64 & 94.58 & 128.58 & 43.62 & 29.55 & 36.91 & 28.68 & 543733.12 & 0.00 & 69180.69 & 247083.31 \\
\hline 7528.51 & 140.51 & 98.64 & 0.00 & 234.16 & 89.99 & 125.68 & 0.00 & 0.00 & 30.06 & 25.27 & 597122.66 & 0.00 & 59341.00 & 219215.25 \\
\hline 7528.57 & 129.44 & 82.40 & 0.00 & 242.45 & 90.06 & 129.20 & 0.00 & 0.00 & 34.31 & 24.39 & 607568.68 & 0.00 & 54960.36 & 202931.57 \\
\hline 7528.64 & 131.02 & 81.69 & 0.00 & 222.51 & 90.29 & 126.75 & 58.70 & 0.00 & 30.10 & 24.16 & 599271.11 & 0.00 & 54993.69 & 203317.74 \\
\hline 7528.71 & 135.95 & 93.07 & 0.00 & 254.23 & 100.88 & 125.65 & 51.01 & 0.00 & 28.90 & 25.28 & 613533.14 & 0.00 & 50144.35 & 190154.07 \\
\hline 7528.77 & 134.43 & 73.89 & 0.00 & 250.07 & 96.11 & 137.78 & 42.63 & 0.00 & 32.56 & 22.87 & 627958.91 & 0.00 & 49736.43 & 192387.19 \\
\hline 7528.84 & 124.64 & 64.35 & 0.00 & 234.79 & 102.08 & 136.63 & 0.00 & 0.00 & 28.31 & 28.48 & 632176.36 & 0.00 & 41947.05 & 165949.64 \\
\hline 7528.90 & 119.68 & 66.67 & 0.00 & 248.21 & 94.36 & 140.95 & 0.00 & 0.00 & 31.26 & 22.99 & 671268.99 & 0.00 & 41107.20 & 161563.57 \\
\hline 7528.97 & 127.46 & 75.83 & 0.00 & 266.44 & 101.20 & 139.08 & 0.00 & 0.00 & 33.00 & 20.87 & 700245.73 & 0.00 & 36848.08 & 147822.74 \\
\hline 7529.03 & 137.19 & 97.93 & 0.00 & 257.27 & 98.69 & 140.79 & 56.19 & 0.00 & 30.17 & 26.69 & 628872.44 & 0.00 & 51811.57 & 178770.49 \\
\hline 7529.10 & 131.60 & 123.07 & 0.00 & 263.77 & 91.12 & 129.70 & 0.00 & 0.00 & 38.07 & 28.53 & 594094.96 & 0.00 & 53239.64 & 207188.56 \\
\hline 7529.17 & 122.77 & 103.99 & 0.00 & 220.20 & 88.88 & 110.70 & 0.00 & 0.00 & 33.95 & 30.38 & 534019.89 & 0.00 & 61931.10 & 230566.65 \\
\hline 7529.23 & 127.48 & 96.21 & 0.00 & 239.87 & 90.90 & 124.73 & 0.00 & 0.00 & 33.28 & 24.73 & 553251.12 & 0.00 & 69674.57 & 257742.75 \\
\hline 7529.30 & 127.54 & 123.70 & 0.00 & 222.32 & 93.54 & 123.83 & 0.00 & 27.33 & 29.68 & 21.11 & 596140.38 & 0.00 & 56323.25 & 206978.17 \\
\hline 7529.36 & 132.29 & 100.48 & 0.00 & 255.75 & 92.16 & 120.06 & 60.76 & 0.00 & 33.78 & 27.53 & 589676.83 & 0.00 & 64436.27 & 230943.27 \\
\hline 7529.43 & 231.99 & 78.96 & 0.00 & 181.89 & 76.28 & 98.09 & 56.85 & 39.17 & 40.79 & 21.97 & 539696.26 & 0.00 & 45676.78 & 172206.43 \\
\hline 7529.49 & 369.38 & 25.72 & 0.00 & 237.06 & 103.18 & 126.63 & 66.30 & 79.55 & 43.04 & 27.38 & 387315.27 & 0.00 & 29357.56 & 155702.06 \\
\hline 7529.56 & 157.20 & 81.08 & 0.00 & 231.53 & 87.26 & 115.56 & 53.06 & 23.24 & 24.68 & 23.30 & 535504.61 & 0.00 & 74599.06 & 272815.38 \\
\hline 7529.62 & 224.80 & 40.69 & 0.00 & 212.11 & 80.23 & 94.83 & 56.37 & 51.87 & 28.50 & 26.35 & 484054.45 & 0.00 & 29870.38 & 202092.69 \\
\hline 7529.69 & 115.65 & 59.82 & 0.00 & 268.17 & 100.93 & 127.88 & 0.00 & 0.00 & 22.97 & 20.60 & 565737.62 & 0.00 & 52536.76 & 241929.17 \\
\hline
\end{tabular}




\begin{tabular}{|c|c|c|c|c|c|c|c|c|c|c|c|c|c|c|}
\hline Depth & $\mathrm{Zr}$ & Mo & $\mathrm{Ag}$ & $\mathrm{Cd}$ & $\mathrm{Sn}$ & $\mathrm{Sb}$ & Hf & $\mathrm{W}$ & $\mathrm{Pb}$ & $\mathrm{Bi}$ & LE & $\mathrm{Mg}$ & $\mathrm{Al}$ & $\mathrm{Si}$ \\
\hline & ppm & ppm & ppm & ppm & $\mathrm{ppm}$ & ppm & ppm & ppm & $\mathrm{ppm}$ & ppm & ppm & $\mathrm{ppm}$ & ppm & ppm \\
\hline 7529.76 & 151.36 & 66.33 & 0.00 & 228.69 & 93.24 & 122.73 & 0.00 & 0.00 & 27.04 & 25.55 & 562994.65 & 0.00 & 59972.97 & 243358.37 \\
\hline 7529.82 & 132.37 & 67.82 & 0.00 & 255.21 & 94.68 & 128.21 & 39.31 & 0.00 & 26.95 & 23.10 & 559964.81 & 0.00 & 65349.37 & 274568.75 \\
\hline 7529.89 & 145.48 & 72.44 & 0.00 & 241.02 & 93.63 & 118.53 & 0.00 & 0.00 & 27.35 & 25.03 & 570341.15 & 0.00 & 65501.28 & 255452.96 \\
\hline 7529.95 & 138.82 & 77.27 & 0.00 & 238.78 & 94.20 & 128.89 & 38.49 & 0.00 & 31.11 & 22.69 & 605899.58 & 0.00 & 57016.32 & 220412.02 \\
\hline 7530.02 & 145.73 & 83.10 & 0.00 & 258.92 & 95.27 & 125.29 & 45.38 & 0.00 & 32.30 & 29.30 & 618517.06 & 0.00 & 53786.41 & 210266.71 \\
\hline 7530.08 & 171.85 & 72.88 & 0.00 & 294.17 & 124.16 & 155.34 & 0.00 & 0.00 & 28.42 & 23.27 & 587855.83 & 0.00 & 43822.45 & 164841.79 \\
\hline 7530.15 & 154.38 & 83.25 & 0.00 & 184.14 & 69.23 & 85.94 & 54.72 & 0.00 & 33.60 & 29.53 & 515594.13 & 0.00 & 49375.08 & 165073.56 \\
\hline 7530.22 & 132.14 & 83.00 & 0.00 & 238.42 & 99.06 & 129.40 & 49.53 & 0.00 & 30.18 & 23.78 & 569333.37 & 0.00 & 65306.63 & 255081.70 \\
\hline 7530.28 & 207.73 & 59.53 & 0.00 & 227.00 & 98.03 & 123.15 & 47.45 & 34.32 & 22.60 & 26.17 & 516100.45 & 0.00 & 56519.40 & 255509.19 \\
\hline 7530.35 & 147.04 & 82.88 & 0.00 & 260.35 & 97.41 & 131.86 & 36.81 & 0.00 & 28.16 & 25.95 & 551631.39 & 0.00 & 62958.38 & 273318.72 \\
\hline 7530.41 & 154.74 & 96.37 & 0.00 & 259.44 & 102.06 & 127.22 & 49.95 & 29.13 & 24.17 & 25.00 & 475719.82 & 0.00 & 79492.07 & 310608.07 \\
\hline 7530.48 & 177.32 & 89.66 & 0.00 & 271.75 & 106.68 & 130.73 & 44.78 & 0.00 & 25.28 & 24.42 & 574535.90 & 0.00 & 55171.61 & 232543.19 \\
\hline 7530.54 & 246.41 & 57.93 & 0.00 & 279.74 & 109.58 & 131.18 & 44.21 & 35.57 & 28.10 & 30.90 & 502200.19 & 0.00 & 56292.67 & 240305.51 \\
\hline 7530.61 & 141.08 & 81.27 & 0.00 & 238.34 & 97.38 & 126.89 & 48.77 & 0.00 & 30.62 & 24.80 & 527301.76 & 0.00 & 72155.66 & 292573.05 \\
\hline 7530.67 & 144.46 & 78.09 & 0.00 & 244.60 & 88.75 & 123.08 & 37.85 & 0.00 & 26.80 & 22.96 & 553262.50 & 0.00 & 69439.60 & 272769.60 \\
\hline 7530.74 & 375.67 & 40.91 & 0.00 & 241.29 & 94.35 & 114.36 & 75.51 & 67.95 & 42.63 & 41.07 & 478181.94 & 0.00 & 29071.90 & 112689.54 \\
\hline 7530.81 & 159.18 & 79.28 & 0.00 & 249.84 & 94.83 & 132.61 & 36.41 & 31.37 & 25.21 & 25.33 & 574985.75 & 0.00 & 58055.25 & 235114.29 \\
\hline 7530.87 & 120.70 & 70.61 & 0.00 & 238.18 & 99.04 & 127.96 & 37.12 & 0.00 & 25.30 & 21.76 & 600739.49 & 0.00 & 55239.50 & 246430.69 \\
\hline 7530.94 & 119.87 & 64.29 & 0.00 & 244.24 & 93.85 & 118.36 & 42.97 & 22.73 & 22.74 & 22.03 & 553636.05 & 0.00 & 63276.96 & 267776.87 \\
\hline 7531.00 & 132.27 & 68.91 & 0.00 & 302.53 & 111.35 & 141.21 & 53.95 & 0.00 & 24.10 & 28.64 & 594607.38 & 0.00 & 53795.79 & 219569.20 \\
\hline 7531.07 & 131.68 & 65.18 & 0.00 & 248.89 & 96.21 & 129.31 & 0.00 & 30.16 & 29.49 & 19.43 & 598084.27 & 0.00 & 52367.02 & 229413.53 \\
\hline 7531.13 & 156.94 & 54.22 & 0.00 & 240.96 & 90.75 & 114.28 & 0.00 & 0.00 & 25.86 & 26.46 & 573810.74 & 0.00 & 49862.57 & 204989.53 \\
\hline 7531.20 & 98.84 & 48.44 & 0.00 & 277.65 & 112.60 & 141.32 & 0.00 & 0.00 & 10.51 & 0.00 & 613817.12 & 0.00 & 30477.96 & 151429.67 \\
\hline 7531.26 & 306.10 & 29.52 & 0.00 & 254.68 & 106.32 & 110.63 & 63.28 & 45.35 & 25.51 & 29.57 & 403728.61 & 0.00 & 26568.86 & 131712.37 \\
\hline 7531.33 & 97.63 & 39.46 & 0.00 & 259.23 & 108.29 & 142.91 & 0.00 & 0.00 & 17.90 & 15.93 & 612227.92 & 0.00 & 28553.26 & 138618.19 \\
\hline 7531.39 & 129.62 & 36.46 & 0.00 & 236.95 & 92.80 & 129.93 & 0.00 & 0.00 & 16.93 & 0.00 & 603574.40 & 0.00 & 25904.80 & 123030.92 \\
\hline 7531.46 & 74.13 & 29.02 & 0.00 & 249.49 & 94.51 & 126.89 & 0.00 & 0.00 & 15.61 & 0.00 & 558507.61 & 0.00 & 29156.56 & 157926.91 \\
\hline 7531.52 & 80.64 & 39.57 & 0.00 & 242.73 & 100.74 & 125.87 & 0.00 & 0.00 & 9.97 & 0.00 & 566431.92 & 0.00 & 35162.34 & 171076.59 \\
\hline 7531.59 & 105.41 & 60.67 & 0.00 & 237.28 & 96.90 & 127.63 & 0.00 & 0.00 & 16.72 & 18.58 & 608998.11 & 0.00 & 41273.63 & 187513.58 \\
\hline 7531.66 & 124.09 & 70.94 & 0.00 & 255.85 & 99.58 & 127.05 & 45.90 & 25.43 & 24.76 & 23.13 & 513431.38 & 0.00 & 69228.57 & 266673.34 \\
\hline 7531.72 & 122.43 & 76.85 & 0.00 & 250.17 & 96.99 & 129.66 & 39.30 & 0.00 & 22.69 & 23.02 & 530101.84 & 0.00 & 67077.78 & 269102.98 \\
\hline 7531.79 & 126.87 & 73.80 & 0.00 & 256.15 & 97.25 & 130.03 & 43.87 & 25.55 & 29.95 & 19.93 & 530654.78 & 0.00 & 67907.80 & 268459.78 \\
\hline
\end{tabular}




\begin{tabular}{|c|c|c|c|c|c|c|c|c|c|c|c|c|c|c|}
\hline Depth & $\mathrm{Zr}$ & Mo & $\mathrm{Ag}$ & $\mathrm{Cd}$ & $\mathrm{Sn}$ & $\mathrm{Sb}$ & $\mathrm{Hf}$ & $\mathrm{W}$ & $\mathrm{Pb}$ & $\mathrm{Bi}$ & LE & $\mathrm{Mg}$ & $\mathrm{Al}$ & $\mathrm{Si}$ \\
\hline & ppm & ppm & $\mathrm{ppm}$ & $\mathrm{ppm}$ & $\mathrm{ppm}$ & $\mathrm{ppm}$ & $\mathrm{ppm}$ & $\mathrm{ppm}$ & $\mathrm{ppm}$ & ppm & $\mathrm{ppm}$ & $\mathrm{ppm}$ & ppm & ppm \\
\hline 7531.85 & 122.64 & 76.87 & 0.00 & 228.28 & 86.16 & 119.80 & 45.37 & 0.00 & 23.66 & 19.55 & 539559.38 & 0.00 & 67933.24 & 270588.97 \\
\hline 7531.92 & 162.21 & 66.48 & 0.00 & 200.42 & 81.46 & 104.27 & 0.00 & 33.11 & 22.93 & 24.38 & 531877.17 & 0.00 & 39045.62 & 152359.70 \\
\hline 7531.98 & 125.18 & 80.98 & 0.00 & 230.29 & 97.45 & 123.38 & 38.19 & 0.00 & 24.91 & 26.09 & 579239.67 & 0.00 & 68783.40 & 236955.53 \\
\hline 7532.05 & 123.22 & 77.71 & 0.00 & 228.88 & 90.31 & 123.00 & 32.97 & 22.87 & 22.90 & 23.64 & 551034.96 & 0.00 & 65842.39 & 258972.18 \\
\hline 7532.12 & 126.30 & 78.64 & 0.00 & 251.97 & 102.19 & 125.46 & 39.89 & 0.00 & 25.93 & 21.86 & 527333.02 & 0.00 & 68954.50 & 269437.72 \\
\hline 7532.18 & 117.37 & 72.94 & 0.00 & 237.78 & 93.65 & 129.03 & 47.12 & 0.00 & 23.61 & 24.20 & 572788.26 & 0.00 & 58385.54 & 235228.67 \\
\hline 7532.25 & 119.01 & 76.68 & 0.00 & 250.57 & 94.61 & 128.02 & 40.44 & 0.00 & 23.65 & 22.49 & 620374.20 & 0.00 & 50103.19 & 203921.17 \\
\hline 7532.31 & 122.77 & 82.62 & 0.00 & 253.60 & 96.49 & 121.56 & 52.31 & 0.00 & 26.29 & 23.98 & 528833.61 & 0.00 & 64303.29 & 254206.66 \\
\hline 7532.38 & 124.78 & 84.83 & 0.00 & 255.09 & 104.83 & 127.06 & 41.97 & 0.00 & 28.17 & 20.24 & 539270.89 & 0.00 & 67191.48 & 253213.13 \\
\hline 7532.44 & 115.46 & 85.38 & 0.00 & 243.17 & 94.88 & 120.20 & 49.89 & 0.00 & 21.25 & 20.70 & 592506.77 & 0.00 & 50437.51 & 209752.11 \\
\hline 7532.51 & 114.60 & 83.71 & 0.00 & 247.68 & 94.03 & 122.53 & 39.72 & 0.00 & 20.32 & 21.46 & 578601.03 & 0.00 & 54442.64 & 224320.82 \\
\hline 7532.57 & 119.39 & 94.62 & 0.00 & 256.84 & 100.77 & 137.29 & 38.18 & 27.24 & 25.11 & 19.77 & 578230.04 & 0.00 & 53148.95 & 217524.55 \\
\hline 7532.64 & 119.91 & 90.18 & 0.00 & 243.11 & 95.02 & 125.50 & 45.30 & 0.00 & 24.12 & 20.83 & 579435.87 & 0.00 & 52166.28 & 211231.23 \\
\hline 7532.71 & 116.01 & 85.96 & 0.00 & 246.49 & 98.22 & 127.99 & 37.83 & 0.00 & 27.84 & 21.09 & 582882.80 & 0.00 & 57624.82 & 229724.09 \\
\hline 7532.77 & 120.97 & 82.09 & 0.00 & 254.85 & 96.93 & 134.21 & 37.77 & 0.00 & 28.43 & 20.05 & 518326.92 & 0.00 & 67277.21 & 259138.63 \\
\hline 7532.84 & 119.52 & 68.45 & 0.00 & 243.55 & 94.12 & 123.28 & 44.59 & 27.22 & 24.43 & 21.35 & 522509.31 & 0.00 & 64230.08 & 255937.93 \\
\hline 7532.90 & 121.50 & 83.12 & 0.00 & 249.17 & 96.00 & 126.74 & 37.38 & 0.00 & 22.45 & 25.17 & 525819.48 & 0.00 & 65273.66 & 256633.98 \\
\hline 7532.97 & 117.20 & 96.63 & 0.00 & 255.00 & 99.03 & 131.53 & 0.00 & 0.00 & 28.83 & 20.42 & 576936.45 & 0.00 & 58955.03 & 229815.09 \\
\hline 7533.03 & 115.01 & 112.33 & 0.00 & 257.51 & 97.02 & 125.42 & 37.61 & 0.00 & 30.89 & 22.85 & 559290.82 & 0.00 & 60360.27 & 244182.03 \\
\hline 7533.10 & 115.97 & 98.01 & 0.00 & 251.26 & 98.57 & 126.67 & 0.00 & 40.53 & 33.81 & 22.69 & 555040.91 & 0.00 & 55016.17 & 218499.30 \\
\hline 7533.17 & 110.73 & 74.63 & 0.00 & 250.77 & 94.75 & 127.74 & 0.00 & 0.00 & 18.33 & 17.20 & 555180.24 & 0.00 & 52632.38 & 205327.00 \\
\hline 7533.23 & 83.89 & 60.36 & 0.00 & 259.53 & 94.99 & 129.20 & 43.82 & 0.00 & 17.39 & 14.54 & 527412.57 & 0.00 & 38570.78 & 186431.84 \\
\hline 7533.30 & 80.95 & 51.30 & 0.00 & 239.47 & 96.22 & 133.38 & 0.00 & 30.50 & 14.10 & 0.00 & 541795.66 & 0.00 & 35141.91 & 166589.38 \\
\hline 7533.36 & 90.34 & 68.79 & 0.00 & 246.83 & 90.58 & 128.83 & 0.00 & 0.00 & 17.82 & 15.06 & 567713.85 & 0.00 & 38231.25 & 168506.65 \\
\hline 7533.43 & 96.85 & 77.63 & 0.00 & 247.43 & 91.58 & 122.43 & 0.00 & 0.00 & 21.80 & 0.00 & 578776.50 & 0.00 & 39495.58 & 174858.27 \\
\hline 7533.49 & 110.31 & 91.76 & 0.00 & 249.71 & 96.34 & 123.77 & 0.00 & 28.09 & 22.19 & 20.13 & 565919.57 & 0.00 & 51084.36 & 203297.24 \\
\hline 7533.56 & 110.91 & 97.44 & 0.00 & 282.75 & 111.99 & 149.20 & 0.00 & 0.00 & 23.18 & 18.25 & 577773.19 & 0.00 & 50198.04 & 195778.45 \\
\hline 7533.62 & 118.28 & 92.18 & 0.00 & 234.61 & 92.69 & 128.03 & 37.93 & 27.94 & 24.93 & 23.13 & 554475.05 & 0.00 & 61020.11 & 234149.74 \\
\hline 7533.69 & 121.53 & 93.13 & 0.00 & 243.63 & 94.00 & 125.01 & 37.11 & 0.00 & 23.85 & 24.26 & 582254.45 & 0.00 & 52406.99 & 210783.12 \\
\hline 7533.76 & 118.22 & 89.05 & 0.00 & 242.06 & 96.59 & 121.28 & 0.00 & 0.00 & 28.14 & 22.51 & 567441.36 & 0.00 & 57675.39 & 225179.27 \\
\hline 7533.82 & 120.09 & 98.28 & 0.00 & 252.42 & 94.44 & 124.11 & 40.09 & 0.00 & 25.83 & 22.26 & 504072.28 & 0.00 & 73546.52 & 270551.91 \\
\hline 7533.89 & 125.63 & 90.50 & 0.00 & 255.14 & 97.99 & 130.16 & 0.00 & 0.00 & 24.66 & 24.43 & 536745.85 & 0.00 & 67594.36 & 243290.95 \\
\hline
\end{tabular}




\begin{tabular}{|c|c|c|c|c|c|c|c|c|c|c|c|c|c|c|}
\hline Depth & $\mathrm{Zr}$ & Mo & $\mathrm{Ag}$ & $\mathrm{Cd}$ & $\mathrm{Sn}$ & $\mathrm{Sb}$ & $\mathrm{Hf}$ & $\mathrm{W}$ & $\mathrm{Pb}$ & $\mathrm{Bi}$ & LE & $\mathrm{Mg}$ & $\mathrm{Al}$ & $\mathrm{Si}$ \\
\hline & ppm & ppm & $\mathrm{ppm}$ & $\mathrm{ppm}$ & $\mathrm{ppm}$ & $\mathrm{ppm}$ & $\mathrm{ppm}$ & $\mathrm{ppm}$ & $\mathrm{ppm}$ & ppm & $\mathrm{ppm}$ & $\mathrm{ppm}$ & ppm & ppm \\
\hline 7533.95 & 124.68 & 80.85 & 0.00 & 251.64 & 95.26 & 125.79 & 0.00 & 32.00 & 20.54 & 20.04 & 559170.93 & 0.00 & 56696.78 & 222055.90 \\
\hline 7534.02 & 114.55 & 91.57 & 0.00 & 228.56 & 95.93 & 123.75 & 44.20 & 24.55 & 28.51 & 22.60 & 586057.55 & 0.00 & 58499.12 & 231397.42 \\
\hline 7534.08 & 118.44 & 111.55 & 0.00 & 248.25 & 97.51 & 119.98 & 43.21 & 0.00 & 29.52 & 21.25 & 599714.91 & 0.00 & 52519.26 & 216659.40 \\
\hline 7534.15 & 114.22 & 102.78 & 0.00 & 250.02 & 93.61 & 128.78 & 0.00 & 0.00 & 31.96 & 22.25 & 606955.68 & 0.00 & 54324.91 & 225672.30 \\
\hline 7534.22 & 113.43 & 105.95 & 0.00 & 248.58 & 99.53 & 142.90 & 0.00 & 0.00 & 30.54 & 19.92 & 573008.93 & 0.00 & 63826.27 & 253337.98 \\
\hline 7534.28 & 115.70 & 112.29 & 0.00 & 240.92 & 93.64 & 128.12 & 34.21 & 26.28 & 28.48 & 22.57 & 535282.19 & 0.00 & 71273.80 & 284195.84 \\
\hline 7534.35 & 114.93 & 122.19 & 0.00 & 257.55 & 99.75 & 130.47 & 44.43 & 0.00 & 27.66 & 21.45 & 576294.38 & 0.00 & 58333.52 & 239316.48 \\
\hline 7534.41 & 111.16 & 141.66 & 0.00 & 245.84 & 97.88 & 128.33 & 0.00 & 31.61 & 32.29 & 26.09 & 588291.00 & 0.00 & 58574.30 & 247237.32 \\
\hline 7534.48 & 121.26 & 139.80 & 0.00 & 250.33 & 90.89 & 124.79 & 43.29 & 47.25 & 31.81 & 23.85 & 520627.29 & 0.00 & 81792.70 & 283271.56 \\
\hline 7534.54 & 124.31 & 130.36 & 0.00 & 246.63 & 100.56 & 127.33 & 38.76 & 0.00 & 28.08 & 24.46 & 544870.28 & 0.00 & 73385.31 & 281426.00 \\
\hline 7534.61 & 103.19 & 129.08 & 0.00 & 271.49 & 98.63 & 138.31 & 41.26 & 53.13 & 33.64 & 19.94 & 544020.65 & 0.00 & 71705.68 & 277996.93 \\
\hline 7534.67 & 114.53 & 155.53 & 0.00 & 249.59 & 95.41 & 122.81 & 0.00 & 27.21 & 24.96 & 23.98 & 529334.55 & 0.00 & 71413.77 & 296087.51 \\
\hline 7534.74 & 119.24 & 114.88 & 0.00 & 249.08 & 97.92 & 131.41 & 41.37 & 23.96 & 28.96 & 21.29 & 561309.50 & 0.00 & 71345.94 & 277549.83 \\
\hline 7534.81 & 116.32 & 102.75 & 0.00 & 248.20 & 97.42 & 123.53 & 33.40 & 0.00 & 26.97 & 23.72 & 588869.71 & 0.00 & 57633.01 & 247289.31 \\
\hline 7534.87 & 116.21 & 104.50 & 0.00 & 262.45 & 95.72 & 122.90 & 41.62 & 0.00 & 24.91 & 20.79 & 586118.09 & 0.00 & 48812.79 & 214148.32 \\
\hline 7535.07 & 111.00 & 142.09 & 0.00 & 233.22 & 92.04 & 120.46 & 0.00 & 0.00 & 28.62 & 19.58 & 555723.80 & 0.00 & 62971.17 & 257262.97 \\
\hline 7535.13 & 117.63 & 181.68 & 0.00 & 251.24 & 95.27 & 125.66 & 36.61 & 35.23 & 31.55 & 23.67 & 508902.87 & 0.00 & 73105.19 & 288510.79 \\
\hline 7535.20 & 117.47 & 148.84 & 0.00 & 249.17 & 91.02 & 122.78 & 45.28 & 31.20 & 27.98 & 28.53 & 552432.35 & 0.00 & 68380.52 & 265872.77 \\
\hline 7535.26 & 109.79 & 141.53 & 0.00 & 309.72 & 116.77 & 153.75 & 0.00 & 51.41 & 21.07 & 18.34 & 577769.28 & 0.00 & 69818.30 & 268248.97 \\
\hline 7535.33 & 123.82 & 120.77 & 0.00 & 274.95 & 100.81 & 127.19 & 36.64 & 0.00 & 34.16 & 29.05 & 570100.73 & 0.00 & 66964.58 & 270115.28 \\
\hline 7535.39 & 120.06 & 117.04 & 0.00 & 270.10 & 92.88 & 127.73 & 48.77 & 0.00 & 31.60 & 23.25 & 553980.41 & 0.00 & 73937.47 & 289866.47 \\
\hline 7535.46 & 104.13 & 114.20 & 0.00 & 293.10 & 107.47 & 140.20 & 0.00 & 34.73 & 26.45 & 22.51 & 526270.38 & 0.00 & 78068.34 & 306776.29 \\
\hline 7535.52 & 119.80 & 117.60 & 0.00 & 230.93 & 97.01 & 128.03 & 40.39 & 0.00 & 26.19 & 23.88 & 508087.04 & 0.00 & 78107.37 & 319567.72 \\
\hline 7535.59 & 125.76 & 131.19 & 0.00 & 243.52 & 98.03 & 126.24 & 41.52 & 35.78 & 32.88 & 25.04 & 508762.45 & 0.00 & 79382.93 & 308926.47 \\
\hline 7535.66 & 109.87 & 120.52 & 0.00 & 231.07 & 85.66 & 124.07 & 39.60 & 0.00 & 31.13 & 20.79 & 574016.54 & 0.00 & 57282.52 & 225407.39 \\
\hline 7535.72 & 105.92 & 108.25 & 0.00 & 237.99 & 97.33 & 125.14 & 0.00 & 0.00 & 30.70 & 21.01 & 577872.60 & 0.00 & 53084.76 & 230554.21 \\
\hline 7535.79 & 112.06 & 143.51 & 0.00 & 233.73 & 90.64 & 128.73 & 43.29 & 32.32 & 30.84 & 22.18 & 564476.90 & 0.00 & 57946.15 & 261491.54 \\
\hline 7535.85 & 117.63 & 149.60 & 0.00 & 257.37 & 91.91 & 123.67 & 35.21 & 32.20 & 23.43 & 23.41 & 544472.05 & 0.00 & 65251.11 & 283470.34 \\
\hline 7535.92 & 115.48 & 155.20 & 0.00 & 236.27 & 96.98 & 124.16 & 0.00 & 33.67 & 24.76 & 21.57 & 573177.24 & 0.00 & 59763.79 & 260716.85 \\
\hline 7535.98 & 112.47 & 123.28 & 0.00 & 234.19 & 91.32 & 122.81 & 36.39 & 0.00 & 28.40 & 20.83 & 516884.82 & 0.00 & 67714.91 & 306700.63 \\
\hline 7536.05 & 109.96 & 113.33 & 0.00 & 262.71 & 97.07 & 124.28 & 47.47 & 30.61 & 25.84 & 20.14 & 531738.32 & 0.00 & 67775.27 & 309823.91 \\
\hline 7536.12 & 111.20 & 117.02 & 0.00 & 268.06 & 98.23 & 127.79 & 36.27 & 34.37 & 28.51 & 24.37 & 562265.69 & 0.00 & 59798.29 & 271900.11 \\
\hline
\end{tabular}




\begin{tabular}{|c|c|c|c|c|c|c|c|c|c|c|c|c|c|c|}
\hline Depth & $\mathrm{Zr}$ & Mo & $\mathrm{Ag}$ & $\mathrm{Cd}$ & $\mathrm{Sn}$ & $\mathrm{Sb}$ & $\mathrm{Hf}$ & $\mathrm{W}$ & $\mathrm{Pb}$ & $\mathrm{Bi}$ & LE & $\mathrm{Mg}$ & $\mathrm{Al}$ & $\mathrm{Si}$ \\
\hline & ppm & ppm & ppm & $\mathrm{ppm}$ & $\mathrm{ppm}$ & $\mathrm{ppm}$ & $\mathrm{ppm}$ & $\mathrm{ppm}$ & $\mathrm{ppm}$ & ppm & $\mathrm{ppm}$ & $\mathrm{ppm}$ & ppm & ppm \\
\hline 7536.18 & 105.67 & 123.11 & 0.00 & 262.30 & 96.94 & 132.57 & 59.64 & 0.00 & 28.44 & 22.45 & 592912.59 & 0.00 & 55029.37 & 236813.80 \\
\hline 7536.25 & 110.74 & 111.34 & 0.00 & 257.44 & 100.31 & 128.55 & 44.07 & 47.46 & 30.27 & 22.55 & 546987.86 & 0.00 & 65424.79 & 285960.55 \\
\hline 7536.31 & 107.76 & 115.99 & 0.00 & 250.29 & 92.50 & 123.77 & 35.00 & 28.17 & 24.87 & 20.13 & 566848.72 & 0.00 & 59334.13 & 269289.93 \\
\hline 7536.38 & 110.13 & 121.28 & 0.00 & 241.81 & 95.25 & 126.11 & 34.62 & 0.00 & 33.86 & 25.31 & 542272.35 & 0.00 & 68742.02 & 300509.91 \\
\hline 7536.44 & 97.53 & 129.61 & 0.00 & 293.77 & 113.51 & 148.88 & 48.78 & 0.00 & 24.17 & 17.41 & 628668.62 & 0.00 & 50289.32 & 226149.30 \\
\hline 7536.51 & 105.70 & 108.54 & 0.00 & 265.32 & 95.65 & 132.58 & 35.81 & 29.69 & 28.41 & 21.99 & 513824.83 & 0.00 & 67528.22 & 297736.53 \\
\hline 7536.57 & 116.32 & 132.42 & 0.00 & 249.97 & 95.10 & 128.57 & 42.46 & 0.00 & 27.11 & 24.68 & 527460.98 & 0.00 & 67154.20 & 290342.19 \\
\hline 7536.64 & 111.53 & 139.81 & 0.00 & 246.29 & 91.28 & 126.25 & 0.00 & 23.91 & 29.06 & 21.80 & 529981.05 & 0.00 & 66325.52 & 282658.52 \\
\hline 7536.71 & 114.36 & 154.61 & 0.00 & 266.66 & 100.60 & 130.29 & 41.88 & 33.49 & 28.60 & 16.90 & 554707.03 & 0.00 & 60659.23 & 254858.58 \\
\hline 7536.77 & 112.09 & 140.35 & 0.00 & 260.96 & 95.31 & 122.15 & 38.41 & 41.59 & 28.70 & 26.25 & 554889.47 & 0.00 & 60598.19 & 260462.87 \\
\hline 7536.84 & 111.51 & 146.88 & 0.00 & 245.56 & 94.26 & 128.51 & 51.16 & 30.52 & 32.99 & 22.66 & 545506.97 & 0.00 & 62354.10 & 275005.48 \\
\hline 7536.90 & 111.07 & 139.17 & 0.00 & 252.36 & 96.19 & 125.03 & 45.27 & 39.11 & 33.65 & 20.41 & 570942.31 & 0.00 & 59213.97 & 250488.05 \\
\hline 7536.97 & 111.59 & 128.92 & 0.00 & 219.06 & 87.54 & 118.50 & 0.00 & 0.00 & 31.43 & 31.13 & 619183.18 & 0.00 & 54529.79 & 213067.06 \\
\hline 7537.07 & 113.30 & 166.57 & 0.00 & 296.41 & 105.60 & 141.97 & 44.58 & 39.36 & 31.32 & 26.23 & 581847.14 & 0.00 & 64956.48 & 229031.94 \\
\hline 7537.13 & 117.60 & 154.23 & 0.00 & 256.03 & 103.48 & 136.79 & 54.33 & 40.64 & 33.55 & 23.00 & 531227.25 & 0.00 & 66994.51 & 255028.64 \\
\hline 7537.20 & 116.05 & 145.35 & 0.00 & 249.63 & 90.20 & 122.88 & 35.15 & 35.09 & 29.32 & 24.78 & 542801.83 & 0.00 & 65191.62 & 266267.12 \\
\hline 7537.26 & 108.76 & 110.11 & 0.00 & 229.38 & 90.39 & 123.29 & 42.88 & 0.00 & 27.70 & 20.88 & 565023.00 & 0.00 & 58809.02 & 242358.26 \\
\hline 7537.33 & 122.56 & 138.48 & 0.00 & 226.26 & 91.15 & 128.15 & 41.78 & 0.00 & 34.50 & 28.71 & 565205.54 & 0.00 & 60762.47 & 251468.00 \\
\hline 7537.39 & 116.48 & 143.17 & 0.00 & 235.50 & 89.37 & 120.74 & 46.67 & 0.00 & 38.06 & 25.26 & 567703.27 & 0.00 & 59589.12 & 242355.05 \\
\hline 7537.46 & 123.21 & 136.66 & 0.00 & 237.75 & 90.86 & 123.02 & 43.50 & 28.23 & 38.76 & 25.46 & 485225.74 & 0.00 & 77043.31 & 282008.72 \\
\hline 7537.52 & 122.33 & 136.07 & 0.00 & 239.87 & 91.92 & 127.03 & 48.70 & 0.00 & 40.56 & 22.72 & 530798.80 & 0.00 & 73344.61 & 269287.48 \\
\hline 7537.59 & 120.54 & 133.70 & 0.00 & 245.12 & 91.29 & 118.44 & 41.41 & 0.00 & 35.73 & 26.68 & 561028.72 & 0.00 & 59972.16 & 239948.87 \\
\hline 7537.66 & 114.85 & 162.81 & 0.00 & 252.98 & 92.10 & 126.29 & 50.97 & 60.35 & 32.06 & 24.07 & 552518.19 & 0.00 & 56165.28 & 215985.64 \\
\hline 7537.72 & 122.15 & 216.15 & 0.00 & 240.23 & 89.34 & 128.47 & 37.14 & 41.03 & 32.06 & 26.86 & 577616.45 & 0.00 & 57555.43 & 229087.40 \\
\hline 7537.79 & 118.44 & 184.15 & 0.00 & 257.06 & 95.48 & 134.24 & 49.66 & 38.36 & 41.90 & 21.68 & 577009.63 & 0.00 & 61477.51 & 245693.08 \\
\hline 7537.85 & 114.55 & 197.85 & 0.00 & 228.08 & 85.10 & 125.78 & 42.40 & 0.00 & 43.33 & 20.93 & 578563.37 & 0.00 & 54166.63 & 208416.98 \\
\hline 7537.92 & 125.96 & 246.13 & 0.00 & 253.76 & 89.05 & 129.35 & 46.51 & 50.66 & 35.01 & 23.77 & 584707.38 & 0.00 & 57016.93 & 219074.62 \\
\hline 7537.98 & 122.00 & 191.21 & 0.00 & 265.60 & 93.30 & 135.22 & 0.00 & 49.98 & 39.01 & 30.01 & 549202.39 & 0.00 & 73117.93 & 260730.26 \\
\hline 7538.05 & 119.98 & 218.88 & 0.00 & 253.50 & 103.43 & 126.84 & 0.00 & 72.17 & 51.07 & 26.39 & 528847.93 & 0.00 & 78682.72 & 253108.42 \\
\hline 7538.12 & 124.53 & 229.61 & 0.00 & 250.36 & 94.76 & 121.26 & 46.74 & 50.33 & 43.19 & 27.24 & 553139.93 & 0.00 & 67325.58 & 249103.69 \\
\hline 7538.18 & 96.65 & 206.69 & 0.00 & 259.66 & 96.41 & 126.89 & 0.00 & 70.08 & 50.18 & 24.99 & 581927.25 & 0.00 & 47571.93 & 168189.19 \\
\hline 7538.25 & 121.19 & 245.31 & 0.00 & 236.21 & 88.36 & 125.45 & 0.00 & 0.00 & 33.71 & 21.58 & 613301.81 & 0.00 & 53487.98 & 212845.58 \\
\hline
\end{tabular}




\begin{tabular}{|c|c|c|c|c|c|c|c|c|c|c|c|c|c|c|}
\hline Depth & $\mathrm{Zr}$ & Mo & $\mathrm{Ag}$ & $\mathrm{Cd}$ & Sn & $\mathrm{Sb}$ & $\mathrm{Hf}$ & W & $\mathrm{Pb}$ & $\mathrm{Bi}$ & LE & $\mathrm{Mg}$ & $\mathrm{Al}$ & $\mathrm{Si}$ \\
\hline & $\mathrm{ppm}$ & $\mathrm{ppm}$ & $\mathrm{ppm}$ & $\mathrm{ppm}$ & $\mathrm{ppm}$ & $\mathrm{ppm}$ & ppm & $\mathrm{ppm}$ & $\mathrm{ppm}$ & $\mathrm{ppm}$ & ppm & $\mathrm{ppm}$ & $\mathrm{ppm}$ & $\mathrm{ppm}$ \\
\hline 7538.31 & 118.41 & 236.51 & 0.00 & 238.84 & 94.20 & 125.48 & 0.00 & 40.45 & 35.12 & 26.13 & 592573.30 & 0.00 & 59948.23 & 228820.17 \\
\hline 7538.38 & 122.88 & 281.08 & 0.00 & 226.46 & 93.55 & 122.91 & 37.54 & 0.00 & 38.94 & 23.55 & 587487.92 & 0.00 & 52571.01 & 211034.97 \\
\hline 7538.44 & 121.00 & 207.23 & 0.00 & 244.70 & 103.57 & 130.03 & 0.00 & 0.00 & 41.81 & 22.02 & 587078.48 & 0.00 & 57421.48 & 231669.84 \\
\hline 7538.51 & 116.95 & 188.73 & 0.00 & 233.46 & 93.88 & 129.29 & 48.58 & 0.00 & 40.41 & 24.00 & 682182.84 & 0.00 & 34045.93 & 141998.02 \\
\hline 7538.57 & 113.86 & 174.78 & 0.00 & 277.75 & 103.96 & 139.26 & 0.00 & 0.00 & 37.87 & 24.78 & 588203.98 & 0.00 & 59266.70 & 228411.62 \\
\hline 7538.64 & 121.70 & 187.13 & 0.00 & 251.13 & 93.73 & 127.73 & 51.89 & 43.67 & 41.16 & 25.78 & 590190.96 & 0.00 & 58293.95 & 217539.21 \\
\hline 7538.71 & 124.84 & 187.47 & 0.00 & 261.16 & 96.04 & 124.54 & 55.82 & 0.00 & 37.91 & 25.17 & 580320.13 & 0.00 & 62344.93 & 237692.94 \\
\hline 7538.77 & 119.27 & 133.04 & 0.00 & 254.59 & 99.72 & 128.64 & 44.53 & 30.58 & 29.50 & 29.06 & 613150.83 & 0.00 & 54269.21 & 224407.59 \\
\hline 7538.84 & 118.37 & 118.05 & 0.00 & 252.23 & 98.52 & 125.40 & 49.12 & 0.00 & 36.06 & 23.30 & 638818.12 & 0.00 & 46190.49 & 213081.40 \\
\hline 7538.90 & 117.09 & 117.81 & 0.00 & 235.19 & 94.44 & 124.92 & 38.05 & 37.65 & 36.00 & 25.45 & 561985.67 & 0.00 & 64167.17 & 270544.61 \\
\hline 7538.97 & 116.76 & 130.13 & 0.00 & 235.37 & 93.03 & 129.25 & 37.21 & 29.25 & 33.71 & 21.16 & 551277.12 & 0.00 & 65448.88 & 268976.16 \\
\hline 7539.03 & 116.43 & 124.61 & 0.00 & 255.06 & 92.31 & 128.12 & 38.20 & 52.54 & 31.89 & 28.01 & 593819.55 & 0.00 & 57017.83 & 222196.74 \\
\hline 7539.10 & 106.93 & 130.15 & 0.00 & 270.69 & 101.75 & 135.37 & 39.33 & 0.00 & 34.21 & 20.25 & 569148.19 & 0.00 & 58553.86 & 254801.60 \\
\hline 7539.17 & 124.49 & 186.08 & 0.00 & 246.81 & 97.84 & 129.66 & 42.96 & 28.09 & 38.13 & 22.48 & 612425.26 & 0.00 & 56703.21 & 206430.01 \\
\hline 7539.23 & 113.94 & 167.47 & 0.00 & 242.08 & 89.94 & 120.26 & 37.78 & 0.00 & 35.12 & 22.74 & 599549.51 & 0.00 & 51429.12 & 216415.81 \\
\hline 7539.30 & 115.73 & 135.49 & 0.00 & 236.53 & 92.76 & 119.93 & 49.11 & 31.83 & 34.07 & 19.54 & 571047.94 & 0.00 & 58470.02 & 255952.00 \\
\hline 7539.36 & 111.88 & 132.62 & 0.00 & 275.96 & 100.61 & 141.68 & 39.77 & 32.90 & 34.26 & 19.53 & 538530.22 & 0.00 & 64367.25 & 274731.80 \\
\hline 7539.43 & 132.00 & 194.13 & 0.00 & 259.62 & 94.00 & 128.04 & 58.77 & 62.39 & 35.08 & 21.50 & 554306.67 & 0.00 & 62898.44 & 229801.15 \\
\hline 7539.49 & 120.23 & 167.07 & 0.00 & 266.86 & 99.73 & 126.15 & 41.92 & 0.00 & 40.33 & 22.10 & 625178.04 & 0.00 & 41492.15 & 181268. \\
\hline 7539.56 & 112.66 & 135.80 & 0.00 & 255.26 & 92.33 & 126.36 & 37.49 & 28.28 & 31.94 & 18.84 & 578398.46 & 0.00 & 49090.49 & 232647.02 \\
\hline 7539.62 & 146.44 & 99.93 & 0.00 & 294.08 & 112.75 & 144.72 & 0.00 & 50.92 & 27.92 & 0.00 & 692316.30 & 0.00 & 24487.36 & 83686.29 \\
\hline 7539.69 & 136.49 & 219.50 & 0.00 & 244.50 & 96.66 & 127.48 & 51.77 & 0.00 & 30.83 & 21.89 & 722156.44 & 0.00 & 26644.67 & 121502.41 \\
\hline 7539.76 & 150.34 & 43.84 & 0.00 & 253.89 & 94.25 & 128.39 & 0.00 & 44.09 & 41.12 & 27.67 & 642008.56 & 0.00 & 55352.01 & 85294.11 \\
\hline 7539.82 & 141.75 & 246.00 & 0.00 & 229.54 & 87.42 & 125.00 & 46.60 & 0.00 & 44.35 & 23.37 & 530149.34 & 0.00 & 59922.65 & 235121.56 \\
\hline 7539.89 & 122.92 & 235.21 & 0.00 & 251.74 & 95.18 & 132.40 & 42.67 & 0.00 & 44.43 & 15.19 & 512812.82 & 0.00 & 68392.42 & 261666.91 \\
\hline 7539.95 & 110.65 & 176.93 & 0.00 & 204.41 & 87.36 & 118.40 & 53.83 & 33.99 & 54.84 & 20.22 & 463982.56 & 0.00 & 52514.66 & 199649.89 \\
\hline 7540.02 & 113.03 & 213.17 & 0.00 & 254.96 & 106.61 & 134.60 & 0.00 & 0.00 & 37.86 & 0.00 & 714341.39 & 0.00 & 20840.85 & 107322.39 \\
\hline 7540.07 & 120.72 & 325.92 & 0.00 & 252.70 & 93.42 & 136.67 & 0.00 & 0.00 & 44.86 & 23.93 & 661067.35 & 0.00 & 34668.47 & 151816.74 \\
\hline 7540.13 & 133.36 & 321.49 & 0.00 & 255.89 & 100.78 & 140.58 & 0.00 & 37.96 & 38.34 & 17.89 & 663187.84 & 0.00 & 32218.12 & 149271.60 \\
\hline 7540.20 & 115.99 & 236.97 & 0.00 & 278.14 & 98.29 & 127.44 & 0.00 & 67.31 & 40.16 & 20.20 & 655405.64 & 0.00 & 34108.34 & 152811.65 \\
\hline 7540.26 & 123.17 & 247.13 & 0.00 & 245.70 & 104.17 & 125.98 & 0.00 & 0.00 & 35.32 & 21.88 & 680404.11 & 0.00 & 26860.48 & 128497.61 \\
\hline 7540.33 & 118.77 & 218.32 & 0.00 & 277.20 & 102.37 & 142.99 & 0.00 & 0.00 & 50.34 & 0.00 & 675896.32 & 0.00 & 24277.27 & 119920.11 \\
\hline
\end{tabular}




\begin{tabular}{|c|c|c|c|c|c|c|c|c|c|c|c|c|c|c|}
\hline Depth & $\mathrm{Zr}$ & Mo & $\mathrm{Ag}$ & $\mathrm{Cd}$ & $\mathrm{Sn}$ & $\mathrm{Sb}$ & $\mathrm{Hf}$ & $\mathrm{W}$ & $\mathrm{Pb}$ & $\mathrm{Bi}$ & LE & $\mathrm{Mg}$ & $\mathrm{Al}$ & $\mathrm{Si}$ \\
\hline & ppm & ppm & $\mathrm{ppm}$ & $\mathrm{ppm}$ & $\mathrm{ppm}$ & $\mathrm{ppm}$ & $\mathrm{ppm}$ & $\mathrm{ppm}$ & $\mathrm{ppm}$ & ppm & $\mathrm{ppm}$ & $\mathrm{ppm}$ & ppm & ppm \\
\hline 7540.39 & 126.24 & 214.76 & 0.00 & 263.80 & 104.18 & 150.83 & 0.00 & 0.00 & 43.63 & 0.00 & 707458.98 & 0.00 & 30316.02 & 123486.55 \\
\hline 7540.46 & 138.37 & 245.35 & 0.00 & 251.47 & 95.73 & 128.34 & 48.61 & 0.00 & 46.60 & 23.33 & 564669.01 & 0.00 & 45052.21 & 190315.23 \\
\hline 7540.52 & 115.40 & 288.30 & 0.00 & 213.29 & 82.59 & 118.67 & 0.00 & 0.00 & 51.10 & 16.86 & 640151.21 & 0.00 & 28784.20 & 132594.24 \\
\hline 7540.59 & 104.49 & 164.13 & 0.00 & 251.29 & 102.04 & 136.31 & 0.00 & 0.00 & 57.97 & 27.88 & 672270.40 & 0.00 & 18871.41 & 93204.75 \\
\hline 7540.66 & 104.66 & 177.96 & 0.00 & 248.58 & 103.28 & 126.52 & 0.00 & 0.00 & 47.34 & 0.00 & 623312.45 & 0.00 & 27561.12 & 141037.72 \\
\hline 7540.72 & 123.02 & 294.00 & 0.00 & 227.25 & 90.99 & 126.92 & 0.00 & 50.30 & 44.82 & 23.84 & 648955.71 & 0.00 & 29617.16 & 141419.50 \\
\hline 7540.79 & 122.25 & 287.10 & 0.00 & 255.62 & 90.38 & 131.26 & 0.00 & 0.00 & 47.87 & 0.00 & 672475.27 & 0.00 & 26106.15 & 120507.49 \\
\hline 7540.85 & 108.91 & 162.43 & 0.00 & 232.36 & 86.12 & 130.45 & 52.72 & 0.00 & 45.08 & 18.00 & 620812.95 & 0.00 & 31947.02 & 156018.78 \\
\hline 7540.92 & 118.67 & 217.41 & 0.00 & 241.93 & 84.89 & 122.65 & 0.00 & 50.17 & 49.80 & 22.92 & 608900.57 & 0.00 & 40680.31 & 168496.43 \\
\hline 7540.98 & 121.48 & 203.99 & 0.00 & 275.03 & 97.08 & 129.92 & 0.00 & 93.17 & 54.38 & 18.84 & 662896.97 & 0.00 & 33205.51 & 125523.23 \\
\hline 7541.05 & 119.43 & 215.79 & 0.00 & 263.45 & 95.20 & 130.42 & 0.00 & 66.44 & 43.19 & 0.00 & 659477.28 & 0.00 & 28329.64 & 146145.12 \\
\hline 7541.12 & 99.36 & 247.53 & 0.00 & 298.67 & 113.11 & 140.37 & 0.00 & 0.00 & 40.45 & 0.00 & 557502.82 & 0.00 & 39717.38 & 200279.80 \\
\hline 7541.18 & 114.68 & 201.91 & 0.00 & 264.17 & 85.03 & 119.60 & 0.00 & 98.79 & 49.31 & 15.61 & 544939.15 & 0.00 & 39667.97 & 206198.05 \\
\hline 7541.25 & 119.05 & 159.40 & 0.00 & 301.29 & 90.08 & 130.29 & 0.00 & 149.72 & 40.05 & 19.19 & 594530.24 & 0.00 & 38380.25 & 179731.71 \\
\hline 7541.31 & 105.73 & 142.85 & 0.00 & 245.22 & 92.04 & 122.88 & 0.00 & 57.92 & 30.15 & 17.91 & 614647.96 & 0.00 & 29858.69 & 165625.41 \\
\hline 7541.38 & 115.43 & 160.72 & 0.00 & 270.47 & 95.01 & 128.30 & 0.00 & 50.12 & 37.86 & 0.00 & 633467.11 & 0.00 & 26624.53 & 170747.64 \\
\hline 7541.44 & 92.76 & 87.79 & 0.00 & 284.84 & 106.68 & 140.36 & 0.00 & 40.79 & 29.97 & 0.00 & 535937.42 & 0.00 & 29210.47 & 204829.59 \\
\hline 7541.51 & 43.04 & 0.00 & 0.00 & 262.55 & 101.95 & 125.96 & 0.00 & 0.00 & 0.00 & 0.00 & 575832.61 & 0.00 & 3114.39 & 93926.27 \\
\hline 7541.57 & 48.43 & 0.00 & 0.00 & 269.82 & 97.17 & 126.03 & 0.00 & 0.00 & 7.49 & 0.00 & 607037.17 & 0.00 & 2541.27 & 23875.87 \\
\hline 7541.64 & 43.13 & 0.00 & 0.00 & 267.17 & 101.56 & 125.58 & 0.00 & 0.00 & 0.00 & 0.00 & 593875.35 & 0.00 & 2492.01 & 13340.77 \\
\hline 7541.71 & 37.91 & 0.00 & 0.00 & 284.12 & 96.34 & 132.03 & 0.00 & 0.00 & 0.00 & 0.00 & 592461.37 & 0.00 & 2368.96 & 14211.05 \\
\hline 7541.77 & 36.84 & 0.00 & 0.00 & 266.58 & 97.50 & 129.16 & 0.00 & 0.00 & 0.00 & 0.00 & 592994.38 & 0.00 & 3361.54 & 16451.95 \\
\hline 7541.84 & 37.52 & 0.00 & 0.00 & 284.88 & 101.36 & 134.87 & 0.00 & 29.98 & 0.00 & 0.00 & 594199.41 & 0.00 & 1968.30 & 22545.20 \\
\hline 7541.90 & 33.84 & 0.00 & 0.00 & 243.07 & 95.41 & 128.76 & 0.00 & 0.00 & 0.00 & 0.00 & 589374.12 & 0.00 & 2388.52 & 25564.90 \\
\hline 7541.97 & 33.20 & 0.00 & 0.00 & 274.09 & 99.96 & 124.77 & 0.00 & 0.00 & 7.25 & 0.00 & 611996.88 & 0.00 & 3619.65 & 22619.68 \\
\hline 7542.03 & 32.81 & 0.00 & 0.00 & 259.52 & 100.08 & 125.50 & 0.00 & 0.00 & 0.00 & 0.00 & 624943.67 & 0.00 & 4024.56 & 36156.41 \\
\hline 7542.10 & 42.15 & 0.00 & 0.00 & 266.11 & 97.08 & 135.18 & 0.00 & 0.00 & 0.00 & 0.00 & 564747.51 & 0.00 & 4430.04 & 111408.34 \\
\hline 7542.17 & 43.59 & 15.76 & 0.00 & 235.74 & 101.48 & 123.24 & 0.00 & 0.00 & 0.00 & 0.00 & 547224.15 & 0.00 & 5230.68 & 189587.71 \\
\hline 7542.23 & 45.23 & 31.95 & 0.00 & 265.42 & 94.24 & 135.49 & 0.00 & 0.00 & 0.00 & 0.00 & 654767.89 & 0.00 & 5870.65 & 161563.01 \\
\hline 7542.30 & 55.51 & 82.26 & 0.00 & 260.84 & 102.07 & 133.54 & 0.00 & 0.00 & 23.04 & 0.00 & 644642.46 & 0.00 & 10200.27 & 110635.64 \\
\hline 7542.36 & 116.55 & 278.49 & 0.00 & 256.25 & 99.61 & 136.35 & 0.00 & 0.00 & 36.51 & 0.00 & 649232.75 & 0.00 & 34673.35 & 129938.06 \\
\hline 7542.43 & 126.09 & 279.35 & 0.00 & 269.08 & 85.95 & 122.47 & 45.89 & 59.44 & 36.95 & 20.35 & 611604.93 & 0.00 & 38194.33 & 190734.18 \\
\hline
\end{tabular}




\begin{tabular}{|c|c|c|c|c|c|c|c|c|c|c|c|c|c|c|}
\hline Depth & $\mathrm{Zr}$ & Mo & $\mathrm{Ag}$ & $\mathrm{Cd}$ & $\mathrm{Sn}$ & $\mathrm{Sb}$ & $\mathrm{Hf}$ & $\mathrm{W}$ & $\mathrm{Pb}$ & $\mathrm{Bi}$ & LE & $\mathrm{Mg}$ & $\mathrm{Al}$ & $\mathrm{Si}$ \\
\hline & ppm & ppm & $\mathrm{ppm}$ & $\mathrm{ppm}$ & $\mathrm{ppm}$ & $\mathrm{ppm}$ & $\mathrm{ppm}$ & $\mathrm{ppm}$ & $\mathrm{ppm}$ & ppm & $\mathrm{ppm}$ & $\mathrm{ppm}$ & ppm & ppm \\
\hline 7542.49 & 113.32 & 259.13 & 0.00 & 261.46 & 93.07 & 120.74 & 0.00 & 82.88 & 34.88 & 0.00 & 653601.84 & 0.00 & 30388.58 & 170579.66 \\
\hline 7542.56 & 123.23 & 238.40 & 0.00 & 247.12 & 92.44 & 128.34 & 0.00 & 56.84 & 43.49 & 16.62 & 615562.76 & 0.00 & 37441.23 & 183200.91 \\
\hline 7542.62 & 129.44 & 294.11 & 0.00 & 261.26 & 91.58 & 127.22 & 0.00 & 59.07 & 40.43 & 0.00 & 680276.92 & 0.00 & 28302.13 & 136915.15 \\
\hline 7542.69 & 108.11 & 235.36 & 0.00 & 268.67 & 98.16 & 127.65 & 0.00 & 72.10 & 35.71 & 18.78 & 653501.37 & 0.00 & 29396.52 & 179151.26 \\
\hline 7542.76 & 81.04 & 136.78 & 0.00 & 185.83 & 84.01 & 108.43 & 0.00 & 0.00 & 44.47 & 0.00 & 584178.67 & 0.00 & 19116.08 & 110554.03 \\
\hline 7542.82 & 120.36 & 260.02 & 0.00 & 267.92 & 106.69 & 142.94 & 0.00 & 0.00 & 39.46 & 21.81 & 669649.37 & 0.00 & 28345.93 & 154658.62 \\
\hline 7542.89 & 132.09 & 220.35 & 0.00 & 267.09 & 101.25 & 129.14 & 0.00 & 51.94 & 38.55 & 0.00 & 557745.56 & 0.00 & 53690.87 & 224500.93 \\
\hline 7542.95 & 87.93 & 89.36 & 0.00 & 261.98 & 98.87 & 125.78 & 39.06 & 50.87 & 23.21 & 13.91 & 524222.86 & 0.00 & 28856.33 & 194014.63 \\
\hline 7543.07 & 121.19 & 250.64 & 0.00 & 242.03 & 84.91 & 118.02 & 0.00 & 43.09 & 42.31 & 21.79 & 537923.22 & 0.00 & 54690.12 & 246284.48 \\
\hline 7543.13 & 123.46 & 272.27 & 0.00 & 237.96 & 87.39 & 120.56 & 49.75 & 42.21 & 37.69 & 17.36 & 583471.26 & 0.00 & 47504.92 & 202805.59 \\
\hline 7543.20 & 106.67 & 136.05 & 0.00 & 277.66 & 100.46 & 129.37 & 0.00 & 0.00 & 21.88 & 0.00 & 563631.39 & 0.00 & 25980.98 & 147460.88 \\
\hline 7543.26 & 127.07 & 178.61 & 0.00 & 267.47 & 89.60 & 129.32 & 0.00 & 53.72 & 34.93 & 16.82 & 533172.59 & 0.00 & 39895.66 & 174642.31 \\
\hline 7543.33 & 124.30 & 132.33 & 0.00 & 252.59 & 91.24 & 118.16 & 0.00 & 0.00 & 37.62 & 15.79 & 530301.07 & 0.00 & 45328.35 & 195437.46 \\
\hline 7543.39 & 129.07 & 240.01 & 0.00 & 224.40 & 87.47 & 118.02 & 43.78 & 0.00 & 44.05 & 18.63 & 566179.65 & 0.00 & 43709.20 & 198093.19 \\
\hline 7543.46 & 117.53 & 231.03 & 0.00 & 236.09 & 87.90 & 117.78 & 41.21 & 0.00 & 38.16 & 18.36 & 582009.08 & 0.00 & 49104.41 & 221487.73 \\
\hline 7543.52 & 103.65 & 157.12 & 0.00 & 227.90 & 94.61 & 116.18 & 45.25 & 0.00 & 43.60 & 19.38 & 522782.01 & 0.00 & 39658.10 & 184987.47 \\
\hline 7543.59 & 129.30 & 118.37 & 0.00 & 266.51 & 88.96 & 118.78 & 0.00 & 0.00 & 34.09 & 19.54 & 571085.57 & 0.00 & 43936.35 & 231942.70 \\
\hline 7543.66 & 111.86 & 185.58 & 0.00 & 238.15 & 91.14 & 117.69 & 0.00 & 55.74 & 54.10 & 21.14 & 552007.05 & 0.00 & 43962.86 & 224449.05 \\
\hline 7543.72 & 110.35 & 238.62 & 0.00 & 225.93 & 87.93 & 117.79 & 0.00 & 33.89 & 43.64 & 17.39 & 543195.68 & 0.00 & 46047.50 & 238838.30 \\
\hline 7543.79 & 118.91 & 195.61 & 0.00 & 234.68 & 96.23 & 123.07 & 0.00 & 44.43 & 50.87 & 18.70 & 517549.18 & 0.00 & 47298.86 & 246547.82 \\
\hline 7543.85 & 111.51 & 172.95 & 0.00 & 234.76 & 87.31 & 123.04 & 0.00 & 57.89 & 46.00 & 14.61 & 552094.45 & 0.00 & 44366.47 & 210433.38 \\
\hline 7543.92 & 118.91 & 169.44 & 0.00 & 281.65 & 92.68 & 130.57 & 0.00 & 57.70 & 48.54 & 0.00 & 565467.32 & 0.00 & 43973.57 & 204348.41 \\
\hline 7543.98 & 108.21 & 134.13 & 0.00 & 224.32 & 90.80 & 133.18 & 0.00 & 0.00 & 43.83 & 0.00 & 580584.84 & 0.00 & 29826.49 & 176158.76 \\
\hline 7544.05 & 106.81 & 169.64 & 0.00 & 242.10 & 92.98 & 120.38 & 0.00 & 39.16 & 50.22 & 16.03 & 575603.43 & 0.00 & 41964.53 & 200648.25 \\
\hline 7544.12 & 96.99 & 169.13 & 0.00 & 242.98 & 89.98 & 113.71 & 0.00 & 41.26 & 40.19 & 0.00 & 570242.84 & 0.00 & 39729.35 & 215275.41 \\
\hline 7544.18 & 96.86 & 212.13 & 0.00 & 249.10 & 93.34 & 122.67 & 49.69 & 0.00 & 49.32 & 22.59 & 560728.65 & 0.00 & 39644.05 & 250664.59 \\
\hline 7544.25 & 96.62 & 170.44 & 0.00 & 264.85 & 97.33 & 131.65 & 43.65 & 0.00 & 39.86 & 0.00 & 604816.54 & 0.00 & 31442.83 & 233434.55 \\
\hline 7544.31 & 151.12 & 141.32 & 0.00 & 260.65 & 97.67 & 131.35 & 0.00 & 34.29 & 46.65 & 19.90 & 567307.45 & 0.00 & 36124.84 & 225939.19 \\
\hline 7544.38 & 440.07 & 89.25 & 0.00 & 286.35 & 116.80 & 132.86 & 70.75 & 73.41 & 42.31 & 32.27 & 439126.14 & 0.00 & 32339.71 & 164250.71 \\
\hline 7544.44 & 318.06 & 96.19 & 0.00 & 298.51 & 116.92 & 144.85 & 0.00 & 52.07 & 46.21 & 28.06 & 547514.87 & 0.00 & 27388.36 & 154325.92 \\
\hline 7544.51 & 170.37 & 114.61 & 0.00 & 263.81 & 97.53 & 127.40 & 0.00 & 38.87 & 44.99 & 17.09 & 585239.17 & 0.00 & 33825.20 & 204509.45 \\
\hline 7544.57 & 222.66 & 140.85 & 0.00 & 251.66 & 105.92 & 133.67 & 0.00 & 53.79 & 46.86 & 0.00 & 556543.57 & 0.00 & 31455.46 & 182896.31 \\
\hline
\end{tabular}




\begin{tabular}{|c|c|c|c|c|c|c|c|c|c|c|c|c|c|c|}
\hline Depth & $\mathrm{Zr}$ & Mo & $\mathrm{Ag}$ & $\mathrm{Cd}$ & Sn & $\mathrm{Sb}$ & $\mathrm{Hf}$ & W & $\mathrm{Pb}$ & $\mathrm{Bi}$ & LE & $\mathrm{Mg}$ & $\mathrm{Al}$ & $\mathrm{Si}$ \\
\hline & $\mathrm{ppm}$ & $\mathrm{ppm}$ & $\mathrm{ppm}$ & $\mathrm{ppm}$ & $\mathrm{ppm}$ & $\mathrm{ppm}$ & ppm & $\mathrm{ppm}$ & $\mathrm{ppm}$ & $\mathrm{ppm}$ & ppm & $\mathrm{ppm}$ & $\mathrm{ppm}$ & $\mathrm{ppm}$ \\
\hline 7544.64 & 176.98 & 159.00 & 0.00 & 269.92 & 107.00 & 127.33 & 58.35 & 0.00 & 26.00 & 18.37 & 587820.77 & 0.00 & 27482.50 & 201404.09 \\
\hline 7544.71 & 150.97 & 158.22 & 0.00 & 263.18 & 102.65 & 128.71 & 0.00 & 37.24 & 40.68 & 18.70 & 613006.28 & 0.00 & 30510.21 & 201875.27 \\
\hline 7544.77 & 225.48 & 120.80 & 0.00 & 248.26 & 100.58 & 137.10 & 0.00 & 0.00 & 42.66 & 23.84 & 476991.73 & 0.00 & 44467.00 & 258602.15 \\
\hline 7544.84 & 205.63 & 91.41 & 0.00 & 248.48 & 104.87 & 121.03 & 56.38 & 35.00 & 40.73 & 22.14 & 504654.27 & 0.00 & 43840.55 & 244050.70 \\
\hline 7544.90 & 265.19 & 86.24 & 0.00 & 261.82 & 98.21 & 128.52 & 58.82 & 33.41 & 39.96 & 28.59 & 496515.61 & 0.00 & 29283.38 & 166346.54 \\
\hline 7544.97 & 175.61 & 78.47 & 0.00 & 246.20 & 99.13 & 131.53 & 0.00 & 33.49 & 39.50 & 21.97 & 553248.63 & 0.00 & 42853.56 & 242757.04 \\
\hline 7545.07 & 151.60 & 124.73 & 0.00 & 264.88 & 103.39 & 139.76 & 50.20 & 0.00 & 34.01 & 17.05 & 508632.22 & 0.00 & 50280.26 & 272933.44 \\
\hline 7545.13 & 124.83 & 262.40 & 0.00 & 219.46 & 86.19 & 117.48 & 48.24 & 27.82 & 46.49 & 20.05 & 523804.46 & 0.00 & 58310.80 & 267264.76 \\
\hline 7545.20 & 117.36 & 217.34 & 0.00 & 244.34 & 88.80 & 128.78 & 47.57 & 0.00 & 53.73 & 16.15 & 534490.26 & 0.00 & 51946.23 & 261061.53 \\
\hline 7545.26 & 120.92 & 234.37 & 0.00 & 240.51 & 84.29 & 119.61 & 46.86 & 0.00 & 45.73 & 19.63 & 558632.17 & 0.00 & 45858.82 & 234537.27 \\
\hline 7545.33 & 107.54 & 221.52 & 0.00 & 239.04 & 91.05 & 129.25 & 44.99 & 0.00 & 45.08 & 14.08 & 566893.47 & 0.00 & 42275.45 & 227240.13 \\
\hline 7545.39 & 125.58 & 321.24 & 0.00 & 232.21 & 89.53 & 122.05 & 40.42 & 0.00 & 35.67 & 16.67 & 556878.47 & 0.00 & 42332.35 & 228194.79 \\
\hline 7545.46 & 119.92 & 125.59 & 0.00 & 254.55 & 90.89 & 128.63 & 0.00 & 34.25 & 20.99 & 0.00 & 547890.34 & 0.00 & 16076.64 & 162957.71 \\
\hline 7545.52 & 182.93 & 113.79 & 0.00 & 268.39 & 95.55 & 131.51 & 51.61 & 0.00 & 28.67 & 0.00 & 481550.93 & 0.00 & 23077.56 & 180366.53 \\
\hline 7545.59 & 218.64 & 196.49 & 0.00 & 271.77 & 97.55 & 123.32 & 60.00 & 32.89 & 42.07 & 19.26 & 454944.16 & 0.00 & 42191.77 & 177164.37 \\
\hline 7545.66 & 174.57 & 251.26 & 0.00 & 236.97 & 88.16 & 121.80 & 62.42 & 28.73 & 43.07 & 20.22 & 512355.79 & 0.00 & 38048.89 & 176481.01 \\
\hline 7545.72 & 108.67 & 317.99 & 0.00 & 240.13 & 93.61 & 119.09 & 41.34 & 0.00 & 48.46 & 19.12 & 578124.49 & 0.00 & 43695.22 & 253197.89 \\
\hline 7545.79 & 105.65 & 316.39 & 0.00 & 224.27 & 90.58 & 125.60 & 0.00 & 0.00 & 49.05 & 18.17 & 596391.06 & 0.00 & 41818.45 & 202958.80 \\
\hline 7545.85 & 107.23 & 289.37 & 0.00 & 240.59 & 95.79 & 120.69 & 48.71 & 48.31 & 44.49 & 15.41 & 567657.29 & 0.00 & 43684.64 & 220021.10 \\
\hline 7545.92 & 98.54 & 295.40 & 0.00 & 242.70 & 88.28 & 120.34 & 0.00 & 48.88 & 43.80 & 16.30 & 540096.50 & 0.00 & 41686.39 & 269196.68 \\
\hline 7545.98 & 97.14 & 275.26 & 0.00 & 258.20 & 98.13 & 127.92 & 0.00 & 46.07 & 37.64 & 13.82 & 545056.84 & 0.00 & 41362.74 & 265895.13 \\
\hline 7546.05 & 83.22 & 208.31 & 0.00 & 216.32 & 89.06 & 122.13 & 0.00 & 0.00 & 48.90 & 17.65 & 493924.70 & 0.00 & 37315.82 & 195728.58 \\
\hline 7546.12 & 94.20 & 244.23 & 0.00 & 245.90 & 82.22 & 122.06 & 0.00 & 50.91 & 49.50 & 19.84 & 558110.19 & 0.00 & 39125.38 & 247136.47 \\
\hline 7546.18 & 106.78 & 320.28 & 0.00 & 250.84 & 90.08 & 121.44 & 0.00 & 51.92 & 41.43 & 19.94 & 589496.12 & 0.00 & 35799.41 & 216624.46 \\
\hline 7546.25 & 111.99 & 308.24 & 0.00 & 237.42 & 92.39 & 120.54 & 44.58 & 47.89 & 40.27 & 15.83 & 544022.89 & 0.00 & 43283.74 & 233696.30 \\
\hline 7546.31 & 120.85 & 265.34 & 0.00 & 256.67 & 86.80 & 117.11 & 0.00 & 75.16 & 35.55 & 16.37 & 568643.37 & 0.00 & 34675.86 & 161273.04 \\
\hline 7546.38 & 117.17 & 273.11 & 0.00 & 246.91 & 90.56 & 119.77 & 0.00 & 61.79 & 31.48 & 15.01 & 595805.61 & 0.00 & 29152.27 & 179822.66 \\
\hline 7546.44 & 104.65 & 269.07 & 0.00 & 265.93 & 93.06 & 129.69 & 0.00 & 68.13 & 30.04 & 0.00 & 669982.33 & 0.00 & 19005.81 & 141539.15 \\
\hline 7546.51 & 105.68 & 276.44 & 0.00 & 267.61 & 109.34 & 151.55 & 0.00 & 60.28 & 33.52 & 0.00 & 717252.01 & 0.00 & 13485.63 & 105491.91 \\
\hline 7546.57 & 109.71 & 254.33 & 0.00 & 233.85 & 85.27 & 123.76 & 0.00 & 0.00 & 43.18 & 16.77 & 645902.01 & 0.00 & 24985.36 & 164711.72 \\
\hline 7546.64 & 94.62 & 258.60 & 0.00 & 272.21 & 90.93 & 129.58 & 0.00 & 68.60 & 54.21 & 0.00 & 650961.24 & 0.00 & 26176.74 & 160400.58 \\
\hline 7546.71 & 120.98 & 356.77 & 0.00 & 258.33 & 103.18 & 135.30 & 0.00 & 84.60 & 45.77 & 22.91 & 672925.64 & 29981.44 & 22957.60 & 109808.18 \\
\hline
\end{tabular}




\begin{tabular}{|c|c|c|c|c|c|c|c|c|c|c|c|c|c|c|}
\hline Depth & $\mathrm{Zr}$ & Mo & $\mathrm{Ag}$ & $\mathrm{Cd}$ & $\mathrm{Sn}$ & $\mathrm{Sb}$ & $\mathrm{Hf}$ & $\mathrm{W}$ & $\mathrm{Pb}$ & $\mathrm{Bi}$ & LE & $\mathrm{Mg}$ & $\mathrm{Al}$ & $\mathrm{Si}$ \\
\hline & ppm & ppm & ppm & $\mathrm{ppm}$ & $\mathrm{ppm}$ & $\mathrm{ppm}$ & $\mathrm{ppm}$ & $\mathrm{ppm}$ & $\mathrm{ppm}$ & ppm & $\mathrm{ppm}$ & $\mathrm{ppm}$ & ppm & ppm \\
\hline 7546.77 & 123.45 & 365.01 & 0.00 & 282.73 & 92.31 & 125.61 & 46.90 & 149.13 & 47.69 & 21.52 & 514764.28 & 0.00 & 61308.56 & 278195.36 \\
\hline 7546.84 & 94.52 & 263.76 & 0.00 & 276.23 & 103.15 & 135.90 & 0.00 & 0.00 & 35.05 & 0.00 & 729579.02 & 42915.30 & 13776.78 & 117105.20 \\
\hline 7546.90 & 97.76 & 238.00 & 0.00 & 287.92 & 101.98 & 138.03 & 0.00 & 0.00 & 50.71 & 0.00 & 698866.17 & 0.00 & 16216.06 & 110215.03 \\
\hline 7546.97 & 110.96 & 378.25 & 0.00 & 440.42 & 85.17 & 125.00 & 77.24 & 862.43 & 63.75 & 19.40 & 544068.62 & 0.00 & 45118.18 & 196546.78 \\
\hline 7547.03 & 103.31 & 300.20 & 0.00 & 264.90 & 90.20 & 124.68 & 0.00 & 218.25 & 45.96 & 16.92 & 562444.20 & 0.00 & 42311.24 & 242043.63 \\
\hline 7547.10 & 103.06 & 353.30 & 0.00 & 249.15 & 86.05 & 122.92 & 44.64 & 49.78 & 41.82 & 13.63 & 550228.39 & 0.00 & 38577.60 & 255724.03 \\
\hline 7547.17 & 100.79 & 240.69 & 0.00 & 225.49 & 92.28 & 119.39 & 0.00 & 0.00 & 32.95 & 0.00 & 584971.16 & 0.00 & 31151.71 & 201624.40 \\
\hline 7547.23 & 96.91 & 208.31 & 0.00 & 232.80 & 91.24 & 122.10 & 0.00 & 55.01 & 45.05 & 16.49 & 556855.61 & 0.00 & 36087.73 & 245284.71 \\
\hline 7547.30 & 107.98 & 358.55 & 0.00 & 255.40 & 90.84 & 130.76 & 46.38 & 53.03 & 36.07 & 16.43 & 607687.54 & 0.00 & 34676.47 & 227604.70 \\
\hline 7547.36 & 112.04 & 341.55 & 0.00 & 298.91 & 86.95 & 126.59 & 0.00 & 222.19 & 58.06 & 20.57 & 594129.34 & 0.00 & 40450.70 & 213385.07 \\
\hline 7547.43 & 96.97 & 220.90 & 0.00 & 245.78 & 93.53 & 129.25 & 47.45 & 96.27 & 56.29 & 14.48 & 610022.98 & 0.00 & 34832.89 & 231142.34 \\
\hline 7547.49 & 103.77 & 358.48 & 0.00 & 244.10 & 89.44 & 125.70 & 0.00 & 81.71 & 49.24 & 17.35 & 575181.43 & 0.00 & 37931.11 & 255227.46 \\
\hline 7547.56 & 100.60 & 223.21 & 0.00 & 270.18 & 86.18 & 111.52 & 55.58 & 228.93 & 46.89 & 18.30 & 532610.70 & 0.00 & 39828.51 & 230642.30 \\
\hline 7547.62 & 90.26 & 215.68 & 0.00 & 245.47 & 86.81 & 127.67 & 46.43 & 69.96 & 47.17 & 14.58 & 576852.57 & 0.00 & 37608.83 & 269395.71 \\
\hline 7547.69 & 76.88 & 199.02 & 0.00 & 262.30 & 91.14 & 123.00 & 42.90 & 52.80 & 41.50 & 13.78 & 582214.96 & 0.00 & 30400.28 & 276197.16 \\
\hline 7547.76 & 86.93 & 191.35 & 0.00 & 278.92 & 92.23 & 119.58 & 0.00 & 173.41 & 47.79 & 14.82 & 584515.01 & 0.00 & 31320.09 & 274880.77 \\
\hline 7547.82 & 99.97 & 215.35 & 0.00 & 350.79 & 95.27 & 120.86 & 0.00 & 402.13 & 48.88 & 17.32 & 602897.46 & 0.00 & 37167.20 & 241123.19 \\
\hline 7547.89 & 85.89 & 157.39 & 0.00 & 243.77 & 92.48 & 122.99 & 0.00 & 0.00 & 38.40 & 15.56 & 586869.42 & 0.00 & 32228.91 & 273763.22 \\
\hline 7547.95 & 96.06 & 331.72 & 0.00 & 241.22 & 92.75 & 117.50 & 0.00 & 67.52 & 42.78 & 0.00 & 586931.87 & 0.00 & 38526.00 & 268735.57 \\
\hline 7548.02 & 98.53 & 267.86 & 0.00 & 230.45 & 86.73 & 115.88 & 0.00 & 48.78 & 53.73 & 17.67 & 547937.84 & 0.00 & 42981.08 & 259116.12 \\
\hline 7548.08 & 93.83 & 261.85 & 0.00 & 257.04 & 87.67 & 119.25 & 41.75 & 109.00 & 43.39 & 19.17 & 542452.41 & 0.00 & 44236.75 & 295295.99 \\
\hline 7548.15 & 108.10 & 410.96 & 0.00 & 247.31 & 84.55 & 120.64 & 44.69 & 55.67 & 59.44 & 17.91 & 561236.58 & 0.00 & 47759.49 & 260253.98 \\
\hline 7548.22 & 113.89 & 193.09 & 0.00 & 235.64 & 87.89 & 124.53 & 0.00 & 0.00 & 44.04 & 16.82 & 546755.04 & 0.00 & 41576.76 & 283830.03 \\
\hline 7548.28 & 105.26 & 281.11 & 0.00 & 250.76 & 95.91 & 123.56 & 0.00 & 0.00 & 42.91 & 18.10 & 560948.53 & 0.00 & 43028.95 & 287697.76 \\
\hline 7548.35 & 110.50 & 340.79 & 0.00 & 218.44 & 94.07 & 117.38 & 50.14 & 0.00 & 51.04 & 15.34 & 547966.42 & 0.00 & 44147.70 & 259297.54 \\
\hline 7548.41 & 86.13 & 196.23 & 0.00 & 227.51 & 92.57 & 131.28 & 0.00 & 0.00 & 39.95 & 16.75 & 555001.64 & 0.00 & 36987.13 & 292401.09 \\
\hline 7548.48 & 80.25 & 288.70 & 0.00 & 244.99 & 90.01 & 120.72 & 0.00 & 26.60 & 36.31 & 13.54 & 571190.99 & 0.00 & 28359.75 & 309145.00 \\
\hline 7548.54 & 81.81 & 262.18 & 0.00 & 248.34 & 90.16 & 121.39 & 43.44 & 30.62 & 39.88 & 12.30 & 558261.86 & 0.00 & 28774.77 & 304350.44 \\
\hline 7548.61 & 78.51 & 231.00 & 0.00 & 241.38 & 89.85 & 124.59 & 0.00 & 42.33 & 30.94 & 12.89 & 564472.08 & 0.00 & 28584.52 & 295513.07 \\
\hline 7548.67 & 88.02 & 223.15 & 0.00 & 249.58 & 90.76 & 127.37 & 0.00 & 29.80 & 33.84 & 16.11 & 565921.11 & 0.00 & 35343.49 & 279233.02 \\
\hline 7548.74 & 66.87 & 196.88 & 0.00 & 240.15 & 88.37 & 120.75 & 0.00 & 38.19 & 22.99 & 0.00 & 572208.71 & 0.00 & 26667.22 & 305672.47 \\
\hline 7548.81 & 77.93 & 231.80 & 0.00 & 242.25 & 89.62 & 114.68 & 31.22 & 0.00 & 27.32 & 11.92 & 568682.19 & 0.00 & 29590.73 & 302657.31 \\
\hline
\end{tabular}




\begin{tabular}{|c|c|c|c|c|c|c|c|c|c|c|c|c|c|c|}
\hline Depth & $\mathrm{Zr}$ & Mo & $\mathrm{Ag}$ & $\mathrm{Cd}$ & $\mathrm{Sn}$ & $\mathrm{Sb}$ & Hf & $\mathrm{W}$ & $\mathrm{Pb}$ & $\mathrm{Bi}$ & LE & $\mathrm{Mg}$ & $\mathrm{Al}$ & $\mathrm{Si}$ \\
\hline & ppm & ppm & $\mathrm{ppm}$ & ppm & $\mathrm{ppm}$ & ppm & $\mathrm{ppm}$ & $\mathrm{ppm}$ & $\mathrm{ppm}$ & $\mathrm{ppm}$ & ppm & $\mathrm{ppm}$ & $\mathrm{ppm}$ & ppm \\
\hline 7548.87 & 87.93 & 190.75 & 0.00 & 225.97 & 90.73 & 119.67 & 43.84 & 0.00 & 34.22 & 14.97 & 572757.28 & 0.00 & 36262.47 & 273490.78 \\
\hline 7548.94 & 90.25 & 129.53 & 0.00 & 252.63 & 99.83 & 138.14 & 0.00 & 0.00 & 35.07 & 14.38 & 587116.67 & 0.00 & 30123.43 & 264211.95 \\
\hline 7549.07 & 107.38 & 141.27 & 0.00 & 245.68 & 103.19 & 134.28 & 0.00 & 0.00 & 42.35 & 0.00 & 670897.09 & 27966.79 & 24763.19 & 150708.91 \\
\hline 7549.13 & 88.89 & 153.54 & 0.00 & 252.16 & 95.13 & 133.23 & 0.00 & 0.00 & 27.48 & 0.00 & 620632.05 & 0.00 & 26056.17 & 191486.15 \\
\hline 7549.20 & 90.22 & 173.46 & 0.00 & 225.83 & 89.06 & 113.42 & 37.40 & 0.00 & 30.17 & 0.00 & 523160.43 & 0.00 & 37238.11 & 289553.67 \\
\hline 7549.26 & 108.50 & 148.26 & 0.00 & 237.21 & 90.51 & 123.30 & 0.00 & 0.00 & 39.79 & 16.56 & 526137.31 & 0.00 & 46653.41 & 298527.77 \\
\hline 7549.33 & 107.79 & 234.18 & 0.00 & 240.19 & 99.05 & 124.04 & 36.95 & 0.00 & 37.93 & 14.47 & 538201.32 & 0.00 & 46379.68 & 308423.42 \\
\hline 7549.39 & 101.80 & 164.09 & 0.00 & 240.19 & 93.90 & 125.32 & 36.21 & 22.08 & 36.48 & 17.39 & 517028.48 & 0.00 & 44714.00 & 305023.86 \\
\hline 7549.46 & 92.44 & 91.27 & 0.00 & 256.15 & 90.70 & 116.56 & 0.00 & 28.22 & 39.05 & 15.04 & 496570.93 & 0.00 & 43142.11 & 268135.53 \\
\hline 7549.52 & 89.63 & 112.59 & 0.00 & 246.15 & 93.12 & 123.06 & 33.52 & 0.00 & 31.53 & 15.78 & 564249.07 & 0.00 & 37269.96 & 280167.38 \\
\hline 7549.59 & 64.21 & 175.79 & 0.00 & 289.13 & 103.60 & 142.82 & 0.00 & 0.00 & 18.96 & 0.00 & 591113.58 & 0.00 & 23900.09 & 285769.28 \\
\hline 7549.66 & 53.66 & 121.78 & 0.00 & 238.12 & 91.97 & 115.32 & 0.00 & 0.00 & 18.92 & 0.00 & 594703.72 & 0.00 & 18656.60 & 291189.83 \\
\hline 7549.72 & 66.95 & 138.83 & 0.00 & 211.95 & 80.50 & 122.13 & 0.00 & 0.00 & 28.37 & 0.00 & 633909.66 & 0.00 & 18737.20 & 219482.26 \\
\hline 7549.79 & 84.26 & 153.73 & 0.00 & 236.08 & 89.40 & 126.26 & 0.00 & 0.00 & 28.29 & 0.00 & 630375.39 & 0.00 & 26211.34 & 233676.37 \\
\hline 7549.85 & 92.70 & 254.28 & 0.00 & 251.54 & 93.40 & 128.07 & 35.09 & 0.00 & 30.83 & 0.00 & 569714.91 & 0.00 & 32592.63 & 287971.48 \\
\hline 7549.92 & 87.07 & 167.66 & 0.00 & 240.14 & 98.13 & 123.52 & 0.00 & 0.00 & 33.37 & 16.49 & 584246.45 & 0.00 & 31857.50 & 259293.57 \\
\hline 7549.98 & 93.05 & 110.98 & 0.00 & 262.35 & 92.84 & 124.57 & 0.00 & 0.00 & 31.15 & 13.07 & 582075.86 & 0.00 & 35621.49 & 285917.50 \\
\hline 7550.05 & 94.75 & 198.34 & 0.00 & 246.08 & 84.78 & 120.56 & 38.10 & 34.38 & 29.57 & 12.20 & 558794.07 & 0.00 & 33793.35 & 273181.90 \\
\hline 7550.12 & 128.18 & 228.94 & 0.00 & 255.15 & 99.82 & 120.60 & 0.00 & 51.38 & 48.22 & 17.68 & 518583.74 & 0.00 & 36301.21 & 256230.02 \\
\hline 7550.18 & 94.34 & 200.60 & 0.00 & 234.75 & 77.93 & 122.08 & 0.00 & 32.35 & 32.99 & 14.82 & 589928.36 & 0.00 & 31564.38 & 231344.75 \\
\hline 7550.25 & 88.13 & 107.74 & 0.00 & 239.77 & 93.54 & 124.88 & 0.00 & 0.00 & 33.40 & 0.00 & 584425.77 & 0.00 & 33801.48 & 269589.78 \\
\hline 7550.31 & 66.10 & 118.02 & 0.00 & 255.29 & 103.56 & 136.32 & 0.00 & 0.00 & 24.90 & 0.00 & 602876.38 & 0.00 & 26474.08 & 283431.74 \\
\hline 7550.38 & 82.78 & 179.82 & 0.00 & 244.67 & 86.93 & 122.27 & 0.00 & 33.40 & 34.69 & 14.85 & 575314.28 & 0.00 & 27379.71 & 292452.28 \\
\hline 7550.44 & 55.88 & 83.55 & 0.00 & 287.91 & 95.21 & 127.61 & 0.00 & 98.56 & 24.00 & 0.00 & 625975.53 & 0.00 & 13966.78 & 243469.92 \\
\hline 7550.51 & 72.20 & 110.20 & 0.00 & 238.47 & 94.68 & 123.20 & 0.00 & 0.00 & 24.69 & 0.00 & 635945.57 & 0.00 & 20903.31 & 241993.61 \\
\hline 7550.57 & 121.90 & 283.56 & 0.00 & 289.60 & 89.14 & 122.54 & 0.00 & 125.72 & 44.30 & 19.72 & 543902.36 & 0.00 & 42399.58 & 254241.01 \\
\hline 7550.64 & 89.20 & 94.98 & 0.00 & 253.24 & 95.24 & 119.15 & 0.00 & 0.00 & 41.80 & 17.96 & 572513.39 & 0.00 & 31512.48 & 251122.65 \\
\hline 7550.71 & 94.08 & 151.98 & 0.00 & 245.06 & 95.13 & 122.75 & 0.00 & 0.00 & 27.34 & 0.00 & 623223.51 & 0.00 & 23566.12 & 212665.25 \\
\hline 7550.77 & 75.04 & 147.10 & 0.00 & 235.62 & 88.48 & 120.45 & 0.00 & 31.78 & 25.79 & 0.00 & 606057.37 & 0.00 & 24172.92 & 278310.87 \\
\hline 7550.84 & 78.91 & 157.46 & 0.00 & 284.93 & 96.77 & 133.40 & 0.00 & 0.00 & 28.90 & 0.00 & 647189.13 & 0.00 & 24787.34 & 213266.67 \\
\hline 7550.90 & 99.80 & 211.25 & 0.00 & 246.52 & 91.32 & 118.67 & 39.22 & 40.68 & 38.15 & 13.50 & 632491.65 & 0.00 & 26501.66 & 208202.90 \\
\hline 7550.97 & 130.32 & 266.73 & 0.00 & 348.11 & 82.43 & 114.59 & 54.23 & 340.36 & 43.86 & 17.72 & 570911.69 & 0.00 & 45989.90 & 240989.35 \\
\hline
\end{tabular}




\begin{tabular}{|c|c|c|c|c|c|c|c|c|c|c|c|c|c|c|}
\hline Depth & $\mathrm{Zr}$ & Mo & $\mathrm{Ag}$ & $\mathrm{Cd}$ & $\mathrm{Sn}$ & $\mathrm{Sb}$ & $\mathrm{Hf}$ & $\mathrm{W}$ & $\mathrm{Pb}$ & $\mathrm{Bi}$ & $\mathrm{LE}$ & $\mathrm{Mg}$ & $\mathrm{Al}$ & $\mathrm{Si}$ \\
\hline & ppm & $\mathrm{ppm}$ & ppm & $\mathrm{ppm}$ & $\mathrm{ppm}$ & $\mathrm{ppm}$ & $\mathrm{ppm}$ & $\mathrm{ppm}$ & $\mathrm{ppm}$ & ppm & $\mathrm{ppm}$ & $\mathrm{ppm}$ & $\mathrm{ppm}$ & ppm \\
\hline 7551.03 & 40.46 & 33.67 & 0.00 & 296.84 & 111.41 & 139.29 & 0.00 & 0.00 & 10.01 & 0.00 & 649602.75 & 0.00 & 7122.45 & 131282.25 \\
\hline 7551.10 & 32.28 & 0.00 & 0.00 & 277.05 & 105.83 & 147.47 & 0.00 & 0.00 & 0.00 & 0.00 & 647890.41 & 0.00 & 2503.34 & 36896.01 \\
\hline 7551.17 & 31.73 & 0.00 & 0.00 & 264.60 & 102.56 & 127.86 & 0.00 & 0.00 & 0.00 & 0.00 & 564458.91 & 0.00 & 5047.22 & 39376.45 \\
\hline 7551.23 & 28.77 & 0.00 & 0.00 & 264.19 & 95.86 & 122.46 & 0.00 & 0.00 & 0.00 & 0.00 & 559649.00 & 0.00 & 3875.12 & 35038.68 \\
\hline 7551.30 & 25.71 & 0.00 & 0.00 & 275.25 & 97.99 & 129.18 & 0.00 & 0.00 & 0.00 & 0.00 & 587803.81 & 0.00 & 0.00 & 26303.97 \\
\hline 7551.36 & 32.94 & 0.00 & 0.00 & 287.86 & 95.08 & 129.37 & 0.00 & 0.00 & 0.00 & 0.00 & 587971.20 & 0.00 & 2299.26 & 20204.10 \\
\hline 7551.43 & 29.21 & 0.00 & 0.00 & 233.61 & 99.66 & 121.04 & 0.00 & 0.00 & 0.00 & 0.00 & 586574.87 & 0.00 & 2254.48 & 20454.71 \\
\hline 7551.49 & 26.50 & 0.00 & 0.00 & 245.09 & 95.65 & 127.49 & 0.00 & 0.00 & 0.00 & 0.00 & 574132.69 & 0.00 & 3358.49 & 19743.23 \\
\hline 7551.56 & 33.74 & 0.00 & 0.00 & 279.43 & 101.42 & 124.86 & 0.00 & 0.00 & 0.00 & 0.00 & 576587.10 & 0.00 & 3247.55 & 19796.24 \\
\hline 7551.62 & 33.88 & 0.00 & 0.00 & 255.53 & 95.72 & 126.11 & 0.00 & 0.00 & 0.00 & 0.00 & 562523.55 & 0.00 & 3745.30 & 20147.75 \\
\hline 7551.69 & 31.56 & 0.00 & 0.00 & 246.17 & 91.45 & 126.50 & 0.00 & 0.00 & 0.00 & 0.00 & 578557.95 & 0.00 & 2981.87 & 17539.24 \\
\hline 7551.76 & 57.86 & 0.00 & 0.00 & 273.34 & 105.44 & 141.80 & 0.00 & 0.00 & 0.00 & 0.00 & 575424.95 & 0.00 & 1602.36 & 24276.25 \\
\hline 7551.82 & 52.60 & 0.00 & 0.00 & 266.99 & 99.02 & 126.22 & 0.00 & 0.00 & 0.00 & 0.00 & 582739.47 & 0.00 & 0.00 & 16849.31 \\
\hline 7551.89 & 37.71 & 0.00 & 0.00 & 275.48 & 95.38 & 125.20 & 0.00 & 0.00 & 0.00 & 0.00 & 590908.62 & 0.00 & 0.00 & 10405.74 \\
\hline 7551.95 & 27.94 & 0.00 & 0.00 & 276.02 & 99.64 & 124.51 & 0.00 & 0.00 & 0.00 & 0.00 & 595549.07 & 0.00 & 0.00 & 9626.87 \\
\hline 7552.02 & 33.26 & 0.00 & 0.00 & 262.00 & 107.28 & 131.66 & 0.00 & 0.00 & 0.00 & 0.00 & 563796.69 & 23048.04 & 7450.54 & 15733.45 \\
\hline 7552.08 & 51.85 & 0.00 & 0.00 & 265.08 & 97.25 & 120.18 & 0.00 & 0.00 & 0.00 & 0.00 & 590949.46 & 0.00 & 0.00 & 8212.47 \\
\hline 7552.15 & 62.94 & 0.00 & 0.00 & 246.17 & 88.24 & 133.14 & 0.00 & 0.00 & 9.16 & 0.00 & 576840.39 & 0.00 & 4247.64 & 29946.23 \\
\hline 7552.22 & 47.69 & 0.00 & 0.00 & 265.64 & 94.01 & 131.11 & 0.00 & 0.00 & 0.00 & 0.00 & 602616.48 & 0.00 & 1458.46 & 70346.06 \\
\hline 7552.28 & 55.67 & 0.00 & 0.00 & 251.92 & 91.99 & 128.27 & 0.00 & 0.00 & 9.12 & 0.00 & 520808.20 & 0.00 & 2825.53 & 74402.28 \\
\hline 7552.35 & 39.51 & 20.68 & 0.00 & 250.63 & 95.85 & 129.15 & 0.00 & 28.99 & 19.49 & 0.00 & 508689.35 & 0.00 & 4424.75 & 95214.11 \\
\hline 7552.41 & 115.18 & 158.50 & 0.00 & 240.44 & 92.23 & 131.04 & 0.00 & 0.00 & 52.93 & 20.94 & 644640.60 & 0.00 & 35830.88 & 172704.35 \\
\hline 7552.48 & 145.41 & 392.31 & 0.00 & 259.65 & 101.53 & 143.15 & 48.13 & 0.00 & 34.03 & 21.45 & 611248.70 & 0.00 & 42007.90 & 207016.60 \\
\hline 7552.54 & 119.48 & 194.10 & 0.00 & 225.26 & 88.39 & 122.02 & 48.69 & 23.10 & 51.66 & 18.44 & 563308.36 & 0.00 & 45632.45 & 247720.57 \\
\hline 7552.61 & 200.91 & 230.37 & 0.00 & 236.53 & 93.21 & 117.43 & 48.64 & 25.79 & 45.40 & 30.50 & 595374.41 & 0.00 & 48557.31 & 213511.69 \\
\hline 7552.67 & 122.94 & 167.68 & 0.00 & 232.28 & 93.52 & 131.33 & 0.00 & 0.00 & 54.24 & 21.70 & 597053.24 & 0.00 & 38440.21 & 215691.34 \\
\hline 7552.74 & 145.47 & 82.25 & 0.00 & 250.99 & 98.24 & 137.16 & 0.00 & 0.00 & 48.86 & 25.41 & 564480.66 & 0.00 & 69666.08 & 178511.10 \\
\hline 7552.81 & 408.69 & 0.00 & 0.00 & 244.33 & 102.75 & 135.27 & 53.16 & 0.00 & 27.89 & 43.84 & 653702.17 & 0.00 & 92231.67 & 172420.81 \\
\hline 7552.87 & 515.56 & 0.00 & 0.00 & 279.35 & 111.13 & 144.75 & 56.37 & 0.00 & 35.54 & 36.06 & 548781.23 & 0.00 & 125259.99 & 219442.68 \\
\hline 7552.94 & 88.82 & 116.25 & 0.00 & 255.91 & 103.68 & 126.45 & 0.00 & 0.00 & 27.02 & 0.00 & 645203.99 & 0.00 & 24534.18 & 253689.01 \\
\hline 7553.07 & 92.26 & 140.40 & 0.00 & 254.41 & 94.39 & 133.15 & 0.00 & 0.00 & 35.94 & 15.26 & 548409.49 & 0.00 & 38732.54 & 304052.89 \\
\hline 7553.13 & 108.82 & 149.94 & 0.00 & 248.46 & 94.33 & 130.69 & 0.00 & 25.89 & 43.31 & 19.30 & 524795.19 & 0.00 & 47499.04 & 307392.44 \\
\hline
\end{tabular}




\begin{tabular}{|c|c|c|c|c|c|c|c|c|c|c|c|c|c|c|}
\hline Depth & $\mathrm{Zr}$ & Mo & $\mathrm{Ag}$ & $\mathrm{Cd}$ & Sn & $\mathrm{Sb}$ & $\mathrm{Hf}$ & $\mathrm{W}$ & $\mathrm{Pb}$ & $\mathrm{Bi}$ & LE & $\mathrm{Mg}$ & $\mathrm{Al}$ & $\mathrm{Si}$ \\
\hline & ppm & ppm & $\mathrm{ppm}$ & ppm & $\mathrm{ppm}$ & ppm & ppm & ppm & $\mathrm{ppm}$ & ppm & ppm & ppm & ppm & ppm \\
\hline 7553.20 & 93.63 & 156.84 & 0.00 & 220.40 & 87.57 & 110.67 & 0.00 & 0.00 & 33.14 & 13.30 & 568884.25 & 0.00 & 33013.19 & 267880.28 \\
\hline 7553.26 & 80.09 & 72.20 & 0.00 & 242.43 & 93.15 & 125.18 & 0.00 & 0.00 & 34.96 & 13.36 & 605747.98 & 0.00 & 34708.93 & 282309.01 \\
\hline 7553.33 & 111.77 & 200.24 & 0.00 & 235.48 & 93.61 & 121.30 & 36.56 & 23.73 & 34.76 & 19.94 & 563680.35 & 0.00 & 44641.90 & 273632.34 \\
\hline 7553.39 & 136.96 & 260.36 & 0.00 & 244.56 & 92.61 & 123.44 & 50.40 & 0.00 & 44.89 & 17.94 & 514880.66 & 0.00 & 55340.46 & 301584.40 \\
\hline 7553.46 & 98.72 & 84.53 & 0.00 & 234.57 & 93.13 & 119.23 & 0.00 & 21.02 & 47.81 & 17.95 & 614555.32 & 0.00 & 33576.40 & 192653.33 \\
\hline 7553.52 & 117.43 & 195.74 & 0.00 & 238.51 & 93.46 & 121.82 & 40.34 & 0.00 & 35.77 & 19.51 & 627799.79 & 0.00 & 35772.17 & 209348.08 \\
\hline 7553.59 & 135.87 & 137.84 & 0.00 & 244.03 & 91.48 & 115.65 & 56.03 & 23.43 & 50.39 & 19.63 & 624596.91 & 0.00 & 46829.57 & 210272.00 \\
\hline 7553.66 & 109.96 & 0.00 & 0.00 & 264.86 & 108.24 & 143.33 & 43.04 & 0.00 & 36.72 & 20.41 & 644347.37 & 0.00 & 86690.66 & 185474.11 \\
\hline 7553.72 & 101.86 & 63.00 & 0.00 & 285.18 & 104.00 & 133.04 & 33.69 & 0.00 & 32.31 & 18.89 & 617661.80 & 0.00 & 45227.09 & 261699.09 \\
\hline 7553.79 & 104.41 & 138.10 & 0.00 & 241.24 & 94.33 & 123.12 & 42.16 & 26.76 & 30.57 & 14.16 & 571941.60 & 0.00 & 41516.85 & 302584.87 \\
\hline 7553.85 & 117.38 & 160.32 & 0.00 & 228.89 & 89.47 & 118.48 & 39.11 & 0.00 & 42.65 & 17.68 & 567360.38 & 0.00 & 49101.55 & 262976.56 \\
\hline 7553.92 & 83.72 & 56.88 & 0.00 & 267.54 & 95.01 & 125.22 & 0.00 & 24.53 & 25.85 & 0.00 & 586149.48 & 0.00 & 28818.61 & 290204.50 \\
\hline 7553.98 & 107.52 & 74.79 & 0.00 & 243.46 & 98.56 & 126.82 & 35.28 & 0.00 & 38.32 & 17.52 & 601741.29 & 0.00 & 41280.58 & 253881.04 \\
\hline 7554.05 & 99.75 & 141.71 & 0.00 & 248.63 & 95.79 & 132.77 & 0.00 & 0.00 & 31.31 & 16.05 & 594108.82 & 0.00 & 38401.90 & 195176.78 \\
\hline 7554.12 & 117.29 & 99.69 & 0.00 & 257.86 & 93.95 & 129.95 & 41.81 & 25.22 & 44.62 & 17.23 & 551195.84 & 0.00 & 48153.35 & 240397.68 \\
\hline 7554.18 & 47.46 & 0.00 & 0.00 & 281.17 & 95.23 & 131.15 & 0.00 & 0.00 & 7.95 & 0.00 & 567153.76 & 0.00 & 3290.49 & 21332.66 \\
\hline 7554.25 & 53.67 & 33.04 & 0.00 & 257.07 & 104.20 & 129.86 & 0.00 & 29.92 & 9.34 & 0.00 & 579033.35 & 0.00 & 5762.26 & 52939.98 \\
\hline 7554.31 & 52.47 & 45.65 & 0.00 & 259.05 & 93.17 & 125.03 & 0.00 & 0.00 & 0.00 & 0.00 & 572140.47 & 0.00 & 5224.04 & 118756.29 \\
\hline 7554.38 & 39.93 & 0.00 & 0.00 & 241.20 & 90.20 & 126.03 & 0.00 & 0.00 & 0.00 & 0.00 & 558384.63 & 0.00 & 2064.35 & 93140.51 \\
\hline 7554.44 & 51.53 & 44.89 & 0.00 & 246.47 & 96.66 & 123.83 & 0.00 & 0.00 & 8.40 & 0.00 & 582353.97 & 0.00 & 5801.17 & 114495.95 \\
\hline 7554.51 & 156.30 & 119.92 & 0.00 & 236.84 & 89.86 & 121.98 & 0.00 & 33.86 & 52.46 & 24.82 & 575486.45 & 0.00 & 49457.67 & 171972.63 \\
\hline 7554.57 & 55.55 & 24.06 & 0.00 & 260.67 & 94.36 & 118.84 & 0.00 & 0.00 & 7.97 & 0.00 & 566449.05 & 0.00 & 4822.93 & 64080.54 \\
\hline 7554.64 & 151.88 & 199.63 & 0.00 & 267.25 & 98.70 & 123.71 & 0.00 & 77.23 & 30.52 & 13.78 & 598898.95 & 0.00 & 28378.52 & 140725.97 \\
\hline 7554.71 & 164.52 & 518.60 & 0.00 & 255.83 & 92.60 & 121.87 & 0.00 & 73.88 & 39.35 & 16.00 & 645584.64 & 0.00 & 27620.99 & 125987.77 \\
\hline 7554.77 & 175.54 & 349.19 & 0.00 & 297.60 & 93.71 & 125.75 & 57.11 & 144.48 & 52.84 & 19.37 & 657796.48 & 0.00 & 41418.10 & 134763.42 \\
\hline 7554.84 & 44.25 & 27.00 & 0.00 & 348.67 & 91.45 & 122.21 & 0.00 & 212.88 & 7.69 & 0.00 & 529280.85 & 0.00 & 7502.75 & 106682.32 \\
\hline 7554.90 & 31.47 & 25.57 & 0.00 & 262.09 & 103.51 & 119.45 & 0.00 & 0.00 & 8.12 & 0.00 & 566695.67 & 0.00 & 1683.78 & 104361.44 \\
\hline 7554.97 & 36.78 & 23.94 & 0.00 & 253.92 & 98.13 & 124.04 & 0.00 & 0.00 & 0.00 & 0.00 & 582618.24 & 0.00 & 1940.59 & 91654.15 \\
\hline
\end{tabular}




\begin{tabular}{|c|c|c|c|c|c|}
\hline Depth & $\mathrm{P}$ & S & $\mathrm{Cl}$ & K & $\mathrm{Ca}$ \\
\hline & ppm & ppm & $\mathrm{ppm}$ & ppm & $\mathrm{ppm}$ \\
\hline 7517.07 & 0.00 & 37401.19 & 0.00 & 7854.59 & 44657.53 \\
\hline 7517.13 & 0.00 & 24566.31 & 0.00 & 5461.12 & 74697.90 \\
\hline 7517.20 & 0.00 & 61434.06 & 1601.89 & 0.00 & 64262.97 \\
\hline 7517.26 & 0.00 & 44557.35 & 3080.00 & 0.00 & 59105.32 \\
\hline 7517.33 & 0.00 & 25631.18 & 0.00 & 9252.64 & 48921.81 \\
\hline 7517.39 & 0.00 & 3627.91 & 0.00 & 0.00 & 344985.76 \\
\hline 7517.46 & 0.00 & 3131.50 & 0.00 & 0.00 & 382689.41 \\
\hline 7517.52 & 0.00 & 2025.61 & 0.00 & 0.00 & 365039.34 \\
\hline 7517.59 & 0.00 & 5247.14 & 889.16 & 0.00 & 302507.30 \\
\hline 7517.66 & 0.00 & 11783.01 & 100.40 & 0.00 & 254788.40 \\
\hline 7517.72 & 0.00 & 20855.76 & 0.00 & 0.00 & 411534.61 \\
\hline 7517.79 & 0.00 & 21496.89 & 885.85 & 0.00 & 409064.42 \\
\hline 7517.85 & 0.00 & 3010.07 & 0.00 & 0.00 & 385298.51 \\
\hline 7517.92 & 0.00 & 4068.44 & 701.82 & 0.00 & 336722.36 \\
\hline 7517.98 & 0.00 & 2733.02 & 355.93 & 0.00 & 359638.35 \\
\hline 7518.05 & 0.00 & 2046.64 & 1449.35 & 0.00 & 355249.66 \\
\hline 7518.12 & 0.00 & 2257.89 & 0.00 & 0.00 & 379470.08 \\
\hline 7518.18 & 0.00 & 3159.62 & 1227.37 & 0.00 & 358984.80 \\
\hline 7518.25 & 0.00 & 2593.11 & 0.00 & 0.00 & 334927.28 \\
\hline 7518.31 & 0.00 & 2488.69 & 0.00 & 0.00 & 336094.64 \\
\hline 7518.38 & 0.00 & 2237.98 & 119.67 & 0.00 & 330607.97 \\
\hline 7518.44 & 0.00 & 2874.81 & 0.00 & 0.00 & 325960.20 \\
\hline 7518.51 & 0.00 & 2584.86 & 48.51 & 0.00 & 312682.11 \\
\hline 7518.57 & 0.00 & 3326.31 & 0.00 & 0.00 & 364972.47 \\
\hline 7518.64 & 0.00 & 3781.09 & 0.00 & 0.00 & 360264.77 \\
\hline 7518.71 & 0.00 & 3025.13 & 0.00 & 0.00 & 356101.43 \\
\hline 7518.77 & 0.00 & 6043.52 & 177.17 & 0.00 & 332903.76 \\
\hline 7518.84 & 0.00 & 6468.64 & 0.00 & 0.00 & 303283.19 \\
\hline 7518.90 & 0.00 & 13928.65 & 144.94 & 0.00 & 267943.10 \\
\hline 7518.97 & 0.00 & 30337.11 & 0.00 & 11413.75 & 85819.66 \\
\hline 7519.03 & 0.00 & 34808.77 & 0.00 & 8108.18 & 55085.39 \\
\hline 7519.10 & 0.00 & 61858.17 & 0.00 & 12311.11 & 50426.64 \\
\hline
\end{tabular}




\begin{tabular}{|c|c|c|c|c|c|}
\hline Depth & $\mathrm{P}$ & S & $\mathrm{Cl}$ & K & $\mathrm{Ca}$ \\
\hline & ppm & ppm & $\mathrm{ppm}$ & ppm & ppm \\
\hline 7519.17 & 0.00 & 36158.24 & 3025.18 & 0.00 & 74699.45 \\
\hline 7519.23 & 0.00 & 27465.00 & 67.71 & 0.00 & 74215.22 \\
\hline 7519.30 & 0.00 & 67375.91 & 0.00 & 0.00 & 120873.15 \\
\hline 7519.36 & 0.00 & 16018.54 & 0.00 & 0.00 & 159794.52 \\
\hline 7519.43 & 0.00 & 22161.55 & 0.00 & 7113.50 & 89156.16 \\
\hline 7519.49 & 0.00 & 18352.32 & 0.00 & 3875.23 & 155066.81 \\
\hline 7519.56 & 0.00 & 11012.51 & 0.00 & 0.00 & 275215.12 \\
\hline 7519.62 & 0.00 & 8259.01 & 0.00 & 0.00 & 280044.46 \\
\hline 7519.69 & 488.02 & 10370.35 & 0.00 & 0.00 & 258698.59 \\
\hline 7519.76 & 302.08 & 13996.42 & 0.00 & 0.00 & 199932.08 \\
\hline 7519.82 & 0.00 & 20333.96 & 0.00 & 4443.43 & 101893.20 \\
\hline 7519.89 & 0.00 & 28650.05 & 0.00 & 4325.10 & 30058.71 \\
\hline 7519.95 & 0.00 & 31677.75 & 0.00 & 0.00 & 17951.56 \\
\hline 7520.02 & 0.00 & 23553.69 & 0.00 & 0.00 & 21175.33 \\
\hline 7520.08 & 0.00 & 25597.20 & 0.00 & 6755.23 & 28747.11 \\
\hline 7520.15 & 0.00 & 26122.96 & 0.00 & 9639.75 & 45150.76 \\
\hline 7520.22 & 0.00 & 24792.23 & 0.00 & 11993.59 & 19531.39 \\
\hline 7520.28 & 0.00 & 25309.63 & 0.00 & 17526.55 & 8834.72 \\
\hline 7520.35 & 0.00 & 32388.62 & 0.00 & 11578.80 & 11981.02 \\
\hline 7520.41 & 0.00 & 42136.61 & 0.00 & 15930.05 & 10661.08 \\
\hline 7520.48 & 353.43 & 43454.85 & 0.00 & 8455.14 & 16123.23 \\
\hline 7520.54 & 271.89 & 34898.54 & 0.00 & 13605.81 & 12990.78 \\
\hline 7520.61 & 405.79 & 25246.56 & 0.00 & 18458.79 & 10182.29 \\
\hline 7520.67 & 0.00 & 34976.18 & 0.00 & 17406.66 & 8529.59 \\
\hline 7520.74 & 0.00 & 22630.33 & 0.00 & 16117.30 & 5857.13 \\
\hline 7520.81 & 0.00 & 41740.86 & 0.00 & 16250.79 & 14204.01 \\
\hline 7520.87 & 0.00 & 45210.88 & 0.00 & 15218.54 & 22507.38 \\
\hline 7520.94 & 0.00 & 32418.00 & 0.00 & 18832.64 & 18737.69 \\
\hline 7521.00 & 297.07 & 25543.11 & 0.00 & 17058.92 & 13408.53 \\
\hline 7521.07 & 189.72 & 25644.89 & 0.00 & 14098.33 & 11788.17 \\
\hline 7521.13 & 237.20 & 33959.29 & 0.00 & 12880.48 & 11986.57 \\
\hline 7521.20 & 0.00 & 41873.46 & 0.00 & 13261.62 & 13488.51 \\
\hline
\end{tabular}




\begin{tabular}{|c|c|c|c|c|c|}
\hline Depth & $\mathrm{P}$ & S & $\mathrm{Cl}$ & K & $\mathrm{Ca}$ \\
\hline & ppm & ppm & $\mathrm{ppm}$ & ppm & ppm \\
\hline 7521.26 & 0.00 & 23641.28 & 0.00 & 19427.83 & 13517.38 \\
\hline 7521.33 & 213.15 & 64629.75 & 0.00 & 10798.18 & 17084.23 \\
\hline 7521.39 & 0.00 & 23883.80 & 0.00 & 18796.32 & 17573.75 \\
\hline 7521.46 & 222.69 & 27221.91 & 387.25 & 12560.67 & 23755.70 \\
\hline 7521.52 & 200.44 & 47045.51 & 0.00 & 10801.32 & 29614.92 \\
\hline 7521.59 & 0.00 & 40673.16 & 0.00 & 15675.02 & 31518.85 \\
\hline 7521.66 & 325.58 & 28343.51 & 0.00 & 16729.44 & 21826.59 \\
\hline 7521.72 & 199.00 & 31109.43 & 0.00 & 16088.30 & 16978.35 \\
\hline 7521.79 & 0.00 & 48545.37 & 0.00 & 15056.22 & 15137.22 \\
\hline 7521.85 & 0.00 & 46749.22 & 0.00 & 7304.12 & 25539.58 \\
\hline 7521.92 & 0.00 & 34089.79 & 0.00 & 10724.56 & 21966.25 \\
\hline 7521.98 & 0.00 & 35592.53 & 0.00 & 13007.94 & 32783.80 \\
\hline 7522.05 & 0.00 & 22784.54 & 0.00 & 15023.89 & 21547.29 \\
\hline 7522.12 & 0.00 & 31201.94 & 0.00 & 15737.69 & 26112.39 \\
\hline 7522.18 & 0.00 & 32509.47 & 0.00 & 15715.74 & 33965.97 \\
\hline 7522.25 & 0.00 & 29583.80 & 0.00 & 12879.84 & 51887.81 \\
\hline 7522.31 & 234.15 & 43936.40 & 0.00 & 8347.85 & 55242.13 \\
\hline 7522.38 & 0.00 & 7557.88 & 0.00 & 0.00 & 280987.18 \\
\hline 7522.44 & 0.00 & 26057.08 & 0.00 & 5128.66 & 142745.90 \\
\hline 7522.51 & 0.00 & 18776.41 & 0.00 & 6618.07 & 130461.53 \\
\hline 7522.57 & 0.00 & 15434.85 & 0.00 & 11863.39 & 76161.61 \\
\hline 7522.64 & 0.00 & 26380.83 & 0.00 & 7459.05 & 82384.14 \\
\hline 7522.71 & 0.00 & 28258.39 & 0.00 & 10806.08 & 64203.56 \\
\hline 7522.77 & 0.00 & 22788.11 & 0.00 & 16572.79 & 43003.98 \\
\hline 7522.84 & 0.00 & 23930.33 & 0.00 & 18038.07 & 43429.49 \\
\hline 7522.90 & 0.00 & 23233.92 & 0.00 & 19425.62 & 33121.22 \\
\hline 7522.97 & 0.00 & 18120.82 & 0.00 & 17670.69 & 42049.71 \\
\hline 7523.03 & 0.00 & 20078.30 & 0.00 & 14235.88 & 40371.51 \\
\hline 7523.10 & 0.00 & 18155.93 & 0.00 & 15596.55 & 47106.70 \\
\hline 7523.17 & 0.00 & 15515.77 & 0.00 & 10859.00 & 57364.53 \\
\hline 7523.23 & 0.00 & 9347.33 & 0.00 & 10840.48 & 74513.11 \\
\hline 7523.30 & 0.00 & 22604.66 & 0.00 & 9189.58 & 99378.40 \\
\hline
\end{tabular}




\begin{tabular}{|c|c|c|c|c|c|}
\hline Depth & $\mathrm{P}$ & $S$ & $\mathrm{Cl}$ & K & $\mathrm{Ca}$ \\
\hline & ppm & $\mathrm{ppm}$ & $\mathrm{ppm}$ & ppm & $\mathrm{ppm}$ \\
\hline 7523.36 & 0.00 & 14215.11 & 0.00 & 16918.91 & 59010.83 \\
\hline 7523.43 & 0.00 & 9119.13 & 0.00 & 18241.22 & 49464.33 \\
\hline 7523.49 & 0.00 & 8668.63 & 0.00 & 21421.27 & 38785.85 \\
\hline 7523.56 & 0.00 & 6048.34 & 0.00 & 16777.80 & 73565.77 \\
\hline 7523.62 & 0.00 & 6161.65 & 0.00 & 2740.39 & 157657.27 \\
\hline 7523.69 & 0.00 & 4252.73 & 0.00 & 0.00 & 281243.14 \\
\hline 7523.76 & 0.00 & 4855.25 & 0.00 & 0.00 & 319277.98 \\
\hline 7523.82 & 0.00 & 7896.39 & 0.00 & 0.00 & 350125.43 \\
\hline 7523.89 & 0.00 & 12644.75 & 108.92 & 0.00 & 358802.41 \\
\hline 7523.95 & 0.00 & 12026.45 & 32.07 & 0.00 & 345878.77 \\
\hline 7524.02 & 0.00 & 2603.42 & 333.60 & 0.00 & 321574.68 \\
\hline 7524.08 & 341.64 & 3112.25 & 0.00 & 0.00 & 350609.32 \\
\hline 7524.15 & 0.00 & 3051.86 & 109.43 & 0.00 & 272140.04 \\
\hline 7524.22 & 342.56 & 4550.98 & 0.00 & 0.00 & 240323.24 \\
\hline 7524.28 & 417.83 & 8905.39 & 0.00 & 6425.98 & 130634.09 \\
\hline 7524.35 & 530.60 & 6949.16 & 0.00 & 0.00 & 159201.36 \\
\hline 7524.41 & 355.93 & 9432.99 & 0.00 & 9239.83 & 103594.22 \\
\hline 7524.48 & 225.56 & 6908.42 & 76.67 & 17031.99 & 66324.86 \\
\hline 7524.54 & 0.00 & 7151.93 & 0.00 & 18650.76 & 70324.32 \\
\hline 7524.61 & 0.00 & 7186.61 & 0.00 & 14891.63 & 74165.47 \\
\hline 7524.67 & 291.65 & 16059.88 & 4551.50 & 0.00 & 108868.20 \\
\hline 7524.74 & 292.36 & 7461.95 & 3186.19 & 0.00 & 171144.10 \\
\hline 7524.81 & 0.00 & 6950.91 & 0.00 & 0.00 & 169341.42 \\
\hline 7524.87 & 0.00 & 6190.29 & 0.00 & 9906.16 & 120760.99 \\
\hline 7524.94 & 214.88 & 5708.68 & 0.00 & 15596.48 & 64939.65 \\
\hline 7525.00 & 0.00 & 15942.16 & 0.00 & 11609.39 & 51947.84 \\
\hline 7525.07 & 0.00 & 7497.19 & 0.00 & 18539.75 & 62857.45 \\
\hline 7525.13 & 0.00 & 8314.16 & 0.00 & 16350.86 & 58726.94 \\
\hline 7525.20 & 0.00 & 7597.19 & 0.00 & 16289.52 & 60065.66 \\
\hline 7525.26 & 0.00 & 11091.15 & 0.00 & 15223.34 & 66787.65 \\
\hline 7525.33 & 478.62 & 9705.12 & 0.00 & 3167.04 & 141548.50 \\
\hline 7525.39 & 1041.17 & 12424.06 & 0.00 & 0.00 & 138092.64 \\
\hline
\end{tabular}




\begin{tabular}{|c|c|c|c|c|c|}
\hline Depth & $\mathrm{P}$ & S & $\mathrm{Cl}$ & K & $\mathrm{Ca}$ \\
\hline & ppm & ppm & $\mathrm{ppm}$ & ppm & ppm \\
\hline 7525.46 & 2116.90 & 28640.09 & 0.00 & 0.00 & 172393.51 \\
\hline 7525.52 & 0.00 & 14700.76 & 0.00 & 0.00 & 233707.45 \\
\hline 7525.59 & 227.89 & 29712.12 & 0.00 & 15071.31 & 62153.60 \\
\hline 7525.66 & 613.08 & 16524.46 & 0.00 & 16904.57 & 66166.94 \\
\hline 7525.72 & 2930.50 & 17552.69 & 0.00 & 0.00 & 185872.72 \\
\hline 7525.79 & 267.96 & 50736.96 & 0.00 & 11121.61 & 62274.80 \\
\hline 7525.85 & 0.00 & 58653.81 & 0.00 & 10002.29 & 42500.61 \\
\hline 7525.92 & 0.00 & 80862.19 & 0.00 & 0.00 & 36733.63 \\
\hline 7525.98 & 0.00 & 67233.66 & 0.00 & 6249.14 & 22106.70 \\
\hline 7526.05 & 0.00 & 29936.59 & 0.00 & 6371.26 & 17805.71 \\
\hline 7526.12 & 0.00 & 37840.91 & 4663.14 & 4889.41 & 19675.41 \\
\hline 7526.18 & 0.00 & 50197.10 & 0.00 & 0.00 & 20591.78 \\
\hline 7526.25 & 0.00 & 46682.67 & 0.00 & 0.00 & 22128.19 \\
\hline 7526.31 & 0.00 & 44710.22 & 2789.50 & 0.00 & 21748.87 \\
\hline 7526.38 & 0.00 & 43162.61 & 1790.10 & 0.00 & 46063.29 \\
\hline 7526.44 & 0.00 & 53922.65 & 4084.31 & 0.00 & 31273.40 \\
\hline 7526.51 & 0.00 & 44661.09 & 0.00 & 0.00 & 30973.59 \\
\hline 7526.57 & 0.00 & 39085.23 & 0.00 & 0.00 & 27344.86 \\
\hline 7526.64 & 0.00 & 47937.61 & 0.00 & 3396.71 & 31587.88 \\
\hline 7526.71 & 0.00 & 42789.50 & 0.00 & 0.00 & 41354.25 \\
\hline 7526.77 & 0.00 & 45324.11 & 0.00 & 0.00 & 33435.53 \\
\hline 7526.84 & 0.00 & 39659.68 & 0.00 & 0.00 & 31547.49 \\
\hline 7526.90 & 0.00 & 43826.56 & 0.00 & 0.00 & 24828.59 \\
\hline 7527.07 & 0.00 & 35147.09 & 0.00 & 0.00 & 39815.58 \\
\hline 7527.13 & 0.00 & 31201.49 & 0.00 & 0.00 & 19141.53 \\
\hline 7527.20 & 0.00 & 31208.43 & 0.00 & 3350.76 & 21356.72 \\
\hline 7527.26 & 0.00 & 39940.04 & 0.00 & 7814.45 & 23906.18 \\
\hline 7527.33 & 0.00 & 33788.19 & 0.00 & 3860.44 & 29815.17 \\
\hline 7527.39 & 0.00 & 32960.34 & 0.00 & 5668.03 & 35863.01 \\
\hline 7527.46 & 0.00 & 32554.09 & 0.00 & 10475.94 & 44061.36 \\
\hline 7527.52 & 0.00 & 39045.94 & 0.00 & 10995.47 & 29232.84 \\
\hline 7527.59 & 0.00 & 27480.19 & 0.00 & 0.00 & 102210.63 \\
\hline
\end{tabular}




\begin{tabular}{|c|c|c|c|c|c|}
\hline Depth & $\mathrm{P}$ & S & $\mathrm{Cl}$ & K & $\mathrm{Ca}$ \\
\hline & ppm & $\mathrm{ppm}$ & $\mathrm{ppm}$ & ppm & ppm \\
\hline 7527.66 & 0.00 & 16876.89 & 0.00 & 0.00 & 135411.17 \\
\hline 7527.72 & 0.00 & 28143.38 & 0.00 & 0.00 & 67606.91 \\
\hline 7527.79 & 0.00 & 24378.78 & 0.00 & 0.00 & 128799.18 \\
\hline 7527.85 & 0.00 & 36230.05 & 85.42 & 0.00 & 67845.47 \\
\hline 7527.92 & 0.00 & 35098.30 & 0.00 & 0.00 & 76630.26 \\
\hline 7527.98 & 0.00 & 34653.77 & 0.00 & 5150.61 & 52444.98 \\
\hline 7528.05 & 0.00 & 36321.19 & 0.00 & 8474.27 & 34121.85 \\
\hline 7528.12 & 0.00 & 30754.52 & 0.00 & 10387.25 & 33975.44 \\
\hline 7528.18 & 0.00 & 33429.20 & 0.00 & 9050.32 & 31640.85 \\
\hline 7528.25 & 0.00 & 59246.66 & 0.00 & 8194.31 & 15330.25 \\
\hline 7528.31 & 0.00 & 60306.86 & 0.00 & 11335.87 & 19749.13 \\
\hline 7528.38 & 0.00 & 63540.06 & 0.00 & 8013.59 & 19494.29 \\
\hline 7528.44 & 0.00 & 51558.72 & 0.00 & 11061.35 & 16540.09 \\
\hline 7528.51 & 0.00 & 44774.60 & 0.00 & 15050.23 & 19803.14 \\
\hline 7528.57 & 0.00 & 52211.11 & 5170.48 & 0.00 & 29343.45 \\
\hline 7528.64 & 0.00 & 47443.36 & 479.66 & 12252.69 & 34552.12 \\
\hline 7528.71 & 0.00 & 48398.02 & 334.61 & 12510.91 & 42210.52 \\
\hline 7528.77 & 0.00 & 33210.79 & 96.59 & 17331.76 & 35162.63 \\
\hline 7528.84 & 0.00 & 49093.16 & 0.00 & 14622.67 & 38158.20 \\
\hline 7528.90 & 0.00 & 34250.94 & 1386.65 & 13868.76 & 32949.86 \\
\hline 7528.97 & 0.00 & 30425.85 & 0.00 & 19770.68 & 20618.82 \\
\hline 7529.03 & 0.00 & 35924.71 & 1135.36 & 11840.11 & 24326.90 \\
\hline 7529.10 & 0.00 & 43273.52 & 1934.84 & 11650.83 & 36423.07 \\
\hline 7529.17 & 0.00 & 68186.22 & 6098.40 & 0.00 & 38084.17 \\
\hline 7529.23 & 0.00 & 41901.79 & 0.00 & 11204.89 & 16684.35 \\
\hline 7529.30 & 0.00 & 49958.53 & 1029.13 & 10283.50 & 27065.00 \\
\hline 7529.36 & 0.00 & 36954.67 & 0.00 & 14588.70 & 19171.48 \\
\hline 7529.43 & 0.00 & 83073.07 & 0.00 & 11835.27 & 10098.45 \\
\hline 7529.49 & 0.00 & 106077.62 & 0.00 & 41765.72 & 1268.58 \\
\hline 7529.56 & 0.00 & 42494.24 & 0.00 & 7404.88 & 20134.33 \\
\hline 7529.62 & 0.00 & 80430.03 & 0.00 & 0.00 & 44180.11 \\
\hline 7529.69 & 0.00 & 27941.62 & 0.00 & 0.00 & 79419.55 \\
\hline
\end{tabular}




\begin{tabular}{|c|c|c|c|c|c|}
\hline Depth & $\mathrm{P}$ & $S$ & $\mathrm{Cl}$ & K & $\mathrm{Ca}$ \\
\hline & ppm & $\mathrm{ppm}$ & $\mathrm{ppm}$ & ppm & $\mathrm{ppm}$ \\
\hline 7529.76 & 0.00 & 45677.39 & 0.00 & 10437.06 & 25222.10 \\
\hline 7529.82 & 0.00 & 31434.02 & 0.00 & 7504.90 & 22007.49 \\
\hline 7529.89 & 0.00 & 35335.74 & 0.00 & 13215.43 & 16745.33 \\
\hline 7529.95 & 0.00 & 42387.41 & 1318.40 & 11104.27 & 16195.44 \\
\hline 7530.02 & 0.00 & 37634.95 & 85.39 & 16498.35 & 16552.31 \\
\hline 7530.08 & 0.00 & 74396.91 & 1234.70 & 7454.22 & 9968.52 \\
\hline 7530.15 & 0.00 & 136434.66 & 0.00 & 0.00 & 11865.66 \\
\hline 7530.22 & 0.00 & 47277.79 & 2637.70 & 0.00 & 17481.51 \\
\hline 7530.28 & 0.00 & 61935.95 & 119.19 & 5908.28 & 19145.03 \\
\hline 7530.35 & 0.00 & 34308.83 & 189.74 & 3583.93 & 32552.46 \\
\hline 7530.41 & 0.00 & 39811.11 & 0.00 & 0.00 & 49172.07 \\
\hline 7530.48 & 0.00 & 31921.23 & 1024.66 & 6785.14 & 48983.78 \\
\hline 7530.54 & 0.00 & 37607.37 & 254.20 & 7292.38 & 82438.82 \\
\hline 7530.61 & 0.00 & 37784.78 & 0.00 & 0.00 & 27668.60 \\
\hline 7530.67 & 0.00 & 38557.53 & 1343.62 & 0.00 & 21284.63 \\
\hline 7530.74 & 0.00 & 111582.13 & 816.60 & 0.00 & 14262.46 \\
\hline 7530.81 & 0.00 & 39534.97 & 429.54 & 11704.71 & 20035.53 \\
\hline 7530.87 & 0.00 & 32161.13 & 2027.63 & 0.00 & 27237.20 \\
\hline 7530.94 & 0.00 & 34478.57 & 671.62 & 0.00 & 42984.77 \\
\hline 7531.00 & 0.00 & 33338.80 & 0.00 & 5009.98 & 37315.02 \\
\hline 7531.07 & 0.00 & 35882.13 & 3583.52 & 0.00 & 42679.18 \\
\hline 7531.13 & 0.00 & 42643.92 & 655.63 & 0.00 & 47683.95 \\
\hline 7531.20 & 0.00 & 38340.89 & 1374.30 & 3606.92 & 109773.16 \\
\hline 7531.26 & 725.19 & 80627.49 & 1489.86 & 3640.58 & 144458.01 \\
\hline 7531.33 & 595.87 & 13421.97 & 1279.06 & 0.00 & 179928.25 \\
\hline 7531.39 & 528.00 & 24775.41 & 1383.69 & 0.00 & 171223.78 \\
\hline 7531.46 & 0.00 & 13708.28 & 1716.36 & 0.00 & 219285.48 \\
\hline 7531.52 & 0.00 & 16593.26 & 2177.04 & 0.00 & 187066.97 \\
\hline 7531.59 & 0.00 & 33571.76 & 2622.50 & 0.00 & 98121.46 \\
\hline 7531.66 & 0.00 & 34294.85 & 563.93 & 0.00 & 81891.41 \\
\hline 7531.72 & 0.00 & 34172.82 & 1823.91 & 0.00 & 62014.02 \\
\hline 7531.79 & 0.00 & 32622.42 & 2758.80 & 0.00 & 61457.30 \\
\hline
\end{tabular}




\begin{tabular}{|c|c|c|c|c|c|}
\hline Depth & $\mathrm{P}$ & S & $\mathrm{Cl}$ & K & $\mathrm{Ca}$ \\
\hline & ppm & $\mathrm{ppm}$ & $\mathrm{ppm}$ & ppm & $\mathrm{ppm}$ \\
\hline 7531.85 & 0.00 & 36471.82 & 0.00 & 0.00 & 48931.31 \\
\hline 7531.92 & 0.00 & 119519.61 & 0.00 & 0.00 & 34212.05 \\
\hline 7531.98 & 0.00 & 24731.59 & 74.15 & 0.00 & 45679.36 \\
\hline 7532.05 & 0.00 & 28212.12 & 264.59 & 4339.17 & 55892.59 \\
\hline 7532.12 & 0.00 & 29475.92 & 0.00 & 0.00 & 68311.05 \\
\hline 7532.18 & 0.00 & 32047.48 & 4445.58 & 0.00 & 62090.14 \\
\hline 7532.25 & 0.00 & 28886.98 & 3956.16 & 0.00 & 55995.10 \\
\hline 7532.31 & 0.00 & 41796.42 & 2942.00 & 0.00 & 70386.93 \\
\hline 7532.38 & 0.00 & 30024.96 & 1316.12 & 0.00 & 72449.87 \\
\hline 7532.44 & 0.00 & 38263.38 & 5760.16 & 0.00 & 69013.36 \\
\hline 7532.51 & 0.00 & 26906.63 & 2136.52 & 0.00 & 79353.39 \\
\hline 7532.57 & 0.00 & 35393.30 & 975.77 & 0.00 & 77891.83 \\
\hline 7532.64 & 0.00 & 36687.27 & 1034.18 & 0.00 & 86022.17 \\
\hline 7532.71 & 0.00 & 31218.79 & 355.02 & 0.00 & 60184.90 \\
\hline 7532.77 & 225.27 & 28653.43 & 0.00 & 0.00 & 88543.66 \\
\hline 7532.84 & 0.00 & 31683.36 & 0.00 & 0.00 & 88432.36 \\
\hline 7532.90 & 0.00 & 37702.42 & 0.00 & 0.00 & 77276.75 \\
\hline 7532.97 & 0.00 & 39685.08 & 609.55 & 3913.00 & 49200.84 \\
\hline 7533.03 & 0.00 & 34466.45 & 0.00 & 6403.62 & 55653.09 \\
\hline 7533.10 & 0.00 & 34805.92 & 0.00 & 4958.66 & 91908.75 \\
\hline 7533.17 & 0.00 & 24293.86 & 0.00 & 0.00 & 130766.26 \\
\hline 7533.23 & 0.00 & 17243.82 & 0.00 & 0.00 & 203398.29 \\
\hline 7533.30 & 332.25 & 18106.41 & 0.00 & 0.00 & 210564.54 \\
\hline 7533.36 & 844.95 & 20244.79 & 886.61 & 0.00 & 174784.31 \\
\hline 7533.43 & 329.31 & 21927.29 & 2269.64 & 0.00 & 154623.01 \\
\hline 7533.49 & 0.00 & 23400.19 & 3088.94 & 0.00 & 121392.16 \\
\hline 7533.56 & 0.00 & 32141.48 & 3267.36 & 0.00 & 104378.81 \\
\hline 7533.62 & 0.00 & 27008.89 & 1281.89 & 0.00 & 88029.27 \\
\hline 7533.69 & 0.00 & 27891.35 & 3096.10 & 0.00 & 89263.12 \\
\hline 7533.76 & 0.00 & 30657.41 & 481.02 & 5121.60 & 73327.36 \\
\hline 7533.82 & 0.00 & 31486.30 & 0.00 & 0.00 & 81702.06 \\
\hline 7533.89 & 0.00 & 34719.20 & 0.00 & 5012.18 & 74177.74 \\
\hline
\end{tabular}




\begin{tabular}{|c|c|c|c|c|c|}
\hline Depth & $\mathrm{P}$ & S & $\mathrm{Cl}$ & K & $\mathrm{Ca}$ \\
\hline & ppm & ppm & $\mathrm{ppm}$ & ppm & $\mathrm{ppm}$ \\
\hline 7533.95 & 0.00 & 36482.70 & 2682.16 & 0.00 & 86683.17 \\
\hline 7534.02 & 0.00 & 45229.86 & 3345.13 & 0.00 & 38117.14 \\
\hline 7534.08 & 0.00 & 49785.47 & 1929.96 & 3742.65 & 36646.39 \\
\hline 7534.15 & 0.00 & 39609.12 & 1452.35 & 7696.98 & 23164.30 \\
\hline 7534.22 & 0.00 & 42067.49 & 1487.90 & 0.00 & 23912.18 \\
\hline 7534.28 & 0.00 & 39490.68 & 0.00 & 3846.41 & 22626.71 \\
\hline 7534.35 & 0.00 & 44494.05 & 0.00 & 6467.05 & 31937.11 \\
\hline 7534.41 & 0.00 & 36163.06 & 0.00 & 9247.85 & 17056.52 \\
\hline 7534.48 & 0.00 & 47966.73 & 0.00 & 7294.23 & 9396.33 \\
\hline 7534.54 & 0.00 & 40016.99 & 0.00 & 7577.85 & 9946.05 \\
\hline 7534.61 & 0.00 & 48128.41 & 0.00 & 0.00 & 15192.90 \\
\hline 7534.67 & 0.00 & 47473.42 & 0.00 & 0.00 & 13996.58 \\
\hline 7534.74 & 0.00 & 36808.25 & 0.00 & 3839.31 & 8515.21 \\
\hline 7534.81 & 0.00 & 48624.23 & 1519.45 & 0.00 & 17017.13 \\
\hline 7534.87 & 0.00 & 66906.05 & 1104.02 & 0.00 & 46891.90 \\
\hline 7535.07 & 0.00 & 55029.00 & 178.63 & 0.00 & 25652.23 \\
\hline 7535.13 & 0.00 & 65676.27 & 0.00 & 0.00 & 18622.66 \\
\hline 7535.20 & 0.00 & 50697.21 & 0.00 & 7371.35 & 11951.82 \\
\hline 7535.26 & 0.00 & 37884.61 & 0.00 & 0.00 & 7115.69 \\
\hline 7535.33 & 0.00 & 35087.95 & 0.00 & 10795.60 & 7141.12 \\
\hline 7535.39 & 0.00 & 30531.70 & 0.00 & 8442.25 & 5352.15 \\
\hline 7535.46 & 0.00 & 40613.87 & 0.00 & 0.00 & 11506.65 \\
\hline 7535.52 & 0.00 & 43588.00 & 0.00 & 0.00 & 10931.70 \\
\hline 7535.59 & 0.00 & 48797.40 & 0.00 & 0.00 & 11221.90 \\
\hline 7535.66 & 0.00 & 54241.73 & 0.00 & 0.00 & 45725.51 \\
\hline 7535.72 & 0.00 & 62224.65 & 2562.57 & 0.00 & 19972.70 \\
\hline 7535.79 & 0.00 & 50550.79 & 0.00 & 0.00 & 20530.51 \\
\hline 7535.85 & 0.00 & 37173.74 & 0.00 & 0.00 & 29834.01 \\
\hline 7535.92 & 0.00 & 34113.59 & 0.00 & 0.00 & 30757.79 \\
\hline 7535.98 & 0.00 & 39497.80 & 0.00 & 0.00 & 30538.22 \\
\hline 7536.05 & 0.00 & 32427.51 & 0.00 & 0.00 & 20982.94 \\
\hline 7536.12 & 0.00 & 39694.70 & 0.00 & 5078.83 & 19499.74 \\
\hline
\end{tabular}




\begin{tabular}{|c|c|c|c|c|c|}
\hline Depth & $\mathrm{P}$ & S & $\mathrm{Cl}$ & K & $\mathrm{Ca}$ \\
\hline & ppm & ppm & $\mathrm{ppm}$ & ppm & $\mathrm{ppm}$ \\
\hline 7536.18 & 0.00 & 48252.00 & 848.74 & 0.00 & 26653.64 \\
\hline 7536.25 & 0.00 & 39604.17 & 0.00 & 3900.88 & 15780.56 \\
\hline 7536.31 & 0.00 & 46518.99 & 0.00 & 0.00 & 20992.44 \\
\hline 7536.38 & 0.00 & 35601.51 & 0.00 & 0.00 & 13806.09 \\
\hline 7536.44 & 0.00 & 34833.63 & 3817.32 & 0.00 & 21227.00 \\
\hline 7536.51 & 0.00 & 48178.70 & 0.00 & 0.00 & 25174.31 \\
\hline 7536.57 & 0.00 & 47135.33 & 0.00 & 0.00 & 28669.87 \\
\hline 7536.64 & 0.00 & 50096.44 & 0.00 & 0.00 & 32143.76 \\
\hline 7536.71 & 0.00 & 56065.31 & 0.00 & 0.00 & 30955.17 \\
\hline 7536.77 & 0.00 & 53078.56 & 0.00 & 0.00 & 27888.63 \\
\hline 7536.84 & 0.00 & 48082.68 & 0.00 & 0.00 & 24540.71 \\
\hline 7536.90 & 0.00 & 47155.20 & 0.00 & 0.00 & 30393.97 \\
\hline 7536.97 & 0.00 & 41381.13 & 0.00 & 0.00 & 25601.51 \\
\hline 7537.07 & 0.00 & 40474.21 & 0.00 & 11173.85 & 23775.48 \\
\hline 7537.13 & 0.00 & 58875.96 & 0.00 & 7004.20 & 30340.44 \\
\hline 7537.20 & 0.00 & 49872.12 & 0.00 & 11023.50 & 23107.01 \\
\hline 7537.26 & 0.00 & 49875.10 & 0.00 & 12397.10 & 22013.90 \\
\hline 7537.33 & 0.00 & 41857.32 & 0.00 & 12653.16 & 24616.34 \\
\hline 7537.39 & 0.00 & 44789.58 & 0.00 & 14411.82 & 20802.02 \\
\hline 7537.46 & 0.00 & 71661.63 & 0.00 & 3573.39 & 21308.09 \\
\hline 7537.52 & 0.00 & 48610.43 & 0.00 & 8336.38 & 23054.33 \\
\hline 7537.59 & 0.00 & 51396.96 & 0.00 & 9462.97 & 28534.57 \\
\hline 7537.66 & 0.00 & 64145.31 & 0.00 & 8235.06 & 37177.51 \\
\hline 7537.72 & 0.00 & 55593.69 & 0.00 & 12504.96 & 23238.43 \\
\hline 7537.79 & 0.00 & 41831.10 & 0.00 & 12072.94 & 18267.80 \\
\hline 7537.85 & 0.00 & 70352.81 & 3353.92 & 3999.68 & 25478.70 \\
\hline 7537.92 & 214.28 & 47589.16 & 0.00 & 14741.84 & 30641.74 \\
\hline 7537.98 & 0.00 & 39128.57 & 0.00 & 13669.08 & 15338.26 \\
\hline 7538.05 & 0.00 & 53704.77 & 0.00 & 6215.35 & 16431.98 \\
\hline 7538.12 & 0.00 & 50760.74 & 0.00 & 12872.39 & 17232.14 \\
\hline 7538.18 & 0.00 & 81209.58 & 11017.97 & 0.00 & 17711.41 \\
\hline 7538.25 & 0.00 & 46092.27 & 0.00 & 16658.05 & 11927.53 \\
\hline
\end{tabular}




\begin{tabular}{|c|c|c|c|c|c|}
\hline Depth & $\mathrm{P}$ & S & $\mathrm{Cl}$ & K & $\mathrm{Ca}$ \\
\hline & ppm & $\mathrm{ppm}$ & $\mathrm{ppm}$ & ppm & $\mathrm{ppm}$ \\
\hline 7538.31 & 0.00 & 47358.99 & 0.00 & 13603.51 & 12463.10 \\
\hline 7538.38 & 0.00 & 58941.96 & 0.00 & 10114.87 & 34885.09 \\
\hline 7538.44 & 0.00 & 50369.45 & 0.00 & 10285.68 & 14628.56 \\
\hline 7538.51 & 0.00 & 53879.03 & 18184.12 & 0.00 & 15723.66 \\
\hline 7538.57 & 0.00 & 44299.21 & 0.00 & 11636.03 & 14217.99 \\
\hline 7538.64 & 0.00 & 52957.66 & 453.53 & 6863.78 & 23554.07 \\
\hline 7538.71 & 0.00 & 40621.00 & 687.42 & 13696.86 & 18599.62 \\
\hline 7538.77 & 0.00 & 32432.32 & 0.00 & 18903.43 & 14854.28 \\
\hline 7538.84 & 0.00 & 31869.63 & 0.00 & 15937.05 & 13404.05 \\
\hline 7538.90 & 0.00 & 41101.62 & 0.00 & 3815.75 & 12916.59 \\
\hline 7538.97 & 0.00 & 39590.69 & 0.00 & 9967.46 & 19766.05 \\
\hline 7539.03 & 0.00 & 47487.04 & 0.00 & 5275.63 & 20816.85 \\
\hline 7539.10 & 0.00 & 46603.66 & 681.32 & 0.00 & 21651.98 \\
\hline 7539.17 & 0.00 & 49310.30 & 11052.49 & 0.00 & 20611.59 \\
\hline 7539.23 & 0.00 & 60686.19 & 8034.29 & 0.00 & 17546.96 \\
\hline 7539.30 & 0.00 & 41733.68 & 230.61 & 6828.63 & 21761.56 \\
\hline 7539.36 & 0.00 & 45787.76 & 0.00 & 0.00 & 29092.54 \\
\hline 7539.43 & 0.00 & 55588.67 & 481.53 & 7330.14 & 42430.74 \\
\hline 7539.49 & 0.00 & 51102.06 & 12346.89 & 0.00 & 45282.30 \\
\hline 7539.56 & 0.00 & 49488.13 & 4012.30 & 0.00 & 37565.49 \\
\hline 7539.62 & 0.00 & 25660.01 & 9060.55 & 0.00 & 92102.35 \\
\hline 7539.69 & 0.00 & 29352.16 & 13533.10 & 0.00 & 48367.46 \\
\hline 7539.76 & 0.00 & 60194.02 & 24499.15 & 0.00 & 14750.21 \\
\hline 7539.82 & 0.00 & 65767.99 & 5351.31 & 0.00 & 44438.07 \\
\hline 7539.89 & 0.00 & 58274.85 & 0.00 & 0.00 & 41953.19 \\
\hline 7539.95 & 0.00 & 131774.60 & 1124.72 & 0.00 & 32744.30 \\
\hline 7540.02 & 0.00 & 40166.91 & 273.04 & 12329.89 & 45427.43 \\
\hline 7540.07 & 0.00 & 49100.27 & 0.00 & 12020.62 & 28864.65 \\
\hline 7540.13 & 0.00 & 48585.08 & 0.00 & 11234.53 & 32639.04 \\
\hline 7540.20 & 0.00 & 53145.26 & 0.00 & 11971.26 & 27262.41 \\
\hline 7540.26 & 0.00 & 50946.37 & 537.34 & 10519.23 & 37161.92 \\
\hline 7540.33 & 0.00 & 57023.00 & 0.00 & 8911.15 & 25492.74 \\
\hline
\end{tabular}




\begin{tabular}{|c|c|c|c|c|c|}
\hline Depth & $\mathrm{P}$ & S & $\mathrm{Cl}$ & K & $\mathrm{Ca}$ \\
\hline & ppm & ppm & $\mathrm{ppm}$ & ppm & ppm \\
\hline 7540.39 & 0.00 & 46478.06 & 113.38 & 4297.84 & 15544.20 \\
\hline 7540.46 & 447.22 & 91736.55 & 556.12 & 0.00 & 24638.95 \\
\hline 7540.52 & 250.55 & 90335.40 & 1663.73 & 0.00 & 31235.87 \\
\hline 7540.59 & 0.00 & 82381.27 & 10988.41 & 0.00 & 33472.65 \\
\hline 7540.66 & 0.00 & 79443.75 & 0.00 & 4268.34 & 40301.17 \\
\hline 7540.72 & 0.00 & 66602.30 & 0.00 & 10249.72 & 29498.03 \\
\hline 7540.79 & 0.00 & 72485.34 & 0.00 & 7816.05 & 28401.56 \\
\hline 7540.85 & 256.18 & 67222.21 & 0.00 & 5021.65 & 51927.45 \\
\hline 7540.92 & 0.00 & 64483.11 & 0.00 & 8078.06 & 38653.47 \\
\hline 7540.98 & 0.00 & 59293.90 & 0.00 & 6463.19 & 23981.57 \\
\hline 7541.05 & 0.00 & 58712.93 & 0.00 & 7661.18 & 38143.56 \\
\hline 7541.12 & 0.00 & 80150.24 & 0.00 & 0.00 & 52793.07 \\
\hline 7541.18 & 225.26 & 82248.12 & 0.00 & 0.00 & 57734.66 \\
\hline 7541.25 & 0.00 & 51156.40 & 0.00 & 0.00 & 78353.93 \\
\hline 7541.31 & 0.00 & 59486.78 & 0.00 & 0.00 & 84631.00 \\
\hline 7541.38 & 0.00 & 33349.32 & 0.00 & 0.00 & 91953.51 \\
\hline 7541.44 & 0.00 & 39098.28 & 0.00 & 0.00 & 144839.29 \\
\hline 7541.51 & 0.00 & 7725.40 & 2466.59 & 0.00 & 309949.50 \\
\hline 7541.57 & 0.00 & 4137.38 & 3387.49 & 0.00 & 353455.17 \\
\hline 7541.64 & 0.00 & 2304.19 & 330.18 & 0.00 & 383520.52 \\
\hline 7541.71 & 0.00 & 2235.58 & 93.47 & 0.00 & 383919.98 \\
\hline 7541.77 & 0.00 & 3045.47 & 0.00 & 0.00 & 377882.52 \\
\hline 7541.84 & 0.00 & 3467.69 & 247.05 & 0.00 & 370497.98 \\
\hline 7541.90 & 0.00 & 3591.11 & 0.00 & 0.00 & 371652.20 \\
\hline 7541.97 & 0.00 & 9228.03 & 10048.79 & 0.00 & 334484.28 \\
\hline 7542.03 & 0.00 & 7531.05 & 8857.41 & 0.00 & 307708.74 \\
\hline 7542.10 & 0.00 & 10747.47 & 0.00 & 0.00 & 291641.23 \\
\hline 7542.17 & 0.00 & 17216.72 & 0.00 & 0.00 & 214342.26 \\
\hline 7542.23 & 0.00 & 9098.35 & 0.00 & 0.00 & 154143.97 \\
\hline 7542.30 & 0.00 & 31421.27 & 1186.40 & 0.00 & 167212.57 \\
\hline 7542.36 & 0.00 & 53310.99 & 0.00 & 10257.10 & 65016.59 \\
\hline 7542.43 & 0.00 & 56323.99 & 0.00 & 4751.57 & 41222.68 \\
\hline
\end{tabular}




\begin{tabular}{|c|c|c|c|c|c|}
\hline Depth & $\mathrm{P}$ & S & $\mathrm{Cl}$ & K & $\mathrm{Ca}$ \\
\hline & ppm & $\mathrm{ppm}$ & $\mathrm{ppm}$ & ppm & ppm \\
\hline 7542.49 & 0.00 & 54612.36 & 0.00 & 0.00 & 35468.49 \\
\hline 7542.56 & 235.01 & 53662.64 & 0.00 & 6621.68 & 51022.04 \\
\hline 7542.62 & 0.00 & 46889.12 & 0.00 & 8910.55 & 47327.42 \\
\hline 7542.69 & 260.22 & 47304.45 & 0.00 & 5212.02 & 30864.45 \\
\hline 7542.76 & 0.00 & 119234.05 & 348.70 & 0.00 & 26762.14 \\
\hline 7542.82 & 0.00 & 49695.30 & 0.00 & 9207.65 & 28346.71 \\
\hline 7542.89 & 0.00 & 56417.27 & 0.00 & 0.00 & 53131.42 \\
\hline 7542.95 & 0.00 & 48093.01 & 0.00 & 0.00 & 162969.75 \\
\hline 7543.07 & 772.34 & 65822.88 & 0.00 & 0.00 & 30362.98 \\
\hline 7543.13 & 432.61 & 56572.87 & 0.00 & 4576.51 & 42422.77 \\
\hline 7543.20 & 0.00 & 26961.64 & 0.00 & 0.00 & 203860.15 \\
\hline 7543.26 & 0.00 & 58018.28 & 0.00 & 0.00 & 135032.45 \\
\hline 7543.33 & 0.00 & 63726.21 & 0.00 & 0.00 & 95565.64 \\
\hline 7543.39 & 275.23 & 73475.50 & 0.00 & 0.00 & 49214.20 \\
\hline 7543.46 & 265.39 & 57685.19 & 0.00 & 6549.34 & 30025.24 \\
\hline 7543.52 & 265.68 & 117474.71 & 0.00 & 0.00 & 38597.46 \\
\hline 7543.59 & 0.00 & 40872.25 & 0.00 & 0.00 & 66698.58 \\
\hline 7543.66 & 0.00 & 72215.74 & 0.00 & 0.00 & 30345.35 \\
\hline 7543.72 & 0.00 & 62122.43 & 0.00 & 0.00 & 50441.61 \\
\hline 7543.79 & 0.00 & 65452.52 & 0.00 & 0.00 & 55352.69 \\
\hline 7543.85 & 0.00 & 67141.90 & 0.00 & 0.00 & 61561.75 \\
\hline 7543.92 & 0.00 & 59256.63 & 318.64 & 0.00 & 64412.72 \\
\hline 7543.98 & 0.00 & 55406.49 & 64.65 & 0.00 & 103200.44 \\
\hline 7544.05 & 0.00 & 55405.13 & 0.00 & 0.00 & 47196.50 \\
\hline 7544.12 & 0.00 & 81578.84 & 0.00 & 0.00 & 30608.15 \\
\hline 7544.18 & 0.00 & 55303.61 & 0.00 & 0.00 & 31302.27 \\
\hline 7544.25 & 0.00 & 42991.61 & 0.00 & 0.00 & 38039.20 \\
\hline 7544.31 & 0.00 & 58285.04 & 0.00 & 0.00 & 48505.40 \\
\hline 7544.38 & 0.00 & 104329.05 & 0.00 & 12470.96 & 42141.94 \\
\hline 7544.44 & 0.00 & 83046.02 & 0.00 & 0.00 & 32727.70 \\
\hline 7544.51 & 0.00 & 59977.75 & 0.00 & 0.00 & 39714.17 \\
\hline 7544.57 & 0.00 & 80506.97 & 2733.02 & 0.00 & 40699.99 \\
\hline
\end{tabular}




\begin{tabular}{|c|c|c|c|c|c|}
\hline Depth & $\mathrm{P}$ & S & $\mathrm{Cl}$ & K & $\mathrm{Ca}$ \\
\hline & ppm & $\mathrm{ppm}$ & $\mathrm{ppm}$ & ppm & ppm \\
\hline 7544.64 & 0.00 & 52205.49 & 0.00 & 0.00 & 59794.07 \\
\hline 7544.71 & 0.00 & 49145.45 & 0.00 & 5315.53 & 38907.03 \\
\hline 7544.77 & 0.00 & 70795.88 & 0.00 & 0.00 & 43139.43 \\
\hline 7544.84 & 0.00 & 73368.61 & 0.00 & 0.00 & 44242.41 \\
\hline 7544.90 & 307.67 & 121666.92 & 0.00 & 4686.71 & 56443.39 \\
\hline 7544.97 & 0.00 & 48526.45 & 0.00 & 6386.11 & 44907.54 \\
\hline 7545.07 & 0.00 & 49261.44 & 0.00 & 0.00 & 63083.79 \\
\hline 7545.13 & 0.00 & 65653.37 & 0.00 & 0.00 & 23478.75 \\
\hline 7545.20 & 0.00 & 66053.34 & 0.00 & 0.00 & 24856.45 \\
\hline 7545.26 & 0.00 & 67878.54 & 0.00 & 0.00 & 31629.98 \\
\hline 7545.33 & 0.00 & 62369.44 & 0.00 & 0.00 & 38925.43 \\
\hline 7545.39 & 0.00 & 51527.59 & 0.00 & 0.00 & 67360.07 \\
\hline 7545.46 & 0.00 & 40944.74 & 0.00 & 0.00 & 192225.50 \\
\hline 7545.52 & 0.00 & 57255.71 & 0.00 & 0.00 & 174184.03 \\
\hline 7545.59 & 0.00 & 99007.53 & 0.00 & 0.00 & 118780.32 \\
\hline 7545.66 & 0.00 & 113266.96 & 0.00 & 4825.04 & 37658.05 \\
\hline 7545.72 & 0.00 & 53985.24 & 0.00 & 0.00 & 16336.05 \\
\hline 7545.79 & 0.00 & 60706.36 & 0.00 & 4446.13 & 30878.96 \\
\hline 7545.85 & 0.00 & 69962.30 & 0.00 & 0.00 & 31754.70 \\
\hline 7545.92 & 0.00 & 61139.52 & 0.00 & 0.00 & 26209.68 \\
\hline 7545.98 & 0.00 & 59505.50 & 0.00 & 0.00 & 29189.87 \\
\hline 7546.05 & 0.00 & 116469.94 & 0.00 & 0.00 & 21487.49 \\
\hline 7546.12 & 0.00 & 68437.53 & 0.00 & 0.00 & 20853.74 \\
\hline 7546.18 & 0.00 & 52705.38 & 0.00 & 0.00 & 50548.08 \\
\hline 7546.25 & 0.00 & 59549.75 & 0.00 & 0.00 & 69628.85 \\
\hline 7546.31 & 0.00 & 56319.04 & 0.00 & 0.00 & 127319.47 \\
\hline 7546.38 & 0.00 & 51256.17 & 0.00 & 0.00 & 96701.77 \\
\hline 7546.44 & 0.00 & 50731.07 & 0.00 & 0.00 & 79408.57 \\
\hline 7546.51 & 0.00 & 40725.04 & 0.00 & 3920.19 & 67605.58 \\
\hline 7546.57 & 0.00 & 41276.68 & 0.00 & 0.00 & 67445.58 \\
\hline 7546.64 & 0.00 & 58556.21 & 0.00 & 4886.90 & 33459.40 \\
\hline 7546.71 & 0.00 & 49471.43 & 0.00 & 5201.53 & 54974.04 \\
\hline
\end{tabular}




\begin{tabular}{|c|c|c|c|c|c|}
\hline Depth & $\mathrm{P}$ & S & $\mathrm{Cl}$ & K & $\mathrm{Ca}$ \\
\hline & ppm & ppm & $\mathrm{ppm}$ & ppm & $\mathrm{ppm}$ \\
\hline 7546.77 & 354.26 & 63144.23 & 0.00 & 0.00 & 17897.51 \\
\hline 7546.84 & 0.00 & 25730.51 & 0.00 & 7449.96 & 17136.49 \\
\hline 7546.90 & 0.00 & 70835.30 & 0.00 & 0.00 & 38318.67 \\
\hline 7546.97 & 0.00 & 75128.87 & 0.00 & 0.00 & 28513.62 \\
\hline 7547.03 & 0.00 & 57709.62 & 0.00 & 0.00 & 31872.41 \\
\hline 7547.10 & 0.00 & 54285.46 & 0.00 & 0.00 & 48785.29 \\
\hline 7547.17 & 0.00 & 69827.41 & 0.00 & 0.00 & 57705.13 \\
\hline 7547.23 & 0.00 & 55715.18 & 0.00 & 0.00 & 49025.07 \\
\hline 7547.30 & 0.00 & 37339.44 & 0.00 & 0.00 & 47141.44 \\
\hline 7547.36 & 0.00 & 58112.21 & 0.00 & 0.00 & 23240.57 \\
\hline 7547.43 & 0.00 & 47783.57 & 0.00 & 0.00 & 19875.25 \\
\hline 7547.49 & 0.00 & 56431.33 & 0.00 & 0.00 & 26465.50 \\
\hline 7547.56 & 0.00 & 80964.27 & 0.00 & 0.00 & 29503.35 \\
\hline 7547.62 & 0.00 & 51408.85 & 0.00 & 0.00 & 16760.85 \\
\hline 7547.69 & 0.00 & 46151.32 & 0.00 & 0.00 & 19592.44 \\
\hline 7547.76 & 0.00 & 46638.59 & 0.00 & 0.00 & 14536.20 \\
\hline 7547.82 & 0.00 & 40605.45 & 0.00 & 0.00 & 18561.46 \\
\hline 7547.89 & 0.00 & 38355.83 & 0.00 & 0.00 & 23260.45 \\
\hline 7547.95 & 0.00 & 44491.36 & 0.00 & 0.00 & 12752.12 \\
\hline 7548.02 & 0.00 & 61600.52 & 0.00 & 0.00 & 17652.61 \\
\hline 7548.08 & 0.00 & 50858.48 & 0.00 & 0.00 & 14507.86 \\
\hline 7548.15 & 214.00 & 57240.57 & 0.00 & 0.00 & 17317.35 \\
\hline 7548.22 & 0.00 & 52779.91 & 0.00 & 0.00 & 18643.09 \\
\hline 7548.28 & 0.00 & 46188.94 & 0.00 & 0.00 & 14069.92 \\
\hline 7548.35 & 0.00 & 68295.88 & 0.00 & 0.00 & 11921.12 \\
\hline 7548.41 & 0.00 & 51627.47 & 0.00 & 0.00 & 12495.08 \\
\hline 7548.48 & 0.00 & 43921.57 & 0.00 & 0.00 & 9298.16 \\
\hline 7548.54 & 0.00 & 48997.23 & 0.00 & 0.00 & 17188.64 \\
\hline 7548.61 & 0.00 & 48251.39 & 0.00 & 0.00 & 21172.39 \\
\hline 7548.67 & 0.00 & 57517.56 & 0.00 & 0.00 & 18429.39 \\
\hline 7548.74 & 0.00 & 47436.95 & 0.00 & 0.00 & 17104.15 \\
\hline 7548.81 & 0.00 & 53661.30 & 0.00 & 0.00 & 12645.60 \\
\hline
\end{tabular}




\begin{tabular}{|c|c|c|c|c|c|}
\hline Depth & $\mathrm{P}$ & S & $\mathrm{Cl}$ & K & $\mathrm{Ca}$ \\
\hline & ppm & $\mathrm{ppm}$ & $\mathrm{ppm}$ & ppm & ppm \\
\hline 7548.87 & 0.00 & 48370.01 & 0.00 & 0.00 & 24201.06 \\
\hline 7548.94 & 0.00 & 39005.32 & 0.00 & 0.00 & 38675.92 \\
\hline 7549.07 & 0.00 & 30626.63 & 4440.80 & 0.00 & 55222.36 \\
\hline 7549.13 & 0.00 & 25707.08 & 0.00 & 0.00 & 105726.58 \\
\hline 7549.20 & 0.00 & 64571.20 & 0.00 & 0.00 & 35577.87 \\
\hline 7549.26 & 0.00 & 48487.80 & 0.00 & 0.00 & 36434.28 \\
\hline 7549.33 & 0.00 & 37236.95 & 0.00 & 0.00 & 30453.37 \\
\hline 7549.39 & 0.00 & 52270.58 & 0.00 & 0.00 & 33223.35 \\
\hline 7549.46 & 0.00 & 76575.15 & 0.00 & 0.00 & 49906.53 \\
\hline 7549.52 & 0.00 & 38740.29 & 0.00 & 0.00 & 44142.14 \\
\hline 7549.59 & 0.00 & 30334.69 & 0.00 & 0.00 & 45315.58 \\
\hline 7549.66 & 0.00 & 40685.92 & 0.00 & 0.00 & 27592.84 \\
\hline 7549.72 & 0.00 & 51927.06 & 0.00 & 0.00 & 31838.31 \\
\hline 7549.79 & 0.00 & 42116.72 & 0.00 & 0.00 & 33534.54 \\
\hline 7549.85 & 0.00 & 48108.00 & 0.00 & 0.00 & 25220.70 \\
\hline 7549.92 & 0.00 & 55852.43 & 0.00 & 0.00 & 29498.88 \\
\hline 7549.98 & 0.00 & 27697.50 & 0.00 & 0.00 & 37246.86 \\
\hline 7550.05 & 0.00 & 40738.18 & 0.00 & 0.00 & 54263.48 \\
\hline 7550.12 & 7579.19 & 64925.07 & 0.00 & 0.00 & 50209.37 \\
\hline 7550.18 & 0.00 & 68717.70 & 0.00 & 0.00 & 33856.45 \\
\hline 7550.25 & 0.00 & 51758.89 & 0.00 & 0.00 & 22655.31 \\
\hline 7550.31 & 0.00 & 46078.63 & 0.00 & 0.00 & 16247.85 \\
\hline 7550.38 & 0.00 & 48545.41 & 0.00 & 0.00 & 14149.25 \\
\hline 7550.44 & 0.00 & 58989.12 & 963.44 & 0.00 & 25451.48 \\
\hline 7550.51 & 0.00 & 44132.48 & 1303.92 & 0.00 & 25892.40 \\
\hline 7550.57 & 0.00 & 71776.74 & 0.00 & 0.00 & 29291.94 \\
\hline 7550.64 & 0.00 & 63495.10 & 0.00 & 0.00 & 40400.72 \\
\hline 7550.71 & 0.00 & 50830.01 & 2337.03 & 0.00 & 57302.90 \\
\hline 7550.77 & 0.00 & 36214.02 & 0.00 & 0.00 & 24508.09 \\
\hline 7550.84 & 0.00 & 49327.98 & 0.00 & 0.00 & 30687.42 \\
\hline 7550.90 & 0.00 & 64464.69 & 0.00 & 0.00 & 32525.85 \\
\hline 7550.97 & 0.00 & 46162.21 & 0.00 & 0.00 & 40929.33 \\
\hline
\end{tabular}




\begin{tabular}{|c|c|c|c|c|c|}
\hline Depth & $\mathrm{P}$ & S & $\mathrm{Cl}$ & K & $\mathrm{Ca}$ \\
\hline & ppm & $\mathrm{ppm}$ & $\mathrm{ppm}$ & ppm & $\mathrm{ppm}$ \\
\hline 7551.03 & 0.00 & 14819.59 & 0.00 & 0.00 & 175965.60 \\
\hline 7551.10 & 0.00 & 3517.55 & 0.00 & 0.00 & 304731.25 \\
\hline 7551.17 & 0.00 & 3171.74 & 0.00 & 0.00 & 382921.68 \\
\hline 7551.23 & 0.00 & 4006.08 & 0.00 & 0.00 & 392340.05 \\
\hline 7551.30 & 0.00 & 4319.57 & 0.00 & 0.00 & 375722.36 \\
\hline 7551.36 & 0.00 & 3230.65 & 0.00 & 0.00 & 380905.09 \\
\hline 7551.43 & 0.00 & 3188.01 & 0.00 & 0.00 & 382762.80 \\
\hline 7551.49 & 0.00 & 1788.46 & 0.00 & 0.00 & 397239.91 \\
\hline 7551.56 & 0.00 & 4002.42 & 260.68 & 0.00 & 391697.18 \\
\hline 7551.62 & 0.00 & 2717.31 & 230.23 & 0.00 & 406455.01 \\
\hline 7551.69 & 0.00 & 3201.28 & 283.36 & 0.00 & 393469.65 \\
\hline 7551.76 & 0.00 & 1831.89 & 30.41 & 0.00 & 393572.92 \\
\hline 7551.82 & 0.00 & 1099.52 & 52.07 & 0.00 & 396131.59 \\
\hline 7551.89 & 0.00 & 648.42 & 0.00 & 0.00 & 395611.17 \\
\hline 7551.95 & 253.89 & 1320.76 & 0.00 & 0.00 & 390021.76 \\
\hline 7552.02 & 0.00 & 1194.22 & 0.00 & 0.00 & 376630.10 \\
\hline 7552.08 & 0.00 & 1655.35 & 0.00 & 0.00 & 390714.79 \\
\hline 7552.15 & 0.00 & 12590.84 & 0.00 & 0.00 & 351031.14 \\
\hline 7552.22 & 295.51 & 11666.42 & 284.43 & 0.00 & 301358.01 \\
\hline 7552.28 & 0.00 & 11157.29 & 171.29 & 0.00 & 374796.16 \\
\hline 7552.35 & 0.00 & 52138.09 & 1156.55 & 0.00 & 291402.79 \\
\hline 7552.41 & 0.00 & 52904.13 & 1043.66 & 0.00 & 52648.26 \\
\hline 7552.48 & 221.76 & 49087.19 & 0.00 & 0.00 & 48590.47 \\
\hline 7552.54 & 0.00 & 69519.08 & 759.01 & 0.00 & 30591.66 \\
\hline 7552.61 & 0.00 & 61135.90 & 0.00 & 3066.44 & 32667.09 \\
\hline 7552.67 & 0.00 & 67580.48 & 0.00 & 0.00 & 32919.57 \\
\hline 7552.74 & 0.00 & 32793.17 & 1428.13 & 5148.95 & 108510.33 \\
\hline 7552.81 & 359.77 & 17090.09 & 0.00 & 31420.93 & 654.60 \\
\hline 7552.87 & 1207.44 & 38228.17 & 0.00 & 10467.47 & 3928.65 \\
\hline 7552.94 & 0.00 & 19712.07 & 0.00 & 0.00 & 29164.45 \\
\hline 7553.07 & 0.00 & 44039.72 & 0.00 & 0.00 & 27884.13 \\
\hline 7553.13 & 0.00 & 44267.90 & 0.00 & 0.00 & 36315.95 \\
\hline
\end{tabular}




\begin{tabular}{|c|c|c|c|c|c|}
\hline Depth & $\mathrm{P}$ & $S$ & $\mathrm{Cl}$ & $\mathrm{K}$ & $\mathrm{Ca}$ \\
\hline & $\mathrm{ppm}$ & $\mathrm{ppm}$ & $\mathrm{ppm}$ & $\mathrm{ppm}$ & ppm \\
\hline 7553.20 & 0.00 & 62112.43 & 0.00 & 0.00 & 23091.90 \\
\hline 7553.26 & 0.00 & 33534.45 & 0.00 & 0.00 & 14200.04 \\
\hline 7553.33 & 0.00 & 61764.54 & 0.00 & 0.00 & 22187.55 \\
\hline 7553.39 & 317.41 & 54336.83 & 0.00 & 0.00 & 23053.58 \\
\hline 7553.46 & 0.00 & 83049.21 & 1343.75 & 0.00 & 36922.62 \\
\hline 7553.52 & 0.00 & 60624.80 & 3719.78 & 0.00 & 29869.32 \\
\hline 7553.59 & 0.00 & 52620.02 & 2665.09 & 0.00 & 30798.69 \\
\hline 7553.66 & 0.00 & 18808.58 & 0.00 & 33802.32 & 2450.89 \\
\hline 7553.72 & 0.00 & 27685.70 & 8543.48 & 0.00 & 14294.23 \\
\hline 7553.79 & 0.00 & 38887.79 & 0.00 & 0.00 & 13749.98 \\
\hline 7553.85 & 0.00 & 44533.62 & 0.00 & 0.00 & 32525.22 \\
\hline 7553.92 & 0.00 & 22108.45 & 0.00 & 0.00 & 50873.03 \\
\hline 7553.98 & 0.00 & 35786.20 & 0.00 & 0.00 & 34326.68 \\
\hline 7554.05 & 0.00 & 32608.40 & 0.00 & 0.00 & 107441.41 \\
\hline 7554.12 & 0.00 & 34985.19 & 0.00 & 0.00 & 89481.51 \\
\hline 7554.18 & 0.00 & 22792.99 & 909.71 & 0.00 & 371490.57 \\
\hline 7554.25 & 0.00 & 15627.65 & 1001.34 & 0.00 & 335159.55 \\
\hline 7554.31 & 0.00 & 10825.84 & 935.96 & 0.00 & 284167.78 \\
\hline 7554.38 & 1355.74 & 2255.44 & 0.00 & 0.00 & 339518.08 \\
\hline 7554.44 & 775.65 & 12866.48 & 189.51 & 0.00 & 273218.30 \\
\hline 7554.51 & 0.00 & 77093.98 & 516.73 & 2873.39 & 80213.40 \\
\hline 7554.57 & 0.00 & 26118.27 & 2010.36 & 0.00 & 329193.45 \\
\hline 7554.64 & 0.00 & 44587.22 & 1040.88 & 0.00 & 156086.63 \\
\hline 7554.71 & 436.39 & 54261.30 & 0.00 & 4156.68 & 104924.29 \\
\hline 7554.77 & 0.00 & 45443.37 & 657.27 & 15282.16 & 67565.99 \\
\hline 7554.84 & 0.00 & 10110.32 & 0.00 & 0.00 & 327828.27 \\
\hline 7554.90 & 0.00 & 4912.31 & 390.58 & 0.00 & 316463.75 \\
\hline 7554.97 & 0.00 & 6054.44 & 0.00 & 0.00 & 311953.54 \\
\hline
\end{tabular}


Table D-6, MSCL Soil Suite:

\begin{tabular}{|c|c|c|c|c|c|c|c|c|c|c|c|c|c|c|}
\hline Depth & XRF & XRF Live Time & $\mathrm{Ti}(\mathrm{ppm})$ & $\mathrm{Cr}(\mathrm{ppm})$ & $\mathrm{Mn}$ (ppm) & $\mathrm{Fe}(\mathrm{ppm})$ & $\mathrm{Ni}(\mathrm{ppm})$ & $\mathrm{Cu}(\mathrm{ppm})$ & $\mathrm{Zn}(\mathrm{ppm})$ & As (ppm) & $\mathrm{Rb}(\mathrm{ppm})$ & $\mathrm{Sr}(\mathrm{ppm})$ & $\mathrm{Y}(\mathrm{ppm})$ & $\mathrm{Zr}(\mathrm{ppm})$ \\
\hline 7445.20 & 305810.60 & 17.20 & 12045.03 & 54.75 & 362.97 & 16377.47 & 42.02 & 55.96 & 16.97 & 1.45 & 128.70 & 55.44 & 61.41 & 12.34 \\
\hline 7445.26 & 371646.40 & 16.60 & 11942.49 & 65.67 & 382.13 & 22401.76 & 50.71 & 96.31 & 18.42 & 5.31 & 154.16 & 57.77 & 89.71 & 10.99 \\
\hline 7445.33 & 323820.60 & 17.00 & 2389.07 & 72.13 & 381.61 & 23426.99 & 58.41 & 238.86 & 18.62 & 5.39 & 150.38 & 56.00 & 92.73 & 10.65 \\
\hline 7445.39 & 323086.40 & 17.00 & 2357.99 & 74.85 & 379.55 & 23070.21 & 60.45 & 409.51 & 18.03 & 5.17 & 155.05 & 57.13 & 91.26 & 10.81 \\
\hline 7445.46 & 320356.60 & 17.00 & 2786.45 & 87.03 & 241.91 & 27111.61 & 73.96 & 657.73 & 22.84 & 6.99 & 147.07 & 30.36 & 116.41 & 13.15 \\
\hline 7445.52 & 312668.20 & 17.20 & 3337.34 & 104.75 & 159.06 & 31012.49 & 82.80 & 704.15 & 26.75 & 7.87 & 142.44 & 27.85 & 137.83 & 16.40 \\
\hline 7445.59 & 314748.00 & 17.20 & 3437.36 & 111.55 & 156.25 & 31162.01 & 81.94 & 706.65 & 28.06 & 7.64 & 145.39 & 27.35 & 136.81 & 16.70 \\
\hline 7445.66 & 377131.00 & 16.60 & 3386.93 & 107.59 & 160.58 & 31924.71 & 79.25 & 623.28 & 28.35 & 8.31 & 151.46 & 29.07 & 136.23 & 16.67 \\
\hline 7445.72 & 386247.20 & 16.40 & 3432.25 & 106.79 & 158.80 & 33636.43 & 82.96 & 447.70 & 30.73 & 9.04 & 152.41 & 28.08 & 136.79 & 16.29 \\
\hline 7445.79 & 390361.80 & 16.40 & 3396.15 & 111.66 & 160.07 & 34533.59 & 79.21 & 201.40 & 33.70 & 8.57 & 147.53 & 27.32 & 135.63 & 16.02 \\
\hline 7445.85 & 401397.20 & 16.20 & 3465.75 & 114.77 & 167.92 & 36158.81 & 81.66 & 151.17 & 35.54 & 8.94 & 150.51 & 29.89 & 135.82 & 14.53 \\
\hline 7445.92 & 414612.00 & 16.00 & 3418.23 & 111.72 & 177.48 & 38330.72 & 89.23 & 102.12 & 36.83 & 9.59 & 154.35 & 32.33 & 137.23 & 15.26 \\
\hline 7445.98 & 422822.60 & 16.00 & 3777.20 & 111.64 & 184.83 & 39784.49 & 91.34 & 35.17 & 39.18 & 9.44 & 157.84 & 31.78 & 137.41 & 15.62 \\
\hline 7446.05 & 428555.80 & 16.00 & 3887.89 & 116.53 & 200.26 & 41097.50 & 87.31 & 33.96 & 40.26 & 9.36 & 157.35 & 31.39 & 138.11 & 16.33 \\
\hline 7446.12 & 429538.00 & 16.00 & 4082.26 & 118.66 & 211.73 & 41841.57 & 89.43 & 33.18 & 39.80 & 9.69 & 162.25 & 33.32 & 142.80 & 16.94 \\
\hline 7446.18 & 424836.80 & 16.00 & 4118.13 & 119.60 & 212.45 & 41929.78 & 92.07 & 30.32 & 39.47 & 9.08 & 162.00 & 32.16 & 141.92 & 17.12 \\
\hline 7446.25 & 430787.80 & 16.00 & 4219.11 & 120.72 & 212.36 & 42145.80 & 89.44 & 29.29 & 38.96 & 8.71 & 160.59 & 30.79 & 144.36 & 15.25 \\
\hline 7446.31 & 437003.40 & 16.00 & 3930.35 & 116.74 & 210.51 & 42029.10 & 91.54 & 30.14 & 37.27 & 8.48 & 160.17 & 32.12 & 144.74 & 14.11 \\
\hline 7446.38 & 435271.20 & 16.00 & 3914.15 & 110.78 & 199.43 & 41438.05 & 98.97 & 29.96 & 35.42 & 8.25 & 158.77 & 34.00 & 145.78 & 14.45 \\
\hline 7446.44 & 439332.40 & 16.00 & 3885.64 & 106.83 & 190.43 & 40721.04 & 99.79 & 30.27 & 33.12 & 7.96 & 160.70 & 32.37 & 143.64 & 14.71 \\
\hline 7446.51 & 446153.40 & 16.00 & 3961.24 & 105.12 & 187.66 & 41199.47 & 98.39 & 33.49 & 31.92 & 8.14 & 160.12 & 33.69 & 146.05 & 14.63 \\
\hline 7446.57 & 438917.80 & 16.00 & 3925.64 & 106.68 & 190.99 & 40314.70 & 98.28 & 34.72 & 30.57 & 7.72 & 161.81 & 33.02 & 143.82 & 15.61 \\
\hline 7446.64 & 401463.20 & 16.40 & 4005.59 & 106.83 & 189.00 & 38717.44 & 93.58 & 60.05 & 30.07 & 7.52 & 155.04 & 31.65 & 143.06 & 16.73 \\
\hline 7446.71 & 405810.60 & 16.40 & 4106.72 & 112.55 & 190.80 & 37303.38 & 85.42 & 171.95 & 29.87 & 7.59 & 155.00 & 32.34 & 145.99 & 15.53 \\
\hline 7446.77 & 408644.00 & 16.40 & 4172.63 & 117.84 & 194.65 & 36717.68 & 85.91 & 189.31 & 29.65 & 7.65 & 149.78 & 33.30 & 149.89 & 16.21 \\
\hline 7446.84 & 408767.00 & 16.40 & 4199.33 & 119.67 & 191.59 & 35113.89 & 90.97 & 199.96 & 55.18 & 12.06 & 157.48 & 58.48 & 126.76 & 43.93 \\
\hline 7446.90 & 408101.80 & 16.40 & 4306.60 & 118.46 & 185.24 & 34189.11 & 87.61 & 207.31 & 54.41 & 12.31 & 152.54 & 60.35 & 129.74 & 44.05 \\
\hline 7446.97 & 435316.20 & 16.00 & 5891.43 & 122.97 & 187.93 & 34675.52 & 87.39 & 182.50 & 55.63 & 12.23 & 155.80 & 59.56 & 131.65 & 44.17 \\
\hline 7447.03 & 432101.60 & 16.00 & 5891.20 & 117.08 & 186.37 & 35871.18 & 86.43 & 71.35 & 57.60 & 12.06 & 152.28 & 57.92 & 130.43 & 45.10 \\
\hline 7447.10 & 414341.20 & 16.20 & 5834.56 & 116.77 & 184.91 & 35876.67 & 79.07 & 53.61 & 59.06 & 12.01 & 154.07 & 56.32 & 127.11 & 44.63 \\
\hline 7447.17 & 380105.80 & 16.60 & 5749.44 & 118.60 & 189.02 & 35779.30 & 66.93 & 41.77 & 33.58 & 7.33 & 138.80 & 31.90 & 145.15 & 17.05 \\
\hline 7447.23 & 347241.40 & 17.00 & 5719.48 & 114.79 & 199.52 & 35371.64 & 64.52 & 32.57 & 33.67 & 6.94 & 130.75 & 30.03 & 140.37 & 16.77 \\
\hline 7447.30 & 323673.00 & 17.20 & 3942.87 & 113.99 & 201.97 & 35564.60 & 64.62 & 28.69 & 33.11 & 6.98 & 129.52 & 31.29 & 135.26 & 15.61 \\
\hline
\end{tabular}




\begin{tabular}{|c|c|c|c|c|c|c|c|c|c|c|c|c|c|c|}
\hline Depth & XRF & XRF Live Time & $\mathrm{Ti}(\mathrm{ppm})$ & $\mathrm{Cr}(\mathrm{ppm})$ & $\mathrm{Mn}(\mathrm{ppm})$ & $\mathrm{Fe}(\mathrm{ppm})$ & $\mathrm{Ni}(\mathrm{ppm})$ & $\mathrm{Cu}(\mathrm{ppm})$ & $\mathrm{Zn}(\mathrm{ppm})$ & As (ppm) & $\mathrm{Rb}(\mathrm{ppm})$ & $\mathrm{Sr}(\mathrm{ppm})$ & $\mathrm{Y}(\mathrm{ppm})$ & $\mathrm{Zr}(\mathrm{ppm})$ \\
\hline 7447.36 & 297025.00 & 17.40 & 3723.76 & 113.88 & 205.07 & 34705.90 & 61.45 & 27.15 & 30.36 & 6.38 & 133.23 & 30.11 & 132.91 & 15.92 \\
\hline 7447.43 & 277757.60 & 17.60 & 3631.21 & 110.11 & 202.28 & 36071.46 & 65.20 & 25.54 & 31.34 & 6.70 & 129.75 & 31.00 & 129.60 & 15.25 \\
\hline 7447.49 & 247958.00 & 17.80 & 3432.90 & 100.74 & 194.16 & 35573.56 & 65.96 & 24.82 & 32.44 & 6.98 & 125.70 & 29.53 & 130.62 & 15.78 \\
\hline 7447.56 & 227731.20 & 18.00 & 3284.47 & 102.38 & 187.08 & 35754.75 & 65.17 & 25.76 & 33.92 & 7.23 & 126.20 & 28.85 & 130.15 & 16.01 \\
\hline 7447.62 & 195750.00 & 18.40 & 3269.45 & 100.33 & 186.31 & 35062.65 & 65.32 & 26.57 & 34.11 & 7.13 & 123.88 & 27.67 & 129.64 & 16.60 \\
\hline 7447.69 & 185697.20 & 18.60 & 3261.52 & 101.76 & 178.60 & 35070.04 & 71.53 & 36.43 & 35.22 & 13.31 & 128.32 & 50.79 & 106.99 & 40.55 \\
\hline 7447.76 & 172000.40 & 18.60 & 3337.22 & 103.26 & 177.78 & 33944.12 & 69.93 & 37.36 & 33.35 & 12.39 & 123.82 & 50.57 & 109.48 & 40.89 \\
\hline 7447.82 & 183755.20 & 18.60 & 3353.45 & 105.52 & 184.43 & 34607.76 & 71.58 & 38.30 & 34.62 & 12.93 & 130.32 & 49.11 & 108.90 & 41.02 \\
\hline 7447.89 & 187026.20 & 18.60 & 3372.22 & 108.41 & 177.82 & 34224.94 & 74.23 & 38.00 & 33.86 & 13.46 & 128.70 & 49.28 & 110.08 & 41.57 \\
\hline 7447.95 & 174821.00 & 18.60 & 3533.06 & 104.28 & 166.73 & 34110.87 & 73.67 & 42.92 & 34.73 & 14.34 & 127.08 & 48.73 & 106.53 & 43.00 \\
\hline 7448.02 & 129653.20 & 19.00 & 6690.12 & 93.55 & 182.08 & 26912.92 & 62.18 & 28.49 & 30.02 & 9.49 & 97.22 & 23.17 & 104.63 & 16.07 \\
\hline 7448.08 & 96745.20 & 19.40 & 6879.53 & 84.72 & 184.93 & 19906.67 & 57.52 & 28.52 & 25.59 & 8.64 & 76.95 & 16.77 & 80.14 & 15.15 \\
\hline 7448.15 & 132691.60 & 19.00 & 7903.72 & 90.07 & 178.64 & 20701.37 & 61.18 & 27.50 & 25.23 & 8.70 & 85.81 & 17.56 & 84.35 & 14.36 \\
\hline 7448.22 & 166648.80 & 18.60 & 7899.52 & 83.66 & 169.50 & 21781.84 & 63.72 & 27.37 & 26.74 & 8.90 & 96.75 & 17.55 & 84.99 & 13.44 \\
\hline 7448.28 & 220271.60 & 18.20 & 7757.27 & 84.11 & 173.81 & 22466.49 & 62.99 & 24.06 & 26.38 & 8.70 & 98.10 & 17.72 & 90.68 & 12.71 \\
\hline 7448.35 & 280514.00 & 17.60 & 4666.06 & 95.46 & 158.39 & 29013.73 & 73.68 & 28.44 & 29.25 & 7.44 & 119.08 & 22.10 & 115.21 & 14.75 \\
\hline 7448.41 & 349415.40 & 17.00 & 4401.14 & 101.67 & 150.86 & 35439.47 & 80.38 & 27.92 & 34.12 & 9.30 & 143.74 & 28.59 & 142.32 & 15.65 \\
\hline 7448.48 & 356069.80 & 16.80 & 3548.20 & 101.45 & 149.32 & 34946.56 & 79.10 & 28.47 & 32.97 & 8.72 & 141.74 & 28.57 & 143.65 & 16.75 \\
\hline 7448.54 & 364561.00 & 16.60 & 3622.44 & 105.62 & 159.94 & 34426.67 & 76.82 & 27.18 & 32.11 & 8.62 & 144.80 & 29.13 & 144.36 & 17.76 \\
\hline 7448.61 & 374352.00 & 16.40 & 3654.43 & 108.26 & 157.81 & 34354.11 & 77.93 & 25.22 & 31.40 & 8.42 & 158.57 & 30.38 & 147.75 & 17.48 \\
\hline 7448.67 & 388896.20 & 16.20 & 3638.44 & 105.71 & 160.83 & 35487.98 & 78.42 & 25.76 & 32.14 & 8.88 & 166.54 & 27.98 & 149.51 & 17.96 \\
\hline 7448.74 & 395260.00 & 16.00 & 3592.99 & 103.99 & 162.77 & 38013.91 & 79.90 & 26.47 & 34.33 & 8.54 & 172.19 & 27.99 & 146.26 & 17.40 \\
\hline 7448.81 & 396206.40 & 16.00 & 3563.26 & 99.86 & 169.88 & 39208.43 & 80.07 & 26.58 & 34.77 & 8.81 & 174.14 & 29.22 & 144.51 & 16.68 \\
\hline 7448.87 & 395120.00 & 16.20 & 3488.45 & 97.29 & 171.26 & 41547.01 & 85.52 & 28.18 & 36.12 & 8.97 & 168.73 & 29.51 & 141.70 & 16.02 \\
\hline 7448.94 & 403281.40 & 16.20 & 3487.92 & 101.28 & 191.57 & 43405.13 & 87.94 & 30.31 & 35.19 & 8.86 & 166.95 & 30.14 & 137.02 & 14.44 \\
\hline 7449.00 & 414548.40 & 16.20 & 3541.67 & 102.94 & 213.23 & 46323.82 & 92.75 & 31.14 & 39.84 & 8.89 & 174.21 & 33.81 & 134.34 & 13.93 \\
\hline 7449.07 & 416971.60 & 16.20 & 3573.80 & 104.55 & 232.37 & 45233.32 & 92.01 & 31.33 & 36.44 & 8.34 & 186.32 & 36.06 & 131.85 & 14.14 \\
\hline 7449.13 & 424317.80 & 16.20 & 6049.20 & 101.92 & 254.18 & 44882.39 & 92.71 & 34.54 & 37.43 & 7.99 & 185.63 & 38.55 & 126.19 & 12.97 \\
\hline 7449.20 & 430918.20 & 16.00 & 6121.34 & 103.09 & 257.40 & 43614.54 & 89.41 & 45.74 & 36.42 & 14.82 & 193.43 & 67.89 & 107.66 & 34.99 \\
\hline 7449.27 & 424115.20 & 16.00 & 6157.29 & 101.96 & 248.55 & 42661.78 & 94.69 & 45.04 & 38.95 & 14.73 & 187.81 & 68.19 & 107.66 & 36.01 \\
\hline 7449.33 & 419627.20 & 16.00 & 6230.05 & 103.81 & 229.28 & 40567.82 & 87.72 & 46.01 & 37.58 & 14.69 & 183.39 & 64.75 & 109.68 & 36.61 \\
\hline 7449.40 & 422775.00 & 16.00 & 6364.02 & 108.85 & 217.55 & 41610.13 & 90.63 & 46.67 & 43.31 & 15.56 & 172.45 & 61.53 & 113.46 & 36.21 \\
\hline 7449.46 & 426573.20 & 16.00 & 3985.89 & 113.29 & 196.69 & 41717.83 & 89.30 & 43.17 & 42.83 & 15.47 & 174.84 & 57.29 & 121.51 & 37.67 \\
\hline
\end{tabular}




\begin{tabular}{|c|c|c|c|c|c|c|c|c|c|c|c|c|c|c|}
\hline Depth & XRF & XRF Live Time & $\mathrm{Ti}(\mathrm{ppm})$ & $\mathrm{Cr}(\mathrm{ppm})$ & $\mathrm{Mn}(\mathrm{ppm})$ & $\mathrm{Fe}(\mathrm{ppm})$ & $\mathrm{Ni}(\mathrm{ppm})$ & $\mathrm{Cu}(\mathrm{ppm})$ & $\mathrm{Zn}(\mathrm{ppm})$ & As (ppm) & $\mathrm{Rb}(\mathrm{ppm})$ & $\mathrm{Sr}(\mathrm{ppm})$ & $\mathrm{Y}(\mathrm{ppm})$ & $\mathrm{Zr}(\mathrm{ppm})$ \\
\hline 7449.53 & 428196.80 & 16.00 & 4019.11 & 117.95 & 191.56 & 41711.42 & 87.65 & 32.53 & 41.87 & 7.73 & 171.05 & 28.84 & 142.89 & 15.61 \\
\hline 7449.59 & 431606.20 & 16.00 & 4019.33 & 120.25 & 187.75 & 41217.46 & 79.65 & 31.12 & 40.40 & 7.41 & 174.92 & 28.24 & 146.05 & 15.59 \\
\hline 7449.66 & 430193.00 & 16.00 & 3978.80 & 123.24 & 189.45 & 40873.05 & 82.81 & 29.41 & 38.84 & 7.10 & 169.98 & 29.11 & 145.26 & 15.74 \\
\hline 7449.72 & 425423.00 & 16.00 & 3966.04 & 123.74 & 186.95 & 39365.93 & 79.76 & 28.37 & 34.47 & 5.95 & 168.81 & 29.57 & 146.21 & 15.99 \\
\hline 7449.79 & 415980.00 & 16.00 & 3836.46 & 123.57 & 181.16 & 38588.71 & 77.78 & 28.16 & 34.24 & 6.07 & 163.34 & 30.47 & 146.15 & 14.99 \\
\hline 7449.86 & 410947.80 & 16.00 & 3847.45 & 122.54 & 180.48 & 38050.55 & 75.42 & 27.48 & 33.97 & 6.31 & 163.11 & 29.53 & 146.63 & 14.58 \\
\hline 7449.92 & 405037.00 & 16.00 & 3827.20 & 121.61 & 175.91 & 37339.54 & 72.92 & 27.21 & 33.18 & 6.47 & 163.66 & 27.78 & 145.54 & 14.96 \\
\hline 7449.99 & 399671.80 & 16.00 & 3754.26 & 115.48 & 173.10 & 36515.41 & 70.31 & 27.46 & 32.21 & 6.45 & 161.40 & 28.08 & 145.35 & 14.16 \\
\hline 7450.05 & 397577.20 & 16.00 & 3749.81 & 112.75 & 172.81 & 36091.43 & 71.15 & 27.20 & 31.92 & 6.79 & 166.79 & 27.84 & 145.27 & 14.34 \\
\hline 7450.12 & 402237.20 & 16.00 & 4588.93 & 115.66 & 179.94 & 35617.16 & 72.30 & 28.05 & 31.06 & 6.60 & 165.73 & 26.60 & 144.73 & 15.71 \\
\hline 7450.18 & 406786.80 & 16.00 & 4606.87 & 117.41 & 184.56 & 36090.67 & 75.80 & 27.83 & 32.82 & 6.44 & 171.41 & 27.05 & 146.03 & 16.32 \\
\hline 7450.25 & 412036.40 & 16.00 & 4634.77 & 117.03 & 188.17 & 36646.72 & 77.20 & 27.99 & 33.59 & 6.52 & 174.90 & 27.02 & 147.67 & 16.46 \\
\hline 7450.31 & 408955.20 & 16.20 & 4759.72 & 123.25 & 188.98 & 36091.90 & 73.97 & 26.15 & 32.60 & 6.92 & 185.97 & 26.94 & 148.12 & 16.74 \\
\hline 7450.38 & 403548.80 & 16.40 & 4686.72 & 127.02 & 187.24 & 36178.74 & 74.19 & 26.88 & 32.98 & 7.23 & 182.85 & 28.32 & 145.77 & 16.21 \\
\hline 7450.45 & 405499.20 & 16.40 & 3997.99 & 127.70 & 188.28 & 38541.59 & 74.52 & 27.15 & 35.65 & 7.72 & 187.18 & 29.01 & 144.98 & 15.39 \\
\hline 7450.51 & 402142.20 & 16.40 & 3948.94 & 122.10 & 186.16 & 38156.56 & 73.73 & 28.40 & 35.10 & 7.93 & 182.92 & 28.26 & 143.84 & 14.78 \\
\hline 7450.58 & 396827.60 & 16.40 & 3983.31 & 121.11 & 185.52 & 37536.49 & 73.10 & 28.39 & 34.66 & 7.58 & 176.42 & 28.40 & 145.35 & 14.56 \\
\hline 7450.64 & 393261.40 & 16.40 & 3940.01 & 117.77 & 188.92 & 36699.93 & 72.66 & 29.83 & 33.23 & 7.13 & 173.58 & 27.19 & 141.75 & 13.52 \\
\hline 7450.71 & 393537.80 & 16.40 & 4083.61 & 118.76 & 190.40 & 36366.61 & 71.86 & 29.88 & 32.65 & 7.08 & 173.89 & 25.86 & 141.86 & 14.27 \\
\hline 7450.77 & 377240.20 & 16.60 & 4027.40 & 114.44 & 185.85 & 33451.06 & 70.78 & 28.20 & 29.35 & 6.12 & 173.08 & 25.72 & 139.92 & 14.39 \\
\hline 7450.84 & 365402.80 & 16.80 & 4111.78 & 114.27 & 184.48 & 32709.71 & 70.56 & 25.72 & 28.20 & 6.30 & 168.81 & 25.58 & 138.75 & 14.26 \\
\hline 7450.91 & 358639.00 & 17.00 & 4156.50 & 116.71 & 183.47 & 34517.87 & 71.68 & 26.07 & 31.06 & 6.83 & 172.99 & 26.66 & 137.30 & 14.54 \\
\hline 7450.97 & 340866.40 & 17.20 & 4141.43 & 113.02 & 180.67 & 33969.30 & 69.29 & 25.97 & 31.30 & 6.70 & 166.19 & 26.13 & 140.12 & 15.04 \\
\hline 7451.04 & 323467.20 & 17.20 & 4018.76 & 109.03 & 175.09 & 33062.19 & 64.26 & 25.01 & 30.30 & 6.22 & 167.48 & 25.88 & 140.80 & 15.06 \\
\hline 7451.10 & 306298.40 & 17.40 & 4055.54 & 109.44 & 172.22 & 33128.51 & 57.58 & 25.61 & 30.44 & 6.10 & 157.95 & 25.37 & 139.16 & 13.77 \\
\hline 7451.17 & 303895.60 & 17.40 & 4019.51 & 112.96 & 172.28 & 32695.52 & 55.61 & 26.75 & 29.58 & 5.54 & 157.96 & 24.69 & 140.22 & 13.60 \\
\hline 7451.23 & 303415.20 & 17.40 & 4008.75 & 110.33 & 177.62 & 30600.16 & 53.69 & 26.61 & 25.42 & 5.15 & 150.99 & 24.52 & 137.39 & 13.21 \\
\hline 7451.30 & 322339.40 & 17.20 & 3957.92 & 115.77 & 175.38 & 32524.27 & 58.80 & 27.37 & 25.98 & 5.34 & 149.00 & 25.00 & 135.50 & 13.09 \\
\hline 7451.36 & 340153.20 & 17.20 & 3921.44 & 111.25 & 184.26 & 34471.35 & 66.60 & 39.22 & 26.14 & 9.08 & 146.66 & 48.88 & 113.72 & 36.93 \\
\hline 7451.43 & 357948.20 & 17.00 & 3811.92 & 107.79 & 189.60 & 35418.98 & 75.75 & 39.38 & 24.68 & 9.87 & 147.10 & 51.22 & 111.61 & 38.60 \\
\hline 7451.50 & 365863.00 & 17.00 & 3733.23 & 105.85 & 193.59 & 37037.17 & 79.12 & 39.01 & 26.63 & 9.61 & 148.73 & 52.70 & 108.61 & 38.09 \\
\hline 7451.56 & 374015.00 & 16.80 & 3712.93 & 105.53 & 194.39 & 38985.24 & 82.91 & 39.98 & 27.56 & 9.73 & 150.10 & 53.37 & 105.93 & 38.16 \\
\hline 7451.63 & 370049.80 & 16.80 & 3765.67 & 100.77 & 204.66 & 39858.03 & 83.12 & 40.65 & 31.14 & 9.83 & 152.98 & 54.63 & 106.61 & 37.83 \\
\hline
\end{tabular}




\begin{tabular}{|c|c|c|c|c|c|c|c|c|c|c|c|c|c|c|}
\hline Depth & XRF & XRF Live Time & $\mathrm{Ti}(\mathrm{ppm})$ & $\mathrm{Cr}(\mathrm{ppm})$ & $\mathrm{Mn}(\mathrm{ppm})$ & $\mathrm{Fe}(\mathrm{ppm})$ & $\mathrm{Ni}(\mathrm{ppm})$ & $\mathrm{Cu}(\mathrm{ppm})$ & $\mathrm{Zn}(\mathrm{ppm})$ & As (ppm) & $\mathrm{Rb}(\mathrm{ppm})$ & $\mathrm{Sr}(\mathrm{ppm})$ & $\mathrm{Y}(\mathrm{ppm})$ & $\mathrm{Zr}(\mathrm{ppm})$ \\
\hline 7451.69 & 354960.60 & 16.80 & 3869.86 & 103.56 & 201.83 & 39759.58 & 81.02 & 29.10 & 32.90 & 6.95 & 149.63 & 31.23 & 125.49 & 13.49 \\
\hline 7451.76 & 373280.00 & 16.60 & 4048.63 & 110.83 & 201.51 & 40883.44 & 81.53 & 29.10 & 37.68 & 7.07 & 157.36 & 30.20 & 131.68 & 13.10 \\
\hline 7451.82 & 385653.20 & 16.40 & 4151.96 & 114.05 & 201.50 & 40909.46 & 82.60 & 29.36 & 38.41 & 7.53 & 156.55 & 30.64 & 134.71 & 13.48 \\
\hline 7451.89 & 389104.20 & 16.40 & 4167.83 & 110.28 & 194.37 & 39068.24 & 80.39 & 28.31 & 38.71 & 7.23 & 159.28 & 29.70 & 140.07 & 13.81 \\
\hline 7451.96 & 398777.00 & 16.20 & 4118.89 & 112.95 & 184.22 & 37180.63 & 81.45 & 27.50 & 36.21 & 7.05 & 159.51 & 29.77 & 142.24 & 14.34 \\
\hline 7452.02 & 421353.80 & 16.00 & 4084.32 & 112.86 & 183.33 & 36675.00 & 79.55 & 28.17 & 36.34 & 7.23 & 163.00 & 29.24 & 145.17 & 14.32 \\
\hline 7452.09 & 413887.40 & 16.00 & 4031.51 & 111.32 & 179.25 & 35288.94 & 77.40 & 29.42 & 34.45 & 7.29 & 157.01 & 28.23 & 145.75 & 14.36 \\
\hline 7452.15 & 412694.80 & 16.00 & 4313.98 & 111.91 & 171.51 & 34761.26 & 78.77 & 30.31 & 34.11 & 7.41 & 164.13 & 27.22 & 146.82 & 15.30 \\
\hline 7452.22 & 414772.00 & 16.00 & 4278.36 & 114.90 & 176.01 & 35595.07 & 80.03 & 30.67 & 34.39 & 7.59 & 160.26 & 26.66 & 149.24 & 15.37 \\
\hline 7452.28 & 414992.80 & 16.00 & 4354.24 & 116.33 & 175.36 & 36732.48 & 77.45 & 29.56 & 36.61 & 7.33 & 156.90 & 25.87 & 150.02 & 15.39 \\
\hline 7452.35 & 411796.60 & 16.00 & 4364.56 & 118.06 & 175.44 & 37662.26 & 76.25 & 29.16 & 37.71 & 6.97 & 153.48 & 26.37 & 149.67 & 15.79 \\
\hline 7452.41 & 418305.40 & 16.00 & 4323.37 & 117.11 & 179.66 & 37884.89 & 72.78 & 28.32 & 36.39 & 6.49 & 157.08 & 26.05 & 149.21 & 16.44 \\
\hline 7452.48 & 420522.00 & 16.00 & 4013.65 & 113.66 & 188.89 & 38127.34 & 67.25 & 28.14 & 35.93 & 6.59 & 149.56 & 24.73 & 148.09 & 15.97 \\
\hline 7452.55 & 423256.80 & 16.00 & 3989.86 & 113.59 & 190.48 & 38387.64 & 67.50 & 34.81 & 35.86 & 7.04 & 154.15 & 24.58 & 145.96 & 15.82 \\
\hline 7452.61 & 428687.80 & 16.00 & 3867.00 & 109.47 & 197.15 & 38533.52 & 68.35 & 47.38 & 33.99 & 8.05 & 161.09 & 25.58 & 141.91 & 15.13 \\
\hline 7452.68 & 453246.80 & 15.80 & 3915.06 & 108.46 & 209.46 & 48201.15 & 83.53 & 63.81 & 41.04 & 9.93 & 162.45 & 25.56 & 137.02 & 14.50 \\
\hline 7452.74 & 448504.00 & 15.80 & 3886.84 & 104.97 & 209.33 & 49051.07 & 88.42 & 80.67 & 41.95 & 10.25 & 159.07 & 25.87 & 133.73 & 13.99 \\
\hline 7452.81 & 441555.60 & 15.80 & 3842.75 & 101.51 & 208.10 & 48670.17 & 90.88 & 114.94 & 40.75 & 9.83 & 159.39 & 26.47 & 132.93 & 14.32 \\
\hline 7452.87 & 434592.20 & 15.80 & 3889.11 & 102.55 & 205.24 & 48018.73 & 92.88 & 123.23 & 39.83 & 9.32 & 153.97 & 26.30 & 133.73 & 14.21 \\
\hline 7452.94 & 438461.40 & 15.80 & 4004.92 & 105.28 & 205.66 & 48286.77 & 99.63 & 129.31 & 39.15 & 8.62 & 155.29 & 25.34 & 135.90 & 15.48 \\
\hline 7453.01 & 420166.20 & 16.00 & 3981.20 & 107.32 & 191.34 & 38674.06 & 89.35 & 142.34 & 31.53 & 6.34 & 160.68 & 24.91 & 140.08 & 16.95 \\
\hline 7453.07 & 421230.60 & 16.00 & 4065.28 & 111.82 & 189.85 & 38518.19 & 89.38 & 150.65 & 31.92 & 7.00 & 167.71 & 24.79 & 142.90 & 16.77 \\
\hline 7453.14 & 425816.20 & 16.00 & 9085.98 & 117.05 & 185.61 & 38250.72 & 92.15 & 134.10 & 32.87 & 7.04 & 169.20 & 24.86 & 145.19 & 15.52 \\
\hline 7453.20 & 431473.80 & 16.00 & 9069.59 & 118.24 & 182.20 & 38125.32 & 91.50 & 127.22 & 33.51 & 7.16 & 175.61 & 25.51 & 143.96 & 14.91 \\
\hline 7453.27 & 408545.80 & 16.20 & 9093.72 & 118.69 & 180.16 & 37019.02 & 86.82 & 116.41 & 33.97 & 7.09 & 166.58 & 25.45 & 143.99 & 14.85 \\
\hline 7453.33 & 397221.80 & 16.40 & 9058.49 & 114.89 & 184.14 & 36534.33 & 86.54 & 96.03 & 33.18 & 7.30 & 155.31 & 24.51 & 144.09 & 14.33 \\
\hline 7453.40 & 384389.20 & 16.60 & 8986.09 & 110.47 & 185.47 & 35957.44 & 88.23 & 75.16 & 31.82 & 6.12 & 147.03 & 24.39 & 142.19 & 14.25 \\
\hline 7453.46 & 374615.40 & 16.80 & 4016.91 & 110.25 & 192.16 & 37448.95 & 84.58 & 61.52 & 33.01 & 6.22 & 141.57 & 23.98 & 135.77 & 15.30 \\
\hline 7453.53 & 365146.60 & 17.00 & 4023.77 & 106.04 & 195.31 & 39038.46 & 84.91 & 61.92 & 33.42 & 6.38 & 130.35 & 22.77 & 129.71 & 15.02 \\
\hline 7453.60 & 376577.60 & 16.80 & 4055.79 & 110.17 & 190.23 & 38979.35 & 84.77 & 55.24 & 32.32 & 5.97 & 130.48 & 22.07 & 130.47 & 14.16 \\
\hline 7453.66 & 375962.00 & 16.80 & 4092.29 & 111.85 & 187.56 & 38142.82 & 83.61 & 46.98 & 31.34 & 5.25 & 131.87 & 23.32 & 132.95 & 14.91 \\
\hline 7453.73 & 369707.40 & 16.80 & 4108.87 & 114.69 & 186.22 & 37710.15 & 80.92 & 42.70 & 30.07 & 5.28 & 137.14 & 23.71 & 132.80 & 13.91 \\
\hline 7453.79 & 368156.00 & 16.80 & 4137.28 & 114.53 & 182.06 & 36971.33 & 86.06 & 39.14 & 28.16 & 4.91 & 138.72 & 23.27 & 139.07 & 14.44 \\
\hline
\end{tabular}




\begin{tabular}{|c|c|c|c|c|c|c|c|c|c|c|c|c|c|c|}
\hline Depth & XRF & XRF Live Time & $\mathrm{Ti}(\mathrm{ppm})$ & $\mathrm{Cr}(\mathrm{ppm})$ & $\mathrm{Mn}(\mathrm{ppm})$ & $\mathrm{Fe}(\mathrm{ppm})$ & $\mathrm{Ni}(\mathrm{ppm})$ & $\mathrm{Cu}(\mathrm{ppm})$ & $\mathrm{Zn}(\mathrm{ppm})$ & As (ppm) & $\mathrm{Rb}(\mathrm{ppm})$ & $\mathrm{Sr}(\mathrm{ppm})$ & $\mathrm{Y}(\mathrm{ppm})$ & $\mathrm{Zr}(\mathrm{ppm})$ \\
\hline 7453.86 & 364386.60 & 16.80 & 4351.35 & 121.16 & 187.60 & 35987.58 & 83.24 & 31.55 & 27.15 & 4.67 & 145.72 & 24.13 & 147.43 & 16.06 \\
\hline 7453.92 & 352407.60 & 17.00 & 4339.47 & 122.73 & 193.40 & 36277.50 & 82.03 & 31.16 & 28.17 & 4.57 & 147.56 & 23.83 & 144.61 & 16.10 \\
\hline 7453.99 & 346169.60 & 17.00 & 4452.42 & 123.76 & 195.00 & 38220.67 & 86.57 & 30.87 & 32.18 & 3.75 & 147.58 & 22.51 & 145.01 & 15.75 \\
\hline 7454.06 & 359065.40 & 16.80 & 4459.97 & 125.99 & 194.54 & 41982.60 & 89.65 & 32.12 & 37.43 & 4.36 & 155.30 & 23.19 & 144.38 & 16.84 \\
\hline 7454.12 & 357663.20 & 16.80 & 4368.71 & 122.81 & 195.29 & 42738.13 & 85.15 & 30.18 & 39.88 & 4.32 & 161.70 & 24.93 & 141.90 & 15.98 \\
\hline 7454.19 & 350443.80 & 16.80 & 4188.62 & 128.19 & 195.14 & 41122.67 & 78.59 & 28.89 & 38.76 & 3.66 & 167.60 & 30.12 & 139.60 & 14.50 \\
\hline 7454.25 & 368377.80 & 16.60 & 4221.22 & 125.80 & 198.15 & 46674.63 & 83.87 & 31.09 & 48.37 & 6.40 & 171.66 & 29.38 & 141.89 & 15.68 \\
\hline 7454.32 & 360778.60 & 16.60 & 4077.35 & 129.71 & 195.85 & 46703.08 & 82.80 & 30.78 & 45.10 & 8.05 & 170.42 & 29.39 & 139.46 & 14.39 \\
\hline 7454.38 & 368649.00 & 16.60 & 4095.27 & 132.75 & 197.82 & 45533.75 & 81.01 & 30.24 & 44.08 & 9.96 & 164.59 & 28.17 & 143.47 & 15.48 \\
\hline 7454.45 & 374142.00 & 16.40 & 4171.80 & 146.23 & 203.32 & 44773.10 & 79.70 & 33.51 & 42.03 & 11.50 & 163.42 & 31.92 & 143.75 & 15.49 \\
\hline 7454.51 & 382602.00 & 16.40 & 4121.70 & 142.45 & 210.20 & 45125.95 & 82.01 & 41.19 & 42.26 & 12.04 & 170.95 & 31.37 & 144.54 & 15.30 \\
\hline 7454.58 & 378471.60 & 16.40 & 3787.12 & 135.64 & 269.47 & 39927.55 & 72.62 & 37.65 & 29.53 & 9.08 & 183.47 & 47.57 & 135.15 & 12.57 \\
\hline 7454.65 & 377585.00 & 16.40 & 3544.90 & 125.57 & 346.75 & 38639.49 & 61.65 & 35.86 & 29.10 & 8.27 & 205.54 & 62.38 & 126.38 & 11.41 \\
\hline 7454.71 & 344021.80 & 16.80 & 3400.22 & 112.86 & 394.83 & 35420.59 & 48.06 & 33.17 & 21.44 & 4.68 & 208.54 & 69.13 & 121.08 & 8.72 \\
\hline 7454.78 & 314559.00 & 17.20 & 3177.30 & 95.54 & 440.91 & 34942.86 & 37.53 & 28.59 & 16.26 & 2.12 & 227.09 & 70.42 & 115.93 & 10.09 \\
\hline 7454.84 & 291113.00 & 17.40 & 3088.34 & 84.72 & 479.32 & 34459.11 & 29.79 & 16.57 & 15.57 & 0.96 & 206.92 & 94.82 & 98.94 & 31.93 \\
\hline 7454.91 & 268420.20 & 17.80 & 3292.99 & 81.50 & 468.26 & 34056.65 & 20.02 & 17.32 & 13.81 & -0.04 & 199.64 & 86.10 & 103.46 & 32.79 \\
\hline 7454.97 & 271542.40 & 17.80 & 3379.52 & 77.01 & 463.15 & 33620.78 & 12.46 & 17.00 & 8.96 & -0.54 & 199.50 & 81.33 & 105.94 & 35.02 \\
\hline 7455.04 & 256956.20 & 17.80 & 3429.19 & 76.24 & 477.05 & 35649.99 & 12.32 & 18.87 & 9.31 & -0.39 & 211.42 & 81.05 & 108.05 & 36.90 \\
\hline 7455.10 & 212348.00 & 18.20 & 6382.98 & 63.34 & 335.13 & 28541.40 & 11.93 & 24.68 & 4.85 & 1.91 & 161.21 & 70.78 & 94.97 & 31.86 \\
\hline 7455.17 & 167782.60 & 18.60 & 9324.39 & 56.90 & 268.89 & 22810.61 & 22.42 & 29.79 & 0.03 & 4.81 & 141.09 & 37.13 & 88.76 & 15.32 \\
\hline 7455.24 & 115666.00 & 19.00 & 12811.36 & 31.27 & 195.91 & 15930.23 & 34.04 & 23.67 & -1.77 & 6.28 & 104.15 & 25.08 & 75.89 & 14.50 \\
\hline 7455.30 & 136805.40 & 18.80 & 13084.36 & 34.32 & 190.92 & 18299.59 & 35.36 & 25.67 & -2.68 & 5.99 & 106.82 & 24.62 & 76.53 & 13.05 \\
\hline 7455.37 & 183948.40 & 18.40 & 13076.77 & 35.59 & 186.49 & 21285.42 & 38.23 & 26.25 & -1.51 & 6.12 & 103.52 & 23.03 & 76.50 & 13.10 \\
\hline 7455.43 & 250880.80 & 17.80 & 10186.09 & 44.92 & 345.62 & 29152.36 & 38.83 & 23.27 & 3.98 & 4.28 & 150.45 & 34.76 & 89.57 & 17.27 \\
\hline 7455.50 & 311136.80 & 17.20 & 7185.79 & 48.78 & 435.33 & 36468.19 & 30.05 & 22.02 & 9.09 & 1.46 & 190.86 & 49.06 & 105.47 & 12.91 \\
\hline 7455.56 & 371356.60 & 16.60 & 3506.68 & 72.32 & 512.43 & 42173.25 & 19.50 & 27.36 & 11.24 & 0.61 & 243.94 & 70.28 & 116.19 & 12.65 \\
\hline 7455.63 & 366456.20 & 16.60 & 3057.72 & 67.82 & 500.98 & 38487.68 & 21.97 & 23.10 & 16.69 & 1.27 & 212.18 & 120.91 & 109.68 & 28.37 \\
\hline 7455.70 & 369151.80 & 16.60 & 2430.08 & 55.89 & 503.68 & 30162.86 & 18.79 & 19.67 & 16.12 & 1.32 & 237.87 & 124.75 & 87.45 & 24.22 \\
\hline 7455.76 & 383275.00 & 16.40 & 1842.97 & 45.06 & 487.56 & 23853.30 & 16.41 & 16.33 & 14.30 & 0.91 & 244.30 & 119.27 & 70.63 & 21.91 \\
\hline 7455.83 & 404490.20 & 16.20 & 1315.75 & 34.05 & 476.55 & 18384.26 & 15.38 & 14.67 & 11.25 & 1.01 & 249.55 & 110.17 & 52.14 & 18.93 \\
\hline 7455.89 & 425809.60 & 16.00 & 865.76 & 20.23 & 481.31 & 14848.87 & 18.02 & 13.27 & 11.50 & 1.24 & 243.50 & 95.81 & 36.69 & 18.27 \\
\hline 7455.96 & 435953.40 & 16.00 & 506.03 & 9.77 & 516.48 & 13137.28 & 16.56 & 14.77 & 7.88 & 0.97 & 273.68 & 41.60 & 26.11 & 0.90 \\
\hline
\end{tabular}




\begin{tabular}{|c|c|c|c|c|c|c|c|c|c|c|c|c|c|c|}
\hline Depth & XRF & XRF Live Time & $\mathrm{Ti}(\mathrm{ppm})$ & $\mathrm{Cr}(\mathrm{ppm})$ & $\mathrm{Mn}(\mathrm{ppm})$ & $\mathrm{Fe}(\mathrm{ppm})$ & $\mathrm{Ni}(\mathrm{ppm})$ & $\mathrm{Cu}(\mathrm{ppm})$ & $\mathrm{Zn}(\mathrm{ppm})$ & As (ppm) & $\mathrm{Rb}(\mathrm{ppm})$ & $\mathrm{Sr}(\mathrm{ppm})$ & $\mathrm{Y}(\mathrm{ppm})$ & $\mathrm{Zr}(\mathrm{ppm})$ \\
\hline 7456.02 & 433669.80 & 16.00 & 1475.72 & 35.84 & 465.62 & 21110.66 & 38.85 & 29.95 & 13.53 & 1.69 & 238.36 & 32.48 & 50.97 & 6.09 \\
\hline 7456.09 & 436307.80 & 16.00 & 2414.50 & 62.47 & 414.10 & 28416.80 & 55.91 & 61.25 & 20.62 & 3.18 & 217.96 & 30.49 & 80.57 & 8.72 \\
\hline 7456.15 & 432736.20 & 16.00 & 3329.73 & 90.83 & 348.08 & 33660.41 & 68.66 & 87.26 & 24.08 & 3.73 & 198.47 & 30.08 & 109.68 & 12.86 \\
\hline 7456.22 & 432084.80 & 16.00 & 4285.55 & 118.21 & 289.09 & 40795.24 & 84.49 & 113.21 & 30.73 & 5.42 & 179.94 & 29.18 & 134.94 & 15.90 \\
\hline 7456.29 & 436436.20 & 16.00 & 5134.82 & 145.38 & 197.68 & 46169.47 & 100.80 & 133.47 & 34.49 & 6.41 & 165.27 & 25.30 & 158.64 & 18.78 \\
\hline 7456.35 & 442431.60 & 16.00 & 8730.13 & 142.78 & 182.38 & 43261.38 & 97.42 & 152.78 & 32.07 & 6.50 & 165.69 & 25.25 & 159.07 & 17.41 \\
\hline 7456.42 & 439925.40 & 16.00 & 8598.15 & 138.37 & 174.37 & 42055.48 & 95.22 & 137.26 & 31.91 & 6.57 & 160.03 & 26.03 & 154.22 & 17.80 \\
\hline 7456.48 & 440590.20 & 16.00 & 8458.70 & 132.61 & 173.72 & 41931.82 & 97.36 & 163.12 & 32.10 & 6.91 & 164.37 & 26.60 & 152.75 & 16.87 \\
\hline 7456.55 & 438009.60 & 16.00 & 8212.67 & 128.83 & 168.66 & 40673.17 & 98.09 & 195.49 & 31.62 & 6.40 & 165.62 & 28.25 & 151.28 & 16.50 \\
\hline 7456.61 & 431057.40 & 16.00 & 8070.54 & 125.07 & 172.03 & 39678.22 & 101.78 & 792.93 & 31.18 & 6.40 & 159.86 & 27.25 & 150.24 & 15.64 \\
\hline 7456.68 & 436236.80 & 15.80 & 4200.57 & 126.13 & 180.65 & 42746.67 & 103.70 & 779.41 & 36.66 & 7.15 & 158.40 & 27.53 & 146.21 & 16.26 \\
\hline 7456.75 & 436824.40 & 15.80 & 4132.08 & 127.27 & 181.74 & 41989.34 & 103.52 & 811.53 & 34.96 & 7.12 & 162.48 & 28.81 & 147.27 & 16.05 \\
\hline 7456.81 & 415588.40 & 16.00 & 3967.88 & 120.96 & 183.77 & 44259.99 & 103.39 & 1006.64 & 36.77 & 6.80 & 159.56 & 30.24 & 144.43 & 15.75 \\
\hline 7456.88 & 385598.80 & 16.40 & 3867.46 & 118.61 & 174.18 & 42328.82 & 99.17 & 1010.26 & 34.78 & 6.74 & 162.38 & 29.75 & 141.90 & 16.28 \\
\hline 7456.94 & 352472.60 & 16.80 & 3804.74 & 117.14 & 159.59 & 40899.67 & 92.85 & 448.50 & 37.05 & 6.95 & 163.48 & 29.39 & 138.41 & 16.83 \\
\hline 7457.01 & 312358.80 & 17.40 & 3728.82 & 108.95 & 152.67 & 36504.67 & 83.34 & 482.55 & 31.55 & 6.67 & 165.22 & 29.31 & 139.54 & 16.57 \\
\hline 7457.07 & 282047.40 & 17.80 & 3535.16 & 106.30 & 148.80 & 35888.22 & 94.56 & 444.42 & 68.16 & 10.61 & 168.53 & 57.21 & 113.62 & 39.04 \\
\hline 7457.14 & 279915.00 & 17.80 & 3498.71 & 105.11 & 140.75 & 33488.35 & 95.20 & 240.77 & 67.59 & 11.61 & 174.88 & 55.65 & 111.38 & 39.89 \\
\hline 7457.20 & 282051.40 & 17.80 & 3453.32 & 103.54 & 145.55 & 33892.00 & 95.58 & 216.60 & 67.89 & 11.67 & 174.57 & 55.18 & 111.46 & 39.36 \\
\hline 7457.27 & 311564.40 & 17.40 & 3952.51 & 103.87 & 151.91 & 35504.54 & 100.66 & 185.08 & 67.41 & 12.19 & 179.66 & 56.31 & 112.11 & 40.07 \\
\hline 7457.34 & 347694.80 & 17.00 & 4060.68 & 110.29 & 153.40 & 38097.27 & 109.79 & 142.51 & 71.69 & 12.81 & 175.12 & 58.13 & 114.28 & 39.64 \\
\hline 7457.40 & 380038.20 & 16.60 & 4264.02 & 116.49 & 159.99 & 40506.70 & 104.59 & 170.25 & 37.73 & 9.13 & 168.41 & 29.86 & 137.33 & 17.00 \\
\hline 7457.47 & 399979.00 & 16.40 & 4350.37 & 123.60 & 165.88 & 40486.61 & 103.74 & 229.55 & 35.95 & 8.91 & 163.90 & 30.04 & 141.16 & 16.59 \\
\hline 7457.53 & 425148.40 & 16.00 & 4516.72 & 128.76 & 167.60 & 41236.96 & 107.49 & 227.36 & 36.39 & 8.93 & 161.45 & 30.24 & 143.21 & 16.97 \\
\hline 7457.60 & 424130.80 & 16.00 & 4111.22 & 126.92 & 182.44 & 40377.60 & 103.61 & 302.66 & 35.31 & 8.71 & 159.06 & 30.53 & 148.58 & 16.73 \\
\hline 7457.66 & 411397.40 & 16.00 & 4052.16 & 123.82 & 179.29 & 38878.95 & 100.15 & 348.62 & 33.02 & 7.84 & 155.39 & 31.71 & 148.71 & 15.80 \\
\hline 7457.73 & 409908.40 & 16.00 & 4071.83 & 119.09 & 179.40 & 38117.99 & 95.81 & 345.19 & 32.07 & 7.56 & 159.95 & 32.16 & 149.10 & 15.31 \\
\hline 7457.80 & 411360.00 & 16.00 & 4155.77 & 118.72 & 181.23 & 38545.40 & 97.60 & 327.24 & 34.35 & 7.62 & 157.89 & 32.38 & 149.65 & 14.56 \\
\hline 7457.86 & 409671.40 & 16.00 & 4137.31 & 120.87 & 187.86 & 38416.67 & 94.10 & 326.98 & 34.31 & 7.76 & 158.65 & 32.33 & 150.65 & 14.96 \\
\hline 7457.93 & 403537.20 & 16.20 & 4037.04 & 120.08 & 171.22 & 37536.18 & 92.05 & 285.11 & 33.60 & 7.60 & 163.68 & 33.26 & 150.69 & 15.09 \\
\hline 7457.99 & 402002.20 & 16.20 & 4014.18 & 116.52 & 169.47 & 37040.68 & 89.77 & 243.90 & 32.75 & 7.98 & 168.26 & 31.46 & 148.25 & 15.94 \\
\hline 7458.06 & 390595.40 & 16.40 & 3918.76 & 118.14 & 169.23 & 36618.71 & 87.99 & 235.00 & 31.45 & 8.14 & 168.77 & 30.72 & 146.60 & 16.15 \\
\hline 7458.12 & 378975.60 & 16.60 & 3830.09 & 111.00 & 168.04 & 36515.90 & 82.95 & 189.93 & 29.88 & 7.93 & 168.78 & 30.50 & 140.94 & 16.07 \\
\hline
\end{tabular}




\begin{tabular}{|c|c|c|c|c|c|c|c|c|c|c|c|c|c|c|}
\hline Depth & XRF & XRF Live Time & $\mathrm{Ti}(\mathrm{ppm})$ & $\mathrm{Cr}(\mathrm{ppm})$ & $\mathrm{Mn}(\mathrm{ppm})$ & $\mathrm{Fe}(\mathrm{ppm})$ & $\mathrm{Ni}(\mathrm{ppm})$ & $\mathrm{Cu}(\mathrm{ppm})$ & $\mathrm{Zn}(\mathrm{ppm})$ & As (ppm) & $\mathrm{Rb}(\mathrm{ppm})$ & $\mathrm{Sr}(\mathrm{ppm})$ & $\mathrm{Y}(\mathrm{ppm})$ & $\mathrm{Zr}(\mathrm{ppm})$ \\
\hline 7458.19 & 366020.40 & 16.80 & 3780.83 & 105.06 & 163.08 & 36705.40 & 82.39 & 169.29 & 28.40 & 7.42 & 166.25 & 30.46 & 138.51 & 15.03 \\
\hline 7458.25 & 365979.80 & 16.80 & 3790.58 & 110.86 & 169.73 & 37892.53 & 83.97 & 162.63 & 29.12 & 7.98 & 165.92 & 30.12 & 134.44 & 14.52 \\
\hline 7458.32 & 360261.40 & 17.00 & 3795.94 & 116.83 & 172.30 & 37214.38 & 83.42 & 198.50 & 27.56 & 7.34 & 168.80 & 31.40 & 136.19 & 14.18 \\
\hline 7458.39 & 361072.00 & 17.00 & 3822.01 & 115.96 & 173.75 & 37508.00 & 86.73 & 233.85 & 28.37 & 7.70 & 171.10 & 30.63 & 137.12 & 14.00 \\
\hline 7458.45 & 366822.60 & 17.00 & 3817.06 & 120.42 & 174.89 & 37326.62 & 93.37 & 304.13 & 29.14 & 7.21 & 173.73 & 32.36 & 139.04 & 14.20 \\
\hline 7458.52 & 379081.60 & 16.80 & 3818.02 & 118.22 & 180.77 & 37828.56 & 93.95 & 378.13 & 30.77 & 7.39 & 177.96 & 31.74 & 140.14 & 14.37 \\
\hline 7458.58 & 385193.60 & 16.60 & 3912.70 & 119.93 & 180.35 & 36238.74 & 95.19 & 356.08 & 28.86 & 6.39 & 171.82 & 31.74 & 142.57 & 13.52 \\
\hline 7458.65 & 396828.20 & 16.40 & 3900.72 & 120.94 & 181.10 & 37325.28 & 96.18 & 365.44 & 30.80 & 6.73 & 173.94 & 31.98 & 144.27 & 14.27 \\
\hline 7458.71 & 408694.20 & 16.20 & 3874.37 & 120.77 & 181.43 & 38424.70 & 98.02 & 309.82 & 31.18 & 6.27 & 175.45 & 32.53 & 143.56 & 15.06 \\
\hline 7458.78 & 420559.60 & 16.00 & 3954.06 & 123.45 & 187.02 & 39656.85 & 99.72 & 205.08 & 31.35 & 6.59 & 174.66 & 30.91 & 146.46 & 14.50 \\
\hline 7458.85 & 426116.00 & 16.00 & 3815.15 & 124.96 & 187.08 & 39331.21 & 105.65 & 120.50 & 29.30 & 6.25 & 176.88 & 33.29 & 142.05 & 14.18 \\
\hline 7458.91 & 445190.60 & 15.80 & 3766.57 & 124.84 & 191.20 & 47210.86 & 115.53 & 95.90 & 39.53 & 6.56 & 188.81 & 33.09 & 139.77 & 14.68 \\
\hline 7458.98 & 451298.60 & 15.80 & 3851.87 & 126.72 & 189.19 & 49289.81 & 116.88 & 38.04 & 42.56 & 7.14 & 185.80 & 32.14 & 136.59 & 14.82 \\
\hline 7459.04 & 453592.20 & 15.80 & 3852.96 & 126.34 & 189.30 & 49181.94 & 119.79 & 38.43 & 46.94 & 6.94 & 182.70 & 32.34 & 137.79 & 14.24 \\
\hline 7459.11 & 469974.40 & 15.60 & 3796.16 & 124.38 & 191.01 & 55408.20 & 130.44 & 38.62 & 62.21 & 7.73 & 183.97 & 33.14 & 136.73 & 14.34 \\
\hline 7459.17 & 484953.20 & 15.40 & 4037.69 & 130.90 & 192.06 & 60540.16 & 144.78 & 67.47 & 64.06 & 22.54 & 186.99 & 61.71 & 120.58 & 39.31 \\
\hline 7459.24 & 471177.40 & 15.60 & 4152.41 & 127.45 & 186.28 & 54874.71 & 139.67 & 66.82 & 58.08 & 22.21 & 176.56 & 61.83 & 124.14 & 39.15 \\
\hline 7459.30 & 466354.40 & 15.60 & 10119.00 & 121.75 & 184.35 & 52385.52 & 142.04 & 72.62 & 58.22 & 21.70 & 175.71 & 62.54 & 127.33 & 38.87 \\
\hline 7459.37 & 468763.60 & 15.60 & 10153.46 & 125.11 & 179.61 & 56764.15 & 151.88 & 88.98 & 54.45 & 42.33 & 181.51 & 92.97 & 103.76 & 61.18 \\
\hline 7459.44 & 448734.20 & 15.80 & 10093.24 & 127.39 & 169.96 & 50226.38 & 137.48 & 90.71 & 42.93 & 41.91 & 188.40 & 93.27 & 103.25 & 61.15 \\
\hline 7459.50 & 443809.60 & 16.00 & 9994.11 & 126.93 & 167.56 & 46593.93 & 123.34 & 61.38 & 46.06 & 27.75 & 187.68 & 63.33 & 122.62 & 37.59 \\
\hline 7459.57 & 446637.00 & 16.00 & 9823.18 & 123.53 & 169.71 & 46736.55 & 123.24 & 61.80 & 46.32 & 28.06 & 190.01 & 63.66 & 122.73 & 37.69 \\
\hline 7459.63 & 450674.80 & 16.00 & 3813.42 & 127.32 & 175.65 & 49232.96 & 127.04 & 56.75 & 46.85 & 28.34 & 192.91 & 63.04 & 119.66 & 37.24 \\
\hline 7459.70 & 444575.20 & 16.00 & 3733.40 & 120.48 & 176.42 & 44746.75 & 117.90 & 40.61 & 48.11 & 8.36 & 187.87 & 33.18 & 143.28 & 14.72 \\
\hline 7459.76 & 445611.80 & 16.00 & 3802.77 & 120.22 & 176.02 & 44817.70 & 121.44 & 36.37 & 46.47 & 8.02 & 181.60 & 33.34 & 144.70 & 15.12 \\
\hline 7459.83 & 437464.80 & 16.00 & 3898.33 & 120.45 & 172.25 & 44244.45 & 119.29 & 37.88 & 43.24 & 6.88 & 173.20 & 33.55 & 144.95 & 14.58 \\
\hline 7459.90 & 440794.00 & 16.00 & 4045.01 & 125.81 & 169.71 & 46062.92 & 118.39 & 37.92 & 45.56 & 7.45 & 173.84 & 33.62 & 142.56 & 14.54 \\
\hline 7459.96 & 435531.00 & 16.00 & 3888.55 & 123.76 & 164.86 & 44664.73 & 115.54 & 36.91 & 45.74 & 7.60 & 179.49 & 33.81 & 144.57 & 15.06 \\
\hline 7460.03 & 439037.80 & 16.00 & 4062.39 & 127.95 & 163.66 & 46044.07 & 113.25 & 37.44 & 47.80 & 7.91 & 180.85 & 33.80 & 142.56 & 14.69 \\
\hline 7460.09 & 432966.40 & 16.00 & 3944.05 & 121.10 & 160.09 & 45896.82 & 110.17 & 39.24 & 48.21 & 8.32 & 188.64 & 33.01 & 141.39 & 14.95 \\
\hline 7460.16 & 425668.00 & 16.00 & 3733.27 & 114.81 & 156.67 & 44897.92 & 110.85 & 37.55 & 48.38 & 9.65 & 200.49 & 33.25 & 140.19 & 14.43 \\
\hline 7460.22 & 389550.80 & 16.20 & 3499.19 & 110.00 & 151.99 & 41591.53 & 113.17 & 49.52 & 43.59 & 14.60 & 207.12 & 59.14 & 119.29 & 40.35 \\
\hline 7460.29 & 394179.80 & 16.20 & 4031.17 & 109.50 & 154.10 & 41315.22 & 111.96 & 50.48 & 40.52 & 14.40 & 206.17 & 59.78 & 117.47 & 40.58 \\
\hline
\end{tabular}




\begin{tabular}{|c|c|c|c|c|c|c|c|c|c|c|c|c|c|c|}
\hline Depth & XRF & XRF Live Time & $\mathrm{Ti}(\mathrm{ppm})$ & $\mathrm{Cr}$ (ppm) & $\mathrm{Mn}$ (ppm) & $\mathrm{Fe}(\mathrm{ppm})$ & $\mathrm{Ni}(\mathrm{ppm})$ & $\mathrm{Cu}(\mathrm{ppm})$ & $\mathrm{Zn}(\mathrm{ppm})$ & As (ppm) & $\mathrm{Rb}(\mathrm{ppm})$ & $\mathrm{Sr}(\mathrm{ppm})$ & $\mathrm{Y}(\mathrm{ppm})$ & $\mathrm{Zr}(\mathrm{ppm})$ \\
\hline 7460.35 & 394409.60 & 16.20 & 3948.14 & 110.61 & 155.90 & 40901.36 & 116.43 & 49.83 & 37.70 & 14.24 & 199.00 & 59.48 & 120.19 & 41.71 \\
\hline 7460.42 & 398612.00 & 16.20 & 4147.41 & 117.41 & 158.47 & 40617.19 & 118.33 & 51.23 & 36.04 & 14.38 & 187.59 & 59.08 & 121.37 & 41.99 \\
\hline 7460.49 & 401408.00 & 16.20 & 4325.06 & 124.06 & 163.81 & 40463.13 & 119.24 & 51.69 & 35.74 & 13.68 & 178.35 & 58.83 & 122.26 & 42.25 \\
\hline 7460.55 & 436599.80 & 16.00 & 4355.03 & 123.02 & 170.08 & 42245.71 & 119.44 & 39.85 & 40.17 & 8.47 & 176.85 & 33.13 & 145.64 & 16.96 \\
\hline 7460.62 & 446534.80 & 15.80 & 3966.10 & 123.24 & 177.94 & 50599.67 & 131.46 & 41.66 & 50.38 & 9.58 & 169.10 & 33.51 & 145.54 & 16.91 \\
\hline 7460.68 & 449410.60 & 15.80 & 3803.93 & 121.22 & 181.56 & 52701.62 & 131.62 & 42.74 & 55.73 & 9.17 & 170.20 & 34.37 & 141.34 & 16.06 \\
\hline 7460.75 & 471788.20 & 15.60 & 3745.64 & 125.62 & 198.60 & 62644.02 & 143.34 & 43.95 & 63.66 & 9.09 & 172.88 & 36.14 & 136.41 & 14.92 \\
\hline 7460.81 & 465653.80 & 15.80 & 3631.14 & 120.07 & 187.29 & 62073.26 & 136.55 & 44.55 & 62.16 & 8.96 & 183.79 & 35.52 & 138.29 & 15.23 \\
\hline 7460.88 & 457138.80 & 15.80 & 3652.88 & 124.35 & 183.11 & 62485.76 & 132.89 & 44.91 & 60.74 & 8.24 & 187.01 & 36.02 & 135.19 & 14.27 \\
\hline 7460.94 & 444978.20 & 16.00 & 3730.04 & 125.88 & 181.26 & 56754.85 & 125.05 & 42.56 & 50.97 & 7.23 & 185.61 & 36.64 & 132.24 & 13.26 \\
\hline 7461.01 & 429100.40 & 16.00 & 3628.72 & 114.67 & 180.71 & 54634.80 & 119.79 & 59.54 & 45.20 & 17.85 & 190.89 & 62.58 & 113.66 & 35.00 \\
\hline 7461.08 & 396821.40 & 16.40 & 3298.05 & 100.26 & 164.21 & 45512.52 & 108.99 & 57.11 & 39.51 & 17.65 & 196.70 & 62.23 & 115.19 & 35.37 \\
\hline 7461.14 & 386169.60 & 16.40 & 3320.41 & 102.85 & 169.05 & 45410.82 & 108.08 & 56.23 & 40.98 & 17.34 & 189.40 & 62.38 & 112.57 & 34.93 \\
\hline 7461.21 & 381464.00 & 16.40 & 3779.95 & 100.97 & 171.23 & 45264.91 & 108.05 & 53.98 & 41.79 & 17.45 & 179.83 & 60.84 & 115.16 & 35.65 \\
\hline 7461.27 & 366808.80 & 16.60 & 3732.72 & 97.05 & 163.73 & 42050.72 & 102.98 & 53.31 & 42.61 & 17.41 & 178.44 & 58.36 & 117.03 & 36.98 \\
\hline 7461.34 & 362658.00 & 16.80 & 3949.51 & 105.98 & 162.03 & 40284.59 & 100.36 & 34.75 & 43.33 & 6.64 & 169.64 & 31.15 & 138.35 & 14.60 \\
\hline 7461.40 & 383382.40 & 16.60 & 4269.47 & 116.11 & 165.76 & 43735.22 & 103.77 & 34.81 & 48.22 & 7.04 & 161.91 & 29.16 & 136.98 & 15.11 \\
\hline 7461.47 & 407213.80 & 16.40 & 4369.64 & 117.21 & 171.87 & 47054.92 & 111.59 & 33.62 & 51.23 & 7.80 & 157.54 & 28.67 & 139.68 & 16.30 \\
\hline 7461.54 & 408899.20 & 16.40 & 3920.71 & 117.41 & 170.57 & 45322.60 & 108.57 & 34.90 & 47.93 & 8.21 & 159.29 & 28.08 & 139.80 & 16.47 \\
\hline 7461.60 & 419956.40 & 16.20 & 3957.59 & 124.86 & 172.68 & 46682.49 & 107.63 & 34.78 & 47.79 & 7.87 & 163.49 & 28.20 & 139.07 & 15.69 \\
\hline 7461.67 & 425737.20 & 16.00 & 3931.51 & 131.38 & 172.64 & 45985.81 & 109.62 & 34.79 & 44.70 & 7.62 & 170.93 & 28.40 & 135.39 & 17.21 \\
\hline 7461.73 & 409807.60 & 16.00 & 3716.67 & 125.38 & 171.10 & 41441.67 & 110.42 & 41.32 & 36.59 & 6.65 & 172.43 & 28.30 & 138.23 & 17.88 \\
\hline 7461.80 & 406060.80 & 16.00 & 3455.57 & 118.56 & 190.48 & 42648.22 & 115.39 & 43.57 & 39.41 & 7.28 & 179.36 & 29.36 & 135.67 & 16.01 \\
\hline 7461.86 & 415243.20 & 16.00 & 3326.42 & 117.72 & 198.52 & 44155.46 & 129.03 & 44.33 & 40.42 & 7.29 & 190.19 & 30.24 & 133.50 & 16.19 \\
\hline 7461.93 & 414571.20 & 16.00 & 3081.22 & 111.31 & 202.09 & 42872.12 & 135.85 & 44.93 & 38.56 & 7.12 & 199.29 & 30.39 & 136.76 & 15.77 \\
\hline 7461.99 & 422782.60 & 16.00 & 3110.36 & 112.13 & 205.42 & 44375.81 & 139.61 & 43.89 & 41.73 & 7.11 & 199.31 & 29.75 & 140.80 & 15.35 \\
\hline 7462.06 & 432421.80 & 16.00 & 3290.29 & 123.13 & 210.51 & 47753.48 & 139.30 & 38.38 & 46.91 & 7.75 & 198.61 & 30.50 & 140.70 & 14.50 \\
\hline 7462.13 & 445082.80 & 15.80 & 4322.00 & 129.17 & 189.21 & 46002.12 & 136.36 & 39.14 & 45.87 & 7.14 & 198.59 & 30.16 & 142.97 & 15.78 \\
\hline 7462.19 & 429777.00 & 16.00 & 4023.30 & 113.69 & 232.63 & 41692.70 & 119.02 & 36.07 & 41.93 & 5.89 & 187.89 & 27.10 & 131.48 & 14.45 \\
\hline 7462.26 & 426697.60 & 16.00 & 4203.38 & 109.06 & 231.68 & 45347.05 & 118.37 & 35.35 & 45.89 & 6.85 & 175.92 & 28.56 & 128.75 & 15.37 \\
\hline 7462.32 & 423844.80 & 16.00 & 4180.91 & 101.78 & 230.52 & 46172.13 & 117.86 & 36.46 & 47.47 & 6.64 & 172.67 & 29.25 & 127.38 & 14.18 \\
\hline 7462.39 & 395997.00 & 16.20 & 4132.34 & 92.19 & 226.07 & 41468.71 & 111.58 & 41.50 & 44.74 & 10.60 & 179.34 & 55.26 & 105.65 & 36.04 \\
\hline 7462.45 & 350730.60 & 16.80 & 3189.49 & 84.61 & 223.27 & 37522.46 & 99.01 & 43.10 & 38.43 & 9.92 & 168.58 & 54.34 & 102.52 & 34.15 \\
\hline
\end{tabular}




\begin{tabular}{|c|c|c|c|c|c|c|c|c|c|c|c|c|c|c|}
\hline Depth & XRF & XRF Live Time & $\mathrm{Ti}(\mathrm{ppm})$ & $\mathrm{Cr}(\mathrm{ppm})$ & $\mathrm{Mn}(\mathrm{ppm})$ & $\mathrm{Fe}(\mathrm{ppm})$ & $\mathrm{Ni}(\mathrm{ppm})$ & $\mathrm{Cu}(\mathrm{ppm})$ & $\mathrm{Zn}(\mathrm{ppm})$ & As (ppm) & $\mathrm{Rb}(\mathrm{ppm})$ & $\mathrm{Sr}(\mathrm{ppm})$ & $\mathrm{Y}(\mathrm{ppm})$ & $\mathrm{Zr}(\mathrm{ppm})$ \\
\hline 7462.52 & 347849.20 & 16.80 & 3695.65 & 97.82 & 173.47 & 38875.60 & 98.37 & 51.37 & 39.98 & 10.35 & 169.01 & 56.42 & 115.34 & 34.89 \\
\hline 7462.59 & 339053.00 & 17.00 & 3672.18 & 103.34 & 173.10 & 33871.62 & 89.38 & 59.71 & 32.93 & 9.06 & 164.60 & 53.95 & 112.12 & 34.42 \\
\hline 7462.65 & 320440.00 & 17.20 & 3597.83 & 96.20 & 168.15 & 30523.03 & 78.61 & 76.27 & 26.43 & 9.16 & 157.61 & 53.88 & 112.42 & 34.61 \\
\hline 7462.72 & 328330.20 & 17.20 & 3581.68 & 95.00 & 170.94 & 30752.19 & 71.73 & 80.36 & 22.06 & 4.69 & 153.89 & 26.50 & 131.13 & 12.90 \\
\hline 7462.78 & 339409.80 & 17.00 & 3582.75 & 99.43 & 175.40 & 30592.42 & 70.33 & 85.19 & 19.40 & 4.58 & 156.55 & 26.57 & 130.46 & 14.45 \\
\hline 7462.85 & 329917.60 & 17.00 & 3616.80 & 110.47 & 180.67 & 30094.73 & 68.88 & 100.76 & 16.73 & 4.06 & 154.28 & 27.15 & 128.69 & 14.98 \\
\hline 7462.91 & 328039.60 & 17.00 & 3643.57 & 106.98 & 178.23 & 29408.73 & 67.06 & 116.10 & 16.61 & 3.61 & 157.82 & 29.66 & 131.52 & 15.72 \\
\hline 7462.98 & 330183.40 & 17.00 & 3586.51 & 109.92 & 174.20 & 29025.46 & 68.79 & 124.35 & 16.19 & 3.00 & 160.55 & 30.12 & 130.98 & 16.94 \\
\hline 7463.04 & 348063.20 & 16.80 & 3721.57 & 115.26 & 179.00 & 29447.34 & 75.54 & 147.75 & 15.41 & 2.84 & 158.00 & 32.37 & 134.79 & 17.93 \\
\hline 7463.11 & 361735.60 & 16.80 & 3769.27 & 116.97 & 178.81 & 30263.19 & 79.58 & 157.12 & 16.31 & 2.92 & 161.77 & 34.47 & 136.26 & 17.20 \\
\hline 7463.18 & 369894.00 & 16.80 & 4965.24 & 113.58 & 175.35 & 30753.16 & 83.10 & 152.88 & 17.43 & 3.30 & 157.47 & 34.86 & 136.11 & 18.01 \\
\hline 7463.24 & 364203.20 & 16.80 & 5067.18 & 118.94 & 174.89 & 30957.17 & 82.75 & 143.51 & 16.47 & 3.23 & 158.67 & 34.18 & 137.45 & 17.78 \\
\hline 7463.31 & 361626.80 & 16.80 & 5223.63 & 129.40 & 180.57 & 30823.83 & 81.51 & 131.52 & 16.49 & 2.92 & 166.91 & 32.63 & 138.61 & 18.64 \\
\hline 7463.37 & 335227.40 & 17.00 & 4931.92 & 119.26 & 165.00 & 29182.69 & 75.05 & 112.32 & 16.18 & 2.75 & 171.07 & 31.52 & 137.38 & 17.73 \\
\hline 7463.44 & 335611.00 & 17.00 & 4924.85 & 121.41 & 159.08 & 29912.61 & 80.55 & 137.04 & 17.41 & 3.21 & 174.27 & 30.57 & 141.58 & 18.10 \\
\hline 7463.50 & 335155.80 & 17.00 & 3560.90 & 111.09 & 155.00 & 30613.60 & 83.95 & 159.05 & 18.25 & 3.47 & 175.78 & 30.63 & 142.46 & 16.42 \\
\hline 7463.57 & 343533.60 & 17.00 & 3380.93 & 104.56 & 199.55 & 34369.99 & 85.59 & 160.42 & 19.38 & 3.57 & 176.61 & 30.87 & 139.52 & 15.75 \\
\hline 7463.64 & 361400.80 & 16.80 & 3212.77 & 96.84 & 202.59 & 36143.01 & 95.20 & 164.58 & 20.30 & 4.20 & 181.14 & 36.49 & 139.79 & 13.58 \\
\hline 7463.70 & 375516.40 & 16.80 & 3363.95 & 104.01 & 206.67 & 37302.40 & 101.64 & 164.21 & 21.10 & 4.18 & 185.33 & 38.42 & 142.01 & 13.68 \\
\hline 7463.77 & 370316.40 & 16.80 & 3517.31 & 104.75 & 209.44 & 36564.87 & 97.53 & 138.03 & 19.68 & 3.89 & 178.66 & 38.26 & 139.31 & 13.41 \\
\hline 7463.83 & 391281.20 & 16.60 & 3584.71 & 107.25 & 213.93 & 38091.95 & 100.97 & 99.67 & 21.80 & 3.90 & 184.32 & 37.51 & 139.38 & 15.29 \\
\hline 7463.90 & 398028.00 & 16.40 & 3691.04 & 106.95 & 179.15 & 35151.67 & 102.75 & 1244.01 & 20.60 & 3.15 & 188.96 & 39.00 & 139.53 & 15.64 \\
\hline 7463.96 & 387174.60 & 16.60 & 3672.24 & 103.56 & 196.82 & 35177.21 & 95.42 & 1370.22 & 20.23 & 2.77 & 211.66 & 40.12 & 133.93 & 16.34 \\
\hline 7464.03 & 378926.20 & 16.60 & 3832.15 & 103.33 & 203.64 & 35695.46 & 91.82 & 1357.80 & 20.47 & 2.46 & 202.54 & 38.63 & 130.03 & 16.04 \\
\hline 7464.09 & 369207.60 & 16.60 & 3792.81 & 98.93 & 208.58 & 36543.87 & 92.22 & 1347.98 & 20.95 & 1.94 & 198.41 & 37.64 & 127.03 & 15.93 \\
\hline 7464.16 & 332300.80 & 17.00 & 6078.86 & 95.56 & 202.70 & 34071.27 & 88.22 & 1352.62 & 17.60 & 1.52 & 189.95 & 38.60 & 124.02 & 15.64 \\
\hline 7464.23 & 330318.20 & 17.20 & 6022.21 & 98.57 & 208.99 & 34220.52 & 86.42 & 247.91 & 19.40 & 2.28 & 196.04 & 37.87 & 124.55 & 15.43 \\
\hline 7464.29 & 334292.40 & 17.20 & 6146.34 & 100.66 & 189.80 & 33503.75 & 95.00 & 111.50 & 20.40 & 2.27 & 164.25 & 31.71 & 126.10 & 15.17 \\
\hline 7464.36 & 347375.60 & 17.00 & 6116.09 & 99.65 & 185.03 & 33878.62 & 103.80 & 111.61 & 20.58 & 2.63 & 160.90 & 30.97 & 127.39 & 15.12 \\
\hline 7464.42 & 359401.40 & 17.00 & 5932.22 & 95.90 & 181.21 & 33193.32 & 107.74 & 105.83 & 20.52 & 2.97 & 171.62 & 32.70 & 127.04 & 15.24 \\
\hline 7464.49 & 380577.60 & 16.80 & 3686.03 & 105.78 & 186.44 & 33933.14 & 113.39 & 109.60 & 21.02 & 2.80 & 171.99 & 34.17 & 130.22 & 13.24 \\
\hline 7464.55 & 389065.60 & 16.60 & 3677.72 & 107.08 & 179.84 & 34546.40 & 126.38 & 54.42 & 21.77 & 3.25 & 165.41 & 32.69 & 132.44 & 12.91 \\
\hline 7464.62 & 403074.40 & 16.40 & 3685.64 & 108.85 & 185.14 & 37316.75 & 133.96 & 48.19 & 26.34 & 3.93 & 169.60 & 32.02 & 133.99 & 13.41 \\
\hline
\end{tabular}




\begin{tabular}{|c|c|c|c|c|c|c|c|c|c|c|c|c|c|c|}
\hline Depth & XRF & XRF Live Time & $\mathrm{Ti}(\mathrm{ppm})$ & $\mathrm{Cr}(\mathrm{ppm})$ & $\mathrm{Mn}(\mathrm{ppm})$ & $\mathrm{Fe}(\mathrm{ppm})$ & $\mathrm{Ni}(\mathrm{ppm})$ & $\mathrm{Cu}(\mathrm{ppm})$ & $\mathrm{Zn}(\mathrm{ppm})$ & As (ppm) & $\mathrm{Rb}(\mathrm{ppm})$ & $\mathrm{Sr}(\mathrm{ppm})$ & $\mathrm{Y}(\mathrm{ppm})$ & $\mathrm{Zr}(\mathrm{ppm})$ \\
\hline 7464.69 & 402938.80 & 16.40 & 3552.83 & 116.24 & 184.06 & 38213.40 & 139.55 & 45.49 & 27.61 & 4.69 & 173.57 & 31.90 & 134.68 & 12.38 \\
\hline 7464.75 & 403498.00 & 16.40 & 3577.61 & 124.70 & 187.05 & 39304.78 & 141.51 & 46.15 & 29.23 & 4.85 & 178.07 & 29.81 & 137.24 & 13.00 \\
\hline 7464.82 & 399011.80 & 16.40 & 3342.86 & 118.36 & 186.94 & 39317.11 & 140.41 & 36.39 & 30.42 & 5.45 & 195.76 & 28.63 & 136.64 & 13.43 \\
\hline 7464.88 & 387128.20 & 16.60 & 3281.33 & 122.04 & 175.97 & 38957.37 & 132.29 & 35.00 & 29.90 & 5.20 & 200.29 & 28.81 & 136.99 & 13.25 \\
\hline 7464.95 & 373824.40 & 16.60 & 3277.66 & 133.89 & 176.79 & 37411.59 & 124.59 & 34.53 & 27.12 & 5.44 & 204.45 & 29.08 & 138.63 & 13.18 \\
\hline 7465.01 & 370986.20 & 16.80 & 3280.43 & 135.61 & 183.93 & 36474.65 & 119.66 & 34.47 & 26.84 & 4.87 & 209.95 & 30.75 & 135.57 & 13.84 \\
\hline 7465.08 & 371114.20 & 16.80 & 4359.47 & 137.22 & 186.28 & 36227.42 & 119.87 & 32.89 & 27.70 & 5.03 & 205.73 & 31.06 & 135.52 & 13.14 \\
\hline 7465.14 & 378079.40 & 16.60 & 4452.09 & 145.72 & 198.69 & 37918.47 & 123.38 & 33.34 & 27.66 & 4.58 & 202.26 & 33.85 & 132.24 & 12.59 \\
\hline 7465.21 & 386357.00 & 16.40 & 4517.43 & 147.02 & 203.86 & 40922.71 & 129.71 & 34.04 & 32.32 & 5.34 & 201.94 & 34.48 & 126.23 & 11.77 \\
\hline 7465.28 & 388212.00 & 16.40 & 4647.49 & 145.05 & 201.81 & 41036.00 & 131.40 & 35.39 & 30.94 & 5.17 & 199.61 & 33.66 & 127.27 & 10.79 \\
\hline 7465.34 & 389927.60 & 16.40 & 4790.79 & 145.67 & 196.23 & 40724.06 & 129.29 & 35.80 & 29.65 & 5.09 & 191.12 & 32.21 & 131.58 & 10.70 \\
\hline 7465.41 & 398888.00 & 16.20 & 3833.81 & 145.89 & 194.34 & 41174.88 & 129.10 & 38.10 & 29.57 & 4.90 & 186.51 & 33.71 & 134.40 & 11.86 \\
\hline 7465.47 & 411177.40 & 16.20 & 3992.14 & 140.90 & 186.19 & 42516.27 & 129.36 & 39.27 & 35.05 & 5.51 & 175.01 & 30.27 & 138.66 & 12.94 \\
\hline 7465.54 & 417253.60 & 16.20 & 4097.30 & 140.81 & 187.87 & 42385.01 & 132.78 & 40.10 & 34.91 & 5.14 & 172.45 & 29.36 & 140.53 & 14.05 \\
\hline 7465.60 & 413492.60 & 16.40 & 4024.27 & 133.34 & 180.84 & 41456.74 & 130.16 & 40.20 & 35.07 & 4.59 & 172.92 & 31.00 & 138.99 & 13.60 \\
\hline 7465.67 & 414872.40 & 16.20 & 3937.88 & 129.23 & 180.72 & 41392.17 & 127.05 & 40.86 & 35.89 & 4.45 & 178.68 & 31.82 & 137.49 & 13.97 \\
\hline 7465.73 & 408599.80 & 16.20 & 3831.23 & 125.71 & 178.04 & 40388.85 & 121.32 & 38.99 & 36.83 & 4.33 & 186.56 & 31.40 & 134.87 & 13.00 \\
\hline 7465.80 & 399763.20 & 16.20 & 3833.54 & 132.36 & 178.79 & 38383.26 & 122.42 & 54.96 & 35.55 & 8.49 & 194.05 & 64.76 & 112.05 & 37.07 \\
\hline 7465.87 & 395865.60 & 16.20 & 3719.41 & 125.81 & 187.75 & 36836.81 & 117.03 & 52.95 & 30.48 & 8.83 & 196.54 & 68.51 & 112.44 & 35.56 \\
\hline 7465.93 & 412569.80 & 16.00 & 3719.67 & 127.28 & 201.37 & 41339.57 & 121.29 & 51.02 & 33.59 & 9.22 & 197.82 & 69.90 & 107.01 & 36.07 \\
\hline 7466.00 & 423464.00 & 16.00 & 3835.20 & 130.02 & 206.79 & 44746.74 & 134.43 & 49.17 & 38.74 & 9.12 & 199.17 & 71.22 & 105.62 & 35.82 \\
\hline 7466.06 & 415990.40 & 16.20 & 3799.77 & 145.39 & 209.47 & 45844.11 & 143.64 & 49.36 & 37.79 & 9.15 & 240.64 & 71.06 & 106.85 & 36.53 \\
\hline 7466.13 & 412343.60 & 16.40 & 3772.63 & 159.16 & 209.72 & 45482.42 & 138.58 & 34.61 & 34.26 & 4.41 & 273.68 & 36.40 & 132.58 & 13.00 \\
\hline 7466.19 & 410227.80 & 16.40 & 3802.27 & 176.43 & 204.24 & 47354.90 & 139.96 & 36.88 & 41.58 & 4.20 & 295.21 & 31.43 & 133.34 & 14.28 \\
\hline 7466.26 & 393064.40 & 16.60 & 3850.22 & 177.48 & 193.44 & 43610.17 & 137.60 & 35.69 & 39.60 & 3.94 & 294.04 & 31.34 & 137.93 & 15.69 \\
\hline 7466.33 & 395522.60 & 16.60 & 3854.32 & 177.72 & 196.37 & 41844.92 & 131.02 & 35.08 & 37.19 & 3.90 & 297.12 & 31.38 & 140.75 & 16.15 \\
\hline 7466.39 & 416754.20 & 16.40 & 4039.02 & 170.86 & 199.31 & 42522.98 & 129.68 & 35.61 & 40.03 & 4.04 & 259.73 & 30.78 & 143.43 & 14.97 \\
\hline 7466.46 & 423730.60 & 16.20 & 4038.97 & 155.84 & 197.95 & 42481.86 & 127.24 & 31.86 & 40.15 & 4.44 & 238.90 & 32.48 & 138.07 & 14.43 \\
\hline 7466.52 & 420810.20 & 16.20 & 4056.42 & 146.26 & 194.42 & 39182.47 & 118.71 & 30.31 & 33.90 & 3.74 & 218.53 & 34.69 & 139.17 & 14.90 \\
\hline 7466.59 & 424339.80 & 16.00 & 3882.43 & 154.61 & 208.02 & 38313.89 & 112.67 & 29.78 & 32.40 & 3.73 & 236.19 & 35.41 & 137.72 & 14.48 \\
\hline 7466.65 & 412296.40 & 16.00 & 3878.63 & 158.56 & 203.60 & 37134.91 & 105.87 & 29.56 & 30.69 & 3.95 & 246.87 & 35.29 & 137.24 & 14.41 \\
\hline 7466.72 & 409706.40 & 16.00 & 3942.75 & 151.47 & 199.13 & 37526.73 & 103.57 & 29.22 & 29.43 & 3.83 & 237.26 & 35.33 & 133.08 & 14.66 \\
\hline 7466.78 & 413970.60 & 16.00 & 4008.11 & 152.68 & 200.68 & 39801.40 & 107.37 & 30.06 & 32.64 & 3.65 & 221.07 & 34.22 & 133.53 & 14.76 \\
\hline
\end{tabular}




\begin{tabular}{|c|c|c|c|c|c|c|c|c|c|c|c|c|c|c|}
\hline Depth & XRF & XRF Live Time & $\mathrm{Ti}(\mathrm{ppm})$ & $\mathrm{Cr}(\mathrm{ppm})$ & $\mathrm{Mn}(\mathrm{ppm})$ & $\mathrm{Fe}(\mathrm{ppm})$ & $\mathrm{Ni}(\mathrm{ppm})$ & $\mathrm{Cu}(\mathrm{ppm})$ & $\mathrm{Zn}(\mathrm{ppm})$ & As (ppm) & $\mathrm{Rb}(\mathrm{ppm})$ & $\mathrm{Sr}(\mathrm{ppm})$ & $\mathrm{Y}(\mathrm{ppm})$ & $\mathrm{Zr}(\mathrm{ppm})$ \\
\hline 7466.85 & 413226.20 & 16.00 & 4022.22 & 148.52 & 209.88 & 40016.37 & 109.53 & 29.19 & 33.77 & 3.55 & 215.85 & 36.99 & 131.07 & 14.55 \\
\hline 7466.92 & 410196.00 & 16.20 & 4199.64 & 144.70 & 205.27 & 41421.00 & 110.64 & 29.17 & 36.12 & 3.05 & 202.65 & 36.48 & 134.53 & 14.39 \\
\hline 7466.98 & 362956.00 & 16.80 & 4169.78 & 127.32 & 220.49 & 40750.02 & 107.69 & 31.75 & 35.21 & 2.55 & 181.33 & 33.88 & 127.26 & 14.52 \\
\hline 7467.05 & 317826.40 & 17.20 & 4050.98 & 122.14 & 230.97 & 39206.39 & 100.37 & 31.15 & 33.48 & 1.76 & 178.43 & 37.69 & 122.27 & 13.53 \\
\hline 7467.11 & 316883.60 & 17.20 & 4024.31 & 115.32 & 242.83 & 36785.95 & 95.16 & 30.00 & 29.25 & 1.19 & 181.93 & 43.24 & 121.98 & 12.30 \\
\hline 7467.18 & 312391.60 & 17.40 & 4084.25 & 113.12 & 241.51 & 36489.76 & 89.60 & 30.67 & 28.00 & 1.32 & 179.11 & 41.71 & 125.02 & 11.96 \\
\hline 7467.24 & 304218.40 & 17.40 & 3708.99 & 96.33 & 307.38 & 34186.77 & 84.12 & 28.73 & 23.60 & 1.60 & 182.82 & 55.63 & 111.24 & 10.45 \\
\hline 7467.31 & 333135.60 & 17.00 & 3615.26 & 100.52 & 290.26 & 35946.78 & 87.95 & 28.52 & 25.86 & 2.06 & 186.23 & 58.09 & 116.60 & 10.94 \\
\hline 7467.38 & 366867.20 & 16.80 & 3329.34 & 99.46 & 341.39 & 36513.05 & 91.75 & 27.08 & 26.44 & 3.00 & 194.51 & 69.73 & 111.56 & 11.26 \\
\hline 7467.44 & 353229.20 & 17.00 & 3301.13 & 95.61 & 344.84 & 36309.71 & 91.37 & 26.73 & 27.05 & 3.06 & 191.64 & 69.07 & 111.26 & 11.62 \\
\hline 7467.51 & 353203.80 & 17.00 & 3263.47 & 96.96 & 334.28 & 37098.60 & 96.21 & 26.76 & 28.14 & 3.33 & 191.67 & 67.66 & 113.20 & 12.66 \\
\hline 7467.57 & 367450.20 & 16.80 & 3686.32 & 106.53 & 279.45 & 41913.32 & 100.64 & 29.63 & 32.47 & 3.51 & 176.71 & 54.11 & 128.55 & 14.66 \\
\hline 7467.64 & 399421.00 & 16.60 & 3140.61 & 91.95 & 378.10 & 40731.24 & 89.89 & 24.75 & 29.17 & 3.54 & 190.60 & 90.00 & 112.17 & 12.58 \\
\hline 7467.70 & 415016.80 & 16.40 & 2801.75 & 79.92 & 442.15 & 39781.20 & 79.06 & 22.76 & 25.51 & 3.23 & 190.95 & 105.24 & 100.99 & 10.59 \\
\hline 7467.77 & 424855.80 & 16.20 & 2150.81 & 66.27 & 551.19 & 38167.31 & 64.64 & 21.58 & 21.33 & 3.47 & 198.29 & 128.28 & 80.03 & 9.62 \\
\hline 7467.83 & 431621.80 & 16.00 & 1501.45 & 48.52 & 663.65 & 36813.06 & 57.65 & 18.77 & 18.32 & 3.04 & 202.43 & 154.25 & 58.28 & 8.25 \\
\hline 7467.90 & 421037.80 & 16.20 & 1113.40 & 40.82 & 714.82 & 33457.17 & 52.51 & 17.81 & 14.55 & 2.22 & 205.55 & 171.25 & 43.96 & 7.08 \\
\hline 7467.97 & 396207.20 & 16.40 & 4603.02 & 60.15 & 626.20 & 34293.74 & 63.80 & 23.81 & 16.61 & 2.22 & 192.22 & 136.28 & 60.71 & 8.42 \\
\hline 7468.03 & 362150.80 & 16.60 & 5325.84 & 80.52 & 499.47 & 34412.57 & 73.29 & 27.80 & 21.54 & 2.11 & 177.95 & 105.53 & 83.79 & 11.58 \\
\hline 7468.10 & 333071.80 & 17.00 & 5977.84 & 94.60 & 377.14 & 36271.29 & 86.03 & 28.61 & 28.19 & 1.98 & 165.71 & 76.07 & 104.53 & 13.14 \\
\hline 7468.16 & 334589.80 & 17.00 & 6728.72 & 112.24 & 273.72 & 39918.55 & 90.97 & 32.08 & 31.79 & 2.33 & 164.51 & 48.77 & 123.36 & 13.50 \\
\hline 7468.23 & 348797.00 & 16.80 & 6898.47 & 116.25 & 220.19 & 43241.23 & 102.88 & 33.61 & 39.23 & 3.95 & 167.13 & 33.65 & 131.82 & 13.04 \\
\hline 7468.29 & 358051.80 & 16.60 & 3988.39 & 114.24 & 209.43 & 43217.36 & 101.40 & 32.13 & 40.38 & 4.05 & 167.67 & 30.25 & 132.72 & 13.39 \\
\hline 7468.36 & 372487.20 & 16.60 & 3982.20 & 110.01 & 204.96 & 42772.20 & 106.84 & 33.51 & 39.92 & 4.76 & 172.08 & 29.04 & 133.09 & 13.37 \\
\hline 7468.43 & 390695.00 & 16.40 & 4035.48 & 110.15 & 197.64 & 41907.36 & 107.49 & 38.58 & 37.90 & 5.61 & 172.79 & 29.69 & 137.60 & 12.56 \\
\hline 7468.49 & 381701.60 & 16.60 & 3950.10 & 114.04 & 181.48 & 38192.89 & 108.38 & 40.55 & 36.62 & 5.68 & 164.66 & 28.51 & 138.82 & 12.94 \\
\hline 7468.56 & 369951.40 & 16.80 & 3971.03 & 115.43 & 164.25 & 33595.71 & 102.82 & 39.82 & 30.41 & 4.93 & 155.62 & 21.95 & 139.76 & 13.63 \\
\hline 7468.62 & 362688.40 & 17.00 & 3877.55 & 113.08 & 171.41 & 33244.77 & 108.49 & 39.17 & 29.96 & 5.19 & 149.44 & 23.57 & 135.69 & 13.38 \\
\hline 7468.69 & 357257.20 & 17.00 & 3694.72 & 113.51 & 180.68 & 34880.67 & 111.16 & 38.02 & 29.35 & 4.93 & 146.48 & 25.31 & 129.03 & 13.13 \\
\hline 7468.75 & 347600.00 & 17.00 & 3557.20 & 112.10 & 187.21 & 36977.10 & 113.46 & 38.83 & 32.77 & 4.94 & 143.09 & 27.05 & 122.40 & 13.34 \\
\hline 7468.82 & 328541.60 & 17.20 & 3417.52 & 110.24 & 204.37 & 36638.58 & 108.91 & 41.89 & 32.22 & 4.69 & 148.67 & 29.73 & 116.62 & 12.71 \\
\hline 7468.88 & 310275.60 & 17.40 & 3390.13 & 111.34 & 211.23 & 38316.23 & 108.53 & 55.08 & 36.84 & 11.89 & 152.18 & 68.23 & 101.24 & 31.28 \\
\hline 7468.95 & 287735.40 & 17.60 & 3348.03 & 111.18 & 199.45 & 36747.69 & 103.91 & 61.66 & 36.19 & 11.79 & 150.25 & 66.50 & 102.64 & 31.99 \\
\hline
\end{tabular}




\begin{tabular}{|c|c|c|c|c|c|c|c|c|c|c|c|c|c|c|}
\hline Depth & XRF & XRF Live Time & $\mathrm{Ti}(\mathrm{ppm})$ & $\mathrm{Cr}$ (ppm) & $\mathrm{Mn}$ (ppm) & $\mathrm{Fe}(\mathrm{ppm})$ & $\mathrm{Ni}(\mathrm{ppm})$ & $\mathrm{Cu}(\mathrm{ppm})$ & $\mathrm{Zn}(\mathrm{ppm})$ & As (ppm) & $\mathrm{Rb}(\mathrm{ppm})$ & $\mathrm{Sr}(\mathrm{ppm})$ & $\mathrm{Y}(\mathrm{ppm})$ & $\mathrm{Zr}(\mathrm{ppm})$ \\
\hline 7469.02 & 267994.40 & 17.80 & 3413.63 & 108.30 & 191.42 & 34695.16 & 95.52 & 66.02 & 35.39 & 11.65 & 146.81 & 64.82 & 105.44 & 32.14 \\
\hline 7469.08 & 264789.60 & 17.80 & 3421.02 & 110.73 & 181.26 & 31638.29 & 91.20 & 66.32 & 30.78 & 10.90 & 146.80 & 62.88 & 108.49 & 32.69 \\
\hline 7469.15 & 292245.00 & 17.40 & 3422.12 & 104.32 & 166.10 & 32534.04 & 98.18 & 62.48 & 31.84 & 11.40 & 142.24 & 60.84 & 115.40 & 33.59 \\
\hline 7469.21 & 326621.00 & 17.00 & 3432.26 & 103.86 & 169.64 & 32599.98 & 100.47 & 48.85 & 27.71 & 4.66 & 138.81 & 24.03 & 131.90 & 15.50 \\
\hline 7469.28 & 365018.40 & 16.60 & 3489.68 & 108.92 & 174.58 & 34290.41 & 104.10 & 43.33 & 27.47 & 5.21 & 143.11 & 23.44 & 134.25 & 14.35 \\
\hline 7469.34 & 405316.60 & 16.20 & 3355.88 & 107.68 & 185.18 & 36353.55 & 112.05 & 37.56 & 28.72 & 5.79 & 152.34 & 26.78 & 132.96 & 13.40 \\
\hline 7469.41 & 430871.60 & 16.00 & 3313.54 & 105.03 & 188.96 & 38163.49 & 118.45 & 36.74 & 30.26 & 6.67 & 164.50 & 28.86 & 134.48 & 13.37 \\
\hline 7469.48 & 435326.60 & 16.00 & 3385.74 & 109.95 & 184.47 & 38350.24 & 118.35 & 38.81 & 29.36 & 6.64 & 166.98 & 29.38 & 135.06 & 13.72 \\
\hline 7469.54 & 432866.40 & 16.00 & 3600.43 & 113.27 & 174.40 & 37413.55 & 118.84 & 43.49 & 29.11 & 6.43 & 171.83 & 29.53 & 137.19 & 14.16 \\
\hline 7469.61 & 431538.60 & 16.00 & 3612.32 & 115.00 & 167.13 & 36725.42 & 117.51 & 51.80 & 30.57 & 6.02 & 179.24 & 29.87 & 140.04 & 14.56 \\
\hline 7469.67 & 427831.80 & 16.00 & 3712.84 & 121.24 & 152.58 & 35119.96 & 111.35 & 58.29 & 29.07 & 5.95 & 179.35 & 28.61 & 143.14 & 15.17 \\
\hline 7469.74 & 408154.40 & 16.20 & 3621.86 & 119.27 & 153.44 & 34666.76 & 106.93 & 64.81 & 28.57 & 5.74 & 175.46 & 28.40 & 140.67 & 15.34 \\
\hline 7469.80 & 404695.40 & 16.20 & 3484.12 & 115.90 & 171.95 & 34884.15 & 108.42 & 68.41 & 28.89 & 5.97 & 187.53 & 32.43 & 135.92 & 13.96 \\
\hline 7469.87 & 402210.20 & 16.20 & 3353.10 & 116.28 & 169.20 & 35537.97 & 107.22 & 71.84 & 29.99 & 6.24 & 187.60 & 33.84 & 132.92 & 13.27 \\
\hline 7469.93 & 399663.40 & 16.20 & 3921.65 & 112.32 & 172.98 & 35587.96 & 104.47 & 65.93 & 28.50 & 6.23 & 179.99 & 34.00 & 130.00 & 14.06 \\
\hline 7470.00 & 397132.80 & 16.20 & 3933.79 & 106.99 & 177.21 & 35969.00 & 106.23 & 69.91 & 27.63 & 6.14 & 177.48 & 32.57 & 129.87 & 14.59 \\
\hline 7470.07 & 414241.40 & 16.00 & 3720.15 & 100.24 & 233.72 & 35398.02 & 102.83 & 82.26 & 25.95 & 6.00 & 190.64 & 35.13 & 128.12 & 13.23 \\
\hline 7470.13 & 417043.40 & 16.00 & 3913.14 & 105.86 & 224.37 & 35937.45 & 103.31 & 108.17 & 26.10 & 5.91 & 180.79 & 31.14 & 133.11 & 14.73 \\
\hline 7470.20 & 411728.40 & 16.00 & 3980.66 & 108.29 & 224.32 & 35216.16 & 99.52 & 113.47 & 27.25 & 5.84 & 186.21 & 29.19 & 135.65 & 15.15 \\
\hline 7470.26 & 407770.80 & 16.00 & 3227.04 & 105.03 & 246.48 & 34791.40 & 99.02 & 132.59 & 26.95 & 5.56 & 197.73 & 38.13 & 128.72 & 13.41 \\
\hline 7470.33 & 403338.00 & 16.20 & 3042.53 & 98.70 & 280.26 & 34737.11 & 97.13 & 127.89 & 27.51 & 5.20 & 216.65 & 47.78 & 122.04 & 13.25 \\
\hline 7470.39 & 399566.20 & 16.20 & 3363.19 & 110.81 & 215.61 & 35378.90 & 101.85 & 104.11 & 31.02 & 5.34 & 205.33 & 44.06 & 126.05 & 14.17 \\
\hline 7470.46 & 393044.40 & 16.20 & 3348.38 & 108.98 & 211.05 & 34636.18 & 99.54 & 69.59 & 33.40 & 5.39 & 205.81 & 43.56 & 122.90 & 14.00 \\
\hline 7470.52 & 396780.60 & 16.20 & 3301.82 & 107.37 & 207.26 & 35835.34 & 105.30 & 58.48 & 34.32 & 5.18 & 199.65 & 44.04 & 121.16 & 13.78 \\
\hline 7470.59 & 400921.00 & 16.20 & 3419.46 & 111.91 & 182.99 & 37830.64 & 107.60 & 37.11 & 38.07 & 5.47 & 201.86 & 34.34 & 128.59 & 14.13 \\
\hline 7470.66 & 407659.60 & 16.00 & 3553.81 & 119.37 & 159.07 & 40093.23 & 112.63 & 32.35 & 43.48 & 6.03 & 186.77 & 26.45 & 132.59 & 13.97 \\
\hline 7470.72 & 417158.00 & 16.00 & 3278.44 & 113.14 & 222.35 & 43197.82 & 114.91 & 33.73 & 44.24 & 5.38 & 212.73 & 42.57 & 122.39 & 13.24 \\
\hline 7470.79 & 421962.40 & 16.00 & 3119.29 & 106.83 & 260.62 & 46943.59 & 120.58 & 34.60 & 48.67 & 5.38 & 219.09 & 48.46 & 115.24 & 11.33 \\
\hline 7470.85 & 427991.40 & 16.00 & 3044.90 & 102.10 & 282.70 & 49646.93 & 126.59 & 33.98 & 52.32 & 5.78 & 223.61 & 53.20 & 113.51 & 10.14 \\
\hline 7470.92 & 417190.40 & 16.20 & 3781.53 & 105.83 & 293.77 & 48183.23 & 125.51 & 32.71 & 49.95 & 5.77 & 216.90 & 53.81 & 113.34 & 10.41 \\
\hline 7470.98 & 394568.40 & 16.40 & 3664.32 & 112.17 & 294.03 & 45322.58 & 118.69 & 31.92 & 45.02 & 4.94 & 226.55 & 52.17 & 114.94 & 11.30 \\
\hline 7471.05 & 336900.20 & 17.00 & 3677.88 & 127.22 & 241.16 & 40706.75 & 110.26 & 33.46 & 41.61 & 5.34 & 232.29 & 36.27 & 117.69 & 12.98 \\
\hline 7471.12 & 324202.60 & 17.20 & 3150.03 & 144.09 & 257.68 & 36108.82 & 97.59 & 42.58 & 33.85 & 5.25 & 293.48 & 35.75 & 116.32 & 14.56 \\
\hline
\end{tabular}




\begin{tabular}{|c|c|c|c|c|c|c|c|c|c|c|c|c|c|c|}
\hline Depth & XRF & XRF Live Time & $\mathrm{Ti}(\mathrm{ppm})$ & $\mathrm{Cr}(\mathrm{ppm})$ & $\mathrm{Mn}(\mathrm{ppm})$ & $\mathrm{Fe}(\mathrm{ppm})$ & $\mathrm{Ni}(\mathrm{ppm})$ & $\mathrm{Cu}(\mathrm{ppm})$ & $\mathrm{Zn}(\mathrm{ppm})$ & As (ppm) & $\mathrm{Rb}(\mathrm{ppm})$ & $\mathrm{Sr}(\mathrm{ppm})$ & $\mathrm{Y}(\mathrm{ppm})$ & $\mathrm{Zr}(\mathrm{ppm})$ \\
\hline 7471.18 & 306767.40 & 17.40 & 2633.87 & 152.41 & 319.40 & 30593.38 & 80.48 & 47.28 & 24.41 & 4.66 & 335.64 & 55.55 & 105.33 & 13.49 \\
\hline 7471.25 & 305659.20 & 17.40 & 1488.87 & 143.02 & 402.20 & 28417.39 & 70.34 & 47.61 & 20.65 & 3.98 & 354.80 & 86.29 & 89.99 & 13.03 \\
\hline 7471.31 & 318115.80 & 17.40 & 1535.21 & 135.69 & 443.55 & 29752.14 & 70.52 & 50.95 & 19.29 & 4.85 & 344.90 & 91.26 & 83.83 & 11.33 \\
\hline 7471.38 & 360035.20 & 17.00 & 1791.95 & 125.34 & 451.25 & 31855.91 & 86.96 & 61.22 & 25.40 & 8.56 & 311.50 & 117.41 & 72.10 & 31.93 \\
\hline 7471.44 & 362207.40 & 17.00 & 2255.25 & 106.82 & 414.87 & 32316.50 & 87.79 & 58.88 & 25.66 & 8.20 & 250.09 & 118.49 & 77.00 & 30.55 \\
\hline 7471.51 & 375636.00 & 16.80 & 2811.93 & 100.85 & 340.50 & 34715.35 & 94.39 & 61.32 & 29.60 & 9.10 & 207.32 & 95.59 & 93.75 & 32.18 \\
\hline 7471.57 & 385845.00 & 16.60 & 3158.01 & 117.94 & 259.44 & 36940.95 & 105.06 & 80.73 & 31.55 & 10.22 & 222.15 & 65.97 & 109.90 & 32.77 \\
\hline 7471.64 & 396336.60 & 16.40 & 2926.04 & 130.94 & 246.41 & 35615.46 & 111.20 & 148.93 & 31.54 & 10.32 & 265.71 & 70.84 & 112.49 & 33.03 \\
\hline 7471.71 & 403458.80 & 16.20 & 2752.98 & 151.96 & 252.01 & 36902.87 & 108.40 & 239.98 & 27.26 & 7.68 & 305.18 & 45.62 & 128.48 & 11.16 \\
\hline 7471.77 & 412525.60 & 16.00 & 2772.53 & 178.31 & 242.76 & 39845.88 & 122.83 & 234.68 & 30.13 & 8.62 & 335.00 & 39.76 & 131.65 & 11.66 \\
\hline 7471.84 & 400593.80 & 16.20 & 2662.46 & 190.41 & 237.07 & 40090.25 & 126.51 & 228.39 & 30.83 & 8.63 & 352.55 & 38.82 & 128.54 & 12.00 \\
\hline 7471.90 & 405710.60 & 16.20 & 6739.01 & 193.32 & 231.59 & 39790.44 & 124.94 & 217.20 & 31.08 & 8.21 & 344.18 & 38.04 & 131.50 & 12.85 \\
\hline 7471.97 & 403494.00 & 16.20 & 7060.64 & 197.41 & 198.96 & 40763.08 & 124.17 & 153.70 & 32.91 & 8.59 & 312.41 & 28.45 & 138.12 & 13.77 \\
\hline 7472.03 & 402930.60 & 16.20 & 7236.27 & 187.73 & 185.76 & 39862.38 & 121.61 & 425.25 & 32.45 & 8.22 & 287.61 & 29.45 & 142.01 & 15.04 \\
\hline 7472.10 & 398760.20 & 16.20 & 7199.15 & 182.91 & 180.94 & 38036.14 & 116.71 & 468.73 & 31.05 & 8.47 & 281.13 & 30.32 & 144.85 & 15.38 \\
\hline 7472.17 & 403291.80 & 16.00 & 7221.91 & 180.95 & 187.24 & 37080.82 & 116.76 & 488.69 & 29.33 & 8.11 & 283.03 & 31.23 & 145.51 & 15.45 \\
\hline 7472.23 & 399201.20 & 16.00 & 3154.41 & 180.59 & 191.22 & 37323.96 & 120.80 & 483.75 & 30.83 & 8.68 & 287.16 & 32.15 & 141.16 & 13.79 \\
\hline 7472.30 & 399384.20 & 16.00 & 3049.04 & 183.54 & 192.13 & 38029.45 & 119.88 & 475.33 & 33.40 & 8.53 & 308.61 & 31.71 & 138.52 & 13.70 \\
\hline 7472.36 & 403963.80 & 16.00 & 3069.87 & 188.97 & 190.20 & 39521.63 & 117.11 & 103.55 & 36.68 & 8.46 & 314.06 & 29.54 & 136.42 & 13.44 \\
\hline 7472.43 & 420295.60 & 16.00 & 3102.09 & 185.10 & 205.17 & 40705.97 & 120.87 & 100.29 & 37.42 & 8.20 & 306.50 & 30.14 & 135.79 & 13.92 \\
\hline 7472.49 & 432226.00 & 16.00 & 3108.10 & 195.90 & 209.68 & 42764.26 & 121.13 & 90.76 & 39.98 & 8.83 & 316.59 & 28.73 & 134.57 & 14.36 \\
\hline 7472.56 & 432912.20 & 16.00 & 3238.26 & 200.98 & 209.85 & 43260.31 & 117.94 & 92.00 & 40.29 & 8.24 & 308.35 & 29.65 & 132.08 & 14.98 \\
\hline 7472.62 & 427935.80 & 16.00 & 3275.38 & 200.71 & 206.98 & 42379.19 & 115.63 & 102.53 & 37.78 & 8.11 & 299.77 & 29.27 & 133.59 & 15.14 \\
\hline 7472.69 & 420779.20 & 16.20 & 3063.57 & 195.82 & 211.84 & 39838.05 & 116.96 & 115.55 & 34.25 & 8.31 & 311.33 & 30.34 & 135.32 & 14.57 \\
\hline 7472.76 & 406963.20 & 16.20 & 3136.43 & 198.84 & 199.93 & 38619.22 & 108.15 & 73.15 & 33.29 & 8.17 & 320.66 & 27.59 & 135.23 & 14.88 \\
\hline 7472.82 & 398229.80 & 16.20 & 3193.65 & 189.85 & 195.55 & 37997.90 & 104.56 & 61.54 & 32.03 & 7.68 & 304.34 & 26.99 & 136.17 & 14.59 \\
\hline 7472.89 & 411701.20 & 16.00 & 6325.59 & 177.55 & 193.82 & 39418.01 & 110.84 & 86.87 & 32.94 & 8.20 & 288.05 & 28.49 & 138.57 & 14.84 \\
\hline 7472.95 & 420472.80 & 16.00 & 6323.85 & 172.29 & 198.24 & 40976.56 & 119.17 & 77.33 & 34.85 & 8.61 & 294.61 & 29.73 & 138.68 & 14.84 \\
\hline 7473.02 & 425694.00 & 15.80 & 6498.27 & 180.14 & 198.87 & 42298.77 & 119.44 & 62.43 & 37.17 & 9.13 & 294.93 & 28.73 & 137.88 & 15.05 \\
\hline 7473.08 & 422481.00 & 16.00 & 6508.54 & 175.19 & 192.64 & 41791.75 & 122.42 & 63.44 & 37.66 & 9.45 & 280.53 & 29.78 & 139.15 & 14.75 \\
\hline 7473.15 & 427241.80 & 16.00 & 6403.26 & 178.09 & 187.14 & 41821.51 & 122.74 & 62.72 & 39.62 & 9.69 & 291.90 & 31.48 & 140.15 & 14.23 \\
\hline 7473.22 & 417557.00 & 16.20 & 3169.07 & 186.33 & 186.40 & 43648.91 & 122.52 & 33.74 & 44.48 & 10.28 & 317.95 & 29.32 & 140.19 & 14.22 \\
\hline 7473.28 & 415780.20 & 16.20 & 3163.99 & 190.08 & 189.69 & 45548.87 & 123.60 & 35.01 & 49.58 & 10.58 & 307.14 & 29.98 & 137.42 & 14.26 \\
\hline
\end{tabular}




\begin{tabular}{|c|c|c|c|c|c|c|c|c|c|c|c|c|c|c|}
\hline Depth & XRF & XRF Live Time & $\mathrm{Ti}(\mathrm{ppm})$ & $\mathrm{Cr}(\mathrm{ppm})$ & $\mathrm{Mn}(\mathrm{ppm})$ & $\mathrm{Fe}(\mathrm{ppm})$ & $\mathrm{Ni}(\mathrm{ppm})$ & $\mathrm{Cu}(\mathrm{ppm})$ & $\mathrm{Zn}(\mathrm{ppm})$ & As (ppm) & $\mathrm{Rb}(\mathrm{ppm})$ & $\mathrm{Sr}(\mathrm{ppm})$ & $\mathrm{Y}(\mathrm{ppm})$ & $\mathrm{Zr}(\mathrm{ppm})$ \\
\hline 7473.35 & 421878.20 & 16.20 & 3271.95 & 189.07 & 189.60 & 48740.21 & 132.46 & 35.25 & 54.38 & 10.43 & 289.27 & 30.04 & 137.17 & 13.73 \\
\hline 7473.41 & 423813.80 & 16.00 & 3310.19 & 194.94 & 194.89 & 50494.84 & 138.64 & 37.56 & 55.31 & 10.11 & 289.33 & 29.88 & 135.66 & 13.17 \\
\hline 7473.48 & 410664.60 & 16.20 & 3323.87 & 204.95 & 199.41 & 49435.13 & 138.29 & 36.09 & 53.03 & 9.78 & 304.40 & 27.66 & 133.53 & 13.26 \\
\hline 7473.54 & 405761.00 & 16.20 & 3313.21 & 203.91 & 195.90 & 46141.63 & 131.94 & 33.98 & 47.19 & 9.17 & 300.18 & 26.70 & 134.68 & 13.48 \\
\hline 7473.61 & 401889.60 & 16.20 & 3335.68 & 197.40 & 193.47 & 44507.87 & 124.48 & 36.50 & 41.88 & 8.50 & 299.15 & 25.79 & 134.70 & 13.20 \\
\hline 7473.67 & 392795.60 & 16.20 & 3116.98 & 194.32 & 191.47 & 42956.20 & 116.23 & 42.75 & 38.26 & 8.69 & 315.06 & 26.59 & 132.53 & 14.29 \\
\hline 7473.74 & 391093.40 & 16.40 & 2875.82 & 210.85 & 198.28 & 45224.96 & 110.57 & 40.42 & 44.43 & 8.84 & 360.37 & 28.46 & 128.78 & 13.38 \\
\hline 7473.81 & 385743.00 & 16.40 & 2773.68 & 208.39 & 201.77 & 45108.56 & 110.95 & 41.68 & 46.52 & 8.22 & 355.02 & 29.04 & 128.85 & 13.04 \\
\hline 7473.87 & 373672.80 & 16.60 & 2816.03 & 209.25 & 202.19 & 44382.40 & 111.77 & 55.14 & 44.89 & 7.74 & 356.89 & 28.83 & 127.86 & 11.55 \\
\hline 7473.94 & 357066.20 & 16.80 & 2760.16 & 215.22 & 198.60 & 42061.70 & 107.21 & 69.36 & 43.36 & 7.02 & 358.59 & 29.52 & 128.72 & 11.64 \\
\hline 7474.00 & 340849.40 & 17.00 & 2956.97 & 199.55 & 188.44 & 39737.90 & 100.64 & 105.32 & 40.78 & 6.33 & 319.60 & 29.56 & 130.97 & 11.41 \\
\hline 7474.07 & 336378.80 & 17.00 & 3167.52 & 164.03 & 175.13 & 36453.99 & 98.70 & 115.04 & 35.33 & 6.15 & 252.40 & 27.76 & 137.61 & 12.35 \\
\hline 7474.13 & 355691.80 & 16.80 & 3409.12 & 141.13 & 163.87 & 41730.51 & 99.78 & 117.71 & 39.48 & 6.83 & 207.14 & 27.51 & 139.36 & 12.78 \\
\hline 7474.20 & 360631.00 & 16.80 & 3444.86 & 119.99 & 156.10 & 42481.94 & 96.88 & 118.80 & 40.90 & 6.88 & 170.60 & 28.98 & 138.54 & 14.34 \\
\hline 7474.27 & 369098.60 & 16.80 & 3520.91 & 101.71 & 147.12 & 42388.47 & 95.10 & 109.30 & 39.58 & 6.61 & 142.91 & 27.36 & 140.36 & 15.07 \\
\hline 7474.33 & 376985.40 & 16.80 & 3471.07 & 101.87 & 144.13 & 41541.71 & 95.35 & 95.38 & 38.53 & 6.27 & 148.47 & 26.00 & 140.19 & 16.19 \\
\hline 7474.40 & 382183.00 & 16.60 & 3511.12 & 102.32 & 144.12 & 41445.37 & 107.55 & 102.51 & 87.67 & 12.17 & 158.06 & 54.10 & 115.13 & 40.58 \\
\hline 7474.46 & 367169.80 & 16.80 & 3422.56 & 95.57 & 141.86 & 35417.90 & 104.13 & 130.37 & 81.40 & 11.70 & 164.46 & 57.76 & 115.26 & 40.30 \\
\hline 7474.53 & 371485.80 & 16.60 & 3475.26 & 98.96 & 143.84 & 34785.15 & 107.88 & 137.48 & 79.49 & 12.12 & 164.98 & 59.76 & 117.13 & 41.59 \\
\hline 7474.59 & 378377.80 & 16.40 & 3558.97 & 102.59 & 144.54 & 35804.48 & 110.54 & 166.88 & 81.61 & 12.60 & 167.75 & 63.36 & 113.20 & 40.41 \\
\hline 7474.66 & 385502.40 & 16.20 & 3669.15 & 107.54 & 152.54 & 36454.85 & 121.15 & 149.81 & 82.30 & 18.12 & 178.06 & 90.77 & 97.27 & 65.02 \\
\hline 7474.72 & 360545.40 & 16.60 & 3402.47 & 100.79 & 187.72 & 34200.71 & 104.52 & 133.98 & 30.14 & 11.44 & 169.74 & 67.57 & 114.24 & 39.96 \\
\hline 7474.79 & 368404.20 & 16.40 & 3483.51 & 109.50 & 201.61 & 34812.46 & 106.85 & 107.07 & 30.02 & 11.58 & 174.45 & 69.36 & 111.14 & 40.33 \\
\hline 7474.86 & 339582.00 & 16.80 & 3399.12 & 106.38 & 215.03 & 34285.82 & 101.89 & 95.40 & 30.44 & 10.83 & 187.26 & 73.15 & 103.33 & 39.36 \\
\hline 7474.92 & 330499.20 & 17.00 & 3187.93 & 112.11 & 234.65 & 34181.70 & 105.01 & 65.61 & 29.31 & 10.54 & 221.55 & 76.88 & 104.73 & 40.09 \\
\hline 7474.99 & 321158.80 & 17.20 & 3003.91 & 122.54 & 242.18 & 34365.86 & 98.27 & 57.01 & 29.68 & 4.37 & 251.83 & 56.50 & 119.06 & 14.67 \\
\hline 7475.05 & 338359.20 & 17.00 & 3275.18 & 136.57 & 211.52 & 36025.74 & 103.75 & 98.66 & 30.58 & 4.49 & 264.96 & 56.87 & 121.02 & 15.37 \\
\hline 7475.12 & 342408.80 & 17.00 & 3363.55 & 137.80 & 204.42 & 36809.17 & 102.91 & 99.19 & 31.89 & 4.17 & 270.35 & 51.96 & 127.15 & 15.93 \\
\hline 7475.18 & 371942.40 & 16.60 & 3494.99 & 142.17 & 196.77 & 38702.11 & 106.90 & 89.07 & 34.39 & 4.42 & 269.12 & 44.60 & 135.13 & 14.88 \\
\hline 7475.25 & 381287.40 & 16.40 & 3671.15 & 133.12 & 184.79 & 38612.88 & 104.70 & 81.00 & 35.16 & 4.28 & 242.48 & 39.99 & 137.19 & 13.91 \\
\hline 7475.31 & 391896.80 & 16.20 & 3645.19 & 110.75 & 198.20 & 38241.44 & 99.89 & 76.63 & 33.49 & 4.45 & 213.01 & 40.29 & 132.47 & 13.73 \\
\hline 7475.38 & 401362.00 & 16.00 & 3510.43 & 98.24 & 208.19 & 38533.49 & 99.05 & 36.82 & 33.14 & 4.61 & 211.32 & 41.08 & 132.47 & 13.04 \\
\hline 7475.45 & 394731.40 & 16.20 & 3230.79 & 90.58 & 236.47 & 36827.82 & 94.99 & 38.61 & 29.77 & 4.78 & 222.84 & 52.20 & 122.65 & 12.03 \\
\hline
\end{tabular}




\begin{tabular}{|c|c|c|c|c|c|c|c|c|c|c|c|c|c|c|}
\hline Depth & XRF & XRF Live Time & $\mathrm{Ti}(\mathrm{ppm})$ & $\mathrm{Cr}$ (ppm) & $\mathrm{Mn}$ (ppm) & $\mathrm{Fe}(\mathrm{ppm})$ & $\mathrm{Ni}(\mathrm{ppm})$ & $\mathrm{Cu}(\mathrm{ppm})$ & $\mathrm{Zn}(\mathrm{ppm})$ & As (ppm) & $\mathrm{Rb}(\mathrm{ppm})$ & $\mathrm{Sr}(\mathrm{ppm})$ & $\mathrm{Y}(\mathrm{ppm})$ & $\mathrm{Zr}(\mathrm{ppm})$ \\
\hline 7475.51 & 392981.40 & 16.40 & 2953.91 & 82.76 & 264.03 & 34460.29 & 88.82 & 38.94 & 26.26 & 4.48 & 248.97 & 62.89 & 112.64 & 12.61 \\
\hline 7475.58 & 435587.80 & 16.00 & 1860.74 & 50.20 & 244.71 & 109266.51 & 71.62 & 32.11 & 25.93 & 4.34 & 600.69 & 55.59 & 82.85 & 10.68 \\
\hline 7475.64 & 442466.00 & 16.00 & 2118.87 & 60.37 & 223.61 & 109448.93 & 74.43 & 36.35 & 26.79 & 4.51 & 585.71 & 48.89 & 90.07 & 11.45 \\
\hline 7475.71 & 449288.60 & 16.00 & 2389.36 & 70.39 & 210.34 & 109417.85 & 75.21 & 82.29 & 27.81 & 4.86 & 573.90 & 42.15 & 98.38 & 13.04 \\
\hline 7475.77 & 461685.60 & 15.80 & 2665.78 & 74.35 & 183.21 & 111794.35 & 88.22 & 80.54 & 32.22 & 5.18 & 551.95 & 31.32 & 104.94 & 14.17 \\
\hline 7475.84 & 473684.60 & 15.60 & 2834.91 & 83.10 & 157.43 & 114798.43 & 103.41 & 87.58 & 35.56 & 5.81 & 521.85 & 24.51 & 111.77 & 13.81 \\
\hline 7475.91 & 444850.80 & 16.00 & 7241.20 & 118.35 & 177.31 & 42578.48 & 123.72 & 97.10 & 34.96 & 6.31 & 163.40 & 32.64 & 140.05 & 16.58 \\
\hline 7475.97 & 442534.20 & 16.00 & 7212.45 & 117.65 & 178.61 & 42941.05 & 127.21 & 134.68 & 34.95 & 6.30 & 165.24 & 33.95 & 139.27 & 15.56 \\
\hline 7476.04 & 441556.00 & 16.00 & 7130.39 & 112.67 & 177.16 & 43470.33 & 129.21 & 106.67 & 34.17 & 5.90 & 170.03 & 35.27 & 135.80 & 14.28 \\
\hline 7476.10 & 433924.40 & 16.00 & 7133.72 & 113.93 & 174.55 & 41586.69 & 119.19 & 118.26 & 31.25 & 5.50 & 170.25 & 36.02 & 136.24 & 14.81 \\
\hline 7476.17 & 433642.20 & 16.00 & 7296.28 & 110.60 & 169.60 & 39835.45 & 110.79 & 115.53 & 27.95 & 5.00 & 167.24 & 32.06 & 142.15 & 15.29 \\
\hline 7476.23 & 430775.40 & 16.00 & 4142.88 & 108.51 & 167.27 & 38346.31 & 111.02 & 113.95 & 28.52 & 5.13 & 162.09 & 29.88 & 147.03 & 15.07 \\
\hline 7476.30 & 433624.40 & 16.00 & 4184.01 & 113.76 & 171.35 & 39460.35 & 111.18 & 72.82 & 29.44 & 5.97 & 162.88 & 29.73 & 149.57 & 15.60 \\
\hline 7476.36 & 431395.00 & 16.00 & 4192.72 & 117.88 & 178.41 & 39786.93 & 114.68 & 57.74 & 30.42 & 6.59 & 166.06 & 30.44 & 148.66 & 16.33 \\
\hline 7476.43 & 425840.80 & 16.20 & 4188.01 & 115.43 & 177.15 & 39301.13 & 113.67 & 51.44 & 30.22 & 7.02 & 167.71 & 29.33 & 150.19 & 16.08 \\
\hline 7476.50 & 412487.60 & 16.40 & 4146.93 & 119.57 & 174.99 & 38586.69 & 112.26 & 70.40 & 32.04 & 6.79 & 161.09 & 30.18 & 148.21 & 16.22 \\
\hline 7476.56 & 408808.20 & 16.40 & 4134.60 & 120.49 & 178.27 & 41457.54 & 123.49 & 71.22 & 37.77 & 7.52 & 165.03 & 28.73 & 144.18 & 14.64 \\
\hline 7476.63 & 416096.80 & 16.40 & 4088.05 & 114.59 & 176.50 & 43101.98 & 138.16 & 70.77 & 40.09 & 6.87 & 179.16 & 28.78 & 140.79 & 13.37 \\
\hline 7476.69 & 423935.20 & 16.40 & 4056.23 & 114.22 & 174.09 & 45936.69 & 143.65 & 65.67 & 45.13 & 6.46 & 172.77 & 27.94 & 137.88 & 13.29 \\
\hline 7476.76 & 439730.00 & 16.20 & 4141.34 & 114.20 & 179.80 & 50418.79 & 146.37 & 70.76 & 46.49 & 17.27 & 177.66 & 55.37 & 113.16 & 39.10 \\
\hline 7476.82 & 449982.20 & 16.00 & 4227.48 & 111.05 & 181.14 & 53009.47 & 146.07 & 48.29 & 50.20 & 17.85 & 186.37 & 54.00 & 113.32 & 38.64 \\
\hline 7476.89 & 450770.40 & 16.00 & 7028.93 & 115.01 & 180.68 & 50999.69 & 135.46 & 48.93 & 48.31 & 16.86 & 188.26 & 54.99 & 114.57 & 40.60 \\
\hline 7476.96 & 443762.40 & 16.00 & 7054.43 & 114.52 & 182.41 & 49983.01 & 125.98 & 50.83 & 48.48 & 16.84 & 180.11 & 53.50 & 116.51 & 42.30 \\
\hline 7477.02 & 446491.40 & 16.00 & 7217.97 & 117.86 & 189.06 & 51681.19 & 128.27 & 50.96 & 48.30 & 17.03 & 181.14 & 54.10 & 119.35 & 42.45 \\
\hline 7477.09 & 445508.40 & 16.00 & 7182.44 & 123.28 & 189.40 & 55045.84 & 141.06 & 37.88 & 54.76 & 6.08 & 179.03 & 28.30 & 142.17 & 15.71 \\
\hline 7477.15 & 445018.20 & 16.00 & 7189.38 & 126.22 & 189.73 & 54310.60 & 137.44 & 37.15 & 52.18 & 5.97 & 171.58 & 29.16 & 142.33 & 16.44 \\
\hline 7477.22 & 434980.40 & 16.00 & 4440.94 & 124.57 & 189.83 & 51177.11 & 130.90 & 33.92 & 47.10 & 5.62 & 173.49 & 28.39 & 142.92 & 16.37 \\
\hline 7477.28 & 423113.40 & 16.20 & 4431.04 & 125.36 & 187.05 & 48871.09 & 119.52 & 55.90 & 41.00 & 5.63 & 168.37 & 28.25 & 145.58 & 15.91 \\
\hline 7477.35 & 401500.60 & 16.40 & 4403.86 & 124.70 & 178.82 & 42029.30 & 102.09 & 91.46 & 34.04 & 5.45 & 169.75 & 26.70 & 147.78 & 16.28 \\
\hline 7477.41 & 381190.20 & 16.60 & 4404.14 & 123.73 & 172.83 & 33631.27 & 84.52 & 100.50 & 25.03 & 4.99 & 162.05 & 24.38 & 148.05 & 17.78 \\
\hline 7477.48 & 369767.20 & 16.80 & 4341.41 & 121.55 & 172.32 & 32013.98 & 88.41 & 124.68 & 22.66 & 5.08 & 167.09 & 25.60 & 147.15 & 18.10 \\
\hline 7477.55 & 362129.20 & 17.00 & 4309.38 & 124.59 & 172.94 & 32463.02 & 91.26 & 151.18 & 21.87 & 5.82 & 161.57 & 26.22 & 146.88 & 18.00 \\
\hline 7477.61 & 354263.80 & 17.00 & 4300.97 & 121.55 & 166.98 & 32614.76 & 93.79 & 129.84 & 22.92 & 6.11 & 160.06 & 26.75 & 143.47 & 16.46 \\
\hline
\end{tabular}




\begin{tabular}{|c|c|c|c|c|c|c|c|c|c|c|c|c|c|c|}
\hline Depth & XRF & XRF Live Time & $\mathrm{Ti}(\mathrm{ppm})$ & $\mathrm{Cr}(\mathrm{ppm})$ & $\mathrm{Mn}(\mathrm{ppm})$ & $\mathrm{Fe}(\mathrm{ppm})$ & $\mathrm{Ni}(\mathrm{ppm})$ & $\mathrm{Cu}(\mathrm{ppm})$ & $\mathrm{Zn}(\mathrm{ppm})$ & As (ppm) & $\mathrm{Rb}(\mathrm{ppm})$ & $\mathrm{Sr}(\mathrm{ppm})$ & $\mathrm{Y}(\mathrm{ppm})$ & $\mathrm{Zr}(\mathrm{ppm})$ \\
\hline 7477.68 & 368130.60 & 16.80 & 4275.50 & 121.61 & 170.30 & 40998.24 & 108.35 & 98.02 & 32.94 & 6.52 & 153.84 & 25.77 & 140.55 & 16.38 \\
\hline 7477.74 & 366785.40 & 16.80 & 4228.17 & 120.00 & 173.09 & 43328.22 & 121.04 & 103.15 & 35.62 & 12.90 & 166.95 & 55.68 & 116.73 & 37.83 \\
\hline 7477.81 & 354511.60 & 16.80 & 4174.08 & 115.89 & 170.07 & 43281.16 & 120.29 & 94.78 & 34.87 & 12.52 & 159.56 & 54.34 & 116.39 & 37.30 \\
\hline 7477.87 & 347173.20 & 16.80 & 8085.22 & 109.13 & 159.36 & 43732.14 & 115.80 & 72.81 & 36.88 & 12.48 & 158.59 & 54.49 & 113.51 & 37.29 \\
\hline 7477.94 & 345798.60 & 16.80 & 8136.43 & 109.39 & 154.99 & 43250.38 & 115.82 & 75.76 & 36.75 & 12.15 & 155.64 & 53.21 & 112.34 & 39.25 \\
\hline 7478.01 & 323767.40 & 17.00 & 8076.07 & 102.73 & 143.59 & 34715.98 & 99.24 & 108.21 & 26.31 & 11.38 & 156.72 & 54.42 & 113.63 & 39.37 \\
\hline 7478.07 & 320404.20 & 17.00 & 8111.40 & 103.12 & 139.85 & 32473.57 & 90.00 & 300.43 & 23.44 & 5.21 & 144.47 & 24.35 & 134.23 & 16.28 \\
\hline 7478.14 & 329322.00 & 17.00 & 8166.00 & 105.49 & 147.71 & 33957.20 & 94.76 & 302.95 & 26.26 & 5.21 & 142.70 & 24.47 & 133.92 & 17.05 \\
\hline 7478.20 & 348362.20 & 16.80 & 4183.46 & 111.11 & 148.24 & 36343.36 & 103.81 & 298.88 & 29.01 & 5.19 & 152.75 & 24.20 & 137.59 & 16.77 \\
\hline 7478.27 & 352711.60 & 16.80 & 4012.39 & 113.50 & 157.39 & 35514.36 & 107.66 & 293.25 & 29.48 & 5.31 & 167.49 & 27.05 & 136.78 & 15.37 \\
\hline 7478.33 & 374877.80 & 16.60 & 3957.40 & 117.10 & 178.04 & 39046.02 & 126.12 & 1201.79 & 32.73 & 5.49 & 180.89 & 31.17 & 131.57 & 14.95 \\
\hline 7478.40 & 388577.40 & 16.40 & 3978.20 & 122.06 & 186.18 & 41696.33 & 138.85 & 1002.79 & 37.08 & 5.85 & 182.22 & 30.74 & 136.55 & 15.66 \\
\hline 7478.46 & 369714.20 & 16.60 & 3851.63 & 118.03 & 179.23 & 38398.97 & 126.95 & 985.90 & 35.79 & 5.63 & 178.54 & 28.95 & 136.15 & 15.13 \\
\hline 7478.53 & 315702.60 & 17.20 & 3785.85 & 107.69 & 187.42 & 34654.60 & 120.63 & 989.80 & 31.76 & 5.22 & 156.46 & 29.85 & 133.95 & 15.99 \\
\hline 7478.60 & 288103.40 & 17.40 & 3652.05 & 97.40 & 192.12 & 35338.02 & 118.27 & 1011.73 & 35.12 & 6.02 & 137.67 & 27.55 & 128.63 & 15.58 \\
\hline 7478.66 & 246459.80 & 17.80 & 3667.64 & 91.15 & 177.21 & 32867.31 & 103.71 & 69.65 & 33.41 & 6.04 & 113.12 & 22.40 & 131.89 & 16.02 \\
\hline 7478.73 & 228740.00 & 18.00 & 3516.20 & 78.75 & 170.21 & 30401.99 & 89.65 & 72.87 & 28.91 & 5.76 & 102.54 & 22.68 & 130.93 & 15.75 \\
\hline 7478.79 & 245268.20 & 17.80 & 6746.54 & 85.86 & 174.90 & 31648.22 & 95.98 & 135.56 & 27.49 & 6.49 & 109.91 & 23.50 & 129.24 & 14.76 \\
\hline 7478.86 & 283783.80 & 17.40 & 6779.32 & 86.07 & 168.21 & 32307.60 & 98.37 & 264.69 & 25.76 & 6.76 & 115.19 & 22.75 & 126.99 & 13.21 \\
\hline 7478.92 & 321324.00 & 17.00 & 7170.13 & 96.24 & 161.49 & 32388.09 & 96.43 & 261.97 & 21.60 & 5.97 & 120.61 & 22.79 & 137.43 & 14.91 \\
\hline 7478.99 & 356348.80 & 16.60 & 7247.81 & 104.81 & 167.27 & 32237.24 & 98.37 & 271.89 & 19.88 & 5.73 & 127.84 & 23.39 & 140.15 & 14.82 \\
\hline 7479.06 & 376788.60 & 16.40 & 7401.71 & 112.44 & 173.11 & 34122.49 & 101.63 & 264.04 & 22.33 & 5.97 & 135.95 & 23.32 & 140.54 & 15.00 \\
\hline 7479.12 & 388975.20 & 16.20 & 4355.95 & 110.39 & 180.37 & 36167.52 & 110.66 & 205.69 & 24.44 & 6.61 & 134.13 & 23.47 & 141.64 & 15.19 \\
\hline 7479.19 & 397135.80 & 16.20 & 4513.56 & 118.07 & 181.34 & 36441.01 & 110.24 & 82.80 & 26.76 & 12.18 & 151.63 & 43.57 & 120.28 & 41.99 \\
\hline 7479.25 & 390246.40 & 16.40 & 4387.51 & 119.78 & 176.88 & 36721.40 & 111.08 & 132.93 & 29.52 & 12.52 & 149.75 & 44.02 & 119.60 & 41.55 \\
\hline 7479.32 & 372588.20 & 16.60 & 4373.69 & 117.47 & 169.55 & 35714.53 & 106.66 & 120.15 & 30.96 & 12.72 & 158.21 & 43.51 & 119.67 & 41.40 \\
\hline 7479.38 & 352806.80 & 16.80 & 4368.73 & 115.79 & 163.91 & 34117.63 & 103.20 & 117.39 & 31.34 & 12.66 & 158.77 & 43.75 & 119.21 & 41.90 \\
\hline 7479.45 & 334415.20 & 17.00 & 4358.34 & 114.94 & 153.27 & 31904.23 & 91.50 & 116.97 & 29.77 & 11.86 & 157.71 & 43.95 & 122.63 & 42.74 \\
\hline 7479.51 & 315763.80 & 17.20 & 4199.30 & 110.34 & 151.31 & 30541.31 & 86.76 & 139.60 & 28.29 & 5.84 & 140.80 & 23.66 & 145.55 & 16.93 \\
\hline 7479.58 & 294800.60 & 17.40 & 4162.53 & 102.09 & 154.66 & 28971.34 & 81.65 & 113.94 & 24.82 & 5.36 & 139.13 & 23.04 & 145.52 & 16.40 \\
\hline 7479.65 & 283990.60 & 17.60 & 4163.42 & 96.70 & 153.59 & 28621.73 & 83.06 & 148.35 & 23.46 & 5.65 & 125.98 & 23.72 & 141.32 & 15.12 \\
\hline 7479.71 & 273144.00 & 17.80 & 4138.87 & 91.44 & 149.49 & 27596.61 & 80.42 & 156.83 & 22.30 & 5.40 & 123.06 & 23.69 & 140.34 & 14.56 \\
\hline 7479.78 & 270227.20 & 17.80 & 4098.40 & 90.41 & 149.57 & 27054.10 & 78.09 & 152.79 & 22.67 & 5.03 & 122.09 & 24.05 & 140.32 & 14.32 \\
\hline
\end{tabular}




\begin{tabular}{|c|c|c|c|c|c|c|c|c|c|c|c|c|c|c|}
\hline Depth & XRF & XRF Live Time & $\mathrm{Ti}(\mathrm{ppm})$ & $\mathrm{Cr}(\mathrm{ppm})$ & $\mathrm{Mn}(\mathrm{ppm})$ & $\mathrm{Fe}(\mathrm{ppm})$ & $\mathrm{Ni}(\mathrm{ppm})$ & $\mathrm{Cu}(\mathrm{ppm})$ & $\mathrm{Zn}(\mathrm{ppm})$ & As (ppm) & $\mathrm{Rb}(\mathrm{ppm})$ & $\mathrm{Sr}(\mathrm{ppm})$ & $\mathrm{Y}(\mathrm{ppm})$ & $\mathrm{Zr}(\mathrm{ppm})$ \\
\hline 7479.84 & 267669.00 & 17.80 & 4186.81 & 91.00 & 154.11 & 27331.72 & 75.66 & 121.06 & 24.02 & 5.34 & 126.57 & 24.21 & 144.52 & 14.48 \\
\hline 7479.91 & 285900.20 & 17.60 & 4357.46 & 98.98 & 156.38 & 28469.42 & 77.68 & 76.58 & 25.97 & 5.33 & 133.60 & 24.18 & 144.56 & 15.53 \\
\hline 7479.97 & 304187.40 & 17.40 & 4394.03 & 106.18 & 160.75 & 29572.29 & 73.40 & 40.37 & 27.63 & 5.22 & 147.31 & 24.18 & 147.22 & 16.55 \\
\hline 7480.04 & 312298.20 & 17.20 & 4289.27 & 107.58 & 160.86 & 30792.35 & 72.79 & 29.62 & 28.66 & 5.58 & 153.42 & 24.48 & 146.84 & 18.02 \\
\hline 7480.10 & 333951.20 & 17.00 & 4371.98 & 111.93 & 170.42 & 36008.65 & 79.33 & 27.51 & 35.20 & 5.89 & 169.62 & 22.94 & 144.03 & 17.96 \\
\hline 7480.17 & 351544.40 & 16.80 & 4412.60 & 114.70 & 174.27 & 37615.49 & 84.54 & 24.44 & 35.91 & 6.09 & 169.14 & 21.48 & 142.25 & 17.57 \\
\hline 7480.24 & 346752.60 & 16.80 & 4080.64 & 106.18 & 170.43 & 37677.64 & 88.98 & 23.68 & 37.29 & 6.10 & 169.88 & 22.53 & 141.50 & 16.27 \\
\hline 7480.30 & 341948.20 & 16.80 & 3972.65 & 99.37 & 174.05 & 37664.37 & 92.76 & 24.47 & 38.74 & 6.06 & 164.40 & 23.36 & 140.03 & 16.87 \\
\hline 7480.37 & 366697.40 & 16.60 & 3955.44 & 96.72 & 181.29 & 37050.16 & 99.57 & 23.83 & 39.26 & 5.89 & 169.07 & 26.11 & 141.22 & 15.32 \\
\hline 7480.43 & 371550.20 & 16.60 & 3338.07 & 83.25 & 259.35 & 31244.28 & 93.18 & 22.97 & 30.35 & 5.80 & 187.42 & 42.05 & 128.20 & 12.65 \\
\hline 7480.50 & 388024.00 & 16.40 & 2660.26 & 67.56 & 357.73 & 28536.01 & 86.01 & 21.26 & 26.50 & 4.90 & 218.88 & 57.54 & 109.71 & 10.60 \\
\hline 7480.56 & 403776.80 & 16.20 & 2301.05 & 60.38 & 449.03 & 26407.56 & 77.06 & 20.82 & 23.66 & 4.48 & 238.88 & 72.10 & 93.13 & 8.33 \\
\hline 7480.63 & 421195.80 & 16.00 & 1744.36 & 46.76 & 536.83 & 24013.59 & 68.71 & 18.10 & 19.27 & 3.92 & 260.05 & 88.14 & 74.91 & 5.75 \\
\hline 7480.70 & 399044.20 & 16.20 & 1219.91 & 38.61 & 611.12 & 21554.94 & 56.59 & 16.21 & 14.73 & 3.39 & 277.64 & 102.69 & 54.18 & 3.40 \\
\hline 7480.76 & 371791.60 & 16.40 & 1242.86 & 36.86 & 618.98 & 20856.55 & 53.85 & 15.94 & 15.50 & 3.02 & 269.01 & 100.60 & 50.73 & 4.66 \\
\hline 7480.83 & 340899.20 & 16.80 & 1354.56 & 45.77 & 591.32 & 20407.15 & 50.50 & 17.46 & 16.04 & 3.07 & 264.30 & 97.66 & 52.83 & 5.20 \\
\hline 7480.89 & 302754.60 & 17.20 & 1798.05 & 60.39 & 506.54 & 20204.87 & 53.52 & 15.96 & 14.41 & 2.53 & 242.97 & 83.03 & 67.57 & 7.81 \\
\hline 7480.96 & 279730.40 & 17.40 & 2359.05 & 77.57 & 439.49 & 21439.20 & 60.51 & 18.22 & 15.30 & 2.37 & 237.77 & 68.63 & 83.96 & 8.86 \\
\hline 7481.02 & 278455.60 & 17.40 & 2747.04 & 85.93 & 408.68 & 22957.99 & 63.18 & 18.87 & 17.18 & 2.05 & 235.32 & 53.60 & 100.58 & 11.60 \\
\hline 7481.09 & 280936.20 & 17.40 & 2986.04 & 90.85 & 374.79 & 23435.96 & 54.51 & 18.51 & 14.64 & 1.39 & 233.72 & 47.50 & 112.52 & 11.54 \\
\hline 7481.15 & 283617.00 & 17.20 & 3142.01 & 83.98 & 373.85 & 23903.11 & 51.01 & 15.99 & 13.60 & 1.36 & 233.56 & 44.57 & 118.62 & 12.44 \\
\hline 7481.22 & 295583.60 & 17.20 & 2863.65 & 71.66 & 442.00 & 23335.98 & 38.14 & 16.09 & 12.89 & 1.04 & 256.61 & 51.88 & 106.20 & 11.53 \\
\hline 7481.29 & 266076.20 & 17.60 & 2622.84 & 57.19 & 484.40 & 21888.81 & 23.24 & 14.74 & 9.89 & 1.25 & 261.92 & 55.77 & 101.60 & 10.60 \\
\hline 7481.35 & 259229.00 & 17.80 & 2737.45 & 52.81 & 503.71 & 21986.26 & 13.24 & 13.92 & 6.15 & 0.51 & 247.78 & 56.70 & 97.52 & 9.75 \\
\hline 7481.42 & 263833.00 & 17.80 & 2931.80 & 55.70 & 501.17 & 23889.57 & 18.21 & 14.07 & 9.73 & 0.48 & 232.44 & 51.46 & 99.33 & 11.12 \\
\hline 7481.48 & 266093.00 & 17.80 & 3052.48 & 60.96 & 472.83 & 26146.42 & 25.80 & 15.41 & 11.54 & 0.37 & 224.21 & 44.71 & 101.42 & 10.07 \\
\hline 7481.55 & 283313.40 & 17.60 & 3146.41 & 61.15 & 440.11 & 28585.40 & 39.91 & 16.76 & 14.03 & 0.75 & 220.06 & 41.16 & 109.35 & 9.67 \\
\hline 7481.61 & 325870.80 & 17.20 & 3358.12 & 72.23 & 417.14 & 31607.00 & 54.23 & 18.07 & 17.66 & 0.25 & 211.57 & 37.21 & 112.56 & 11.26 \\
\hline 7481.68 & 339983.20 & 17.00 & 3599.59 & 78.03 & 389.89 & 31506.18 & 65.36 & 19.11 & 20.73 & 0.65 & 219.79 & 35.74 & 114.29 & 11.03 \\
\hline 7481.75 & 351669.60 & 17.00 & 3437.58 & 76.10 & 358.91 & 33073.45 & 78.32 & 20.60 & 20.88 & 0.89 & 244.42 & 37.86 & 114.36 & 10.42 \\
\hline 7481.81 & 372976.60 & 16.80 & 3269.32 & 71.00 & 363.06 & 33347.82 & 79.46 & 20.07 & 19.95 & 0.54 & 275.65 & 42.54 & 110.85 & 10.52 \\
\hline 7481.88 & 372708.00 & 16.80 & 3234.31 & 72.37 & 352.02 & 33071.03 & 74.15 & 30.28 & 21.00 & 3.52 & 249.53 & 85.50 & 97.60 & 27.28 \\
\hline 7481.94 & 358137.20 & 16.80 & 3111.81 & 73.48 & 323.45 & 31768.62 & 73.00 & 29.43 & 18.94 & 3.68 & 240.93 & 86.33 & 97.86 & 26.82 \\
\hline
\end{tabular}




\begin{tabular}{|c|c|c|c|c|c|c|c|c|c|c|c|c|c|c|}
\hline Depth & XRF & XRF Live Time & $\mathrm{Ti}(\mathrm{ppm})$ & $\mathrm{Cr}$ (ppm) & $\mathrm{Mn}$ (ppm) & $\mathrm{Fe}(\mathrm{ppm})$ & $\mathrm{Ni}(\mathrm{ppm})$ & $\mathrm{Cu}(\mathrm{ppm})$ & $\mathrm{Zn}(\mathrm{ppm})$ & As (ppm) & $\mathrm{Rb}(\mathrm{ppm})$ & $\mathrm{Sr}(\mathrm{ppm})$ & $\mathrm{Y}(\mathrm{ppm})$ & $\mathrm{Zr}(\mathrm{ppm})$ \\
\hline 7482.01 & 340885.80 & 17.00 & 2971.31 & 76.39 & 296.32 & 32902.91 & 79.43 & 29.38 & 18.13 & 3.99 & 224.45 & 85.02 & 99.47 & 27.24 \\
\hline 7482.07 & 338982.40 & 17.00 & 1592.55 & 62.38 & 258.61 & 71169.39 & 57.81 & 20.02 & 16.84 & 2.99 & 569.26 & 76.44 & 80.61 & 24.71 \\
\hline 7482.14 & 334258.60 & 17.00 & 1923.97 & 76.04 & 213.03 & 70121.70 & 63.77 & 21.51 & 17.01 & 3.53 & 522.73 & 68.92 & 90.12 & 27.14 \\
\hline 7482.20 & 345436.20 & 16.80 & 2309.41 & 84.45 & 189.59 & 70421.71 & 69.36 & 21.66 & 16.72 & 3.84 & 536.57 & 48.79 & 85.62 & 39.68 \\
\hline 7482.27 & 371271.80 & 16.60 & 2432.68 & 85.65 & 183.85 & 70942.46 & 71.93 & 22.82 & 17.71 & 4.17 & 534.79 & 46.20 & 88.96 & 40.89 \\
\hline 7482.34 & 407785.40 & 16.20 & 2230.92 & 83.09 & 198.73 & 71151.25 & 68.66 & 24.05 & 18.37 & 4.23 & 548.20 & 54.84 & 89.58 & 40.08 \\
\hline 7482.40 & 395754.40 & 16.20 & 3815.48 & 103.27 & 214.72 & 30708.47 & 83.43 & 32.36 & 17.54 & 5.29 & 176.96 & 60.10 & 108.02 & 42.36 \\
\hline 7482.47 & 378549.40 & 16.40 & 3846.51 & 100.98 & 220.03 & 29904.92 & 80.36 & 32.99 & 17.23 & 5.00 & 175.71 & 62.21 & 106.45 & 40.28 \\
\hline 7482.53 & 359511.60 & 16.60 & 3767.01 & 102.25 & 219.36 & 30254.60 & 80.83 & 24.40 & 17.06 & 1.88 & 171.03 & 36.08 & 126.81 & 12.64 \\
\hline 7482.60 & 329277.00 & 17.00 & 3706.11 & 101.30 & 207.61 & 29314.07 & 78.00 & 24.28 & 16.44 & 2.17 & 168.11 & 34.50 & 124.19 & 12.55 \\
\hline 7482.66 & 292171.40 & 17.40 & 3826.48 & 100.62 & 187.22 & 28638.65 & 78.11 & 24.15 & 17.48 & 1.73 & 154.09 & 26.01 & 124.55 & 12.93 \\
\hline 7482.73 & 281596.00 & 17.60 & 3856.47 & 100.77 & 186.41 & 28532.68 & 78.57 & 24.29 & 18.72 & 1.60 & 147.02 & 25.90 & 122.67 & 13.76 \\
\hline 7482.80 & 276160.00 & 17.80 & 3679.00 & 95.03 & 174.93 & 28446.02 & 81.85 & 24.64 & 20.00 & 1.35 & 144.23 & 26.23 & 121.34 & 14.68 \\
\hline 7482.86 & 269885.00 & 17.80 & 3677.32 & 92.54 & 173.46 & 28530.64 & 76.89 & 27.53 & 18.79 & 1.30 & 141.26 & 24.03 & 121.63 & 15.12 \\
\hline 7482.93 & 272060.00 & 17.80 & 3703.98 & 89.06 & 176.10 & 29151.02 & 78.83 & 27.95 & 19.83 & 0.87 & 141.24 & 25.52 & 122.77 & 14.59 \\
\hline 7482.99 & 273785.40 & 17.80 & 3521.89 & 83.10 & 163.26 & 28419.51 & 74.08 & 27.85 & 18.54 & 1.43 & 141.99 & 24.79 & 121.08 & 15.18 \\
\hline 7483.06 & 300356.40 & 17.40 & 3229.20 & 75.67 & 159.22 & 28095.39 & 74.27 & 28.22 & 18.49 & 1.49 & 150.27 & 25.81 & 126.71 & 14.80 \\
\hline 7483.12 & 328844.40 & 17.00 & 3445.40 & 80.41 & 177.82 & 29650.33 & 73.55 & 27.53 & 18.48 & 2.18 & 153.54 & 25.51 & 128.26 & 15.21 \\
\hline 7483.19 & 350977.40 & 16.80 & 3530.32 & 83.73 & 185.76 & 29502.22 & 76.39 & 23.62 & 18.38 & 2.04 & 146.02 & 26.92 & 130.17 & 14.32 \\
\hline 7483.25 & 373131.00 & 16.40 & 3537.74 & 85.25 & 187.68 & 30038.52 & 74.72 & 22.32 & 18.07 & 2.18 & 144.22 & 27.63 & 131.75 & 14.77 \\
\hline 7483.32 & 396068.40 & 16.20 & 3731.87 & 87.85 & 197.14 & 31250.20 & 78.79 & 21.34 & 19.16 & 1.81 & 144.73 & 28.91 & 134.57 & 15.40 \\
\hline 7483.39 & 397404.20 & 16.20 & 3894.88 & 93.14 & 203.52 & 31866.11 & 79.26 & 28.17 & 19.19 & 5.95 & 150.15 & 60.44 & 111.80 & 41.34 \\
\hline 7483.45 & 395835.60 & 16.20 & 3910.32 & 92.56 & 197.39 & 31963.74 & 73.69 & 29.89 & 19.06 & 5.59 & 152.97 & 59.61 & 114.34 & 41.14 \\
\hline 7483.52 & 392722.60 & 16.20 & 3753.25 & 87.40 & 189.96 & 32092.10 & 70.09 & 29.89 & 19.50 & 5.67 & 167.28 & 60.15 & 114.46 & 41.09 \\
\hline 7483.58 & 394145.20 & 16.20 & 3851.67 & 92.33 & 189.56 & 33101.50 & 68.46 & 31.46 & 21.29 & 5.82 & 173.75 & 61.17 & 116.05 & 40.52 \\
\hline 7483.65 & 393529.40 & 16.20 & 3844.15 & 97.85 & 185.81 & 33249.05 & 66.51 & 32.42 & 20.02 & 6.63 & 174.41 & 60.55 & 117.68 & 40.34 \\
\hline 7483.71 & 383902.80 & 16.40 & 7807.16 & 97.61 & 177.43 & 32889.05 & 62.34 & 27.14 & 19.63 & 2.83 & 162.09 & 28.56 & 141.06 & 14.08 \\
\hline 7483.78 & 365306.20 & 16.60 & 7565.35 & 94.82 & 165.10 & 31259.32 & 65.11 & 27.40 & 19.69 & 3.39 & 163.21 & 28.21 & 139.59 & 14.79 \\
\hline 7483.85 & 348434.80 & 16.80 & 7516.54 & 95.76 & 159.92 & 29814.48 & 78.72 & 39.93 & 22.09 & 7.37 & 162.07 & 55.26 & 117.01 & 36.73 \\
\hline 7483.91 & 334537.60 & 17.00 & 7429.11 & 93.20 & 153.93 & 27942.68 & 84.55 & 40.76 & 20.99 & 7.45 & 155.06 & 52.52 & 116.35 & 36.67 \\
\hline 7483.98 & 323595.80 & 17.00 & 7412.49 & 94.17 & 155.12 & 26222.38 & 83.47 & 40.90 & 20.81 & 6.87 & 157.79 & 51.77 & 114.85 & 37.03 \\
\hline 7484.04 & 311084.40 & 17.00 & 3091.74 & 89.40 & 150.17 & 24588.68 & 84.51 & 37.57 & 20.76 & 6.44 & 169.25 & 50.46 & 111.28 & 36.92 \\
\hline 7484.11 & 316853.40 & 17.00 & 3229.08 & 93.99 & 153.76 & 25411.26 & 81.69 & 36.87 & 21.25 & 6.28 & 164.12 & 51.12 & 109.04 & 35.11 \\
\hline
\end{tabular}




\begin{tabular}{|c|c|c|c|c|c|c|c|c|c|c|c|c|c|c|}
\hline Depth & XRF & XRF Live Time & $\mathrm{Ti}(\mathrm{ppm})$ & $\mathrm{Cr}(\mathrm{ppm})$ & $\mathrm{Mn}(\mathrm{ppm})$ & $\mathrm{Fe}(\mathrm{ppm})$ & $\mathrm{Ni}(\mathrm{ppm})$ & $\mathrm{Cu}(\mathrm{ppm})$ & $\mathrm{Zn}(\mathrm{ppm})$ & As (ppm) & $\mathrm{Rb}(\mathrm{ppm})$ & $\mathrm{Sr}(\mathrm{ppm})$ & $\mathrm{Y}(\mathrm{ppm})$ & $\mathrm{Zr}(\mathrm{ppm})$ \\
\hline 7484.17 & 325512.80 & 17.00 & 3321.26 & 99.99 & 156.87 & 26120.85 & 70.03 & 24.20 & 19.29 & 2.65 & 159.35 & 22.69 & 130.40 & 13.85 \\
\hline 7484.24 & 316454.20 & 17.20 & 3202.03 & 97.39 & 161.17 & 26410.64 & 62.34 & 30.93 & 18.44 & 2.47 & 175.21 & 22.75 & 126.03 & 14.69 \\
\hline 7484.30 & 325499.40 & 17.20 & 3331.38 & 97.85 & 164.38 & 28241.89 & 62.99 & 31.90 & 18.97 & 2.74 & 168.90 & 22.77 & 124.81 & 13.63 \\
\hline 7484.37 & 330302.40 & 17.20 & 3622.51 & 102.43 & 165.48 & 29531.39 & 59.96 & 33.95 & 18.37 & 3.28 & 153.78 & 25.16 & 128.50 & 15.22 \\
\hline 7484.44 & 331493.00 & 17.20 & 3456.36 & 98.65 & 155.47 & 28706.65 & 59.73 & 32.87 & 16.91 & 3.21 & 162.32 & 25.94 & 130.30 & 16.09 \\
\hline 7484.50 & 334540.60 & 17.20 & 3447.32 & 93.67 & 151.82 & 28289.69 & 59.14 & 32.92 & 16.73 & 3.22 & 160.60 & 27.65 & 130.00 & 15.12 \\
\hline 7484.57 & 344575.40 & 17.00 & 3248.93 & 90.85 & 141.15 & 26692.90 & 62.20 & 28.19 & 17.16 & 3.50 & 154.31 & 29.27 & 135.73 & 14.10 \\
\hline 7484.63 & 319945.60 & 17.20 & 2774.86 & 79.28 & 125.58 & 24792.90 & 61.49 & 27.16 & 17.83 & 3.69 & 174.80 & 30.74 & 139.34 & 12.76 \\
\hline 7484.70 & 323219.80 & 17.20 & 3789.06 & 78.98 & 128.51 & 24558.68 & 62.31 & 29.07 & 18.87 & 3.20 & 179.21 & 29.33 & 138.26 & 12.53 \\
\hline 7484.76 & 316537.80 & 17.20 & 3928.90 & 80.49 & 134.75 & 24574.27 & 64.58 & 281.69 & 19.32 & 3.09 & 168.90 & 28.55 & 137.62 & 12.13 \\
\hline 7484.83 & 303932.80 & 17.20 & 3911.71 & 78.01 & 138.29 & 24362.73 & 63.99 & 387.04 & 20.41 & 3.03 & 162.64 & 27.66 & 135.92 & 12.21 \\
\hline 7484.90 & 297361.20 & 17.20 & 4175.94 & 76.84 & 149.77 & 26025.49 & 62.54 & 385.59 & 19.48 & 2.88 & 148.82 & 27.56 & 132.11 & 13.17 \\
\hline 7484.96 & 309680.60 & 17.00 & 4565.77 & 84.50 & 169.25 & 28137.97 & 63.82 & 386.08 & 20.64 & 3.23 & 127.88 & 25.66 & 131.44 & 15.14 \\
\hline 7485.03 & 302150.80 & 17.00 & 3657.66 & 84.67 & 179.38 & 28528.05 & 72.10 & 391.86 & 23.85 & 7.12 & 136.68 & 47.96 & 109.91 & 39.30 \\
\hline 7485.09 & 298850.60 & 17.00 & 3691.45 & 90.54 & 180.05 & 28351.29 & 70.58 & 145.73 & 23.93 & 7.42 & 138.72 & 48.47 & 110.10 & 39.39 \\
\hline 7485.16 & 305860.80 & 17.00 & 3739.54 & 91.40 & 175.75 & 28430.17 & 72.11 & 42.86 & 22.28 & 7.31 & 137.06 & 48.57 & 116.06 & 39.87 \\
\hline 7485.22 & 304615.80 & 17.00 & 3881.18 & 100.56 & 175.47 & 27851.57 & 72.53 & 41.23 & 23.06 & 6.98 & 137.25 & 49.13 & 118.38 & 39.70 \\
\hline 7485.29 & 280100.00 & 17.40 & 3923.48 & 101.28 & 166.13 & 26152.69 & 71.23 & 41.30 & 22.27 & 5.97 & 130.63 & 49.53 & 114.12 & 38.46 \\
\hline 7485.35 & 267959.80 & 17.60 & 3906.66 & 104.24 & 155.92 & 25414.25 & 65.42 & 37.55 & 18.65 & 2.18 & 119.55 & 27.27 & 133.51 & 13.46 \\
\hline 7485.42 & 257303.80 & 17.80 & 3854.05 & 94.43 & 159.47 & 25714.92 & 65.92 & 46.79 & 19.28 & 1.76 & 117.17 & 27.05 & 133.64 & 13.36 \\
\hline 7485.49 & 240846.20 & 18.00 & 3869.38 & 92.23 & 168.76 & 26524.00 & 66.94 & 138.97 & 20.64 & 1.77 & 117.72 & 28.34 & 127.11 & 13.23 \\
\hline 7485.55 & 234152.60 & 18.20 & 3884.74 & 89.66 & 169.82 & 27660.57 & 66.18 & 142.67 & 21.21 & 2.06 & 118.21 & 27.64 & 125.84 & 11.87 \\
\hline 7485.62 & 245927.40 & 18.00 & 3847.17 & 87.54 & 175.12 & 28610.33 & 62.60 & 143.47 & 21.04 & 2.55 & 122.63 & 27.54 & 126.53 & 13.59 \\
\hline 7485.68 & 242004.00 & 18.00 & 4044.04 & 83.83 & 177.39 & 29125.48 & 69.15 & 144.63 & 24.57 & 5.76 & 130.59 & 45.63 & 106.39 & 34.39 \\
\hline 7485.75 & 235792.20 & 18.00 & 4151.92 & 89.96 & 178.82 & 28877.36 & 67.05 & 130.44 & 24.46 & 6.41 & 125.88 & 44.19 & 105.96 & 34.97 \\
\hline 7485.81 & 231605.20 & 18.00 & 4179.28 & 91.52 & 180.18 & 28436.57 & 65.37 & 40.37 & 23.34 & 6.36 & 134.44 & 43.75 & 108.53 & 34.26 \\
\hline 7485.88 & 213896.00 & 18.20 & 4168.71 & 88.07 & 186.80 & 26925.57 & 64.65 & 50.84 & 22.22 & 6.41 & 130.53 & 43.59 & 105.59 & 34.64 \\
\hline 7485.94 & 226400.40 & 18.00 & 4284.13 & 95.29 & 192.11 & 26422.92 & 69.77 & 54.05 & 21.24 & 6.21 & 130.77 & 44.98 & 106.71 & 33.21 \\
\hline 7486.01 & 241863.20 & 17.80 & 4188.28 & 95.80 & 198.10 & 26209.03 & 61.46 & 124.42 & 17.57 & 2.66 & 122.87 & 27.79 & 127.85 & 12.40 \\
\hline 7486.08 & 254970.80 & 17.80 & 4173.34 & 95.02 & 205.09 & 26240.53 & 61.25 & 145.72 & 16.72 & 1.85 & 131.74 & 29.44 & 127.14 & 12.04 \\
\hline 7486.14 & 263624.40 & 17.80 & 4114.97 & 98.51 & 209.44 & 25953.47 & 58.50 & 152.01 & 17.23 & 1.93 & 128.36 & 27.71 & 125.31 & 12.84 \\
\hline 7486.21 & 308547.40 & 17.20 & 4168.97 & 99.67 & 215.40 & 26910.25 & 59.90 & 139.16 & 18.17 & 1.77 & 138.17 & 28.39 & 131.61 & 14.54 \\
\hline 7486.27 & 329743.80 & 17.00 & 4076.39 & 100.66 & 212.21 & 27393.94 & 61.24 & 135.03 & 18.85 & 2.01 & 147.85 & 27.77 & 136.56 & 15.72 \\
\hline
\end{tabular}




\begin{tabular}{|c|c|c|c|c|c|c|c|c|c|c|c|c|c|c|}
\hline Depth & XRF & XRF Live Time & $\mathrm{Ti}(\mathrm{ppm})$ & $\mathrm{Cr}(\mathrm{ppm})$ & $\mathrm{Mn}(\mathrm{ppm})$ & $\mathrm{Fe}(\mathrm{ppm})$ & $\mathrm{Ni}(\mathrm{ppm})$ & $\mathrm{Cu}(\mathrm{ppm})$ & $\mathrm{Zn}(\mathrm{ppm})$ & As (ppm) & $\mathrm{Rb}(\mathrm{ppm})$ & $\mathrm{Sr}(\mathrm{ppm})$ & $\mathrm{Y}(\mathrm{ppm})$ & $\mathrm{Zr}(\mathrm{ppm})$ \\
\hline 7486.34 & 350004.80 & 16.80 & 4135.41 & 105.55 & 212.02 & 27980.37 & 63.98 & 64.67 & 18.92 & 2.49 & 153.78 & 28.17 & 139.83 & 16.76 \\
\hline 7486.40 & 371441.60 & 16.40 & 4174.95 & 104.40 & 209.50 & 28857.96 & 64.84 & 46.35 & 20.45 & 3.34 & 155.09 & 28.00 & 145.08 & 17.66 \\
\hline 7486.47 & 395881.40 & 16.00 & 4263.53 & 104.62 & 210.56 & 29743.74 & 66.88 & 44.40 & 21.19 & 3.44 & 163.20 & 28.79 & 148.43 & 18.44 \\
\hline 7486.54 & 401384.00 & 16.00 & 4301.59 & 110.86 & 199.68 & 29832.49 & 68.25 & 50.66 & 21.80 & 2.99 & 164.66 & 28.22 & 150.13 & 17.67 \\
\hline 7486.60 & 400576.60 & 16.00 & 4340.99 & 106.24 & 197.11 & 29708.83 & 67.77 & 70.00 & 22.08 & 2.85 & 164.63 & 28.58 & 148.51 & 16.55 \\
\hline 7486.67 & 358007.20 & 16.40 & 4339.95 & 108.29 & 192.38 & 30407.03 & 75.96 & 78.15 & 31.35 & 8.24 & 175.80 & 60.24 & 125.82 & 40.77 \\
\hline 7486.73 & 355547.80 & 16.60 & 5060.93 & 114.97 & 183.87 & 30151.80 & 78.28 & 76.23 & 30.88 & 7.78 & 171.23 & 61.34 & 123.46 & 39.96 \\
\hline 7486.80 & 349471.00 & 16.80 & 5101.81 & 116.74 & 170.02 & 29334.89 & 78.43 & 67.52 & 29.63 & 7.63 & 163.47 & 62.48 & 123.18 & 38.92 \\
\hline 7486.86 & 328607.80 & 17.00 & 5012.67 & 109.33 & 166.43 & 29651.68 & 82.85 & 67.68 & 29.50 & 12.18 & 171.33 & 87.25 & 97.31 & 62.38 \\
\hline 7486.93 & 328483.80 & 17.00 & 5144.76 & 114.62 & 172.77 & 31313.43 & 80.30 & 47.96 & 29.52 & 11.86 & 165.93 & 85.98 & 95.81 & 63.94 \\
\hline 7486.99 & 371066.00 & 16.60 & 5205.22 & 112.49 & 181.03 & 32381.69 & 71.06 & 34.66 & 20.97 & 6.25 & 151.45 & 53.45 & 116.67 & 38.53 \\
\hline 7487.06 & 370155.80 & 16.60 & 4631.17 & 113.41 & 190.10 & 33277.93 & 69.77 & 30.84 & 21.87 & 6.46 & 156.40 & 51.93 & 117.47 & 39.07 \\
\hline 7487.13 & 374600.20 & 16.60 & 4726.23 & 112.67 & 198.31 & 34812.28 & 69.08 & 31.13 & 23.00 & 6.52 & 162.60 & 51.45 & 117.33 & 40.30 \\
\hline 7487.19 & 388267.40 & 16.60 & 4770.88 & 117.39 & 200.19 & 35161.21 & 65.16 & 22.60 & 23.73 & 2.31 & 161.23 & 25.51 & 139.65 & 16.50 \\
\hline 7487.26 & 386687.40 & 16.60 & 4747.43 & 117.82 & 195.64 & 34595.21 & 63.51 & 22.64 & 25.21 & 2.44 & 168.82 & 27.40 & 142.84 & 15.50 \\
\hline 7487.32 & 383842.20 & 16.80 & 4740.84 & 118.23 & 197.94 & 33407.32 & 64.35 & 22.90 & 24.80 & 2.45 & 171.53 & 27.96 & 145.47 & 16.61 \\
\hline 7487.39 & 379571.00 & 16.80 & 4486.38 & 107.45 & 196.77 & 32819.18 & 62.90 & 24.13 & 23.86 & 2.28 & 171.46 & 28.49 & 144.78 & 16.66 \\
\hline 7487.45 & 367113.00 & 16.80 & 4340.72 & 108.72 & 194.89 & 31557.68 & 61.58 & 24.12 & 21.35 & 2.16 & 164.84 & 28.25 & 146.75 & 15.78 \\
\hline 7487.52 & 348629.80 & 16.80 & 4474.02 & 110.85 & 198.08 & 30217.87 & 55.61 & 22.87 & 19.60 & 1.89 & 158.65 & 30.62 & 146.97 & 17.00 \\
\hline 7487.59 & 327757.00 & 17.00 & 4410.34 & 105.38 & 210.94 & 29169.68 & 54.79 & 23.03 & 16.37 & 1.66 & 149.75 & 30.31 & 142.37 & 15.83 \\
\hline 7487.65 & 303196.00 & 17.20 & 4343.99 & 105.29 & 207.44 & 28386.50 & 56.75 & 29.70 & 19.70 & 5.71 & 156.91 & 55.58 & 117.08 & 38.98 \\
\hline 7487.72 & 282803.80 & 17.40 & 9802.07 & 109.29 & 204.80 & 27348.08 & 53.70 & 35.42 & 19.51 & 5.44 & 151.45 & 54.67 & 116.06 & 40.33 \\
\hline 7487.78 & 297263.60 & 17.20 & 9805.19 & 106.74 & 220.96 & 29826.47 & 55.98 & 36.80 & 20.42 & 5.64 & 148.78 & 53.86 & 114.26 & 40.23 \\
\hline 7487.85 & 306759.60 & 17.20 & 9715.00 & 102.40 & 218.02 & 30220.83 & 57.48 & 38.06 & 20.38 & 5.93 & 142.86 & 53.06 & 113.85 & 39.45 \\
\hline 7487.91 & 309339.80 & 17.20 & 9785.90 & 101.93 & 201.96 & 30652.65 & 53.65 & 37.72 & 21.54 & 6.07 & 142.71 & 52.04 & 115.89 & 40.25 \\
\hline 7487.98 & 328483.40 & 17.00 & 9761.52 & 97.98 & 196.41 & 31236.58 & 48.73 & 29.72 & 18.64 & 2.03 & 133.09 & 27.50 & 142.69 & 15.86 \\
\hline 7488.04 & 347245.80 & 16.80 & 4382.86 & 100.87 & 194.14 & 32184.99 & 51.38 & 26.44 & 18.02 & 2.09 & 138.04 & 28.42 & 142.80 & 13.85 \\
\hline 7488.11 & 340321.20 & 17.00 & 4435.67 & 101.56 & 178.70 & 30954.71 & 51.23 & 42.09 & 19.99 & 1.65 & 150.59 & 29.30 & 144.44 & 14.00 \\
\hline 7488.18 & 346071.40 & 17.00 & 4427.46 & 106.10 & 178.90 & 32374.92 & 53.88 & 42.48 & 21.19 & 1.42 & 156.21 & 29.58 & 144.86 & 13.55 \\
\hline 7488.24 & 353667.40 & 17.00 & 4275.98 & 109.56 & 177.27 & 31189.58 & 60.27 & 47.95 & 21.65 & 1.84 & 166.03 & 30.41 & 145.71 & 12.97 \\
\hline 7488.31 & 339742.60 & 17.20 & 4192.24 & 108.90 & 166.76 & 30115.40 & 63.06 & 52.18 & 20.63 & 1.70 & 173.81 & 28.27 & 139.18 & 13.60 \\
\hline 7488.37 & 345607.00 & 17.20 & 4264.42 & 107.19 & 167.09 & 29748.61 & 64.28 & 62.75 & 21.32 & 1.59 & 168.67 & 28.14 & 141.44 & 13.89 \\
\hline 7488.44 & 345121.20 & 17.20 & 4086.30 & 102.64 & 161.24 & 28048.88 & 65.01 & 52.75 & 19.23 & 1.69 & 164.69 & 29.50 & 139.94 & 13.41 \\
\hline
\end{tabular}




\begin{tabular}{|c|c|c|c|c|c|c|c|c|c|c|c|c|c|c|}
\hline Depth & XRF & XRF Live Time & $\mathrm{Ti}(\mathrm{ppm})$ & $\mathrm{Cr}(\mathrm{ppm})$ & $\mathrm{Mn}(\mathrm{ppm})$ & $\mathrm{Fe}(\mathrm{ppm})$ & $\mathrm{Ni}(\mathrm{ppm})$ & $\mathrm{Cu}(\mathrm{ppm})$ & $\mathrm{Zn}(\mathrm{ppm})$ & As (ppm) & $\mathrm{Rb}(\mathrm{ppm})$ & $\mathrm{Sr}(\mathrm{ppm})$ & $\mathrm{Y}(\mathrm{ppm})$ & $\mathrm{Zr}(\mathrm{ppm})$ \\
\hline 7488.50 & 344985.20 & 17.20 & 4155.53 & 103.41 & 167.12 & 26859.78 & 64.23 & 56.33 & 17.64 & 2.12 & 163.03 & 29.58 & 139.02 & 13.50 \\
\hline 7488.57 & 353876.00 & 17.20 & 4201.69 & 102.23 & 169.66 & 28376.17 & 63.18 & 84.25 & 18.17 & 1.96 & 155.31 & 30.57 & 137.80 & 13.55 \\
\hline 7488.64 & 374036.00 & 17.00 & 4380.18 & 106.37 & 184.28 & 29894.82 & 60.94 & 86.36 & 18.56 & 2.25 & 147.56 & 31.00 & 139.20 & 14.27 \\
\hline 7488.70 & 367212.20 & 17.00 & 4413.66 & 105.08 & 180.00 & 30042.34 & 60.41 & 75.18 & 17.93 & 2.29 & 155.26 & 30.26 & 137.45 & 14.81 \\
\hline 7488.77 & 378104.00 & 16.80 & 4591.99 & 109.28 & 186.31 & 33680.50 & 58.48 & 68.75 & 20.02 & 2.56 & 154.88 & 28.41 & 138.03 & 15.59 \\
\hline 7488.83 & 385343.40 & 16.60 & 4513.04 & 107.93 & 185.75 & 34223.67 & 56.65 & 65.63 & 20.82 & 2.34 & 151.99 & 29.00 & 140.12 & 16.13 \\
\hline 7488.90 & 387850.60 & 16.40 & 4596.45 & 108.17 & 194.62 & 34272.46 & 54.43 & 36.17 & 19.36 & 2.28 & 150.72 & 28.26 & 140.23 & 17.17 \\
\hline 7488.96 & 384338.60 & 16.40 & 4530.16 & 107.66 & 199.38 & 33658.50 & 54.01 & 46.38 & 19.44 & 2.22 & 154.59 & 30.33 & 141.65 & 15.69 \\
\hline 7489.03 & 389656.40 & 16.40 & 4493.95 & 107.98 & 208.90 & 33577.20 & 50.59 & 46.51 & 19.74 & 2.33 & 146.59 & 30.87 & 140.94 & 16.10 \\
\hline 7489.09 & 389411.40 & 16.40 & 4485.93 & 109.66 & 211.40 & 30444.46 & 50.76 & 56.58 & 17.70 & 2.44 & 146.11 & 31.20 & 143.68 & 16.08 \\
\hline 7489.16 & 388405.60 & 16.40 & 4465.01 & 109.33 & 207.89 & 30108.63 & 51.87 & 58.58 & 17.59 & 2.49 & 151.85 & 30.30 & 144.03 & 15.09 \\
\hline 7489.23 & 388277.80 & 16.40 & 4434.12 & 110.17 & 204.86 & 29850.41 & 54.14 & 56.40 & 17.84 & 2.39 & 156.30 & 30.94 & 143.97 & 14.98 \\
\hline 7489.29 & 394587.20 & 16.20 & 4512.47 & 113.77 & 199.07 & 30229.95 & 58.30 & 45.40 & 18.45 & 2.54 & 155.19 & 29.51 & 145.70 & 16.28 \\
\hline 7489.36 & 397504.40 & 16.20 & 4474.54 & 114.02 & 195.32 & 30791.63 & 62.15 & 64.29 & 19.28 & 2.53 & 162.28 & 30.15 & 147.11 & 14.94 \\
\hline 7489.42 & 392626.40 & 16.20 & 4501.84 & 116.86 & 192.58 & 31529.63 & 59.91 & 55.54 & 20.92 & 2.49 & 168.76 & 30.03 & 142.89 & 14.77 \\
\hline 7489.49 & 392317.20 & 16.20 & 4580.24 & 115.64 & 196.76 & 31859.55 & 61.87 & 55.22 & 21.32 & 2.41 & 170.61 & 29.30 & 142.57 & 14.87 \\
\hline 7489.55 & 394994.00 & 16.20 & 4624.69 & 117.34 & 193.50 & 31821.48 & 60.99 & 55.85 & 21.98 & 1.95 & 169.54 & 27.86 & 145.62 & 14.72 \\
\hline 7489.62 & 385802.40 & 16.40 & 4640.04 & 117.25 & 195.91 & 32971.42 & 59.27 & 51.68 & 21.76 & 1.49 & 169.65 & 27.41 & 142.02 & 15.05 \\
\hline 7489.69 & 390290.40 & 16.20 & 4692.89 & 118.39 & 199.35 & 33814.66 & 60.33 & 32.23 & 22.34 & 1.73 & 166.62 & 25.59 & 144.37 & 16.55 \\
\hline 7489.75 & 392974.20 & 16.20 & 5843.43 & 114.85 & 203.82 & 34408.83 & 59.06 & 31.04 & 21.44 & 1.47 & 155.17 & 24.98 & 145.21 & 16.78 \\
\hline 7489.82 & 388667.60 & 16.40 & 5868.45 & 117.00 & 199.96 & 34465.19 & 54.85 & 29.33 & 20.92 & 1.18 & 153.70 & 26.96 & 145.86 & 17.84 \\
\hline 7489.88 & 382803.40 & 16.60 & 5770.00 & 113.39 & 198.40 & 33796.37 & 54.52 & 26.94 & 19.71 & 1.43 & 156.32 & 28.22 & 145.44 & 18.69 \\
\hline 7489.95 & 392876.60 & 16.40 & 5762.31 & 105.60 & 205.86 & 33278.26 & 51.89 & 26.16 & 19.59 & 1.64 & 153.30 & 29.82 & 144.19 & 18.47 \\
\hline 7490.01 & 396701.00 & 16.40 & 5820.02 & 107.84 & 224.60 & 34135.42 & 47.23 & 23.68 & 18.21 & 1.21 & 157.13 & 32.82 & 140.36 & 17.63 \\
\hline 7490.08 & 400335.60 & 16.40 & 4695.81 & 108.63 & 240.06 & 34796.88 & 47.39 & 23.45 & 18.21 & 1.34 & 159.27 & 36.40 & 138.83 & 16.76 \\
\hline 7490.14 & 404224.00 & 16.20 & 4624.93 & 106.42 & 259.45 & 35149.05 & 46.59 & 25.82 & 19.38 & 1.86 & 155.39 & 36.25 & 137.00 & 15.59 \\
\hline 7490.21 & 409199.00 & 16.00 & 4670.51 & 108.31 & 267.58 & 35790.63 & 48.04 & 27.08 & 21.45 & 2.40 & 158.64 & 35.86 & 138.31 & 15.25 \\
\hline 7490.28 & 406130.80 & 16.20 & 4699.45 & 115.28 & 256.91 & 35348.41 & 49.39 & 28.27 & 22.21 & 2.84 & 160.09 & 36.06 & 144.35 & 15.22 \\
\hline 7490.34 & 401828.80 & 16.20 & 4761.68 & 114.65 & 241.21 & 35513.38 & 53.78 & 30.15 & 24.20 & 3.49 & 157.26 & 36.32 & 150.80 & 15.03 \\
\hline 7490.41 & 383549.00 & 16.40 & 4757.12 & 113.92 & 224.54 & 35805.12 & 59.27 & 29.92 & 25.69 & 3.94 & 149.98 & 32.70 & 152.10 & 16.06 \\
\hline 7490.47 & 377062.60 & 16.60 & 4692.92 & 111.93 & 207.52 & 35648.77 & 66.63 & 28.63 & 25.46 & 3.80 & 157.81 & 32.93 & 149.68 & 15.40 \\
\hline 7490.54 & 367313.60 & 16.80 & 4531.46 & 110.04 & 195.43 & 34952.84 & 67.22 & 28.12 & 24.26 & 3.48 & 157.36 & 33.03 & 146.43 & 15.33 \\
\hline 7490.60 & 351246.00 & 16.80 & 4412.79 & 103.48 & 194.89 & 34079.32 & 66.98 & 26.63 & 23.95 & 2.77 & 156.40 & 32.74 & 141.56 & 13.66 \\
\hline
\end{tabular}




\begin{tabular}{|c|c|c|c|c|c|c|c|c|c|c|c|c|c|c|}
\hline Depth & XRF & XRF Live Time & $\mathrm{Ti}(\mathrm{ppm})$ & $\mathrm{Cr}$ (ppm) & $\mathrm{Mn}$ (ppm) & $\mathrm{Fe}(\mathrm{ppm})$ & $\mathrm{Ni}(\mathrm{ppm})$ & $\mathrm{Cu}(\mathrm{ppm})$ & $\mathrm{Zn}(\mathrm{ppm})$ & As (ppm) & $\mathrm{Rb}(\mathrm{ppm})$ & $\mathrm{Sr}(\mathrm{ppm})$ & $\mathrm{Y}(\mathrm{ppm})$ & $\mathrm{Zr}(\mathrm{ppm})$ \\
\hline 7490.67 & 323097.60 & 17.20 & 3986.08 & 97.68 & 185.07 & 31386.36 & 63.08 & 31.93 & 22.76 & 2.15 & 159.31 & 31.08 & 136.72 & 13.17 \\
\hline 7490.73 & 262819.60 & 17.80 & 4544.17 & 109.34 & 187.82 & 24507.97 & 63.67 & 41.20 & 16.11 & 0.03 & 145.85 & 26.69 & 112.37 & 9.15 \\
\hline 7490.80 & 194957.00 & 18.40 & 7137.78 & 108.03 & 180.07 & 17982.63 & 54.05 & 41.06 & 13.43 & 2.17 & 112.66 & 20.13 & 94.02 & 9.57 \\
\hline 7490.87 & 190716.00 & 18.40 & 8580.89 & 108.70 & 188.42 & 18831.02 & 52.57 & 80.91 & 13.65 & 2.03 & 100.82 & 20.22 & 92.30 & 8.96 \\
\hline 7490.93 & 193913.60 & 18.40 & 8553.56 & 112.12 & 192.63 & 18982.25 & 53.96 & 80.80 & 12.53 & 2.59 & 100.74 & 20.40 & 95.20 & 9.52 \\
\hline 7491.00 & 206498.60 & 18.20 & 8830.80 & 120.61 & 196.85 & 19445.08 & 57.68 & 75.83 & 11.96 & 2.91 & 94.85 & 20.51 & 96.84 & 10.26 \\
\hline 7491.06 & 269749.60 & 17.60 & 8293.73 & 109.83 & 185.31 & 25044.97 & 56.45 & 66.78 & 17.60 & 4.53 & 112.62 & 25.56 & 127.23 & 13.50 \\
\hline 7491.13 & 336632.80 & 17.00 & 5805.20 & 111.91 & 193.75 & 32457.54 & 65.70 & 65.11 & 21.45 & 2.75 & 141.03 & 29.88 & 149.08 & 14.07 \\
\hline 7491.19 & 343733.40 & 17.00 & 4593.76 & 114.08 & 186.17 & 33048.64 & 69.83 & 24.04 & 22.81 & 3.51 & 147.41 & 29.33 & 151.99 & 14.38 \\
\hline 7491.26 & 355618.20 & 17.00 & 4594.87 & 112.68 & 186.82 & 34688.60 & 72.24 & 25.06 & 24.37 & 3.60 & 149.72 & 29.49 & 153.10 & 14.83 \\
\hline 7491.33 & 372923.80 & 16.80 & 4427.40 & 106.25 & 190.74 & 37165.25 & 73.44 & 25.28 & 26.34 & 4.07 & 153.49 & 30.60 & 150.80 & 14.84 \\
\hline 7491.39 & 392970.20 & 16.60 & 4187.65 & 101.33 & 218.45 & 40303.43 & 74.96 & 25.18 & 27.22 & 4.62 & 157.44 & 36.28 & 141.10 & 14.46 \\
\hline 7491.46 & 407589.20 & 16.40 & 4037.64 & 104.85 & 221.07 & 42724.10 & 74.97 & 27.56 & 28.10 & 4.46 & 154.11 & 38.19 & 139.47 & 14.04 \\
\hline 7491.52 & 409209.60 & 16.40 & 3953.29 & 106.02 & 224.11 & 42557.88 & 76.57 & 40.59 & 27.95 & 9.44 & 158.13 & 59.09 & 116.54 & 41.33 \\
\hline 7491.59 & 409426.60 & 16.40 & 4042.57 & 110.84 & 221.81 & 42236.47 & 74.23 & 40.66 & 27.85 & 9.27 & 155.86 & 57.17 & 117.07 & 42.49 \\
\hline 7491.65 & 406646.20 & 16.40 & 4272.41 & 115.63 & 217.47 & 41882.81 & 75.74 & 39.17 & 29.34 & 9.06 & 153.68 & 57.22 & 118.25 & 42.00 \\
\hline 7491.72 & 398081.00 & 16.40 & 4357.21 & 118.98 & 196.02 & 39939.79 & 76.90 & 40.11 & 30.67 & 8.76 & 152.69 & 52.44 & 123.33 & 41.91 \\
\hline 7491.78 & 389315.80 & 16.40 & 4315.79 & 121.07 & 192.12 & 36373.05 & 73.97 & 41.21 & 30.01 & 8.40 & 187.86 & 50.71 & 124.59 & 42.32 \\
\hline 7491.85 & 389362.20 & 16.40 & 4089.45 & 136.52 & 203.17 & 36286.38 & 70.86 & 40.55 & 28.26 & 2.57 & 276.48 & 29.67 & 146.12 & 15.02 \\
\hline 7491.92 & 388529.60 & 16.40 & 3839.78 & 145.76 & 207.06 & 35775.72 & 72.26 & 62.65 & 27.78 & 2.67 & 330.73 & 30.37 & 144.79 & 13.34 \\
\hline 7491.98 & 383697.80 & 16.60 & 3634.54 & 153.42 & 211.43 & 33860.12 & 70.94 & 67.83 & 25.76 & 2.14 & 364.76 & 29.80 & 145.68 & 14.75 \\
\hline 7492.05 & 377827.20 & 16.80 & 3721.50 & 162.05 & 208.30 & 31803.14 & 67.50 & 68.21 & 23.82 & 2.03 & 375.47 & 28.73 & 145.62 & 14.63 \\
\hline 7492.11 & 375252.40 & 17.00 & 3901.79 & 162.74 & 208.23 & 31757.36 & 67.52 & 64.93 & 22.84 & 2.33 & 354.97 & 29.24 & 146.65 & 15.12 \\
\hline 7492.18 & 382410.20 & 16.80 & 4180.84 & 152.13 & 199.20 & 31822.33 & 65.99 & 56.90 & 25.82 & 2.70 & 278.19 & 31.45 & 146.92 & 15.52 \\
\hline 7492.24 & 389625.00 & 16.60 & 4418.99 & 150.88 & 201.13 & 32666.14 & 63.40 & 37.09 & 27.13 & 2.75 & 247.22 & 31.00 & 148.80 & 16.45 \\
\hline 7492.31 & 392977.00 & 16.40 & 4553.07 & 146.08 & 199.26 & 33028.50 & 61.83 & 33.95 & 26.85 & 2.95 & 231.86 & 29.63 & 148.15 & 14.82 \\
\hline 7492.38 & 396762.40 & 16.20 & 4474.32 & 141.73 & 200.40 & 33408.81 & 61.19 & 37.90 & 28.41 & 3.07 & 237.96 & 30.86 & 148.72 & 15.17 \\
\hline 7492.44 & 400093.20 & 16.00 & 4437.92 & 139.69 & 201.25 & 33331.82 & 61.00 & 55.95 & 29.20 & 3.28 & 236.18 & 30.32 & 150.12 & 15.12 \\
\hline 7492.51 & 396969.20 & 16.00 & 4338.83 & 140.81 & 204.92 & 33322.31 & 58.60 & 58.84 & 28.36 & 3.14 & 240.07 & 28.54 & 151.62 & 14.75 \\
\hline 7492.57 & 392825.00 & 16.00 & 4228.66 & 139.09 & 199.79 & 32609.50 & 58.12 & 60.96 & 27.68 & 3.14 & 246.72 & 30.55 & 149.45 & 13.75 \\
\hline 7492.64 & 390522.80 & 16.20 & 4253.71 & 139.05 & 200.12 & 32215.24 & 56.24 & 65.01 & 27.09 & 2.92 & 238.20 & 30.37 & 148.66 & 13.88 \\
\hline 7492.70 & 389032.20 & 16.40 & 4300.36 & 143.92 & 201.41 & 31842.84 & 56.55 & 60.92 & 25.49 & 2.93 & 234.48 & 30.13 & 149.28 & 13.95 \\
\hline 7492.77 & 388446.20 & 16.40 & 4287.76 & 146.61 & 198.37 & 31637.85 & 54.66 & 46.18 & 25.23 & 2.68 & 231.84 & 30.63 & 147.86 & 13.56 \\
\hline
\end{tabular}




\begin{tabular}{|c|c|c|c|c|c|c|c|c|c|c|c|c|c|c|}
\hline Depth & XRF & XRF Live Time & $\mathrm{Ti}(\mathrm{ppm})$ & $\mathrm{Cr}(\mathrm{ppm})$ & $\mathrm{Mn}(\mathrm{ppm})$ & $\mathrm{Fe}(\mathrm{ppm})$ & $\mathrm{Ni}(\mathrm{ppm})$ & $\mathrm{Cu}(\mathrm{ppm})$ & $\mathrm{Zn}(\mathrm{ppm})$ & As (ppm) & $\mathrm{Rb}(\mathrm{ppm})$ & $\mathrm{Sr}(\mathrm{ppm})$ & $\mathrm{Y}(\mathrm{ppm})$ & $\mathrm{Zr}(\mathrm{ppm})$ \\
\hline 7492.83 & 387798.00 & 16.40 & 4500.56 & 143.59 & 192.72 & 31507.84 & 57.25 & 40.36 & 25.21 & 2.72 & 217.01 & 30.95 & 148.60 & 13.18 \\
\hline 7492.90 & 386178.80 & 16.60 & 4488.45 & 134.59 & 193.02 & 31301.47 & 57.56 & 37.80 & 25.08 & 2.47 & 197.48 & 30.62 & 148.89 & 13.04 \\
\hline 7492.97 & 387182.60 & 16.60 & 4506.49 & 133.78 & 189.66 & 32019.68 & 59.22 & 32.07 & 27.65 & 2.93 & 193.50 & 31.10 & 150.45 & 14.04 \\
\hline 7493.03 & 386849.40 & 16.60 & 4404.30 & 128.16 & 186.75 & 33437.43 & 62.82 & 32.44 & 29.96 & 2.85 & 192.64 & 30.54 & 151.08 & 14.57 \\
\hline 7493.10 & 381795.80 & 16.80 & 4367.61 & 129.42 & 181.83 & 33478.46 & 63.59 & 30.00 & 30.76 & 2.79 & 192.06 & 30.95 & 150.51 & 13.79 \\
\hline 7493.16 & 373013.80 & 17.00 & 4009.73 & 120.91 & 178.39 & 32422.47 & 64.51 & 35.56 & 29.81 & 2.71 & 201.91 & 31.12 & 147.80 & 14.18 \\
\hline 7493.23 & 366320.80 & 17.00 & 4054.42 & 125.63 & 175.16 & 31805.75 & 63.14 & 34.16 & 30.74 & 2.62 & 199.56 & 28.94 & 147.82 & 16.12 \\
\hline 7493.29 & 360071.20 & 17.00 & 3961.70 & 123.36 & 173.92 & 31334.09 & 62.68 & 34.42 & 29.51 & 2.60 & 201.74 & 28.98 & 148.06 & 15.74 \\
\hline 7493.36 & 349453.00 & 17.00 & 4104.34 & 126.63 & 176.73 & 32044.60 & 59.94 & 34.30 & 28.58 & 2.48 & 195.26 & 27.23 & 146.34 & 15.96 \\
\hline 7493.43 & 343657.60 & 17.00 & 4025.84 & 118.77 & 181.09 & 31799.96 & 61.16 & 32.82 & 27.77 & 2.31 & 183.06 & 26.85 & 145.52 & 16.59 \\
\hline 7493.49 & 337453.80 & 17.00 & 4060.28 & 117.83 & 178.64 & 31491.08 & 60.40 & 27.89 & 27.74 & 2.73 & 161.59 & 28.20 & 144.50 & 15.29 \\
\hline 7493.56 & 332103.60 & 17.00 & 4042.92 & 113.48 & 178.37 & 31405.15 & 62.93 & 33.23 & 26.87 & 3.95 & 153.67 & 28.95 & 143.25 & 13.91 \\
\hline 7493.62 & 322902.40 & 17.00 & 4098.94 & 114.81 & 175.46 & 31042.72 & 64.44 & 54.87 & 26.29 & 4.05 & 139.40 & 28.53 & 141.49 & 14.15 \\
\hline 7493.69 & 319827.60 & 17.00 & 3805.88 & 103.17 & 168.93 & 28820.49 & 64.85 & 91.84 & 24.56 & 4.60 & 135.25 & 31.35 & 136.54 & 13.00 \\
\hline 7493.75 & 316273.60 & 17.00 & 3839.34 & 100.43 & 167.95 & 29834.79 & 64.85 & 212.52 & 24.44 & 5.09 & 134.95 & 31.18 & 135.33 & 12.19 \\
\hline 7493.82 & 304033.60 & 17.20 & 3460.80 & 92.88 & 225.42 & 28710.87 & 63.78 & 216.19 & 30.42 & 7.38 & 131.38 & 61.83 & 131.19 & 28.02 \\
\hline 7493.88 & 303202.80 & 17.20 & 3422.86 & 91.71 & 221.74 & 28006.69 & 64.89 & 216.38 & 29.86 & 6.88 & 132.22 & 61.17 & 130.42 & 27.46 \\
\hline 7493.95 & 301576.20 & 17.20 & 3288.76 & 85.79 & 222.42 & 27404.29 & 65.35 & 230.36 & 30.01 & 6.76 & 134.06 & 61.65 & 130.31 & 26.55 \\
\hline 7494.02 & 294475.80 & 17.40 & 3445.86 & 89.38 & 220.88 & 27005.46 & 64.70 & 212.70 & 30.73 & 6.66 & 127.81 & 60.49 & 130.64 & 27.42 \\
\hline 7494.08 & 282844.20 & 17.60 & 3218.70 & 86.34 & 219.68 & 25156.81 & 64.73 & 108.10 & 30.16 & 6.25 & 134.40 & 60.72 & 129.20 & 28.62 \\
\hline 7494.15 & 280763.20 & 17.60 & 3585.57 & 95.23 & 161.27 & 26560.34 & 68.66 & 136.67 & 24.45 & 3.91 & 139.06 & 27.61 & 133.71 & 14.10 \\
\hline 7494.21 & 254729.60 & 17.80 & 3392.30 & 92.49 & 147.94 & 26423.22 & 68.14 & 145.22 & 24.65 & 3.70 & 132.35 & 26.25 & 133.34 & 14.67 \\
\hline 7494.28 & 257788.40 & 17.80 & 3357.86 & 95.32 & 145.86 & 27005.72 & 69.02 & 123.97 & 23.76 & 4.10 & 133.89 & 26.26 & 132.68 & 13.93 \\
\hline 7494.34 & 268010.40 & 17.60 & 4200.83 & 96.70 & 148.43 & 27504.89 & 72.01 & 116.96 & 23.96 & 4.33 & 136.62 & 26.81 & 135.52 & 13.45 \\
\hline 7494.41 & 282374.60 & 17.40 & 4286.03 & 103.13 & 147.09 & 30384.37 & 76.35 & 133.64 & 26.15 & 5.67 & 136.72 & 26.44 & 136.32 & 12.70 \\
\hline 7494.48 & 289682.60 & 17.40 & 4282.70 & 98.83 & 151.64 & 30179.52 & 76.49 & 111.15 & 25.38 & 5.62 & 136.94 & 27.75 & 135.10 & 12.54 \\
\hline 7494.54 & 310579.80 & 17.20 & 4395.10 & 99.25 & 164.30 & 30544.63 & 77.10 & 98.94 & 25.28 & 5.75 & 142.81 & 30.49 & 136.08 & 12.30 \\
\hline 7494.61 & 305692.40 & 17.20 & 4460.22 & 99.99 & 171.84 & 31671.07 & 76.15 & 151.85 & 26.83 & 6.04 & 141.25 & 31.73 & 135.23 & 13.39 \\
\hline 7494.67 & 289967.20 & 17.40 & 3586.35 & 102.08 & 177.60 & 31236.76 & 72.66 & 142.27 & 27.21 & 5.75 & 135.74 & 33.56 & 134.48 & 13.81 \\
\hline 7494.74 & 285644.00 & 17.40 & 3542.15 & 98.12 & 178.26 & 28838.58 & 72.56 & 110.70 & 25.52 & 5.12 & 132.87 & 34.70 & 134.78 & 14.64 \\
\hline 7494.80 & 289951.80 & 17.20 & 3503.96 & 100.50 & 179.96 & 29301.67 & 72.70 & 101.84 & 26.50 & 5.32 & 130.32 & 35.89 & 133.32 & 13.79 \\
\hline 7494.87 & 260928.20 & 17.60 & 3444.65 & 98.58 & 183.56 & 29575.20 & 75.86 & 105.73 & 26.91 & 5.07 & 130.02 & 37.58 & 129.28 & 12.30 \\
\hline 7494.93 & 250564.40 & 17.80 & 3248.83 & 93.84 & 179.20 & 27944.11 & 75.36 & 39.45 & 26.01 & 4.68 & 128.99 & 37.29 & 126.48 & 12.76 \\
\hline
\end{tabular}




\begin{tabular}{|c|c|c|c|c|c|c|c|c|c|c|c|c|c|c|}
\hline Depth & XRF & XRF Live Time & $\mathrm{Ti}(\mathrm{ppm})$ & $\mathrm{Cr}(\mathrm{ppm})$ & $\mathrm{Mn}(\mathrm{ppm})$ & $\mathrm{Fe}(\mathrm{ppm})$ & $\mathrm{Ni}(\mathrm{ppm})$ & $\mathrm{Cu}(\mathrm{ppm})$ & $\mathrm{Zn}(\mathrm{ppm})$ & As (ppm) & $\mathrm{Rb}(\mathrm{ppm})$ & $\mathrm{Sr}(\mathrm{ppm})$ & $\mathrm{Y}(\mathrm{ppm})$ & $\mathrm{Zr}(\mathrm{ppm})$ \\
\hline 7495.00 & 246193.60 & 17.80 & 3133.71 & 88.08 & 167.89 & 27894.77 & 79.50 & 34.88 & 24.48 & 4.31 & 126.87 & 34.72 & 126.93 & 12.70 \\
\hline 7495.07 & 236792.00 & 18.00 & 3299.91 & 87.88 & 165.47 & 28227.18 & 76.70 & 34.08 & 23.05 & 4.34 & 124.27 & 33.80 & 126.12 & 11.86 \\
\hline 7495.13 & 226961.20 & 18.20 & 3360.25 & 91.72 & 159.06 & 27999.44 & 75.97 & 32.07 & 21.83 & 4.04 & 123.84 & 30.12 & 127.29 & 12.87 \\
\hline 7495.20 & 240451.00 & 18.00 & 3398.61 & 89.74 & 153.49 & 27786.31 & 76.47 & 24.54 & 21.31 & 4.46 & 115.90 & 27.39 & 126.82 & 14.58 \\
\hline 7495.26 & 224417.40 & 18.20 & 3422.02 & 87.57 & 141.86 & 27020.00 & 76.73 & 23.78 & 20.47 & 4.45 & 109.51 & 23.64 & 122.64 & 13.97 \\
\hline 7495.33 & 210499.80 & 18.40 & 3572.05 & 82.00 & 142.16 & 26224.19 & 75.33 & 31.47 & 20.03 & 7.85 & 117.88 & 38.96 & 100.61 & 33.05 \\
\hline 7495.39 & 206926.20 & 18.40 & 3496.55 & 79.36 & 141.72 & 26218.47 & 75.14 & 30.94 & 21.26 & 7.93 & 113.37 & 39.14 & 99.15 & 32.82 \\
\hline 7495.46 & 211684.80 & 18.40 & 3389.96 & 79.09 & 147.01 & 27663.36 & 78.09 & 224.41 & 23.52 & 10.13 & 118.32 & 41.22 & 98.52 & 31.60 \\
\hline 7495.52 & 217750.00 & 18.40 & 3342.95 & 83.85 & 146.55 & 27287.93 & 76.09 & 230.01 & 23.25 & 10.01 & 118.62 & 40.73 & 99.39 & 30.99 \\
\hline 7495.59 & 246789.60 & 18.00 & 3318.96 & 85.31 & 174.14 & 28819.43 & 75.64 & 242.92 & 23.19 & 10.43 & 122.39 & 39.91 & 104.50 & 31.14 \\
\hline 7495.66 & 291302.20 & 17.60 & 2960.12 & 85.46 & 217.20 & 28345.78 & 72.44 & 247.63 & 22.39 & 7.57 & 118.38 & 26.12 & 117.28 & 10.98 \\
\hline 7495.72 & 314513.20 & 17.40 & 2950.47 & 93.55 & 217.11 & 28254.69 & 76.26 & 3074.28 & 22.17 & 5.88 & 116.11 & 25.43 & 117.77 & 12.16 \\
\hline 7495.79 & 312787.40 & 17.40 & 2928.21 & 90.79 & 210.10 & 25614.25 & 74.44 & 2924.28 & 19.29 & 4.27 & 111.79 & 24.04 & 116.13 & 12.86 \\
\hline 7495.85 & 316005.20 & 17.40 & 2869.47 & 86.87 & 208.77 & 25192.82 & 83.94 & 2929.24 & 89.34 & 6.56 & 125.20 & 47.32 & 97.45 & 33.44 \\
\hline 7495.92 & 320905.80 & 17.40 & 2972.94 & 88.41 & 187.30 & 24371.71 & 86.51 & 3021.61 & 89.31 & 6.53 & 126.35 & 49.74 & 98.09 & 33.52 \\
\hline 7495.98 & 311808.80 & 17.40 & 3146.87 & 94.66 & 152.88 & 27058.41 & 91.01 & 3035.53 & 92.90 & 7.70 & 128.03 & 49.30 & 105.05 & 33.90 \\
\hline 7496.05 & 303390.80 & 17.40 & 3027.13 & 91.75 & 153.57 & 26978.41 & 89.61 & 550.19 & 92.90 & 9.23 & 134.88 & 49.91 & 105.83 & 32.69 \\
\hline 7496.12 & 298851.40 & 17.40 & 3050.59 & 95.67 & 158.45 & 27849.47 & 92.57 & 570.98 & 94.47 & 9.59 & 147.68 & 49.69 & 108.84 & 32.95 \\
\hline 7496.18 & 296583.80 & 17.40 & 3073.69 & 101.41 & 158.24 & 28178.90 & 81.00 & 588.30 & 25.04 & 8.06 & 144.27 & 27.80 & 128.10 & 12.88 \\
\hline 7496.25 & 252435.00 & 17.80 & 2982.03 & 102.20 & 158.76 & 27187.53 & 81.37 & 491.42 & 24.46 & 8.15 & 152.62 & 27.01 & 126.74 & 12.26 \\
\hline 7496.31 & 276354.80 & 17.60 & 3052.10 & 108.81 & 152.02 & 27834.36 & 86.66 & 475.97 & 26.69 & 7.85 & 161.80 & 27.28 & 132.60 & 13.08 \\
\hline 7496.38 & 281053.40 & 17.60 & 5140.95 & 112.54 & 156.48 & 29194.04 & 87.82 & 136.24 & 30.06 & 8.44 & 167.48 & 27.65 & 133.66 & 12.99 \\
\hline 7496.44 & 275472.20 & 17.60 & 5112.42 & 116.68 & 153.41 & 30341.08 & 83.59 & 89.92 & 32.17 & 9.31 & 168.15 & 29.16 & 134.98 & 12.29 \\
\hline 7496.51 & 292101.20 & 17.40 & 5030.10 & 110.95 & 161.12 & 31772.17 & 90.12 & 70.07 & 35.16 & 8.77 & 177.02 & 31.55 & 137.52 & 11.99 \\
\hline 7496.57 & 318924.80 & 17.20 & 5032.60 & 116.36 & 169.01 & 33391.18 & 93.09 & 66.93 & 37.00 & 8.33 & 184.62 & 35.27 & 137.72 & 11.56 \\
\hline 7496.64 & 295406.00 & 17.40 & 5033.42 & 118.59 & 173.81 & 32908.70 & 91.03 & 57.73 & 34.77 & 8.25 & 187.57 & 34.92 & 134.60 & 10.91 \\
\hline 7496.71 & 294244.00 & 17.40 & 3005.84 & 127.57 & 172.78 & 34172.23 & 94.10 & 63.59 & 34.59 & 8.11 & 204.49 & 37.08 & 130.29 & 11.89 \\
\hline 7496.77 & 305199.40 & 17.40 & 3064.39 & 125.24 & 176.96 & 34161.40 & 96.03 & 53.44 & 34.93 & 7.31 & 201.66 & 38.27 & 126.97 & 11.98 \\
\hline 7496.84 & 293631.40 & 17.60 & 3164.96 & 132.28 & 179.11 & 33151.35 & 95.03 & 68.99 & 32.59 & 7.43 & 191.15 & 37.08 & 126.20 & 12.32 \\
\hline 7496.90 & 305499.00 & 17.40 & 3195.01 & 130.24 & 176.70 & 35229.62 & 92.26 & 89.57 & 36.35 & 8.65 & 191.75 & 35.49 & 128.05 & 12.40 \\
\hline 7496.97 & 290644.00 & 17.60 & 3045.73 & 126.69 & 177.44 & 34254.37 & 91.10 & 102.24 & 36.08 & 8.66 & 195.95 & 34.51 & 123.62 & 12.38 \\
\hline 7497.03 & 271982.20 & 17.80 & 2872.91 & 116.71 & 173.72 & 31463.25 & 85.14 & 101.43 & 32.62 & 7.85 & 183.07 & 31.93 & 122.95 & 12.41 \\
\hline 7497.10 & 266641.20 & 17.80 & 2814.00 & 118.64 & 180.72 & 31228.62 & 86.24 & 133.32 & 30.63 & 7.79 & 189.23 & 31.31 & 123.94 & 12.68 \\
\hline
\end{tabular}




\begin{tabular}{|c|c|c|c|c|c|c|c|c|c|c|c|c|c|c|}
\hline Depth & $\mathrm{XRF}$ & XRF Live Time & $\mathrm{Ti}(\mathrm{ppm})$ & $\mathrm{Cr}$ (ppm) & $\mathrm{Mn}(\mathrm{ppm})$ & $\mathrm{Fe}(\mathrm{ppm})$ & $\mathrm{Ni}$ (ppm) & $\mathrm{Cu}(\mathrm{ppm})$ & $\mathrm{Zn}(\mathrm{ppm})$ & As (ppm) & $\mathrm{Rb}(\mathrm{ppm})$ & $\mathrm{Sr}(\mathrm{ppm})$ & $\mathrm{Y}(\mathrm{ppm})$ & $\mathrm{Zr}(\mathrm{ppm})$ \\
\hline 7497.17 & 256427.20 & 17.80 & 2862.61 & 116.02 & 182.53 & 32800.33 & 87.30 & 124.14 & 31.75 & 8.17 & 193.06 & 29.84 & 124.47 & 12.09 \\
\hline 7497.23 & 247711.20 & 18.00 & 2804.99 & 113.90 & 183.83 & 31334.04 & 85.59 & 101.20 & 29.86 & 7.12 & 201.52 & 29.80 & 120.66 & 12.67 \\
\hline 7497.30 & 238223.20 & 18.00 & 2773.64 & 113.77 & 190.27 & 31343.79 & 83.53 & 95.85 & 30.18 & 7.12 & 208.17 & 30.37 & 124.27 & 13.31 \\
\hline 7497.36 & 238152.40 & 18.00 & 2804.43 & 118.91 & 198.38 & 32567.06 & 85.41 & 103.85 & 33.20 & 8.56 & 224.56 & 30.24 & 129.53 & 13.38 \\
\hline 7497.43 & 253028.40 & 17.80 & 10723.25 & 121.99 & 193.96 & 32573.16 & 85.46 & 80.68 & 36.38 & 9.02 & 234.83 & 29.41 & 131.22 & 13.40 \\
\hline 7497.49 & 270050.00 & 17.60 & 10557.15 & 125.17 & 196.69 & 32860.51 & 84.76 & 75.29 & 38.85 & 9.27 & 251.60 & 31.97 & 127.54 & 12.94 \\
\hline 7497.56 & 283491.20 & 17.40 & 10639.42 & 133.23 & 208.72 & 33919.54 & 95.12 & 84.58 & 43.28 & 11.22 & 248.28 & 33.48 & 130.25 & 11.60 \\
\hline 7497.62 & 310039.60 & 17.20 & 10702.27 & 135.38 & 204.17 & 34440.66 & 102.50 & 83.10 & 45.37 & 11.04 & 236.14 & 35.43 & 130.42 & 10.18 \\
\hline 7497.69 & 326210.20 & 17.00 & 10806.76 & 132.37 & 200.70 & 34168.21 & 110.73 & 72.07 & 42.60 & 11.01 & 220.00 & 37.55 & 131.64 & 10.06 \\
\hline 7497.76 & 332455.80 & 17.00 & 2899.23 & 135.57 & 198.15 & 34400.62 & 120.92 & 61.50 & 38.63 & 10.63 & 209.75 & 39.51 & 132.58 & 9.66 \\
\hline 7497.82 & 344454.40 & 17.00 & 2983.64 & 133.76 & 193.84 & 36645.44 & 126.42 & 53.04 & 39.08 & 11.48 & 193.18 & 38.84 & 135.22 & 10.63 \\
\hline 7497.89 & 354371.80 & 17.00 & 2929.49 & 122.51 & 186.54 & 36380.55 & 124.70 & 41.99 & 34.06 & 11.43 & 175.38 & 38.48 & 133.67 & 12.40 \\
\hline 7497.95 & 351546.80 & 17.00 & 2905.76 & 119.00 & 188.69 & 35716.68 & 123.56 & 37.75 & 30.85 & 12.17 & 165.25 & 41.11 & 130.01 & 12.85 \\
\hline 7498.02 & 352497.40 & 17.00 & 2841.95 & 113.48 & 191.30 & 35230.36 & 119.98 & 65.30 & 31.41 & 12.29 & 155.74 & 42.82 & 127.40 & 12.01 \\
\hline 7498.08 & 351461.80 & 17.00 & 2777.92 & 103.70 & 193.27 & 34143.16 & 114.63 & 91.03 & 31.17 & 12.95 & 144.11 & 46.21 & 123.23 & 11.14 \\
\hline 7498.15 & 347901.40 & 17.00 & 2699.16 & 102.59 & 189.74 & 31211.95 & 115.46 & 130.31 & 29.18 & 12.62 & 137.16 & 45.49 & 121.75 & 11.54 \\
\hline 7498.22 & 344405.60 & 17.00 & 2729.05 & 107.04 & 179.70 & 30034.70 & 115.06 & 129.19 & 28.58 & 12.68 & 134.36 & 45.18 & 125.20 & 10.41 \\
\hline 7498.28 & 354051.40 & 17.00 & 2855.55 & 108.17 & 169.35 & 31957.77 & 118.70 & 128.59 & 31.80 & 13.92 & 132.03 & 42.47 & 128.25 & 10.06 \\
\hline 7498.35 & 360437.60 & 17.00 & 2940.69 & 112.08 & 163.40 & 33021.95 & 119.55 & 97.24 & 33.75 & 14.98 & 129.97 & 40.63 & 128.84 & 11.16 \\
\hline 7498.41 & 369928.20 & 16.80 & 3159.43 & 106.73 & 165.43 & 36649.34 & 120.72 & 63.68 & 37.91 & 16.04 & 124.59 & 35.85 & 131.59 & 12.95 \\
\hline 7498.48 & 392745.40 & 16.60 & 3366.90 & 106.66 & 172.26 & 40600.16 & 127.04 & 25.47 & 40.11 & 17.38 & 123.87 & 36.56 & 139.32 & 12.08 \\
\hline 7498.54 & 404426.00 & 16.40 & 3359.34 & 107.23 & 178.99 & 41432.71 & 136.51 & 45.87 & 39.38 & 20.18 & 129.31 & 56.88 & 123.53 & 35.45 \\
\hline 7498.61 & 408477.00 & 16.20 & 3333.73 & 106.98 & 180.27 & 40150.99 & 134.58 & 43.98 & 37.06 & 19.21 & 131.75 & 58.03 & 125.90 & 36.74 \\
\hline 7498.67 & 413927.60 & 16.20 & 3179.33 & 101.96 & 202.03 & 38562.73 & 144.14 & 62.76 & 54.63 & 20.23 & 134.13 & 85.25 & 118.40 & 54.91 \\
\hline 7498.74 & 411257.20 & 16.40 & 2891.93 & 101.28 & 227.50 & 34631.96 & 144.79 & 77.70 & 49.92 & 18.70 & 144.61 & 102.02 & 113.46 & 52.69 \\
\hline 7498.81 & 397315.00 & 16.40 & 2488.72 & 95.02 & 262.29 & 28996.19 & 130.67 & 83.35 & 44.22 & 17.28 & 157.72 & 125.07 & 98.01 & 51.11 \\
\hline 7498.87 & 398592.60 & 16.40 & 2516.84 & 93.20 & 266.94 & 29483.43 & 124.59 & 62.12 & 45.81 & 14.00 & 154.82 & 106.48 & 112.89 & 29.30 \\
\hline 7498.94 & 397653.60 & 16.40 & 2515.48 & 90.35 & 273.64 & 30810.30 & 124.63 & 60.21 & 45.91 & 14.54 & 155.59 & 104.57 & 112.03 & 28.40 \\
\hline 7499.00 & 406386.20 & 16.20 & 2639.21 & 91.28 & 254.89 & 32653.14 & 121.65 & 44.38 & 27.19 & 12.99 & 156.29 & 78.82 & 120.49 & 9.30 \\
\hline 7499.07 & 415081.20 & 16.00 & 2852.00 & 95.00 & 222.79 & 35791.10 & 121.40 & 31.89 & 30.13 & 14.60 & 147.12 & 61.66 & 128.43 & 11.19 \\
\hline 7499.13 & 416827.00 & 16.00 & 3143.91 & 100.46 & 179.29 & 38847.02 & 131.88 & 25.31 & 33.20 & 15.19 & 134.87 & 38.19 & 138.69 & 12.06 \\
\hline 7499.20 & 376156.00 & 16.40 & 2969.72 & 94.67 & 176.95 & 37222.37 & 128.52 & 24.87 & 29.88 & 15.02 & 133.59 & 36.24 & 132.74 & 11.01 \\
\hline 7499.27 & 327393.40 & 17.00 & 2967.91 & 94.18 & 181.61 & 35905.56 & 127.92 & 26.65 & 28.54 & 14.43 & 127.60 & 35.47 & 125.76 & 8.38 \\
\hline
\end{tabular}




\begin{tabular}{|c|c|c|c|c|c|c|c|c|c|c|c|c|c|c|}
\hline Depth & XRF & XRF Live Time & $\mathrm{Ti}(\mathrm{ppm})$ & $\mathrm{Cr}$ (ppm) & $\mathrm{Mn}(\mathrm{ppm})$ & $\mathrm{Fe}(\mathrm{ppm})$ & $\mathrm{Ni}$ (ppm) & $\mathrm{Cu}(\mathrm{ppm})$ & $\mathrm{Zn}(\mathrm{ppm})$ & As (ppm) & $\mathrm{Rb}(\mathrm{ppm})$ & $\mathrm{Sr}(\mathrm{ppm})$ & $\mathrm{Y}(\mathrm{ppm})$ & $\mathrm{Zr}(\mathrm{ppm})$ \\
\hline 7499.33 & 312283.00 & 17.20 & 2934.74 & 96.62 & 201.59 & 35565.77 & 120.92 & 24.38 & 26.86 & 14.13 & 125.08 & 34.45 & 121.09 & 7.95 \\
\hline 7499.40 & 293669.00 & 17.40 & 2910.52 & 96.79 & 209.63 & 35532.39 & 120.99 & 26.30 & 27.64 & 14.22 & 126.52 & 36.17 & 116.13 & 7.42 \\
\hline 7499.46 & 271127.40 & 17.60 & 2895.44 & 91.21 & 210.01 & 34987.65 & 112.91 & 26.32 & 26.18 & 14.51 & 123.08 & 36.81 & 113.83 & 8.49 \\
\hline 7499.53 & 282653.60 & 17.60 & 3066.80 & 91.32 & 208.31 & 36368.41 & 110.63 & 27.04 & 30.40 & 14.51 & 121.92 & 36.40 & 118.50 & 7.96 \\
\hline 7499.59 & 291362.20 & 17.40 & 2896.79 & 84.68 & 191.04 & 34899.31 & 109.77 & 40.17 & 30.36 & 14.78 & 119.60 & 36.04 & 120.68 & 10.95 \\
\hline 7499.66 & 269619.80 & 17.60 & 2888.25 & 81.26 & 164.56 & 32864.09 & 106.33 & 57.38 & 29.90 & 14.59 & 116.44 & 33.03 & 123.14 & 11.10 \\
\hline 7499.72 & 240482.60 & 17.80 & 2711.74 & 76.84 & 152.03 & 28942.04 & 97.00 & 83.17 & 26.91 & 13.71 & 110.87 & 30.80 & 125.26 & 11.73 \\
\hline 7499.79 & 219149.00 & 18.00 & 2691.09 & 79.61 & 150.42 & 26104.67 & 94.79 & 128.48 & 24.59 & 12.86 & 113.14 & 29.62 & 123.44 & 10.45 \\
\hline 7499.86 & 218843.20 & 18.00 & 2613.15 & 86.48 & 143.86 & 24009.84 & 94.68 & 173.73 & 21.45 & 13.18 & 112.07 & 30.03 & 125.33 & 11.83 \\
\hline 7499.92 & 227947.80 & 18.00 & 2707.69 & 89.72 & 153.86 & 23805.40 & 90.40 & 214.74 & 21.03 & 13.21 & 116.13 & 29.62 & 129.05 & 12.01 \\
\hline 7499.99 & 224760.00 & 18.00 & 1454.16 & 116.17 & 158.81 & 25197.42 & 92.24 & 202.81 & 21.91 & 13.47 & 379.08 & 30.25 & 125.49 & 14.45 \\
\hline 7500.05 & 245435.00 & 17.80 & 282.69 & 150.60 & 170.29 & 28893.49 & 94.37 & 171.64 & 26.04 & 13.85 & 720.04 & 28.42 & 121.08 & 13.52 \\
\hline 7500.12 & 273726.20 & 17.60 & -41.82 & 165.20 & 174.50 & 29341.16 & 97.43 & 123.84 & 27.26 & 14.58 & 761.30 & 28.48 & 125.43 & 13.22 \\
\hline 7500.18 & 283038.40 & 17.40 & -813.20 & 194.62 & 184.44 & 30887.02 & 97.53 & 78.73 & 28.41 & 14.52 & 979.14 & 27.33 & 120.33 & 12.48 \\
\hline 7500.25 & 286618.80 & 17.40 & -1035.28 & 213.69 & 191.04 & 30979.19 & 98.48 & 21.27 & 30.35 & 14.86 & 1025.51 & 26.69 & 118.94 & 11.67 \\
\hline 7500.31 & 286851.40 & 17.40 & -88.92 & 201.33 & 197.14 & 29772.00 & 107.81 & 33.26 & 29.19 & 18.49 & 765.95 & 103.36 & 102.15 & 34.00 \\
\hline 7500.38 & 288425.40 & 17.40 & 834.78 & 183.21 & 197.33 & 30486.46 & 113.31 & 34.92 & 25.76 & 17.72 & 498.42 & 106.51 & 104.09 & 34.67 \\
\hline 7500.45 & 262557.00 & 17.60 & 1013.42 & 172.61 & 194.35 & 29351.22 & 110.91 & 37.06 & 25.47 & 16.73 & 461.81 & 105.30 & 102.05 & 35.25 \\
\hline 7500.51 & 245918.60 & 17.80 & 1584.49 & 148.51 & 187.51 & 26889.30 & 110.15 & 36.41 & 24.41 & 16.37 & 284.36 & 106.40 & 107.97 & 35.64 \\
\hline 7500.58 & 252567.20 & 17.60 & 1593.37 & 137.83 & 176.78 & 26717.57 & 111.66 & 40.01 & 22.41 & 15.09 & 292.99 & 107.45 & 108.82 & 36.64 \\
\hline 7500.64 & 264224.80 & 17.40 & 1627.43 & 140.55 & 172.17 & 26753.83 & 103.66 & 24.58 & 23.82 & 11.31 & 350.59 & 31.39 & 130.93 & 11.90 \\
\hline 7500.71 & 259597.40 & 17.60 & 1600.48 & 133.89 & 167.65 & 22974.96 & 105.52 & 29.62 & 23.93 & 11.35 & 337.87 & 32.77 & 126.23 & 11.30 \\
\hline 7500.77 & 274181.40 & 17.60 & 1285.65 & 148.34 & 169.40 & 24049.95 & 106.13 & 32.18 & 24.70 & 11.60 & 406.42 & 36.14 & 125.08 & 11.11 \\
\hline 7500.84 & 281966.80 & 17.60 & 1299.03 & 147.14 & 171.03 & 24464.18 & 108.17 & 32.12 & 24.26 & 11.79 & 398.91 & 37.81 & 123.81 & 10.58 \\
\hline 7500.91 & 275894.00 & 17.80 & 726.07 & 173.95 & 182.99 & 25166.25 & 108.36 & 31.81 & 25.23 & 12.30 & 556.10 & 39.31 & 119.03 & 10.50 \\
\hline 7500.97 & 274708.00 & 18.00 & -177.27 & 172.52 & 181.57 & 26785.05 & 103.65 & 27.66 & 25.70 & 12.27 & 681.80 & 39.92 & 111.98 & 9.35 \\
\hline 7501.04 & 247084.40 & 18.20 & 186.33 & 167.56 & 177.93 & 26272.19 & 109.69 & 35.95 & 25.91 & 14.60 & 623.27 & 51.30 & 99.87 & 31.88 \\
\hline 7501.10 & 220450.60 & 18.40 & 583.54 & 146.21 & 181.65 & 25758.49 & 109.35 & 40.34 & 24.88 & 14.10 & 544.36 & 48.09 & 97.13 & 32.83 \\
\hline 7501.17 & 220504.80 & 18.40 & 752.61 & 137.62 & 177.43 & 27024.89 & 124.65 & 59.38 & 37.40 & 17.15 & 510.96 & 62.57 & 76.85 & 55.60 \\
\hline 7501.23 & 213925.00 & 18.40 & 1532.56 & 106.11 & 166.94 & 28335.25 & 125.94 & 70.76 & 37.89 & 17.96 & 295.22 & 59.88 & 79.23 & 54.81 \\
\hline 7501.30 & 204096.00 & 18.40 & 2585.00 & 91.61 & 158.97 & 26571.43 & 132.48 & 131.80 & 38.37 & 18.49 & 105.41 & 57.57 & 80.38 & 57.19 \\
\hline 7501.36 & 219909.20 & 18.20 & 2446.74 & 86.64 & 161.42 & 26975.18 & 122.93 & 150.84 & 37.27 & 16.44 & 101.36 & 43.24 & 94.54 & 34.60 \\
\hline 7501.43 & 235114.80 & 18.00 & 2386.13 & 88.28 & 150.14 & 27654.40 & 128.33 & 164.25 & 38.24 & 17.16 & 102.30 & 43.22 & 92.93 & 34.33 \\
\hline
\end{tabular}




\begin{tabular}{|c|c|c|c|c|c|c|c|c|c|c|c|c|c|c|}
\hline Depth & XRF & XRF Live Time & $\mathrm{Ti}(\mathrm{ppm})$ & $\mathrm{Cr}(\mathrm{ppm})$ & $\mathrm{Mn}(\mathrm{ppm})$ & $\mathrm{Fe}(\mathrm{ppm})$ & $\mathrm{Ni}(\mathrm{ppm})$ & $\mathrm{Cu}(\mathrm{ppm})$ & $\mathrm{Zn}(\mathrm{ppm})$ & As (ppm) & $\mathrm{Rb}(\mathrm{ppm})$ & $\mathrm{Sr}(\mathrm{ppm})$ & $\mathrm{Y}(\mathrm{ppm})$ & $\mathrm{Zr}(\mathrm{ppm})$ \\
\hline 7501.50 & 219872.40 & 18.00 & 2308.81 & 85.26 & 151.28 & 26378.10 & 111.07 & 167.25 & 27.45 & 14.97 & 100.51 & 29.81 & 108.58 & 11.28 \\
\hline 7501.56 & 227685.80 & 18.00 & 2239.09 & 81.01 & 148.76 & 24004.05 & 109.19 & 174.65 & 26.12 & 13.70 & 100.47 & 33.26 & 105.79 & 11.84 \\
\hline 7501.63 & 234945.40 & 18.00 & 2291.25 & 81.20 & 156.12 & 23700.61 & 106.58 & 802.37 & 25.33 & 13.24 & 100.81 & 33.68 & 106.86 & 10.82 \\
\hline 7501.69 & 263537.20 & 17.80 & 2363.81 & 86.16 & 151.34 & 23873.06 & 108.04 & 932.60 & 25.68 & 13.03 & 104.05 & 34.48 & 109.37 & 12.00 \\
\hline 7501.76 & 292149.00 & 17.60 & 2411.88 & 90.63 & 156.94 & 23772.47 & 107.63 & 1109.62 & 25.71 & 12.72 & 106.25 & 35.75 & 112.16 & 11.98 \\
\hline 7501.82 & 299730.40 & 17.60 & 2412.62 & 89.87 & 148.25 & 23548.89 & 109.15 & 1103.95 & 24.72 & 11.48 & 105.43 & 31.92 & 111.96 & 11.49 \\
\hline 7501.89 & 290976.00 & 17.60 & 2366.72 & 88.94 & 143.32 & 23541.25 & 109.70 & 1097.14 & 24.95 & 11.75 & 105.69 & 29.02 & 114.05 & 10.63 \\
\hline 7501.96 & 291083.60 & 17.60 & 2327.49 & 84.34 & 140.57 & 23382.11 & 107.89 & 433.06 & 24.44 & 11.36 & 106.46 & 30.17 & 112.78 & 11.19 \\
\hline 7502.02 & 274134.40 & 17.80 & 2329.20 & 78.26 & 137.12 & 23406.46 & 106.92 & 293.31 & 25.60 & 12.05 & 105.13 & 29.81 & 109.80 & 10.17 \\
\hline 7502.09 & 260901.00 & 17.80 & 2299.51 & 73.16 & 133.91 & 24107.83 & 105.54 & 489.10 & 26.59 & 12.56 & 103.60 & 28.22 & 108.11 & 9.27 \\
\hline 7502.15 & 269547.40 & 17.80 & 2285.35 & 78.23 & 137.38 & 25645.54 & 106.75 & 643.29 & 27.40 & 13.97 & 104.81 & 27.44 & 110.34 & 10.14 \\
\hline 7502.22 & 274760.80 & 17.80 & 2313.67 & 80.94 & 140.92 & 26790.23 & 105.63 & 689.02 & 27.86 & 14.89 & 104.62 & 28.53 & 109.29 & 10.30 \\
\hline 7502.28 & 276614.00 & 17.80 & 2288.85 & 85.48 & 140.78 & 27354.62 & 109.29 & 1036.47 & 28.91 & 16.21 & 106.56 & 29.75 & 109.60 & 9.46 \\
\hline 7502.35 & 272082.60 & 17.80 & 3934.20 & 88.16 & 141.84 & 26853.51 & 108.35 & 1035.77 & 27.66 & 15.95 & 107.06 & 29.79 & 113.06 & 10.41 \\
\hline 7502.41 & 269115.00 & 18.00 & 3861.37 & 87.88 & 144.35 & 26270.26 & 106.63 & 666.07 & 27.69 & 15.83 & 109.13 & 31.78 & 115.46 & 11.56 \\
\hline 7502.48 & 291048.40 & 17.60 & 3873.68 & 87.24 & 145.30 & 26119.87 & 108.31 & 560.73 & 28.37 & 15.91 & 111.42 & 33.65 & 116.65 & 11.03 \\
\hline 7502.55 & 316223.20 & 17.40 & 3866.89 & 88.57 & 147.11 & 26153.86 & 114.12 & 812.76 & 29.25 & 15.71 & 114.32 & 34.79 & 117.75 & 11.25 \\
\hline 7502.61 & 332285.80 & 17.20 & 3881.55 & 87.64 & 148.05 & 26298.56 & 114.18 & 904.99 & 28.61 & 14.99 & 112.49 & 35.95 & 122.02 & 9.75 \\
\hline 7502.68 & 350640.40 & 17.00 & 2151.77 & 88.94 & 150.53 & 27466.45 & 116.10 & 918.88 & 31.34 & 15.94 & 113.22 & 37.10 & 120.17 & 8.58 \\
\hline 7502.74 & 359302.80 & 16.80 & 2175.83 & 94.24 & 143.17 & 27046.05 & 115.64 & 907.65 & 29.45 & 16.01 & 113.01 & 35.83 & 117.73 & 8.51 \\
\hline 7502.81 & 344997.80 & 17.00 & 2111.37 & 89.69 & 139.24 & 25950.78 & 110.06 & 860.51 & 27.85 & 15.38 & 111.47 & 35.58 & 115.67 & 8.52 \\
\hline 7502.87 & 334344.20 & 17.00 & 2085.66 & 86.10 & 134.90 & 25329.11 & 104.59 & 583.85 & 26.76 & 15.28 & 112.14 & 34.08 & 115.16 & 8.84 \\
\hline 7502.94 & 314079.00 & 17.20 & 2172.94 & 89.30 & 131.56 & 26170.28 & 107.20 & 326.28 & 29.62 & 16.31 & 116.71 & 31.98 & 113.61 & 11.30 \\
\hline 7503.01 & 315868.00 & 17.20 & 2201.68 & 89.60 & 134.36 & 26451.41 & 106.68 & 311.80 & 27.76 & 15.94 & 117.75 & 32.52 & 113.47 & 11.76 \\
\hline 7503.07 & 313956.60 & 17.20 & 2243.33 & 86.99 & 144.43 & 28057.50 & 108.80 & 801.52 & 29.40 & 16.72 & 117.88 & 35.06 & 113.34 & 12.65 \\
\hline 7503.14 & 313620.60 & 17.20 & 2255.36 & 89.35 & 148.35 & 28466.57 & 111.16 & 944.86 & 28.88 & 16.59 & 116.23 & 35.19 & 114.30 & 13.27 \\
\hline 7503.20 & 307491.60 & 17.20 & 2242.28 & 89.36 & 154.91 & 28697.48 & 112.04 & 941.53 & 28.85 & 17.24 & 114.72 & 36.91 & 116.32 & 12.55 \\
\hline 7503.27 & 314760.00 & 17.00 & 2164.10 & 83.88 & 160.23 & 28601.97 & 108.28 & 1231.92 & 28.22 & 16.83 & 110.98 & 37.74 & 112.94 & 10.95 \\
\hline 7503.33 & 295255.00 & 17.20 & 4202.18 & 79.18 & 157.89 & 26975.51 & 103.57 & 1245.71 & 26.53 & 16.85 & 109.61 & 37.15 & 113.09 & 10.19 \\
\hline 7503.40 & 278789.60 & 17.40 & 4138.53 & 76.16 & 150.23 & 25420.67 & 101.74 & 771.37 & 25.90 & 16.63 & 107.76 & 35.55 & 113.59 & 9.68 \\
\hline 7503.46 & 269836.40 & 17.60 & 4167.79 & 78.91 & 160.55 & 25781.74 & 105.55 & 3504.20 & 27.97 & 15.53 & 110.75 & 36.83 & 111.71 & 8.85 \\
\hline 7503.53 & 270889.20 & 17.60 & 4159.06 & 77.83 & 158.37 & 25223.26 & 100.87 & 3511.86 & 26.89 & 14.92 & 109.68 & 35.31 & 109.08 & 9.91 \\
\hline 7503.60 & 270281.60 & 17.60 & 4114.19 & 78.96 & 154.92 & 23981.92 & 99.76 & 3088.95 & 24.07 & 14.46 & 109.76 & 35.43 & 110.41 & 10.54 \\
\hline
\end{tabular}




\begin{tabular}{|c|c|c|c|c|c|c|c|c|c|c|c|c|c|c|}
\hline Depth & XRF & XRF Live Time & $\mathrm{Ti}(\mathrm{ppm})$ & $\mathrm{Cr}(\mathrm{ppm})$ & $\mathrm{Mn}(\mathrm{ppm})$ & $\mathrm{Fe}(\mathrm{ppm})$ & $\mathrm{Ni}(\mathrm{ppm})$ & $\mathrm{Cu}(\mathrm{ppm})$ & $\mathrm{Zn}(\mathrm{ppm})$ & As (ppm) & $\mathrm{Rb}(\mathrm{ppm})$ & $\mathrm{Sr}(\mathrm{ppm})$ & $\mathrm{Y}(\mathrm{ppm})$ & $\mathrm{Zr}(\mathrm{ppm})$ \\
\hline 7503.66 & 277333.60 & 17.40 & 2043.86 & 77.94 & 152.51 & 24457.56 & 100.99 & 3111.00 & 26.15 & 13.92 & 110.37 & 37.43 & 111.02 & 10.52 \\
\hline 7503.73 & 295956.00 & 17.20 & 2138.68 & 82.33 & 158.18 & 26256.67 & 97.69 & 3111.80 & 26.77 & 13.56 & 112.95 & 37.36 & 114.20 & 10.25 \\
\hline 7503.79 & 303402.20 & 17.00 & 2158.41 & 80.62 & 150.32 & 26283.31 & 93.72 & 264.53 & 26.73 & 15.57 & 112.43 & 37.57 & 118.34 & 10.72 \\
\hline 7503.86 & 305027.80 & 17.00 & 2196.36 & 84.46 & 153.85 & 26846.09 & 96.33 & 746.20 & 28.68 & 15.79 & 116.67 & 39.10 & 117.83 & 9.74 \\
\hline 7503.92 & 305296.60 & 17.00 & 2275.16 & 84.94 & 159.74 & 27017.61 & 104.73 & 689.65 & 52.26 & 18.30 & 118.81 & 57.03 & 106.73 & 32.87 \\
\hline 7503.99 & 305406.80 & 17.00 & 2363.97 & 91.83 & 165.10 & 27049.00 & 105.29 & 641.64 & 51.62 & 18.82 & 120.19 & 56.45 & 108.81 & 33.78 \\
\hline 7504.06 & 302734.60 & 17.00 & 2299.29 & 88.33 & 168.59 & 25659.80 & 106.74 & 640.24 & 51.31 & 18.77 & 123.09 & 55.78 & 108.66 & 33.86 \\
\hline 7504.12 & 304790.00 & 17.00 & 2289.92 & 91.27 & 167.29 & 25473.06 & 108.16 & 615.86 & 51.43 & 18.47 & 127.36 & 57.48 & 108.67 & 34.55 \\
\hline 7504.19 & 312176.80 & 17.00 & 2364.43 & 92.77 & 167.20 & 27252.45 & 109.89 & 115.23 & 51.56 & 18.93 & 124.92 & 57.21 & 114.65 & 35.34 \\
\hline 7504.25 & 315525.00 & 17.00 & 2414.17 & 93.88 & 166.36 & 27445.57 & 100.05 & 196.80 & 29.23 & 16.74 & 126.00 & 39.04 & 127.00 & 12.73 \\
\hline 7504.32 & 318552.20 & 17.00 & 4138.03 & 91.11 & 161.00 & 27185.12 & 99.04 & 212.63 & 28.98 & 16.20 & 126.41 & 38.02 & 125.21 & 12.35 \\
\hline 7504.38 & 313994.80 & 17.00 & 4223.73 & 90.04 & 156.28 & 27804.28 & 101.60 & 201.33 & 30.36 & 15.97 & 123.18 & 38.56 & 125.39 & 12.24 \\
\hline 7504.45 & 304518.20 & 17.20 & 4303.62 & 92.14 & 151.66 & 27339.96 & 97.54 & 182.54 & 29.74 & 15.88 & 116.79 & 37.02 & 124.45 & 12.43 \\
\hline 7504.51 & 293297.60 & 17.20 & 4320.98 & 88.34 & 145.08 & 25400.38 & 95.83 & 164.48 & 28.11 & 14.90 & 115.26 & 36.15 & 120.75 & 12.26 \\
\hline 7504.58 & 293505.80 & 17.20 & 4390.37 & 88.73 & 141.61 & 28187.76 & 102.88 & 72.90 & 28.86 & 15.34 & 112.57 & 36.76 & 124.06 & 11.53 \\
\hline 7504.65 & 292750.20 & 17.20 & 2684.00 & 89.68 & 151.41 & 30420.62 & 113.34 & 66.02 & 32.44 & 18.35 & 111.18 & 54.94 & 109.49 & 30.09 \\
\hline 7504.71 & 309243.00 & 17.20 & 2687.50 & 92.98 & 149.01 & 31965.52 & 121.78 & 54.44 & 33.62 & 20.44 & 112.86 & 56.59 & 111.24 & 31.30 \\
\hline 7504.78 & 333863.40 & 16.80 & 2637.42 & 96.54 & 155.28 & 35619.07 & 139.78 & 62.34 & 34.29 & 21.47 & 114.14 & 59.23 & 116.09 & 29.68 \\
\hline 7504.84 & 349331.20 & 16.80 & 2578.23 & 101.66 & 160.27 & 37952.11 & 153.06 & 95.19 & 37.61 & 23.99 & 115.80 & 63.68 & 120.61 & 29.44 \\
\hline 7504.91 & 359275.40 & 16.80 & 2514.78 & 101.42 & 159.97 & 36984.37 & 154.41 & 104.61 & 37.65 & 24.55 & 114.98 & 63.08 & 126.54 & 30.35 \\
\hline 7504.97 & 372528.80 & 16.80 & 2694.53 & 107.84 & 158.41 & 37903.56 & 156.43 & 102.40 & 35.21 & 23.00 & 113.43 & 45.79 & 152.80 & 12.07 \\
\hline 7505.04 & 371141.00 & 16.80 & 2708.60 & 111.12 & 169.48 & 38953.84 & 157.94 & 100.65 & 35.55 & 22.62 & 113.98 & 47.30 & 154.23 & 10.58 \\
\hline 7505.10 & 366358.20 & 17.00 & 2899.80 & 108.81 & 165.39 & 38227.48 & 148.19 & 105.45 & 31.90 & 25.35 & 120.85 & 59.83 & 132.94 & 34.86 \\
\hline 7505.17 & 370333.00 & 17.00 & 3117.62 & 108.88 & 164.97 & 39722.09 & 138.98 & 71.11 & 32.81 & 23.51 & 118.31 & 54.67 & 130.43 & 34.85 \\
\hline 7505.24 & 368363.60 & 17.00 & 3183.58 & 106.59 & 153.04 & 39872.22 & 131.13 & 56.38 & 33.73 & 22.32 & 118.81 & 52.84 & 122.97 & 34.80 \\
\hline 7505.30 & 358662.60 & 17.00 & 3058.64 & 102.00 & 143.27 & 36833.26 & 121.40 & 47.11 & 33.68 & 21.11 & 120.53 & 52.05 & 111.28 & 34.97 \\
\hline 7505.37 & 348777.20 & 17.00 & 3017.34 & 99.18 & 127.74 & 34010.19 & 118.37 & 72.35 & 31.79 & 20.61 & 117.71 & 49.75 & 109.82 & 34.39 \\
\hline 7505.43 & 348039.20 & 17.00 & 2828.55 & 96.87 & 130.73 & 31187.52 & 117.14 & 147.50 & 34.17 & 17.50 & 111.59 & 32.59 & 127.76 & 11.26 \\
\hline 7505.50 & 344829.40 & 17.00 & 2651.21 & 93.59 & 125.23 & 27821.91 & 122.05 & 195.29 & 31.07 & 18.65 & 115.06 & 34.94 & 130.54 & 11.93 \\
\hline 7505.56 & 347478.60 & 17.00 & 2559.23 & 94.33 & 135.85 & 26578.76 & 131.04 & 278.04 & 28.79 & 20.17 & 120.28 & 39.04 & 132.00 & 11.31 \\
\hline 7505.63 & 357289.60 & 17.00 & 2499.57 & 90.33 & 139.82 & 27143.30 & 135.74 & 299.08 & 27.61 & 20.68 & 120.88 & 43.18 & 133.40 & 11.18 \\
\hline 7505.70 & 361761.60 & 17.00 & 2502.61 & 92.47 & 138.96 & 26051.44 & 129.41 & 296.14 & 26.42 & 20.45 & 123.06 & 45.97 & 133.20 & 11.53 \\
\hline 7505.76 & 361375.60 & 17.00 & 2500.89 & 93.13 & 136.31 & 26638.72 & 130.15 & 273.14 & 27.09 & 20.45 & 126.15 & 49.89 & 133.10 & 12.50 \\
\hline
\end{tabular}




\begin{tabular}{|c|c|c|c|c|c|c|c|c|c|c|c|c|c|c|}
\hline Depth & XRF & XRF Live Time & $\mathrm{Ti}(\mathrm{ppm})$ & $\mathrm{Cr}(\mathrm{ppm})$ & $\mathrm{Mn}(\mathrm{ppm})$ & $\mathrm{Fe}(\mathrm{ppm})$ & $\mathrm{Ni}(\mathrm{ppm})$ & $\mathrm{Cu}(\mathrm{ppm})$ & $\mathrm{Zn}(\mathrm{ppm})$ & As (ppm) & $\mathrm{Rb}(\mathrm{ppm})$ & $\mathrm{Sr}(\mathrm{ppm})$ & $\mathrm{Y}(\mathrm{ppm})$ & $\mathrm{Zr}(\mathrm{ppm})$ \\
\hline 7505.83 & 358866.20 & 17.00 & 2429.16 & 93.72 & 139.68 & 27404.07 & 130.21 & 254.30 & 27.66 & 20.29 & 125.43 & 51.74 & 129.60 & 11.75 \\
\hline 7505.89 & 341931.60 & 17.20 & 2395.30 & 91.71 & 135.60 & 27511.33 & 127.24 & 182.45 & 28.70 & 19.96 & 124.46 & 50.33 & 131.98 & 11.64 \\
\hline 7505.96 & 345214.80 & 17.00 & 2346.99 & 95.58 & 137.04 & 28837.70 & 125.95 & 156.73 & 28.84 & 20.35 & 125.26 & 47.09 & 130.27 & 11.55 \\
\hline 7506.02 & 351786.80 & 17.00 & 2337.27 & 91.58 & 142.56 & 29869.26 & 127.23 & 155.47 & 29.29 & 20.23 & 125.88 & 47.33 & 128.66 & 11.53 \\
\hline 7506.09 & 348143.00 & 17.00 & 2350.70 & 90.07 & 138.40 & 29577.36 & 126.65 & 97.11 & 29.29 & 19.94 & 123.27 & 45.29 & 127.99 & 10.36 \\
\hline 7506.15 & 342595.40 & 17.00 & 2414.15 & 89.94 & 135.23 & 29130.15 & 120.29 & 96.91 & 29.94 & 19.15 & 123.52 & 42.52 & 127.63 & 10.79 \\
\hline 7506.22 & 352656.80 & 16.80 & 2409.44 & 90.78 & 131.78 & 28788.42 & 116.38 & 86.43 & 29.12 & 18.28 & 121.83 & 42.18 & 123.62 & 11.04 \\
\hline 7506.29 & 331619.00 & 17.20 & 2493.90 & 88.69 & 127.38 & 27213.63 & 114.61 & 85.96 & 29.97 & 17.71 & 122.05 & 41.06 & 124.93 & 12.40 \\
\hline 7506.35 & 329396.20 & 17.20 & 2641.26 & 92.03 & 125.11 & 27007.78 & 114.63 & 62.43 & 30.00 & 17.51 & 122.23 & 39.19 & 124.73 & 12.99 \\
\hline 7506.42 & 327802.00 & 17.20 & 2759.10 & 90.56 & 128.37 & 27966.09 & 111.37 & 52.11 & 29.76 & 17.27 & 120.80 & 38.21 & 124.95 & 12.88 \\
\hline 7506.48 & 341579.00 & 17.00 & 2844.53 & 90.12 & 130.93 & 29726.84 & 115.13 & 22.71 & 30.18 & 17.31 & 117.30 & 36.79 & 123.75 & 12.33 \\
\hline 7506.55 & 351555.20 & 17.00 & 2969.65 & 96.14 & 138.11 & 31298.49 & 120.75 & 21.93 & 31.29 & 18.05 & 115.97 & 35.93 & 124.48 & 12.55 \\
\hline 7506.61 & 363789.80 & 16.80 & 3029.52 & 101.90 & 143.02 & 33205.18 & 128.15 & 23.72 & 32.72 & 18.28 & 115.28 & 35.70 & 126.87 & 11.64 \\
\hline 7506.68 & 364098.60 & 16.80 & 2936.99 & 103.20 & 149.45 & 35054.99 & 132.79 & 43.59 & 34.51 & 18.43 & 114.02 & 33.94 & 128.19 & 11.56 \\
\hline 7506.75 & 378884.80 & 16.60 & 2861.31 & 104.90 & 153.29 & 35618.67 & 140.22 & 74.36 & 35.43 & 18.22 & 116.93 & 35.39 & 129.98 & 11.56 \\
\hline 7506.81 & 356808.20 & 16.80 & 2729.28 & 109.26 & 151.54 & 33733.81 & 141.85 & 99.07 & 34.88 & 17.78 & 120.57 & 35.25 & 132.46 & 12.12 \\
\hline 7506.88 & 337475.00 & 17.00 & 2685.65 & 105.67 & 150.48 & 32294.10 & 136.05 & 118.57 & 34.17 & 16.85 & 119.20 & 35.07 & 133.38 & 10.30 \\
\hline 7506.94 & 321411.60 & 17.20 & 2676.01 & 102.80 & 151.62 & 30074.06 & 127.46 & 117.83 & 30.83 & 15.76 & 114.90 & 35.32 & 131.61 & 9.95 \\
\hline 7507.01 & 310686.00 & 17.20 & 2669.68 & 98.11 & 150.27 & 30703.99 & 123.16 & 97.94 & 30.25 & 15.58 & 113.89 & 35.57 & 132.02 & 9.50 \\
\hline 7507.07 & 284280.20 & 17.60 & 2742.76 & 95.35 & 151.66 & 30916.90 & 113.54 & 66.23 & 29.59 & 15.30 & 107.21 & 32.39 & 127.25 & 9.78 \\
\hline 7507.14 & 279967.00 & 17.80 & 2846.03 & 90.44 & 148.21 & 31271.30 & 105.38 & 44.47 & 29.07 & 15.00 & 104.29 & 33.69 & 121.02 & 8.58 \\
\hline 7507.20 & 273909.40 & 17.80 & 2901.90 & 89.54 & 146.12 & 31324.70 & 105.63 & 23.89 & 28.40 & 14.29 & 103.09 & 32.23 & 117.18 & 10.07 \\
\hline 7507.27 & 270229.00 & 17.80 & 3147.51 & 82.25 & 141.11 & 31871.95 & 103.51 & 20.67 & 29.29 & 13.90 & 102.16 & 29.56 & 112.01 & 9.95 \\
\hline 7507.34 & 272883.00 & 17.80 & 3223.35 & 84.92 & 132.76 & 29318.19 & 97.62 & 19.88 & 28.47 & 12.97 & 103.03 & 28.77 & 107.26 & 10.54 \\
\hline 7507.40 & 280017.20 & 17.60 & 3162.00 & 84.45 & 126.26 & 27938.54 & 99.99 & 20.46 & 28.93 & 12.31 & 111.49 & 30.36 & 107.61 & 10.20 \\
\hline 7507.47 & 278876.20 & 17.60 & 3133.12 & 83.21 & 131.78 & 27005.35 & 103.06 & 18.84 & 28.13 & 12.58 & 115.14 & 30.40 & 111.21 & 11.60 \\
\hline 7507.53 & 287557.40 & 17.40 & 3041.06 & 81.63 & 136.57 & 26511.51 & 100.47 & 18.29 & 28.69 & 13.22 & 120.37 & 33.76 & 112.22 & 12.01 \\
\hline 7507.60 & 276514.00 & 17.40 & 2625.15 & 81.48 & 152.46 & 25385.29 & 97.91 & 24.87 & 27.92 & 13.64 & 135.17 & 35.29 & 115.80 & 13.11 \\
\hline 7507.66 & 262420.60 & 17.60 & 2544.41 & 79.05 & 159.41 & 24473.22 & 100.53 & 27.68 & 25.85 & 13.65 & 133.97 & 34.23 & 118.77 & 13.18 \\
\hline 7507.73 & 247223.40 & 17.80 & 2519.01 & 79.07 & 163.75 & 23449.28 & 97.36 & 29.07 & 24.63 & 13.78 & 130.14 & 32.24 & 118.22 & 12.66 \\
\hline 7507.80 & 240741.40 & 17.80 & 2409.35 & 75.02 & 161.38 & 23328.86 & 92.10 & 27.89 & 25.18 & 13.63 & 127.79 & 29.79 & 120.23 & 12.64 \\
\hline 7507.86 & 222344.80 & 18.00 & 2394.54 & 74.77 & 157.21 & 22087.97 & 88.14 & 30.53 & 23.70 & 12.63 & 123.87 & 25.86 & 118.06 & 11.69 \\
\hline 7507.93 & 233703.40 & 18.00 & 2435.11 & 77.61 & 135.32 & 22068.89 & 89.83 & 29.61 & 23.96 & 12.76 & 114.58 & 26.54 & 117.93 & 11.28 \\
\hline
\end{tabular}




\begin{tabular}{|c|c|c|c|c|c|c|c|c|c|c|c|c|c|c|}
\hline Depth & XRF & XRF Live Time & $\mathrm{Ti}(\mathrm{ppm})$ & $\mathrm{Cr}(\mathrm{ppm})$ & $\mathrm{Mn}(\mathrm{ppm})$ & $\mathrm{Fe}(\mathrm{ppm})$ & $\mathrm{Ni}(\mathrm{ppm})$ & $\mathrm{Cu}(\mathrm{ppm})$ & $\mathrm{Zn}(\mathrm{ppm})$ & As (ppm) & $\mathrm{Rb}(\mathrm{ppm})$ & $\mathrm{Sr}(\mathrm{ppm})$ & $\mathrm{Y}(\mathrm{ppm})$ & $\mathrm{Zr}(\mathrm{ppm})$ \\
\hline 7507.99 & 252167.80 & 17.80 & 2341.58 & 75.06 & 133.72 & 21937.80 & 87.80 & 41.63 & 24.18 & 13.00 & 118.33 & 27.16 & 119.41 & 10.60 \\
\hline 7508.06 & 268313.60 & 17.60 & 2239.51 & 72.81 & 139.37 & 21819.36 & 86.23 & 77.45 & 23.06 & 13.33 & 123.60 & 29.44 & 120.58 & 11.38 \\
\hline 7508.12 & 288509.60 & 17.40 & 2263.79 & 76.99 & 144.16 & 21582.80 & 90.48 & 96.25 & 21.23 & 13.27 & 126.63 & 34.41 & 117.17 & 9.45 \\
\hline 7508.19 & 312634.20 & 17.20 & 2263.33 & 76.87 & 147.84 & 22619.92 & 97.78 & 116.33 & 21.53 & 14.03 & 130.14 & 38.11 & 117.64 & 9.84 \\
\hline 7508.25 & 323352.00 & 17.00 & 2170.51 & 75.39 & 176.88 & 24217.28 & 96.50 & 112.47 & 22.52 & 14.23 & 140.07 & 53.58 & 112.24 & 8.44 \\
\hline 7508.32 & 306007.00 & 17.20 & 11490.53 & 79.10 & 182.26 & 24534.58 & 99.29 & 117.23 & 22.01 & 14.27 & 143.05 & 58.41 & 105.40 & 9.17 \\
\hline 7508.39 & 296444.00 & 17.40 & 11546.89 & 81.20 & 185.96 & 24225.74 & 103.28 & 90.01 & 22.89 & 14.56 & 143.23 & 65.94 & 103.03 & 7.62 \\
\hline 7508.45 & 303856.60 & 17.40 & 11517.92 & 83.33 & 196.31 & 24097.81 & 103.69 & 80.30 & 24.32 & 14.49 & 153.39 & 71.28 & 102.69 & 9.76 \\
\hline 7508.52 & 307545.40 & 17.40 & 11415.02 & 83.22 & 198.70 & 23513.54 & 101.90 & 68.33 & 25.41 & 14.70 & 160.33 & 75.84 & 105.60 & 9.93 \\
\hline 7508.58 & 314846.40 & 17.40 & 11523.78 & 85.39 & 176.50 & 22753.56 & 107.70 & 75.93 & 25.37 & 14.71 & 151.38 & 63.84 & 110.75 & 10.13 \\
\hline 7508.65 & 323260.60 & 17.20 & 2245.32 & 87.14 & 172.28 & 23510.45 & 109.22 & 64.70 & 27.64 & 14.93 & 148.31 & 62.71 & 114.33 & 9.36 \\
\hline 7508.71 & 326253.20 & 17.00 & 2207.23 & 88.78 & 160.26 & 24378.25 & 108.34 & 82.76 & 28.03 & 14.89 & 142.99 & 58.03 & 117.33 & 10.98 \\
\hline 7508.78 & 299767.80 & 17.20 & 2266.19 & 84.69 & 151.39 & 24643.06 & 108.78 & 144.13 & 27.43 & 14.94 & 131.50 & 52.71 & 119.76 & 9.81 \\
\hline 7508.85 & 321191.40 & 17.00 & 2224.52 & 81.36 & 153.98 & 32372.96 & 111.67 & 246.38 & 30.76 & 15.36 & 126.73 & 51.28 & 112.15 & 10.76 \\
\hline 7508.91 & 333906.80 & 16.80 & 2223.94 & 83.04 & 156.17 & 33200.56 & 112.68 & 258.50 & 31.47 & 15.60 & 125.84 & 49.25 & 115.53 & 11.10 \\
\hline 7508.98 & 328477.20 & 17.00 & 2242.61 & 80.47 & 149.63 & 32550.40 & 110.07 & 265.10 & 30.61 & 14.88 & 121.10 & 45.12 & 118.46 & 11.96 \\
\hline 7509.04 & 321981.00 & 17.20 & 2313.12 & 77.47 & 143.30 & 31469.12 & 109.41 & 249.54 & 28.81 & 13.70 & 120.20 & 41.42 & 121.66 & 12.30 \\
\hline 7509.11 & 326271.40 & 17.20 & 2371.14 & 80.70 & 134.70 & 30356.80 & 104.15 & 184.06 & 27.91 & 12.46 & 117.43 & 37.17 & 121.47 & 12.74 \\
\hline 7509.17 & 328500.60 & 17.20 & 2280.27 & 82.48 & 145.18 & 36076.89 & 98.03 & 74.23 & 28.79 & 11.20 & 117.32 & 35.16 & 113.42 & 11.63 \\
\hline 7509.24 & 320769.00 & 17.40 & 2350.86 & 84.67 & 143.28 & 34560.25 & 95.20 & 63.66 & 27.35 & 10.51 & 115.88 & 37.68 & 112.14 & 11.30 \\
\hline 7509.30 & 332479.80 & 17.20 & 6974.14 & 87.60 & 147.21 & 33855.03 & 91.66 & 96.13 & 27.23 & 11.38 & 120.50 & 52.66 & 110.05 & 9.54 \\
\hline 7509.37 & 340543.80 & 17.00 & 6934.18 & 85.98 & 151.11 & 33952.74 & 91.88 & 429.77 & 28.18 & 12.74 & 120.33 & 53.94 & 106.17 & 9.05 \\
\hline 7509.44 & 360986.20 & 16.80 & 6891.25 & 84.37 & 149.22 & 34715.70 & 94.35 & 559.08 & 29.48 & 14.31 & 123.40 & 54.43 & 107.05 & 9.40 \\
\hline 7509.50 & 326283.20 & 17.00 & 7135.81 & 87.29 & 128.13 & 21135.08 & 94.22 & 936.33 & 23.95 & 14.88 & 118.91 & 52.08 & 123.48 & 9.53 \\
\hline 7509.57 & 302746.00 & 17.20 & 7018.23 & 86.84 & 120.49 & 20809.47 & 89.55 & 981.85 & 21.97 & 14.53 & 117.42 & 48.36 & 122.00 & 9.89 \\
\hline 7509.63 & 307614.40 & 17.20 & 2405.78 & 84.42 & 120.27 & 21886.99 & 91.74 & 958.24 & 22.56 & 13.44 & 113.81 & 35.23 & 125.01 & 11.00 \\
\hline 7509.70 & 312974.00 & 17.20 & 2415.45 & 86.60 & 118.64 & 23510.96 & 92.35 & 649.25 & 23.88 & 13.14 & 113.90 & 33.44 & 126.45 & 10.48 \\
\hline 7509.76 & 309866.40 & 17.20 & 2423.63 & 85.98 & 126.85 & 24623.05 & 95.20 & 545.73 & 25.73 & 14.08 & 113.17 & 33.82 & 128.12 & 10.83 \\
\hline 7509.83 & 318731.40 & 17.20 & 2397.78 & 85.82 & 132.04 & 26077.41 & 103.21 & 1221.95 & 28.83 & 14.99 & 112.07 & 37.87 & 127.80 & 11.53 \\
\hline 7509.90 & 351418.60 & 16.80 & 2513.45 & 87.29 & 137.36 & 31320.92 & 111.49 & 1579.29 & 34.23 & 16.66 & 113.86 & 38.85 & 128.32 & 11.46 \\
\hline 7509.96 & 346326.00 & 16.80 & 2440.29 & 88.49 & 132.91 & 32033.55 & 114.90 & 1609.12 & 33.31 & 17.81 & 112.92 & 38.85 & 125.55 & 11.09 \\
\hline 7510.03 & 345114.80 & 16.80 & 2449.29 & 89.23 & 134.20 & 31502.83 & 114.72 & 1977.23 & 31.18 & 18.07 & 111.74 & 42.04 & 123.74 & 12.28 \\
\hline 7510.09 & 345462.80 & 16.80 & 2396.58 & 90.95 & 131.37 & 31555.28 & 119.25 & 2972.70 & 28.99 & 17.66 & 110.02 & 47.11 & 123.03 & 10.66 \\
\hline
\end{tabular}




\begin{tabular}{|c|c|c|c|c|c|c|c|c|c|c|c|c|c|c|}
\hline Depth & XRF & XRF Live Time & $\mathrm{Ti}(\mathrm{ppm})$ & $\mathrm{Cr}$ (ppm) & $\mathrm{Mn}(\mathrm{ppm})$ & $\mathrm{Fe}(\mathrm{ppm})$ & $\mathrm{Ni}(\mathrm{ppm})$ & $\mathrm{Cu}(\mathrm{ppm})$ & $\mathrm{Zn}(\mathrm{ppm})$ & As (ppm) & $\mathrm{Rb}$ (ppm) & $\mathrm{Sr}(\mathrm{ppm})$ & $\mathrm{Y}(\mathrm{ppm})$ & $\mathrm{Zr}(\mathrm{ppm})$ \\
\hline 7510.16 & 342228.60 & 16.80 & 2382.32 & 93.43 & 129.30 & 30867.54 & 113.47 & 2352.72 & 25.62 & 17.39 & 108.60 & 44.55 & 123.11 & 10.04 \\
\hline 7510.22 & 316088.20 & 17.20 & 2275.45 & 85.81 & 128.93 & 26605.10 & 102.68 & 2021.55 & 20.53 & 16.93 & 107.97 & 46.49 & 125.80 & 9.79 \\
\hline 7510.29 & 306574.80 & 17.40 & 2446.74 & 85.13 & 135.11 & 26530.28 & 99.39 & 2000.37 & 22.81 & 18.33 & 106.55 & 47.52 & 127.65 & 10.35 \\
\hline 7510.35 & 304215.20 & 17.40 & 2445.51 & 86.33 & 137.79 & 27141.63 & 103.00 & 1634.01 & 25.66 & 19.58 & 107.18 & 48.11 & 128.51 & 9.30 \\
\hline 7510.42 & 289164.80 & 17.60 & 2482.31 & 85.80 & 134.80 & 25823.47 & 92.60 & 633.32 & 25.16 & 19.14 & 106.95 & 44.59 & 127.17 & 10.37 \\
\hline 7510.49 & 291863.80 & 17.60 & 2510.92 & 84.61 & 135.59 & 29926.76 & 102.47 & 204.92 & 27.97 & 20.51 & 108.02 & 43.81 & 124.13 & 9.91 \\
\hline 7510.55 & 293744.20 & 17.40 & 2586.86 & 91.77 & 135.93 & 30473.09 & 106.45 & 130.03 & 29.47 & 20.47 & 107.08 & 44.06 & 123.51 & 10.49 \\
\hline 7510.62 & 273681.20 & 17.40 & 2283.51 & 87.95 & 127.95 & 28611.85 & 103.97 & 102.62 & 26.63 & 18.58 & 102.34 & 41.81 & 116.08 & 9.44 \\
\hline 7510.68 & 291516.20 & 17.20 & 2275.32 & 86.87 & 131.12 & 28283.86 & 101.87 & 76.41 & 26.54 & 18.56 & 104.11 & 41.37 & 120.53 & 9.20 \\
\hline 7510.75 & 313794.20 & 17.00 & 2244.28 & 91.26 & 134.60 & 29227.92 & 106.84 & 64.94 & 27.51 & 19.25 & 105.86 & 43.45 & 122.83 & 9.31 \\
\hline 7510.81 & 321380.60 & 17.00 & 2228.54 & 94.58 & 135.73 & 25172.68 & 98.89 & 78.93 & 24.55 & 17.50 & 109.37 & 46.72 & 126.09 & 8.85 \\
\hline 7510.88 & 341912.60 & 17.00 & 2187.90 & 96.28 & 145.63 & 25673.04 & 103.96 & 87.53 & 26.57 & 18.60 & 111.91 & 47.26 & 125.63 & 8.69 \\
\hline 7510.94 & 371093.40 & 16.80 & 2318.55 & 101.91 & 151.01 & 27158.12 & 109.71 & 119.40 & 27.58 & 19.41 & 119.39 & 49.66 & 133.73 & 9.61 \\
\hline 7511.01 & 359767.40 & 17.00 & 2275.33 & 102.53 & 146.69 & 27676.71 & 108.63 & 106.14 & 26.43 & 20.02 & 119.84 & 48.88 & 127.05 & 9.84 \\
\hline 7511.08 & 369601.80 & 16.80 & 2380.00 & 101.59 & 158.83 & 35859.13 & 117.08 & 96.40 & 34.48 & 20.98 & 115.64 & 45.14 & 123.94 & 8.67 \\
\hline 7511.14 & 366663.00 & 16.80 & 2388.00 & 96.88 & 164.70 & 39021.47 & 137.59 & 100.76 & 36.97 & 27.21 & 113.40 & 57.28 & 105.10 & 28.82 \\
\hline 7511.21 & 349683.60 & 16.80 & 2326.95 & 91.99 & 155.39 & 38124.68 & 132.26 & 83.28 & 33.82 & 26.12 & 110.99 & 55.16 & 104.15 & 28.29 \\
\hline 7511.27 & 354576.80 & 16.80 & 3179.79 & 89.20 & 157.35 & 39891.30 & 131.62 & 51.51 & 34.68 & 27.08 & 108.69 & 52.97 & 100.80 & 28.17 \\
\hline 7511.34 & 343801.40 & 17.00 & 3116.18 & 84.81 & 155.10 & 39096.98 & 134.31 & 67.07 & 34.79 & 26.24 & 109.19 & 54.29 & 104.41 & 28.37 \\
\hline 7511.40 & 322676.00 & 17.20 & 2991.12 & 83.61 & 141.14 & 30601.96 & 128.58 & 80.14 & 26.96 & 24.93 & 113.93 & 58.03 & 104.59 & 29.31 \\
\hline 7511.47 & 316432.00 & 17.20 & 2987.29 & 83.93 & 136.32 & 27241.20 & 103.79 & 74.43 & 24.48 & 18.57 & 115.41 & 43.02 & 123.83 & 10.93 \\
\hline 7511.54 & 323214.80 & 17.20 & 3011.40 & 90.16 & 141.53 & 27602.54 & 109.65 & 80.92 & 26.14 & 19.54 & 117.34 & 43.43 & 121.91 & 11.33 \\
\hline 7511.60 & 318625.40 & 17.20 & 2192.87 & 89.04 & 139.56 & 25291.67 & 103.63 & 81.13 & 24.19 & 17.89 & 119.38 & 45.37 & 123.41 & 11.48 \\
\hline 7511.67 & 326795.00 & 17.00 & 2225.40 & 92.09 & 140.51 & 25003.75 & 100.57 & 81.34 & 24.77 & 17.28 & 115.77 & 43.74 & 121.21 & 11.25 \\
\hline 7511.73 & 328109.40 & 17.00 & 2266.51 & 92.33 & 140.96 & 24537.57 & 98.73 & 113.12 & 24.34 & 18.02 & 113.79 & 40.05 & 123.47 & 9.62 \\
\hline 7511.80 & 328335.60 & 17.00 & 2237.53 & 94.25 & 131.44 & 25459.41 & 108.62 & 225.57 & 28.32 & 19.73 & 109.31 & 38.31 & 122.59 & 8.76 \\
\hline 7511.86 & 334668.60 & 17.00 & 2197.52 & 87.06 & 124.30 & 25985.07 & 118.91 & 238.81 & 169.18 & 20.66 & 109.70 & 51.41 & 106.10 & 31.13 \\
\hline 7511.93 & 339254.20 & 17.00 & 2172.42 & 93.38 & 114.61 & 26010.14 & 119.11 & 233.98 & 169.14 & 21.61 & 108.83 & 48.50 & 108.53 & 32.03 \\
\hline 7511.99 & 347262.80 & 17.00 & 2275.38 & 96.98 & 109.74 & 28040.35 & 123.14 & 222.37 & 169.39 & 21.91 & 108.80 & 46.04 & 109.78 & 32.64 \\
\hline 7512.06 & 352473.00 & 17.00 & 2334.67 & 96.71 & 110.32 & 29011.90 & 125.43 & 178.69 & 171.14 & 22.43 & 109.32 & 46.60 & 108.07 & 35.31 \\
\hline 7512.13 & 352448.60 & 17.00 & 2365.66 & 98.86 & 110.50 & 27952.17 & 119.95 & 52.25 & 169.16 & 21.40 & 111.43 & 48.19 & 108.06 & 34.80 \\
\hline 7512.19 & 352588.60 & 17.00 & 2381.08 & 99.20 & 112.68 & 27765.06 & 110.02 & 44.75 & 28.79 & 21.03 & 111.39 & 34.66 & 126.43 & 11.77 \\
\hline 7512.26 & 364199.00 & 16.80 & 2531.27 & 94.71 & 136.05 & 30274.97 & 122.90 & 92.90 & 36.64 & 23.72 & 115.62 & 37.84 & 123.71 & 10.82 \\
\hline
\end{tabular}




\begin{tabular}{|c|c|c|c|c|c|c|c|c|c|c|c|c|c|c|}
\hline Depth & XRF & XRF Live Time & $\mathrm{Ti}(\mathrm{ppm})$ & $\mathrm{Cr}(\mathrm{ppm})$ & $\mathrm{Mn}(\mathrm{ppm})$ & $\mathrm{Fe}(\mathrm{ppm})$ & $\mathrm{Ni}(\mathrm{ppm})$ & $\mathrm{Cu}(\mathrm{ppm})$ & $\mathrm{Zn}(\mathrm{ppm})$ & As (ppm) & $\mathrm{Rb}(\mathrm{ppm})$ & $\mathrm{Sr}(\mathrm{ppm})$ & $\mathrm{Y}(\mathrm{ppm})$ & $\mathrm{Zr}(\mathrm{ppm})$ \\
\hline 7512.32 & 362680.40 & 16.80 & 2401.82 & 94.39 & 139.20 & 28420.30 & 122.27 & 142.19 & 35.10 & 24.43 & 120.64 & 42.11 & 125.55 & 10.14 \\
\hline 7512.39 & 358131.20 & 16.80 & 2347.47 & 95.43 & 132.83 & 27616.27 & 114.23 & 154.21 & 33.39 & 23.23 & 120.61 & 41.95 & 130.14 & 9.60 \\
\hline 7512.45 & 364265.80 & 16.80 & 2344.63 & 95.50 & 137.29 & 28595.99 & 114.82 & 364.74 & 33.15 & 24.36 & 122.40 & 42.64 & 129.81 & 9.64 \\
\hline 7512.52 & 354238.80 & 16.80 & 2413.15 & 99.05 & 130.87 & 27896.19 & 113.01 & 360.59 & 33.67 & 23.03 & 122.85 & 41.61 & 129.44 & 9.75 \\
\hline 7512.59 & 338948.20 & 17.00 & 2236.93 & 98.63 & 110.01 & 25383.08 & 103.47 & 317.88 & 27.80 & 19.84 & 120.75 & 41.34 & 130.76 & 9.87 \\
\hline 7512.65 & 339716.20 & 17.00 & 2301.97 & 99.86 & 109.19 & 25266.08 & 117.69 & 283.48 & 43.27 & 19.72 & 120.02 & 56.39 & 112.46 & 38.22 \\
\hline 7512.72 & 340728.40 & 17.00 & 2314.58 & 103.37 & 114.09 & 25706.22 & 126.36 & 387.59 & 44.89 & 20.46 & 119.17 & 55.85 & 108.74 & 37.96 \\
\hline 7512.78 & 332788.60 & 17.00 & 2311.33 & 98.33 & 115.07 & 24556.93 & 125.97 & 192.29 & 44.74 & 19.39 & 120.84 & 53.19 & 107.69 & 38.46 \\
\hline 7512.85 & 329630.20 & 17.00 & 2330.05 & 95.52 & 117.34 & 24077.30 & 123.29 & 184.57 & 46.25 & 18.84 & 120.25 & 52.82 & 106.47 & 40.41 \\
\hline 7512.91 & 338837.80 & 17.00 & 2491.81 & 95.74 & 127.10 & 25596.41 & 126.14 & 179.51 & 47.78 & 19.02 & 124.84 & 54.12 & 106.07 & 39.99 \\
\hline 7512.98 & 335852.20 & 17.00 & 2507.26 & 94.31 & 128.76 & 27031.89 & 111.14 & 161.55 & 35.69 & 18.19 & 129.45 & 38.44 & 120.71 & 11.27 \\
\hline 7513.04 & 331346.40 & 17.00 & 2504.67 & 92.32 & 129.11 & 27140.55 & 108.81 & 40.70 & 36.49 & 17.58 & 132.21 & 40.28 & 119.99 & 11.63 \\
\hline 7513.11 & 329910.80 & 17.00 & 2460.71 & 95.63 & 130.43 & 27726.13 & 109.08 & 50.47 & 36.74 & 18.23 & 136.93 & 42.75 & 120.29 & 11.05 \\
\hline 7513.18 & 322831.00 & 17.20 & 2418.42 & 94.52 & 135.29 & 29000.28 & 116.27 & 62.03 & 38.15 & 20.70 & 138.23 & 42.27 & 119.96 & 9.82 \\
\hline 7513.24 & 300936.00 & 17.40 & 3051.97 & 91.59 & 138.14 & 27805.29 & 114.56 & 333.33 & 37.27 & 21.29 & 138.38 & 38.87 & 119.05 & 10.19 \\
\hline 7513.31 & 270299.80 & 17.60 & 3042.57 & 88.48 & 135.63 & 24734.38 & 107.62 & 339.93 & 33.34 & 19.47 & 132.43 & 35.42 & 117.96 & 10.19 \\
\hline 7513.37 & 246202.80 & 17.80 & 3077.71 & 84.45 & 131.98 & 23122.01 & 98.54 & 348.76 & 30.88 & 17.82 & 131.35 & 33.44 & 115.23 & 9.24 \\
\hline 7513.44 & 259860.60 & 17.80 & 2998.51 & 82.41 & 140.24 & 22172.25 & 93.18 & 318.30 & 30.09 & 15.75 & 143.40 & 51.31 & 110.90 & 9.53 \\
\hline 7513.50 & 266941.00 & 17.60 & 2938.43 & 81.36 & 141.63 & 20634.45 & 82.03 & 306.70 & 26.46 & 12.42 & 150.76 & 53.77 & 109.66 & 10.21 \\
\hline 7513.57 & 271485.20 & 17.40 & 2191.64 & 85.21 & 133.54 & 19784.58 & 81.44 & 34.38 & 24.52 & 10.18 & 146.09 & 53.16 & 108.47 & 10.26 \\
\hline 7513.64 & 287767.40 & 17.40 & 2242.98 & 86.83 & 138.12 & 20744.52 & 83.13 & 26.53 & 26.52 & 10.37 & 148.37 & 52.27 & 107.91 & 10.89 \\
\hline 7513.70 & 309044.40 & 17.20 & 2135.70 & 85.31 & 150.10 & 22560.91 & 88.29 & 15.78 & 28.61 & 10.74 & 150.41 & 52.57 & 106.35 & 10.25 \\
\hline 7513.77 & 303078.60 & 17.20 & 2263.46 & 85.51 & 142.98 & 23986.57 & 94.64 & 150.56 & 31.54 & 11.49 & 143.26 & 33.57 & 110.13 & 10.76 \\
\hline 7513.83 & 280332.80 & 17.40 & 2179.79 & 82.68 & 156.23 & 22778.95 & 93.13 & 154.00 & 32.01 & 10.72 & 148.76 & 32.77 & 108.79 & 9.94 \\
\hline 7513.90 & 290200.40 & 17.40 & 2180.61 & 85.04 & 155.30 & 23983.54 & 97.63 & 156.50 & 32.54 & 11.46 & 154.65 & 33.68 & 111.34 & 9.60 \\
\hline 7513.96 & 300013.60 & 17.20 & 2077.76 & 82.50 & 158.48 & 25497.79 & 112.18 & 179.69 & 39.66 & 17.79 & 150.82 & 65.99 & 98.44 & 33.39 \\
\hline 7514.03 & 294985.00 & 17.40 & 2167.36 & 84.46 & 147.26 & 27090.62 & 114.84 & 182.56 & 40.37 & 18.16 & 158.32 & 66.25 & 100.95 & 34.33 \\
\hline 7514.09 & 305879.00 & 17.20 & 2206.95 & 84.52 & 146.14 & 29186.38 & 111.58 & 49.81 & 41.64 & 17.82 & 161.65 & 65.93 & 101.92 & 33.89 \\
\hline 7514.16 & 338813.00 & 17.00 & 2279.93 & 87.50 & 141.77 & 30523.27 & 118.70 & 46.09 & 42.30 & 18.75 & 172.78 & 66.80 & 105.25 & 34.06 \\
\hline 7514.23 & 276452.40 & 17.60 & 4542.37 & 90.96 & 165.00 & 25277.47 & 101.05 & 50.38 & 36.53 & 16.94 & 146.96 & 57.73 & 81.64 & 32.01 \\
\hline 7514.29 & 249665.40 & 17.80 & 5871.79 & 89.25 & 159.27 & 25702.86 & 86.48 & 29.02 & 29.67 & 10.33 & 160.58 & 29.62 & 94.25 & 7.51 \\
\hline 7514.36 & 255148.60 & 17.60 & 6007.98 & 93.06 & 165.10 & 24049.50 & 85.03 & 26.88 & 28.49 & 9.90 & 162.83 & 29.36 & 98.52 & 7.08 \\
\hline 7514.42 & 233501.00 & 17.80 & 6133.53 & 94.23 & 158.91 & 21538.64 & 86.53 & 24.61 & 27.38 & 10.22 & 151.37 & 27.62 & 103.38 & 8.40 \\
\hline
\end{tabular}




\begin{tabular}{|c|c|c|c|c|c|c|c|c|c|c|c|c|c|c|}
\hline Depth & XRF & XRF Live Time & $\mathrm{Ti}(\mathrm{ppm})$ & $\mathrm{Cr}(\mathrm{ppm})$ & $\mathrm{Mn}(\mathrm{ppm})$ & $\mathrm{Fe}(\mathrm{ppm})$ & $\mathrm{Ni}(\mathrm{ppm})$ & $\mathrm{Cu}(\mathrm{ppm})$ & $\mathrm{Zn}(\mathrm{ppm})$ & As (ppm) & $\mathrm{Rb}(\mathrm{ppm})$ & $\mathrm{Sr}(\mathrm{ppm})$ & $\mathrm{Y}(\mathrm{ppm})$ & $\mathrm{Zr}(\mathrm{ppm})$ \\
\hline 7514.49 & 228791.40 & 17.80 & 6158.77 & 93.01 & 157.75 & 21990.28 & 81.46 & 25.30 & 28.25 & 10.95 & 140.51 & 25.57 & 103.55 & 9.00 \\
\hline 7514.55 & 287682.20 & 17.20 & 3802.11 & 80.82 & 136.39 & 26852.67 & 95.79 & 17.82 & 34.61 & 12.94 & 166.58 & 35.00 & 128.56 & 11.14 \\
\hline 7514.62 & 315508.80 & 17.00 & 2648.29 & 82.28 & 141.38 & 25908.23 & 99.14 & 16.30 & 35.01 & 15.06 & 159.15 & 31.41 & 138.12 & 12.54 \\
\hline 7514.69 & 318780.00 & 17.00 & 2582.23 & 79.15 & 136.11 & 25627.43 & 99.10 & 16.20 & 34.73 & 16.15 & 153.80 & 28.84 & 138.46 & 13.84 \\
\hline 7514.75 & 322613.80 & 17.00 & 2625.52 & 81.73 & 134.25 & 25470.89 & 98.16 & 15.20 & 33.26 & 17.11 & 153.91 & 29.74 & 135.74 & 13.19 \\
\hline 7514.82 & 320329.40 & 17.00 & 2787.19 & 85.34 & 118.35 & 25747.33 & 101.28 & 15.17 & 33.99 & 17.89 & 141.53 & 27.93 & 141.93 & 12.15 \\
\hline 7514.88 & 316671.40 & 17.00 & 3037.30 & 91.33 & 117.36 & 28421.38 & 100.30 & 18.58 & 37.54 & 18.69 & 138.01 & 24.72 & 143.21 & 12.84 \\
\hline 7514.95 & 313713.40 & 17.00 & 3105.00 & 99.99 & 110.01 & 28104.49 & 97.78 & 18.36 & 38.72 & 18.63 & 134.91 & 23.98 & 141.95 & 12.86 \\
\hline 7515.01 & 306287.20 & 17.00 & 3096.31 & 97.26 & 105.49 & 27162.70 & 93.13 & 17.70 & 38.98 & 18.75 & 131.56 & 25.90 & 143.77 & 12.01 \\
\hline 7515.08 & 301071.60 & 17.00 & 3087.16 & 97.40 & 105.03 & 26433.96 & 92.46 & 17.77 & 37.95 & 18.45 & 131.43 & 25.09 & 145.37 & 11.61 \\
\hline 7515.14 & 323685.80 & 16.80 & 3148.32 & 99.25 & 113.96 & 28599.47 & 104.64 & 22.28 & 36.94 & 20.57 & 129.08 & 24.08 & 147.84 & 12.30 \\
\hline 7515.21 & 345088.40 & 16.60 & 3050.76 & 101.59 & 114.55 & 29169.89 & 117.68 & 21.45 & 37.87 & 22.64 & 126.09 & 25.75 & 149.29 & 11.58 \\
\hline 7515.28 & 353024.40 & 16.60 & 3061.24 & 98.11 & 118.62 & 31661.38 & 126.00 & 21.63 & 37.94 & 24.15 & 125.04 & 25.24 & 148.52 & 11.43 \\
\hline 7515.34 & 365798.00 & 16.60 & 3144.48 & 102.51 & 122.39 & 34332.35 & 140.23 & 23.09 & 39.11 & 26.14 & 121.95 & 23.27 & 148.41 & 11.31 \\
\hline 7515.41 & 369122.60 & 16.60 & 3229.57 & 101.93 & 131.05 & 38250.90 & 149.37 & 25.71 & 44.33 & 27.60 & 120.68 & 23.04 & 150.27 & 12.67 \\
\hline 7515.47 & 352684.80 & 16.80 & 3358.55 & 106.19 & 132.63 & 38978.19 & 151.72 & 21.54 & 45.77 & 27.27 & 125.55 & 22.51 & 149.70 & 13.26 \\
\hline 7515.54 & 356711.00 & 16.80 & 3400.19 & 106.23 & 134.03 & 38135.60 & 154.69 & 21.33 & 46.03 & 27.63 & 130.86 & 22.54 & 151.54 & 14.72 \\
\hline 7515.60 & 372449.00 & 16.60 & 2998.04 & 99.68 & 175.89 & 35807.95 & 153.48 & 22.41 & 44.25 & 24.62 & 171.32 & 33.86 & 137.81 & 13.49 \\
\hline 7515.67 & 380762.00 & 16.40 & 2848.88 & 96.28 & 227.09 & 34029.70 & 143.80 & 21.00 & 41.68 & 21.09 & 189.42 & 38.42 & 130.39 & 13.95 \\
\hline 7515.73 & 411887.40 & 16.20 & 2292.58 & 84.21 & 306.42 & 31601.64 & 136.03 & 17.52 & 35.53 & 17.64 & 234.13 & 52.12 & 115.50 & 10.08 \\
\hline 7515.80 & 437351.60 & 16.00 & 1576.64 & 67.79 & 367.41 & 31453.06 & 115.93 & 16.03 & 32.16 & 13.54 & 272.35 & 73.38 & 87.95 & 7.43 \\
\hline 7515.87 & 440234.80 & 16.00 & 1234.58 & 58.21 & 414.13 & 30079.02 & 102.81 & 13.65 & 27.16 & 9.05 & 301.90 & 86.04 & 70.60 & 4.32 \\
\hline 7515.93 & 422108.00 & 16.20 & 1405.18 & 58.22 & 384.06 & 29895.76 & 95.94 & 14.05 & 28.06 & 9.90 & 286.74 & 75.32 & 79.18 & 4.71 \\
\hline 7516.00 & 429928.60 & 16.20 & 1569.81 & 65.53 & 351.90 & 32928.41 & 105.01 & 17.10 & 32.63 & 11.45 & 281.91 & 73.68 & 91.12 & 4.80 \\
\hline 7516.06 & 412702.40 & 16.40 & 2091.08 & 85.50 & 271.96 & 33676.83 & 115.28 & 20.68 & 36.61 & 13.37 & 240.24 & 59.72 & 107.85 & 7.66 \\
\hline 7516.13 & 377791.00 & 16.60 & 2761.13 & 104.31 & 205.69 & 32625.61 & 129.26 & 24.06 & 38.99 & 15.68 & 200.40 & 38.98 & 136.19 & 10.44 \\
\hline 7516.19 & 368904.60 & 16.60 & 3204.48 & 117.89 & 165.73 & 33571.44 & 134.11 & 30.71 & 41.76 & 18.77 & 170.32 & 27.14 & 156.54 & 12.49 \\
\hline 7516.26 & 374905.80 & 16.60 & 3437.64 & 128.52 & 157.70 & 35085.15 & 143.24 & 40.05 & 42.27 & 20.07 & 149.65 & 27.06 & 166.05 & 14.27 \\
\hline 7516.33 & 361139.00 & 16.80 & 4173.34 & 131.80 & 145.81 & 32631.39 & 141.39 & 189.36 & 39.66 & 20.69 & 147.16 & 25.02 & 167.36 & 15.41 \\
\hline 7516.39 & 348256.20 & 16.80 & 3964.20 & 124.10 & 150.46 & 30229.59 & 132.35 & 210.58 & 36.58 & 20.69 & 165.22 & 26.45 & 165.17 & 14.99 \\
\hline 7516.46 & 350179.60 & 16.80 & 3545.10 & 110.77 & 151.09 & 27011.65 & 125.46 & 244.49 & 33.84 & 20.57 & 190.79 & 27.67 & 157.43 & 14.67 \\
\hline 7516.52 & 336528.20 & 17.00 & 3364.78 & 109.15 & 147.86 & 24866.56 & 121.14 & 256.72 & 33.31 & 19.76 & 213.10 & 27.23 & 150.99 & 14.49 \\
\hline 7516.59 & 315814.80 & 17.20 & 3274.13 & 108.11 & 142.18 & 22920.65 & 117.35 & 248.88 & 31.31 & 18.21 & 227.84 & 28.79 & 148.85 & 12.66 \\
\hline
\end{tabular}




\begin{tabular}{|c|c|c|c|c|c|c|c|c|c|c|c|c|c|c|}
\hline Depth & XRF & XRF Live Time & $\mathrm{Ti}(\mathrm{ppm})$ & $\mathrm{Cr}(\mathrm{ppm})$ & $\mathrm{Mn}(\mathrm{ppm})$ & $\mathrm{Fe}(\mathrm{ppm})$ & $\mathrm{Ni}(\mathrm{ppm})$ & $\mathrm{Cu}(\mathrm{ppm})$ & $\mathrm{Zn}(\mathrm{ppm})$ & As (ppm) & $\mathrm{Rb}(\mathrm{ppm})$ & $\mathrm{Sr}(\mathrm{ppm})$ & $\mathrm{Y}(\mathrm{ppm})$ & $\mathrm{Zr}(\mathrm{ppm})$ \\
\hline 7516.65 & 298331.60 & 17.40 & 1928.15 & 84.43 & 146.40 & 18075.35 & 94.21 & 95.54 & 25.12 & 14.66 & 238.64 & 32.34 & 118.99 & 9.48 \\
\hline 7516.72 & 299872.40 & 17.40 & 1364.05 & 61.91 & 154.73 & 14371.10 & 74.63 & 70.15 & 20.95 & 11.61 & 348.48 & 37.98 & 91.85 & 6.79 \\
\hline 7516.78 & 308444.60 & 17.40 & 1060.06 & 51.29 & 164.04 & 11068.10 & 59.05 & 32.22 & 16.46 & 8.38 & 341.29 & 40.11 & 69.63 & 3.93 \\
\hline 7516.85 & 308665.80 & 17.40 & 435.26 & 33.63 & 183.96 & 7284.74 & 40.71 & 11.70 & 9.81 & 4.99 & 442.41 & 46.03 & 47.76 & 1.31 \\
\hline 7516.92 & 312771.60 & 17.20 & -177.07 & 17.03 & 205.19 & 4105.29 & 22.96 & 6.83 & 5.39 & 2.92 & 508.86 & 48.40 & 24.36 & 0.32 \\
\hline 7516.98 & 332255.40 & 17.00 & -450.98 & 13.24 & 223.02 & 5887.86 & 22.46 & 7.08 & 5.30 & 2.99 & 626.98 & 47.85 & 26.51 & -0.02 \\
\hline 7517.05 & 331437.80 & 17.00 & -876.55 & 16.85 & 212.31 & 6987.64 & 24.83 & 8.80 & 5.57 & 3.20 & 686.66 & 42.38 & 31.11 & 1.21 \\
\hline 7517.11 & 323620.20 & 17.00 & -868.08 & 12.34 & 226.93 & 7192.60 & 24.45 & 9.57 & 6.34 & 3.56 & 680.16 & 39.23 & 30.59 & 1.27 \\
\hline 7517.18 & 298481.00 & 17.20 & -633.94 & 11.42 & 226.02 & 6965.44 & 24.75 & 10.82 & 6.89 & 3.80 & 567.29 & 33.05 & 26.92 & 2.13 \\
\hline 7517.24 & 293598.40 & 17.40 & -561.10 & 11.88 & 230.24 & 6162.03 & 17.56 & 10.93 & 6.23 & 3.30 & 487.92 & 27.67 & 21.57 & 1.48 \\
\hline 7517.31 & 225024.00 & 18.00 & 2136.31 & 29.77 & 205.53 & 3620.92 & 13.07 & 14.19 & 6.82 & 3.45 & 331.07 & 19.49 & 13.76 & 3.08 \\
\hline 7517.38 & 221799.60 & 18.00 & 3689.48 & 30.14 & 219.07 & 2366.40 & 12.12 & 13.03 & 5.58 & 3.61 & 144.24 & 17.40 & 7.76 & 3.71 \\
\hline 7517.44 & 215424.40 & 18.00 & 3706.53 & 31.63 & 216.73 & 2269.13 & 11.15 & 12.73 & 5.23 & 3.68 & 132.83 & 18.39 & 10.12 & 3.93 \\
\hline 7517.51 & 228415.60 & 18.00 & 3642.18 & 29.07 & 217.00 & 2091.28 & 10.54 & 12.93 & 4.71 & 4.35 & 123.61 & 19.21 & 9.27 & 3.60 \\
\hline 7517.57 & 225668.40 & 18.00 & 3665.78 & 23.62 & 223.59 & 2254.72 & 13.74 & 12.93 & 5.14 & 4.50 & 122.25 & 20.56 & 10.73 & 3.91 \\
\hline 7517.64 & 267673.80 & 17.60 & 1209.57 & 9.93 & 257.81 & 2750.92 & 18.00 & 8.91 & 4.09 & 4.20 & 146.97 & 27.71 & 13.81 & 1.97 \\
\hline 7517.70 & 254247.20 & 17.80 & 182.69 & 11.17 & 285.85 & 2943.97 & 19.37 & 8.77 & 4.97 & 4.08 & 144.80 & 29.00 & 16.16 & 0.98 \\
\hline 7517.77 & 243826.00 & 18.00 & 206.69 & 12.92 & 311.43 & 3184.55 & 19.33 & 8.20 & 5.02 & 3.58 & 147.44 & 27.90 & 15.60 & 1.96 \\
\hline 7517.83 & 232566.60 & 18.00 & 206.64 & 14.40 & 336.71 & 3262.23 & 18.96 & 7.79 & 5.44 & 2.84 & 151.30 & 27.71 & 17.12 & 2.41 \\
\hline 7517.90 & 254430.20 & 17.80 & 212.16 & 16.84 & 353.91 & 3311.38 & 20.75 & 6.68 & 5.13 & 2.64 & 158.72 & 29.50 & 16.23 & 2.20 \\
\hline 7517.97 & 277627.80 & 17.60 & 224.96 & 15.53 & 346.29 & 3587.71 & 23.46 & 7.69 & 5.97 & 3.06 & 161.09 & 29.37 & 18.08 & 2.01 \\
\hline 7518.03 & 291732.20 & 17.40 & 208.98 & 13.10 & 318.93 & 3599.99 & 24.62 & 6.38 & 6.23 & 2.94 & 169.33 & 30.64 & 16.58 & 1.59 \\
\hline 7518.10 & 308845.40 & 17.20 & 148.60 & 15.36 & 286.35 & 3675.46 & 26.04 & 6.55 & 7.47 & 3.48 & 220.06 & 34.31 & 17.09 & 1.10 \\
\hline 7518.16 & 325471.20 & 17.00 & 111.27 & 18.75 & 244.28 & 3931.00 & 26.71 & 5.75 & 6.43 & 3.83 & 251.46 & 36.46 & 18.84 & 1.33 \\
\hline 7518.23 & 303468.20 & 17.20 & 99.87 & 22.80 & 198.23 & 4745.48 & 28.76 & 7.81 & 9.00 & 4.68 & 276.97 & 42.16 & 28.35 & 2.65 \\
\hline 7518.29 & 302607.80 & 17.20 & 585.11 & 41.82 & 174.27 & 8108.09 & 50.06 & 9.08 & 14.13 & 8.20 & 293.17 & 43.70 & 58.19 & 5.84 \\
\hline 7518.36 & 292541.40 & 17.40 & 1016.97 & 60.93 & 155.55 & 11520.84 & 70.13 & 11.41 & 17.83 & 11.78 & 300.03 & 42.78 & 88.41 & 7.39 \\
\hline 7518.43 & 299801.60 & 17.40 & 1258.24 & 91.73 & 148.44 & 16821.02 & 92.81 & 13.59 & 23.06 & 14.61 & 347.29 & 40.38 & 113.09 & 8.88 \\
\hline 7518.49 & 302413.40 & 17.40 & 1536.37 & 102.34 & 139.23 & 19320.57 & 110.86 & 15.06 & 28.65 & 17.48 & 339.71 & 37.87 & 136.62 & 10.97 \\
\hline 7518.56 & 309617.00 & 17.40 & 1794.13 & 112.28 & 135.87 & 21228.80 & 127.01 & 15.65 & 29.04 & 19.83 & 322.50 & 31.64 & 152.33 & 12.07 \\
\hline 7518.62 & 324483.00 & 17.20 & 1350.07 & 117.06 & 144.89 & 24450.95 & 123.30 & 16.84 & 30.66 & 19.79 & 428.06 & 32.89 & 143.48 & 10.97 \\
\hline 7518.69 & 344963.40 & 17.00 & 1164.13 & 114.55 & 159.01 & 23418.75 & 116.49 & 17.41 & 29.26 & 18.83 & 454.64 & 39.22 & 134.33 & 10.93 \\
\hline 7518.75 & 341144.00 & 17.00 & 1361.60 & 100.03 & 160.51 & 21381.97 & 114.22 & 19.10 & 27.71 & 19.08 & 368.10 & 41.34 & 134.45 & 11.08 \\
\hline
\end{tabular}




\begin{tabular}{|c|c|c|c|c|c|c|c|c|c|c|c|c|c|c|}
\hline Depth & XRF & XRF Live Time & $\mathrm{Ti}(\mathrm{ppm})$ & $\mathrm{Cr}(\mathrm{ppm})$ & $\mathrm{Mn}(\mathrm{ppm})$ & $\mathrm{Fe}(\mathrm{ppm})$ & $\mathrm{Ni}(\mathrm{ppm})$ & $\mathrm{Cu}(\mathrm{ppm})$ & $\mathrm{Zn}(\mathrm{ppm})$ & As (ppm) & $\mathrm{Rb}(\mathrm{ppm})$ & $\mathrm{Sr}(\mathrm{ppm})$ & $\mathrm{Y}(\mathrm{ppm})$ & $\mathrm{Zr}(\mathrm{ppm})$ \\
\hline 7518.82 & 339252.00 & 17.00 & 1383.95 & 99.87 & 178.65 & 21022.93 & 109.44 & 25.17 & 26.14 & 18.72 & 362.64 & 46.83 & 129.24 & 10.34 \\
\hline 7518.88 & 364811.00 & 16.60 & 1236.51 & 89.61 & 195.61 & 19790.60 & 100.32 & 82.05 & 25.17 & 17.48 & 376.54 & 53.95 & 115.78 & 7.85 \\
\hline 7518.95 & 356826.20 & 16.80 & 1224.30 & 68.47 & 191.94 & 13824.00 & 86.20 & 238.28 & 18.04 & 14.56 & 271.57 & 54.53 & 100.21 & 7.09 \\
\hline 7519.02 & 365307.00 & 16.60 & 1041.25 & 55.91 & 188.70 & 12571.60 & 79.27 & 606.75 & 15.74 & 13.38 & 261.82 & 53.01 & 88.81 & 6.58 \\
\hline 7519.08 & 373977.20 & 16.60 & 852.63 & 49.21 & 194.80 & 10988.74 & 74.48 & 622.36 & 435.57 & 13.55 & 236.62 & 113.92 & 73.99 & 23.51 \\
\hline 7519.15 & 382667.40 & 16.60 & 917.96 & 48.72 & 187.81 & 11385.58 & 85.15 & 929.55 & 435.62 & 13.48 & 235.93 & 111.64 & 77.20 & 23.47 \\
\hline 7519.21 & 372891.60 & 16.80 & 1279.28 & 64.88 & 170.76 & 13639.61 & 102.44 & 1813.59 & 437.91 & 15.66 & 204.51 & 103.25 & 93.17 & 25.39 \\
\hline 7519.28 & 362019.80 & 16.80 & 1662.43 & 78.19 & 155.27 & 16406.09 & 119.77 & 1771.94 & 443.33 & 18.63 & 179.81 & 98.11 & 113.81 & 26.92 \\
\hline 7519.34 & 349944.40 & 17.00 & 2123.02 & 93.93 & 141.07 & 18530.87 & 131.15 & 1448.26 & 446.31 & 20.47 & 144.53 & 91.11 & 131.40 & 29.53 \\
\hline 7519.41 & 337089.80 & 17.00 & 2390.70 & 100.85 & 121.13 & 19565.74 & 134.27 & 1533.59 & 25.49 & 20.16 & 156.24 & 27.85 & 150.23 & 13.00 \\
\hline 7519.48 & 337860.00 & 17.00 & 2604.88 & 107.69 & 120.29 & 20794.02 & 132.24 & 1294.84 & 27.64 & 21.87 & 132.79 & 24.07 & 159.00 & 12.87 \\
\hline 7519.54 & 332568.60 & 17.00 & 2744.16 & 111.26 & 123.21 & 21236.13 & 135.04 & 418.69 & 28.66 & 22.11 & 129.83 & 24.56 & 168.18 & 14.45 \\
\hline 7519.61 & 327496.60 & 17.00 & 2971.94 & 122.50 & 127.16 & 21017.95 & 126.20 & 302.57 & 27.68 & 20.79 & 128.95 & 25.10 & 172.06 & 14.70 \\
\hline 7519.67 & 323603.40 & 17.00 & 3094.29 & 129.03 & 135.45 & 21799.06 & 123.12 & 257.29 & 28.34 & 20.50 & 129.45 & 26.42 & 175.28 & 13.90 \\
\hline 7519.74 & 327029.00 & 17.00 & 3232.54 & 134.69 & 140.24 & 24875.99 & 125.57 & 156.08 & 30.90 & 19.98 & 124.89 & 26.06 & 174.32 & 14.23 \\
\hline 7519.80 & 327511.60 & 17.00 & 3370.25 & 143.19 & 143.15 & 25935.83 & 124.24 & 81.68 & 30.29 & 17.34 & 122.09 & 27.22 & 174.34 & 16.27 \\
\hline 7519.87 & 335276.20 & 17.00 & 3508.88 & 148.57 & 157.55 & 27455.58 & 120.54 & 18.10 & 30.81 & 16.05 & 123.54 & 29.57 & 171.11 & 16.25 \\
\hline 7519.93 & 337735.20 & 17.00 & 3639.14 & 150.00 & 160.62 & 28040.42 & 128.12 & 18.71 & 29.69 & 15.22 & 124.60 & 30.54 & 171.55 & 16.54 \\
\hline 7520.00 & 334483.00 & 17.00 & 3719.50 & 152.43 & 159.29 & 29652.85 & 135.20 & 19.05 & 29.78 & 14.34 & 128.55 & 31.14 & 174.66 & 17.99 \\
\hline 7520.07 & 326601.40 & 17.00 & 3873.73 & 159.86 & 154.29 & 26607.69 & 131.76 & 16.88 & 26.14 & 13.15 & 130.81 & 32.04 & 180.03 & 17.08 \\
\hline 7520.13 & 307363.00 & 17.20 & 3695.33 & 147.88 & 136.10 & 23953.32 & 125.65 & 18.95 & 23.58 & 13.07 & 132.17 & 31.36 & 180.13 & 16.65 \\
\hline 7520.20 & 290337.20 & 17.20 & 3605.57 & 143.63 & 131.95 & 23729.94 & 127.07 & 17.44 & 20.81 & 13.04 & 132.48 & 30.17 & 179.63 & 16.74 \\
\hline 7520.26 & 277407.20 & 17.40 & 3544.44 & 142.67 & 133.95 & 23932.06 & 129.91 & 15.64 & 19.84 & 13.10 & 133.73 & 30.21 & 181.02 & 16.88 \\
\hline 7520.33 & 264227.40 & 17.60 & 3499.07 & 141.88 & 137.18 & 21988.72 & 127.34 & 16.90 & 17.67 & 12.58 & 131.33 & 30.34 & 178.66 & 16.93 \\
\hline 7520.39 & 211027.20 & 18.20 & 4468.72 & 120.27 & 139.67 & 18500.74 & 115.74 & 27.02 & 20.83 & 9.29 & 109.78 & 26.98 & 140.19 & 17.68 \\
\hline 7520.46 & 215626.40 & 18.20 & 4699.23 & 128.17 & 148.17 & 19177.14 & 122.99 & 27.05 & 20.18 & 8.79 & 109.55 & 27.04 & 140.36 & 16.89 \\
\hline 7520.52 & 220490.60 & 18.20 & 4811.29 & 132.93 & 140.44 & 18361.10 & 124.12 & 28.38 & 19.80 & 7.87 & 108.76 & 28.56 & 140.60 & 15.85 \\
\hline 7520.59 & 235270.80 & 18.00 & 4823.61 & 132.89 & 145.36 & 19921.59 & 125.72 & 44.57 & 20.79 & 8.84 & 107.45 & 28.50 & 139.09 & 16.13 \\
\hline 7520.66 & 250389.40 & 17.80 & 4882.10 & 136.76 & 144.82 & 20680.13 & 127.98 & 43.07 & 20.74 & 9.29 & 107.68 & 27.52 & 141.05 & 15.73 \\
\hline 7520.72 & 323803.60 & 17.00 & 3858.87 & 157.45 & 159.23 & 30927.28 & 146.16 & 36.15 & 20.32 & 12.90 & 130.67 & 32.06 & 179.01 & 16.18 \\
\hline 7520.79 & 326283.20 & 16.80 & 3920.68 & 159.86 & 166.43 & 31271.28 & 142.58 & 35.27 & 20.36 & 12.82 & 132.63 & 32.05 & 177.96 & 16.55 \\
\hline 7520.85 & 327098.00 & 16.80 & 3850.83 & 157.58 & 172.79 & 31489.90 & 142.85 & 35.39 & 21.11 & 12.64 & 133.98 & 30.99 & 177.66 & 16.71 \\
\hline 7520.92 & 338671.40 & 16.80 & 3796.08 & 157.80 & 179.98 & 32914.32 & 145.92 & 23.47 & 21.01 & 11.93 & 142.70 & 32.46 & 177.75 & 17.75 \\
\hline
\end{tabular}




\begin{tabular}{|c|c|c|c|c|c|c|c|c|c|c|c|c|c|c|}
\hline Depth & XRF & XRF Live Time & $\mathrm{Ti}(\mathrm{ppm})$ & $\mathrm{Cr}(\mathrm{ppm})$ & $\mathrm{Mn}(\mathrm{ppm})$ & $\mathrm{Fe}(\mathrm{ppm})$ & $\mathrm{Ni}(\mathrm{ppm})$ & $\mathrm{Cu}(\mathrm{ppm})$ & $\mathrm{Zn}(\mathrm{ppm})$ & As (ppm) & $\mathrm{Rb}(\mathrm{ppm})$ & $\mathrm{Sr}(\mathrm{ppm})$ & $\mathrm{Y}(\mathrm{ppm})$ & $\mathrm{Zr}(\mathrm{ppm})$ \\
\hline 7520.98 & 351791.40 & 16.80 & 3757.78 & 160.71 & 181.73 & 34445.49 & 150.41 & 38.75 & 21.98 & 12.00 & 144.70 & 34.38 & 174.78 & 18.32 \\
\hline 7521.05 & 336401.60 & 17.00 & 3711.55 & 159.80 & 171.96 & 28216.06 & 147.32 & 36.92 & 18.65 & 12.06 & 142.69 & 34.88 & 172.48 & 18.14 \\
\hline 7521.12 & 346028.20 & 17.00 & 3596.06 & 158.05 & 171.29 & 29508.16 & 153.89 & 38.41 & 21.00 & 14.46 & 143.57 & 35.30 & 175.22 & 17.62 \\
\hline 7521.18 & 354653.40 & 17.00 & 3581.54 & 151.72 & 166.52 & 31832.51 & 148.19 & 38.15 & 25.06 & 15.53 & 142.82 & 32.43 & 175.89 & 18.37 \\
\hline 7521.25 & 343187.40 & 17.00 & 3577.75 & 146.00 & 156.88 & 29907.67 & 146.49 & 39.85 & 26.28 & 16.75 & 134.04 & 30.39 & 181.66 & 16.29 \\
\hline 7521.31 & 345733.00 & 16.80 & 3527.96 & 133.98 & 150.62 & 29384.80 & 143.15 & 29.75 & 29.17 & 18.09 & 132.91 & 30.74 & 188.83 & 14.97 \\
\hline 7521.38 & 355521.60 & 16.80 & 3487.05 & 130.43 & 154.66 & 30677.28 & 147.04 & 58.66 & 32.12 & 19.48 & 136.79 & 29.91 & 193.30 & 14.19 \\
\hline 7521.44 & 357816.40 & 16.80 & 8548.16 & 125.83 & 143.16 & 29379.11 & 141.64 & 63.44 & 30.98 & 18.18 & 135.69 & 28.48 & 195.69 & 14.98 \\
\hline 7521.51 & 353882.80 & 16.80 & 8553.93 & 124.39 & 139.12 & 26652.64 & 135.50 & 61.86 & 26.85 & 18.38 & 138.48 & 31.26 & 195.72 & 14.85 \\
\hline 7521.57 & 358299.60 & 16.80 & 8544.64 & 123.04 & 138.01 & 26128.62 & 131.90 & 58.98 & 26.19 & 17.47 & 143.94 & 32.48 & 193.01 & 14.80 \\
\hline 7521.64 & 353597.80 & 17.00 & 8445.44 & 120.94 & 149.67 & 25594.19 & 133.21 & 54.78 & 24.18 & 17.53 & 148.72 & 31.07 & 191.89 & 14.76 \\
\hline 7521.71 & 360747.20 & 16.80 & 8348.91 & 116.83 & 149.17 & 26196.75 & 140.78 & 28.80 & 23.59 & 17.93 & 153.74 & 30.33 & 192.35 & 15.10 \\
\hline 7521.77 & 374084.20 & 16.60 & 2810.25 & 105.69 & 178.03 & 25015.06 & 132.67 & 20.33 & 22.31 & 16.49 & 220.68 & 40.41 & 168.75 & 12.53 \\
\hline 7521.84 & 375461.00 & 16.60 & 2288.57 & 90.87 & 180.68 & 22429.06 & 129.30 & 20.71 & 20.28 & 14.53 & 280.77 & 47.63 & 154.77 & 10.97 \\
\hline 7521.90 & 379564.80 & 16.60 & 2122.93 & 84.54 & 189.06 & 21064.63 & 131.83 & 56.38 & 18.77 & 13.94 & 317.17 & 51.45 & 151.47 & 11.73 \\
\hline 7521.97 & 369664.20 & 16.60 & 2158.80 & 86.30 & 171.69 & 18163.13 & 120.18 & 55.54 & 16.71 & 11.89 & 333.23 & 53.54 & 151.61 & 11.35 \\
\hline 7522.03 & 360415.20 & 16.80 & 2235.18 & 85.51 & 167.41 & 15685.42 & 104.65 & 51.31 & 15.52 & 10.18 & 345.58 & 56.33 & 151.01 & 11.39 \\
\hline 7522.10 & 355800.20 & 16.80 & 2591.54 & 96.42 & 154.20 & 17748.92 & 118.28 & 64.94 & 17.23 & 12.00 & 302.02 & 49.75 & 174.40 & 12.81 \\
\hline 7522.17 & 361885.00 & 16.80 & 3044.13 & 118.16 & 157.38 & 19954.00 & 142.66 & 234.58 & 20.26 & 14.50 & 244.10 & 42.54 & 194.01 & 13.73 \\
\hline 7522.23 & 355114.40 & 16.80 & 3195.29 & 124.32 & 147.18 & 19507.43 & 139.07 & 201.68 & 20.77 & 14.61 & 212.76 & 38.01 & 200.20 & 14.05 \\
\hline 7522.30 & 359107.00 & 16.80 & 3221.56 & 130.12 & 149.82 & 20504.01 & 149.89 & 205.84 & 21.85 & 15.41 & 198.32 & 36.26 & 199.24 & 14.84 \\
\hline 7522.36 & 353833.00 & 16.80 & 3295.97 & 128.83 & 150.44 & 20427.37 & 153.25 & 230.00 & 21.04 & 15.50 & 192.11 & 34.71 & 199.61 & 15.81 \\
\hline 7522.43 & 341172.20 & 17.00 & 5945.67 & 127.96 & 146.78 & 18808.69 & 141.47 & 223.85 & 19.37 & 13.92 & 182.28 & 31.89 & 193.02 & 15.91 \\
\hline 7522.49 & 329229.20 & 17.00 & 6023.09 & 121.15 & 147.88 & 17241.27 & 116.85 & 56.87 & 17.68 & 11.61 & 191.65 & 32.09 & 187.32 & 16.23 \\
\hline 7522.56 & 320158.00 & 17.00 & 6034.26 & 117.48 & 141.53 & 15746.18 & 105.15 & 52.57 & 16.35 & 10.63 & 199.25 & 32.60 & 182.45 & 16.92 \\
\hline 7522.62 & 313650.40 & 17.00 & 6111.30 & 109.52 & 145.20 & 14214.56 & 94.73 & 48.91 & 13.85 & 9.01 & 207.92 & 34.25 & 181.82 & 16.67 \\
\hline 7522.69 & 319166.20 & 17.00 & 6077.34 & 106.05 & 161.02 & 15452.40 & 90.54 & 50.97 & 13.12 & 8.66 & 223.56 & 34.24 & 178.02 & 15.58 \\
\hline 7522.76 & 311278.80 & 17.00 & 3524.38 & 103.42 & 160.58 & 14858.99 & 92.21 & 46.36 & 12.94 & 8.66 & 226.06 & 35.78 & 182.38 & 16.43 \\
\hline 7522.82 & 296862.60 & 17.20 & 3517.76 & 101.58 & 149.02 & 13416.19 & 88.75 & 45.79 & 11.44 & 7.83 & 228.57 & 34.83 & 181.35 & 16.83 \\
\hline 7522.89 & 288451.40 & 17.40 & 3663.24 & 104.01 & 150.63 & 13213.31 & 83.92 & 46.60 & 10.83 & 6.89 & 223.27 & 34.46 & 180.77 & 16.11 \\
\hline 7522.95 & 277644.40 & 17.60 & 3676.22 & 103.28 & 148.50 & 12784.10 & 75.32 & 52.77 & 12.24 & 6.58 & 226.65 & 34.52 & 181.46 & 16.83 \\
\hline 7523.02 & 264141.80 & 17.60 & 3601.48 & 100.67 & 143.04 & 9870.59 & 61.32 & 35.34 & 11.18 & 5.20 & 229.72 & 36.49 & 179.03 & 16.87 \\
\hline 7523.08 & 281165.80 & 17.60 & 3168.14 & 87.79 & 173.90 & 8464.61 & 46.93 & 32.93 & 9.45 & 3.87 & 276.81 & 42.44 & 166.38 & 14.80 \\
\hline
\end{tabular}




\begin{tabular}{|c|c|c|c|c|c|c|c|c|c|c|c|c|c|c|}
\hline Depth & XRF & XRF Live Time & $\mathrm{Ti}(\mathrm{ppm})$ & $\mathrm{Cr}(\mathrm{ppm})$ & $\mathrm{Mn}(\mathrm{ppm})$ & $\mathrm{Fe}(\mathrm{ppm})$ & $\mathrm{Ni}(\mathrm{ppm})$ & $\mathrm{Cu}(\mathrm{ppm})$ & $\mathrm{Zn}(\mathrm{ppm})$ & As (ppm) & $\mathrm{Rb}(\mathrm{ppm})$ & $\mathrm{Sr}(\mathrm{ppm})$ & $\mathrm{Y}(\mathrm{ppm})$ & $\mathrm{Zr}(\mathrm{ppm})$ \\
\hline 7523.15 & 306622.80 & 17.40 & 2595.50 & 71.32 & 216.40 & 8248.57 & 38.44 & 40.96 & 8.93 & 3.25 & 326.89 & 49.01 & 150.22 & 12.23 \\
\hline 7523.22 & 328455.00 & 17.20 & 1596.15 & 47.13 & 206.99 & 7344.92 & 32.23 & 36.77 & 8.44 & 2.92 & 403.88 & 45.74 & 119.03 & 10.23 \\
\hline 7523.28 & 351750.60 & 17.00 & 689.83 & 29.05 & 197.44 & 7540.14 & 25.19 & 28.57 & 8.62 & 2.48 & 495.96 & 40.03 & 89.71 & 6.96 \\
\hline 7523.35 & 299810.20 & 17.60 & 1011.39 & 23.06 & 173.44 & 5834.65 & 21.79 & 26.87 & 7.67 & 2.42 & 440.40 & 29.93 & 60.33 & 4.52 \\
\hline 7523.41 & 294957.80 & 17.60 & 2415.18 & 19.01 & 142.97 & 5121.05 & 24.38 & 27.20 & 7.16 & 2.67 & 385.60 & 21.54 & 41.40 & 3.43 \\
\hline 7523.48 & 289829.00 & 17.60 & 2352.86 & 13.54 & 112.85 & 4159.73 & 22.90 & 15.47 & 6.30 & 2.43 & 341.25 & 14.68 & 27.74 & 2.84 \\
\hline 7523.54 & 288008.20 & 17.60 & 2657.09 & 20.12 & 146.84 & 4089.76 & 22.09 & 19.32 & 5.33 & 2.10 & 271.95 & 21.98 & 32.44 & 1.47 \\
\hline 7523.61 & 272150.40 & 17.80 & 3200.13 & 26.16 & 174.07 & 3701.78 & 25.91 & 22.74 & 2.93 & 1.91 & 200.48 & 30.32 & 48.26 & 3.10 \\
\hline 7523.67 & 320758.00 & 17.40 & 2741.49 & 26.98 & 199.43 & 5659.30 & 31.97 & 27.22 & 3.90 & 1.43 & 251.27 & 40.17 & 72.29 & 4.04 \\
\hline 7523.74 & 312916.40 & 17.40 & 1745.80 & 42.64 & 207.69 & 7797.62 & 39.20 & 68.71 & 6.51 & 1.53 & 271.50 & 45.07 & 102.99 & 6.35 \\
\hline 7523.81 & 303121.40 & 17.40 & 1923.13 & 49.70 & 211.10 & 9254.63 & 47.24 & 450.24 & 7.61 & 1.71 & 314.27 & 50.26 & 121.18 & 8.36 \\
\hline 7523.87 & 285518.60 & 17.60 & 2463.11 & 61.96 & 190.20 & 10294.80 & 56.83 & 458.03 & 8.58 & 1.93 & 314.54 & 47.67 & 148.93 & 11.90 \\
\hline 7523.94 & 281837.40 & 17.60 & 2731.21 & 67.38 & 176.79 & 11236.68 & 64.58 & 461.79 & 11.10 & 2.05 & 301.45 & 45.59 & 160.71 & 12.98 \\
\hline 7524.00 & 270327.00 & 17.60 & 2812.41 & 72.59 & 160.25 & 11175.75 & 69.94 & 467.40 & 10.99 & 2.37 & 299.13 & 44.51 & 170.32 & 14.75 \\
\hline 7524.07 & 262943.80 & 17.80 & 2930.99 & 77.78 & 153.87 & 10563.17 & 70.45 & 544.24 & 10.37 & 1.98 & 294.62 & 43.60 & 171.45 & 15.80 \\
\hline 7524.13 & 253311.40 & 18.00 & 3198.09 & 86.89 & 146.59 & 10158.75 & 69.36 & 177.87 & 9.21 & 1.91 & 270.61 & 41.92 & 184.97 & 16.58 \\
\hline 7524.20 & 270268.60 & 17.80 & 2976.05 & 82.77 & 164.30 & 9901.47 & 66.45 & 238.34 & 8.66 & 1.83 & 309.72 & 48.28 & 179.29 & 13.74 \\
\hline 7524.27 & 278652.80 & 17.60 & 2776.39 & 83.55 & 173.56 & 9837.55 & 62.97 & 236.66 & 6.93 & 2.09 & 319.77 & 50.11 & 170.89 & 13.31 \\
\hline 7524.33 & 296503.00 & 17.40 & 3033.29 & 89.10 & 189.16 & 10724.05 & 64.73 & 253.94 & 7.50 & 2.06 & 319.59 & 49.56 & 168.35 & 13.17 \\
\hline 7524.40 & 247085.20 & 17.80 & 3557.03 & 65.49 & 166.49 & 8628.84 & 68.17 & 146.23 & 13.19 & 0.36 & 272.97 & 45.63 & 129.64 & 13.84 \\
\hline 7524.46 & 246990.20 & 17.80 & 6687.87 & 69.22 & 164.13 & 9415.33 & 70.31 & 142.58 & 15.03 & 0.35 & 252.68 & 42.68 & 126.49 & 13.97 \\
\hline 7524.53 & 223119.80 & 18.00 & 6967.76 & 76.29 & 146.71 & 9782.32 & 72.08 & 80.45 & 15.99 & 0.45 & 214.13 & 35.98 & 129.69 & 16.59 \\
\hline 7524.59 & 214728.80 & 18.20 & 7274.97 & 83.76 & 134.68 & 9320.62 & 72.86 & 78.59 & 16.42 & 0.23 & 197.44 & 31.58 & 137.95 & 17.04 \\
\hline 7524.66 & 205009.80 & 18.40 & 7139.76 & 81.48 & 121.65 & 8339.97 & 64.64 & 46.20 & 15.56 & -0.41 & 201.52 & 31.87 & 138.08 & 17.71 \\
\hline 7524.72 & 258076.20 & 17.80 & 6469.66 & 100.64 & 145.33 & 10813.98 & 57.49 & 52.85 & 9.46 & 1.35 & 262.43 & 36.63 & 175.04 & 16.48 \\
\hline 7524.79 & 274047.00 & 17.60 & 3199.55 & 92.68 & 158.76 & 10222.86 & 50.59 & 57.18 & 8.41 & 1.20 & 329.28 & 42.51 & 175.67 & 15.48 \\
\hline 7524.86 & 295467.60 & 17.40 & 3069.85 & 87.76 & 173.56 & 11584.62 & 49.19 & 55.80 & 8.98 & 1.18 & 361.71 & 45.51 & 179.68 & 14.98 \\
\hline 7524.92 & 309757.80 & 17.20 & 2828.28 & 78.77 & 180.00 & 14580.51 & 48.70 & 141.15 & 12.73 & 1.84 & 416.95 & 52.32 & 176.48 & 14.77 \\
\hline 7524.99 & 334450.60 & 17.00 & 2412.10 & 72.50 & 210.92 & 15130.60 & 49.34 & 148.89 & 13.68 & 2.42 & 492.48 & 58.83 & 163.94 & 12.43 \\
\hline 7525.05 & 347738.80 & 17.00 & 2441.07 & 80.93 & 204.21 & 17890.21 & 69.30 & 147.99 & 15.67 & 3.61 & 490.23 & 57.26 & 169.53 & 13.20 \\
\hline 7525.12 & 349834.60 & 17.00 & 2551.32 & 96.46 & 196.54 & 20244.46 & 86.52 & 135.25 & 17.68 & 5.08 & 482.77 & 56.66 & 175.68 & 13.14 \\
\hline 7525.18 & 354836.40 & 17.00 & 1957.87 & 96.50 & 198.63 & 19934.44 & 83.91 & 129.70 & 16.84 & 5.59 & 645.47 & 74.93 & 162.61 & 11.54 \\
\hline 7525.25 & 359978.40 & 17.00 & 1927.06 & 107.03 & 192.96 & 22747.57 & 100.11 & 47.13 & 20.56 & 7.77 & 578.62 & 68.56 & 162.97 & 11.51 \\
\hline
\end{tabular}




\begin{tabular}{|c|c|c|c|c|c|c|c|c|c|c|c|c|c|c|}
\hline Depth & XRF & XRF Live Time & $\mathrm{Ti}(\mathrm{ppm})$ & $\mathrm{Cr}(\mathrm{ppm})$ & $\mathrm{Mn}(\mathrm{ppm})$ & $\mathrm{Fe}(\mathrm{ppm})$ & $\mathrm{Ni}(\mathrm{ppm})$ & $\mathrm{Cu}(\mathrm{ppm})$ & $\mathrm{Zn}(\mathrm{ppm})$ & As (ppm) & $\mathrm{Rb}(\mathrm{ppm})$ & $\mathrm{Sr}(\mathrm{ppm})$ & $\mathrm{Y}(\mathrm{ppm})$ & $\mathrm{Zr}(\mathrm{ppm})$ \\
\hline 7525.31 & 362261.20 & 16.80 & 2049.71 & 122.27 & 157.96 & 27880.52 & 117.08 & 42.33 & 28.33 & 10.53 & 480.48 & 61.84 & 168.50 & 13.51 \\
\hline 7525.38 & 361004.00 & 16.80 & 1760.25 & 118.12 & 147.70 & 29232.66 & 112.81 & 58.31 & 34.21 & 12.53 & 445.96 & 59.05 & 157.54 & 12.43 \\
\hline 7525.45 & 354672.40 & 16.80 & 1676.55 & 112.23 & 133.22 & 32051.46 & 115.98 & 96.92 & 39.96 & 15.20 & 365.89 & 53.17 & 147.72 & 13.95 \\
\hline 7525.51 & 353059.80 & 16.80 & 4400.27 & 120.37 & 109.30 & 34078.72 & 130.54 & 182.94 & 45.35 & 18.24 & 151.12 & 29.80 & 151.48 & 15.12 \\
\hline 7525.58 & 350266.60 & 16.80 & 4246.68 & 116.81 & 103.13 & 31976.88 & 126.22 & 250.88 & 43.36 & 18.56 & 143.01 & 30.03 & 148.36 & 14.26 \\
\hline 7525.64 & 347816.40 & 17.00 & 4170.98 & 110.57 & 100.18 & 29801.80 & 121.46 & 323.94 & 40.88 & 18.33 & 136.88 & 27.20 & 145.31 & 12.97 \\
\hline 7525.71 & 348443.20 & 17.00 & 4080.26 & 104.68 & 103.62 & 28809.11 & 114.99 & 488.15 & 40.03 & 17.85 & 134.46 & 25.70 & 138.63 & 13.16 \\
\hline 7525.77 & 339697.80 & 17.20 & 3832.79 & 99.91 & 101.71 & 26793.45 & 111.74 & 782.58 & 38.47 & 16.77 & 131.46 & 24.98 & 128.96 & 12.50 \\
\hline 7525.84 & 332010.00 & 17.20 & 1259.31 & 90.11 & 102.54 & 25588.22 & 107.83 & 762.37 & 37.42 & 14.97 & 142.18 & 27.91 & 122.59 & 11.60 \\
\hline 7525.91 & 336515.60 & 17.20 & 1252.55 & 86.68 & 98.81 & 24708.81 & 101.43 & 720.68 & 35.93 & 13.66 & 147.51 & 27.59 & 116.81 & 11.42 \\
\hline 7525.97 & 325014.40 & 17.20 & 1329.94 & 84.00 & 104.47 & 24347.66 & 98.43 & 665.47 & 34.53 & 12.91 & 151.14 & 29.11 & 114.63 & 11.80 \\
\hline 7526.04 & 326525.60 & 17.20 & 1412.43 & 83.71 & 102.79 & 24039.48 & 96.46 & 500.82 & 32.97 & 12.48 & 150.89 & 30.64 & 117.37 & 10.87 \\
\hline 7526.10 & 344045.60 & 17.00 & 1519.00 & 85.51 & 104.54 & 24086.09 & 95.59 & 205.57 & 32.69 & 11.63 & 159.36 & 30.81 & 121.95 & 10.49 \\
\hline 7526.17 & 346028.80 & 17.00 & 1663.97 & 89.13 & 106.85 & 25138.51 & 95.31 & 196.38 & 32.45 & 12.09 & 153.61 & 28.88 & 120.12 & 10.80 \\
\hline 7526.23 & 347401.40 & 17.00 & 1691.64 & 87.16 & 108.80 & 25221.90 & 96.75 & 264.43 & 32.47 & 11.98 & 156.05 & 29.10 & 122.72 & 11.40 \\
\hline 7526.30 & 353455.20 & 17.00 & 1662.66 & 82.12 & 106.20 & 24869.42 & 95.09 & 406.80 & 31.99 & 11.71 & 158.24 & 28.53 & 124.34 & 11.44 \\
\hline 7526.36 & 354217.60 & 17.00 & 1631.52 & 76.22 & 107.03 & 25241.46 & 97.97 & 450.29 & 32.54 & 11.83 & 158.13 & 27.58 & 121.12 & 12.05 \\
\hline 7526.43 & 361134.40 & 16.80 & 10744.40 & 70.86 & 108.12 & 24857.92 & 95.55 & 464.07 & 30.98 & 11.80 & 152.29 & 27.43 & 117.26 & 11.71 \\
\hline 7526.50 & 363861.20 & 16.80 & 10788.72 & 70.43 & 107.42 & 24026.64 & 92.89 & 430.13 & 30.28 & 11.36 & 152.52 & 26.48 & 119.36 & 11.94 \\
\hline 7526.56 & 357079.40 & 16.80 & 10870.48 & 74.80 & 111.67 & 24038.30 & 91.70 & 352.20 & 30.39 & 10.81 & 142.59 & 25.86 & 117.22 & 11.35 \\
\hline 7526.63 & 358077.40 & 16.80 & 10950.43 & 81.17 & 111.15 & 24277.18 & 94.99 & 235.19 & 30.81 & 10.97 & 137.18 & 27.07 & 115.87 & 11.22 \\
\hline 7526.69 & 354612.40 & 16.80 & 11047.53 & 86.88 & 113.35 & 23708.63 & 93.35 & 202.11 & 28.11 & 10.71 & 141.91 & 29.30 & 118.39 & 11.73 \\
\hline 7526.76 & 346660.20 & 17.00 & 2068.60 & 89.79 & 121.22 & 24803.19 & 91.88 & 201.17 & 28.81 & 10.89 & 146.34 & 29.41 & 119.96 & 11.32 \\
\hline 7526.82 & 345340.20 & 17.00 & 2126.97 & 89.75 & 123.09 & 25553.20 & 91.03 & 194.00 & 29.06 & 10.84 & 155.05 & 31.28 & 119.79 & 12.82 \\
\hline 7526.89 & 339714.60 & 17.00 & 2081.53 & 87.04 & 120.27 & 25084.10 & 88.22 & 189.36 & 28.49 & 11.72 & 171.46 & 31.76 & 119.37 & 14.59 \\
\hline 7526.96 & 329395.00 & 17.00 & 2055.41 & 78.78 & 124.47 & 24549.34 & 81.92 & 153.37 & 26.44 & 11.68 & 186.33 & 33.35 & 120.28 & 15.29 \\
\hline 7527.02 & 318258.20 & 17.00 & 2008.43 & 72.80 & 124.14 & 22849.89 & 73.84 & 121.31 & 25.37 & 10.27 & 195.15 & 36.17 & 114.47 & 14.17 \\
\hline 7527.09 & 309815.60 & 17.00 & 2046.72 & 71.74 & 123.48 & 22094.16 & 66.79 & 75.75 & 23.83 & 9.59 & 204.69 & 39.22 & 116.61 & 14.62 \\
\hline 7527.15 & 300217.60 & 17.20 & 2096.37 & 69.39 & 131.09 & 21182.98 & 62.89 & 83.76 & 22.96 & 9.22 & 201.32 & 40.75 & 115.93 & 13.91 \\
\hline 7527.22 & 289737.00 & 17.40 & 2177.28 & 69.27 & 134.25 & 21157.77 & 61.51 & 96.23 & 22.58 & 8.05 & 198.87 & 43.97 & 114.38 & 12.51 \\
\hline 7527.28 & 288231.80 & 17.40 & 2269.13 & 71.41 & 140.63 & 21223.86 & 59.92 & 112.54 & 22.95 & 7.88 & 192.29 & 43.87 & 113.55 & 12.58 \\
\hline 7527.35 & 284540.60 & 17.60 & 2381.92 & 75.72 & 148.64 & 22386.34 & 59.31 & 107.69 & 23.91 & 8.78 & 187.00 & 42.65 & 114.12 & 13.09 \\
\hline 7527.41 & 274738.20 & 17.80 & 2585.32 & 78.05 & 147.83 & 22059.13 & 62.23 & 102.10 & 24.96 & 8.96 & 179.69 & 41.09 & 112.76 & 12.36 \\
\hline
\end{tabular}




\begin{tabular}{|c|c|c|c|c|c|c|c|c|c|c|c|c|c|c|}
\hline Depth & XRF & XRF Live Time & $\mathrm{Ti}(\mathrm{ppm})$ & $\mathrm{Cr}(\mathrm{ppm})$ & $\mathrm{Mn}(\mathrm{ppm})$ & $\mathrm{Fe}(\mathrm{ppm})$ & $\mathrm{Ni}(\mathrm{ppm})$ & $\mathrm{Cu}(\mathrm{ppm})$ & $\mathrm{Zn}(\mathrm{ppm})$ & As (ppm) & $\mathrm{Rb}(\mathrm{ppm})$ & $\mathrm{Sr}(\mathrm{ppm})$ & $\mathrm{Y}(\mathrm{ppm})$ & $\mathrm{Zr}(\mathrm{ppm})$ \\
\hline 7527.48 & 225187.40 & 18.20 & 3168.20 & 61.16 & 121.08 & 18210.40 & 64.33 & 83.63 & 21.88 & 10.20 & 146.43 & 31.16 & 94.14 & 10.92 \\
\hline 7527.55 & 230518.60 & 18.00 & 3275.26 & 63.09 & 121.47 & 18743.74 & 65.09 & 53.56 & 22.91 & 11.22 & 139.40 & 27.33 & 96.56 & 11.19 \\
\hline 7527.61 & 226857.00 & 18.20 & 3272.43 & 67.29 & 112.33 & 18934.66 & 62.93 & 25.14 & 23.12 & 10.96 & 135.10 & 24.94 & 99.32 & 11.02 \\
\hline 7527.68 & 232256.20 & 18.00 & 3357.14 & 72.82 & 104.61 & 20613.43 & 72.89 & 21.18 & 26.17 & 11.05 & 122.20 & 21.84 & 108.20 & 12.45 \\
\hline 7527.74 & 235657.00 & 18.00 & 3391.51 & 76.41 & 105.37 & 23266.95 & 82.29 & 22.01 & 28.94 & 10.83 & 113.76 & 19.39 & 109.37 & 12.82 \\
\hline 7527.81 & 305760.60 & 17.40 & 3006.32 & 96.67 & 125.13 & 30521.95 & 90.13 & 18.15 & 38.00 & 10.22 & 135.67 & 25.51 & 133.90 & 13.67 \\
\hline 7527.87 & 325592.80 & 17.40 & 3195.16 & 98.63 & 125.30 & 34356.26 & 95.25 & 19.07 & 42.38 & 10.10 & 132.09 & 24.45 & 136.78 & 12.82 \\
\hline 7527.94 & 333159.20 & 17.20 & 3351.18 & 99.23 & 125.33 & 35567.53 & 102.68 & 19.79 & 45.77 & 10.37 & 131.28 & 24.14 & 139.92 & 12.47 \\
\hline 7528.01 & 334959.20 & 17.20 & 3423.24 & 93.66 & 129.68 & 36401.46 & 99.84 & 20.07 & 47.33 & 10.14 & 141.07 & 24.88 & 138.34 & 11.64 \\
\hline 7528.07 & 346788.40 & 17.00 & 3318.61 & 89.06 & 129.09 & 35598.94 & 95.36 & 18.93 & 48.39 & 10.30 & 149.47 & 27.03 & 139.10 & 12.47 \\
\hline 7528.14 & 328176.00 & 17.00 & 3244.93 & 88.76 & 123.06 & 33144.17 & 87.84 & 17.36 & 44.25 & 9.92 & 159.23 & 29.55 & 140.98 & 12.20 \\
\hline 7528.20 & 309262.00 & 17.00 & 3067.80 & 86.50 & 120.98 & 30028.85 & 84.99 & 17.09 & 39.19 & 9.19 & 166.47 & 32.02 & 140.93 & 13.41 \\
\hline 7528.27 & 306562.00 & 17.00 & 2901.83 & 81.04 & 124.58 & 31844.79 & 84.52 & 16.16 & 39.65 & 9.39 & 167.22 & 33.12 & 135.41 & 13.94 \\
\hline 7528.33 & 297778.40 & 17.20 & 2848.76 & 81.65 & 121.11 & 30544.37 & 82.87 & 15.68 & 35.69 & 9.16 & 167.11 & 33.65 & 132.80 & 13.69 \\
\hline 7528.40 & 278591.00 & 17.40 & 2847.88 & 85.01 & 117.44 & 30245.71 & 80.07 & 15.50 & 31.87 & 9.11 & 161.88 & 34.65 & 131.30 & 13.04 \\
\hline 7528.46 & 267684.20 & 17.60 & 4006.82 & 88.14 & 118.75 & 31366.07 & 83.76 & 17.26 & 32.39 & 9.51 & 155.55 & 32.61 & 130.82 & 12.97 \\
\hline 7528.53 & 253857.40 & 17.80 & 4052.41 & 86.28 & 123.30 & 32369.41 & 84.46 & 18.79 & 33.96 & 9.74 & 149.56 & 30.16 & 128.16 & 11.50 \\
\hline 7528.60 & 235162.80 & 18.00 & 4020.99 & 87.23 & 122.21 & 32062.80 & 83.03 & 20.65 & 32.33 & 9.60 & 150.95 & 28.07 & 125.99 & 10.78 \\
\hline 7528.66 & 245286.40 & 17.80 & 4040.46 & 89.55 & 118.85 & 32649.97 & 102.85 & 32.94 & 30.20 & 14.65 & 149.04 & 48.08 & 104.23 & 36.57 \\
\hline 7528.73 & 258965.20 & 17.60 & 4032.54 & 87.47 & 120.95 & 33506.15 & 108.78 & 33.05 & 31.34 & 14.79 & 148.74 & 45.67 & 103.40 & 36.49 \\
\hline 7528.79 & 272376.40 & 17.40 & 2966.62 & 88.57 & 123.51 & 33122.78 & 108.75 & 31.96 & 30.98 & 14.30 & 146.71 & 46.51 & 101.86 & 37.57 \\
\hline 7528.86 & 294502.80 & 17.20 & 2346.65 & 73.51 & 111.95 & 34953.26 & 104.42 & 30.37 & 30.08 & 14.41 & 304.30 & 46.35 & 101.63 & 37.95 \\
\hline 7528.92 & 344748.40 & 16.80 & 749.03 & 8.30 & 65.53 & 31657.66 & 83.12 & 22.37 & 24.12 & 10.80 & 891.19 & 40.98 & 87.33 & 36.43 \\
\hline 7528.99 & 361492.00 & 16.60 & 589.59 & 6.86 & 64.57 & 30367.45 & 61.90 & 9.78 & 25.58 & 5.64 & 921.76 & 20.72 & 116.12 & 10.35 \\
\hline 7529.06 & 369581.20 & 16.60 & -467.81 & -6.78 & 48.44 & 27182.40 & 58.13 & 16.48 & 20.09 & 7.91 & 915.36 & 203.42 & 97.65 & 28.06 \\
\hline 7529.12 & 370559.40 & 16.60 & -721.44 & -11.40 & 48.54 & 24863.41 & 52.73 & 15.85 & 16.77 & 7.17 & 927.82 & 203.70 & 92.50 & 27.13 \\
\hline 7529.19 & 363414.00 & 16.60 & -569.33 & 4.16 & 50.51 & 20260.94 & 51.93 & 14.65 & 15.37 & 6.72 & 809.14 & 204.80 & 92.01 & 27.94 \\
\hline 7529.25 & 333015.20 & 16.80 & 1070.85 & 71.85 & 94.19 & 20751.33 & 72.66 & 20.12 & 19.59 & 9.47 & 229.39 & 211.47 & 107.70 & 29.46 \\
\hline 7529.32 & 311740.60 & 17.00 & 1117.77 & 76.63 & 94.62 & 20481.39 & 71.75 & 18.99 & 19.04 & 9.39 & 219.33 & 211.87 & 101.99 & 30.57 \\
\hline 7529.38 & 298802.60 & 17.00 & 2084.12 & 89.47 & 105.78 & 21040.27 & 69.45 & 12.56 & 22.27 & 6.58 & 233.50 & 29.92 & 120.71 & 13.02 \\
\hline 7529.45 & 288980.00 & 17.20 & 3562.27 & 92.55 & 100.42 & 22855.97 & 76.81 & 13.85 & 23.80 & 7.34 & 225.91 & 29.80 & 124.52 & 14.15 \\
\hline 7529.51 & 266698.40 & 17.40 & 3914.35 & 97.23 & 99.64 & 24266.13 & 77.98 & 17.94 & 23.59 & 7.28 & 195.07 & 28.38 & 125.90 & 14.80 \\
\hline 7529.58 & 298364.00 & 17.20 & 3428.78 & 99.81 & 107.77 & 48279.76 & 77.18 & 19.80 & 28.84 & 7.65 & 302.02 & 26.23 & 120.10 & 15.92 \\
\hline
\end{tabular}




\begin{tabular}{|c|c|c|c|c|c|c|c|c|c|c|c|c|c|c|}
\hline Depth & XRF & XRF Live Time & $\mathrm{Ti}(\mathrm{ppm})$ & $\mathrm{Cr}(\mathrm{ppm})$ & $\mathrm{Mn}(\mathrm{ppm})$ & $\mathrm{Fe}(\mathrm{ppm})$ & $\mathrm{Ni}(\mathrm{ppm})$ & $\mathrm{Cu}(\mathrm{ppm})$ & $\mathrm{Zn}(\mathrm{ppm})$ & As (ppm) & $\mathrm{Rb}(\mathrm{ppm})$ & $\mathrm{Sr}(\mathrm{ppm})$ & $\mathrm{Y}(\mathrm{ppm})$ & $\mathrm{Zr}(\mathrm{ppm})$ \\
\hline 7529.65 & 289353.60 & 17.40 & 3335.29 & 94.29 & 110.77 & 47997.89 & 78.77 & 20.30 & 28.93 & 8.18 & 293.58 & 25.95 & 119.65 & 14.98 \\
\hline 7529.71 & 280817.80 & 17.60 & 3044.73 & 92.64 & 111.75 & 48023.08 & 74.64 & 18.58 & 28.08 & 7.27 & 367.45 & 23.88 & 114.98 & 14.32 \\
\hline 7529.78 & 270646.00 & 17.60 & 1501.83 & 86.16 & 113.07 & 46422.79 & 66.76 & 16.07 & 27.47 & 6.81 & 386.99 & 23.65 & 111.76 & 13.61 \\
\hline 7529.84 & 273084.40 & 17.60 & 1354.65 & 93.28 & 126.02 & 45248.38 & 69.46 & 14.79 & 27.95 & 6.50 & 436.11 & 26.74 & 109.49 & 12.66 \\
\hline 7529.91 & 214459.40 & 18.20 & 1502.65 & 108.11 & 130.00 & 20599.24 & 70.04 & 13.46 & 22.29 & 6.74 & 408.87 & 30.18 & 114.40 & 11.69 \\
\hline 7529.97 & 201716.20 & 18.20 & 922.16 & 129.50 & 137.94 & 19280.34 & 65.73 & 13.67 & 21.20 & 5.88 & 558.07 & 30.58 & 107.69 & 10.85 \\
\hline 7530.04 & 194351.80 & 18.20 & 1149.68 & 134.63 & 140.37 & 18585.44 & 67.57 & 14.39 & 21.73 & 6.14 & 505.91 & 32.45 & 113.88 & 12.28 \\
\hline 7530.10 & 194248.40 & 18.20 & 1253.05 & 141.64 & 141.96 & 19454.58 & 70.22 & 16.59 & 22.71 & 6.33 & 497.56 & 30.34 & 116.78 & 11.92 \\
\hline 7530.17 & 206105.40 & 18.20 & 212.03 & 109.48 & 120.40 & 27607.18 & 54.34 & 20.25 & 20.38 & 5.05 & 839.29 & 26.98 & 116.56 & 8.93 \\
\hline 7530.24 & 211296.20 & 18.00 & 476.76 & 87.45 & 105.23 & 27456.97 & 49.47 & 20.03 & 20.25 & 4.21 & 768.31 & 24.58 & 116.55 & 8.03 \\
\hline 7530.30 & 209001.60 & 18.00 & 1023.44 & 61.52 & 96.23 & 27569.59 & 49.51 & 21.00 & 20.85 & 3.74 & 623.33 & 23.30 & 115.52 & 8.26 \\
\hline 7530.37 & 214012.00 & 18.00 & 1066.46 & 61.13 & 95.18 & 27689.17 & 48.61 & 22.12 & 21.75 & 3.94 & 608.37 & 23.29 & 111.36 & 7.13 \\
\hline 7530.43 & 191441.80 & 18.20 & 748.65 & 66.39 & 112.08 & 27768.14 & 59.19 & 37.06 & 22.47 & 6.48 & 589.47 & 94.60 & 92.70 & 24.38 \\
\hline 7530.50 & 187195.20 & 18.20 & 1844.69 & 85.83 & 126.77 & 19544.20 & 69.37 & 32.18 & 25.36 & 7.95 & 205.63 & 94.67 & 90.80 & 27.16 \\
\hline 7530.56 & 173658.00 & 18.40 & 1802.43 & 96.76 & 136.52 & 19681.97 & 71.16 & 33.70 & 24.96 & 8.17 & 241.60 & 93.96 & 87.85 & 27.94 \\
\hline 7530.63 & 144370.20 & 18.80 & 2330.46 & 108.02 & 135.09 & 16480.20 & 76.18 & 37.12 & 20.38 & 7.65 & 213.06 & 91.18 & 73.03 & 27.08 \\
\hline 7530.70 & 149544.80 & 18.80 & 1883.47 & 98.70 & 127.24 & 17528.68 & 69.02 & 35.21 & 18.34 & 7.18 & 279.94 & 91.67 & 72.81 & 26.76 \\
\hline 7530.76 & 163928.20 & 18.80 & 1965.63 & 85.63 & 117.93 & 15936.95 & 54.20 & 18.47 & 15.28 & 3.83 & 322.41 & 26.07 & 81.95 & 7.83 \\
\hline 7530.83 & 177278.80 & 18.60 & 849.69 & 63.55 & 115.29 & 22027.81 & 49.35 & 15.74 & 12.49 & 2.84 & 697.32 & 29.92 & 70.83 & 5.61 \\
\hline 7530.89 & 182110.80 & 18.60 & 718.73 & 47.36 & 116.20 & 20076.78 & 46.94 & 14.31 & 10.26 & 2.62 & 656.09 & 35.52 & 61.71 & 4.26 \\
\hline 7530.96 & 222044.40 & 18.20 & -266.30 & 37.07 & 122.29 & 22495.94 & 37.49 & 8.41 & 13.87 & 3.72 & 810.87 & 46.32 & 72.37 & 5.12 \\
\hline 7531.02 & 220969.20 & 18.20 & -102.70 & 36.13 & 141.03 & 19424.89 & 35.49 & 7.86 & 12.81 & 3.79 & 744.56 & 50.77 & 62.10 & 4.10 \\
\hline 7531.09 & 234642.00 & 18.00 & -46.62 & 34.53 & 140.14 & 18749.14 & 32.45 & 7.84 & 13.32 & 3.64 & 718.91 & 50.49 & 59.51 & 5.88 \\
\hline 7531.15 & 214354.20 & 18.20 & 988.56 & 49.61 & 142.69 & 11699.90 & 37.29 & 9.89 & 14.38 & 4.49 & 340.11 & 49.03 & 69.56 & 7.50 \\
\hline 7531.22 & 248873.00 & 17.80 & 1202.04 & 58.71 & 141.20 & 13477.27 & 42.16 & 10.77 & 17.35 & 4.77 & 343.22 & 47.09 & 81.91 & 8.69 \\
\hline 7531.29 & 272362.00 & 17.60 & 1777.77 & 57.01 & 139.70 & 15139.79 & 47.93 & 11.84 & 18.71 & 4.89 & 211.67 & 41.14 & 91.10 & 9.07 \\
\hline 7531.35 & 293439.20 & 17.40 & 2016.98 & 60.96 & 131.55 & 17368.37 & 58.52 & 15.72 & 20.99 & 5.54 & 216.42 & 38.59 & 103.59 & 10.45 \\
\hline 7531.42 & 315644.00 & 17.20 & 2208.68 & 60.23 & 125.07 & 19411.75 & 68.34 & 15.88 & 21.94 & 6.66 & 215.93 & 34.55 & 115.47 & 11.33 \\
\hline 7531.48 & 373917.40 & 16.60 & 1747.06 & 48.73 & 117.98 & 32445.16 & 69.97 & 19.39 & 25.16 & 6.41 & 334.20 & 33.64 & 112.83 & 12.07 \\
\hline 7531.55 & 381678.80 & 16.60 & 6896.40 & 48.46 & 114.80 & 32909.35 & 67.07 & 23.96 & 24.33 & 6.15 & 328.99 & 32.82 & 116.64 & 13.12 \\
\hline 7531.61 & 380112.80 & 16.60 & 6901.24 & 52.01 & 112.93 & 32882.89 & 67.79 & 24.23 & 23.43 & 5.97 & 328.73 & 33.10 & 118.48 & 13.74 \\
\hline 7531.68 & 376661.40 & 16.60 & 6971.02 & 53.98 & 115.54 & 33048.49 & 67.46 & 21.96 & 23.46 & 5.96 & 324.74 & 32.13 & 117.77 & 13.94 \\
\hline 7531.75 & 373759.20 & 16.60 & 6947.39 & 58.25 & 120.39 & 32926.38 & 64.49 & 22.38 & 23.36 & 5.59 & 327.43 & 34.15 & 115.04 & 13.09 \\
\hline
\end{tabular}




\begin{tabular}{|c|c|c|c|c|c|c|c|c|c|c|c|c|c|c|}
\hline Depth & XRF & XRF Live Time & $\mathrm{Ti}(\mathrm{ppm})$ & $\mathrm{Cr}(\mathrm{ppm})$ & $\mathrm{Mn}(\mathrm{ppm})$ & $\mathrm{Fe}(\mathrm{ppm})$ & $\mathrm{Ni}(\mathrm{ppm})$ & $\mathrm{Cu}(\mathrm{ppm})$ & $\mathrm{Zn}(\mathrm{ppm})$ & As (ppm) & $\mathrm{Rb}(\mathrm{ppm})$ & $\mathrm{Sr}(\mathrm{ppm})$ & $\mathrm{Y}(\mathrm{ppm})$ & $\mathrm{Zr}(\mathrm{ppm})$ \\
\hline 7531.81 & 337033.40 & 17.00 & 7485.66 & 74.04 & 126.26 & 21077.01 & 68.59 & 23.38 & 20.74 & 6.07 & 214.66 & 36.17 & 121.06 & 12.80 \\
\hline 7531.88 & 336462.40 & 17.00 & 2274.35 & 71.30 & 127.97 & 21249.98 & 72.38 & 18.76 & 20.72 & 6.65 & 221.30 & 35.93 & 117.22 & 11.34 \\
\hline 7531.94 & 343906.00 & 17.00 & 2234.21 & 71.87 & 136.45 & 21458.87 & 75.13 & 20.11 & 22.56 & 7.13 & 225.22 & 37.94 & 115.20 & 11.13 \\
\hline 7532.01 & 342897.80 & 17.00 & 2025.73 & 68.92 & 130.11 & 20926.24 & 75.04 & 19.95 & 22.80 & 7.33 & 229.91 & 38.33 & 116.16 & 10.54 \\
\hline 7532.07 & 346952.60 & 17.00 & 2046.78 & 68.90 & 132.41 & 20947.45 & 77.78 & 20.22 & 22.77 & 7.30 & 234.34 & 40.31 & 115.06 & 10.52 \\
\hline 7532.14 & 346850.60 & 17.00 & 1960.01 & 65.06 & 136.20 & 20974.91 & 77.64 & 17.48 & 23.53 & 7.44 & 238.26 & 41.15 & 114.01 & 10.78 \\
\hline 7532.20 & 333252.00 & 17.00 & 1794.83 & 60.26 & 134.01 & 19905.91 & 75.39 & 16.97 & 22.84 & 7.13 & 246.01 & 42.26 & 111.92 & 11.04 \\
\hline 7532.27 & 319622.20 & 17.00 & 1818.64 & 59.51 & 126.88 & 20059.23 & 74.88 & 16.32 & 21.77 & 7.34 & 243.17 & 40.75 & 111.89 & 10.87 \\
\hline 7532.34 & 329567.60 & 17.00 & 1951.06 & 63.53 & 133.20 & 20829.41 & 75.81 & 17.23 & 22.58 & 7.71 & 245.55 & 43.14 & 110.49 & 12.26 \\
\hline 7532.40 & 333378.40 & 17.00 & 1900.57 & 64.52 & 132.18 & 21068.68 & 73.23 & 17.02 & 23.29 & 7.95 & 249.96 & 41.74 & 111.68 & 12.78 \\
\hline 7532.47 & 346323.00 & 17.00 & 2071.53 & 71.24 & 134.39 & 21563.56 & 73.18 & 24.12 & 23.44 & 8.26 & 245.22 & 40.92 & 111.67 & 12.63 \\
\hline 7532.53 & 359274.40 & 17.00 & 3073.63 & 80.37 & 131.63 & 22960.18 & 75.89 & 61.34 & 25.42 & 8.08 & 228.64 & 41.15 & 116.29 & 12.19 \\
\hline 7532.60 & 367305.40 & 17.00 & 3041.87 & 81.53 & 129.91 & 23363.93 & 78.97 & 91.32 & 27.47 & 8.42 & 227.13 & 39.39 & 114.62 & 12.17 \\
\hline 7532.66 & 364458.40 & 17.00 & 3051.44 & 82.55 & 135.38 & 23608.84 & 83.13 & 158.16 & 27.79 & 8.25 & 229.05 & 39.22 & 114.88 & 12.52 \\
\hline 7532.73 & 359112.00 & 17.00 & 3005.64 & 81.41 & 139.87 & 22927.97 & 84.54 & 162.80 & 26.59 & 8.11 & 227.80 & 43.01 & 109.78 & 11.08 \\
\hline 7532.80 & 357448.00 & 17.00 & 2700.28 & 72.67 & 145.24 & 20750.95 & 76.00 & 157.19 & 23.77 & 7.09 & 235.36 & 52.27 & 98.47 & 10.05 \\
\hline 7532.86 & 361993.60 & 16.80 & 1676.79 & 63.59 & 157.43 & 19521.05 & 69.39 & 130.94 & 22.06 & 6.77 & 252.88 & 61.56 & 86.70 & 9.61 \\
\hline 7532.93 & 362654.20 & 16.80 & 1508.98 & 59.44 & 167.74 & 17986.22 & 62.61 & 130.88 & 19.53 & 5.51 & 267.63 & 72.60 & 81.42 & 8.02 \\
\hline 7532.99 & 357139.00 & 16.80 & 1358.55 & 51.78 & 167.08 & 16462.14 & 54.20 & 86.61 & 17.26 & 4.90 & 269.13 & 78.94 & 75.46 & 6.14 \\
\hline 7533.06 & 356607.20 & 16.80 & 1385.62 & 50.64 & 170.23 & 16563.71 & 52.88 & 95.85 & 18.08 & 5.09 & 267.49 & 79.47 & 79.35 & 5.52 \\
\hline 7533.12 & 339986.00 & 17.00 & 1554.85 & 56.33 & 166.11 & 18532.96 & 61.38 & 119.10 & 20.54 & 5.73 & 265.59 & 72.71 & 88.79 & 6.12 \\
\hline 7533.19 & 328437.40 & 17.20 & 1781.88 & 61.29 & 161.09 & 19102.78 & 67.96 & 124.77 & 21.64 & 6.40 & 256.38 & 65.42 & 96.16 & 7.42 \\
\hline 7533.25 & 321068.80 & 17.20 & 1945.59 & 63.27 & 157.85 & 19797.31 & 68.94 & 106.69 & 22.38 & 6.82 & 253.09 & 57.46 & 102.10 & 9.37 \\
\hline 7533.32 & 312388.60 & 17.20 & 2188.86 & 66.89 & 157.07 & 21238.92 & 71.93 & 83.80 & 23.38 & 7.46 & 244.94 & 52.30 & 105.97 & 10.17 \\
\hline 7533.39 & 319803.80 & 17.20 & 2286.57 & 69.44 & 150.19 & 22166.53 & 73.95 & 85.63 & 24.08 & 7.88 & 241.64 & 49.52 & 106.69 & 11.97 \\
\hline 7533.45 & 324792.00 & 17.00 & 2406.80 & 70.75 & 146.35 & 22126.86 & 71.88 & 72.06 & 24.55 & 7.68 & 241.53 & 49.21 & 108.77 & 11.36 \\
\hline 7533.52 & 325318.00 & 17.00 & 2714.48 & 72.54 & 147.40 & 22429.17 & 69.56 & 65.05 & 25.60 & 7.90 & 245.38 & 46.80 & 107.64 & 11.41 \\
\hline 7533.58 & 317303.20 & 17.20 & 2591.93 & 68.97 & 137.19 & 22247.74 & 70.43 & 65.56 & 25.46 & 8.31 & 230.98 & 42.14 & 108.33 & 10.98 \\
\hline 7533.65 & 304262.60 & 17.40 & 2350.90 & 63.43 & 120.14 & 21194.70 & 68.75 & 68.64 & 25.76 & 7.88 & 219.96 & 36.42 & 109.48 & 11.40 \\
\hline 7533.71 & 235103.60 & 18.00 & 2971.40 & 64.22 & 122.36 & 16514.83 & 67.58 & 55.65 & 19.77 & 5.86 & 175.42 & 25.07 & 94.77 & 11.25 \\
\hline 7533.78 & 227587.20 & 18.20 & 3027.17 & 70.07 & 120.90 & 17058.56 & 83.23 & 53.71 & 17.26 & 9.61 & 152.93 & 43.96 & 76.78 & 34.41 \\
\hline 7533.85 & 204753.80 & 18.40 & 2860.29 & 74.07 & 112.09 & 18458.85 & 84.65 & 44.45 & 17.24 & 9.85 & 129.66 & 41.14 & 78.72 & 34.58 \\
\hline 7533.91 & 212317.40 & 18.20 & 3011.00 & 81.08 & 115.05 & 19827.08 & 89.11 & 32.16 & 19.08 & 9.99 & 117.34 & 39.81 & 80.43 & 33.86 \\
\hline
\end{tabular}




\begin{tabular}{|c|c|c|c|c|c|c|c|c|c|c|c|c|c|c|}
\hline Depth & XRF & XRF Live Time & $\mathrm{Ti}(\mathrm{ppm})$ & $\mathrm{Cr}(\mathrm{ppm})$ & $\mathrm{Mn}(\mathrm{ppm})$ & $\mathrm{Fe}(\mathrm{ppm})$ & $\mathrm{Ni}(\mathrm{ppm})$ & $\mathrm{Cu}(\mathrm{ppm})$ & $\mathrm{Zn}(\mathrm{ppm})$ & As (ppm) & $\mathrm{Rb}(\mathrm{ppm})$ & $\mathrm{Sr}(\mathrm{ppm})$ & $\mathrm{Y}(\mathrm{ppm})$ & $\mathrm{Zr}(\mathrm{ppm})$ \\
\hline 7533.98 & 227008.40 & 18.00 & 3222.10 & 91.81 & 121.91 & 21465.53 & 97.22 & 35.91 & 19.26 & 10.39 & 109.38 & 38.58 & 79.82 & 33.05 \\
\hline 7534.04 & 280719.60 & 17.40 & 2557.54 & 93.25 & 114.85 & 26893.95 & 105.45 & 69.27 & 25.22 & 12.04 & 130.22 & 45.43 & 96.36 & 33.22 \\
\hline 7534.11 & 306261.80 & 17.00 & 2663.49 & 91.72 & 117.16 & 28742.74 & 97.01 & 195.07 & 28.60 & 9.00 & 129.79 & 19.73 & 119.84 & 11.80 \\
\hline 7534.17 & 329013.60 & 16.80 & 2722.56 & 88.35 & 120.35 & 28434.72 & 102.46 & 226.10 & 28.48 & 9.08 & 126.13 & 20.51 & 124.78 & 12.53 \\
\hline 7534.24 & 309165.00 & 17.00 & 2593.86 & 84.71 & 114.92 & 27938.13 & 101.58 & 340.70 & 27.83 & 9.43 & 125.47 & 21.09 & 123.72 & 13.10 \\
\hline 7534.30 & 315707.60 & 17.00 & 2538.00 & 80.90 & 117.11 & 27712.28 & 100.92 & 403.60 & 27.94 & 9.44 & 122.69 & 20.98 & 126.70 & 12.73 \\
\hline 7534.37 & 313892.60 & 17.00 & 2677.01 & 79.67 & 114.28 & 27271.37 & 95.19 & 376.73 & 28.00 & 9.15 & 123.45 & 20.78 & 126.72 & 11.87 \\
\hline 7534.44 & 302063.00 & 17.20 & 2579.40 & 74.52 & 109.35 & 25477.40 & 91.53 & 247.38 & 27.14 & 8.84 & 121.01 & 22.78 & 126.03 & 12.16 \\
\hline 7534.50 & 290486.60 & 17.40 & 2147.77 & 68.67 & 100.00 & 23445.85 & 84.96 & 227.83 & 25.48 & 8.45 & 135.88 & 23.92 & 120.80 & 11.62 \\
\hline 7534.57 & 252841.80 & 17.80 & 3296.72 & 50.96 & 91.34 & 18653.72 & 90.80 & 119.25 & 23.35 & 7.89 & 117.32 & 20.03 & 99.01 & 11.02 \\
\hline 7534.63 & 236486.00 & 18.00 & 3317.58 & 43.37 & 77.31 & 17802.67 & 88.81 & 81.71 & 23.22 & 8.03 & 115.17 & 21.47 & 96.09 & 10.87 \\
\hline 7534.70 & 233564.60 & 18.20 & 3097.33 & 42.33 & 69.34 & 17890.42 & 93.92 & 138.26 & 23.71 & 8.58 & 115.72 & 20.28 & 94.71 & 11.21 \\
\hline 7534.76 & 239083.20 & 18.20 & 2941.99 & 45.56 & 64.59 & 18669.87 & 101.76 & 187.19 & 24.74 & 8.96 & 116.44 & 21.09 & 90.64 & 10.52 \\
\hline 7534.83 & 254482.40 & 18.00 & 3235.85 & 54.36 & 67.03 & 20740.84 & 109.31 & 199.63 & 26.67 & 8.55 & 104.15 & 20.71 & 94.79 & 10.04 \\
\hline 7534.90 & 297712.20 & 17.60 & 2240.89 & 71.62 & 77.06 & 25719.01 & 105.50 & 261.23 & 29.15 & 8.07 & 118.84 & 25.50 & 119.09 & 12.16 \\
\hline 7534.96 & 301579.00 & 17.60 & 2222.27 & 74.55 & 83.92 & 25972.52 & 102.94 & 246.18 & 29.83 & 7.86 & 123.77 & 24.98 & 123.57 & 13.53 \\
\hline 7535.03 & 309471.40 & 17.40 & 2366.20 & 75.46 & 90.88 & 25259.66 & 99.48 & 201.13 & 29.70 & 7.31 & 124.93 & 27.89 & 124.83 & 14.23 \\
\hline 7535.09 & 306697.80 & 17.40 & 2487.95 & 76.00 & 90.43 & 24232.35 & 92.35 & 154.81 & 28.49 & 6.87 & 126.09 & 28.49 & 127.53 & 14.29 \\
\hline 7535.16 & 312885.00 & 17.40 & 2529.05 & 75.53 & 94.54 & 23788.26 & 89.12 & 136.42 & 28.62 & 7.61 & 128.85 & 27.40 & 127.67 & 13.88 \\
\hline 7535.22 & 340656.40 & 17.20 & 2493.88 & 80.62 & 89.99 & 23971.49 & 90.64 & 100.13 & 28.54 & 8.38 & 137.52 & 29.46 & 127.21 & 12.20 \\
\hline 7535.29 & 348292.20 & 17.00 & 2298.69 & 76.52 & 87.26 & 23638.76 & 93.73 & 97.30 & 27.53 & 8.85 & 148.47 & 30.51 & 122.79 & 11.94 \\
\hline 7535.35 & 362589.40 & 16.80 & 2171.85 & 71.62 & 83.90 & 27268.71 & 99.72 & 84.73 & 29.05 & 9.36 & 150.46 & 31.77 & 122.29 & 12.07 \\
\hline 7535.42 & 363657.40 & 16.80 & 2080.95 & 70.76 & 88.12 & 28470.78 & 104.70 & 131.48 & 31.30 & 9.92 & 149.46 & 30.83 & 121.71 & 10.97 \\
\hline 7535.49 & 355970.60 & 16.80 & 1924.96 & 68.31 & 86.02 & 28498.31 & 107.97 & 166.64 & 31.09 & 9.61 & 157.35 & 33.82 & 118.41 & 11.04 \\
\hline 7535.55 & 345417.40 & 16.80 & 1880.02 & 63.99 & 91.92 & 28370.24 & 107.95 & 168.94 & 30.97 & 9.52 & 164.13 & 35.55 & 114.31 & 10.47 \\
\hline 7535.62 & 355632.80 & 16.80 & 2011.84 & 73.14 & 96.47 & 28995.18 & 106.02 & 177.92 & 32.02 & 9.15 & 164.57 & 37.02 & 114.13 & 11.03 \\
\hline 7535.68 & 349723.20 & 17.00 & 2185.07 & 79.25 & 95.43 & 25574.47 & 98.67 & 214.21 & 30.05 & 8.95 & 173.48 & 37.39 & 113.25 & 10.59 \\
\hline 7535.75 & 349378.20 & 17.00 & 2231.00 & 81.97 & 93.62 & 24764.75 & 98.33 & 239.30 & 28.37 & 9.30 & 178.64 & 38.65 & 111.23 & 12.10 \\
\hline 7535.81 & 340231.60 & 17.00 & 2219.34 & 82.71 & 88.09 & 24488.78 & 109.58 & 214.90 & 91.98 & 13.01 & 167.70 & 60.38 & 95.78 & 32.44 \\
\hline 7535.88 & 348742.60 & 17.00 & 2258.32 & 85.84 & 88.73 & 25134.98 & 110.93 & 261.66 & 92.83 & 13.12 & 163.65 & 57.94 & 96.78 & 32.55 \\
\hline 7535.94 & 345858.60 & 17.00 & 2291.27 & 84.32 & 88.59 & 25162.86 & 114.18 & 274.08 & 93.10 & 13.21 & 155.90 & 57.42 & 98.16 & 32.35 \\
\hline 7536.01 & 344694.80 & 17.00 & 2129.71 & 78.35 & 90.17 & 25639.19 & 115.30 & 253.44 & 94.48 & 13.48 & 145.92 & 55.84 & 101.95 & 31.95 \\
\hline 7536.08 & 330388.60 & 17.20 & 2041.53 & 76.21 & 88.22 & 25803.02 & 111.74 & 209.09 & 94.87 & 13.02 & 149.49 & 56.44 & 103.44 & 31.69 \\
\hline
\end{tabular}




\begin{tabular}{|c|c|c|c|c|c|c|c|c|c|c|c|c|c|c|}
\hline Depth & XRF & XRF Live Time & $\mathrm{Ti}(\mathrm{ppm})$ & $\mathrm{Cr}(\mathrm{ppm})$ & $\mathrm{Mn}(\mathrm{ppm})$ & $\mathrm{Fe}(\mathrm{ppm})$ & $\mathrm{Ni}(\mathrm{ppm})$ & $\mathrm{Cu}(\mathrm{ppm})$ & $\mathrm{Zn}(\mathrm{ppm})$ & As (ppm) & $\mathrm{Rb}(\mathrm{ppm})$ & $\mathrm{Sr}(\mathrm{ppm})$ & $\mathrm{Y}(\mathrm{ppm})$ & $\mathrm{Zr}(\mathrm{ppm})$ \\
\hline 7536.14 & 365591.40 & 17.00 & 2122.91 & 73.23 & 105.67 & 35009.98 & 97.60 & 231.71 & 32.84 & 9.33 & 152.47 & 32.11 & 117.88 & 11.13 \\
\hline 7536.21 & 363552.20 & 17.00 & 2047.70 & 68.26 & 101.47 & 33854.40 & 92.09 & 174.81 & 32.84 & 9.26 & 156.28 & 32.33 & 118.65 & 12.10 \\
\hline 7536.27 & 371845.20 & 16.80 & 2084.92 & 70.27 & 104.83 & 34470.29 & 107.88 & 154.51 & 53.60 & 13.86 & 154.71 & 62.56 & 103.66 & 32.19 \\
\hline 7536.34 & 381581.80 & 16.60 & 2117.10 & 73.62 & 107.62 & 34982.59 & 112.86 & 186.97 & 53.72 & 14.23 & 159.07 & 62.38 & 101.18 & 31.93 \\
\hline 7536.40 & 401012.40 & 16.40 & 2119.16 & 71.71 & 112.41 & 34665.68 & 115.00 & 188.43 & 54.44 & 14.15 & 157.03 & 60.40 & 100.42 & 32.08 \\
\hline 7536.47 & 385087.60 & 16.40 & 2108.88 & 72.58 & 101.04 & 27167.35 & 119.17 & 196.54 & 53.98 & 14.63 & 161.10 & 61.45 & 102.08 & 32.61 \\
\hline 7536.54 & 383549.00 & 16.40 & 2108.33 & 79.31 & 102.34 & 27856.36 & 123.87 & 219.14 & 54.48 & 14.92 & 156.88 & 61.86 & 102.69 & 31.88 \\
\hline 7536.60 & 388037.40 & 16.40 & 2137.72 & 81.61 & 108.72 & 31040.60 & 113.33 & 364.48 & 36.29 & 11.36 & 161.53 & 31.25 & 117.07 & 10.83 \\
\hline 7536.67 & 354404.40 & 16.80 & 2516.88 & 86.68 & 101.89 & 30332.72 & 120.79 & 330.03 & 82.47 & 15.13 & 157.74 & 49.30 & 100.28 & 31.86 \\
\hline 7536.73 & 333826.80 & 17.00 & 2547.08 & 91.04 & 98.84 & 31176.51 & 127.33 & 326.05 & 82.76 & 15.86 & 160.55 & 53.19 & 101.52 & 31.08 \\
\hline 7536.80 & 332127.80 & 17.20 & 2584.96 & 97.38 & 97.67 & 29962.03 & 127.60 & 321.84 & 83.43 & 15.51 & 158.41 & 53.69 & 105.10 & 30.26 \\
\hline 7536.86 & 342405.40 & 17.00 & 2689.81 & 99.22 & 105.12 & 31824.88 & 125.42 & 314.08 & 84.03 & 15.61 & 156.37 & 54.55 & 104.86 & 31.02 \\
\hline 7536.93 & 331451.80 & 17.20 & 2683.44 & 101.19 & 98.99 & 29043.65 & 125.78 & 185.98 & 82.58 & 14.92 & 155.48 & 58.17 & 110.08 & 31.50 \\
\hline 7536.99 & 357773.80 & 17.00 & 2388.20 & 104.09 & 111.42 & 31406.74 & 119.53 & 220.24 & 38.20 & 11.25 & 158.72 & 42.19 & 126.59 & 10.44 \\
\hline 7537.06 & 393141.80 & 16.60 & 2530.08 & 105.32 & 115.74 & 34741.24 & 115.01 & 213.13 & 40.21 & 11.35 & 155.06 & 40.53 & 129.16 & 10.99 \\
\hline 7537.13 & 397280.40 & 16.40 & 2543.63 & 107.33 & 117.73 & 35767.20 & 119.86 & 203.42 & 41.60 & 11.77 & 155.18 & 41.13 & 131.13 & 11.90 \\
\hline 7537.19 & 396000.60 & 16.40 & 2494.78 & 107.52 & 118.10 & 35654.90 & 125.92 & 228.28 & 42.68 & 12.25 & 159.69 & 41.84 & 133.78 & 11.80 \\
\hline 7537.26 & 406938.20 & 16.20 & 2414.61 & 112.03 & 120.67 & 39606.91 & 131.93 & 384.19 & 48.97 & 12.86 & 167.22 & 41.27 & 131.45 & 12.61 \\
\hline 7537.32 & 402246.80 & 16.20 & 2115.57 & 104.61 & 109.53 & 37499.41 & 135.18 & 420.40 & 56.91 & 13.58 & 165.78 & 38.58 & 134.83 & 13.44 \\
\hline 7537.39 & 373333.00 & 16.40 & 1844.37 & 103.26 & 102.19 & 33580.62 & 138.21 & 485.49 & 62.12 & 14.04 & 161.04 & 36.28 & 131.26 & 13.97 \\
\hline 7537.45 & 358778.40 & 16.60 & 1544.22 & 94.80 & 95.78 & 34443.27 & 136.71 & 550.89 & 68.83 & 14.98 & 158.20 & 35.36 & 126.58 & 14.20 \\
\hline 7537.52 & 337569.00 & 16.80 & 1363.63 & 93.21 & 84.68 & 33262.68 & 142.72 & 658.47 & 72.06 & 15.66 & 156.43 & 35.12 & 125.63 & 14.27 \\
\hline 7537.59 & 306201.80 & 17.20 & 1297.52 & 87.18 & 78.13 & 29367.47 & 137.45 & 571.35 & 68.99 & 15.99 & 139.95 & 32.24 & 125.12 & 11.89 \\
\hline 7537.65 & 312384.20 & 17.20 & 5320.18 & 93.47 & 83.01 & 32233.35 & 138.46 & 618.16 & 63.63 & 16.23 & 134.80 & 33.20 & 124.11 & 10.97 \\
\hline 7537.72 & 256105.80 & 17.80 & 6075.47 & 87.34 & 86.36 & 27011.05 & 122.98 & 525.46 & 57.69 & 13.86 & 117.13 & 32.05 & 98.37 & 10.59 \\
\hline 7537.78 & 248182.40 & 17.80 & 6052.57 & 93.92 & 84.11 & 25829.03 & 124.26 & 609.44 & 49.94 & 13.87 & 114.38 & 30.78 & 98.65 & 10.16 \\
\hline 7537.85 & 261286.00 & 17.80 & 6005.25 & 101.53 & 91.67 & 27291.16 & 122.61 & 816.23 & 47.72 & 13.84 & 105.53 & 29.93 & 98.54 & 9.64 \\
\hline 7537.91 & 275216.00 & 17.60 & 5979.98 & 105.22 & 90.96 & 27495.39 & 125.79 & 806.82 & 45.76 & 13.93 & 106.52 & 31.05 & 99.71 & 11.02 \\
\hline 7537.98 & 277822.20 & 17.60 & 2051.19 & 101.35 & 93.41 & 29901.67 & 127.09 & 760.66 & 46.01 & 14.81 & 108.41 & 32.31 & 98.12 & 11.69 \\
\hline 7538.04 & 351773.00 & 16.80 & 1312.21 & 108.28 & 92.85 & 37177.44 & 144.43 & 954.56 & 47.39 & 17.56 & 129.74 & 35.33 & 123.09 & 12.16 \\
\hline 7538.11 & 371827.40 & 16.60 & 1504.81 & 112.95 & 103.54 & 38735.74 & 149.67 & 854.91 & 49.43 & 17.36 & 131.13 & 36.54 & 125.65 & 12.38 \\
\hline 7538.18 & 374357.80 & 16.60 & 1602.73 & 112.42 & 102.92 & 39313.00 & 151.93 & 575.93 & 49.85 & 16.78 & 136.09 & 38.18 & 126.41 & 11.14 \\
\hline 7538.24 & 383745.40 & 16.60 & 1484.09 & 110.55 & 104.44 & 40286.31 & 157.80 & 605.60 & 50.52 & 17.58 & 148.06 & 40.03 & 126.87 & 10.98 \\
\hline
\end{tabular}




\begin{tabular}{|c|c|c|c|c|c|c|c|c|c|c|c|c|c|c|}
\hline Depth & XRF & XRF Live Time & $\mathrm{Ti}(\mathrm{ppm})$ & $\mathrm{Cr}(\mathrm{ppm})$ & $\mathrm{Mn}(\mathrm{ppm})$ & $\mathrm{Fe}(\mathrm{ppm})$ & $\mathrm{Ni}(\mathrm{ppm})$ & $\mathrm{Cu}(\mathrm{ppm})$ & $\mathrm{Zn}(\mathrm{ppm})$ & As (ppm) & $\mathrm{Rb}(\mathrm{ppm})$ & $\mathrm{Sr}(\mathrm{ppm})$ & $\mathrm{Y}(\mathrm{ppm})$ & $\mathrm{Zr}(\mathrm{ppm})$ \\
\hline 7538.31 & 354831.20 & 16.80 & 1485.30 & 110.46 & 103.56 & 35452.76 & 156.14 & 584.44 & 47.67 & 15.65 & 154.87 & 39.56 & 125.51 & 9.91 \\
\hline 7538.37 & 333492.20 & 17.00 & 1489.97 & 108.59 & 105.37 & 33439.38 & 154.62 & 461.13 & 44.95 & 14.57 & 157.23 & 40.20 & 127.26 & 8.46 \\
\hline 7538.44 & 320175.20 & 17.20 & 1592.57 & 101.95 & 102.69 & 31281.30 & 159.21 & 397.06 & 76.86 & 18.64 & 160.73 & 60.57 & 107.23 & 33.47 \\
\hline 7538.50 & 310044.80 & 17.20 & 1758.20 & 98.70 & 101.59 & 28809.46 & 146.54 & 353.72 & 74.64 & 18.81 & 159.30 & 60.41 & 105.67 & 34.88 \\
\hline 7538.57 & 312639.00 & 17.00 & 2027.78 & 95.82 & 102.83 & 28193.90 & 137.06 & 383.39 & 74.94 & 17.12 & 150.99 & 58.62 & 104.33 & 35.46 \\
\hline 7538.64 & 341377.40 & 16.80 & 2184.15 & 96.69 & 102.02 & 28558.67 & 133.07 & 356.40 & 75.61 & 16.84 & 151.57 & 58.89 & 107.24 & 37.18 \\
\hline 7538.70 & 356862.00 & 16.80 & 3211.78 & 94.26 & 96.17 & 28941.68 & 127.47 & 376.21 & 77.94 & 16.39 & 151.24 & 59.00 & 106.01 & 38.02 \\
\hline 7538.77 & 361465.20 & 16.80 & 3141.91 & 91.37 & 93.51 & 30357.08 & 117.75 & 444.31 & 45.76 & 11.27 & 151.53 & 38.93 & 122.48 & 12.56 \\
\hline 7538.83 & 373775.80 & 16.80 & 2948.57 & 82.94 & 90.92 & 30333.32 & 120.99 & 445.32 & 46.05 & 11.03 & 151.70 & 37.44 & 123.88 & 13.27 \\
\hline 7538.90 & 377151.40 & 16.80 & 2883.86 & 82.19 & 91.96 & 31233.87 & 136.74 & 316.21 & 88.99 & 19.15 & 153.00 & 56.63 & 105.19 & 36.04 \\
\hline 7538.96 & 371854.60 & 16.80 & 2772.05 & 85.28 & 91.41 & 30768.27 & 136.02 & 309.76 & 89.55 & 19.51 & 153.94 & 57.32 & 102.54 & 35.19 \\
\hline 7539.03 & 371340.20 & 16.80 & 1821.36 & 87.78 & 98.17 & 31348.80 & 141.90 & 291.33 & 89.15 & 19.39 & 159.91 & 59.96 & 101.63 & 34.61 \\
\hline 7539.09 & 385089.80 & 16.60 & 1741.33 & 92.11 & 98.04 & 30606.39 & 142.66 & 374.31 & 88.48 & 19.67 & 169.90 & 68.32 & 104.36 & 34.60 \\
\hline 7539.16 & 377965.40 & 16.60 & 1658.86 & 91.71 & 102.16 & 30711.20 & 147.41 & 319.71 & 90.15 & 18.85 & 188.45 & 79.37 & 101.27 & 32.81 \\
\hline 7539.23 & 377738.80 & 16.60 & 1587.17 & 94.23 & 104.19 & 30263.74 & 134.28 & 306.21 & 48.37 & 10.26 & 200.77 & 65.54 & 118.59 & 8.63 \\
\hline 7539.29 & 353391.20 & 16.80 & 1473.06 & 103.91 & 113.29 & 36654.13 & 184.77 & 295.99 & 56.57 & 22.92 & 187.94 & 90.17 & 106.04 & 26.23 \\
\hline 7539.36 & 321083.60 & 17.00 & 1178.45 & 103.77 & 128.15 & 39213.04 & 181.26 & 235.20 & 59.51 & 24.80 & 225.71 & 86.63 & 98.29 & 25.05 \\
\hline 7539.42 & 300479.40 & 17.20 & 1075.54 & 93.24 & 129.04 & 38778.67 & 181.04 & 66.91 & 59.70 & 24.11 & 233.26 & 81.24 & 100.30 & 25.56 \\
\hline 7539.49 & 283962.20 & 17.40 & 1177.38 & 94.77 & 128.17 & 39282.29 & 176.77 & 66.00 & 58.07 & 24.10 & 242.45 & 71.04 & 102.50 & 26.74 \\
\hline 7539.55 & 255115.80 & 17.80 & 1154.20 & 95.59 & 126.76 & 38743.52 & 176.58 & 59.00 & 56.26 & 24.73 & 253.14 & 70.26 & 107.41 & 27.66 \\
\hline 7539.62 & 290406.00 & 17.40 & 1277.91 & 88.51 & 123.87 & 35545.56 & 138.18 & 38.15 & 51.10 & 12.30 & 273.30 & 48.86 & 123.73 & 10.06 \\
\hline 7539.69 & 314852.60 & 17.20 & 1369.23 & 90.68 & 107.90 & 37687.40 & 149.46 & 71.63 & 56.83 & 12.51 & 232.38 & 49.21 & 134.77 & 9.91 \\
\hline 7539.75 & 301161.80 & 17.40 & 1246.43 & 106.74 & 118.13 & 41957.07 & 161.65 & 152.37 & 61.74 & 15.69 & 219.83 & 47.65 & 132.96 & 9.39 \\
\hline 7539.82 & 306593.00 & 17.20 & 1068.45 & 118.58 & 123.72 & 49746.65 & 179.23 & 382.12 & 72.99 & 20.33 & 199.55 & 46.50 & 130.70 & 10.04 \\
\hline 7539.88 & 305598.60 & 17.20 & 825.92 & 119.35 & 123.23 & 52459.68 & 188.08 & 469.05 & 79.50 & 22.63 & 172.26 & 40.88 & 125.71 & 11.31 \\
\hline 7539.95 & 284149.80 & 17.40 & 450.58 & 122.81 & 119.43 & 55171.66 & 188.22 & 758.86 & 89.12 & 25.85 & 156.76 & 36.25 & 124.16 & 10.64 \\
\hline 7540.01 & 271298.20 & 17.60 & 160.50 & 127.43 & 122.26 & 53973.10 & 196.69 & 804.07 & 87.10 & 27.87 & 154.08 & 35.97 & 123.48 & 11.77 \\
\hline 7540.08 & 264068.20 & 17.60 & 167.22 & 127.71 & 120.65 & 54943.67 & 196.82 & 762.03 & 90.90 & 27.73 & 148.65 & 34.32 & 120.71 & 12.07 \\
\hline 7540.14 & 256121.40 & 17.80 & 292.27 & 132.91 & 126.22 & 53802.02 & 199.36 & 613.56 & 92.10 & 27.40 & 151.93 & 34.50 & 123.15 & 11.60 \\
\hline 7540.21 & 272989.20 & 17.60 & 164.29 & 143.10 & 135.39 & 56064.11 & 207.01 & 539.78 & 94.87 & 28.49 & 152.58 & 40.92 & 127.97 & 11.52 \\
\hline 7540.28 & 280050.40 & 17.60 & 211.53 & 141.24 & 137.41 & 58867.52 & 209.86 & 250.82 & 91.88 & 27.90 & 149.44 & 41.84 & 127.67 & 10.79 \\
\hline 7540.34 & 287641.60 & 17.40 & 423.79 & 135.14 & 140.45 & 68680.66 & 197.36 & 179.86 & 105.38 & 26.78 & 142.44 & 40.92 & 117.94 & 10.57 \\
\hline 7540.41 & 310619.80 & 17.20 & 369.05 & 128.37 & 142.29 & 71333.68 & 192.30 & 159.30 & 106.66 & 27.41 & 137.93 & 43.43 & 119.52 & 10.31 \\
\hline
\end{tabular}




\begin{tabular}{|c|c|c|c|c|c|c|c|c|c|c|c|c|c|c|}
\hline Depth & XRF & XRF Live Time & $\mathrm{Ti}(\mathrm{ppm})$ & $\mathrm{Cr}(\mathrm{ppm})$ & $\mathrm{Mn}(\mathrm{ppm})$ & $\mathrm{Fe}(\mathrm{ppm})$ & $\mathrm{Ni}(\mathrm{ppm})$ & $\mathrm{Cu}(\mathrm{ppm})$ & $\mathrm{Zn}(\mathrm{ppm})$ & As (ppm) & $\mathrm{Rb}(\mathrm{ppm})$ & $\mathrm{Sr}(\mathrm{ppm})$ & $\mathrm{Y}(\mathrm{ppm})$ & $\mathrm{Zr}(\mathrm{ppm})$ \\
\hline 7540.47 & 317747.20 & 17.00 & 118.53 & 123.48 & 127.99 & 70572.53 & 190.24 & 113.57 & 103.24 & 27.15 & 120.88 & 45.57 & 124.84 & 8.99 \\
\hline 7540.54 & 299494.00 & 17.20 & 304.62 & 112.38 & 124.96 & 77648.71 & 187.49 & 127.18 & 111.04 & 26.42 & 131.97 & 38.41 & 112.71 & 7.62 \\
\hline 7540.60 & 300340.60 & 17.20 & 320.40 & 108.86 & 125.72 & 73370.78 & 184.42 & 195.45 & 107.65 & 25.55 & 145.27 & 39.27 & 110.25 & 7.38 \\
\hline 7540.67 & 289950.60 & 17.40 & 346.92 & 109.47 & 122.71 & 66176.37 & 199.22 & 310.29 & 96.28 & 27.24 & 149.57 & 41.05 & 119.43 & 6.90 \\
\hline 7540.73 & 292657.60 & 17.40 & 3422.78 & 118.08 & 116.13 & 64311.47 & 214.57 & 653.22 & 95.15 & 28.57 & 162.21 & 39.15 & 119.71 & 6.03 \\
\hline 7540.80 & 290703.60 & 17.60 & 3520.36 & 114.23 & 123.10 & 60927.16 & 209.59 & 785.84 & 90.15 & 27.48 & 178.57 & 36.25 & 116.05 & 6.19 \\
\hline 7540.87 & 292015.80 & 17.60 & 3451.96 & 111.33 & 114.64 & 50955.70 & 210.62 & 929.65 & 77.21 & 27.47 & 181.03 & 36.71 & 126.44 & 6.28 \\
\hline 7540.93 & 283956.00 & 17.60 & 3554.77 & 122.96 & 111.79 & 52701.93 & 218.70 & 1103.98 & 77.89 & 28.26 & 180.41 & 36.85 & 127.84 & 7.61 \\
\hline 7541.00 & 291971.60 & 17.40 & 3498.56 & 119.87 & 111.02 & 49773.03 & 208.70 & 1210.69 & 73.24 & 27.38 & 180.16 & 36.32 & 123.61 & 8.23 \\
\hline 7541.06 & 299930.60 & 17.40 & 430.78 & 110.74 & 111.49 & 47088.87 & 195.47 & 1096.77 & 67.53 & 25.44 & 180.74 & 35.37 & 119.44 & 7.60 \\
\hline 7541.13 & 323499.40 & 17.00 & 437.41 & 107.10 & 110.90 & 45942.94 & 194.84 & 1156.30 & 68.45 & 25.10 & 180.78 & 35.41 & 118.09 & 8.02 \\
\hline 7541.19 & 346589.40 & 16.80 & 469.97 & 107.92 & 117.30 & 43826.78 & 186.18 & 1302.00 & 66.13 & 23.46 & 190.58 & 38.32 & 112.56 & 7.28 \\
\hline 7541.26 & 338932.60 & 17.00 & 426.69 & 80.08 & 121.85 & 36030.86 & 172.10 & 1128.24 & 54.15 & 19.56 & 237.96 & 43.39 & 100.71 & 5.18 \\
\hline 7541.33 & 350093.00 & 17.00 & 363.24 & 65.81 & 126.25 & 27106.04 & 138.72 & 888.98 & 42.23 & 14.13 & 269.57 & 44.27 & 80.67 & 3.14 \\
\hline 7541.39 & 347560.20 & 17.00 & 311.52 & 48.23 & 138.53 & 18430.00 & 104.33 & 639.07 & 30.27 & 10.07 & 293.38 & 46.11 & 60.45 & 3.33 \\
\hline 7541.46 & 349397.80 & 17.00 & 220.39 & 28.40 & 134.33 & 11270.75 & 71.85 & 407.94 & 17.68 & 6.14 & 322.71 & 44.48 & 35.39 & 1.16 \\
\hline 7541.52 & 354163.00 & 16.80 & 171.16 & 12.55 & 131.56 & 5421.46 & 42.98 & 86.46 & 7.91 & 3.15 & 323.97 & 41.06 & 16.96 & -0.45 \\
\hline 7541.59 & 381171.40 & 16.40 & 73.59 & 7.93 & 130.65 & 2067.53 & 16.41 & 6.68 & 4.46 & 2.26 & 286.25 & 32.65 & 6.66 & -1.00 \\
\hline 7541.65 & 386401.40 & 16.40 & 95.33 & 6.66 & 124.52 & 1937.26 & 15.97 & 3.51 & 3.61 & 2.34 & 272.24 & 28.69 & 7.54 & -0.88 \\
\hline 7541.72 & 394765.80 & 16.20 & 110.25 & 5.56 & 114.01 & 2349.47 & 19.78 & 3.09 & 3.97 & 2.37 & 249.17 & 27.32 & 7.24 & -0.45 \\
\hline 7541.78 & 395578.80 & 16.20 & 103.56 & 7.24 & 119.86 & 2900.21 & 19.70 & 3.88 & 4.11 & 2.23 & 227.24 & 30.43 & 8.09 & 0.33 \\
\hline 7541.85 & 396789.60 & 16.20 & 2759.27 & 7.63 & 121.48 & 3123.38 & 19.21 & 8.57 & 4.00 & 2.45 & 222.59 & 33.01 & 6.42 & 1.14 \\
\hline 7541.92 & 397563.00 & 16.20 & 2759.54 & 7.43 & 122.39 & 4834.49 & 21.77 & 9.80 & 4.96 & 2.43 & 221.96 & 34.49 & 6.76 & 2.52 \\
\hline 7541.98 & 389586.40 & 16.20 & 2746.80 & 8.97 & 121.28 & 6486.84 & 24.94 & 9.78 & 6.63 & 2.48 & 229.50 & 36.89 & 8.58 & 3.39 \\
\hline 7542.05 & 349413.20 & 16.60 & 2747.56 & 13.56 & 116.09 & 7512.13 & 24.31 & 11.18 & 8.00 & 2.65 & 228.29 & 40.09 & 14.49 & 2.56 \\
\hline 7542.11 & 313117.40 & 17.00 & 2809.40 & 14.29 & 111.36 & 9969.26 & 28.67 & 17.71 & 11.21 & 2.87 & 222.40 & 46.36 & 20.12 & 2.46 \\
\hline 7542.18 & 285862.60 & 17.40 & 407.87 & 23.39 & 114.51 & 17030.65 & 53.82 & 102.15 & 19.78 & 4.90 & 218.75 & 48.47 & 38.29 & 3.39 \\
\hline 7542.24 & 272223.60 & 17.60 & 515.40 & 44.71 & 108.26 & 23255.74 & 88.07 & 331.57 & 28.91 & 8.83 & 205.60 & 50.12 & 58.55 & 4.82 \\
\hline 7542.31 & 267349.60 & 17.60 & 560.51 & 64.19 & 105.85 & 29670.07 & 122.09 & 599.33 & 40.14 & 13.01 & 184.94 & 50.90 & 77.92 & 6.04 \\
\hline 7542.38 & 285620.00 & 17.40 & 596.87 & 79.28 & 104.20 & 35140.66 & 153.60 & 770.73 & 49.66 & 17.04 & 188.37 & 50.55 & 94.15 & 6.06 \\
\hline 7542.44 & 296130.20 & 17.40 & 571.09 & 96.40 & 99.76 & 39054.41 & 181.85 & 956.71 & 55.41 & 20.66 & 193.20 & 46.44 & 110.18 & 7.06 \\
\hline 7542.51 & 304943.80 & 17.20 & 362.38 & 105.66 & 88.06 & 39371.24 & 186.79 & 1123.27 & 57.45 & 22.47 & 183.99 & 45.21 & 115.89 & 7.39 \\
\hline 7542.57 & 316723.40 & 17.00 & 367.89 & 103.00 & 91.61 & 48611.63 & 182.85 & 1096.71 & 67.61 & 21.83 & 184.84 & 43.98 & 112.90 & 6.96 \\
\hline
\end{tabular}




\begin{tabular}{|c|c|c|c|c|c|c|c|c|c|c|c|c|c|c|}
\hline Depth & XRF & XRF Live Time & $\mathrm{Ti}(\mathrm{ppm})$ & $\mathrm{Cr}(\mathrm{ppm})$ & $\mathrm{Mn}(\mathrm{ppm})$ & $\mathrm{Fe}(\mathrm{ppm})$ & $\mathrm{Ni}(\mathrm{ppm})$ & $\mathrm{Cu}(\mathrm{ppm})$ & $\mathrm{Zn}(\mathrm{ppm})$ & As (ppm) & $\mathrm{Rb}(\mathrm{ppm})$ & $\mathrm{Sr}(\mathrm{ppm})$ & $\mathrm{Y}(\mathrm{ppm})$ & $\mathrm{Zr}(\mathrm{ppm})$ \\
\hline 7542.64 & 312846.60 & 17.00 & 405.90 & 98.10 & 99.34 & 49424.91 & 182.05 & 983.73 & 67.18 & 21.53 & 180.46 & 45.08 & 113.14 & 7.42 \\
\hline 7542.70 & 310533.20 & 17.20 & 482.72 & 99.56 & 99.08 & 49403.75 & 178.35 & 950.96 & 67.60 & 20.02 & 183.06 & 43.10 & 114.64 & 9.18 \\
\hline 7542.77 & 322431.60 & 17.00 & 459.42 & 92.19 & 105.94 & 47383.31 & 160.88 & 858.34 & 64.94 & 17.49 & 199.81 & 49.48 & 104.09 & 8.16 \\
\hline 7542.83 & 345309.00 & 16.80 & 1806.87 & 95.87 & 111.26 & 48744.06 & 172.51 & 720.21 & 67.21 & 16.78 & 199.53 & 51.77 & 110.30 & 8.53 \\
\hline 7542.90 & 347140.00 & 16.80 & 1707.00 & 97.26 & 105.21 & 40829.09 & 179.25 & 672.77 & 60.80 & 17.67 & 203.25 & 51.82 & 113.88 & 8.72 \\
\hline 7542.97 & 376476.00 & 16.60 & 1632.96 & 103.17 & 104.02 & 42413.33 & 188.93 & 611.49 & 59.74 & 17.77 & 205.10 & 50.84 & 115.01 & 7.51 \\
\hline 7543.03 & 402295.20 & 16.20 & 1459.86 & 93.24 & 120.35 & 38593.64 & 175.34 & 554.73 & 54.21 & 16.41 & 253.59 & 71.38 & 100.44 & 7.17 \\
\hline 7543.10 & 412928.00 & 16.00 & 1401.00 & 91.76 & 127.05 & 40038.93 & 187.07 & 595.89 & 58.02 & 17.98 & 274.97 & 75.17 & 104.21 & 6.92 \\
\hline 7543.16 & 418111.60 & 16.00 & 23.68 & 90.42 & 125.96 & 40751.30 & 181.19 & 663.59 & 60.73 & 18.75 & 290.87 & 73.17 & 95.51 & 6.45 \\
\hline 7543.23 & 425042.20 & 16.00 & 95.13 & 88.88 & 132.84 & 41307.87 & 183.99 & 579.74 & 59.80 & 18.69 & 299.14 & 76.33 & 95.08 & 5.65 \\
\hline 7543.29 & 402271.00 & 16.20 & 185.70 & 88.83 & 129.26 & 39931.11 & 177.85 & 528.55 & 60.13 & 18.58 & 296.75 & 81.22 & 98.36 & 6.69 \\
\hline 7543.36 & 415416.40 & 16.20 & 283.73 & 101.54 & 115.02 & 48394.35 & 196.47 & 495.44 & 70.18 & 20.72 & 235.00 & 61.31 & 110.23 & 7.09 \\
\hline 7543.43 & 417450.00 & 16.20 & 363.16 & 111.59 & 99.95 & 49162.33 & 202.68 & 413.29 & 73.10 & 21.36 & 196.78 & 51.37 & 116.29 & 8.17 \\
\hline 7543.49 & 408757.60 & 16.20 & 276.34 & 109.21 & 93.15 & 47733.52 & 208.01 & 302.25 & 72.29 & 21.97 & 180.44 & 51.13 & 115.90 & 7.95 \\
\hline 7543.56 & 409825.40 & 16.20 & 72.63 & 111.99 & 90.36 & 46876.56 & 207.18 & 299.50 & 73.96 & 22.60 & 175.05 & 48.09 & 116.75 & 8.57 \\
\hline 7543.62 & 432019.80 & 16.00 & -150.44 & 115.58 & 84.51 & 47831.50 & 211.66 & 392.99 & 78.93 & 23.47 & 183.78 & 42.95 & 113.85 & 7.89 \\
\hline 7543.69 & 408265.40 & 16.20 & -299.37 & 114.00 & 84.25 & 45424.00 & 235.82 & 384.71 & 199.08 & 34.58 & 169.66 & 71.40 & 101.91 & 27.98 \\
\hline 7543.75 & 394083.20 & 16.40 & -441.05 & 112.96 & 86.03 & 46201.49 & 237.29 & 446.92 & 200.98 & 35.41 & 172.23 & 71.51 & 99.50 & 27.28 \\
\hline 7543.82 & 349104.40 & 16.80 & -446.15 & 113.29 & 82.58 & 44386.28 & 222.24 & 427.23 & 198.46 & 34.05 & 207.05 & 75.88 & 94.07 & 26.58 \\
\hline 7543.88 & 266952.60 & 17.60 & 617.37 & 104.36 & 72.27 & 35201.06 & 193.49 & 374.08 & 196.66 & 30.27 & 177.53 & 75.62 & 69.26 & 26.91 \\
\hline 7543.95 & 250492.40 & 17.80 & 671.60 & 102.35 & 77.46 & 33451.74 & 186.78 & 322.95 & 193.70 & 29.85 & 169.92 & 76.91 & 64.78 & 26.57 \\
\hline 7544.02 & 256344.20 & 17.60 & 668.69 & 96.36 & 72.02 & 33538.69 & 170.82 & 378.21 & 69.54 & 19.85 & 181.17 & 49.19 & 78.08 & 6.14 \\
\hline 7544.08 & 250759.20 & 17.60 & 707.55 & 87.28 & 67.93 & 32657.37 & 191.74 & 289.18 & 104.23 & 26.60 & 151.12 & 66.77 & 66.33 & 24.78 \\
\hline 7544.15 & 275996.00 & 17.40 & 646.10 & 82.30 & 73.35 & 32465.81 & 191.62 & 302.40 & 103.60 & 26.26 & 126.64 & 61.26 & 68.08 & 24.26 \\
\hline 7544.21 & 321006.80 & 17.00 & -1014.13 & 118.18 & 93.74 & 38573.76 & 205.36 & 308.32 & 100.73 & 28.15 & 327.48 & 62.71 & 90.71 & 23.28 \\
\hline 7544.28 & 308640.40 & 17.00 & -2088.49 & 122.03 & 101.04 & 45862.80 & 190.28 & 233.10 & 105.12 & 26.30 & 876.52 & 57.91 & 74.47 & 23.08 \\
\hline 7544.34 & 275893.40 & 17.40 & -2713.05 & 157.36 & 125.35 & 42059.79 & 172.03 & 143.00 & 102.67 & 24.60 & 1046.19 & 56.30 & 72.36 & 23.50 \\
\hline 7544.41 & 261860.80 & 17.60 & -3157.48 & 202.34 & 149.75 & 41440.66 & 147.28 & 222.94 & 62.00 & 16.17 & 1184.42 & 35.65 & 85.90 & 5.70 \\
\hline 7544.48 & 241361.60 & 17.80 & -3682.06 & 239.78 & 167.93 & 40939.18 & 146.75 & 228.09 & 59.33 & 15.85 & 1327.46 & 34.72 & 83.00 & 7.32 \\
\hline 7544.54 & 229125.00 & 17.80 & -3334.21 & 243.69 & 169.01 & 39111.40 & 146.57 & 245.32 & 57.29 & 14.95 & 1242.32 & 33.59 & 76.04 & 7.42 \\
\hline 7544.61 & 212636.80 & 18.00 & -2571.62 & 268.35 & 179.64 & 28915.42 & 155.20 & 270.63 & 49.00 & 15.45 & 786.76 & 37.01 & 90.02 & 7.97 \\
\hline 7544.67 & 223564.20 & 18.00 & -2225.20 & 266.38 & 172.69 & 28569.17 & 155.39 & 284.00 & 48.50 & 15.42 & 738.97 & 37.80 & 89.78 & 7.69 \\
\hline 7544.74 & 237486.40 & 17.80 & -2647.25 & 259.23 & 165.27 & 30666.27 & 146.15 & 183.55 & 52.60 & 14.05 & 904.99 & 37.53 & 85.77 & 6.40 \\
\hline
\end{tabular}




\begin{tabular}{|c|c|c|c|c|c|c|c|c|c|c|c|c|c|c|}
\hline Depth & XRF & XRF Live Time & $\mathrm{Ti}(\mathrm{ppm})$ & $\mathrm{Cr}$ (ppm) & $\mathrm{Mn}$ (ppm) & $\mathrm{Fe}(\mathrm{ppm})$ & $\mathrm{Ni}(\mathrm{ppm})$ & $\mathrm{Cu}(\mathrm{ppm})$ & $\mathrm{Zn}(\mathrm{ppm})$ & As (ppm) & $\mathrm{Rb}(\mathrm{ppm})$ & $\mathrm{Sr}(\mathrm{ppm})$ & $\mathrm{Y}(\mathrm{ppm})$ & $\mathrm{Zr}(\mathrm{ppm})$ \\
\hline 7544.80 & 249747.40 & 17.80 & -2592.43 & 266.60 & 156.52 & 29642.25 & 145.96 & 116.85 & 51.31 & 13.68 & 992.28 & 41.31 & 88.22 & 6.29 \\
\hline 7544.87 & 216169.20 & 18.20 & -618.55 & 234.80 & 134.96 & 25124.12 & 125.35 & 87.68 & 43.43 & 10.92 & 870.59 & 32.45 & 76.56 & 12.85 \\
\hline 7544.93 & 211174.80 & 18.20 & -376.18 & 218.59 & 130.67 & 23121.76 & 126.49 & 65.99 & 40.07 & 9.75 & 910.70 & 36.10 & 75.69 & 12.49 \\
\hline 7545.00 & 211441.60 & 18.00 & -59.84 & 194.48 & 122.15 & 22262.41 & 132.82 & 66.20 & 38.53 & 9.34 & 845.06 & 36.18 & 72.95 & 11.26 \\
\hline 7545.07 & 217835.80 & 18.00 & 698.34 & 182.26 & 125.00 & 23406.84 & 147.55 & 90.82 & 35.77 & 11.88 & 603.01 & 36.32 & 76.45 & 12.88 \\
\hline 7545.13 & 242942.60 & 17.60 & 1323.46 & 145.30 & 119.77 & 27396.01 & 162.35 & 172.61 & 39.55 & 14.05 & 357.99 & 33.62 & 81.73 & 13.25 \\
\hline 7545.20 & 309283.40 & 17.00 & -222.84 & 142.59 & 123.25 & 36401.25 & 227.11 & 202.27 & 107.78 & 25.27 & 374.34 & 75.78 & 87.78 & 28.70 \\
\hline 7545.26 & 345767.60 & 16.80 & -11.99 & 121.84 & 113.28 & 40733.58 & 226.62 & 273.65 & 108.80 & 27.06 & 247.27 & 72.77 & 91.80 & 29.27 \\
\hline 7545.33 & 354649.60 & 16.80 & 46.81 & 122.87 & 114.76 & 43688.73 & 229.20 & 264.74 & 110.80 & 28.01 & 215.73 & 74.64 & 96.61 & 29.93 \\
\hline 7545.39 & 344015.80 & 17.00 & -83.58 & 112.47 & 110.16 & 36449.74 & 201.57 & 232.11 & 102.94 & 24.92 & 287.47 & 93.72 & 81.26 & 28.78 \\
\hline 7545.46 & 328355.80 & 17.20 & -525.13 & 125.53 & 122.88 & 33178.29 & 185.96 & 148.34 & 99.87 & 21.97 & 420.69 & 103.63 & 74.60 & 26.71 \\
\hline 7545.52 & 338674.80 & 17.00 & -1027.82 & 143.53 & 142.64 & 31472.50 & 147.29 & 112.48 & 40.72 & 14.45 & 567.22 & 86.74 & 86.19 & 4.44 \\
\hline 7545.59 & 348734.00 & 16.80 & -1084.39 & 152.99 & 142.23 & 31624.45 & 157.27 & 33.65 & 44.75 & 15.77 & 567.60 & 90.30 & 87.97 & 4.20 \\
\hline 7545.66 & 364341.00 & 16.60 & -1103.53 & 147.59 & 137.73 & 32400.48 & 159.74 & 58.43 & 44.88 & 16.05 & 539.72 & 88.94 & 85.76 & 4.15 \\
\hline 7545.72 & 390378.60 & 16.20 & -860.82 & 144.03 & 134.78 & 40181.97 & 191.18 & 177.23 & 53.81 & 19.39 & 415.07 & 70.08 & 101.37 & 4.78 \\
\hline 7545.79 & 411936.20 & 16.00 & -512.60 & 127.22 & 119.37 & 44703.87 & 205.47 & 325.99 & 57.99 & 23.37 & 282.15 & 59.61 & 104.88 & 6.34 \\
\hline 7545.85 & 414654.00 & 16.00 & -199.08 & 112.14 & 97.68 & 46338.16 & 213.76 & 455.41 & 59.42 & 25.04 & 142.09 & 44.26 & 105.06 & 6.46 \\
\hline 7545.92 & 416499.20 & 16.00 & -333.60 & 112.10 & 93.97 & 46847.08 & 213.08 & 550.97 & 59.69 & 24.46 & 132.79 & 41.75 & 103.66 & 5.25 \\
\hline 7545.98 & 438364.20 & 15.80 & -384.59 & 113.51 & 100.49 & 68975.32 & 218.80 & 554.58 & 76.66 & 26.46 & 129.24 & 42.04 & 98.69 & 4.41 \\
\hline 7546.05 & 437418.00 & 15.80 & -343.05 & 112.36 & 95.66 & 70199.36 & 220.63 & 529.94 & 80.51 & 26.64 & 131.06 & 44.22 & 97.73 & 4.12 \\
\hline 7546.12 & 429126.60 & 16.00 & -316.86 & 110.22 & 97.84 & 67529.13 & 216.37 & 516.17 & 76.85 & 25.38 & 152.20 & 47.48 & 96.07 & 2.59 \\
\hline 7546.18 & 421277.00 & 16.20 & -246.51 & 100.40 & 107.37 & 65448.90 & 246.41 & 417.96 & 198.81 & 30.33 & 144.50 & 88.38 & 89.76 & 20.99 \\
\hline 7546.25 & 411093.80 & 16.40 & -232.04 & 94.30 & 111.01 & 64124.09 & 241.71 & 566.21 & 197.02 & 30.02 & 181.36 & 100.00 & 86.82 & 20.90 \\
\hline 7546.31 & 370388.20 & 16.80 & -216.83 & 87.01 & 107.86 & 39639.11 & 225.98 & 743.64 & 177.86 & 27.56 & 221.17 & 109.11 & 87.46 & 21.27 \\
\hline 7546.38 & 332914.60 & 17.20 & -248.55 & 78.48 & 103.49 & 33874.85 & 215.49 & 835.26 & 174.28 & 27.74 & 251.74 & 118.67 & 84.34 & 19.81 \\
\hline 7546.44 & 290804.00 & 17.60 & -273.97 & 80.46 & 105.23 & 31084.85 & 207.73 & 814.33 & 171.69 & 26.42 & 269.96 & 124.10 & 81.42 & 20.06 \\
\hline 7546.51 & 263259.40 & 17.80 & -305.27 & 89.50 & 107.39 & 32903.15 & 178.14 & 906.53 & 52.44 & 21.27 & 298.82 & 89.04 & 85.45 & 0.23 \\
\hline 7546.57 & 227412.60 & 18.00 & -293.47 & 91.63 & 102.11 & 33690.79 & 183.61 & 882.81 & 55.67 & 22.17 & 273.29 & 79.54 & 84.42 & 2.53 \\
\hline 7546.64 & 183272.20 & 18.40 & -261.93 & 107.81 & 103.55 & 36324.96 & 193.45 & 970.38 & 60.15 & 23.31 & 246.87 & 70.86 & 84.36 & 3.90 \\
\hline 7546.71 & 198087.80 & 18.20 & -329.91 & 119.74 & 102.30 & 40814.67 & 209.88 & 1087.22 & 62.87 & 23.64 & 217.39 & 65.31 & 87.78 & 4.84 \\
\hline 7546.77 & 251834.00 & 17.60 & -437.23 & 116.76 & 92.07 & 45462.77 & 223.72 & 1301.94 & 70.22 & 25.01 & 178.78 & 59.39 & 93.26 & 6.02 \\
\hline 7546.84 & 264565.00 & 17.40 & -560.61 & 113.62 & 81.68 & 43789.32 & 216.20 & 1474.16 & 66.96 & 25.27 & 155.53 & 54.93 & 93.77 & 7.82 \\
\hline 7546.90 & 264277.60 & 17.40 & -568.86 & 108.54 & 85.51 & 46180.40 & 212.39 & 1378.35 & 67.78 & 23.44 & 160.96 & 51.60 & 92.81 & 5.74 \\
\hline
\end{tabular}




\begin{tabular}{|c|c|c|c|c|c|c|c|c|c|c|c|c|c|c|}
\hline Depth & XRF & XRF Live Time & $\mathrm{Ti}(\mathrm{ppm})$ & $\mathrm{Cr}(\mathrm{ppm})$ & $\mathrm{Mn}(\mathrm{ppm})$ & $\mathrm{Fe}(\mathrm{ppm})$ & $\mathrm{Ni}(\mathrm{ppm})$ & $\mathrm{Cu}(\mathrm{ppm})$ & $\mathrm{Zn}(\mathrm{ppm})$ & As (ppm) & $\mathrm{Rb}(\mathrm{ppm})$ & $\mathrm{Sr}(\mathrm{ppm})$ & $\mathrm{Y}(\mathrm{ppm})$ & $\mathrm{Zr}(\mathrm{ppm})$ \\
\hline 7546.97 & 325860.20 & 16.80 & 4948.44 & 101.95 & 88.64 & 52005.22 & 230.95 & 4433.29 & 72.37 & 24.20 & 146.92 & 53.76 & 96.21 & 4.21 \\
\hline 7547.03 & 322993.40 & 17.00 & 4960.61 & 94.44 & 90.61 & 48468.26 & 221.07 & 4470.49 & 66.93 & 23.44 & 150.53 & 51.61 & 92.92 & 4.77 \\
\hline 7547.10 & 290532.40 & 17.40 & 5027.97 & 94.09 & 96.39 & 45385.32 & 210.50 & 4239.29 & 63.16 & 23.19 & 173.76 & 54.81 & 86.35 & 4.58 \\
\hline 7547.17 & 300743.20 & 17.40 & 5294.86 & 96.90 & 99.27 & 46091.54 & 210.87 & 4032.81 & 63.42 & 21.90 & 196.23 & 56.99 & 88.22 & 4.56 \\
\hline 7547.23 & 334347.80 & 17.20 & 5399.16 & 95.44 & 96.34 & 45715.91 & 206.11 & 4051.82 & 59.22 & 21.52 & 202.24 & 63.20 & 84.14 & 4.33 \\
\hline 7547.30 & 329378.00 & 17.40 & -196.12 & 90.27 & 79.43 & 37442.15 & 185.74 & 844.41 & 51.87 & 19.92 & 231.18 & 67.31 & 84.79 & 3.73 \\
\hline 7547.36 & 361373.60 & 17.00 & -139.22 & 96.04 & 76.61 & 41374.31 & 199.88 & 951.55 & 58.69 & 21.00 & 225.03 & 68.22 & 88.54 & 3.59 \\
\hline 7547.43 & 376778.40 & 16.80 & -145.84 & 99.40 & 72.05 & 41643.57 & 206.48 & 1142.39 & 60.20 & 20.55 & 199.28 & 63.82 & 93.42 & 4.43 \\
\hline 7547.49 & 380420.00 & 16.80 & -207.62 & 92.91 & 66.57 & 40521.13 & 212.19 & 1601.78 & 60.50 & 21.13 & 190.38 & 62.58 & 92.36 & 2.62 \\
\hline 7547.56 & 381205.80 & 16.80 & -261.04 & 97.25 & 59.96 & 36800.64 & 216.99 & 1993.47 & 62.83 & 21.99 & 166.19 & 60.56 & 98.15 & 3.03 \\
\hline 7547.62 & 371893.40 & 16.80 & -199.42 & 95.52 & 58.35 & 36621.92 & 213.60 & 2022.10 & 64.00 & 21.66 & 139.59 & 54.03 & 94.91 & 4.66 \\
\hline 7547.69 & 342331.60 & 17.00 & -155.32 & 91.13 & 57.97 & 31326.16 & 187.94 & 1798.58 & 58.50 & 19.99 & 135.12 & 51.75 & 90.33 & 5.52 \\
\hline 7547.76 & 334970.20 & 17.00 & -220.09 & 91.31 & 58.55 & 31293.15 & 185.36 & 2895.69 & 58.71 & 19.29 & 128.44 & 55.53 & 90.61 & 5.62 \\
\hline 7547.82 & 311219.20 & 17.20 & -275.12 & 95.74 & 57.89 & 30051.89 & 171.08 & 2494.21 & 58.48 & 18.58 & 114.91 & 54.78 & 86.81 & 7.96 \\
\hline 7547.89 & 299830.60 & 17.20 & -232.83 & 95.68 & 63.11 & 31411.21 & 164.11 & 2038.81 & 61.83 & 18.91 & 110.13 & 53.91 & 85.98 & 7.91 \\
\hline 7547.95 & 300878.40 & 17.20 & -95.79 & 102.01 & 73.48 & 33645.28 & 169.43 & 2050.24 & 62.73 & 19.94 & 108.46 & 57.70 & 92.81 & 8.03 \\
\hline 7548.02 & 248768.20 & 17.80 & 743.26 & 86.49 & 86.88 & 28295.97 & 143.53 & 1838.28 & 52.39 & 17.21 & 93.19 & 46.66 & 78.59 & 10.89 \\
\hline 7548.08 & 252914.00 & 17.80 & 901.56 & 86.65 & 91.82 & 29087.99 & 145.36 & 657.29 & 52.07 & 18.06 & 92.87 & 43.90 & 76.87 & 10.53 \\
\hline 7548.15 & 263855.00 & 17.60 & 870.48 & 89.02 & 92.06 & 30578.98 & 165.24 & 641.61 & 50.96 & 18.70 & 91.69 & 47.83 & 81.62 & 10.34 \\
\hline 7548.22 & 270260.40 & 17.60 & 987.39 & 84.78 & 89.91 & 30249.39 & 172.42 & 593.07 & 45.35 & 17.81 & 95.03 & 47.04 & 87.86 & 11.94 \\
\hline 7548.28 & 268278.20 & 17.60 & 986.11 & 75.33 & 85.08 & 28664.24 & 171.55 & 433.88 & 47.20 & 17.04 & 95.14 & 42.94 & 85.33 & 11.21 \\
\hline 7548.35 & 333311.00 & 17.00 & 174.99 & 90.59 & 75.02 & 37155.15 & 211.13 & 441.01 & 57.31 & 20.29 & 111.85 & 54.54 & 104.35 & 9.19 \\
\hline 7548.41 & 333489.80 & 17.00 & 226.80 & 85.48 & 69.58 & 37469.68 & 207.26 & 241.14 & 57.00 & 19.85 & 112.54 & 55.05 & 103.75 & 9.09 \\
\hline 7548.48 & 332471.60 & 17.00 & 240.29 & 78.09 & 68.47 & 35765.45 & 192.15 & 134.68 & 54.28 & 18.68 & 107.74 & 50.34 & 100.30 & 7.85 \\
\hline 7548.54 & 323065.40 & 17.00 & 123.85 & 80.38 & 63.69 & 33455.64 & 184.36 & 136.67 & 51.92 & 19.13 & 101.16 & 47.66 & 92.85 & 6.41 \\
\hline 7548.61 & 317136.60 & 17.00 & -8.68 & 76.12 & 58.83 & 31848.32 & 169.43 & 166.27 & 45.90 & 17.82 & 99.02 & 45.62 & 86.49 & 6.09 \\
\hline 7548.67 & 313423.40 & 17.00 & 94.87 & 75.73 & 58.12 & 30112.57 & 162.78 & 224.35 & 44.79 & 17.14 & 97.32 & 44.84 & 85.69 & 5.75 \\
\hline 7548.74 & 310812.60 & 17.00 & 64.60 & 76.43 & 58.70 & 26997.00 & 155.63 & 250.61 & 38.37 & 16.60 & 98.81 & 41.12 & 85.28 & 5.52 \\
\hline 7548.81 & 315734.60 & 17.00 & 110.21 & 76.21 & 57.42 & 26274.45 & 149.15 & 246.81 & 37.20 & 16.69 & 101.12 & 40.92 & 84.78 & 6.06 \\
\hline 7548.87 & 324982.60 & 17.00 & 190.24 & 74.73 & 62.45 & 26668.88 & 147.95 & 211.59 & 38.53 & 15.82 & 109.13 & 41.87 & 87.21 & 6.17 \\
\hline 7548.94 & 333875.80 & 17.00 & 325.99 & 77.06 & 67.69 & 26848.30 & 150.25 & 149.48 & 39.81 & 16.30 & 124.92 & 44.54 & 88.42 & 6.69 \\
\hline 7549.00 & 334745.40 & 17.00 & 1640.00 & 68.63 & 67.65 & 24699.20 & 131.06 & 70.14 & 38.01 & 14.47 & 161.04 & 50.24 & 77.47 & 5.15 \\
\hline 7549.07 & 327648.20 & 17.20 & 1649.90 & 64.56 & 78.71 & 23635.29 & 117.81 & 37.98 & 39.44 & 13.60 & 195.62 & 59.16 & 75.72 & 4.49 \\
\hline
\end{tabular}




\begin{tabular}{|c|c|c|c|c|c|c|c|c|c|c|c|c|c|c|}
\hline Depth & XRF & XRF Live Time & $\mathrm{Ti}(\mathrm{ppm})$ & $\mathrm{Cr}(\mathrm{ppm})$ & $\mathrm{Mn}(\mathrm{ppm})$ & $\mathrm{Fe}(\mathrm{ppm})$ & $\mathrm{Ni}(\mathrm{ppm})$ & $\mathrm{Cu}(\mathrm{ppm})$ & $\mathrm{Zn}(\mathrm{ppm})$ & As (ppm) & $\mathrm{Rb}(\mathrm{ppm})$ & $\mathrm{Sr}(\mathrm{ppm})$ & $\mathrm{Y}(\mathrm{ppm})$ & $\mathrm{Zr}(\mathrm{ppm})$ \\
\hline 7549.13 & 340994.00 & 17.00 & 1794.73 & 68.39 & 83.28 & 26450.02 & 122.96 & 15.34 & 43.60 & 13.36 & 206.96 & 56.25 & 78.17 & 5.32 \\
\hline 7549.20 & 347242.80 & 17.00 & 1922.16 & 70.46 & 85.49 & 26421.67 & 121.59 & 15.83 & 43.92 & 13.55 & 213.37 & 56.88 & 79.61 & 5.52 \\
\hline 7549.27 & 348707.00 & 17.00 & 2022.97 & 73.39 & 86.97 & 26350.14 & 125.48 & 18.41 & 42.95 & 13.45 & 212.74 & 57.35 & 83.61 & 5.41 \\
\hline 7549.33 & 366044.60 & 16.80 & 755.29 & 78.97 & 87.13 & 28649.13 & 141.90 & 19.47 & 43.54 & 14.76 & 189.61 & 51.02 & 92.63 & 7.62 \\
\hline 7549.40 & 402872.60 & 16.40 & 1037.50 & 81.11 & 84.86 & 34853.47 & 149.47 & 19.14 & 48.87 & 15.13 & 178.19 & 45.68 & 97.57 & 8.34 \\
\hline 7549.46 & 393799.60 & 16.60 & 1142.73 & 76.71 & 87.72 & 32406.49 & 142.59 & 20.37 & 45.34 & 14.93 & 186.18 & 49.60 & 95.04 & 8.21 \\
\hline 7549.53 & 348055.80 & 17.00 & 961.10 & 69.17 & 75.61 & 29527.46 & 136.51 & 26.40 & 39.24 & 13.47 & 185.64 & 51.49 & 88.78 & 8.46 \\
\hline 7549.59 & 321705.40 & 17.20 & 759.28 & 61.38 & 69.54 & 27659.37 & 122.44 & 31.90 & 36.15 & 12.00 & 170.96 & 46.56 & 78.81 & 7.08 \\
\hline 7549.66 & 283191.40 & 17.60 & 658.17 & 64.89 & 69.95 & 25278.63 & 105.08 & 41.16 & 34.21 & 11.19 & 160.39 & 43.87 & 71.79 & 6.02 \\
\hline 7549.72 & 229998.00 & 18.00 & 446.99 & 65.02 & 62.71 & 19481.20 & 101.48 & 46.00 & 28.87 & 10.96 & 141.36 & 39.32 & 67.01 & 5.35 \\
\hline 7549.79 & 229603.00 & 18.00 & 289.22 & 69.23 & 59.37 & 19286.67 & 105.67 & 120.02 & 29.00 & 11.26 & 127.65 & 35.96 & 70.23 & 5.44 \\
\hline 7549.86 & 273021.00 & 17.60 & 368.24 & 74.94 & 67.49 & 21825.27 & 112.82 & 143.74 & 35.09 & 12.46 & 124.01 & 30.88 & 76.30 & 6.08 \\
\hline 7549.92 & 290796.80 & 17.40 & 519.27 & 81.60 & 70.52 & 22474.10 & 117.90 & 151.63 & 38.20 & 13.30 & 139.97 & 33.14 & 82.97 & 7.86 \\
\hline 7549.99 & 317410.80 & 17.20 & 1538.29 & 80.04 & 67.35 & 22170.52 & 129.28 & 233.35 & 38.36 & 13.48 & 159.66 & 35.62 & 89.27 & 7.41 \\
\hline 7550.05 & 374548.40 & 16.60 & 1458.69 & 98.55 & 88.89 & 30341.97 & 144.64 & 377.72 & 50.73 & 15.14 & 223.76 & 85.30 & 98.06 & 5.88 \\
\hline 7550.12 & 377823.00 & 16.60 & 1469.82 & 95.06 & 91.20 & 31710.06 & 146.24 & 354.53 & 53.23 & 15.78 & 230.17 & 85.65 & 99.20 & 6.90 \\
\hline 7550.18 & 374629.80 & 16.60 & 1469.46 & 95.89 & 92.54 & 31780.85 & 142.93 & 355.52 & 52.48 & 15.57 & 225.43 & 84.29 & 99.83 & 7.69 \\
\hline 7550.25 & 358972.40 & 16.80 & 1431.60 & 94.62 & 86.59 & 31046.93 & 140.35 & 370.29 & 50.97 & 15.24 & 209.74 & 84.40 & 97.30 & 7.05 \\
\hline 7550.31 & 353442.60 & 16.80 & 387.01 & 90.41 & 81.85 & 32474.91 & 143.36 & 401.13 & 52.03 & 15.14 & 186.71 & 80.55 & 98.17 & 6.49 \\
\hline 7550.38 & 300396.60 & 17.40 & 347.60 & 68.30 & 55.15 & 23319.65 & 121.48 & 673.13 & 39.48 & 12.63 & 115.17 & 29.26 & 84.95 & 7.72 \\
\hline 7550.45 & 266541.60 & 17.60 & 331.30 & 61.89 & 49.62 & 20557.73 & 109.76 & 642.24 & 35.58 & 11.31 & 108.88 & 26.85 & 78.35 & 6.56 \\
\hline 7550.51 & 283958.00 & 17.40 & 297.90 & 67.82 & 50.02 & 22518.72 & 123.66 & 1367.03 & 36.62 & 12.33 & 112.41 & 30.08 & 85.73 & 6.65 \\
\hline 7550.58 & 306041.00 & 17.20 & 321.14 & 71.62 & 50.92 & 24975.89 & 128.64 & 1360.86 & 39.56 & 13.14 & 123.32 & 29.76 & 89.62 & 7.77 \\
\hline 7550.64 & 294468.00 & 17.20 & 365.77 & 72.88 & 53.74 & 22566.33 & 116.30 & 1264.60 & 37.04 & 12.78 & 154.13 & 33.97 & 84.31 & 8.00 \\
\hline 7550.71 & 309010.20 & 17.00 & 391.27 & 74.27 & 56.26 & 23014.03 & 123.87 & 890.66 & 36.88 & 13.68 & 156.24 & 35.02 & 89.57 & 8.41 \\
\hline 7550.77 & 309347.60 & 17.00 & 407.83 & 75.11 & 59.03 & 24596.63 & 128.95 & 898.85 & 38.21 & 14.07 & 158.61 & 34.51 & 91.89 & 9.02 \\
\hline 7550.84 & 263291.00 & 17.40 & 361.14 & 65.22 & 54.24 & 20615.44 & 111.22 & 207.49 & 33.39 & 12.78 & 151.82 & 31.92 & 81.69 & 7.92 \\
\hline 7550.91 & 250985.20 & 17.60 & 325.71 & 67.21 & 62.04 & 20610.09 & 122.66 & 1982.75 & 30.87 & 12.04 & 157.50 & 32.75 & 85.70 & 5.95 \\
\hline 7550.97 & 220401.20 & 18.00 & 504.07 & 66.53 & 68.03 & 21529.23 & 137.26 & 1979.92 & 71.40 & 16.63 & 127.50 & 64.43 & 79.21 & 26.58 \\
\hline 7551.04 & 211738.00 & 18.20 & 4870.56 & 62.58 & 72.00 & 18419.53 & 118.26 & 2071.50 & 66.17 & 14.73 & 148.46 & 65.56 & 69.36 & 26.56 \\
\hline 7551.10 & 176997.00 & 18.60 & 5684.16 & 61.06 & 80.97 & 13608.49 & 106.97 & 2044.79 & 66.54 & 12.26 & 125.96 & 61.57 & 53.84 & 28.28 \\
\hline 7551.17 & 209006.20 & 18.40 & 5661.20 & 49.27 & 89.11 & 10516.94 & 88.26 & 1982.34 & 62.52 & 10.30 & 147.52 & 62.30 & 37.40 & 26.64 \\
\hline 7551.23 & 219358.20 & 18.20 & 5605.96 & 32.60 & 89.05 & 5384.22 & 56.45 & 181.33 & 56.68 & 8.27 & 149.84 & 60.40 & 16.81 & 26.52 \\
\hline
\end{tabular}




\begin{tabular}{|c|c|c|c|c|c|c|c|c|c|c|c|c|c|c|}
\hline Depth & XRF & XRF Live Time & $\mathrm{Ti}(\mathrm{ppm})$ & $\mathrm{Cr}(\mathrm{ppm})$ & $\mathrm{Mn}(\mathrm{ppm})$ & $\mathrm{Fe}(\mathrm{ppm})$ & $\mathrm{Ni}(\mathrm{ppm})$ & $\mathrm{Cu}(\mathrm{ppm})$ & $\mathrm{Zn}(\mathrm{ppm})$ & As (ppm) & $\mathrm{Rb}(\mathrm{ppm})$ & $\mathrm{Sr}(\mathrm{ppm})$ & $\mathrm{Y}(\mathrm{ppm})$ & $\mathrm{Zr}(\mathrm{ppm})$ \\
\hline 7551.30 & 264826.60 & 17.80 & 5347.83 & 20.18 & 88.99 & 1810.61 & 25.50 & 157.18 & 11.87 & 1.94 & 166.74 & 24.24 & 10.39 & 5.33 \\
\hline 7551.36 & 286170.20 & 17.60 & 984.79 & 12.67 & 92.08 & 1829.35 & 26.39 & 15.42 & 10.89 & 2.01 & 170.96 & 22.50 & 6.48 & 4.61 \\
\hline 7551.43 & 347045.00 & 17.00 & 101.51 & 5.05 & 86.67 & 2148.34 & 16.02 & 10.69 & 4.50 & 2.39 & 205.23 & 27.44 & 6.79 & 1.51 \\
\hline 7551.50 & 336487.20 & 17.00 & 92.65 & 5.45 & 81.96 & 2043.20 & 12.67 & 4.73 & 4.11 & 1.81 & 199.93 & 26.37 & 6.40 & 1.94 \\
\hline 7551.56 & 319662.20 & 17.20 & 61.31 & 3.69 & 84.47 & 1869.15 & 12.42 & 4.95 & 3.11 & 1.44 & 205.89 & 25.31 & 7.12 & 1.55 \\
\hline 7551.63 & 294543.20 & 17.40 & 69.02 & 2.08 & 82.20 & 1389.93 & 10.91 & 4.10 & 2.97 & 1.25 & 212.59 & 24.04 & 8.87 & 1.46 \\
\hline 7551.69 & 274451.40 & 17.60 & 86.33 & 2.74 & 82.39 & 1142.57 & 10.65 & 6.25 & 3.65 & 0.68 & 215.61 & 23.41 & 8.55 & 1.13 \\
\hline 7551.76 & 244490.20 & 17.80 & 100.94 & 6.71 & 81.80 & 967.64 & 12.66 & 6.71 & 3.10 & 0.47 & 256.38 & 24.19 & 7.78 & -0.20 \\
\hline 7551.82 & 272499.80 & 17.60 & 87.07 & 5.22 & 85.67 & 862.04 & 13.90 & 7.43 & 3.03 & 0.87 & 299.33 & 25.46 & 6.88 & -0.55 \\
\hline 7551.89 & 311986.60 & 17.20 & 63.80 & 5.25 & 86.62 & 749.75 & 11.96 & 6.30 & 3.00 & 1.85 & 317.72 & 29.89 & 5.15 & -0.27 \\
\hline 7551.96 & 352636.80 & 16.80 & 35.97 & 5.51 & 85.05 & 737.30 & 11.92 & 5.97 & 2.80 & 2.01 & 317.63 & 29.60 & 3.87 & -0.75 \\
\hline 7552.02 & 384748.60 & 16.40 & 730.58 & 4.50 & 79.65 & 721.51 & 11.69 & 6.64 & 2.80 & 2.35 & 316.02 & 29.62 & 3.73 & -0.17 \\
\hline 7552.09 & 432131.60 & 16.00 & 683.70 & 0.82 & 79.32 & 685.93 & 10.37 & 6.80 & 2.96 & 1.87 & 235.71 & 89.21 & 8.07 & -0.10 \\
\hline 7552.15 & 425567.60 & 16.00 & 628.33 & 2.63 & 84.77 & 3259.25 & 8.21 & 6.20 & 5.65 & 1.90 & 284.13 & 91.36 & 9.29 & 0.04 \\
\hline 7552.22 & 405503.40 & 16.20 & 625.45 & 1.43 & 79.55 & 4193.66 & 9.01 & 8.37 & 6.60 & 2.25 & 221.01 & 148.18 & 15.60 & -0.25 \\
\hline 7552.28 & 420282.40 & 16.00 & 627.97 & 1.88 & 88.39 & 6628.14 & 13.41 & 7.36 & 6.96 & 2.10 & 263.40 & 150.85 & 16.57 & -0.23 \\
\hline 7552.35 & 421771.60 & 16.00 & -72.58 & 2.42 & 93.79 & 12773.74 & 23.12 & 6.15 & 10.61 & 2.67 & 271.47 & 153.14 & 19.89 & -0.22 \\
\hline 7552.41 & 385592.40 & 16.40 & 97.55 & 19.71 & 93.38 & 17622.55 & 53.43 & 7.26 & 18.18 & 6.28 & 308.01 & 93.68 & 35.81 & 2.81 \\
\hline 7552.48 & 383992.60 & 16.40 & 359.05 & 30.06 & 85.43 & 20729.29 & 92.37 & 11.28 & 24.89 & 10.13 & 222.53 & 95.96 & 65.60 & 4.93 \\
\hline 7552.55 & 395860.80 & 16.20 & 522.73 & 43.16 & 81.62 & 25749.97 & 131.72 & 11.04 & 33.18 & 12.79 & 258.85 & 37.56 & 86.05 & 7.33 \\
\hline 7552.61 & 379185.60 & 16.40 & 698.86 & 60.61 & 73.22 & 30790.33 & 167.91 & 16.66 & 41.43 & 16.31 & 221.56 & 39.83 & 130.52 & 10.48 \\
\hline 7552.68 & 363977.80 & 16.60 & 789.77 & 71.43 & 67.20 & 30079.75 & 190.13 & 18.53 & 45.73 & 18.49 & 196.23 & 39.38 & 154.38 & 12.51 \\
\hline 7552.74 & 383223.20 & 16.40 & 811.56 & 64.17 & 74.82 & 28336.05 & 175.17 & 18.11 & 44.05 & 16.62 & 251.04 & 53.48 & 157.81 & 11.98 \\
\hline 7552.81 & 324156.20 & 17.00 & 660.14 & 58.54 & 64.34 & 24313.74 & 141.76 & 16.05 & 39.09 & 12.92 & 274.20 & 45.54 & 160.99 & 12.71 \\
\hline 7552.87 & 304320.60 & 17.20 & 1059.55 & 55.46 & 73.73 & 21522.68 & 105.04 & 14.67 & 40.22 & 10.07 & 293.30 & 44.90 & 243.79 & 13.81 \\
\hline 7552.94 & 278620.00 & 17.40 & 998.99 & 47.28 & 68.09 & 17192.60 & 81.98 & 10.94 & 36.91 & 7.76 & 288.26 & 43.33 & 221.60 & 12.85 \\
\hline 7553.01 & 228668.00 & 17.80 & 1136.24 & 48.13 & 66.62 & 15091.36 & 70.18 & 33.46 & 34.36 & 7.32 & 285.55 & 42.19 & 209.59 & 12.05 \\
\hline 7553.07 & 226510.40 & 17.80 & 1119.21 & 53.32 & 54.01 & 16692.33 & 94.03 & 61.45 & 31.46 & 15.08 & 212.45 & 52.31 & 191.30 & 29.81 \\
\hline 7553.14 & 271922.40 & 17.40 & 1180.31 & 60.58 & 59.04 & 19926.24 & 115.83 & 61.53 & 33.97 & 17.96 & 177.91 & 56.74 & 175.52 & 28.34 \\
\hline 7553.20 & 279140.40 & 17.40 & 683.82 & 63.86 & 49.32 & 23477.01 & 148.53 & 65.23 & 30.71 & 20.06 & 141.78 & 54.96 & 84.08 & 26.26 \\
\hline 7553.27 & 272129.20 & 17.60 & 731.70 & 64.88 & 49.72 & 23483.34 & 147.65 & 67.06 & 31.01 & 20.23 & 120.83 & 51.37 & 76.10 & 26.06 \\
\hline 7553.33 & 322677.00 & 17.20 & 671.72 & 66.42 & 47.71 & 24057.13 & 151.31 & 45.07 & 31.69 & 19.80 & 119.68 & 52.93 & 84.17 & 25.79 \\
\hline 7553.40 & 337060.80 & 17.00 & 693.84 & 65.70 & 48.84 & 25874.18 & 150.25 & 20.02 & 37.82 & 14.25 & 133.48 & 32.90 & 106.16 & 6.99 \\
\hline
\end{tabular}




\begin{tabular}{|c|c|c|c|c|c|c|c|c|c|c|c|c|c|c|}
\hline Depth & XRF & XRF Live Time & $\mathrm{Ti}(\mathrm{ppm})$ & $\mathrm{Cr}(\mathrm{ppm})$ & $\mathrm{Mn}(\mathrm{ppm})$ & $\mathrm{Fe}(\mathrm{ppm})$ & $\mathrm{Ni}(\mathrm{ppm})$ & $\mathrm{Cu}(\mathrm{ppm})$ & $\mathrm{Zn}(\mathrm{ppm})$ & As (ppm) & $\mathrm{Rb}$ (ppm) & $\mathrm{Sr}(\mathrm{ppm})$ & $\mathrm{Y}(\mathrm{ppm})$ & $\mathrm{Zr}(\mathrm{ppm})$ \\
\hline 7553.46 & 346883.60 & 16.80 & 866.50 & 66.08 & 52.73 & 28563.62 & 152.51 & 21.30 & 38.91 & 13.69 & 130.84 & 31.98 & 107.96 & 8.00 \\
\hline 7553.53 & 346374.00 & 16.80 & 954.35 & 67.50 & 51.43 & 25961.29 & 145.10 & 19.99 & 38.81 & 13.79 & 140.76 & 34.65 & 113.15 & 11.60 \\
\hline 7553.60 & 349327.80 & 16.80 & 820.23 & 69.50 & 49.84 & 25915.34 & 152.28 & 18.21 & 39.38 & 14.58 & 170.30 & 36.54 & 124.11 & 13.28 \\
\hline 7553.66 & 315589.80 & 17.00 & 713.64 & 64.32 & 49.76 & 23604.50 & 131.38 & 15.64 & 39.91 & 13.08 & 194.31 & 31.20 & 123.02 & 13.53 \\
\hline 7553.73 & 291206.60 & 17.40 & 733.19 & 63.51 & 46.45 & 19909.84 & 111.46 & 12.59 & 36.74 & 10.98 & 188.05 & 24.08 & 113.75 & 15.52 \\
\hline 7553.79 & 270577.00 & 17.60 & 574.12 & 65.16 & 39.47 & 16059.85 & 108.82 & 22.69 & 34.42 & 10.94 & 181.94 & 24.49 & 116.98 & 15.62 \\
\hline 7553.86 & 271992.00 & 17.60 & 566.10 & 69.15 & 45.73 & 18219.08 & 118.14 & 47.68 & 37.69 & 11.68 & 180.05 & 23.56 & 119.85 & 12.71 \\
\hline 7553.92 & 257876.00 & 17.60 & 733.23 & 66.63 & 46.56 & 17280.48 & 111.00 & 47.39 & 35.71 & 10.89 & 159.65 & 22.61 & 108.95 & 10.85 \\
\hline 7553.99 & 262259.60 & 17.60 & 967.71 & 66.64 & 47.01 & 19211.96 & 127.41 & 53.39 & 34.93 & 11.90 & 143.15 & 28.49 & 104.87 & 10.89 \\
\hline 7554.06 & 244140.00 & 17.60 & 1161.28 & 66.48 & 54.58 & 20119.86 & 138.07 & 70.36 & 34.29 & 12.17 & 151.45 & 32.66 & 106.62 & 11.76 \\
\hline 7554.12 & 232917.00 & 17.80 & 1383.48 & 58.01 & 61.67 & 20108.36 & 143.40 & 87.43 & 34.57 & 16.76 & 146.38 & 70.83 & 91.22 & 27.76 \\
\hline 7554.19 & 172498.20 & 18.40 & 1647.07 & 39.64 & 62.53 & 14048.39 & 118.54 & 67.16 & 25.24 & 14.19 & 158.58 & 68.47 & 64.96 & 27.23 \\
\hline 7554.25 & 183970.40 & 18.40 & 1497.77 & 33.04 & 69.53 & 12982.31 & 108.51 & 68.00 & 22.39 & 13.33 & 184.04 & 71.70 & 53.29 & 26.39 \\
\hline 7554.32 & 199989.60 & 18.20 & 1233.79 & 27.21 & 68.23 & 10177.17 & 92.72 & 62.12 & 17.59 & 11.75 & 200.83 & 68.87 & 36.63 & 25.23 \\
\hline 7554.38 & 218575.00 & 18.20 & 830.36 & 11.99 & 65.86 & 6589.28 & 64.72 & 43.86 & 12.44 & 9.69 & 222.03 & 70.46 & 17.97 & 22.27 \\
\hline 7554.45 & 228355.60 & 18.20 & 480.31 & 6.35 & 63.92 & 3556.99 & 32.95 & 13.40 & 6.69 & 2.29 & 271.12 & 37.60 & 12.10 & 3.00 \\
\hline 7554.51 & 285740.80 & 17.60 & 511.53 & 18.28 & 65.21 & 9536.06 & 63.90 & 39.34 & 13.40 & 8.02 & 247.81 & 94.12 & 20.59 & 24.11 \\
\hline 7554.58 & 301712.60 & 17.40 & 576.86 & 19.64 & 65.64 & 10292.03 & 76.71 & 43.65 & 16.72 & 9.01 & 266.37 & 95.38 & 25.36 & 24.63 \\
\hline 7554.65 & 305783.20 & 17.40 & 618.75 & 26.53 & 70.01 & 11860.57 & 100.82 & 71.73 & 20.24 & 10.96 & 274.85 & 99.55 & 44.44 & 25.18 \\
\hline 7554.71 & 308088.80 & 17.20 & 804.99 & 46.09 & 65.92 & 15868.31 & 152.21 & 128.90 & 30.36 & 15.04 & 261.88 & 97.77 & 71.11 & 27.35 \\
\hline 7554.78 & 306908.00 & 17.20 & 939.02 & 55.60 & 62.02 & 18104.61 & 206.12 & 153.43 & 37.37 & 18.65 & 238.45 & 91.15 & 100.70 & 31.43 \\
\hline 7554.84 & 314530.00 & 17.20 & 529.27 & 43.83 & 68.11 & 12092.59 & 163.95 & 146.98 & 31.09 & 12.12 & 283.70 & 38.64 & 93.76 & 9.25 \\
\hline 7554.91 & 316759.60 & 17.20 & 431.50 & 38.29 & 80.47 & 10498.65 & 147.33 & 141.94 & 26.38 & 10.55 & 257.14 & 35.08 & 87.23 & 7.59 \\
\hline 7554.97 & 324904.00 & 17.20 & 329.27 & 30.44 & 92.71 & 8455.80 & 121.20 & 113.98 & 22.78 & 8.31 & 248.48 & 31.72 & 66.31 & 6.18 \\
\hline 7555.04 & 272998.60 & 17.80 & 979.05 & 17.44 & 114.77 & 4289.10 & 79.21 & 61.57 & 10.83 & 5.46 & 211.46 & 25.34 & 42.82 & 4.47 \\
\hline 7555.10 & 284978.20 & 17.60 & 1295.73 & 11.49 & 124.89 & 2476.45 & 53.31 & 41.30 & 5.94 & 2.91 & 242.37 & 27.69 & 26.30 & 2.33 \\
\hline 7555.17 & 261512.20 & 17.80 & 1355.84 & 15.85 & 124.36 & 2744.41 & 55.10 & 17.07 & 6.56 & 2.99 & 228.57 & 30.31 & 37.51 & 3.74 \\
\hline 7555.24 & 202914.60 & 18.20 & 1894.73 & 19.41 & 116.81 & 4457.82 & 58.28 & 16.20 & 12.58 & 3.97 & 219.19 & 27.36 & 77.67 & 6.65 \\
\hline 7555.30 & 178524.40 & 18.40 & 2721.94 & 22.90 & 109.50 & 7040.14 & 60.65 & 15.86 & 16.29 & 4.83 & 198.81 & 28.22 & 161.92 & 9.77 \\
\hline 7555.37 & 199978.20 & 18.20 & 2367.43 & 20.57 & 90.70 & 9252.48 & 54.57 & 11.52 & 22.85 & 4.95 & 225.36 & 32.37 & 230.81 & 10.76 \\
\hline 7555.43 & 171936.40 & 18.40 & 2039.86 & 22.04 & 83.87 & 9179.44 & 42.91 & 6.52 & 21.97 & 4.75 & 189.56 & 30.67 & 235.93 & 11.40 \\
\hline 7555.50 & 178870.20 & 18.40 & 1846.11 & 29.34 & 70.76 & 11632.93 & 88.54 & 46.94 & 26.07 & 7.03 & 188.36 & 27.35 & 245.40 & 12.21 \\
\hline 7555.56 & 225019.20 & 18.00 & 1199.26 & 37.63 & 57.72 & 12172.91 & 128.60 & 51.06 & 24.48 & 8.60 & 191.47 & 33.81 & 222.53 & 12.13 \\
\hline
\end{tabular}




\begin{tabular}{|c|c|c|c|c|c|c|c|c|c|c|c|c|c|c|}
\hline Depth & XRF & XRF Live Time & $\mathrm{Ti}(\mathrm{ppm})$ & $\mathrm{Cr}(\mathrm{ppm})$ & $\mathrm{Mn}(\mathrm{ppm})$ & $\mathrm{Fe}(\mathrm{ppm})$ & $\mathrm{Ni}(\mathrm{ppm})$ & $\mathrm{Cu}(\mathrm{ppm})$ & $\mathrm{Zn}(\mathrm{ppm})$ & As (ppm) & $\mathrm{Rb}$ (ppm) & $\mathrm{Sr}(\mathrm{ppm})$ & $\mathrm{Y}(\mathrm{ppm})$ & $\mathrm{Zr}(\mathrm{ppm})$ \\
\hline 7555.63 & 234912.00 & 18.00 & 241.03 & 45.77 & 47.55 & 11692.55 & 164.79 & 61.80 & 23.96 & 10.36 & 200.73 & 34.03 & 154.21 & 11.04 \\
\hline 7555.70 & 256553.40 & 17.80 & -318.96 & 50.03 & 42.49 & 11672.65 & 198.54 & 64.98 & 21.94 & 11.32 & 211.80 & 35.68 & 98.08 & 9.36 \\
\hline 7555.76 & 274370.20 & 17.80 & -487.10 & 57.49 & 39.99 & 13414.58 & 224.14 & 68.34 & 24.58 & 13.85 & 227.82 & 38.82 & 96.27 & 8.90 \\
\hline 7555.83 & 271544.40 & 17.80 & -435.67 & 52.49 & 37.98 & 12162.05 & 193.28 & 26.59 & 21.59 & 12.64 & 260.01 & 42.55 & 88.83 & 7.04 \\
\hline 7555.89 & 273309.40 & 17.80 & -383.57 & 47.51 & 39.78 & 15655.42 & 156.79 & 38.22 & 17.88 & 11.52 & 273.06 & 40.64 & 73.40 & 4.42 \\
\hline 7555.96 & 275566.20 & 17.60 & -347.02 & 51.00 & 41.82 & 16430.56 & 172.14 & 28.64 & 18.51 & 11.84 & 290.57 & 40.96 & 81.87 & 3.50 \\
\hline 7556.02 & 290284.40 & 17.40 & -330.82 & 55.72 & 45.01 & 17368.49 & 168.58 & 26.76 & 20.89 & 12.52 & 308.05 & 40.78 & 84.76 & 3.85 \\
\hline 7556.09 & 296820.40 & 17.20 & -329.81 & 45.50 & 48.47 & 15875.90 & 140.36 & 24.66 & 18.38 & 9.92 & 329.33 & 37.42 & 70.07 & 3.51 \\
\hline 7556.15 & 303572.00 & 17.00 & -235.73 & 47.86 & 47.92 & 16839.51 & 146.38 & 51.60 & 20.48 & 11.37 & 305.24 & 31.23 & 70.44 & 4.21 \\
\hline 7556.22 & 314484.00 & 17.00 & -469.08 & 62.08 & 44.01 & 15326.36 & 186.75 & 44.58 & 30.95 & 15.18 & 325.85 & 31.13 & 99.27 & 6.33 \\
\hline 7556.29 & 318397.00 & 17.00 & -210.48 & 59.47 & 43.92 & 15529.55 & 179.55 & 77.86 & 34.27 & 16.03 & 314.08 & 29.15 & 98.11 & 7.19 \\
\hline 7556.35 & 325544.00 & 17.00 & -93.59 & 65.76 & 49.80 & 17864.29 & 199.24 & 97.21 & 40.57 & 18.41 & 303.99 & 28.91 & 107.35 & 6.94 \\
\hline 7556.42 & 306111.20 & 17.20 & -8.59 & 72.94 & 45.72 & 19241.46 & 234.11 & 118.88 & 43.52 & 21.37 & 274.40 & 27.46 & 130.52 & 7.76 \\
\hline 7556.48 & 318911.60 & 17.20 & -10.38 & 69.80 & 45.03 & 24749.27 & 213.66 & 90.35 & 39.46 & 18.76 & 248.70 & 22.12 & 116.21 & 7.52 \\
\hline 7556.55 & 289246.40 & 17.40 & 270.56 & 52.00 & 44.34 & 20135.72 & 163.61 & 80.46 & 27.17 & 13.32 & 218.75 & 16.68 & 84.39 & 5.47 \\
\hline 7556.61 & 268039.20 & 17.60 & 118.38 & 40.46 & 39.38 & 16698.60 & 114.85 & 45.93 & 18.22 & 9.78 & 221.67 & 14.11 & 60.76 & 3.86 \\
\hline 7556.68 & 238536.40 & 17.80 & 3.15 & 24.07 & 27.94 & 12216.62 & 59.05 & 24.09 & 6.57 & 4.56 & 230.51 & 8.13 & 31.79 & 2.99 \\
\hline 7556.75 & 250483.20 & 17.80 & -84.41 & 15.65 & 34.39 & 9588.35 & 10.73 & 2.05 & 1.82 & 1.00 & 245.42 & 7.32 & 4.91 & 0.79 \\
\hline 7556.81 & 249865.60 & 17.80 & -121.36 & 10.48 & 39.07 & 1518.87 & 5.68 & 1.54 & 1.47 & 0.48 & 263.01 & 9.47 & 6.55 & -0.17 \\
\hline 7556.88 & 285911.60 & 17.60 & -130.38 & 9.81 & 54.77 & 1651.18 & 5.58 & 1.86 & 2.39 & 0.51 & 256.88 & 12.18 & 3.58 & -0.20 \\
\hline 7556.94 & 324541.60 & 17.20 & -157.76 & 8.00 & 74.60 & 1740.30 & 3.91 & 2.14 & 2.93 & 0.92 & 259.34 & 15.94 & 4.60 & -0.97 \\
\hline 7557.01 & 360147.80 & 17.00 & -102.25 & 9.22 & 85.32 & 801.06 & 7.54 & 106.86 & 2.80 & 0.90 & 245.16 & 20.94 & 5.52 & -0.84 \\
\hline
\end{tabular}




\begin{tabular}{|c|c|c|c|c|c|c|c|c|c|c|c|c|c|}
\hline Depth & $\mathrm{XRF}$ & XRF Live Time & $\mathrm{Nb}(\mathrm{ppm})$ & Mo (ppm) & $\mathrm{Ba}(\mathrm{ppm})$ & $\mathrm{Pb}(\mathrm{ppm})$ & Th (ppm) & $\mathrm{U}(\mathrm{ppm})$ & $\mathrm{P}(\mathrm{ppm})$ & $\mathrm{S}(\%)$ & $\mathrm{Cl}(\%)$ & $\mathrm{K}(\%)$ & $\mathrm{Ca}(\%)$ \\
\hline 7445.20 & 305810.60 & 17.20 & 24.11 & 65.99 & 86.95 & 9.32 & 4.62 & -138.90 & -5013.98 & 1.98 & 10.00 & 1.39 & 19.98 \\
\hline 7445.26 & 371646.40 & 16.60 & 36.15 & 72.06 & 1.78 & -2.39 & 5.05 & -177.20 & -3943.92 & 2.46 & 6.70 & 1.99 & 19.94 \\
\hline 7445.33 & 323820.60 & 17.00 & 35.73 & 99.99 & 5.12 & 3.95 & 6.11 & -223.29 & -2690.28 & 2.75 & 7.22 & 2.22 & 20.01 \\
\hline 7445.39 & 323086.40 & 17.00 & 40.92 & 100.01 & 7.64 & 5.80 & 5.33 & -208.12 & -3229.68 & 2.73 & 7.25 & 2.27 & 19.98 \\
\hline 7445.46 & 320356.60 & 17.00 & 55.39 & 88.16 & 9.63 & 6.89 & 6.91 & -176.13 & 301.06 & 2.78 & 7.06 & 2.72 & 8.06 \\
\hline 7445.52 & 312668.20 & 17.20 & 66.98 & 69.41 & 11.04 & 3.98 & 8.43 & -217.11 & -1294.89 & 2.63 & 1.57 & 3.31 & 0.42 \\
\hline 7445.59 & 314748.00 & 17.20 & 66.92 & 64.07 & 10.70 & 3.84 & 7.71 & -197.31 & -816.43 & 2.69 & 1.55 & 3.38 & 0.40 \\
\hline 7445.66 & 377131.00 & 16.60 & 74.16 & 40.94 & 9.12 & -2.95 & 6.84 & -164.66 & -1193.89 & 2.66 & 0.99 & 3.38 & 0.30 \\
\hline 7445.72 & 386247.20 & 16.40 & 75.99 & 41.74 & 7.59 & -4.49 & 6.48 & -183.75 & -531.89 & 2.99 & 1.07 & 3.42 & 0.37 \\
\hline 7445.79 & 390361.80 & 16.40 & 71.63 & 40.41 & 4.87 & -5.13 & 5.86 & -215.50 & -169.69 & 3.06 & 0.96 & 3.46 & 0.44 \\
\hline 7445.85 & 401397.20 & 16.20 & 67.19 & 41.25 & 1.40 & -3.07 & 5.77 & -219.73 & -89.39 & 3.27 & 0.71 & 3.51 & 0.53 \\
\hline 7445.92 & 414612.00 & 16.00 & 65.02 & 59.50 & 2.63 & -2.10 & 6.48 & -253.16 & 283.36 & 3.65 & 0.61 & 3.52 & 0.64 \\
\hline 7445.98 & 422822.60 & 16.00 & 57.95 & 66.03 & 1.36 & -7.99 & 7.18 & -268.06 & -159.72 & 3.87 & 0.74 & 3.54 & 0.77 \\
\hline 7446.05 & 428555.80 & 16.00 & 50.69 & 72.11 & 1.39 & -8.75 & 7.74 & -264.57 & -73.95 & 3.97 & 0.81 & 3.63 & 0.80 \\
\hline 7446.12 & 429538.00 & 16.00 & 48.18 & 73.51 & 0.60 & -9.64 & 8.89 & -272.07 & -938.31 & 4.05 & 0.99 & 3.68 & 0.81 \\
\hline 7446.18 & 424836.80 & 16.00 & 46.20 & 76.92 & 1.73 & -10.21 & 7.93 & -247.61 & -166.32 & 4.12 & 1.20 & 3.71 & 0.80 \\
\hline 7446.25 & 430787.80 & 16.00 & 44.97 & 72.29 & 2.70 & -12.98 & 8.34 & -253.80 & -427.96 & 3.96 & 1.25 & 3.74 & 0.77 \\
\hline 7446.31 & 437003.40 & 16.00 & 48.41 & 66.79 & 2.76 & -7.89 & 7.89 & -256.35 & -591.86 & 3.90 & 1.05 & 3.83 & 0.70 \\
\hline 7446.38 & 435271.20 & 16.00 & 52.53 & 63.35 & 1.86 & -4.49 & 9.29 & -263.53 & -1175.38 & 3.92 & 0.83 & 3.82 & 0.68 \\
\hline 7446.44 & 439332.40 & 16.00 & 58.66 & 64.90 & 2.90 & -1.81 & 9.13 & -240.60 & -289.38 & 3.90 & 0.72 & 3.86 & 0.67 \\
\hline 7446.51 & 446153.40 & 16.00 & 64.94 & 63.54 & 4.84 & -1.90 & 12.89 & -280.70 & 111.53 & 4.01 & 0.71 & 3.91 & 0.63 \\
\hline 7446.57 & 438917.80 & 16.00 & 71.15 & 59.89 & 4.47 & 2.61 & 14.22 & -277.31 & -379.47 & 4.33 & 1.08 & 3.89 & 0.89 \\
\hline 7446.64 & 401463.20 & 16.40 & 72.52 & 52.77 & 3.67 & 5.22 & 14.21 & -278.03 & -373.92 & 4.15 & 1.15 & 3.85 & 0.82 \\
\hline 7446.71 & 405810.60 & 16.40 & 74.29 & 55.82 & 5.64 & 4.48 & 12.61 & -289.09 & -232.32 & 3.76 & 1.26 & 3.90 & 0.71 \\
\hline 7446.77 & 408644.00 & 16.40 & 77.11 & 56.09 & 6.94 & 5.17 & 11.60 & -281.86 & 356.45 & 3.64 & 1.18 & 3.96 & 0.63 \\
\hline 7446.84 & 408767.00 & 16.40 & 65.34 & 59.60 & 298.44 & 8.93 & 11.04 & -208.72 & 591.92 & 3.28 & 1.06 & 3.97 & 0.57 \\
\hline 7446.90 & 408101.80 & 16.40 & 63.96 & 59.65 & 297.26 & 8.56 & 8.88 & -193.47 & 508.09 & 2.78 & 0.59 & 4.05 & 0.23 \\
\hline 7446.97 & 435316.20 & 16.00 & 62.77 & 65.11 & 298.18 & 6.44 & 8.28 & -189.14 & 203.20 & 2.87 & 0.60 & 4.04 & 0.26 \\
\hline 7447.03 & 432101.60 & 16.00 & 56.09 & 58.10 & 295.53 & 4.47 & 8.40 & -196.67 & 1262.70 & 3.13 & 0.42 & 4.01 & 0.27 \\
\hline 7447.10 & 414341.20 & 16.20 & 45.45 & 52.34 & 294.86 & 1.43 & 9.59 & -204.32 & 507.44 & 3.24 & 0.86 & 3.87 & 0.34 \\
\hline 7447.17 & 380105.80 & 16.60 & 48.71 & 38.34 & 1.21 & -2.31 & 6.64 & -268.13 & -1565.56 & 3.28 & 0.71 & 3.76 & 0.42 \\
\hline 7447.23 & 347241.40 & 17.00 & 38.78 & 33.46 & 1.39 & -4.21 & 8.24 & -269.53 & -2013.56 & 3.17 & 0.71 & 3.67 & 0.51 \\
\hline 7447.30 & 323673.00 & 17.20 & 34.50 & 29.95 & 0.36 & -8.46 & 7.51 & -284.28 & -2143.21 & 3.34 & 0.97 & 3.53 & 0.74 \\
\hline
\end{tabular}




\begin{tabular}{|c|c|c|c|c|c|c|c|c|c|c|c|c|c|}
\hline Depth & $\mathrm{XRF}$ & XRF Live Time & $\mathrm{Nb}(\mathrm{ppm})$ & Mo (ppm) & $\mathrm{Ba}(\mathrm{ppm})$ & $\mathrm{Pb}(\mathrm{ppm})$ & Th (ppm) & $\mathrm{U}(\mathrm{ppm})$ & $\mathrm{P}(\mathrm{ppm})$ & $\mathrm{S}(\%)$ & $\mathrm{Cl}(\%)$ & $\mathrm{K}(\%)$ & $\mathrm{Ca}(\%)$ \\
\hline 7447.36 & 297025.00 & 17.40 & 31.46 & 30.43 & 1.93 & -5.53 & 6.24 & -262.74 & -1935.08 & 3.37 & 1.56 & 3.33 & 0.92 \\
\hline 7447.43 & 277757.60 & 17.60 & 30.51 & 35.37 & 1.85 & -9.25 & 4.45 & -246.99 & -3496.64 & 3.56 & 2.14 & 3.22 & 0.89 \\
\hline 7447.49 & 247958.00 & 17.80 & 28.66 & 56.09 & -0.04 & -6.57 & 4.80 & -205.46 & -1591.17 & 3.46 & 3.83 & 2.97 & 0.91 \\
\hline 7447.56 & 227731.20 & 18.00 & 28.51 & 70.50 & -0.47 & -6.37 & 2.66 & -226.36 & -1487.87 & 3.59 & 3.92 & 2.89 & 0.84 \\
\hline 7447.62 & 195750.00 & 18.40 & 27.90 & 89.86 & -2.03 & -2.72 & 3.57 & -196.78 & -431.83 & 3.47 & 3.76 & 2.91 & 0.64 \\
\hline 7447.69 & 185697.20 & 18.60 & 25.68 & 92.02 & 273.67 & -0.47 & 6.46 & -144.32 & -1624.99 & 3.32 & 4.24 & 2.91 & 0.52 \\
\hline 7447.76 & 172000.40 & 18.60 & 28.65 & 91.95 & 273.61 & 0.77 & 5.70 & -169.33 & -2168.29 & 3.05 & 3.31 & 2.96 & 0.48 \\
\hline 7447.82 & 183755.20 & 18.60 & 31.56 & 99.25 & 275.31 & -5.36 & 7.40 & -184.95 & -4430.35 & 3.20 & 1.84 & 3.11 & 0.45 \\
\hline 7447.89 & 187026.20 & 18.60 & 36.33 & 81.13 & 275.50 & -4.16 & 8.00 & -129.51 & -3690.01 & 3.25 & 3.19 & 3.05 & 0.45 \\
\hline 7447.95 & 174821.00 & 18.60 & 37.00 & 74.53 & 277.02 & -6.98 & 6.90 & -139.42 & -5850.13 & 3.37 & 5.37 & 2.93 & 0.63 \\
\hline 7448.02 & 129653.20 & 19.00 & 36.23 & 162.73 & 1.76 & -5.22 & 7.92 & -136.78 & -5115.41 & 2.95 & 20.17 & 2.33 & 1.18 \\
\hline 7448.08 & 96745.20 & 19.40 & 27.95 & 189.16 & 2.61 & -10.71 & 7.53 & -72.28 & -678.47 & 2.47 & 24.21 & 1.69 & 1.26 \\
\hline 7448.15 & 132691.60 & 19.00 & 32.03 & 163.32 & 2.25 & -7.91 & 6.35 & -77.50 & -122.68 & 2.63 & 24.07 & 1.77 & 1.26 \\
\hline 7448.22 & 166648.80 & 18.60 & 35.71 & 168.72 & 1.75 & -8.91 & 6.12 & -110.10 & -30.48 & 2.83 & 22.69 & 1.87 & 1.35 \\
\hline 7448.28 & 220271.60 & 18.20 & 39.98 & 156.08 & 0.70 & -6.48 & 6.83 & -118.12 & 575.21 & 2.77 & 21.03 & 1.99 & 1.14 \\
\hline 7448.35 & 280514.00 & 17.60 & 52.17 & 62.41 & 0.70 & -10.51 & 3.31 & -146.44 & 207.94 & 3.09 & 5.93 & 2.64 & 0.55 \\
\hline 7448.41 & 349415.40 & 17.00 & 62.46 & 29.43 & -1.41 & 0.81 & 4.55 & -195.53 & -2814.06 & 3.48 & 1.82 & 3.33 & 0.42 \\
\hline 7448.48 & 356069.80 & 16.80 & 63.81 & 35.24 & -0.32 & 3.10 & 4.99 & -212.29 & -1703.96 & 3.35 & 1.81 & 3.38 & 0.38 \\
\hline 7448.54 & 364561.00 & 16.60 & 61.13 & 36.72 & 0.57 & 7.47 & 4.69 & -197.43 & -1389.37 & 3.16 & 1.85 & 3.43 & 0.26 \\
\hline 7448.61 & 374352.00 & 16.40 & 55.56 & 37.51 & 1.29 & 9.71 & 4.91 & -200.09 & -1244.12 & 3.10 & 1.37 & 3.46 & 0.26 \\
\hline 7448.67 & 388896.20 & 16.20 & 47.67 & 45.63 & -0.39 & 7.61 & 4.66 & -215.41 & -1762.66 & 3.59 & 0.72 & 3.52 & 0.40 \\
\hline 7448.74 & 395260.00 & 16.00 & 44.24 & 48.16 & -0.54 & 4.91 & 6.27 & -240.85 & -725.00 & 4.13 & 1.02 & 3.49 & 0.57 \\
\hline 7448.81 & 396206.40 & 16.00 & 40.59 & 46.92 & 0.86 & 3.44 & 4.39 & -220.35 & -1049.36 & 4.40 & 1.34 & 3.44 & 0.80 \\
\hline 7448.87 & 395120.00 & 16.20 & 40.41 & 44.77 & 0.59 & -0.17 & 4.29 & -259.99 & -491.65 & 4.78 & 1.50 & 3.39 & 0.96 \\
\hline 7448.94 & 403281.40 & 16.20 & 42.82 & 50.36 & 0.07 & -1.90 & 4.33 & -273.69 & -584.18 & 5.17 & 1.48 & 3.41 & 1.30 \\
\hline 7449.00 & 414548.40 & 16.20 & 45.03 & 50.22 & -0.01 & -3.06 & 5.69 & -281.14 & 2219.58 & 5.60 & 1.46 & 3.49 & 1.75 \\
\hline 7449.07 & 416971.60 & 16.20 & 43.79 & 54.89 & 0.40 & -2.79 & 5.21 & -264.28 & 1990.74 & 5.23 & 1.24 & 3.50 & 2.38 \\
\hline 7449.13 & 424317.80 & 16.20 & 41.15 & 63.94 & -2.33 & -4.53 & 6.63 & -285.10 & 2335.66 & 4.94 & 0.90 & 3.55 & 2.79 \\
\hline 7449.20 & 430918.20 & 16.00 & 32.79 & 63.96 & 302.64 & 0.65 & 7.27 & -219.56 & 2338.90 & 4.64 & 0.73 & 3.59 & 2.89 \\
\hline 7449.27 & 424115.20 & 16.00 & 31.96 & 61.41 & 304.34 & 0.37 & 7.78 & -212.75 & 3731.16 & 4.26 & 0.56 & 3.65 & 2.64 \\
\hline 7449.33 & 419627.20 & 16.00 & 34.25 & 55.84 & 303.85 & -0.62 & 8.71 & -228.20 & 519.77 & 3.75 & 0.52 & 3.63 & 2.08 \\
\hline 7449.40 & 422775.00 & 16.00 & 37.86 & 52.34 & 303.29 & -0.87 & 9.05 & -217.94 & 1035.82 & 4.13 & 0.62 & 3.69 & 1.35 \\
\hline 7449.46 & 426573.20 & 16.00 & 37.84 & 47.45 & 304.93 & 0.48 & 7.88 & -193.70 & 1583.22 & 4.34 & 0.83 & 3.68 & 0.79 \\
\hline
\end{tabular}




\begin{tabular}{|c|c|c|c|c|c|c|c|c|c|c|c|c|c|}
\hline Depth & $\mathrm{XRF}$ & XRF Live Time & $\mathrm{Nb}(\mathrm{ppm})$ & Mo (ppm) & $\mathrm{Ba}(\mathrm{ppm})$ & $\mathrm{Pb}(\mathrm{ppm})$ & Th (ppm) & $\mathrm{U}(\mathrm{ppm})$ & $\mathrm{P}(\mathrm{ppm})$ & $\mathrm{S}(\%)$ & $\mathrm{Cl}(\%)$ & $\mathrm{K}(\%)$ & $\mathrm{Ca}(\%)$ \\
\hline 7449.53 & 428196.80 & 16.00 & 42.52 & 51.68 & -0.11 & -2.09 & 8.88 & -228.84 & 725.38 & 4.46 & 1.24 & 3.64 & 0.60 \\
\hline 7449.59 & 431606.20 & 16.00 & 39.93 & 47.22 & -0.61 & -2.21 & 7.78 & -225.13 & 1669.33 & 4.53 & 1.33 & 3.61 & 0.54 \\
\hline 7449.66 & 430193.00 & 16.00 & 36.72 & 46.07 & 0.44 & -0.84 & 6.17 & -194.07 & 2440.81 & 4.52 & 1.47 & 3.60 & 0.62 \\
\hline 7449.72 & 425423.00 & 16.00 & 31.79 & 50.03 & 3.47 & 0.34 & 6.93 & -191.31 & 2415.13 & 4.14 & 1.77 & 3.55 & 0.62 \\
\hline 7449.79 & 415980.00 & 16.00 & 30.76 & 54.36 & 3.96 & -1.46 & 7.35 & -166.67 & 2053.20 & 4.18 & 1.71 & 3.51 & 0.70 \\
\hline 7449.86 & 410947.80 & 16.00 & 31.12 & 49.04 & 4.04 & -4.56 & 6.83 & -173.96 & 2017.86 & 4.09 & 1.50 & 3.54 & 0.68 \\
\hline 7449.92 & 405037.00 & 16.00 & 31.65 & 57.64 & 4.59 & -4.81 & 8.79 & -120.52 & 949.98 & 4.33 & 2.11 & 3.45 & 0.78 \\
\hline 7449.99 & 399671.80 & 16.00 & 30.24 & 59.17 & 3.95 & -1.98 & 9.44 & -121.29 & 1604.63 & 4.23 & 2.48 & 3.36 & 0.81 \\
\hline 7450.05 & 397577.20 & 16.00 & 32.35 & 61.99 & 0.38 & -2.85 & 6.70 & -128.36 & 3488.65 & 4.35 & 2.16 & 3.41 & 0.80 \\
\hline 7450.12 & 402237.20 & 16.00 & 31.65 & 56.17 & -0.83 & -0.06 & 6.45 & -163.68 & 3436.51 & 4.04 & 2.02 & 3.47 & 0.66 \\
\hline 7450.18 & 406786.80 & 16.00 & 29.25 & 56.16 & 0.84 & 1.09 & 5.61 & -147.92 & 3873.94 & 4.21 & 2.17 & 3.49 & 0.68 \\
\hline 7450.25 & 412036.40 & 16.00 & 28.70 & 53.94 & 1.14 & 1.05 & 4.20 & -175.24 & 4386.48 & 4.45 & 1.90 & 3.51 & 0.76 \\
\hline 7450.31 & 408955.20 & 16.20 & 26.50 & 53.55 & 2.63 & 1.48 & 3.66 & -195.39 & 2999.59 & 4.44 & 1.48 & 3.58 & 0.67 \\
\hline 7450.38 & 403548.80 & 16.40 & 25.23 & 42.48 & 3.53 & 3.87 & 4.94 & -200.29 & 707.44 & 4.46 & 1.93 & 3.52 & 0.64 \\
\hline 7450.45 & 405499.20 & 16.40 & 29.04 & 44.78 & 3.76 & 0.01 & 6.17 & -212.85 & 2151.43 & 5.24 & 2.28 & 3.51 & 0.78 \\
\hline 7450.51 & 402142.20 & 16.40 & 30.79 & 47.55 & 0.94 & -0.25 & 7.15 & -218.44 & 2881.60 & 5.04 & 2.21 & 3.47 & 0.73 \\
\hline 7450.58 & 396827.60 & 16.40 & 29.85 & 38.50 & -1.07 & 0.45 & 7.19 & -221.16 & 1060.41 & 4.40 & 1.89 & 3.53 & 0.46 \\
\hline 7450.64 & 393261.40 & 16.40 & 29.93 & 32.46 & -2.60 & 0.50 & 8.21 & -196.64 & 2620.01 & 4.18 & 1.91 & 3.50 & 0.44 \\
\hline 7450.71 & 393537.80 & 16.40 & 29.84 & 37.43 & -2.48 & -0.81 & 8.41 & -185.83 & 1688.14 & 4.15 & 1.71 & 3.53 & 0.44 \\
\hline 7450.77 & 377240.20 & 16.60 & 27.89 & 35.27 & -3.01 & 0.41 & 7.14 & -166.64 & 101.22 & 3.54 & 1.90 & 3.45 & 0.36 \\
\hline 7450.84 & 365402.80 & 16.80 & 29.96 & 32.17 & -2.03 & 2.21 & 5.77 & -163.48 & -367.22 & 3.44 & 1.68 & 3.49 & 0.33 \\
\hline 7450.91 & 358639.00 & 17.00 & 31.98 & 38.67 & -2.00 & 2.10 & 5.79 & -182.77 & 262.71 & 3.98 & 2.07 & 3.44 & 0.45 \\
\hline 7450.97 & 340866.40 & 17.20 & 31.49 & 41.75 & -1.50 & 1.30 & 4.35 & -185.41 & -824.85 & 3.71 & 2.07 & 3.40 & 0.41 \\
\hline 7451.04 & 323467.20 & 17.20 & 30.06 & 36.34 & -1.40 & -0.18 & 3.32 & -186.60 & 382.32 & 3.45 & 2.27 & 3.34 & 0.38 \\
\hline 7451.10 & 306298.40 & 17.40 & 27.30 & 33.09 & -0.88 & 2.27 & 3.20 & -182.25 & -898.04 & 3.32 & 2.21 & 3.35 & 0.32 \\
\hline 7451.17 & 303895.60 & 17.40 & 22.11 & 32.38 & -1.65 & -0.96 & 3.75 & -189.64 & -1179.78 & 3.23 & 2.23 & 3.29 & 0.34 \\
\hline 7451.23 & 303415.20 & 17.40 & 19.13 & 35.60 & -1.19 & 0.79 & 4.22 & -167.68 & -1774.14 & 2.67 & 1.69 & 3.33 & 0.27 \\
\hline 7451.30 & 322339.40 & 17.20 & 20.97 & 36.59 & -0.89 & -1.52 & 5.83 & -177.90 & -1190.66 & 2.99 & 1.57 & 3.35 & 0.31 \\
\hline 7451.36 & 340153.20 & 17.20 & 20.08 & 42.88 & 231.86 & 5.02 & 6.06 & -140.66 & -1025.95 & 3.27 & 1.14 & 3.38 & 0.43 \\
\hline 7451.43 & 357948.20 & 17.00 & 27.00 & 47.68 & 231.91 & 3.80 & 5.97 & -151.03 & -196.44 & 3.39 & 1.35 & 3.34 & 0.54 \\
\hline 7451.50 & 365863.00 & 17.00 & 34.30 & 48.13 & 231.32 & 3.91 & 6.38 & -171.81 & -1322.42 & 3.75 & 1.54 & 3.34 & 0.74 \\
\hline 7451.56 & 374015.00 & 16.80 & 39.64 & 40.27 & 231.35 & 3.80 & 5.88 & -202.87 & -1035.36 & 4.16 & 1.78 & 3.33 & 0.90 \\
\hline 7451.63 & 370049.80 & 16.80 & 41.27 & 37.49 & 231.66 & 2.73 & 5.64 & -181.21 & -938.47 & 4.52 & 2.42 & 3.29 & 1.14 \\
\hline
\end{tabular}




\begin{tabular}{|c|c|c|c|c|c|c|c|c|c|c|c|c|c|}
\hline Depth & $\mathrm{XRF}$ & XRF Live Time & $\mathrm{Nb}(\mathrm{ppm})$ & Mo (ppm) & $\mathrm{Ba}(\mathrm{ppm})$ & $\mathrm{Pb}(\mathrm{ppm})$ & Th (ppm) & $\mathrm{U}(\mathrm{ppm})$ & $\mathrm{P}(\mathrm{ppm})$ & $\mathrm{S}(\%)$ & $\mathrm{Cl}(\%)$ & $\mathrm{K}(\%)$ & $\mathrm{Ca}(\%)$ \\
\hline 7451.69 & 354960.60 & 16.80 & 47.61 & 39.71 & -0.74 & -1.35 & 5.84 & -226.20 & -525.19 & 4.68 & 2.60 & 3.30 & 1.14 \\
\hline 7451.76 & 373280.00 & 16.60 & 44.89 & 35.84 & -0.91 & 0.94 & 5.06 & -232.36 & 245.02 & 4.86 & 1.95 & 3.42 & 1.08 \\
\hline 7451.82 & 385653.20 & 16.40 & 42.38 & 39.10 & 1.18 & 3.55 & 6.00 & -218.55 & 1389.69 & 4.81 & 1.69 & 3.51 & 0.86 \\
\hline 7451.89 & 389104.20 & 16.40 & 39.13 & 40.87 & 2.36 & 2.58 & 5.63 & -182.21 & 1701.50 & 4.52 & 1.55 & 3.50 & 0.73 \\
\hline 7451.96 & 398777.00 & 16.20 & 37.82 & 47.96 & 1.39 & 3.65 & 6.02 & -224.03 & 930.34 & 4.05 & 1.48 & 3.52 & 0.47 \\
\hline 7452.02 & 421353.80 & 16.00 & 36.06 & 45.88 & 1.06 & -1.11 & 6.78 & -225.05 & 625.91 & 3.84 & 1.28 & 3.55 & 0.33 \\
\hline 7452.09 & 413887.40 & 16.00 & 36.98 & 47.67 & -0.13 & -1.85 & 9.27 & -220.50 & 353.99 & 3.61 & 1.18 & 3.56 & 0.26 \\
\hline 7452.15 & 412694.80 & 16.00 & 38.63 & 45.23 & -0.87 & -7.21 & 9.34 & -235.92 & -191.20 & 3.68 & 1.24 & 3.53 & 0.37 \\
\hline 7452.22 & 414772.00 & 16.00 & 41.13 & 52.36 & 0.27 & -6.40 & 9.49 & -247.35 & 518.18 & 3.72 & 1.28 & 3.57 & 0.31 \\
\hline 7452.28 & 414992.80 & 16.00 & 41.42 & 55.81 & 0.86 & -6.52 & 8.05 & -227.66 & 424.87 & 3.91 & 0.85 & 3.60 & 0.28 \\
\hline 7452.35 & 411796.60 & 16.00 & 40.25 & 55.94 & 0.76 & -6.49 & 8.03 & -223.55 & 37.11 & 4.07 & 0.77 & 3.60 & 0.28 \\
\hline 7452.41 & 418305.40 & 16.00 & 35.89 & 64.57 & 2.55 & -6.51 & 7.38 & -230.49 & 850.10 & 3.97 & 1.22 & 3.53 & 0.28 \\
\hline 7452.48 & 420522.00 & 16.00 & 30.61 & 66.28 & 3.85 & -2.20 & 6.62 & -219.47 & 732.39 & 3.78 & 1.31 & 3.49 & 0.20 \\
\hline 7452.55 & 423256.80 & 16.00 & 29.29 & 64.03 & 2.68 & -5.48 & 7.19 & -210.39 & 1000.37 & 3.76 & 1.44 & 3.42 & 0.25 \\
\hline 7452.61 & 428687.80 & 16.00 & 31.21 & 60.81 & 3.07 & -3.01 & 7.05 & -213.71 & 2806.95 & 4.42 & 1.89 & 3.31 & 0.60 \\
\hline 7452.68 & 453246.80 & 15.80 & 40.62 & 72.36 & 2.28 & -5.00 & 6.05 & -288.16 & 5052.13 & 7.07 & 2.43 & 3.29 & 1.02 \\
\hline 7452.74 & 448504.00 & 15.80 & 45.14 & 60.30 & 1.96 & -5.50 & 4.42 & -286.17 & 3836.20 & 7.38 & 2.15 & 3.31 & 1.08 \\
\hline 7452.81 & 441555.60 & 15.80 & 50.69 & 63.43 & 2.15 & -8.38 & 3.71 & -285.64 & 5204.44 & 7.38 & 2.02 & 3.32 & 1.09 \\
\hline 7452.87 & 434592.20 & 15.80 & 49.26 & 66.05 & 2.38 & -7.36 & 3.90 & -295.88 & 3619.84 & 7.31 & 1.77 & 3.35 & 1.13 \\
\hline 7452.94 & 438461.40 & 15.80 & 48.08 & 68.50 & 2.62 & -9.77 & 4.53 & -301.90 & 2705.86 & 7.03 & 1.32 & 3.48 & 0.91 \\
\hline 7453.01 & 420166.20 & 16.00 & 39.87 & 56.79 & 4.86 & -5.29 & 6.04 & -224.61 & 556.56 & 4.53 & 0.99 & 3.53 & 0.56 \\
\hline 7453.07 & 421230.60 & 16.00 & 40.28 & 62.10 & 3.71 & -5.37 & 7.21 & -217.55 & 1325.14 & 4.66 & 0.94 & 3.60 & 0.55 \\
\hline 7453.14 & 425816.20 & 16.00 & 40.92 & 58.55 & 3.44 & -4.16 & 8.87 & -196.06 & 947.45 & 4.57 & 1.07 & 3.57 & 0.53 \\
\hline 7453.20 & 431473.80 & 16.00 & 47.48 & 57.70 & 3.93 & -3.31 & 8.20 & -196.51 & 1958.66 & 4.86 & 1.19 & 3.60 & 0.58 \\
\hline 7453.27 & 408545.80 & 16.20 & 51.57 & 49.47 & 2.37 & -1.41 & 8.32 & -178.44 & 1107.18 & 4.36 & 1.16 & 3.61 & 0.46 \\
\hline 7453.33 & 397221.80 & 16.40 & 56.70 & 47.05 & 0.61 & -1.08 & 6.32 & -187.04 & 440.66 & 4.14 & 0.85 & 3.58 & 0.41 \\
\hline 7453.40 & 384389.20 & 16.60 & 57.32 & 40.08 & 0.99 & -3.48 & 7.00 & -203.47 & -218.64 & 3.88 & 0.72 & 3.55 & 0.37 \\
\hline 7453.46 & 374615.40 & 16.80 & 54.31 & 37.64 & -0.22 & -6.88 & 5.69 & -203.59 & 122.08 & 4.15 & 0.46 & 3.64 & 0.37 \\
\hline 7453.53 & 365146.60 & 17.00 & 48.36 & 33.14 & 0.06 & -9.18 & 5.24 & -191.41 & -1153.78 & 4.18 & 0.87 & 3.60 & 0.31 \\
\hline 7453.60 & 376577.60 & 16.80 & 40.85 & 35.42 & 1.59 & -10.90 & 2.82 & -200.75 & -1485.39 & 4.15 & 0.84 & 3.61 & 0.30 \\
\hline 7453.66 & 375962.00 & 16.80 & 30.90 & 35.44 & 2.93 & -13.66 & 4.11 & -198.63 & -412.52 & 3.92 & 0.89 & 3.66 & 0.30 \\
\hline 7453.73 & 369707.40 & 16.80 & 23.46 & 33.99 & 3.45 & -9.43 & 3.64 & -191.17 & 631.64 & 3.99 & 1.51 & 3.60 & 0.40 \\
\hline 7453.79 & 368156.00 & 16.80 & 21.04 & 38.17 & 3.60 & -5.18 & 4.50 & -212.21 & -541.06 & 3.95 & 2.08 & 3.55 & 0.41 \\
\hline
\end{tabular}




\begin{tabular}{|c|c|c|c|c|c|c|c|c|c|c|c|c|c|}
\hline Depth & $\mathrm{XRF}$ & XRF Live Time & $\mathrm{Nb}(\mathrm{ppm})$ & Mo (ppm) & $\mathrm{Ba}(\mathrm{ppm})$ & $\mathrm{Pb}(\mathrm{ppm})$ & Th (ppm) & $\mathrm{U}(\mathrm{ppm})$ & $\mathrm{P}(\mathrm{ppm})$ & $\mathrm{S}(\%)$ & $\mathrm{Cl}(\%)$ & $\mathrm{K}(\%)$ & $\mathrm{Ca}(\%)$ \\
\hline 7453.86 & 364386.60 & 16.80 & 17.61 & 36.90 & 1.81 & -2.77 & 4.55 & -231.57 & 518.52 & 3.69 & 1.79 & 3.65 & 0.40 \\
\hline 7453.92 & 352407.60 & 17.00 & 16.35 & 33.50 & 2.17 & -1.29 & 6.47 & -211.15 & 1381.18 & 3.83 & 1.94 & 3.65 & 0.54 \\
\hline 7453.99 & 346169.60 & 17.00 & 17.35 & 33.82 & 2.62 & -2.38 & 6.71 & -196.85 & -399.86 & 4.15 & 1.99 & 3.69 & 0.61 \\
\hline 7454.06 & 359065.40 & 16.80 & 22.89 & 42.22 & 2.35 & -3.26 & 6.55 & -196.28 & -2047.93 & 5.24 & 1.99 & 3.69 & 0.82 \\
\hline 7454.12 & 357663.20 & 16.80 & 22.72 & 41.06 & 3.60 & -3.06 & 6.60 & -193.91 & -1446.06 & 5.73 & 1.90 & 3.66 & 1.15 \\
\hline 7454.19 & 350443.80 & 16.80 & 20.60 & 39.92 & 4.66 & -3.51 & 7.21 & -190.68 & -869.87 & 5.38 & 1.91 & 3.63 & 1.24 \\
\hline 7454.25 & 368377.80 & 16.60 & 28.93 & 52.93 & 3.12 & -4.34 & 7.33 & -231.99 & 33.02 & 6.54 & 2.32 & 3.64 & 1.20 \\
\hline 7454.32 & 360778.60 & 16.60 & 34.07 & 64.45 & 1.06 & 5.25 & 5.86 & -275.62 & 1098.97 & 6.73 & 2.30 & 3.65 & 1.16 \\
\hline 7454.38 & 368649.00 & 16.60 & 43.41 & 63.85 & 1.24 & 1.35 & 6.48 & -270.73 & 2081.27 & 6.19 & 1.89 & 3.81 & 1.05 \\
\hline 7454.45 & 374142.00 & 16.40 & 45.15 & 56.33 & -0.08 & 1.75 & 5.38 & -275.03 & 1712.66 & 5.71 & 1.69 & 3.92 & 0.91 \\
\hline 7454.51 & 382602.00 & 16.40 & 45.27 & 60.95 & -0.17 & 0.25 & 5.45 & -282.07 & 309.40 & 5.91 & 1.88 & 3.87 & 1.40 \\
\hline 7454.58 & 378471.60 & 16.40 & 36.62 & 48.16 & 0.68 & -0.42 & 4.65 & -258.81 & -1224.76 & 4.69 & 1.20 & 3.75 & 5.29 \\
\hline 7454.65 & 377585.00 & 16.40 & 29.23 & 33.46 & 1.94 & -4.55 & 5.93 & -223.25 & -1770.86 & 4.33 & 1.37 & 3.54 & 9.42 \\
\hline 7454.71 & 344021.80 & 16.80 & 11.87 & 34.09 & 2.34 & -4.23 & 5.63 & -257.39 & -2496.13 & 3.10 & 1.44 & 3.29 & 11.46 \\
\hline 7454.78 & 314559.00 & 17.20 & 4.62 & 37.91 & 3.49 & -5.96 & 4.16 & -266.44 & -2861.36 & 2.81 & 2.50 & 3.00 & 13.75 \\
\hline 7454.84 & 291113.00 & 17.40 & 5.61 & 28.05 & 211.63 & -2.95 & 3.60 & -217.17 & -1104.40 & 2.29 & 2.95 & 2.82 & 15.11 \\
\hline 7454.91 & 268420.20 & 17.80 & 4.31 & 27.43 & 212.20 & -4.89 & 3.67 & -233.55 & -1481.34 & 1.83 & 3.51 & 2.74 & 13.43 \\
\hline 7454.97 & 271542.40 & 17.80 & 3.47 & 28.92 & 212.50 & -7.80 & 2.57 & -268.23 & 734.93 & 1.32 & 4.67 & 2.64 & 12.57 \\
\hline 7455.04 & 256956.20 & 17.80 & 3.15 & 26.27 & 212.64 & -8.32 & 1.12 & -277.98 & 872.26 & 1.45 & 5.41 & 2.58 & 13.60 \\
\hline 7455.10 & 212348.00 & 18.20 & 6.43 & 111.80 & 215.85 & -6.50 & 8.44 & -206.40 & 4570.19 & 0.76 & 18.42 & 2.03 & 11.64 \\
\hline 7455.17 & 167782.60 & 18.60 & 11.49 & 233.22 & 7.77 & -7.27 & 3.00 & -188.03 & 11298.11 & 0.92 & 32.63 & 1.52 & 10.28 \\
\hline 7455.24 & 115666.00 & 19.00 & 10.43 & 325.34 & 17.77 & 1.98 & 4.82 & -135.74 & 15736.19 & 0.90 & 49.94 & 0.99 & 8.63 \\
\hline 7455.30 & 136805.40 & 18.80 & 11.41 & 339.81 & 17.32 & 0.33 & 4.64 & -119.81 & 15220.16 & 0.98 & 48.79 & 1.06 & 9.16 \\
\hline 7455.37 & 183948.40 & 18.40 & 12.35 & 342.91 & 16.64 & 2.19 & 4.42 & -112.84 & 15382.32 & 1.55 & 49.10 & 1.07 & 8.77 \\
\hline 7455.43 & 250880.80 & 17.80 & 10.78 & 264.22 & 11.34 & -1.40 & -2.10 & -177.60 & 11343.63 & 1.92 & 35.08 & 1.66 & 11.95 \\
\hline 7455.50 & 311136.80 & 17.20 & 4.13 & 155.73 & 10.24 & -4.39 & 1.95 & -259.64 & 3399.38 & 2.01 & 20.42 & 2.16 & 14.90 \\
\hline 7455.56 & 371356.60 & 16.60 & 4.62 & 76.64 & -0.86 & -12.13 & 0.04 & -316.05 & -2217.45 & 1.95 & 3.29 & 2.65 & 18.37 \\
\hline 7455.63 & 366456.20 & 16.60 & 5.21 & 57.33 & 135.52 & -8.13 & 4.53 & -259.84 & -1369.01 & 1.83 & 3.82 & 2.61 & 19.69 \\
\hline 7455.70 & 369151.80 & 16.60 & 4.44 & 61.62 & 135.04 & -13.97 & 6.27 & -195.86 & 118.38 & 1.33 & 2.78 & 2.26 & 30.13 \\
\hline 7455.76 & 383275.00 & 16.40 & 3.55 & 59.12 & 134.82 & -11.45 & 6.21 & -163.78 & -927.35 & 1.14 & 2.56 & 1.97 & 40.77 \\
\hline 7455.83 & 404490.20 & 16.20 & 4.08 & 62.16 & 135.09 & -10.17 & 6.79 & -112.92 & -1357.44 & 1.11 & 2.16 & 1.70 & 51.81 \\
\hline 7455.89 & 425809.60 & 16.00 & 3.91 & 64.56 & 135.03 & -12.61 & 6.86 & -69.32 & -1257.95 & 1.24 & 1.75 & 1.41 & 61.54 \\
\hline 7455.96 & 435953.40 & 16.00 & 4.32 & 81.03 & -1.51 & -15.95 & 3.90 & -106.60 & -4920.03 & 1.54 & 1.24 & 1.12 & 68.15 \\
\hline
\end{tabular}




\begin{tabular}{|c|c|c|c|c|c|c|c|c|c|c|c|c|c|}
\hline Depth & $\mathrm{XRF}$ & XRF Live Time & $\mathrm{Nb}(\mathrm{ppm})$ & Mo (ppm) & $\mathrm{Ba}(\mathrm{ppm})$ & $\mathrm{Pb}(\mathrm{ppm})$ & Th (ppm) & $\mathrm{U}(\mathrm{ppm})$ & $\mathrm{P}(\mathrm{ppm})$ & $\mathrm{S}(\%)$ & $\mathrm{Cl}(\%)$ & $\mathrm{K}(\%)$ & $\mathrm{Ca}(\%)$ \\
\hline 7456.02 & 433669.80 & 16.00 & 21.53 & 80.00 & -2.07 & -8.94 & 4.57 & -143.61 & -6337.40 & 2.50 & 1.03 & 1.91 & 55.21 \\
\hline 7456.09 & 436307.80 & 16.00 & 45.45 & 78.60 & -1.81 & -11.60 & 5.94 & -162.73 & -5340.22 & 3.39 & 1.09 & 2.68 & 40.85 \\
\hline 7456.15 & 432736.20 & 16.00 & 67.83 & 69.97 & -0.98 & -10.32 & 6.96 & -193.48 & -4563.29 & 3.80 & 0.99 & 3.41 & 26.23 \\
\hline 7456.22 & 432084.80 & 16.00 & 95.02 & 62.08 & -1.84 & -9.44 & 8.59 & -237.79 & -1224.30 & 4.51 & 0.77 & 4.14 & 12.34 \\
\hline 7456.29 & 436436.20 & 16.00 & 115.10 & 56.60 & -3.54 & -8.00 & 9.83 & -237.50 & 587.62 & 5.23 & 0.58 & 4.86 & 0.65 \\
\hline 7456.35 & 442431.60 & 16.00 & 120.77 & 50.51 & -1.76 & -6.41 & 10.78 & -234.80 & 732.79 & 4.88 & 0.65 & 4.71 & 0.39 \\
\hline 7456.42 & 439925.40 & 16.00 & 123.41 & 48.17 & -1.68 & -6.68 & 11.69 & -251.27 & 2375.19 & 4.67 & 0.51 & 4.60 & 0.35 \\
\hline 7456.48 & 440590.20 & 16.00 & 123.94 & 47.93 & -1.65 & -6.70 & 12.63 & -245.56 & 3028.50 & 4.88 & 0.61 & 4.50 & 0.45 \\
\hline 7456.55 & 438009.60 & 16.00 & 120.91 & 47.92 & -0.78 & -6.15 & 13.77 & -230.76 & 2084.08 & 5.00 & 1.02 & 4.32 & 0.60 \\
\hline 7456.61 & 431057.40 & 16.00 & 116.11 & 42.00 & 4.52 & -7.15 & 12.34 & -227.24 & 2453.89 & 4.74 & 0.93 & 4.27 & 0.69 \\
\hline 7456.68 & 436236.80 & 15.80 & 123.31 & 47.27 & 2.79 & -9.13 & 12.00 & -234.99 & 4479.57 & 5.91 & 1.05 & 4.24 & 0.98 \\
\hline 7456.75 & 436824.40 & 15.80 & 116.10 & 51.56 & 1.80 & -4.63 & 11.84 & -218.40 & 3861.05 & 6.17 & 1.09 & 4.10 & 1.20 \\
\hline 7456.81 & 415588.40 & 16.00 & 115.13 & 54.23 & 3.76 & -4.12 & 11.09 & -238.31 & 3228.62 & 6.81 & 1.35 & 3.92 & 1.49 \\
\hline 7456.88 & 385598.80 & 16.40 & 110.50 & 46.87 & 4.40 & -6.01 & 11.21 & -231.83 & 2050.08 & 6.67 & 1.16 & 3.84 & 1.52 \\
\hline 7456.94 & 352472.60 & 16.80 & 111.94 & 42.27 & -1.50 & -4.10 & 11.21 & -225.03 & -357.10 & 6.67 & 1.57 & 3.64 & 1.49 \\
\hline 7457.01 & 312358.80 & 17.40 & 99.34 & 33.11 & -1.45 & -2.97 & 10.99 & -204.61 & -2363.77 & 5.54 & 1.46 & 3.57 & 1.29 \\
\hline 7457.07 & 282047.40 & 17.80 & 81.57 & 37.72 & 326.13 & 1.87 & 8.75 & -164.46 & -3324.53 & 5.56 & 2.29 & 3.39 & 1.37 \\
\hline 7457.14 & 279915.00 & 17.80 & 77.48 & 34.47 & 322.18 & 0.26 & 8.11 & -162.32 & -3794.51 & 5.10 & 2.14 & 3.37 & 1.23 \\
\hline 7457.20 & 282051.40 & 17.80 & 72.59 & 40.57 & 322.62 & 2.86 & 6.60 & -152.29 & -3017.05 & 5.02 & 2.96 & 3.30 & 1.22 \\
\hline 7457.27 & 311564.40 & 17.40 & 71.26 & 52.42 & 324.71 & 3.67 & 6.66 & -169.24 & -1117.18 & 4.96 & 2.65 & 3.43 & 1.13 \\
\hline 7457.34 & 347694.80 & 17.00 & 76.04 & 62.38 & 325.55 & 3.43 & 7.24 & -181.89 & -289.71 & 4.95 & 2.49 & 3.55 & 0.96 \\
\hline 7457.40 & 380038.20 & 16.60 & 97.39 & 58.51 & 0.05 & -2.17 & 9.65 & -219.22 & 1044.76 & 4.98 & 1.66 & 3.77 & 0.75 \\
\hline 7457.47 & 399979.00 & 16.40 & 97.37 & 61.68 & 1.26 & 1.85 & 12.00 & -221.82 & 1888.79 & 4.73 & 1.65 & 3.87 & 0.61 \\
\hline 7457.53 & 425148.40 & 16.00 & 102.45 & 61.84 & 0.91 & 4.00 & 13.01 & -239.37 & 1892.55 & 4.69 & 0.59 & 4.05 & 0.49 \\
\hline 7457.60 & 424130.80 & 16.00 & 104.62 & 57.68 & -0.13 & 1.82 & 12.66 & -245.79 & 2719.62 & 4.65 & 0.75 & 4.00 & 0.83 \\
\hline 7457.66 & 411397.40 & 16.00 & 101.59 & 47.69 & 0.56 & 2.38 & 12.22 & -257.01 & 2707.91 & 4.42 & 0.86 & 3.96 & 0.81 \\
\hline 7457.73 & 409908.40 & 16.00 & 98.72 & 42.16 & -0.51 & 1.20 & 12.21 & -238.76 & 2515.11 & 4.21 & 0.81 & 3.99 & 0.75 \\
\hline 7457.80 & 411360.00 & 16.00 & 95.81 & 45.13 & -1.36 & -1.18 & 11.55 & -217.79 & 2691.31 & 4.16 & 0.93 & 4.02 & 0.71 \\
\hline 7457.86 & 409671.40 & 16.00 & 93.61 & 41.40 & -1.63 & -1.93 & 9.74 & -195.26 & 2965.53 & 4.17 & 1.31 & 3.97 & 0.76 \\
\hline 7457.93 & 403537.20 & 16.20 & 91.76 & 38.77 & 0.58 & 0.60 & 11.03 & -172.40 & 1195.16 & 4.03 & 1.30 & 3.95 & 0.44 \\
\hline 7457.99 & 402002.20 & 16.20 & 89.76 & 35.06 & 0.37 & -4.04 & 11.28 & -145.57 & 1199.11 & 4.29 & 1.70 & 3.84 & 0.59 \\
\hline 7458.06 & 390595.40 & 16.40 & 88.23 & 37.54 & 0.86 & -4.58 & 10.91 & -164.24 & 708.83 & 4.48 & 1.95 & 3.75 & 0.82 \\
\hline 7458.12 & 378975.60 & 16.60 & 90.84 & 29.72 & 1.90 & -5.65 & 10.85 & -157.30 & -623.17 & 4.49 & 2.22 & 3.64 & 0.87 \\
\hline
\end{tabular}




\begin{tabular}{|c|c|c|c|c|c|c|c|c|c|c|c|c|c|}
\hline Depth & $\mathrm{XRF}$ & XRF Live Time & $\mathrm{Nb}(\mathrm{ppm})$ & Mo (ppm) & $\mathrm{Ba}(\mathrm{ppm})$ & $\mathrm{Pb}(\mathrm{ppm})$ & Th (ppm) & $\mathrm{U}(\mathrm{ppm})$ & $\mathrm{P}(\mathrm{ppm})$ & $\mathrm{S}(\%)$ & $\mathrm{Cl}(\%)$ & $\mathrm{K}(\%)$ & $\mathrm{Ca}(\%)$ \\
\hline 7458.19 & 366020.40 & 16.80 & 91.14 & 25.95 & 1.96 & -7.03 & 10.17 & -180.22 & -966.84 & 4.40 & 2.06 & 3.64 & 0.87 \\
\hline 7458.25 & 365979.80 & 16.80 & 90.75 & 31.52 & 1.95 & -7.31 & 10.07 & -192.98 & -141.39 & 4.73 & 2.50 & 3.67 & 0.97 \\
\hline 7458.32 & 360261.40 & 17.00 & 92.89 & 38.19 & 4.69 & -3.94 & 8.84 & -202.19 & 319.02 & 4.66 & 2.46 & 3.73 & 0.99 \\
\hline 7458.39 & 361072.00 & 17.00 & 92.40 & 37.39 & 5.83 & -3.71 & 9.48 & -198.95 & 792.48 & 4.69 & 2.98 & 3.73 & 0.91 \\
\hline 7458.45 & 366822.60 & 17.00 & 93.04 & 33.78 & 5.11 & -5.22 & 10.17 & -196.29 & 1444.78 & 4.87 & 3.07 & 3.81 & 0.91 \\
\hline 7458.52 & 379081.60 & 16.80 & 103.15 & 38.74 & 4.44 & -6.70 & 13.25 & -176.36 & 1753.16 & 5.22 & 4.05 & 3.73 & 1.04 \\
\hline 7458.58 & 385193.60 & 16.60 & 106.99 & 33.94 & 3.40 & -6.61 & 13.91 & -175.24 & 1563.95 & 4.78 & 3.86 & 3.82 & 0.90 \\
\hline 7458.65 & 396828.20 & 16.40 & 110.05 & 37.68 & 0.46 & -10.30 & 15.21 & -166.85 & 407.51 & 5.13 & 4.96 & 3.75 & 0.95 \\
\hline 7458.71 & 408694.20 & 16.20 & 114.29 & 42.89 & 0.17 & -8.91 & 14.78 & -164.18 & 1262.96 & 5.42 & 5.94 & 3.68 & 1.00 \\
\hline 7458.78 & 420559.60 & 16.00 & 111.32 & 53.09 & 1.36 & -12.02 & 14.57 & -188.36 & 2044.24 & 5.41 & 5.97 & 3.72 & 0.94 \\
\hline 7458.85 & 426116.00 & 16.00 & 95.34 & 53.94 & 2.29 & -8.66 & 14.00 & -208.05 & 2437.81 & 5.42 & 5.84 & 3.70 & 1.22 \\
\hline 7458.91 & 445190.60 & 15.80 & 91.96 & 65.82 & 3.71 & -9.54 & 12.00 & -245.18 & 3365.93 & 7.28 & 5.47 & 3.70 & 1.43 \\
\hline 7458.98 & 451298.60 & 15.80 & 90.51 & 65.93 & 2.73 & -7.97 & 11.47 & -253.50 & 5408.07 & 7.58 & 4.62 & 3.85 & 1.40 \\
\hline 7459.04 & 453592.20 & 15.80 & 81.23 & 60.62 & 0.79 & -7.73 & 12.20 & -280.11 & 4117.27 & 7.79 & 3.18 & 3.97 & 1.54 \\
\hline 7459.11 & 469974.40 & 15.60 & 83.44 & 68.21 & 1.22 & -5.72 & 14.06 & -305.57 & 3443.74 & 8.98 & 4.07 & 3.96 & 1.65 \\
\hline 7459.17 & 484953.20 & 15.40 & 75.84 & 82.21 & 318.94 & 1.40 & 15.85 & -254.94 & 3433.28 & 9.66 & 3.43 & 4.17 & 1.30 \\
\hline 7459.24 & 471177.40 & 15.60 & 81.00 & 69.29 & 316.92 & 1.90 & 17.68 & -228.16 & 3025.85 & 8.12 & 3.29 & 4.26 & 1.11 \\
\hline 7459.30 & 466354.40 & 15.60 & 79.39 & 62.46 & 317.03 & 3.96 & 17.85 & -203.59 & 1091.44 & 7.43 & 3.43 & 4.13 & 1.03 \\
\hline 7459.37 & 468763.60 & 15.60 & 68.57 & 72.86 & 871.63 & 11.28 & 17.92 & -145.93 & 2484.20 & 7.86 & 4.02 & 4.09 & 0.87 \\
\hline 7459.44 & 448734.20 & 15.80 & 67.80 & 65.73 & 871.19 & 9.86 & 15.16 & -102.74 & 3175.23 & 6.93 & 2.93 & 4.03 & 0.91 \\
\hline 7459.50 & 443809.60 & 16.00 & 80.67 & 56.47 & 553.16 & 1.45 & 11.75 & -137.55 & 2369.18 & 6.25 & 3.26 & 3.93 & 0.95 \\
\hline 7459.57 & 446637.00 & 16.00 & 76.44 & 61.70 & 554.00 & 1.29 & 11.05 & -146.84 & 2383.51 & 6.29 & 3.66 & 3.81 & 1.08 \\
\hline 7459.63 & 450674.80 & 16.00 & 75.62 & 68.17 & 553.25 & 1.78 & 9.08 & -160.77 & 2198.46 & 6.75 & 3.80 & 3.87 & 1.12 \\
\hline 7459.70 & 444575.20 & 16.00 & 91.00 & 66.65 & -0.55 & -3.82 & 8.63 & -196.98 & 1700.76 & 5.68 & 4.85 & 3.77 & 1.02 \\
\hline 7459.76 & 445611.80 & 16.00 & 88.69 & 60.88 & -1.65 & 1.22 & 9.65 & -213.60 & 556.64 & 5.52 & 4.60 & 3.85 & 0.96 \\
\hline 7459.83 & 437464.80 & 16.00 & 90.59 & 61.51 & -1.04 & -0.24 & 11.21 & -224.10 & 292.61 & 5.35 & 4.16 & 3.95 & 0.78 \\
\hline 7459.90 & 440794.00 & 16.00 & 91.16 & 62.00 & -1.24 & -1.55 & 11.01 & -218.85 & 1198.68 & 5.62 & 3.78 & 4.02 & 0.66 \\
\hline 7459.96 & 435531.00 & 16.00 & 90.85 & 61.42 & 1.23 & -0.80 & 12.04 & -210.26 & 2555.80 & 5.94 & 5.34 & 3.87 & 0.85 \\
\hline 7460.03 & 439037.80 & 16.00 & 92.56 & 62.00 & 2.26 & -4.19 & 11.47 & -218.41 & 2142.97 & 6.10 & 3.60 & 4.03 & 0.85 \\
\hline 7460.09 & 432966.40 & 16.00 & 93.25 & 69.41 & 4.05 & -1.92 & 11.55 & -182.94 & 2333.69 & 6.21 & 6.93 & 3.71 & 0.90 \\
\hline 7460.16 & 425668.00 & 16.00 & 91.55 & 65.15 & 2.95 & 0.97 & 11.14 & -155.66 & 2849.05 & 6.37 & 7.93 & 3.48 & 1.10 \\
\hline 7460.22 & 389550.80 & 16.20 & 73.84 & 67.98 & 370.75 & 7.85 & 11.58 & -98.75 & 2234.69 & 5.96 & 9.96 & 3.20 & 1.14 \\
\hline 7460.29 & 394179.80 & 16.20 & 74.77 & 67.88 & 369.43 & 8.04 & 11.87 & -100.41 & 1385.52 & 5.38 & 9.42 & 3.24 & 0.95 \\
\hline
\end{tabular}




\begin{tabular}{|c|c|c|c|c|c|c|c|c|c|c|c|c|c|}
\hline Depth & $\mathrm{XRF}$ & XRF Live Time & $\mathrm{Nb}(\mathrm{ppm})$ & Mo (ppm) & $\mathrm{Ba}(\mathrm{ppm})$ & $\mathrm{Pb}(\mathrm{ppm})$ & Th (ppm) & $\mathrm{U}(\mathrm{ppm})$ & $\mathrm{P}(\mathrm{ppm})$ & $\mathrm{S}(\%)$ & $\mathrm{Cl}(\%)$ & $\mathrm{K}(\%)$ & $\mathrm{Ca}(\%)$ \\
\hline 7460.35 & 394409.60 & 16.20 & 82.06 & 59.77 & 368.99 & 7.67 & 12.40 & -106.84 & 1543.37 & 5.46 & 10.29 & 3.19 & 0.93 \\
\hline 7460.42 & 398612.00 & 16.20 & 92.54 & 54.76 & 367.40 & 8.30 & 12.41 & -128.91 & 2053.27 & 5.05 & 6.72 & 3.54 & 0.72 \\
\hline 7460.49 & 401408.00 & 16.20 & 93.59 & 54.01 & 368.37 & 8.74 & 13.60 & -155.26 & 1652.26 & 4.88 & 5.62 & 3.71 & 0.59 \\
\hline 7460.55 & 436599.80 & 16.00 & 110.03 & 52.38 & 1.43 & 1.77 & 14.46 & -189.08 & 1961.35 & 5.91 & 5.53 & 3.74 & 0.98 \\
\hline 7460.62 & 446534.80 & 15.80 & 113.54 & 58.10 & 3.45 & 2.72 & 15.40 & -178.80 & 4203.34 & 7.38 & 5.90 & 3.73 & 1.21 \\
\hline 7460.68 & 449410.60 & 15.80 & 104.16 & 63.98 & 2.79 & 3.46 & 13.97 & -164.80 & 4914.68 & 7.81 & 8.70 & 3.48 & 1.41 \\
\hline 7460.75 & 471788.20 & 15.60 & 98.01 & 70.40 & 2.96 & -0.87 & 14.31 & -202.18 & 6179.45 & 9.72 & 10.17 & 3.43 & 1.85 \\
\hline 7460.81 & 465653.80 & 15.80 & 101.50 & 69.47 & 2.69 & -5.08 & 11.53 & -188.43 & 7752.04 & 9.93 & 11.39 & 3.29 & 1.99 \\
\hline 7460.88 & 457138.80 & 15.80 & 103.00 & 66.36 & 2.50 & -4.97 & 11.32 & -201.09 & 6397.66 & 9.34 & 11.31 & 3.31 & 1.66 \\
\hline 7460.94 & 444978.20 & 16.00 & 98.66 & 58.70 & 0.98 & -5.08 & 11.01 & -212.96 & 3745.54 & 8.04 & 9.22 & 3.51 & 1.46 \\
\hline 7461.01 & 429100.40 & 16.00 & 83.02 & 59.69 & 385.05 & 2.92 & 13.12 & -176.46 & 3158.46 & 8.12 & 9.70 & 3.41 & 1.67 \\
\hline 7461.08 & 396821.40 & 16.40 & 75.11 & 52.74 & 384.60 & 4.02 & 13.02 & -117.29 & 2005.67 & 7.02 & 12.94 & 3.03 & 1.68 \\
\hline 7461.14 & 386169.60 & 16.40 & 70.86 & 53.15 & 384.70 & 9.19 & 16.17 & -112.11 & 355.10 & 6.66 & 12.71 & 3.10 & 1.54 \\
\hline 7461.21 & 381464.00 & 16.40 & 72.10 & 52.66 & 384.53 & 7.50 & 15.76 & -143.01 & 1702.23 & 6.54 & 10.97 & 3.30 & 1.46 \\
\hline 7461.27 & 366808.80 & 16.60 & 75.70 & 53.01 & 383.33 & 7.01 & 16.41 & -150.46 & 1038.46 & 6.02 & 11.05 & 3.27 & 1.29 \\
\hline 7461.34 & 362658.00 & 16.80 & 91.54 & 38.25 & -0.69 & -0.46 & 15.37 & -218.80 & 767.59 & 5.06 & 6.66 & 3.70 & 0.81 \\
\hline 7461.40 & 383382.40 & 16.60 & 102.44 & 49.32 & 1.23 & 1.70 & 13.73 & -243.29 & 572.87 & 5.05 & 1.99 & 4.23 & 0.38 \\
\hline 7461.47 & 407213.80 & 16.40 & 110.13 & 50.30 & 0.59 & -0.99 & 12.58 & -288.65 & 1357.48 & 5.42 & 0.93 & 4.42 & 0.31 \\
\hline 7461.54 & 408899.20 & 16.40 & 110.37 & 47.61 & -0.79 & -1.21 & 13.32 & -254.08 & -347.69 & 5.40 & 1.42 & 4.35 & 0.45 \\
\hline 7461.60 & 419956.40 & 16.20 & 111.26 & 45.02 & 1.84 & -3.46 & 13.67 & -276.35 & 80.41 & 5.54 & 1.08 & 4.42 & 0.44 \\
\hline 7461.67 & 425737.20 & 16.00 & 114.31 & 44.08 & 2.57 & -1.26 & 14.12 & -262.55 & 41.56 & 5.48 & 1.31 & 4.38 & 0.48 \\
\hline 7461.73 & 409807.60 & 16.00 & 109.44 & 37.54 & 0.91 & -3.39 & 14.53 & -240.68 & -1034.99 & 5.01 & 4.29 & 4.00 & 0.68 \\
\hline 7461.80 & 406060.80 & 16.00 & 101.70 & 51.93 & 1.74 & 0.48 & 14.63 & -197.94 & -2532.29 & 5.51 & 6.34 & 3.67 & 2.69 \\
\hline 7461.86 & 415243.20 & 16.00 & 100.97 & 58.57 & 2.48 & 0.70 & 12.84 & -228.12 & -1086.02 & 5.56 & 7.09 & 3.56 & 3.05 \\
\hline 7461.93 & 414571.20 & 16.00 & 97.33 & 62.17 & 1.32 & -0.72 & 13.64 & -210.22 & -979.55 & 5.76 & 9.60 & 3.25 & 3.49 \\
\hline 7461.99 & 422782.60 & 16.00 & 95.63 & 69.12 & 1.92 & -3.54 & 12.22 & -217.41 & -951.30 & 6.09 & 10.04 & 3.22 & 3.55 \\
\hline 7462.06 & 432421.80 & 16.00 & 100.20 & 77.49 & 1.91 & -3.58 & 13.00 & -231.36 & 1677.36 & 6.48 & 7.63 & 3.48 & 3.65 \\
\hline 7462.13 & 445082.80 & 15.80 & 106.00 & 74.28 & 2.08 & -4.99 & 12.46 & -246.86 & 2734.12 & 6.49 & 6.72 & 3.60 & 2.01 \\
\hline 7462.19 & 429777.00 & 16.00 & 93.57 & 78.11 & 3.01 & -4.01 & 12.39 & -190.66 & 3181.45 & 6.22 & 10.81 & 3.14 & 4.62 \\
\hline 7462.26 & 426697.60 & 16.00 & 90.88 & 81.30 & 1.49 & -4.30 & 9.99 & -203.46 & 5260.29 & 6.56 & 9.98 & 3.27 & 4.43 \\
\hline 7462.32 & 423844.80 & 16.00 & 82.74 & 85.19 & 1.14 & -0.87 & 12.14 & -179.31 & 4681.09 & 6.71 & 9.73 & 3.24 & 4.54 \\
\hline 7462.39 & 395997.00 & 16.20 & 62.14 & 79.12 & 413.01 & 3.15 & 12.05 & -143.18 & 1991.98 & 5.84 & 10.29 & 3.07 & 4.33 \\
\hline 7462.45 & 350730.60 & 16.80 & 55.87 & 72.17 & 412.42 & 1.73 & 12.22 & -105.17 & 2127.76 & 4.70 & 9.92 & 3.04 & 4.02 \\
\hline
\end{tabular}




\begin{tabular}{|c|c|c|c|c|c|c|c|c|c|c|c|c|c|}
\hline Depth & $\mathrm{XRF}$ & XRF Live Time & $\mathrm{Nb}(\mathrm{ppm})$ & Mo (ppm) & $\mathrm{Ba}(\mathrm{ppm})$ & $\mathrm{Pb}(\mathrm{ppm})$ & Th (ppm) & $\mathrm{U}(\mathrm{ppm})$ & $\mathrm{P}(\mathrm{ppm})$ & $\mathrm{S}(\%)$ & $\mathrm{Cl}(\%)$ & $\mathrm{K}(\%)$ & $\mathrm{Ca}(\%)$ \\
\hline 7462.52 & 347849.20 & 16.80 & 66.31 & 59.97 & 411.22 & 0.54 & 12.06 & -134.14 & 529.94 & 4.53 & 4.84 & 3.58 & 0.94 \\
\hline 7462.59 & 339053.00 & 17.00 & 64.03 & 49.82 & 412.42 & 2.31 & 12.44 & -109.82 & -1598.40 & 3.55 & 3.40 & 3.65 & 0.72 \\
\hline 7462.65 & 320440.00 & 17.20 & 67.59 & 42.04 & 412.40 & 0.65 & 11.52 & -102.72 & -1458.04 & 3.14 & 4.18 & 3.52 & 0.67 \\
\hline 7462.72 & 328330.20 & 17.20 & 79.10 & 35.25 & 0.75 & -3.33 & 10.86 & -140.15 & -892.48 & 3.34 & 3.57 & 3.56 & 0.78 \\
\hline 7462.78 & 339409.80 & 17.00 & 79.36 & 25.12 & 1.04 & -1.63 & 10.96 & -177.14 & -1288.37 & 3.25 & 3.92 & 3.50 & 0.83 \\
\hline 7462.85 & 329917.60 & 17.00 & 76.18 & 21.11 & 1.35 & -0.35 & 13.75 & -162.71 & -785.02 & 2.99 & 3.55 & 3.54 & 0.79 \\
\hline 7462.91 & 328039.60 & 17.00 & 79.55 & 20.81 & 2.26 & 1.58 & 14.90 & -176.32 & -1309.72 & 2.98 & 3.36 & 3.55 & 0.74 \\
\hline 7462.98 & 330183.40 & 17.00 & 80.41 & 22.92 & 3.67 & 0.42 & 14.88 & -183.22 & -403.45 & 2.94 & 3.52 & 3.51 & 0.70 \\
\hline 7463.04 & 348063.20 & 16.80 & 84.52 & 30.77 & 4.24 & 1.62 & 13.74 & -169.91 & 380.16 & 2.69 & 2.98 & 3.69 & 0.59 \\
\hline 7463.11 & 361735.60 & 16.80 & 86.84 & 35.15 & 2.90 & -1.10 & 13.86 & -173.17 & 51.34 & 3.37 & 2.48 & 3.77 & 0.82 \\
\hline 7463.18 & 369894.00 & 16.80 & 90.73 & 35.59 & 2.73 & -0.33 & 10.88 & -187.89 & 623.53 & 3.42 & 2.51 & 3.78 & 0.84 \\
\hline 7463.24 & 364203.20 & 16.80 & 95.05 & 39.26 & 1.68 & -0.72 & 9.05 & -172.73 & 655.44 & 3.44 & 2.55 & 3.81 & 0.83 \\
\hline 7463.31 & 361626.80 & 16.80 & 98.83 & 37.53 & 0.14 & -0.31 & 7.60 & -157.41 & -478.36 & 3.22 & 1.83 & 3.94 & 0.74 \\
\hline 7463.37 & 335227.40 & 17.00 & 98.65 & 28.69 & 0.02 & -3.74 & 9.84 & -126.21 & -2230.79 & 3.25 & 4.99 & 3.50 & 0.76 \\
\hline 7463.44 & 335611.00 & 17.00 & 104.91 & 27.15 & 2.75 & -2.97 & 10.76 & -124.51 & -1552.24 & 2.83 & 5.83 & 3.48 & 0.47 \\
\hline 7463.50 & 335155.80 & 17.00 & 109.97 & 34.67 & 4.31 & -3.77 & 12.76 & -127.66 & -2114.94 & 3.24 & 8.02 & 3.29 & 0.59 \\
\hline 7463.57 & 343533.60 & 17.00 & 112.31 & 46.80 & 5.02 & -1.82 & 14.09 & -147.87 & -920.85 & 3.48 & 8.96 & 3.13 & 1.82 \\
\hline 7463.64 & 361400.80 & 16.80 & 118.96 & 54.15 & 4.73 & -3.46 & 16.57 & -169.90 & -775.17 & 4.42 & 10.27 & 2.99 & 2.34 \\
\hline 7463.70 & 375516.40 & 16.80 & 130.81 & 53.87 & 5.30 & -3.05 & 16.21 & -187.89 & 849.36 & 5.07 & 9.04 & 3.19 & 2.52 \\
\hline 7463.77 & 370316.40 & 16.80 & 131.65 & 52.10 & 2.03 & -4.56 & 14.78 & -160.43 & 1654.33 & 4.87 & 7.66 & 3.30 & 2.49 \\
\hline 7463.83 & 391281.20 & 16.60 & 117.82 & 57.68 & 0.05 & -4.20 & 13.33 & -168.28 & 1005.14 & 4.91 & 6.31 & 3.41 & 2.60 \\
\hline 7463.90 & 398028.00 & 16.40 & 98.79 & 41.93 & 15.67 & -5.83 & 12.92 & -152.15 & 280.63 & 4.65 & 5.91 & 3.48 & 1.81 \\
\hline 7463.96 & 387174.60 & 16.60 & 80.71 & 40.13 & 17.12 & -3.93 & 13.12 & -148.79 & 226.64 & 4.05 & 5.16 & 3.49 & 3.30 \\
\hline 7464.03 & 378926.20 & 16.60 & 70.89 & 41.66 & 17.38 & -1.52 & 14.15 & -179.52 & -954.45 & 3.35 & 3.64 & 3.64 & 3.22 \\
\hline 7464.09 & 369207.60 & 16.60 & 63.91 & 47.35 & 18.71 & -4.69 & 16.03 & -207.71 & -2423.71 & 3.48 & 3.44 & 3.67 & 3.38 \\
\hline 7464.16 & 332300.80 & 17.00 & 71.71 & 36.23 & 18.91 & -5.47 & 16.79 & -176.95 & -2848.26 & 3.28 & 3.31 & 3.60 & 3.38 \\
\hline 7464.23 & 330318.20 & 17.20 & 71.79 & 42.00 & 4.63 & -9.95 & 15.72 & -182.07 & -3136.14 & 3.33 & 3.11 & 3.60 & 3.80 \\
\hline 7464.29 & 334292.40 & 17.20 & 73.99 & 41.91 & 2.77 & -9.09 & 13.14 & -168.05 & -2407.23 & 3.07 & 3.35 & 3.67 & 2.07 \\
\hline 7464.36 & 347375.60 & 17.00 & 75.08 & 39.79 & 1.98 & -10.18 & 10.48 & -194.00 & -2097.56 & 3.20 & 3.01 & 3.73 & 1.96 \\
\hline 7464.42 & 359401.40 & 17.00 & 78.99 & 35.71 & 2.27 & -5.96 & 9.37 & -178.06 & -1418.30 & 3.64 & 4.50 & 3.55 & 2.14 \\
\hline 7464.49 & 380577.60 & 16.80 & 82.40 & 39.17 & 2.55 & -7.21 & 11.70 & -201.41 & 79.68 & 3.56 & 4.13 & 3.71 & 2.11 \\
\hline 7464.55 & 389065.60 & 16.60 & 99.85 & 43.46 & 0.70 & -4.99 & 12.43 & -195.17 & 80.46 & 4.03 & 5.96 & 3.67 & 1.46 \\
\hline 7464.62 & 403074.40 & 16.40 & 106.90 & 48.78 & 1.39 & -5.67 & 15.33 & -178.54 & -635.40 & 4.93 & 6.99 & 3.72 & 1.41 \\
\hline
\end{tabular}




\begin{tabular}{|c|c|c|c|c|c|c|c|c|c|c|c|c|c|}
\hline Depth & $\mathrm{XRF}$ & XRF Live Time & $\mathrm{Nb}(\mathrm{ppm})$ & Mo (ppm) & $\mathrm{Ba}(\mathrm{ppm})$ & $\mathrm{Pb}(\mathrm{ppm})$ & Th (ppm) & $\mathrm{U}(\mathrm{ppm})$ & $\mathrm{P}(\mathrm{ppm})$ & $\mathrm{S}(\%)$ & $\mathrm{Cl}(\%)$ & $\mathrm{K}(\%)$ & $\mathrm{Ca}(\%)$ \\
\hline 7464.69 & 402938.80 & 16.40 & 110.94 & 47.66 & 0.82 & -5.00 & 18.15 & -155.49 & -718.83 & 5.19 & 7.92 & 3.61 & 1.48 \\
\hline 7464.75 & 403498.00 & 16.40 & 115.50 & 46.73 & 0.65 & -3.37 & 17.12 & -175.46 & -2376.60 & 4.94 & 6.89 & 3.72 & 1.25 \\
\hline 7464.82 & 399011.80 & 16.40 & 121.41 & 46.16 & 1.56 & -4.48 & 15.80 & -164.76 & -3949.30 & 5.52 & 8.62 & 3.47 & 1.41 \\
\hline 7464.88 & 387128.20 & 16.60 & 124.35 & 37.07 & 0.76 & -5.91 & 18.20 & -164.49 & -2627.61 & 5.35 & 7.39 & 3.51 & 1.36 \\
\hline 7464.95 & 373824.40 & 16.60 & 131.83 & 29.28 & 0.18 & -8.71 & 19.79 & -200.60 & -2423.24 & 4.79 & 4.94 & 3.62 & 1.39 \\
\hline 7465.01 & 370986.20 & 16.80 & 137.40 & 37.81 & 0.40 & -7.43 & 18.69 & -209.63 & -2125.24 & 4.54 & 4.18 & 3.70 & 1.44 \\
\hline 7465.08 & 371114.20 & 16.80 & 132.30 & 41.35 & 0.80 & -11.19 & 21.90 & -196.92 & -346.95 & 4.45 & 4.15 & 3.71 & 1.36 \\
\hline 7465.14 & 378079.40 & 16.60 & 127.17 & 45.98 & -1.25 & -6.49 & 21.69 & -220.18 & 2538.29 & 4.15 & 2.27 & 3.92 & 1.65 \\
\hline 7465.21 & 386357.00 & 16.40 & 123.03 & 59.49 & -0.68 & -4.94 & 20.43 & -241.35 & 2385.63 & 4.55 & 1.53 & 4.01 & 1.79 \\
\hline 7465.28 & 388212.00 & 16.40 & 128.53 & 64.68 & -1.58 & -1.95 & 20.60 & -257.80 & 2474.13 & 4.51 & 1.56 & 4.03 & 1.53 \\
\hline 7465.34 & 389927.60 & 16.40 & 126.42 & 59.66 & -1.48 & -4.30 & 21.36 & -253.89 & 3003.23 & 4.51 & 1.41 & 4.06 & 1.37 \\
\hline 7465.41 & 398888.00 & 16.20 & 126.49 & 64.96 & -1.62 & -2.63 & 17.83 & -288.66 & 2565.76 & 4.54 & 0.88 & 4.13 & 1.33 \\
\hline 7465.47 & 411177.40 & 16.20 & 117.44 & 69.26 & -1.62 & -3.89 & 16.99 & -293.70 & 1437.79 & 4.70 & 0.74 & 4.19 & 0.84 \\
\hline 7465.54 & 417253.60 & 16.20 & 111.24 & 64.96 & -1.57 & -1.38 & 17.10 & -298.57 & 1257.24 & 4.72 & 0.69 & 4.29 & 0.89 \\
\hline 7465.60 & 413492.60 & 16.40 & 105.45 & 64.18 & 0.19 & -3.79 & 14.27 & -279.26 & 128.59 & 4.65 & 1.15 & 4.21 & 1.08 \\
\hline 7465.67 & 414872.40 & 16.20 & 101.89 & 66.57 & 0.03 & -4.29 & 12.54 & -276.55 & -1058.78 & 4.64 & 1.08 & 4.18 & 1.10 \\
\hline 7465.73 & 408599.80 & 16.20 & 97.06 & 67.75 & 1.19 & -3.00 & 14.85 & -217.02 & -913.61 & 4.61 & 2.42 & 4.03 & 1.22 \\
\hline 7465.80 & 399763.20 & 16.20 & 86.23 & 70.53 & 417.25 & 2.27 & 15.55 & -149.47 & -1510.21 & 4.35 & 2.58 & 4.01 & 1.19 \\
\hline 7465.87 & 395865.60 & 16.20 & 81.08 & 67.10 & 417.04 & -2.29 & 14.11 & -136.64 & -3371.10 & 4.04 & 2.37 & 3.90 & 1.91 \\
\hline 7465.93 & 412569.80 & 16.00 & 66.24 & 68.40 & 417.96 & -3.48 & 13.81 & -172.61 & -3251.56 & 4.92 & 1.84 & 3.93 & 2.67 \\
\hline 7466.00 & 423464.00 & 16.00 & 54.82 & 64.73 & 417.36 & -1.35 & 13.57 & -181.64 & -1858.33 & 5.55 & 1.95 & 4.00 & 2.79 \\
\hline 7466.06 & 415990.40 & 16.20 & 61.56 & 66.62 & 416.34 & -1.99 & 12.72 & -235.83 & -1848.46 & 5.66 & 0.81 & 4.12 & 2.67 \\
\hline 7466.13 & 412343.60 & 16.40 & 85.08 & 61.87 & 1.87 & -6.50 & 12.88 & -286.53 & -1585.92 & 5.62 & 0.55 & 4.15 & 2.56 \\
\hline 7466.19 & 410227.80 & 16.40 & 95.57 & 61.87 & 1.68 & -3.00 & 13.87 & -318.18 & -1090.88 & 6.24 & 0.70 & 4.16 & 1.62 \\
\hline 7466.26 & 393064.40 & 16.60 & 95.09 & 58.71 & 0.00 & -1.27 & 14.35 & -289.56 & 775.63 & 5.49 & 0.69 & 4.18 & 0.85 \\
\hline 7466.33 & 395522.60 & 16.60 & 89.94 & 71.54 & 0.94 & -2.38 & 15.88 & -282.58 & 1583.47 & 5.20 & 0.55 & 4.22 & 1.00 \\
\hline 7466.39 & 416754.20 & 16.40 & 79.32 & 71.37 & 0.28 & -4.20 & 16.67 & -297.67 & 2437.94 & 5.32 & 0.50 & 4.31 & 1.11 \\
\hline 7466.46 & 423730.60 & 16.20 & 73.68 & 66.65 & 0.26 & -5.50 & 14.88 & -314.45 & 1848.50 & 5.44 & 0.56 & 4.27 & 1.41 \\
\hline 7466.52 & 420810.20 & 16.20 & 71.37 & 63.65 & 1.72 & -7.09 & 15.83 & -293.91 & 2727.06 & 4.51 & 0.36 & 4.38 & 1.78 \\
\hline 7466.59 & 424339.80 & 16.00 & 76.81 & 68.80 & 1.27 & -6.02 & 15.78 & -294.01 & 1946.25 & 4.39 & 0.35 & 4.42 & 2.29 \\
\hline 7466.65 & 412296.40 & 16.00 & 84.73 & 51.05 & 1.50 & -7.74 & 15.15 & -300.43 & 1540.09 & 4.31 & 0.39 & 4.37 & 2.16 \\
\hline 7466.72 & 409706.40 & 16.00 & 85.86 & 44.69 & 2.64 & -7.32 & 14.46 & -260.28 & 930.47 & 4.25 & 0.26 & 4.37 & 2.08 \\
\hline 7466.78 & 413970.60 & 16.00 & 78.39 & 47.87 & 2.31 & -7.49 & 14.58 & -264.06 & 409.65 & 4.37 & 0.20 & 4.45 & 2.11 \\
\hline
\end{tabular}




\begin{tabular}{|c|c|c|c|c|c|c|c|c|c|c|c|c|c|}
\hline Depth & $\mathrm{XRF}$ & XRF Live Time & $\mathrm{Nb}(\mathrm{ppm})$ & Mo (ppm) & $\mathrm{Ba}(\mathrm{ppm})$ & $\mathrm{Pb}(\mathrm{ppm})$ & Th (ppm) & $\mathrm{U}(\mathrm{ppm})$ & $\mathrm{P}(\mathrm{ppm})$ & $\mathrm{S}(\%)$ & $\mathrm{Cl}(\%)$ & $\mathrm{K}(\%)$ & $\mathrm{Ca}(\%)$ \\
\hline 7466.85 & 413226.20 & 16.00 & 66.02 & 51.18 & 0.96 & -9.16 & 13.38 & -250.68 & -38.22 & 4.46 & 0.22 & 4.36 & 2.65 \\
\hline 7466.92 & 410196.00 & 16.20 & 67.68 & 48.39 & 1.66 & -7.78 & 13.05 & -270.36 & 44.23 & 4.57 & 0.28 & 4.37 & 2.24 \\
\hline 7466.98 & 362956.00 & 16.80 & 68.85 & 59.65 & 1.49 & -7.14 & 12.07 & -274.57 & -1003.59 & 4.48 & 0.56 & 4.35 & 2.73 \\
\hline 7467.05 & 317826.40 & 17.20 & 68.01 & 62.85 & 1.53 & -4.75 & 11.47 & -315.08 & -1512.13 & 4.27 & 0.78 & 4.20 & 3.69 \\
\hline 7467.11 & 316883.60 & 17.20 & 74.46 & 61.41 & 0.88 & -8.36 & 11.05 & -281.81 & -221.63 & 3.98 & 0.80 & 4.19 & 4.35 \\
\hline 7467.18 & 312391.60 & 17.40 & 88.22 & 57.64 & 1.20 & -6.15 & 10.08 & -291.39 & 781.63 & 3.95 & 0.95 & 4.22 & 3.80 \\
\hline 7467.24 & 304218.40 & 17.40 & 86.15 & 55.73 & 2.34 & -10.54 & 10.73 & -280.36 & 1670.56 & 3.78 & 0.93 & 3.98 & 8.14 \\
\hline 7467.31 & 333135.60 & 17.00 & 93.74 & 49.05 & 0.65 & -11.49 & 11.29 & -267.30 & 2599.90 & 3.86 & 0.92 & 3.92 & 7.98 \\
\hline 7467.38 & 366867.20 & 16.80 & 96.27 & 47.05 & -0.64 & -12.26 & 11.68 & -268.34 & 2959.16 & 4.06 & 0.75 & 3.88 & 10.84 \\
\hline 7467.44 & 353229.20 & 17.00 & 96.62 & 47.30 & 0.60 & -8.65 & 13.01 & -283.93 & 3202.08 & 4.02 & 0.76 & 3.84 & 10.84 \\
\hline 7467.51 & 353203.80 & 17.00 & 100.19 & 43.78 & 0.56 & -6.63 & 12.86 & -294.50 & 1479.65 & 4.03 & 0.63 & 3.80 & 10.61 \\
\hline 7467.57 & 367450.20 & 16.80 & 103.65 & 42.40 & 1.17 & -6.09 & 10.69 & -335.78 & 259.09 & 4.45 & 1.28 & 3.84 & 6.72 \\
\hline 7467.64 & 399421.00 & 16.60 & 90.23 & 50.49 & 4.18 & -8.96 & 10.96 & -365.93 & -597.20 & 4.51 & 1.71 & 3.42 & 13.69 \\
\hline 7467.70 & 415016.80 & 16.40 & 78.74 & 62.27 & 4.94 & -10.80 & 9.90 & -342.44 & 525.41 & 4.59 & 1.73 & 3.05 & 21.14 \\
\hline 7467.77 & 424855.80 & 16.20 & 64.04 & 64.71 & 4.29 & -11.44 & 7.92 & -343.72 & -4621.38 & 4.68 & 1.99 & 2.46 & 31.78 \\
\hline 7467.83 & 431621.80 & 16.00 & 46.82 & 77.95 & 3.31 & -16.65 & 7.48 & -334.64 & -2994.00 & 4.69 & 2.28 & 1.97 & 41.19 \\
\hline 7467.90 & 421037.80 & 16.20 & 46.62 & 78.86 & 2.94 & -16.28 & 8.86 & -280.50 & -2812.26 & 4.21 & 1.66 & 1.90 & 44.62 \\
\hline 7467.97 & 396207.20 & 16.40 & 63.33 & 75.77 & 1.69 & -13.05 & 9.19 & -244.56 & -1932.09 & 3.96 & 1.32 & 2.25 & 37.91 \\
\hline 7468.03 & 362150.80 & 16.60 & 78.84 & 56.61 & 1.85 & -12.32 & 9.90 & -232.61 & -3684.12 & 3.69 & 1.34 & 2.80 & 26.67 \\
\hline 7468.10 & 333071.80 & 17.00 & 91.71 & 55.73 & 2.97 & -12.95 & 11.54 & -225.55 & 740.04 & 3.65 & 1.14 & 3.30 & 15.37 \\
\hline 7468.16 & 334589.80 & 17.00 & 97.96 & 54.33 & 3.93 & -7.97 & 13.04 & -230.12 & 219.10 & 4.16 & 0.88 & 3.85 & 6.07 \\
\hline 7468.23 & 348797.00 & 16.80 & 99.98 & 56.92 & 2.13 & -9.48 & 15.83 & -223.42 & 560.96 & 5.18 & 1.05 & 4.00 & 3.08 \\
\hline 7468.29 & 358051.80 & 16.60 & 96.62 & 53.75 & 1.68 & -10.21 & 15.41 & -226.97 & 589.31 & 5.33 & 0.72 & 4.12 & 2.38 \\
\hline 7468.36 & 372487.20 & 16.60 & 105.30 & 63.82 & 0.38 & -8.92 & 15.14 & -225.41 & 1248.77 & 5.35 & 0.85 & 4.04 & 2.35 \\
\hline 7468.43 & 390695.00 & 16.40 & 119.11 & 53.07 & 0.67 & -7.10 & 15.81 & -224.39 & 2273.95 & 5.47 & 0.91 & 4.14 & 2.12 \\
\hline 7468.49 & 381701.60 & 16.60 & 132.29 & 45.94 & 0.70 & -7.97 & 17.37 & -206.99 & 2163.07 & 5.02 & 0.87 & 4.09 & 1.77 \\
\hline 7468.56 & 369951.40 & 16.80 & 136.18 & 40.49 & -0.11 & -4.53 & 12.76 & -211.42 & 1911.70 & 4.14 & 1.27 & 4.03 & 0.83 \\
\hline 7468.62 & 362688.40 & 17.00 & 130.54 & 40.48 & -0.72 & -2.22 & 13.80 & -220.79 & 874.54 & 4.42 & 1.71 & 3.94 & 1.14 \\
\hline 7468.69 & 357257.20 & 17.00 & 113.59 & 33.28 & 0.50 & -0.66 & 14.09 & -246.81 & 45.14 & 4.89 & 1.97 & 3.91 & 1.64 \\
\hline 7468.75 & 347600.00 & 17.00 & 91.88 & 41.64 & -1.89 & -2.07 & 13.87 & -228.66 & -668.50 & 5.52 & 2.64 & 3.78 & 1.98 \\
\hline 7468.82 & 328541.60 & 17.20 & 72.04 & 41.82 & -1.24 & -4.42 & 10.06 & -210.99 & -648.70 & 5.38 & 2.83 & 3.70 & 2.79 \\
\hline 7468.88 & 310275.60 & 17.40 & 50.60 & 45.25 & 232.77 & 1.19 & 11.68 & -163.94 & -2064.04 & 5.58 & 2.59 & 3.71 & 3.86 \\
\hline 7468.95 & 287735.40 & 17.60 & 55.94 & 44.47 & 233.07 & 2.57 & 11.05 & -140.78 & -2979.63 & 5.02 & 3.38 & 3.62 & 3.59 \\
\hline
\end{tabular}




\begin{tabular}{|c|c|c|c|c|c|c|c|c|c|c|c|c|c|}
\hline Depth & $\mathrm{XRF}$ & XRF Live Time & $\mathrm{Nb}(\mathrm{ppm})$ & Mo (ppm) & $\mathrm{Ba}(\mathrm{ppm})$ & $\mathrm{Pb}(\mathrm{ppm})$ & Th (ppm) & $\mathrm{U}(\mathrm{ppm})$ & $\mathrm{P}(\mathrm{ppm})$ & $\mathrm{S}(\%)$ & $\mathrm{Cl}(\%)$ & $\mathrm{K}(\%)$ & $\mathrm{Ca}(\%)$ \\
\hline 7469.02 & 267994.40 & 17.80 & 73.42 & 47.65 & 232.24 & 1.77 & 10.57 & -120.17 & -2306.27 & 4.36 & 3.58 & 3.59 & 3.13 \\
\hline 7469.08 & 264789.60 & 17.80 & 95.34 & 32.42 & 233.15 & 3.09 & 11.60 & -129.38 & -3809.35 & 3.62 & 4.10 & 3.52 & 2.84 \\
\hline 7469.15 & 292245.00 & 17.40 & 124.22 & 29.55 & 232.06 & 4.40 & 13.93 & -132.43 & -3355.45 & 3.88 & 4.41 & 3.55 & 2.10 \\
\hline 7469.21 & 326621.00 & 17.00 & 151.01 & 32.89 & -1.22 & -2.92 & 14.05 & -198.19 & -1385.31 & 4.40 & 4.45 & 3.58 & 1.37 \\
\hline 7469.28 & 365018.40 & 16.60 & 155.36 & 35.06 & 0.39 & -3.89 & 14.63 & -193.05 & 546.35 & 5.22 & 4.13 & 3.65 & 1.55 \\
\hline 7469.34 & 405316.60 & 16.20 & 148.88 & 36.33 & 0.83 & -5.37 & 17.44 & -199.43 & 163.68 & 5.75 & 4.13 & 3.68 & 2.06 \\
\hline 7469.41 & 430871.60 & 16.00 & 145.91 & 51.97 & 1.50 & -3.46 & 17.42 & -195.21 & 2523.17 & 6.24 & 4.03 & 3.73 & 2.42 \\
\hline 7469.48 & 435326.60 & 16.00 & 147.45 & 56.37 & 1.75 & -4.32 & 18.85 & -204.58 & 3176.90 & 6.38 & 3.64 & 3.81 & 2.47 \\
\hline 7469.54 & 432866.40 & 16.00 & 159.66 & 52.79 & 2.70 & -1.74 & 21.44 & -177.85 & 2570.62 & 5.76 & 3.25 & 3.98 & 2.00 \\
\hline 7469.61 & 431538.60 & 16.00 & 174.99 & 50.76 & 1.61 & -0.79 & 22.59 & -212.83 & 2902.01 & 5.54 & 2.97 & 4.01 & 1.89 \\
\hline 7469.67 & 427831.80 & 16.00 & 184.37 & 50.26 & 1.34 & -0.09 & 19.99 & -190.93 & 2999.78 & 5.57 & 2.77 & 4.05 & 1.47 \\
\hline 7469.74 & 408154.40 & 16.20 & 184.74 & 53.68 & 0.59 & -0.99 & 20.96 & -208.02 & 2846.47 & 5.48 & 3.32 & 4.01 & 1.59 \\
\hline 7469.80 & 404695.40 & 16.20 & 171.79 & 56.68 & 0.89 & -2.33 & 20.12 & -216.97 & 2190.77 & 5.78 & 4.10 & 3.89 & 2.58 \\
\hline 7469.87 & 402210.20 & 16.20 & 157.42 & 57.72 & -1.44 & -5.63 & 17.07 & -218.92 & 2834.55 & 5.91 & 4.60 & 3.80 & 2.86 \\
\hline 7469.93 & 399663.40 & 16.20 & 139.61 & 55.18 & -0.89 & -8.04 & 16.13 & -218.97 & 2103.23 & 5.40 & 4.17 & 3.84 & 2.68 \\
\hline 7470.00 & 397132.80 & 16.20 & 134.07 & 53.19 & -1.90 & -5.56 & 19.13 & -214.70 & 3027.26 & 5.36 & 4.45 & 3.82 & 2.62 \\
\hline 7470.07 & 414241.40 & 16.00 & 129.96 & 57.93 & -1.23 & -11.84 & 15.93 & -199.00 & 2113.35 & 5.08 & 4.19 & 3.59 & 5.85 \\
\hline 7470.13 & 417043.40 & 16.00 & 136.12 & 53.35 & 0.04 & -8.11 & 16.37 & -194.42 & 1983.80 & 4.63 & 4.10 & 3.69 & 4.76 \\
\hline 7470.20 & 411728.40 & 16.00 & 132.00 & 47.54 & 1.19 & -5.94 & 18.67 & -211.44 & 1127.23 & 4.40 & 4.26 & 3.67 & 4.40 \\
\hline 7470.26 & 407770.80 & 16.00 & 125.51 & 52.73 & 1.92 & -5.15 & 20.10 & -205.77 & -855.29 & 4.46 & 4.24 & 3.63 & 6.33 \\
\hline 7470.33 & 403338.00 & 16.20 & 115.54 & 59.46 & 3.42 & -8.80 & 20.70 & -219.31 & -1716.02 & 4.30 & 4.11 & 3.55 & 8.93 \\
\hline 7470.39 & 399566.20 & 16.20 & 112.10 & 47.85 & 2.41 & -1.49 & 22.31 & -208.21 & -1549.12 & 4.63 & 3.80 & 3.82 & 5.24 \\
\hline 7470.46 & 393044.40 & 16.20 & 105.81 & 47.09 & 1.53 & -4.93 & 22.13 & -202.53 & -341.99 & 4.84 & 3.46 & 3.83 & 5.37 \\
\hline 7470.52 & 396780.60 & 16.20 & 108.93 & 52.71 & 2.38 & -5.35 & 21.46 & -190.76 & 35.66 & 5.23 & 3.13 & 3.86 & 5.43 \\
\hline 7470.59 & 400921.00 & 16.20 & 110.92 & 45.63 & 1.79 & -6.65 & 20.43 & -167.50 & 2155.90 & 5.93 & 3.81 & 3.80 & 3.67 \\
\hline 7470.66 & 407659.60 & 16.00 & 111.86 & 41.40 & 0.82 & -5.20 & 16.75 & -185.03 & 3510.44 & 6.32 & 3.30 & 3.96 & 1.58 \\
\hline 7470.72 & 417158.00 & 16.00 & 100.92 & 46.11 & 1.40 & -6.79 & 18.07 & -238.88 & 2073.39 & 6.76 & 2.29 & 3.86 & 6.47 \\
\hline 7470.79 & 421962.40 & 16.00 & 98.41 & 52.29 & 0.63 & -4.61 & 18.83 & -283.93 & 800.25 & 7.74 & 2.38 & 3.82 & 8.32 \\
\hline 7470.85 & 427991.40 & 16.00 & 100.38 & 48.55 & 0.83 & -6.91 & 17.28 & -296.83 & 1115.92 & 8.83 & 2.29 & 3.85 & 9.29 \\
\hline 7470.92 & 417190.40 & 16.20 & 112.54 & 51.82 & 1.20 & -6.02 & 15.77 & -327.44 & 1066.89 & 8.17 & 1.84 & 3.90 & 9.30 \\
\hline 7470.98 & 394568.40 & 16.40 & 120.25 & 53.09 & 1.70 & -5.93 & 15.99 & -291.57 & 381.82 & 7.59 & 2.52 & 3.75 & 9.04 \\
\hline 7471.05 & 336900.20 & 17.00 & 139.85 & 60.59 & 2.20 & -6.77 & 16.39 & -253.46 & 491.50 & 6.62 & 2.38 & 3.77 & 4.10 \\
\hline 7471.12 & 324202.60 & 17.20 & 142.30 & 65.42 & 3.44 & -11.67 & 13.58 & -200.81 & 1752.20 & 5.48 & 3.19 & 3.50 & 4.79 \\
\hline
\end{tabular}




\begin{tabular}{|c|c|c|c|c|c|c|c|c|c|c|c|c|c|}
\hline Depth & $\mathrm{XRF}$ & XRF Live Time & $\mathrm{Nb}(\mathrm{ppm})$ & Mo (ppm) & $\mathrm{Ba}(\mathrm{ppm})$ & $\mathrm{Pb}(\mathrm{ppm})$ & Th (ppm) & $\mathrm{U}(\mathrm{ppm})$ & $\mathrm{P}(\mathrm{ppm})$ & $\mathrm{S}(\%)$ & $\mathrm{Cl}(\%)$ & $\mathrm{K}(\%)$ & $\mathrm{Ca}(\%)$ \\
\hline 7471.18 & 306767.40 & 17.40 & 128.81 & 73.06 & 2.97 & -11.22 & 18.49 & -213.11 & 667.68 & 3.84 & 2.93 & 3.15 & 9.21 \\
\hline 7471.25 & 305659.20 & 17.40 & 104.09 & 75.03 & 2.39 & -14.20 & 20.94 & -214.68 & 405.75 & 3.54 & 2.49 & 2.88 & 15.93 \\
\hline 7471.31 & 318115.80 & 17.40 & 103.52 & 70.16 & 0.94 & -14.54 & 22.43 & -268.26 & 393.28 & 3.64 & 2.48 & 2.83 & 17.33 \\
\hline 7471.38 & 360035.20 & 17.00 & 76.32 & 78.89 & 194.10 & -8.03 & 18.69 & -227.35 & 100.87 & 3.60 & 2.54 & 2.97 & 17.91 \\
\hline 7471.44 & 362207.40 & 17.00 & 83.26 & 75.70 & 192.90 & -5.71 & 21.01 & -246.84 & 139.66 & 3.48 & 1.58 & 3.19 & 16.54 \\
\hline 7471.51 & 375636.00 & 16.80 & 106.15 & 70.84 & 192.33 & -0.04 & 15.85 & -224.85 & -188.22 & 3.83 & 1.84 & 3.51 & 11.41 \\
\hline 7471.57 & 385845.00 & 16.60 & 131.73 & 73.67 & 192.03 & 0.76 & 14.77 & -214.11 & -233.80 & 4.25 & 1.91 & 3.85 & 4.69 \\
\hline 7471.64 & 396336.60 & 16.40 & 132.69 & 84.88 & 193.46 & -2.85 & 14.23 & -194.67 & -1105.21 & 4.19 & 1.55 & 3.81 & 6.99 \\
\hline 7471.71 & 403458.80 & 16.20 & 160.81 & 70.45 & 1.75 & -7.70 & 19.07 & -270.39 & -784.13 & 4.73 & 1.53 & 3.86 & 6.95 \\
\hline 7471.77 & 412525.60 & 16.00 & 170.76 & 70.05 & 0.78 & -4.54 & 20.02 & -281.37 & -2303.80 & 5.23 & 1.14 & 4.00 & 5.59 \\
\hline 7471.84 & 400593.80 & 16.20 & 168.11 & 66.68 & 0.84 & -11.23 & 22.49 & -284.57 & -2501.24 & 5.22 & 1.23 & 3.99 & 5.22 \\
\hline 7471.90 & 405710.60 & 16.20 & 165.22 & 69.98 & 1.30 & -9.47 & 23.68 & -289.70 & -2575.10 & 5.01 & 1.15 & 3.99 & 5.03 \\
\hline 7471.97 & 403494.00 & 16.20 & 172.43 & 61.59 & 1.30 & -6.59 & 23.86 & -306.92 & -1722.29 & 5.09 & 0.70 & 4.28 & 0.91 \\
\hline 7472.03 & 402930.60 & 16.20 & 166.99 & 56.83 & 6.92 & -11.59 & 22.04 & -282.19 & -1168.63 & 5.02 & 1.09 & 4.26 & 0.35 \\
\hline 7472.10 & 398760.20 & 16.20 & 158.14 & 49.75 & 9.25 & -13.64 & 21.79 & -285.41 & -314.09 & 4.80 & 1.61 & 4.19 & 0.56 \\
\hline 7472.17 & 403291.80 & 16.00 & 158.03 & 50.43 & 10.67 & -8.55 & 23.11 & -319.80 & -45.70 & 4.71 & 1.40 & 4.20 & 0.72 \\
\hline 7472.23 & 399201.20 & 16.00 & 164.89 & 43.38 & 11.15 & -6.63 & 24.61 & -287.14 & -702.76 & 4.93 & 1.72 & 4.18 & 0.93 \\
\hline 7472.30 & 399384.20 & 16.00 & 165.40 & 42.09 & 12.53 & -6.82 & 26.77 & -295.03 & -1358.41 & 5.43 & 2.35 & 4.09 & 1.20 \\
\hline 7472.36 & 403963.80 & 16.00 & 170.47 & 42.39 & 4.24 & -4.36 & 27.20 & -306.96 & -1328.44 & 5.70 & 2.07 & 4.16 & 1.13 \\
\hline 7472.43 & 420295.60 & 16.00 & 170.92 & 50.40 & 3.92 & -6.93 & 27.01 & -306.73 & -1255.52 & 5.71 & 1.64 & 4.23 & 1.55 \\
\hline 7472.49 & 432226.00 & 16.00 & 169.80 & 57.24 & 2.67 & -10.16 & 25.48 & -292.18 & -1032.32 & 6.08 & 1.59 & 4.29 & 1.52 \\
\hline 7472.56 & 432912.20 & 16.00 & 165.90 & 66.24 & 0.79 & -11.15 & 23.32 & -324.77 & -1277.59 & 6.20 & 1.52 & 4.39 & 1.52 \\
\hline 7472.62 & 427935.80 & 16.00 & 167.57 & 70.50 & -0.84 & -14.27 & 23.52 & -290.42 & -901.88 & 5.70 & 1.50 & 4.37 & 1.38 \\
\hline 7472.69 & 420779.20 & 16.20 & 172.44 & 71.42 & -1.22 & -17.78 & 24.46 & -271.07 & -945.44 & 5.58 & 2.86 & 4.15 & 1.47 \\
\hline 7472.76 & 406963.20 & 16.20 & 174.16 & 67.73 & -2.70 & -13.31 & 24.02 & -253.52 & -1714.95 & 5.63 & 3.46 & 4.03 & 0.97 \\
\hline 7472.82 & 398229.80 & 16.20 & 170.03 & 56.71 & -2.65 & -12.77 & 24.39 & -252.84 & -793.75 & 5.47 & 3.58 & 4.01 & 0.87 \\
\hline 7472.89 & 411701.20 & 16.00 & 163.61 & 53.14 & -0.38 & -13.71 & 23.99 & -252.77 & 67.27 & 5.58 & 3.33 & 3.95 & 1.05 \\
\hline 7472.95 & 420472.80 & 16.00 & 166.10 & 54.76 & 0.30 & -12.85 & 23.65 & -280.44 & -344.68 & 6.07 & 2.99 & 3.99 & 1.05 \\
\hline 7473.02 & 425694.00 & 15.80 & 162.63 & 52.89 & 1.95 & -11.04 & 23.95 & -274.84 & -361.87 & 5.84 & 1.78 & 4.19 & 0.91 \\
\hline 7473.08 & 422481.00 & 16.00 & 160.20 & 57.43 & 3.13 & -11.37 & 23.55 & -277.71 & -1114.97 & 5.52 & 1.69 & 4.23 & 0.81 \\
\hline 7473.15 & 427241.80 & 16.00 & 157.66 & 70.18 & 4.78 & -9.04 & 23.97 & -265.81 & -1321.74 & 5.47 & 1.47 & 4.22 & 0.77 \\
\hline 7473.22 & 417557.00 & 16.20 & 149.82 & 68.98 & 1.94 & -8.67 & 23.72 & -271.69 & -712.88 & 5.79 & 2.08 & 4.18 & 0.47 \\
\hline 7473.28 & 415780.20 & 16.20 & 130.96 & 70.96 & 2.95 & -6.98 & 20.49 & -297.77 & 609.19 & 5.76 & 1.97 & 4.20 & 0.39 \\
\hline
\end{tabular}




\begin{tabular}{|c|c|c|c|c|c|c|c|c|c|c|c|c|c|}
\hline Depth & $\mathrm{XRF}$ & XRF Live Time & $\mathrm{Nb}(\mathrm{ppm})$ & Mo (ppm) & $\mathrm{Ba}(\mathrm{ppm})$ & $\mathrm{Pb}(\mathrm{ppm})$ & Th (ppm) & $\mathrm{U}(\mathrm{ppm})$ & $\mathrm{P}(\mathrm{ppm})$ & $\mathrm{S}(\%)$ & $\mathrm{Cl}(\%)$ & $\mathrm{K}(\%)$ & $\mathrm{Ca}(\%)$ \\
\hline 7473.35 & 421878.20 & 16.20 & 123.65 & 76.60 & 1.57 & -7.00 & 18.56 & -348.76 & 1929.02 & 6.33 & 1.71 & 4.25 & 0.37 \\
\hline 7473.41 & 423813.80 & 16.00 & 121.62 & 71.90 & 0.50 & -8.53 & 19.64 & -351.38 & 2648.35 & 6.84 & 1.35 & 4.30 & 0.40 \\
\hline 7473.48 & 410664.60 & 16.20 & 123.50 & 62.81 & 0.42 & -9.41 & 18.29 & -359.14 & 3132.92 & 6.73 & 1.54 & 4.26 & 0.39 \\
\hline 7473.54 & 405761.00 & 16.20 & 130.61 & 62.07 & 0.93 & -5.71 & 19.24 & -340.58 & 2011.76 & 6.08 & 1.45 & 4.25 & 0.28 \\
\hline 7473.61 & 401889.60 & 16.20 & 131.66 & 53.37 & 0.37 & -6.50 & 19.53 & -329.72 & 2161.38 & 5.82 & 1.46 & 4.21 & 0.56 \\
\hline 7473.67 & 392795.60 & 16.20 & 127.52 & 60.30 & 1.17 & -5.35 & 18.40 & -351.34 & 1496.58 & 5.43 & 1.43 & 4.12 & 0.77 \\
\hline 7473.74 & 391093.40 & 16.40 & 124.52 & 67.12 & 0.58 & -5.72 & 18.11 & -374.28 & 663.72 & 5.75 & 1.23 & 4.11 & 0.84 \\
\hline 7473.81 & 385743.00 & 16.40 & 125.45 & 68.14 & -0.80 & -8.57 & 19.31 & -375.22 & 800.85 & 5.72 & 1.46 & 4.09 & 0.90 \\
\hline 7473.87 & 373672.80 & 16.60 & 129.83 & 63.46 & -0.30 & -12.41 & 18.19 & -383.59 & 1452.82 & 5.60 & 1.51 & 4.01 & 1.03 \\
\hline 7473.94 & 357066.20 & 16.80 & 135.75 & 63.63 & -1.07 & -14.66 & 19.10 & -334.95 & -287.31 & 5.20 & 1.89 & 3.96 & 0.79 \\
\hline 7474.00 & 340849.40 & 17.00 & 143.61 & 56.77 & -0.76 & -10.61 & 21.45 & -260.95 & -348.49 & 4.75 & 2.11 & 3.93 & 0.55 \\
\hline 7474.07 & 336378.80 & 17.00 & 143.82 & 48.90 & -1.58 & -7.87 & 21.24 & -234.98 & 952.06 & 4.17 & 2.47 & 3.82 & 0.54 \\
\hline 7474.13 & 355691.80 & 16.80 & 140.61 & 47.37 & -0.51 & -6.61 & 20.93 & -257.17 & 947.75 & 5.58 & 2.09 & 3.84 & 0.50 \\
\hline 7474.20 & 360631.00 & 16.80 & 135.86 & 44.48 & 0.16 & -4.19 & 21.54 & -247.49 & 1560.06 & 5.74 & 2.01 & 3.80 & 0.44 \\
\hline 7474.27 & 369098.60 & 16.80 & 133.97 & 43.79 & 1.38 & 0.80 & 21.55 & -245.06 & 2971.56 & 5.65 & 1.57 & 3.81 & 0.37 \\
\hline 7474.33 & 376985.40 & 16.80 & 128.77 & 39.11 & 2.31 & -0.59 & 17.70 & -244.68 & 2938.71 & 5.68 & 2.06 & 3.73 & 0.44 \\
\hline 7474.40 & 382183.00 & 16.60 & 106.14 & 58.75 & 255.87 & 3.79 & 16.94 & -194.96 & 3350.54 & 5.58 & 1.84 & 3.73 & 0.47 \\
\hline 7474.46 & 367169.80 & 16.80 & 109.27 & 60.81 & 254.70 & 5.46 & 18.39 & -113.60 & 1005.39 & 3.98 & 2.52 & 3.59 & 0.54 \\
\hline 7474.53 & 371485.80 & 16.60 & 110.46 & 68.86 & 251.91 & 3.68 & 18.85 & -130.66 & -183.75 & 3.77 & 2.03 & 3.72 & 0.57 \\
\hline 7474.59 & 378377.80 & 16.40 & 112.14 & 77.61 & 251.85 & 1.05 & 21.38 & -127.46 & -666.33 & 4.08 & 2.72 & 3.66 & 0.70 \\
\hline 7474.66 & 385502.40 & 16.20 & 91.14 & 93.26 & 499.41 & 6.09 & 22.70 & -91.92 & -1582.23 & 4.10 & 2.28 & 3.79 & 0.67 \\
\hline 7474.72 & 360545.40 & 16.60 & 100.59 & 70.87 & 249.96 & 2.12 & 21.82 & -99.46 & -3325.01 & 3.61 & 5.12 & 3.47 & 3.87 \\
\hline 7474.79 & 368404.20 & 16.40 & 87.79 & 73.41 & 249.86 & 2.24 & 21.11 & -130.18 & -892.20 & 3.69 & 4.42 & 3.67 & 4.55 \\
\hline 7474.86 & 339582.00 & 16.80 & 80.06 & 81.48 & 251.91 & 4.22 & 18.57 & -131.12 & -1913.76 & 3.79 & 4.40 & 3.58 & 5.80 \\
\hline 7474.92 & 330499.20 & 17.00 & 78.71 & 79.29 & 249.88 & 5.35 & 16.23 & -151.36 & -2153.39 & 3.90 & 3.60 & 3.62 & 6.94 \\
\hline 7474.99 & 321158.80 & 17.20 & 104.15 & 61.33 & 0.57 & 2.71 & 18.36 & -206.53 & -768.59 & 3.96 & 3.26 & 3.61 & 6.98 \\
\hline 7475.05 & 338359.20 & 17.00 & 119.33 & 67.49 & -1.01 & 2.35 & 19.40 & -262.77 & -213.82 & 4.31 & 0.18 & 3.99 & 3.97 \\
\hline 7475.12 & 342408.80 & 17.00 & 129.89 & 65.70 & 0.39 & 4.01 & 17.72 & -277.15 & -1290.33 & 4.43 & 0.15 & 3.99 & 3.22 \\
\hline 7475.18 & 371942.40 & 16.60 & 133.11 & 56.79 & -0.45 & 5.36 & 20.26 & -256.91 & -431.06 & 4.67 & 0.17 & 4.09 & 1.94 \\
\hline 7475.25 & 381287.40 & 16.40 & 128.91 & 51.12 & 0.25 & 5.82 & 19.82 & -261.09 & 35.92 & 4.67 & 0.26 & 4.14 & 1.23 \\
\hline 7475.31 & 391896.80 & 16.20 & 120.61 & 49.74 & 1.15 & 2.83 & 16.89 & -229.70 & -261.60 & 4.72 & 0.50 & 4.04 & 2.98 \\
\hline 7475.38 & 401362.00 & 16.00 & 115.27 & 46.78 & -0.79 & 2.32 & 16.20 & -239.60 & 229.38 & 5.02 & 0.57 & 3.98 & 4.19 \\
\hline 7475.45 & 394731.40 & 16.20 & 108.37 & 51.83 & -2.16 & -1.21 & 15.66 & -228.93 & -1051.14 & 4.90 & 0.61 & 3.88 & 7.05 \\
\hline
\end{tabular}




\begin{tabular}{|c|c|c|c|c|c|c|c|c|c|c|c|c|c|}
\hline Depth & XRF & XRF Live Time & $\mathrm{Nb}(\mathrm{ppm})$ & Mo (ppm) & $\mathrm{Ba}(\mathrm{ppm})$ & $\mathrm{Pb}(\mathrm{ppm})$ & Th (ppm) & $\mathrm{U}(\mathrm{ppm})$ & $\mathrm{P}(\mathrm{ppm})$ & $\mathrm{S}(\%)$ & $\mathrm{Cl}(\%)$ & $\mathrm{K}(\%)$ & $\mathrm{Ca}(\%)$ \\
\hline 7475.51 & 392981.40 & 16.40 & 99.47 & 56.00 & -2.12 & -6.90 & 17.27 & -246.15 & -853.16 & 4.55 & 0.83 & 3.74 & 10.68 \\
\hline 7475.58 & 435587.80 & 16.00 & 91.48 & 179.39 & -14.46 & 16.84 & 7.04 & -1232.84 & 10631.64 & 16.27 & 0.84 & 2.99 & 10.02 \\
\hline 7475.64 & 442466.00 & 16.00 & 106.63 & 186.86 & -13.50 & 17.91 & 9.26 & -1260.53 & 10177.37 & 16.11 & 0.59 & 3.17 & 8.34 \\
\hline 7475.71 & 449288.60 & 16.00 & 111.17 & 187.52 & -13.33 & 19.50 & 9.10 & -1249.34 & 9596.28 & 15.70 & 0.52 & 3.28 & 6.83 \\
\hline 7475.77 & 461685.60 & 15.80 & 119.87 & 186.74 & -12.64 & 23.32 & 9.76 & -1259.91 & 12239.00 & 15.80 & 0.51 & 3.39 & 4.18 \\
\hline 7475.84 & 473684.60 & 15.60 & 127.75 & 179.09 & -11.53 & 27.89 & 7.49 & -1249.00 & 13665.34 & 16.28 & 0.29 & 3.52 & 1.26 \\
\hline 7475.91 & 444850.80 & 16.00 & 138.51 & 57.40 & 1.84 & 5.63 & 18.97 & -235.56 & 3003.76 & 4.64 & 0.19 & 4.24 & 1.89 \\
\hline 7475.97 & 442534.20 & 16.00 & 129.03 & 53.96 & 2.19 & 7.43 & 17.30 & -244.34 & 4197.58 & 4.70 & 0.19 & 4.23 & 1.95 \\
\hline 7476.04 & 441556.00 & 16.00 & 128.08 & 57.08 & 3.97 & 8.27 & 16.91 & -243.35 & 5186.35 & 4.90 & 0.21 & 4.22 & 2.46 \\
\hline 7476.10 & 433924.40 & 16.00 & 128.19 & 52.01 & 3.70 & 4.62 & 16.19 & -231.82 & 4020.94 & 4.65 & 0.18 & 4.20 & 2.34 \\
\hline 7476.17 & 433642.20 & 16.00 & 141.52 & 56.53 & 4.01 & -0.05 & 15.86 & -229.33 & 2983.87 & 4.39 & 0.27 & 4.28 & 1.67 \\
\hline 7476.23 & 430775.40 & 16.00 & 142.18 & 59.41 & 3.78 & -5.72 & 14.50 & -243.90 & 2869.47 & 4.21 & 0.26 & 4.39 & 1.12 \\
\hline 7476.30 & 433624.40 & 16.00 & 140.94 & 59.44 & 2.52 & -12.26 & 14.19 & -251.70 & 1259.46 & 4.47 & 0.25 & 4.48 & 0.90 \\
\hline 7476.36 & 431395.00 & 16.00 & 139.64 & 56.84 & 2.21 & -13.60 & 14.63 & -252.85 & 1098.00 & 4.63 & 0.28 & 4.46 & 0.62 \\
\hline 7476.43 & 425840.80 & 16.20 & 135.47 & 55.97 & 1.38 & -11.93 & 12.57 & -269.01 & 862.67 & 4.71 & 0.54 & 4.43 & 0.56 \\
\hline 7476.50 & 412487.60 & 16.40 & 123.45 & 53.83 & 3.98 & -12.91 & 12.69 & -261.03 & 1227.86 & 4.51 & 0.78 & 4.36 & 0.53 \\
\hline 7476.56 & 408808.20 & 16.40 & 130.57 & 57.86 & 3.62 & -7.74 & 13.17 & -295.59 & 1465.47 & 5.36 & 0.79 & 4.39 & 0.67 \\
\hline 7476.63 & 416096.80 & 16.40 & 122.95 & 62.65 & 2.43 & -4.28 & 14.67 & -281.33 & 3632.23 & 5.92 & 0.87 & 4.31 & 1.18 \\
\hline 7476.69 & 423935.20 & 16.40 & 112.24 & 65.86 & 2.03 & -4.75 & 12.63 & -266.49 & 3437.87 & 6.95 & 1.74 & 4.24 & 1.32 \\
\hline 7476.76 & 439730.00 & 16.20 & 90.12 & 74.14 & 246.93 & 0.56 & 15.54 & -209.61 & 3977.31 & 7.87 & 1.52 & 4.29 & 1.36 \\
\hline 7476.82 & 449982.20 & 16.00 & 87.17 & 75.17 & 243.23 & 4.16 & 15.28 & -247.15 & 3634.78 & 8.65 & 1.26 & 4.33 & 1.48 \\
\hline 7476.89 & 450770.40 & 16.00 & 76.79 & 65.51 & 242.27 & 2.75 & 13.24 & -235.47 & 3718.79 & 7.98 & 1.27 & 4.27 & 1.56 \\
\hline 7476.96 & 443762.40 & 16.00 & 82.27 & 60.10 & 242.16 & 1.39 & 12.11 & -218.86 & 2964.70 & 7.49 & 1.22 & 4.26 & 1.42 \\
\hline 7477.02 & 446491.40 & 16.00 & 88.24 & 58.92 & 243.23 & 0.38 & 15.67 & -272.11 & 2740.33 & 7.24 & 0.46 & 4.39 & 1.30 \\
\hline 7477.09 & 445508.40 & 16.00 & 105.52 & 64.02 & -1.90 & -4.11 & 15.43 & -351.07 & 4747.55 & 7.52 & 0.54 & 4.41 & 1.35 \\
\hline 7477.15 & 445018.20 & 16.00 & 98.88 & 65.91 & -0.89 & -5.01 & 13.95 & -345.64 & 4235.95 & 6.95 & 0.45 & 4.45 & 1.16 \\
\hline 7477.22 & 434980.40 & 16.00 & 90.75 & 66.31 & 0.24 & -3.52 & 14.69 & -314.81 & 1707.86 & 6.49 & 0.44 & 4.44 & 0.96 \\
\hline 7477.28 & 423113.40 & 16.20 & 84.84 & 67.99 & 0.84 & -2.04 & 14.71 & -329.10 & 1904.10 & 6.05 & 0.88 & 4.38 & 0.72 \\
\hline 7477.35 & 401500.60 & 16.40 & 82.27 & 55.08 & -1.07 & -2.86 & 12.48 & -273.93 & 1617.77 & 4.81 & 0.78 & 4.33 & 0.47 \\
\hline 7477.41 & 381190.20 & 16.60 & 86.58 & 42.16 & -0.69 & -4.01 & 11.35 & -234.82 & -206.31 & 3.44 & 0.66 & 4.35 & 0.34 \\
\hline 7477.48 & 369767.20 & 16.80 & 97.68 & 34.82 & -1.12 & -0.34 & 13.02 & -209.97 & 400.52 & 3.30 & 0.70 & 4.32 & 0.37 \\
\hline 7477.55 & 362129.20 & 17.00 & 108.92 & 28.07 & -2.72 & 0.97 & 14.01 & -198.09 & 1301.02 & 3.32 & 0.71 & 4.36 & 0.42 \\
\hline 7477.61 & 354263.80 & 17.00 & 115.23 & 18.35 & -1.74 & 1.68 & 14.24 & -202.28 & 28.78 & 3.45 & 0.38 & 4.38 & 0.33 \\
\hline
\end{tabular}




\begin{tabular}{|c|c|c|c|c|c|c|c|c|c|c|c|c|c|}
\hline Depth & $\mathrm{XRF}$ & XRF Live Time & $\mathrm{Nb}(\mathrm{ppm})$ & Mo (ppm) & $\mathrm{Ba}(\mathrm{ppm})$ & $\mathrm{Pb}(\mathrm{ppm})$ & Th (ppm) & $\mathrm{U}(\mathrm{ppm})$ & $\mathrm{P}(\mathrm{ppm})$ & $\mathrm{S}(\%)$ & $\mathrm{Cl}(\%)$ & $\mathrm{K}(\%)$ & $\mathrm{Ca}(\%)$ \\
\hline 7477.68 & 368130.60 & 16.80 & 125.66 & 27.60 & 0.53 & 0.75 & 15.78 & -245.40 & 1069.32 & 5.12 & 0.33 & 4.42 & 0.36 \\
\hline 7477.74 & 366785.40 & 16.80 & 104.24 & 44.33 & 337.48 & 5.95 & 16.65 & -205.13 & 434.87 & 5.53 & 0.39 & 4.36 & 0.37 \\
\hline 7477.81 & 354511.60 & 16.80 & 98.14 & 44.92 & 336.82 & 3.28 & 15.59 & -202.35 & 590.76 & 5.47 & 0.35 & 4.31 & 0.36 \\
\hline 7477.87 & 347173.20 & 16.80 & 93.08 & 47.93 & 338.05 & 2.17 & 13.61 & -216.89 & 606.06 & 5.40 & 0.34 & 4.11 & 0.31 \\
\hline 7477.94 & 345798.60 & 16.80 & 93.28 & 54.64 & 336.89 & 4.77 & 13.17 & -207.99 & 441.94 & 5.21 & 0.21 & 4.07 & 0.24 \\
\hline 7478.01 & 323767.40 & 17.00 & 88.91 & 53.31 & 336.96 & 6.20 & 12.67 & -144.22 & -999.82 & 3.48 & 0.20 & 3.96 & 0.21 \\
\hline 7478.07 & 320404.20 & 17.00 & 114.03 & 37.14 & 0.87 & 1.87 & 12.50 & -191.08 & -1007.66 & 3.12 & 0.14 & 3.94 & 0.21 \\
\hline 7478.14 & 329322.00 & 17.00 & 120.61 & 40.08 & 2.79 & 2.24 & 12.75 & -203.55 & 108.13 & 3.47 & 0.46 & 3.99 & 0.21 \\
\hline 7478.20 & 348362.20 & 16.80 & 119.72 & 44.87 & 2.61 & 0.40 & 14.15 & -228.86 & 765.22 & 4.18 & 0.49 & 4.13 & 0.32 \\
\hline 7478.27 & 352711.60 & 16.80 & 102.04 & 38.01 & 1.85 & -4.07 & 14.88 & -221.08 & 114.98 & 4.20 & 0.60 & 4.10 & 0.81 \\
\hline 7478.33 & 374877.80 & 16.60 & 95.04 & 36.93 & 3.11 & -3.83 & 14.87 & -264.84 & -824.75 & 4.77 & 0.60 & 4.18 & 2.10 \\
\hline 7478.40 & 388577.40 & 16.40 & 90.46 & 36.86 & 2.74 & -3.00 & 14.26 & -286.69 & -804.35 & 5.25 & 0.72 & 4.25 & 2.15 \\
\hline 7478.46 & 369714.20 & 16.60 & 82.92 & 29.81 & 2.18 & -4.61 & 12.93 & -276.77 & -1121.33 & 4.91 & 1.90 & 4.02 & 2.36 \\
\hline 7478.53 & 315702.60 & 17.20 & 88.47 & 35.51 & 3.46 & -2.46 & 12.59 & -224.42 & -4399.37 & 4.27 & 2.12 & 3.88 & 2.34 \\
\hline 7478.60 & 288103.40 & 17.40 & 100.77 & 54.04 & 7.42 & -1.68 & 10.34 & -200.39 & -5881.63 & 4.81 & 3.19 & 3.67 & 3.39 \\
\hline 7478.66 & 246459.80 & 17.80 & 105.93 & 50.58 & 5.64 & -7.32 & 10.33 & -173.05 & -4960.94 & 4.70 & 3.91 & 3.50 & 2.47 \\
\hline 7478.73 & 228740.00 & 18.00 & 108.45 & 45.51 & 7.38 & -9.87 & 10.89 & -131.04 & -4256.30 & 4.44 & 4.28 & 3.32 & 2.65 \\
\hline 7478.79 & 245268.20 & 17.80 & 113.63 & 45.13 & 6.87 & -8.83 & 12.17 & -110.42 & -4745.13 & 4.37 & 2.80 & 3.47 & 2.45 \\
\hline 7478.86 & 283783.80 & 17.40 & 113.55 & 45.56 & 8.62 & -9.73 & 13.71 & -125.40 & -1616.35 & 4.38 & 2.63 & 3.56 & 2.43 \\
\hline 7478.92 & 321324.00 & 17.00 & 119.18 & 36.94 & 5.71 & -7.60 & 14.53 & -172.91 & -57.15 & 3.98 & 1.52 & 3.84 & 0.95 \\
\hline 7478.99 & 356348.80 & 16.60 & 119.88 & 40.57 & 5.29 & -2.92 & 14.44 & -195.51 & 272.36 & 3.67 & 0.80 & 4.04 & 0.63 \\
\hline 7479.06 & 376788.60 & 16.40 & 117.88 & 44.66 & 4.03 & -1.16 & 13.77 & -210.23 & -251.28 & 3.89 & 0.38 & 4.24 & 0.47 \\
\hline 7479.12 & 388975.20 & 16.20 & 114.40 & 53.73 & 2.72 & -0.65 & 12.04 & -253.16 & -48.28 & 4.29 & 0.38 & 4.37 & 0.50 \\
\hline 7479.19 & 397135.80 & 16.20 & 94.39 & 52.76 & 228.34 & 4.45 & 10.32 & -219.97 & -668.93 & 4.34 & 0.30 & 4.44 & 0.43 \\
\hline 7479.25 & 390246.40 & 16.40 & 86.10 & 48.84 & 228.11 & 1.94 & 11.20 & -206.25 & 1091.52 & 4.32 & 0.38 & 4.41 & 0.44 \\
\hline 7479.32 & 372588.20 & 16.60 & 75.27 & 46.90 & 228.50 & 2.08 & 9.69 & -172.93 & 866.71 & 4.27 & 0.58 & 4.27 & 0.41 \\
\hline 7479.38 & 352806.80 & 16.80 & 62.85 & 45.46 & 229.65 & 0.95 & 9.88 & -169.16 & 759.87 & 3.86 & 0.78 & 4.13 & 0.34 \\
\hline 7479.45 & 334415.20 & 17.00 & 54.60 & 41.00 & 231.10 & -0.57 & 10.35 & -141.47 & -480.42 & 3.37 & 0.79 & 3.95 & 0.27 \\
\hline 7479.51 & 315763.80 & 17.20 & 62.71 & 31.06 & 4.12 & -6.56 & 9.79 & -172.42 & -1179.33 & 3.11 & 1.04 & 3.79 & 0.29 \\
\hline 7479.58 & 294800.60 & 17.40 & 60.18 & 30.60 & 5.25 & -4.63 & 8.54 & -172.19 & -2807.05 & 2.83 & 1.07 & 3.68 & 0.28 \\
\hline 7479.65 & 283990.60 & 17.60 & 62.54 & 26.73 & 5.60 & -1.53 & 8.38 & -185.74 & -3594.79 & 2.66 & 1.05 & 3.65 & 0.27 \\
\hline 7479.71 & 273144.00 & 17.80 & 65.30 & 28.43 & 3.43 & -2.20 & 7.24 & -177.12 & -4679.56 & 2.40 & 0.95 & 3.57 & 0.27 \\
\hline 7479.78 & 270227.20 & 17.80 & 65.68 & 32.47 & 2.91 & 0.15 & 8.11 & -151.44 & -4450.66 & 2.39 & 1.29 & 3.53 & 0.29 \\
\hline
\end{tabular}




\begin{tabular}{|c|c|c|c|c|c|c|c|c|c|c|c|c|c|}
\hline Depth & $\mathrm{XRF}$ & XRF Live Time & $\mathrm{Nb}(\mathrm{ppm})$ & Mo (ppm) & $\mathrm{Ba}(\mathrm{ppm})$ & $\mathrm{Pb}(\mathrm{ppm})$ & Th (ppm) & $\mathrm{U}(\mathrm{ppm})$ & $\mathrm{P}(\mathrm{ppm})$ & $\mathrm{S}(\%)$ & $\mathrm{Cl}(\%)$ & $\mathrm{K}(\%)$ & $\mathrm{Ca}(\%)$ \\
\hline 7479.84 & 267669.00 & 17.80 & 63.63 & 34.60 & -0.39 & -2.72 & 8.50 & -165.75 & -3235.92 & 2.44 & 1.55 & 3.55 & 0.31 \\
\hline 7479.91 & 285900.20 & 17.60 & 62.90 & 41.21 & -2.27 & -2.62 & 8.15 & -177.84 & -2338.26 & 2.68 & 1.52 & 3.65 & 0.36 \\
\hline 7479.97 & 304187.40 & 17.40 & 58.41 & 41.00 & -2.80 & -4.96 & 7.77 & -165.26 & 142.34 & 2.92 & 1.78 & 3.68 & 0.39 \\
\hline 7480.04 & 312298.20 & 17.20 & 54.56 & 41.34 & -3.05 & -3.48 & 5.64 & -169.74 & 873.25 & 3.43 & 4.55 & 3.56 & 0.48 \\
\hline 7480.10 & 333951.20 & 17.00 & 52.50 & 44.83 & -2.83 & -4.37 & 3.09 & -226.68 & 2454.98 & 4.63 & 4.92 & 3.68 & 0.65 \\
\hline 7480.17 & 351544.40 & 16.80 & 52.14 & 50.30 & -1.27 & -1.28 & 1.53 & -217.05 & 2421.39 & 4.84 & 4.71 & 3.75 & 0.70 \\
\hline 7480.24 & 346752.60 & 16.80 & 50.13 & 42.52 & 0.50 & -3.65 & 1.26 & -168.41 & 1323.41 & 4.97 & 6.91 & 3.51 & 0.96 \\
\hline 7480.30 & 341948.20 & 16.80 & 49.28 & 47.12 & 0.18 & -4.09 & 1.13 & -176.59 & 630.42 & 4.90 & 7.11 & 3.47 & 1.29 \\
\hline 7480.37 & 366697.40 & 16.60 & 48.58 & 53.14 & 1.87 & -6.37 & 3.01 & -168.01 & -405.79 & 4.53 & 6.14 & 3.53 & 1.70 \\
\hline 7480.43 & 371550.20 & 16.60 & 41.16 & 56.76 & 1.36 & -5.93 & 5.75 & -141.39 & -2751.89 & 3.33 & 6.55 & 3.18 & 7.70 \\
\hline 7480.50 & 388024.00 & 16.40 & 35.34 & 63.58 & 0.61 & -8.30 & 5.49 & -149.19 & -4234.39 & 3.00 & 6.31 & 2.79 & 15.98 \\
\hline 7480.56 & 403776.80 & 16.20 & 30.05 & 74.57 & 0.51 & -8.18 & 5.80 & -170.16 & -3299.36 & 2.59 & 4.86 & 2.64 & 22.73 \\
\hline 7480.63 & 421195.80 & 16.00 & 24.76 & 78.21 & 1.21 & -10.41 & 4.91 & -160.45 & -6849.66 & 2.32 & 4.45 & 2.29 & 30.87 \\
\hline 7480.70 & 399044.20 & 16.20 & 18.84 & 77.84 & 1.24 & -12.83 & 5.51 & -172.28 & -7489.34 & 2.12 & 2.48 & 2.05 & 38.71 \\
\hline 7480.76 & 371791.60 & 16.40 & 18.16 & 68.19 & 1.62 & -19.24 & 2.20 & -165.40 & -4502.97 & 2.07 & 1.35 & 2.07 & 39.57 \\
\hline 7480.83 & 340899.20 & 16.80 & 16.30 & 61.23 & 3.84 & -20.14 & 1.86 & -160.30 & -4712.74 & 1.97 & 1.29 & 2.15 & 36.85 \\
\hline 7480.89 & 302754.60 & 17.20 & 13.95 & 51.14 & 2.82 & -17.50 & 1.13 & -165.14 & -6387.79 & 1.83 & 0.68 & 2.41 & 30.28 \\
\hline 7480.96 & 279730.40 & 17.40 & 12.57 & 43.95 & 0.07 & -15.96 & 2.38 & -170.68 & -3350.58 & 2.01 & 0.59 & 2.83 & 23.01 \\
\hline 7481.02 & 278455.60 & 17.40 & 10.61 & 44.20 & -1.75 & -14.03 & 1.94 & -176.53 & -805.47 & 2.02 & 1.63 & 3.06 & 16.68 \\
\hline 7481.09 & 280936.20 & 17.40 & 7.91 & 50.75 & -2.38 & -11.88 & 3.59 & -196.57 & -3519.18 & 1.90 & 1.63 & 3.15 & 13.41 \\
\hline 7481.15 & 283617.00 & 17.20 & 5.96 & 51.58 & -3.77 & -9.28 & 3.50 & -187.90 & -1863.60 & 2.03 & 1.66 & 3.20 & 12.99 \\
\hline 7481.22 & 295583.60 & 17.20 & 5.04 & 58.66 & -2.98 & -14.51 & 3.19 & -186.36 & -1287.61 & 1.90 & 1.37 & 3.08 & 17.87 \\
\hline 7481.29 & 266076.20 & 17.60 & 3.14 & 84.07 & -0.56 & -11.39 & 2.85 & -157.44 & -1334.35 & 1.62 & 2.11 & 2.84 & 20.85 \\
\hline 7481.35 & 259229.00 & 17.80 & 2.25 & 77.26 & 0.32 & -8.96 & 2.58 & -173.51 & -1178.70 & 1.52 & 1.46 & 2.89 & 21.30 \\
\hline 7481.42 & 263833.00 & 17.80 & 3.24 & 74.65 & 0.67 & -6.33 & 1.73 & -169.11 & -305.61 & 1.77 & 1.56 & 3.00 & 19.05 \\
\hline 7481.48 & 266093.00 & 17.80 & 3.64 & 65.60 & 2.27 & -7.69 & 1.76 & -177.15 & -260.17 & 2.20 & 2.13 & 3.06 & 15.95 \\
\hline 7481.55 & 283313.40 & 17.60 & 4.32 & 60.54 & 2.69 & -5.78 & 2.90 & -168.79 & 1377.07 & 2.76 & 2.65 & 3.07 & 14.66 \\
\hline 7481.61 & 325870.80 & 17.20 & 6.51 & 48.54 & 2.89 & -11.19 & 1.90 & -234.59 & 2471.04 & 3.20 & 1.83 & 3.28 & 12.48 \\
\hline 7481.68 & 339983.20 & 17.00 & 6.36 & 48.71 & 3.27 & -15.37 & 2.38 & -229.83 & 828.84 & 3.33 & 1.48 & 3.36 & 12.43 \\
\hline 7481.75 & 351669.60 & 17.00 & 8.65 & 52.00 & 3.76 & -17.86 & 3.65 & -223.05 & 1223.51 & 3.93 & 3.39 & 3.23 & 13.04 \\
\hline 7481.81 & 372976.60 & 16.80 & 10.97 & 59.61 & 2.69 & -16.71 & 4.75 & -261.45 & 2711.39 & 4.07 & 2.98 & 3.12 & 16.51 \\
\hline 7481.88 & 372708.00 & 16.80 & 11.48 & 54.69 & 131.81 & -9.45 & 6.47 & -234.00 & 1491.64 & 4.72 & 3.02 & 3.13 & 15.90 \\
\hline 7481.94 & 358137.20 & 16.80 & 15.70 & 40.76 & 129.58 & -3.92 & 8.45 & -183.54 & -185.80 & 4.88 & 3.70 & 2.97 & 15.79 \\
\hline
\end{tabular}




\begin{tabular}{|c|c|c|c|c|c|c|c|c|c|c|c|c|c|}
\hline Depth & $\mathrm{XRF}$ & XRF Live Time & $\mathrm{Nb}(\mathrm{ppm})$ & Mo (ppm) & $\mathrm{Ba}(\mathrm{ppm})$ & $\mathrm{Pb}(\mathrm{ppm})$ & Th (ppm) & $\mathrm{U}(\mathrm{ppm})$ & $\mathrm{P}(\mathrm{ppm})$ & $\mathrm{S}(\%)$ & $\mathrm{Cl}(\%)$ & $\mathrm{K}(\%)$ & $\mathrm{Ca}(\%)$ \\
\hline 7482.01 & 340885.80 & 17.00 & 21.10 & 35.13 & 129.51 & -0.35 & 8.41 & -162.30 & 1396.32 & 5.44 & 3.99 & 3.01 & 13.97 \\
\hline 7482.07 & 338982.40 & 17.00 & 22.76 & 127.12 & 128.18 & 15.36 & 8.66 & -732.04 & 8597.69 & 12.72 & 2.59 & 2.55 & 11.81 \\
\hline 7482.14 & 334258.60 & 17.00 & 31.81 & 121.77 & 127.33 & 13.38 & 8.82 & -692.16 & 6876.27 & 12.54 & 3.13 & 2.76 & 6.46 \\
\hline 7482.20 & 345436.20 & 16.80 & 32.66 & 131.02 & 225.10 & 14.46 & 7.53 & -690.96 & 8482.81 & 12.12 & 3.21 & 2.92 & 3.54 \\
\hline 7482.27 & 371271.80 & 16.60 & 40.20 & 137.97 & 225.74 & 13.61 & 6.85 & -702.46 & 8554.32 & 12.52 & 3.82 & 2.97 & 2.59 \\
\hline 7482.34 & 407785.40 & 16.20 & 52.58 & 151.96 & 227.61 & 13.10 & 12.00 & -713.88 & 7209.68 & 12.53 & 4.25 & 2.85 & 4.15 \\
\hline 7482.40 & 395754.40 & 16.20 & 63.83 & 49.92 & 228.30 & 0.19 & 13.85 & -106.08 & 17.48 & 4.79 & 4.49 & 3.44 & 4.26 \\
\hline 7482.47 & 378549.40 & 16.40 & 67.53 & 43.58 & 230.47 & 1.38 & 16.25 & -120.76 & 278.28 & 4.59 & 4.21 & 3.44 & 4.29 \\
\hline 7482.53 & 359511.60 & 16.60 & 85.91 & 35.76 & 2.05 & -3.71 & 17.61 & -171.05 & -1293.09 & 4.77 & 4.55 & 3.38 & 4.17 \\
\hline 7482.60 & 329277.00 & 17.00 & 92.71 & 34.55 & 3.48 & -4.23 & 19.19 & -165.17 & 490.05 & 4.19 & 5.98 & 3.25 & 3.29 \\
\hline 7482.66 & 292171.40 & 17.40 & 93.81 & 22.07 & 1.32 & -3.26 & 14.24 & -159.81 & 711.86 & 4.10 & 6.32 & 3.23 & 1.28 \\
\hline 7482.73 & 281596.00 & 17.60 & 97.03 & 25.44 & 2.03 & -3.16 & 14.27 & -160.14 & 417.44 & 4.07 & 6.07 & 3.24 & 1.27 \\
\hline 7482.80 & 276160.00 & 17.80 & 99.38 & 31.39 & 1.26 & -0.04 & 11.77 & -135.78 & -890.24 & 4.06 & 8.00 & 3.03 & 1.19 \\
\hline 7482.86 & 269885.00 & 17.80 & 94.43 & 33.84 & 1.93 & -2.64 & 10.88 & -139.39 & -886.79 & 3.62 & 7.05 & 3.11 & 0.75 \\
\hline 7482.93 & 272060.00 & 17.80 & 88.60 & 31.16 & 0.16 & -4.45 & 11.31 & -147.85 & -2946.28 & 3.50 & 6.05 & 3.14 & 0.68 \\
\hline 7482.99 & 273785.40 & 17.80 & 86.28 & 35.93 & 0.71 & -6.11 & 11.27 & -133.67 & -3208.62 & 3.26 & 8.60 & 2.88 & 0.61 \\
\hline 7483.06 & 300356.40 & 17.40 & 85.07 & 36.60 & 1.84 & -5.33 & 10.11 & -125.33 & -3340.47 & 3.37 & 12.40 & 2.56 & 0.71 \\
\hline 7483.12 & 328844.40 & 17.00 & 87.63 & 40.83 & 1.16 & -6.57 & 11.65 & -144.56 & -1685.25 & 3.48 & 10.02 & 2.85 & 0.95 \\
\hline 7483.19 & 350977.40 & 16.80 & 92.80 & 37.51 & 2.27 & -4.54 & 13.38 & -131.48 & -1833.77 & 3.50 & 9.99 & 2.92 & 1.19 \\
\hline 7483.25 & 373131.00 & 16.40 & 99.62 & 40.37 & 4.27 & -1.34 & 11.82 & -131.80 & -810.51 & 3.60 & 9.59 & 3.00 & 1.25 \\
\hline 7483.32 & 396068.40 & 16.20 & 105.86 & 37.58 & 4.24 & -0.10 & 11.70 & -140.09 & 130.66 & 3.58 & 7.74 & 3.26 & 1.25 \\
\hline 7483.39 & 397404.20 & 16.20 & 91.00 & 50.18 & 224.19 & 4.21 & 11.31 & -141.22 & 1209.26 & 3.78 & 5.40 & 3.48 & 1.30 \\
\hline 7483.45 & 395835.60 & 16.20 & 92.66 & 51.05 & 223.50 & 3.16 & 11.13 & -144.11 & 631.22 & 3.95 & 5.56 & 3.44 & 1.08 \\
\hline 7483.52 & 392722.60 & 16.20 & 92.78 & 55.25 & 221.93 & -1.05 & 10.45 & -145.89 & 1901.63 & 4.38 & 6.81 & 3.27 & 1.05 \\
\hline 7483.58 & 394145.20 & 16.20 & 97.02 & 55.99 & 221.24 & -3.90 & 12.48 & -159.83 & 1996.27 & 4.57 & 6.19 & 3.35 & 1.00 \\
\hline 7483.65 & 393529.40 & 16.20 & 100.47 & 53.46 & 220.95 & -1.94 & 13.76 & -163.97 & 1920.86 & 4.74 & 4.86 & 3.46 & 0.99 \\
\hline 7483.71 & 383902.80 & 16.40 & 118.73 & 34.07 & 1.33 & -5.44 & 15.84 & -190.09 & -654.70 & 4.27 & 3.32 & 3.45 & 0.79 \\
\hline 7483.78 & 365306.20 & 16.60 & 118.91 & 29.66 & 1.39 & -2.50 & 16.35 & -173.28 & -510.12 & 4.16 & 4.85 & 3.31 & 0.66 \\
\hline 7483.85 & 348434.80 & 16.80 & 99.74 & 45.33 & 244.24 & 5.49 & 14.54 & -131.91 & -1661.35 & 3.75 & 4.74 & 3.26 & 0.57 \\
\hline 7483.91 & 334537.60 & 17.00 & 100.28 & 40.99 & 243.24 & 6.16 & 15.14 & -117.79 & -2654.76 & 3.43 & 4.47 & 3.24 & 0.56 \\
\hline 7483.98 & 323595.80 & 17.00 & 97.07 & 42.31 & 243.17 & 2.86 & 14.25 & -122.57 & -3427.45 & 3.20 & 4.98 & 3.16 & 0.51 \\
\hline 7484.04 & 311084.40 & 17.00 & 96.29 & 43.01 & 241.88 & 2.94 & 12.95 & -84.22 & -2993.25 & 3.57 & 11.20 & 2.78 & 0.67 \\
\hline 7484.11 & 316853.40 & 17.00 & 95.01 & 46.67 & 240.95 & 0.89 & 12.99 & -91.79 & -3004.60 & 3.33 & 10.23 & 2.88 & 0.59 \\
\hline
\end{tabular}




\begin{tabular}{|c|c|c|c|c|c|c|c|c|c|c|c|c|c|}
\hline Depth & $\mathrm{XRF}$ & XRF Live Time & $\mathrm{Nb}(\mathrm{ppm})$ & Mo (ppm) & $\mathrm{Ba}(\mathrm{ppm})$ & $\mathrm{Pb}(\mathrm{ppm})$ & Th (ppm) & $\mathrm{U}(\mathrm{ppm})$ & $\mathrm{P}(\mathrm{ppm})$ & $\mathrm{S}(\%)$ & $\mathrm{Cl}(\%)$ & $\mathrm{K}(\%)$ & $\mathrm{Ca}(\%)$ \\
\hline 7484.17 & 325512.80 & 17.00 & 110.68 & 28.30 & -1.69 & -6.73 & 15.68 & -112.99 & -3003.02 & 3.45 & 9.40 & 3.03 & 0.58 \\
\hline 7484.24 & 316454.20 & 17.20 & 102.34 & 35.58 & -0.73 & -3.14 & 15.71 & -87.16 & -293.09 & 4.17 & 12.07 & 2.86 & 0.87 \\
\hline 7484.30 & 325499.40 & 17.20 & 97.37 & 39.31 & -1.72 & -0.22 & 16.08 & -92.60 & 91.79 & 4.28 & 11.37 & 2.98 & 0.85 \\
\hline 7484.37 & 330302.40 & 17.20 & 95.12 & 44.31 & -1.77 & 1.91 & 16.46 & -121.39 & 1452.45 & 3.92 & 7.16 & 3.26 & 0.68 \\
\hline 7484.44 & 331493.00 & 17.20 & 92.73 & 46.00 & -0.12 & 5.19 & 15.11 & -80.75 & 782.86 & 4.06 & 9.09 & 3.09 & 0.72 \\
\hline 7484.50 & 334540.60 & 17.20 & 93.43 & 48.63 & -0.18 & 7.50 & 12.71 & -96.33 & 856.94 & 3.76 & 10.14 & 3.04 & 0.64 \\
\hline 7484.57 & 344575.40 & 17.00 & 99.19 & 43.68 & 0.26 & 4.14 & 10.61 & -82.63 & -481.15 & 3.13 & 13.26 & 2.86 & 0.41 \\
\hline 7484.63 & 319945.60 & 17.20 & 101.26 & 51.46 & 2.01 & 5.34 & 12.22 & -29.16 & -938.18 & 3.10 & 19.29 & 2.31 & 0.51 \\
\hline 7484.70 & 323219.80 & 17.20 & 103.51 & 46.30 & 2.31 & 1.76 & 12.46 & -43.66 & -1046.27 & 3.03 & 16.74 & 2.55 & 0.52 \\
\hline 7484.76 & 316537.80 & 17.20 & 109.26 & 35.49 & 6.51 & 0.70 & 12.84 & -87.34 & -533.14 & 2.91 & 14.43 & 2.69 & 0.52 \\
\hline 7484.83 & 303932.80 & 17.20 & 114.93 & 31.77 & 6.39 & -0.63 & 12.72 & -86.78 & -103.96 & 2.92 & 13.50 & 2.68 & 0.60 \\
\hline 7484.90 & 297361.20 & 17.20 & 112.97 & 29.60 & 4.98 & 0.86 & 12.29 & -141.87 & -446.27 & 2.74 & 7.65 & 3.01 & 0.63 \\
\hline 7484.96 & 309680.60 & 17.00 & 115.74 & 29.65 & 4.90 & -0.06 & 12.80 & -188.13 & 64.12 & 2.70 & 1.92 & 3.45 & 0.60 \\
\hline 7485.03 & 302150.80 & 17.00 & 97.83 & 50.08 & 243.49 & 4.77 & 12.63 & -157.26 & -1060.32 & 2.66 & 2.15 & 3.38 & 0.67 \\
\hline 7485.09 & 298850.60 & 17.00 & 96.13 & 58.32 & 238.32 & 5.09 & 12.77 & -145.34 & -1440.51 & 2.64 & 2.04 & 3.43 & 0.67 \\
\hline 7485.16 & 305860.80 & 17.00 & 96.56 & 65.13 & 239.73 & 6.99 & 12.44 & -146.81 & -1881.59 & 2.50 & 1.82 & 3.50 & 0.58 \\
\hline 7485.22 & 304615.80 & 17.00 & 95.57 & 58.49 & 240.13 & 6.24 & 12.26 & -147.35 & -2970.87 & 2.52 & 1.52 & 3.59 & 0.46 \\
\hline 7485.29 & 280100.00 & 17.40 & 88.48 & 56.51 & 239.70 & 6.00 & 10.44 & -143.31 & -4087.39 & 2.42 & 1.13 & 3.64 & 0.37 \\
\hline 7485.35 & 267959.80 & 17.60 & 107.46 & 40.10 & 0.86 & 0.60 & 8.91 & -168.18 & -3619.37 & 2.54 & 1.77 & 3.62 & 0.36 \\
\hline 7485.42 & 257303.80 & 17.80 & 106.23 & 37.09 & 1.41 & -3.89 & 7.99 & -168.65 & -3950.98 & 2.49 & 1.87 & 3.53 & 0.39 \\
\hline 7485.49 & 240846.20 & 18.00 & 99.65 & 33.63 & 1.94 & -6.05 & 9.17 & -193.80 & -4022.63 & 2.59 & 1.86 & 3.48 & 0.44 \\
\hline 7485.55 & 234152.60 & 18.20 & 100.69 & 40.53 & 3.66 & -6.98 & 9.62 & -192.11 & -5030.32 & 2.61 & 1.86 & 3.46 & 0.49 \\
\hline 7485.62 & 245927.40 & 18.00 & 106.14 & 36.53 & 4.28 & -9.21 & 9.40 & -189.54 & -4224.84 & 2.69 & 1.92 & 3.45 & 0.59 \\
\hline 7485.68 & 242004.00 & 18.00 & 86.49 & 47.06 & 205.96 & -3.62 & 9.46 & -164.85 & -5252.81 & 2.64 & 1.26 & 3.44 & 0.64 \\
\hline 7485.75 & 235792.20 & 18.00 & 83.81 & 48.10 & 204.75 & -1.56 & 11.66 & -194.59 & -5143.54 & 2.56 & 1.24 & 3.53 & 0.57 \\
\hline 7485.81 & 231605.20 & 18.00 & 85.92 & 48.78 & 202.62 & 0.97 & 13.11 & -181.30 & -4426.77 & 2.76 & 1.28 & 3.51 & 0.69 \\
\hline 7485.88 & 213896.00 & 18.20 & 81.54 & 54.13 & 199.84 & 3.85 & 14.08 & -170.43 & -3084.10 & 2.66 & 1.98 & 3.43 & 0.85 \\
\hline 7485.94 & 226400.40 & 18.00 & 78.71 & 50.51 & 197.34 & 8.00 & 13.22 & -185.59 & -2322.16 & 2.51 & 2.05 & 3.48 & 0.91 \\
\hline 7486.01 & 241863.20 & 17.80 & 94.85 & 39.09 & -1.34 & 2.81 & 14.06 & -229.36 & -502.55 & 2.54 & 2.16 & 3.56 & 0.90 \\
\hline 7486.08 & 254970.80 & 17.80 & 95.57 & 30.80 & -0.39 & 2.93 & 14.11 & -194.33 & -159.12 & 2.53 & 2.04 & 3.58 & 1.02 \\
\hline 7486.14 & 263624.40 & 17.80 & 95.10 & 23.46 & 1.55 & 2.19 & 11.35 & -179.91 & 35.86 & 2.26 & 2.22 & 3.62 & 1.06 \\
\hline 7486.21 & 308547.40 & 17.20 & 100.91 & 23.20 & 3.71 & -1.27 & 9.66 & -196.13 & 935.95 & 2.30 & 1.50 & 3.77 & 1.05 \\
\hline 7486.27 & 329743.80 & 17.00 & 103.37 & 27.86 & 7.28 & -4.63 & 11.11 & -194.67 & 462.51 & 2.52 & 1.32 & 3.76 & 1.14 \\
\hline
\end{tabular}




\begin{tabular}{|c|c|c|c|c|c|c|c|c|c|c|c|c|c|}
\hline Depth & $\mathrm{XRF}$ & XRF Live Time & $\mathrm{Nb}(\mathrm{ppm})$ & Mo (ppm) & $\mathrm{Ba}(\mathrm{ppm})$ & $\mathrm{Pb}(\mathrm{ppm})$ & Th (ppm) & $\mathrm{U}(\mathrm{ppm})$ & $\mathrm{P}(\mathrm{ppm})$ & $\mathrm{S}(\%)$ & $\mathrm{Cl}(\%)$ & $\mathrm{K}(\%)$ & $\mathrm{Ca}(\%)$ \\
\hline 7486.34 & 350004.80 & 16.80 & 107.55 & 31.47 & 5.08 & -5.50 & 10.43 & -186.84 & -323.69 & 2.53 & 1.01 & 3.83 & 1.11 \\
\hline 7486.40 & 371441.60 & 16.40 & 109.98 & 39.74 & 6.10 & -8.21 & 9.33 & -215.37 & 143.81 & 2.75 & 0.94 & 3.85 & 1.15 \\
\hline 7486.47 & 395881.40 & 16.00 & 106.90 & 47.17 & 5.55 & -8.16 & 10.95 & -224.28 & -370.44 & 3.09 & 0.79 & 3.87 & 1.18 \\
\hline 7486.54 & 401384.00 & 16.00 & 102.95 & 45.79 & 4.71 & -7.40 & 12.96 & -207.73 & 440.96 & 3.23 & 0.69 & 3.90 & 1.07 \\
\hline 7486.60 & 400576.60 & 16.00 & 101.70 & 43.62 & 2.98 & -6.01 & 10.65 & -177.67 & 1430.94 & 3.46 & 0.69 & 3.92 & 1.07 \\
\hline 7486.67 & 358007.20 & 16.40 & 81.57 & 55.64 & 300.55 & 0.82 & 10.18 & -141.48 & 2256.28 & 3.73 & 0.79 & 3.94 & 1.05 \\
\hline 7486.73 & 355547.80 & 16.60 & 79.04 & 57.85 & 300.30 & 5.23 & 9.43 & -132.45 & 1892.17 & 3.63 & 0.67 & 4.02 & 0.89 \\
\hline 7486.80 & 349471.00 & 16.80 & 80.13 & 56.32 & 299.80 & 6.35 & 8.01 & -135.23 & 2457.98 & 3.44 & 0.38 & 4.09 & 0.70 \\
\hline 7486.86 & 328607.80 & 17.00 & 64.07 & 64.26 & 526.23 & 11.58 & 7.46 & -97.43 & 294.02 & 3.36 & 0.54 & 4.05 & 0.69 \\
\hline 7486.93 & 328483.80 & 17.00 & 60.33 & 60.59 & 526.96 & 9.50 & 8.00 & -130.00 & -151.57 & 3.23 & 0.46 & 4.14 & 0.56 \\
\hline 7486.99 & 371066.00 & 16.60 & 75.52 & 46.32 & 228.66 & 3.75 & 6.80 & -180.78 & -587.39 & 3.32 & 0.38 & 4.19 & 0.57 \\
\hline 7487.06 & 370155.80 & 16.60 & 72.18 & 44.82 & 227.98 & -0.99 & 7.32 & -181.90 & -35.44 & 3.51 & 0.38 & 4.22 & 0.59 \\
\hline 7487.13 & 374600.20 & 16.60 & 70.60 & 41.31 & 227.28 & 0.34 & 7.47 & -170.40 & -1019.09 & 3.72 & 0.42 & 4.24 & 0.56 \\
\hline 7487.19 & 388267.40 & 16.60 & 84.69 & 28.22 & 0.34 & -2.85 & 6.18 & -223.62 & 474.73 & 4.47 & 0.64 & 4.21 & 0.70 \\
\hline 7487.26 & 386687.40 & 16.60 & 84.09 & 38.16 & -0.41 & -5.75 & 6.44 & -240.96 & 568.02 & 4.47 & 0.71 & 4.17 & 0.65 \\
\hline 7487.32 & 383842.20 & 16.80 & 83.31 & 40.02 & -1.11 & -6.40 & 8.18 & -227.86 & -39.10 & 4.18 & 0.78 & 4.13 & 0.76 \\
\hline 7487.39 & 379571.00 & 16.80 & 83.35 & 33.89 & -1.38 & -0.73 & 8.13 & -216.18 & -67.69 & 4.04 & 1.94 & 3.89 & 1.04 \\
\hline 7487.45 & 367113.00 & 16.80 & 83.14 & 36.55 & -1.53 & -1.79 & 8.24 & -201.08 & 227.00 & 3.84 & 1.94 & 3.84 & 1.08 \\
\hline 7487.52 & 348629.80 & 16.80 & 79.72 & 40.42 & 0.02 & -4.01 & 8.35 & -202.08 & -713.34 & 3.12 & 1.59 & 3.84 & 0.94 \\
\hline 7487.59 & 327757.00 & 17.00 & 81.54 & 29.43 & -0.43 & 0.53 & 8.77 & -188.57 & -1295.66 & 2.95 & 1.51 & 3.80 & 1.23 \\
\hline 7487.65 & 303196.00 & 17.20 & 67.23 & 38.39 & 179.76 & 6.39 & 9.35 & -151.41 & -10.27 & 2.83 & 1.56 & 3.74 & 1.13 \\
\hline 7487.72 & 282803.80 & 17.40 & 67.90 & 46.65 & 180.20 & 2.87 & 9.05 & -141.95 & -1021.33 & 2.63 & 0.37 & 3.81 & 0.91 \\
\hline 7487.78 & 297263.60 & 17.20 & 67.44 & 45.08 & 179.82 & 2.28 & 10.23 & -176.22 & 424.59 & 3.88 & 0.66 & 3.75 & 1.64 \\
\hline 7487.85 & 306759.60 & 17.20 & 71.20 & 41.17 & 179.86 & 2.50 & 9.95 & -150.03 & 585.01 & 3.81 & 0.62 & 3.76 & 1.69 \\
\hline 7487.91 & 309339.80 & 17.20 & 70.78 & 42.17 & 179.43 & 3.32 & 9.80 & -143.02 & 592.86 & 3.91 & 0.57 & 3.78 & 1.43 \\
\hline 7487.98 & 328483.40 & 17.00 & 81.98 & 30.60 & -0.44 & -1.26 & 9.68 & -189.97 & -462.60 & 4.35 & 0.61 & 3.76 & 1.53 \\
\hline 7488.04 & 347245.80 & 16.80 & 79.67 & 30.55 & 0.07 & 2.37 & 9.29 & -192.82 & 1083.81 & 4.77 & 0.68 & 3.80 & 1.60 \\
\hline 7488.11 & 340321.20 & 17.00 & 78.71 & 36.36 & 1.58 & 2.35 & 8.11 & -189.63 & 549.81 & 4.17 & 0.62 & 3.85 & 1.06 \\
\hline 7488.18 & 346071.40 & 17.00 & 76.24 & 36.08 & 0.28 & 1.25 & 7.40 & -213.55 & 1619.31 & 4.52 & 0.98 & 3.82 & 1.08 \\
\hline 7488.24 & 353667.40 & 17.00 & 72.76 & 43.39 & 0.76 & -1.99 & 7.35 & -174.25 & 1370.46 & 4.47 & 1.58 & 3.76 & 1.10 \\
\hline 7488.31 & 339742.60 & 17.20 & 74.84 & 45.89 & 1.24 & -2.09 & 6.25 & -138.09 & 966.76 & 4.31 & 2.14 & 3.71 & 1.03 \\
\hline 7488.37 & 345607.00 & 17.20 & 77.10 & 39.10 & 1.12 & -2.27 & 6.35 & -149.64 & 1264.26 & 4.00 & 2.08 & 3.77 & 0.88 \\
\hline 7488.44 & 345121.20 & 17.20 & 80.58 & 32.93 & 0.70 & -0.39 & 7.28 & -133.70 & 1025.07 & 3.83 & 3.04 & 3.62 & 0.91 \\
\hline
\end{tabular}




\begin{tabular}{|c|c|c|c|c|c|c|c|c|c|c|c|c|c|}
\hline Depth & $\mathrm{XRF}$ & XRF Live Time & $\mathrm{Nb}(\mathrm{ppm})$ & Mo (ppm) & $\mathrm{Ba}(\mathrm{ppm})$ & $\mathrm{Pb}(\mathrm{ppm})$ & Th (ppm) & $\mathrm{U}(\mathrm{ppm})$ & $\mathrm{P}(\mathrm{ppm})$ & $\mathrm{S}(\%)$ & $\mathrm{Cl}(\%)$ & $\mathrm{K}(\%)$ & $\mathrm{Ca}(\%)$ \\
\hline 7488.50 & 344985.20 & 17.20 & 82.62 & 30.23 & 1.50 & 1.07 & 7.93 & -111.66 & 96.88 & 3.58 & 2.95 & 3.71 & 0.89 \\
\hline 7488.57 & 353876.00 & 17.20 & 83.91 & 26.41 & 2.87 & 4.15 & 10.32 & -148.88 & 328.20 & 3.49 & 2.56 & 3.75 & 0.88 \\
\hline 7488.64 & 374036.00 & 17.00 & 85.62 & 21.53 & 2.58 & 3.25 & 11.64 & -175.29 & 398.55 & 3.37 & 1.84 & 3.88 & 0.84 \\
\hline 7488.70 & 367212.20 & 17.00 & 85.95 & 28.87 & 3.38 & 3.69 & 10.28 & -164.46 & -983.57 & 3.51 & 2.14 & 3.79 & 0.93 \\
\hline 7488.77 & 378104.00 & 16.80 & 88.47 & 36.52 & 4.56 & 0.35 & 10.85 & -158.99 & 125.56 & 3.68 & 1.49 & 3.90 & 0.85 \\
\hline 7488.83 & 385343.40 & 16.60 & 89.65 & 42.73 & 3.38 & -1.15 & 11.10 & -181.85 & 420.59 & 3.64 & 1.32 & 3.88 & 0.90 \\
\hline 7488.90 & 387850.60 & 16.40 & 93.75 & 45.20 & 2.32 & -3.98 & 8.98 & -196.15 & 848.64 & 3.71 & 1.18 & 3.91 & 1.01 \\
\hline 7488.96 & 384338.60 & 16.40 & 92.87 & 37.14 & 3.21 & -3.64 & 7.71 & -202.18 & 1969.38 & 3.72 & 1.72 & 3.84 & 1.19 \\
\hline 7489.03 & 389656.40 & 16.40 & 89.71 & 34.78 & 2.89 & -7.38 & 7.94 & -204.14 & 1202.37 & 3.51 & 1.44 & 3.91 & 1.26 \\
\hline 7489.09 & 389411.40 & 16.40 & 83.16 & 30.79 & 2.37 & -7.42 & 7.29 & -213.39 & 469.19 & 2.76 & 1.14 & 3.97 & 1.22 \\
\hline 7489.16 & 388405.60 & 16.40 & 86.50 & 29.78 & 3.05 & -7.63 & 6.81 & -196.11 & 737.78 & 2.83 & 1.23 & 3.99 & 1.20 \\
\hline 7489.23 & 388277.80 & 16.40 & 86.79 & 28.78 & 3.41 & -4.62 & 6.66 & -190.85 & 1022.20 & 2.96 & 1.68 & 3.97 & 1.16 \\
\hline 7489.29 & 394587.20 & 16.20 & 91.09 & 44.15 & 3.06 & -5.22 & 7.06 & -191.55 & 847.98 & 3.10 & 1.18 & 4.08 & 0.98 \\
\hline 7489.36 & 397504.40 & 16.20 & 96.15 & 43.96 & 3.29 & -4.15 & 8.29 & -195.82 & 2520.41 & 3.36 & 2.15 & 4.02 & 0.90 \\
\hline 7489.42 & 392626.40 & 16.20 & 99.09 & 42.06 & 3.08 & -1.35 & 8.28 & -198.81 & 1995.13 & 3.84 & 2.16 & 4.01 & 0.86 \\
\hline 7489.49 & 392317.20 & 16.20 & 95.81 & 44.11 & 3.00 & -0.67 & 9.93 & -176.92 & 2083.25 & 4.12 & 2.25 & 3.96 & 0.84 \\
\hline 7489.55 & 394994.00 & 16.20 & 95.71 & 40.35 & 2.29 & -0.55 & 11.27 & -167.14 & 2071.18 & 4.00 & 1.75 & 4.01 & 0.74 \\
\hline 7489.62 & 385802.40 & 16.40 & 94.14 & 36.60 & 1.51 & 1.24 & 11.29 & -189.04 & 1350.12 & 4.10 & 2.16 & 3.98 & 0.75 \\
\hline 7489.69 & 390290.40 & 16.20 & 94.07 & 37.94 & 0.14 & 0.38 & 10.16 & -189.62 & 138.33 & 4.19 & 1.26 & 4.08 & 0.73 \\
\hline 7489.75 & 392974.20 & 16.20 & 95.66 & 38.69 & -0.58 & -3.17 & 10.06 & -188.75 & -183.97 & 3.96 & 1.10 & 4.10 & 0.69 \\
\hline 7489.82 & 388667.60 & 16.40 & 92.90 & 39.98 & 0.08 & 1.11 & 9.38 & -209.50 & -752.29 & 3.77 & 0.87 & 4.16 & 0.66 \\
\hline 7489.88 & 382803.40 & 16.60 & 89.74 & 46.15 & 1.13 & 1.58 & 8.59 & -196.64 & -1521.51 & 3.66 & 0.90 & 4.12 & 0.81 \\
\hline 7489.95 & 392876.60 & 16.40 & 85.90 & 44.96 & 2.56 & -0.75 & 8.87 & -177.64 & -73.86 & 3.44 & 0.44 & 4.17 & 0.99 \\
\hline 7490.01 & 396701.00 & 16.40 & 82.70 & 45.08 & 3.49 & -0.27 & 9.30 & -201.47 & 2023.02 & 3.48 & 0.54 & 4.18 & 1.47 \\
\hline 7490.08 & 400335.60 & 16.40 & 78.43 & 49.86 & 4.07 & 2.11 & 9.64 & -215.66 & 1854.25 & 3.32 & 0.43 & 4.20 & 1.99 \\
\hline 7490.14 & 404224.00 & 16.20 & 78.31 & 48.36 & 2.46 & -2.80 & 8.32 & -243.96 & 1898.40 & 3.17 & 0.41 & 4.16 & 2.41 \\
\hline 7490.21 & 409199.00 & 16.00 & 78.88 & 53.28 & 1.60 & -6.00 & 8.28 & -246.41 & 2351.93 & 3.48 & 0.48 & 4.17 & 2.63 \\
\hline 7490.28 & 406130.80 & 16.20 & 79.76 & 52.91 & 0.35 & -4.47 & 8.05 & -240.31 & 1239.19 & 3.47 & 0.48 & 4.15 & 2.50 \\
\hline 7490.34 & 401828.80 & 16.20 & 79.86 & 55.70 & -1.22 & -4.07 & 8.57 & -237.32 & 897.48 & 3.59 & 0.43 & 4.18 & 2.09 \\
\hline 7490.41 & 383549.00 & 16.40 & 82.69 & 49.02 & -1.15 & -4.26 & 8.19 & -225.55 & 2249.83 & 4.03 & 0.86 & 4.13 & 1.70 \\
\hline 7490.47 & 377062.60 & 16.60 & 87.39 & 46.60 & 0.92 & -4.92 & 10.37 & -203.78 & 1460.17 & 4.72 & 1.59 & 4.08 & 1.62 \\
\hline 7490.54 & 367313.60 & 16.80 & 87.41 & 35.17 & 0.34 & -4.19 & 9.51 & -196.91 & 2123.92 & 5.16 & 2.58 & 3.92 & 1.65 \\
\hline 7490.60 & 351246.00 & 16.80 & 86.84 & 29.12 & 0.43 & -6.29 & 10.75 & -201.92 & 1545.62 & 5.08 & 2.81 & 3.83 & 1.65 \\
\hline
\end{tabular}




\begin{tabular}{|c|c|c|c|c|c|c|c|c|c|c|c|c|c|}
\hline Depth & $\mathrm{XRF}$ & XRF Live Time & $\mathrm{Nb}(\mathrm{ppm})$ & Mo (ppm) & $\mathrm{Ba}(\mathrm{ppm})$ & $\mathrm{Pb}(\mathrm{ppm})$ & Th (ppm) & $\mathrm{U}(\mathrm{ppm})$ & $\mathrm{P}(\mathrm{ppm})$ & $\mathrm{S}(\%)$ & $\mathrm{Cl}(\%)$ & $\mathrm{K}(\%)$ & $\mathrm{Ca}(\%)$ \\
\hline 7490.67 & 323097.60 & 17.20 & 92.45 & 26.36 & 1.13 & -10.43 & 11.43 & -168.97 & 106.46 & 5.21 & 6.48 & 3.48 & 1.77 \\
\hline 7490.73 & 262819.60 & 17.80 & 73.48 & 97.23 & 2.62 & -10.91 & 22.24 & -121.61 & -4330.56 & 4.59 & 8.73 & 2.91 & 2.28 \\
\hline 7490.80 & 194957.00 & 18.40 & 52.78 & 181.22 & -2.74 & -6.45 & 17.71 & -82.60 & -15151.48 & 3.57 & 21.12 & 2.21 & 2.39 \\
\hline 7490.87 & 190716.00 & 18.40 & 59.13 & 182.41 & -0.59 & -4.82 & 19.67 & -104.76 & -16578.34 & 2.86 & 20.01 & 2.33 & 2.07 \\
\hline 7490.93 & 193913.60 & 18.40 & 72.89 & 183.71 & -0.61 & -2.00 & 19.54 & -99.25 & -14772.10 & 2.87 & 19.77 & 2.36 & 2.31 \\
\hline 7491.00 & 206498.60 & 18.20 & 73.28 & 182.81 & -1.32 & 0.44 & 20.05 & -92.62 & -15398.61 & 2.48 & 16.08 & 2.64 & 2.20 \\
\hline 7491.06 & 269749.60 & 17.60 & 98.60 & 114.91 & -4.62 & 0.85 & 9.60 & -125.08 & -11564.29 & 2.86 & 13.44 & 3.22 & 1.53 \\
\hline 7491.13 & 336632.80 & 17.00 & 122.97 & 39.16 & -0.74 & -0.04 & 13.61 & -178.18 & -588.11 & 3.53 & 0.37 & 3.97 & 1.05 \\
\hline 7491.19 & 343733.40 & 17.00 & 120.45 & 37.74 & -2.50 & -0.50 & 12.60 & -167.60 & -1296.44 & 3.79 & 0.39 & 4.03 & 1.04 \\
\hline 7491.26 & 355618.20 & 17.00 & 107.94 & 44.76 & -3.13 & 3.85 & 12.03 & -176.49 & -3530.65 & 4.08 & 0.40 & 4.06 & 0.91 \\
\hline 7491.33 & 372923.80 & 16.80 & 107.90 & 44.36 & -1.59 & 4.44 & 13.14 & -194.65 & -1021.98 & 4.80 & 1.67 & 3.96 & 1.22 \\
\hline 7491.39 & 392970.20 & 16.60 & 103.73 & 47.79 & -1.07 & 5.92 & 14.81 & -248.46 & -1543.09 & 5.21 & 1.63 & 3.94 & 2.24 \\
\hline 7491.46 & 407589.20 & 16.40 & 102.11 & 49.05 & -1.49 & 3.69 & 16.65 & -218.76 & -851.98 & 5.55 & 1.59 & 3.96 & 2.59 \\
\hline 7491.52 & 409209.60 & 16.40 & 84.65 & 63.86 & 202.23 & 8.30 & 16.16 & -182.41 & 542.05 & 5.48 & 1.60 & 3.95 & 2.56 \\
\hline 7491.59 & 409426.60 & 16.40 & 90.78 & 57.37 & 203.20 & 3.08 & 17.31 & -158.17 & 2386.49 & 5.35 & 1.59 & 3.97 & 2.43 \\
\hline 7491.65 & 406646.20 & 16.40 & 89.52 & 55.16 & 202.60 & 3.88 & 14.76 & -186.60 & 417.19 & 4.81 & 0.24 & 4.09 & 2.24 \\
\hline 7491.72 & 398081.00 & 16.40 & 92.75 & 51.80 & 202.71 & 4.01 & 13.43 & -170.51 & -21.59 & 4.52 & 0.24 & 4.08 & 1.48 \\
\hline 7491.78 & 389315.80 & 16.40 & 92.50 & 44.96 & 204.60 & 4.46 & 10.92 & -193.80 & 86.89 & 4.06 & 0.42 & 4.03 & 1.22 \\
\hline 7491.85 & 389362.20 & 16.40 & 111.51 & 45.53 & 1.80 & -3.37 & 10.42 & -248.01 & 301.01 & 4.12 & 0.39 & 4.02 & 1.18 \\
\hline 7491.92 & 388529.60 & 16.40 & 109.91 & 60.78 & 1.45 & -5.23 & 7.91 & -273.34 & -720.37 & 4.18 & 0.40 & 3.96 & 1.22 \\
\hline 7491.98 & 383697.80 & 16.60 & 111.31 & 70.29 & 2.19 & -7.61 & 8.96 & -244.28 & -640.37 & 3.92 & 0.38 & 3.97 & 1.09 \\
\hline 7492.05 & 377827.20 & 16.80 & 106.66 & 69.29 & 3.90 & -8.79 & 8.28 & -231.77 & -461.26 & 3.63 & 0.40 & 4.07 & 0.78 \\
\hline 7492.11 & 375252.40 & 17.00 & 102.78 & 75.25 & 3.03 & -6.32 & 9.45 & -216.55 & -35.15 & 3.63 & 0.33 & 4.10 & 0.74 \\
\hline 7492.18 & 382410.20 & 16.80 & 98.09 & 67.10 & 3.21 & -2.73 & 10.03 & -197.15 & -211.62 & 3.70 & 0.45 & 4.15 & 0.83 \\
\hline 7492.24 & 389625.00 & 16.60 & 93.55 & 64.78 & 3.21 & -3.74 & 10.83 & -203.24 & -48.07 & 3.78 & 0.45 & 4.27 & 0.78 \\
\hline 7492.31 & 392977.00 & 16.40 & 87.84 & 59.55 & 3.75 & -1.47 & 9.68 & -201.22 & 1023.31 & 4.09 & 0.84 & 4.27 & 0.85 \\
\hline 7492.38 & 396762.40 & 16.20 & 88.16 & 62.74 & 1.80 & -1.83 & 10.39 & -179.62 & 1196.64 & 4.57 & 1.13 & 4.19 & 1.09 \\
\hline 7492.44 & 400093.20 & 16.00 & 87.75 & 56.36 & 1.20 & -3.36 & 7.90 & -159.77 & 365.88 & 4.44 & 1.02 & 4.24 & 1.07 \\
\hline 7492.51 & 396969.20 & 16.00 & 90.10 & 56.92 & 1.12 & -4.88 & 8.33 & -169.43 & 88.84 & 4.47 & 1.15 & 4.20 & 1.02 \\
\hline 7492.57 & 392825.00 & 16.00 & 89.95 & 53.53 & 2.27 & -1.41 & 7.79 & -156.24 & 239.56 & 4.36 & 1.15 & 4.14 & 0.95 \\
\hline 7492.64 & 390522.80 & 16.20 & 90.61 & 53.07 & 2.51 & 2.38 & 8.87 & -177.85 & -741.34 & 4.07 & 0.77 & 4.20 & 0.80 \\
\hline 7492.70 & 389032.20 & 16.40 & 90.74 & 47.62 & 2.89 & 4.19 & 8.35 & -187.93 & -219.70 & 3.65 & 0.49 & 4.20 & 0.62 \\
\hline 7492.77 & 388446.20 & 16.40 & 88.69 & 45.19 & 3.65 & 2.18 & 10.93 & -221.93 & -262.84 & 3.66 & 0.43 & 4.22 & 0.58 \\
\hline
\end{tabular}




\begin{tabular}{|c|c|c|c|c|c|c|c|c|c|c|c|c|c|}
\hline Depth & $\mathrm{XRF}$ & XRF Live Time & $\mathrm{Nb}(\mathrm{ppm})$ & Mo (ppm) & $\mathrm{Ba}(\mathrm{ppm})$ & $\mathrm{Pb}(\mathrm{ppm})$ & Th (ppm) & $\mathrm{U}(\mathrm{ppm})$ & $\mathrm{P}(\mathrm{ppm})$ & $\mathrm{S}(\%)$ & $\mathrm{Cl}(\%)$ & $\mathrm{K}(\%)$ & $\mathrm{Ca}(\%)$ \\
\hline 7492.83 & 387798.00 & 16.40 & 86.61 & 49.78 & 3.62 & 4.19 & 11.79 & -212.81 & -652.39 & 3.47 & 0.23 & 4.21 & 0.56 \\
\hline 7492.90 & 386178.80 & 16.60 & 85.31 & 49.80 & 2.32 & 3.37 & 13.10 & -210.81 & -645.61 & 3.51 & 0.58 & 4.16 & 0.59 \\
\hline 7492.97 & 387182.60 & 16.60 & 83.66 & 47.99 & 1.31 & -1.16 & 13.91 & -175.35 & 285.35 & 3.60 & 0.58 & 4.15 & 0.61 \\
\hline 7493.03 & 386849.40 & 16.60 & 82.33 & 51.54 & 2.53 & -4.39 & 15.04 & -162.20 & -1251.28 & 3.95 & 1.92 & 4.07 & 0.63 \\
\hline 7493.10 & 381795.80 & 16.80 & 81.71 & 50.93 & 2.99 & -2.22 & 15.09 & -137.82 & -1296.57 & 3.93 & 2.07 & 4.01 & 0.60 \\
\hline 7493.16 & 373013.80 & 17.00 & 80.09 & 40.30 & 4.75 & -5.85 & 15.88 & -117.42 & -747.71 & 4.08 & 4.00 & 3.84 & 0.68 \\
\hline 7493.23 & 366320.80 & 17.00 & 83.88 & 38.47 & 5.66 & -5.22 & 15.65 & -118.30 & -765.19 & 3.86 & 3.66 & 3.88 & 0.66 \\
\hline 7493.29 & 360071.20 & 17.00 & 87.44 & 42.49 & 5.38 & -6.31 & 13.54 & -139.34 & -1904.62 & 3.78 & 3.66 & 3.82 & 0.65 \\
\hline 7493.36 & 349453.00 & 17.00 & 88.80 & 44.67 & 6.14 & -3.24 & 13.66 & -134.06 & -622.47 & 3.73 & 2.46 & 3.91 & 0.56 \\
\hline 7493.43 & 343657.60 & 17.00 & 91.82 & 47.76 & 5.74 & -4.93 & 12.73 & -152.04 & -795.48 & 3.61 & 2.39 & 3.85 & 0.67 \\
\hline 7493.49 & 337453.80 & 17.00 & 97.84 & 44.07 & 3.65 & -2.56 & 10.99 & -176.68 & -1245.49 & 3.20 & 0.72 & 3.90 & 0.61 \\
\hline 7493.56 & 332103.60 & 17.00 & 102.84 & 40.90 & 3.02 & -2.80 & 12.50 & -156.70 & -2170.67 & 3.28 & 0.74 & 3.85 & 0.77 \\
\hline 7493.62 & 322902.40 & 17.00 & 106.69 & 32.03 & 4.50 & -2.26 & 12.35 & -158.76 & -3688.81 & 3.27 & 0.81 & 3.80 & 0.83 \\
\hline 7493.69 & 319827.60 & 17.00 & 110.21 & 28.38 & 3.82 & -1.00 & 11.41 & -134.82 & -3679.50 & 3.48 & 3.15 & 3.48 & 1.25 \\
\hline 7493.75 & 316273.60 & 17.00 & 116.43 & 30.16 & 2.59 & 1.60 & 11.02 & -128.05 & -3549.33 & 3.84 & 3.66 & 3.46 & 1.29 \\
\hline 7493.82 & 304033.60 & 17.20 & 95.94 & 40.87 & 161.56 & 3.66 & 10.00 & -86.94 & -5462.95 & 4.20 & 6.38 & 3.20 & 3.79 \\
\hline 7493.88 & 303202.80 & 17.20 & 89.12 & 39.98 & 161.85 & 4.40 & 8.57 & -83.51 & -4701.06 & 4.04 & 6.34 & 3.19 & 3.65 \\
\hline 7493.95 & 301576.20 & 17.20 & 89.44 & 47.20 & 160.64 & 4.15 & 9.46 & -61.78 & -3091.02 & 3.93 & 6.56 & 3.15 & 3.63 \\
\hline 7494.02 & 294475.80 & 17.40 & 93.89 & 43.50 & 160.02 & 1.38 & 9.27 & -88.14 & -3213.86 & 3.26 & 4.31 & 3.36 & 3.28 \\
\hline 7494.08 & 282844.20 & 17.60 & 95.53 & 34.67 & 161.23 & -0.33 & 9.33 & -54.17 & -3998.60 & 3.09 & 6.41 & 3.12 & 3.33 \\
\hline 7494.15 & 280763.20 & 17.60 & 122.65 & 29.48 & 2.19 & -3.03 & 10.79 & -91.06 & -2616.97 & 2.80 & 4.64 & 3.30 & 0.81 \\
\hline 7494.21 & 254729.60 & 17.80 & 129.73 & 25.19 & 1.94 & -1.51 & 10.30 & -106.24 & -2961.54 & 2.84 & 6.39 & 3.09 & 0.77 \\
\hline 7494.28 & 257788.40 & 17.80 & 135.31 & 19.47 & 1.71 & 3.95 & 10.49 & -110.10 & -3313.35 & 2.92 & 6.13 & 3.08 & 0.75 \\
\hline 7494.34 & 268010.40 & 17.60 & 133.02 & 19.50 & 1.04 & 6.18 & 9.08 & -115.46 & -3059.86 & 3.00 & 5.88 & 3.08 & 0.74 \\
\hline 7494.41 & 282374.60 & 17.40 & 133.32 & 23.29 & 0.75 & 4.69 & 10.11 & -170.54 & -1465.57 & 3.22 & 3.48 & 3.30 & 0.61 \\
\hline 7494.48 & 289682.60 & 17.40 & 128.19 & 29.66 & -0.17 & 5.85 & 8.37 & -171.35 & -162.35 & 3.24 & 2.42 & 3.42 & 0.68 \\
\hline 7494.54 & 310579.80 & 17.20 & 129.57 & 32.16 & 0.09 & 5.68 & 9.68 & -161.40 & 89.18 & 3.41 & 1.05 & 3.54 & 0.79 \\
\hline 7494.61 & 305692.40 & 17.20 & 127.86 & 32.05 & 0.33 & -0.62 & 9.37 & -169.96 & 579.58 & 3.57 & 1.18 & 3.53 & 0.98 \\
\hline 7494.67 & 289967.20 & 17.40 & 126.13 & 41.18 & 1.13 & -6.81 & 12.72 & -165.16 & 1130.81 & 3.55 & 1.35 & 3.51 & 1.31 \\
\hline 7494.74 & 285644.00 & 17.40 & 126.50 & 47.03 & 1.01 & -3.92 & 13.29 & -136.99 & 876.98 & 3.41 & 2.75 & 3.35 & 1.53 \\
\hline 7494.80 & 289951.80 & 17.20 & 133.93 & 37.10 & 1.95 & -4.49 & 16.49 & -131.04 & 536.63 & 3.57 & 2.90 & 3.27 & 1.77 \\
\hline 7494.87 & 260928.20 & 17.60 & 132.33 & 42.86 & 1.17 & -5.84 & 13.81 & -134.12 & 324.80 & 3.49 & 2.92 & 3.19 & 1.96 \\
\hline 7494.93 & 250564.40 & 17.80 & 129.00 & 49.49 & 0.04 & -6.34 & 13.38 & -151.36 & 534.98 & 3.38 & 3.42 & 3.08 & 2.07 \\
\hline
\end{tabular}




\begin{tabular}{|c|c|c|c|c|c|c|c|c|c|c|c|c|c|}
\hline Depth & $\mathrm{XRF}$ & XRF Live Time & $\mathrm{Nb}(\mathrm{ppm})$ & Mo (ppm) & $\mathrm{Ba}(\mathrm{ppm})$ & $\mathrm{Pb}(\mathrm{ppm})$ & Th (ppm) & $\mathrm{U}(\mathrm{ppm})$ & $\mathrm{P}(\mathrm{ppm})$ & $\mathrm{S}(\%)$ & $\mathrm{Cl}(\%)$ & $\mathrm{K}(\%)$ & $\mathrm{Ca}(\%)$ \\
\hline 7495.00 & 246193.60 & 17.80 & 133.08 & 45.50 & -0.72 & -3.45 & 10.59 & -162.90 & -1430.19 & 3.36 & 3.57 & 2.98 & 1.74 \\
\hline 7495.07 & 236792.00 & 18.00 & 126.56 & 44.02 & -0.52 & -4.85 & 9.40 & -167.78 & -2370.23 & 3.19 & 2.14 & 3.09 & 1.50 \\
\hline 7495.13 & 226961.20 & 18.20 & 119.23 & 45.38 & -0.33 & -5.16 & 8.44 & -189.28 & -2786.22 & 2.99 & 1.95 & 3.10 & 1.18 \\
\hline 7495.20 & 240451.00 & 18.00 & 115.78 & 43.24 & 1.79 & -6.87 & 11.70 & -202.24 & -3633.67 & 2.89 & 1.80 & 3.13 & 0.93 \\
\hline 7495.26 & 224417.40 & 18.20 & 117.06 & 47.49 & 3.70 & -4.92 & 11.31 & -179.54 & -3833.33 & 2.83 & 2.45 & 3.05 & 0.73 \\
\hline 7495.33 & 210499.80 & 18.40 & 93.12 & 63.52 & 206.24 & 0.01 & 13.34 & -142.20 & -2605.79 & 2.72 & 2.43 & 3.06 & 0.74 \\
\hline 7495.39 & 206926.20 & 18.40 & 101.68 & 64.88 & 206.29 & -0.23 & 12.74 & -159.61 & -1844.91 & 2.77 & 2.44 & 3.03 & 0.75 \\
\hline 7495.46 & 211684.80 & 18.40 & 104.31 & 64.97 & 209.33 & 1.73 & 13.25 & -157.84 & -2001.44 & 3.08 & 2.42 & 3.07 & 0.78 \\
\hline 7495.52 & 217750.00 & 18.40 & 106.96 & 57.68 & 208.91 & 2.13 & 10.97 & -139.12 & -2682.35 & 3.10 & 2.18 & 3.05 & 0.84 \\
\hline 7495.59 & 246789.60 & 18.00 & 103.42 & 54.02 & 208.04 & 2.79 & 11.67 & -144.31 & -3709.09 & 3.29 & 1.03 & 3.13 & 1.57 \\
\hline 7495.66 & 291302.20 & 17.60 & 120.07 & 40.74 & 6.28 & -2.35 & 9.58 & -167.70 & -3393.55 & 3.57 & 1.21 & 2.96 & 5.83 \\
\hline 7495.72 & 314513.20 & 17.40 & 121.24 & 37.71 & 34.66 & -4.03 & 9.96 & -159.47 & -3207.66 & 3.77 & 1.27 & 3.01 & 5.91 \\
\hline 7495.79 & 312787.40 & 17.40 & 122.90 & 33.92 & 31.81 & -5.59 & 9.19 & -133.94 & -3052.38 & 3.60 & 1.44 & 2.93 & 5.97 \\
\hline 7495.85 & 316005.20 & 17.40 & 102.29 & 65.09 & 214.78 & -0.47 & 9.08 & -112.17 & -1069.32 & 4.06 & 1.42 & 2.97 & 6.10 \\
\hline 7495.92 & 320905.80 & 17.40 & 112.13 & 57.83 & 216.48 & -0.09 & 10.60 & -134.94 & 545.82 & 3.86 & 1.26 & 3.10 & 5.30 \\
\hline 7495.98 & 311808.80 & 17.40 & 127.38 & 51.72 & 215.72 & 1.38 & 14.11 & -142.09 & 875.43 & 4.31 & 1.23 & 3.27 & 1.33 \\
\hline 7496.05 & 303390.80 & 17.40 & 125.76 & 51.86 & 190.53 & 2.59 & 16.25 & -140.11 & 618.25 & 4.09 & 0.91 & 3.26 & 1.38 \\
\hline 7496.12 & 298851.40 & 17.40 & 127.09 & 52.56 & 191.03 & 6.34 & 16.84 & -158.69 & 12.53 & 4.03 & 0.86 & 3.28 & 1.34 \\
\hline 7496.18 & 296583.80 & 17.40 & 152.80 & 28.77 & 6.20 & 1.57 & 18.86 & -184.39 & -656.77 & 3.95 & 1.18 & 3.25 & 1.33 \\
\hline 7496.25 & 252435.00 & 17.80 & 151.01 & 58.69 & 5.74 & 5.63 & 16.96 & -141.71 & -1425.18 & 4.30 & 2.27 & 3.20 & 1.71 \\
\hline 7496.31 & 276354.80 & 17.60 & 148.30 & 71.06 & 6.23 & 6.04 & 17.00 & -157.21 & -296.55 & 4.18 & 1.99 & 3.30 & 1.50 \\
\hline 7496.38 & 281053.40 & 17.60 & 148.91 & 72.79 & 2.73 & 3.71 & 18.69 & -140.56 & -1160.88 & 4.25 & 2.00 & 3.33 & 1.39 \\
\hline 7496.44 & 275472.20 & 17.60 & 148.39 & 82.78 & -0.97 & 2.58 & 19.25 & -111.60 & -32.70 & 4.41 & 2.40 & 3.38 & 1.38 \\
\hline 7496.51 & 292101.20 & 17.40 & 151.94 & 77.08 & 0.23 & 2.05 & 19.36 & -113.26 & 926.94 & 4.31 & 2.48 & 3.40 & 1.41 \\
\hline 7496.57 & 318924.80 & 17.20 & 158.38 & 46.85 & -2.74 & -1.29 & 22.16 & -152.59 & 910.49 & 4.11 & 1.53 & 3.46 & 1.16 \\
\hline 7496.64 & 295406.00 & 17.40 & 162.81 & 45.28 & -3.44 & -1.90 & 23.42 & -160.11 & -1552.57 & 3.86 & 1.35 & 3.46 & 1.13 \\
\hline 7496.71 & 294244.00 & 17.40 & 159.50 & 39.80 & -4.25 & 2.66 & 19.96 & -201.03 & 469.86 & 4.20 & 1.36 & 3.46 & 1.31 \\
\hline 7496.77 & 305199.40 & 17.40 & 158.42 & 31.96 & -0.72 & 0.31 & 19.53 & -214.35 & 1510.52 & 4.14 & 0.87 & 3.46 & 1.31 \\
\hline 7496.84 & 293631.40 & 17.60 & 161.32 & 39.91 & -3.10 & 3.55 & 20.27 & -239.03 & 1777.79 & 4.25 & 1.21 & 3.47 & 1.40 \\
\hline 7496.90 & 305499.00 & 17.40 & 153.59 & 47.82 & -1.60 & 3.22 & 19.55 & -241.92 & 2648.04 & 4.58 & 1.06 & 3.46 & 1.48 \\
\hline 7496.97 & 290644.00 & 17.60 & 146.89 & 34.16 & -1.76 & 2.73 & 17.38 & -233.30 & 3513.72 & 4.47 & 1.25 & 3.37 & 1.53 \\
\hline 7497.03 & 271982.20 & 17.80 & 150.80 & 34.52 & -0.46 & 2.23 & 18.15 & -188.20 & 2274.47 & 4.17 & 1.68 & 3.25 & 1.48 \\
\hline 7497.10 & 266641.20 & 17.80 & 153.62 & 35.64 & -1.07 & -1.37 & 18.71 & -181.96 & 747.16 & 4.36 & 1.53 & 3.24 & 1.63 \\
\hline
\end{tabular}




\begin{tabular}{|c|c|c|c|c|c|c|c|c|c|c|c|c|c|}
\hline Depth & $\mathrm{XRF}$ & XRF Live Time & $\mathrm{Nb}(\mathrm{ppm})$ & Mo (ppm) & $\mathrm{Ba}(\mathrm{ppm})$ & $\mathrm{Pb}(\mathrm{ppm})$ & Th (ppm) & $\mathrm{U}(\mathrm{ppm})$ & $\mathrm{P}(\mathrm{ppm})$ & $\mathrm{S}(\%)$ & $\mathrm{Cl}(\%)$ & $\mathrm{K}(\%)$ & $\mathrm{Ca}(\%)$ \\
\hline 7497.17 & 256427.20 & 17.80 & 149.40 & 34.19 & -1.35 & -5.20 & 17.48 & -175.06 & -386.51 & 4.29 & 0.78 & 3.27 & 1.36 \\
\hline 7497.23 & 247711.20 & 18.00 & 147.83 & 37.78 & 0.01 & -5.58 & 17.71 & -170.80 & -1378.31 & 4.14 & 0.82 & 3.23 & 1.30 \\
\hline 7497.30 & 238223.20 & 18.00 & 144.48 & 44.85 & 0.22 & -7.33 & 17.76 & -167.39 & -1959.29 & 4.28 & 0.97 & 3.18 & 1.46 \\
\hline 7497.36 & 238152.40 & 18.00 & 141.69 & 53.35 & 0.38 & -10.03 & 18.91 & -204.41 & -2372.68 & 4.42 & 0.69 & 3.25 & 1.46 \\
\hline 7497.43 & 253028.40 & 17.80 & 140.06 & 52.39 & -0.94 & -8.78 & 17.91 & -186.23 & -3138.92 & 4.36 & 1.00 & 3.14 & 1.49 \\
\hline 7497.49 & 270050.00 & 17.60 & 139.37 & 52.34 & -0.31 & -9.74 & 18.73 & -191.23 & -1451.19 & 4.52 & 1.01 & 3.14 & 1.73 \\
\hline 7497.56 & 283491.20 & 17.40 & 142.78 & 43.63 & -1.89 & -13.35 & 19.41 & -199.37 & -1490.72 & 4.78 & 0.97 & 3.20 & 1.94 \\
\hline 7497.62 & 310039.60 & 17.20 & 159.72 & 42.65 & 0.04 & -9.93 & 21.00 & -199.52 & -489.49 & 4.95 & 0.63 & 3.28 & 1.89 \\
\hline 7497.69 & 326210.20 & 17.00 & 173.52 & 39.13 & 0.51 & -5.51 & 21.08 & -195.72 & 609.64 & 5.15 & 0.52 & 3.30 & 2.07 \\
\hline 7497.76 & 332455.80 & 17.00 & 177.66 & 41.23 & 1.51 & 0.01 & 24.61 & -242.55 & 2017.56 & 5.35 & 0.22 & 3.46 & 2.00 \\
\hline 7497.82 & 344454.40 & 17.00 & 182.98 & 45.00 & 3.35 & 1.87 & 27.23 & -241.32 & 1087.30 & 5.95 & 0.21 & 3.49 & 1.99 \\
\hline 7497.89 & 354371.80 & 17.00 & 195.60 & 44.50 & 2.65 & 7.38 & 28.21 & -247.53 & 1835.56 & 5.94 & 0.22 & 3.44 & 2.16 \\
\hline 7497.95 & 351546.80 & 17.00 & 192.04 & 40.37 & 2.34 & 8.29 & 27.74 & -227.28 & -224.05 & 5.83 & 0.25 & 3.42 & 2.71 \\
\hline 7498.02 & 352497.40 & 17.00 & 188.72 & 34.78 & 0.60 & 7.09 & 29.87 & -197.80 & -32.93 & 5.56 & 0.18 & 3.39 & 2.81 \\
\hline 7498.08 & 351461.80 & 17.00 & 192.63 & 33.59 & 2.46 & 4.54 & 28.11 & -174.75 & -1563.60 & 5.32 & 0.18 & 3.33 & 3.36 \\
\hline 7498.15 & 347901.40 & 17.00 & 192.42 & 24.87 & 2.06 & 5.36 & 27.29 & -164.40 & -1723.58 & 4.51 & 0.16 & 3.30 & 3.30 \\
\hline 7498.22 & 344405.60 & 17.00 & 186.45 & 26.72 & 4.26 & 5.12 & 26.41 & -125.14 & -2433.53 & 4.25 & 0.15 & 3.32 & 3.00 \\
\hline 7498.28 & 354051.40 & 17.00 & 183.07 & 26.25 & 3.46 & 2.12 & 27.43 & -150.90 & -914.18 & 4.62 & 0.13 & 3.37 & 2.52 \\
\hline 7498.35 & 360437.60 & 17.00 & 180.06 & 33.07 & 3.82 & 1.70 & 28.62 & -160.65 & -1785.84 & 4.71 & 0.13 & 3.39 & 2.23 \\
\hline 7498.41 & 369928.20 & 16.80 & 183.88 & 30.29 & 4.16 & 0.44 & 29.06 & -169.89 & 115.76 & 5.28 & 0.13 & 3.45 & 1.64 \\
\hline 7498.48 & 392745.40 & 16.60 & 192.53 & 40.35 & 3.86 & -0.71 & 28.03 & -184.94 & 1664.71 & 5.98 & 0.14 & 3.57 & 1.55 \\
\hline 7498.54 & 404426.00 & 16.40 & 158.13 & 66.88 & 235.01 & 3.48 & 26.76 & -155.94 & 2600.67 & 6.40 & 0.16 & 3.60 & 1.86 \\
\hline 7498.61 & 408477.00 & 16.20 & 160.98 & 66.66 & 234.40 & 6.24 & 24.63 & -135.40 & 3217.52 & 6.12 & 0.16 & 3.56 & 1.82 \\
\hline 7498.67 & 413927.60 & 16.20 & 128.91 & 91.26 & 421.68 & 10.29 & 19.09 & -97.88 & 3844.65 & 5.90 & 0.15 & 3.53 & 3.04 \\
\hline 7498.74 & 411257.20 & 16.40 & 123.60 & 98.60 & 419.72 & 11.80 & 21.32 & -102.46 & 1698.34 & 5.24 & 0.20 & 3.42 & 4.90 \\
\hline 7498.81 & 397315.00 & 16.40 & 106.86 & 95.29 & 419.69 & 12.11 & 27.31 & -76.52 & 394.23 & 4.46 & 0.19 & 3.19 & 8.29 \\
\hline 7498.87 & 398592.60 & 16.40 & 143.95 & 70.48 & 187.55 & 7.51 & 28.45 & -147.67 & -700.41 & 4.25 & 0.18 & 3.16 & 8.16 \\
\hline 7498.94 & 397653.60 & 16.40 & 146.66 & 79.58 & 188.19 & 6.08 & 29.08 & -165.24 & -41.44 & 4.74 & 0.72 & 3.13 & 8.28 \\
\hline 7499.00 & 406386.20 & 16.20 & 195.82 & 58.10 & -0.48 & 3.11 & 31.61 & -203.61 & -783.13 & 5.08 & 0.73 & 3.20 & 7.15 \\
\hline 7499.07 & 415081.20 & 16.00 & 205.89 & 58.41 & -2.22 & 4.82 & 29.88 & -216.07 & 2200.75 & 5.61 & 0.69 & 3.28 & 5.36 \\
\hline 7499.13 & 416827.00 & 16.00 & 219.77 & 57.09 & -1.54 & 4.76 & 26.82 & -233.88 & 4439.02 & 6.08 & 0.93 & 3.41 & 2.10 \\
\hline 7499.20 & 376156.00 & 16.40 & 220.25 & 54.51 & -3.62 & -0.58 & 25.61 & -197.79 & 7108.51 & 5.89 & 1.61 & 3.25 & 3.56 \\
\hline 7499.27 & 327393.40 & 17.00 & 219.71 & 45.49 & -3.16 & -1.24 & 25.64 & -178.01 & 6632.35 & 5.53 & 1.47 & 3.26 & 4.00 \\
\hline
\end{tabular}




\begin{tabular}{|c|c|c|c|c|c|c|c|c|c|c|c|c|c|}
\hline Depth & $\mathrm{XRF}$ & XRF Live Time & $\mathrm{Nb}(\mathrm{ppm})$ & Mo (ppm) & $\mathrm{Ba}(\mathrm{ppm})$ & $\mathrm{Pb}(\mathrm{ppm})$ & Th (ppm) & $\mathrm{U}(\mathrm{ppm})$ & $\mathrm{P}(\mathrm{ppm})$ & $\mathrm{S}(\%)$ & $\mathrm{Cl}(\%)$ & $\mathrm{K}(\%)$ & $\mathrm{Ca}(\%)$ \\
\hline 7499.33 & 312283.00 & 17.20 & 210.09 & 37.64 & -0.34 & -4.67 & 28.35 & -189.29 & 7791.14 & 5.62 & 1.89 & 3.17 & 5.77 \\
\hline 7499.40 & 293669.00 & 17.40 & 204.18 & 27.59 & 0.86 & -9.02 & 29.24 & -183.68 & 6695.80 & 5.66 & 1.90 & 3.22 & 5.79 \\
\hline 7499.46 & 271127.40 & 17.60 & 206.88 & 27.41 & 1.20 & -5.68 & 29.54 & -171.60 & 5011.77 & 5.93 & 2.85 & 3.11 & 5.80 \\
\hline 7499.53 & 282653.60 & 17.60 & 205.07 & 28.11 & 2.89 & -3.33 & 34.05 & -167.08 & 2093.18 & 6.35 & 2.50 & 3.22 & 4.13 \\
\hline 7499.59 & 291362.20 & 17.40 & 200.00 & 32.87 & 3.96 & 1.12 & 34.02 & -180.17 & -35.78 & 6.06 & 3.78 & 2.98 & 3.47 \\
\hline 7499.66 & 269619.80 & 17.60 & 195.33 & 34.53 & 2.79 & 3.14 & 31.94 & -144.14 & -1325.15 & 5.72 & 3.61 & 2.91 & 1.62 \\
\hline 7499.72 & 240482.60 & 17.80 & 191.07 & 37.17 & 3.60 & 5.14 & 29.63 & -106.80 & -2764.07 & 5.04 & 3.78 & 2.68 & 1.64 \\
\hline 7499.79 & 219149.00 & 18.00 & 179.96 & 35.13 & 1.69 & 0.72 & 26.16 & -107.86 & -4801.18 & 4.09 & 2.75 & 2.66 & 1.51 \\
\hline 7499.86 & 218843.20 & 18.00 & 178.16 & 29.23 & 0.86 & 2.95 & 22.42 & -115.73 & -3588.81 & 3.60 & 2.48 & 2.61 & 1.47 \\
\hline 7499.92 & 227947.80 & 18.00 & 172.38 & 25.94 & -0.27 & -1.71 & 22.43 & -103.09 & -1463.56 & 3.59 & 1.12 & 2.72 & 1.54 \\
\hline 7499.99 & 224760.00 & 18.00 & 164.15 & 65.74 & -0.32 & 3.68 & 16.96 & -160.22 & 1209.27 & 4.16 & 0.95 & 2.64 & 1.48 \\
\hline 7500.05 & 245435.00 & 17.80 & 156.72 & 114.89 & -0.78 & -2.43 & 12.73 & -251.89 & 2965.91 & 5.64 & 0.91 & 2.54 & 1.43 \\
\hline 7500.12 & 273726.20 & 17.60 & 158.54 & 118.32 & 1.61 & -0.40 & 12.44 & -249.01 & 5074.74 & 6.08 & 1.34 & 2.55 & 1.53 \\
\hline 7500.18 & 283038.40 & 17.40 & 160.09 & 147.92 & 1.61 & -4.08 & 10.77 & -280.42 & 6038.32 & 6.96 & 1.44 & 2.45 & 1.42 \\
\hline 7500.25 & 286618.80 & 17.40 & 161.64 & 149.61 & 1.13 & -5.73 & 9.19 & -287.62 & 4764.38 & 7.00 & 1.15 & 2.46 & 1.47 \\
\hline 7500.31 & 286851.40 & 17.40 & 135.05 & 137.79 & 1228.84 & -7.76 & 10.94 & -221.13 & 3481.55 & 6.64 & 1.81 & 2.46 & 1.61 \\
\hline 7500.38 & 288425.40 & 17.40 & 146.88 & 96.02 & 1228.98 & -0.22 & 16.03 & -199.04 & 4390.43 & 7.42 & 1.69 & 2.53 & 1.60 \\
\hline 7500.45 & 262557.00 & 17.60 & 145.39 & 85.52 & 1227.00 & 1.08 & 16.52 & -213.09 & 4053.75 & 6.96 & 1.43 & 2.49 & 1.42 \\
\hline 7500.51 & 245918.60 & 17.80 & 137.29 & 53.89 & 1227.99 & 4.60 & 19.33 & -168.11 & 2650.95 & 5.78 & 1.45 & 2.53 & 1.45 \\
\hline 7500.58 & 252567.20 & 17.60 & 137.61 & 49.45 & 1227.83 & 5.64 & 18.94 & -171.80 & 3439.72 & 5.59 & 1.47 & 2.49 & 1.27 \\
\hline 7500.64 & 264224.80 & 17.40 & 166.30 & 31.39 & -0.71 & -0.67 & 20.77 & -212.26 & 3133.56 & 5.34 & 1.01 & 2.52 & 1.17 \\
\hline 7500.71 & 259597.40 & 17.60 & 154.65 & 31.30 & 0.22 & -3.80 & 19.82 & -157.34 & 1578.40 & 3.29 & 1.55 & 2.50 & 1.33 \\
\hline 7500.77 & 274181.40 & 17.60 & 149.47 & 46.48 & 1.79 & -7.01 & 19.98 & -140.88 & 1446.01 & 3.60 & 1.73 & 2.48 & 1.58 \\
\hline 7500.84 & 281966.80 & 17.60 & 152.57 & 52.35 & 0.59 & -9.34 & 18.46 & -153.52 & 2601.92 & 3.84 & 1.69 & 2.53 & 1.66 \\
\hline 7500.91 & 275894.00 & 17.80 & 149.37 & 82.05 & -1.58 & -12.09 & 16.31 & -163.27 & 3326.19 & 4.35 & 1.71 & 2.53 & 1.77 \\
\hline 7500.97 & 274708.00 & 18.00 & 136.66 & 92.34 & -3.20 & -13.39 & 19.80 & -203.33 & 3137.09 & 5.57 & 1.41 & 2.48 & 1.86 \\
\hline 7501.04 & 247084.40 & 18.20 & 108.96 & 115.68 & 215.10 & -8.11 & 18.49 & -175.53 & 2042.99 & 5.39 & 0.81 & 2.54 & 1.74 \\
\hline 7501.10 & 220450.60 & 18.40 & 110.06 & 113.15 & 215.18 & -8.23 & 15.96 & -146.84 & 1224.74 & 5.24 & 0.53 & 2.53 & 1.55 \\
\hline 7501.17 & 220504.80 & 18.40 & 80.18 & 141.08 & 423.35 & -0.62 & 14.74 & -111.48 & 557.88 & 5.38 & 0.42 & 2.52 & 1.61 \\
\hline 7501.23 & 213925.00 & 18.40 & 80.44 & 123.21 & 426.74 & 4.42 & 18.31 & -110.89 & -201.53 & 5.29 & 0.44 & 2.55 & 1.62 \\
\hline 7501.30 & 204096.00 & 18.40 & 94.54 & 92.82 & 430.10 & 8.26 & 13.03 & -59.15 & 741.99 & 4.22 & 0.70 & 2.60 & 1.66 \\
\hline 7501.36 & 219909.20 & 18.20 & 127.89 & 59.82 & 210.36 & 4.63 & 14.71 & -83.25 & 1421.37 & 4.36 & 1.16 & 2.53 & 1.63 \\
\hline 7501.43 & 235114.80 & 18.00 & 134.39 & 48.78 & 209.32 & 7.35 & 17.80 & -129.27 & 1344.51 & 4.42 & 0.96 & 2.55 & 1.66 \\
\hline
\end{tabular}




\begin{tabular}{|c|c|c|c|c|c|c|c|c|c|c|c|c|c|}
\hline Depth & $\mathrm{XRF}$ & XRF Live Time & $\mathrm{Nb}(\mathrm{ppm})$ & Mo (ppm) & $\mathrm{Ba}(\mathrm{ppm})$ & $\mathrm{Pb}(\mathrm{ppm})$ & Th (ppm) & $\mathrm{U}(\mathrm{ppm})$ & $\mathrm{P}(\mathrm{ppm})$ & $\mathrm{S}(\%)$ & $\mathrm{Cl}(\%)$ & $\mathrm{K}(\%)$ & $\mathrm{Ca}(\%)$ \\
\hline 7501.50 & 219872.40 & 18.00 & 168.76 & 19.70 & 1.77 & -1.05 & 20.31 & -157.40 & 1072.12 & 4.22 & 0.92 & 2.52 & 1.65 \\
\hline 7501.56 & 227685.80 & 18.00 & 170.95 & 4.58 & 1.88 & -1.43 & 20.80 & -129.47 & 802.12 & 3.73 & 0.90 & 2.49 & 1.61 \\
\hline 7501.63 & 234945.40 & 18.00 & 171.43 & 8.16 & 7.38 & -2.57 & 23.15 & -130.75 & -656.70 & 3.55 & 0.74 & 2.55 & 1.54 \\
\hline 7501.69 & 263537.20 & 17.80 & 172.15 & 11.54 & 9.24 & -4.78 & 22.80 & -151.66 & -448.63 & 3.52 & 0.30 & 2.62 & 1.53 \\
\hline 7501.76 & 292149.00 & 17.60 & 171.36 & 14.24 & 12.08 & -7.18 & 21.16 & -140.22 & 350.58 & 3.47 & 0.30 & 2.67 & 1.47 \\
\hline 7501.82 & 299730.40 & 17.60 & 162.60 & 13.05 & 13.26 & -3.68 & 20.30 & -138.22 & -747.56 & 3.56 & 0.31 & 2.65 & 1.40 \\
\hline 7501.89 & 290976.00 & 17.60 & 159.66 & 14.67 & 11.19 & -2.97 & 19.85 & -131.39 & -699.72 & 3.73 & 0.68 & 2.58 & 1.54 \\
\hline 7501.96 & 291083.60 & 17.60 & 155.55 & 19.16 & 5.06 & -5.00 & 18.49 & -120.91 & -221.59 & 3.59 & 0.61 & 2.53 & 1.47 \\
\hline 7502.02 & 274134.40 & 17.80 & 147.62 & 12.44 & 4.37 & -2.32 & 17.30 & -104.69 & 1057.27 & 3.72 & 0.61 & 2.49 & 1.49 \\
\hline 7502.09 & 260901.00 & 17.80 & 139.84 & 8.13 & 3.44 & -0.90 & 18.33 & -106.21 & 724.74 & 3.85 & 0.67 & 2.42 & 1.53 \\
\hline 7502.15 & 269547.40 & 17.80 & 137.38 & 12.09 & 0.12 & 0.12 & 17.82 & -105.72 & 2043.00 & 4.22 & 0.68 & 2.44 & 1.63 \\
\hline 7502.22 & 274760.80 & 17.80 & 138.10 & 13.04 & -0.12 & -0.29 & 16.44 & -110.70 & 1260.20 & 4.29 & 0.32 & 2.51 & 1.55 \\
\hline 7502.28 & 276614.00 & 17.80 & 136.65 & 11.82 & 3.63 & 3.07 & 16.94 & -122.78 & 1144.53 & 4.50 & 0.34 & 2.52 & 1.77 \\
\hline 7502.35 & 272082.60 & 17.80 & 140.80 & 15.17 & 2.79 & 2.03 & 17.70 & -114.91 & -1470.23 & 4.38 & 0.37 & 2.46 & 1.87 \\
\hline 7502.41 & 269115.00 & 18.00 & 144.85 & 22.99 & 1.21 & 3.01 & 17.94 & -95.66 & -231.21 & 4.27 & 0.31 & 2.49 & 2.04 \\
\hline 7502.48 & 291048.40 & 17.60 & 157.16 & 31.95 & 5.08 & 2.77 & 19.53 & -99.57 & 1068.20 & 4.11 & 0.28 & 2.53 & 2.01 \\
\hline 7502.55 & 316223.20 & 17.40 & 164.09 & 37.47 & 9.65 & 1.62 & 22.94 & -126.93 & 3341.30 & 4.09 & 0.21 & 2.56 & 2.03 \\
\hline 7502.61 & 332285.80 & 17.20 & 175.44 & 34.25 & 11.21 & 1.20 & 25.96 & -132.46 & 3432.02 & 4.19 & 0.18 & 2.58 & 1.93 \\
\hline 7502.68 & 350640.40 & 17.00 & 182.57 & 37.63 & 10.43 & 2.78 & 26.53 & -144.04 & 4800.65 & 4.33 & 0.14 & 2.63 & 1.83 \\
\hline 7502.74 & 359302.80 & 16.80 & 186.93 & 34.77 & 9.27 & 3.86 & 26.24 & -161.15 & 3858.63 & 4.35 & 0.15 & 2.62 & 1.71 \\
\hline 7502.81 & 344997.80 & 17.00 & 182.13 & 20.97 & 7.98 & 1.48 & 26.65 & -165.21 & 3782.15 & 4.11 & 0.50 & 2.54 & 1.72 \\
\hline 7502.87 & 334344.20 & 17.00 & 179.12 & 18.98 & 4.29 & 0.85 & 25.27 & -155.45 & 2979.10 & 4.29 & 0.59 & 2.46 & 1.82 \\
\hline 7502.94 & 314079.00 & 17.20 & 171.20 & 19.45 & 0.66 & 2.44 & 22.78 & -154.15 & 2611.05 & 4.37 & 0.61 & 2.48 & 1.84 \\
\hline 7503.01 & 315868.00 & 17.20 & 164.30 & 14.82 & 0.49 & 1.96 & 22.75 & -175.41 & 3508.92 & 4.31 & 0.61 & 2.50 & 1.96 \\
\hline 7503.07 & 313956.60 & 17.20 & 159.68 & 16.94 & 5.14 & -1.38 & 21.23 & -187.23 & 4578.69 & 4.54 & 0.60 & 2.54 & 2.05 \\
\hline 7503.14 & 313620.60 & 17.20 & 157.32 & 20.22 & 3.73 & 1.41 & 19.82 & -177.34 & 2805.07 & 4.54 & 0.25 & 2.59 & 2.16 \\
\hline 7503.20 & 307491.60 & 17.20 & 157.79 & 20.70 & 4.63 & 4.82 & 19.14 & -172.18 & 1711.86 & 4.25 & 0.15 & 2.64 & 2.24 \\
\hline 7503.27 & 314760.00 & 17.00 & 153.98 & 18.33 & 7.21 & 3.24 & 19.43 & -170.97 & 1014.42 & 4.15 & 0.12 & 2.58 & 2.37 \\
\hline 7503.33 & 295255.00 & 17.20 & 152.47 & 17.74 & 5.86 & 3.61 & 20.52 & -143.47 & -446.01 & 3.91 & 0.12 & 2.53 & 2.45 \\
\hline 7503.40 & 278789.60 & 17.40 & 155.69 & 18.13 & 1.21 & 5.55 & 21.39 & -120.20 & -2189.98 & 3.53 & 0.12 & 2.44 & 2.52 \\
\hline 7503.46 & 269836.40 & 17.60 & 155.55 & 23.07 & 32.58 & 3.44 & 20.49 & -116.82 & 276.46 & 3.67 & 0.13 & 2.47 & 2.71 \\
\hline 7503.53 & 270889.20 & 17.60 & 157.23 & 19.41 & 31.07 & 1.06 & 20.48 & -94.78 & 879.74 & 3.64 & 0.12 & 2.48 & 2.76 \\
\hline 7503.60 & 270281.60 & 17.60 & 166.39 & 24.68 & 27.57 & -0.02 & 20.68 & -97.05 & 1551.33 & 3.47 & 0.13 & 2.47 & 2.64 \\
\hline
\end{tabular}




\begin{tabular}{|c|c|c|c|c|c|c|c|c|c|c|c|c|c|}
\hline Depth & $\mathrm{XRF}$ & XRF Live Time & $\mathrm{Nb}(\mathrm{ppm})$ & Mo (ppm) & $\mathrm{Ba}(\mathrm{ppm})$ & $\mathrm{Pb}(\mathrm{ppm})$ & Th (ppm) & $\mathrm{U}(\mathrm{ppm})$ & $\mathrm{P}(\mathrm{ppm})$ & $\mathrm{S}(\%)$ & $\mathrm{Cl}(\%)$ & $\mathrm{K}(\%)$ & $\mathrm{Ca}(\%)$ \\
\hline 7503.66 & 277333.60 & 17.40 & 173.64 & 29.75 & 29.40 & -3.52 & 19.26 & -102.03 & 3563.31 & 3.55 & 0.14 & 2.49 & 2.55 \\
\hline 7503.73 & 295956.00 & 17.20 & 174.33 & 22.27 & 30.17 & -5.96 & 18.84 & -113.00 & 5210.74 & 4.16 & 0.14 & 2.62 & 2.52 \\
\hline 7503.79 & 303402.20 & 17.00 & 179.16 & 12.15 & -0.96 & -5.93 & 20.30 & -120.39 & 3169.78 & 4.03 & 0.12 & 2.58 & 2.30 \\
\hline 7503.86 & 305027.80 & 17.00 & 169.92 & 15.48 & 3.97 & -7.59 & 19.69 & -142.35 & 1938.27 & 4.40 & 0.50 & 2.53 & 2.44 \\
\hline 7503.92 & 305296.60 & 17.00 & 135.40 & 42.24 & 226.77 & -1.76 & 17.52 & -104.31 & 2554.14 & 4.49 & 0.49 & 2.58 & 2.67 \\
\hline 7503.99 & 305406.80 & 17.00 & 135.39 & 34.71 & 226.99 & -3.79 & 17.14 & -117.44 & 544.98 & 4.55 & 0.49 & 2.62 & 2.78 \\
\hline 7504.06 & 302734.60 & 17.00 & 133.15 & 38.74 & 228.98 & 1.48 & 18.28 & -100.45 & -544.34 & 4.26 & 1.16 & 2.51 & 2.99 \\
\hline 7504.12 & 304790.00 & 17.00 & 130.62 & 46.86 & 229.66 & 0.04 & 16.93 & -98.92 & -502.59 & 4.33 & 1.16 & 2.52 & 3.09 \\
\hline 7504.19 & 312176.80 & 17.00 & 137.96 & 44.51 & 226.03 & -0.60 & 17.50 & -122.82 & 2178.44 & 4.39 & 0.79 & 2.60 & 2.84 \\
\hline 7504.25 & 315525.00 & 17.00 & 168.67 & 19.89 & 1.49 & -6.35 & 19.61 & -152.35 & 960.89 & 4.52 & 0.86 & 2.63 & 2.70 \\
\hline 7504.32 & 318552.20 & 17.00 & 168.58 & 25.03 & 0.92 & -2.70 & 21.39 & -130.66 & 2033.63 & 4.45 & 0.87 & 2.62 & 2.61 \\
\hline 7504.38 & 313994.80 & 17.00 & 171.84 & 23.22 & -2.65 & -6.13 & 22.36 & -126.73 & 2242.38 & 4.46 & 0.20 & 2.69 & 2.45 \\
\hline 7504.45 & 304518.20 & 17.20 & 174.68 & 21.55 & -1.31 & -2.56 & 23.48 & -135.09 & 2585.20 & 4.33 & 0.23 & 2.70 & 2.20 \\
\hline 7504.51 & 293297.60 & 17.20 & 180.56 & 21.51 & -1.05 & 1.55 & 24.34 & -127.00 & 432.36 & 3.92 & 0.23 & 2.69 & 2.10 \\
\hline 7504.58 & 293505.80 & 17.20 & 187.77 & 23.76 & -0.70 & 1.53 & 26.03 & -142.58 & 2161.88 & 4.26 & 0.28 & 2.68 & 1.92 \\
\hline 7504.65 & 292750.20 & 17.20 & 153.96 & 54.44 & 241.84 & 6.60 & 23.25 & -118.20 & 2242.05 & 4.70 & 0.29 & 2.72 & 2.18 \\
\hline 7504.71 & 309243.00 & 17.20 & 167.06 & 62.57 & 242.66 & 7.78 & 24.82 & -147.33 & 2909.18 & 4.93 & 0.33 & 2.79 & 2.14 \\
\hline 7504.78 & 333863.40 & 16.80 & 215.95 & 61.30 & 240.96 & 5.66 & 30.19 & -168.11 & 2150.19 & 5.70 & 0.54 & 2.86 & 2.30 \\
\hline 7504.84 & 349331.20 & 16.80 & 251.20 & 63.81 & 239.62 & 3.70 & 34.93 & -169.71 & 2077.81 & 6.29 & 0.60 & 2.94 & 2.37 \\
\hline 7504.91 & 359275.40 & 16.80 & 276.16 & 61.39 & 240.35 & 4.14 & 36.68 & -143.63 & 1614.09 & 6.48 & 0.91 & 2.94 & 2.38 \\
\hline 7504.97 & 372528.80 & 16.80 & 330.52 & 40.55 & -2.48 & 0.58 & 42.10 & -196.35 & 2721.73 & 6.73 & 0.92 & 2.97 & 1.95 \\
\hline 7505.04 & 371141.00 & 16.80 & 325.68 & 37.36 & -2.21 & 1.11 & 41.46 & -203.32 & 1672.33 & 7.34 & 1.40 & 2.94 & 2.06 \\
\hline 7505.10 & 366358.20 & 17.00 & 242.93 & 62.62 & 264.33 & 6.97 & 36.89 & -147.81 & 1564.06 & 6.96 & 1.16 & 2.99 & 1.74 \\
\hline 7505.17 & 370333.00 & 17.00 & 197.14 & 61.89 & 265.07 & 8.98 & 30.53 & -131.14 & 3251.78 & 6.95 & 1.11 & 3.04 & 1.43 \\
\hline 7505.24 & 368363.60 & 17.00 & 155.80 & 61.41 & 264.93 & 8.55 & 25.20 & -126.52 & 4072.12 & 6.53 & 0.70 & 3.07 & 1.23 \\
\hline 7505.30 & 358662.60 & 17.00 & 131.57 & 50.87 & 266.06 & 8.32 & 20.50 & -109.26 & 2634.49 & 5.85 & 0.68 & 3.03 & 1.23 \\
\hline 7505.37 & 348777.20 & 17.00 & 133.55 & 49.57 & 267.48 & 7.78 & 18.47 & -85.16 & 2272.23 & 4.92 & 0.28 & 3.00 & 0.91 \\
\hline 7505.43 & 348039.20 & 17.00 & 173.03 & 21.44 & 3.50 & 2.23 & 18.24 & -116.90 & 3773.49 & 4.60 & 0.31 & 2.91 & 1.09 \\
\hline 7505.50 & 344829.40 & 17.00 & 196.54 & 24.56 & 3.29 & -0.60 & 19.84 & -125.08 & 3269.94 & 4.21 & 0.41 & 2.85 & 1.32 \\
\hline 7505.56 & 347478.60 & 17.00 & 230.15 & 24.77 & 2.90 & 2.02 & 22.50 & -146.57 & 1879.87 & 4.25 & 0.67 & 2.83 & 1.57 \\
\hline 7505.63 & 357289.60 & 17.00 & 238.78 & 29.52 & 2.50 & 0.73 & 25.21 & -151.77 & 1303.59 & 4.48 & 0.70 & 2.84 & 1.66 \\
\hline 7505.70 & 361761.60 & 17.00 & 223.43 & 32.46 & 1.19 & 2.76 & 24.21 & -145.98 & 1701.12 & 4.26 & 0.59 & 2.85 & 1.74 \\
\hline 7505.76 & 361375.60 & 17.00 & 228.57 & 34.01 & -1.96 & 2.61 & 24.27 & -152.87 & 668.66 & 4.32 & 0.57 & 2.84 & 1.77 \\
\hline
\end{tabular}




\begin{tabular}{|c|c|c|c|c|c|c|c|c|c|c|c|c|c|}
\hline Depth & XRF & XRF Live Time & $\mathrm{Nb}(\mathrm{ppm})$ & Mo (ppm) & $\mathrm{Ba}(\mathrm{ppm})$ & $\mathrm{Pb}(\mathrm{ppm})$ & Th (ppm) & $\mathrm{U}(\mathrm{ppm})$ & $\mathrm{P}(\mathrm{ppm})$ & $\mathrm{S}(\%)$ & $\mathrm{Cl}(\%)$ & $\mathrm{K}(\%)$ & $\mathrm{Ca}(\%)$ \\
\hline 7505.83 & 358866.20 & 17.00 & 223.80 & 32.16 & -2.01 & 2.33 & 23.47 & -149.90 & -158.40 & 4.50 & 0.51 & 2.80 & 1.83 \\
\hline 7505.89 & 341931.60 & 17.20 & 206.60 & 27.39 & -2.88 & 0.97 & 23.06 & -141.90 & -855.01 & 4.22 & 0.86 & 2.75 & 1.75 \\
\hline 7505.96 & 345214.80 & 17.00 & 201.96 & 25.93 & -3.63 & 4.29 & 20.55 & -149.08 & -177.65 & 4.36 & 0.86 & 2.72 & 1.76 \\
\hline 7506.02 & 351786.80 & 17.00 & 207.51 & 23.80 & -3.71 & 2.68 & 21.54 & -163.56 & 254.49 & 4.50 & 0.85 & 2.69 & 1.88 \\
\hline 7506.09 & 348143.00 & 17.00 & 198.34 & 29.77 & -2.01 & 4.50 & 20.29 & -167.78 & 2002.31 & 4.43 & 0.83 & 2.66 & 1.79 \\
\hline 7506.15 & 342595.40 & 17.00 & 188.25 & 26.14 & -1.99 & 7.72 & 19.12 & -141.59 & 2107.06 & 4.27 & 0.80 & 2.66 & 1.73 \\
\hline 7506.22 & 352656.80 & 16.80 & 185.01 & 33.63 & -1.26 & 8.67 & 17.73 & -128.25 & 3863.06 & 4.31 & 0.55 & 2.69 & 1.70 \\
\hline 7506.29 & 331619.00 & 17.20 & 183.55 & 31.14 & -0.09 & 7.77 & 18.42 & -125.17 & 3298.94 & 3.99 & 0.54 & 2.70 & 1.59 \\
\hline 7506.35 & 329396.20 & 17.20 & 183.97 & 31.59 & 1.45 & 8.46 & 17.57 & -125.32 & 1589.22 & 4.03 & 0.54 & 2.81 & 1.42 \\
\hline 7506.42 & 327802.00 & 17.20 & 177.01 & 23.85 & 0.76 & 8.15 & 16.61 & -130.74 & 303.49 & 4.12 & 0.53 & 2.84 & 1.30 \\
\hline 7506.48 & 341579.00 & 17.00 & 171.21 & 30.92 & 1.94 & 3.93 & 17.71 & -165.57 & 920.65 & 4.49 & 0.53 & 2.91 & 1.08 \\
\hline 7506.55 & 351555.20 & 17.00 & 172.32 & 25.75 & 0.61 & 1.48 & 18.76 & -179.53 & 950.15 & 4.86 & 0.13 & 3.00 & 1.05 \\
\hline 7506.61 & 363789.80 & 16.80 & 182.71 & 29.16 & -0.06 & -1.79 & 19.15 & -190.66 & 1405.02 & 5.40 & 0.11 & 3.07 & 1.15 \\
\hline 7506.68 & 364098.60 & 16.80 & 200.97 & 22.03 & -2.10 & -3.62 & 21.78 & -191.16 & 3188.12 & 5.74 & 0.11 & 3.05 & 1.33 \\
\hline 7506.75 & 378884.80 & 16.60 & 233.84 & 27.09 & -0.86 & -4.63 & 25.10 & -183.43 & 2875.78 & 5.92 & 0.12 & 3.10 & 1.68 \\
\hline 7506.81 & 356808.20 & 16.80 & 257.08 & 22.36 & -1.95 & -5.05 & 26.78 & -181.10 & 2000.03 & 5.68 & 0.12 & 3.05 & 1.92 \\
\hline 7506.88 & 337475.00 & 17.00 & 265.80 & 24.68 & 0.15 & -2.05 & 28.53 & -202.85 & 1872.05 & 5.43 & 0.16 & 3.00 & 1.96 \\
\hline 7506.94 & 321411.60 & 17.20 & 257.23 & 21.10 & 0.07 & 1.79 & 28.50 & -180.98 & 2042.88 & 4.95 & 0.15 & 2.89 & 1.87 \\
\hline 7507.01 & 310686.00 & 17.20 & 236.02 & 28.84 & 0.37 & 3.93 & 25.50 & -163.01 & 1697.36 & 5.09 & 0.16 & 2.80 & 1.70 \\
\hline 7507.07 & 284280.20 & 17.60 & 197.31 & 21.15 & -0.74 & 3.87 & 23.65 & -148.54 & 984.29 & 5.01 & 0.17 & 2.70 & 1.39 \\
\hline 7507.14 & 279967.00 & 17.80 & 168.45 & 19.68 & -1.28 & 5.93 & 20.73 & -158.49 & 1103.74 & 5.04 & 0.15 & 2.63 & 1.24 \\
\hline 7507.20 & 273909.40 & 17.80 & 149.26 & 14.28 & -1.80 & 5.21 & 18.36 & -138.10 & -70.91 & 4.99 & 0.12 & 2.59 & 1.10 \\
\hline 7507.27 & 270229.00 & 17.80 & 141.12 & 17.96 & 0.89 & 0.20 & 18.41 & -121.49 & -566.29 & 5.07 & 0.14 & 2.57 & 1.01 \\
\hline 7507.34 & 272883.00 & 17.80 & 139.23 & 18.64 & 1.26 & -3.32 & 18.59 & -122.85 & -45.23 & 4.56 & 0.12 & 2.62 & 0.99 \\
\hline 7507.40 & 280017.20 & 17.60 & 152.02 & 24.21 & 0.53 & -1.04 & 19.52 & -136.51 & 1248.22 & 4.37 & 0.12 & 2.66 & 1.06 \\
\hline 7507.47 & 278876.20 & 17.60 & 164.66 & 28.73 & 0.92 & 0.71 & 21.07 & -99.89 & 3326.58 & 4.13 & 0.20 & 2.71 & 1.37 \\
\hline 7507.53 & 287557.40 & 17.40 & 178.98 & 37.09 & 0.31 & 0.21 & 23.20 & -91.81 & 3188.36 & 4.08 & 0.24 & 2.72 & 1.92 \\
\hline 7507.60 & 276514.00 & 17.40 & 184.90 & 38.43 & -0.86 & -0.43 & 22.05 & -97.26 & 5148.64 & 3.83 & 0.32 & 2.67 & 3.95 \\
\hline 7507.66 & 262420.60 & 17.60 & 184.36 & 34.75 & -0.80 & -0.31 & 22.59 & -92.09 & 4669.20 & 3.74 & 0.41 & 2.57 & 4.25 \\
\hline 7507.73 & 247223.40 & 17.80 & 172.23 & 23.63 & 0.04 & -6.44 & 21.62 & -71.36 & 4261.07 & 3.66 & 0.49 & 2.47 & 4.64 \\
\hline 7507.80 & 240741.40 & 17.80 & 156.91 & 18.54 & 1.32 & -8.39 & 20.62 & -73.15 & 1722.73 & 3.60 & 0.65 & 2.34 & 5.02 \\
\hline 7507.86 & 222344.80 & 18.00 & 140.94 & 15.69 & 1.59 & -8.06 & 19.73 & -64.87 & 1408.86 & 3.41 & 0.86 & 2.22 & 5.13 \\
\hline 7507.93 & 233703.40 & 18.00 & 139.61 & 8.23 & 0.45 & -4.79 & 20.29 & -59.74 & -1214.34 & 3.39 & 1.67 & 2.17 & 3.34 \\
\hline
\end{tabular}




\begin{tabular}{|c|c|c|c|c|c|c|c|c|c|c|c|c|c|}
\hline Depth & $\mathrm{XRF}$ & XRF Live Time & $\mathrm{Nb}(\mathrm{ppm})$ & Mo (ppm) & $\mathrm{Ba}(\mathrm{ppm})$ & $\mathrm{Pb}(\mathrm{ppm})$ & Th (ppm) & $\mathrm{U}(\mathrm{ppm})$ & $\mathrm{P}(\mathrm{ppm})$ & $\mathrm{S}(\%)$ & $\mathrm{Cl}(\%)$ & $\mathrm{K}(\%)$ & $\mathrm{Ca}(\%)$ \\
\hline 7507.99 & 252167.80 & 17.80 & 152.54 & 12.85 & 0.71 & -2.32 & 22.63 & -48.99 & -1111.42 & 3.38 & 2.17 & 2.14 & 4.25 \\
\hline 7508.06 & 268313.60 & 17.60 & 174.65 & 25.53 & 1.71 & 2.43 & 23.79 & -48.86 & -2585.79 & 3.47 & 2.60 & 2.11 & 5.78 \\
\hline 7508.12 & 288509.60 & 17.40 & 197.87 & 24.76 & 0.98 & 3.56 & 27.73 & -51.98 & -1611.53 & 3.61 & 2.84 & 2.21 & 5.93 \\
\hline 7508.19 & 312634.20 & 17.20 & 213.90 & 21.85 & 0.78 & 3.65 & 30.57 & -66.77 & -518.20 & 3.85 & 2.88 & 2.31 & 5.72 \\
\hline 7508.25 & 323352.00 & 17.00 & 213.74 & 27.59 & -0.01 & 3.20 & 33.58 & -88.38 & 1604.45 & 4.34 & 1.98 & 2.39 & 7.72 \\
\hline 7508.32 & 306007.00 & 17.20 & 207.39 & 21.98 & 1.08 & -0.15 & 33.20 & -106.22 & -407.40 & 4.26 & 1.41 & 2.37 & 7.07 \\
\hline 7508.39 & 296444.00 & 17.40 & 197.03 & 25.79 & -1.79 & -1.47 & 35.18 & -120.74 & 611.34 & 4.29 & 0.91 & 2.50 & 6.11 \\
\hline 7508.45 & 303856.60 & 17.40 & 194.36 & 27.76 & -1.09 & -5.06 & 34.60 & -116.26 & 1100.92 & 4.22 & 0.42 & 2.56 & 6.48 \\
\hline 7508.52 & 307545.40 & 17.40 & 198.57 & 29.47 & -0.48 & -6.71 & 33.54 & -103.72 & 159.15 & 4.08 & 0.14 & 2.57 & 7.05 \\
\hline 7508.58 & 314846.40 & 17.40 & 212.29 & 23.32 & 0.44 & -5.65 & 32.28 & -91.23 & -1241.84 & 3.80 & 0.16 & 2.64 & 5.18 \\
\hline 7508.65 & 323260.60 & 17.20 & 217.25 & 26.06 & -0.45 & -3.81 & 34.74 & -90.37 & 1014.83 & 3.96 & 0.15 & 2.76 & 5.08 \\
\hline 7508.71 & 326253.20 & 17.00 & 223.86 & 20.64 & 1.30 & -3.15 & 35.29 & -85.39 & 2544.49 & 3.97 & 0.17 & 2.71 & 4.52 \\
\hline 7508.78 & 299767.80 & 17.20 & 219.43 & 19.07 & -1.86 & 2.33 & 35.21 & -101.81 & 2209.04 & 4.01 & 0.18 & 2.65 & 3.79 \\
\hline 7508.85 & 321191.40 & 17.00 & 213.75 & 22.80 & -0.63 & 2.89 & 35.38 & -173.02 & 5462.24 & 6.01 & 0.20 & 2.57 & 4.54 \\
\hline 7508.91 & 333906.80 & 16.80 & 213.15 & 29.24 & 0.06 & 3.97 & 36.53 & -167.78 & 6497.97 & 6.27 & 0.30 & 2.57 & 4.59 \\
\hline 7508.98 & 328477.20 & 17.00 & 213.26 & 19.97 & -1.38 & 2.95 & 33.83 & -176.23 & 5533.02 & 6.35 & 0.49 & 2.51 & 4.17 \\
\hline 7509.04 & 321981.00 & 17.20 & 203.76 & 18.46 & -1.00 & 2.08 & 31.67 & -163.81 & 4122.67 & 6.22 & 0.63 & 2.47 & 3.74 \\
\hline 7509.11 & 326271.40 & 17.20 & 192.62 & 20.72 & 1.85 & -1.42 & 28.39 & -145.81 & 3483.95 & 5.93 & 0.61 & 2.49 & 3.26 \\
\hline 7509.17 & 328500.60 & 17.20 & 172.97 & 30.39 & 0.71 & -4.16 & 23.54 & -173.50 & 5383.12 & 7.11 & 0.62 & 2.39 & 4.41 \\
\hline 7509.24 & 320769.00 & 17.40 & 152.73 & 28.58 & 1.03 & -4.82 & 21.08 & -179.48 & 4973.51 & 6.67 & 0.50 & 2.39 & 4.14 \\
\hline 7509.30 & 332479.80 & 17.20 & 134.10 & 42.96 & 3.53 & -4.49 & 22.67 & -173.90 & 5601.27 & 6.33 & 0.31 & 2.38 & 4.42 \\
\hline 7509.37 & 340543.80 & 17.00 & 123.04 & 40.79 & 7.48 & -4.35 & 22.29 & -175.87 & 6289.76 & 6.21 & 0.14 & 2.39 & 4.45 \\
\hline 7509.44 & 360986.20 & 16.80 & 123.96 & 44.35 & 8.06 & -1.29 & 22.34 & -185.58 & 7163.81 & 6.24 & 0.13 & 2.39 & 4.47 \\
\hline 7509.50 & 326283.20 & 17.00 & 139.35 & 25.38 & 12.01 & 1.98 & 26.48 & -109.13 & 1941.01 & 2.93 & 0.11 & 2.58 & 1.73 \\
\hline 7509.57 & 302746.00 & 17.20 & 155.46 & 26.49 & 10.42 & 4.12 & 26.61 & -107.83 & 2514.84 & 2.92 & 0.12 & 2.58 & 1.69 \\
\hline 7509.63 & 307614.40 & 17.20 & 180.96 & 19.12 & 9.31 & 3.30 & 25.34 & -115.02 & 2309.45 & 3.17 & 0.10 & 2.68 & 1.40 \\
\hline 7509.70 & 312974.00 & 17.20 & 206.43 & 24.42 & 5.36 & 4.54 & 27.87 & -131.62 & 912.72 & 3.59 & 0.29 & 2.72 & 1.38 \\
\hline 7509.76 & 309866.40 & 17.20 & 226.81 & 20.39 & 2.57 & 3.24 & 30.28 & -140.67 & 1027.47 & 3.94 & 0.29 & 2.73 & 1.32 \\
\hline 7509.83 & 318731.40 & 17.20 & 236.47 & 28.07 & 9.92 & 1.32 & 31.28 & -128.66 & 2737.31 & 4.24 & 0.28 & 2.71 & 1.24 \\
\hline 7509.90 & 351418.60 & 16.80 & 237.73 & 26.43 & 10.95 & -3.85 & 33.67 & -180.08 & 3286.06 & 5.45 & 0.30 & 2.75 & 1.11 \\
\hline 7509.96 & 346326.00 & 16.80 & 226.79 & 28.62 & 11.74 & -3.97 & 32.85 & -202.18 & 3290.30 & 5.68 & 0.50 & 2.65 & 0.99 \\
\hline 7510.03 & 345114.80 & 16.80 & 220.77 & 20.92 & 17.16 & -2.91 & 33.50 & -189.21 & 3972.61 & 5.52 & 0.32 & 2.64 & 0.93 \\
\hline 7510.09 & 345462.80 & 16.80 & 226.20 & 17.52 & 30.66 & -4.23 & 36.48 & -200.98 & 3452.06 & 5.47 & 0.35 & 2.63 & 1.03 \\
\hline
\end{tabular}




\begin{tabular}{|c|c|c|c|c|c|c|c|c|c|c|c|c|c|}
\hline Depth & $\mathrm{XRF}$ & XRF Live Time & $\mathrm{Nb}(\mathrm{ppm})$ & Mo (ppm) & $\mathrm{Ba}(\mathrm{ppm})$ & $\mathrm{Pb}(\mathrm{ppm})$ & Th (ppm) & $\mathrm{U}(\mathrm{ppm})$ & $\mathrm{P}(\mathrm{ppm})$ & $\mathrm{S}(\%)$ & $\mathrm{Cl}(\%)$ & $\mathrm{K}(\%)$ & $\mathrm{Ca}(\%)$ \\
\hline 7510.16 & 342228.60 & 16.80 & 252.22 & 10.39 & 27.80 & -3.00 & 39.95 & -204.49 & 1728.26 & 5.44 & 0.40 & 2.64 & 1.11 \\
\hline 7510.22 & 316088.20 & 17.20 & 295.75 & 6.66 & 28.88 & 0.11 & 44.30 & -157.95 & -965.23 & 4.40 & 0.46 & 2.58 & 1.24 \\
\hline 7510.29 & 306574.80 & 17.40 & 322.83 & 5.56 & 29.80 & 1.13 & 48.70 & -140.36 & -682.90 & 4.34 & 0.27 & 2.63 & 1.26 \\
\hline 7510.35 & 304215.20 & 17.40 & 337.80 & 9.93 & 24.85 & -3.62 & 50.10 & -146.14 & 451.73 & 4.51 & 0.32 & 2.64 & 1.27 \\
\hline 7510.42 & 289164.80 & 17.60 & 323.05 & 10.48 & 13.08 & -3.54 & 49.61 & -144.97 & 305.32 & 4.33 & 0.31 & 2.62 & 1.15 \\
\hline 7510.49 & 291863.80 & 17.60 & 294.34 & 13.97 & 4.61 & -4.64 & 46.55 & -154.41 & -104.51 & 4.98 & 0.29 & 2.65 & 1.04 \\
\hline 7510.55 & 293744.20 & 17.40 & 247.97 & 14.44 & 2.40 & -7.14 & 41.61 & -155.23 & 1215.78 & 5.06 & 0.32 & 2.66 & 1.34 \\
\hline 7510.62 & 273681.20 & 17.40 & 214.09 & 5.13 & 0.66 & -8.95 & 36.76 & -140.36 & 257.45 & 4.64 & 0.34 & 2.48 & 1.34 \\
\hline 7510.68 & 291516.20 & 17.20 & 209.71 & 9.92 & 0.31 & -4.23 & 35.42 & -121.47 & 57.33 & 4.48 & 0.28 & 2.53 & 1.51 \\
\hline 7510.75 & 313794.20 & 17.00 & 227.71 & 11.74 & 0.03 & -1.78 & 35.06 & -100.56 & 760.52 & 4.59 & 0.27 & 2.60 & 1.72 \\
\hline 7510.81 & 321380.60 & 17.00 & 242.22 & 9.65 & -0.54 & -2.10 & 39.07 & -89.80 & 2215.55 & 3.91 & 0.25 & 2.64 & 2.02 \\
\hline 7510.88 & 341912.60 & 17.00 & 265.09 & 11.65 & -0.12 & -0.43 & 42.02 & -95.73 & 3053.52 & 4.04 & 0.15 & 2.68 & 1.92 \\
\hline 7510.94 & 371093.40 & 16.80 & 284.60 & 19.71 & 0.00 & 1.00 & 47.69 & -90.28 & 3985.06 & 4.42 & 0.46 & 2.87 & 2.01 \\
\hline 7511.01 & 359767.40 & 17.00 & 275.91 & 12.16 & -0.55 & 2.78 & 48.95 & -103.08 & 3229.45 & 4.56 & 0.48 & 2.82 & 1.86 \\
\hline 7511.08 & 369601.80 & 16.80 & 262.13 & 14.45 & -1.86 & -3.09 & 47.93 & -186.20 & 3732.03 & 7.42 & 0.49 & 2.83 & 1.70 \\
\hline 7511.14 & 366663.00 & 16.80 & 208.10 & 66.08 & 326.05 & 6.33 & 36.31 & -155.86 & 4893.28 & 8.05 & 0.48 & 2.80 & 1.54 \\
\hline 7511.21 & 349683.60 & 16.80 & 193.24 & 61.08 & 326.49 & 6.47 & 34.02 & -157.09 & 4787.68 & 7.97 & 0.56 & 2.75 & 1.36 \\
\hline 7511.27 & 354576.80 & 16.80 & 184.54 & 62.72 & 326.50 & 6.45 & 30.14 & -183.62 & 5423.79 & 8.23 & 0.25 & 2.74 & 1.30 \\
\hline 7511.34 & 343801.40 & 17.00 & 186.94 & 66.03 & 326.61 & 3.81 & 27.23 & -168.23 & 4584.08 & 8.07 & 1.27 & 2.66 & 1.35 \\
\hline 7511.40 & 322676.00 & 17.20 & 183.35 & 56.77 & 327.21 & 6.89 & 27.37 & -84.71 & 4076.58 & 5.18 & 1.28 & 2.63 & 1.45 \\
\hline 7511.47 & 316432.00 & 17.20 & 228.59 & 8.01 & 0.51 & -0.42 & 36.73 & -118.60 & 2833.27 & 4.55 & 1.38 & 2.67 & 1.51 \\
\hline 7511.54 & 323214.80 & 17.20 & 228.39 & 11.57 & -0.11 & 0.56 & 36.40 & -98.98 & 2953.93 & 4.62 & 1.33 & 2.72 & 1.57 \\
\hline 7511.60 & 318625.40 & 17.20 & 229.27 & 4.67 & -0.64 & 2.27 & 37.67 & -89.79 & 1607.44 & 4.18 & 1.32 & 2.75 & 1.56 \\
\hline 7511.67 & 326795.00 & 17.00 & 224.69 & 1.87 & -0.33 & 1.28 & 37.60 & -120.61 & 2631.62 & 4.13 & 0.28 & 2.84 & 1.53 \\
\hline 7511.73 & 328109.40 & 17.00 & 225.72 & 5.84 & 0.87 & 3.66 & 36.19 & -111.99 & 2426.26 & 4.11 & 0.29 & 2.87 & 1.35 \\
\hline 7511.80 & 328335.60 & 17.00 & 217.44 & 3.28 & -1.63 & 1.23 & 32.65 & -107.94 & 3825.14 & 4.31 & 0.19 & 2.82 & 1.10 \\
\hline 7511.86 & 334668.60 & 17.00 & 170.97 & 39.86 & 241.25 & 3.80 & 28.11 & -90.21 & 3036.11 & 4.39 & 2.62 & 2.70 & 0.86 \\
\hline 7511.93 & 339254.20 & 17.00 & 163.79 & 50.04 & 240.57 & 3.10 & 25.73 & -88.18 & 3680.98 & 4.53 & 2.74 & 2.70 & 0.81 \\
\hline 7511.99 & 347262.80 & 17.00 & 159.63 & 57.02 & 242.15 & 4.64 & 25.81 & -104.28 & 4651.17 & 4.91 & 2.75 & 2.72 & 0.76 \\
\hline 7512.06 & 352473.00 & 17.00 & 163.88 & 58.38 & 241.52 & 1.54 & 26.16 & -116.58 & 4429.52 & 5.02 & 2.73 & 2.71 & 0.82 \\
\hline 7512.13 & 352448.60 & 17.00 & 175.34 & 64.09 & 243.28 & 3.55 & 28.12 & -107.25 & 2113.73 & 4.74 & 2.76 & 2.73 & 1.00 \\
\hline 7512.19 & 352588.60 & 17.00 & 232.84 & 31.35 & 1.79 & 0.98 & 34.07 & -156.62 & 2091.66 & 4.65 & 0.35 & 2.85 & 1.27 \\
\hline 7512.26 & 364199.00 & 16.80 & 260.54 & 24.89 & 2.90 & -0.35 & 37.79 & -170.18 & 1980.45 & 5.11 & 0.24 & 2.90 & 1.57 \\
\hline
\end{tabular}




\begin{tabular}{|c|c|c|c|c|c|c|c|c|c|c|c|c|c|}
\hline Depth & $\mathrm{XRF}$ & XRF Live Time & $\mathrm{Nb}(\mathrm{ppm})$ & Mo (ppm) & $\mathrm{Ba}(\mathrm{ppm})$ & $\mathrm{Pb}(\mathrm{ppm})$ & Th (ppm) & $\mathrm{U}(\mathrm{ppm})$ & $\mathrm{P}(\mathrm{ppm})$ & $\mathrm{S}(\%)$ & $\mathrm{Cl}(\%)$ & $\mathrm{K}(\%)$ & $\mathrm{Ca}(\%)$ \\
\hline 7512.32 & 362680.40 & 16.80 & 276.09 & 21.06 & 3.01 & -2.43 & 41.13 & -151.96 & 1286.66 & 4.85 & 0.26 & 2.91 & 1.89 \\
\hline 7512.39 & 358131.20 & 16.80 & 276.85 & 20.70 & 3.75 & -1.18 & 41.17 & -139.23 & 797.18 & 4.68 & 0.25 & 2.91 & 1.93 \\
\hline 7512.45 & 364265.80 & 16.80 & 268.08 & 15.65 & 5.29 & -0.83 & 42.42 & -135.55 & 2184.43 & 4.98 & 0.22 & 2.93 & 1.92 \\
\hline 7512.52 & 354238.80 & 16.80 & 251.11 & 18.17 & 4.31 & -3.28 & 41.31 & -124.86 & 3339.75 & 4.86 & 0.19 & 2.91 & 1.75 \\
\hline 7512.59 & 338948.20 & 17.00 & 228.80 & 21.29 & 5.16 & 0.04 & 38.91 & -117.05 & 3598.10 & 4.28 & 0.16 & 2.86 & 1.54 \\
\hline 7512.65 & 339716.20 & 17.00 & 177.83 & 70.35 & 337.64 & 3.71 & 30.49 & -79.59 & 2488.18 & 4.22 & 0.13 & 2.86 & 1.39 \\
\hline 7512.72 & 340728.40 & 17.00 & 165.45 & 68.53 & 335.33 & 3.66 & 30.56 & -100.39 & 2804.94 & 4.31 & 0.12 & 2.85 & 1.43 \\
\hline 7512.78 & 332788.60 & 17.00 & 156.19 & 68.56 & 335.04 & 2.52 & 26.23 & -101.50 & 2199.42 & 4.14 & 0.51 & 2.83 & 1.42 \\
\hline 7512.85 & 329630.20 & 17.00 & 140.12 & 63.19 & 335.40 & 1.54 & 22.34 & -98.81 & 883.33 & 4.12 & 0.51 & 2.82 & 1.46 \\
\hline 7512.91 & 338837.80 & 17.00 & 130.51 & 62.80 & 334.08 & 1.79 & 20.72 & -104.29 & 1441.57 & 4.47 & 0.52 & 2.88 & 1.70 \\
\hline 7512.98 & 335852.20 & 17.00 & 159.52 & 14.64 & 0.48 & -3.67 & 24.88 & -144.87 & 2203.33 & 4.78 & 0.68 & 2.85 & 1.94 \\
\hline 7513.04 & 331346.40 & 17.00 & 160.05 & 14.93 & 1.43 & -4.73 & 24.07 & -154.50 & 2493.47 & 4.83 & 0.68 & 2.87 & 2.09 \\
\hline 7513.11 & 329910.80 & 17.00 & 176.75 & 14.63 & 0.52 & -6.11 & 24.84 & -165.62 & 2314.86 & 4.84 & 0.36 & 2.90 & 2.52 \\
\hline 7513.18 & 322831.00 & 17.20 & 192.32 & 10.70 & 0.08 & -4.69 & 26.57 & -161.03 & 2848.41 & 4.94 & 0.58 & 2.87 & 2.72 \\
\hline 7513.24 & 300936.00 & 17.40 & 201.66 & 13.26 & 2.07 & -10.99 & 27.53 & -158.70 & 1580.88 & 4.67 & 0.58 & 2.76 & 3.00 \\
\hline 7513.31 & 270299.80 & 17.60 & 197.72 & 10.64 & 0.57 & -9.44 & 25.14 & -146.62 & 1893.33 & 4.12 & 0.57 & 2.69 & 2.92 \\
\hline 7513.37 & 246202.80 & 17.80 & 184.55 & 13.14 & 0.93 & -8.97 & 22.49 & -123.02 & 1851.51 & 3.86 & 0.57 & 2.63 & 2.95 \\
\hline 7513.44 & 259860.60 & 17.80 & 154.96 & 19.30 & 1.28 & -8.66 & 22.31 & -112.71 & 3102.21 & 3.74 & 0.50 & 2.57 & 3.99 \\
\hline 7513.50 & 266941.00 & 17.60 & 131.99 & 23.52 & 1.99 & -7.26 & 19.09 & -96.50 & 2786.41 & 3.72 & 1.06 & 2.52 & 4.45 \\
\hline 7513.57 & 271485.20 & 17.40 & 108.51 & 16.39 & -0.99 & -3.35 & 15.73 & -75.95 & 2952.29 & 3.63 & 2.36 & 2.45 & 4.07 \\
\hline 7513.64 & 287767.40 & 17.40 & 96.95 & 16.33 & -0.92 & -2.21 & 14.62 & -77.46 & 3174.22 & 3.67 & 2.52 & 2.47 & 4.11 \\
\hline 7513.70 & 309044.40 & 17.20 & 94.94 & 23.57 & -1.07 & -3.87 & 15.06 & -72.02 & 4200.98 & 4.04 & 2.86 & 2.44 & 4.18 \\
\hline 7513.77 & 303078.60 & 17.20 & 96.14 & 22.63 & -0.19 & -0.32 & 12.95 & -83.09 & 2936.92 & 4.46 & 3.52 & 2.46 & 4.01 \\
\hline 7513.83 & 280332.80 & 17.40 & 88.98 & 35.21 & -1.92 & 0.84 & 15.08 & -69.19 & 60.66 & 4.22 & 3.60 & 2.38 & 6.60 \\
\hline 7513.90 & 290200.40 & 17.40 & 121.88 & 39.77 & -0.10 & 1.39 & 14.16 & -80.86 & 134.59 & 4.68 & 3.34 & 2.40 & 7.48 \\
\hline 7513.96 & 300013.60 & 17.20 & 107.77 & 87.51 & 230.27 & 5.26 & 15.41 & -52.42 & 217.84 & 5.37 & 3.90 & 2.36 & 8.14 \\
\hline 7514.03 & 294985.00 & 17.40 & 112.76 & 79.41 & 231.04 & 7.05 & 15.20 & -54.36 & -130.78 & 5.69 & 4.53 & 2.32 & 8.71 \\
\hline 7514.09 & 305879.00 & 17.20 & 109.90 & 81.33 & 229.37 & 5.53 & 15.34 & -62.31 & 811.20 & 6.10 & 4.40 & 2.34 & 9.07 \\
\hline 7514.16 & 338813.00 & 17.00 & 113.73 & 79.21 & 231.80 & 4.02 & 13.77 & -79.80 & 3344.89 & 6.65 & 5.22 & 2.37 & 8.70 \\
\hline 7514.23 & 276452.40 & 17.60 & 64.65 & 126.28 & 229.10 & -1.82 & 16.44 & -52.98 & 5776.43 & 5.84 & 15.46 & 1.92 & 7.83 \\
\hline 7514.29 & 249665.40 & 17.80 & 77.52 & 95.30 & 0.23 & -10.00 & 16.31 & -82.49 & 6288.05 & 5.88 & 14.61 & 2.00 & 8.20 \\
\hline 7514.36 & 255148.60 & 17.60 & 72.20 & 102.14 & 1.01 & -7.27 & 16.51 & -87.24 & 4986.21 & 5.73 & 14.15 & 2.14 & 7.74 \\
\hline 7514.42 & 233501.00 & 17.80 & 81.13 & 98.26 & 1.07 & -7.62 & 17.75 & -102.67 & 3350.05 & 4.95 & 14.09 & 2.21 & 6.26 \\
\hline
\end{tabular}




\begin{tabular}{|c|c|c|c|c|c|c|c|c|c|c|c|c|c|}
\hline Depth & $\mathrm{XRF}$ & XRF Live Time & $\mathrm{Nb}(\mathrm{ppm})$ & Mo (ppm) & $\mathrm{Ba}(\mathrm{ppm})$ & $\mathrm{Pb}(\mathrm{ppm})$ & Th (ppm) & $\mathrm{U}(\mathrm{ppm})$ & $\mathrm{P}(\mathrm{ppm})$ & $\mathrm{S}(\%)$ & $\mathrm{Cl}(\%)$ & $\mathrm{K}(\%)$ & $\mathrm{Ca}(\%)$ \\
\hline 7514.49 & 228791.40 & 17.80 & 94.18 & 98.38 & 0.67 & -8.92 & 18.68 & -99.20 & 3148.15 & 4.73 & 16.35 & 2.14 & 5.17 \\
\hline 7514.55 & 287682.20 & 17.20 & 125.19 & 47.87 & 2.91 & -5.67 & 17.72 & -113.96 & 2035.52 & 5.29 & 9.90 & 2.44 & 5.41 \\
\hline 7514.62 & 315508.80 & 17.00 & 142.98 & 37.55 & 0.81 & -0.80 & 20.36 & -117.68 & -810.97 & 4.86 & 12.28 & 2.41 & 4.20 \\
\hline 7514.69 & 318780.00 & 17.00 & 155.07 & 30.54 & -0.99 & -4.72 & 20.97 & -103.50 & -207.53 & 4.60 & 13.78 & 2.32 & 3.84 \\
\hline 7514.75 & 322613.80 & 17.00 & 150.44 & 35.08 & -0.23 & -6.27 & 20.12 & -84.57 & 1410.04 & 4.54 & 13.47 & 2.40 & 3.60 \\
\hline 7514.82 & 320329.40 & 17.00 & 144.95 & 25.00 & -0.70 & -7.02 & 21.03 & -89.74 & 2095.24 & 4.59 & 11.50 & 2.57 & 1.80 \\
\hline 7514.88 & 316671.40 & 17.00 & 134.96 & 29.92 & -0.82 & -7.92 & 19.48 & -81.50 & 420.99 & 5.10 & 8.44 & 2.79 & 1.33 \\
\hline 7514.95 & 313713.40 & 17.00 & 122.26 & 25.22 & 0.30 & -9.10 & 17.03 & -69.59 & 2445.60 & 4.99 & 6.24 & 2.95 & 1.19 \\
\hline 7515.01 & 306287.20 & 17.00 & 111.98 & 28.82 & 1.06 & -7.18 & 15.39 & -69.18 & 1968.66 & 4.82 & 6.80 & 2.90 & 1.08 \\
\hline 7515.08 & 301071.60 & 17.00 & 114.81 & 23.57 & 0.77 & -7.30 & 14.23 & -36.43 & 1051.19 & 4.81 & 7.29 & 2.83 & 1.10 \\
\hline 7515.14 & 323685.80 & 16.80 & 169.75 & 27.34 & -1.72 & -3.33 & 15.69 & -59.49 & 830.70 & 5.08 & 5.61 & 3.02 & 1.21 \\
\hline 7515.21 & 345088.40 & 16.60 & 210.42 & 25.18 & -2.60 & 2.49 & 18.64 & -70.56 & 2288.01 & 5.07 & 7.23 & 2.94 & 1.33 \\
\hline 7515.28 & 353024.40 & 16.60 & 247.89 & 30.77 & -2.72 & 5.60 & 21.02 & -90.79 & 2015.69 & 5.70 & 7.69 & 2.89 & 1.42 \\
\hline 7515.34 & 365798.00 & 16.60 & 277.13 & 30.48 & -4.11 & 7.04 & 23.85 & -119.28 & 2277.42 & 6.28 & 6.35 & 3.02 & 1.55 \\
\hline 7515.41 & 369122.60 & 16.60 & 300.05 & 37.38 & -5.13 & 11.04 & 26.60 & -212.34 & 1989.14 & 6.99 & 6.04 & 3.16 & 1.72 \\
\hline 7515.47 & 352684.80 & 16.80 & 286.80 & 34.52 & -3.54 & 8.48 & 24.03 & -214.46 & 2963.78 & 7.14 & 5.89 & 3.24 & 1.93 \\
\hline 7515.54 & 356711.00 & 16.80 & 290.80 & 40.74 & -3.17 & 5.77 & 22.32 & -206.10 & 3676.11 & 7.37 & 5.23 & 3.30 & 2.19 \\
\hline 7515.60 & 372449.00 & 16.60 & 247.75 & 45.86 & -2.84 & 1.22 & 22.22 & -192.19 & 3692.20 & 7.22 & 4.92 & 3.13 & 8.17 \\
\hline 7515.67 & 380762.00 & 16.40 & 205.07 & 50.50 & -1.32 & -2.56 & 19.41 & -188.01 & 4437.96 & 6.89 & 4.47 & 3.12 & 11.46 \\
\hline 7515.73 & 411887.40 & 16.20 & 164.43 & 53.66 & -0.67 & -5.91 & 17.51 & -132.01 & 1967.81 & 6.84 & 4.21 & 2.72 & 19.67 \\
\hline 7515.80 & 437351.60 & 16.00 & 107.32 & 63.93 & -0.60 & -10.92 & 18.09 & -162.52 & 2157.18 & 7.51 & 4.49 & 2.15 & 29.38 \\
\hline 7515.87 & 440234.80 & 16.00 & 58.63 & 71.83 & 1.07 & -11.80 & 19.37 & -218.15 & 2223.29 & 7.50 & 1.90 & 2.06 & 36.57 \\
\hline 7515.93 & 422108.00 & 16.20 & 71.49 & 75.90 & 0.22 & -11.37 & 17.77 & -208.72 & 5075.85 & 7.73 & 6.48 & 1.95 & 33.12 \\
\hline 7516.00 & 429928.60 & 16.20 & 107.73 & 72.70 & 0.51 & -11.15 & 20.83 & -239.61 & 5911.12 & 8.27 & 5.73 & 2.15 & 31.13 \\
\hline 7516.06 & 412702.40 & 16.40 & 136.91 & 66.52 & 1.45 & -11.05 & 20.57 & -267.11 & 7616.75 & 7.95 & 5.63 & 2.56 & 22.90 \\
\hline 7516.13 & 377791.00 & 16.60 & 165.38 & 62.19 & 3.20 & -4.12 & 21.37 & -221.25 & 6635.00 & 6.85 & 5.47 & 3.10 & 12.89 \\
\hline 7516.19 & 368904.60 & 16.60 & 187.38 & 47.13 & 2.56 & -2.73 & 20.88 & -192.54 & 5947.58 & 6.36 & 5.55 & 3.48 & 5.51 \\
\hline 7516.26 & 374905.80 & 16.60 & 197.41 & 33.58 & 3.76 & 0.97 & 19.16 & -220.53 & 4191.01 & 5.92 & 0.63 & 3.93 & 3.24 \\
\hline 7516.33 & 361139.00 & 16.80 & 188.90 & 30.64 & 4.28 & 0.99 & 17.87 & -194.46 & 3179.80 & 5.35 & 0.61 & 3.87 & 2.65 \\
\hline 7516.39 & 348256.20 & 16.80 & 176.48 & 24.16 & 3.48 & 0.40 & 19.22 & -152.95 & 4154.17 & 4.93 & 0.63 & 3.76 & 3.70 \\
\hline 7516.46 & 350179.60 & 16.80 & 175.95 & 17.70 & 3.03 & -2.58 & 15.55 & -129.50 & 3257.42 & 4.81 & 4.39 & 3.39 & 4.84 \\
\hline 7516.52 & 336528.20 & 17.00 & 165.24 & 20.41 & 2.40 & -6.70 & 15.51 & -144.43 & 3445.60 & 4.63 & 6.28 & 3.13 & 5.66 \\
\hline 7516.59 & 315814.80 & 17.20 & 161.37 & 33.12 & 1.48 & -10.35 & 15.80 & -136.68 & 1890.68 & 4.38 & 6.43 & 3.07 & 6.25 \\
\hline
\end{tabular}




\begin{tabular}{|c|c|c|c|c|c|c|c|c|c|c|c|c|c|}
\hline Depth & $\mathrm{XRF}$ & XRF Live Time & $\mathrm{Nb}(\mathrm{ppm})$ & Mo (ppm) & $\mathrm{Ba}(\mathrm{ppm})$ & $\mathrm{Pb}(\mathrm{ppm})$ & Th (ppm) & $\mathrm{U}(\mathrm{ppm})$ & $\mathrm{P}(\mathrm{ppm})$ & $\mathrm{S}(\%)$ & $\mathrm{Cl}(\%)$ & $\mathrm{K}(\%)$ & $\mathrm{Ca}(\%)$ \\
\hline 7516.65 & 298331.60 & 17.40 & 131.40 & 41.41 & 0.76 & -10.80 & 13.28 & -113.42 & 41.59 & 3.66 & 6.91 & 2.53 & 15. \\
\hline 7516.72 & 299872.40 & 17.40 & 107.92 & 46.48 & 0.16 & -19.09 & 8.36 & -104.31 & -1386.13 & 3.26 & 6.97 & 1.94 & 26.43 \\
\hline 7516.78 & 308444.60 & 17.40 & 74.22 & 50.46 & -0.32 & -16.10 & 8.44 & -102.36 & 3104.66 & 2.39 & 3.00 & 1.65 & 39.62 \\
\hline 7516.85 & 308665.80 & 17.40 & 46.30 & 59.94 & 0.79 & -15.48 & 4.33 & -58.60 & 53.84 & 1.66 & 1.05 & 1.17 & 47.38 \\
\hline 7516.92 & 312771.60 & 17.20 & 18.61 & 66.04 & 2.83 & -19.66 & 2.11 & -24.70 & -754.76 & 1.26 & 1.65 & 0.57 & 52.11 \\
\hline 7516.98 & 332255.40 & 17.00 & 15.20 & 83.34 & 2.07 & -24.78 & 0.83 & -40.72 & 799.65 & 2.26 & 1.24 & 0.49 & 53.75 \\
\hline 7517.05 & 331437.80 & 17.00 & 16.72 & 119.70 & 1.09 & -20.65 & 0.31 & -47.07 & 153.80 & 3.00 & 3.01 & 0.51 & 49.38 \\
\hline 7517.11 & 323620.20 & 17.00 & 17.44 & 122.72 & -0.10 & -28.33 & -0.62 & -51.72 & -1918.78 & 3.20 & 3.03 & 0.56 & 49.40 \\
\hline 7517.18 & 298481.00 & 17.20 & 18.98 & 126.07 & -0.92 & -27.76 & 0.84 & -57.66 & -3487.76 & 3.17 & 3.64 & 0.65 & 54.07 \\
\hline 7517.24 & 293598.40 & 17.40 & 14.33 & 115.83 & -2.79 & -24.08 & 1.19 & -68.00 & 1691.58 & 2.97 & 3.02 & 0.65 & 62.83 \\
\hline 7517.31 & 225024.00 & 18.00 & 14.10 & 155.62 & 3.87 & -28.75 & 0.27 & -46.32 & -5423.84 & 1.75 & 13.00 & 0.59 & 51.24 \\
\hline 7517.38 & 221799.60 & 18.00 & 13.24 & 124.79 & 4.19 & -25.39 & 1.77 & -29.69 & -3846.67 & 0.90 & 11.37 & 0.61 & 57.39 \\
\hline 7517.44 & 215424.40 & 18.00 & 11.54 & 131.82 & 4.06 & -19.59 & 1.99 & -24.63 & -3963.32 & 0.80 & 11.35 & 0.58 & 57.09 \\
\hline 7517.51 & 228415.60 & 18.00 & 10.89 & 129.12 & 4.07 & -18.83 & 3.07 & -15.44 & 1852.87 & 0.78 & 11.19 & 0.52 & 56.33 \\
\hline 7517.57 & 225668.40 & 18.00 & 12.83 & 123.27 & 6.02 & -17.89 & 3.26 & -13.04 & -3143.57 & 0.75 & 11.22 & 0.53 & 54.78 \\
\hline 7517.64 & 267673.80 & 17.60 & 14.06 & 69.88 & 0.74 & -6.93 & 4.87 & -11.54 & 3732.36 & 0.93 & 1.56 & 0.60 & 67.57 \\
\hline 7517.70 & 254247.20 & 17.80 & 15.01 & 66.27 & 0.03 & -6.19 & 4.32 & -19.12 & 11509.36 & 0.94 & 2.58 & 0.64 & 65.51 \\
\hline 7517.77 & 243826.00 & 18.00 & 16.45 & 66.81 & 2.09 & -10.53 & 5.16 & -20.48 & 14063.78 & 0.97 & 2.63 & 0.67 & 63.46 \\
\hline 7517.83 & 232566.60 & 18.00 & 18.17 & 67.90 & 3.61 & -17.00 & 2.17 & -20.23 & 12777.30 & 1.03 & 2.82 & 0.68 & 62.30 \\
\hline 7517.90 & 254430.20 & 17.80 & 18.58 & 73.29 & 2.79 & -18.20 & 0.87 & -22.87 & 11692.80 & 1.10 & 2.78 & 0.71 & 62.19 \\
\hline 7517.97 & 277627.80 & 17.60 & 19.85 & 72.40 & 1.18 & -15.61 & 0.86 & -34.99 & 12312.57 & 1.21 & 2.70 & 0.72 & 61.21 \\
\hline 7518.03 & 291732.20 & 17.40 & 21.16 & 79.99 & 2.59 & -19.11 & 1.96 & -38.53 & 2073.97 & 1.28 & 1.90 & 0.71 & 60.93 \\
\hline 7518.10 & 308845.40 & 17.20 & 24.43 & 79.45 & 2.49 & -17.22 & 0.55 & -40.88 & -4261.44 & 1.42 & 4.26 & 0.67 & 57.42 \\
\hline 7518.16 & 325471.20 & 17.00 & 27.69 & 77.44 & -0.13 & -9.76 & 2.14 & -43.37 & -4550.82 & 1.50 & 5.51 & 0.68 & 53.85 \\
\hline 7518.23 & 303468.20 & 17.20 & 37.77 & 72.62 & -1.30 & -9.18 & 2.78 & -44.73 & -3163.64 & 1.77 & 9.11 & 0.72 & 45.89 \\
\hline 7518.29 & 302607.80 & 17.20 & 65.63 & 68.59 & 0.04 & -9.99 & 4.52 & -63.03 & -4335.14 & 2.25 & 9.10 & 1.36 & 35.59 \\
\hline 7518.36 & 292541.40 & 17.40 & 89.91 & 66.37 & 1.37 & -3.65 & 7.39 & -78.46 & -1470.51 & 2.79 & 10.50 & 1.81 & 24.83 \\
\hline 7518.43 & 299801.60 & 17.40 & 110.56 & 61.90 & 2.27 & -7.87 & 10.65 & -128.61 & 1734.18 & 3.87 & 8.62 & 2.34 & 16.98 \\
\hline 7518.49 & 302413.40 & 17.40 & 128.88 & 55.91 & 3.75 & -6.62 & 12.18 & -133.70 & 448.08 & 4.23 & 10.62 & 2.59 & 9.87 \\
\hline 7518.56 & 309617.00 & 17.40 & 145.22 & 53.70 & 4.00 & -3.36 & 15.36 & -149.57 & 929.18 & 4.32 & 9.44 & 2.83 & 6.03 \\
\hline 7518.62 & 324483.00 & 17.20 & 140.23 & 73.46 & 4.14 & -7.53 & 15.86 & -175.68 & 3646.08 & 5.53 & 9.24 & 2.60 & 6.59 \\
\hline 7518.69 & 344963.40 & 17.00 & 132.96 & 69.42 & 2.85 & -7.65 & 14.22 & -180.75 & 2451.58 & 5.30 & 7.48 & 2.57 & 9.46 \\
\hline 7518.75 & 341144.00 & 17.00 & 131.09 & 64.07 & 1.60 & -1.53 & 12.90 & -156.02 & 1677.44 & 4.61 & 6.93 & 2.59 & 10.62 \\
\hline
\end{tabular}




\begin{tabular}{|c|c|c|c|c|c|c|c|c|c|c|c|c|c|}
\hline Depth & $\mathrm{XRF}$ & XRF Live Time & $\mathrm{Nb}(\mathrm{ppm})$ & Mo (ppm) & $\mathrm{Ba}(\mathrm{ppm})$ & $\mathrm{Pb}(\mathrm{ppm})$ & Th (ppm) & $\mathrm{U}(\mathrm{ppm})$ & $\mathrm{P}(\mathrm{ppm})$ & $\mathrm{S}(\%)$ & $\mathrm{Cl}(\%)$ & $\mathrm{K}(\%)$ & $\mathrm{Ca}(\%)$ \\
\hline 7518.82 & 339252.00 & 17.00 & 123.09 & 64.06 & 1.09 & -0.43 & 13.53 & -140.58 & 1312.42 & 4.50 & 2.98 & 2.81 & 13.23 \\
\hline 7518.88 & 364811.00 & 16.60 & 107.21 & 72.95 & 2.17 & -4.84 & 12.85 & -128.94 & -1569.99 & 4.43 & 0.47 & 2.76 & 20.39 \\
\hline 7518.95 & 356826.20 & 16.80 & 90.81 & 58.53 & 1.04 & -1.04 & 10.54 & -90.24 & -1557.22 & 2.86 & 1.40 & 2.45 & 26.33 \\
\hline 7519.02 & 365307.00 & 16.60 & 82.63 & 56.93 & 3.74 & -1.15 & 8.73 & -79.10 & 1221.09 & 2.76 & 1.59 & 2.23 & 30.25 \\
\hline 7519.08 & 373977.20 & 16.60 & 59.49 & 70.22 & 184.98 & 1.22 & 8.30 & -45.84 & 505.49 & 2.55 & 1.78 & 2.07 & 33.75 \\
\hline 7519.15 & 382667.40 & 16.60 & 70.71 & 69.56 & 187.88 & -2.74 & 7.71 & -58.29 & 1785.88 & 2.62 & 2.06 & 2.03 & 32.36 \\
\hline 7519.21 & 372891.60 & 16.80 & 86.26 & 58.66 & 194.27 & -0.23 & 7.25 & -60.15 & 6134.55 & 2.82 & 2.23 & 2.22 & 24.63 \\
\hline 7519.28 & 362019.80 & 16.80 & 104.95 & 51.85 & 195.63 & 0.62 & 9.57 & -49.69 & 4389.89 & 3.09 & 2.52 & 2.46 & 16.55 \\
\hline 7519.34 & 349944.40 & 17.00 & 112.83 & 51.94 & 194.76 & 3.72 & 11.64 & -52.03 & 2860.16 & 3.18 & 2.25 & 2.74 & 9.18 \\
\hline 7519.41 & 337089.80 & 17.00 & 131.76 & 29.72 & 13.12 & 2.87 & 11.93 & -71.15 & 2720.25 & 3.21 & 3.14 & 2.81 & 4.08 \\
\hline 7519.48 & 337860.00 & 17.00 & 130.86 & 31.08 & 11.05 & 4.59 & 13.78 & -73.56 & 1393.38 & 3.23 & 2.74 & 2.92 & 2.74 \\
\hline 7519.54 & 332568.60 & 17.00 & 128.22 & 35.08 & 3.16 & 5.96 & 14.70 & -68.31 & -24.74 & 3.13 & 2.55 & 2.99 & 2.58 \\
\hline 7519.61 & 327496.60 & 17.00 & 109.88 & 29.53 & 2.30 & 7.40 & 13.95 & -87.29 & 26.36 & 3.02 & 1.72 & 3.23 & 2.44 \\
\hline 7519.67 & 323603.40 & 17.00 & 100.11 & 25.98 & -0.03 & 3.30 & 15.14 & -70.00 & 298.36 & 3.16 & 1.72 & 3.32 & 2.25 \\
\hline 7519.74 & 327029.00 & 17.00 & 89.55 & 35.06 & 0.54 & 0.42 & 16.43 & -84.48 & 1157.05 & 3.52 & 1.75 & 3.40 & 1.93 \\
\hline 7519.80 & 327511.60 & 17.00 & 68.92 & 31.89 & 0.40 & -0.66 & 15.17 & -114.98 & 2247.31 & 3.86 & 1.88 & 3.44 & 1.32 \\
\hline 7519.87 & 335276.20 & 17.00 & 51.33 & 26.26 & 2.40 & -1.05 & 14.46 & -159.46 & 2216.47 & 4.28 & 1.91 & 3.50 & 1.12 \\
\hline 7519.93 & 337735.20 & 17.00 & 45.59 & 27.74 & 2.40 & -5.28 & 14.55 & -166.41 & 1971.77 & 4.42 & 1.48 & 3.51 & 1.20 \\
\hline 7520.00 & 334483.00 & 17.00 & 43.31 & 25.22 & 3.43 & -7.27 & 13.41 & -202.47 & 1601.31 & 4.84 & 1.64 & 3.54 & 1.10 \\
\hline 7520.07 & 326601.40 & 17.00 & 41.76 & 18.64 & 2.79 & -6.42 & 12.50 & -196.77 & 1017.60 & 4.39 & 0.51 & 3.60 & 1.08 \\
\hline 7520.13 & 307363.00 & 17.20 & 42.93 & 11.12 & 1.43 & -5.69 & 11.58 & -153.65 & 296.94 & 4.06 & 3.87 & 3.31 & 0.93 \\
\hline 7520.20 & 290337.20 & 17.20 & 42.43 & 10.33 & -0.36 & -3.78 & 10.96 & -123.90 & -72.65 & 4.08 & 5.53 & 3.21 & 1.07 \\
\hline 7520.26 & 277407.20 & 17.40 & 44.18 & 7.39 & -0.47 & -3.11 & 11.33 & -106.87 & -889.30 & 4.12 & 5.69 & 3.18 & 1.21 \\
\hline 7520.33 & 264227.40 & 17.60 & 42.87 & 8.97 & -1.02 & -3.13 & 10.23 & -95.78 & -2200.31 & 3.66 & 5.55 & 3.16 & 1.35 \\
\hline 7520.39 & 211027.20 & 18.20 & 36.10 & 6.53 & 91.46 & 8.97 & 9.84 & -70.61 & -1799.76 & 3.45 & 13.05 & 2.44 & 1.52 \\
\hline 7520.46 & 215626.40 & 18.20 & 34.34 & 8.72 & 91.58 & 7.80 & 9.99 & -102.00 & -1652.44 & 3.24 & 9.66 & 2.72 & 1.63 \\
\hline 7520.52 & 220490.60 & 18.20 & 35.78 & 7.34 & 92.12 & 8.07 & 10.11 & -100.37 & -482.22 & 2.99 & 7.97 & 2.84 & 1.54 \\
\hline 7520.59 & 235270.80 & 18.00 & 39.51 & 14.74 & 91.53 & 9.01 & 9.23 & -107.34 & 676.42 & 3.27 & 9.06 & 2.82 & 1.48 \\
\hline 7520.66 & 250389.40 & 17.80 & 40.37 & 17.87 & 92.23 & 11.79 & 10.17 & -127.21 & 2024.03 & 3.42 & 9.05 & 2.89 & 1.50 \\
\hline 7520.72 & 323803.60 & 17.00 & 48.21 & 23.19 & -0.12 & -1.48 & 11.23 & -188.40 & 2436.87 & 6.68 & 3.67 & 3.50 & 1.53 \\
\hline 7520.79 & 326283.20 & 16.80 & 48.80 & 21.08 & 1.29 & 0.54 & 9.89 & -171.27 & 1941.74 & 6.76 & 3.82 & 3.54 & 1.64 \\
\hline 7520.85 & 327098.00 & 16.80 & 48.48 & 25.28 & 0.25 & -2.51 & 10.61 & -157.41 & 33.57 & 6.80 & 5.34 & 3.46 & 1.81 \\
\hline 7520.92 & 338671.40 & 16.80 & 49.34 & 31.76 & 2.53 & -3.56 & 11.51 & -159.23 & 77.83 & 7.23 & 5.75 & 3.42 & 2.12 \\
\hline
\end{tabular}




\begin{tabular}{|c|c|c|c|c|c|c|c|c|c|c|c|c|c|}
\hline Depth & $\mathrm{XRF}$ & XRF Live Time & $\mathrm{Nb}(\mathrm{ppm})$ & Mo (ppm) & $\mathrm{Ba}(\mathrm{ppm})$ & $\mathrm{Pb}(\mathrm{ppm})$ & Th (ppm) & $\mathrm{U}(\mathrm{ppm})$ & $\mathrm{P}(\mathrm{ppm})$ & $\mathrm{S}(\%)$ & $\mathrm{Cl}(\%)$ & $\mathrm{K}(\%)$ & $\mathrm{Ca}(\%)$ \\
\hline 7520.98 & 351791.40 & 16.80 & 51.65 & 31.44 & 3.00 & -6.15 & 11.38 & -138.32 & 660.76 & 7.57 & 5.75 & 3.46 & 2.42 \\
\hline 7521.05 & 336401.60 & 17.00 & 52.57 & 31.43 & 2.90 & -5.04 & 12.36 & -97.25 & 188.87 & 4.73 & 3.64 & 3.57 & 2.52 \\
\hline 7521.12 & 346028.20 & 17.00 & 63.32 & 33.37 & 2.09 & -5.17 & 14.73 & -108.94 & 1362.68 & 5.06 & 3.60 & 3.51 & 2.51 \\
\hline 7521.18 & 354653.40 & 17.00 & 70.94 & 27.26 & 2.66 & -6.45 & 14.49 & -156.95 & 2806.25 & 6.02 & 2.45 & 3.55 & 2.32 \\
\hline 7521.25 & 343187.40 & 17.00 & 82.84 & 20.02 & 0.41 & -5.37 & 15.12 & -153.47 & 1868.96 & 5.59 & 0.78 & 3.65 & 2.30 \\
\hline 7521.31 & 345733.00 & 16.80 & 89.79 & 25.86 & 0.22 & -4.00 & 16.17 & -148.42 & 877.26 & 5.35 & 0.76 & 3.59 & 2.10 \\
\hline 7521.38 & 355521.60 & 16.80 & 107.57 & 30.58 & -1.13 & -1.46 & 17.91 & -145.16 & -235.73 & 5.55 & 0.75 & 3.60 & 2.38 \\
\hline 7521.44 & 357816.40 & 16.80 & 106.39 & 36.96 & -1.08 & -2.77 & 17.60 & -145.73 & -542.19 & 5.18 & 0.65 & 3.50 & 2.48 \\
\hline 7521.51 & 353882.80 & 16.80 & 104.45 & 44.57 & -0.35 & 1.36 & 16.65 & -125.62 & -1653.22 & 4.10 & 0.39 & 3.51 & 2.70 \\
\hline 7521.57 & 358299.60 & 16.80 & 104.67 & 47.02 & 0.30 & 0.07 & 16.04 & -132.06 & -1979.36 & 3.88 & 0.37 & 3.52 & 2.74 \\
\hline 7521.64 & 353597.80 & 17.00 & 110.82 & 42.30 & 0.97 & 0.57 & 16.14 & -149.05 & -1957.56 & 3.82 & 0.37 & 3.48 & 3.32 \\
\hline 7521.71 & 360747.20 & 16.80 & 125.54 & 44.76 & 2.02 & -3.64 & 14.52 & -153.50 & -681.13 & 3.96 & 1.17 & 3.41 & 3.83 \\
\hline 7521.77 & 374084.20 & 16.60 & 120.11 & 55.45 & 2.14 & -5.28 & 15.44 & -151.14 & 528.02 & 4.01 & 1.90 & 3.15 & 10.77 \\
\hline 7521.84 & 375461.00 & 16.60 & 112.51 & 57.22 & 2.56 & -9.08 & 17.74 & -121.24 & 2543.75 & 4.01 & 6.85 & 2.73 & 14.26 \\
\hline 7521.90 & 379564.80 & 16.60 & 100.41 & 56.50 & 1.83 & -7.66 & 18.35 & -106.49 & 5200.78 & 3.94 & 7.03 & 2.63 & 17.03 \\
\hline 7521.97 & 369664.20 & 16.60 & 84.37 & 58.08 & 1.30 & -6.51 & 17.74 & -73.32 & 5818.12 & 3.49 & 7.03 & 2.62 & 17.42 \\
\hline 7522.03 & 360415.20 & 16.80 & 50.96 & 55.33 & 2.14 & -5.87 & 16.73 & -84.16 & 6334.58 & 3.05 & 6.36 & 2.66 & 17.66 \\
\hline 7522.10 & 355800.20 & 16.80 & 50.70 & 47.17 & 1.14 & -3.56 & 15.86 & -79.55 & 6015.46 & 3.41 & 5.57 & 3.03 & 11.92 \\
\hline 7522.17 & 361885.00 & 16.80 & 60.02 & 48.37 & 3.02 & -2.12 & 15.20 & -102.65 & 5126.45 & 3.47 & 0.53 & 3.52 & 8.72 \\
\hline 7522.23 & 355114.40 & 16.80 & 58.85 & 48.18 & 3.17 & -2.62 & 13.39 & -98.41 & 3745.19 & 3.27 & 0.42 & 3.63 & 6.18 \\
\hline 7522.30 & 359107.00 & 16.80 & 60.30 & 45.43 & 2.09 & -1.17 & 12.11 & -108.09 & 3971.41 & 3.53 & 0.69 & 3.69 & 5.52 \\
\hline 7522.36 & 353833.00 & 16.80 & 57.44 & 44.56 & 2.26 & 0.20 & 10.91 & -94.14 & 3071.79 & 3.48 & 0.65 & 3.74 & 4.92 \\
\hline 7522.43 & 341172.20 & 17.00 & 48.90 & 42.51 & 4.09 & 1.80 & 10.24 & -84.19 & 2639.28 & 3.10 & 1.33 & 3.67 & 4.09 \\
\hline 7522.49 & 329229.20 & 17.00 & 34.13 & 45.99 & 2.41 & 0.87 & 8.32 & -63.01 & 2423.11 & 2.82 & 1.37 & 3.61 & 4.26 \\
\hline 7522.56 & 320158.00 & 17.00 & 26.61 & 43.51 & 2.80 & 1.66 & 6.79 & -56.01 & 805.47 & 2.55 & 2.10 & 3.50 & 4.39 \\
\hline 7522.62 & 313650.40 & 17.00 & 19.86 & 38.04 & 2.75 & -2.71 & 6.65 & -49.73 & -690.66 & 2.22 & 1.88 & 3.43 & 4.98 \\
\hline 7522.69 & 319166.20 & 17.00 & 17.12 & 36.33 & 1.00 & -2.77 & 7.60 & -70.47 & -111.14 & 2.61 & 2.64 & 3.29 & 6.07 \\
\hline 7522.76 & 311278.80 & 17.00 & 17.48 & 39.04 & 0.67 & -4.58 & 5.94 & -87.21 & 33.67 & 2.50 & 2.47 & 3.31 & 6.36 \\
\hline 7522.82 & 296862.60 & 17.20 & 16.19 & 28.05 & 0.20 & -2.73 & 5.17 & -85.50 & -593.40 & 2.17 & 2.53 & 3.29 & 6.27 \\
\hline 7522.89 & 288451.40 & 17.40 & 13.51 & 24.04 & 0.54 & -4.32 & 7.03 & -100.34 & 762.11 & 2.09 & 1.76 & 3.43 & 6.05 \\
\hline 7522.95 & 277644.40 & 17.60 & 11.53 & 25.16 & 1.98 & -3.08 & 7.66 & -96.89 & 824.34 & 1.97 & 1.80 & 3.44 & 5.95 \\
\hline 7523.02 & 264141.80 & 17.60 & 8.01 & 27.14 & 2.08 & -1.56 & 6.41 & -67.75 & 308.71 & 1.30 & 1.98 & 3.35 & 6.64 \\
\hline 7523.08 & 281165.80 & 17.60 & 5.16 & 23.23 & 2.10 & -3.81 & 6.21 & -51.73 & -1152.14 & 1.08 & 1.67 & 2.96 & 14.67 \\
\hline
\end{tabular}




\begin{tabular}{|c|c|c|c|c|c|c|c|c|c|c|c|c|c|}
\hline Depth & $\mathrm{XRF}$ & XRF Live Time & $\mathrm{Nb}(\mathrm{ppm})$ & Mo (ppm) & $\mathrm{Ba}(\mathrm{ppm})$ & $\mathrm{Pb}(\mathrm{ppm})$ & Th (ppm) & $\mathrm{U}(\mathrm{ppm})$ & $\mathrm{P}(\mathrm{ppm})$ & $\mathrm{S}(\%)$ & $\mathrm{Cl}(\%)$ & $\mathrm{K}(\%)$ & $\mathrm{Ca}(\%)$ \\
\hline 7523.15 & 306622.80 & 17.40 & 4.62 & 30.45 & 4.02 & -7.50 & 7.08 & -48.42 & -1815.51 & 1.23 & 1.68 & 2.46 & 24. \\
\hline 7523.22 & 328455.00 & 17.20 & 4.54 & 48.88 & 4.81 & -7.64 & 4.20 & -44.15 & -553.37 & 1.55 & 2.11 & 1.77 & 36.81 \\
\hline 7523.28 & 351750.60 & 17.00 & 4.69 & 72.51 & 5.41 & -8.61 & 2.78 & -47.14 & 919.83 & 2.11 & 2.10 & 1.19 & 48.98 \\
\hline 7523.35 & 299810.20 & 17.60 & 4.01 & 121.09 & 6.73 & -12.50 & 6.00 & -42.03 & 411.98 & 1.95 & 5.69 & 0.69 & 46.54 \\
\hline 7523.41 & 294957.80 & 17.60 & 3.73 & 127.24 & 5.55 & -13.21 & 6.01 & -38.90 & -314.04 & 1.96 & 5.64 & 0.59 & 51.21 \\
\hline 7523.48 & 289829.00 & 17.60 & 3.06 & 128.96 & 3.62 & -14.30 & 4.77 & -29.62 & -850.00 & 1.82 & 5.69 & 0.54 & 53.16 \\
\hline 7523.54 & 288008.20 & 17.60 & 3.85 & 116.39 & 2.53 & -16.42 & 6.00 & -20.44 & -4701.30 & 1.54 & 5.36 & 0.64 & 53.10 \\
\hline 7523.61 & 272150.40 & 17.80 & 3.05 & 98.72 & 2.13 & -13.03 & 6.82 & -13.82 & -5095.88 & 1.11 & 5.27 & 0.91 & 46.18 \\
\hline 7523.67 & 320758.00 & 17.40 & 4.21 & 47.99 & 1.70 & -13.20 & 2.52 & -26.57 & -2453.46 & 1.40 & 0.65 & 1.34 & 50.88 \\
\hline 7523.74 & 312916.40 & 17.40 & 4.80 & 46.13 & 2.73 & -7.59 & 2.30 & -29.09 & 470.59 & 1.57 & 0.49 & 1.78 & 39.67 \\
\hline 7523.81 & 303121.40 & 17.40 & 4.31 & 39.69 & 6.29 & -8.00 & 1.81 & -31.60 & 2586.25 & 1.74 & 5.42 & 1.84 & 30.14 \\
\hline 7523.87 & 285518.60 & 17.60 & 3.12 & 32.73 & 6.38 & -7.09 & 2.04 & -26.96 & 4869.60 & 1.70 & 7.66 & 2.18 & 18.57 \\
\hline 7523.94 & 281837.40 & 17.60 & 4.21 & 30.95 & 6.44 & -11.21 & 1.52 & -35.79 & 5286.85 & 1.76 & 10.13 & 2.37 & 13.35 \\
\hline 7524.00 & 270327.00 & 17.60 & 4.26 & 33.42 & 5.95 & -5.71 & 3.03 & -21.04 & 2901.82 & 1.64 & 13.38 & 2.39 & 9.44 \\
\hline 7524.07 & 262943.80 & 17.80 & 2.37 & 30.43 & 6.27 & -10.36 & 4.68 & -22.54 & 181.57 & 1.58 & 13.40 & 2.48 & 8.72 \\
\hline 7524.13 & 253311.40 & 18.00 & 2.65 & 32.94 & 1.63 & -7.01 & 4.91 & -31.51 & -48.42 & 1.42 & 9.49 & 2.77 & 7.71 \\
\hline 7524.20 & 270268.60 & 17.80 & 2.20 & 42.41 & 2.31 & -6.12 & 5.65 & -36.84 & 5053.88 & 1.45 & 7.13 & 2.70 & 12.51 \\
\hline 7524.27 & 278652.80 & 17.60 & 1.82 & 47.48 & 2.41 & -4.45 & 5.75 & -24.18 & 7312.87 & 1.49 & 6.58 & 2.64 & 15.33 \\
\hline 7524.33 & 296503.00 & 17.40 & 0.95 & 46.28 & 3.12 & -5.58 & 5.45 & -39.06 & 8563.13 & 1.65 & 3.67 & 2.87 & 15.87 \\
\hline 7524.40 & 247085.20 & 17.80 & 2.45 & 41.74 & 77.97 & 7.49 & 10.67 & -31.20 & 12338.51 & 1.35 & 8.84 & 2.16 & 14.15 \\
\hline 7524.46 & 246990.20 & 17.80 & 2.22 & 36.84 & 78.63 & 8.36 & 11.93 & -25.63 & 10633.88 & 1.46 & 7.89 & 2.33 & 12.73 \\
\hline 7524.53 & 223119.80 & 18.00 & 4.85 & 25.59 & 78.66 & 12.05 & 10.39 & -24.85 & 5769.04 & 1.44 & 7.89 & 2.55 & 8.13 \\
\hline 7524.59 & 214728.80 & 18.20 & 4.06 & 19.43 & 78.09 & 12.10 & 10.60 & -40.58 & 2376.33 & 1.25 & 5.94 & 2.78 & 5.21 \\
\hline 7524.66 & 205009.80 & 18.40 & 4.43 & 17.99 & 78.84 & 10.59 & 9.64 & -37.14 & 469.21 & 1.07 & 6.00 & 2.71 & 5.02 \\
\hline 7524.72 & 258076.20 & 17.80 & 4.33 & 20.28 & 2.79 & -0.24 & 3.44 & -44.89 & -2424.30 & 1.36 & 1.06 & 3.28 & 6.87 \\
\hline 7524.79 & 274047.00 & 17.60 & 5.12 & 30.11 & 1.81 & -3.17 & 1.78 & -51.17 & -705.41 & 1.38 & 0.79 & 3.00 & 11.23 \\
\hline 7524.86 & 295467.60 & 17.40 & 3.89 & 39.02 & 3.32 & -6.33 & 1.62 & -68.96 & 1386.97 & 1.82 & 0.80 & 2.86 & 13.36 \\
\hline 7524.92 & 309757.80 & 17.20 & 7.04 & 48.09 & 1.11 & -8.46 & 1.77 & -93.59 & 1703.95 & 2.51 & 1.00 & 2.65 & 15.82 \\
\hline 7524.99 & 334450.60 & 17.00 & 9.25 & 63.20 & 1.47 & -11.03 & 3.34 & -91.54 & 1517.62 & 2.88 & 0.82 & 2.46 & 20.92 \\
\hline 7525.05 & 347738.80 & 17.00 & 21.88 & 68.16 & 3.30 & -11.91 & 4.05 & -108.94 & 2326.74 & 3.47 & 0.56 & 2.64 & 19.78 \\
\hline 7525.12 & 349834.60 & 17.00 & 27.43 & 72.34 & 4.45 & -10.62 & 7.85 & -115.82 & 3363.20 & 3.84 & 0.56 & 3.01 & 16.65 \\
\hline 7525.18 & 354836.40 & 17.00 & 28.25 & 96.57 & 2.40 & -16.53 & 10.32 & -115.30 & 6587.20 & 4.06 & 0.61 & 2.87 & 18.59 \\
\hline 7525.25 & 359978.40 & 17.00 & 61.49 & 93.72 & 3.68 & -14.91 & 12.53 & -111.94 & 6658.99 & 4.91 & 0.46 & 3.07 & 16.15 \\
\hline
\end{tabular}




\begin{tabular}{|c|c|c|c|c|c|c|c|c|c|c|c|c|c|}
\hline Depth & $\mathrm{XRF}$ & XRF Live Time & $\mathrm{Nb}(\mathrm{ppm})$ & Mo (ppm) & $\mathrm{Ba}(\mathrm{ppm})$ & $\mathrm{Pb}(\mathrm{ppm})$ & Th (ppm) & $\mathrm{U}(\mathrm{ppm})$ & $\mathrm{P}(\mathrm{ppm})$ & $\mathrm{S}(\%)$ & $\mathrm{Cl}(\%)$ & $\mathrm{K}(\%)$ & $\mathrm{Ca}(\%)$ \\
\hline 7525.31 & 362261.20 & 16.80 & 98.12 & 79.26 & 2.21 & -12.52 & 13.65 & -157.56 & 11227.90 & 5.80 & 0.61 & 3.34 & 10.57 \\
\hline 7525.38 & 361004.00 & 16.80 & 123.25 & 75.65 & 1.35 & -9.18 & 15.72 & -161.94 & 11601.59 & 6.77 & 1.19 & 3.18 & 10.31 \\
\hline 7525.45 & 354672.40 & 16.80 & 157.23 & 69.37 & -1.41 & -5.21 & 16.78 & -182.36 & 10453.70 & 7.39 & 1.67 & 3.12 & 8.37 \\
\hline 7525.51 & 353059.80 & 16.80 & 190.60 & 40.82 & -3.34 & 2.17 & 19.23 & -176.77 & 4014.82 & 7.26 & 2.13 & 3.33 & 3.50 \\
\hline 7525.58 & 350266.60 & 16.80 & 187.16 & 36.54 & -1.37 & 3.47 & 19.58 & -173.32 & 4881.91 & 6.47 & 3.02 & 3.20 & 2.70 \\
\hline 7525.64 & 347816.40 & 17.00 & 175.67 & 37.44 & -0.51 & 2.93 & 19.02 & -146.24 & 1688.33 & 6.06 & 3.62 & 3.00 & 2.30 \\
\hline 7525.71 & 348443.20 & 17.00 & 162.13 & 36.66 & -0.94 & 1.62 & 17.85 & -117.33 & 2051.33 & 5.44 & 4.82 & 2.84 & 2.12 \\
\hline 7525.77 & 339697.80 & 17.20 & 148.24 & 32.52 & 4.09 & -0.98 & 14.92 & -92.76 & 1448.82 & 5.41 & 7.51 & 2.49 & 2.20 \\
\hline 7525.84 & 332010.00 & 17.20 & 138.88 & 36.36 & 6.50 & -3.03 & 16.75 & -78.96 & 2559.13 & 5.53 & 11.72 & 2.14 & 2.52 \\
\hline 7525.91 & 336515.60 & 17.20 & 133.82 & 35.13 & 4.59 & -2.19 & 16.49 & -56.14 & 2361.04 & 5.62 & 12.30 & 2.02 & 2.70 \\
\hline 7525.97 & 325014.40 & 17.20 & 131.07 & 29.87 & 4.36 & -1.26 & 17.73 & -54.49 & 2655.81 & 5.45 & 11.42 & 2.03 & 2.89 \\
\hline 7526.04 & 326525.60 & 17.20 & 129.89 & 32.73 & 4.80 & -2.94 & 17.93 & -67.40 & 2113.11 & 5.27 & 10.00 & 2.10 & 2.91 \\
\hline 7526.10 & 344045.60 & 17.00 & 127.06 & 39.19 & 3.62 & -5.60 & 19.51 & -57.97 & 2807.25 & 5.20 & 7.21 & 2.26 & 3.11 \\
\hline 7526.17 & 346028.80 & 17.00 & 125.00 & 39.22 & 4.24 & -3.16 & 15.01 & -79.66 & 1078.36 & 5.18 & 2.49 & 2.52 & 3.25 \\
\hline 7526.23 & 347401.40 & 17.00 & 128.56 & 43.36 & 7.92 & -2.41 & 16.01 & -113.39 & 359.46 & 5.06 & 1.06 & 2.61 & 3.37 \\
\hline 7526.30 & 353455.20 & 17.00 & 133.83 & 52.67 & 8.47 & -1.31 & 15.52 & -111.24 & -623.19 & 4.99 & 0.99 & 2.60 & 3.30 \\
\hline 7526.36 & 354217.60 & 17.00 & 140.08 & 56.85 & 8.33 & 0.90 & 13.18 & -137.81 & 40.83 & 5.20 & 0.82 & 2.57 & 3.36 \\
\hline 7526.43 & 361134.40 & 16.80 & 141.45 & 55.05 & 6.96 & 3.28 & 12.00 & -162.26 & -529.32 & 4.53 & 0.43 & 2.49 & 3.10 \\
\hline 7526.50 & 363861.20 & 16.80 & 136.86 & 58.26 & 5.00 & 3.97 & 13.69 & -165.09 & 995.17 & 4.41 & 0.52 & 2.43 & 2.99 \\
\hline 7526.56 & 357079.40 & 16.80 & 129.37 & 60.85 & 2.02 & 3.23 & 11.00 & -146.96 & 1825.20 & 4.20 & 0.42 & 2.42 & 2.74 \\
\hline 7526.63 & 358077.40 & 16.80 & 124.67 & 56.31 & 1.17 & 4.59 & 11.94 & -142.89 & 3551.35 & 4.06 & 0.41 & 2.47 & 2.61 \\
\hline 7526.69 & 354612.40 & 16.80 & 123.86 & 50.83 & 0.12 & 4.62 & 15.87 & -132.85 & 1875.75 & 3.77 & 0.81 & 2.50 & 2.67 \\
\hline 7526.76 & 346660.20 & 17.00 & 119.43 & 52.36 & 0.55 & 4.81 & 16.75 & -146.68 & 2626.21 & 4.17 & 0.95 & 2.62 & 2.92 \\
\hline 7526.82 & 345340.20 & 17.00 & 121.19 & 50.43 & 2.48 & 2.38 & 15.92 & -161.46 & 2194.00 & 4.20 & 0.89 & 2.71 & 3.38 \\
\hline 7526.89 & 339714.60 & 17.00 & 121.00 & 45.39 & 2.40 & 1.45 & 16.56 & -152.49 & 2606.70 & 4.54 & 2.60 & 2.63 & 3.69 \\
\hline 7526.96 & 329395.00 & 17.00 & 115.63 & 48.38 & 2.19 & -0.60 & 16.27 & -166.09 & 789.20 & 4.89 & 3.65 & 2.53 & 4.57 \\
\hline 7527.02 & 318258.20 & 17.00 & 102.13 & 48.02 & 2.78 & -2.20 & 15.90 & -149.40 & 698.88 & 4.53 & 3.58 & 2.45 & 5.76 \\
\hline 7527.09 & 309815.60 & 17.00 & 94.28 & 46.80 & 2.80 & -3.58 & 15.81 & -136.29 & 522.15 & 4.55 & 3.62 & 2.53 & 6.44 \\
\hline 7527.15 & 300217.60 & 17.20 & 92.31 & 42.41 & 1.29 & -4.38 & 14.47 & -120.23 & -485.97 & 4.43 & 3.66 & 2.53 & 6.73 \\
\hline 7527.22 & 289737.00 & 17.40 & 87.04 & 40.24 & 1.31 & -7.00 & 14.32 & -119.84 & -1213.93 & 4.16 & 2.15 & 2.62 & 7.36 \\
\hline 7527.28 & 288231.80 & 17.40 & 81.40 & 36.92 & 0.15 & -8.34 & 13.44 & -119.99 & -1443.96 & 3.77 & 1.18 & 2.74 & 7.33 \\
\hline 7527.35 & 284540.60 & 17.60 & 82.85 & 40.61 & 0.90 & -9.16 & 11.81 & -128.87 & -128.27 & 4.04 & 0.85 & 2.86 & 6.80 \\
\hline 7527.41 & 274738.20 & 17.80 & 86.59 & 32.60 & 0.22 & -8.13 & 11.56 & -139.03 & -898.57 & 3.77 & 0.68 & 2.89 & 6.20 \\
\hline
\end{tabular}




\begin{tabular}{|c|c|c|c|c|c|c|c|c|c|c|c|c|c|}
\hline Depth & $\mathrm{XRF}$ & XRF Live Time & $\mathrm{Nb}(\mathrm{ppm})$ & Mo (ppm) & $\mathrm{Ba}(\mathrm{ppm})$ & $\mathrm{Pb}(\mathrm{ppm})$ & Th (ppm) & $\mathrm{U}(\mathrm{ppm})$ & $\mathrm{P}(\mathrm{ppm})$ & $\mathrm{S}(\%)$ & $\mathrm{Cl}(\%)$ & $\mathrm{K}(\%)$ & $\mathrm{Ca}(\%)$ \\
\hline 7527.48 & 225187.40 & 18.20 & 69.78 & 84.22 & -2.46 & -16.92 & 11.71 & -93.06 & -885.09 & 3.18 & 4.63 & 2.33 & 4.87 \\
\hline 7527.55 & 230518.60 & 18.00 & 67.05 & 79.60 & -1.83 & -15.31 & 11.02 & -85.05 & -1127.13 & 3.11 & 4.45 & 2.40 & 4.13 \\
\hline 7527.61 & 226857.00 & 18.20 & 69.09 & 77.83 & -0.43 & -12.22 & 11.31 & -91.80 & -1149.23 & 3.18 & 4.57 & 2.42 & 3.46 \\
\hline 7527.68 & 232256.20 & 18.00 & 77.82 & 75.57 & -1.56 & -12.45 & 11.30 & -123.29 & -603.93 & 3.37 & 4.38 & 2.52 & 2.39 \\
\hline 7527.74 & 235657.00 & 18.00 & 94.18 & 80.15 & -0.67 & -8.96 & 11.65 & -96.11 & -907.72 & 4.00 & 4.37 & 2.59 & 1.92 \\
\hline 7527.81 & 305760.60 & 17.40 & 125.65 & 30.22 & 1.78 & 3.33 & 15.46 & -131.30 & 1452.92 & 5.49 & 1.47 & 3.23 & 2.14 \\
\hline 7527.87 & 325592.80 & 17.40 & 135.94 & 32.86 & 0.29 & 4.88 & 18.17 & -157.05 & 2070.20 & 6.13 & 1.48 & 3.33 & 1.90 \\
\hline 7527.94 & 333159.20 & 17.20 & 142.30 & 34.39 & -0.45 & 4.24 & 19.33 & -161.59 & 2104.43 & 6.40 & 1.96 & 3.38 & 1.60 \\
\hline 7528.01 & 334959.20 & 17.20 & 135.90 & 29.80 & -0.62 & 2.38 & 19.92 & -139.65 & 1515.39 & 6.43 & 2.36 & 3.38 & 1.98 \\
\hline 7528.07 & 346788.40 & 17.00 & 124.12 & 28.43 & -1.17 & -4.54 & 21.22 & -169.96 & 1279.04 & 6.23 & 3.66 & 3.27 & 2.33 \\
\hline 7528.14 & 328176.00 & 17.00 & 112.96 & 30.94 & -2.25 & -3.49 & 19.33 & -170.69 & -233.24 & 5.83 & 5.03 & 3.15 & 2.66 \\
\hline 7528.20 & 309262.00 & 17.00 & 105.45 & 33.17 & -1.83 & -3.48 & 19.05 & -164.14 & -1254.28 & 5.48 & 6.63 & 2.97 & 3.02 \\
\hline 7528.27 & 306562.00 & 17.00 & 101.32 & 38.84 & -1.93 & -6.47 & 18.22 & -114.00 & -746.32 & 5.88 & 9.73 & 2.75 & 3.35 \\
\hline 7528.33 & 297778.40 & 17.20 & 98.17 & 41.55 & -2.64 & -3.46 & 19.73 & -117.79 & -1584.23 & 5.53 & 10.46 & 2.64 & 3.30 \\
\hline 7528.40 & 278591.00 & 17.40 & 96.48 & 44.95 & -3.25 & -2.10 & 18.93 & -104.20 & -1464.20 & 5.31 & 10.00 & 2.63 & 3.17 \\
\hline 7528.46 & 267684.20 & 17.60 & 96.19 & 47.22 & -1.89 & -2.47 & 19.85 & -115.69 & -2090.22 & 5.06 & 8.28 & 2.73 & 2.87 \\
\hline 7528.53 & 253857.40 & 17.80 & 102.92 & 45.50 & -2.52 & -2.02 & 20.00 & -144.46 & -2235.68 & 5.07 & 6.84 & 2.82 & 2.69 \\
\hline 7528.60 & 235162.80 & 18.00 & 104.93 & 37.86 & -2.06 & -0.06 & 21.66 & -214.59 & -1993.65 & 4.76 & 4.13 & 2.89 & 2.51 \\
\hline 7528.66 & 245286.40 & 17.80 & 90.98 & 52.72 & 242.02 & 4.63 & 19.43 & -174.98 & -2509.26 & 4.91 & 3.21 & 2.98 & 2.35 \\
\hline 7528.73 & 258965.20 & 17.60 & 99.18 & 57.77 & 241.48 & 5.80 & 19.78 & -191.88 & -1407.64 & 5.12 & 3.21 & 2.95 & 2.38 \\
\hline 7528.79 & 272376.40 & 17.40 & 100.18 & 49.97 & 241.53 & 2.61 & 20.60 & -213.01 & 397.71 & 5.03 & 2.46 & 3.04 & 2.37 \\
\hline 7528.86 & 294502.80 & 17.20 & 94.08 & 70.10 & 245.67 & -4.03 & 18.90 & -226.77 & 1654.33 & 6.33 & 3.35 & 2.73 & 1.94 \\
\hline 7528.92 & 344748.40 & 16.80 & 78.55 & 191.78 & 238.71 & 25.60 & 3.98 & -222.59 & 2496.96 & 8.28 & 2.25 & 2.31 & 1.46 \\
\hline 7528.99 & 361492.00 & 16.60 & 97.78 & 189.43 & -4.63 & 22.70 & 6.21 & -233.57 & 3245.65 & 8.26 & 2.54 & 2.30 & 1.55 \\
\hline 7529.06 & 369581.20 & 16.60 & 72.01 & 188.46 & 6004.51 & 25.49 & 5.59 & -190.97 & 4026.66 & 8.68 & 4.63 & 1.92 & 1.62 \\
\hline 7529.12 & 370559.40 & 16.60 & 62.24 & 189.75 & 6003.91 & 29.17 & 5.37 & -147.35 & 1685.85 & 8.44 & 5.59 & 1.72 & 2.94 \\
\hline 7529.19 & 363414.00 & 16.60 & 58.69 & 175.78 & 6001.90 & 34.21 & 4.07 & -78.32 & 542.57 & 7.02 & 8.09 & 1.72 & 3.17 \\
\hline 7529.25 & 333015.20 & 16.80 & 71.04 & 54.53 & 6008.38 & 4.61 & 16.09 & -41.87 & -752.11 & 4.64 & 8.24 & 2.23 & 3.64 \\
\hline 7529.32 & 311740.60 & 17.00 & 67.42 & 47.27 & 6009.48 & 1.78 & 14.87 & -60.16 & -1139.95 & 4.40 & 8.00 & 2.21 & 3.53 \\
\hline 7529.38 & 298802.60 & 17.00 & 85.77 & 37.92 & 0.56 & -3.49 & 15.14 & -66.60 & -1905.35 & 3.63 & 7.20 & 2.50 & 3.33 \\
\hline 7529.45 & 288980.00 & 17.20 & 90.73 & 42.26 & 2.28 & -7.26 & 11.83 & -86.46 & 347.32 & 3.79 & 6.80 & 2.61 & 1.88 \\
\hline 7529.51 & 266698.40 & 17.40 & 92.94 & 48.82 & -1.38 & -6.87 & 11.68 & -102.82 & 655.73 & 3.84 & 3.35 & 2.84 & 1.80 \\
\hline 7529.58 & 298364.00 & 17.20 & 91.97 & 80.86 & -4.19 & -11.20 & 10.39 & -367.93 & 4460.39 & 11.45 & 5.58 & 2.62 & 1.46 \\
\hline
\end{tabular}




\begin{tabular}{|c|c|c|c|c|c|c|c|c|c|c|c|c|c|}
\hline Depth & $\mathrm{XRF}$ & XRF Live Time & $\mathrm{Nb}(\mathrm{ppm})$ & Mo (ppm) & $\mathrm{Ba}(\mathrm{ppm})$ & $\mathrm{Pb}(\mathrm{ppm})$ & Th (ppm) & $\mathrm{U}(\mathrm{ppm})$ & $\mathrm{P}(\mathrm{ppm})$ & $\mathrm{S}(\%)$ & $\mathrm{Cl}(\%)$ & $\mathrm{K}(\%)$ & $\mathrm{Ca}(\%)$ \\
\hline 7529.65 & 289353.60 & 17.40 & 93.32 & 69.78 & -5.05 & -7.68 & 10.31 & -368.17 & 5422.99 & 11.43 & 7.41 & 2.47 & 1.39 \\
\hline 7529.71 & 280817.80 & 17.60 & 88.11 & 81.80 & -4.02 & -8.82 & 7.38 & -380.86 & 5309.65 & 11.73 & 5.48 & 2.44 & 1.36 \\
\hline 7529.78 & 270646.00 & 17.60 & 89.25 & 88.71 & -4.92 & -8.75 & 7.65 & -366.30 & 3992.88 & 11.56 & 6.29 & 2.31 & 1.71 \\
\hline 7529.84 & 273084.40 & 17.60 & 93.82 & 89.57 & -3.03 & -11.55 & 8.87 & -349.28 & 4552.99 & 11.34 & 7.10 & 2.24 & 2.24 \\
\hline 7529.91 & 214459.40 & 18.20 & 97.75 & 70.06 & 0.10 & -9.90 & 10.52 & -98.84 & 1074.73 & 3.77 & 4.85 & 2.38 & 3.03 \\
\hline 7529.97 & 201716.20 & 18.20 & 93.97 & 91.89 & 0.55 & -19.26 & 8.98 & -103.68 & 1240.73 & 3.82 & 3.37 & 2.35 & 4.21 \\
\hline 7530.04 & 194351.80 & 18.20 & 97.37 & 87.45 & -0.07 & -13.52 & 11.77 & -106.18 & 702.61 & 3.26 & 4.49 & 2.37 & 4.28 \\
\hline 7530.10 & 194248.40 & 18.20 & 97.05 & 75.83 & -1.45 & -12.72 & 13.47 & -138.73 & 1218.51 & 3.31 & 4.23 & 2.42 & 4.03 \\
\hline 7530.17 & 206105.40 & 18.20 & 84.58 & 151.40 & -3.44 & -2.34 & 10.57 & -324.22 & 2896.03 & 5.86 & 4.27 & 2.04 & 3.35 \\
\hline 7530.24 & 211296.20 & 18.00 & 82.67 & 133.45 & -4.14 & -0.57 & 11.23 & -304.52 & 2698.94 & 5.76 & 5.75 & 1.97 & 2.98 \\
\hline 7530.30 & 209001.60 & 18.00 & 84.95 & 123.25 & -4.81 & 3.08 & 11.45 & -289.80 & 1347.52 & 5.54 & 7.26 & 1.88 & 2.35 \\
\hline 7530.37 & 214012.00 & 18.00 & 81.39 & 117.88 & -4.51 & -1.28 & 10.26 & -269.00 & 1153.18 & 5.62 & 7.72 & 1.84 & 2.69 \\
\hline 7530.43 & 191441.80 & 18.20 & 63.10 & 126.24 & 2950.81 & 5.04 & 8.73 & -222.31 & -150.54 & 5.74 & 7.07 & 1.78 & 3.50 \\
\hline 7530.50 & 187195.20 & 18.20 & 70.66 & 35.72 & 2951.41 & -2.77 & 11.49 & -57.36 & -2266.70 & 3.12 & 7.78 & 2.11 & 4.11 \\
\hline 7530.56 & 173658.00 & 18.40 & 66.47 & 52.23 & 2952.52 & -6.74 & 10.04 & -69.29 & -1971.04 & 3.16 & 6.73 & 2.21 & 4.39 \\
\hline 7530.63 & 144370.20 & 18.80 & 49.53 & 89.32 & 2956.45 & 0.79 & 9.65 & -35.84 & -2608.62 & 2.68 & 9.17 & 1.87 & 3.74 \\
\hline 7530.70 & 149544.80 & 18.80 & 46.91 & 104.49 & 2955.79 & 5.94 & 8.25 & -77.88 & -728.73 & 3.53 & 8.48 & 1.78 & 3.76 \\
\hline 7530.76 & 163928.20 & 18.80 & 57.90 & 108.03 & 1.80 & 4.65 & 9.35 & -109.47 & -878.34 & 3.52 & 8.75 & 1.72 & 5.45 \\
\hline 7530.83 & 177278.80 & 18.60 & 47.69 & 176.53 & 3.04 & 1.14 & 2.46 & -212.11 & -177.42 & 5.98 & 7.25 & 1.45 & 7.12 \\
\hline 7530.89 & 182110.80 & 18.60 & 41.46 & 181.27 & 3.03 & 5.26 & 1.73 & -198.02 & 882.70 & 5.78 & 7.02 & 1.33 & 10.76 \\
\hline 7530.96 & 222044.40 & 18.20 & 51.10 & 158.83 & -1.30 & -1.91 & 0.86 & -234.95 & 650.19 & 6.75 & 3.51 & 1.63 & 14.19 \\
\hline 7531.02 & 220969.20 & 18.20 & 48.51 & 141.40 & -1.21 & -4.47 & 2.71 & -195.12 & -1568.83 & 5.70 & 2.92 & 1.63 & 19.10 \\
\hline 7531.09 & 234642.00 & 18.00 & 47.38 & 133.75 & -1.24 & -10.38 & 1.59 & -183.83 & 578.01 & 5.40 & 2.26 & 1.64 & 20.61 \\
\hline 7531.15 & 214354.20 & 18.20 & 57.77 & 65.30 & -0.36 & -9.35 & 9.29 & -59.01 & -127.24 & 3.00 & 3.46 & 1.87 & 19.82 \\
\hline 7531.22 & 248873.00 & 17.80 & 66.40 & 51.71 & -0.98 & -11.53 & 9.95 & -68.47 & -1957.56 & 3.19 & 3.91 & 1.99 & 16.86 \\
\hline 7531.29 & 272362.00 & 17.60 & 74.43 & 36.87 & 0.34 & -7.86 & 13.20 & -63.81 & -1705.85 & 2.87 & 3.20 & 2.29 & 14.46 \\
\hline 7531.35 & 293439.20 & 17.40 & 84.72 & 43.64 & 0.37 & -10.77 & 12.52 & -89.64 & -893.79 & 3.10 & 4.14 & 2.44 & 9.75 \\
\hline 7531.42 & 315644.00 & 17.20 & 93.97 & 43.52 & 0.89 & -10.02 & 11.41 & -97.79 & -1095.32 & 3.32 & 5.16 & 2.56 & 6.21 \\
\hline 7531.48 & 373917.40 & 16.60 & 94.55 & 74.55 & 0.50 & -6.23 & 8.82 & -274.82 & 3858.93 & 8.45 & 4.33 & 2.50 & 5.23 \\
\hline 7531.55 & 381678.80 & 16.60 & 95.71 & 74.30 & 1.17 & -2.59 & 9.67 & -270.47 & 4552.65 & 8.42 & 3.68 & 2.57 & 4.70 \\
\hline 7531.61 & 380112.80 & 16.60 & 98.46 & 70.87 & 0.23 & -2.21 & 7.58 & -265.99 & 6400.30 & 8.35 & 3.52 & 2.58 & 4.61 \\
\hline 7531.68 & 376661.40 & 16.60 & 97.26 & 66.56 & -0.09 & 2.54 & 7.57 & -261.91 & 4861.28 & 8.40 & 2.28 & 2.69 & 4.88 \\
\hline 7531.75 & 373759.20 & 16.60 & 98.79 & 61.42 & 0.18 & 0.26 & 8.59 & -280.87 & 4108.09 & 8.39 & 1.88 & 2.72 & 5.33 \\
\hline
\end{tabular}




\begin{tabular}{|c|c|c|c|c|c|c|c|c|c|c|c|c|c|}
\hline Depth & $\mathrm{XRF}$ & XRF Live Time & $\mathrm{Nb}(\mathrm{ppm})$ & Mo (ppm) & $\mathrm{Ba}(\mathrm{ppm})$ & $\mathrm{Pb}(\mathrm{ppm})$ & Th (ppm) & $\mathrm{U}(\mathrm{ppm})$ & $\mathrm{P}(\mathrm{ppm})$ & $\mathrm{S}(\%)$ & $\mathrm{Cl}(\%)$ & $\mathrm{K}(\%)$ & $\mathrm{Ca}(\%)$ \\
\hline 7531.81 & 337033.40 & 17.00 & 98.56 & 33.92 & 1.09 & -0.81 & 12.24 & -133.36 & -1512.10 & 3.16 & 2.93 & 2.83 & 5.8 \\
\hline 7531.88 & 336462.40 & 17.00 & 102.95 & 40.00 & 0.77 & 2.45 & 12.71 & -127.45 & 93.41 & 3.51 & 4.11 & 2.76 & 6.13 \\
\hline 7531.94 & 343906.00 & 17.00 & 104.05 & 42.44 & 3.09 & 0.94 & 15.01 & -146.16 & -737.49 & 3.61 & 3.97 & 2.79 & 6.53 \\
\hline 7532.01 & 342897.80 & 17.00 & 107.15 & 38.63 & 4.92 & -0.24 & 16.76 & -137.90 & 1493.97 & 3.68 & 8.18 & 2.51 & 6.23 \\
\hline 7532.07 & 346952.60 & 17.00 & 107.93 & 45.96 & 5.42 & 1.92 & 17.34 & -124.67 & 3819.70 & 3.77 & 7.48 & 2.58 & 6.71 \\
\hline 7532.14 & 346850.60 & 17.00 & 114.17 & 47.78 & 5.92 & 2.63 & 16.01 & -100.91 & 5834.71 & 4.08 & 7.84 & 2.59 & 7.05 \\
\hline 7532.20 & 333252.00 & 17.00 & 115.77 & 49.92 & 7.00 & -1.15 & 15.93 & -85.56 & 4266.05 & 3.92 & 11.96 & 2.31 & 6.73 \\
\hline 7532.27 & 319622.20 & 17.00 & 114.76 & 51.69 & 6.15 & -0.19 & 13.89 & -79.43 & 5539.13 & 3.92 & 12.05 & 2.27 & 6.53 \\
\hline 7532.34 & 329567.60 & 17.00 & 114.39 & 62.17 & 3.70 & -0.25 & 14.29 & -84.31 & 3663.33 & 3.93 & 7.63 & 2.58 & 7.50 \\
\hline 7532.40 & 333378.40 & 17.00 & 109.06 & 67.76 & 3.27 & 0.80 & 14.90 & -75.00 & 2789.61 & 4.04 & 7.60 & 2.58 & 7.48 \\
\hline 7532.47 & 346323.00 & 17.00 & 106.23 & 66.59 & 2.28 & -2.82 & 14.25 & -88.22 & 1880.43 & 3.98 & 5.70 & 2.74 & 7.52 \\
\hline 7532.53 & 359274.40 & 17.00 & 104.38 & 59.97 & 2.33 & -4.68 & 14.74 & -102.19 & 3021.77 & 4.08 & 1.59 & 3.01 & 7.46 \\
\hline 7532.60 & 367305.40 & 17.00 & 109.07 & 58.67 & 2.34 & -8.44 & 15.34 & -101.80 & 4008.33 & 4.21 & 1.53 & 3.06 & 7.35 \\
\hline 7532.66 & 364458.40 & 17.00 & 113.31 & 59.47 & 5.42 & -10.32 & 13.38 & -117.54 & 5402.40 & 4.38 & 1.58 & 3.08 & 7.57 \\
\hline 7532.73 & 359112.00 & 17.00 & 114.59 & 51.34 & 3.90 & -10.06 & 14.69 & -102.49 & 5201.45 & 4.26 & 1.59 & 3.06 & 8.99 \\
\hline 7532.80 & 357448.00 & 17.00 & 107.54 & 55.90 & 4.36 & -8.80 & 14.67 & -101.56 & 5027.30 & 3.95 & 1.54 & 2.84 & 12.99 \\
\hline 7532.86 & 361993.60 & 16.80 & 101.48 & 58.26 & 5.99 & -8.40 & 15.60 & -113.55 & 5478.01 & 3.81 & 0.32 & 2.78 & 17.39 \\
\hline 7532.93 & 362654.20 & 16.80 & 94.14 & 56.06 & 6.46 & -7.63 & 17.77 & -114.05 & 4703.58 & 3.65 & 0.67 & 2.63 & 20.07 \\
\hline 7532.99 & 357139.00 & 16.80 & 88.75 & 57.89 & 6.68 & -8.88 & 20.77 & -88.39 & 2154.36 & 3.36 & 1.21 & 2.46 & 21.69 \\
\hline 7533.06 & 356607.20 & 16.80 & 94.63 & 62.70 & 7.42 & -12.03 & 21.19 & -104.17 & 1025.97 & 3.34 & 1.21 & 2.47 & 21.27 \\
\hline 7533.12 & 339986.00 & 17.00 & 106.65 & 63.23 & 6.99 & -9.63 & 24.91 & -95.73 & -126.82 & 3.56 & 3.23 & 2.53 & 17.61 \\
\hline 7533.19 & 328437.40 & 17.20 & 112.86 & 59.71 & 6.07 & -8.38 & 23.45 & -92.44 & -1801.18 & 3.47 & 3.19 & 2.67 & 14.15 \\
\hline 7533.25 & 321068.80 & 17.20 & 117.12 & 52.95 & 5.29 & -8.93 & 23.03 & -90.08 & -3835.67 & 3.45 & 2.95 & 2.80 & 12.16 \\
\hline 7533.32 & 312388.60 & 17.20 & 116.99 & 41.94 & 2.90 & -5.53 & 22.41 & -103.35 & 340.95 & 3.55 & 2.42 & 2.95 & 10.05 \\
\hline 7533.39 & 319803.80 & 17.20 & 115.31 & 34.06 & 3.69 & -5.25 & 21.52 & -101.32 & -1298.61 & 3.62 & 2.44 & 2.99 & 8.92 \\
\hline 7533.45 & 324792.00 & 17.00 & 110.24 & 28.41 & 4.26 & -7.05 & 19.93 & -106.47 & -196.23 & 3.61 & 0.86 & 3.13 & 8.70 \\
\hline 7533.52 & 325318.00 & 17.00 & 109.06 & 27.75 & 3.86 & -8.75 & 20.42 & -96.57 & -531.36 & 3.68 & 1.84 & 3.08 & 8.39 \\
\hline 7533.58 & 317303.20 & 17.20 & 107.15 & 24.39 & 2.69 & -5.59 & 19.42 & -78.50 & -440.47 & 3.81 & 6.52 & 2.78 & 7.00 \\
\hline 7533.65 & 304262.60 & 17.40 & 112.03 & 25.05 & 2.83 & -5.90 & 17.85 & -48.96 & -3532.82 & 3.93 & 12.27 & 2.38 & 5.71 \\
\hline 7533.71 & 235103.60 & 18.00 & 93.90 & 63.12 & 9.15 & -10.39 & 15.00 & -29.23 & 1474.39 & 3.22 & 17.59 & 1.74 & 4.27 \\
\hline 7533.78 & 227587.20 & 18.20 & 75.30 & 81.73 & 258.68 & -5.09 & 13.38 & -11.93 & 962.87 & 3.26 & 17.77 & 1.72 & 3.10 \\
\hline 7533.85 & 204753.80 & 18.40 & 78.65 & 78.33 & 258.52 & -3.10 & 13.75 & -39.88 & -53.71 & 3.45 & 16.89 & 1.79 & 2.01 \\
\hline 7533.91 & 212317.40 & 18.20 & 82.62 & 82.74 & 258.64 & -2.82 & 15.18 & -53.53 & -75.50 & 3.48 & 12.02 & 2.11 & 1.96 \\
\hline
\end{tabular}




\begin{tabular}{|c|c|c|c|c|c|c|c|c|c|c|c|c|c|}
\hline Depth & $\mathrm{XRF}$ & XRF Live Time & $\mathrm{Nb}(\mathrm{ppm})$ & Mo (ppm) & $\mathrm{Ba}(\mathrm{ppm})$ & $\mathrm{Pb}(\mathrm{ppm})$ & Th (ppm) & $\mathrm{U}(\mathrm{ppm})$ & $\mathrm{P}(\mathrm{ppm})$ & $\mathrm{S}(\%)$ & $\mathrm{Cl}(\%)$ & $\mathrm{K}(\%)$ & $\mathrm{Ca}(\%)$ \\
\hline 7533.98 & 227008.40 & 18.00 & 83.27 & 82.74 & 257.64 & -1.82 & 17.71 & -74.89 & 1962.54 & 3.52 & 6.31 & 2.51 & 2.28 \\
\hline 7534.04 & 280719.60 & 17.40 & 108.95 & 44.83 & 250.88 & 6.28 & 22.09 & -104.09 & -310.58 & 4.31 & 0.99 & 3.12 & 2.44 \\
\hline 7534.11 & 306261.80 & 17.00 & 140.65 & 30.30 & 2.72 & 2.16 & 25.76 & -150.82 & 759.84 & 4.65 & 0.38 & 3.32 & 2.16 \\
\hline 7534.17 & 329013.60 & 16.80 & 148.03 & 34.88 & 0.82 & 1.98 & 25.07 & -124.60 & 2161.08 & 4.58 & 0.27 & 3.37 & 1.87 \\
\hline 7534.24 & 309165.00 & 17.00 & 155.39 & 44.88 & 2.72 & 6.26 & 24.02 & -111.31 & 3405.46 & 4.83 & 3.49 & 3.15 & 1.68 \\
\hline 7534.30 & 315707.60 & 17.00 & 163.88 & 37.26 & 5.83 & 7.17 & 22.21 & -116.07 & 2832.99 & 4.85 & 4.89 & 3.08 & 1.27 \\
\hline 7534.37 & 313892.60 & 17.00 & 161.87 & 44.36 & 5.77 & 5.34 & 20.05 & -124.61 & 1796.94 & 4.83 & 5.09 & 3.11 & 1.09 \\
\hline 7534.44 & 302063.00 & 17.20 & 152.88 & 39.44 & 2.65 & 5.40 & 17.74 & -112.89 & 398.72 & 4.60 & 6.56 & 2.86 & 1.16 \\
\hline 7534.50 & 290486.60 & 17.40 & 146.72 & 38.34 & 3.81 & 4.52 & 17.85 & -88.18 & 517.07 & 4.73 & 13.05 & 2.36 & 1.57 \\
\hline 7534.57 & 252841.80 & 17.80 & 113.62 & 79.44 & -1.33 & 0.72 & 13.00 & -76.58 & -4822.47 & 3.73 & 16.51 & 1.94 & 1.58 \\
\hline 7534.63 & 236486.00 & 18.00 & 109.40 & 91.63 & -0.63 & -1.97 & 12.45 & -52.50 & -4884.80 & 3.46 & 19.20 & 1.61 & 1.50 \\
\hline 7534.70 & 233564.60 & 18.20 & 113.31 & 86.10 & -0.04 & -2.57 & 12.99 & -41.59 & -4753.38 & 3.55 & 20.88 & 1.34 & 1.68 \\
\hline 7534.76 & 239083.20 & 18.20 & 133.24 & 89.44 & 1.62 & -2.18 & 14.63 & -27.59 & -4063.45 & 3.68 & 20.68 & 1.27 & 1.65 \\
\hline 7534.83 & 254482.40 & 18.00 & 142.20 & 90.92 & 1.62 & -4.35 & 17.49 & -54.82 & -4863.99 & 3.70 & 14.18 & 1.70 & 1.23 \\
\hline 7534.90 & 297712.20 & 17.60 & 174.61 & 42.06 & 4.51 & -3.56 & 23.13 & -89.51 & -885.89 & 4.34 & 7.50 & 2.27 & 1.08 \\
\hline 7534.96 & 301579.00 & 17.60 & 172.26 & 40.18 & 2.08 & -0.10 & 25.34 & -81.05 & -1777.98 & 4.30 & 4.09 & 2.55 & 1.01 \\
\hline 7535.03 & 309471.40 & 17.40 & 169.09 & 44.32 & 2.08 & -2.28 & 24.95 & -56.81 & -874.12 & 3.97 & 2.25 & 2.78 & 0.76 \\
\hline 7535.09 & 306697.80 & 17.40 & 152.00 & 44.58 & 0.29 & -2.54 & 23.83 & -84.77 & -478.45 & 3.58 & 1.42 & 2.88 & 0.71 \\
\hline 7535.16 & 312885.00 & 17.40 & 145.39 & 42.69 & 0.57 & 2.66 & 22.38 & -90.79 & 1774.40 & 3.41 & 1.45 & 2.89 & 0.76 \\
\hline 7535.22 & 340656.40 & 17.20 & 144.28 & 43.83 & 1.59 & 2.43 & 22.08 & -89.01 & 3024.40 & 3.54 & 2.55 & 2.85 & 0.83 \\
\hline 7535.29 & 348292.20 & 17.00 & 144.56 & 41.96 & 3.00 & 2.29 & 21.92 & -83.58 & 3092.57 & 3.97 & 7.24 & 2.51 & 1.95 \\
\hline 7535.35 & 362589.40 & 16.80 & 143.31 & 40.52 & 2.73 & 5.72 & 23.34 & -110.20 & 3237.61 & 4.70 & 10.38 & 2.30 & 2.07 \\
\hline 7535.42 & 363657.40 & 16.80 & 147.10 & 36.63 & 4.48 & 6.05 & 22.75 & -87.82 & 3025.12 & 5.27 & 10.58 & 2.23 & 2.28 \\
\hline 7535.49 & 355970.60 & 16.80 & 154.78 & 35.95 & 4.71 & 3.20 & 21.39 & -102.58 & 841.17 & 5.19 & 10.54 & 2.17 & 2.64 \\
\hline 7535.55 & 345417.40 & 16.80 & 159.17 & 31.14 & 5.03 & 2.89 & 21.44 & -101.52 & 413.55 & 5.13 & 9.70 & 2.18 & 3.05 \\
\hline 7535.62 & 355632.80 & 16.80 & 163.80 & 34.82 & 5.10 & 1.30 & 19.51 & -142.32 & 56.62 & 4.80 & 5.22 & 2.48 & 2.33 \\
\hline 7535.68 & 349723.20 & 17.00 & 165.80 & 28.28 & 4.88 & 0.73 & 18.82 & -129.57 & -342.43 & 4.18 & 2.13 & 2.65 & 2.58 \\
\hline 7535.75 & 349378.20 & 17.00 & 163.48 & 27.69 & 4.52 & 0.24 & 19.99 & -129.25 & -669.16 & 3.72 & 1.48 & 2.75 & 2.57 \\
\hline 7535.81 & 340231.60 & 17.00 & 129.48 & 50.56 & 198.73 & 5.31 & 18.65 & -84.78 & 971.85 & 3.85 & 3.71 & 2.60 & 2.32 \\
\hline 7535.88 & 348742.60 & 17.00 & 123.27 & 55.40 & 197.27 & 2.77 & 20.21 & -83.86 & 76.39 & 3.89 & 3.44 & 2.67 & 2.03 \\
\hline 7535.94 & 345858.60 & 17.00 & 118.36 & 54.05 & 195.98 & 3.26 & 21.08 & -76.39 & 944.64 & 3.96 & 2.73 & 2.73 & 1.81 \\
\hline 7536.01 & 344694.80 & 17.00 & 118.46 & 58.19 & 195.18 & 2.08 & 21.34 & -95.62 & 383.00 & 4.04 & 3.53 & 2.70 & 1.58 \\
\hline 7536.08 & 330388.60 & 17.20 & 130.18 & 64.93 & 193.65 & 4.14 & 21.60 & -97.48 & -647.81 & 4.06 & 4.76 & 2.53 & 1.57 \\
\hline
\end{tabular}




\begin{tabular}{|c|c|c|c|c|c|c|c|c|c|c|c|c|c|}
\hline Depth & $\mathrm{XRF}$ & XRF Live Time & $\mathrm{Nb}(\mathrm{ppm})$ & Mo (ppm) & $\mathrm{Ba}(\mathrm{ppm})$ & $\mathrm{Pb}(\mathrm{ppm})$ & Th (ppm) & $\mathrm{U}(\mathrm{ppm})$ & $\mathrm{P}(\mathrm{ppm})$ & $\mathrm{S}(\%)$ & $\mathrm{Cl}(\%)$ & $\mathrm{K}(\%)$ & $\mathrm{Ca}(\%)$ \\
\hline 7536.14 & 365591.40 & 17.00 & 154.20 & 54.95 & -1.60 & -2.85 & 24.07 & -183.29 & -517.79 & 5.67 & 2.72 & 2.72 & 1.79 \\
\hline 7536.21 & 363552.20 & 17.00 & 152.78 & 56.67 & -1.33 & -1.13 & 22.92 & -182.77 & -153.64 & 5.74 & 4.06 & 2.54 & 1.94 \\
\hline 7536.27 & 371845.20 & 16.80 & 127.20 & 80.90 & 219.67 & 2.18 & 21.16 & -158.78 & 244.07 & 5.80 & 3.81 & 2.60 & 2.18 \\
\hline 7536.34 & 381581.80 & 16.60 & 130.86 & 83.09 & 219.45 & 3.98 & 21.40 & -162.14 & 829.81 & 6.03 & 2.92 & 2.67 & 2.44 \\
\hline 7536.40 & 401012.40 & 16.40 & 121.61 & 83.61 & 219.88 & 0.91 & 21.10 & -151.26 & 2622.32 & 6.35 & 3.44 & 2.69 & 2.58 \\
\hline 7536.47 & 385087.60 & 16.40 & 134.92 & 73.83 & 220.63 & 1.77 & 20.10 & -95.52 & 818.62 & 5.03 & 3.95 & 2.64 & 2.54 \\
\hline 7536.54 & 383549.00 & 16.40 & 143.90 & 74.14 & 222.90 & 1.76 & 20.12 & -96.58 & 1008.31 & 5.16 & 3.21 & 2.74 & 2.56 \\
\hline 7536.60 & 388037.40 & 16.40 & 173.34 & 54.03 & 1.49 & -3.59 & 24.79 & -159.28 & 854.03 & 5.81 & 3.20 & 2.83 & 2.62 \\
\hline 7536.67 & 354404.40 & 16.80 & 140.93 & 77.50 & 310.02 & 0.19 & 21.64 & -114.89 & -257.02 & 5.55 & 3.89 & 2.82 & 2.48 \\
\hline 7536.73 & 333826.80 & 17.00 & 147.34 & 82.27 & 310.10 & 4.12 & 21.54 & -152.35 & -173.21 & 5.51 & 3.59 & 2.92 & 2.44 \\
\hline 7536.80 & 332127.80 & 17.20 & 141.14 & 86.60 & 310.49 & 5.88 & 23.96 & -142.10 & 887.84 & 5.28 & 3.19 & 3.01 & 2.32 \\
\hline 7536.86 & 342405.40 & 17.00 & 132.69 & 86.50 & 309.49 & 7.06 & 23.67 & -145.28 & 1942.11 & 5.53 & 3.06 & 3.12 & 2.20 \\
\hline 7536.93 & 331451.80 & 17.20 & 134.44 & 81.59 & 309.90 & 5.32 & 20.98 & -118.01 & 2569.54 & 4.98 & 3.11 & 3.07 & 2.05 \\
\hline 7536.99 & 357773.80 & 17.00 & 167.81 & 52.46 & 0.59 & 0.22 & 25.52 & -163.78 & 4454.93 & 5.44 & 2.42 & 3.16 & 2.11 \\
\hline 7537.06 & 393141.80 & 16.60 & 162.55 & 49.29 & -0.44 & -4.63 & 26.34 & -177.44 & 3934.21 & 5.96 & 0.97 & 3.31 & 2.01 \\
\hline 7537.13 & 397280.40 & 16.40 & 161.31 & 44.66 & -1.22 & -4.00 & 25.23 & -198.92 & 3028.52 & 6.08 & 0.82 & 3.35 & 2.08 \\
\hline 7537.19 & 396000.60 & 16.40 & 167.84 & 47.51 & -1.75 & -8.08 & 27.08 & -208.32 & 2695.44 & 6.14 & 0.36 & 3.37 & 2.27 \\
\hline 7537.26 & 406938.20 & 16.20 & 175.05 & 53.73 & -0.01 & -5.55 & 30.24 & -213.97 & 4613.17 & 7.67 & 1.31 & 3.30 & 2.55 \\
\hline 7537.32 & 402246.80 & 16.20 & 193.05 & 61.61 & 1.49 & -1.79 & 33.55 & -194.74 & 2636.68 & 7.62 & 5.04 & 3.00 & 2.40 \\
\hline 7537.39 & 373333.00 & 16.40 & 201.29 & 47.68 & 3.03 & -2.00 & 35.89 & -158.18 & 3058.10 & 7.11 & 6.09 & 2.83 & 2.37 \\
\hline 7537.45 & 358778.40 & 16.60 & 216.77 & 47.84 & 3.98 & -3.32 & 39.84 & -158.55 & 3838.73 & 7.45 & 8.67 & 2.58 & 2.24 \\
\hline 7537.52 & 337569.00 & 16.80 & 240.72 & 38.69 & 3.53 & -2.80 & 44.07 & -165.72 & 4577.89 & 7.28 & 9.24 & 2.50 & 2.21 \\
\hline 7537.59 & 306201.80 & 17.20 & 244.97 & 37.63 & 1.20 & 0.08 & 44.95 & -155.82 & 1590.30 & 5.69 & 8.25 & 2.58 & 1.75 \\
\hline 7537.65 & 312384.20 & 17.20 & 241.78 & 34.30 & 4.02 & -2.37 & 44.59 & -175.24 & 2254.40 & 5.81 & 4.68 & 2.85 & 1.77 \\
\hline 7537.72 & 256105.80 & 17.80 & 199.88 & 40.46 & 59.64 & 9.26 & 43.55 & -146.94 & 3933.12 & 4.75 & 7.94 & 2.29 & 1.61 \\
\hline 7537.78 & 248182.40 & 17.80 & 202.90 & 36.41 & 60.86 & 12.67 & 42.04 & -129.90 & 3979.81 & 4.56 & 7.30 & 2.34 & 1.56 \\
\hline 7537.85 & 261286.00 & 17.80 & 199.37 & 40.60 & 65.24 & 13.54 & 41.00 & -134.93 & 3305.84 & 4.79 & 7.17 & 2.45 & 1.25 \\
\hline 7537.91 & 275216.00 & 17.60 & 209.55 & 32.31 & 66.50 & 9.42 & 41.39 & -159.58 & 3614.60 & 5.04 & 7.19 & 2.48 & 1.19 \\
\hline 7537.98 & 277822.20 & 17.60 & 217.21 & 39.89 & 63.15 & 6.24 & 40.19 & -184.73 & 3413.41 & 6.13 & 9.27 & 2.40 & 1.15 \\
\hline 7538.04 & 351773.00 & 16.80 & 279.10 & 43.57 & 7.75 & -1.72 & 42.49 & -195.55 & 1390.91 & 7.71 & 6.20 & 2.97 & 1.35 \\
\hline 7538.11 & 371827.40 & 16.60 & 275.36 & 44.44 & 4.45 & -4.67 & 42.64 & -218.26 & 1839.92 & 7.82 & 4.19 & 3.22 & 1.34 \\
\hline 7538.18 & 374357.80 & 16.60 & 266.26 & 42.63 & 2.04 & -4.38 & 42.83 & -222.18 & 1195.68 & 7.82 & 4.05 & 3.18 & 1.37 \\
\hline 7538.24 & 383745.40 & 16.60 & 260.26 & 49.70 & 1.23 & -0.63 & 41.18 & -204.22 & 1475.66 & 7.89 & 8.06 & 2.91 & 1.57 \\
\hline
\end{tabular}




\begin{tabular}{|c|c|c|c|c|c|c|c|c|c|c|c|c|c|}
\hline Depth & $\mathrm{XRF}$ & XRF Live Time & $\mathrm{Nb}(\mathrm{ppm})$ & Mo (ppm) & $\mathrm{Ba}(\mathrm{ppm})$ & $\mathrm{Pb}(\mathrm{ppm})$ & Th (ppm) & $\mathrm{U}(\mathrm{ppm})$ & $\mathrm{P}(\mathrm{ppm})$ & $\mathrm{S}(\%)$ & $\mathrm{Cl}(\%)$ & $\mathrm{K}(\%)$ & $\mathrm{Ca}(\%)$ \\
\hline 7538.31 & 354831.20 & 16.80 & 245.28 & 30.81 & -0.85 & 1.38 & 40.22 & -157.54 & 2061.09 & 6.42 & 7.90 & 2.84 & 1.65 \\
\hline 7538.37 & 333492.20 & 17.00 & 225.56 & 22.62 & 0.34 & 2.12 & 38.38 & -154.83 & 1421.86 & 5.65 & 8.79 & 2.79 & 1.60 \\
\hline 7538.44 & 320175.20 & 17.20 & 181.30 & 48.51 & 307.06 & 7.20 & 32.90 & -106.86 & 664.76 & 5.15 & 8.74 & 2.77 & 1.65 \\
\hline 7538.50 & 310044.80 & 17.20 & 162.67 & 53.28 & 304.68 & 7.02 & 28.25 & -94.19 & 689.83 & 4.56 & 8.90 & 2.75 & 1.63 \\
\hline 7538.57 & 312639.00 & 17.00 & 144.27 & 55.73 & 304.59 & 4.31 & 27.12 & -86.57 & 1653.91 & 4.39 & 5.06 & 2.97 & 1.49 \\
\hline 7538.64 & 341377.40 & 16.80 & 132.61 & 66.27 & 306.91 & 6.36 & 26.25 & -97.70 & 1237.13 & 4.36 & 2.97 & 3.18 & 1.58 \\
\hline 7538.70 & 356862.00 & 16.80 & 121.68 & 71.19 & 306.58 & 1.08 & 22.58 & -106.44 & 1305.97 & 4.52 & 3.86 & 3.07 & 1.58 \\
\hline 7538.77 & 361465.20 & 16.80 & 151.90 & 53.26 & 2.73 & -5.42 & 24.53 & -129.82 & 1442.51 & 4.85 & 4.64 & 2.95 & 1.65 \\
\hline 7538.83 & 373775.80 & 16.80 & 169.95 & 56.93 & 5.84 & -3.46 & 25.18 & -91.08 & 2039.74 & 4.80 & 9.07 & 2.68 & 1.64 \\
\hline 7538.90 & 377151.40 & 16.80 & 142.48 & 81.36 & 277.61 & 1.75 & 22.49 & -61.43 & 1585.66 & 5.05 & 11.47 & 2.56 & 1.55 \\
\hline 7538.96 & 371854.60 & 16.80 & 145.77 & 77.42 & 277.11 & 0.63 & 20.94 & -46.70 & 2100.28 & 5.20 & 12.78 & 2.43 & 1.57 \\
\hline 7539.03 & 371340.20 & 16.80 & 150.93 & 90.19 & 277.91 & 3.54 & 22.57 & -68.33 & 3465.88 & 5.28 & 9.75 & 2.66 & 1.92 \\
\hline 7539.09 & 385089.80 & 16.60 & 169.22 & 88.94 & 275.62 & 2.46 & 25.12 & -82.55 & 4067.87 & 5.24 & 11.53 & 2.57 & 2.14 \\
\hline 7539.16 & 377965.40 & 16.60 & 167.28 & 88.61 & 274.12 & 0.42 & 26.10 & -69.08 & 3126.73 & 5.56 & 12.53 & 2.49 & 2.48 \\
\hline 7539.23 & 377738.80 & 16.60 & 198.12 & 65.07 & 3.21 & -5.19 & 30.63 & -117.48 & 4150.85 & 5.61 & 12.11 & 2.44 & 2.92 \\
\hline 7539.29 & 353391.20 & 16.80 & 166.76 & 121.09 & 1721.13 & -0.82 & 29.87 & -94.14 & 1540.92 & 6.56 & 11.01 & 2.40 & 2.97 \\
\hline 7539.36 & 321083.60 & 17.00 & 171.53 & 109.51 & 1719.35 & -1.67 & 29.60 & -99.59 & 1605.18 & 6.94 & 14.11 & 2.15 & 5.26 \\
\hline 7539.42 & 300479.40 & 17.20 & 171.62 & 101.05 & 1720.16 & 1.49 & 29.95 & -82.07 & 197.74 & 6.58 & 17.77 & 1.91 & 5.12 \\
\hline 7539.49 & 283962.20 & 17.40 & 165.11 & 92.28 & 1720.77 & 1.06 & 28.73 & -112.34 & -372.86 & 6.45 & 15.20 & 2.03 & 5.57 \\
\hline 7539.55 & 255115.80 & 17.80 & 182.80 & 88.77 & 1720.46 & 4.25 & 28.39 & -96.61 & -2706.97 & 6.16 & 14.68 & 2.05 & 5.99 \\
\hline 7539.62 & 290406.00 & 17.40 & 237.33 & 34.62 & 4.28 & 0.73 & 32.83 & -160.41 & 1830.54 & 5.89 & 15.13 & 2.16 & 6.57 \\
\hline 7539.69 & 314852.60 & 17.20 & 242.48 & 43.13 & 5.29 & -2.36 & 37.22 & -176.82 & 538.81 & 6.13 & 14.23 & 2.26 & 4.13 \\
\hline 7539.75 & 301161.80 & 17.40 & 262.71 & 47.87 & 5.49 & -4.47 & 37.62 & -253.84 & 490.84 & 7.06 & 8.82 & 2.62 & 4.40 \\
\hline 7539.82 & 306593.00 & 17.20 & 287.28 & 55.09 & 5.84 & -2.52 & 41.47 & -317.91 & 2517.71 & 8.78 & 5.72 & 2.83 & 4.20 \\
\hline 7539.88 & 305598.60 & 17.20 & 309.69 & 52.00 & 8.10 & -2.70 & 41.15 & -353.27 & 3789.39 & 9.52 & 4.95 & 2.89 & 3.69 \\
\hline 7539.95 & 284149.80 & 17.40 & 295.31 & 55.74 & 11.50 & -4.71 & 41.65 & -360.28 & 1127.23 & 10.03 & 4.90 & 2.83 & 3.32 \\
\hline 7540.01 & 271298.20 & 17.60 & 313.53 & 54.82 & 7.66 & -2.11 & 37.35 & -352.44 & 828.89 & 10.06 & 3.77 & 2.93 & 3.66 \\
\hline 7540.08 & 264068.20 & 17.60 & 298.68 & 56.75 & 8.55 & 0.18 & 37.56 & -325.66 & 1340.23 & 10.13 & 3.25 & 2.84 & 3.40 \\
\hline 7540.14 & 256121.40 & 17.80 & 278.48 & 65.28 & 2.81 & 2.45 & 36.43 & -314.33 & 4827.34 & 9.76 & 3.74 & 2.77 & 3.22 \\
\hline 7540.21 & 272989.20 & 17.60 & 281.88 & 63.72 & -0.66 & 0.87 & 41.34 & -318.03 & 7841.93 & 10.64 & 4.53 & 2.78 & 3.15 \\
\hline 7540.28 & 280050.40 & 17.60 & 312.93 & 64.99 & -5.71 & -0.35 & 44.23 & -304.29 & 10134.48 & 11.50 & 5.36 & 2.69 & 2.97 \\
\hline 7540.34 & 287641.60 & 17.40 & 279.33 & 76.31 & -2.12 & 0.67 & 46.27 & -366.83 & 12881.10 & 13.87 & 5.77 & 2.64 & 2.83 \\
\hline 7540.41 & 310619.80 & 17.20 & 274.73 & 78.81 & -3.42 & 0.62 & 48.22 & -388.42 & 17287.38 & 14.97 & 6.59 & 2.59 & 3.20 \\
\hline
\end{tabular}




\begin{tabular}{|c|c|c|c|c|c|c|c|c|c|c|c|c|c|}
\hline Depth & $\mathrm{XRF}$ & XRF Live Time & $\mathrm{Nb}(\mathrm{ppm})$ & Mo (ppm) & $\mathrm{Ba}(\mathrm{ppm})$ & $\mathrm{Pb}(\mathrm{ppm})$ & Th (ppm) & $\mathrm{U}(\mathrm{ppm})$ & $\mathrm{P}(\mathrm{ppm})$ & $\mathrm{S}(\%)$ & $\mathrm{Cl}(\%)$ & $\mathrm{K}(\%)$ & $\mathrm{Ca}(\%)$ \\
\hline 7540.47 & 317747.20 & 17.00 & 292.76 & 68.62 & -1.23 & -0.79 & 50.25 & -346.68 & 14022.97 & 15.11 & 7.67 & 2.47 & 3.15 \\
\hline 7540.54 & 299494.00 & 17.20 & 248.19 & 87.35 & -2.01 & -6.36 & 44.58 & -394.04 & 10513.06 & 15.80 & 8.41 & 2.29 & 3.48 \\
\hline 7540.60 & 300340.60 & 17.20 & 216.37 & 86.07 & -0.12 & -6.66 & 41.03 & -413.55 & 10255.88 & 14.65 & 7.67 & 2.34 & 3.80 \\
\hline 7540.67 & 289950.60 & 17.40 & 244.83 & 70.63 & 2.61 & -6.93 & 43.58 & -376.55 & 7404.18 & 12.91 & 7.06 & 2.36 & 3.73 \\
\hline 7540.73 & 292657.60 & 17.40 & 250.55 & 71.86 & 0.91 & -5.32 & 42.16 & -368.61 & 6211.26 & 12.37 & 6.23 & 2.41 & 3.90 \\
\hline 7540.80 & 290703.60 & 17.60 & 243.61 & 74.22 & 3.32 & -5.96 & 39.62 & -378.59 & 4657.70 & 11.90 & 4.68 & 2.51 & 4.37 \\
\hline 7540.87 & 292015.80 & 17.60 & 264.62 & 62.41 & 4.92 & 2.28 & 38.26 & -293.43 & 6818.95 & 10.33 & 3.13 & 2.62 & 4.61 \\
\hline 7540.93 & 283956.00 & 17.60 & 270.89 & 66.15 & 3.86 & 4.72 & 39.32 & -345.79 & 7383.35 & 10.91 & 2.49 & 2.70 & 4.53 \\
\hline 7541.00 & 291971.60 & 17.40 & 258.95 & 57.12 & 0.67 & 4.27 & 33.45 & -336.58 & 8768.81 & 10.65 & 2.13 & 2.57 & 4.99 \\
\hline 7541.06 & 299930.60 & 17.40 & 239.34 & 56.91 & 3.33 & 5.13 & 30.95 & -321.31 & 7139.69 & 10.24 & 1.90 & 2.54 & 5.51 \\
\hline 7541.13 & 323499.40 & 17.00 & 224.20 & 51.51 & 4.89 & 6.59 & 28.58 & -313.47 & 8387.84 & 10.24 & 2.13 & 2.51 & 6.17 \\
\hline 7541.19 & 346589.40 & 16.80 & 208.22 & 49.85 & 6.35 & 2.66 & 27.57 & -327.27 & 6680.52 & 9.78 & 1.33 & 2.42 & 7.67 \\
\hline 7541.26 & 338932.60 & 17.00 & 178.06 & 55.94 & 7.81 & 4.58 & 23.59 & -225.06 & 6103.87 & 8.18 & 1.44 & 2.11 & 11.53 \\
\hline 7541.33 & 350093.00 & 17.00 & 134.81 & 62.75 & 7.32 & 1.88 & 20.07 & -160.94 & 7624.04 & 6.31 & 1.41 & 1.71 & 20.07 \\
\hline 7541.39 & 347560.20 & 17.00 & 99.85 & 59.51 & 5.47 & -2.68 & 15.55 & -95.24 & 7548.12 & 4.54 & 1.88 & 1.24 & 33.35 \\
\hline 7541.46 & 349397.80 & 17.00 & 64.18 & 65.62 & 2.38 & -7.56 & 10.46 & -71.67 & 6126.34 & 2.70 & 1.63 & 0.78 & 48.79 \\
\hline 7541.52 & 354163.00 & 16.80 & 28.26 & 65.78 & 1.00 & -10.27 & 7.11 & -31.88 & 5249.13 & 1.64 & 1.74 & 0.41 & 62.37 \\
\hline 7541.59 & 381171.40 & 16.40 & 12.24 & 58.75 & 0.55 & -16.23 & 3.14 & -14.29 & 4497.53 & 1.01 & 1.63 & 0.19 & 73.85 \\
\hline 7541.65 & 386401.40 & 16.40 & 9.17 & 60.95 & 0.51 & -12.12 & 2.15 & -8.77 & 4530.85 & 0.94 & 2.12 & 0.20 & 78.48 \\
\hline 7541.72 & 394765.80 & 16.20 & 8.81 & 62.58 & 2.69 & -11.53 & 3.89 & -17.64 & 2857.39 & 1.06 & 1.61 & 0.25 & 78.05 \\
\hline 7541.78 & 395578.80 & 16.20 & 9.98 & 62.73 & 4.33 & -10.78 & 3.78 & -19.80 & 3608.88 & 1.40 & 3.93 & 0.28 & 72.66 \\
\hline 7541.85 & 396789.60 & 16.20 & 12.21 & 69.32 & 4.81 & -13.31 & 4.07 & -25.25 & 878.57 & 1.49 & 6.43 & 0.28 & 67.94 \\
\hline 7541.92 & 397563.00 & 16.20 & 14.63 & 68.14 & 4.77 & -13.89 & 3.52 & -30.22 & -2488.18 & 1.88 & 6.43 & 0.29 & 62.44 \\
\hline 7541.98 & 389586.40 & 16.20 & 18.28 & 62.42 & 5.65 & -16.34 & 5.92 & -48.83 & -5481.66 & 2.19 & 5.42 & 0.32 & 54.29 \\
\hline 7542.05 & 349413.20 & 16.60 & 23.46 & 57.21 & 2.89 & -14.17 & 6.30 & -57.37 & -5685.11 & 2.34 & 5.41 & 0.34 & 43.71 \\
\hline 7542.11 & 313117.40 & 17.00 & 36.09 & 55.06 & 1.91 & -15.61 & 10.23 & -66.27 & -4488.77 & 2.68 & 4.19 & 0.43 & 35.98 \\
\hline 7542.18 & 285862.60 & 17.40 & 78.28 & 49.63 & 0.80 & -11.27 & 17.06 & -80.37 & -64.72 & 4.08 & 2.17 & 0.86 & 26.91 \\
\hline 7542.24 & 272223.60 & 17.60 & 139.10 & 42.95 & 1.93 & -4.58 & 25.01 & -119.78 & 2045.45 & 4.93 & 2.37 & 1.32 & 17.11 \\
\hline 7542.31 & 267349.60 & 17.60 & 194.39 & 43.10 & 3.84 & -1.29 & 31.51 & -137.19 & 4059.45 & 6.26 & 2.55 & 1.74 & 11.79 \\
\hline 7542.38 & 285620.00 & 17.40 & 239.10 & 39.12 & 7.07 & -5.51 & 37.55 & -172.85 & 5614.79 & 7.29 & 2.58 & 2.15 & 9.03 \\
\hline 7542.44 & 296130.20 & 17.40 & 291.39 & 32.60 & 5.28 & -4.26 & 40.26 & -210.17 & 2429.28 & 8.05 & 1.55 & 2.51 & 6.06 \\
\hline 7542.51 & 304943.80 & 17.20 & 300.12 & 26.81 & 5.73 & -4.65 & 41.50 & -221.70 & 2938.85 & 7.94 & 0.93 & 2.51 & 4.51 \\
\hline 7542.57 & 316723.40 & 17.00 & 272.00 & 38.56 & 7.10 & -10.00 & 37.76 & -269.62 & 5579.12 & 10.86 & 1.15 & 2.41 & 4.57 \\
\hline
\end{tabular}




\begin{tabular}{|c|c|c|c|c|c|c|c|c|c|c|c|c|c|}
\hline Depth & XRF & XRF Live Time & $\mathrm{Nb}(\mathrm{ppm})$ & Mo (ppm) & $\mathrm{Ba}(\mathrm{ppm})$ & $\mathrm{Pb}(\mathrm{ppm})$ & Th (ppm) & $\mathrm{U}(\mathrm{ppm})$ & $\mathrm{P}(\mathrm{ppm})$ & $\mathrm{S}(\%)$ & $\mathrm{Cl}(\%)$ & $\mathrm{K}(\%)$ & $\mathrm{Ca}(\%)$ \\
\hline 7542.64 & 312846.60 & 17.00 & 271.92 & 40.24 & 3.46 & -12.85 & 38.67 & -249.95 & 6110.72 & 11.03 & 1.13 & 2.46 & 4.43 \\
\hline 7542.70 & 310533.20 & 17.20 & 264.89 & 46.64 & 3.51 & -7.28 & 38.63 & -228.54 & 5837.14 & 11.35 & 1.31 & 2.52 & 4.61 \\
\hline 7542.77 & 322431.60 & 17.00 & 220.85 & 49.51 & 6.52 & -6.52 & 37.88 & -223.14 & 9966.84 & 11.12 & 2.00 & 2.32 & 7.17 \\
\hline 7542.83 & 345309.00 & 16.80 & 231.06 & 46.97 & 6.38 & -3.93 & 39.62 & -234.10 & 7823.18 & 11.27 & 1.95 & 2.41 & 7.06 \\
\hline 7542.90 & 347140.00 & 16.80 & 252.92 & 39.60 & 4.07 & 1.55 & 44.82 & -211.67 & 6842.21 & 8.69 & 1.50 & 2.55 & 7.24 \\
\hline 7542.97 & 376476.00 & 16.60 & 256.43 & 42.48 & 5.45 & 4.12 & 44.22 & -284.54 & 6862.18 & 9.03 & 1.36 & 2.63 & 7.47 \\
\hline 7543.03 & 402295.20 & 16.20 & 235.64 & 48.84 & 3.72 & -0.65 & 47.08 & -274.90 & 9364.11 & 8.23 & 1.15 & 2.37 & 12.99 \\
\hline 7543.10 & 412928.00 & 16.00 & 246.85 & 52.43 & 3.88 & -0.31 & 48.96 & -256.01 & 9458.42 & 8.87 & 2.19 & 2.44 & 12.84 \\
\hline 7543.16 & 418111.60 & 16.00 & 221.15 & 63.78 & 5.97 & -0.91 & 45.69 & -266.60 & 11892.19 & 9.21 & 2.48 & 2.46 & 13.68 \\
\hline 7543.23 & 425042.20 & 16.00 & 214.42 & 62.48 & 6.26 & -0.86 & 44.06 & -259.22 & 12159.69 & 9.86 & 2.97 & 2.42 & 13.70 \\
\hline 7543.29 & 402271.00 & 16.20 & 209.96 & 58.72 & 7.55 & -1.38 & 44.13 & -214.21 & 10672.46 & 9.40 & 3.00 & 2.38 & 13.27 \\
\hline 7543.36 & 415416.40 & 16.20 & 227.32 & 59.33 & 6.41 & 0.79 & 39.48 & -248.60 & 8242.54 & 11.17 & 3.07 & 2.62 & 7.14 \\
\hline 7543.43 & 417450.00 & 16.20 & 221.14 & 50.46 & 4.73 & 2.68 & 38.49 & -271.19 & 6053.34 & 10.52 & 1.02 & 2.74 & 4.63 \\
\hline 7543.49 & 408757.60 & 16.20 & 231.80 & 40.44 & 4.08 & -0.65 & 41.95 & -245.71 & 5055.21 & 10.02 & 1.09 & 2.65 & 3.75 \\
\hline 7543.56 & 409825.40 & 16.20 & 236.55 & 46.91 & 2.54 & -2.51 & 43.49 & -237.53 & 5171.57 & 9.54 & 0.62 & 2.64 & 3.91 \\
\hline 7543.62 & 432019.80 & 16.00 & 223.98 & 53.35 & 3.76 & -1.20 & 42.64 & -251.64 & 3481.71 & 9.72 & 0.61 & 2.57 & 4.68 \\
\hline 7543.69 & 408265.40 & 16.20 & 184.05 & 77.82 & 482.54 & 4.93 & 37.12 & -189.99 & 3632.40 & 9.21 & 1.05 & 2.53 & 5.51 \\
\hline 7543.75 & 394083.20 & 16.40 & 190.24 & 83.79 & 483.24 & 5.26 & 40.11 & -181.30 & 4252.87 & 9.37 & 1.38 & 2.51 & 6.04 \\
\hline 7543.82 & 349104.40 & 16.80 & 170.28 & 93.70 & 481.92 & 7.33 & 37.04 & -209.31 & 4317.76 & 9.23 & 1.40 & 2.39 & 7.87 \\
\hline 7543.88 & 266952.60 & 17.60 & 115.15 & 85.18 & 541.41 & 19.91 & 30.74 & -152.55 & 2173.64 & 7.26 & 5.35 & 1.90 & 6.84 \\
\hline 7543.95 & 250492.40 & 17.80 & 111.07 & 80.61 & 537.95 & 17.26 & 33.31 & -174.14 & 3632.59 & 6.97 & 5.59 & 1.82 & 6.37 \\
\hline 7544.02 & 256344.20 & 17.60 & 161.88 & 48.88 & 60.88 & 9.83 & 41.27 & -245.01 & 3698.79 & 6.23 & 5.07 & 1.78 & 5.50 \\
\hline 7544.08 & 250759.20 & 17.60 & 128.16 & 79.31 & 420.91 & 14.37 & 32.23 & -193.56 & 3191.96 & 5.91 & 4.74 & 1.66 & 4.61 \\
\hline 7544.15 & 275996.00 & 17.40 & 134.30 & 71.60 & 421.01 & 14.35 & 31.70 & -168.37 & 2322.32 & 5.63 & 4.37 & 1.64 & 3.05 \\
\hline 7544.21 & 321006.80 & 17.00 & 162.33 & 97.30 & 360.55 & -0.97 & 32.86 & -247.02 & 3358.88 & 7.11 & 0.99 & 1.93 & 3.65 \\
\hline 7544.28 & 308640.40 & 17.00 & 147.07 & 165.70 & 357.59 & -4.66 & 15.33 & -361.84 & 3377.13 & 8.59 & 1.26 & 1.69 & 3.18 \\
\hline 7544.34 & 275893.40 & 17.40 & 120.76 & 185.37 & 355.11 & -13.84 & 9.13 & -325.80 & 2618.28 & 8.60 & 2.40 & 1.53 & 3.30 \\
\hline 7544.41 & 261860.80 & 17.60 & 152.00 & 166.02 & -3.55 & -20.15 & 15.27 & -389.30 & 2431.95 & 9.01 & 2.52 & 1.54 & 3.37 \\
\hline 7544.48 & 241361.60 & 17.80 & 150.55 & 192.48 & -3.58 & -19.28 & 10.98 & -410.44 & 3513.39 & 9.28 & 2.86 & 1.48 & 2.98 \\
\hline 7544.54 & 229125.00 & 17.80 & 154.70 & 180.91 & -0.97 & -19.15 & 11.83 & -376.19 & 4449.54 & 8.88 & 3.59 & 1.47 & 3.19 \\
\hline 7544.61 & 212636.80 & 18.00 & 156.06 & 135.04 & 2.46 & -17.88 & 26.26 & -228.88 & 5802.03 & 6.88 & 3.21 & 1.64 & 3.40 \\
\hline 7544.67 & 223564.20 & 18.00 & 149.91 & 132.30 & 3.91 & -10.34 & 29.57 & -247.28 & 6472.41 & 6.56 & 2.07 & 1.69 & 3.38 \\
\hline 7544.74 & 237486.40 & 17.80 & 137.66 & 154.62 & 2.12 & -13.34 & 27.36 & -279.69 & 6939.72 & 7.08 & 2.36 & 1.65 & 3.20 \\
\hline
\end{tabular}




\begin{tabular}{|c|c|c|c|c|c|c|c|c|c|c|c|c|c|}
\hline Depth & $\mathrm{XRF}$ & XRF Live Time & $\mathrm{Nb}(\mathrm{ppm})$ & Mo (ppm) & $\mathrm{Ba}(\mathrm{ppm})$ & $\mathrm{Pb}(\mathrm{ppm})$ & Th (ppm) & $\mathrm{U}(\mathrm{ppm})$ & $\mathrm{P}(\mathrm{ppm})$ & $\mathrm{S}(\%)$ & $\mathrm{Cl}(\%)$ & $\mathrm{K}(\%)$ & $\mathrm{Ca}(\%)$ \\
\hline 7544.80 & 249747.40 & 17.80 & 126.55 & 156.59 & 1.22 & -15.53 & 28.18 & -285.03 & 3836.38 & 7.06 & 2.76 & 1.73 & 3.42 \\
\hline 7544.87 & 216169.20 & 18.20 & 94.70 & 192.83 & 1.13 & -16.35 & 25.63 & -246.97 & 2803.85 & 6.03 & 7.81 & 1.47 & 2.68 \\
\hline 7544.93 & 211174.80 & 18.20 & 84.06 & 188.11 & 0.83 & -15.99 & 24.29 & -249.32 & 869.37 & 5.70 & 7.65 & 1.49 & 2.77 \\
\hline 7545.00 & 211441.60 & 18.00 & 101.40 & 170.27 & 0.60 & -14.40 & 23.60 & -225.04 & 246.57 & 5.58 & 7.69 & 1.54 & 3.21 \\
\hline 7545.07 & 217835.80 & 18.00 & 130.13 & 144.13 & 1.27 & -13.33 & 28.44 & -222.44 & -859.42 & 5.41 & 7.27 & 1.71 & 3.21 \\
\hline 7545.13 & 242942.60 & 17.60 & 156.00 & 116.13 & 2.16 & -9.00 & 33.38 & -224.29 & 155.74 & 5.68 & 6.53 & 1.86 & 2.99 \\
\hline 7545.20 & 309283.40 & 17.00 & 154.62 & 106.35 & 407.71 & 0.85 & 30.57 & -209.87 & -1764.53 & 7.34 & 0.14 & 2.31 & 3.55 \\
\hline 7545.26 & 345767.60 & 16.80 & 186.93 & 82.64 & 406.44 & 6.19 & 33.34 & -206.84 & 517.13 & 8.01 & 0.15 & 2.42 & 3.70 \\
\hline 7545.33 & 354649.60 & 16.80 & 220.35 & 77.76 & 405.61 & 6.74 & 37.16 & -213.12 & -298.75 & 8.36 & 0.11 & 2.47 & 3.14 \\
\hline 7545.39 & 344015.80 & 17.00 & 204.61 & 77.43 & 406.47 & 5.52 & 35.19 & -140.29 & -1890.44 & 7.48 & 1.08 & 2.14 & 6.41 \\
\hline 7545.46 & 328355.80 & 17.20 & 189.08 & 92.19 & 406.34 & -2.64 & 34.71 & -132.43 & -1504.74 & 7.34 & 1.16 & 1.93 & 8.85 \\
\hline 7545.52 & 338674.80 & 17.00 & 240.99 & 73.40 & 0.61 & -11.95 & 45.54 & -197.97 & -465.91 & 7.81 & 1.47 & 1.85 & 10.35 \\
\hline 7545.59 & 348734.00 & 16.80 & 262.86 & 80.36 & 0.16 & -11.95 & 48.55 & -196.70 & -1900.46 & 8.08 & 1.49 & 1.95 & 10.16 \\
\hline 7545.66 & 364341.00 & 16.60 & 264.87 & 79.40 & 1.52 & -11.57 & 49.90 & -208.27 & -663.11 & 8.37 & 1.55 & 2.01 & 10.03 \\
\hline 7545.72 & 390378.60 & 16.20 & 301.06 & 73.42 & 2.72 & -5.97 & 54.42 & -263.57 & 998.36 & 9.33 & 0.58 & 2.37 & 6.88 \\
\hline 7545.79 & 411936.20 & 16.00 & 333.58 & 56.10 & 2.32 & 0.19 & 56.14 & -258.17 & 3320.51 & 9.94 & 0.63 & 2.59 & 4.67 \\
\hline 7545.85 & 414654.00 & 16.00 & 346.33 & 37.24 & 2.12 & 6.02 & 55.91 & -233.84 & 3845.52 & 9.45 & 0.32 & 2.63 & 2.89 \\
\hline 7545.92 & 416499.20 & 16.00 & 343.49 & 30.53 & 5.73 & 3.61 & 58.07 & -229.59 & 4079.71 & 9.33 & 0.30 & 2.51 & 2.88 \\
\hline 7545.98 & 438364.20 & 15.80 & 320.27 & 56.18 & 3.14 & 3.31 & 59.28 & -429.53 & 3603.13 & 14.49 & 0.25 & 2.51 & 3.03 \\
\hline 7546.05 & 437418.00 & 15.80 & 311.83 & 58.50 & 0.86 & 5.94 & 59.68 & -434.74 & 3420.52 & 14.86 & 0.28 & 2.48 & 2.94 \\
\hline 7546.12 & 429126.60 & 16.00 & 318.24 & 55.61 & -0.03 & 6.07 & 61.86 & -431.25 & 3345.40 & 14.29 & 0.22 & 2.40 & 3.42 \\
\hline 7546.18 & 421277.00 & 16.20 & 253.34 & 112.28 & 385.13 & 7.48 & 51.56 & -388.80 & 4322.21 & 14.25 & 0.61 & 2.40 & 4.33 \\
\hline 7546.25 & 411093.80 & 16.40 & 250.15 & 115.13 & 384.03 & 5.87 & 50.13 & -382.49 & 3938.03 & 14.25 & 0.99 & 2.42 & 6.45 \\
\hline 7546.31 & 370388.20 & 16.80 & 255.63 & 96.97 & 388.87 & 7.14 & 51.20 & -182.05 & 5049.15 & 8.91 & 1.65 & 2.27 & 7.97 \\
\hline 7546.38 & 332914.60 & 17.20 & 249.91 & 94.13 & 390.70 & 1.37 & 51.54 & -118.34 & 5726.17 & 7.86 & 3.78 & 2.06 & 8.86 \\
\hline 7546.44 & 290804.00 & 17.60 & 237.11 & 111.77 & 392.07 & 2.29 & 53.06 & -104.67 & 2011.67 & 7.60 & 4.70 & 1.97 & 9.87 \\
\hline 7546.51 & 263259.40 & 17.80 & 299.64 & 51.15 & 6.41 & -3.02 & 65.63 & -169.10 & -603.46 & 7.84 & 5.30 & 1.96 & 10.03 \\
\hline 7546.57 & 227412.60 & 18.00 & 290.26 & 62.08 & 6.45 & 0.93 & 63.20 & -178.26 & -2142.46 & 7.68 & 6.36 & 1.86 & 8.33 \\
\hline 7546.64 & 183272.20 & 18.40 & 295.60 & 92.73 & 4.27 & 0.37 & 57.86 & -204.24 & -5718.16 & 7.63 & 5.80 & 1.96 & 7.30 \\
\hline 7546.71 & 198087.80 & 18.20 & 319.64 & 91.70 & 0.20 & 2.26 & 58.20 & -252.51 & -4537.79 & 8.20 & 4.16 & 2.13 & 6.34 \\
\hline 7546.77 & 251834.00 & 17.60 & 325.68 & 80.24 & 4.70 & 3.08 & 56.48 & -291.57 & -1255.53 & 8.71 & 3.86 & 2.19 & 4.82 \\
\hline 7546.84 & 264565.00 & 17.40 & 345.41 & 73.03 & 6.64 & 4.09 & 59.72 & -272.21 & -503.03 & 8.13 & 3.09 & 2.13 & 3.64 \\
\hline 7546.90 & 264277.60 & 17.40 & 343.14 & 84.40 & 10.21 & 3.65 & 64.15 & -249.46 & 657.55 & 8.62 & 3.92 & 2.00 & 3.17 \\
\hline
\end{tabular}




\begin{tabular}{|c|c|c|c|c|c|c|c|c|c|c|c|c|c|}
\hline Depth & $\mathrm{XRF}$ & XRF Live Time & $\mathrm{Nb}(\mathrm{ppm})$ & Mo (ppm) & $\mathrm{Ba}(\mathrm{ppm})$ & $\mathrm{Pb}(\mathrm{ppm})$ & Th (ppm) & $\mathrm{U}(\mathrm{ppm})$ & $\mathrm{P}(\mathrm{ppm})$ & $\mathrm{S}(\%)$ & $\mathrm{Cl}(\%)$ & $\mathrm{K}(\%)$ & $\mathrm{Ca}(\%)$ \\
\hline 7546.97 & 325860.20 & 16.80 & 354.57 & 54.45 & 36.13 & 9.96 & 75.23 & -240.60 & 3753.38 & 9.35 & 3.83 & 2.05 & 2.70 \\
\hline 7547.03 & 322993.40 & 17.00 & 344.02 & 51.02 & 40.25 & 10.24 & 75.25 & -214.86 & 2630.81 & 8.65 & 3.31 & 2.00 & 2.80 \\
\hline 7547.10 & 290532.40 & 17.40 & 341.46 & 53.99 & 37.95 & 7.77 & 77.77 & -181.03 & 470.54 & 8.11 & 2.62 & 1.94 & 3.59 \\
\hline 7547.17 & 300743.20 & 17.40 & 310.96 & 63.34 & 37.56 & 6.80 & 74.75 & -215.53 & 1076.81 & 8.21 & 2.40 & 2.01 & 4.37 \\
\hline 7547.23 & 334347.80 & 17.20 & 295.26 & 39.15 & 35.12 & 4.73 & 76.46 & -247.09 & 1778.47 & 8.46 & 0.86 & 2.09 & 5.21 \\
\hline 7547.30 & 329378.00 & 17.40 & 297.57 & 32.08 & 10.07 & -0.18 & 74.84 & -234.08 & 922.49 & 7.46 & 0.83 & 1.97 & 5.91 \\
\hline 7547.36 & 361373.60 & 17.00 & 301.06 & 31.39 & 12.43 & -0.54 & 76.93 & -264.00 & 1241.01 & 8.46 & 0.89 & 2.04 & 5.81 \\
\hline 7547.43 & 376778.40 & 16.80 & 301.50 & 19.87 & 14.54 & 3.15 & 75.58 & -249.35 & 2816.43 & 8.39 & 0.99 & 2.11 & 4.74 \\
\hline 7547.49 & 380420.00 & 16.80 & 319.72 & 21.86 & 19.31 & 4.95 & 78.92 & -204.48 & 3083.56 & 8.51 & 2.20 & 2.01 & 4.27 \\
\hline 7547.56 & 381205.80 & 16.80 & 334.91 & 24.86 & 23.99 & 5.99 & 76.96 & -184.87 & 2993.46 & 7.55 & 2.07 & 2.03 & 3.35 \\
\hline 7547.62 & 371893.40 & 16.80 & 306.63 & 22.46 & 26.15 & 6.49 & 69.58 & -183.91 & 2477.51 & 7.51 & 2.34 & 1.99 & 2.47 \\
\hline 7547.69 & 342331.60 & 17.00 & 279.09 & 19.20 & 24.09 & 7.24 & 65.37 & -152.10 & 2717.06 & 6.35 & 2.60 & 1.84 & 2.34 \\
\hline 7547.76 & 334970.20 & 17.00 & 270.71 & 12.27 & 36.87 & 5.87 & 66.85 & -177.06 & 2116.33 & 6.34 & 2.53 & 1.82 & 2.24 \\
\hline 7547.82 & 311219.20 & 17.20 & 241.69 & 8.26 & 31.72 & 3.73 & 58.72 & -177.52 & 2364.35 & 5.60 & 1.34 & 1.80 & 1.97 \\
\hline 7547.89 & 299830.60 & 17.20 & 219.68 & 10.29 & 23.06 & 4.89 & 54.58 & -182.24 & 1021.24 & 5.61 & 0.70 & 1.82 & 1.83 \\
\hline 7547.95 & 300878.40 & 17.20 & 245.48 & 13.20 & 22.21 & 1.34 & 63.52 & -206.08 & 817.57 & 5.93 & 0.44 & 1.91 & 1.79 \\
\hline 7548.02 & 248768.20 & 17.80 & 203.09 & 70.49 & 18.92 & 0.55 & 50.37 & -175.33 & -2579.78 & 4.96 & 4.24 & 1.60 & 1.59 \\
\hline 7548.08 & 252914.00 & 17.80 & 200.34 & 81.35 & 6.42 & -1.69 & 49.87 & -177.16 & -3012.78 & 5.00 & 4.20 & 1.61 & 1.62 \\
\hline 7548.15 & 263855.00 & 17.60 & 238.93 & 85.51 & 2.60 & 1.73 & 60.45 & -190.91 & -3497.03 & 5.46 & 4.20 & 1.71 & 1.45 \\
\hline 7548.22 & 270260.40 & 17.60 & 252.37 & 83.37 & 4.47 & 0.98 & 60.97 & -205.37 & -3208.25 & 5.45 & 4.21 & 1.74 & 1.47 \\
\hline 7548.28 & 268278.20 & 17.60 & 226.63 & 80.07 & 2.63 & 1.34 & 53.33 & -183.82 & -1609.62 & 5.06 & 4.22 & 1.75 & 1.33 \\
\hline 7548.35 & 333311.00 & 17.00 & 300.25 & 28.54 & 2.29 & 3.48 & 69.06 & -232.12 & 2327.92 & 6.61 & 0.11 & 2.20 & 1.43 \\
\hline 7548.41 & 333489.80 & 17.00 & 292.66 & 26.33 & -2.29 & 1.41 & 67.05 & -219.18 & 2692.81 & 6.71 & 0.14 & 2.16 & 1.37 \\
\hline 7548.48 & 332471.60 & 17.00 & 277.46 & 20.76 & -1.36 & -0.47 & 60.38 & -210.54 & 2983.80 & 6.31 & 0.29 & 2.00 & 1.25 \\
\hline 7548.54 & 323065.40 & 17.00 & 291.45 & 15.22 & -0.56 & -0.42 & 57.82 & -170.41 & 5262.79 & 5.91 & 0.36 & 1.87 & 1.13 \\
\hline 7548.61 & 317136.60 & 17.00 & 289.57 & 19.36 & -0.43 & 3.12 & 52.52 & -159.17 & 4943.06 & 5.82 & 0.80 & 1.62 & 1.26 \\
\hline 7548.67 & 313423.40 & 17.00 & 266.22 & 20.04 & 2.62 & 0.55 & 49.64 & -126.35 & 3817.39 & 5.53 & 0.80 & 1.57 & 1.32 \\
\hline 7548.74 & 310812.60 & 17.00 & 271.04 & 24.65 & 4.21 & 5.05 & 43.15 & -120.86 & 3510.76 & 5.13 & 0.99 & 1.49 & 1.42 \\
\hline 7548.81 & 315734.60 & 17.00 & 260.27 & 26.41 & 3.91 & 6.28 & 37.92 & -111.62 & 2917.06 & 5.14 & 0.84 & 1.50 & 1.47 \\
\hline 7548.87 & 324982.60 & 17.00 & 244.47 & 32.45 & 3.88 & 2.33 & 35.32 & -141.15 & 680.28 & 5.51 & 0.87 & 1.57 & 1.65 \\
\hline 7548.94 & 333875.80 & 17.00 & 233.70 & 34.47 & 3.60 & -0.42 & 33.01 & -139.73 & -44.68 & 5.56 & 0.60 & 1.67 & 2.10 \\
\hline 7549.00 & 334745.40 & 17.00 & 201.07 & 37.09 & 1.60 & -2.99 & 29.64 & -153.72 & 852.07 & 5.19 & 0.59 & 1.53 & 5.21 \\
\hline 7549.07 & 327648.20 & 17.20 & 187.55 & 36.25 & -0.23 & -4.67 & 33.22 & -149.82 & 1205.88 & 5.00 & 0.62 & 1.66 & 8.04 \\
\hline
\end{tabular}




\begin{tabular}{|c|c|c|c|c|c|c|c|c|c|c|c|c|c|}
\hline Depth & $\mathrm{XRF}$ & XRF Live Time & $\mathrm{Nb}(\mathrm{ppm})$ & Mo (ppm) & $\mathrm{Ba}(\mathrm{ppm})$ & $\mathrm{Pb}(\mathrm{ppm})$ & Th (ppm) & $\mathrm{U}(\mathrm{ppm})$ & $\mathrm{P}(\mathrm{ppm})$ & $\mathrm{S}(\%)$ & $\mathrm{Cl}(\%)$ & $\mathrm{K}(\%)$ & $\mathrm{Ca}(\%)$ \\
\hline 7549.13 & 340994.00 & 17.00 & 174.14 & 39.00 & 0.62 & -7.24 & 29.77 & -176.31 & 2238.66 & 5.79 & 0.61 & 1.74 & 8.5 \\
\hline 7549.20 & 347242.80 & 17.00 & 165.25 & 36.45 & -1.07 & -5.99 & 30.40 & -165.99 & 4022.81 & 5.59 & 0.51 & 1.85 & 8.89 \\
\hline 7549.27 & 348707.00 & 17.00 & 181.32 & 32.80 & -2.44 & -5.40 & 33.32 & -134.80 & 5632.98 & 5.46 & 0.34 & 1.99 & 8.74 \\
\hline 7549.33 & 366044.60 & 16.80 & 202.90 & 31.08 & -1.50 & -1.12 & 30.59 & -138.53 & 4183.43 & 5.81 & 0.33 & 2.12 & 5.98 \\
\hline 7549.40 & 402872.60 & 16.40 & 191.30 & 42.01 & -0.79 & -2.50 & 27.69 & -158.04 & 6952.95 & 7.53 & 0.47 & 2.19 & 3.85 \\
\hline 7549.46 & 393799.60 & 16.60 & 178.59 & 42.67 & -1.06 & -1.34 & 30.28 & -135.29 & 6544.45 & 6.73 & 0.47 & 2.21 & 3.99 \\
\hline 7549.53 & 348055.80 & 17.00 & 184.82 & 56.15 & 1.54 & 3.62 & 28.86 & -111.70 & 6087.90 & 6.33 & 0.48 & 2.03 & 4.02 \\
\hline 7549.59 & 321705.40 & 17.20 & 163.49 & 47.29 & 2.88 & 2.22 & 24.43 & -126.52 & 4881.34 & 6.15 & 0.58 & 1.75 & 3.85 \\
\hline 7549.66 & 283191.40 & 17.60 & 150.86 & 45.45 & 2.23 & 2.24 & 22.41 & -124.53 & 4897.49 & 6.18 & 0.99 & 1.54 & 3.80 \\
\hline 7549.72 & 229998.00 & 18.00 & 159.01 & 28.94 & 1.61 & 3.45 & 22.04 & -78.35 & 1764.07 & 4.60 & 0.82 & 1.36 & 3.30 \\
\hline 7549.79 & 229603.00 & 18.00 & 194.26 & 27.60 & 4.02 & 2.72 & 22.32 & -57.49 & 2287.92 & 4.75 & 1.06 & 1.31 & 2.90 \\
\hline 7549.86 & 273021.00 & 17.60 & 195.97 & 21.44 & 2.59 & -3.72 & 22.80 & -66.36 & 909.58 & 5.24 & 1.34 & 1.39 & 2.64 \\
\hline 7549.92 & 290796.80 & 17.40 & 192.61 & 31.48 & 1.96 & -2.01 & 26.14 & -81.00 & 1835.89 & 5.15 & 1.33 & 1.57 & 2.89 \\
\hline 7549.99 & 317410.80 & 17.20 & 210.83 & 38.09 & 1.84 & -1.51 & 35.18 & -76.88 & 3301.72 & 4.79 & 1.41 & 1.65 & 3.41 \\
\hline 7550.05 & 374548.40 & 16.60 & 230.83 & 50.12 & 1.90 & -0.02 & 40.68 & -151.92 & 14882.55 & 6.96 & 1.49 & 1.77 & 4.51 \\
\hline 7550.12 & 377823.00 & 16.60 & 220.38 & 48.51 & 0.02 & 1.88 & 43.12 & -164.80 & 14262.66 & 7.57 & 2.99 & 1.72 & 4.57 \\
\hline 7550.18 & 374629.80 & 16.60 & 205.48 & 48.00 & 1.92 & 5.23 & 43.41 & -152.31 & 15416.83 & 7.60 & 3.41 & 1.70 & 4.45 \\
\hline 7550.25 & 358972.40 & 16.80 & 209.09 & 45.37 & 2.70 & 6.94 & 43.45 & -144.58 & 14545.52 & 7.56 & 3.65 & 1.64 & 3.96 \\
\hline 7550.31 & 353442.60 & 16.80 & 206.28 & 41.10 & 3.11 & 5.23 & 38.27 & -156.72 & 14050.91 & 7.69 & 3.49 & 1.61 & 3.04 \\
\hline 7550.38 & 300396.60 & 17.40 & 171.04 & 25.40 & 7.76 & 3.50 & 31.99 & -72.60 & 3884.79 & 5.55 & 5.02 & 1.34 & 1.70 \\
\hline 7550.45 & 266541.60 & 17.60 & 142.43 & 28.26 & 7.70 & 0.27 & 26.79 & -68.56 & 4268.88 & 4.62 & 4.44 & 1.25 & 1.54 \\
\hline 7550.51 & 283958.00 & 17.40 & 184.76 & 27.55 & 15.96 & -2.21 & 32.52 & -91.66 & 5708.09 & 5.18 & 4.55 & 1.36 & 1.55 \\
\hline 7550.58 & 306041.00 & 17.20 & 180.15 & 31.72 & 15.62 & -4.54 & 33.29 & -97.59 & 7027.65 & 5.87 & 4.63 & 1.43 & 1.94 \\
\hline 7550.64 & 294468.00 & 17.20 & 163.29 & 28.05 & 15.09 & -4.83 & 33.67 & -58.71 & 6474.32 & 5.76 & 6.60 & 1.38 & 2.65 \\
\hline 7550.71 & 309010.20 & 17.00 & 176.71 & 33.02 & 10.30 & -4.97 & 33.07 & -76.59 & 6453.38 & 5.46 & 4.78 & 1.50 & 2.80 \\
\hline 7550.77 & 309347.60 & 17.00 & 187.28 & 37.99 & 10.65 & -5.44 & 33.37 & -82.80 & 6832.69 & 5.83 & 4.37 & 1.58 & 2.97 \\
\hline 7550.84 & 263291.00 & 17.40 & 154.89 & 23.90 & 0.16 & -2.31 & 28.55 & -78.79 & 3791.55 & 5.02 & 5.24 & 1.36 & 2.89 \\
\hline 7550.91 & 250985.20 & 17.60 & 193.46 & 20.12 & 18.68 & 0.25 & 30.44 & -81.37 & 4147.60 & 4.96 & 5.19 & 1.47 & 3.02 \\
\hline 7550.97 & 220401.20 & 18.00 & 167.00 & 45.68 & 313.18 & 6.87 & 25.60 & -71.32 & 3680.70 & 4.67 & 3.12 & 1.66 & 2.77 \\
\hline 7551.04 & 211738.00 & 18.20 & 140.24 & 57.70 & 312.51 & 7.50 & 20.96 & -67.10 & -778.61 & 4.13 & 3.13 & 1.48 & 12.46 \\
\hline 7551.10 & 176997.00 & 18.60 & 107.61 & 44.16 & 388.41 & 19.03 & 19.40 & -48.14 & -1232.36 & 3.08 & 6.35 & 1.19 & 12.06 \\
\hline 7551.17 & 209006.20 & 18.40 & 74.47 & 60.32 & 389.31 & 12.90 & 14.27 & -25.16 & -6736.05 & 2.21 & 4.75 & 1.05 & 25.01 \\
\hline 7551.23 & 219358.20 & 18.20 & 14.40 & 62.52 & 371.17 & 9.49 & 5.98 & 9.95 & -9356.45 & 1.22 & 5.57 & 0.65 & 36.00 \\
\hline
\end{tabular}




\begin{tabular}{|c|c|c|c|c|c|c|c|c|c|c|c|c|c|}
\hline Depth & $\mathrm{XRF}$ & XRF Live Time & $\mathrm{Nb}(\mathrm{ppm})$ & Mo (ppm) & $\mathrm{Ba}(\mathrm{ppm})$ & $\mathrm{Pb}(\mathrm{ppm})$ & Th (ppm) & $\mathrm{U}(\mathrm{ppm})$ & $\mathrm{P}(\mathrm{ppm})$ & $\mathrm{S}(\%)$ & $\mathrm{Cl}(\%)$ & $\mathrm{K}(\%)$ & $\mathrm{Ca}(\%)$ \\
\hline 7551.30 & 264826.60 & 17.80 & 16.39 & 47.66 & 76.95 & -0.72 & 5.73 & -5.11 & -6362.78 & 0.73 & 6.02 & 0.27 & 47.35 \\
\hline 7551.36 & 286170.20 & 17.60 & 14.62 & 41.81 & 78.60 & -2.62 & 5.19 & 0.25 & -4790.71 & 0.83 & 6.78 & 0.25 & 50.26 \\
\hline 7551.43 & 347045.00 & 17.00 & 15.37 & 50.86 & 1.69 & -17.38 & 4.21 & -3.61 & -8183.93 & 0.97 & 4.47 & 0.31 & 61.58 \\
\hline 7551.50 & 336487.20 & 17.00 & 13.81 & 48.97 & 2.12 & -16.32 & 3.73 & -7.85 & -1207.61 & 1.00 & 4.58 & 0.26 & 62.58 \\
\hline 7551.56 & 319662.20 & 17.20 & 15.29 & 60.42 & 2.44 & -14.50 & 4.71 & -13.33 & -3919.71 & 1.02 & 6.71 & 0.23 & 60.64 \\
\hline 7551.63 & 294543.20 & 17.40 & 15.27 & 54.34 & 2.38 & -11.43 & 2.48 & -11.64 & -4168.81 & 0.93 & 9.12 & 0.19 & 58.60 \\
\hline 7551.69 & 274451.40 & 17.60 & 14.25 & 68.48 & 1.48 & -14.79 & 4.72 & -10.85 & -2750.01 & 0.88 & 9.24 & 0.19 & 58.42 \\
\hline 7551.76 & 244490.20 & 17.80 & 13.30 & 87.60 & 1.49 & -6.42 & 1.86 & -11.48 & 5766.86 & 0.88 & 10.27 & 0.17 & 57.87 \\
\hline 7551.82 & 272499.80 & 17.60 & 12.39 & 95.30 & 2.12 & -3.92 & 0.70 & -5.26 & 6515.92 & 0.78 & 10.08 & 0.15 & 59.10 \\
\hline 7551.89 & 311986.60 & 17.20 & 10.37 & 97.93 & 1.57 & -6.91 & 0.70 & -2.53 & 9290.79 & 0.66 & 6.81 & 0.14 & 65.59 \\
\hline 7551.96 & 352636.80 & 16.80 & 8.40 & 92.22 & 1.78 & -6.80 & 2.28 & 2.78 & 6093.26 & 0.61 & 3.72 & 0.13 & 71.46 \\
\hline 7552.02 & 384748.60 & 16.40 & 7.94 & 75.20 & 1.93 & -7.33 & 2.10 & 0.48 & 6460.99 & 0.55 & 3.03 & 0.13 & 73.77 \\
\hline 7552.09 & 432131.60 & 16.00 & 6.40 & 49.08 & 307.96 & -8.98 & 6.56 & 4.89 & -1777.59 & 0.50 & 0.34 & 0.12 & 78.47 \\
\hline 7552.15 & 425567.60 & 16.00 & 6.01 & 49.14 & 308.27 & -14.12 & 6.26 & -24.20 & -6255.17 & 1.36 & 0.33 & 0.18 & 77.08 \\
\hline 7552.22 & 405503.40 & 16.20 & 4.77 & 31.59 & 426.45 & -11.31 & 7.08 & -24.29 & -7136.90 & 1.73 & 1.69 & 0.20 & 71.09 \\
\hline 7552.28 & 420282.40 & 16.00 & 6.37 & 45.02 & 425.82 & -11.62 & 7.56 & -38.77 & -8372.20 & 2.18 & 1.98 & 0.20 & 67.89 \\
\hline 7552.35 & 421771.60 & 16.00 & 16.29 & 62.83 & 426.71 & -7.54 & 9.61 & -33.89 & -6247.73 & 3.70 & 8.73 & 0.21 & 57.08 \\
\hline 7552.41 & 385592.40 & 16.40 & 47.11 & 68.45 & 121.41 & -6.79 & 12.06 & -55.03 & -4873.47 & 4.66 & 9.30 & 0.64 & 42.34 \\
\hline 7552.48 & 383992.60 & 16.40 & 121.95 & 59.61 & 119.79 & 1.55 & 21.55 & -39.18 & -634.81 & 4.93 & 10.41 & 1.03 & 29.01 \\
\hline 7552.55 & 395860.80 & 16.20 & 172.24 & 74.98 & 1.60 & 1.82 & 27.89 & -61.78 & 1756.20 & 5.68 & 11.20 & 1.37 & 19.20 \\
\hline 7552.61 & 379185.60 & 16.40 & 242.06 & 71.54 & 2.12 & 3.98 & 37.02 & -70.25 & 3675.89 & 6.53 & 11.11 & 1.85 & 7.19 \\
\hline 7552.68 & 363977.80 & 16.60 & 273.95 & 55.17 & 1.48 & 7.59 & 40.49 & -74.73 & 1175.22 & 6.24 & 6.36 & 2.12 & 3.22 \\
\hline 7552.74 & 383223.20 & 16.40 & 266.81 & 59.97 & 2.52 & 3.05 & 43.94 & -67.32 & 3320.03 & 5.89 & 7.27 & 2.08 & 5.01 \\
\hline 7552.81 & 324156.20 & 17.00 & 195.12 & 63.57 & 3.00 & 4.49 & 37.07 & -46.81 & 641.75 & 4.89 & 12.83 & 1.72 & 4.24 \\
\hline 7552.87 & 304320.60 & 17.20 & 145.93 & 62.80 & 2.98 & 5.75 & 30.35 & -14.84 & -913.70 & 3.98 & 14.89 & 1.72 & 3.78 \\
\hline 7552.94 & 278620.00 & 17.40 & 95.87 & 57.24 & 2.95 & 2.23 & 27.10 & -4.58 & -1027.59 & 3.12 & 17.59 & 1.40 & 3.60 \\
\hline 7553.01 & 228668.00 & 17.80 & 79.57 & 79.65 & 5.02 & 4.34 & 26.24 & -27.71 & -1626.14 & 2.31 & 15.57 & 1.51 & 3.90 \\
\hline 7553.07 & 226510.40 & 17.80 & 57.62 & 100.12 & 281.51 & 11.83 & 19.22 & -6.01 & -1607.50 & 2.42 & 14.08 & 1.48 & 1.94 \\
\hline 7553.14 & 271922.40 & 17.40 & 88.47 & 91.71 & 282.28 & 10.12 & 24.94 & -28.91 & 585.52 & 3.18 & 7.40 & 1.68 & 2.50 \\
\hline 7553.20 & 279140.40 & 17.40 & 121.65 & 81.73 & 281.43 & 8.58 & 30.70 & -32.53 & 1940.51 & 4.13 & 3.36 & 1.64 & 2.74 \\
\hline 7553.27 & 272129.20 & 17.60 & 117.61 & 78.79 & 282.03 & 13.63 & 28.86 & -29.41 & 2291.06 & 4.20 & 1.40 & 1.72 & 2.51 \\
\hline 7553.33 & 322677.00 & 17.20 & 140.45 & 50.31 & 279.55 & 11.57 & 30.00 & -3.79 & 3367.13 & 4.71 & 2.27 & 1.66 & 2.09 \\
\hline 7553.40 & 337060.80 & 17.00 & 201.51 & 30.32 & 2.12 & 9.33 & 41.34 & -31.69 & 2688.57 & 5.19 & 3.62 & 1.65 & 1.81 \\
\hline
\end{tabular}




\begin{tabular}{|c|c|c|c|c|c|c|c|c|c|c|c|c|c|}
\hline Depth & $\mathrm{XRF}$ & XRF Live Time & $\mathrm{Nb}(\mathrm{ppm})$ & Mo (ppm) & $\mathrm{Ba}(\mathrm{ppm})$ & $\mathrm{Pb}(\mathrm{ppm})$ & Th (ppm) & $\mathrm{U}(\mathrm{ppm})$ & $\mathrm{P}(\mathrm{ppm})$ & $\mathrm{S}(\%)$ & $\mathrm{Cl}(\%)$ & $\mathrm{K}(\%)$ & $\mathrm{Ca}(\%)$ \\
\hline 7553.46 & 346883.60 & 16.80 & 186.88 & 31.89 & -0.90 & 7.12 & 40.69 & -39.48 & 3149.43 & 6.16 & 4.37 & 1.84 & 1.48 \\
\hline 7553.53 & 346374.00 & 16.80 & 196.05 & 29.03 & -0.86 & 5.66 & 41.84 & -64.82 & 2684.02 & 5.73 & 5.44 & 1.91 & 1.53 \\
\hline 7553.60 & 349327.80 & 16.80 & 209.61 & 25.04 & -1.18 & 3.65 & 42.16 & -43.74 & 3389.29 & 5.68 & 9.85 & 1.81 & 1.53 \\
\hline 7553.66 & 315589.80 & 17.00 & 163.69 & 32.61 & -1.83 & 1.20 & 34.95 & -33.48 & 2560.62 & 4.85 & 13.87 & 1.71 & 1.27 \\
\hline 7553.73 & 291206.60 & 17.40 & 117.29 & 24.50 & -2.40 & -1.15 & 25.65 & -46.34 & 2093.68 & 3.97 & 12.90 & 1.71 & 1.16 \\
\hline 7553.79 & 270577.00 & 17.60 & 135.98 & 21.89 & -0.45 & 0.93 & 25.36 & -38.86 & 2106.47 & 2.68 & 12.16 & 1.53 & 1.13 \\
\hline 7553.86 & 271992.00 & 17.60 & 135.27 & 21.53 & 0.86 & 1.27 & 24.29 & -61.28 & 2223.88 & 2.97 & 10.91 & 1.65 & 1.58 \\
\hline 7553.92 & 257876.00 & 17.60 & 117.31 & 28.99 & 0.62 & 3.99 & 20.26 & -73.10 & 1200.54 & 2.83 & 5.62 & 1.76 & 2.22 \\
\hline 7553.99 & 262259.60 & 17.60 & 133.62 & 23.80 & 0.93 & 0.97 & 24.50 & -102.23 & 367.19 & 3.25 & 0.59 & 1.92 & 2.80 \\
\hline 7554.06 & 244140.00 & 17.60 & 151.23 & 33.76 & 1.40 & 1.67 & 23.04 & -95.25 & 1290.00 & 3.50 & 0.59 & 1.99 & 4.08 \\
\hline 7554.12 & 232917.00 & 17.80 & 111.67 & 49.04 & 178.97 & 4.62 & 17.47 & -77.25 & 1622.77 & 3.57 & 0.61 & 2.07 & 5.90 \\
\hline 7554.19 & 172498.20 & 18.40 & 70.98 & 90.63 & 180.65 & 3.11 & 13.14 & -45.13 & -1977.66 & 2.61 & 2.40 & 1.59 & 6.07 \\
\hline 7554.25 & 183970.40 & 18.40 & 71.02 & 88.02 & 180.42 & -2.39 & 12.87 & -58.42 & -3646.61 & 2.58 & 4.06 & 1.39 & 11.68 \\
\hline 7554.32 & 199989.60 & 18.20 & 62.52 & 91.13 & 180.18 & -2.46 & 10.58 & -52.44 & 867.85 & 2.23 & 5.36 & 1.09 & 17.76 \\
\hline 7554.38 & 218575.00 & 18.20 & 35.18 & 84.72 & 180.45 & -5.67 & 9.58 & -30.66 & -727.79 & 1.77 & 8.22 & 0.67 & 22.97 \\
\hline 7554.45 & 228355.60 & 18.20 & 38.02 & 69.72 & 2.49 & -12.61 & 8.89 & -35.35 & -898.36 & 1.49 & 10.55 & 0.28 & 27.26 \\
\hline 7554.51 & 285740.80 & 17.60 & 37.31 & 80.12 & 198.18 & -1.93 & 6.63 & -22.66 & 2685.70 & 2.81 & 9.02 & 0.78 & 29.47 \\
\hline 7554.58 & 301712.60 & 17.40 & 40.87 & 86.72 & 197.58 & -1.67 & 7.58 & -15.72 & 5436.23 & 2.99 & 9.48 & 0.92 & 29.62 \\
\hline 7554.65 & 305783.20 & 17.40 & 78.43 & 88.65 & 198.15 & 0.33 & 9.51 & -21.39 & 3397.32 & 3.56 & 10.97 & 1.03 & 26.42 \\
\hline 7554.71 & 308088.80 & 17.20 & 133.77 & 89.59 & 197.84 & 2.90 & 10.58 & -17.08 & 5119.70 & 4.54 & 9.47 & 1.41 & 20.50 \\
\hline 7554.78 & 306908.00 & 17.20 & 176.26 & 89.44 & 197.82 & 5.65 & 12.83 & -16.96 & 3403.89 & 5.00 & 9.93 & 1.75 & 14.81 \\
\hline 7554.84 & 314530.00 & 17.20 & 184.23 & 40.90 & -0.04 & -7.88 & 14.08 & -18.50 & 1254.06 & 3.91 & 12.53 & 1.27 & 19.92 \\
\hline 7554.91 & 316759.60 & 17.20 & 176.25 & 35.60 & 0.86 & -6.99 & 12.64 & -16.72 & 785.58 & 3.55 & 11.36 & 1.09 & 22.62 \\
\hline 7554.97 & 324904.00 & 17.20 & 133.64 & 35.42 & 1.71 & -9.88 & 9.29 & -2.62 & 2712.99 & 2.84 & 8.71 & 0.89 & 29.88 \\
\hline 7555.04 & 272998.60 & 17.80 & 79.42 & 56.78 & 9.50 & -5.74 & 9.27 & 13.14 & 1115.22 & 1.71 & 10.79 & 0.48 & 29.15 \\
\hline 7555.10 & 284978.20 & 17.60 & 60.24 & 51.95 & 10.00 & -6.93 & 9.27 & 4.21 & 2303.78 & 1.08 & 8.01 & 0.38 & 33.53 \\
\hline 7555.17 & 261512.20 & 17.80 & 56.28 & 57.16 & 11.06 & -2.41 & 9.72 & -2.96 & 4707.11 & 0.83 & 7.13 & 0.48 & 30.01 \\
\hline 7555.24 & 202914.60 & 18.20 & 49.39 & 69.04 & 14.13 & 1.45 & 12.61 & -35.61 & 2285.81 & 0.90 & 6.74 & 1.11 & 20.69 \\
\hline 7555.30 & 178524.40 & 18.40 & 44.46 & 83.12 & 14.21 & 8.14 & 12.30 & -32.08 & -1633.74 & 1.29 & 6.58 & 1.86 & 9.97 \\
\hline 7555.37 & 199978.20 & 18.20 & 39.43 & 63.27 & 5.74 & 3.76 & 10.82 & -44.58 & -2624.74 & 1.53 & 5.39 & 2.36 & 9.85 \\
\hline 7555.43 & 171936.40 & 18.40 & 35.51 & 62.46 & 6.47 & 4.42 & 10.89 & -34.76 & -4036.10 & 1.54 & 6.46 & 2.31 & 5.29 \\
\hline 7555.50 & 178870.20 & 18.40 & 100.44 & 52.00 & 6.24 & 5.13 & 15.06 & -34.09 & -4203.65 & 2.09 & 4.83 & 2.49 & 1.66 \\
\hline 7555.56 & 225019.20 & 18.00 & 173.69 & 38.33 & 2.34 & 3.17 & 17.85 & -1.18 & -1480.99 & 2.29 & 4.22 & 2.16 & 2.78 \\
\hline
\end{tabular}




\begin{tabular}{|c|c|c|c|c|c|c|c|c|c|c|c|c|c|}
\hline Depth & XRF & XRF Live Time & $\mathrm{Nb}(\mathrm{ppm})$ & Mo (ppm) & $\mathrm{Ba}(\mathrm{ppm})$ & $\mathrm{Pb}(\mathrm{ppm})$ & Th (ppm) & $\mathrm{U}(\mathrm{ppm})$ & $\mathrm{P}(\mathrm{ppm})$ & $\mathrm{S}(\%)$ & $\mathrm{Cl}(\%)$ & $\mathrm{K}(\%)$ & $\mathrm{Ca}(\%)$ \\
\hline 7555.63 & 234912.00 & 18.00 & 230.32 & 13.95 & 1.56 & -0.38 & 23.81 & -18.30 & -289.91 & 2.27 & 4.27 & 1.65 & 3.81 \\
\hline 7555.70 & 256553.40 & 17.80 & 281.71 & 6.25 & 2.54 & 2.48 & 28.58 & -34.58 & 1595.42 & 2.42 & 2.03 & 1.37 & 5.01 \\
\hline 7555.76 & 274370.20 & 17.80 & 342.75 & 9.48 & 1.61 & 5.34 & 35.26 & -42.97 & 3271.15 & 2.79 & 0.98 & 1.49 & 6.29 \\
\hline 7555.83 & 271544.40 & 17.80 & 319.48 & 16.16 & 1.04 & 4.79 & 35.26 & -49.17 & 2604.16 & 2.46 & 0.69 & 1.37 & 8.30 \\
\hline 7555.89 & 273309.40 & 17.80 & 268.65 & 12.42 & 1.77 & -2.38 & 31.82 & -72.33 & 3510.91 & 3.78 & 0.98 & 1.10 & 10.30 \\
\hline 7555.96 & 275566.20 & 17.60 & 304.67 & 16.71 & 2.32 & -0.84 & 31.24 & -62.91 & 3197.47 & 4.14 & 1.21 & 1.22 & 11.33 \\
\hline 7556.02 & 290284.40 & 17.40 & 362.97 & 15.69 & 1.49 & -3.91 & 29.69 & -56.54 & 4385.27 & 4.42 & 0.84 & 1.33 & 11.96 \\
\hline 7556.09 & 296820.40 & 17.20 & 306.84 & 26.73 & 3.22 & -10.63 & 23.20 & -40.75 & 6003.32 & 4.89 & 3.47 & 1.09 & 15.59 \\
\hline 7556.15 & 303572.00 & 17.00 & 308.90 & 24.09 & 3.79 & -9.92 & 20.99 & -39.35 & 7172.39 & 5.20 & 3.95 & 1.17 & 13.96 \\
\hline 7556.22 & 314484.00 & 17.00 & 383.00 & 27.52 & 3.61 & -5.41 & 23.34 & -34.03 & 4297.81 & 4.29 & 3.76 & 1.81 & 12.58 \\
\hline 7556.29 & 318397.00 & 17.00 & 323.57 & 27.32 & 3.78 & -6.47 & 21.46 & -57.08 & 2680.11 & 4.16 & 3.53 & 1.94 & 11.95 \\
\hline 7556.35 & 325544.00 & 17.00 & 290.24 & 33.18 & 5.17 & -6.63 & 23.90 & -89.59 & 1482.57 & 4.59 & 3.52 & 2.18 & 12.79 \\
\hline 7556.42 & 306111.20 & 17.20 & 371.87 & 20.20 & 2.95 & 1.58 & 25.92 & -101.64 & -1384.40 & 4.17 & 5.32 & 2.29 & 8.69 \\
\hline 7556.48 & 318911.60 & 17.20 & 327.32 & 14.88 & 2.17 & 0.60 & 22.30 & -125.24 & 368.77 & 6.41 & 6.59 & 2.04 & 7.68 \\
\hline 7556.55 & 289246.40 & 17.40 & 230.50 & 7.60 & 2.34 & 4.64 & 17.52 & -105.37 & 3175.14 & 5.63 & 7.93 & 1.33 & 8.11 \\
\hline 7556.61 & 268039.20 & 17.60 & 197.48 & 8.42 & 0.82 & 3.76 & 14.93 & -76.83 & 5411.45 & 5.07 & 10.15 & 0.84 & 8.48 \\
\hline 7556.68 & 238536.40 & 17.80 & 120.85 & 7.15 & 0.26 & 2.93 & 7.12 & -45.73 & 4956.97 & 4.38 & 12.81 & 0.28 & 6.42 \\
\hline 7556.75 & 250483.20 & 17.80 & 15.10 & 18.72 & -0.65 & -4.13 & 3.22 & -41.00 & 5412.10 & 3.99 & 14.51 & 0.08 & 11.78 \\
\hline 7556.81 & 249865.60 & 17.80 & 13.73 & 25.73 & -0.54 & -7.05 & 2.87 & -7.41 & 3528.14 & 1.14 & 13.29 & 0.08 & 22.64 \\
\hline 7556.88 & 285911.60 & 17.60 & 14.09 & 38.24 & -0.01 & -13.95 & 1.90 & -6.19 & 1027.53 & 1.04 & 13.12 & 0.09 & 33.62 \\
\hline 7556.94 & 324541.60 & 17.20 & 14.09 & 48.34 & 0.69 & -17.00 & 1.94 & -6.95 & -213.32 & 0.93 & 11.58 & 0.07 & 45.78 \\
\hline 7557.01 & 360147.80 & 17.00 & 14.91 & 54.89 & -0.97 & -18.49 & 3.83 & -2.56 & 171.55 & 0.51 & 9.06 & 0.07 & 57.69 \\
\hline
\end{tabular}


Table D-7, MSCL Mining Suite:

\begin{tabular}{|c|c|c|c|c|c|c|c|c|c|c|c|}
\hline True Depth & XRF tCOUNTS & XRF Live Time & $\mathrm{Ti}(\mathrm{ppm})$ & $\mathrm{V}(\mathrm{ppm})$ & $\mathrm{Fe}(\mathrm{ppm})$ & $\mathrm{Ni}(\mathrm{ppm})$ & $\mathrm{Cu}(\mathrm{ppm})$ & $\mathrm{Pb}(\mathrm{ppm})$ & $\mathrm{LE}(\mathrm{ppm})$ & $\mathrm{Mg}(\mathrm{ppm})$ & $\mathrm{Cl}(\mathrm{ppm})$ \\
\hline $\mathrm{Ft}$ & Total Counts & secs & $\mathrm{ppm}$ & $\mathrm{ppm}$ & $\mathrm{ppm}$ & $\mathrm{ppm}$ & ppm & ppm & $\mathrm{ppm}$ & $\mathrm{ppm}$ & $\mathrm{ppm}$ \\
\hline 7445.07 & 170922 & 9 & 1597.2 & 5088.57 & 34184.5 & 5378.53 & 14024.57 & 821.93 & 459570.41 & 0 & 303946.67 \\
\hline 7445.13 & 165018 & 9 & 1654.09 & 6005.35 & 42607.76 & 6511.16 & 17017.13 & 1039.31 & 455609.84 & 0 & 132384.62 \\
\hline 7445.20 & 273658 & 7 & 1481.46 & 1723.83 & 14393.68 & 1053.71 & 2772.97 & 150.43 & 281487.36 & 0 & 388742.65 \\
\hline 7445.26 & 335892 & 7 & 339.14 & 728.82 & 5282.67 & 435.12 & 1083.76 & 56.4 & 332717.9 & 0 & 520114.24 \\
\hline 7445.33 & 177759 & 9 & 1706.06 & 5723.97 & 34623.91 & 5447.48 & 14211.09 & 840.1 & 476421.98 & 0 & 315058.62 \\
\hline 7445.39 & 94792 & 9 & 1635.23 & 5306.94 & 32619.97 & 4778.94 & 12450.96 & 725.42 & 475688.82 & 0 & 376509.15 \\
\hline 7445.46 & 144070 & 9 & 1997.35 & 6735.64 & 41025.35 & 7588.48 & 19748.36 & 1260.13 & 527104.64 & 0 & 15067.26 \\
\hline 7445.52 & 176220 & 9 & 1795.51 & 6179.59 & 40849.98 & 6485.58 & 16854.93 & 1023.99 & 460070.21 & 0 & 159035.04 \\
\hline 7445.59 & 160496 & 9 & 1749.01 & 6447.33 & 42627.1 & 7410.99 & 19329.06 & 1231.11 & 488567 & $\overline{0}$ & 0 \\
\hline 7445.66 & 162045 & 9 & 1698.35 & 6737.92 & 44581.36 & 7898.59 & 20578.98 & 1317.3 & 470875.4 & 0 & 0 \\
\hline 7445.72 & 196806 & 8 & 1110.77 & 5911.32 & 40438.64 & 6400.35 & 16679.42 & 1006.81 & 420790.68 & 0 & 280516.45 \\
\hline 7445.79 & 172408 & 9 & 1476.37 & 6210.43 & 48935.63 & 7194.67 & 18775.83 & 1177.71 & 461768.56 & 0 & 35183.74 \\
\hline 7445.85 & 169505 & 9 & 1517.23 & 5987.3 & 50542.43 & 7219.91 & 18806.3 & 1179.78 & 453713.77 & 0 & 0 \\
\hline 7445.92 & 177975 & 9 & 1366.08 & 6496.18 & 51014.52 & 7602.91 & 19844.83 & 1252.85 & 451727.33 & 0 & 21898.48 \\
\hline 7445.98 & 187336 & 8 & 1533.1 & 5601.71 & 48917.43 & 6145.9 & 16025.88 & 973.49 & 438023.88 & 0 & 172412.48 \\
\hline 7446.05 & 173576 & 9 & 2148.14 & 6079.08 & 51501.71 & 7582.54 & 19894.7 & 1269.24 & 479302 & 0 & 0 \\
\hline 7446.12 & 169737 & 9 & 1470.45 & 5790.98 & 54358.26 & 7158.86 & 18723.11 & 1178.86 & 465957.1 & 0 & 22523.71 \\
\hline 7446.18 & 165540 & 9 & 1458.13 & 6226.15 & 51050.21 & 7519.99 & 19709.26 & 1245.04 & 465385.77 & 0 & 22003.48 \\
\hline 7446.25 & 173445 & 9 & 1092.11 & 6023.74 & 47923.09 & 6480.79 & 17002.47 & 1035.71 & 460741.32 & 0 & 183563.71 \\
\hline 7446.31 & 173943 & 9 & 1636.49 & 6355.46 & 53092.18 & 7583.1 & 19866.68 & 1248.04 & 439440.56 & 0 & 55169.27 \\
\hline 7446.38 & 176698 & 9 & 1149.48 & 6178.16 & 54435.97 & 7441.29 & 19358.51 & 1203.71 & 332330.45 & 77030.33 & 0 \\
\hline 7446.44 & 168545 & 9 & 1561.43 & 6380.42 & 52765.1 & 7835.4 & 20498.18 & 1303.36 & 467899.07 & 0 & 0 \\
\hline 7446.51 & 170287 & 9 & 1073.82 & 6054.79 & 48649.52 & 7191.11 & 18747.45 & 1178.67 & 474185.14 & 0 & 12568.38 \\
\hline 7446.57 & 165904 & 9 & 1732.41 & 5917.52 & 53411.77 & 7401.75 & 19308.26 & 1218.88 & 472116.31 & 0 & 15027.12 \\
\hline 7446.64 & 218623 & 8 & 927.24 & 5043.22 & 39513.68 & 5377.63 & 13951.9 & 820.88 & 402567.8 & 0 & 363726.98 \\
\hline 7446.71 & 121559 & 9 & 1065.4 & 6337.98 & 43742.66 & 7773.73 & 20338.05 & 1318.63 & 558955.45 & 0 & 9316.79 \\
\hline 7446.77 & 170805 & 9 & 1472.47 & 7020.65 & 47581.49 & 8145.79 & 21303.95 & 1345.48 & 358954.25 & 57199.93 & 0 \\
\hline 7446.84 & 169237 & 9 & 1281.29 & 7041.47 & 44097.45 & 8388.42 & 21940.71 & 1407.29 & 450436.94 & 0 & 0 \\
\hline 7446.90 & 263464 & 8 & 475.6 & 3054.52 & 17874.67 & 2774.32 & 7202.12 & 402.04 & 383828.49 & 0 & 493213.73 \\
\hline 7446.97 & 160042 & 9 & 1367.73 & 6313.47 & 44947.05 & 7484.85 & 19519.42 & 1236.78 & 476931.1 & 0 & 0 \\
\hline 7447.03 & 52256 & 10 & 2849.95 & 0 & 26683.76 & 2533.1 & 0 & 359.93 & 8064.2 & 378085.33 & 395543.79 \\
\hline 7447.10 & 130645 & 9 & 1828.52 & 6291.8 & 44811.57 & 7141.02 & 18692.45 & 1169.14 & 461518.94 & 0 & 0 \\
\hline
\end{tabular}




\begin{tabular}{|c|c|c|c|c|c|c|c|c|c|c|c|}
\hline True Depth & XRF tCOUNTS & XRF Live Time & $\mathrm{Ti}(\mathrm{ppm})$ & V (ppm) & $\mathrm{Fe}(\mathrm{ppm})$ & $\mathrm{Ni}(\mathrm{ppm})$ & $\mathrm{Cu}(\mathrm{ppm})$ & $\mathrm{Pb}(\mathrm{ppm})$ & LE (ppm) & $\mathrm{Mg}(\mathrm{ppm})$ & $\mathrm{Cl}(\mathrm{ppm})$ \\
\hline 7447.17 & 161022 & 9 & 2044.46 & 6062.34 & 50934.45 & 7460.58 & 19488.29 & 1233.69 & 472670.08 & 0 & 0 \\
\hline 7447.23 & 137003 & 9 & 2366.56 & 6247.99 & 45070.75 & 7439.48 & 19408.99 & 1252.13 & 554284.7 & 0 & 17084.82 \\
\hline 7447.30 & 125621 & 9 & 2194.85 & 5793.77 & 46032.72 & 7271.03 & 19092.29 & 1230.95 & 584565.59 & 0 & 0 \\
\hline 7447.36 & 125198 & 9 & 1622.76 & 6120.34 & 43476.3 & 7201.01 & 18879.97 & 1217.96 & 585281.66 & 0 & 0 \\
\hline 7447.43 & 193161 & 8 & 2133.4 & 4864.59 & 33190.37 & 4421.42 & 11534.72 & 665.66 & 427151.17 & 0 & 422852.17 \\
\hline 7447.49 & 244533 & 8 & 1098.97 & 3904.18 & 23858.09 & 3307.39 & 8604.37 & 489.5 & 377267.99 & 0 & 469690.03 \\
\hline 7447.56 & 140276 & 9 & 1487.85 & 5212.05 & 49056.41 & 5610.43 & 14693.32 & 900.72 & 506076.72 & 0 & 236857.49 \\
\hline 7447.62 & 74098 & 9 & 1931.9 & 5821.69 & 40427.22 & 6144.25 & 16206.38 & 1028.58 & 661690.53 & 0 & 167903.02 \\
\hline 7447.69 & 72833 & 9 & 2030.66 & 5019.46 & 40735.41 & 5776.78 & 15075.09 & 957.89 & 698098.55 & 0 & 103181.7 \\
\hline 7447.76 & 179338 & 8 & 1599.11 & 5295.23 & 41452.3 & 5059.04 & 13212.85 & 787.74 & 447875.96 & 0 & 333857.96 \\
\hline 7447.82 & 148904 & 9 & 2004.66 & 5986.69 & 46493.49 & 7082.73 & 18604.8 & 1171.06 & 518014.76 & 0 & 37945.06 \\
\hline 7447.89 & 179840 & 8 & 1872.31 & 6314.56 & 50933.96 & 6904.42 & 18019.9 & 1114.53 & 442866.15 & 0 & 77513.62 \\
\hline 7447.95 & 114874 & 9 & 2285.5 & 5873.06 & 45482.42 & 6932.17 & 18191.53 & 1154.97 & 594548.37 & 0 & 36390.85 \\
\hline 7448.02 & 113571 & 9 & 1150.78 & 5404.2 & 48887.15 & 6541.7 & 17132.58 & 1076.77 & 575567.71 & 0 & 69243.17 \\
\hline 7448.08 & 194260 & 8 & 2163.75 & 6058.55 & 47219.84 & 6586.96 & 17140.72 & 1043.56 & 422748.57 & 0 & 210299.65 \\
\hline 7448.15 & 239453 & 8 & 1367.11 & 5007.21 & 34367.6 & 4595.24 & 11941.59 & 689.12 & 394478.43 & 0 & 427964.77 \\
\hline 7448.22 & 176814 & 9 & 1919.65 & 5649.23 & 35993.53 & 4864.61 & 12668.98 & 734.66 & 347332.92 & 51798.16 & 406844.94 \\
\hline 7448.28 & 150670 & 9 & 1864.81 & 6278.43 & 46181.05 & 6957.46 & 18145.89 & 1128.13 & 394161.25 & 93476.23 & 26782.01 \\
\hline 7448.35 & 154185 & 9 & 1773.25 & 6617.64 & 44553.39 & 7719.33 & 20138.61 & 1283 & 465536.16 & 0 & 29282.81 \\
\hline 7448.41 & 195635 & 8 & 4094.41 & 7615.12 & 39374.2 & 6641.77 & 17338.32 & 1051.74 & 407809.1 & 47303.95 & 255180.16 \\
\hline 7448.48 & 192996 & 8 & 4572.49 & 7416.01 & 36737.1 & 6080.59 & 15836.18 & 957.58 & 469517.79 & 0 & 254628.71 \\
\hline 7448.54 & 172854 & 9 & 4795.23 & 8262.25 & 43762.65 & 7297.44 & 19072.93 & 1195.42 & 487300.68 & 0 & 112419.74 \\
\hline 7448.61 & 179107 & 8 & 1651.31 & 5890.81 & 50312.9 & 6411.33 & 16765.08 & 1020.11 & 462941.87 & 0 & 178255.71 \\
\hline 7448.67 & 189592 & 8 & 2769.15 & 6391.56 & 46073.63 & 6047.69 & 15827.75 & 945.92 & 449758.81 & 0 & 288179.28 \\
\hline 7448.74 & 215322 & 8 & 2122.05 & 5872.58 & 39425.79 & 5482.68 & 14335.61 & 840.42 & 411451.76 & 0 & 367809.18 \\
\hline 7448.81 & 250585 & 8 & 1009.52 & 4659.83 & 40123.08 & 4612.58 & 12002.77 & 693.32 & 360456.85 & 0 & 463552.2 \\
\hline 7448.87 & 261608 & 8 & 873.56 & 4013 & 30835.08 & 3799.79 & 9879.76 & 564.9 & 355442.38 & 0 & 474499.58 \\
\hline 7448.94 & 296772 & 7 & 417.71 & 2302.64 & 17820.83 & 2016.41 & 5219.91 & 291.56 & 403536.46 & 0 & 507159.04 \\
\hline 7449.00 & 266326 & 8 & 0 & 3462.39 & 27607.38 & 3161.92 & 8247.06 & 461.39 & 356477.43 & 0 & 482852.18 \\
\hline 7449.07 & 109662 & 9 & 1356.82 & 5564.61 & 53159.96 & 6075.63 & 15946.38 & 956.52 & 411790.39 & 0 & 37804.28 \\
\hline 7449.13 & 236288 & 8 & 1753.26 & 5150.61 & 33342.96 & 4522.59 & 11791.02 & 673.63 & 398548.67 & 0 & 440032.94 \\
\hline 7449.20 & 160888 & 9 & 2155.64 & 6147.38 & 46489.13 & 6924.57 & 18129.89 & 1134.09 & 497036.04 & 0 & 0 \\
\hline 7449.27 & 214744 & 8 & 2300.11 & 5472.09 & 42974.1 & 4955.6 & 12949.7 & 756.19 & 353538.28 & 42411.99 & 393157.97 \\
\hline
\end{tabular}




\begin{tabular}{|c|c|c|c|c|c|c|c|c|c|c|c|}
\hline True Depth & XRF tCOUNTS & XRF Live Time & $\mathrm{Ti}(\mathrm{ppm})$ & V (ppm) & $\mathrm{Fe}(\mathrm{ppm})$ & $\mathrm{Ni}(\mathrm{ppm})$ & $\mathrm{Cu}(\mathrm{ppm})$ & $\mathrm{Pb}(\mathrm{ppm})$ & LE (ppm) & $\mathrm{Mg}(\mathrm{ppm})$ & $\mathrm{Cl}(\mathrm{ppm})$ \\
\hline 7449.33 & 197198 & 8 & 2271.61 & 6006.26 & 43046.55 & 5926.13 & 15445.33 & 923.27 & 444936.86 & 0 & 293618.66 \\
\hline 7449.40 & 173301 & 9 & 2537.33 & 6734.31 & 53001.28 & 7747.44 & 20297.96 & 1277.74 & 449252.02 & 0 & 0 \\
\hline 7449.46 & 172660 & 9 & 4171.71 & 7478.03 & 48529.07 & 7562.58 & 19820.15 & 1250.58 & 474864.21 & 0 & 30423.66 \\
\hline 7449.53 & 197068 & 8 & 1754.76 & 6192.73 & 41855.66 & 6217.12 & 16273.02 & 972.72 & 430137.42 & 0 & 262608.48 \\
\hline 7449.59 & 232560 & 8 & 2278.19 & 5675.45 & 33409.33 & 4775.57 & 12489.58 & 726.57 & 385012.22 & 0 & 443504.84 \\
\hline 7449.66 & 201972 & 8 & 2219.89 & 5926.28 & 41011.96 & 5830.52 & 15188.21 & 901.05 & 411945.3 & 0 & 325125.03 \\
\hline 7449.72 & 178913 & 9 & 3019.69 & 6507.63 & 47932.6 & 6811.05 & 17868.44 & 1098.71 & 454492.5 & 0 & 135110.83 \\
\hline 7449.79 & 204963 & 8 & 1912.29 & 5742.78 & 38465.15 & 5480.27 & 14360.23 & 845.03 & 414892.64 & 0 & 346132.71 \\
\hline 7449.86 & 170674 & 9 & 3660.42 & 6773.73 & 47842.27 & 6923.18 & 18064.22 & 1119.34 & 485358.84 & 0 & 117191.41 \\
\hline 7449.92 & 216279 & 8 & 2536.66 & 5386.93 & 34904.33 & 4972.87 & 12929.98 & 755.23 & 415313.12 & 0 & 407830.37 \\
\hline 7449.99 & 215148 & 8 & 2305.66 & 5526.29 & 39448.81 & 5204.28 & 13595.7 & 799.83 & 410516.07 & 0 & 384218.46 \\
\hline 7450.05 & 218043 & 8 & 2499.71 & 5634.41 & 31078.01 & 4683.42 & 12217.63 & 706.2 & 400906.75 & 0 & 425302.22 \\
\hline 7450.12 & 213812 & 8 & 5318.95 & 6250.31 & 33977.44 & 5823.36 & 15236.09 & 912.26 & 412591.39 & 0 & 354275.02 \\
\hline 7450.18 & 207701 & 8 & 2031.47 & 6137.19 & 40707.21 & 6006.16 & 15689.13 & 941.8 & 415719.14 & 0 & 315314.69 \\
\hline 7450.25 & 233191 & 8 & 1754.83 & 5250.85 & 31292.3 & 4823.82 & 12614.39 & 729.24 & 392888.87 & 0 & 443306.6 \\
\hline 7450.31 & 218845 & 8 & 3577.18 & 6614.11 & 33375.6 & 5303.15 & 13834.03 & 817.23 & 420207.64 & 0 & 406244.63 \\
\hline 7450.38 & 183218 & 8 & 2717.29 & 5305.64 & 67147.23 & 5787.2 & 15015.57 & 895.84 & 384364.49 & 0 & 248435.7 \\
\hline 7450.45 & 182249 & 8 & 1462.59 & 6291.89 & 48184.07 & 6724.6 & 17565.39 & 1077.5 & 451060.76 & 0 & 157229.07 \\
\hline 7450.51 & 206715 & 8 & 1760.28 & 5588.4 & 38372.01 & 5512.08 & 14406.42 & 852.05 & 410084.43 & 0 & 334360.55 \\
\hline 7450.58 & 190640 & 8 & 1994.37 & 6073.48 & 38644.07 & 6012.6 & 15726.95 & 942.41 & 445024.57 & 0 & 277630.63 \\
\hline 7450.64 & 190098 & 8 & 1603.54 & 5381.63 & 37359.4 & 5492.08 & 14372.41 & 856.27 & 435987.08 & 0 & 286823.13 \\
\hline 7450.71 & 233946 & 8 & 1542.47 & 5038.48 & 31775.86 & 4664.27 & 12195.09 & 706.76 & 345143.96 & 37097.09 & 437290.36 \\
\hline 7450.77 & 205947 & 8 & 1021.46 & 5640.51 & 37282.78 & 5779.79 & 15110.59 & 893.36 & 405248.28 & 0 & 360242.03 \\
\hline 7450.84 & 174777 & 9 & 1688.78 & 5967.59 & 55414.54 & 7350.81 & 19199.29 & 1198.08 & 441231.79 & 0 & 70239.07 \\
\hline 7450.91 & 255251 & 8 & 674.12 & 3684.94 & 25596.05 & 3382.28 & 8785.99 & 499.12 & 366244.15 & 0 & 481958.63 \\
\hline 7450.97 & 249644 & 8 & 772.47 & 3166.68 & 20131.6 & 2858.25 & 7457.5 & 417.2 & 386868.21 & 0 & 477374.9 \\
\hline 7451.04 & 243829 & 8 & 515.31 & 3042.5 & 16832.07 & 2737.03 & 7091.9 & 396.87 & 411083.22 & 0 & 486996.28 \\
\hline 7451.10 & 81674 & 9 & 0 & 4693.87 & 34323.32 & 4711.94 & 12259.57 & 694.44 & 293625.59 & 0 & 301888.48 \\
\hline 7451.17 & 200915 & 8 & 2093.06 & 5617.76 & 37372.18 & 5636.75 & 14732.42 & 873.23 & 424927.83 & 0 & 292369.56 \\
\hline 7451.23 & 174319 & 9 & 2634.23 & 6170.67 & 42702.66 & 6450.83 & 16931.06 & 1049.56 & 474179.72 & 0 & 158121.19 \\
\hline 7451.30 & 203195 & 8 & 2422.24 & 5621.8 & 37741.89 & 5320.12 & 13879.14 & 824.73 & 423753.44 & 0 & 339536.91 \\
\hline 7451.36 & 175768 & 9 & 4195.18 & 6432.88 & 47781.65 & 6393.78 & 16752.84 & 1024.61 & 485625.05 & 0 & 141784.58 \\
\hline 7451.43 & 194837 & 8 & 1359.28 & 5126.52 & 39917.95 & 5052.04 & 13140.18 & 776.62 & 437157.79 & 0 & 327819.23 \\
\hline
\end{tabular}




\begin{tabular}{|c|c|c|c|c|c|c|c|c|c|c|c|}
\hline True Depth & XRF tCOUNTS & XRF Live Time & $\mathrm{Ti}(\mathrm{ppm})$ & $\mathrm{V}(\mathrm{ppm})$ & $\mathrm{Fe}(\mathrm{ppm})$ & $\mathrm{Ni}(\mathrm{ppm})$ & $\mathrm{Cu}(\mathrm{ppm})$ & $\mathrm{Pb}(\mathrm{ppm})$ & $\mathrm{LE}(\mathrm{ppm})$ & $\mathrm{Mg}(\mathrm{ppm})$ & $\mathrm{Cl}(\mathrm{ppm})$ \\
\hline 7451.50 & 205807 & 8 & 1740.49 & 5309.23 & 40941.74 & 5199.75 & 13533.73 & 791.57 & 423959.64 & 0 & 344583.2 \\
\hline 7451.56 & 225623 & 8 & 2284.83 & 5222.51 & 38684.95 & 4536.77 & 11792.5 & 685.87 & 393310.71 & 0 & 424981.34 \\
\hline 7451.63 & 219805 & 8 & 2240.11 & 5558.31 & 38815.03 & 4939.38 & 12868.91 & 748.18 & 406081.1 & 0 & 406608.61 \\
\hline 7451.69 & 156633 & 9 & 2115.26 & 6039 & 42229.9 & 5780.61 & 15110.08 & 902.54 & 413372.5 & 0 & 288561.77 \\
\hline 7451.76 & 187391 & 8 & 1533.21 & 6524.7 & 48747.18 & 7058 & 18459.98 & 1134.5 & 413625.09 & 0 & 181804.63 \\
\hline 7451.82 & 224286 & 8 & 2494.88 & 5542.71 & 33849.27 & 5013.32 & 13095.21 & 766.56 & 402246.09 & 0 & 412307.36 \\
\hline 7451.89 & 208009 & 8 & 3619.75 & 6358.97 & 28351.3 & 5066.13 & 13201.11 & 777.11 & 438044.89 & 0 & 373670.5 \\
\hline 7451.96 & 189204 & 8 & 3155.77 & 6020.23 & 32997.01 & 5617.71 & 14706.42 & 879.9 & 451935.26 & 0 & 327977.21 \\
\hline 7452.02 & 163333 & 9 & 4538.24 & 7553.95 & 41802.07 & 6860.23 & 17971.36 & 1111.34 & 508097.94 & 0 & 159369.26 \\
\hline 7452.09 & 157070 & 9 & 2441.01 & 6236.67 & 43573.97 & 7089.32 & 18548.43 & 1163.7 & 501915.21 & 0 & 0 \\
\hline 7452.15 & 239744 & 8 & 2541.14 & 6046.98 & 29611.85 & 5212.35 & 13564.11 & 794.38 & 387603.48 & 0 & 461364.99 \\
\hline 7452.22 & 158193 & 9 & 2438.75 & 6299.87 & 46789.82 & 7200.02 & 18837.28 & 1196.6 & 509537.56 & 0 & 0 \\
\hline 7452.28 & 152835 & 9 & 2557.7 & 6320.22 & 48768.08 & 7174.17 & 18790.81 & 1189.66 & 509114.61 & 0 & 0 \\
\hline 7452.35 & 166375 & 9 & 2359.01 & 6049.84 & 48649.83 & 6425.33 & 16786.42 & 1030.04 & 465433.7 & 0 & 189507.84 \\
\hline 7452.41 & 171250 & 9 & 1655.35 & 6219.33 & 45720.2 & 7262.48 & 19090.58 & 1183.88 & 455131.34 & 0 & 127088.34 \\
\hline 7452.48 & 162478 & 9 & 1852.1 & 6488.74 & 45758.75 & 7271.98 & 18957.15 & 1202.43 & 484555.99 & 0 & 11450.69 \\
\hline 7452.55 & 158491 & 9 & 2618.08 & 6242.11 & 46857.07 & 6798.38 & 17781.41 & 1108.44 & 495407.79 & 0 & 70792.84 \\
\hline 7452.61 & 232578 & 8 & 2080.02 & 5411.39 & 31587.96 & 4743.16 & 12399.4 & 714.85 & 391414.15 & 0 & 461364 \\
\hline 7452.68 & 192125 & 8 & 4729 & 6063.59 & 60623.68 & 5226.92 & 13472.31 & 800.71 & 417630.46 & 0 & 323029.87 \\
\hline 7452.74 & 196685 & 8 & 3958.95 & 5760.35 & 34579 & 4898.96 & 12779.69 & 752.98 & 435200.62 & 0 & 358427.46 \\
\hline 7452.81 & 174186 & 9 & 2062.9 & 6320 & 45932.95 & 6219.17 & 16229.62 & 988.97 & 463494.42 & 0 & 174184.33 \\
\hline 7452.87 & 233967 & 8 & 683.58 & 3170.68 & 19063.34 & 2788.98 & 7221.21 & 406.92 & 388005.19 & 0 & 480465.38 \\
\hline 7452.94 & 259487 & 8 & 927.19 & 3082.4 & 22378.25 & 2796.89 & 7257.59 & 408.04 & 382190.69 & 0 & 486441.17 \\
\hline 7453.01 & 169326 & 9 & 1669.22 & 6179.92 & 51220.8 & 7619.98 & 19859.32 & 1258.96 & 444505.67 & 0 & 22094.36 \\
\hline 7453.07 & 187325 & 8 & 655.36 & 5832.3 & 47013.81 & 6574.51 & 17188.77 & 1040.84 & 430625.47 & 0 & 197432.91 \\
\hline 7453.14 & 80278 & 9 & 0 & 4362.44 & 29534.52 & 4339.66 & 11235.74 & 640.76 & 359936.7 & 0 & 336158.07 \\
\hline 7453.20 & 183311 & 8 & 1264.71 & 5524.6 & 40123.98 & 5803.76 & 15160.98 & 905.3 & 443174.05 & 0 & 285966.66 \\
\hline 7453.27 & 175432 & 9 & 957.28 & 5394.89 & 41522.97 & 5846.34 & 15267.23 & 919.57 & 464303.26 & 0 & 264620.3 \\
\hline 7453.33 & 142420 & 9 & 1405.33 & 5938.93 & 42419.16 & 7048.95 & 18447.17 & 1156.5 & 531632.73 & 0 & 82696.94 \\
\hline 7453.40 & 150409 & 9 & 1620.06 & 5907.93 & 47144.14 & 6836.46 & 17813.98 & 1130.63 & 526433.87 & 0 & 0 \\
\hline 7453.46 & 134299 & 9 & 1953.01 & 5840.88 & 48586.43 & 7354.6 & 19286.91 & 1234.65 & 529796.83 & 0 & 0 \\
\hline 7453.53 & 165831 & 9 & 2344.28 & 5858.57 & 49185.77 & 6847.84 & 17875.54 & 1126.28 & 490974.74 & 0 & 0 \\
\hline 7453.60 & 157350 & 9 & 1972.11 & 6339.64 & 37233.91 & 6611.07 & 17302.93 & 1068.62 & 491712.7 & 0 & 108429.05 \\
\hline
\end{tabular}




\begin{tabular}{|c|c|c|c|c|c|c|c|c|c|c|c|}
\hline True Depth & XRF tCOUNTS & XRF Live Time & $\mathrm{Ti}(\mathrm{ppm})$ & V (ppm) & $\mathrm{Fe}(\mathrm{ppm})$ & $\mathrm{Ni}(\mathrm{ppm})$ & $\mathrm{Cu}(\mathrm{ppm})$ & $\mathrm{Pb}(\mathrm{ppm})$ & LE (ppm) & $\mathrm{Mg}(\mathrm{ppm})$ & $\mathrm{Cl}(\mathrm{ppm})$ \\
\hline 7453.66 & 157706 & 9 & 2567.83 & 6033.13 & 49246.06 & 7189.55 & 18770.33 & 1180.89 & 478874.36 & 0 & 0 \\
\hline 7453.73 & 216884 & 8 & 1384.94 & 5524.43 & 28067.03 & 5049.78 & 13201.96 & 771.16 & 409634.68 & 0 & 418981.13 \\
\hline 7453.79 & 182624 & 8 & 1603.97 & 5529.15 & 43945.36 & 5944.61 & 15613.21 & 940.17 & 433141.59 & 0 & 240693.75 \\
\hline 7453.86 & 163164 & 9 & 2324.86 & 6687.86 & 47750.44 & 7468.45 & 19625.44 & 1243.71 & 480630.51 & 0 & 17427.57 \\
\hline 7453.92 & 226341 & 8 & 1943.48 & 5029.68 & 41021.04 & 5000.13 & 13066.18 & 766.74 & 383447.35 & 0 & 415408.36 \\
\hline 7453.99 & 259182 & 8 & 1461.38 & 3530.2 & 20860.09 & 2836.85 & 7409.38 & 412.86 & 394347.12 & 0 & 487709.12 \\
\hline 7454.06 & 248408 & 8 & 1936.88 & 4146.96 & 37481.01 & 3650.43 & 9527.83 & 544.03 & 349802.13 & 0 & 464767.09 \\
\hline 7454.12 & 262622 & 8 & 639.18 & 2363.19 & 20591.75 & 2016.46 & 5225.04 & 289.49 & 408903.07 & 0 & 512046.6 \\
\hline 7454.19 & 275708 & 7 & 6862.84 & 3313.03 & 21970.68 & 3123.3 & 8118.9 & 458.79 & 384700.08 & 0 & 488015.05 \\
\hline 7454.25 & 237367 & 8 & 1575.52 & 5508.38 & 27087.71 & 5013.47 & 13108.35 & 759.31 & 400951.23 & 0 & 436231.74 \\
\hline 7454.32 & 234048 & 8 & 1682.28 & 3530.22 & 39179.17 & 3381.48 & 8789.74 & 503.56 & 367474.34 & 0 & 456468.49 \\
\hline 7454.38 & 212750 & 8 & 1950.94 & 5441.07 & 45689.61 & 5076.1 & 13036.49 & 759.74 & 397406.19 & 0 & 425058.18 \\
\hline 7454.45 & 221477 & 8 & 1434.36 & 6185.28 & 44780.29 & 6256.94 & 16284.72 & 961.8 & 415472.69 & 0 & 298392.56 \\
\hline 7454.51 & 240127 & 8 & 1529.49 & 5814.94 & 41759.25 & 5851.5 & 15197.75 & 893.84 & 372958.03 & 0 & 418809.89 \\
\hline 7454.58 & 207750 & 8 & 2120.26 & 6219.73 & 28862.25 & 5806.57 & 15212.03 & 898.76 & 436850.53 & 0 & 366960.93 \\
\hline 7454.65 & 219414 & 8 & 1069.36 & 5273.55 & 39295.84 & 5253.08 & 13762.65 & 796.01 & 424366.9 & 0 & 233470.68 \\
\hline 7454.71 & 180455 & 8 & 0 & 2522.4 & 32115.65 & 2362.61 & 6172.21 & 339.54 & 386139.65 & 0 & 463714.24 \\
\hline 7454.78 & 149624 & 9 & 1326.44 & 5090.58 & 35443.83 & 5176.93 & 13545.69 & 780.34 & 461515.78 & 0 & 370485.33 \\
\hline 7454.84 & 157367 & 9 & 1614.77 & 4882.58 & 35200.1 & 4462.8 & 11671.94 & 665.13 & 425661.86 & 0 & 421424.22 \\
\hline 7454.91 & 172788 & 9 & 1379.58 & 5112.54 & 27979.58 & 4526.05 & 11871.93 & 669.47 & 380934.6 & 0 & 456859.93 \\
\hline 7454.97 & 194175 & 8 & 1589.26 & 5746.99 & 41537.72 & 5761.58 & 15153.06 & 889.3 & 455892.06 & 0 & 228083.58 \\
\hline 7455.04 & 234541 & 8 & 638.55 & 3285.43 & 17031.6 & 2721.05 & 7099.06 & 395.59 & 387591.05 & 0 & 482493.69 \\
\hline 7455.10 & 193666 & 8 & 0 & 5518.09 & 55376.48 & 6669.43 & 17591.06 & 1064.39 & 514940.27 & 0 & 70232.2 \\
\hline 7455.17 & 195566 & 8 & 2362.63 & 6064.29 & 55495.82 & 5913.42 & 15499 & 910.13 & 407802 & 0 & 213030.47 \\
\hline 7455.24 & 252674 & 8 & 1830.22 & 5908.87 & 57002.76 & 6023.24 & 15814.13 & 920.15 & 349015.98 & 0 & 300590.27 \\
\hline 7455.30 & 219956 & 8 & 2353.69 & 6144.3 & 37943.92 & 5441.55 & 14287.5 & 824.37 & 402072.11 & 0 & 340333.33 \\
\hline 7455.37 & 191575 & 8 & 1917.85 & 6085.32 & 45670.67 & 5912.21 & 15510.03 & 911.21 & 463405.49 & 0 & 222408.8 \\
\hline 7455.43 & 195416 & 8 & 2025.02 & 5585.44 & 33435.43 & 4739 & 12445.74 & 708.17 & 396670 & 0 & 364395.15 \\
\hline 7455.50 & 239022 & 8 & 2018.9 & 5960.97 & 33561.98 & 4807.6 & 12606.14 & 712.66 & 322689.31 & 0 & 430900.35 \\
\hline 7455.56 & 317691 & 7 & 4110.09 & 5412.73 & 17985.17 & 2929.72 & 7708.19 & 420.15 & 265606.45 & 0 & 333919.69 \\
\hline 7455.63 & 324870 & 7 & 2762.81 & 4173.88 & 13713.5 & 2671.79 & 6974.31 & 379.01 & 264236.84 & 0 & 333625.5 \\
\hline 7455.70 & 291796 & 7 & 6813.32 & 5948.48 & 21097.75 & 2650.71 & 6949.53 & 388.36 & 367000.93 & 0 & 192710.81 \\
\hline 7455.76 & 300163 & 7 & 8245.85 & 6822.39 & 26713.42 & 3324.26 & 8614.94 & 491.7 & 438300.12 & 0 & 33796.39 \\
\hline
\end{tabular}




\begin{tabular}{|c|c|c|c|c|c|c|c|c|c|c|c|}
\hline True Depth & XRF tCOUNTS & XRF Live Time & $\mathrm{Ti}(\mathrm{ppm})$ & V (ppm) & $\mathrm{Fe}(\mathrm{ppm})$ & $\mathrm{Ni}(\mathrm{ppm})$ & $\mathrm{Cu}(\mathrm{ppm})$ & $\mathrm{Pb}(\mathrm{ppm})$ & LE (ppm) & $\mathrm{Mg}(\mathrm{ppm})$ & $\mathrm{Cl}(\mathrm{ppm})$ \\
\hline 7455.83 & 297591 & 7 & 3820.85 & 4926.86 & 20073.79 & 2601.92 & 6788.18 & 368.32 & 303775.58 & 0 & 327268.48 \\
\hline 7455.89 & 186074 & 8 & 2410.83 & 5679.53 & 97274.77 & 8226.52 & 21539.31 & 1355.49 & 469632.07 & 0 & 16287.75 \\
\hline 7455.96 & 303014 & 7 & 462.28 & 2693.9 & 19410.61 & 2350.43 & 6039.86 & 337.58 & 400021.63 & 0 & 513402.99 \\
\hline 7456.02 & 168233 & 9 & 2990.98 & 7871.71 & 43925.95 & 8712.61 & 22752.19 & 1475.87 & 501722.07 & 0 & 20682.91 \\
\hline 7456.09 & 179948 & 8 & 4268.32 & 8196.14 & 44229.53 & 7963.48 & 20801.25 & 1297.24 & 478627.06 & 0 & 151088.63 \\
\hline 7456.15 & 208448 & 8 & 2513.69 & 6121.99 & 51827.25 & 6642.36 & 17100.28 & 1037.36 & 435221.03 & 0 & 261642.46 \\
\hline 7456.22 & 280690 & 7 & 13509.77 & 3755.59 & 22927.15 & 3382.38 & 8704.82 & 491.58 & 394854.99 & 0 & 466068.55 \\
\hline 7456.29 & 206612 & 8 & 2101.15 & 6181.67 & 42358.74 & 6308.47 & 16401.41 & 977.97 & 413394.37 & 0 & 301144.25 \\
\hline 7456.35 & 175778 & 9 & 1806.84 & 7049.25 & 44816.63 & 7790.34 & 20310.52 & 1274.27 & 455075.49 & 0 & 93002.63 \\
\hline 7456.42 & 214585 & 8 & 4296.16 & 6899.82 & 35344.05 & 5874.85 & 15316.54 & 907.18 & 438143.31 & 0 & 361961.06 \\
\hline 7456.48 & 168004 & 9 & 2627.82 & 6657.64 & 61257.44 & 7441.16 & 19374.05 & 1208.84 & 366238.45 & 79035.69 & 0 \\
\hline 7456.55 & 189038 & 8 & 2360 & 5828.61 & 53741.86 & 5805.08 & 15032.34 & 893.79 & 426277.37 & 0 & 265122.72 \\
\hline 7456.61 & 234629 & 8 & 4402.86 & 6800.71 & 38720.82 & 5757.14 & 14904.11 & 870.77 & 349334.48 & 40150.91 & 381697.54 \\
\hline 7456.68 & 207877 & 8 & 3099.95 & 6079.15 & 56594.75 & 5540.67 & 14301.18 & 844.75 & 373004.32 & 57733.16 & 297431.57 \\
\hline 7456.75 & 128757 & 9 & 2309.33 & 5728.53 & 38710.7 & 6342.05 & 16532.9 & 1014.66 & 573637.55 & 0 & 180777.71 \\
\hline 7456.81 & 129530 & 9 & 1743.06 & 6444.28 & 37759.74 & 6707.18 & 17482.15 & 1083.22 & 563787.51 & 0 & 202713.55 \\
\hline 7456.88 & 178226 & 8 & 2159.15 & 5670.67 & 44920.81 & 5851.81 & 15141.95 & 907.67 & 481716.14 & 0 & 279076.44 \\
\hline 7456.94 & 221375 & 8 & 1322.35 & 4733.29 & 32063.87 & 4350.37 & 11262.08 & 651.82 & 415058.97 & 0 & 458547.66 \\
\hline 7457.01 & 207021 & 8 & 1567.17 & 5382.66 & 43048.95 & 5297.27 & 13756.27 & 812.37 & 432054.99 & 0 & 386510.86 \\
\hline 7457.07 & 169687 & 9 & 1178.37 & 4714 & 32791.87 & 4541.55 & 11795.58 & 686.92 & 430630.11 & 0 & 430341.08 \\
\hline 7457.14 & 214375 & 8 & 413.23 & 2608.72 & 18658.48 & 2319.08 & 6003.34 & 336.98 & 420174.25 & 0 & 495286.27 \\
\hline 7457.20 & 121398 & 9 & 1699.56 & 4345.46 & 39568.24 & 4628.47 & 11932.18 & 702.65 & 376838.61 & 0 & 304129.37 \\
\hline 7457.27 & 176205 & 9 & 2498.61 & 5881.95 & 58904.22 & 7065.11 & 18320.71 & 1157.07 & 451429.75 & 0 & 47508.06 \\
\hline 7457.34 & 168315 & 9 & 2723.32 & 6637.68 & 54885.55 & 7445.15 & 19311.24 & 1224.68 & 494441.66 & 0 & 45895.78 \\
\hline 7457.40 & 200087 & 8 & 2541.09 & 5546.62 & 35659.65 & 5212.8 & 13560.51 & 798.27 & 444636.27 & 0 & 341792.53 \\
\hline 7457.47 & 176557 & 9 & 3445.86 & 6434.31 & 45507.49 & 6619.17 & 17267.13 & 1069.42 & 489843.24 & 0 & 128768.84 \\
\hline 7457.53 & 187785 & 8 & 4732.13 & 7128.44 & 42016.19 & 6052.07 & 15728.84 & 944.33 & 472743.58 & 0 & 251857.14 \\
\hline 7457.60 & 165199 & 9 & 2628.97 & 6294.45 & 48987.94 & 7057.94 & 18279.51 & 1148.8 & 486865.87 & 0 & 0 \\
\hline 7457.66 & 179640 & 8 & 3102.89 & 7043.06 & 50205.92 & 7703.75 & 20102.69 & 1273.93 & 467796.11 & 0 & 42683.72 \\
\hline 7457.73 & 179406 & 9 & 4040.66 & 7616.57 & 47090.36 & 7247.91 & 18856.42 & 1168.8 & 410456.43 & 65247.22 & 77080.34 \\
\hline 7457.80 & 182719 & 8 & 4405.47 & 7679.69 & 45747.73 & 7337.38 & 19066.33 & 1193.58 & 477642.63 & 0 & 98800.56 \\
\hline 7457.86 & 169085 & 9 & 4669.85 & 7876.75 & 44988.01 & 7276.42 & 18990.75 & 1201.03 & 511716.67 & 0 & 50376.48 \\
\hline 7457.93 & 226927 & 8 & 2165.05 & 5615.7 & 35965.88 & 5308.79 & 13762.76 & 814.64 & 417877.13 & 0 & 373207.19 \\
\hline
\end{tabular}




\begin{tabular}{|c|c|c|c|c|c|c|c|c|c|c|c|}
\hline True Depth & XRF tCOUNTS & XRF Live Time & $\mathrm{Ti}(\mathrm{ppm})$ & $\mathrm{V}(\mathrm{ppm})$ & $\mathrm{Fe}(\mathrm{ppm})$ & $\mathrm{Ni}(\mathrm{ppm})$ & $\mathrm{Cu}(\mathrm{ppm})$ & $\mathrm{Pb}(\mathrm{ppm})$ & $\mathrm{LE}(\mathrm{ppm})$ & $\mathrm{Mg}(\mathrm{ppm})$ & $\mathrm{Cl}(\mathrm{ppm})$ \\
\hline 7457.99 & 196554 & 8 & 3294.44 & 6761.52 & 43762.64 & 6864.25 & 17866.38 & 1087.54 & 454173.17 & 0 & 241109.39 \\
\hline 7458.06 & 176824 & 9 & 4051.36 & 6991 & 46643.1 & 7314.44 & 19031.66 & 1195.62 & 498967.53 & 0 & 106081.81 \\
\hline 7458.12 & 182244 & 8 & 3125.38 & 6551.53 & 45031.89 & 6791.23 & 17602.15 & 1087.28 & 475242.77 & 0 & 194900.99 \\
\hline 7458.19 & 205804 & 8 & 2168.61 & 5730.36 & 45799.95 & 5758.67 & 14935.74 & 887.78 & 413161.06 & 0 & 303852.15 \\
\hline 7458.25 & 261503 & 8 & 4109.97 & 4298.59 & 20547.12 & 3428.16 & 8889.1 & 505.38 & 389910.92 & 0 & 473182.44 \\
\hline 7458.32 & 204897 & 8 & 1802.7 & 6472.54 & 41645.51 & 6212.3 & 16154.7 & 972.02 & 438636.2 & 0 & 285171.59 \\
\hline 7458.39 & 191456 & 8 & 4363.24 & 7446.46 & 40274.61 & 6588.67 & 17091.49 & 1043.91 & 470925.72 & 0 & 251133.78 \\
\hline 7458.45 & 197284 & 8 & 3851 & 6818.4 & 42886.9 & 6162.17 & 15982.05 & 963.74 & 463212.05 & 0 & 277602.14 \\
\hline 7458.52 & 182063 & 8 & 1773.68 & 6245.3 & 39508.19 & 6659.89 & 17356.55 & 1060.18 & 441125.15 & 0 & 218594.08 \\
\hline 7458.58 & 245912 & 8 & 2031.77 & 5708.63 & 30735.18 & 5056.19 & 13150.82 & 758.89 & 346571.73 & 43269.33 & 442542.19 \\
\hline 7458.65 & 251245 & 8 & 2153.36 & 5345.12 & 37465.79 & 4497.02 & 11651.11 & 676.52 & 371949.48 & 0 & 466533.58 \\
\hline 7458.71 & 174433 & 9 & 2654.62 & 6295.97 & 52331.84 & 7082.92 & 18407.32 & 1147.53 & 482721.63 & 0 & 66058.21 \\
\hline 7458.78 & 194230 & 8 & 5236.94 & 7263.9 & 43270.4 & 6123.63 & 15974.61 & 958.16 & 470328.84 & 0 & 220451.42 \\
\hline 7458.85 & 254246 & 8 & 1845.2 & 4132.14 & 35181.75 & 3464.83 & 8974.59 & 509.93 & 357172.26 & 0 & 461361.69 \\
\hline 7458.91 & 232726 & 8 & 1287.09 & 5058.55 & 43506.67 & 4946.51 & 12857.84 & 746.91 & 390876.39 & 0 & 406992.01 \\
\hline 7458.98 & 250529 & 8 & 1707.5 & 5307.95 & 41611.2 & 5004.49 & 13076.75 & 763.23 & 372707.13 & 0 & 424904 \\
\hline 7459.04 & 220523 & 8 & 2584.75 & 5587.15 & 65171.39 & 5926.59 & 15437.38 & 928.45 & 372556.26 & 0 & 280067.41 \\
\hline 7459.11 & 187700 & 8 & 2267.48 & 5844.15 & 63578.62 & 7225.19 & 18796.84 & 1171.6 & 433530.97 & 0 & 23640.31 \\
\hline 7459.17 & 200905 & 8 & 1097.8 & 6209.82 & 47329.04 & 6925.95 & 18035.13 & 1095.07 & 425753.68 & 0 & 275139.58 \\
\hline 7459.24 & 112820 & 9 & 2141.14 & 5832.62 & 42216.22 & 6277.66 & 16269.01 & 989.1 & 423257.43 & 0 & 143572.92 \\
\hline 7459.30 & 176080 & 9 & 2415.9 & 6294.53 & 51579.5 & 7215.15 & 18765.4 & 1171.79 & 466350.61 & 0 & 83912.47 \\
\hline 7459.37 & 190638 & 8 & 2463.2 & 6044.82 & 44336.02 & 6028.98 & 15722.68 & 940.55 & 461081.77 & 0 & 268327.11 \\
\hline 7459.44 & 173818 & 9 & 1569.39 & 5477.58 & 41155.39 & 5262.21 & 13750.24 & 809.97 & 399795.03 & 0 & 371969.33 \\
\hline 7459.50 & 205407 & 8 & 2684.28 & 6377.72 & 48662.22 & 6166.56 & 16066.61 & 959.58 & 424921.98 & 0 & 285629.25 \\
\hline 7459.57 & 175008 & 9 & 2813.84 & 6702.3 & 56100.09 & 7530.41 & 19721.76 & 1249.56 & 491192.2 & 0 & 26793.78 \\
\hline 7459.63 & 181969 & 8 & 2744.14 & 6761.61 & 54276.99 & 7074.27 & 18412.08 & 1143.36 & 455787.34 & 0 & 124015.39 \\
\hline 7459.70 & 229069 & 8 & 2358.81 & 5650.74 & 40271.12 & 5115.4 & 13265.15 & 775.25 & 397707.53 & 0 & 409757.29 \\
\hline 7459.76 & 191592 & 8 & 3948.23 & 5986.03 & 60587.26 & 6139.76 & 15913.07 & 964.31 & 438869.66 & 0 & 199259.4 \\
\hline 7459.83 & 171624 & 9 & 4262.71 & 7321.87 & 49873.71 & 7613.71 & 19875.84 & 1256.37 & 501871.76 & 0 & 87574.54 \\
\hline 7459.90 & 171071 & 9 & 3205.53 & 6898.65 & 51604.99 & 7410.55 & 19320.54 & 1222.47 & 481923.7 & 0 & 25291.67 \\
\hline 7459.96 & 188227 & 8 & 3258.8 & 6398.52 & 51500.51 & 6418.92 & 16797.75 & 1022.22 & 471814.2 & 0 & 187550.91 \\
\hline 7460.03 & 201296 & 8 & 3620.73 & 6456.61 & 47781.62 & 6053.97 & 15801.79 & 947.43 & 436258.36 & 0 & 300806.79 \\
\hline 7460.09 & 183526 & 8 & 3660.85 & 6896.22 & 52411.75 & 6591.1 & 17201.24 & 1058.62 & 477405.06 & 0 & 192048.43 \\
\hline
\end{tabular}




\begin{tabular}{|c|c|c|c|c|c|c|c|c|c|c|c|}
\hline True Depth & XRF tCOUNTS & XRF Live Time & $\mathrm{Ti}(\mathrm{ppm})$ & V (ppm) & $\mathrm{Fe}(\mathrm{ppm})$ & $\mathrm{Ni}(\mathrm{ppm})$ & $\mathrm{Cu}(\mathrm{ppm})$ & $\mathrm{Pb}(\mathrm{ppm})$ & LE (ppm) & $\mathrm{Mg}(\mathrm{ppm})$ & $\mathrm{Cl}(\mathrm{ppm})$ \\
\hline 7460.16 & 200355 & 8 & 1532.78 & 6000.02 & 43000.05 & 6080.82 & 15842.12 & 947.24 & 449945.82 & 0 & 297757.33 \\
\hline 7460.22 & 195052 & 8 & 3368 & 6557.16 & 49790.65 & 6859.21 & 17807.77 & 1098.82 & 392806.66 & 54620.02 & 137690.55 \\
\hline 7460.29 & 223675 & 8 & 2496.66 & 5504.32 & 41838.94 & 5232.53 & 13581.27 & 805.12 & 409917.3 & 0 & 371173.16 \\
\hline 7460.35 & 166961 & 9 & 1613.15 & 6455.68 & 53781.39 & 7899.19 & 20533.33 & 1311.31 & 479165.35 & 0 & 0 \\
\hline 7460.42 & 169576 & 9 & 2329.9 & 6484.7 & 45283.08 & 6903.23 & 18016.61 & 1120.5 & 496625.1 & 0 & 132546.08 \\
\hline 7460.49 & 153577 & 9 & 2034.44 & 5973.27 & 56939.48 & 6945.2 & 18120.48 & 1136.1 & 507623.12 & 0 & 47509.84 \\
\hline 7460.55 & 202388 & 8 & 2816.77 & 6191.9 & 53812.05 & 6385.88 & 16620.58 & 1007.16 & 425825.49 & 0 & 217190.83 \\
\hline 7460.62 & 215470 & 8 & 3243.9 & 5917.13 & 67568.52 & 5759.66 & 15053.72 & 896.24 & 414883.12 & 0 & 234435.99 \\
\hline 7460.68 & 230638 & 8 & 2537.13 & 4900.63 & 68254.89 & 5158.27 & 13430.33 & 799.32 & 394418.64 & 0 & 301879.1 \\
\hline 7460.75 & 219162 & 8 & 2316.32 & 6170.6 & 42138.21 & 5658.19 & 14743.12 & 881.9 & 433457.69 & 0 & 324455.7 \\
\hline 7460.81 & 205864 & 8 & 1509.65 & 6081.46 & 44018.78 & 6347.6 & 16556.74 & 1001.1 & 440689.34 & 0 & 283933.32 \\
\hline 7460.88 & 191426 & 8 & 2476.5 & 5824.33 & 63664.91 & 6803.94 & 17759.4 & 1086.91 & 433261.18 & 0 & 127812.85 \\
\hline 7460.94 & 285053 & 7 & 1027.2 & 2417.46 & 18887.16 & 1901.78 & 4907.85 & 271.06 & 406511.98 & 0 & 503042.57 \\
\hline 7461.01 & 233253 & 8 & 1621.12 & 4774.07 & 53929.09 & 4836.63 & 12591.42 & 734.44 & 353456.41 & 0 & 398230.92 \\
\hline 7461.08 & 297150 & 7 & 626.94 & 2444.43 & 20499.6 & 2167.61 & 5612.69 & 311.72 & 403249.28 & 0 & 499384.78 \\
\hline 7461.14 & 223568 & 8 & 1188.18 & 5314.32 & 43306.58 & 5385.47 & 14029.11 & 819.7 & 398534.74 & 0 & 372131.46 \\
\hline 7461.21 & 141830 & 9 & 1850.96 & 5348.99 & 74286.67 & 7355.38 & 19063.35 & 1202.33 & 427133 & 0 & 0 \\
\hline 7461.27 & 178373 & 8 & 2979.75 & 5937.65 & 55555.46 & 6994.75 & 18205.84 & 1127.24 & 472453.89 & 0 & 98294.61 \\
\hline 7461.34 & 164578 & 9 & 1781.39 & 6523.49 & 49661.65 & 7558.14 & 19740.69 & 1238.02 & 486817.56 & 0 & 71871.35 \\
\hline 7461.40 & 165000 & 9 & 2303.47 & 6162.1 & 53783.73 & 7222.05 & 18796.15 & 1175.47 & 480674.86 & 0 & 25414.91 \\
\hline 7461.47 & 189331 & 8 & 2055.63 & 6159.61 & 68601.23 & 7482.02 & 19429.45 & 1203.85 & 423406.73 & 0 & 34564.56 \\
\hline 7461.54 & 181543 & 9 & 2919.19 & 6530.61 & 60057.99 & 7510.75 & 19527.2 & 1219.73 & 462966.47 & 0 & 16303.59 \\
\hline 7461.60 & 222308 & 8 & 2773.93 & 5978.18 & 37283.91 & 5578.16 & 14472.9 & 852.64 & 424535.02 & 0 & 360952.28 \\
\hline 7461.67 & 174752 & 9 & 2546.36 & 7053.39 & 55019.49 & 8025.45 & 20959.06 & 1338.11 & 463601.57 & 0 & 22738.43 \\
\hline 7461.73 & 189744 & 8 & 2525.28 & 6937.03 & 43091.46 & 6771.23 & 17528.93 & 1069.21 & 444916.19 & 0 & 190027.1 \\
\hline 7461.80 & 252181 & 8 & 1581.12 & 4298.59 & 26414.3 & 3604.44 & 9363.2 & 536.43 & 370925.21 & 0 & 468461.44 \\
\hline 7461.86 & 191725 & 8 & 3452.33 & 6823.27 & 58957.74 & 6422.23 & 16702.23 & 1017.76 & 415584.86 & 0 & 153577.86 \\
\hline 7461.93 & 225949 & 8 & 2984.56 & 6578.18 & 44020.27 & 5782.4 & 15053.92 & 890.69 & 396244.79 & 0 & 371459.12 \\
\hline 7461.99 & 233875 & 8 & 2394 & 6008.33 & 35767.22 & 5271.54 & 13722.93 & 801.86 & 387730.04 & 0 & 438625.71 \\
\hline 7462.06 & 189169 & 8 & 2982.18 & 7126.7 & 48951.36 & 6648.45 & 17384.83 & 1067.43 & 461955.09 & 0 & 199306.47 \\
\hline 7462.13 & 193759 & 8 & 2983.36 & 6000.9 & 70582.97 & 7051.29 & 18364.45 & 1137.3 & 421474.52 & 0 & 93034.38 \\
\hline 7462.19 & 237221 & 8 & 7614.69 & 5939.65 & 46394.97 & 6126.82 & 15941.25 & 947.8 & 397209.67 & 0 & 333971.21 \\
\hline 7462.26 & 266367 & 8 & 2779.25 & 3005.34 & 15339.44 & 1814.07 & 4699.57 & 259.07 & 356028.06 & 0 & 490413.38 \\
\hline
\end{tabular}




\begin{tabular}{|c|c|c|c|c|c|c|c|c|c|c|c|}
\hline True Depth & XRF tCOUNTS & XRF Live Time & $\mathrm{Ti}(\mathrm{ppm})$ & V (ppm) & $\mathrm{Fe}(\mathrm{ppm})$ & $\mathrm{Ni}(\mathrm{ppm})$ & $\mathrm{Cu}(\mathrm{ppm})$ & $\mathrm{Pb}(\mathrm{ppm})$ & LE (ppm) & $\mathrm{Mg}(\mathrm{ppm})$ & $\mathrm{Cl}(\mathrm{ppm})$ \\
\hline 7462.32 & 216404 & 8 & 2077.43 & 5081.94 & 42825.81 & 4333.94 & 11242.11 & 644.92 & 382933.78 & 0 & 456563.15 \\
\hline 7462.39 & 164156 & 9 & 3196.19 & 6014.46 & 48496.49 & 5919.21 & 15448.72 & 933.71 & 493289.78 & 0 & 251208.39 \\
\hline 7462.45 & 181263 & 8 & 2598.96 & 5420.41 & 31296.39 & 4729.59 & 12289.16 & 724.88 & 453519.65 & 0 & 400214.91 \\
\hline 7462.52 & 170128 & 9 & 1361.14 & 4884.1 & 25741.7 & 4273.9 & 11091.78 & 634.31 & 383981.51 & 0 & 450064.43 \\
\hline 7462.59 & 266173 & 8 & 901.16 & 3547.33 & 19850.91 & 3181.52 & 8266.61 & 462.92 & 381860.32 & 0 & 483316.79 \\
\hline 7462.65 & 170569 & 9 & 2606.68 & 6351.78 & 43490.39 & 6894.13 & 17995.32 & 1115.95 & 465393.17 & 0 & 58590.69 \\
\hline 7462.72 & 206320 & 8 & 623.19 & 3259.19 & 18938.46 & 2804.72 & 7265.46 & 405.8 & 416959.15 & 0 & 471421.17 \\
\hline 7462.78 & 182362 & 8 & 2723.24 & 5292.37 & 31480.54 & 4515.28 & 11754.75 & 682.97 & 449711.04 & 0 & 391780.4 \\
\hline 7462.85 & 243024 & 8 & 720.72 & 2485.7 & 12449.94 & 2021.14 & 5247.22 & 287.33 & 429680.8 & 0 & 493942.29 \\
\hline 7462.91 & 154893 & 9 & 2348.37 & 6825.37 & 39484.25 & 7167.88 & 18688.86 & 1166.54 & 494598.15 & 0 & 117993.91 \\
\hline 7462.98 & 163500 & 9 & 1859.63 & 6884.71 & 39987.03 & 7603.08 & 19860.18 & 1256.98 & 467307.84 & 0 & 76098.92 \\
\hline 7463.04 & 212611 & 8 & 1061.22 & 4788.75 & 31108.75 & 4515.92 & 11747.93 & 682.37 & 413393.46 & 0 & 404923.47 \\
\hline 7463.11 & 173770 & 9 & 1916.63 & 6181.34 & 42914.42 & 7087.92 & 18526.56 & 1156.03 & 453304.06 & 0 & 73690.28 \\
\hline 7463.18 & 225638 & 8 & 0 & 4608.01 & 30764.49 & 4609.93 & 11984.11 & 687.93 & 392582.35 & 0 & 431871.12 \\
\hline 7463.24 & 112805 & 9 & 1554.64 & 5751.13 & 40799.16 & 6701.22 & 17511.77 & 1108.18 & 497454.6 & 0 & 0 \\
\hline 7463.31 & 235450 & 8 & 489.4 & 3706.6 & 19149.87 & 3404.61 & 8840.29 & 503.75 & 397934.71 & 0 & 471901.83 \\
\hline 7463.37 & 261033 & 8 & 0 & 2579.64 & 11116.75 & 2151.07 & 5565.33 & 307.96 & 421327.23 & 0 & 500481.68 \\
\hline 7463.44 & 190504 & 8 & 1523.42 & 5326.92 & 32726.91 & 5215.47 & 13606.37 & 794.15 & 435553.75 & 0 & 383091.12 \\
\hline 7463.50 & 136849 & 9 & 1685.14 & 6444.79 & 39667.99 & 7381.7 & 19266.97 & 1217.81 & 532920.38 & 0 & 48482.06 \\
\hline 7463.57 & 281191 & 7 & 532.81 & 2858.04 & 17800.91 & 2456.55 & 6352.51 & 357.2 & 399688.1 & 0 & 496825.15 \\
\hline 7463.64 & 293794 & 7 & 329.8 & 2007.85 & 14731.93 & 1729.01 & 4463.52 & 247.17 & 425358.56 & 0 & 505562.15 \\
\hline 7463.70 & 178630 & 9 & 2062.79 & 5792.53 & 57582.49 & 6865.4 & 17873.99 & 1109.09 & 474392.73 & 0 & 12651.52 \\
\hline 7463.77 & 175278 & 9 & 1624.46 & 6585.44 & 45405.75 & 7179.47 & 18678.15 & 1163.98 & 466278.43 & 0 & 92518.23 \\
\hline 7463.83 & 201604 & 8 & 1653.79 & 5936.52 & 34042.53 & 5634.07 & 14635.19 & 865.57 & 428367.63 & 0 & 352922.16 \\
\hline 7463.90 & 152362 & 9 & 1471.69 & 5646.56 & 46569.78 & 6392.86 & 16619.57 & 1002.49 & 423762.07 & 0 & 186342.72 \\
\hline 7463.96 & 176252 & 9 & 1874.06 & 6180.56 & 42679.95 & 6731.14 & 17677.27 & 1089.66 & 475361.62 & 0 & 136322.86 \\
\hline 7464.03 & 246423 & 8 & 697.42 & 3568.86 & 24508.61 & 3136.03 & 8206.86 & 459.37 & 372341.5 & 0 & 479933.23 \\
\hline 7464.09 & 222660 & 8 & 1341.2 & 4923.89 & 35793.97 & 4971.43 & 12980.52 & 754.36 & 396249.51 & 0 & 444372.89 \\
\hline 7464.16 & 229486 & 8 & 678.98 & 3445.92 & 18981.63 & 3043.4 & 7883.35 & 444.13 & 398772.38 & 0 & 475906.53 \\
\hline 7464.23 & 226674 & 8 & 4316.58 & 5558.13 & 27378.91 & 5000.92 & 13008.33 & 759.96 & 423734.65 & 0 & 442613.68 \\
\hline 7464.29 & 214002 & 8 & 1414.03 & 4643.76 & 30245.31 & 4499.39 & 11718.35 & 675.32 & 423381.31 & 0 & 452066.58 \\
\hline 7464.36 & 277425 & 7 & 0 & 2232.87 & 13064.07 & 1956.83 & 5089.69 & 278.41 & 418263.58 & 0 & 500418.94 \\
\hline 7464.42 & 250078 & 8 & 664.29 & 3542.8 & 23302.15 & 3233.33 & 8426 & 477.31 & 372515.2 & 0 & 477822.15 \\
\hline
\end{tabular}




\begin{tabular}{|c|c|c|c|c|c|c|c|c|c|c|c|}
\hline True Depth & XRF tCOUNTS & XRF Live Time & $\mathrm{Ti}(\mathrm{ppm})$ & V (ppm) & $\mathrm{Fe}(\mathrm{ppm})$ & $\mathrm{Ni}(\mathrm{ppm})$ & $\mathrm{Cu}(\mathrm{ppm})$ & $\mathrm{Pb}(\mathrm{ppm})$ & LE (ppm) & $\mathrm{Mg}(\mathrm{ppm})$ & $\mathrm{Cl}(\mathrm{ppm})$ \\
\hline 7464.49 & 191907 & 8 & 1544.17 & 5619.34 & 38453.85 & 5636.87 & 14709.7 & 874.55 & 432097.64 & 0 & 297269.7 \\
\hline 7464.55 & 276481 & 7 & 498.47 & 1900.69 & 9103 & 1488.18 & 3841.47 & 209.29 & 445791.26 & 0 & 500355.3 \\
\hline 7464.62 & 157793 & 9 & 1677.11 & 5901.27 & 44679.02 & 6551.19 & 17019.32 & 1052.41 & 508139.72 & 0 & 85419.94 \\
\hline 7464.69 & 245104 & 8 & 843.79 & 3773.73 & 25449.62 & 3375.75 & 8766.38 & 498.57 & 370376.55 & 0 & 464132.15 \\
\hline 7464.75 & 277575 & 7 & 598.5 & 2696.63 & 22502.79 & 2409.82 & 6272.67 & 348.6 & 386618.94 & 0 & 503328.39 \\
\hline 7464.82 & 188425 & 8 & 1880.66 & 5336.77 & 44825.58 & 5500.35 & 14262.67 & 861.04 & 452990.46 & 0 & 311235.15 \\
\hline 7464.88 & 218548 & 8 & 1367.21 & 5471.6 & 33179.26 & 4830.3 & 12626.62 & 732.81 & 408023.11 & 0 & 457617.78 \\
\hline 7464.95 & 249917 & 8 & 562.03 & 2388.81 & 11500.37 & 1827.51 & 4720.42 & 259.38 & 444634.24 & 0 & 494329.38 \\
\hline 7465.01 & 261905 & 8 & 527.74 & 2312.97 & 12761.64 & 1866.79 & 4844.76 & 266.86 & 436342.77 & 0 & 499355.51 \\
\hline 7465.08 & 154179 & 9 & 2859.05 & 7102.8 & 53550.01 & 8030.48 & 20894.11 & 1335.73 & 520537.69 & 0 & 15663.93 \\
\hline 7465.14 & 150400 & 9 & 2954.92 & 6573.33 & 46104.3 & 7054.58 & 18380.64 & 1159.68 & 514347.76 & 0 & 58955.72 \\
\hline 7465.21 & 133131 & 9 & 3033.94 & 6591.09 & 50391.49 & 7804.7 & 20437.56 & 1315.62 & 552407.49 & 0 & 26341.46 \\
\hline 7465.28 & 187988 & 8 & 3348.28 & 7365.02 & 48112.82 & 7597.68 & 19926.42 & 1242.93 & 429864.06 & 0 & 113795.57 \\
\hline 7465.34 & 193991 & 8 & 2381.05 & 6092.99 & 53555.34 & 6578.46 & 17138.27 & 1044.43 & 420184.67 & 0 & 134968.16 \\
\hline 7465.41 & 200637 & 8 & 1625.51 & 5067.47 & 47025.66 & 5007.2 & 13099.97 & 756.66 & 371878.08 & 0 & 358824.11 \\
\hline 7465.47 & 176316 & 9 & 3062.2 & 6385.88 & 47175.26 & 6434.83 & 16809.11 & 1021.76 & 451480.43 & 0 & 206227.48 \\
\hline 7465.54 & 166435 & 9 & 1809.09 & 5585.61 & 39002.83 & 5488.72 & 14252.84 & 851.31 & 440541.85 & 0 & 270676.56 \\
\hline 7465.60 & 163282 & 9 & 2236.41 & 6495.35 & 49120.96 & 7395.87 & 19280.46 & 1217.61 & 477722.93 & 0 & 34632.67 \\
\hline 7465.67 & 159254 & 9 & 1736 & 5290.65 & 55281.41 & 6222.86 & 16262.38 & 988.27 & 448379.99 & 0 & 138901.33 \\
\hline 7465.73 & 212238 & 8 & 1067.29 & 4906.48 & 47769.01 & 5192.27 & 13585.86 & 796.69 & 393468.93 & 0 & 366438.65 \\
\hline 7465.80 & 262494 & 8 & 559.27 & 2743.26 & 15623.64 & 2336.87 & 6049.71 & 336.65 & 403733.29 & 0 & 502490.98 \\
\hline 7465.87 & 191668 & 8 & 1968.02 & 5818.83 & 38721.05 & 5897.26 & 15342.5 & 908.81 & 356040.27 & 52003.5 & 290849.46 \\
\hline 7465.93 & 237242 & 8 & 964.54 & 4035.91 & 22260.5 & 3599.5 & 9363.27 & 536.05 & 380961.75 & 0 & 468670.8 \\
\hline 7466.00 & 220080 & 8 & 2015.52 & 5470.51 & 37922.1 & 5047.96 & 13151.02 & 768.63 & 384771.75 & 0 & 412287.04 \\
\hline 7466.06 & 189284 & 8 & 1195.2 & 5581.59 & 53984.36 & 6507.98 & 17086.28 & 1042.83 & 438384.63 & 0 & 46522.49 \\
\hline 7466.13 & 195076 & 8 & 1532.87 & 5191.41 & 63544.1 & 6168.44 & 16191.3 & 985.96 & 413664.83 & 0 & 114775.34 \\
\hline 7466.19 & 185762 & 8 & 2231.17 & 5590.96 & 52678.91 & 6377.93 & 16651.63 & 1014.58 & 427401.42 & 0 & 166316.78 \\
\hline 7466.26 & 206377 & 8 & 6539.57 & 8608.38 & 39200.54 & 6556.84 & 17117.2 & 1020.72 & 362267.23 & 0 & 411661.8 \\
\hline 7466.33 & 209879 & 8 & 4444 & 7896.73 & 39111.77 & 6563.27 & 17161.92 & 1025.65 & 395850.51 & 0 & 345741.23 \\
\hline 7466.39 & 230787 & 8 & 3659.39 & 6348.15 & 44690.7 & 5293.72 & 13807.45 & 801.87 & 358979.75 & 0 & 454194.51 \\
\hline 7466.46 & 213999 & 8 & 1153.73 & 5299.88 & 38643.39 & 5154.46 & 13511.45 & 783.58 & 381490.19 & 0 & 409170.14 \\
\hline 7466.52 & 218081 & 8 & 718.94 & 4749.35 & 34174.87 & 4533.83 & 11851.14 & 681.44 & 366503.23 & 0 & 439056.11 \\
\hline 7466.59 & 253702 & 8 & 1024.47 & 4242.65 & 25527.52 & 3873.16 & 10083.7 & 574.37 & 351193.77 & 0 & 481435.8 \\
\hline
\end{tabular}




\begin{tabular}{|c|c|c|c|c|c|c|c|c|c|c|c|}
\hline True Depth & XRF tCOUNTS & XRF Live Time & $\mathrm{Ti}(\mathrm{ppm})$ & V (ppm) & $\mathrm{Fe}(\mathrm{ppm})$ & $\mathrm{Ni}(\mathrm{ppm})$ & $\mathrm{Cu}(\mathrm{ppm})$ & $\mathrm{Pb}(\mathrm{ppm})$ & LE (ppm) & $\mathrm{Mg}(\mathrm{ppm})$ & $\mathrm{Cl}(\mathrm{ppm})$ \\
\hline 7466.65 & 280861 & 7 & 545.29 & 2597.4 & 13639.37 & 2122.44 & 5529.51 & 304.47 & 417186.1 & 0 & 504039.17 \\
\hline 7466.72 & 165769 & 9 & 1476.84 & 7071.47 & 45367.93 & 7678.07 & 20084.84 & 1274.37 & 485480.66 & 0 & 0 \\
\hline 7466.78 & 161529 & 9 & 4151.18 & 8416.06 & 47033.44 & 8247.77 & 21579.59 & 1385.9 & 487848.06 & 0 & 9146.42 \\
\hline 7466.85 & 263869 & 8 & 578.1 & 2425.88 & 13028.41 & 2004.73 & 5198.59 & 284.85 & 425942.73 & 0 & 502314.39 \\
\hline 7466.92 & 177763 & 8 & 2649.26 & 6435.13 & 56528.29 & 8020.26 & 21055.74 & 1320.83 & 418018.5 & 0 & 22003.3 \\
\hline 7466.98 & 196489 & 8 & 2197.15 & 5635.31 & 59830 & 6665.66 & 17491.33 & 1068.87 & 397856.74 & 0 & 104929.64 \\
\hline 7467.05 & 248472 & 8 & 1464.77 & 4915.82 & 39735.19 & 4930.93 & 12885.32 & 747.24 & 344005.42 & 0 & 422907.69 \\
\hline 7467.11 & 247755 & 8 & 1330.57 & 4160.23 & 29585.8 & 3515.13 & 9188.3 & 523.12 & 361861.56 & 0 & 479792.63 \\
\hline 7467.18 & 96924 & 9 & 2722.19 & 5235.87 & 43910.66 & 4698.14 & 12324.63 & 716.92 & 432744.8 & 0 & 387281.27 \\
\hline 7467.24 & 198377 & 8 & 4219.38 & 5405.8 & 47499.35 & 5999.86 & 15639.28 & 940.61 & 448379.47 & 0 & 241926.67 \\
\hline 7467.31 & 225162 & 8 & 1112.13 & 4708.48 & 34609.55 & 4775.22 & 12477.58 & 720.06 & 404241.47 & 0 & 391087.98 \\
\hline 7467.38 & 173121 & 9 & 1163.49 & 6070.85 & 47111.14 & 7134.52 & 18667.9 & 1176.12 & 481492.03 & 0 & 18074.68 \\
\hline 7467.44 & 162136 & 9 & 1180.22 & 5864.17 & 46169.17 & 7066.8 & 18525.82 & 1147.79 & 499464.29 & 0 & 54471.91 \\
\hline 7467.51 & 236541 & 8 & 0 & 4047.26 & 41258.38 & 4146.08 & 10836.84 & 618.45 & 395524.81 & 0 & 275784.4 \\
\hline 7467.57 & 195808 & 8 & 1009.7 & 5053.39 & 43086.37 & 5308.07 & 13900.39 & 815.51 & 423318.08 & 0 & 389651.37 \\
\hline 7467.64 & 242470 & 8 & 0 & 4560.37 & 41030.93 & 4550.67 & 11866.7 & 691.59 & 391491.19 & 0 & 278288.9 \\
\hline 7467.70 & 213224 & 8 & 989.77 & 4981.17 & 35668.31 & 5084.97 & 13257.79 & 767.52 & 386337.58 & 0 & 390438.36 \\
\hline 7467.77 & 194402 & 8 & 1081.47 & 5767.23 & 58561.34 & 7066.11 & 18535.88 & 1141.07 & 435772.97 & 0 & 47239.13 \\
\hline 7467.83 & 220327 & 8 & 0 & 4837 & 49876.18 & 5238.57 & 13660.75 & 792.91 & 366714.77 & 0 & 360758.34 \\
\hline 7467.90 & 295690 & 7 & 0 & 2900.37 & 40589.9 & 2780.45 & 7254.51 & 401.2 & 306448.8 & 0 & 325546.31 \\
\hline 7467.97 & 319137 & 7 & 0 & 2181.9 & 34129.3 & 2149.74 & 5612.07 & 310.12 & 251740.78 & 0 & 407529.88 \\
\hline 7468.03 & 320969 & 7 & 0 & 2167.29 & 28540.76 & 1949.23 & 5099.65 & 283.27 & 260606.89 & 0 & 388587.79 \\
\hline 7468.10 & 295070 & 7 & 0 & 2829.56 & 43383.94 & 2842.29 & 7426.99 & 410.59 & 353331.82 & 0 & 209612.39 \\
\hline 7468.16 & 220183 & 8 & 1067.94 & 5058 & 52101.08 & 5543.24 & 14464.41 & 845.13 & 395838.01 & 0 & 235077.89 \\
\hline 7468.23 & 196639 & 8 & 10667.28 & 6100.7 & 47734.22 & 7349.62 & 19264.39 & 1188.08 & 460451.65 & 0 & 84074.93 \\
\hline 7468.29 & 195590 & 8 & 971.84 & 5956.43 & 43901.61 & 6583.42 & 17212.07 & 1037.1 & 404845.4 & 0 & 258963.01 \\
\hline 7468.36 & 198481 & 8 & 871.48 & 5744.06 & 50579.98 & 6384.36 & 16630.92 & 1003.94 & 400343.3 & 0 & 200470.17 \\
\hline 7468.43 & 274900 & 8 & 0 & 2622.93 & 23071.68 & 2463.72 & 6429.42 & 360.03 & 397005.23 & 0 & 508193 \\
\hline 7468.49 & 233718 & 8 & 756.04 & 4786.02 & 52482.26 & 5218.1 & 13591.16 & 789.65 & 380031.91 & 0 & 380544.04 \\
\hline 7468.56 & 174191 & 9 & 1827.58 & 5635.06 & 51064.36 & 6957.92 & 18072.73 & 1108 & 451901.73 & 0 & 101298.88 \\
\hline 7468.62 & 187642 & 8 & 1706.94 & 5277.28 & 40742.81 & 5731.22 & 14913.49 & 892.24 & 444424.82 & 0 & 251325.49 \\
\hline 7468.69 & 207206 & 8 & 836.22 & 5810.02 & 33105.56 & 5790.96 & 15039.97 & 882.66 & 418406.05 & 0 & 363658.38 \\
\hline 7468.75 & 235528 & 8 & 0 & 5161.33 & 28945.08 & 4986.49 & 12919.55 & 744.56 & 386676.94 & 0 & 462683.11 \\
\hline
\end{tabular}




\begin{tabular}{|c|c|c|c|c|c|c|c|c|c|c|c|}
\hline True Depth & XRF tCOUNTS & XRF Live Time & $\mathrm{Ti}(\mathrm{ppm})$ & V (ppm) & $\mathrm{Fe}(\mathrm{ppm})$ & $\mathrm{Ni}(\mathrm{ppm})$ & $\mathrm{Cu}(\mathrm{ppm})$ & $\mathrm{Pb}(\mathrm{ppm})$ & LE (ppm) & $\mathrm{Mg}(\mathrm{ppm})$ & $\mathrm{Cl}(\mathrm{ppm})$ \\
\hline 7468.82 & 197063 & 8 & 908.08 & 5037.55 & 33626.78 & 5081.01 & 13202.9 & 778.99 & 383671.02 & 42987.28 & 317757.36 \\
\hline 7468.88 & 246566 & 8 & 632.8 & 4421.58 & 38792.55 & 4492.04 & 11710.38 & 674.38 & 360871.79 & 0 & 440730.83 \\
\hline 7468.95 & 210814 & 8 & 819.1 & 4929.52 & 50830.21 & 5423.03 & 14126.38 & 835.55 & 392993.61 & 0 & 256188.43 \\
\hline 7469.02 & 198871 & 8 & 0 & 2062.07 & 17413.26 & 1836.54 & 4748.33 & 264.92 & 436192.32 & 0 & 486632.05 \\
\hline 7469.08 & 147532 & 9 & 0 & 4787.67 & 27679.57 & 4923.13 & 12875.5 & 749.44 & 461426.3 & 0 & 404621.94 \\
\hline 7469.15 & 147047 & 9 & 0 & 2450.57 & 19921.02 & 2223.12 & 5767.97 & 318.64 & 438285.56 & 0 & 475587.7 \\
\hline 7469.21 & 153408 & 9 & 1355.63 & 6065.95 & 44947.51 & 6037.38 & 15817.96 & 951.11 & 447366.21 & 0 & 157236.6 \\
\hline 7469.28 & 252618 & 8 & 484.98 & 3219.92 & 20556.91 & 2989.66 & 7740.2 & 435.96 & 393757.23 & 0 & 480114.84 \\
\hline 7469.34 & 174208 & 9 & 1096.8 & 5371.9 & 36455.05 & 5401.06 & 14014.98 & 823.96 & 454113.57 & 0 & 332767.42 \\
\hline 7469.41 & 291436 & 7 & 0 & 2337.36 & 13227.39 & 2092.22 & 5392.65 & 298.52 & 418729.47 & 0 & 498772.69 \\
\hline 7469.48 & 259352 & 8 & 0 & 3634.87 & 20855.45 & 3379.71 & 8736.91 & 493.58 & 384853.1 & 0 & 474054.39 \\
\hline 7469.54 & 197132 & 8 & 802.12 & 5322.77 & 47771.4 & 5884.5 & 15231.7 & 908.72 & 441419.65 & 0 & 185032.36 \\
\hline 7469.61 & 222624 & 8 & 1203.31 & 5034.5 & 42887.8 & 5348.49 & 13844.55 & 815.67 & 388150.37 & 0 & 350400 \\
\hline 7469.67 & 186111 & 8 & 1488.71 & 5939.12 & 55401.67 & 7280.04 & 18984.85 & 1185.54 & 438884.53 & 0 & 15546.14 \\
\hline 7469.74 & 264230 & 8 & 662.44 & 4779.37 & 35316.7 & 4769.76 & 12377.86 & 719.13 & 345344.95 & 0 & 464640.01 \\
\hline 7469.80 & 188216 & 8 & 1214.05 & 6396.07 & 44334.92 & 7048.81 & 18334.41 & 1125.09 & 433725.07 & 0 & 154618.72 \\
\hline 7469.87 & 177026 & 8 & 1269.01 & 6838.57 & 47451.93 & 7919.82 & 20564.09 & 1301.97 & 443149.64 & 0 & 45207.27 \\
\hline 7469.93 & 299568 & 7 & 0 & 2121.39 & 12017.77 & 1865.12 & 4808.86 & 263.3 & 429569.29 & 0 & 502357.11 \\
\hline 7470.00 & 240322 & 8 & 813.9 & 4724.89 & 33624.24 & 4783.92 & 12330.5 & 719.81 & 385471.56 & 0 & 421396.2 \\
\hline 7470.07 & 190162 & 8 & 1352.51 & 5141.86 & 40523.82 & 5342.81 & 13828.18 & 818.47 & 429418.84 & 0 & 261024.66 \\
\hline 7470.13 & 196929 & 8 & 0 & 5561.08 & 46994.01 & 6167.15 & 15984.76 & 964.97 & 461976.04 & 0 & 138255.26 \\
\hline 7470.20 & 174067 & 9 & 956.59 & 5940.59 & 50411.08 & 7108.76 & 18409.39 & 1154.65 & 479391.3 & 0 & 53334.68 \\
\hline 7470.26 & 167869 & 9 & 1323.91 & 6093.71 & 44270 & 6838.17 & 17813.6 & 1099.62 & 463138.73 & 0 & 99734.82 \\
\hline 7470.33 & 165966 & 9 & 1053.82 & 6410.91 & 45629.88 & 7586.5 & 19722.71 & 1246.9 & 497328.55 & 0 & 61197.14 \\
\hline 7470.39 & 295107 & 7 & 969.67 & 3201.88 & 16145.75 & 2492.16 & 6460.14 & 361.24 & 385755.22 & 0 & 499137.63 \\
\hline 7470.46 & 241616 & 8 & 899.82 & 4950.41 & 34038.06 & 4823.15 & 12511.15 & 725.24 & 373177.07 & 0 & 445250.76 \\
\hline 7470.52 & 192446 & 8 & 1544.49 & 5534.1 & 42071.6 & 5918.58 & 15370.67 & 927.85 & 360599.23 & 59706.51 & 258148.98 \\
\hline 7470.59 & 195699 & 8 & 1094.28 & 6121.9 & 47371.44 & 6919.59 & 18054.08 & 1112.08 & 468878.18 & 0 & 33165.53 \\
\hline 7470.66 & 186931 & 8 & 783.95 & 4930.79 & 43392.88 & 5276.88 & 13715.95 & 810.39 & 432795.64 & 0 & 244081.99 \\
\hline 7470.72 & 244909 & 8 & 0 & 3631.22 & 23267.87 & 3480.62 & 9046.42 & 509.92 & 368144.28 & 0 & 474633.49 \\
\hline 7470.79 & 229841 & 8 & 1425.26 & 4869.39 & 34174.43 & 5012.55 & 12978.59 & 756.95 & 387334.43 & 0 & 419958.46 \\
\hline 7470.85 & 202146 & 8 & 981.6 & 5798.34 & 46297.78 & 6105.37 & 15843.6 & 949.08 & 327837.15 & 65716.34 & 263647.53 \\
\hline 7470.92 & 254383 & 8 & 1012.17 & 3894.32 & 30890.19 & 3583.05 & 9291.91 & 533.01 & 360146.35 & 0 & 464221.72 \\
\hline
\end{tabular}




\begin{tabular}{|c|c|c|c|c|c|c|c|c|c|c|c|}
\hline True Depth & XRF tCOUNTS & XRF Live Time & $\mathrm{Ti}(\mathrm{ppm})$ & V (ppm) & $\mathrm{Fe}(\mathrm{ppm})$ & $\mathrm{Ni}(\mathrm{ppm})$ & $\mathrm{Cu}(\mathrm{ppm})$ & $\mathrm{Pb}(\mathrm{ppm})$ & LE (ppm) & $\mathrm{Mg}(\mathrm{ppm})$ & $\mathrm{Cl}(\mathrm{ppm})$ \\
\hline 7470.98 & 259731 & 8 & 633.12 & 3157.83 & 31385.19 & 3022.46 & 7820.11 & 443.36 & 353679.82 & 0 & 472694.73 \\
\hline 7471.05 & 277685 & 7 & 0 & 3918.64 & 54787.26 & 4131.11 & 10796.7 & 620.32 & 307014.47 & 0 & 347935.13 \\
\hline 7471.12 & 289536 & 7 & 0 & 1808.77 & 19442.09 & 1667.61 & 4311.5 & 237.12 & 399073.26 & 0 & 509313.68 \\
\hline 7471.18 & 300517 & 7 & 0 & 2312.62 & 23038.05 & 2190.37 & 5630.65 & 317.16 & 388804.6 & 0 & 506599.78 \\
\hline 7471.25 & 139098 & 9 & 7463.04 & 5679.18 & 41875.63 & 6789.86 & 17676.88 & 1101.49 & 495625.92 & 0 & 19568.29 \\
\hline 7471.31 & 172890 & 9 & 2134.6 & 6236.77 & 45329.84 & 6762.36 & 17600.68 & 1094.58 & 474017.95 & 0 & 41236.31 \\
\hline 7471.38 & 175222 & 9 & 2548.87 & 6505.79 & 49946.19 & 6923.67 & 17977.12 & 1104.63 & 447106.74 & 0 & 114819.94 \\
\hline 7471.44 & 118868 & 9 & 4821.1 & 9064.28 & 42055.26 & 8241.5 & 21523.2 & 1397.67 & 575642.05 & 0 & 99660.44 \\
\hline 7471.51 & 227195 & 8 & 6879.1 & 10269.12 & 40856.65 & 6568.56 & 17157.87 & 1016.64 & 361230.98 & 0 & 324084.18 \\
\hline 7471.57 & 257417 & 8 & 2084.55 & 5819.61 & 44089.24 & 5295.99 & 13765.52 & 818.11 & 469450.32 & 0 & 41689.53 \\
\hline 7471.64 & 293872 & 7 & 0 & 4366.37 & 36152.06 & 3978.45 & 10366.89 & 595.63 & 372977.56 & 0 & 207189.33 \\
\hline 7471.71 & 181499 & 8 & 1378.84 & 5470.6 & 48591.48 & 6473.99 & 16787.16 & 1032.56 & 453056.28 & 0 & 100828.35 \\
\hline 7471.77 & 249245 & 8 & 0 & 3425.77 & 24909.73 & 3269.5 & 8459.8 & 480.48 & 362892.77 & 0 & 465528.32 \\
\hline 7471.84 & 188547 & 8 & 1280.64 & 6120.2 & 47315.15 & 7163.31 & 18621.67 & 1161.25 & 450454.61 & 0 & 0 \\
\hline 7471.90 & 152215 & 9 & 2743.74 & 6646.95 & 46027.49 & 6855.57 & 17737.16 & 1083.32 & 445092.64 & 0 & 93476.3 \\
\hline 7471.97 & 259260 & 8 & 1848.7 & 4403.63 & 22013.32 & 3374.28 & 8704.35 & 491.77 & 376897.04 & 0 & 478830.82 \\
\hline 7472.03 & 264032 & 8 & 1759.27 & 4365.76 & 20912.68 & 3108.34 & 8046.46 & 453.54 & 377546.1 & 0 & 492891.28 \\
\hline 7472.10 & 233011 & 8 & 4852.08 & 7805.34 & 42160.38 & 5914.77 & 15330.02 & 905.08 & 365325.16 & 0 & 426670.8 \\
\hline 7472.17 & 173127 & 9 & 4651.99 & 8506.76 & 55976.68 & 8115.65 & 21109.88 & 1332.4 & 451541.22 & 0 & 101485.84 \\
\hline 7472.23 & 154963 & 9 & 2634.73 & 4883.03 & 24141.91 & 3385.93 & 8728.87 & 494.93 & 364765.4 & 0 & 478249.32 \\
\hline 7472.30 & 234438 & 8 & 2223.93 & 6281.23 & 32652.89 & 5303.18 & 13744.84 & 798.88 & 384590.48 & 0 & 450167.02 \\
\hline 7472.36 & 166202 & 9 & 3282.28 & 7177.38 & 43168.03 & 7078.51 & 18375.52 & 1131.17 & 468469.76 & 0 & 164839.69 \\
\hline 7472.43 & 217174 & 8 & 1124.63 & 4028.07 & 26550.69 & 3440.92 & 8896.72 & 504.88 & 381766.7 & 0 & 467014.81 \\
\hline 7472.49 & 176672 & 8 & 3694.51 & 7831.66 & 42232.97 & 7061.84 & 18404.91 & 1127.02 & 452375.66 & 0 & 223186.66 \\
\hline 7472.56 & 197316 & 8 & 3752.65 & 7483.56 & 43537.11 & 6750.4 & 17527.08 & 1063.94 & 410952.11 & 0 & 244606.97 \\
\hline 7472.62 & 252714 & 8 & 1737.02 & 4565.18 & 21842.11 & 3676.27 & 9520.72 & 541.45 & 378448.72 & 0 & 480800.7 \\
\hline 7472.69 & 234638 & 8 & 3155.26 & 6004.32 & 40082.45 & 4962.98 & 12920.53 & 748.76 & 360697.62 & 0 & 459395.27 \\
\hline 7472.76 & 193160 & 8 & 1641.16 & 5397.85 & 35392.17 & 5064.07 & 13145.48 & 763.06 & 400164.63 & 0 & 413698.03 \\
\hline 7472.82 & 193538 & 8 & 2912.57 & 6743.43 & 53646.45 & 7084.98 & 18363 & 1132.06 & 431714.18 & 0 & 98596.16 \\
\hline 7472.89 & 285426 & 7 & 922.57 & 3391.8 & 18000.57 & 2520.46 & 6527.85 & 364.9 & 392240.37 & 0 & 504723.75 \\
\hline 7472.95 & 179378 & 8 & 4477.54 & 8398.57 & 48298.19 & 7962.28 & 20737.41 & 1304.55 & 434284.12 & 0 & 90743.36 \\
\hline 7473.02 & 227040 & 8 & 3298.51 & 6557.96 & 31652.86 & 5365.24 & 13874.95 & 807.9 & 376367.69 & 0 & 446531.25 \\
\hline 7473.08 & 230385 & 8 & 2761.15 & 7174.75 & 32309.65 & 5716.3 & 14834.28 & 865.24 & 301176.84 & 48943.84 & 460118.3 \\
\hline
\end{tabular}




\begin{tabular}{|c|c|c|c|c|c|c|c|c|c|c|c|}
\hline True Depth & XRF tCOUNTS & XRF Live Time & $\mathrm{Ti}(\mathrm{ppm})$ & V (ppm) & $\mathrm{Fe}(\mathrm{ppm})$ & $\mathrm{Ni}(\mathrm{ppm})$ & $\mathrm{Cu}(\mathrm{ppm})$ & $\mathrm{Pb}(\mathrm{ppm})$ & LE (ppm) & $\mathrm{Mg}(\mathrm{ppm})$ & $\mathrm{Cl}(\mathrm{ppm})$ \\
\hline 7473.15 & 189513 & 8 & 3985.75 & 7634.68 & 49032.1 & 7218.88 & 18802.05 & 1154.76 & 408207 & 0 & 246412.37 \\
\hline 7473.22 & 124250 & 9 & 2896.24 & 7157.3 & 55339.68 & 7375.16 & 19203.7 & 1181.38 & 405303.51 & 0 & 71169.88 \\
\hline 7473.28 & 260366 & 8 & 1007.3 & 3846.25 & 34031.78 & 3391.4 & 8789.94 & 495.42 & 295063.12 & 53091.05 & 486775.17 \\
\hline 7473.35 & 199788 & 8 & 3463.26 & 7550.47 & 44824.5 & 6500.55 & 16942.88 & 1016.49 & 402899.3 & 0 & 342561.55 \\
\hline 7473.41 & 186240 & 8 & 3685.19 & 7342.52 & 38975.59 & 6495.9 & 16899.85 & 1014.7 & 444899.58 & 0 & 286753.4 \\
\hline 7473.48 & 183589 & 8 & 3524.69 & 7553.81 & 43720.9 & 6947.7 & 18046.06 & 1095.63 & 423129.16 & 0 & 243263.09 \\
\hline 7473.54 & 184194 & 8 & 5103.68 & 8657.2 & 54925 & 7687.98 & 20008.61 & 1227.47 & 329428.88 & 67381.64 & 153145.41 \\
\hline 7473.61 & 280533 & 7 & 1309.47 & 3235.75 & 23316.01 & 2435.29 & 6297.34 & 350.41 & 378809.39 & 0 & 508333.53 \\
\hline 7473.67 & 190415 & 8 & 3843.51 & 7614.47 & 52620.37 & 7295.96 & 19143.57 & 1166.13 & 411342.37 & 0 & 189450.19 \\
\hline 7473.74 & 187271 & 8 & 3060.08 & 7359.15 & 57226.9 & 7703.54 & 20089.63 & 1240.46 & 418262.16 & 0 & 152147.46 \\
\hline 7473.81 & 264921 & 8 & 2330.86 & 4633.69 & 26521.11 & 3486.95 & 9048.05 & 509.12 & 364632.55 & 0 & 494127.14 \\
\hline 7473.87 & 193083 & 8 & 4784.91 & 8324.76 & 42573.79 & 6930.67 & 17982.25 & 1086.17 & 395064.42 & 0 & 272881.18 \\
\hline 7473.94 & 262711 & 8 & 1357.46 & 3829.42 & 20714.36 & 3122.58 & 8095.27 & 457.49 & 378391.13 & 0 & 493175.29 \\
\hline 7474.00 & 173176 & 9 & 4049.37 & 8073.91 & 55875.64 & 8013.41 & 20907.33 & 1320.18 & 451020.2 & 0 & 0 \\
\hline 7474.07 & 195158 & 8 & 5390.75 & 8459.55 & 50480.79 & 7081.53 & 18504.29 & 1120.88 & 387245.6 & 0 & 267064.38 \\
\hline 7474.13 & 182733 & 8 & 6671.73 & 9618.48 & 53856.18 & 7863.57 & 20487.75 & 1266.56 & 402247.82 & 0 & 181411.11 \\
\hline 7474.20 & 190792 & 8 & 4611.36 & 8042.61 & 36694.18 & 6206.09 & 16177.37 & 963.05 & 398017.32 & 0 & 337718.1 \\
\hline 7474.27 & 197278 & 8 & 6827 & 8345.22 & 41131.16 & 7276.21 & 18979.69 & 1162.39 & 430175 & 0 & 225837.42 \\
\hline 7474.33 & 168469 & 9 & 4435.79 & 7990.82 & 48526.61 & 8345 & 21689.28 & 1383.19 & 460167.47 & 0 & 13246.44 \\
\hline 7474.40 & 251424 & 8 & 505.22 & 3114.39 & 23295.94 & 2946.73 & 7574.3 & 425.02 & 384232.5 & 0 & 483570.63 \\
\hline 7474.46 & 212085 & 8 & 959.48 & 5338.09 & 42745.26 & 5540.6 & 14326.74 & 843.92 & 389045.54 & 0 & 358401.31 \\
\hline 7474.53 & 310903 & 7 & 0 & 1689.16 & 10944.84 & 1513.17 & 3889.51 & 212.13 & 440816.98 & 0 & 507497.17 \\
\hline 7474.59 & 201037 & 8 & 1542 & 5878.1 & 44293.95 & 6255.51 & 16214.91 & 963.54 & 402811.37 & 0 & 260253.87 \\
\hline 7474.66 & 175618 & 8 & 1044.31 & 6618.56 & 43310.29 & 7655.57 & 19893.97 & 1248.07 & 444569.68 & 0 & 119256.82 \\
\hline 7474.72 & 251172 & 8 & 556.36 & 3768.66 & 25793.81 & 3574.88 & 9237.78 & 523.87 & 374450.48 & 0 & 468798.41 \\
\hline 7474.79 & 221628 & 8 & 713.9 & 5038.71 & 36988.46 & 5170.91 & 13440.92 & 773.04 & 386318.75 & 0 & 404154.02 \\
\hline 7474.86 & 166150 & 9 & 1783.5 & 6385.9 & 46544.77 & 7605.49 & 19712.13 & 1228.72 & 388738.4 & 56377.59 & 27249.87 \\
\hline 7474.92 & 236109 & 8 & 788.79 & 4693.05 & 32377.24 & 4769.22 & 12385.82 & 717.65 & 382304.35 & 0 & 447604.48 \\
\hline 7474.99 & 191362 & 8 & 1461.26 & 5600.89 & 45744.6 & 6230.97 & 16146.11 & 969.9 & 418259.52 & 0 & 272425.09 \\
\hline 7475.05 & 160513 & 9 & 952.55 & 6312.02 & 51495.27 & 7567.39 & 19704.98 & 1246.88 & 467718.45 & 0 & 10617.38 \\
\hline 7475.12 & 166514 & 9 & 1298.54 & 5799.88 & 44400.09 & 6583.82 & 17105.03 & 1041.69 & 449310.99 & 0 & 173774.17 \\
\hline 7475.18 & 233963 & 8 & 917.96 & 5447.41 & 44431.4 & 5517.49 & 14280.27 & 840.07 & 380038.77 & 0 & 320793.88 \\
\hline 7475.25 & 57195 & 10 & 0 & 5209.83 & 28628.96 & 4887.5 & 12809.29 & 729.42 & 198080.18 & 0 & 122085.48 \\
\hline
\end{tabular}




\begin{tabular}{|c|c|c|c|c|c|c|c|c|c|c|c|}
\hline True Depth & XRF tCOUNTS & XRF Live Time & $\mathrm{Ti}(\mathrm{ppm})$ & V (ppm) & $\mathrm{Fe}(\mathrm{ppm})$ & $\mathrm{Ni}(\mathrm{ppm})$ & $\mathrm{Cu}(\mathrm{ppm})$ & $\mathrm{Pb}(\mathrm{ppm})$ & LE (ppm) & $\mathrm{Mg}(\mathrm{ppm})$ & $\mathrm{Cl}(\mathrm{ppm})$ \\
\hline 7475.31 & 203799 & 8 & 3344.81 & 7351.5 & 52198.58 & 7756.03 & 20095.04 & 1262.32 & 452772.48 & 0 & 0 \\
\hline 7475.38 & 170629 & 9 & 5713.13 & 9371.51 & 50969.86 & 8669.33 & 22589.16 & 1431.93 & 439165.61 & 0 & 49555.08 \\
\hline 7475.45 & 114605 & 9 & 3165.21 & 7212.24 & 50375.98 & 7365.93 & 19098.03 & 1194.45 & 406383.67 & 0 & 0 \\
\hline 7475.51 & 178268 & 8 & 2857.16 & 7110.19 & 52593.14 & 7933.24 & 20804.47 & 1313.64 & 443118.01 & 0 & 33967.28 \\
\hline 7475.58 & 172019 & 9 & 2326.3 & 6278.08 & 54433.48 & 7218.12 & 18780.4 & 1165.86 & 443780.67 & 0 & 93985.82 \\
\hline 7475.64 & 183171 & 8 & 2337.56 & 6489.09 & 48185.39 & 7001.71 & 18231.92 & 1103.4 & 429230.37 & 0 & 183093.06 \\
\hline 7475.71 & 205587 & 8 & 1275.83 & 5774.1 & 50284.11 & 6781.45 & 17651.39 & 1071.82 & 447376.96 & 0 & 0 \\
\hline 7475.77 & 261553 & 8 & 0 & 4537.54 & 32662.25 & 4498.04 & 11637.52 & 666.48 & 354300.75 & 0 & 456863.09 \\
\hline 7475.84 & 225342 & 8 & 1454.19 & 5453.19 & 42284.44 & 5876.65 & 15267 & 901.19 & 401141.12 & 0 & 230189.62 \\
\hline 7475.91 & 221474 & 8 & 1130.37 & 6909.8 & 45780.32 & 7640.68 & 19959.77 & 1230.29 & 443563.12 & 0 & 0 \\
\hline 7475.97 & 272999 & 7 & 53338.49 & 31074.34 & 290171.59 & 9754.4 & 25869.17 & 1543.82 & 113745.54 & 156515.34 & 0 \\
\hline 7476.04 & 141849 & 9 & 1952.82 & 6604.06 & 45932.43 & 7688.27 & 19959.25 & 1268.28 & 476140.76 & 0 & 0 \\
\hline 7476.10 & 179327 & 8 & 1322.69 & 6403.92 & 45347.11 & 7311.46 & 19008.54 & 1175.4 & 434106.65 & 0 & 148009.88 \\
\hline 7476.17 & 169316 & 9 & 1611.33 & 6368.77 & 56283.09 & 7674.18 & 20016.11 & 1260.04 & 464390.88 & 0 & 0 \\
\hline 7476.23 & 190483 & 8 & 1529.99 & 5240.38 & 54482.12 & 6171.85 & 16051.06 & 965.38 & 441964.55 & 0 & 120179.45 \\
\hline 7476.30 & 217603 & 8 & 13006.74 & 5333.65 & 53696.46 & 6248.6 & 16240.54 & 972.44 & 427338.85 & 0 & 238348.85 \\
\hline 7476.36 & 186288 & 8 & 1330.72 & 5568.09 & 46820.1 & 6081.12 & 15850.93 & 957.7 & 418794.34 & 0 & 188428.14 \\
\hline 7476.43 & 185511 & 8 & 1640.37 & 5428.84 & 50048.38 & 6280.63 & 16277.47 & 987.37 & 449404.72 & 0 & 131561.39 \\
\hline 7476.50 & 162804 & 9 & 1518.8 & 6148.99 & 46153 & 7255.4 & 18976.45 & 1187.35 & 485588.18 & 0 & 17758.06 \\
\hline 7476.56 & 205068 & 8 & 1176.34 & 5373.51 & 37913.57 & 5673.61 & 14695.32 & 871.16 & 418922.83 & 0 & 305180.48 \\
\hline 7476.63 & 166052 & 9 & 1381.52 & 6038.06 & 52756.26 & 7403.73 & 19222.87 & 1221.93 & 448524.47 & 0 & 0 \\
\hline 7476.69 & 167967 & 9 & 1435.9 & 6026.46 & 55609.31 & 7670.51 & 20013.79 & 1266.03 & 447815.63 & 0 & 0 \\
\hline 7476.76 & 195936 & 8 & 909.85 & 6105.28 & 43261.89 & 6174.45 & 16049.78 & 956.48 & 432985.37 & 0 & 293514.79 \\
\hline 7476.82 & 156481 & 9 & 1140.45 & 6208.21 & 42187.76 & 6863.27 & 17890.33 & 1101.12 & 480807.7 & 0 & 111710.89 \\
\hline 7476.89 & 162228 & 9 & 1492.49 & 6089.56 & 43082.27 & 6808.46 & 17715.6 & 1084.38 & 394875.42 & 59039.38 & 144968.98 \\
\hline 7476.96 & 206764 & 8 & 1533.3 & 5248.82 & 64620.82 & 6424.56 & 16654.63 & 1004.64 & 393199.01 & 0 & 188485.08 \\
\hline 7477.02 & 195082 & 8 & 1094.75 & 5924.01 & 66970.74 & 7441.84 & 19464.98 & 1203.8 & 423190.52 & 0 & 51301.31 \\
\hline 7477.09 & 196168 & 8 & 1280.94 & 4865.8 & 47697.13 & 5334.66 & 13912.08 & 815.71 & 379146.18 & 0 & 386811.78 \\
\hline 7477.15 & 215056 & 8 & 1471.73 & 5484.33 & 56581.94 & 6247.9 & 16280.48 & 974.76 & 381344.17 & 0 & 281816.55 \\
\hline 7477.22 & 225201 & 8 & 895.67 & 5681.83 & 47243.55 & 5918.59 & 15391.53 & 909.03 & 373493.32 & 0 & 368490.2 \\
\hline 7477.28 & 267646 & 18 & 1369.81 & 6216.91 & 51361.14 & 7372.57 & 19253.97 & 1211.23 & 434396.2 & 0 & 18011.34 \\
\hline 7477.35 & 387579 & 17 & 1622.87 & 5806.92 & 62357.36 & 7065.99 & 18414.65 & 1128.71 & 355716.45 & 51676.73 & 81945.84 \\
\hline 7477.41 & 507582 & 15 & 578.19 & 3351.09 & 36388.64 & 3380.94 & 8776.23 & 498.58 & 355349.88 & 0 & 472992.2 \\
\hline
\end{tabular}




\begin{tabular}{|c|c|c|c|c|c|c|c|c|c|c|c|}
\hline True Depth & XRF tCOUNTS & XRF Live Time & $\mathrm{Ti}(\mathrm{ppm})$ & $\mathrm{V}(\mathrm{ppm})$ & $\mathrm{Fe}(\mathrm{ppm})$ & $\mathrm{Ni}(\mathrm{ppm})$ & $\mathrm{Cu}(\mathrm{ppm})$ & $\mathrm{Pb}(\mathrm{ppm})$ & $\mathrm{LE}(\mathrm{ppm})$ & $\mathrm{Mg}(\mathrm{ppm})$ & $\mathrm{Cl}(\mathrm{ppm})$ \\
\hline 7477.48 & 407802 & 16 & 1460.32 & 4893.29 & 63262.09 & 5604.86 & 14619.13 & 866.55 & 402913.48 & 0 & 253337.99 \\
\hline 7477.55 & 414729 & 16 & 1641.12 & 5068.34 & 55464.49 & 5733.29 & 14936.54 & 884.69 & 377129.21 & 0 & 289265.89 \\
\hline 7477.61 & 328666 & 17 & 1933.07 & 6517.58 & 43423.12 & 7720.46 & 20182.64 & 1273.54 & 450732.67 & 0 & 23377.11 \\
\hline 7477.68 & 343761 & 17 & 1489.95 & 6482.55 & 43261.2 & 7317.63 & 19123.29 & 1185.57 & 445727.82 & 0 & 126378.42 \\
\hline 7477.74 & 321716 & 17 & 1946.7 & 6542.41 & 45366.1 & 7922.63 & 20700.79 & 1317.04 & 463667.46 & 0 & 16539.15 \\
\hline 7477.81 & 315071 & 17 & 1920.31 & 6556.64 & 42343.09 & 7608 & 19877 & 1253.83 & 468548.25 & 0 & 27985.66 \\
\hline 7477.87 & 328417 & 17 & 1374.66 & 6157.99 & 39373.55 & 6731.21 & 17492.27 & 1077.01 & 455396.81 & 0 & 149068.87 \\
\hline 7477.94 & 361982 & 17 & 1744.93 & 5817.87 & 39958.59 & 6309.91 & 16392.15 & 990.22 & 444588.83 & 0 & 216003.65 \\
\hline 7478.01 & 326084 & 17 & 1715.59 & 6411.76 & 48883.02 & 7562.86 & 19692.06 & 1227.86 & 400982.65 & 48118.32 & 0 \\
\hline 7478.07 & 343654 & 17 & 2383.49 & 6222.76 & 58740.2 & 7543.24 & 19688.08 & 1237.55 & 444684.84 & 0 & 28667.42 \\
\hline 7478.14 & 369581 & 17 & 1699.87 & 5765.26 & 52389.54 & 6541.15 & 17017.16 & 1036.83 & 436418.97 & 0 & 164635.21 \\
\hline 7478.20 & 359931 & 17 & 1225.9 & 6138.19 & 45170.22 & 6712.39 & 17480.4 & 1065.6 & 454295.81 & 0 & 200849.3 \\
\hline 7478.27 & 311324 & 17 & 1531.41 & 6523.27 & 46125.87 & 7765.47 & 20290.2 & 1289.19 & 474475.48 & 0 & 15987.65 \\
\hline 7478.33 & 435948 & 16 & 1057.51 & 5150.1 & 40394.2 & 5267.68 & 13728.46 & 802.91 & 389953.2 & 0 & 402324.71 \\
\hline 7478.40 & 318878 & 17 & 1487.44 & 6504.37 & 45320.96 & 7366.35 & 19200.31 & 1198.13 & 400920.96 & 53894.75 & 72012.25 \\
\hline 7478.46 & 307283 & 17 & 1611.41 & 6614.72 & 41750.83 & 7538.03 & 19715.42 & 1253.25 & 489469.34 & 0 & 7952.31 \\
\hline 7478.53 & 411832 & 16 & 1028.03 & 5466.4 & 32699.58 & 5482.82 & 14248.14 & 836.53 & 416105.83 & 0 & 342821.88 \\
\hline 7478.60 & 444659 & 16 & 1110.62 & 4971.28 & 30262.34 & 4867.61 & 12644.24 & 737.32 & 401833.84 & 0 & 426851.87 \\
\hline 7478.66 & 593893 & 14 & 210.73 & 2357.06 & 22331.54 & 2194.18 & 5667.16 & 315.38 & 395195.6 & 0 & 507603.52 \\
\hline 7478.73 & 416514 & 16 & 1150.73 & 5343.03 & 57968.25 & 6191.36 & 16138.85 & 962.92 & 398379.62 & 0 & 285234.35 \\
\hline 7478.79 & 329400 & 17 & 1673.45 & 6431.35 & 53476 & 7736.19 & 20278.32 & 1271.93 & 453829.47 & 0 & 0 \\
\hline 7478.86 & 350933 & 17 & 1421.01 & 6134.16 & 54950.88 & 7561.55 & 19723.05 & 1233.04 & 471202.14 & 0 & 17263.67 \\
\hline 7478.92 & 376795 & 17 & 253.74 & 2768.59 & 14716.8 & 2429.84 & 6293.72 & 353.16 & 437707.27 & 0 & 475020.45 \\
\hline 7478.99 & 170630 & 19 & 1193.19 & 5808.49 & 36060.59 & 6033.03 & 15731.99 & 953.42 & 550031.61 & 0 & 272001.18 \\
\hline 7479.06 & 291019 & 18 & 642.41 & 4118.55 & 31040.98 & 3781.76 & 9805.53 & 553.38 & 394248.35 & 0 & 423804.3 \\
\hline 7479.12 & 479066 & 16 & 0 & 1112.94 & 5828.42 & 947.74 & 2442.22 & 130.41 & 474223.67 & 0 & 499675.23 \\
\hline 7479.19 & 506923 & 15 & 0 & 1286.01 & 6742.09 & 1083.9 & 2787.21 & 150.41 & 464033.33 & 0 & 500461.56 \\
\hline 7479.25 & 326543 & 17 & 3443.39 & 5949.21 & 44285.21 & 7077.45 & 18396.73 & 1154.57 & 479307.16 & 0 & 66579.26 \\
\hline 7479.32 & 333204 & 17 & 1984.63 & 6233.35 & 44070.95 & 7019.92 & 18272.66 & 1143.44 & 462097.01 & 0 & 71838.28 \\
\hline 7479.38 & 454988 & 16 & 664.4 & 4736.41 & 31903.77 & 4813.47 & 12526.12 & 726.85 & 396297.26 & 0 & 437574.79 \\
\hline 7479.45 & 318367 & 17 & 1072.98 & 5996.14 & 45547.61 & 7038.4 & 18313.03 & 1148.21 & 473919.98 & 0 & 59812.01 \\
\hline 7479.51 & 342110 & 17 & 1311.41 & 5608.98 & 43253.59 & 6129.16 & 15971.41 & 960.65 & 447720.77 & 0 & 238410.21 \\
\hline 7479.58 & 311033 & 17 & 1475.41 & 5669.51 & 51028.91 & 7074.55 & 18445.02 & 1155.22 & 489532.13 & 0 & 18341.67 \\
\hline
\end{tabular}




\begin{tabular}{|c|c|c|c|c|c|c|c|c|c|c|c|}
\hline True Depth & XRF tCOUNTS & XRF Live Time & $\mathrm{Ti}(\mathrm{ppm})$ & V (ppm) & $\mathrm{Fe}(\mathrm{ppm})$ & $\mathrm{Ni}(\mathrm{ppm})$ & $\mathrm{Cu}(\mathrm{ppm})$ & $\mathrm{Pb}(\mathrm{ppm})$ & LE (ppm) & $\mathrm{Mg}(\mathrm{ppm})$ & $\mathrm{Cl}(\mathrm{ppm})$ \\
\hline 7479.65 & 381084 & 17 & 932.71 & 5662.63 & 34789.05 & 5824.99 & 15198 & 906.16 & 423016.78 & 0 & 336063.3 \\
\hline 7479.71 & 299892 & 17 & 1156.4 & 6371.67 & 43267.88 & 7448.86 & 19501.46 & 1231.42 & 486289.39 & 0 & 26410.31 \\
\hline 7479.78 & 433611 & 16 & 1037.99 & 5296.92 & 30513.39 & 5126.92 & 13375.81 & 784.31 & 408889.41 & 0 & 417724.12 \\
\hline 7479.84 & 482518 & 16 & 545.58 & 3704.87 & 20596.35 & 3405.22 & 8838.13 & 503.76 & 386640.79 & 0 & 469067.94 \\
\hline 7479.91 & 298103 & 17 & 1659.26 & 6141.11 & 41432.75 & 7070.98 & 18507.88 & 1151.23 & 484482.91 & 0 & 78700.72 \\
\hline 7479.97 & 317591 & 17 & 1254 & 5773.68 & 34926.89 & 6254.9 & 16336.22 & 986.78 & 474495.34 & 0 & 207032.99 \\
\hline 7480.04 & 351449 & 17 & 974.34 & 5350.38 & 30156.49 & 5486.74 & 14259.23 & 845.66 & 460241.29 & 0 & 326164.51 \\
\hline 7480.10 & 342546 & 17 & 1204.71 & 5607.05 & 32541.43 & 5938.65 & 15473.26 & 924.69 & 460278.62 & 0 & 280198.22 \\
\hline 7480.17 & 290309 & 18 & 1919.12 & 6098.03 & 34875.97 & 6262.2 & 16350.94 & 987.67 & 496885.22 & 0 & 234411.75 \\
\hline 7480.24 & 446032 & 16 & 2362.14 & 2727 & 14378.38 & 2476.75 & 6421.59 & 359.03 & 426679.68 & 0 & 479839.52 \\
\hline 7480.30 & 374354 & 17 & 1502.26 & 5710.36 & 35395.08 & 5769.62 & 15001.31 & 894.02 & 432137.11 & 0 & 281143.74 \\
\hline 7480.37 & 261238 & 18 & 1548.73 & 5884.83 & 41960.41 & 6936.19 & 18130.46 & 1140.98 & 528751.64 & 0 & 56677.37 \\
\hline 7480.43 & 496983 & 15 & 291 & 2847.24 & 13409.4 & 2510.12 & 6514.87 & 364.36 & 417984.05 & 0 & 493090.11 \\
\hline 7480.50 & 343589 & 17 & 1512.52 & 6111.6 & 48665.07 & 7209.65 & 18795.4 & 1167.46 & 434114.65 & 0 & 74850.59 \\
\hline 7480.56 & 413609 & 16 & 968.23 & 4826.29 & 36524.27 & 4937.2 & 12864.32 & 748.33 & 400328.85 & 0 & 433692.82 \\
\hline 7480.63 & 301714 & 17 & 1427.23 & 5681.46 & 48600.98 & 6387.89 & 16727.28 & 1012.83 & 483345.77 & 0 & 179885.86 \\
\hline 7480.70 & 259652 & 18 & 1013.17 & 5916.66 & 47274.01 & 7070.93 & 18541.8 & 1177.73 & 544152.08 & 0 & 33579.32 \\
\hline 7480.76 & 329053 & 17 & 1626.46 & 5912.2 & 45596.57 & 6971.83 & 18245.31 & 1135.53 & 476131.38 & 0 & 56615.4 \\
\hline 7480.83 & 314678 & 17 & 1387.71 & 6286.03 & 45670.49 & 7316.52 & 19144.76 & 1198.48 & 483043.41 & 0 & 47544.9 \\
\hline 7480.89 & 493359 & 15 & 692.96 & 5158.84 & 34498.29 & 5188.15 & 13531.24 & 785.41 & 363122.7 & 0 & 330345.78 \\
\hline 7480.96 & 578549 & 14 & 0 & 4078.05 & 27962.01 & 3888.21 & 10173.84 & 570.62 & 346414.67 & 0 & 296737.12 \\
\hline 7481.02 & 521460 & 15 & 0 & 4640.56 & 37551.65 & 4893.6 & 12817.57 & 744.53 & 475931.61 & 0 & 57993.09 \\
\hline 7481.09 & 503426 & 15 & 0 & 3969.97 & 27103.34 & 3679.71 & 9605.38 & 538.3 & 366963.44 & 0 & 300644.37 \\
\hline 7481.15 & 481464 & 16 & 0 & 3809.42 & 28635.48 & 3610.82 & 9440 & 535.81 & 422338.34 & 0 & 185650.3 \\
\hline 7481.22 & 389612 & 17 & 0 & 2799.68 & 18589.12 & 2562.91 & 6650.56 & 363.44 & 280259.57 & 0 & 356027.41 \\
\hline 7481.29 & 568582 & 15 & 1168.51 & 5041.78 & 36549.67 & 5117.31 & 13427.75 & 785.52 & 456549.79 & 0 & 14296.26 \\
\hline 7481.35 & 623398 & 14 & 0 & 2806.31 & 19071.91 & 2512.42 & 6536.48 & 362.33 & 304727.61 & 0 & 463315.93 \\
\hline 7481.42 & 523356 & 15 & 501.84 & 3270.45 & 19040.66 & 2939.91 & 7666.9 & 429.06 & 381835.34 & 0 & 482054.01 \\
\hline 7481.48 & 448908 & 16 & 1176.23 & 5458.06 & 27193.78 & 5192.57 & 13621.32 & 786.9 & 396688.72 & 0 & 399170.65 \\
\hline 7481.55 & 451291 & 16 & 976.62 & 4892.59 & 31858.39 & 4835.82 & 12643.31 & 729.26 & 396196.01 & 0 & 403485.04 \\
\hline 7481.61 & 476819 & 16 & 1609.53 & 7168.37 & 39641.87 & 6910.58 & 18174.25 & 1088.13 & 432157.33 & 0 & 137425.82 \\
\hline 7481.68 & 467367 & 16 & 767.81 & 6992.28 & 37086.14 & 7633.19 & 20095.71 & 1237.46 & 498657.47 & 0 & 0 \\
\hline 7481.75 & 564298 & 15 & 0 & 6203.71 & 37709.56 & 6390.9 & 16827.29 & 996.75 & 467417.61 & 0 & 4578.57 \\
\hline
\end{tabular}




\begin{tabular}{|c|c|c|c|c|c|c|c|c|c|c|c|}
\hline True Depth & XRF tCOUNTS & XRF Live Time & $\mathrm{Ti}(\mathrm{ppm})$ & V (ppm) & $\mathrm{Fe}(\mathrm{ppm})$ & $\mathrm{Ni}(\mathrm{ppm})$ & $\mathrm{Cu}(\mathrm{ppm})$ & $\mathrm{Pb}(\mathrm{ppm})$ & LE (ppm) & $\mathrm{Mg}(\mathrm{ppm})$ & $\mathrm{Cl}(\mathrm{ppm})$ \\
\hline 7481.81 & 505681 & 15 & 0 & 5740.57 & 29014.75 & 5846.2 & 15348.7 & 891.32 & 413679.64 & 0 & 219648.77 \\
\hline 7481.88 & 497049 & 15 & 0 & 4181.18 & 24233.35 & 4007.19 & 10496.9 & 591.08 & 352992.34 & 0 & 417266.75 \\
\hline 7481.94 & 229416 & 18 & 1568.63 & 6034.53 & 41402.94 & 5997.21 & 15735.91 & 939.72 & 446882.12 & 0 & 62289.4 \\
\hline 7482.01 & 423804 & 16 & 911.85 & 6628.12 & 41757 & 7325.83 & 19268.8 & 1180.69 & 461441.26 & 0 & 0 \\
\hline 7482.07 & 480056 & 16 & 818 & 6747.5 & 48718.3 & 7209.61 & 18956.55 & 1154.59 & 425784.91 & 0 & 61615.57 \\
\hline 7482.14 & 577072 & 14 & 0 & 3490.37 & 24344.19 & 3310.23 & 8654.13 & 485.24 & 330983.35 & 0 & 465952.93 \\
\hline 7482.20 & 516406 & 15 & 645.99 & 4991.78 & 38080.83 & 4876.67 & 12747.45 & 731.27 & 347754.49 & 0 & 340518.56 \\
\hline 7482.27 & 437063 & 16 & 1264.35 & 5350.83 & 40505.78 & 5676.26 & 14897.33 & 881.73 & 400484.88 & 0 & 268778.03 \\
\hline 7482.34 & 615663 & 14 & 257.63 & 2613.93 & 16230.29 & 2226.44 & 5804.19 & 322.66 & 380967.22 & 0 & 502003.72 \\
\hline 7482.40 & 611492 & 14 & 1101.84 & 3823.66 & 20291.47 & 2919.13 & 7621.17 & 425.39 & 335532.11 & 0 & 484916.27 \\
\hline 7482.47 & 548999 & 15 & 0 & 1451.69 & 8457.3 & 1222.54 & 3176.08 & 172.67 & 446031.69 & 0 & 500415.27 \\
\hline 7482.53 & 399464 & 17 & 802.31 & 4602.26 & 30498.05 & 4490.54 & 11734.73 & 684.38 & 426106.63 & 0 & 423618.61 \\
\hline 7482.60 & 359592 & 17 & 48552.01 & 34439.77 & 88500.91 & 10924.68 & 28831.67 & 1810.33 & 371550.72 & 0 & 293338.89 \\
\hline 7482.66 & 247992 & 18 & 1444.85 & 5105.95 & 31328.26 & 4921.41 & 12849.85 & 748.93 & 447465.57 & 0 & 297235.11 \\
\hline 7482.73 & 471978 & 16 & 1206.62 & 5267.43 & 30950.04 & 5261.96 & 13743.11 & 804.49 & 393779.47 & 0 & 401737.24 \\
\hline 7482.80 & 580204 & 14 & 296.34 & 2462.9 & 14737.45 & 2204.97 & 5718.09 & 317.98 & 410183.19 & 0 & 492761.91 \\
\hline 7482.86 & 584576 & 14 & 0 & 3033.37 & 19636.06 & 2853.38 & 7426.99 & 416.81 & 363754.88 & 0 & 478697.12 \\
\hline 7482.93 & 380759 & 17 & 408.47 & 2651.18 & 18371.07 & 2356.17 & 6118.24 & 338.73 & 440474.46 & 0 & 480799.31 \\
\hline 7482.99 & 297675 & 18 & 822.21 & 5143.56 & 27963.59 & 5076.11 & 13235.01 & 780.86 & 496287.16 & 0 & 354109.57 \\
\hline 7483.06 & 406598 & 16 & 0 & 2112.13 & 11871.29 & 1863.43 & 4822.52 & 265.21 & 452040.5 & 0 & 484857.65 \\
\hline 7483.12 & 424829 & 16 & 287.75 & 2348.64 & 13271.37 & 2083.27 & 5388.86 & 298.08 & 437544.89 & 0 & 483920.64 \\
\hline 7483.19 & 361005 & 17 & 929.47 & 4648.59 & 33420.8 & 4743.13 & 12368.72 & 717.69 & 404325.96 & 35019.5 & 370793.14 \\
\hline 7483.25 & 135056 & 19 & 0 & 2733.85 & 9193.39 & 2612.93 & 6794.8 & 410.17 & 856982.2 & 0 & 72800.41 \\
\hline 7483.32 & 368287 & 17 & 3153.63 & 5163.18 & 41197.2 & 5714.43 & 14883.13 & 891.86 & 458686.25 & 0 & 226791.86 \\
\hline 7483.39 & 465214 & 16 & 724.81 & 4890.58 & 30703.33 & 4809.63 & 12511.9 & 725.06 & 391933.47 & 0 & 430563.29 \\
\hline 7483.45 & 342591 & 17 & 1454.32 & 5559.34 & 40336.8 & 5849.87 & 15275.29 & 921.5 & 454897.98 & 0 & 226091.82 \\
\hline 7483.52 & 423066 & 16 & 1574.83 & 4843.86 & 31264.9 & 4623.15 & 12039.29 & 690.93 & 411737.81 & 0 & 436135.72 \\
\hline 7483.58 & 490563 & 16 & 308.34 & 2425.86 & 13763.11 & 2140.71 & 5554.59 & 307.1 & 427582.82 & 0 & 487874.77 \\
\hline 7483.65 & 581831 & 14 & 0 & 2258.08 & 12690.48 & 2008.73 & 5219 & 287.88 & 418539.56 & 0 & 501354.12 \\
\hline 7483.71 & 356576 & 17 & 1322.57 & 6083.3 & 44931.3 & 6898.15 & 18047.32 & 1114.77 & 396355.92 & 50795.4 & 78455.29 \\
\hline 7483.78 & 346654 & 17 & 1476.94 & 5967.31 & 42603.48 & 6815.18 & 17770.6 & 1096.31 & 464204.72 & 0 & 72205.08 \\
\hline 7483.85 & 319942 & 17 & 1411.96 & 5919.94 & 44340.18 & 7070 & 18503.51 & 1165.64 & 481216.41 & 0 & 0 \\
\hline 7483.91 & 349587 & 17 & 1047.56 & 5192.19 & 38523.03 & 5552.68 & 14479.65 & 861.75 & 449254.03 & 0 & 318871.08 \\
\hline
\end{tabular}




\begin{tabular}{|c|c|c|c|c|c|c|c|c|c|c|c|}
\hline True Depth & XRF tCOUNTS & XRF Live Time & $\mathrm{Ti}(\mathrm{ppm})$ & V (ppm) & $\mathrm{Fe}(\mathrm{ppm})$ & $\mathrm{Ni}(\mathrm{ppm})$ & $\mathrm{Cu}(\mathrm{ppm})$ & $\mathrm{Pb}(\mathrm{ppm})$ & LE (ppm) & $\mathrm{Mg}(\mathrm{ppm})$ & $\mathrm{Cl}(\mathrm{ppm})$ \\
\hline 7483.98 & 503290 & 15 & 663.08 & 3580.59 & 21333.6 & 3312.56 & 8607.08 & 485.37 & 377666.03 & 0 & 477300.73 \\
\hline 7484.04 & 464733 & 16 & 1034.29 & 4875.59 & 32118.34 & 4818.38 & 12563.44 & 727.48 & 398156.96 & 0 & 428877.19 \\
\hline 7484.11 & 576015 & 15 & 316.18 & 2249.36 & 12905.14 & 1970.76 & 5098.89 & 282.06 & 421133.13 & 0 & 502123.04 \\
\hline 7484.17 & 443222 & 16 & 1198.17 & 5331.6 & 37188.72 & 5393.93 & 14039.11 & 827.25 & 390690.37 & 0 & 390126.66 \\
\hline 7484.24 & 437904 & 16 & 762.98 & 4991.39 & 32984.25 & 4920.91 & 12795.09 & 747.1 & 400788.51 & 0 & 404419.27 \\
\hline 7484.30 & 357665 & 17 & 10140.87 & 5874.71 & 37146.8 & 7004.06 & 18268.41 & 1135.49 & 493613.4 & 0 & 152702.54 \\
\hline 7484.37 & 545269 & 15 & 224.83 & 2931.85 & 13853.68 & 2660.93 & 6880.25 & 385.27 & 403005.35 & 0 & 492498.05 \\
\hline 7484.44 & 539014 & 15 & 222.08 & 2871.54 & 13963.56 & 2606.41 & 6729.94 & 376.52 & 408144.98 & 0 & 490587.64 \\
\hline 7484.50 & 359395 & 17 & 1123.28 & 6074.28 & 36655.14 & 6477.6 & 16835.91 & 1022.51 & 426540.7 & 0 & 164385.68 \\
\hline 7484.57 & 396423 & 17 & 930.14 & 5708.53 & 29701.49 & 5679.32 & 14803.51 & 874.66 & 416535.5 & 0 & 317618.28 \\
\hline 7484.63 & 637431 & 14 & 0 & 1455.28 & 6451.64 & 1220.07 & 3145.41 & 169.24 & 458713.4 & 0 & 503285.96 \\
\hline 7484.70 & 450927 & 16 & 1220.59 & 4638.82 & 31637.73 & 4523.12 & 11762.99 & 674.24 & 391368.66 & 0 & 437686.08 \\
\hline 7484.76 & 383836 & 17 & 968.45 & 5750.45 & 33054.15 & 5774.91 & 15061.43 & 898.78 & 431398.71 & 0 & 313137.94 \\
\hline 7484.83 & 641679 & 14 & 0 & 1493.07 & 7002.29 & 1266.13 & 3267.05 & 177.49 & 451290.04 & 0 & 507951.86 \\
\hline 7484.90 & 449190 & 16 & 1172.15 & 4637.44 & 32638.53 & 4585.67 & 11928.62 & 693.8 & 393645.44 & 0 & 421815.35 \\
\hline 7484.96 & 374078 & 17 & 791.97 & 5406.11 & 35112.23 & 5444.38 & 14194.76 & 838.11 & 421297.51 & 0 & 296295.65 \\
\hline 7485.03 & 389090 & 17 & 1162.5 & 5577.25 & 34300.99 & 5749.12 & 14976.04 & 895.23 & 418685.47 & 0 & 275960.65 \\
\hline 7485.09 & 582643 & 14 & 0 & 2485.86 & 11172.71 & 2221.49 & 5762.57 & 318.68 & 405819.94 & 0 & 504921.56 \\
\hline 7485.16 & 593849 & 14 & 0 & 2147.1 & 10403.04 & 1900.21 & 4910.41 & 269.82 & 422761.95 & 0 & 504695.7 \\
\hline 7485.22 & 408582 & 16 & 532.85 & 3423.01 & 17605.72 & 3103.72 & 8059.04 & 451.25 & 379296.66 & 0 & 482066.71 \\
\hline 7485.29 & 218676 & 18 & 1691.2 & 6135.24 & 39032.97 & 6893.09 & 18016.57 & 1118.39 & 397113.62 & 0 & 0 \\
\hline 7485.35 & 479333 & 16 & 642.98 & 3624.75 & 20023.37 & 3407.01 & 8842.6 & 504.39 & 379387.84 & 0 & 464812.18 \\
\hline 7485.42 & 490072 & 16 & 409.18 & 2927.25 & 14703.23 & 2571.24 & 6673.78 & 373.1 & 415035.43 & 0 & 484392.87 \\
\hline 7485.49 & 451040 & 16 & 344.04 & 2801.95 & 16421.33 & 2579.5 & 6660.97 & 372.94 & 416589.78 & 0 & 475895.53 \\
\hline 7485.55 & 430982 & 16 & 908.55 & 4653.67 & 30885.97 & 4442.25 & 11531.94 & 661.82 & 417901.2 & 0 & 441869.73 \\
\hline 7485.62 & 439163 & 16 & 1156.66 & 4946.94 & 29371.85 & 4723.22 & 12290.67 & 709.43 & 417970.24 & 0 & 446261.08 \\
\hline 7485.68 & 253660 & 18 & 1603.75 & 5433.36 & 39222.35 & 5825.53 & 15143.98 & 903.45 & 448453.04 & 0 & 240170.9 \\
\hline 7485.75 & 315172 & 17 & 1295.38 & 6123.05 & 35530.61 & 6675.91 & 17411.13 & 1074.4 & 477340.43 & 0 & 133305.23 \\
\hline 7485.81 & 309864 & 17 & 1193.18 & 5329 & 31846.7 & 5328.11 & 13866.81 & 818.78 & 460453.16 & 0 & 299598.09 \\
\hline 7485.88 & 156723 & 19 & 5050.49 & 6771.35 & 38211.17 & 5836.7 & 15162.83 & 918.41 & 511697.46 & 0 & 234097.51 \\
\hline 7485.94 & 273306 & 18 & 970.33 & 5085.98 & 30006.17 & 5104.2 & 13316.05 & 782.72 & 505877.2 & 0 & 323826.93 \\
\hline 7486.01 & 305161 & 17 & 1179.24 & 5053.12 & 33770.58 & 5215.72 & 13590.44 & 806.93 & 486948.69 & 0 & 278667.32 \\
\hline 7486.08 & 235415 & 18 & 1273.45 & 5427.18 & 38665.42 & 6123.46 & 15962.53 & 988.71 & 541675.36 & 0 & 107395.63 \\
\hline
\end{tabular}




\begin{tabular}{|c|c|c|c|c|c|c|c|c|c|c|c|}
\hline True Depth & XRF tCOUNTS & XRF Live Time & $\mathrm{Ti}(\mathrm{ppm})$ & V (ppm) & $\mathrm{Fe}(\mathrm{ppm})$ & $\mathrm{Ni}(\mathrm{ppm})$ & $\mathrm{Cu}(\mathrm{ppm})$ & $\mathrm{Pb}(\mathrm{ppm})$ & LE (ppm) & $\mathrm{Mg}(\mathrm{ppm})$ & $\mathrm{Cl}(\mathrm{ppm})$ \\
\hline 7486.14 & 250709 & 18 & 1485.15 & 5651.36 & 42508.29 & 6544.6 & 17145.86 & 1069.55 & 546475.98 & 0 & 90422.66 \\
\hline 7486.21 & 262482 & 18 & 1070.13 & 5201.09 & 37850.14 & 5775.63 & 15051.35 & 908.19 & 527214.32 & 0 & 228626.81 \\
\hline 7486.27 & 282354 & 18 & 1190.08 & 5376.22 & 31947.89 & 5490.15 & 14320.75 & 854.58 & 508309.93 & 0 & 318076.46 \\
\hline 7486.34 & 247885 & 18 & 1085.85 & 5172.03 & 32637.45 & 5440.45 & 14196.17 & 850.68 & 509811.15 & 0 & 243772.57 \\
\hline 7486.40 & 351206 & 17 & 904.12 & 4750.27 & 28610.32 & 4620.81 & 12031.25 & 697.71 & 434349.17 & 0 & 435289.71 \\
\hline 7486.47 & 304232 & 17 & 411.81 & 2492.99 & 15844.23 & 2324.1 & 6037.91 & 333.29 & 405073.35 & 0 & 472781.63 \\
\hline 7486.54 & 358918 & 17 & 1085.14 & 5335.42 & 33168.31 & 5528.49 & 14413.45 & 856.33 & 452476.83 & 0 & 290975.7 \\
\hline 7486.60 & 340067 & 17 & 1099.09 & 5465.84 & 34006.58 & 5761.27 & 15028.16 & 898.33 & 450861.79 & 0 & 233191.97 \\
\hline 7486.67 & 306578 & 17 & 1417.24 & 6185.45 & 35786.41 & 6698.95 & 17510.81 & 1075.79 & 492120.42 & 0 & 145670.78 \\
\hline 7486.73 & 286391 & 18 & 1077.08 & 5237.82 & 31780.39 & 5422.15 & 14150.88 & 836.92 & 469340.34 & 0 & 328875.41 \\
\hline 7486.80 & 382084 & 17 & 1346.02 & 5797.17 & 35169.29 & 6207.28 & 16151.87 & 965.55 & 386103.77 & 31286.24 & 255995.57 \\
\hline 7486.86 & 499716 & 15 & 463.21 & 4996.32 & 25305.54 & 4789.64 & 12454.66 & 715.54 & 378293.15 & 0 & 465840.7 \\
\hline 7486.93 & 338441 & 17 & 1193.89 & 6488.11 & 38930.06 & 7077.01 & 18452.16 & 1137.44 & 397312.57 & 41474.66 & 88776.89 \\
\hline 7486.99 & 430970 & 16 & 553.23 & 5707.47 & 30288.67 & 5766.08 & 15013.88 & 889.64 & 406777.21 & 0 & 365599.56 \\
\hline 7487.06 & 497310 & 15 & 503.97 & 3285.86 & 19988.55 & 3105.83 & 8063.08 & 453.17 & 387859.06 & 0 & 473156.92 \\
\hline 7487.13 & 349334 & 17 & 1094.36 & 6185.06 & 38146.26 & 6904.63 & 18000.82 & 1108.73 & 437601.07 & 0 & 114007.77 \\
\hline 7487.19 & 382872 & 17 & 1081.45 & 6016.39 & 35444.03 & 6302.65 & 16422.11 & 989.46 & 418216.31 & 0 & 266693.15 \\
\hline 7487.26 & 131478 & 19 & 1034.36 & 0 & 3732.28 & 309.66 & 0 & 49.55 & 457465.3 & 0 & 508225.1 \\
\hline 7487.32 & 114705 & 19 & 0 & 2950.89 & 16166.42 & 2617.03 & 6760.2 & 366.28 & 308076.82 & 0 & 407918.02 \\
\hline 7487.39 & 325665 & 17 & 2248.77 & 6240.69 & 39861.42 & 7133.22 & 18649.26 & 1160.74 & 470787.42 & 0 & 42217.87 \\
\hline 7487.45 & 350651 & 17 & 1205.65 & 5709.47 & 33119.88 & 6234 & 16288.87 & 984.31 & 447718.73 & 0 & 233005.47 \\
\hline 7487.52 & 368508 & 17 & 1001.25 & 5181.06 & 34566.64 & 5236.24 & 13687.99 & 802.47 & 431839.77 & 0 & 329094.64 \\
\hline 7487.59 & 328290 & 17 & 1295.36 & 5509.2 & 46416.6 & 6426.75 & 16835.66 & 1037.27 & 468604.77 & 0 & 82007.67 \\
\hline 7487.65 & 349189 & 17 & 1554.53 & 5955.52 & 47695.83 & 6990.22 & 18284.94 & 1126.26 & 436180.2 & 0 & 108399.13 \\
\hline 7487.72 & 378959 & 17 & 1666.78 & 5920.8 & 40110.42 & 6239.78 & 16302.54 & 977.02 & 368375.94 & 36392.93 & 251069.29 \\
\hline 7487.78 & 413485 & 16 & 1512.73 & 5284.99 & 37987.72 & 5553.7 & 14521.62 & 857.06 & 402154.61 & 0 & 324684.28 \\
\hline 7487.85 & 603048 & 14 & 210.36 & 2213.04 & 11709.17 & 1939.75 & 5031.95 & 277.31 & 427040.32 & 0 & 503417.9 \\
\hline 7487.91 & 530833 & 15 & 500.11 & 3518.95 & 20366.27 & 3252.6 & 8454.09 & 475.3 & 381622.94 & 0 & 485241.89 \\
\hline 7487.98 & 405975 & 16 & 1109.68 & 5558.51 & 34403.5 & 5883.56 & 15347.08 & 911.3 & 425590.55 & 0 & 317776.03 \\
\hline 7488.04 & 475231 & 16 & 702.81 & 4908.72 & 29963.98 & 4783.34 & 12477.75 & 716.94 & 380743.03 & 0 & 426666.94 \\
\hline 7488.11 & 401935 & 16 & 1492.72 & 5449.68 & 33563.98 & 5630.23 & 14681.25 & 870.09 & 411626.87 & 0 & 320843 \\
\hline 7488.18 & 435699 & 16 & 1063.55 & 5407.51 & 28337.97 & 5383.45 & 14077.87 & 822.44 & 420330.86 & 0 & 397128.15 \\
\hline 7488.24 & 351501 & 17 & 1165.69 & 5888.12 & 40390.3 & 6543.49 & 17085.88 & 1037.3 & 455743.74 & 0 & 156416.38 \\
\hline
\end{tabular}




\begin{tabular}{|c|c|c|c|c|c|c|c|c|c|c|c|}
\hline True Depth & XRF tCOUNTS & XRF Live Time & $\mathrm{Ti}(\mathrm{ppm})$ & V (ppm) & $\mathrm{Fe}(\mathrm{ppm})$ & $\mathrm{Ni}(\mathrm{ppm})$ & $\mathrm{Cu}(\mathrm{ppm})$ & $\mathrm{Pb}(\mathrm{ppm})$ & LE (ppm) & $\mathrm{Mg}(\mathrm{ppm})$ & $\mathrm{Cl}(\mathrm{ppm})$ \\
\hline 7488.31 & 323964 & 17 & 1215.52 & 6216.57 & 37552.69 & 6961.49 & 18199 & 1123.33 & 475883.5 & 0 & 119212.13 \\
\hline 7488.37 & 329337 & 17 & 9997.18 & 5802.44 & 36891.13 & 6998.7 & 18326.17 & 1148.34 & 521741.48 & 0 & 69698.74 \\
\hline 7488.44 & 388282 & 17 & 1665.66 & 5584.52 & 56053.88 & 6644.65 & 17386.76 & 1059.43 & 353518.74 & 60062.1 & 57083.29 \\
\hline 7488.50 & 323990 & 17 & 1455.6 & 6699.48 & 41225.08 & 7907.45 & 20672.6 & 1307.29 & 393528.71 & 47025.24 & 0 \\
\hline 7488.57 & 356970 & 17 & 1592.61 & 6021.98 & 44817.37 & 6926.03 & 18040.64 & 1106.58 & 432955.51 & 0 & 134341.84 \\
\hline 7488.64 & 371681 & 17 & 1321.26 & 5848.15 & 37197.19 & 6465.15 & 16881.83 & 1016.3 & 438532.13 & 0 & 233029.26 \\
\hline 7488.70 & 385995 & 17 & 1268.09 & 5834.86 & 39266.82 & 6299.17 & 16412.61 & 989.36 & 433255.73 & 0 & 239733.69 \\
\hline 7488.77 & 415457 & 16 & 1199.46 & 5515.57 & 36979.4 & 5796.72 & 15132.97 & 897.4 & 409082.35 & 0 & 326472.97 \\
\hline 7488.83 & 426065 & 16 & 951 & 5174.87 & 31838.03 & 5155.43 & 13436.95 & 782.44 & 410209.61 & 0 & 377390.46 \\
\hline 7488.90 & 339711 & 17 & 1470.69 & 6016.85 & 35802.36 & 6529.31 & 17076.74 & 1038.33 & 454341.46 & 0 & 149628.64 \\
\hline 7488.96 & 556944 & 15 & 346.32 & 3069.72 & 14498.14 & 2816.8 & 7317.49 & 408.53 & 397292.04 & 0 & 492030.67 \\
\hline 7489.03 & 348149 & 17 & 1219.04 & 6334.97 & 38457.56 & 6984.16 & 18246.22 & 1114.71 & 365841.53 & 50344.88 & 109519.32 \\
\hline 7489.09 & 425609 & 16 & 1219.88 & 5589.38 & 32994.71 & 5661.1 & 14784.72 & 872.62 & 410487.55 & 0 & 336429.43 \\
\hline 7489.16 & 436013 & 16 & 826.3 & 5233.52 & 29384.68 & 5105.88 & 13306.19 & 770.4 & 397871.06 & 0 & 394395.77 \\
\hline 7489.23 & 337776 & 17 & 1095.03 & 6035.93 & 45108.26 & 6967.99 & 18232.88 & 1124.54 & 446920.79 & 0 & 73970.58 \\
\hline 7489.29 & 389172 & 17 & 1083.37 & 5971.14 & 37793.45 & 6312.13 & 16469.29 & 987.48 & 425992.16 & 0 & 273289.46 \\
\hline 7489.36 & 300026 & 17 & 766.6 & 4815.65 & 26313.42 & 4666.78 & 12170.68 & 702.97 & 416839.47 & 0 & 391972.57 \\
\hline 7489.42 & 512200 & 15 & 647.22 & 3242.53 & 23838.03 & 3038.84 & 7879.72 & 441.74 & 380402.6 & 0 & 482233.35 \\
\hline 7489.49 & 347259 & 17 & 1186.93 & 5939.73 & 39621.04 & 6571.17 & 17108.07 & 1039.52 & 453383.08 & 0 & 185656.22 \\
\hline 7489.55 & 324538 & 17 & 1560.08 & 5771.31 & 42954.16 & 6600.47 & 17232.04 & 1058.72 & 482858.8 & 0 & 105178.83 \\
\hline 7489.62 & 391032 & 17 & 1219.12 & 5064.18 & 31123.57 & 5151.87 & 13450.16 & 784.2 & 436033.75 & 0 & 377655.97 \\
\hline 7489.69 & 298330 & 17 & 1574.02 & 6011.51 & 37928.92 & 6982.34 & 18287.96 & 1142.64 & 500057.17 & 0 & 68752.02 \\
\hline 7489.75 & 438999 & 16 & 1451.69 & 5609.27 & 30711.28 & 5652.58 & 14774.74 & 868.89 & 402160.84 & 0 & 362876.85 \\
\hline 7489.82 & 340431 & 17 & 1513.6 & 6544.82 & 39965.49 & 7314.45 & 19126.35 & 1180.68 & 396726.91 & 38693.04 & 101561.53 \\
\hline 7489.88 & 515576 & 15 & 0 & 3276.42 & 17881.37 & 3116.91 & 8094.53 & 453.66 & 378616.3 & 0 & 488056.45 \\
\hline 7489.95 & 388707 & 17 & 1017.99 & 5738.41 & 36623.3 & 6041.67 & 15757.69 & 939.22 & 427948.59 & 0 & 289968.43 \\
\hline 7490.01 & 427378 & 16 & 973.68 & 4887.18 & 31082.66 & 5017.27 & 13078.42 & 758.26 & 410911.34 & 0 & 392794.63 \\
\hline 7490.08 & 488306 & 16 & 629.54 & 3749.05 & 21155.56 & 3443.9 & 8937.81 & 505.3 & 385587.12 & 0 & 472862.29 \\
\hline 7490.14 & 402650 & 16 & 1019.06 & 5492.75 & 32467.13 & 5532.88 & 14413.42 & 851.77 & 423083.18 & 0 & 340415.44 \\
\hline 7490.21 & 353030 & 17 & 1094.67 & 6397.75 & 41385.15 & 7261.92 & 18987.26 & 1166.18 & 429419.69 & 0 & 130571.9 \\
\hline 7490.28 & 374535 & 17 & 1431.37 & 5529.77 & 41261.85 & 6012.74 & 15706.52 & 934.59 & 407771.84 & 0 & 310683.29 \\
\hline 7490.34 & 359582 & 17 & 1375.24 & 5642.17 & 42328.71 & 6207.85 & 16218.59 & 977.6 & 440362.84 & 0 & 208072.93 \\
\hline 7490.41 & 370559 & 17 & 3561.36 & 5830.64 & 43102.16 & 6551.95 & 17092.02 & 1033.1 & 434404.64 & 0 & 196973.65 \\
\hline
\end{tabular}




\begin{tabular}{|c|c|c|c|c|c|c|c|c|c|c|c|}
\hline True Depth & XRF tCOUNTS & XRF Live Time & $\mathrm{Ti}(\mathrm{ppm})$ & V (ppm) & $\mathrm{Fe}(\mathrm{ppm})$ & $\mathrm{Ni}(\mathrm{ppm})$ & $\mathrm{Cu}(\mathrm{ppm})$ & $\mathrm{Pb}(\mathrm{ppm})$ & LE (ppm) & $\mathrm{Mg}(\mathrm{ppm})$ & $\mathrm{Cl}(\mathrm{ppm})$ \\
\hline 7490.47 & 363920 & 17 & 1381.29 & 6043.11 & 39246.82 & 6558.21 & 17123.57 & 1036.31 & 422847.19 & 0 & 223174.72 \\
\hline 7490.54 & 452348 & 16 & 1195.51 & 5137.44 & 28806.61 & 5137.07 & 13420.66 & 782.1 & 400903.93 & 0 & 413304.84 \\
\hline 7490.60 & 358677 & 17 & 1219.45 & 5589.31 & 45145.96 & 6420.41 & 16755.5 & 1016.86 & 442483.61 & 0 & 117266.22 \\
\hline 7490.67 & 477986 & 16 & 981.37 & 4730.66 & 36375.7 & 4808.1 & 12549.25 & 725.22 & 377154.44 & 0 & 429368.46 \\
\hline 7490.73 & 361669 & 17 & 1314.38 & 5893.44 & 49199.45 & 6674.29 & 17449.35 & 1055.45 & 397552.38 & 44761.38 & 115227.66 \\
\hline 7490.80 & 342714 & 17 & 1427.9 & 6038.4 & 43596.96 & 6856.57 & 17967.5 & 1100.6 & 473085.26 & 0 & 105149.17 \\
\hline 7490.87 & 519208 & 15 & 0 & 3625.79 & 19101.55 & 3395.32 & 8844.75 & 499.47 & 391824.5 & 0 & 472239.9 \\
\hline 7490.93 & 386605 & 17 & 1312.27 & 5958.62 & 37482.03 & 6323.84 & 16518.45 & 989.95 & 414172.37 & 0 & 258713.5 \\
\hline 7491.00 & 373050 & 17 & 1224.33 & 6009.2 & 47663.77 & 6726.41 & 17575.9 & 1067.64 & 426652.16 & 0 & 208065.79 \\
\hline 7491.06 & 428089 & 16 & 1245.71 & 4616.64 & 38421.9 & 4749.9 & 12361.48 & 713.46 & 391083.62 & 0 & 434305.71 \\
\hline 7491.13 & 454881 & 16 & 957.31 & 5392.89 & 33241.74 & 5371.89 & 13982.52 & 816.7 & 390833.13 & 0 & 412649.55 \\
\hline 7491.19 & 636220 & 14 & 0 & 1499.61 & 6815.44 & 1277.25 & 3300.87 & 179.3 & 450690.56 & 0 & 505966.91 \\
\hline 7491.26 & 335936 & 17 & 1151.91 & 6386.22 & 40156.8 & 7206.52 & 18861.19 & 1172.02 & 453013.89 & 0 & 81886.92 \\
\hline 7491.33 & 554117 & 15 & 0 & 1708.66 & 7600.91 & 1509.76 & 3903.76 & 212.92 & 452537.67 & 0 & 501853.4 \\
\hline 7491.39 & 123129 & 19 & 0 & 3782.94 & 19471.53 & 3498.36 & 8996.18 & 509.93 & 362083.39 & 0 & 84174.7 \\
\hline 7491.46 & 348801 & 17 & 1448.69 & 6350.32 & 42346.11 & 7389.22 & 19269.62 & 1199.2 & 452194.81 & 0 & 80519.51 \\
\hline 7491.52 & 345934 & 17 & 1287.11 & 6532.71 & 40669.29 & 7465.62 & 19491.33 & 1207.17 & 462644.89 & 0 & 126604.53 \\
\hline 7491.59 & 319984 & 17 & 1432.74 & 6806.82 & 45251.93 & 8387.28 & 21950.23 & 1414.83 & 480220.09 & 0 & 0 \\
\hline 7491.65 & 339560 & 17 & 1536.81 & 6299.31 & 49500.43 & 7526.61 & 19707.84 & 1236.06 & 455955.08 & 0 & 81898.16 \\
\hline 7491.72 & 324756 & 17 & 1768.51 & 6492.66 & 49749.46 & 7956.24 & 20793.37 & 1309.11 & 393640.5 & 57596.03 & 16074 \\
\hline 7491.78 & 393860 & 17 & 1060.55 & 5848.27 & 43438.33 & 6471.63 & 16918.94 & 1016.14 & 427332 & 0 & 223203.69 \\
\hline 7491.85 & 394296 & 17 & 1208.44 & 4986.56 & 45074.12 & 5409.28 & 14115.94 & 832.29 & 419159.82 & 0 & 312045.99 \\
\hline 7491.92 & 384253 & 17 & 1604.84 & 5411.51 & 62923.13 & 6809.58 & 17857.68 & 1103.39 & 436306.91 & 0 & 0 \\
\hline 7491.98 & 357318 & 17 & 1476.81 & 5498.66 & 61633.11 & 7077.44 & 18554.34 & 1154.28 & 450986.86 & 0 & 0 \\
\hline 7492.05 & 347838 & 17 & 1145.29 & 5297.26 & 60520.83 & 6768.13 & 17685.1 & 1099.86 & 458321.01 & 0 & 0 \\
\hline 7492.11 & 374265 & 17 & 871.38 & 5729.05 & 40609.34 & 6218.6 & 16276.14 & 975.91 & 432660.51 & 0 & 250475.16 \\
\hline 7492.18 & 547265 & 15 & 0 & 2290.92 & 15049.29 & 2098.62 & 5435.68 & 300.18 & 416527.04 & 0 & 498505.31 \\
\hline 7492.24 & 340798 & 17 & 1249.96 & 5747.29 & 55795.89 & 7070.58 & 18500.54 & 1148.95 & 472127.65 & 0 & 21441.22 \\
\hline 7492.31 & 454157 & 16 & 2188.32 & 6416.55 & 31390.63 & 5790.91 & 15103.36 & 886.8 & 375734.73 & 0 & 403993.82 \\
\hline 7492.38 & 343090 & 17 & 8454.54 & 12108.12 & 47617.6 & 10292.75 & 27041.79 & 1749.64 & 341258.41 & 47631.18 & 0 \\
\hline 7492.44 & 409464 & 16 & 6440.61 & 10591.92 & 35792.58 & 7703.59 & 20133.11 & 1220.56 & 373325.96 & 0 & 324586.25 \\
\hline 7492.51 & 362429 & 17 & 4188.73 & 8639.26 & 38798.61 & 7575.15 & 19779.07 & 1218.33 & 423573.66 & 0 & 212703.63 \\
\hline 7492.57 & 321355 & 17 & 3099.55 & 7367.9 & 39196.15 & 7722 & 20195.21 & 1274.01 & 466776.4 & 0 & 67487.63 \\
\hline
\end{tabular}




\begin{tabular}{|c|c|c|c|c|c|c|c|c|c|c|c|}
\hline True Depth & XRF tCOUNTS & XRF Live Time & $\mathrm{Ti}(\mathrm{ppm})$ & V (ppm) & $\mathrm{Fe}(\mathrm{ppm})$ & $\mathrm{Ni}(\mathrm{ppm})$ & $\mathrm{Cu}(\mathrm{ppm})$ & $\mathrm{Pb}(\mathrm{ppm})$ & LE (ppm) & $\mathrm{Mg}(\mathrm{ppm})$ & $\mathrm{Cl}(\mathrm{ppm})$ \\
\hline 7492.64 & 283655 & 18 & 2549.37 & 6468.02 & 39401.27 & 6622.02 & 17292.51 & 1052.3 & 442579.88 & 0 & 199496.32 \\
\hline 7492.70 & 390581 & 17 & 2287.02 & 7049.18 & 40641.76 & 7050.64 & 18435.58 & 1119.39 & 396140.14 & 0 & 265019.4 \\
\hline 7492.77 & 341350 & 17 & 2613.57 & 7502.7 & 46184.61 & 7778.59 & 20329.22 & 1264.7 & 439429.41 & 0 & 95765.91 \\
\hline 7492.83 & 408057 & 16 & 2195.58 & 6080.16 & 34875.49 & 5825.55 & 15216.62 & 899.63 & 402832.17 & 0 & 348436.16 \\
\hline 7492.90 & 401508 & 16 & 2240.03 & 6433.24 & 34639.12 & 5942.08 & 15487.15 & 918.39 & 414236.98 & 0 & 334708.08 \\
\hline 7492.97 & 409134 & 16 & 2196.31 & 6489.87 & 33238.78 & 6118.13 & 15993.06 & 946.93 & 367894.19 & 29874.14 & 339240.23 \\
\hline 7493.03 & 364567 & 17 & 2568.47 & 7439.16 & 39813.23 & 7371.89 & 19286.21 & 1187.17 & 421365.85 & 0 & 189740 \\
\hline 7493.10 & 327284 & 17 & 2622.66 & 7711.29 & 44537.1 & 8029.39 & 20975.55 & 1323.23 & 462402.63 & 0 & 73740.67 \\
\hline 7493.16 & 358162 & 17 & 1928.19 & 6766.56 & 33188.34 & 6462.02 & 16871.13 & 1020.11 & 432637.45 & 0 & 270344.04 \\
\hline 7493.23 & 373714 & 17 & 1639.15 & 6519.16 & 34865.84 & 6323.68 & 16496.96 & 985.78 & 432365.08 & 0 & 280515.56 \\
\hline 7493.29 & 379753 & 17 & 1400.88 & 6619.17 & 35653.43 & 6699.21 & 17504.52 & 1054.18 & 414928.89 & 0 & 243317.06 \\
\hline 7493.36 & 293319 & 18 & 2301.39 & 7184.84 & 45561.13 & 8354.56 & 21883.37 & 1407.19 & 474992.87 & 0 & 0 \\
\hline 7493.43 & 328626 & 17 & 2799.03 & 6976.7 & 44732.17 & 7878.96 & 20600.75 & 1302.88 & 455964.88 & 0 & 13684.4 \\
\hline 7493.49 & 516793 & 15 & 575.5 & 3529.67 & 19214.64 & 3203.27 & 8329.11 & 466.39 & 383580.29 & 0 & 486508.02 \\
\hline 7493.56 & 369950 & 17 & 2227.13 & 6167.48 & 39362.43 & 6308.65 & 16447.5 & 990.64 & 433192.31 & 0 & 249172.46 \\
\hline 7493.62 & 470030 & 16 & 1518.57 & 5338.73 & 32566.17 & 4969.02 & 12949.91 & 744.96 & 371636.11 & 0 & 466823.67 \\
\hline 7493.69 & 391902 & 17 & 1832.18 & 6104.04 & 33972.81 & 5891.15 & 15347.57 & 908.89 & 409681.59 & 0 & 317979.36 \\
\hline 7493.75 & 628296 & 14 & 0 & 1434.13 & 5628.26 & 1182.74 & 3060.7 & 164.92 & 459652.91 & 0 & 507061.08 \\
\hline 7493.82 & 304990 & 17 & 2052.75 & 6994.9 & 41083.52 & 7983.52 & 20826.35 & 1332.46 & 484770.86 & 0 & 0 \\
\hline 7493.88 & 322825 & 17 & 2267.71 & 7074.98 & 40870.19 & 7493.43 & 19609.11 & 1220.27 & 466364.49 & 0 & 111379.27 \\
\hline 7493.95 & 318635 & 17 & 1764.56 & 6165.44 & 47794.28 & 6615.47 & 17308.45 & 1050.92 & 424681.04 & 0 & 174832.22 \\
\hline 7494.02 & 365216 & 17 & 2085.42 & 6649.49 & 52814.4 & 7421.1 & 19419.56 & 1195.09 & 364986.05 & 54961.7 & 66757.97 \\
\hline 7494.08 & 461730 & 16 & 1861.18 & 5435.61 & 37152.83 & 5066.6 & 13216.55 & 765.29 & 376017.52 & 0 & 417740.62 \\
\hline 7494.15 & 556272 & 15 & 334.05 & 2738.62 & 13948.52 & 2287.3 & 5922.96 & 327.44 & 412894.09 & 0 & 505755.62 \\
\hline 7494.21 & 348260 & 17 & 1653.41 & 6053.1 & 41072.97 & 6323.98 & 16522.49 & 999.86 & 449900.85 & 0 & 222163.67 \\
\hline 7494.28 & 437236 & 16 & 1170.09 & 4953.82 & 35684.27 & 4965.02 & 12932.57 & 753.13 & 396906.01 & 0 & 403735.65 \\
\hline 7494.34 & 372797 & 17 & 1095.87 & 5869.09 & 45198.52 & 6496.02 & 16968.1 & 1017.88 & 428458.26 & 0 & 258376.7 \\
\hline 7494.41 & 484103 & 16 & 525.88 & 3150.22 & 21524.32 & 2965.85 & 7697.02 & 430.8 & 390582.4 & 0 & 482561.17 \\
\hline 7494.48 & 378818 & 17 & 3287.34 & 5968.32 & 37833.76 & 6472.46 & 16934.19 & 1029.94 & 448435.79 & 0 & 192260.91 \\
\hline 7494.54 & 537885 & 15 & 297.67 & 2951.19 & 15353.63 & 2688.19 & 6981.17 & 389.42 & 399908.65 & 0 & 491936.58 \\
\hline 7494.61 & 570226 & 15 & 0 & 2008.37 & 9797.16 & 1731.93 & 4478.51 & 245.22 & 432245.11 & 0 & 504326.06 \\
\hline 7494.67 & 371013 & 17 & 1265.66 & 5971.1 & 33640.32 & 6014.78 & 15689.81 & 934.64 & 435665.31 & 0 & 304226.15 \\
\hline 7494.74 & 446246 & 16 & 598.74 & 5112.36 & 35118.36 & 5289.68 & 13777.76 & 804.92 & 387508.55 & 0 & 348329.37 \\
\hline
\end{tabular}




\begin{tabular}{|c|c|c|c|c|c|c|c|c|c|c|c|}
\hline True Depth & XRF tCOUNTS & XRF Live Time & $\mathrm{Ti}(\mathrm{ppm})$ & V (ppm) & $\mathrm{Fe}(\mathrm{ppm})$ & $\mathrm{Ni}(\mathrm{ppm})$ & $\mathrm{Cu}(\mathrm{ppm})$ & $\mathrm{Pb}(\mathrm{ppm})$ & LE (ppm) & $\mathrm{Mg}(\mathrm{ppm})$ & $\mathrm{Cl}(\mathrm{ppm})$ \\
\hline 7494.80 & 494945 & 15 & 0 & 3012.14 & 18550.88 & 2730.19 & 7091.88 & 398.1 & 392986.35 & 0 & 488203.92 \\
\hline 7494.87 & 525859 & 15 & 0 & 2965.08 & 15291.7 & 2681.37 & 6958.23 & 387.47 & 410392.49 & 0 & 489398.98 \\
\hline 7494.93 & 386860 & 17 & 911.78 & 5661.3 & 37706.4 & 6010.66 & 15686.28 & 940.09 & 451676.38 & 0 & 126698.93 \\
\hline 7495.00 & 488006 & 16 & 305.94 & 3699.02 & 17522.78 & 3518.1 & 9125.45 & 515.72 & 375741.55 & 0 & 474877.14 \\
\hline 7495.07 & 359862 & 17 & 779.78 & 5469.63 & 30112.16 & 5562.29 & 14488.86 & 857.9 & 456140.65 & 0 & 343219.5 \\
\hline 7495.13 & 339477 & 17 & 777.75 & 5187.86 & 31029.07 & 5199.25 & 13551.84 & 798.22 & 456029.79 & 0 & 371215.43 \\
\hline 7495.20 & 337820 & 17 & 866.11 & 5042.61 & 33573.29 & 5253.75 & 13617.78 & 797.55 & 459900.28 & 0 & 349825.11 \\
\hline 7495.26 & 266316 & 18 & 1356.26 & 5826.03 & 40336.2 & 6956.65 & 18139.85 & 1142.86 & 538685.84 & 0 & 58458.16 \\
\hline 7495.33 & 376398 & 17 & 0 & 2420.1 & 12943.38 & 2178.48 & 5627.21 & 310.46 & 439954.28 & 0 & 485466.96 \\
\hline 7495.39 & 201604 & 18 & 2008.14 & 6349.01 & 42443.06 & 6827.01 & 17756.12 & 1089.67 & 458555.77 & 0 & 217841.29 \\
\hline 7495.46 & 334957 & 17 & 2696.36 & 6733.86 & 44380.63 & 7536.18 & 19580.38 & 1237.43 & 459030.04 & 0 & 0 \\
\hline 7495.52 & 377333 & 17 & 1438.45 & 5719.22 & 39050 & 5918 & 15449.72 & 918.54 & 430864.96 & 0 & 272747.14 \\
\hline 7495.59 & 362418 & 17 & 1247.44 & 6263.63 & 36350.57 & 6792.56 & 17720.19 & 1078.54 & 434988.44 & 0 & 195754.39 \\
\hline 7495.66 & 503191 & 15 & 349.9 & 3547.27 & 21151.8 & 3372.17 & 8764.16 & 497.61 & 380244.85 & 0 & 471675.23 \\
\hline 7495.72 & 402568 & 16 & 1119.22 & 5168.51 & 35813.3 & 5333.49 & 13867.69 & 812.73 & 414638.35 & 0 & 283771.1 \\
\hline 7495.79 & 301073 & 17 & 1057.26 & 5307.76 & 35153.46 & 5615.49 & 14632.32 & 868.84 & 449294.38 & 0 & 282717.76 \\
\hline 7495.85 & 371351 & 17 & 844.42 & 5502.97 & 34503.09 & 5643.09 & 14690.82 & 869.51 & 444765.89 & 0 & 321012.02 \\
\hline 7495.92 & 351529 & 17 & 901.36 & 4576.61 & 34663.58 & 4709.8 & 12233.92 & 711.93 & 448244.69 & 0 & 371161.15 \\
\hline 7495.98 & 307813 & 17 & 823.03 & 5039.85 & 31961.79 & 5261.62 & 13651.78 & 804.42 & 492395.92 & 0 & 311230.51 \\
\hline 7496.05 & 318582 & 17 & 619.61 & 5125.46 & 32770.37 & 5314.08 & 13861.42 & 822.52 & 483659.73 & 0 & 290941.8 \\
\hline 7496.12 & 322890 & 17 & 502.22 & 4836.31 & 31317.3 & 4860.9 & 12641.66 & 736.09 & 468566.2 & 0 & 355738.65 \\
\hline 7496.18 & 374280 & 17 & 1015.44 & 5108.13 & 32859.04 & 5165.47 & 13412.68 & 783.05 & 429386.48 & 0 & 361661.66 \\
\hline 7496.25 & 335368 & 17 & 1117.56 & 5048.04 & 33361.43 & 5248.48 & 13622.67 & 808.15 & 467383.56 & 0 & 309535.99 \\
\hline 7496.31 & 289049 & 18 & 0 & 4075.86 & 24925.43 & 3898.33 & 10117.23 & 576.02 & 465739.2 & 0 & 447419.22 \\
\hline 7496.38 & 238933 & 18 & 700.48 & 4898.26 & 26857.23 & 4793.5 & 12476.56 & 729.47 & 503150.18 & 0 & 390488.81 \\
\hline 7496.44 & 364362 & 17 & 3295.03 & 4174.73 & 30755.53 & 4309.34 & 11201.88 & 652.05 & 457560.8 & 0 & 404290.31 \\
\hline 7496.51 & 484035 & 16 & 607.35 & 4312.71 & 31661.77 & 4243.26 & 11005.12 & 633.03 & 382124.53 & 0 & 466873.11 \\
\hline 7496.57 & 300452 & 17 & 1335.43 & 5157.04 & 43864.96 & 5578.27 & 14477.39 & 876.28 & 503847.79 & 0 & 99815.73 \\
\hline 7496.64 & 570112 & 15 & 246.06 & 1921.67 & 21673.96 & 1759.45 & 4525.83 & 248.94 & 388369.03 & 0 & 486035.19 \\
\hline 7496.71 & 538732 & 15 & 0 & 1995.76 & 10546.48 & 1758.19 & 4525.87 & 249.4 & 423717 & 0 & 492359.81 \\
\hline 7496.77 & 414260 & 16 & 945.63 & 4199.84 & 35843.71 & 4184.09 & 10864.51 & 619.23 & 358577.53 & 0 & 435701.49 \\
\hline 7496.84 & 366151 & 17 & 833.33 & 5365.47 & 29423.99 & 5466.11 & 14162.96 & 843.08 & 453165.51 & 0 & 259772.31 \\
\hline 7496.90 & 371678 & 17 & 561.11 & 5589.95 & 30247.66 & 5653.19 & 14679.27 & 880.21 & 452000.48 & 0 & 279290.47 \\
\hline
\end{tabular}




\begin{tabular}{|c|c|c|c|c|c|c|c|c|c|c|c|}
\hline True Depth & XRF tCOUNTS & XRF Live Time & $\mathrm{Ti}(\mathrm{ppm})$ & V (ppm) & $\mathrm{Fe}(\mathrm{ppm})$ & $\mathrm{Ni}(\mathrm{ppm})$ & $\mathrm{Cu}(\mathrm{ppm})$ & $\mathrm{Pb}(\mathrm{ppm})$ & LE (ppm) & $\mathrm{Mg}(\mathrm{ppm})$ & $\mathrm{Cl}(\mathrm{ppm})$ \\
\hline 7496.97 & 277682 & 18 & 1196.93 & 5564.27 & 34569.07 & 6084.47 & 15753.47 & 965.51 & 516551.1 & 0 & 160735.29 \\
\hline 7497.03 & 331903 & 17 & 674.38 & 5177.16 & 37975.62 & 5456.47 & 14152.26 & 842.29 & 471916.55 & 0 & 260020.98 \\
\hline 7497.10 & 379155 & 17 & 876.51 & 5348.63 & 37669.94 & 5648.99 & 14613.26 & 863.99 & 397198.48 & 0 & 274532.81 \\
\hline 7497.17 & 341778 & 17 & 1622.45 & 6049.82 & 33918.5 & 6149.16 & 15969.3 & 968.69 & 456513.89 & 0 & 188568.05 \\
\hline 7497.23 & 432164 & 16 & 0 & 2572.36 & 12207.17 & 2225.81 & 5746.17 & 320.15 & 428409.17 & 0 & 482716.68 \\
\hline 7497.30 & 294263 & 18 & 0 & 4744.35 & 26448.33 & 4364.64 & 11343.68 & 651.4 & 365939.07 & 0 & 448016.12 \\
\hline 7497.36 & 502317 & 15 & 772.81 & 3579.64 & 25263.34 & 3325.36 & 8617.09 & 490.01 & 371721.83 & 0 & 469243.38 \\
\hline 7497.43 & 290450 & 18 & 3722.02 & 6770.84 & 48101.59 & 7781.04 & 20269.77 & 1299.25 & 466142.58 & 0 & 0 \\
\hline 7497.49 & 363740 & 17 & 2524.38 & 6503.11 & 49711.6 & 6873.14 & 17836.38 & 1091.93 & 423417.88 & 0 & 154722.48 \\
\hline 7497.56 & 380096 & 17 & 1601.31 & 6289 & 38756.57 & 6188.09 & 16126.57 & 970.44 & 442922.41 & 0 & 260903.09 \\
\hline 7497.62 & 319265 & 17 & 2428.18 & 7010.46 & 42336.06 & 7093.51 & 18490.71 & 1145.55 & 487319.24 & 0 & 120842.13 \\
\hline 7497.69 & 337624 & 17 & 2905.87 & 7108.75 & 46591.19 & 7499.03 & 19529.05 & 1219.96 & 462815.3 & 0 & 112342.47 \\
\hline 7497.76 & 425361 & 16 & 2326.34 & 5704.17 & 41513.37 & 5221.45 & 13545.43 & 797.52 & 384417.16 & 0 & 400574.43 \\
\hline 7497.82 & 313708 & 17 & 2372.6 & 6903.72 & 46195.23 & 7395.93 & 19288.46 & 1212.73 & 482046.32 & 0 & 47139 \\
\hline 7497.89 & 341077 & 17 & 843.43 & 5101.26 & 33330.03 & 5079.22 & 13237 & 774.12 & 437755.76 & 0 & 346338.46 \\
\hline 7497.95 & 356978 & 17 & 2339.82 & 7047.84 & 47245.01 & 7793.21 & 20233.72 & 1271.18 & 434366.82 & 0 & 24705.75 \\
\hline 7498.02 & 364276 & 17 & 2597.98 & 7828.44 & 45698.81 & 7942.14 & 20687.19 & 1300.29 & 424657.95 & 0 & 37429.87 \\
\hline 7498.08 & 335433 & 17 & 1269.61 & 5363.31 & 27700.77 & 4823.27 & 12556.47 & 730.06 & 411159.75 & 0 & 399550.79 \\
\hline 7498.15 & 367878 & 17 & 1956.28 & 7101.87 & 42418.53 & 7242.02 & 18883.02 & 1162.28 & 441324.67 & 0 & 112550.71 \\
\hline 7498.22 & 336141 & 17 & 2179.35 & 7072.43 & 48122.47 & 7611.13 & 19826.4 & 1244.84 & 460701.31 & 0 & 103627.67 \\
\hline 7498.28 & 307433 & 17 & 2658.06 & 6895.7 & 43249.04 & 6846.54 & 17889.9 & 1104.47 & 499948.34 & 0 & 163797.22 \\
\hline 7498.35 & 284042 & 18 & 3408.91 & 7633.27 & 41468.56 & 7264 & 18940.98 & 1179.41 & 513766.03 & 0 & 156083.52 \\
\hline 7498.41 & 301965 & 17 & 3155.23 & 7656.06 & 44381.68 & 7513.68 & 19607.88 & 1235.03 & 501954.51 & 0 & 137030.47 \\
\hline 7498.48 & 475615 & 16 & 8384.92 & 6548.01 & 35183.24 & 5928.18 & 15434.29 & 917.16 & 412494.95 & 0 & 378494.2 \\
\hline 7498.54 & 410355 & 16 & 3059.84 & 7219.49 & 41064.82 & 6527.63 & 17021.88 & 1022.99 & 399433.1 & 0 & 254702.1 \\
\hline 7498.61 & 416153 & 16 & 2003.11 & 6457.75 & 43093.47 & 6351.88 & 16505.37 & 992.83 & 401692.85 & 0 & 274743.36 \\
\hline 7498.67 & 374559 & 17 & 1243.96 & 5972.61 & 35042.39 & 5373.16 & 14003.38 & 823.13 & 393524.69 & 0 & 381650.12 \\
\hline 7498.74 & 360183 & 17 & 2483.86 & 6825.54 & 49536.56 & 7437.3 & 19315.89 & 1206.99 & 441575.21 & 0 & 51074.05 \\
\hline 7498.81 & 404745 & 16 & 2328.48 & 6556.5 & 37622.5 & 6022.83 & 15653.33 & 933.92 & 409794.99 & 0 & 306409.53 \\
\hline 7498.87 & 365862 & 17 & 2099.22 & 5944.15 & 55762.4 & 6886.46 & 17857.03 & 1107.37 & 447670.12 & 0 & 104739.28 \\
\hline 7498.94 & 384972 & 17 & 530.3 & 5207.43 & 49241.74 & 5800.55 & 14988.75 & 907.82 & 438078.9 & 0 & 155619.88 \\
\hline 7499.00 & 359842 & 17 & 841.61 & 6121.24 & 42633.18 & 6819.52 & 17687.31 & 1097.53 & 474870.63 & 0 & 45528 \\
\hline 7499.07 & 354186 & 17 & 617.34 & 5978.29 & 43134.5 & 6706.71 & 17401.95 & 1080.37 & 467190.13 & 0 & 40783.24 \\
\hline
\end{tabular}




\begin{tabular}{|c|c|c|c|c|c|c|c|c|c|c|c|}
\hline True Depth & XRF tCOUNTS & XRF Live Time & $\mathrm{Ti}(\mathrm{ppm})$ & V (ppm) & $\mathrm{Fe}(\mathrm{ppm})$ & $\mathrm{Ni}(\mathrm{ppm})$ & $\mathrm{Cu}(\mathrm{ppm})$ & $\mathrm{Pb}(\mathrm{ppm})$ & LE (ppm) & $\mathrm{Mg}(\mathrm{ppm})$ & $\mathrm{Cl}(\mathrm{ppm})$ \\
\hline 7499.13 & 368072 & 17 & 822.31 & 5925.88 & 41205.51 & 6493.87 & 16821.67 & 1039.96 & 465098.04 & 0 & 60844.23 \\
\hline 7499.20 & 337184 & 17 & 772.25 & 6180.47 & 43673.56 & 7338.43 & 19033.59 & 1205.83 & 471392.72 & 0 & 0 \\
\hline 7499.27 & 338476 & 17 & 1058.63 & 6230.28 & 42271.09 & 7218.9 & 18716.2 & 1180.57 & 482530.99 & 0 & 16744.61 \\
\hline 7499.33 & 356098 & 17 & 1078.37 & 6034.38 & 45610.29 & 6826.76 & 17696.01 & 1097.78 & 465772.32 & 0 & 129508.11 \\
\hline 7499.40 & 359853 & 17 & 1222.35 & 5615.2 & 53103.57 & 6516.43 & 16899.78 & 1040.02 & 450687.92 & 0 & 100602.27 \\
\hline 7499.46 & 336992 & 17 & 1383.94 & 6015.29 & 48820.33 & 7181.25 & 18630.62 & 1181.86 & 468120.65 & 0 & 0 \\
\hline 7499.53 & 345115 & 17 & 1233.78 & 5519.8 & 57605.22 & 6680.2 & 17332.27 & 1068.8 & 458224.98 & 0 & 114517.75 \\
\hline 7499.59 & 392368 & 17 & 766.36 & 5281.12 & 49050.17 & 5980.96 & 15456.29 & 932.68 & 427140.81 & 0 & 228093.41 \\
\hline 7499.66 & 408116 & 16 & 690.38 & 5025.7 & 39925.9 & 5313.35 & 13753.77 & 816.51 & 419105.39 & 0 & 308083.4 \\
\hline 7499.72 & 377406 & 17 & 0 & 5147.89 & 35700.55 & 5307.15 & 13701.35 & 817.79 & 442323.19 & 0 & 282826.31 \\
\hline 7499.79 & 363327 & 17 & 520.71 & 5250.86 & 38590.81 & 6005.2 & 15519.97 & 953.47 & 509541.54 & 0 & 0 \\
\hline 7499.86 & 393502 & 17 & 590.39 & 5279.39 & 38347.55 & 5662.91 & 14626.1 & 879.99 & 469554.55 & 0 & 64390.46 \\
\hline 7499.92 & 444709 & 16 & 0 & 5299.44 & 39702.31 & 5577.84 & 14468.55 & 858.81 & 411450.41 & 0 & 183575.94 \\
\hline 7499.99 & 436492 & 16 & 609.55 & 5033.19 & 43905.02 & 5378.3 & 13951.99 & 825.79 & 387514.95 & 0 & 322804.99 \\
\hline 7500.05 & 622815 & 14 & 0 & 1585.41 & 10973.45 & 1409.68 & 3608.33 & 198.18 & 435393.23 & 0 & 506115.59 \\
\hline 7500.12 & 368021 & 17 & 978.08 & 5645.23 & 46682.72 & 6524.7 & 16851.59 & 1033.04 & 443191.7 & 0 & 141699.25 \\
\hline 7500.18 & 340507 & 17 & 1212.41 & 5647.41 & 53713.29 & 7179.13 & 18559.07 & 1166.77 & 462080.21 & 0 & 0 \\
\hline 7500.25 & 335206 & 17 & 1147.82 & 5109.13 & 62618.87 & 6579.7 & 17009.15 & 1042.03 & 467020.65 & 0 & 107841.6 \\
\hline 7500.31 & 299299 & 17 & 0 & 5146.16 & 38287.1 & 5557.07 & 14350.05 & 871.25 & 518438.37 & 0 & 254263.05 \\
\hline 7500.38 & 354709 & 17 & 768.29 & 4857.12 & 41834.71 & 5320.64 & 13731.71 & 816.21 & 423082.5 & 0 & 281143.16 \\
\hline 7500.45 & 413137 & 16 & 1477.74 & 4842.57 & 32181.32 & 4229.5 & 10906.57 & 628.03 & 391449.06 & 0 & 360425.18 \\
\hline 7500.51 & 360790 & 17 & 508.77 & 4547.06 & 40606.28 & 4644.34 & 11995.74 & 704.58 & 422001.47 & 0 & 366191.73 \\
\hline 7500.58 & 247711 & 18 & 0 & 4547.81 & 44220.77 & 5070.37 & 13110.28 & 787.36 & 488475.43 & 0 & 313797.05 \\
\hline 7500.64 & 301489 & 17 & 0 & 4453.32 & 34051.43 & 4608.76 & 11945.97 & 699.76 & 466191.93 & 0 & 399235.32 \\
\hline 7500.71 & 302536 & 17 & 0 & 5239.11 & 29887.12 & 5293.9 & 13709.96 & 808.88 & 487544.39 & 0 & 335249.41 \\
\hline 7500.77 & 337508 & 17 & 0 & 4975.69 & 29039.81 & 4946.86 & 12768.59 & 746.94 & 445723.46 & 0 & 375023.71 \\
\hline 7500.84 & 292986 & 18 & 807.9 & 5579.88 & 34521.38 & 6137.53 & 15905.92 & 978.16 & 527896.93 & 0 & 139812.7 \\
\hline 7500.91 & 333375 & 17 & 480.27 & 4669.62 & 26377.49 & 4587.57 & 11838.11 & 690.64 & 459358.59 & 0 & 368429.9 \\
\hline 7500.97 & 288562 & 18 & 0 & 5596.47 & 32765.32 & 6047.74 & 15638.94 & 949.22 & 508416.21 & 0 & 222778.43 \\
\hline 7501.04 & 349020 & 17 & 6216.4 & 9925.51 & 29194.44 & 6454.01 & 16750.99 & 999.26 & 411821.84 & 0 & 373253.12 \\
\hline 7501.10 & 424956 & 16 & 15175.47 & 16683.4 & 26537.37 & 8387.01 & 21925.55 & 1322.21 & 314977.44 & 0 & 449331.95 \\
\hline 7501.17 & 426880 & 16 & 13028.92 & 13657.08 & 20581.03 & 6036.83 & 15741.12 & 915.59 & 281822.35 & 0 & 506568.21 \\
\hline 7501.23 & 474101 & 16 & 32618.09 & 28441.45 & 33545.96 & 10500.87 & 27502.83 & 1692.27 & 236123.01 & 0 & 427392.45 \\
\hline
\end{tabular}




\begin{tabular}{|c|c|c|c|c|c|c|c|c|c|c|c|}
\hline True Depth & XRF tCOUNTS & XRF Live Time & $\mathrm{Ti}(\mathrm{ppm})$ & V (ppm) & $\mathrm{Fe}(\mathrm{ppm})$ & $\mathrm{Ni}(\mathrm{ppm})$ & $\mathrm{Cu}(\mathrm{ppm})$ & $\mathrm{Pb}(\mathrm{ppm})$ & LE (ppm) & $\mathrm{Mg}(\mathrm{ppm})$ & $\mathrm{Cl}(\mathrm{ppm})$ \\
\hline 7501.30 & 321113 & 17 & 2484.9 & 6431.22 & 24817.45 & 5213.83 & 13561.55 & 791.32 & 432165.84 & 0 & 422749.42 \\
\hline 7501.36 & 393828 & 17 & 21228.53 & 18071.41 & 40187.01 & 7462.39 & 19484.14 & 1164.64 & 348482.99 & 0 & 432553 \\
\hline 7501.43 & 189049 & 18 & 2436.07 & 3282.08 & 7773.69 & 1845.84 & 4776.62 & 264.35 & 431036.44 & 0 & 507965.55 \\
\hline 7501.50 & 332354 & 17 & 7134.35 & 11416.58 & 39187.97 & 9428.29 & 24564.09 & 1596.88 & 434842.37 & 0 & 37268.35 \\
\hline 7501.56 & 497699 & 15 & 10302.9 & 10784.23 & 70063.99 & 6335.2 & 16551.04 & 979.49 & 364629.64 & 0 & 388726.15 \\
\hline 7501.63 & 317592 & 17 & 1717.93 & 5913.56 & 23855.66 & 5032.57 & 13022.66 & 762.85 & 463502.4 & 0 & 367712.44 \\
\hline 7501.69 & 308756 & 17 & 3234.84 & 8680.51 & 29615.36 & 7422.29 & 19320.76 & 1201.21 & 475033.51 & 0 & 191792.2 \\
\hline 7501.76 & 332552 & 17 & 5693.69 & 10721.8 & 35556.97 & 8920.79 & 23268.97 & 1503.52 & 429664.24 & 0 & 21726.2 \\
\hline 7501.82 & 384625 & 17 & 4710.43 & 9816.41 & 31732.67 & 7342.09 & 19072.68 & 1156.74 & 396284.42 & 0 & 290402.79 \\
\hline 7501.89 & 442580 & 16 & 3638.96 & 7837.68 & 25513.03 & 5559.08 & 14390.25 & 839.28 & 357819.61 & 0 & 459983.81 \\
\hline 7501.96 & 407355 & 16 & 6208.12 & 9639.6 & 26666.37 & 6388.93 & 16635.45 & 987.65 & 382468.75 & 0 & 395481.85 \\
\hline 7502.02 & 333427 & 17 & 2714.52 & 7888.09 & 32317.85 & 6884.52 & 17874.3 & 1098.04 & 458186.66 & 0 & 180161.74 \\
\hline 7502.09 & 374359 & 17 & 18954.45 & 20271.81 & 34068.36 & 10263.78 & 26846.79 & 1685.78 & 346614.47 & 0 & 260065.23 \\
\hline 7502.15 & 369409 & 17 & 39651.45 & 32374.93 & 48786.82 & 14355.58 & 37835.79 & 2577.72 & 369615.81 & 0 & 0 \\
\hline 7502.22 & 177311 & 19 & 0 & 5319.84 & 31447.32 & 6110 & 15814.94 & 1004.98 & 649953.81 & 0 & 77321.75 \\
\hline 7502.28 & 177221 & 19 & 0 & 4728.84 & 30722.07 & 5120.72 & 13234.57 & 807.34 & 603426.29 & 0 & 130809.32 \\
\hline 7502.35 & 229716 & 18 & 0 & 5396.73 & 40640.29 & 6404.83 & 16597.83 & 1052.07 & 590781.52 & 0 & 37522.78 \\
\hline 7502.41 & 246996 & 18 & 816.37 & 5009.16 & 48501.36 & 6205.68 & 16058.21 & 996.49 & 502736.87 & 0 & 34956.76 \\
\hline 7502.48 & 284276 & 18 & 0 & 4614.34 & 32089.79 & 4844.35 & 12522.42 & 741.25 & 497928.82 & 0 & 234358.21 \\
\hline 7502.55 & 291006 & 18 & 0 & 4446.58 & 32072.73 & 4601.58 & 11870.76 & 702.2 & 493398.76 & 0 & 294365.38 \\
\hline 7502.61 & 237225 & 18 & 0 & 4911.68 & 36846.16 & 5421.17 & 13925.24 & 851.72 & 544881.89 & 0 & 79463.79 \\
\hline 7502.68 & 231419 & 18 & 997.3 & 5260.26 & 35925.97 & 6045.43 & 15629.02 & 978.74 & 493955.73 & 69765.9 & 17911.3 \\
\hline 7502.74 & 349863 & 17 & 532.8 & 5361.27 & 33084.37 & 5573.01 & 14437.67 & 866.1 & 426065.52 & 0 & 142531.61 \\
\hline 7502.81 & 538836 & 15 & 0 & 2527.18 & 14155.52 & 2267.66 & 5844.45 & 325.63 & 381548.62 & 0 & 497890.83 \\
\hline 7502.87 & 309250 & 17 & 0 & 5573.47 & 35167.42 & 6158.88 & 15957.17 & 986.83 & 483489.4 & 0 & 6870.41 \\
\hline 7502.94 & 336984 & 17 & 612.08 & 5594.49 & 35052.11 & 5947.3 & 15382.05 & 933.7 & 447318.25 & 0 & 92270.42 \\
\hline 7503.01 & 341176 & 17 & 0 & 4836.74 & 27493.72 & 4827.41 & 12498.61 & 731.1 & 467839.24 & 0 & 308641.61 \\
\hline 7503.07 & 337410 & 17 & 505.1 & 4619.59 & 27338.89 & 4560.27 & 11794.95 & 686.22 & 472651.2 & 0 & 311415.79 \\
\hline 7503.14 & 316510 & 17 & 659.58 & 5868.38 & 36465.66 & 6485.92 & 16864.35 & 1047.89 & 458421.64 & 0 & 0 \\
\hline 7503.20 & 356830 & 17 & 638.75 & 5044.11 & 34742.87 & 5197.83 & 13447.57 & 799.44 & 432536.67 & 0 & 235688.13 \\
\hline 7503.27 & 318474 & 17 & 856.68 & 5187.97 & 40438.94 & 5677.12 & 14659.45 & 889.52 & 461725.6 & 0 & 95495.79 \\
\hline 7503.33 & 339858 & 17 & 810.84 & 4604.87 & 38803.37 & 4880.28 & 12593.47 & 747.13 & 461225.49 & 0 & 216290.75 \\
\hline 7503.40 & 278199 & 18 & 787.87 & 5067.39 & 39872.2 & 5617.83 & 14540.53 & 885.12 & 516215.1 & 0 & 81959.84 \\
\hline
\end{tabular}




\begin{tabular}{|c|c|c|c|c|c|c|c|c|c|c|c|}
\hline True Depth & XRF tCOUNTS & XRF Live Time & $\mathrm{Ti}(\mathrm{ppm})$ & V (ppm) & $\mathrm{Fe}(\mathrm{ppm})$ & $\mathrm{Ni}(\mathrm{ppm})$ & $\mathrm{Cu}(\mathrm{ppm})$ & $\mathrm{Pb}(\mathrm{ppm})$ & LE (ppm) & $\mathrm{Mg}(\mathrm{ppm})$ & $\mathrm{Cl}(\mathrm{ppm})$ \\
\hline 7503.46 & 311687 & 17 & 952.54 & 5367.31 & 37579.42 & 5849.15 & 15094.69 & 925.42 & 481731.82 & 0 & 84852.43 \\
\hline 7503.53 & 272733 & 18 & 673.7 & 5968.38 & 35933.27 & 6547.47 & 16896.72 & 1041.65 & 452236.94 & 0 & 0 \\
\hline 7503.60 & 334159 & 17 & 681.13 & 5573.13 & 37198.65 & 5958.01 & 15390.81 & 935.02 & 462565.86 & 0 & 75265.06 \\
\hline 7503.66 & 404928 & 16 & 556.31 & 4648.19 & 31667.86 & 4705.13 & 12095.87 & 708.25 & 416636.8 & 0 & 322482.33 \\
\hline 7503.73 & 306747 & 17 & 936.63 & 5721.73 & 38842.39 & 6363.12 & 16439.26 & 1018.78 & 467759.96 & 0 & 17879.7 \\
\hline 7503.79 & 355707 & 17 & 0 & 5060.21 & 34157.95 & 5135.68 & 13285.74 & 781.79 & 436236.22 & 0 & 247939.9 \\
\hline 7503.86 & 353570 & 17 & 551.74 & 5145.76 & 31592.93 & 5348.14 & 13833.27 & 818.76 & 453386.41 & 0 & 210593.01 \\
\hline 7503.92 & 489676 & 16 & 0 & 4210.65 & 23140.85 & 4070.06 & 10499.2 & 599.16 & 389093.16 & 0 & 455518.63 \\
\hline 7503.99 & 320528 & 17 & 662.38 & 5620.72 & 35499.7 & 6243.38 & 16139.76 & 991.32 & 457333.41 & 0 & 0 \\
\hline 7504.06 & 341094 & 17 & 497.3 & 5973.23 & 35885.24 & 6348.15 & 16450.07 & 1002.69 & 447590.41 & 0 & 62841.39 \\
\hline 7504.12 & 491206 & 16 & 0 & 3051.31 & 15693.2 & 2820.53 & 7263.52 & 406.2 & 384807.64 & 0 & 472199.6 \\
\hline 7504.19 & 345119 & 17 & 619.75 & 5847.52 & 41625.84 & 6568.53 & 17028.44 & 1050.18 & 435502.93 & 0 & 35254.68 \\
\hline 7504.25 & 334955 & 17 & 866.72 & 5539.51 & 48376.33 & 6325 & 16419 & 1006.04 & 364121.68 & 52221.12 & 0 \\
\hline 7504.32 & 343566 & 17 & 0 & 5131.86 & 37438.04 & 5337.55 & 13813.22 & 822.66 & 432641.26 & 0 & 179241.65 \\
\hline 7504.38 & 321735 & 17 & 484.58 & 5652.61 & 39430.38 & 6184.71 & 16025.68 & 986.83 & 477721.02 & 0 & 24679.64 \\
\hline 7504.45 & 335250 & 17 & 525.6 & 5163.71 & 35426.57 & 5496.86 & 14223.97 & 857.33 & 447216.27 & 0 & 143524.59 \\
\hline 7504.51 & 316697 & 17 & 636.47 & 5639.84 & 37198.94 & 6304.87 & 16272.67 & 1008.99 & 479131.3 & 0 & 0 \\
\hline 7504.58 & 307540 & 17 & 541.72 & 5599.7 & 34688.03 & 5949.69 & 15405.11 & 944.51 & 490158.7 & 0 & 0 \\
\hline 7504.65 & 316281 & 17 & 619.4 & 5533.34 & 39077.9 & 6168.03 & 15890.01 & 973.58 & 483248.51 & 0 & 0 \\
\hline 7504.71 & 305602 & 17 & 892.7 & 5534.91 & 36752.48 & 6122.05 & 15823.18 & 967.34 & 459702.54 & 0 & 0 \\
\hline 7504.78 & 320161 & 17 & 548.88 & 5998.53 & 36266.05 & 6623 & 17093.59 & 1069.08 & 475280.18 & 0 & 0 \\
\hline 7504.84 & 319535 & 17 & 826.15 & 5976.67 & 39419.19 & 6710.03 & 17375.36 & 1085.63 & 474369.64 & 0 & 0 \\
\hline 7504.91 & 325366 & 17 & 932.96 & 5894.57 & 42578.73 & 6749.28 & 17466.09 & 1087.54 & 457702.19 & 0 & 0 \\
\hline 7504.97 & 317230 & 17 & 888.21 & 6209.22 & 38905.73 & 6879.88 & 17879.64 & 1112.95 & 479587.88 & 0 & 0 \\
\hline 7505.04 & 456916 & 16 & 519.29 & 4127.38 & 26030.17 & 3908.61 & 10080.82 & 573.12 & 380365.61 & 0 & 451161.4 \\
\hline 7505.10 & 300669 & 17 & 572.67 & 6148.92 & 31867.54 & 6508.74 & 16885.23 & 1047.05 & 502872.08 & 0 & 43310.33 \\
\hline 7505.17 & 343844 & 17 & 803.73 & 5828.6 & 33374.48 & 5958.59 & 15419.39 & 932.14 & 455921.31 & 0 & 163662.94 \\
\hline 7505.24 & 486753 & 16 & 391.11 & 4470.39 & 27866.9 & 4348.78 & 11247.39 & 648.1 & 367567.88 & 0 & 442809.38 \\
\hline 7505.30 & 342936 & 17 & 649.34 & 6459.23 & 41048.88 & 7100.39 & 18398.65 & 1137.88 & 399312.72 & 43044.69 & 0 \\
\hline 7505.37 & 382501 & 17 & 619.82 & 5325.91 & 34470.3 & 5472.57 & 14170.89 & 841.12 & 425286.61 & 0 & 280233.73 \\
\hline 7505.43 & 384702 & 17 & 675.59 & 4985.4 & 38554.86 & 5192.54 & 13443.88 & 797.49 & 424943.67 & 0 & 283774.12 \\
\hline 7505.50 & 366361 & 17 & 0 & 5517.94 & 28380.21 & 5392.39 & 13918.59 & 823.68 & 439268.06 & 0 & 274670.53 \\
\hline 7505.56 & 163885 & 19 & 0 & 5691.7 & 28544.55 & 5842.81 & 15104.83 & 919.02 & 458260.41 & 0 & 0 \\
\hline
\end{tabular}




\begin{tabular}{|c|c|c|c|c|c|c|c|c|c|c|c|}
\hline True Depth & XRF tCOUNTS & XRF Live Time & $\mathrm{Ti}(\mathrm{ppm})$ & V (ppm) & $\mathrm{Fe}(\mathrm{ppm})$ & $\mathrm{Ni}(\mathrm{ppm})$ & $\mathrm{Cu}(\mathrm{ppm})$ & $\mathrm{Pb}(\mathrm{ppm})$ & LE (ppm) & $\mathrm{Mg}(\mathrm{ppm})$ & $\mathrm{Cl}(\mathrm{ppm})$ \\
\hline 7505.63 & 306873 & 17 & 1201.49 & 5991.97 & 40676.14 & 6751.37 & 17508.85 & 1101.89 & 499439.68 & 0 & 0 \\
\hline 7505.70 & 282074 & 18 & 647.38 & 5397.89 & 42222.37 & 6300.36 & 16359.8 & 1018.72 & 528702.95 & 0 & 35559.21 \\
\hline 7505.76 & 272395 & 18 & 1195.35 & 5692.42 & 34157.19 & 6170.93 & 15944.15 & 989.44 & 535298.92 & 0 & 43280.65 \\
\hline 7505.83 & 286107 & 18 & 1038.32 & 5987.93 & 47307.08 & 7411.99 & 19312.45 & 1232.02 & 521179.55 & 0 & 0 \\
\hline 7505.89 & 350296 & 17 & 1746.25 & 5427.92 & 53476.17 & 6146.41 & 15936.39 & 969.82 & 450330.49 & 0 & 119613.56 \\
\hline 7505.96 & 362185 & 17 & 618.95 & 5047.72 & 38070.23 & 5205.82 & 13468.7 & 792.64 & 403533.61 & 0 & 334404.47 \\
\hline 7506.02 & 365072 & 17 & 846.83 & 6677.34 & 53051.4 & 8316.23 & 21449.04 & 1367.2 & 467633.93 & 0 & 0 \\
\hline 7506.09 & 389669 & 17 & 870 & 5872.84 & 44331.33 & 6249.72 & 16085.48 & 984.54 & 435058.16 & 0 & 266220.45 \\
\hline 7506.15 & 387526 & 17 & 908.3 & 5818.73 & 47543.66 & 6487.04 & 16731.08 & 1018.99 & 435191.78 & 0 & 216180.07 \\
\hline 7506.22 & 346442 & 17 & 620.37 & 6321.84 & 50187.23 & 7755.21 & 20077.96 & 1269.76 & 471988.91 & 0 & 65997.29 \\
\hline 7506.29 & 373374 & 17 & 1066.91 & 5777.26 & 61271.05 & 7172.37 & 18590.71 & 1157.63 & 432135.41 & 0 & 72449.33 \\
\hline 7506.35 & 344524 & 17 & 1334.3 & 5519.99 & 65967.07 & 7105.4 & 18376.62 & 1146.35 & 445808.48 & 0 & 49529.42 \\
\hline 7506.42 & 348530 & 17 & 1294.89 & 5612.86 & 63721.13 & 7236.21 & 18849.44 & 1186.17 & 406994.67 & 0 & 0 \\
\hline 7506.48 & 339254 & 17 & 1132.85 & 5667.31 & 53077.15 & 6667.27 & 17280.05 & 1076.51 & 366417.18 & 46047.08 & 0 \\
\hline 7506.55 & 318728 & 17 & 1119.1 & 6344.89 & 36804.17 & 7059.48 & 18293.99 & 1158.72 & 465769.69 & 0 & 0 \\
\hline 7506.61 & 322791 & 17 & 784.27 & 5574.66 & 33572.07 & 5874.06 & 15257.55 & 924.83 & 482829.07 & 0 & 151022.4 \\
\hline 7506.68 & 326105 & 17 & 1005 & 6136.45 & 36154.56 & 6726.17 & 17387.71 & 1088.29 & 453750.08 & 0 & 0 \\
\hline 7506.75 & 374651 & 17 & 462.77 & 5801.82 & 32285.14 & 5924.32 & 15303.06 & 917.72 & 444404.67 & 0 & 249125.17 \\
\hline 7506.81 & 364981 & 17 & 737.02 & 5676.53 & 37567.51 & 5973.92 & 15399.98 & 925.99 & 448730.98 & 0 & 218176.96 \\
\hline 7506.88 & 350276 & 17 & 1053.38 & 5902.59 & 38923.88 & 6431.76 & 16619.87 & 1026.57 & 444516.13 & 0 & 73409.7 \\
\hline 7506.94 & 313753 & 17 & 879.58 & 6283.31 & 34787.91 & 6944.1 & 18068.8 & 1143.46 & 472137.59 & 0 & 0 \\
\hline 7507.01 & 357484 & 17 & 592.91 & 5865.36 & 35681.47 & 6265.17 & 16245.79 & 987.46 & 450105.77 & 0 & 157309.71 \\
\hline 7507.07 & 328953 & 17 & 711.8 & 6036.36 & 45919.07 & 7023.66 & 18229.75 & 1154.05 & 470777 & 0 & 0 \\
\hline 7507.14 & 410679 & 16 & 624.05 & 5220.22 & 32261.92 & 5118.51 & 13295.48 & 784.66 & 399804.91 & 0 & 344040.61 \\
\hline 7507.20 & 372087 & 17 & 736.98 & 5599.1 & 44290.94 & 6098.43 & 15820.91 & 955.06 & 410931 & 0 & 156481.3 \\
\hline 7507.27 & 322518 & 17 & 1068.43 & 5802.1 & 41165.11 & 6821.04 & 17684.31 & 1107.86 & 469462.89 & 0 & 0 \\
\hline 7507.34 & 312620 & 17 & 896.46 & 6168.05 & 37717.09 & 6839.09 & 17805.52 & 1117.61 & 473308.63 & 0 & 0 \\
\hline 7507.40 & 307728 & 17 & 812.41 & 5834.25 & 41939.5 & 6637.37 & 17218.03 & 1081.29 & 481717 & 0 & 0 \\
\hline 7507.47 & 307607 & 17 & 1319.62 & 5153.76 & 36879.7 & 5571.5 & 14440.79 & 863.54 & 443754.19 & 0 & 177826.87 \\
\hline 7507.53 & 305349 & 17 & 1158.55 & 5648.1 & 36363.8 & 6089.11 & 15802.24 & 971.58 & 490328.37 & 0 & 110123.85 \\
\hline 7507.60 & 321783 & 17 & 1618.15 & 5917 & 39187.49 & 6636.72 & 17230.74 & 1081.45 & 468353.83 & 0 & 0 \\
\hline 7507.66 & 297194 & 17 & 1113.83 & 6048.64 & 35428 & 6620.89 & 17226.15 & 1080.18 & 505133.47 & 0 & 16054.49 \\
\hline 7507.73 & 263582 & 18 & 905.22 & 5397.8 & 43607.22 & 6352.01 & 16456.85 & 1026.25 & 458305.35 & 51514.63 & 0 \\
\hline
\end{tabular}




\begin{tabular}{|c|c|c|c|c|c|c|c|c|c|c|c|}
\hline True Depth & XRF tCOUNTS & XRF Live Time & $\mathrm{Ti}(\mathrm{ppm})$ & V (ppm) & $\mathrm{Fe}(\mathrm{ppm})$ & $\mathrm{Ni}(\mathrm{ppm})$ & $\mathrm{Cu}(\mathrm{ppm})$ & $\mathrm{Pb}(\mathrm{ppm})$ & LE (ppm) & $\mathrm{Mg}(\mathrm{ppm})$ & $\mathrm{Cl}(\mathrm{ppm})$ \\
\hline 7507.80 & 339400 & 17 & 913.16 & 5104.16 & 52962.95 & 5906.86 & 15359.34 & 933.32 & 440955.35 & 0 & 92847.96 \\
\hline 7507.86 & 317655 & 17 & 932.1 & 5900.62 & 49852.07 & 7265.59 & 18892.36 & 1190.61 & 472472.89 & 0 & 0 \\
\hline 7507.93 & 297460 & 17 & 850.17 & 6158.32 & 46830.16 & 7337.13 & 19019.99 & 1214.2 & 503443.6 & 0 & 0 \\
\hline 7507.99 & 298580 & 17 & 768.16 & 5992.07 & 46621.23 & 7168.46 & 18612.86 & 1178.62 & 519763.1 & 0 & 0 \\
\hline 7508.06 & 342116 & 17 & 540.07 & 6319.33 & 45830.1 & 7594.81 & 19651.29 & 1248.12 & 459526.96 & 0 & 0 \\
\hline 7508.12 & 436330 & 16 & 0 & 4950.34 & 32212.13 & 5114.89 & 13202.12 & 774.03 & 405987.22 & 0 & 368321.47 \\
\hline 7508.19 & 295250 & 17 & 0 & 5078.89 & 34134.08 & 5445.89 & 14092.77 & 849.64 & 501837.32 & 0 & 220659.91 \\
\hline 7508.25 & 253731 & 18 & 0 & 5620.53 & 36632.79 & 6470.62 & 16835.39 & 1060.64 & 548575.82 & 0 & 40299.52 \\
\hline 7508.32 & 357660 & 17 & 0 & 4447.53 & 43351.57 & 4802.11 & 12416.7 & 728.7 & 430588 & 0 & 365289.01 \\
\hline 7508.39 & 272435 & 18 & 928.85 & 5069.39 & 45201.3 & 6256.52 & 16223.01 & 1009.81 & 502922.01 & 0 & 0 \\
\hline 7508.45 & 279583 & 18 & 647.52 & 5272.54 & 41127.13 & 6051.51 & 15705.17 & 954.14 & 499073.6 & 0 & 80580.45 \\
\hline 7508.52 & 260883 & 18 & 876.73 & 5251.47 & 43100.06 & 6137.29 & 15991.53 & 993.54 & 515331.08 & 0 & 0 \\
\hline 7508.58 & 278852 & 18 & 11067.96 & 4561.79 & 38834.12 & 5879.44 & 15231.83 & 959.7 & 569082.76 & 0 & 0 \\
\hline 7508.65 & 295724 & 18 & 592.64 & 5581.73 & 38167.85 & 6354.93 & 16484.12 & 1027.57 & 488386.96 & 0 & 0 \\
\hline 7508.71 & 272786 & 18 & 853.65 & 5533.84 & 39341.65 & 6479.41 & 16811.43 & 1056.27 & 520802.08 & 0 & 0 \\
\hline 7508.78 & 286857 & 18 & 557.23 & 5244.97 & 32513.25 & 5590.63 & 14475.72 & 886.12 & 507995.82 & 0 & 212629.45 \\
\hline 7508.85 & 256449 & 18 & 877.45 & 5197.88 & 37948.85 & 5978.52 & 15501.29 & 965.79 & 557456.77 & 0 & 78257.49 \\
\hline 7508.91 & 219121 & 18 & 1043.95 & 5459.51 & 33608.23 & 6401.61 & 16590.35 & 1055.55 & 588634.43 & 0 & 8222.17 \\
\hline 7508.98 & 262868 & 18 & 524.6 & 5681.09 & 30738.93 & 6142.25 & 15925.5 & 978.15 & 531452.72 & 0 & 167767.77 \\
\hline 7509.04 & 277572 & 18 & 0 & 5318.16 & 30831.62 & 5693.51 & 14789.85 & 889.32 & 508219.41 & 0 & 237903.01 \\
\hline 7509.11 & 242842 & 18 & 0 & 4995.57 & 32738.12 & 5223.69 & 13583.6 & 814.8 & 533054.99 & 0 & 180535.3 \\
\hline 7509.17 & 255715 & 18 & 0 & 5752.8 & 30728.02 & 6106.31 & 15909.95 & 985.42 & 544286.58 & 0 & 131023.36 \\
\hline 7509.24 & 518077 & 15 & 0 & 3301.63 & 16358.93 & 3057.96 & 7896.55 & 442.59 & 375621.13 & 0 & 480136.86 \\
\hline 7509.30 & 408820 & 16 & 0 & 4798.75 & 23457.69 & 4669.12 & 12097.49 & 698.02 & 401520.06 & 0 & 408805.11 \\
\hline 7509.37 & 451192 & 16 & 1183.2 & 5294.87 & 25360.18 & 4654.55 & 12032.51 & 692.73 & 387799.46 & 0 & 440839.71 \\
\hline 7509.44 & 355042 & 17 & 614.44 & 5128.8 & 30559.57 & 5172.06 & 13341.61 & 786.53 & 432228.66 & 0 & 306138.02 \\
\hline 7509.50 & 356984 & 17 & 0 & 5092.55 & 31585.97 & 5248.12 & 13583.12 & 809.79 & 445511.33 & 0 & 270843.66 \\
\hline 7509.57 & 296667 & 17 & 664.46 & 4967.64 & 35982.65 & 5364.96 & 13884.44 & 833.02 & 522180.24 & 0 & 109857.39 \\
\hline 7509.63 & 365018 & 17 & 23513.16 & 4676.6 & 36131.24 & 6197.48 & 16115.11 & 1003.21 & 541680.69 & 0 & 0 \\
\hline 7509.70 & 343595 & 17 & 643.81 & 6083.9 & 36309.52 & 6622.87 & 17111.26 & 1056.51 & 475110.29 & 0 & 0 \\
\hline 7509.76 & 367696 & 17 & 0 & 6201.07 & 34704.9 & 6660.58 & 17253.1 & 1068.17 & 464528.38 & 0 & 64341.79 \\
\hline 7509.83 & 332760 & 17 & 0 & 6247.65 & 33565.6 & 6912.7 & 17872.48 & 1121.5 & 501402.15 & 0 & 0 \\
\hline 7509.90 & 314925 & 17 & 785.89 & 5430.34 & 35388.5 & 5939 & 15272.99 & 924.61 & 490227.72 & 0 & 111260.85 \\
\hline
\end{tabular}




\begin{tabular}{|c|c|c|c|c|c|c|c|c|c|c|c|}
\hline True Depth & XRF tCOUNTS & XRF Live Time & $\mathrm{Ti}(\mathrm{ppm})$ & $\mathrm{V}(\mathrm{ppm})$ & $\mathrm{Fe}(\mathrm{ppm})$ & $\mathrm{Ni}(\mathrm{ppm})$ & $\mathrm{Cu}(\mathrm{ppm})$ & $\mathrm{Pb}(\mathrm{ppm})$ & $\mathrm{LE}(\mathrm{ppm})$ & $\mathrm{Mg}(\mathrm{ppm})$ & $\mathrm{Cl}(\mathrm{ppm})$ \\
\hline 7509.96 & 315346 & 17 & 740.16 & 5888.16 & 38679.42 & 6739.38 & 17454.39 & 1095.35 & 490704.77 & 0 & 0 \\
\hline 7510.03 & 520290 & 15 & 0 & 3167.53 & 18428.85 & 2936.89 & 7542.6 & 424.62 & 373763.23 & 0 & 478716.9 \\
\hline 7510.09 & 312576 & 17 & 711.78 & 6283.68 & 38115.88 & 6819.51 & 17571.71 & 1088.03 & 403668.96 & 56279.68 & 43486.42 \\
\hline 7510.16 & 475082 & 16 & 0 & 2800.05 & 83357.1 & 3329.69 & 8596.29 & 505.51 & 322766.26 & 0 & 296925.19 \\
\hline 7510.22 & 340061 & 17 & 566.63 & 5935.78 & 39049.06 & 6689.18 & 17237.97 & 1069.93 & 470260.88 & 0 & 61516.1 \\
\hline 7510.29 & 372939 & 17 & 0 & 5310.12 & 28171.22 & 5308.22 & 13746.14 & 813.86 & 447551.95 & 0 & 307491.3 \\
\hline 7510.35 & 430592 & 16 & 0 & 2433.6 & 10921.63 & 2148.78 & 5531.49 & 308.29 & 429374.29 & 0 & 478866.48 \\
\hline 7510.42 & 262171 & 18 & 548.63 & 6040.74 & 29354.47 & 6680.76 & 17380.18 & 1108.27 & 526338.25 & 0 & 0 \\
\hline 7510.49 & 404077 & 16 & 0 & 2330.26 & 99434.82 & 3199.07 & 8267.17 & 482.59 & 378156.01 & 0 & 84622.44 \\
\hline 7510.55 & 313809 & 17 & 582.81 & 6239.51 & 32392.48 & 6662.65 & 17280.03 & 1078.69 & 455920.19 & 0 & 0 \\
\hline 7510.62 & 317062 & 17 & 5631.68 & 5793.67 & 28665.75 & 6446.71 & 16766.11 & 1052.37 & 531050.34 & 0 & 0 \\
\hline 7510.68 & 239591 & 18 & 1024.33 & 5963.14 & 29795.97 & 6441.04 & 16733.75 & 1057.65 & 556256.91 & 0 & 0 \\
\hline 7510.75 & 292471 & 18 & 603.66 & 6073.71 & 33848.8 & 6738.87 & 17471.23 & 1098.22 & 487421.99 & 0 & 0 \\
\hline 7510.81 & 256301 & 18 & 1019.13 & 5989.76 & 32011.01 & 6710.91 & 17381.65 & 1094.48 & 550635.58 & 0 & 34539.47 \\
\hline 7510.88 & 258577 & 18 & 539.52 & 6183.82 & 31098.48 & 6713.85 & 17382.61 & 1085.35 & 506762.22 & 0 & 0 \\
\hline 7510.94 & 325959 & 17 & 1372.92 & 6970.39 & 36026.41 & 7535.13 & 19436.87 & 1231.29 & 481265.84 & 0 & 0 \\
\hline 7511.01 & 324308 & 17 & 827.87 & 5521.19 & 35670.67 & 6176.83 & 15923.06 & 966.96 & 463104.01 & 0 & 144202.53 \\
\hline 7511.08 & 373820 & 17 & 501.79 & 5154.23 & 34952.85 & 5423.29 & 14000.43 & 838.12 & 438708.9 & 0 & 262942.38 \\
\hline 7511.14 & 307453 & 17 & 491.12 & 6154.46 & 35559 & 6969.89 & 18024.66 & 1127.46 & 478898.23 & 0 & 0 \\
\hline 7511.21 & 331281 & 17 & 863.3 & 5856.39 & 35850.87 & 6383.92 & 16559.19 & 1015 & 453944.62 & 0 & 97632.71 \\
\hline 7511.27 & 412980 & 16 & 387.81 & 4609.6 & 32101.18 & 4618.19 & 11957.09 & 697.53 & 414167.14 & 0 & 363426.45 \\
\hline 7511.34 & 296448 & 17 & 864.04 & 5908.58 & 37022.25 & 6668.62 & 17224.82 & 1076.9 & 487666.3 & 0 & 0 \\
\hline 7511.40 & 332728 & 17 & 0 & 6117.52 & 36555.63 & 6716.48 & 17383.64 & 1071.45 & 472884.72 & 0 & 127155.7 \\
\hline 7511.47 & 382513 & 17 & 0 & 5853.42 & 32722.26 & 6117.7 & 15733.48 & 936.22 & 440322.2 & 0 & 269591.91 \\
\hline 7511.54 & 482714 & 16 & 0 & 5118.5 & 25474.49 & 4993.05 & 12819.19 & 741.4 & 396910.52 & 0 & 439443.62 \\
\hline 7511.60 & 158570 & 19 & 0 & 4544.43 & 33347.23 & 4820.79 & 12265.62 & 733.33 & 404568.57 & 0 & 0 \\
\hline 7511.67 & 331817 & 17 & 816.72 & 4785.54 & 47526.05 & 5509.59 & 14178.63 & 850.42 & 465307.22 & 0 & 222847.07 \\
\hline 7511.73 & 271067 & 18 & 957.5 & 6187.15 & 32313.25 & 6853.74 & 17730.06 & 1123 & 539293.67 & 0 & 0 \\
\hline 7511.80 & 322076 & 17 & 842.48 & 5084.64 & 35679.97 & 5227.8 & 13505.12 & 816.95 & 484726.61 & 0 & 223041.67 \\
\hline 7511.86 & 387508 & 17 & 669.95 & 4419.99 & 42630.3 & 4670.08 & 12087.47 & 705.07 & 422052.08 & 0 & 358037.44 \\
\hline 7511.93 & 289106 & 18 & 861.59 & 4832.81 & 38170.79 & 5080.29 & 13071.16 & 783.11 & 510578.4 & 0 & 251089.17 \\
\hline 7511.99 & 295801 & 17 & 827.95 & 5475.4 & 37275.46 & 6229.41 & 16073.31 & 1006.9 & 515124.52 & 0 & 0 \\
\hline 7512.06 & 303836 & 17 & 0 & 6029.41 & 36261.47 & 6840.05 & 17577.12 & 1104.54 & 513024.12 & 0 & 0 \\
\hline
\end{tabular}




\begin{tabular}{|c|c|c|c|c|c|c|c|c|c|c|c|}
\hline True Depth & XRF tCOUNTS & XRF Live Time & $\mathrm{Ti}(\mathrm{ppm})$ & V (ppm) & $\mathrm{Fe}(\mathrm{ppm})$ & $\mathrm{Ni}(\mathrm{ppm})$ & $\mathrm{Cu}(\mathrm{ppm})$ & $\mathrm{Pb}(\mathrm{ppm})$ & LE (ppm) & $\mathrm{Mg}(\mathrm{ppm})$ & $\mathrm{Cl}(\mathrm{ppm})$ \\
\hline 7512.13 & 298387 & 17 & 551.67 & 5567.78 & 43269.59 & 6702.15 & 17200.33 & 1089.59 & 524426.34 & 0 & 0 \\
\hline 7512.19 & 290741 & 17 & 0 & 5553.14 & 45181.71 & 6660.86 & 17049.06 & 1084.14 & 520779.61 & 0 & 0 \\
\hline 7512.26 & 207264 & 18 & 844.44 & 6002.07 & 37205.56 & 7016.52 & 18148.33 & 1173.47 & 591349.57 & 0 & 7612.07 \\
\hline 7512.32 & 418214 & 16 & 0 & 2441.9 & 15523.19 & 2231.55 & 5709.11 & 319.83 & 415896.01 & 0 & 482442.08 \\
\hline 7512.39 & 270668 & 18 & 866.23 & 5298.13 & 44387.3 & 6424.94 & 16592.43 & 1044.98 & 522610.69 & 0 & 0 \\
\hline 7512.45 & 340366 & 17 & 684.24 & 5095.43 & 66695.98 & 6698.15 & 17263.3 & 1086.25 & 473722.2 & 0 & 0 \\
\hline 7512.52 & 301788 & 17 & 770.63 & 5354.98 & 47977.06 & 6396.76 & 16520.82 & 1033.86 & 508675.95 & 0 & 0 \\
\hline 7512.59 & 394971 & 16 & 434.22 & 5132.45 & 39124.02 & 5223.83 & 13483.72 & 795.84 & 409295.14 & 0 & 319621.16 \\
\hline 7512.65 & 293022 & 17 & 550.39 & 5570.3 & 40148.77 & 6506.17 & 16772.55 & 1053.69 & 496884.34 & 0 & 0 \\
\hline 7512.72 & 472865 & 16 & 0 & 4178.89 & 25149.19 & 3993.64 & 10298.75 & 591.63 & 377368.56 & 0 & 452605.48 \\
\hline 7512.78 & 455678 & 16 & 414.23 & 4595.79 & 27500.15 & 4474.54 & 11556.21 & 668.99 & 397565.28 & 0 & 427447.96 \\
\hline 7512.85 & 321357 & 17 & 831.87 & 6281.43 & 37236.88 & 7068.95 & 18342.01 & 1154.81 & 489061.03 & 0 & 0 \\
\hline 7512.91 & 308282 & 17 & 634.42 & 5529.56 & 36181.26 & 6066.27 & 15724.9 & 961 & 498998.57 & 0 & 61143.48 \\
\hline 7512.98 & 376690 & 17 & 0 & 5266.38 & 29691.07 & 5316.42 & 13725.29 & 810.16 & 445762.92 & 0 & 287247.76 \\
\hline 7513.04 & 304927 & 17 & 546.37 & 5735.88 & 27640.37 & 5747.66 & 14864.47 & 893.83 & 472273.66 & 0 & 168584.98 \\
\hline 7513.11 & 373966 & 17 & 453.18 & 4749.07 & 28696.25 & 4677.26 & 12096.08 & 708.27 & 445627.59 & 0 & 329145.31 \\
\hline 7513.18 & 268383 & 18 & 820.96 & 5810.83 & 41176.76 & 6702.88 & 17396.66 & 1100.81 & 541671.7 & 0 & 0 \\
\hline 7513.24 & 407062 & 16 & 0 & 5283.96 & 27560.38 & 5164.94 & 13369.9 & 780.17 & 414972.38 & 0 & 344724.74 \\
\hline 7513.31 & 473128 & 16 & 0 & 4455.49 & 28518.3 & 4316.46 & 11137.56 & 643.44 & 374153.32 & 0 & 458955.25 \\
\hline 7513.37 & 313501 & 17 & 937.93 & 5610.81 & 38263.28 & 6351.08 & 16475.02 & 1019.43 & 468262.25 & 0 & 0 \\
\hline 7513.44 & 308212 & 17 & 993.76 & 6101.27 & 35114.57 & 6441.09 & 16637.67 & 1025.64 & 439549.68 & 45088.11 & 50294.75 \\
\hline 7513.50 & 341287 & 17 & 522.25 & 5583.55 & 34683.09 & 5902.03 & 15226.46 & 919.16 & 465359.78 & 0 & 195913.83 \\
\hline 7513.57 & 339182 & 17 & 954.47 & 5984.08 & 40326.65 & 6879.17 & 17733 & 1109.89 & 468425.38 & 0 & 0 \\
\hline 7513.64 & 253899 & 18 & 903.67 & 5824.01 & 39572.66 & 6434.05 & 16531.6 & 1026.18 & 459744.35 & 0 & 0 \\
\hline 7513.70 & 347644 & 17 & 806.79 & 5591.29 & 34322.11 & 6001.72 & 15469.13 & 936.95 & 478796.06 & 0 & 139066.63 \\
\hline 7513.77 & 295351 & 17 & 798.34 & 5887.35 & 36983.76 & 6558.23 & 16927.63 & 1059.77 & 514102.6 & 0 & 0 \\
\hline 7513.83 & 302580 & 17 & 849.92 & 5837.67 & 42319.23 & 6672.72 & 17249.83 & 1090.24 & 485146.35 & 0 & 0 \\
\hline 7513.90 & 332202 & 17 & 1084.96 & 5300.57 & 60196.65 & 6623.98 & 17078.88 & 1059.93 & 456154.07 & 0 & 38518.06 \\
\hline 7513.96 & 279779 & 18 & 899.93 & 5298.38 & 39103.76 & 5972.06 & 15421.73 & 960.43 & 517957.69 & 0 & 0 \\
\hline 7514.03 & 347684 & 17 & 722.5 & 6166.78 & 33352.14 & 6487.91 & 16763.93 & 1029.84 & 463663.58 & 0 & 129347.05 \\
\hline 7514.09 & 350248 & 17 & 504.05 & 5360.61 & 53751.86 & 6266.34 & 16142.26 & 982.33 & 438194.46 & 0 & 147119.07 \\
\hline 7514.16 & 467273 & 16 & 353.19 & 4078.65 & 22219.07 & 3742.57 & 9659.12 & 553.54 & 393492.37 & 0 & 464682.45 \\
\hline 7514.23 & 303695 & 17 & 760.5 & 6428.05 & 33946.34 & 6992.8 & 18141.04 & 1139.9 & 476145.22 & 0 & 0 \\
\hline
\end{tabular}




\begin{tabular}{|c|c|c|c|c|c|c|c|c|c|c|c|}
\hline True Depth & XRF tCOUNTS & XRF Live Time & $\mathrm{Ti}(\mathrm{ppm})$ & $\mathrm{V}(\mathrm{ppm})$ & $\mathrm{Fe}(\mathrm{ppm})$ & $\mathrm{Ni}(\mathrm{ppm})$ & $\mathrm{Cu}(\mathrm{ppm})$ & $\mathrm{Pb}(\mathrm{ppm})$ & $\mathrm{LE}(\mathrm{ppm})$ & $\mathrm{Mg}(\mathrm{ppm})$ & $\mathrm{Cl}(\mathrm{ppm})$ \\
\hline 7514.29 & 377717 & 17 & 1134.3 & 4558.54 & 81627.5 & 6260.53 & 16280.56 & 991.4 & 395122.37 & 0 & 0 \\
\hline 7514.36 & 358937 & 17 & 610.53 & 5742.84 & 38970.26 & 6136.3 & 15913.47 & 975.74 & 448677.96 & 0 & 132317.09 \\
\hline 7514.42 & 408519 & 16 & 0 & 4978.98 & 27407.16 & 4938.19 & 12784.77 & 746.62 & 405311.81 & 0 & 387027.47 \\
\hline 7514.49 & 552620 & 15 & 0 & 2250.02 & 10565.65 & 1963.56 & 5046.51 & 278.13 & 423580.28 & 0 & 501062.42 \\
\hline 7514.55 & 319540 & 17 & 560.58 & 6061.58 & 37500.78 & 6550.43 & 16877.16 & 1051.05 & 485036.02 & 0 & 85125.75 \\
\hline 7514.62 & 314216 & 17 & 742.52 & 6251.78 & 41880.28 & 7309.82 & 18960.38 & 1196.34 & 484453.69 & 0 & 0 \\
\hline 7514.69 & 303286 & 17 & 702.05 & 6128.27 & 29783.54 & 6468.42 & 16803.14 & 1041.38 & 484603.66 & 0 & 29091.28 \\
\hline 7514.75 & 309340 & 17 & 614.7 & 6020.44 & 30792.12 & 6599.49 & 17114.62 & 1069.24 & 484509.41 & 0 & 0 \\
\hline 7514.82 & 344517 & 17 & 0 & 5641.15 & 33745.89 & 6021.37 & 15666.79 & 951.62 & 452350.6 & 0 & 0 \\
\hline 7514.88 & 370704 & 17 & 0 & 6274.96 & 31665.19 & 6413.46 & 16641.23 & 1021.51 & 470391.14 & 0 & 0 \\
\hline 7514.95 & 599599 & 14 & 0 & 1678.27 & 8259.83 & 1435.23 & 3699.99 & 203.19 & 428962.77 & 0 & 504092.35 \\
\hline 7515.01 & 394837 & 17 & 0 & 4379.26 & 23091.98 & 4192.26 & 10893.47 & 626.44 & 426760.07 & 0 & 400043.91 \\
\hline 7515.08 & 311998 & 17 & 0 & 4504.36 & 25427.67 & 4305.89 & 11190.23 & 650.37 & 462898.72 & 0 & 339538.01 \\
\hline 7515.14 & 356446 & 17 & 0 & 3885.22 & 26744.35 & 3689.37 & 9563.83 & 543.9 & 412990.11 & 0 & 452655.93 \\
\hline 7515.21 & 636904 & 14 & 0 & 1410.84 & 7756.65 & 1226.39 & 3164.26 & 171.11 & 437642.53 & 0 & 510290.77 \\
\hline 7515.28 & 643500 & 14 & 0 & 1231.06 & 5311.29 & 1031.82 & 2652.45 & 143.46 & 452415.24 & 0 & 504467.81 \\
\hline 7515.34 & 664732 & 13 & 0 & 1236.56 & 6968.99 & 1063.3 & 2723.99 & 147.51 & 454548.38 & 0 & 508787.54 \\
\hline 7515.41 & 626438 & 14 & 0 & 1329.12 & 6156 & 1152.82 & 2961.05 & 159.33 & 460773.15 & 0 & 503002.65 \\
\hline 7515.47 & 431113 & 16 & 0 & 2673.86 & 22191.75 & 2448.88 & 6319.22 & 355.08 & 397733.24 & 0 & 485130.59 \\
\hline 7515.54 & 619384 & 14 & 0 & 1924.39 & 8851.04 & 1695.77 & 4372.85 & 239.97 & 424291.28 & 0 & 508518.5 \\
\hline 7515.60 & 645312 & 14 & 0 & 1761.2 & 8783.38 & 1535.31 & 3954.34 & 216.49 & 418572.67 & 0 & 515210.12 \\
\hline 7515.67 & 114084 & 19 & 0 & 4699.87 & 31070.6 & 4697.63 & 12194.62 & 714.98 & 580329.23 & 0 & 137172.26 \\
\hline 7515.73 & 489214 & 16 & 0 & 4950.01 & 23803.92 & 4731.45 & 12297.8 & 710.63 & 359786.32 & 0 & 419334.39 \\
\hline 7515.80 & 324001 & 17 & 1013.36 & 6434.43 & 39062.22 & 7162.01 & 18571.3 & 1162.55 & 469071.91 & 0 & 107295.44 \\
\hline 7515.87 & 468890 & 16 & 313.04 & 3081.57 & 18133.37 & 2891.65 & 7477.77 & 420.5 & 386702.59 & 0 & 480338.68 \\
\hline 7515.93 & 582443 & 14 & 0 & 2349.96 & 11962.44 & 2104.41 & 5426.84 & 301.52 & 415052.46 & 0 & 500758.7 \\
\hline 7516.00 & 497286 & 15 & 0 & 3967.32 & 20638.29 & 3743.17 & 9664.69 & 549.56 & 345402.17 & 29125.91 & 477328.63 \\
\hline 7516.06 & 426813 & 16 & 559.14 & 5497.08 & 28853.83 & 5427.93 & 14059.51 & 826.02 & 404463.87 & 0 & 388983.79 \\
\hline 7516.13 & 348626 & 17 & 474.95 & 6018.03 & 32231.38 & 6348.36 & 16521.82 & 1000.23 & 432416.83 & 0 & 252889.57 \\
\hline 7516.19 & 549494 & 15 & 0 & 2660.64 & 11553.93 & 2377.66 & 6134.26 & 340.26 & 409533.42 & 0 & 495336.62 \\
\hline 7516.26 & 548876 & 15 & 330.33 & 2602.81 & 42553.32 & 2729.53 & 7043.79 & 396.47 & 354212.5 & 0 & 502150.43 \\
\hline 7516.33 & 372035 & 17 & 943.46 & 5884.25 & 31660.69 & 5956.27 & 15475 & 929.15 & 425505.42 & 0 & 265900.64 \\
\hline 7516.39 & 527741 & 15 & 0 & 2505.08 & 10731.6 & 2191.35 & 5664.78 & 313.93 & 417784.87 & 0 & 497486.18 \\
\hline
\end{tabular}




\begin{tabular}{|c|c|c|c|c|c|c|c|c|c|c|c|}
\hline True Depth & XRF tCOUNTS & XRF Live Time & $\mathrm{Ti}(\mathrm{ppm})$ & V (ppm) & $\mathrm{Fe}(\mathrm{ppm})$ & $\mathrm{Ni}(\mathrm{ppm})$ & $\mathrm{Cu}(\mathrm{ppm})$ & $\mathrm{Pb}(\mathrm{ppm})$ & LE (ppm) & $\mathrm{Mg}(\mathrm{ppm})$ & $\mathrm{Cl}(\mathrm{ppm})$ \\
\hline 7516.46 & 422332 & 16 & 0 & 5124.2 & 23448.8 & 4955.24 & 12867.99 & 746.7 & 406354.78 & 0 & 438296.72 \\
\hline 7516.52 & 400342 & 16 & 529.04 & 6182.04 & 44072.69 & 6947.39 & 17888.98 & 1089.59 & 426727.48 & 0 & 207801.2 \\
\hline 7516.59 & 497255 & 15 & 0 & 4556.25 & 38190.98 & 4639.2 & 11906.26 & 688.87 & 358776.5 & 0 & 476840.34 \\
\hline 7516.65 & 365720 & 17 & 736.21 & 5337.09 & 42343.22 & 5834.43 & 15015.27 & 896.53 & 438242.12 & 0 & 311695.42 \\
\hline 7516.72 & 370791 & 17 & 1422.83 & 5163.94 & 38196.41 & 5630.58 & 14501.68 & 863.17 & 447104.77 & 0 & 327652.64 \\
\hline 7516.78 & 301737 & 17 & 555.52 & 5508.93 & 48978.15 & 6102.53 & 15810.95 & 954.28 & 430420.48 & 0 & 235694.45 \\
\hline 7516.85 & 302406 & 17 & 929.51 & 6423.12 & 48690.58 & 7624.49 & 19761.12 & 1238.01 & 516844.7 & 0 & 129526.78 \\
\hline 7516.92 & 561168 & 15 & 0 & 3070.07 & 19875.69 & 2914.84 & 7477.66 & 422.98 & 388323.85 & 0 & 493771.79 \\
\hline 7516.98 & 536661 & 15 & 0 & 4981.43 & 33803.95 & 5075.16 & 13250.85 & 766.72 & 377407.51 & 0 & 195178.19 \\
\hline 7517.05 & 462345 & 16 & 553.67 & 5131.24 & 33205.94 & 5137.3 & 13485.4 & 790.86 & 430253.91 & 0 & 208730.58 \\
\hline 7517.11 & 556827 & 15 & 0 & 4925.94 & 44379.39 & 5269.25 & 13797.67 & 802.18 & 433467.24 & 0 & 104873.66 \\
\hline 7517.18 & 580098 & 14 & 0 & 3680.78 & 48240.69 & 3904.98 & 10243.86 & 586.97 & 372506.1 & 0 & 155713.6 \\
\hline 7517.24 & 561972 & 15 & 0 & 5061.62 & 43972.57 & 5265 & 13713.08 & 809.21 & 423746.66 & 0 & 79507.39 \\
\hline 7517.31 & 522081 & 15 & 0 & 2619.45 & 14924.43 & 2442.66 & 6304.48 & 354.17 & 375994.12 & 0 & 491775.72 \\
\hline 7517.38 & 374299 & 17 & 673.74 & 7128.98 & 52558.58 & 8594 & 22402.59 & 1423.29 & 479065.53 & 0 & 0 \\
\hline 7517.44 & 329585 & 17 & 689.01 & 7327.75 & 43836.15 & 8826.69 & 22955.55 & 1503.39 & 480172.03 & 0 & 0 \\
\hline 7517.51 & 311347 & 17 & 1028.19 & 6677.66 & 42330.14 & 7379.37 & 19130.74 & 1195.04 & 465832.5 & 0 & 174728.7 \\
\hline 7517.57 & 360611 & 17 & 1253.94 & 7134.82 & 43443.61 & 7969.29 & 20696.95 & 1299.18 & 448531.32 & 0 & 135040.83 \\
\hline 7517.64 & 322004 & 17 & 1162.73 & 7167.32 & 41124.76 & 8211.71 & 21325.96 & 1373.59 & 504861.45 & 0 & 0 \\
\hline 7517.70 & 315120 & 17 & 1143.71 & 6270.33 & 32035.7 & 6239.01 & 16210.74 & 969.7 & 432860.32 & 0 & 294945.3 \\
\hline 7517.77 & 278812 & 18 & 980.16 & 7811.93 & 34702.05 & 8691.86 & 22630.07 & 1460.79 & 448039.77 & 68143.48 & 12888.03 \\
\hline 7517.83 & 326000 & 17 & 0 & 7778.29 & 30505.58 & 8253.01 & 21532.08 & 1372.66 & 516903.75 & 0 & 66703.01 \\
\hline 7517.90 & 474119 & 16 & 0 & 5673.57 & 22611.93 & 5370.39 & 13895.45 & 805.06 & 385118.44 & 0 & 441844.32 \\
\hline 7517.97 & 549272 & 15 & 0 & 2829.39 & 11405.24 & 2550.77 & 6579.68 & 366.92 & 411405.9 & 0 & 490859.96 \\
\hline 7518.03 & 379103 & 17 & 607.04 & 7546.98 & 32744.4 & 8090.93 & 21037.71 & 1325.69 & 464295.96 & 0 & 102203.11 \\
\hline 7518.10 & 616642 & 14 & 802.2 & 3688.97 & 7788.04 & 2619.34 & 6812.33 & 374.49 & 314540.72 & 0 & 295432.81 \\
\hline 7518.16 & 635620 & 14 & 4271.95 & 9417.87 & 7661.06 & 5911.72 & 15507.33 & 905.91 & 461111.03 & 0 & 16265.7 \\
\hline 7518.23 & 639114 & 14 & 6078.34 & 9892.23 & 8394.31 & 6131.6 & 16112.35 & 942.57 & 437793.13 & 0 & 0 \\
\hline 7518.29 & 616558 & 14 & 1900.58 & 6595.51 & 9505.83 & 4465.97 & 11688.81 & 653.16 & 256356.19 & 0 & 418442.72 \\
\hline 7518.36 & 545921 & 15 & 22259.48 & 22802.1 & 22830.51 & 9683.78 & 25453.02 & 1549.98 & 359177.46 & 0 & 0 \\
\hline 7518.43 & 620536 & 14 & 41140.57 & 30968.05 & 17271.6 & 7658.74 & 20142.6 & 1177.45 & 213815.07 & 0 & 235010.87 \\
\hline 7518.49 & 632466 & 14 & 14189.77 & 12662.27 & 8950.65 & 4481.97 & 11710.34 & 666.36 & 351256.96 & 0 & 127445.12 \\
\hline 7518.56 & 637098 & 14 & 0 & 2877.89 & 7122.36 & 2208.14 & 5751.52 & 318.3 & 473211.1 & 0 & 32246.93 \\
\hline
\end{tabular}




\begin{tabular}{|c|c|c|c|c|c|c|c|c|c|c|c|}
\hline True Depth & XRF tCOUNTS & XRF Live Time & $\mathrm{Ti}(\mathrm{ppm})$ & $\mathrm{V}(\mathrm{ppm})$ & $\mathrm{Fe}(\mathrm{ppm})$ & $\mathrm{Ni}(\mathrm{ppm})$ & $\mathrm{Cu}(\mathrm{ppm})$ & $\mathrm{Pb}(\mathrm{ppm})$ & $\mathrm{LE}(\mathrm{ppm})$ & $\mathrm{Mg}(\mathrm{ppm})$ & $\mathrm{Cl}(\mathrm{ppm})$ \\
\hline 7518.62 & 302806 & 17 & 0 & 2662.66 & 7299.09 & 2156.25 & 5597.77 & 312.42 & 400776.4 & 0 & 103265.6 \\
\hline 7518.69 & 542088 & 15 & 0 & 2012.04 & 4795.86 & 1576.99 & 4125.25 & 222.64 & 360888.58 & 0 & 237610.65 \\
\hline 7518.75 & 517061 & 15 & 25783.37 & 1745.66 & 5834.82 & 2102.7 & 5508.79 & 310.44 & 528216.98 & 0 & 30057.64 \\
\hline 7518.82 & 513099 & 15 & 0 & 2089.5 & 5559.88 & 1741.24 & 4553.24 & 250.04 & 535570.38 & 0 & 31860.38 \\
\hline 7518.88 & 451361 & 16 & 0 & 1938.77 & 5342.25 & 1456.35 & 3779.91 & 212.26 & 408554.03 & 0 & 208007.77 \\
\hline 7518.95 & 520598 & 15 & 0 & 2512.68 & 7205.19 & 2005.49 & 5198.51 & 286.48 & 508622.82 & 0 & 44037.69 \\
\hline 7519.02 & 531156 & 15 & 0 & 2004.21 & 6100.73 & 1678.13 & 4398.89 & 242.63 & 430097.48 & 0 & 137176.32 \\
\hline 7519.08 & 574427 & 15 & 0 & 1876.3 & 5414.34 & 1560.61 & 4057.28 & 220.22 & 301411.09 & 0 & 332611.56 \\
\hline 7519.15 & 555803 & 15 & 0 & 2662.39 & 8900.17 & 2364.75 & 6116.65 & 342.01 & 524601.39 & 0 & 18486.49 \\
\hline 7519.21 & 469131 & 16 & 0 & 2469.64 & 6877.18 & 1943.1 & 4998.5 & 271.62 & 430897.79 & 0 & 187990.43 \\
\hline 7519.28 & 620432 & 14 & 0 & 2056.21 & 5588.67 & 1721.79 & 4459.97 & 239.5 & 310696.49 & 0 & 295992.18 \\
\hline 7519.34 & 620402 & 14 & 0 & 2068.1 & 7600.49 & 1710.64 & 4439.19 & 242.59 & 359885.74 & 0 & 187793.59 \\
\hline 7519.41 & 627859 & 14 & 0 & 2469.28 & 8350.86 & 2030.71 & 5266.07 & 286.72 & 465605.02 & 0 & 50789.79 \\
\hline 7519.48 & 659809 & 13 & 1349.74 & 3440.79 & 5394.13 & 2138.53 & 5567.11 & 304.73 & 267288.4 & 0 & 458535.06 \\
\hline 7519.54 & 594526 & 14 & 1346.88 & 4417.43 & 8488.7 & 3049.53 & 7925.94 & 441.93 & 320780.81 & 0 & 286642.69 \\
\hline 7519.61 & 492364 & 15 & 329.26 & 2883.42 & 7775.74 & 2156.68 & 5583.04 & 306.27 & 378446.38 & 0 & 472525.02 \\
\hline 7519.67 & 404871 & 16 & 662.1 & 7947.68 & 30483.98 & 8281.88 & 21561.51 & 1352.89 & 458350.62 & 0 & 108070.69 \\
\hline 7519.74 & 209358 & 18 & 1156.44 & 7655.34 & 28106.86 & 7724.61 & 20096.86 & 1264.58 & 449838.06 & 0 & 0 \\
\hline 7519.80 & 476854 & 16 & 10633.08 & 13880.46 & 45997.88 & 8077.9 & 21089.92 & 1263.07 & 283094.89 & 0 & 419669.95 \\
\hline 7519.87 & 452488 & 16 & 0 & 6497.21 & 22303.54 & 6087.95 & 15843.9 & 940.06 & 396881.52 & 0 & 382733.78 \\
\hline 7519.93 & 321586 & 17 & 575.19 & 7122.97 & 28088.88 & 7127.73 & 18528.71 & 1126.93 & 434283.39 & 0 & 213628.26 \\
\hline 7520.00 & 459834 & 16 & 16953.45 & 18165.55 & 85654.42 & 11758.96 & 30890.33 & 1965.01 & 330219.51 & 0 & 38532.46 \\
\hline 7520.07 & 444424 & 16 & 0 & 7316.37 & 24172.19 & 7363.96 & 19210.01 & 1182.17 & 481921.4 & 0 & 35487.33 \\
\hline 7520.13 & 361345 & 17 & 658.1 & 7370.83 & 31335.96 & 7920.53 & 20680.03 & 1310.36 & 512459.09 & 0 & 0 \\
\hline 7520.20 & 417863 & 16 & 0 & 7315.47 & 26443.68 & 7443.5 & 19463.95 & 1203.47 & 486872.16 & 0 & 0 \\
\hline 7520.26 & 565307 & 15 & 0 & 5207.02 & 18928.15 & 4752.92 & 12420.84 & 720.27 & 480816.17 & 0 & 0 \\
\hline 7520.33 & 548158 & 15 & 0 & 3983.32 & 14437.67 & 3757.53 & 9783.19 & 554.35 & 413555.81 & 0 & 148329.92 \\
\hline 7520.39 & 530922 & 15 & 0 & 4725 & 14476.09 & 4394.54 & 11458.64 & 662.57 & 438142.53 & 0 & 112866.24 \\
\hline 7520.46 & 459453 & 16 & 0 & 7006.12 & 21613 & 6782.14 & 17722.01 & 1077.21 & 498750.55 & 0 & 29119.83 \\
\hline 7520.52 & 361309 & 17 & 0 & 7193.05 & 26616.43 & 7503.08 & 19577.15 & 1217.14 & 494732.77 & 0 & 100828.88 \\
\hline 7520.59 & 312423 & 17 & 0 & 6532.18 & 28281.19 & 6599.93 & 17156.65 & 1055.94 & 489463.78 & 0 & 179433.46 \\
\hline 7520.66 & 346560 & 17 & 576 & 6284.77 & 28271.87 & 6416.91 & 16687.45 & 1010.03 & 451487.35 & 0 & 249018.48 \\
\hline 7520.72 & 307116 & 17 & 1031.47 & 7072.95 & 31959.86 & 7804.99 & 20391.56 & 1311.25 & 489146.54 & 0 & 6974.84 \\
\hline
\end{tabular}




\begin{tabular}{|c|c|c|c|c|c|c|c|c|c|c|c|}
\hline True Depth & XRF tCOUNTS & XRF Live Time & $\mathrm{Ti}(\mathrm{ppm})$ & V (ppm) & $\mathrm{Fe}(\mathrm{ppm})$ & $\mathrm{Ni}(\mathrm{ppm})$ & $\mathrm{Cu}(\mathrm{ppm})$ & $\mathrm{Pb}(\mathrm{ppm})$ & LE (ppm) & $\mathrm{Mg}(\mathrm{ppm})$ & $\mathrm{Cl}(\mathrm{ppm})$ \\
\hline 7520.79 & 365211 & 17 & 0 & 7097.7 & 24300.67 & 7061.22 & 18380.48 & 1125.23 & 451778.39 & 0 & 224988.49 \\
\hline 7520.85 & 345491 & 17 & 561.38 & 8200.27 & 34038.14 & 9155.07 & 23979.59 & 1573.94 & 494588.08 & 0 & 0 \\
\hline 7520.92 & 298323 & 17 & 0 & 8537.62 & 32834.34 & 9811.77 & 25673.09 & 1720.2 & 508104.05 & 0 & 0 \\
\hline 7520.98 & 411741 & 16 & 576.93 & 5734.14 & 20071.76 & 5427.24 & 14156.09 & 829.03 & 434757.25 & 0 & 406301.6 \\
\hline 7521.05 & 307956 & 17 & 1000.26 & 7363.39 & 42552.86 & 8730.48 & 22818.83 & 1482.35 & 480183.9 & 0 & 0 \\
\hline 7521.12 & 304664 & 17 & 1324.8 & 7005.22 & 42526.92 & 8080.54 & 21132.54 & 1346.4 & 492439.31 & 0 & 48852.25 \\
\hline 7521.18 & 389002 & 17 & 1171.47 & 6490.53 & 57110.07 & 7785.16 & 20343.39 & 1247.95 & 423198.6 & 0 & 181400.76 \\
\hline 7521.25 & 366340 & 17 & 798.09 & 6919.52 & 34279.13 & 7496.07 & 19619.13 & 1205.92 & 457443.16 & 0 & 232707.47 \\
\hline 7521.31 & 294533 & 18 & 1003.99 & 7424.39 & 31941.39 & 8225.05 & 21574.07 & 1393.86 & 525164.78 & 0 & 9812.93 \\
\hline 7521.38 & 276033 & 18 & 748.47 & 6930.04 & 34368.22 & 7719.34 & 20217.28 & 1276.61 & 487904.43 & 0 & 43878.07 \\
\hline 7521.44 & 304849 & 17 & 1024.35 & 8308.86 & 29890.48 & 9214.69 & 24163.08 & 1591.2 & 490620.03 & 0 & 0 \\
\hline 7521.51 & 475936 & 16 & 416.75 & 5602.95 & 23663.32 & 5445.29 & 14177.09 & 826.58 & 388406.87 & 0 & 464452.55 \\
\hline 7521.57 & 380439 & 17 & 1074.46 & 6334.28 & 36918.88 & 6749.13 & 17665.26 & 1057.37 & 438903.11 & 0 & 295574.88 \\
\hline 7521.64 & 327209 & 17 & 1071.32 & 7020.13 & 33001.64 & 7576.37 & 19788.27 & 1230.3 & 497488.21 & 0 & 172197.34 \\
\hline 7521.71 & 235365 & 18 & 1211.81 & 7868.74 & 31971.84 & 8913.3 & 23332.1 & 1547.73 & 560101.98 & 0 & 6694.17 \\
\hline 7521.77 & 288730 & 18 & 1359.74 & 7572.16 & 36170.74 & 8484.57 & 22253.25 & 1415.12 & 412141.22 & 64100.34 & 83713.67 \\
\hline 7521.84 & 333205 & 17 & 927.82 & 7661.36 & 27899.52 & 8310.01 & 21737.8 & 1381.28 & 474472.17 & 0 & 95835.26 \\
\hline 7521.90 & 327307 & 17 & 1049.11 & 7500.01 & 40601.4 & 8916.59 & 23409.9 & 1519.42 & 489179.22 & 0 & 0 \\
\hline 7521.97 & 383437 & 17 & 858.21 & 6495.48 & 37798.03 & 6938.71 & 18140.59 & 1095.6 & 427628.98 & 0 & 288234.84 \\
\hline 7522.03 & 319970 & 17 & 1320.37 & 7070.81 & 36852.44 & 7785.79 & 20339.12 & 1282.55 & 490825.22 & 0 & 101239.14 \\
\hline 7522.10 & 464196 & 16 & 489.42 & 5057.68 & 60596.25 & 5802.2 & 15197.28 & 889.09 & 377438.89 & 0 & 411368.58 \\
\hline 7522.17 & 337002 & 17 & 1006.67 & 7749.33 & 30093.07 & 8337.15 & 21824.79 & 1380.14 & 468211.38 & 0 & 109975.36 \\
\hline 7522.23 & 365727 & 17 & 1180.86 & 6998.89 & 33205.46 & 7431.52 & 19441.86 & 1192.05 & 441498.81 & 0 & 215063.61 \\
\hline 7522.30 & 445261 & 16 & 813.76 & 6297.11 & 35456.33 & 6354.28 & 16629.57 & 983.09 & 397232.68 & 0 & 372416.95 \\
\hline 7522.36 & 407197 & 16 & 733.89 & 6460.91 & 37364.94 & 6713.49 & 17547.35 & 1051.56 & 419670.31 & 0 & 288609.79 \\
\hline 7522.43 & 328262 & 17 & 540.18 & 7613.09 & 30107.78 & 8065.89 & 21132.91 & 1328.78 & 494127.59 & 0 & 118342.56 \\
\hline 7522.49 & 322874 & 17 & 919.65 & 7883.96 & 33130.85 & 8475.65 & 22165.73 & 1403.32 & 433104.04 & 46983.03 & 114965.57 \\
\hline 7522.56 & 496493 & 15 & 0 & 3223.5 & 35746.51 & 3305 & 8564.01 & 485.26 & 378649.38 & 0 & 491220.81 \\
\hline 7522.62 & 538087 & 15 & 0 & 3061.59 & 14428.7 & 2846.3 & 7359.36 & 412.57 & 411359.36 & 0 & 496267.18 \\
\hline 7522.69 & 325489 & 17 & 943.25 & 7909.99 & 39595.24 & 9243.01 & 24131.94 & 1586.17 & 488832.02 & 0 & 0 \\
\hline 7522.76 & 411183 & 16 & 0 & 6521.25 & 32603.04 & 6586.79 & 17131.94 & 1027.22 & 416634.98 & 0 & 374996.14 \\
\hline 7522.82 & 327299 & 17 & 6048.98 & 7354.26 & 30728.69 & 8259.91 & 21595.8 & 1386.65 & 527714.1 & 0 & 136268.37 \\
\hline 7522.89 & 309108 & 17 & 622.97 & 7737.11 & 40735.52 & 9040.7 & 23691.07 & 1553.41 & 514903.44 & 0 & 0 \\
\hline
\end{tabular}




\begin{tabular}{|c|c|c|c|c|c|c|c|c|c|c|c|}
\hline True Depth & XRF tCOUNTS & XRF Live Time & $\mathrm{Ti}(\mathrm{ppm})$ & V (ppm) & $\mathrm{Fe}(\mathrm{ppm})$ & $\mathrm{Ni}(\mathrm{ppm})$ & $\mathrm{Cu}(\mathrm{ppm})$ & $\mathrm{Pb}(\mathrm{ppm})$ & LE (ppm) & $\mathrm{Mg}(\mathrm{ppm})$ & $\mathrm{Cl}(\mathrm{ppm})$ \\
\hline 7522.95 & 389833 & 17 & 681.54 & 6678.27 & 26600.48 & 6770.66 & 17621.9 & 1062.77 & 442352.68 & 0 & 320506.5 \\
\hline 7523.02 & 329726 & 17 & 794.37 & 8075.69 & 34666.95 & 9069.77 & 23738.89 & 1557.8 & 528016.28 & 0 & 0 \\
\hline 7523.08 & 411237 & 16 & 481.1 & 7679.46 & 40473.39 & 8304.69 & 21590.6 & 1341.75 & 423354.5 & 0 & 220103.74 \\
\hline 7523.15 & 595153 & 14 & 0 & 6479.32 & 15464.07 & 6244.53 & 16265.13 & 954.41 & 439659.66 & 0 & 51362.98 \\
\hline 7523.22 & 491609 & 16 & 0 & 6416.35 & 25408.2 & 6377.97 & 16676.68 & 983.09 & 353343.22 & 0 & 435781.48 \\
\hline 7523.28 & 421604 & 16 & 0 & 8354.92 & 30294.84 & 9041.62 & 23700.29 & 1501.16 & 492080.62 & 0 & 20628.09 \\
\hline 7523.35 & 340200 & 17 & 559.61 & 8576.53 & 27506.74 & 9175.08 & 23996.71 & 1546.99 & 498714.45 & 0 & 9719.46 \\
\hline 7523.41 & 473099 & 16 & 0 & 7127.62 & 22653.79 & 6936.68 & 18103.43 & 1075.35 & 389764.13 & 0 & 367951.04 \\
\hline 7523.48 & 432365 & 16 & 0 & 7539.84 & 26855.8 & 7573.19 & 19716.66 & 1194.47 & 410444.56 & 0 & 312860.27 \\
\hline 7523.54 & 331793 & 17 & 0 & 8679.32 & 28120.51 & 9583.65 & 25073.2 & 1647.92 & 507046.42 & 0 & 0 \\
\hline 7523.61 & 450373 & 16 & 0 & 6711.51 & 17334.72 & 6415.02 & 16754.2 & 988.91 & 405015.09 & 0 & 433191.4 \\
\hline 7523.67 & 384240 & 17 & 0 & 6884.86 & 22482.73 & 6623.82 & 17237.44 & 1025.42 & 382772.43 & 38847.29 & 398788.58 \\
\hline 7523.74 & 449453 & 16 & 0 & 6847.13 & 17527.65 & 6518.81 & 17015.95 & 1006.27 & 399391.73 & 0 & 390929.96 \\
\hline 7523.81 & 98472 & 19 & 0 & 4910.74 & 8022.96 & 4831.73 & 12618.02 & 762.33 & 702708.65 & 0 & 105840.89 \\
\hline 7523.87 & 382782 & 17 & 454.41 & 7600.7 & 15439.27 & 7369.72 & 19287.34 & 1171.96 & 448460.4 & 0 & 265669.92 \\
\hline 7523.94 & 284936 & 18 & 852.01 & 7795.16 & 25249.99 & 7989.97 & 20791.11 & 1291.65 & 474582.93 & 0 & 170937.87 \\
\hline 7524.00 & 349772 & 17 & 592.82 & 9258.58 & 20582.85 & 9837.07 & 25879.57 & 1698.85 & 492986.72 & 0 & 0 \\
\hline 7524.07 & 420483 & 16 & 0 & 6989.81 & 24888.3 & 7062.85 & 18458.01 & 1102.36 & 419024.27 & 0 & 314392.07 \\
\hline 7524.13 & 374084 & 17 & 0 & 8906.74 & 28466.94 & 9693.67 & 25453.18 & 1641.2 & 487048.01 & 0 & 14660.96 \\
\hline 7524.20 & 317854 & 17 & 672.27 & 8500.6 & 21150.65 & 9045.51 & 23718.09 & 1538.2 & 509209.06 & 0 & 27929.08 \\
\hline 7524.27 & 416171 & 16 & 0 & 6997.33 & 17894.31 & 6812.55 & 17816.12 & 1062.72 & 412073.11 & 0 & 367725.99 \\
\hline 7524.33 & 467490 & 16 & 0 & 6184.08 & 11197.64 & 5764.41 & 15048.2 & 871.29 & 393707.26 & 0 & 464609.1 \\
\hline 7524.40 & 415087 & 16 & 0 & 8764.4 & 14165.92 & 8502.93 & 22373.1 & 1386.43 & 492566.84 & 0 & 68165.15 \\
\hline 7524.46 & 628671 & 14 & 0 & 8724.49 & 14865.16 & 8470.87 & 22257.46 & 1336.47 & 421733.19 & 0 & 72919.67 \\
\hline 7524.53 & 541271 & 15 & 0 & 8930.72 & 17129.03 & 9025.36 & 23741.63 & 1453.54 & 439634.41 & 0 & 109186.5 \\
\hline 7524.59 & 551232 & 15 & 60282.12 & 42919.47 & 134377 & 15328.03 & 40634.79 & 2546.77 & 222097.56 & 0 & 100166.26 \\
\hline 7524.66 & 600176 & 14 & 9786.82 & 11649.23 & 14739.31 & 5418.05 & 14213.8 & 820.85 & 408627.99 & 0 & 96607.68 \\
\hline 7524.72 & 576497 & 14 & 40860.43 & 33977.02 & 22390.85 & 10728.02 & 28256.85 & 1712.26 & 327842.5 & 0 & 106575.03 \\
\hline 7524.79 & 642939 & 14 & 0 & 3200.72 & 6887.2 & 2842.74 & 7430.28 & 407.77 & 432814.53 & 0 & 86943.2 \\
\hline 7524.86 & 638326 & 14 & 0 & 4084.07 & 7352.56 & 3797.12 & 9968.96 & 565.97 & 446867.57 & 0 & 69307.97 \\
\hline 7524.92 & 638049 & 14 & 0 & 4003.92 & 8622.34 & 3627.23 & 9509.7 & 532.68 & 473864.31 & 0 & 32866.63 \\
\hline 7524.99 & 512080 & 15 & 0 & 9224.1 & 15829.42 & 9242.19 & 24292.46 & 1517.04 & 500565.32 & 0 & 4131.21 \\
\hline 7525.05 & 463900 & 16 & 0 & 9564.4 & 16330.43 & 9902.04 & 26067.45 & 1656.67 & 521309 & 0 & 9162.62 \\
\hline
\end{tabular}




\begin{tabular}{|c|c|c|c|c|c|c|c|c|c|c|c|}
\hline True Depth & XRF tCOUNTS & XRF Live Time & $\mathrm{Ti}(\mathrm{ppm})$ & V (ppm) & $\mathrm{Fe}(\mathrm{ppm})$ & $\mathrm{Ni}(\mathrm{ppm})$ & $\mathrm{Cu}(\mathrm{ppm})$ & $\mathrm{Pb}(\mathrm{ppm})$ & LE (ppm) & $\mathrm{Mg}(\mathrm{ppm})$ & $\mathrm{Cl}(\mathrm{ppm})$ \\
\hline 7525.12 & 428051 & 16 & 0 & 9582.48 & 21778.08 & 10130.34 & 26641.52 & 1708.12 & 470637.08 & 0 & 60015.7 \\
\hline 7525.18 & 616466 & 14 & 0 & 4333.37 & 8429.58 & 3946.14 & 10312.3 & 578.06 & 340066.84 & 0 & 490816.96 \\
\hline 7525.25 & 470522 & 16 & 0 & 7008.63 & 13061.44 & 6643.27 & 17395.08 & 1027.77 & 394559.14 & 0 & 414120.34 \\
\hline 7525.31 & 482479 & 16 & 0 & 6763.43 & 16457.98 & 6451.04 & 16884.33 & 989.59 & 363958.14 & 0 & 451438.29 \\
\hline 7525.38 & 396154 & 17 & 0 & 7068.73 & 13694.09 & 6776.27 & 17754.31 & 1057.69 & 427874.55 & 0 & 361475.76 \\
\hline 7525.45 & 363356 & 17 & 0 & 9189.93 & 19537.12 & 9627.18 & 25337.88 & 1637.12 & 493667.6 & 0 & 57027.95 \\
\hline 7525.51 & 471889 & 16 & 0 & 7371.86 & 12926.66 & 7060.87 & 18496.85 & 1094.65 & 383364.92 & 0 & 389693.8 \\
\hline 7525.58 & 495175 & 15 & 0 & 10057.54 & 17182.32 & 10641.86 & 27995.45 & 1795.9 & 483513.17 & 0 & 0 \\
\hline 7525.64 & 485819 & 16 & 0 & 6434.55 & 19258.92 & 6236.93 & 16322.53 & 952.2 & 371439.47 & 0 & 321142.35 \\
\hline 7525.71 & 368672 & 17 & 0 & 8378.32 & 24209.91 & 8929.12 & 23419.57 & 1484.28 & 482089.26 & 0 & 92856.14 \\
\hline 7525.77 & 336431 & 17 & 607.3 & 9035.57 & 17584.69 & 9507.73 & 25006.33 & 1633.7 & 499277.21 & 0 & 0 \\
\hline 7525.84 & 372477 & 17 & 346.24 & 3917.85 & 10592.14 & 3461.31 & 9001.77 & 506.95 & 405023.69 & 0 & 472697.14 \\
\hline 7525.91 & 310349 & 17 & 1179.54 & 8904.91 & 18104.19 & 9091.45 & 23938.25 & 1559.44 & 535180.25 & 0 & 0 \\
\hline 7525.97 & 365225 & 17 & 704.11 & 8016.78 & 15848.2 & 7663.08 & 20091.38 & 1230.29 & 467742.36 & 0 & 227191.92 \\
\hline 7526.04 & 424053 & 16 & 596.45 & 6981.9 & 13115.78 & 6542.65 & 17140.68 & 1014.08 & 420055.42 & 0 & 388927.46 \\
\hline 7526.10 & 355027 & 17 & 0 & 8232.12 & 23260.57 & 8232.06 & 21539.38 & 1334.23 & 457835.81 & 0 & 224716.33 \\
\hline 7526.17 & 399735 & 16 & 0 & 9823.51 & 18894.9 & 10174.71 & 26737.1 & 1745.66 & 523724.73 & 0 & 0 \\
\hline 7526.23 & 413206 & 16 & 0 & 10222.3 & 23302.11 & 10899.95 & 28683.73 & 1875.05 & 491713.51 & 0 & 30327.57 \\
\hline 7526.30 & 631716 & 14 & 0 & 2471.57 & 6220.73 & 2189.05 & 5697.32 & 312.08 & 422316.1 & 0 & 515983.92 \\
\hline 7526.36 & 598639 & 14 & 1293.61 & 6346.8 & 12450.06 & 4751.22 & 12432.74 & 704.16 & 303500.55 & 0 & 464506.18 \\
\hline 7526.43 & 385067 & 17 & 1581.19 & 9055.86 & 32828.66 & 8931.25 & 23356.89 & 1468.01 & 444052.98 & 0 & 169896.81 \\
\hline 7526.50 & 341917 & 17 & 1839.94 & 10124.32 & 28444.66 & 10833.48 & 28451.17 & 1903.1 & 506882.33 & 0 & 0 \\
\hline 7526.56 & 553589 & 15 & 3884.89 & 8968.1 & 8526.54 & 5520.56 & 14435.01 & 831.66 & 328323.8 & 0 & 498251.5 \\
\hline 7526.63 & 408389 & 16 & 943.33 & 5878.59 & 47385.02 & 6075.44 & 15682.12 & 936.79 & 414995.81 & 0 & 288418.74 \\
\hline 7526.69 & 350194 & 17 & 1394.68 & 6350.67 & 58145.45 & 7495.73 & 19305.27 & 1218.93 & 499028.83 & 0 & 28591.74 \\
\hline 7526.76 & 510337 & 15 & 428.53 & 3198.5 & 18762.19 & 2815.09 & 7189.79 & 406.8 & 404137.94 & 0 & 482242.88 \\
\hline 7526.82 & 374521 & 17 & 708.8 & 5918.98 & 40317.68 & 6010.84 & 15459.24 & 920.9 & 452496.73 & 0 & 305762.67 \\
\hline 7526.89 & 307837 & 17 & 5571.35 & 6221.35 & 33260.96 & 6480.04 & 16689.74 & 1035.46 & 535725.03 & 0 & 157868.67 \\
\hline 7526.96 & 332339 & 17 & 727.5 & 5533.95 & 29005.84 & 5283.49 & 13590.11 & 799.34 & 459239.95 & 0 & 360325.17 \\
\hline 7527.02 & 413486 & 16 & 394.43 & 4584.83 & 27520.57 & 4318.17 & 11107.35 & 646.67 & 416383.3 & 0 & 425074.9 \\
\hline 7527.09 & 370225 & 17 & 539.68 & 4662.49 & 27551.88 & 4345.96 & 11138.99 & 643.34 & 432522.21 & 0 & 402715.32 \\
\hline 7527.15 & 351607 & 17 & 435.08 & 4229.2 & 26068.5 & 3909.4 & 10038.39 & 576.85 & 436693.71 & 0 & 439598.66 \\
\hline 7527.22 & 481290 & 16 & 422.17 & 3170.01 & 15544.76 & 2727.24 & 6986.5 & 393.36 & 387094.9 & 0 & 466515.87 \\
\hline
\end{tabular}




\begin{tabular}{|c|c|c|c|c|c|c|c|c|c|c|c|}
\hline True Depth & XRF tCOUNTS & XRF Live Time & $\mathrm{Ti}(\mathrm{ppm})$ & V (ppm) & $\mathrm{Fe}(\mathrm{ppm})$ & $\mathrm{Ni}(\mathrm{ppm})$ & $\mathrm{Cu}(\mathrm{ppm})$ & $\mathrm{Pb}(\mathrm{ppm})$ & LE (ppm) & $\mathrm{Mg}(\mathrm{ppm})$ & $\mathrm{Cl}(\mathrm{ppm})$ \\
\hline 7527.28 & 487293 & 16 & 0 & 4521.41 & 24790.73 & 4145.33 & 10668.51 & 610.92 & 374865.08 & 0 & 442713.39 \\
\hline 7527.35 & 306173 & 17 & 1033.04 & 6229.49 & 37681.45 & 6614.45 & 17068.21 & 1067.84 & 531571.52 & 0 & 0 \\
\hline 7527.41 & 331972 & 17 & 668.99 & 6569.21 & 37408.03 & 6954.42 & 17938.52 & 1124.65 & 475947.71 & 0 & 0 \\
\hline 7527.48 & 370060 & 17 & 799.89 & 5906.15 & 32904.27 & 5786.37 & 14975.05 & 898.38 & 447022.11 & 0 & 214008.49 \\
\hline 7527.55 & 336765 & 17 & 661.91 & 5809.34 & 34299.06 & 5898.74 & 15167.7 & 917.05 & 475684.89 & 0 & 117930.64 \\
\hline 7527.61 & 357131 & 17 & 756.4 & 6233.82 & 35954.96 & 6376.22 & 16406.11 & 1001.64 & 446294.63 & 0 & 89668.99 \\
\hline 7527.68 & 361058 & 17 & 964.44 & 6077.55 & 31346.64 & 6080.32 & 15657.1 & 950.48 & 445852.67 & 0 & 145801.71 \\
\hline 7527.74 & 349396 & 17 & 1034.76 & 5691.47 & 33560.96 & 5878.34 & 15114.03 & 913.94 & 451804.39 & 0 & 160771.04 \\
\hline 7527.81 & 269628 & 18 & 5061.7 & 4605.3 & 40077.62 & 4940.15 & 12642.64 & 758.55 & 497629.83 & 0 & 120377.12 \\
\hline 7527.87 & 338202 & 17 & 1039.73 & 5591.72 & 29646.23 & 5363.56 & 13811.1 & 820.6 & 460655.15 & 0 & 154701.23 \\
\hline 7527.94 & 297455 & 17 & 1061.63 & 6203.37 & 35981.94 & 6830 & 17717.9 & 1110.45 & 500386.03 & 0 & 0 \\
\hline 7528.01 & 315283 & 17 & 1235.13 & 6455.94 & 35247.35 & 6819.25 & 17628.85 & 1099.04 & 487837.84 & 0 & 11679.16 \\
\hline 7528.07 & 360924 & 17 & 908.84 & 5883.88 & 36102.69 & 6113.46 & 15730.28 & 950.99 & 453006.43 & 0 & 189381.37 \\
\hline 7528.14 & 349713 & 17 & 1230.53 & 5716.84 & 36889.19 & 5980.12 & 15417.96 & 932.96 & 454627.26 & 0 & 123178.28 \\
\hline 7528.20 & 367220 & 17 & 1016.54 & 5825.47 & 37933.84 & 6159.86 & 15886.49 & 952.7 & 436141.69 & 0 & 146203.16 \\
\hline 7528.27 & 363545 & 17 & 1427.83 & 6943.97 & 39346.29 & 7561.94 & 19565.37 & 1225.96 & 451820.75 & 0 & 0 \\
\hline 7528.33 & 361699 & 17 & 1077.14 & 6982.18 & 38875.1 & 7586.25 & 19659.84 & 1232.73 & 443300.49 & 0 & 49266.23 \\
\hline 7528.40 & 469072 & 16 & 504.05 & 4908.66 & 20411.8 & 4554.08 & 11806.51 & 675.11 & 370824.68 & 0 & 350313.89 \\
\hline 7528.46 & 417557 & 16 & 624.92 & 6361.59 & 25550.19 & 6297.54 & 16290.24 & 981.32 & 406491.83 & 50762.04 & 0 \\
\hline 7528.53 & 377792 & 17 & 732.14 & 5779.29 & 29756.77 & 5680.38 & 14690.91 & 875.73 & 441393.56 & 0 & 185469.54 \\
\hline 7528.60 & 409774 & 16 & 704.84 & 5861.43 & 27646.74 & 5649.52 & 14610.2 & 872.04 & 462370.1 & 0 & 69662.02 \\
\hline 7528.66 & 350216 & 17 & 682.05 & 5429.07 & 28025.58 & 5312.21 & 13764.23 & 813.72 & 429801.12 & 0 & 205628.99 \\
\hline 7528.73 & 452587 & 16 & 722.72 & 5145.86 & 27168.69 & 5036.49 & 13028.86 & 760.61 & 391874.8 & 0 & 334537.17 \\
\hline 7528.79 & 362403 & 17 & 1289.27 & 6536.62 & 34538.36 & 6720.81 & 17444.27 & 1071.77 & 453599.22 & 0 & 111561.49 \\
\hline 7528.86 & 398286 & 16 & 8182.36 & 5231.41 & 67681.91 & 7229.95 & 18735.5 & 1164.02 & 485557.02 & 0 & 12694.17 \\
\hline 7528.92 & 320270 & 17 & 943.53 & 6760.59 & 36333.27 & 7326.12 & 19005.56 & 1201.62 & 490055.4 & 0 & 0 \\
\hline 7528.99 & 392416 & 17 & 754.76 & 5602.81 & 28889.73 & 5510.43 & 14236.82 & 843.72 & 428686.06 & 0 & 318755.79 \\
\hline 7529.06 & 329409 & 17 & 1124.43 & 6190.8 & 44220.26 & 7214.82 & 18634.84 & 1171.61 & 488924.47 & 0 & 47573.03 \\
\hline 7529.12 & 280240 & 18 & 1257.1 & 5915.57 & 50127.24 & 7257.65 & 18645.75 & 1172.47 & 486246.8 & 0 & 43753.18 \\
\hline 7529.19 & 494519 & 15 & 781.2 & 4702.35 & 35957.52 & 4802.98 & 12382.03 & 718.91 & 371563.95 & 0 & 441826.93 \\
\hline 7529.25 & 348542 & 17 & 1141.07 & 6094.38 & 56724.6 & 7608.34 & 19753.27 & 1246.97 & 458760.13 & 0 & 7295.99 \\
\hline 7529.32 & 295551 & 17 & 836.94 & 6309.94 & 38605.04 & 6909.9 & 17968.5 & 1117 & 512868.22 & 0 & 164707.09 \\
\hline 7529.38 & 320087 & 17 & 798.85 & 5047.07 & 37833.33 & 5257.7 & 13586.55 & 798.73 & 454728.71 & 0 & 350524.34 \\
\hline
\end{tabular}




\begin{tabular}{|c|c|c|c|c|c|c|c|c|c|c|c|}
\hline True Depth & XRF tCOUNTS & XRF Live Time & $\mathrm{Ti}(\mathrm{ppm})$ & V (ppm) & $\mathrm{Fe}(\mathrm{ppm})$ & $\mathrm{Ni}(\mathrm{ppm})$ & $\mathrm{Cu}(\mathrm{ppm})$ & $\mathrm{Pb}(\mathrm{ppm})$ & LE (ppm) & $\mathrm{Mg}(\mathrm{ppm})$ & $\mathrm{Cl}(\mathrm{ppm})$ \\
\hline 7529.45 & 415794 & 16 & 854.38 & 4934.42 & 33904.48 & 5031.28 & 12960.21 & 753.99 & 401215.11 & 0 & 398745.01 \\
\hline 7529.51 & 530647 & 15 & 0 & 2130.68 & 9944.09 & 1855.38 & 4774.65 & 262.68 & 434008.55 & 0 & 500150.63 \\
\hline 7529.58 & 459789 & 16 & 353.39 & 3463.5 & 19416.1 & 3235.97 & 8348.25 & 471.72 & 396675.75 & 0 & 472360.4 \\
\hline 7529.65 & 443352 & 16 & 0 & 2283.19 & 19129.46 & 2120.67 & 5461.7 & 303.12 & 421770.76 & 0 & 489061.99 \\
\hline 7529.71 & 333208 & 17 & 1118.4 & 5153.47 & 33218.64 & 5128.9 & 13310.98 & 781.73 & 455338.16 & 0 & 359020.56 \\
\hline 7529.78 & 289556 & 18 & 1456.91 & 5188.5 & 31795.88 & 4827.88 & 12477.61 & 719.38 & 399241.04 & 0 & 448066.59 \\
\hline 7529.84 & 256343 & 18 & 2226.26 & 5188.76 & 61407.92 & 5470.18 & 13937.31 & 826.97 & 265412.29 & 73380.11 & 153824.83 \\
\hline 7529.91 & 440944 & 16 & 1039.71 & 5134.12 & 31016.34 & 4871.32 & 12597.7 & 732.38 & 392957.1 & 0 & 457673.6 \\
\hline 7529.97 & 368822 & 17 & 3304.35 & 6235.37 & 35632.56 & 4861.75 & 12501.78 & 730.66 & 423897.43 & 0 & 426457.84 \\
\hline 7530.04 & 262001 & 18 & 1078.55 & 6417.97 & 35676.83 & 6862.78 & 17713.96 & 1090.64 & 439696.59 & 0 & 90832.11 \\
\hline 7530.10 & 376478 & 17 & 1297.36 & 5979.3 & 48261.02 & 6736.48 & 17377.21 & 1067.53 & 425692.51 & 0 & 126577.6 \\
\hline 7530.17 & 339122 & 17 & 1595.32 & 6645.14 & 40373.52 & 7274.59 & 18874.25 & 1179.37 & 467305.48 & 0 & 135178.23 \\
\hline 7530.24 & 338100 & 17 & 11657.61 & 14008.36 & 27351.91 & 7850.89 & 20486.48 & 1248.13 & 409285.01 & 0 & 367895.95 \\
\hline 7530.30 & 367693 & 17 & 44804.43 & 33003.77 & 46855.51 & 10361.31 & 27175.02 & 1727.35 & 370642.31 & 68266.02 & 160104.46 \\
\hline 7530.37 & 550849 & 15 & 3524.38 & 4991.07 & 10363.34 & 2610.18 & 6749.35 & 378.83 & 418552.62 & 0 & 495963.23 \\
\hline 7530.43 & 355231 & 17 & 1674.27 & 6609.09 & 32289.33 & 6535.64 & 16984.49 & 1031.57 & 442847.58 & 0 & 184066.89 \\
\hline 7530.50 & 399852 & 16 & 4554.14 & 9548.21 & 25635.68 & 7274.59 & 18985.84 & 1156.77 & 454386.2 & 0 & 52050.97 \\
\hline 7530.56 & 399351 & 16 & 2414.68 & 7111.52 & 26181.85 & 5881.83 & 15265.4 & 900.15 & 408461.63 & 0 & 344430.19 \\
\hline 7530.63 & 443799 & 16 & 554.59 & 5254.67 & 23027.57 & 4938.07 & 12794.24 & 740.99 & 383454.42 & 0 & 402341.61 \\
\hline 7530.70 & 436174 & 16 & 2814.12 & 7010.58 & 25788.12 & 5676.95 & 14722.97 & 865.91 & 384290.99 & 0 & 425371.5 \\
\hline 7530.76 & 453535 & 16 & 146033.42 & 91954.93 & 40908.33 & 19918.34 & 52686.72 & 3588.59 & 284408.36 & 0 & 137048.1 \\
\hline 7530.83 & 454042 & 16 & 1945.37 & 4567.79 & 15023.37 & 3333.93 & 8607.91 & 486.23 & 397217.38 & 0 & 487168.1 \\
\hline 7530.89 & 453830 & 16 & 135018.76 & 83452.18 & 44714.12 & 18561.33 & 49168.41 & 3354.92 & 327707.78 & 0 & 0 \\
\hline 7530.96 & 339725 & 17 & 1733.98 & 7114.74 & 39824.97 & 7804.31 & 20275.13 & 1289.66 & 457480.02 & 0 & 46041.69 \\
\hline 7531.02 & 633272 & 14 & 19670.83 & 15536.02 & 18805.3 & 3537.27 & 9232.68 & 516.93 & 330088.21 & 0 & 583413.03 \\
\hline 7531.09 & 364493 & 17 & 1614.74 & 5810.27 & 24996.88 & 4838.15 & 12551.74 & 729.54 & 439805.59 & 0 & 400696.65 \\
\hline 7531.15 & 263056 & 18 & 2536.15 & 7547.07 & 30005 & 7050.95 & 18308.3 & 1150.4 & 539177.15 & 0 & 73327.16 \\
\hline 7531.22 & 329758 & 17 & 1775.34 & 6894.11 & 27873.58 & 6429.01 & 16681.75 & 1016.48 & 466412.95 & 0 & 192545.91 \\
\hline 7531.29 & 436727 & 16 & 4641.47 & 9736.36 & 24133.48 & 6902.95 & 17954.42 & 1068.1 & 360144.38 & 0 & 422296.8 \\
\hline 7531.35 & 436543 & 16 & 87650.97 & 58312.82 & 50061.01 & 17300.47 & 45702.57 & 3086.77 & 316154.48 & 0 & 84837.34 \\
\hline 7531.42 & 485534 & 16 & 6849.26 & 9250.57 & 14236.35 & 4650.46 & 12080.68 & 696.34 & 356858.25 & 0 & 500565.63 \\
\hline 7531.48 & 352685 & 17 & 19682.86 & 18284.87 & 31009.53 & 8554.06 & 22340.4 & 1381.45 & 420953.09 & 0 & 339099.98 \\
\hline 7531.55 & 364774 & 17 & 24697.3 & 21252.01 & 22068.74 & 7355.29 & 19231.49 & 1149.11 & 378631.22 & 0 & 448492.45 \\
\hline
\end{tabular}




\begin{tabular}{|c|c|c|c|c|c|c|c|c|c|c|c|}
\hline True Depth & XRF tCOUNTS & XRF Live Time & $\mathrm{Ti}(\mathrm{ppm})$ & V (ppm) & $\mathrm{Fe}(\mathrm{ppm})$ & $\mathrm{Ni}(\mathrm{ppm})$ & $\mathrm{Cu}(\mathrm{ppm})$ & $\mathrm{Pb}(\mathrm{ppm})$ & LE (ppm) & $\mathrm{Mg}(\mathrm{ppm})$ & $\mathrm{Cl}(\mathrm{ppm})$ \\
\hline 7531.61 & 362992 & 17 & 4444.29 & 8380.93 & 25029.5 & 5858.59 & 15242.71 & 896.96 & 423755.01 & 0 & 406689.3 \\
\hline 7531.68 & 467359 & 16 & 0 & 2769.77 & 11723.16 & 2457.51 & 6344.85 & 353.31 & 412832.03 & 0 & 483800.35 \\
\hline 7531.75 & 304817 & 17 & 702.05 & 5395.44 & 25042.37 & 5136.85 & 13332.45 & 788.7 & 471119.74 & 0 & 303054.37 \\
\hline 7531.81 & 430795 & 16 & 0 & 2349.44 & 9527.37 & 2017.58 & 5200.69 & 286.16 & 437214.73 & 0 & 486304.55 \\
\hline 7531.88 & 254710 & 18 & 2150.14 & 6577.16 & 27739.27 & 6173.45 & 16074.69 & 978.67 & 584280.14 & 0 & 153780.34 \\
\hline 7531.94 & 512970 & 15 & 913.77 & 2719.01 & 12402.19 & 2276.23 & 5884.68 & 326.67 & 405283.51 & 0 & 490815.04 \\
\hline 7532.01 & 306823 & 17 & 2542.2 & 6691.59 & 28528.85 & 6071.38 & 15786.65 & 948.87 & 484128.64 & 0 & 224611.91 \\
\hline 7532.07 & 298107 & 18 & 4614.78 & 8238.36 & 30163.6 & 6751.98 & 17640.76 & 1095.7 & 540988.85 & 0 & 96787.68 \\
\hline 7532.14 & 317668 & 17 & 1513.37 & 5379.73 & 20930.97 & 4685.14 & 12135.9 & 702.34 & 431950.86 & 0 & 259967.89 \\
\hline 7532.20 & 372300 & 17 & 24060.17 & 20572.03 & 23249.62 & 6804.72 & 17813.69 & 1054.97 & 407094.02 & 0 & 252508.9 \\
\hline 7532.27 & 457665 & 16 & 0 & 5777.23 & 21590.55 & 5552.04 & 14437.4 & 858.58 & 469940.11 & 0 & 45709.75 \\
\hline 7532.34 & 464291 & 16 & 42320.26 & 33909.49 & 27264.33 & 11750.36 & 30993.07 & 1955.24 & 364145.02 & 0 & 96598.67 \\
\hline 7532.40 & 519335 & 15 & 0 & 4419.36 & 17095.75 & 3818.77 & 9954.08 & 567.64 & 388622.79 & 0 & 210290.69 \\
\hline 7532.47 & 447598 & 16 & 0 & 4903.93 & 21257.63 & 4694.73 & 12219.15 & 709.27 & 448379.83 & 0 & 96289.55 \\
\hline 7532.53 & 394404 & 17 & 605.73 & 4116.39 & 18238.11 & 3881.66 & 10049.97 & 571.82 & 386279.95 & 0 & 414766.03 \\
\hline 7532.60 & 422578 & 16 & 467.75 & 5879.87 & 25576.37 & 5796.86 & 15104.54 & 892.52 & 403675.56 & 0 & 241046.44 \\
\hline 7532.66 & 384918 & 17 & 517.45 & 6207.72 & 30928.88 & 6332.49 & 16454.16 & 987.15 & 432225.94 & 0 & 127454.1 \\
\hline 7532.73 & 507656 & 15 & 0 & 3059.46 & 14077.49 & 2728.54 & 7051.89 & 392.88 & 386944.05 & 0 & 486510.67 \\
\hline 7532.80 & 352194 & 17 & 631.55 & 6364.85 & 30413.76 & 6558.91 & 17005.99 & 1035.78 & 464305.99 & 0 & 68207.38 \\
\hline 7532.86 & 552054 & 15 & 82057.96 & 52535.44 & 183513.43 & 14683.39 & 38957.84 & 2452.52 & 222877.92 & 0 & 160296.17 \\
\hline 7532.93 & 378869 & 17 & 9555.53 & 5455.28 & 29205.2 & 5903.65 & 15346.66 & 927.19 & 464971.89 & 0 & 123800.96 \\
\hline 7532.99 & 516066 & 15 & 0 & 2948.16 & 12042.82 & 2600.29 & 6714.71 & 374.3 & 395170.45 & 0 & 488720.07 \\
\hline 7533.06 & 419392 & 16 & 564.47 & 4974.29 & 25835.52 & 4761.66 & 12350.26 & 722.08 & 403955.79 & 0 & 350214.27 \\
\hline 7533.12 & 449683 & 16 & 0 & 4751.23 & 22057.06 & 4408.12 & 11422.14 & 652.81 & 375068.76 & 0 & 422990.38 \\
\hline 7533.19 & 383749 & 17 & 733.66 & 5778.5 & 28400.29 & 5671.56 & 14686.34 & 869.5 & 408451.63 & 0 & 246732.04 \\
\hline 7533.25 & 465276 & 16 & 0 & 5284.64 & 25531.67 & 5298.85 & 13731.76 & 799.4 & 363615.43 & 0 & 363797.77 \\
\hline 7533.32 & 412397 & 16 & 722.57 & 6255.7 & 36250.47 & 6527.01 & 16982.87 & 1022.97 & 420363.11 & 0 & 100667.13 \\
\hline 7533.39 & 588372 & 14 & 0 & 1789.54 & 7121.18 & 1510.76 & 3887.56 & 210.36 & 432738.07 & 0 & 504206.3 \\
\hline 7533.45 & 381325 & 17 & 918.55 & 6244.33 & 32431.67 & 6463.12 & 16775.85 & 1022.32 & 465256.6 & 0 & 54710.71 \\
\hline 7533.52 & 393983 & 17 & 640.26 & 5757.31 & 29427.94 & 5696.34 & 14729.4 & 875.58 & 434444.36 & 0 & 211381.88 \\
\hline 7533.58 & 508638 & 15 & 0 & 2783.94 & 11535.23 & 2471.58 & 6391.03 & 354.77 & 393218.43 & 0 & 487168.89 \\
\hline 7533.65 & 372589 & 17 & 636.86 & 5876.8 & 36289.52 & 5961.47 & 15432.02 & 926.19 & 414339 & 0 & 138909.09 \\
\hline 7533.71 & 501844 & 15 & 0 & 4793.01 & 21693.52 & 4430.27 & 11474.27 & 653.81 & 356334.41 & 0 & 419860.21 \\
\hline
\end{tabular}




\begin{tabular}{|c|c|c|c|c|c|c|c|c|c|c|c|}
\hline True Depth & XRF tCOUNTS & XRF Live Time & $\mathrm{Ti}(\mathrm{ppm})$ & $\mathrm{V}(\mathrm{ppm})$ & $\mathrm{Fe}(\mathrm{ppm})$ & $\mathrm{Ni}(\mathrm{ppm})$ & $\mathrm{Cu}(\mathrm{ppm})$ & $\mathrm{Pb}(\mathrm{ppm})$ & $\mathrm{LE}(\mathrm{ppm})$ & $\mathrm{Mg}(\mathrm{ppm})$ & $\mathrm{Cl}(\mathrm{ppm})$ \\
\hline 7533.78 & 450388 & 16 & 470.68 & 5283.26 & 25042.7 & 5109.69 & 13254.46 & 774.01 & 369422.48 & 0 & 334224.45 \\
\hline 7533.85 & 371307 & 17 & 0 & 6812 & 35481.85 & 7153.14 & 18565.54 & 1152.35 & 445597.95 & 0 & 0 \\
\hline 7533.91 & 304571 & 17 & 853.58 & 4856.28 & 28219.74 & 4720.5 & 12240.93 & 712.22 & 410926.31 & 0 & 337852.3 \\
\hline 7533.98 & 351580 & 17 & 1122.78 & 6372.53 & 36633.22 & 6916.84 & 17922.71 & 1114.2 & 480939.11 & 0 & 0 \\
\hline 7534.04 & 420587 & 16 & 569.9 & 4903.83 & 29458.46 & 4801.94 & 12394.94 & 722.86 & 409312.73 & 0 & 321446.63 \\
\hline 7534.11 & 389112 & 17 & 618.67 & 5930.1 & 34987.88 & 6252.29 & 16225.46 & 991.53 & 494227.27 & 0 & 0 \\
\hline 7534.17 & 453300 & 16 & 0 & 5364.22 & 29388.75 & 5335.46 & 13866.47 & 822.27 & 481986.53 & 0 & 0 \\
\hline 7534.24 & 506902 & 15 & 0 & 5221.73 & 24981.42 & 4937.36 & 12895.08 & 748.8 & 443014.27 & 0 & 53311.7 \\
\hline 7534.30 & 479480 & 16 & 0 & 5451.56 & 26182.91 & 5316.18 & 13780.61 & 806.67 & 468224.03 & 0 & 56456.27 \\
\hline 7534.37 & 463943 & 16 & 0 & 5148.85 & 22748.27 & 4940.72 & 12801.52 & 744.07 & 402802.02 & 0 & 248611.58 \\
\hline 7534.44 & 405999 & 16 & 0 & 6222.86 & 31808.25 & 6424.25 & 16625.45 & 1016.23 & 475868.21 & 0 & 0 \\
\hline 7534.50 & 361622 & 17 & 822.81 & 5655.29 & 30629.2 & 5814.65 & 15072.45 & 906.58 & 488495.21 & 0 & 134148.93 \\
\hline 7534.57 & 400168 & 16 & 505.23 & 5804.31 & 29171.38 & 5795.33 & 15046.26 & 896.46 & 436334.6 & 0 & 183790.86 \\
\hline 7534.63 & 381511 & 17 & 849.77 & 6595.59 & 32329.67 & 7002.66 & 18210.68 & 1126.39 & 474347.19 & 0 & 45219.12 \\
\hline 7534.70 & 363123 & 17 & 844.64 & 5713.89 & 35915.08 & 5851.01 & 15213.64 & 912.05 & 457446.52 & 0 & 161138.54 \\
\hline 7534.76 & 351280 & 17 & 706.46 & 6316.16 & 33931.54 & 6785.8 & 17671.83 & 1081.91 & 465550.38 & 0 & 12108.21 \\
\hline 7534.83 & 366819 & 17 & 720.89 & 6073.73 & 32533.33 & 6280.39 & 16315.43 & 979.47 & 463784.38 & 0 & 141080.4 \\
\hline 7534.90 & 523090 & 15 & 0 & 3399.41 & 14609.96 & 3060.24 & 7932.86 & 446.05 & 368504.5 & 0 & 476949.67 \\
\hline 7534.96 & 416528 & 16 & 580.84 & 4813.18 & 24950.79 & 4772.81 & 12363.81 & 717.48 & 410666.62 & 0 & 396605.99 \\
\hline 7535.03 & 391302 & 17 & 687.5 & 4682.85 & 26795.81 & 4625.23 & 11956.86 & 695.55 & 419431.29 & 0 & 391426.21 \\
\hline 7535.09 & 332813 & 17 & 963.68 & 4886.25 & 30767.11 & 5038.67 & 13030.45 & 764.98 & 461371.43 & 0 & 307134.33 \\
\hline 7535.16 & 259650 & 18 & 1020.03 & 5451.27 & 38889.03 & 5881.95 & 15247.41 & 925.99 & 468216.39 & 0 & 109353.07 \\
\hline 7535.22 & 342677 & 17 & 1172.65 & 5880.27 & 38923.45 & 6370.16 & 16471.99 & 1004.79 & 453472.74 & 0 & 100159.28 \\
\hline 7535.29 & 324701 & 17 & 1154.76 & 6034.8 & 38805.51 & 6620.56 & 17156.89 & 1058.55 & 479891.85 & 0 & 48563.25 \\
\hline 7535.35 & 291522 & 18 & 777.15 & 5923.43 & 39556.93 & 6727.46 & 17397.96 & 1088.95 & 517896.18 & 0 & 0 \\
\hline 7535.42 & 438341 & 16 & 713.84 & 4811.66 & 30389.35 & 4639.95 & 11955.96 & 691.06 & 394503.43 & 0 & 386539.84 \\
\hline 7535.49 & 431299 & 16 & 444.84 & 4425.9 & 25351.1 & 4248.54 & 10947.91 & 627.51 & 412001.5 & 0 & 438719.17 \\
\hline 7535.55 & 335596 & 17 & 1211.79 & 6183.12 & 44060.76 & 7263.15 & 18764.09 & 1170.03 & 447376.96 & 0 & 16066.83 \\
\hline 7535.62 & 295975 & 17 & 1162.74 & 6282.68 & 38417.96 & 7203.35 & 18665.24 & 1180.68 & 490599.95 & 0 & 0 \\
\hline 7535.68 & 374276 & 17 & 489.46 & 5551.23 & 31034.71 & 5606.18 & 14516.55 & 864.44 & 423757.22 & 0 & 292817.66 \\
\hline 7535.75 & 376888 & 17 & 426.56 & 5221.78 & 28146.81 & 5031.97 & 13052.55 & 766.96 & 441458.23 & 0 & 344896.77 \\
\hline 7535.81 & 601458 & 14 & 0 & 1313.32 & 5751.19 & 1103.24 & 2832.8 & 152.29 & 459713.91 & 0 & 502840.2 \\
\hline 7535.88 & 221840 & 18 & 762.22 & 5276.36 & 34550.77 & 5656.34 & 14605.14 & 890.91 & 554940.72 & 0 & 208546.31 \\
\hline
\end{tabular}




\begin{tabular}{|c|c|c|c|c|c|c|c|c|c|c|c|}
\hline True Depth & XRF tCOUNTS & XRF Live Time & $\mathrm{Ti}(\mathrm{ppm})$ & $\mathrm{V}(\mathrm{ppm})$ & $\mathrm{Fe}(\mathrm{ppm})$ & $\mathrm{Ni}(\mathrm{ppm})$ & $\mathrm{Cu}(\mathrm{ppm})$ & $\mathrm{Pb}(\mathrm{ppm})$ & $\mathrm{LE}(\mathrm{ppm})$ & $\mathrm{Mg}(\mathrm{ppm})$ & $\mathrm{Cl}(\mathrm{ppm})$ \\
\hline 7535.94 & 297170 & 18 & 0 & 860.7 & 4245.18 & 685 & 1763.4 & 92.42 & 453301.17 & 0 & 511784.15 \\
\hline 7536.01 & 434922 & 16 & 497.54 & 4278.5 & 28554.12 & 4111.71 & 10601.21 & 607.94 & 408337.18 & 0 & 429903.42 \\
\hline 7536.08 & 512604 & 15 & 0 & 2923.69 & 18109.41 & 2659.63 & 6816.69 & 383.42 & 382248.51 & 0 & 482591.66 \\
\hline 7536.14 & 323625 & 17 & 979.6 & 5805.24 & 38576.44 & 6232.7 & 16102.67 & 984.48 & 481602.84 & 0 & 128141.04 \\
\hline 7536.21 & 253259 & 18 & 753.41 & 4803.3 & 33836.71 & 4989.6 & 12875.95 & 767.9 & 515939.11 & 0 & 239898.09 \\
\hline 7536.27 & 303368 & 17 & 827.65 & 5260.09 & 29404.04 & 5322.99 & 13744.75 & 815.31 & 483485.94 & 0 & 280162.86 \\
\hline 7536.34 & 309048 & 17 & 562.98 & 5556.24 & 28432.72 & 5618.46 & 14513.14 & 870.7 & 477216.2 & 0 & 256940.64 \\
\hline 7536.40 & 318970 & 17 & 1014.27 & 6084.97 & 33150.73 & 6281.01 & 16287.93 & 991.88 & 462681.08 & 0 & 141069.17 \\
\hline 7536.47 & 329687 & 17 & 898 & 6007.27 & 34957.33 & 6442.24 & 16718.35 & 1021.78 & 455276.54 & 0 & 86059.36 \\
\hline 7536.54 & 445441 & 16 & 383.13 & 4462.14 & 26054.46 & 4315.95 & 11175.99 & 637.22 & 396614.38 & 0 & 425704.73 \\
\hline 7536.60 & 595190 & 14 & 0 & 1293.52 & 6715.22 & 1113.35 & 2854.22 & 154.13 & 448652.53 & 0 & 503562.17 \\
\hline 7536.67 & 511303 & 15 & 303.46 & 2145.67 & 20209.08 & 1915.23 & 4913.6 & 273.29 & 406005.64 & 0 & 494856.26 \\
\hline 7536.73 & 370496 & 17 & 563.66 & 5114.17 & 36447.32 & 5244.33 & 13535.64 & 794.92 & 434023.44 & 0 & 303464.79 \\
\hline 7536.80 & 300456 & 17 & 523.22 & 6081.1 & 36492.27 & 6695.61 & 17332.02 & 1079.12 & 504112.01 & 0 & 0 \\
\hline 7536.86 & 335831 & 17 & 765.79 & 5182.63 & 31903.85 & 5299.87 & 13674.36 & 805.84 & 459664.99 & 0 & 257844.1 \\
\hline 7536.93 & 431698 & 16 & 490.04 & 4851.85 & 26310.92 & 4630.46 & 11990.51 & 691.65 & 382051.31 & 0 & 379238.89 \\
\hline 7536.99 & 336816 & 17 & 706.94 & 5728.16 & 32916.48 & 5969.08 & 15466.28 & 938.17 & 445194.23 & 0 & 95753.82 \\
\hline 7537.06 & 321527 & 17 & 653.27 & 6013.91 & 36932.71 & 6505.67 & 16806.24 & 1034.17 & 468905.69 & 0 & 105384.62 \\
\hline 7537.13 & 398077 & 17 & 388.2 & 2786.33 & 14769.03 & 2440.33 & 6279.03 & 348.06 & 406768.94 & 0 & 479788.06 \\
\hline 7537.19 & 308552 & 17 & 1064.43 & 5848.05 & 41125.07 & 6637.53 & 17140.65 & 1062.38 & 479494.65 & 0 & 0 \\
\hline 7537.26 & 352906 & 17 & 740.33 & 5123.31 & 29694.89 & 5082.51 & 13148.09 & 774.73 & 443215.41 & 0 & 280358.95 \\
\hline 7537.32 & 313851 & 17 & 904.77 & 5320.88 & 33180.43 & 5595.02 & 14480.71 & 873.24 & 473776.02 & 0 & 187102.54 \\
\hline 7537.39 & 360646 & 17 & 0 & 4931.23 & 29383.97 & 4781.3 & 12306.79 & 716.37 & 450028.65 & 0 & 388415.48 \\
\hline 7537.45 & 361836 & 17 & 842.73 & 4653.36 & 68440.28 & 5762.37 & 14896.28 & 905.56 & 415310.14 & 0 & 69002.97 \\
\hline 7537.52 & 465048 & 16 & 0 & 4490.73 & 24139.96 & 4173.2 & 10790.03 & 618.79 & 380310.78 & 0 & 448091.93 \\
\hline 7537.59 & 339631 & 17 & 824.68 & 5687.29 & 36993.52 & 6093.43 & 15687.78 & 959.34 & 453108 & 0 & 56880.59 \\
\hline 7537.65 & 345738 & 17 & 1074.19 & 5394.93 & 37241.24 & 5749.68 & 14879.75 & 894.37 & 454937.95 & 0 & 152297.66 \\
\hline 7537.72 & 471118 & 16 & 585.93 & 4243.02 & 26741.22 & 4035.69 & 10404.17 & 596.49 & 385677.88 & 0 & 435689.99 \\
\hline 7537.78 & 375908 & 17 & 725.4 & 5437.51 & 38281.16 & 5616.02 & 14491.13 & 862.82 & 423859.43 & 0 & 190416.29 \\
\hline 7537.85 & 344122 & 17 & 774.14 & 5336.83 & 36076.91 & 5448.18 & 14112.25 & 840.62 & 443326.04 & 0 & 201079.14 \\
\hline 7537.91 & 310216 & 17 & 1034.52 & 5653.3 & 52416.19 & 6674.43 & 17277.12 & 1072.62 & 424488.43 & 51488.25 & 0 \\
\hline 7537.98 & 179536 & 19 & 1008.73 & 4933.77 & 27287.87 & 4823.25 & 12524.96 & 757.54 & 561182.34 & 0 & 30047.65 \\
\hline 7538.04 & 347000 & 17 & 1199.55 & 5931.6 & 39764.77 & 6295.38 & 16318.77 & 1000.69 & 448464.7 & 0 & 38560.49 \\
\hline
\end{tabular}




\begin{tabular}{|c|c|c|c|c|c|c|c|c|c|c|c|}
\hline True Depth & XRF tCOUNTS & XRF Live Time & $\mathrm{Ti}(\mathrm{ppm})$ & V (ppm) & $\mathrm{Fe}(\mathrm{ppm})$ & $\mathrm{Ni}(\mathrm{ppm})$ & $\mathrm{Cu}(\mathrm{ppm})$ & $\mathrm{Pb}(\mathrm{ppm})$ & LE (ppm) & $\mathrm{Mg}(\mathrm{ppm})$ & $\mathrm{Cl}(\mathrm{ppm})$ \\
\hline 7538.11 & 422081 & 16 & 659.97 & 4502.49 & 28672.31 & 4340.12 & 11210.58 & 647.23 & 416136.59 & 0 & 380745.6 \\
\hline 7538.18 & 348365 & 17 & 1314.35 & 5460.39 & 40190.6 & 5551 & 14403.52 & 857.35 & 449718.6 & 0 & 204542.21 \\
\hline 7538.24 & 310488 & 17 & 993.83 & 6046.4 & 39527.06 & 6602.05 & 17052.05 & 1057.69 & 510742.66 & 0 & 73832.94 \\
\hline 7538.31 & 328845 & 17 & 1478.53 & 4799.1 & 79437.13 & 5921.24 & 15360.53 & 925.38 & 364855.98 & 0 & 79451.65 \\
\hline 7538.37 & 428090 & 16 & 953.24 & 5360.52 & 49127.98 & 5504.5 & 14219.76 & 836.32 & 373326.12 & 0 & 310269.23 \\
\hline 7538.44 & 365350 & 17 & 834.91 & 6039.02 & 42258.25 & 6655.35 & 17193.47 & 1055.15 & 434031.49 & 0 & 77176.38 \\
\hline 7538.50 & 355659 & 17 & 578.69 & 6008.46 & 43179.33 & 6777.15 & 17475.28 & 1080.13 & 464453.44 & 0 & 52979.55 \\
\hline 7538.57 & 440424 & 16 & 0 & 4987.43 & 31237.11 & 4974.2 & 12770.16 & 743.1 & 413512.9 & 0 & 385642.25 \\
\hline 7538.64 & 340797 & 17 & 904.2 & 5698.03 & 35443.72 & 5999.61 & 15383.97 & 929.3 & 477392.74 & 0 & 163421.11 \\
\hline 7538.70 & 334267 & 17 & 962.15 & 6200.6 & 40836.53 & 6814.48 & 17490.19 & 1081.7 & 472606.65 & 0 & 24044.51 \\
\hline 7538.77 & 390751 & 17 & 818.44 & 5487.49 & 52938.46 & 5815.05 & 14923.72 & 882.49 & 406818.87 & 0 & 285631.56 \\
\hline 7538.83 & 386713 & 17 & 512.61 & 5656.89 & 36330.51 & 5614.03 & 14376.17 & 853.92 & 445167.74 & 0 & 285163.59 \\
\hline 7538.90 & 357471 & 17 & 1114.9 & 6147.46 & 41961.99 & 6428.4 & 16456.33 & 1001.41 & 453013.15 & 0 & 161716.65 \\
\hline 7538.96 & 379035 & 17 & 5025.22 & 5869.71 & 43928.9 & 6236.64 & 15978.77 & 979.3 & 465262.53 & 0 & 160034.84 \\
\hline 7539.03 & 410788 & 16 & 727.12 & 5188.07 & 38940.94 & 5028.42 & 12881.48 & 756.02 & 430268.09 & 0 & 372275.58 \\
\hline 7539.09 & 420478 & 16 & 789.22 & 5010.92 & 39512.59 & 5005.04 & 12751.42 & 750.71 & 411809.05 & 0 & 361757.47 \\
\hline 7539.16 & 332743 & 17 & 1147.61 & 6169.73 & 40051.33 & 6524.18 & 16662.63 & 1029.37 & 488709.43 & 0 & 81433.71 \\
\hline 7539.23 & 338174 & 17 & 1418.93 & 4535.98 & 79207.87 & 5680.05 & 14461.42 & 862.02 & 394010.85 & 0 & 161191.84 \\
\hline 7539.29 & 424909 & 16 & 431.38 & 5759.47 & 34400.42 & 5651.75 & 14494.9 & 860.24 & 412029.09 & 0 & 318515.14 \\
\hline 7539.36 & 332403 & 17 & 1181.68 & 6418.05 & 48248.1 & 7238.05 & 18586.98 & 1160.41 & 476474.55 & 0 & 36879.97 \\
\hline 7539.42 & 416316 & 16 & 473.17 & 4700.39 & 37217.4 & 4601.34 & 11746.79 & 680.7 & 401183.54 & 0 & 438404.5 \\
\hline 7539.49 & 390478 & 17 & 628.56 & 5446.98 & 38842.49 & 5250.41 & 13455.41 & 787.73 & 421021.07 & 0 & 357951.08 \\
\hline 7539.55 & 323170 & 17 & 687.87 & 4495.07 & 29890.73 & 4088.06 & 10408.49 & 605.3 & 449924.14 & 0 & 416081.03 \\
\hline 7539.62 & 475293 & 16 & 727.58 & 5036.89 & 29840.75 & 4783.5 & 12275.89 & 711.91 & 390250.02 & 0 & 452536.56 \\
\hline 7539.69 & 384045 & 17 & 936.14 & 5891.56 & 33576.55 & 5947.56 & 15363.92 & 923.42 & 416990.73 & 0 & 224321.64 \\
\hline 7539.75 & 416779 & 16 & 543.52 & 4863.52 & 29089.94 & 4687.02 & 12045.67 & 699.52 & 399071.51 & 0 & 377560.73 \\
\hline 7539.82 & 360306 & 17 & 831.21 & 4779.54 & 31139.13 & 4627.91 & 11916.73 & 688.87 & 383217.38 & 0 & 369852.95 \\
\hline 7539.88 & 346952 & 17 & 984.41 & 6414.27 & 44347.76 & 7182.69 & 18516.68 & 1159.19 & 429860.1 & 0 & 0 \\
\hline 7539.95 & 175871 & 19 & 1134.46 & 4290.07 & 24354.41 & 4187.95 & 10823.47 & 615.15 & 366068.17 & 0 & 293674.1 \\
\hline 7540.01 & 533369 & 15 & 0 & 3337.75 & 19574.63 & 3018.64 & 7750.54 & 438.31 & 370268.36 & 0 & 484134.69 \\
\hline 7540.08 & 350719 & 17 & 856.09 & 5841.12 & 48724.38 & 6755.37 & 17327.8 & 1070.94 & 438972.67 & 0 & 38233.53 \\
\hline 7540.14 & 519779 & 15 & 340.62 & 2925.57 & 19038.22 & 2643.85 & 6755.95 & 377.63 & 387670.95 & 0 & 487243.57 \\
\hline 7540.21 & 419986 & 16 & 684.21 & 4305.83 & 42822.92 & 4332.24 & 11141.14 & 648.57 & 385727.98 & 0 & 410760.93 \\
\hline
\end{tabular}




\begin{tabular}{|c|c|c|c|c|c|c|c|c|c|c|c|}
\hline True Depth & XRF tCOUNTS & XRF Live Time & $\mathrm{Ti}(\mathrm{ppm})$ & V (ppm) & $\mathrm{Fe}(\mathrm{ppm})$ & $\mathrm{Ni}(\mathrm{ppm})$ & $\mathrm{Cu}(\mathrm{ppm})$ & $\mathrm{Pb}(\mathrm{ppm})$ & LE (ppm) & $\mathrm{Mg}(\mathrm{ppm})$ & $\mathrm{Cl}(\mathrm{ppm})$ \\
\hline 7540.28 & 447332 & 16 & 0 & 3221.66 & 21496.69 & 2819.62 & 7211.25 & 407.86 & 382960.36 & 0 & 476299.73 \\
\hline 7540.34 & 358579 & 17 & 978.58 & 6310.51 & 42395.51 & 6951.05 & 17917.81 & 1117.88 & 444001.44 & 0 & 7210.36 \\
\hline 7540.41 & 409113 & 16 & 559.16 & 5581.24 & 35377.71 & 5682.44 & 14677.34 & 873.32 & 402839.4 & 0 & 275243.35 \\
\hline 7540.47 & 586321 & 14 & 0 & 2301.98 & 22280.77 & 2086.72 & 5355.19 & 297.25 & 380187.78 & 0 & 506034.11 \\
\hline 7540.54 & 665217 & 13 & 0 & 1074.22 & 6157.6 & 875.33 & 2228.49 & 120.31 & 458614.75 & 0 & 510448.42 \\
\hline 7540.60 & 277124 & 18 & 1211.79 & 6275.81 & 17981.74 & 5143.24 & 13407.66 & 764.09 & 383441.06 & 0 & 351591.18 \\
\hline 7540.67 & 383519 & 17 & 0 & 2868.07 & 14643.06 & 2562.12 & 6566.94 & 369.09 & 418874.97 & 0 & 476625.62 \\
\hline 7540.73 & 410896 & 16 & 423.99 & 1846.67 & 26497.93 & 1533.81 & 3983.84 & 219.67 & 425290.07 & 0 & 510925.37 \\
\hline 7540.80 & 610446 & 14 & 0 & 2391.09 & 16448.51 & 2186.68 & 5589.4 & 309.89 & 396995.86 & 0 & 515587.73 \\
\hline 7540.87 & 313227 & 17 & 0 & 4447.73 & 35876 & 4423.8 & 11324.87 & 650.73 & 378722.38 & 0 & 429871.98 \\
\hline 7540.93 & 603410 & 14 & 0 & 2786.41 & 43515.69 & 2710.04 & 6916.8 & 387.94 & 349033.71 & 0 & 502443.76 \\
\hline 7541.00 & 266998 & 18 & 3030.51 & 6541.7 & 60488.89 & 5638.57 & 14346.78 & 854.73 & 420010.91 & 62683.26 & 241629.3 \\
\hline 7541.06 & 272582 & 18 & 1326.34 & 5993.34 & 54327.52 & 5861.61 & 14916.32 & 896.08 & 488774.47 & 0 & 280394.02 \\
\hline 7541.13 & 284771 & 18 & 608.75 & 6229.59 & 63154.55 & 7184.49 & 18250.58 & 1149.94 & 530936.39 & 0 & 65461.31 \\
\hline 7541.19 & 411888 & 16 & 720.44 & 5289.36 & 52475.14 & 5386.8 & 13580.11 & 797.49 & 408362.75 & 0 & 369429.84 \\
\hline 7541.26 & 284665 & 18 & 1457.7 & 6103.5 & 61243.79 & 6562.79 & 16646.03 & 1012.01 & 475580.8 & 0 & 208661.59 \\
\hline 7541.33 & 392343 & 17 & 710.89 & 5024.74 & 58127.92 & 4984.83 & 12649.45 & 742.09 & 395220.68 & 0 & 386997.46 \\
\hline 7541.39 & 288663 & 18 & 2939.64 & 6836.49 & 72263.88 & 6824.32 & 17352.03 & 1062.81 & 483546.14 & 0 & 167083.29 \\
\hline 7541.46 & 307316 & 17 & 0 & 5365 & 56438.6 & 5260.16 & 13423.19 & 793.58 & 373147.81 & 70486.59 & 368928.56 \\
\hline 7541.52 & 309772 & 17 & 621 & 4483.29 & 64022.3 & 4791.1 & 12034.87 & 698.33 & 440054.1 & 0 & 389178.81 \\
\hline 7541.59 & 302030 & 17 & 0 & 2433.9 & 50274.2 & 2295.75 & 5838.82 & 332.74 & 406058.51 & 0 & 463460.87 \\
\hline 7541.65 & 307072 & 17 & 1059.82 & 4161.17 & 78484.49 & 5138.36 & 12820.06 & 774.35 & 498921.08 & 0 & 178917.86 \\
\hline 7541.72 & 284227 & 18 & 0 & 4921.03 & 66441.27 & 5385.38 & 13494.61 & 812.2 & 488194.42 & 0 & 284962.49 \\
\hline 7541.78 & 265464 & 18 & 0 & 4414.86 & 60125.98 & 4741.62 & 12006.49 & 708.6 & 481521.36 & 0 & 352315.86 \\
\hline 7541.85 & 365919 & 17 & 0 & 4464.93 & 53558.83 & 4672.58 & 11847.57 & 696.28 & 410463.69 & 0 & 334187.83 \\
\hline 7541.92 & 439763 & 16 & 1020.41 & 5646.43 & 67677.75 & 6206.69 & 15778.19 & 948.27 & 397345.35 & 0 & 217502.19 \\
\hline 7541.98 & 360428 & 17 & 777.49 & 5923.79 & 62148.68 & 6094.4 & 15454.76 & 921.92 & 402379.23 & 0 & 299217.94 \\
\hline 7542.05 & 349909 & 17 & 928.61 & 6126.2 & 71487.45 & 7418.04 & 18818.12 & 1181.9 & 504583.43 & 0 & 52385.08 \\
\hline 7542.11 & 333450 & 17 & 916.57 & 5428.38 & 65427.58 & 6403.82 & 16191.15 & 987.93 & 509110.39 & 0 & 114694.69 \\
\hline 7542.18 & 332229 & 17 & 519.83 & 5244.96 & 53530.39 & 5732.98 & 14551.28 & 871.87 & 477174.14 & 0 & 258175.59 \\
\hline 7542.24 & 320211 & 17 & 693.74 & 5661.44 & 60460.22 & 6678.89 & 16986.43 & 1048.79 & 509837.02 & 0 & 34877.46 \\
\hline 7542.31 & 388161 & 17 & 0 & 5108.04 & 38676.53 & 5079.84 & 12961.69 & 757.7 & 433775.78 & 0 & 335594.92 \\
\hline 7542.38 & 341012 & 17 & 0 & 5707.14 & 40963.94 & 5941.43 & 15215.2 & 912.94 & 502884.85 & 0 & 154536.87 \\
\hline
\end{tabular}




\begin{tabular}{|c|c|c|c|c|c|c|c|c|c|c|c|}
\hline True Depth & XRF tCOUNTS & XRF Live Time & $\mathrm{Ti}(\mathrm{ppm})$ & $\mathrm{V}(\mathrm{ppm})$ & $\mathrm{Fe}(\mathrm{ppm})$ & $\mathrm{Ni}(\mathrm{ppm})$ & $\mathrm{Cu}(\mathrm{ppm})$ & $\mathrm{Pb}(\mathrm{ppm})$ & $\mathrm{LE}(\mathrm{ppm})$ & $\mathrm{Mg}(\mathrm{ppm})$ & $\mathrm{Cl}(\mathrm{ppm})$ \\
\hline 7542.44 & 377708 & 17 & 0 & 5243.36 & 30239.08 & 5023.53 & 13046.09 & 752.94 & 454636.28 & 0 & 145562.33 \\
\hline 7542.51 & 562030 & 15 & 0 & 2377.09 & 4988.17 & 1831.49 & 4770.53 & 259.48 & 333538.56 & 0 & 307397.23 \\
\hline 7542.57 & 627316 & 14 & 0 & 2902.32 & 3324.06 & 2538.56 & 6623.81 & 365.47 & 357041.25 & 0 & 186909.61 \\
\hline 7542.64 & 690021 & 13 & 0 & 2911.35 & 2946.25 & 2427.75 & 6320.5 & 347.61 & 350096.78 & 0 & 155250.13 \\
\hline 7542.70 & 682211 & 13 & 0 & 2725.41 & 5557.83 & 2327.81 & 6074.37 & 335.99 & 427323.82 & 0 & 67488.49 \\
\hline 7542.77 & 701062 & 13 & 0 & 3382.71 & 6996.16 & 2856.84 & 7476.01 & 414.36 & 467930.33 & 0 & 15276.14 \\
\hline 7542.83 & 642887 & 14 & 0 & 2000.15 & 4586.9 & 1684.81 & 4395.93 & 244.35 & 333136.35 & 0 & 183747.76 \\
\hline 7542.90 & 678580 & 13 & 0 & 2524.46 & 7678.33 & 2256.8 & 5848.81 & 322.83 & 490574 & 0 & 5326.22 \\
\hline 7542.97 & 696120 & 13 & 0 & 1688.06 & 6003.46 & 1338.6 & 3469.9 & 188.28 & 221559.98 & 0 & 430238.54 \\
\hline 7543.03 & 703070 & 13 & 6596.19 & 1571.46 & 4464.72 & 1454.47 & 3795.59 & 206.43 & 219762.8 & 0 & 425190.61 \\
\hline 7543.10 & 610544 & 14 & 0 & 3076.74 & 21067.39 & 2818.99 & 7367.82 & 412.26 & 427769.62 & 0 & 30247.91 \\
\hline 7543.16 & 506214 & 15 & 0 & 3382.37 & 20417.69 & 3168.17 & 8270.7 & 465.98 & 435202.28 & 0 & 0 \\
\hline 7543.23 & 349425 & 17 & 0 & 3250.69 & 16707.5 & 3136.53 & 8116.69 & 459.98 & 526938.2 & 0 & 7177.51 \\
\hline 7543.29 & 464204 & 16 & 0 & 2527.91 & 21068.89 & 2352.99 & 6097.37 & 336.32 & 356452.43 & 0 & 385597.18 \\
\hline 7543.36 & 419979 & 16 & 0 & 4795.75 & 52086.59 & 5036.88 & 13048.56 & 763.2 & 419967.88 & 0 & 261280.05 \\
\hline 7543.43 & 376387 & 17 & 492.16 & 6083.23 & 55694.63 & 7025.14 & 17846.6 & 1095.36 & 470280.09 & 0 & 126501.86 \\
\hline 7543.49 & 338436 & 17 & 0 & 5702.32 & 54407.68 & 6412.11 & 16275.45 & 987.62 & 443707.69 & 52950.47 & 118036.72 \\
\hline 7543.56 & 323357 & 17 & 0 & 6142.88 & 48460.72 & 7039.63 & 18009.28 & 1132.08 & 550229.12 & 0 & 36388.22 \\
\hline 7543.62 & 337011 & 17 & 0 & 6289.5 & 49352.87 & 7157.24 & 18292.11 & 1140.54 & 525922.65 & 0 & 68166.7 \\
\hline 7543.69 & 341500 & 17 & 573.7 & 5834.17 & 55133.73 & 6618.69 & 16834.59 & 1046.13 & 502272.25 & 0 & 19431.68 \\
\hline 7543.75 & 467016 & 16 & 731.83 & 2741.48 & 176500.85 & 4223.7 & 10724.36 & 629.37 & 261643.64 & 67126.48 & 237651.81 \\
\hline 7543.82 & 302062 & 17 & 613.86 & 5764.43 & 60900.9 & 6919.3 & 17590.53 & 1103.76 & 551066.77 & 0 & 24857.69 \\
\hline 7543.88 & 360487 & 17 & 0 & 6340.62 & 50435.55 & 7207.75 & 18369.63 & 1143.24 & 492254.11 & 0 & 92658.3 \\
\hline 7543.95 & 445080 & 16 & 0 & 4720.83 & 38088.6 & 4753.96 & 12236.07 & 715.32 & 415395.35 & 0 & 175444.42 \\
\hline 7544.02 & 319105 & 17 & 0 & 5387.91 & 68861.37 & 6525.78 & 16812.4 & 1023.4 & 421256.29 & 0 & 13252.4 \\
\hline 7544.08 & 384806 & 17 & 737.52 & 5823.37 & 69675.75 & 7034.3 & 17913.52 & 1116.21 & 478436.59 & 0 & 0 \\
\hline 7544.15 & 342837 & 17 & 966.52 & 6270.35 & 62124.46 & 7059.66 & 17954.63 & 1112.65 & 407461.5 & 104917.14 & 0 \\
\hline 7544.21 & 417313 & 16 & 0 & 6453.08 & 38180.83 & 6305.57 & 16241.58 & 981.95 & 492355.89 & 0 & 33290.44 \\
\hline 7544.28 & 483777 & 16 & 0 & 5673.71 & 51760.96 & 6018.17 & 15384.64 & 914.19 & 399613.01 & 0 & 206900.64 \\
\hline 7544.34 & 423108 & 16 & 0 & 6237.79 & 67121.12 & 7113.47 & 18315.08 & 1122 & 460531.94 & 0 & 0 \\
\hline 7544.41 & 396142 & 17 & 739.07 & 6254.53 & 68514.38 & 7428.78 & 18983.49 & 1173.81 & 474596.27 & 0 & 39394.68 \\
\hline 7544.48 & 383653 & 17 & 809.87 & 5389.96 & 42523.85 & 5333.68 & 13583.73 & 807 & 469848.51 & 0 & 257832.46 \\
\hline 7544.54 & 453067 & 16 & 890.2 & 4023.21 & 103418.96 & 5037.72 & 12891.61 & 772.12 & 406791.97 & 0 & 200707.99 \\
\hline
\end{tabular}




\begin{tabular}{|c|c|c|c|c|c|c|c|c|c|c|c|}
\hline True Depth & XRF tCOUNTS & XRF Live Time & $\mathrm{Ti}(\mathrm{ppm})$ & V (ppm) & $\mathrm{Fe}(\mathrm{ppm})$ & $\mathrm{Ni}(\mathrm{ppm})$ & $\mathrm{Cu}(\mathrm{ppm})$ & $\mathrm{Pb}(\mathrm{ppm})$ & LE (ppm) & $\mathrm{Mg}(\mathrm{ppm})$ & $\mathrm{Cl}(\mathrm{ppm})$ \\
\hline 7544.61 & 359974 & 17 & 0 & 6766.74 & 40913.71 & 7121.11 & 18318.45 & 1139.32 & 511874.24 & 0 & 0 \\
\hline 7544.67 & 375621 & 17 & 529.13 & 5259.91 & 81768.71 & 6484.28 & 16662.86 & 1023.66 & 451131.28 & 0 & 67555.38 \\
\hline 7544.74 & 376409 & 17 & 0 & 6030.32 & 58931.97 & 6954.07 & 17706.15 & 1092.18 & 477472.35 & 0 & 0 \\
\hline 7544.80 & 394440 & 17 & 571.79 & 5836.28 & 67830.91 & 6758.9 & 17227.05 & 1068.68 & 462647.07 & 0 & 6522.1 \\
\hline 7544.87 & 399479 & 16 & 0 & 5958.5 & 57906.49 & 6265.47 & 16012.96 & 977.09 & 462668.42 & 0 & 140956.75 \\
\hline 7544.93 & 379347 & 17 & 0 & 5870.7 & 55002.78 & 6103.72 & 15591.06 & 936.65 & 453357.95 & 0 & 188976.11 \\
\hline 7545.00 & 395249 & 17 & 0 & 5375.3 & 48705.85 & 5381.65 & 13806.23 & 820.1 & 451244.03 & 0 & 223618.51 \\
\hline 7545.07 & 393200 & 17 & 8687.45 & 5263.95 & 67123.92 & 6595.22 & 16696.71 & 1039.16 & 507126.42 & 0 & 31225.04 \\
\hline 7545.13 & 335749 & 17 & 0 & 4970.63 & 53882.08 & 5131.05 & 12994.24 & 774.34 & 464813.98 & 0 & 204555.78 \\
\hline 7545.20 & 307639 & 17 & 0 & 5038.37 & 61628.71 & 6080.53 & 15328.2 & 954.05 & 530521.97 & 0 & 0 \\
\hline 7545.26 & 282291 & 18 & 0 & 5123.36 & 48531.96 & 5288.74 & 13521.37 & 821.37 & 521126.49 & 0 & 0 \\
\hline 7545.33 & 391510 & 17 & 6817.38 & 10438.4 & 40361.57 & 7085.38 & 18195.23 & 1112.12 & 420788.74 & 0 & 223212.92 \\
\hline 7545.39 & 480716 & 16 & 49512.76 & 38964.04 & 32454.27 & 12357.09 & 32407.32 & 2022.22 & 224869.06 & 0 & 457207.47 \\
\hline 7545.46 & 375430 & 17 & 48480.15 & 38620.07 & 55106.99 & 15534.23 & 40837.1 & 2796.74 & 402320.33 & 0 & 27942.5 \\
\hline 7545.52 & 336817 & 17 & 12535.91 & 15376.5 & 44892.2 & 9242.59 & 23911.75 & 1536.42 & 478848.31 & 0 & 63342.68 \\
\hline 7545.59 & 424141 & 16 & 19026.82 & 19049.25 & 36023.05 & 8046.87 & 20890.67 & 1257.32 & 318004.2 & 0 & 423167.8 \\
\hline 7545.66 & 435006 & 16 & 12104.67 & 13939.59 & 27641.93 & 6790.75 & 17600.47 & 1047.55 & 352267.34 & 0 & 364080.88 \\
\hline 7545.72 & 392903 & 17 & 7507.94 & 10201.82 & 30369.32 & 5995.3 & 15530.37 & 917.03 & 406208.32 & 0 & 359803.37 \\
\hline 7545.79 & 405717 & 16 & 26325.49 & 24453.18 & 46196.86 & 11930.11 & 31061.21 & 2041.69 & 367571.16 & 0 & 0 \\
\hline 7545.85 & 427772 & 16 & 18273.12 & 18608.25 & 35013.8 & 9245.56 & 23959.92 & 1477.99 & 345001.28 & 0 & 263250.89 \\
\hline 7545.92 & 602420 & 14 & 9561.02 & 10079.27 & 14169.45 & 3737.81 & 9725.84 & 549.58 & 349522.2 & 0 & 551398.12 \\
\hline 7545.98 & 346451 & 17 & 12291.71 & 15928.14 & 32886.9 & 9765.98 & 25400.24 & 1647.54 & 456224.38 & 0 & 21871.38 \\
\hline 7546.05 & 142576 & 19 & 1041.44 & 1580.49 & 1899.08 & 1292.42 & 3358.39 & 193.64 & 835365.22 & 0 & 102705.11 \\
\hline 7546.12 & 346017 & 17 & 30617.37 & 19033.4 & 47362.52 & 11557.03 & 30202.87 & 2026.17 & 511769.72 & 0 & 0 \\
\hline 7546.18 & 361851 & 17 & 2412.29 & 7406.43 & 50581.14 & 7167.92 & 18245.11 & 1145.46 & 478204.91 & 0 & 0 \\
\hline 7546.25 & 362520 & 17 & 1245.25 & 5817.62 & 60116.94 & 6724.16 & 17054.46 & 1063.86 & 479972.2 & 0 & 0 \\
\hline 7546.31 & 365462 & 17 & 1218.69 & 5387.14 & 67348.99 & 6531.45 & 16517.26 & 1028 & 482017.43 & 0 & 40463.01 \\
\hline 7546.38 & 376612 & 17 & 767.22 & 6489.22 & 54643.92 & 7459.48 & 19053.65 & 1193.41 & 491358.99 & 0 & 0 \\
\hline 7546.44 & 391287 & 17 & 627.45 & 6645.49 & 44997.13 & 7196.15 & 18403.74 & 1155.53 & 489524.56 & 0 & 0 \\
\hline 7546.51 & 487723 & 16 & 4853.68 & 9430.35 & 20548.5 & 5604.46 & 14481.15 & 840.31 & 356955.51 & 0 & 322421.24 \\
\hline 7546.57 & 519133 & 15 & 18136.59 & 18917.78 & 74119.38 & 9309.65 & 24359.24 & 1469.34 & 270518.13 & 0 & 187154.8 \\
\hline 7546.64 & 378586 & 17 & 14228.46 & 15849.86 & 47364.92 & 8509.15 & 22130.64 & 1343.88 & 336835.25 & 0 & 199612.15 \\
\hline 7546.71 & 545124 & 15 & 40664.93 & 29529.57 & 68968.24 & 7204.74 & 18815.14 & 1103.59 & 228359.73 & 0 & 458560.21 \\
\hline
\end{tabular}




\begin{tabular}{|c|c|c|c|c|c|c|c|c|c|c|c|}
\hline True Depth & XRF tCOUNTS & XRF Live Time & $\mathrm{Ti}(\mathrm{ppm})$ & $\mathrm{V}$ (ppm) & $\mathrm{Fe}(\mathrm{ppm})$ & $\mathrm{Ni}(\mathrm{ppm})$ & $\mathrm{Cu}(\mathrm{ppm})$ & $\mathrm{Pb}(\mathrm{ppm})$ & LE (ppm) & $\mathrm{Mg}(\mathrm{ppm})$ & $\mathrm{Cl}(\mathrm{ppm})$ \\
\hline 7546.77 & 354766 & 17 & 703.71 & 6077.09 & 61835.13 & 6773.52 & 16995.34 & 1043.96 & 397054.18 & 93566.91 & 6067.4 \\
\hline 7546.84 & 336188 & 17 & 603.81 & 5159.88 & 64947.6 & 5953.1 & 14970.07 & 914.48 & 422799.41 & 59162.22 & 0 \\
\hline 7546.90 & 372301 & 17 & 760.11 & 5403.06 & 65224.53 & 6256.59 & 15728.9 & 964.51 & 451995.87 & 0 & 0 \\
\hline 7546.97 & 354393 & 17 & 0 & 5413.32 & 61112.44 & 6378.7 & 16168.95 & 993.49 & 472920 & 0 & 0 \\
\hline 7547.03 & 446132 & 16 & 11467.06 & 3838.15 & 122311.67 & 5930.55 & 15137.06 & 922.81 & 403365.21 & 0 & 0 \\
\hline 7547.10 & 358018 & 17 & 955.8 & 4984.34 & 66625.92 & 5822.84 & 14621.05 & 899.65 & 469891.94 & 0 & 0 \\
\hline 7547.17 & 327083 & 17 & 0 & 5507.82 & 55356.5 & 6090.42 & 15405.1 & 937.55 & 456444.14 & 56149.8 & 41788.29 \\
\hline 7547.23 & 348609 & 17 & 0 & 5892.05 & 42154.8 & 6037.62 & 15362.8 & 922.2 & 457120.48 & 0 & 111699.99 \\
\hline 7547.30 & 464646 & 16 & 0 & 5239.52 & 38049.58 & 5195.05 & 13216.2 & 774.45 & 413532.01 & 0 & 264780.12 \\
\hline 7547.36 & 354266 & 17 & 0 & 6248.96 & 45431.99 & 6826.61 & 17306.6 & 1062.79 & 516001.6 & 0 & 77865.13 \\
\hline 7547.43 & 438651 & 16 & 0 & 4124.58 & 25541.68 & 3855.4 & 9849.15 & 561.53 & 380070.4 & 0 & 453786.85 \\
\hline 7547.49 & 426688 & 16 & 0 & 6003.93 & 57740.42 & 6564.91 & 16699.78 & 1011.59 & 444198.74 & 0 & 82207.94 \\
\hline 7547.56 & 337922 & 17 & 0 & 5797.41 & 56909.77 & 6776.06 & 17290.49 & 1071.82 & 535434.36 & 0 & 9992.33 \\
\hline 7547.62 & 367860 & 17 & 0 & 5995.04 & 44236.29 & 6414.06 & 16318.74 & 991.99 & 511485.65 & 0 & 88388.22 \\
\hline 7547.69 & 368096 & 17 & 0 & 4881.55 & 49089.61 & 4725.3 & 11999.98 & 700 & 439245.18 & 0 & 363147.49 \\
\hline 7547.76 & 335700 & 17 & 0 & 5391.93 & 36846.97 & 5314.72 & 13571.54 & 800.07 & 468145.14 & 0 & 309789.16 \\
\hline 7547.82 & 216191 & 18 & 7648.99 & 8608.63 & 36488.6 & 4597.16 & 11731.69 & 694.95 & 525298.48 & 0 & 323050.43 \\
\hline 7547.89 & 206146 & 18 & 7306.83 & 8869.95 & 55780.05 & 6163.33 & 15523.59 & 948.69 & 508363.21 & 0 & 243777.69 \\
\hline 7547.95 & 252465 & 18 & 0 & 4108.18 & 61164.04 & 4284.99 & 10890.35 & 635.87 & 423612.81 & 0 & 382748.51 \\
\hline 7548.02 & 309434 & 17 & 879.56 & 5557.99 & 65337.73 & 6339.82 & 15845.84 & 978.78 & 444275.17 & 0 & 0 \\
\hline 7548.08 & 379521 & 17 & 914.44 & 5510.34 & 69191.95 & 6339.83 & 15977.12 & 989.67 & 470694.97 & 0 & 0 \\
\hline 7548.15 & 323139 & 17 & 0 & 6118.99 & 41424.79 & 6491.55 & 16405.08 & 1013.91 & 526854.12 & 0 & 12006.18 \\
\hline 7548.22 & 378285 & 17 & 1189.02 & 4564.99 & 94002.29 & 5427.77 & 13863.14 & 835.69 & 463588.21 & 0 & 89264.02 \\
\hline 7548.28 & 386686 & 17 & 943.08 & 6120.07 & 52794.67 & 6575.94 & 16712.28 & 1031.72 & 444986.33 & 0 & 0 \\
\hline 7548.35 & 373841 & 17 & 595.93 & 5381.57 & 42479.94 & 5245.7 & 13265.06 & 788.57 & 455546.84 & 0 & 162076.41 \\
\hline 7548.41 & 388108 & 17 & 0 & 6128.22 & 50338.07 & 6682.3 & 16859.92 & 1041.71 & 478842.93 & 0 & 0 \\
\hline 7548.48 & 343644 & 17 & 774.52 & 5593.44 & 59765.65 & 6398.09 & 16188.15 & 1006.86 & 481621.62 & 0 & 9235.53 \\
\hline 7548.54 & 388029 & 17 & 0 & 5746.11 & 40671.4 & 5543.76 & 13954.79 & 834.44 & 450218.21 & 0 & 222009.36 \\
\hline 7548.61 & 361954 & 17 & 602.18 & 5840.34 & 56452.98 & 6321.46 & 16065.37 & 991.19 & 453470.96 & 0 & 11894.69 \\
\hline 7548.67 & 316792 & 17 & 761.1 & 4587.02 & 43543.27 & 4509.43 & 11426.08 & 675.13 & 486548.02 & 0 & 120297.9 \\
\hline 7548.74 & 340851 & 17 & 480.33 & 4794.73 & 44084.18 & 4751.44 & 12005.66 & 716.05 & 470047.4 & 0 & 163538.91 \\
\hline 7548.81 & 323392 & 17 & 0 & 4884.4 & 39051.66 & 4472.73 & 11324.98 & 675.35 & 485720.55 & 0 & 114946.9 \\
\hline 7548.87 & 294573 & 17 & 642.57 & 5322.86 & 43261.41 & 5294.18 & 13379.53 & 807.56 & 475058.93 & 0 & 107572.98 \\
\hline
\end{tabular}




\begin{tabular}{|c|c|c|c|c|c|c|c|c|c|c|c|}
\hline True Depth & XRF tCOUNTS & XRF Live Time & $\mathrm{Ti}(\mathrm{ppm})$ & $\mathrm{V}(\mathrm{ppm})$ & $\mathrm{Fe}(\mathrm{ppm})$ & $\mathrm{Ni}(\mathrm{ppm})$ & $\mathrm{Cu}(\mathrm{ppm})$ & $\mathrm{Pb}(\mathrm{ppm})$ & $\mathrm{LE}(\mathrm{ppm})$ & $\mathrm{Mg}(\mathrm{ppm})$ & $\mathrm{Cl}(\mathrm{ppm})$ \\
\hline 7548.94 & 325716 & 17 & 1071.71 & 5692.47 & 35684.61 & 5235.81 & 13440.1 & 815.05 & 479190.44 & 0 & 0 \\
\hline 7549.00 & 343257 & 17 & 1327.84 & 6103.66 & 47635.66 & 6017.67 & 15240.46 & 936.98 & 449760.03 & 0 & 0 \\
\hline 7549.07 & 411155 & 16 & 13451.42 & 5230.4 & 61086.01 & 6359.76 & 16137.85 & 1003.08 & 478240.78 & $\overline{0}$ & 0 \\
\hline 7549.13 & 346720 & 17 & 1054.33 & 5215.66 & 58515 & 5707.89 & 14381.87 & 876.43 & 477446.01 & 0 & 0 \\
\hline 7549.20 & 320219 & 17 & 486.32 & 5712.86 & 45941.62 & 6130.4 & 15524.53 & 971.68 & 505242.83 & 0 & 0 \\
\hline 7549.27 & 331519 & 17 & 634.36 & 5746.29 & 53361.72 & 6572.49 & 16812.66 & 1040.22 & 466655.95 & 0 & 0 \\
\hline 7549.33 & 322074 & 17 & 1307.55 & 5286.41 & 49756.95 & 5770.64 & 14677.11 & 904.15 & 473796.22 & 0 & 0 \\
\hline 7549.40 & 340123 & 17 & 0 & 5890.86 & 55269.71 & 6766.53 & 17193.31 & 1072.77 & 479101.71 & 0 & 11317.9 \\
\hline 7549.46 & 332217 & 17 & 840.34 & 4959.25 & 57941.77 & 5682.16 & 14380.46 & 879.15 & 481190.96 & 0 & 12609.75 \\
\hline 7549.53 & 337687 & 17 & 571.02 & 4479.02 & 38947.66 & 4458.5 & 11159.68 & 658.09 & 463557.66 & 0 & 144217.79 \\
\hline 7549.59 & 315617 & 17 & 0 & 4637.67 & 39104.66 & 4631.03 & 11681.13 & 698.53 & 476606.61 & 0 & 60453.94 \\
\hline 7549.66 & 341552 & 17 & 0 & 4031.47 & 34043.86 & 3759.38 & 9498.48 & 552.64 & 458885.54 & 0 & 188968.78 \\
\hline 7549.72 & 332828 & 17 & 805.74 & 5252.05 & 49041.93 & 5937.16 & 15139.23 & 942.04 & 472653.07 & 0 & 0 \\
\hline 7549.79 & 348902 & 17 & 0 & 4571.17 & 33550.81 & 4371.35 & 11064.64 & 642.81 & 456692.96 & 0 & 229055.33 \\
\hline 7549.86 & 240287 & 18 & 0 & 5412.17 & 37948.01 & 5686 & 14388.11 & 880 & 464359.78 & 0 & 0 \\
\hline 7549.92 & 398178 & 17 & $\overline{0}$ & 4402.4 & 33450.68 & 4235.3 & 10866.2 & 628.06 & 448687.45 & $\overline{0}$ & 280527.97 \\
\hline 7549.99 & 361801 & 17 & 0 & 4399.86 & 34365.52 & 4202.69 & 10758.68 & 629.6 & 435036.25 & 0 & 188875.72 \\
\hline 7550.05 & 334540 & 17 & 516.69 & 5640.1 & 42883.18 & 6145.27 & 15760.96 & 971 & 487525.18 & 0 & 0 \\
\hline 7550.12 & 352080 & 17 & 1485.9 & 4479.36 & 32584.88 & 4526.43 & 11756.57 & 691.32 & 527983.89 & 0 & 40202.44 \\
\hline 7550.18 & 308908 & 17 & 937.82 & 5487.2 & 43944.15 & 5568.5 & 14293.78 & 857.03 & 412268.41 & 63978.86 & 80585.54 \\
\hline 7550.25 & 345583 & 17 & 892.53 & 5757.12 & 49936.03 & 6626.3 & 16992.65 & 1063.67 & 494970.72 & 0 & 0 \\
\hline 7550.31 & 326988 & 17 & 693.54 & 5800.48 & 38934.29 & 6099.26 & 15736.6 & 977.3 & 495024.59 & 0 & 35539.78 \\
\hline 7550.38 & 285002 & 18 & 583.48 & 5895.93 & 43704.81 & 6850.84 & 17517.23 & 1104.65 & 523719.86 & 0 & 0 \\
\hline 7550.45 & 410633 & 16 & 0 & 5166.22 & 41015.91 & 5288.64 & 13608.37 & 803.18 & 408779.59 & 0 & 249152.48 \\
\hline 7550.51 & 357005 & 17 & 0 & 5120.74 & 44459.79 & 5675.76 & 14636.42 & 900.16 & 446636.78 & 0 & 0 \\
\hline 7550.58 & 331350 & 17 & 0 & 4932.16 & 26869.33 & 4870.92 & 12584.87 & 745.97 & 458169.75 & 0 & 143555.2 \\
\hline 7550.64 & 307575 & 17 & 0 & 5199.63 & 42970.52 & 5586.97 & 14154.54 & 864.79 & 521078.32 & 0 & 66353.33 \\
\hline 7550.71 & 298388 & 17 & 423.96 & 3360.68 & 25043.59 & 3059.21 & 7779.53 & 445.12 & 494145.96 & 0 & 281018.61 \\
\hline 7550.77 & 288072 & 18 & 557.04 & 4506.57 & 31174.1 & 4351.85 & 11103.92 & 654.8 & 507473.66 & 0 & 185147.9 \\
\hline 7550.84 & 297542 & 17 & 0 & 4707.71 & 28220.6 & 4503.83 & 11502.36 & 679.06 & 477426.73 & 0 & 108863.56 \\
\hline 7550.91 & 356169 & 17 & 0 & 4552.04 & 35151.23 & 4562.47 & 11521.16 & 680.06 & 455400.7 & 0 & 219056.01 \\
\hline 7550.97 & 439871 & 16 & 0 & 3839.16 & 45024.3 & 3704.37 & 9532.09 & 551.05 & 391768.91 & 0 & 404554.32 \\
\hline 7551.04 & 284262 & 18 & 538.82 & 5247.74 & 29307.49 & 5257.75 & 13555.51 & 824.52 & 521721.89 & 0 & 0 \\
\hline
\end{tabular}




\begin{tabular}{|c|c|c|c|c|c|c|c|c|c|c|c|}
\hline True Depth & XRF tCOUNTS & XRF Live Time & $\mathrm{Ti}(\mathrm{ppm})$ & V (ppm) & $\mathrm{Fe}(\mathrm{ppm})$ & $\mathrm{Ni}(\mathrm{ppm})$ & $\mathrm{Cu}(\mathrm{ppm})$ & $\mathrm{Pb}(\mathrm{ppm})$ & LE (ppm) & $\mathrm{Mg}(\mathrm{ppm})$ & $\mathrm{Cl}(\mathrm{ppm})$ \\
\hline 7551.10 & 387980 & 17 & 1373.69 & 4832.52 & 32442.18 & 4600.62 & 11758.48 & 691.57 & 427968.91 & 0 & 203732.53 \\
\hline 7551.17 & 390662 & 17 & 0 & 5074.52 & 38089.16 & 4921.43 & 12551.93 & 734.15 & 424059.43 & 0 & 299473.01 \\
\hline 7551.23 & 384290 & 17 & 900.91 & 4905.26 & 45268.72 & 4677.64 & 11955.8 & 706.34 & 434021.49 & 0 & 240433.84 \\
\hline 7551.30 & 343324 & 17 & 0 & 4155.28 & 23504.25 & 3831.4 & 9778.96 & 565.82 & 468404.43 & 0 & 292090.46 \\
\hline 7551.36 & 400252 & 16 & 0 & 3986.89 & 39778.95 & 3822.41 & 9666.34 & 552.28 & 394197.49 & 0 & 300165.72 \\
\hline 7551.43 & 294144 & 18 & 0 & 3460.55 & 24654.94 & 3052.16 & 7766.11 & 448.46 & 498417.96 & 0 & 209627.67 \\
\hline 7551.50 & 281646 & 18 & 0 & 3189.92 & 18929.72 & 2780.34 & 7145.95 & 403.97 & 516745.64 & 0 & 283471.53 \\
\hline 7551.56 & 207154 & 18 & 0 & 4536.22 & 27255.25 & 4506.18 & 11418.93 & 678.3 & 514442.74 & 0 & 140776.85 \\
\hline 7551.63 & 373687 & 17 & 0 & 5216.1 & 38373.32 & 5442.14 & 14016.12 & 844.38 & 439711.44 & 0 & 87712.87 \\
\hline 7551.69 & 522992 & 15 & 0 & 2653.78 & 41717.97 & 2591.01 & 6691.08 & 373.05 & 339567.12 & 0 & 476441.28 \\
\hline 7551.76 & 466215 & 16 & 0 & 4010.66 & 21992.82 & 3682 & 9379.84 & 534.12 & 406298.51 & 0 & 438144.67 \\
\hline 7551.82 & 305425 & 17 & 0 & 4248.74 & 67697.91 & 5070.14 & 13078.45 & 782.51 & 508487.97 & 0 & 158419.9 \\
\hline 7551.89 & 326390 & 17 & 0 & 3897.57 & 21782.1 & 3600.56 & 9147.43 & 522.36 & 475940.11 & 0 & 304951.66 \\
\hline 7551.96 & 382728 & 17 & 0 & 5752.19 & 37866.8 & 5804.97 & 14749.46 & 881.13 & 413521.95 & 0 & 258184.64 \\
\hline 7552.02 & 331394 & 17 & 0 & 6077.27 & 34427.05 & 6225.44 & 16021.01 & 975.02 & 484985.74 & 0 & 133051.33 \\
\hline 7552.09 & 280862 & 18 & 674.9 & 5603.1 & 30194.44 & 5557.93 & 14296.98 & 868.11 & 496585.96 & 0 & 220101.2 \\
\hline 7552.15 & 540508 & 15 & 8655.41 & 2655.88 & 5612.6 & 2140.02 & 5575.39 & 313.54 & 507682.29 & 0 & 18084.68 \\
\hline 7552.22 & 654116 & 14 & 0 & 2901.59 & 4632.81 & 2296.6 & 5943.24 & 329.92 & 460723.01 & 0 & 40124.32 \\
\hline 7552.28 & 651836 & 14 & 593.54 & 2088.16 & 3935.72 & 1647.92 & 4237.91 & 232.02 & 337817.65 & 0 & 203940.19 \\
\hline 7552.35 & 590349 & 14 & 0 & 2059.93 & 7876.55 & 1593.17 & 4145.21 & 222.79 & 361404.94 & 0 & 189665.87 \\
\hline 7552.41 & 630531 & 14 & 0 & 3018.22 & 5122.36 & 2249.63 & 5854.59 & 322.81 & 356335.1 & 0 & 186974.01 \\
\hline 7552.48 & 659914 & 13 & 0 & 1769.83 & 3604.99 & 1383.47 & 3607.14 & 195.6 & 295241.02 & 0 & 274385.82 \\
\hline 7552.55 & 612197 & 14 & 0 & 2022.96 & 2907.85 & 1511.55 & 3936.22 & 215.01 & 386272.2 & 0 & 147046.08 \\
\hline 7552.61 & 623372 & 14 & 274.28 & 1431.27 & 1441.68 & 956.61 & 2487.42 & 134.01 & 292579.09 & 0 & 473433.51 \\
\hline 7552.68 & 641246 & 14 & 804.79 & 1912.55 & 2172.31 & 1254.18 & 3275.97 & 179.8 & 231798.38 & 0 & 411659.73 \\
\hline 7552.74 & 648064 & 14 & 1064.16 & 2331.13 & 2906.42 & 1440.42 & 3731.59 & 203.81 & 291686.47 & 0 & 277271.6 \\
\hline 7552.81 & 518325 & 15 & 0 & 2279.37 & 2115.71 & 1708.6 & 4445.9 & 238.63 & 214736 & 0 & 434626.68 \\
\hline 7552.87 & 698043 & 13 & 0 & 3932.1 & 2898.72 & 3459.51 & 9070.96 & 506.85 & 503020.66 & 0 & 0 \\
\hline 7552.94 & 680298 & 13 & 0 & 3538.43 & 2467.09 & 2897 & 7598.6 & 433.39 & 515365.22 & 0 & 0 \\
\hline 7553.01 & 666638 & 13 & 0 & 2574.54 & 2885.45 & 2099.92 & 5532.11 & 307.94 & 517163.47 & 0 & 0 \\
\hline 7553.07 & 660865 & 13 & 2443.89 & 2543.04 & 2825.65 & 2054.15 & 5334.27 & 295.99 & 475395.25 & 0 & 38761.64 \\
\hline 7553.14 & 661870 & 13 & 0 & 2165.82 & 2471.96 & 1779.36 & 4621.9 & 253.08 & 518802.17 & 0 & 0 \\
\hline 7553.20 & 619399 & 14 & 1290.29 & 7426.01 & 22916.78 & 5814.53 & 15252.04 & 903.62 & 498524.83 & 0 & 0 \\
\hline
\end{tabular}




\begin{tabular}{|c|c|c|c|c|c|c|c|c|c|c|c|}
\hline True Depth & XRF tCOUNTS & XRF Live Time & $\mathrm{Ti}(\mathrm{ppm})$ & $\mathrm{V}(\mathrm{ppm})$ & $\mathrm{Fe}(\mathrm{ppm})$ & $\mathrm{Ni}(\mathrm{ppm})$ & $\mathrm{Cu}(\mathrm{ppm})$ & $\mathrm{Pb}(\mathrm{ppm})$ & $\mathrm{LE}(\mathrm{ppm})$ & $\mathrm{Mg}(\mathrm{ppm})$ & $\mathrm{Cl}(\mathrm{ppm})$ \\
\hline 7553.27 & 549064 & 15 & 0 & 2756.48 & 7933.61 & 2344.58 & 6127.3 & 332.64 & 329981.64 & 0 & 283022.5 \\
\hline 7553.33 & 630129 & 14 & 0 & 5528.29 & 14652.46 & 5444.52 & 14268.22 & 824.96 & 416473.29 & 0 & 71399.02 \\
\hline 7553.40 & 702584 & 13 & 0 & 686.1 & 13734.96 & 594.16 & 1526.4 & 83.24 & 372964.18 & 0 & 539344.06 \\
\hline 7553.46 & 428979 & 16 & 0 & 4842.29 & 27365.84 & 4595.1 & 11855.07 & 691.76 & 408144.14 & 0 & 442626.51 \\
\hline 7553.53 & 309440 & 17 & 0 & 6609.77 & 35119.83 & 6982.26 & 17842.95 & 1096.23 & 513303 & 0 & 161495.59 \\
\hline 7553.60 & 531825 & 15 & 0 & 1766.12 & 9993.79 & 1517.92 & 3878.73 & 214.27 & 442852.85 & 0 & 497611.2 \\
\hline 7553.66 & 568001 & 15 & 0 & 3442.62 & 17260.7 & 2927.61 & 7564.76 & 426.49 & 416780.76 & 0 & 512392.24 \\
\hline 7553.73 & 472000 & 16 & 0 & 4724.46 & 25444.64 & 4597.98 & 11818.97 & 686.88 & 390371.5 & 0 & 463506.95 \\
\hline 7553.79 & 559909 & 15 & 0 & 2989.56 & 12346.78 & 2527.73 & 6563.61 & 368.45 & 392191.54 & 0 & 499171.57 \\
\hline 7553.86 & 373558 & 17 & 693.81 & 3689.43 & 5090.11 & 2705.19 & 7031.47 & 393.78 & 469383.35 & 0 & 489307.46 \\
\hline 7553.92 & 488440 & 16 & 0 & 7407.75 & 10376.68 & 6797.25 & 17801.42 & 1084.82 & 432806.42 & 0 & 489568.75 \\
\hline 7553.99 & 474909 & 16 & 0 & 3291.67 & 12699.25 & 2979.55 & 7687.61 & 434.97 & 392834.56 & 0 & 473108 \\
\hline 7554.06 & 103833 & 19 & 3413.46 & 0 & 11264.9 & 1335.64 & 0 & 206.4 & 366902.45 & 0 & 480156.62 \\
\hline 7554.12 & 234252 & 18 & 0 & 5396.32 & 31594.89 & 5473.5 & 14138.51 & 859.72 & 532017.49 & 0 & 115196.34 \\
\hline 7554.19 & 308455 & 17 & 5560.26 & 5423.61 & 34195.88 & 5741.62 & 14781.89 & 920.5 & 533349.94 & 0 & 14649.75 \\
\hline 7554.25 & 300956 & 17 & 0 & 5903.43 & 34230.89 & 5902.62 & 15113.28 & 928.35 & 515931.9 & 0 & 0 \\
\hline 7554.32 & 349789 & 17 & 635.85 & 5532.22 & 29549.63 & 5330.52 & 13689.22 & 813.02 & 473304.2 & 0 & 273713.09 \\
\hline 7554.38 & 334600 & 17 & 0 & 3802.21 & 23554.14 & 3470.1 & 8916.84 & 511.36 & 466798.18 & 0 & 300745.11 \\
\hline 7554.45 & 416475 & 16 & 448.79 & 4443.39 & 21066.8 & 4087.48 & 10493.93 & 602.86 & 424972.01 & 0 & 370643.6 \\
\hline 7554.51 & 431868 & 16 & 0 & 5266.95 & 34234.24 & 5193.34 & 13191.04 & 771.26 & 398493.7 & 0 & 343914.76 \\
\hline 7554.58 & 442751 & 16 & 627.95 & 4200.9 & 49036.27 & 4267.76 & 11009.79 & 645.03 & 388451.12 & 0 & 371449.08 \\
\hline 7554.65 & 439788 & 16 & 0 & 4663.33 & 19063.2 & 4227.1 & 10828.72 & 622.98 & 383605.77 & 24736.68 & 412527.72 \\
\hline 7554.71 & 428671 & 16 & 0 & 5734.51 & 24820.26 & 5475.36 & 14083.91 & 830.74 & 414322.23 & 0 & 415784.61 \\
\hline 7554.78 & 467874 & 16 & 0 & 2035.9 & 15115.26 & 1560.19 & 4063.13 & 224.28 & 425503.9 & 0 & 505605.9 \\
\hline 7554.84 & 209670 & 18 & 511.95 & 5139 & 12193.17 & 4216.11 & 10939.89 & 627.9 & 398938.36 & 0 & 466703.46 \\
\hline 7554.91 & 297736 & 17 & 0 & 5287.58 & 27308.3 & 5386.52 & 13765.89 & 829.8 & 480653.22 & 0 & 57549.45 \\
\hline 7554.97 & 324677 & 17 & 0 & 6232 & 36196.93 & 6680.19 & 17155.18 & 1072.76 & 490139.36 & 0 & 0 \\
\hline 7555.04 & 311056 & 17 & 0 & 4954.56 & 19574.03 & 4720.7 & 12206.91 & 730.28 & 482491.19 & 0 & 83905.35 \\
\hline 7555.10 & 346152 & 17 & 0 & 5124.5 & 24977.98 & 4927.5 & 12762.31 & 756.89 & 467559.32 & 0 & 243484.5 \\
\hline 7555.17 & 449410 & 16 & 0 & 3657.8 & 16675.14 & 3325.01 & 8533.25 & 485.69 & 384326.87 & 0 & 410090.23 \\
\hline 7555.24 & 396367 & 17 & 0 & 6005.92 & 30822.55 & 6062 & 15744.8 & 944.89 & 434539.19 & 0 & 166181.34 \\
\hline 7555.30 & 637873 & 14 & 0 & 1546.17 & 23390.21 & 1308.61 & 3412.62 & 184.55 & 253767.86 & 0 & 468529.18 \\
\hline 7555.37 & 652761 & 14 & 192.29 & 1276.22 & 1809.31 & 980.15 & 2522.47 & 133.64 & 346453.33 & 0 & 512233.6 \\
\hline
\end{tabular}




\begin{tabular}{|c|c|c|c|c|c|c|c|c|c|c|c|}
\hline True Depth & XRF tCOUNTS & XRF Live Time & $\mathrm{Ti}(\mathrm{ppm})$ & V (ppm) & $\mathrm{Fe}(\mathrm{ppm})$ & $\mathrm{Ni}(\mathrm{ppm})$ & $\mathrm{Cu}(\mathrm{ppm})$ & $\mathrm{Pb}(\mathrm{ppm})$ & LE (ppm) & $\mathrm{Mg}(\mathrm{ppm})$ & $\mathrm{Cl}(\mathrm{ppm})$ \\
\hline 7555.43 & 648299 & 14 & 0 & 1576.73 & 3493.6 & 1277.12 & 3288.46 & 178.78 & 323886.3 & 0 & 487612.33 \\
\hline 7555.50 & 641791 & 14 & 0 & 2612.72 & 2812.52 & 2067.35 & 5373.61 & 294.85 & 316755.78 & 0 & 234029.83 \\
\hline 7555.56 & 515587 & 15 & 0 & 2061.18 & 21022.99 & 1793.12 & 4653.33 & 253.29 & 334248.64 & 0 & 354263.88 \\
\hline 7555.63 & 580364 & 14 & 0 & 3036.89 & 12755.96 & 2790.19 & 7207.03 & 407.96 & 399606.75 & 0 & 494329.17 \\
\hline 7555.70 & 654100 & 14 & 0 & 1262.34 & 2164.32 & 1022.16 & 2627.51 & 141.72 & 324881.53 & 0 & 493232.8 \\
\hline 7555.76 & 523233 & 15 & 0 & 6030.88 & 18621.2 & 5714.69 & 14771.63 & 860.39 & 352480.54 & 0 & 429542.19 \\
\hline 7555.83 & 633206 & 14 & 0 & 2485.59 & 7450.08 & 2146.13 & 5499.99 & 302.61 & 430775.62 & 0 & 505973.12 \\
\hline 7555.89 & 578099 & 14 & 0 & 3826.64 & 9578.44 & 3307.43 & 8569.5 & 483.81 & 392914.85 & 0 & 500819.06 \\
\hline 7555.96 & 604713 & 14 & 0 & 3372.63 & 12707.54 & 2927.36 & 7520.31 & 421.92 & 402740.47 & 0 & 24151.5 \\
\hline 7556.02 & 574704 & 15 & 0 & 2696.62 & 5532.73 & 2291.39 & 5947.93 & 329.25 & 400831.89 & 0 & 117114.47 \\
\hline 7556.09 & 559671 & 15 & 0 & 2051.35 & 4194.88 & 1717.62 & 4461.34 & 243.41 & 333598.14 & 0 & 285513.84 \\
\hline 7556.15 & 243095 & 18 & 0 & 8144.18 & 23709.68 & 8458.53 & 21937.51 & 1389.33 & 488520.48 & 0 & 51434.71 \\
\hline 7556.22 & 481452 & 16 & 0 & 4131.89 & 4868.31 & 3455.11 & 9020.15 & 504.52 & 477248.86 & 0 & 73216.93 \\
\hline 7556.29 & 181733 & 19 & 0 & 9669.78 & 15473.9 & 9253.4 & 24360.72 & 1574.13 & 526589.68 & 0 & 326485.61 \\
\hline 7556.35 & 248799 & 18 & 0 & 10527.66 & 21095.61 & 11017.24 & 29062.95 & 1947.4 & 537360.75 & 0 & 250269.59 \\
\hline 7556.42 & 219315 & 18 & 1018.72 & 7842.68 & 108875.63 & 11715.21 & 30804.16 & 2053.71 & 382854.17 & 0 & 26796.26 \\
\hline 7556.48 & 281531 & 18 & 0 & 5103.45 & 9339.4 & 4704.67 & 12175.99 & 705.69 & 476930.8 & 0 & 341660.9 \\
\hline 7556.55 & 352126 & 17 & 0 & 6607.1 & 22120.34 & 6381.67 & 16340.33 & 991.13 & 467239.03 & 0 & 162315.52 \\
\hline 7556.61 & 334439 & 17 & 0 & 7148.66 & 23260.27 & 6958.32 & 17778.84 & 1101.35 & 491903.42 & 0 & 0 \\
\hline 7556.68 & 332439 & 17 & 0 & 5939.13 & 21629.35 & 5505.65 & 13987.6 & 827.94 & 483068.74 & 0 & 52621.38 \\
\hline 7556.75 & 344621 & 17 & 0 & 5856.78 & 18747.19 & 5476.66 & 13967.85 & 816.15 & 476091.3 & 0 & 162217.86 \\
\hline 7556.81 & 367617 & 17 & 0 & 6950.55 & 23935.63 & 6622.36 & 16969.29 & 1033.98 & 481883.19 & 0 & 78284.72 \\
\hline 7556.88 & 379264 & 17 & 0 & 6604.37 & 18595.16 & 6416.13 & 16550.39 & 1007.73 & 484922.24 & 0 & 32673.84 \\
\hline 7556.94 & 430301 & 16 & 0 & 2989.07 & 48314.29 & 3153.97 & 8128.83 & 468.51 & 385026.8 & 0 & 141127.72 \\
\hline 7557.01 & 378212 & 17 & 0 & 7414.57 & 25623.69 & 7564.21 & 19359.9 & 1184.18 & 493921.12 & 0 & 121452.31 \\
\hline 7557.07 & 398513 & 17 & 0 & 7425.73 & 26017.7 & 7334.47 & 18730.81 & 1159.29 & 462790.54 & 0 & 27781.57 \\
\hline 7557.14 & 612431 & 14 & 0 & 2661.5 & 10283.38 & 2344.79 & 5982.89 & 325.62 & 297458.49 & 0 & 434214.61 \\
\hline 7557.20 & 413155 & 16 & 0 & 5320.38 & 19216.53 & 4903.73 & 12619.05 & 739.99 & 426823.52 & 0 & 281259.99 \\
\hline 7557.27 & 433351 & 16 & 0 & 9148.81 & 30742.29 & 8756.19 & 22642.97 & 1433.54 & 438179.14 & 0 & 227834.97 \\
\hline 7557.34 & 348719 & 17 & 0 & 6465.92 & 26278.13 & 6530.69 & 16816.96 & 1039.78 & 500921.72 & 0 & 60195.11 \\
\hline 7557.40 & 415157 & 16 & 0 & 8113.05 & 40956.14 & 8926.29 & 23014.97 & 1479.95 & 492900.47 & 0 & 0 \\
\hline 7557.47 & 481344 & 16 & 0 & 2551.89 & 6818.25 & 2187.64 & 5633.77 & 311.77 & 446808.79 & 0 & 489818.21 \\
\hline 7557.53 & 462535 & 16 & 0 & 982.04 & 20820.2 & 935.92 & 2418.67 & 134.05 & 375069 & 0 & 460815.42 \\
\hline
\end{tabular}




\begin{tabular}{|c|c|c|c|c|c|c|c|c|c|c|c|}
\hline True Depth & XRF tCOUNTS & XRF Live Time & $\mathrm{Ti}(\mathrm{ppm})$ & $\mathrm{V}(\mathrm{ppm})$ & $\mathrm{Fe}(\mathrm{ppm})$ & $\mathrm{Ni}(\mathrm{ppm})$ & $\mathrm{Cu}(\mathrm{ppm})$ & $\mathrm{Pb}(\mathrm{ppm})$ & LE (ppm) & $\mathrm{Mg}(\mathrm{ppm})$ & $\mathrm{Cl}(\mathrm{ppm})$ \\
\hline 7557.60 & 450726 & 16 & 0 & 1761.95 & 1166.77 & 1488.36 & 3871.2 & 205.68 & 400326.18 & 0 & 417960.37 \\
\hline 7557.66 & 481631 & 16 & 0 & 836.96 & 771.88 & 615.3 & 1601.18 & 84.02 & 394118.94 & 0 & 478842.86 \\
\hline 7557.73 & 498842 & 15 & 175.14 & 1059.55 & 2063.57 & 736.7 & 1915.53 & 101.1 & 401802.49 & 0 & 476870.95 \\
\hline 7557.80 & 606336 & 14 & 0 & 678.17 & 562.24 & 464.6 & 1201.61 & 63.66 & 418536.85 & 0 & 512692.15 \\
\hline 7557.86 & 587553 & 14 & 0 & 2324.43 & 2276.42 & 2074.9 & 5400.95 & 295.88 & 353304.5 & 0 & 219520.14 \\
\hline 7557.93 & 648111 & 14 & 0 & 2153.54 & 2337.93 & 1762.63 & 4589.61 & 246.79 & 327745.06 & 0 & 207452.05 \\
\hline 7557.99 & 664767 & 13 & 0 & 2241.27 & 2354.97 & 1743.64 & 4532.11 & 245.17 & 350150.32 & 0 & 174244.37 \\
\hline 7558.06 & 623808 & 14 & 0 & 2331.16 & 2421.94 & 1954.34 & 5091.4 & 282.14 & 447025.6 & 0 & 45367.58 \\
\hline 7558.12 & 573901 & 15 & 396.89 & 962.29 & 620.84 & 554.61 & 1442.69 & 77.22 & 403550.79 & 0 & 513964.03 \\
\hline
\end{tabular}




\begin{tabular}{|c|c|c|c|c|c|c|c|c|}
\hline True Depth & XRF tCOUNTS & XRF Live Time & $\mathrm{Si}(\mathrm{ppm})$ & $\mathrm{Al}(\mathrm{ppm})$ & $\mathrm{P}(\mathrm{ppm})$ & $\mathrm{S}(\mathrm{ppm})$ & $\mathrm{K}(\mathrm{ppm})$ & $\mathrm{Ca}(\mathrm{ppm})$ \\
\hline $\mathrm{Ft}$ & Total Counts & secs & ppm & $\mathrm{ppm}$ & ppm & $\mathrm{ppm}$ & $\mathrm{ppm}$ & $\mathrm{ppm}$ \\
\hline 7445.07 & 170922 & 9 & 135411.58 & 31362.57 & 0 & 4931.72 & 0 & 3681.76 \\
\hline 7445.13 & 165018 & 9 & 261516.62 & 60076.39 & 0 & 10954.84 & 0 & 4622.89 \\
\hline 7445.20 & 273658 & 7 & 26390.73 & 6193.41 & 0 & 3138.73 & 0 & 272471.03 \\
\hline 7445.26 & 335892 & 7 & 14093.65 & 5657.76 & 0 & 2809.08 & 0 & 116681.47 \\
\hline 7445.33 & 177759 & 9 & 107210.8 & 23310.03 & 0 & 4848.47 & 0 & 10597.49 \\
\hline 7445.39 & 94792 & 9 & 66298.13 & 14873.91 & 0 & 3534.81 & 0 & 5577.71 \\
\hline 7445.46 & 144070 & 9 & 284884.25 & 61312.36 & 0 & 12818.63 & 15312.03 & 5145.53 \\
\hline 7445.52 & 176220 & 9 & 226064.09 & 56636.93 & 0 & 11793.59 & 6700.14 & 6510.42 \\
\hline 7445.59 & 160496 & 9 & 329754.19 & 71688.69 & 0 & 13115.27 & 14387.02 & 3693.24 \\
\hline 7445.66 & 162045 & 9 & 338153.61 & 79763.15 & 0 & 14624.5 & 10328.78 & 3442.04 \\
\hline 7445.72 & 196806 & 8 & 170086.85 & 43241.74 & 0 & 8918.95 & 0 & 4898.03 \\
\hline 7445.79 & 172408 & 9 & 306697.39 & 75620.45 & 0 & 19526.25 & 9563.6 & 7869.37 \\
\hline 7445.85 & 169505 & 9 & 347241.37 & 79510.26 & 0 & 16521.17 & 10361.46 & 7399.02 \\
\hline 7445.92 & 177975 & 9 & 328875.98 & 70807.38 & 0 & 21237.58 & 7243.16 & 10632.72 \\
\hline 7445.98 & 187336 & 8 & 228175.1 & 54519.67 & 0 & 16432.37 & 0 & 11238.98 \\
\hline 7446.05 & 173576 & 9 & 316894.28 & 72441.81 & 0 & 21601.1 & 9948.47 & 11336.92 \\
\hline 7446.12 & 169737 & 9 & 314583.43 & 71152.48 & 0 & 19803.02 & 7919.88 & 9379.88 \\
\hline 7446.18 & 165540 & 9 & 319997.93 & 73476.45 & 0 & 15548.92 & 8284.23 & 8094.45 \\
\hline 7446.25 & 173445 & 9 & 205442.92 & 49679.7 & 0 & 12073.07 & 0 & 8941.38 \\
\hline 7446.31 & 173943 & 9 & 313909.16 & 75281.07 & 0 & 18723.6 & 0 & 7694.38 \\
\hline 7446.38 & 176698 & 9 & 371365.4 & 90687.15 & 0 & 19286.2 & 13121.56 & 6411.8 \\
\hline 7446.44 & 168545 & 9 & 317950.32 & 77299.79 & 0 & 23950.58 & 12065.38 & 10490.98 \\
\hline 7446.51 & 170287 & 9 & 314274.65 & 73915.75 & 0 & 18424 & 15383.83 & 8352.89 \\
\hline 7446.57 & 165904 & 9 & 308626.13 & 75567.78 & 0 & 17380.46 & 16773.82 & 5517.79 \\
\hline 7446.64 & 218623 & 8 & 112595.61 & 31115.12 & 0 & 8760.58 & 0 & 15599.37 \\
\hline 7446.71 & 121559 & 9 & 253673.91 & 65755.07 & 0 & 10528.79 & 18735.87 & 2457.69 \\
\hline 7446.77 & 170805 & 9 & 365013.78 & 95147.55 & 0 & 14190.92 & 20948.4 & 1675.36 \\
\hline 7446.84 & 169237 & 9 & 340846.63 & 88018.47 & 0 & 12468.5 & 22115.7 & 1957.13 \\
\hline 7446.90 & 263464 & 8 & 69523.75 & 19498.46 & 0 & 2152.31 & 0 & 0 \\
\hline 7446.97 & 160042 & 9 & 329061.57 & 78548.13 & 0 & 12827.44 & 19238.54 & 2523.93 \\
\hline 7447.03 & 52256 & 10 & 140227.17 & 32561.63 & 0 & 6735.98 & 0 & 14419.37 \\
\hline 7447.10 & 130645 & 9 & 350211.32 & 89791.82 & 0 & 16244.04 & 0 & 2299.38 \\
\hline
\end{tabular}




\begin{tabular}{|c|c|c|c|c|c|c|c|c|}
\hline True Depth & XRF tCOUNTS & XRF Live Time & $\mathrm{Si}(\mathrm{ppm})$ & $\mathrm{Al}(\mathrm{ppm})$ & $\mathrm{P}(\mathrm{ppm})$ & $\mathrm{S}(\mathrm{ppm})$ & $\mathrm{K}(\mathrm{ppm})$ & $\mathrm{Ca}(\mathrm{ppm})$ \\
\hline 7447.17 & 161022 & 9 & 325743.55 & 78093.75 & 0 & 16744.26 & 17092.63 & 2431.92 \\
\hline 7447.23 & 137003 & 9 & 255898.12 & 56356.2 & 0 & 12449.19 & 15759.95 & 6381.12 \\
\hline 7447.30 & 125621 & 9 & 243760.3 & 54144.7 & 0 & 11944.33 & 17973.48 & 5996 \\
\hline 7447.36 & 125198 & 9 & 245424.27 & 55904.54 & 0 & 11183.05 & 15942.9 & 7745.24 \\
\hline 7447.43 & 193161 & 8 & 63873.43 & 14681.09 & 0 & 5060.14 & 0 & 9571.84 \\
\hline 7447.49 & 244533 & 8 & 80152.24 & 18788.02 & 0 & 6070.14 & 0 & 6769.09 \\
\hline 7447.56 & 140276 & 9 & 136833.15 & 31328.19 & 0 & 7576.1 & 0 & 4367.57 \\
\hline 7447.62 & 74098 & 9 & 71623.63 & 15351 & 0 & 5185.66 & 0 & 6686.13 \\
\hline 7447.69 & 72833 & 9 & 100275.46 & 15987.96 & 0 & 6931.23 & 0 & 5929.8 \\
\hline 7447.76 & 179338 & 8 & 109302.53 & 28781.95 & 0 & 6761.95 & 0 & 6013.38 \\
\hline 7447.82 & 148904 & 9 & 265159.88 & 63804.58 & 0 & 13676.7 & 15677.96 & 4377.63 \\
\hline 7447.89 & 179840 & 8 & 290873.79 & 72298.14 & 0 & 16698.74 & 9166.94 & 5422.94 \\
\hline 7447.95 & 114874 & 9 & 207975.25 & 46644.58 & 0 & 10639.36 & 18695.32 & 5186.63 \\
\hline 7448.02 & 113571 & 9 & 203342.31 & 40263.59 & 0 & 11817.12 & 13944.61 & 5628.31 \\
\hline 7448.08 & 194260 & 8 & 211935.51 & 53828.92 & 0 & 10885.58 & 7536.29 & 2552.11 \\
\hline 7448.15 & 239453 & 8 & 82437.09 & 23243.78 & 0 & 6531.77 & 0 & 7376.29 \\
\hline 7448.22 & 176814 & 9 & 97120.98 & 22173.54 & 0 & 7462.19 & 0 & 5436.61 \\
\hline 7448.28 & 150670 & 9 & 291624.94 & 73276.79 & 0 & 13462.12 & 23903 & 2757.89 \\
\hline 7448.35 & 154185 & 9 & 317985.07 & 78738.56 & 0 & 13008.39 & 11320.76 & 2043.02 \\
\hline 7448.41 & 195635 & 8 & 154357.39 & 39047.36 & 0 & 10651.44 & 0 & 9535.05 \\
\hline 7448.48 & 192996 & 8 & 148775.2 & 41903.35 & 0 & 7929.41 & 0 & 5645.6 \\
\hline 7448.54 & 172854 & 9 & 227447.82 & 60551.46 & 0 & 12079.87 & 10899.96 & 4914.56 \\
\hline 7448.61 & 179107 & 8 & 197775.96 & 51157.59 & 0 & 17057.24 & 0 & 10760.1 \\
\hline 7448.67 & 189592 & 8 & 129335.01 & 34012.1 & 0 & 9836.09 & 0 & 10823.01 \\
\hline 7448.74 & 215322 & 8 & 111068.19 & 27508.81 & 0 & 6521.63 & 0 & 7561.31 \\
\hline 7448.81 & 250585 & 8 & 70790.61 & 19249.19 & 0 & 7954.35 & 0 & 14895.7 \\
\hline 7448.87 & 261608 & 8 & 84751.41 & 22678.16 & 0 & 5228.8 & 0 & 7433.57 \\
\hline 7448.94 & 296772 & 7 & 38931.44 & 9433.47 & 0 & 4648.48 & 0 & 8222.05 \\
\hline 7449.00 & 266326 & 8 & 80603.92 & 19845.31 & 0 & 4411.93 & 0 & 12869.09 \\
\hline 7449.07 & 109662 & 9 & 340192.4 & 79901.93 & 0 & 17082 & 0 & 30169.07 \\
\hline 7449.13 & 236288 & 8 & 70072.57 & 18090.96 & 0 & 4003.25 & 0 & 12017.54 \\
\hline 7449.20 & 160888 & 9 & 304465.61 & 74068.93 & 0 & 13344.63 & 19173.7 & 10930.38 \\
\hline 7449.27 & 214744 & 8 & 98616.12 & 26949.02 & 0 & 8342.8 & 0 & 7576.01 \\
\hline
\end{tabular}




\begin{tabular}{|c|c|c|c|c|c|c|c|c|}
\hline True Depth & XRF tCOUNTS & XRF Live Time & $\mathrm{Si}(\mathrm{ppm})$ & $\mathrm{Al}(\mathrm{ppm})$ & $\mathrm{P}(\mathrm{ppm})$ & $\mathrm{S}(\mathrm{ppm})$ & $\mathrm{K}(\mathrm{ppm})$ & $\mathrm{Ca}(\mathrm{ppm})$ \\
\hline 7449.33 & 197198 & 8 & 132125.67 & 35030.39 & 0 & 11170.65 & 0 & 9498.63 \\
\hline 7449.40 & 173301 & 9 & 340579.31 & 83015.09 & 0 & 17824.92 & 14950.7 & 2781.89 \\
\hline 7449.46 & 172660 & 9 & 295434.83 & 75229.37 & 0 & 16963.42 & 13012.69 & 5259.69 \\
\hline 7449.53 & 197068 & 8 & 172967.37 & 44113.67 & 0 & 10114.19 & 0 & 6792.86 \\
\hline 7449.59 & 232560 & 8 & 78485.82 & 21100.68 & 0 & 5443.21 & 0 & 7098.54 \\
\hline 7449.66 & 201972 & 8 & 139230.2 & 39205.53 & 0 & 7356.82 & 0 & 6059.22 \\
\hline 7449.72 & 178913 & 9 & 237056.03 & 59420.54 & 0 & 14300.99 & 8694.29 & 7686.71 \\
\hline 7449.79 & 204963 & 8 & 127443.52 & 33707.79 & 0 & 6316.67 & 0 & 4700.92 \\
\hline 7449.86 & 170674 & 9 & 225251.91 & 53849.49 & 0 & 16470.77 & 8139.41 & 9355.01 \\
\hline 7449.92 & 216279 & 8 & 85062.52 & 21982.72 & 0 & 4677.43 & 0 & 3647.85 \\
\hline 7449.99 & 215148 & 8 & 98606.42 & 24813.32 & 0 & 7299.55 & 0 & 7665.62 \\
\hline 7450.05 & 218043 & 8 & 84943.95 & 23232.26 & 0 & 4867.9 & 0 & 3927.55 \\
\hline 7450.12 & 213812 & 8 & 122997.02 & 33441.99 & 0 & 5474.24 & 0 & 3701.93 \\
\hline 7450.18 & 207701 & 8 & 142866.1 & 37218.76 & 0 & 10130.85 & 0 & 7237.51 \\
\hline 7450.25 & 233191 & 8 & 78947.39 & 19422.9 & 0 & 4926.68 & 0 & 4042.12 \\
\hline 7450.31 & 218845 & 8 & 77062.53 & 19878.3 & 0 & 5838.89 & 0 & 7246.71 \\
\hline 7450.38 & 183218 & 8 & 196583.23 & 48502.26 & 0 & 18493.77 & 0 & 6751.78 \\
\hline 7450.45 & 182249 & 8 & 231396.52 & 56112.45 & 0 & 16727.22 & 0 & 6167.94 \\
\hline 7450.51 & 206715 & 8 & 142523.8 & 34576.5 & 0 & 7849.32 & 0 & 4114.16 \\
\hline 7450.58 & 190640 & 8 & 156861.26 & 39160.42 & 0 & 8225.51 & 0 & 3703.73 \\
\hline 7450.64 & 190098 & 8 & 159375.45 & 41452 & 0 & 7830.78 & 0 & 3466.23 \\
\hline 7450.71 & 233946 & 8 & 93351.73 & 21588.87 & 0 & 5624.41 & 0 & 3980.65 \\
\hline 7450.77 & 205947 & 8 & 129195.25 & 30929.17 & 0 & 5914.92 & 0 & 2741.87 \\
\hline 7450.84 & 174777 & 9 & 311283.56 & 65613.59 & 0 & 18817.38 & 0 & 1995.52 \\
\hline 7450.91 & 255251 & 8 & 82910.82 & 21742.41 & 0 & 3725.02 & 0 & 796.46 \\
\hline 7450.97 & 249644 & 8 & 77953.75 & 20655.68 & 0 & 2343.77 & 0 & 0 \\
\hline 7451.04 & 243829 & 8 & 55153.76 & 12597.22 & 0 & 2616.89 & 0 & 936.95 \\
\hline 7451.10 & 81674 & 9 & 269848.86 & 63476.7 & 0 & 14477.23 & 0 & 0 \\
\hline 7451.17 & 200915 & 8 & 163861.62 & 42554.11 & 0 & 6700.2 & 0 & 3261.28 \\
\hline 7451.23 & 174319 & 9 & 223095.33 & 51140.05 & 0 & 11611.94 & 0 & 5912.76 \\
\hline 7451.30 & 203195 & 8 & 126828.16 & 30312.49 & 0 & 7427.57 & 0 & 6331.51 \\
\hline 7451.36 & 175768 & 9 & 209178.26 & 50612.9 & 0 & 13325.91 & 6774.1 & 10118.27 \\
\hline 7451.43 & 194837 & 8 & 124093.17 & 29830.54 & 0 & 7751.54 & 0 & 7975.15 \\
\hline
\end{tabular}




\begin{tabular}{|c|c|c|c|c|c|c|c|c|}
\hline True Depth & XRF tCOUNTS & XRF Live Time & $\mathrm{Si}(\mathrm{ppm})$ & $\mathrm{Al}(\mathrm{ppm})$ & $\mathrm{P}(\mathrm{ppm})$ & $\mathrm{S}(\mathrm{ppm})$ & $\mathrm{K}(\mathrm{ppm})$ & $\mathrm{Ca}(\mathrm{ppm})$ \\
\hline 7451.50 & 205807 & 8 & 115814.95 & 28052.03 & 0 & 8349.99 & 0 & 11723.67 \\
\hline 7451.56 & 225623 & 8 & 84389.54 & 21520.42 & 0 & 5434.93 & 0 & 7155.64 \\
\hline 7451.63 & 219805 & 8 & 85267.94 & 19922.23 & 0 & 6783.1 & 0 & 10167.11 \\
\hline 7451.69 & 156633 & 9 & 168006.32 & 39209.54 & 0 & 10266.08 & 0 & 8406.38 \\
\hline 7451.76 & 187391 & 8 & 245729.2 & 55941.76 & 0 & 13456.63 & 0 & 5985.11 \\
\hline 7451.82 & 224286 & 8 & 92626.95 & 23329.49 & 0 & 5262.67 & 0 & 3465.49 \\
\hline 7451.89 & 208009 & 8 & 94229.43 & 25363.44 & 0 & 5448.79 & 0 & 5868.58 \\
\hline 7451.96 & 189204 & 8 & 116388.25 & 31218.21 & 0 & 5312.13 & 0 & 3791.89 \\
\hline 7452.02 & 163333 & 9 & 184428.32 & 47281.52 & 0 & 10497.38 & 6061.13 & 4427.27 \\
\hline 7452.09 & 157070 & 9 & 316706.52 & 72692.17 & 0 & 14724.98 & 12961.15 & 1946.88 \\
\hline 7452.15 & 239744 & 8 & 66317.97 & 17913 & 0 & 4189.5 & 0 & 4840.25 \\
\hline 7452.22 & 158193 & 9 & 310421.56 & 67166.15 & 0 & 16199.15 & 11747.91 & 2165.32 \\
\hline 7452.28 & 152835 & 9 & 312414.24 & 64846.24 & 0 & 16355.21 & 10296.59 & 2172.47 \\
\hline 7452.35 & 166375 & 9 & 204012.47 & 45255.72 & 0 & 11306.4 & 0 & 3183.4 \\
\hline 7452.41 & 171250 & 9 & 262597.54 & 61525.56 & 0 & 10337.81 & 0 & 2187.6 \\
\hline 7452.48 & 162478 & 9 & 329733.01 & 71803.9 & 0 & 17142.33 & 0 & 3782.94 \\
\hline 7452.55 & 158491 & 9 & 271402.97 & 61921.59 & 0 & 14467.86 & 0 & 4601.46 \\
\hline 7452.61 & 232578 & 8 & 57483.5 & 14906.73 & 0 & 6347.7 & 0 & 11547.13 \\
\hline 7452.68 & 192125 & 8 & 116426.67 & 30689.56 & 0 & 11500.83 & 0 & 9806.4 \\
\hline 7452.74 & 196685 & 8 & 105874.72 & 26153.7 & 0 & 5954.9 & 0 & 5658.67 \\
\hline 7452.81 & 174186 & 9 & 215947.01 & 50792.33 & 0 & 12822.41 & 0 & 5005.89 \\
\hline 7452.87 & 233967 & 8 & 72203.09 & 18747.87 & 0 & 3977.56 & 0 & 3266.18 \\
\hline 7452.94 & 259487 & 8 & 68409.71 & 16718.46 & 0 & 5219.06 & 0 & 4170.56 \\
\hline 7453.01 & 169326 & 9 & 343943.06 & 75751.33 & 0 & 22309.87 & 0 & 3587.51 \\
\hline 7453.07 & 187325 & 8 & 216837.83 & 55835.49 & 0 & 15595.59 & 0 & 5367.11 \\
\hline 7453.14 & 80278 & 9 & 189118.74 & 47699.84 & 0 & 11674.48 & 0 & 5299.04 \\
\hline 7453.20 & 183311 & 8 & 155001.02 & 36921.04 & 0 & 7512.55 & 0 & 2641.34 \\
\hline 7453.27 & 175432 & 9 & 154265.82 & 34631.68 & 0 & 8599.03 & 0 & 3671.61 \\
\hline 7453.33 & 142420 & 9 & 232855.44 & 52065.96 & 0 & 10133.74 & 12015.1 & 2184.04 \\
\hline 7453.40 & 150409 & 9 & 298740.27 & 65829.35 & 0 & 16101.16 & 9783.42 & 2658.73 \\
\hline 7453.46 & 134299 & 9 & 296880.39 & 66010.43 & 0 & 18460.43 & 0 & 4595.45 \\
\hline 7453.53 & 165831 & 9 & 328902.46 & 68129.91 & 0 & 21255.4 & 0 & 7499.22 \\
\hline 7453.60 & 157350 & 9 & 244439.33 & 59548.6 & 0 & 9982.1 & 11577.81 & 3782.12 \\
\hline
\end{tabular}




\begin{tabular}{|c|c|c|c|c|c|c|c|c|}
\hline True Depth & XRF tCOUNTS & XRF Live Time & $\mathrm{Si}(\mathrm{ppm})$ & $\mathrm{Al}(\mathrm{ppm})$ & $\mathrm{P}(\mathrm{ppm})$ & $\mathrm{S}(\mathrm{ppm})$ & $\mathrm{K}(\mathrm{ppm})$ & $\mathrm{Ca}(\mathrm{ppm})$ \\
\hline 7453.66 & 157706 & 9 & 327381.87 & 74953.57 & 0 & 16295.29 & 15213.55 & 2293.59 \\
\hline 7453.73 & 216884 & 8 & 87095.03 & 23004.83 & 0 & 4036.9 & 0 & 3248.13 \\
\hline 7453.79 & 182624 & 8 & 190021.78 & 50127.78 & 0 & 9183.99 & 0 & 3254.64 \\
\hline 7453.86 & 163164 & 9 & 302923.5 & 73038.77 & 0 & 19581.33 & 15405.69 & 5891.87 \\
\hline 7453.92 & 226341 & 8 & 97112.51 & 24012.48 & 0 & 6441.97 & 0 & 6750.08 \\
\hline 7453.99 & 259182 & 8 & 56892.84 & 15925.81 & 0 & 4358.1 & 0 & 4256.26 \\
\hline 7454.06 & 248408 & 8 & 87835.87 & 24953.18 & 0 & 7721.71 & 0 & 7632.89 \\
\hline 7454.12 & 262622 & 8 & 33407.42 & 8233.47 & 0 & 3918.11 & 0 & 2366.21 \\
\hline 7454.19 & 275708 & 7 & 59115.03 & 17622.67 & 0 & 3931.9 & 0 & 2767.73 \\
\hline 7454.25 & 237367 & 8 & 77752.27 & 19186.07 & 0 & 4532.97 & 0 & 8292.99 \\
\hline 7454.32 & 234048 & 8 & 74241.01 & 20865 & 0 & 11564.89 & 0 & 12319.82 \\
\hline 7454.38 & 212750 & 8 & 64054.49 & 17233.32 & 0 & 10482.43 & 0 & 13811.43 \\
\hline 7454.45 & 221477 & 8 & 131908.88 & 38907.43 & 0 & 17511.05 & 0 & 21904 \\
\hline 7454.51 & 240127 & 8 & 93833.52 & 25692.46 & 0 & 8102.46 & 0 & 9556.87 \\
\hline 7454.58 & 207750 & 8 & 98999.75 & 25028.57 & 0 & 2806.3 & 0 & 10234.33 \\
\hline 7454.65 & 219414 & 8 & 130531.78 & 33345.95 & 0 & 5041.83 & 0 & 107792.37 \\
\hline 7454.71 & 180455 & 8 & 30361.2 & 4797.05 & 0 & 4916.26 & 0 & 66559.19 \\
\hline 7454.78 & 149624 & 9 & 41764.5 & 7699.3 & 0 & 1853.78 & 0 & 55317.51 \\
\hline 7454.84 & 157367 & 9 & 29350.21 & 3220.53 & 0 & 1546.25 & 0 & 60299.61 \\
\hline 7454.91 & 172788 & 9 & 44595.64 & 10468.83 & 0 & 787 & 0 & 54814.85 \\
\hline 7454.97 & 194175 & 8 & 119521.1 & 28851.47 & 0 & 847.49 & 0 & 96126.38 \\
\hline 7455.04 & 234541 & 8 & 49322.41 & 9090.74 & 0 & 1230.76 & 0 & 39100.08 \\
\hline 7455.10 & 193666 & 8 & 164444.16 & 33481.09 & 0 & 2173.01 & 0 & 128509.82 \\
\hline 7455.17 & 195566 & 8 & 135200.61 & 38650.2 & 0 & 4410.12 & 0 & 114661.3 \\
\hline 7455.24 & 252674 & 8 & 116937.35 & 31740.72 & 0 & 6196.37 & 0 & 108019.93 \\
\hline 7455.30 & 219956 & 8 & 65121.73 & 16176.41 & 0 & 2157.63 & 0 & 107143.45 \\
\hline 7455.37 & 191575 & 8 & 97752.22 & 21023.9 & 0 & 3563.8 & 0 & 115838.5 \\
\hline 7455.43 & 195416 & 8 & 55686.13 & 17921.18 & 0 & 2049.95 & 0 & 104338.79 \\
\hline 7455.50 & 239022 & 8 & 61674.39 & 18470.37 & 0 & 3029.97 & 0 & 103567.36 \\
\hline 7455.56 & 317691 & 7 & 54956.35 & 14620.09 & 0 & 3548.18 & 0 & 288783.18 \\
\hline 7455.63 & 324870 & 7 & 41998.49 & 11140.3 & 0 & 2522.64 & 0 & 315800.93 \\
\hline 7455.70 & 291796 & 7 & 81327.57 & 24462.36 & 0 & 5579.53 & 0 & 285070.67 \\
\hline 7455.76 & 300163 & 7 & 104717.08 & 33007.28 & 0 & 6946.74 & 0 & 329019.83 \\
\hline
\end{tabular}




\begin{tabular}{|c|c|c|c|c|c|c|c|c|}
\hline True Depth & XRF tCOUNTS & XRF Live Time & $\mathrm{Si}(\mathrm{ppm})$ & $\mathrm{Al}(\mathrm{ppm})$ & $\mathrm{P}(\mathrm{ppm})$ & $\mathrm{S}(\mathrm{ppm})$ & $\mathrm{K}(\mathrm{ppm})$ & $\mathrm{Ca}(\mathrm{ppm})$ \\
\hline 7455.83 & 297591 & 7 & 50854.26 & 15891.1 & 0 & 4206.24 & 0 & 259424.42 \\
\hline 7455.89 & 186074 & 8 & 219128 & 72244.52 & 0 & 36766.22 & 25050.26 & 24404.74 \\
\hline 7455.96 & 303014 & 7 & 36710.69 & 12299.96 & 0 & 3800.73 & 0 & 2469.33 \\
\hline 7456.02 & 168233 & 9 & 255155.19 & 84402.56 & 0 & 12272.19 & 34319.05 & 3716.74 \\
\hline 7456.09 & 179948 & 8 & 181591.74 & 59807.03 & 0 & 11701.82 & 23748.98 & 6678.77 \\
\hline 7456.15 & 208448 & 8 & 139162.49 & 42811.54 & 0 & 19148.43 & 0 & 16771.13 \\
\hline 7456.22 & 280690 & 7 & 51178.13 & 18245.39 & 0 & 6415.6 & 0 & 10466.04 \\
\hline 7456.29 & 206612 & 8 & 143324.62 & 43660.75 & 0 & 8552.41 & 10406.75 & 5187.44 \\
\hline 7456.35 & 175778 & 9 & 247230.08 & 75007.21 & 0 & 13028.53 & 30708.89 & 2899.32 \\
\hline 7456.42 & 214585 & 8 & 83946.78 & 28090.57 & 0 & 8321.32 & 0 & 10898.35 \\
\hline 7456.48 & 168004 & 9 & 315590.29 & 85552.05 & 0 & 22468.02 & 23600.79 & 8947.77 \\
\hline 7456.55 & 189038 & 8 & 145870.8 & 43189.88 & 0 & 18784.68 & 0 & 17092.88 \\
\hline 7456.61 & 234629 & 8 & 100111.92 & 31550.25 & 0 & 11054.82 & 0 & 14643.66 \\
\hline 7456.68 & 207877 & 8 & 105448.23 & 32632.24 & 0 & 13781.42 & 0 & 33508.63 \\
\hline 7456.75 & 128757 & 9 & 112682.67 & 27666.31 & 0 & 11161.16 & 13002.02 & 10434.41 \\
\hline 7456.81 & 129530 & 9 & 105693.43 & 26403.57 & 0 & 9882.06 & 10369.19 & 9931.06 \\
\hline 7456.88 & 178226 & 8 & 105479.37 & 30847.17 & 0 & 13276.21 & 0 & 14952.62 \\
\hline 7456.94 & 221375 & 8 & 44018.7 & 10308.29 & 0 & 6016.56 & 0 & 11666.04 \\
\hline 7457.01 & 207021 & 8 & 65828.75 & 16980.28 & 0 & 11355.62 & 0 & 17404.8 \\
\hline 7457.07 & 169687 & 9 & 57590.58 & 14887.91 & 0 & 4272.74 & 0 & 6569.29 \\
\hline 7457.14 & 214375 & 8 & 39874.03 & 11441.03 & 0 & 2316.8 & 0 & 567.79 \\
\hline 7457.20 & 121398 & 9 & 186738.02 & 54190.21 & 0 & 11096.76 & 0 & 4130.49 \\
\hline 7457.27 & 176205 & 9 & 284541.11 & 76477.28 & 0 & 21838.14 & 18705.21 & 5672.78 \\
\hline 7457.34 & 168315 & 9 & 256529.06 & 64119.23 & 0 & 20427.62 & 18090.18 & 8268.85 \\
\hline 7457.40 & 200087 & 8 & 105547.45 & 29480.78 & 0 & 7410.78 & 0 & 7813.27 \\
\hline 7457.47 & 176557 & 9 & 208873 & 53275.94 & 0 & 14694.3 & 17080.26 & 7121.04 \\
\hline 7457.53 & 187785 & 8 & 135453.49 & 37082.96 & 0 & 9065.76 & 6456.82 & 10738.26 \\
\hline 7457.60 & 165199 & 9 & 307550.98 & 79010.93 & 0 & 17943.5 & 20010.59 & 4220.52 \\
\hline 7457.66 & 179640 & 8 & 280719.72 & 76909.95 & 0 & 18007.57 & 18705.53 & 5745.16 \\
\hline 7457.73 & 179406 & 9 & 252223.81 & 65382.15 & 0 & 16352.95 & 18214.18 & 9022.18 \\
\hline 7457.80 & 182719 & 8 & 226440.5 & 66086.71 & 0 & 16740.98 & 18359.65 & 10498.78 \\
\hline 7457.86 & 169085 & 9 & 240591.47 & 67100.07 & 0 & 16994.1 & 19559.15 & 8659.25 \\
\hline 7457.93 & 226927 & 8 & 97026.1 & 27460.95 & 0 & 8966.88 & 0 & 11828.94 \\
\hline
\end{tabular}




\begin{tabular}{|c|c|c|c|c|c|c|c|c|}
\hline True Depth & XRF tCOUNTS & XRF Live Time & $\mathrm{Si}(\mathrm{ppm})$ & $\mathrm{Al}(\mathrm{ppm})$ & $\mathrm{P}(\mathrm{ppm})$ & $\mathrm{S}(\mathrm{ppm})$ & $\mathrm{K}(\mathrm{ppm})$ & $\mathrm{Ca}(\mathrm{ppm})$ \\
\hline 7457.99 & 196554 & 8 & 153938.51 & 42117.57 & 0 & 11530.11 & 6917.48 & 10576.99 \\
\hline 7458.06 & 176824 & 9 & 211807.27 & 54516.52 & 0 & 15387.11 & 16958.61 & 11053.98 \\
\hline 7458.12 & 182244 & 8 & 168839.92 & 46356.68 & 0 & 11784.75 & 14783.37 & 7902.08 \\
\hline 7458.19 & 205804 & 8 & 149046.72 & 41374.7 & 0 & 10546.69 & 0 & 6737.58 \\
\hline 7458.25 & 261503 & 8 & 63855.69 & 20715.21 & 0 & 4891.87 & 0 & 5665.54 \\
\hline 7458.32 & 204897 & 8 & 132631.56 & 41098.4 & 0 & 11719.73 & 6817.84 & 10664.9 \\
\hline 7458.39 & 191456 & 8 & 128767.07 & 40474.48 & 0 & 11336.43 & 8938.95 & 11615.2 \\
\hline 7458.45 & 197284 & 8 & 118900.19 & 35684.23 & 0 & 10430.99 & 6827.1 & 10679.05 \\
\hline 7458.52 & 182063 & 8 & 186976.08 & 58959 & 0 & 7437.99 & 11753.48 & 2550.44 \\
\hline 7458.58 & 245912 & 8 & 73199 & 21408.26 & 0 & 6721.35 & 0 & 8846.65 \\
\hline 7458.65 & 251245 & 8 & 66283.2 & 19865.89 & 0 & 6206.2 & 0 & 7372.73 \\
\hline 7458.71 & 174433 & 9 & 245224.68 & 67819.35 & 0 & 19428.75 & 22516.45 & 8310.72 \\
\hline 7458.78 & 194230 & 8 & 147797.29 & 43652.63 & 0 & 13136.17 & 7429.61 & 18376.4 \\
\hline 7458.85 & 254246 & 8 & 85529.88 & 25424.98 & 0 & 8492.5 & 0 & 7910.26 \\
\hline 7458.91 & 232726 & 8 & 84057.18 & 25736.86 & 0 & 10438.91 & 0 & 13495.09 \\
\hline 7458.98 & 250529 & 8 & 83017.34 & 27048.74 & 0 & 10432.21 & 0 & 14419.45 \\
\hline 7459.04 & 220523 & 8 & 166769.78 & 48162.57 & 0 & 19281.12 & 6715.38 & 10811.79 \\
\hline 7459.11 & 187700 & 8 & 300906.7 & 85380.38 & 0 & 27609.04 & 22605.28 & 7443.45 \\
\hline 7459.17 & 200905 & 8 & 145492.92 & 43013.43 & 0 & 11145.97 & 12007.15 & 6754.45 \\
\hline 7459.24 & 112820 & 9 & 256286.03 & 83206.27 & 0 & 16007.61 & 0 & 3944 \\
\hline 7459.30 & 176080 & 9 & 243572.2 & 73777.37 & 0 & 14630.83 & 26102.65 & 4211.61 \\
\hline 7459.37 & 190638 & 8 & 127216.88 & 37991.64 & 0 & 9917.2 & 10567.75 & 9361.4 \\
\hline 7459.44 & 173818 & 9 & 106202.37 & 32974.58 & 0 & 9413.64 & 0 & 11620.27 \\
\hline 7459.50 & 205407 & 8 & 134437.18 & 42129.33 & 0 & 10967.8 & 9940.27 & 11057.23 \\
\hline 7459.57 & 175008 & 9 & 255721.67 & 76403.49 & 0 & 18772.08 & 26796.31 & 10202.52 \\
\hline 7459.63 & 181969 & 8 & 216761.59 & 65859.8 & 0 & 14468.54 & 23007.78 & 9687.12 \\
\hline 7459.70 & 229069 & 8 & 81435.67 & 26126.6 & 0 & 7530.51 & 0 & 10005.94 \\
\hline 7459.76 & 191592 & 8 & 173030.75 & 53077.71 & 0 & 14914.77 & 17539.54 & 9769.52 \\
\hline 7459.83 & 171624 & 9 & 210467.03 & 65997.95 & 0 & 14281.06 & 21470.2 & 8133.25 \\
\hline 7459.90 & 171071 & 9 & 274131.68 & 78304.15 & 0 & 16527.98 & 30007.15 & 4150.95 \\
\hline 7459.96 & 188227 & 8 & 163007.48 & 50112.27 & 0 & 16234.4 & 14769.8 & 11114.22 \\
\hline 7460.03 & 201296 & 8 & 122250.68 & 38650.44 & 0 & 10278.77 & 0 & 11092.83 \\
\hline 7460.09 & 183526 & 8 & 155627.1 & 46965.72 & 0 & 13508.56 & 15590.59 & 11034.76 \\
\hline
\end{tabular}




\begin{tabular}{|c|c|c|c|c|c|c|c|c|}
\hline True Depth & XRF tCOUNTS & XRF Live Time & $\mathrm{Si}(\mathrm{ppm})$ & $\mathrm{Al}(\mathrm{ppm})$ & $\mathrm{P}(\mathrm{ppm})$ & $\mathrm{S}(\mathrm{ppm})$ & $\mathrm{K}(\mathrm{ppm})$ & $\mathrm{Ca}(\mathrm{ppm})$ \\
\hline 7460.16 & 200355 & 8 & 113819.27 & 34504.4 & 0 & 14076.29 & 0 & 16493.87 \\
\hline 7460.22 & 195052 & 8 & 220266.82 & 67476 & 0 & 13777.29 & 22913.92 & 4967.14 \\
\hline 7460.29 & 223675 & 8 & 101462.13 & 31068.64 & 0 & 8451.37 & 0 & 8468.55 \\
\hline 7460.35 & 166961 & 9 & 291292.94 & 85973.51 & 0 & 18701.34 & 31969.51 & 1303.32 \\
\hline 7460.42 & 169576 & 9 & 190808.03 & 59398.6 & 0 & 12690.3 & 21723.41 & 6070.45 \\
\hline 7460.49 & 153577 & 9 & 239239.27 & 69390.59 & 0 & 17852.54 & 24021.54 & 3214.1 \\
\hline 7460.55 & 202388 & 8 & 183045.72 & 55328.98 & 0 & 13815.53 & 11566.77 & 6392.32 \\
\hline 7460.62 & 215470 & 8 & 163128.72 & 46437.26 & 0 & 22654.08 & 0 & 20021.65 \\
\hline 7460.68 & 230638 & 8 & 121793.99 & 35773.03 & 0 & 22991.91 & 0 & 28062.75 \\
\hline 7460.75 & 219162 & 8 & 103236.51 & 33680.13 & 0 & 14388.87 & 0 & 18872.75 \\
\hline 7460.81 & 205864 & 8 & 123656.64 & 36622.1 & 0 & 14596.95 & 10122.24 & 14864.09 \\
\hline 7460.88 & 191426 & 8 & 224711.36 & 65328.79 & 0 & 19948.38 & 20426.87 & 10894.57 \\
\hline 7460.94 & 285053 & 7 & 37773.11 & 12253.13 & 0 & 4500.78 & 0 & 6505.92 \\
\hline 7461.01 & 233253 & 8 & 112028.55 & 36242.25 & 0 & 9110.77 & 0 & 12444.34 \\
\hline 7461.08 & 297150 & 7 & 39763.63 & 11088.7 & 0 & 6921.28 & 0 & 7929.34 \\
\hline 7461.14 & 223568 & 8 & 113587.87 & 32830.95 & 0 & 8859.56 & 0 & 4012.06 \\
\hline 7461.21 & 141830 & 9 & 321882.51 & 91987.98 & 0 & 36526.27 & 11131.34 & 2231.23 \\
\hline 7461.27 & 178373 & 8 & 222439.99 & 65381.54 & 0 & 18458.91 & 26801.47 & 5368.89 \\
\hline 7461.34 & 164578 & 9 & 233887.23 & 70555.94 & 0 & 13924.26 & 32325.86 & 4114.42 \\
\hline 7461.40 & 165000 & 9 & 268469.98 & 80553.38 & 0 & 16702.52 & 33567.94 & 5173.43 \\
\hline 7461.47 & 189331 & 8 & 290853.13 & 86170.72 & 0 & 26368.19 & 29297.12 & 4407.77 \\
\hline 7461.54 & 181543 & 9 & 282055.46 & 82659.23 & 0 & 21435.61 & 29523.74 & 7290.44 \\
\hline 7461.60 & 222308 & 8 & 96414.36 & 30387.1 & 0 & 8700.67 & 0 & 12070.85 \\
\hline 7461.67 & 174752 & 9 & 285395.8 & 81081.77 & 0 & 19046.77 & 28300.78 & 4893.02 \\
\hline 7461.73 & 189744 & 8 & 190074.56 & 56946.2 & 0 & 10914.97 & 23083.2 & 6114.66 \\
\hline 7461.80 & 252181 & 8 & 78392.1 & 26380.79 & 0 & 5258.92 & 0 & 4783.48 \\
\hline 7461.86 & 191725 & 8 & 203699.96 & 62747.35 & 0 & 17990.09 & 11707.87 & 41316.47 \\
\hline 7461.93 & 225949 & 8 & 98679.74 & 30675.57 & 0 & 9696.41 & 0 & 17934.35 \\
\hline 7461.99 & 233875 & 8 & 68070.19 & 21742.73 & 0 & 6481.87 & 0 & 13383.59 \\
\hline 7462.06 & 189169 & 8 & 165323.2 & 49646.45 & 0 & 11472.22 & 18702.81 & 9432.81 \\
\hline 7462.13 & 193759 & 8 & 251171.39 & 74592.02 & 0 & 25730.8 & 19009.36 & 8867.26 \\
\hline 7462.19 & 237221 & 8 & 114377.36 & 38550.41 & 0 & 14192.64 & 0 & 18733.52 \\
\hline 7462.26 & 266367 & 8 & 50476.68 & 17343.98 & 0 & 4413.48 & 0 & 53427.68 \\
\hline
\end{tabular}




\begin{tabular}{|c|c|c|c|c|c|c|c|c|}
\hline True Depth & XRF tCOUNTS & XRF Live Time & $\mathrm{Si}(\mathrm{ppm})$ & $\mathrm{Al}(\mathrm{ppm})$ & $\mathrm{P}(\mathrm{ppm})$ & $\mathrm{S}(\mathrm{ppm})$ & $\mathrm{K}(\mathrm{ppm})$ & $\mathrm{Ca}(\mathrm{ppm})$ \\
\hline 7462.32 & 216404 & 8 & 61365.62 & 17546.07 & 0 & 5389.15 & 0 & 9996.07 \\
\hline 7462.39 & 164156 & 9 & 115128.52 & 32335.72 & 0 & 8451.82 & 7955.19 & 11621.81 \\
\hline 7462.45 & 181263 & 8 & 61428.21 & 18436.06 & 0 & 3917.53 & 0 & 5424.25 \\
\hline 7462.52 & 170128 & 9 & 82741.62 & 25093.01 & 0 & 5360.62 & 0 & 4771.88 \\
\hline 7462.59 & 266173 & 8 & 68574.75 & 21519.1 & 0 & 4407.73 & 0 & 4110.85 \\
\hline 7462.65 & 170569 & 9 & 280777.3 & 75962.45 & 0 & 11918.43 & 22951.07 & 5952.64 \\
\hline 7462.72 & 206320 & 8 & 53528.67 & 14001.43 & 0 & 4984.73 & 0 & 5808.03 \\
\hline 7462.78 & 182362 & 8 & 67908.81 & 19856.72 & 0 & 4907.19 & 0 & 9386.71 \\
\hline 7462.85 & 243024 & 8 & 38419.48 & 9420.86 & 0 & 2307.57 & 0 & 3016.96 \\
\hline 7462.91 & 154893 & 9 & 216173.74 & 61172.4 & 0 & 8052.8 & 22182.79 & 4144.95 \\
\hline 7462.98 & 163500 & 9 & 265642.43 & 74840.34 & 0 & 9812.94 & 25967.38 & 2878.54 \\
\hline 7463.04 & 212611 & 8 & 89772.92 & 26220.68 & 0 & 5180.92 & 0 & 6603.61 \\
\hline 7463.11 & 173770 & 9 & 275698.7 & 77267.49 & 0 & 10986.4 & 25301.73 & 5968.44 \\
\hline 7463.18 & 225638 & 8 & 81095.93 & 23237.65 & 0 & 6576.32 & 0 & 11982.16 \\
\hline 7463.24 & 112805 & 9 & 319640.22 & 80522.15 & 0 & 12968.51 & 10496.88 & 5491.55 \\
\hline 7463.31 & 235450 & 8 & 68015.09 & 19559.24 & 0 & 3610.47 & 0 & 2884.14 \\
\hline 7463.37 & 261033 & 8 & 39715.62 & 12418.68 & 0 & 2508.71 & 0 & 1827.33 \\
\hline 7463.44 & 190504 & 8 & 83721.44 & 26412.91 & 0 & 4069.03 & 0 & 7958.52 \\
\hline 7463.50 & 136849 & 9 & 236905.4 & 67318.15 & 0 & 9579.14 & 27112.11 & 2018.36 \\
\hline 7463.57 & 281191 & 7 & 50828.04 & 16296.87 & 0 & 3420.63 & 0 & 2583.19 \\
\hline 7463.64 & 293794 & 7 & 30659.37 & 6376.61 & 0 & 4269.93 & 0 & 4264.11 \\
\hline 7463.70 & 178630 & 9 & 279283.34 & 76665.55 & 0 & 14802.16 & 23545.87 & 27372.55 \\
\hline 7463.77 & 175278 & 9 & 238184.3 & 70305.9 & 0 & 12954.21 & 24725.69 & 14396 \\
\hline 7463.83 & 201604 & 8 & 102803.57 & 31456.85 & 0 & 5194.92 & 7061.84 & 9425.35 \\
\hline 7463.90 & 152362 & 9 & 213684.43 & 64167.07 & 0 & 12346.39 & 15407.73 & 6586.64 \\
\hline 7463.96 & 176252 & 9 & 202565.68 & 56509.4 & 0 & 10188.19 & 16673.76 & 26145.83 \\
\hline 7464.03 & 246423 & 8 & 60412.42 & 14659.01 & 0 & 2495.59 & 0 & 29581.1 \\
\hline 7464.09 & 222660 & 8 & 62519.1 & 18063.64 & 0 & 4774.76 & 0 & 13254.74 \\
\hline 7464.16 & 229486 & 8 & 61417.26 & 21576.09 & 0 & 2933.26 & 0 & 4917.09 \\
\hline 7464.23 & 226674 & 8 & 48901.74 & 14607.45 & 0 & 4123.75 & 0 & 9995.91 \\
\hline 7464.29 & 214002 & 8 & 43333.79 & 11969.7 & 0 & 4733.21 & 0 & 11319.25 \\
\hline 7464.36 & 277425 & 7 & 38396.97 & 12109.02 & 0 & 1833.35 & 0 & 6356.28 \\
\hline 7464.42 & 250078 & 8 & 74774.88 & 22610.74 & 0 & 3701.62 & 0 & 8929.53 \\
\hline
\end{tabular}




\begin{tabular}{|c|c|c|c|c|c|c|c|c|}
\hline True Depth & XRF tCOUNTS & XRF Live Time & $\mathrm{Si}(\mathrm{ppm})$ & $\mathrm{Al}(\mathrm{ppm})$ & $\mathrm{P}(\mathrm{ppm})$ & $\mathrm{S}(\mathrm{ppm})$ & $\mathrm{K}(\mathrm{ppm})$ & $\mathrm{Ca}(\mathrm{ppm})$ \\
\hline 7464.49 & 191907 & 8 & 136762.94 & 42704.94 & 0 & 6548.7 & 11159.71 & 6617.89 \\
\hline 7464.55 & 276481 & 7 & 24683.35 & 6478.56 & 0 & 2725.78 & 0 & 2924.66 \\
\hline 7464.62 & 157793 & 9 & 218823.68 & 62036.93 & 0 & 11163.39 & 25789.93 & 11746.09 \\
\hline 7464.69 & 245104 & 8 & 84720.63 & 27616.79 & 0 & 4480.16 & 0 & 5965.87 \\
\hline 7464.75 & 277575 & 7 & 51684.61 & 16650.82 & 0 & 3520.73 & 0 & 3367.49 \\
\hline 7464.82 & 188425 & 8 & 110427.27 & 33346.43 & 0 & 8810.14 & 0 & 10523.48 \\
\hline 7464.88 & 218548 & 8 & 50510.6 & 12451.67 & 0 & 4356.96 & 0 & 8832.07 \\
\hline 7464.95 & 249917 & 8 & 26340.51 & 5471.56 & 0 & 3552.95 & 0 & 4412.84 \\
\hline 7465.01 & 261905 & 8 & 29227.27 & 6680.2 & 0 & 3029.53 & 0 & 2783.97 \\
\hline 7465.08 & 154179 & 9 & 241106.99 & 67344.33 & 0 & 16440.62 & 27060 & 18074.25 \\
\hline 7465.14 & 150400 & 9 & 226055.26 & 64255.67 & 0 & 12109.1 & 28822.76 & 13226.29 \\
\hline 7465.21 & 133131 & 9 & 222213.88 & 63162.7 & 0 & 13133.47 & 26640.73 & 6525.87 \\
\hline 7465.28 & 187988 & 8 & 251622.44 & 75732.5 & 0 & 13076.92 & 23677.4 & 4637.96 \\
\hline 7465.34 & 193991 & 8 & 226586.67 & 66160.52 & 0 & 15609.15 & 16557.59 & 33142.71 \\
\hline 7465.41 & 200637 & 8 & 131644.06 & 38847.45 & 0 & 9702.05 & 0 & 16521.78 \\
\hline 7465.47 & 176316 & 9 & 173794.12 & 53186.57 & 0 & 10887.72 & 19882.19 & 3652.46 \\
\hline 7465.54 & 166435 & 9 & 149677.44 & 45973.67 & 0 & 7280.38 & 14224.13 & 4635.57 \\
\hline 7465.60 & 163282 & 9 & 270714.65 & 80522.11 & 0 & 18319.05 & 27003.12 & 5338.83 \\
\hline 7465.67 & 159254 & 9 & 227332.53 & 63654.06 & 0 & 15819.12 & 9910.78 & 10220.6 \\
\hline 7465.73 & 212238 & 8 & 110614.6 & 33513.24 & 0 & 8978.84 & 0 & 13668.13 \\
\hline 7465.80 & 262494 & 8 & 45351.65 & 14283.61 & 0 & 2812.9 & 0 & 3678.18 \\
\hline 7465.87 & 191668 & 8 & 153904.08 & 50124.6 & 0 & 7841.1 & 14205.02 & 6375.49 \\
\hline 7465.93 & 237242 & 8 & 77471.76 & 22582.91 & 0 & 4557.05 & 0 & 4995.96 \\
\hline 7466.00 & 220080 & 8 & 95314.73 & 28879.44 & 0 & 5867.05 & 0 & 8504.27 \\
\hline 7466.06 & 189284 & 8 & 275411.81 & 73840.04 & 0 & 17213.4 & 14432.23 & 48797.17 \\
\hline 7466.13 & 195076 & 8 & 234385.38 & 64079.15 & 0 & 21619.41 & 14103.15 & 43758.65 \\
\hline 7466.19 & 185762 & 8 & 215467.57 & 61646.03 & 0 & 15149.75 & 16718.15 & 12755.11 \\
\hline 7466.26 & 206377 & 8 & 102507.78 & 34909.01 & 0 & 6565.01 & 0 & 3045.92 \\
\hline 7466.33 & 209879 & 8 & 123800.09 & 36959.27 & 0 & 7589.92 & 8466.8 & 5388.86 \\
\hline 7466.39 & 230787 & 8 & 73522.19 & 22199.28 & 0 & 8182.19 & 0 & 8320.8 \\
\hline 7466.46 & 213999 & 8 & 100742.65 & 30393.53 & 0 & 5525.31 & 0 & 8131.68 \\
\hline 7466.52 & 218081 & 8 & 88751.75 & 29184.01 & 0 & 6530.32 & 0 & 13265.02 \\
\hline 7466.59 & 253702 & 8 & 83892.11 & 27309.09 & 0 & 5555.56 & 0 & 5287.79 \\
\hline
\end{tabular}




\begin{tabular}{|c|c|c|c|c|c|c|c|c|}
\hline True Depth & XRF tCOUNTS & XRF Live Time & $\mathrm{Si}(\mathrm{ppm})$ & $\mathrm{Al}(\mathrm{ppm})$ & $\mathrm{P}(\mathrm{ppm})$ & $\mathrm{S}(\mathrm{ppm})$ & $\mathrm{K}(\mathrm{ppm})$ & $\mathrm{Ca}(\mathrm{ppm})$ \\
\hline 7466.65 & 280861 & 7 & 34527.29 & 10971.88 & 0 & 3785.53 & 0 & 4751.54 \\
\hline 7466.72 & 165769 & 9 & 277106.59 & 82094.01 & 0 & 14007.35 & 29853.53 & 28504.34 \\
\hline 7466.78 & 161529 & 9 & 269399.56 & 73689.4 & 0 & 15492.78 & 23691.32 & 29918.52 \\
\hline 7466.85 & 263869 & 8 & 33075.51 & 8812.76 & 0 & 2984.88 & 0 & 3349.18 \\
\hline 7466.92 & 177763 & 8 & 318043.69 & 93932.59 & 0 & 20046.55 & 26686.17 & 5259.71 \\
\hline 7466.98 & 196489 & 8 & 270493.55 & 77851.17 & 0 & 19338.19 & 16348.47 & 20293.93 \\
\hline 7467.05 & 248472 & 8 & 100495.76 & 30274.04 & 0 & 5547.57 & 0 & 32090.24 \\
\hline 7467.11 & 247755 & 8 & 70182.16 & 21803.7 & 0 & 5778.43 & 0 & 12278.37 \\
\hline 7467.18 & 96924 & 9 & 54909.72 & 14586.06 & 0 & 5884.89 & 0 & 34984.85 \\
\hline 7467.24 & 198377 & 8 & 137167.95 & 42078.73 & 0 & 7712.97 & 10580.61 & 32449.31 \\
\hline 7467.31 & 225162 & 8 & 86750.35 & 25366.11 & 0 & 3949.17 & 0 & 30201.92 \\
\hline 7467.38 & 173121 & 9 & 270798.79 & 71963.99 & 0 & 12922.46 & 23704.89 & 39719.12 \\
\hline 7467.44 & 162136 & 9 & 230868.69 & 62267.93 & 0 & 11723.08 & 27765.09 & 33485.03 \\
\hline 7467.51 & 236541 & 8 & 85328.03 & 23467.98 & 0 & 5280.8 & 0 & 153706.97 \\
\hline 7467.57 & 195808 & 8 & 76289.01 & 21727.87 & 0 & 5158.5 & 0 & 14681.75 \\
\hline 7467.64 & 242470 & 8 & 110048.88 & 28874.52 & 0 & 6519 & 0 & 122077.25 \\
\hline 7467.70 & 213224 & 8 & 110603.62 & 35170.08 & 0 & 6543.8 & 0 & 10157.04 \\
\hline 7467.77 & 194402 & 8 & 264094.29 & 75010.59 & 0 & 16167.56 & 17815.62 & 51746.73 \\
\hline 7467.83 & 220327 & 8 & 110352.09 & 30996.42 & 0 & 7426.12 & 0 & 49346.84 \\
\hline 7467.90 & 295690 & 7 & 51804.2 & 11609.74 & 0 & 6276.99 & 0 & 244387.55 \\
\hline 7467.97 & 319137 & 7 & 29670.69 & 4911.88 & 0 & 5182.45 & 0 & 256581.18 \\
\hline 7468.03 & 320969 & 7 & 29686.82 & 4119.47 & 0 & 3315.7 & 0 & 275643.15 \\
\hline 7468.10 & 295070 & 7 & 62447.18 & 12079.19 & 0 & 5205.05 & 0 & 300431.01 \\
\hline 7468.16 & 220183 & 8 & 144871.56 & 40935.39 & 0 & 11974.63 & 0 & 92222.73 \\
\hline 7468.23 & 196639 & 8 & 246892.84 & 74970.63 & 0 & 12049.52 & 14942.4 & 14313.74 \\
\hline 7468.29 & 195590 & 8 & 181022.69 & 55026.03 & 0 & 9730.78 & 9254.2 & 5495.4 \\
\hline 7468.36 & 198481 & 8 & 212940.13 & 63091 & 0 & 12209.26 & 8216.72 & 21514.68 \\
\hline 7468.43 & 274900 & 8 & 36949.69 & 12563.02 & 0 & 4417.31 & 0 & 5923.98 \\
\hline 7468.49 & 233718 & 8 & 90873.16 & 23031.67 & 0 & 13835.94 & 0 & 34060.04 \\
\hline 7468.56 & 174191 & 9 & 247596.37 & 67239.67 & 0 & 16680.11 & 21641 & 8976.58 \\
\hline 7468.62 & 187642 & 8 & 160609.18 & 46484.79 & 0 & 9796.4 & 13015.41 & 5079.94 \\
\hline 7468.69 & 207206 & 8 & 110619.28 & 32431.71 & 0 & 6652.31 & 0 & 6766.89 \\
\hline 7468.75 & 235528 & 8 & 71653.79 & 20057.1 & 0 & 3688.62 & 0 & 2483.44 \\
\hline
\end{tabular}




\begin{tabular}{|c|c|c|c|c|c|c|c|c|}
\hline True Depth & XRF tCOUNTS & XRF Live Time & $\mathrm{Si}(\mathrm{ppm})$ & $\mathrm{Al}(\mathrm{ppm})$ & $\mathrm{P}(\mathrm{ppm})$ & $\mathrm{S}(\mathrm{ppm})$ & $\mathrm{K}(\mathrm{ppm})$ & $\mathrm{Ca}(\mathrm{ppm})$ \\
\hline 7468.82 & 197063 & 8 & 140622 & 40609.28 & 0 & 8728.79 & 0 & 6988.93 \\
\hline 7468.88 & 246566 & 8 & 89513.98 & 26542.65 & 0 & 6907 & 0 & 14710.01 \\
\hline 7468.95 & 210814 & 8 & 183822.84 & 47618.77 & 0 & 13187.3 & 0 & 29225.25 \\
\hline 7469.02 & 198871 & 8 & 33997.8 & 7744.33 & 0 & 3806.05 & 0 & 5302.33 \\
\hline 7469.08 & 147532 & 9 & 46793.52 & 11471.15 & 0 & 2570.48 & 0 & 22101.31 \\
\hline 7469.15 & 147047 & 9 & 30857.67 & 6763.32 & 0 & 3999.92 & 0 & 13824.52 \\
\hline 7469.21 & 153408 & 9 & 194353.71 & 57997.45 & 0 & 10779.55 & 0 & 57090.95 \\
\hline 7469.28 & 252618 & 8 & 64365.5 & 19855.52 & 0 & 3450.4 & 0 & 3028.89 \\
\hline 7469.34 & 174208 & 9 & 103121.07 & 31056.53 & 0 & 4822.72 & 6707.21 & 4247.72 \\
\hline 7469.41 & 291436 & 7 & 41638.61 & 11285.79 & 0 & 3506.53 & 0 & 2718.78 \\
\hline 7469.48 & 259352 & 8 & 73046.02 & 22011.41 & 0 & 5341.68 & 0 & 3592.88 \\
\hline 7469.54 & 197132 & 8 & 200223.41 & 51031.51 & 0 & 15899.98 & 7748.16 & 22723.71 \\
\hline 7469.61 & 222624 & 8 & 127367.12 & 35497.98 & 0 & 12881.63 & 0 & 16568.6 \\
\hline 7469.67 & 186111 & 8 & 307113.79 & 76744.04 & 0 & 24300.89 & 15639.04 & 31491.65 \\
\hline 7469.74 & 264230 & 8 & 76291.97 & 22316.81 & 0 & 9142.34 & 0 & 23638.65 \\
\hline 7469.80 & 188216 & 8 & 224037.22 & 67531.69 & 0 & 15302.57 & 18139.33 & 8192.06 \\
\hline 7469.87 & 177026 & 8 & 291993.93 & 79188.35 & 0 & 18153.63 & 28283.23 & 8678.56 \\
\hline 7469.93 & 299568 & 7 & 31370.36 & 8197.89 & 0 & 4089.07 & 0 & 3339.83 \\
\hline 7470.00 & 240322 & 8 & 90438.14 & 26359.05 & 0 & 7926.21 & 0 & 11411.58 \\
\hline 7470.07 & 190162 & 8 & 152148.67 & 41875.41 & 0 & 11575.22 & 0 & 36949.55 \\
\hline 7470.13 & 196929 & 8 & 186836.57 & 51052.22 & 0 & 22890.3 & 11072.05 & 52245.59 \\
\hline 7470.20 & 174067 & 9 & 247785.6 & 67362.2 & 0 & 25292.53 & 19119.37 & 23733.27 \\
\hline 7470.26 & 167869 & 9 & 247580.24 & 70481.72 & 0 & 14043.13 & 24386.79 & 3195.57 \\
\hline 7470.33 & 165966 & 9 & 234520.56 & 67520.87 & 0 & 22668.84 & 25274.8 & 9838.51 \\
\hline 7470.39 & 295107 & 7 & 40985.25 & 12118.39 & 0 & 4822.22 & 0 & 27550.44 \\
\hline 7470.46 & 241616 & 8 & 86301.13 & 25730.56 & 0 & 6167.73 & 0 & 5424.93 \\
\hline 7470.52 & 192446 & 8 & 169238.56 & 49303.13 & 0 & 12860.54 & 10507.03 & 8268.72 \\
\hline 7470.59 & 195699 & 8 & 242972.28 & 61278.73 & 0 & 15950.09 & 13347.86 & 83733.96 \\
\hline 7470.66 & 186931 & 8 & 151996.9 & 43782.96 & 0 & 9368.22 & 0 & 49063.44 \\
\hline 7470.72 & 244909 & 8 & 80864.15 & 26042.03 & 0 & 5532.04 & 0 & 4847.96 \\
\hline 7470.79 & 229841 & 8 & 94001.82 & 28180.01 & 0 & 6055.72 & 0 & 5252.38 \\
\hline 7470.85 & 202146 & 8 & 181453.64 & 50931.45 & 0 & 15323.09 & 11816.79 & 7298.24 \\
\hline 7470.92 & 254383 & 8 & 82831.6 & 26359.97 & 0 & 9468.42 & 0 & 7767.29 \\
\hline
\end{tabular}




\begin{tabular}{|c|c|c|c|c|c|c|c|c|}
\hline True Depth & XRF tCOUNTS & XRF Live Time & $\mathrm{Si}(\mathrm{ppm})$ & $\mathrm{Al}(\mathrm{ppm})$ & $\mathrm{P}(\mathrm{ppm})$ & $\mathrm{S}(\mathrm{ppm})$ & $\mathrm{K}(\mathrm{ppm})$ & $\mathrm{Ca}(\mathrm{ppm})$ \\
\hline 7470.98 & 259731 & 8 & 75369.18 & 20763.73 & 0 & 6128.23 & 0 & 24902.25 \\
\hline 7471.05 & 277685 & 7 & 82157.59 & 23225.52 & 0 & 10867.99 & 0 & 154545.27 \\
\hline 7471.12 & 289536 & 7 & 33742.58 & 8579.37 & 0 & 5265.33 & 0 & 16558.68 \\
\hline 7471.18 & 300517 & 7 & 39477.01 & 11517.6 & 0 & 6816.15 & 0 & 13296 \\
\hline 7471.25 & 139098 & 9 & 286118.53 & 84031.04 & 0 & 13969.91 & 10768.54 & 9331.7 \\
\hline 7471.31 & 172890 & 9 & 278408.77 & 70853.53 & 0 & 15089.47 & 19787.04 & 21448.1 \\
\hline 7471.38 & 175222 & 9 & 238799.05 & 63635.29 & 0 & 16832.69 & 14586.19 & 19213.82 \\
\hline 7471.44 & 118868 & 9 & 162795.18 & 38282.72 & 0 & 11660.29 & 16227.53 & 8628.79 \\
\hline 7471.51 & 227195 & 8 & 118454.46 & 30300.48 & 0 & 8008.53 & 0 & 75173.43 \\
\hline 7471.57 & 257417 & 8 & 143386.41 & 29370.01 & 0 & 7491.16 & 0 & 236739.54 \\
\hline 7471.64 & 293872 & 7 & 79148.43 & 17332.65 & 0 & 5025.66 & 0 & 262866.97 \\
\hline 7471.71 & 181499 & 8 & 244159.02 & 68467.83 & 0 & 11866.31 & 18267.56 & 23620.02 \\
\hline 7471.77 & 249245 & 8 & 77377.83 & 23416.75 & 0 & 4787.67 & 0 & 25451.39 \\
\hline 7471.84 & 188547 & 8 & 295645.25 & 85884.12 & 0 & 14646.22 & 21914.83 & 49792.75 \\
\hline 7471.90 & 152215 & 9 & 260466.09 & 74273.37 & 0 & 16623.74 & 17204.79 & 11768.84 \\
\hline 7471.97 & 259260 & 8 & 63728.91 & 16768.55 & 0 & 6016.77 & 0 & 16921.85 \\
\hline 7472.03 & 264032 & 8 & 51905.59 & 13052.39 & 0 & 6392.21 & 0 & 19566.37 \\
\hline 7472.10 & 233011 & 8 & 79675.25 & 22669.8 & 0 & 11191.54 & 0 & 17499.8 \\
\hline 7472.17 & 173127 & 9 & 229360.62 & 66084.05 & 0 & 23385.4 & 23519.88 & 4929.62 \\
\hline 7472.23 & 154963 & 9 & 79269.33 & 25216.19 & 0 & 5350.94 & 0 & 2879.43 \\
\hline 7472.30 & 234438 & 8 & 73061.23 & 21804.15 & 0 & 5482.01 & 0 & 3890.17 \\
\hline 7472.36 & 166202 & 9 & 192490.22 & 56421.93 & 0 & 12268.18 & 22081.51 & 3215.83 \\
\hline 7472.43 & 217174 & 8 & 72726.35 & 18921.93 & 0 & 5580.75 & 0 & 9443.54 \\
\hline 7472.49 & 176672 & 8 & 160395.33 & 48942.89 & 0 & 11903.25 & 16642.42 & 6200.89 \\
\hline 7472.56 & 197316 & 8 & 172182.82 & 51975.02 & 0 & 11865.94 & 15499.57 & 12802.83 \\
\hline 7472.62 & 252714 & 8 & 65271.2 & 20472.81 & 0 & 5535.87 & 0 & 7587.95 \\
\hline 7472.69 & 234638 & 8 & 75977.59 & 23628.84 & 0 & 6505.1 & 0 & 5921.29 \\
\hline 7472.76 & 193160 & 8 & 87934.57 & 26894.76 & 0 & 5878.87 & 0 & 4025.35 \\
\hline 7472.82 & 193538 & 8 & 242605.21 & 63526.29 & 0 & 17047.04 & 16661.45 & 39967.19 \\
\hline 7472.89 & 285426 & 7 & 46255.26 & 14158.22 & 0 & 4835.23 & 0 & 6059.02 \\
\hline 7472.95 & 179378 & 8 & 258383.85 & 75694.39 & 0 & 17188.27 & 23244.9 & 9282.57 \\
\hline 7473.02 & 227040 & 8 & 80186.85 & 24681.69 & 0 & 5816.79 & 0 & 4858.32 \\
\hline 7473.08 & 230385 & 8 & 89636.89 & 29257.12 & 0 & 5250.03 & 0 & 1955.6 \\
\hline
\end{tabular}




\begin{tabular}{|c|c|c|c|c|c|c|c|c|}
\hline True Depth & XRF tCOUNTS & XRF Live Time & $\mathrm{Si}(\mathrm{ppm})$ & $\mathrm{Al}(\mathrm{ppm})$ & $\mathrm{P}(\mathrm{ppm})$ & $\mathrm{S}(\mathrm{ppm})$ & $\mathrm{K}(\mathrm{ppm})$ & $\mathrm{Ca}(\mathrm{ppm})$ \\
\hline 7473.15 & 189513 & 8 & 175554.1 & 52007.76 & 0 & 11947.54 & 16336.71 & 1706.3 \\
\hline 7473.22 & 124250 & 9 & 310900.46 & 87101.13 & 0 & 24812.37 & 0 & 7559.2 \\
\hline 7473.28 & 260366 & 8 & 66553.45 & 20132.06 & 0 & 6798.85 & 0 & 20024.2 \\
\hline 7473.35 & 199788 & 8 & 125969.84 & 35542.18 & 0 & 9044.12 & 0 & 3684.87 \\
\hline 7473.41 & 186240 & 8 & 130695.68 & 35744.28 & 0 & 7764.48 & 16334.89 & 3393.93 \\
\hline 7473.48 & 183589 & 8 & 174337.22 & 51729.01 & 0 & 10067.23 & 15059.59 & 1525.91 \\
\hline 7473.54 & 184194 & 8 & 243641.35 & 68541.5 & 0 & 18657.82 & 19188.24 & 2405.21 \\
\hline 7473.61 & 280533 & 7 & 50001.16 & 15924.6 & 0 & 5532.6 & 0 & 4454.45 \\
\hline 7473.67 & 190415 & 8 & 210509.37 & 58512.47 & 0 & 15918.38 & 17815.14 & 4768.07 \\
\hline 7473.74 & 187271 & 8 & 224764.72 & 67833.68 & 0 & 20189.93 & 18076.11 & 2046.19 \\
\hline 7473.81 & 264921 & 8 & 65165.47 & 21056.17 & 0 & 5859.97 & 0 & 2628.92 \\
\hline 7473.87 & 193083 & 8 & 168755.82 & 51119.54 & 0 & 10801.74 & 17464.3 & 2230.44 \\
\hline 7473.94 & 262711 & 8 & 64587.99 & 19912.25 & 0 & 4140.43 & 0 & 2216.33 \\
\hline 7474.00 & 173176 & 9 & 302975.56 & 82728.75 & 0 & 22808.6 & 25354.54 & 16872.51 \\
\hline 7474.07 & 195158 & 8 & 169455.07 & 49264.91 & 0 & 12922 & 13000.38 & 10009.88 \\
\hline 7474.13 & 182733 & 8 & 212329.47 & 64938.25 & 0 & 17750.44 & 18510.3 & 3048.34 \\
\hline 7474.20 & 190792 & 8 & 135908.52 & 41436.22 & 0 & 7687.2 & 0 & 6537.96 \\
\hline 7474.27 & 197278 & 8 & 176232.96 & 55729.14 & 0 & 10003.07 & 12849.56 & 5451.17 \\
\hline 7474.33 & 168469 & 9 & 300612.09 & 86142.8 & 0 & 17645.74 & 26638.79 & 3175.96 \\
\hline 7474.40 & 251424 & 8 & 66220.03 & 20930.7 & 0 & 4511.86 & 0 & 2672.67 \\
\hline 7474.46 & 212085 & 8 & 131189.93 & 41918.4 & 0 & 7628.5 & 0 & 2062.24 \\
\hline 7474.53 & 310903 & 7 & 21803.23 & 4991.98 & 0 & 4527.08 & 0 & 2114.77 \\
\hline 7474.59 & 201037 & 8 & 177706.6 & 56367.17 & 0 & 11116.97 & 13263.62 & 3332.38 \\
\hline 7474.66 & 175618 & 8 & 240683.2 & 75282.4 & 0 & 11026.46 & 27330.64 & 2080.03 \\
\hline 7474.72 & 251172 & 8 & 78979.22 & 23984.39 & 0 & 4102.7 & 0 & 6229.43 \\
\hline 7474.79 & 221628 & 8 & 104638.02 & 33642.48 & 0 & 6037.86 & 0 & 3082.93 \\
\hline 7474.86 & 166150 & 9 & 305442.51 & 88914.21 & 0 & 14882.17 & 29891.5 & 5243.24 \\
\hline 7474.92 & 236109 & 8 & 79461.41 & 25995.61 & 0 & 4395.27 & 0 & 4507.12 \\
\hline 7474.99 & 191362 & 8 & 153762.95 & 49185.76 & 0 & 9499.68 & 15143.56 & 5569.71 \\
\hline 7475.05 & 160513 & 9 & 295837.19 & 85604 & 0 & 17672.42 & 31779.61 & 3491.87 \\
\hline 7475.12 & 166514 & 9 & 201980.73 & 60656.19 & 0 & 9958.53 & 17827.28 & 10263.05 \\
\hline 7475.18 & 233963 & 8 & 119519.44 & 31936.1 & 0 & 10321.53 & 0 & 65955.68 \\
\hline 7475.25 & 57195 & 10 & 376653.12 & 101394.8 & 0 & 28511.17 & 0 & 121010.26 \\
\hline
\end{tabular}




\begin{tabular}{|c|c|c|c|c|c|c|c|c|}
\hline True Depth & XRF tCOUNTS & XRF Live Time & $\mathrm{Si}(\mathrm{ppm})$ & $\mathrm{Al}(\mathrm{ppm})$ & $\mathrm{P}(\mathrm{ppm})$ & $\mathrm{S}(\mathrm{ppm})$ & $\mathrm{K}(\mathrm{ppm})$ & $\mathrm{Ca}(\mathrm{ppm})$ \\
\hline 7475.31 & 203799 & 8 & 268870.87 & 72920.8 & 0 & 19813.06 & 14092.12 & 79522.4 \\
\hline 7475.38 & 170629 & 9 & 273239.89 & 78802.12 & 866.76 & 20858.87 & 30982.22 & 7784.53 \\
\hline 7475.45 & 114605 & 9 & 340928.62 & 96612.21 & 0 & 24862.66 & 18350.65 & 24450.35 \\
\hline 7475.51 & 178268 & 8 & 283631.43 & 87270.27 & 0 & 24646.14 & 26931.13 & 7823.9 \\
\hline 7475.58 & 172019 & 9 & 251410.96 & 73192.78 & 0 & 19717.17 & 23290.43 & 4419.91 \\
\hline 7475.64 & 183171 & 8 & 199402.07 & 56884.8 & 0 & 13107.89 & 17065.86 & 17866.88 \\
\hline 7475.71 & 205587 & 8 & 267561.1 & 73259 & 0 & 18275.02 & 16778.35 & 93910.87 \\
\hline 7475.77 & 261553 & 8 & 62955.7 & 17629.51 & 0 & 7149.19 & 0 & 47099.94 \\
\hline 7475.84 & 225342 & 8 & 145573.99 & 42478.55 & 0 & 11810.69 & 0 & 97569.36 \\
\hline 7475.91 & 221474 & 8 & 247276.25 & 75100.73 & 0 & 11857.3 & 16878.58 & 122672.79 \\
\hline 7475.97 & 272999 & 7 & 82769.89 & 31447.23 & 0 & 203770.18 & 0 & 0 \\
\hline 7476.04 & 141849 & 9 & 292220.55 & 94459.73 & 0 & 16740.26 & 26304.25 & 10729.34 \\
\hline 7476.10 & 179327 & 8 & 224315.57 & 72034.8 & 0 & 13042.74 & 23862.31 & 4058.93 \\
\hline 7476.17 & 169316 & 9 & 303367.83 & 80473.4 & 0 & 23257.52 & 24470.12 & 10826.73 \\
\hline 7476.23 & 190483 & 8 & 218098.5 & 63812.39 & 0 & 18099.31 & 17791.76 & 35613.25 \\
\hline 7476.30 & 217603 & 8 & 144399.76 & 45582.56 & 0 & 13581.53 & 6971.56 & 28278.46 \\
\hline 7476.36 & 186288 & 8 & 205911.82 & 63251.56 & 0 & 11878.11 & 21697.13 & 13430.23 \\
\hline 7476.43 & 185511 & 8 & 210583.37 & 65045.7 & 0 & 14356.6 & 23418.06 & 24967.08 \\
\hline 7476.50 & 162804 & 9 & 277800.78 & 80026 & 0 & 14655.06 & 32757.33 & 10174.62 \\
\hline 7476.56 & 205068 & 8 & 136731.51 & 43333.88 & 0 & 9830.64 & 12308.09 & 7989.05 \\
\hline 7476.63 & 166052 & 9 & 320979.01 & 91590.64 & 0 & 20195.17 & 29671.55 & 1014.79 \\
\hline 7476.69 & 167967 & 9 & 317330.31 & 87085.88 & 0 & 21503.08 & 31958.43 & 2284.67 \\
\hline 7476.76 & 195936 & 8 & 128150.76 & 38667.43 & 0 & 11509.73 & 9923.65 & 11790.55 \\
\hline 7476.82 & 156481 & 9 & 221685.71 & 66328.99 & 0 & 11439.75 & 28494.6 & 4141.21 \\
\hline 7476.89 & 162228 & 9 & 220000.96 & 66539.22 & 0 & 10899.18 & 24304.01 & 3100.1 \\
\hline 7476.96 & 206764 & 8 & 210085.05 & 64605.44 & 0 & 25572.88 & 12085.32 & 10480.46 \\
\hline 7477.02 & 195082 & 8 & 272641.36 & 77073.22 & 0 & 32048.54 & 15905.36 & 25739.58 \\
\hline 7477.09 & 196168 & 8 & 101334.23 & 30789.15 & 0 & 12833 & 0 & 15179.34 \\
\hline 7477.15 & 215056 & 8 & 166999.16 & 50181.72 & 0 & 20671.23 & 0 & 11946.04 \\
\hline 7477.22 & 225201 & 8 & 127505.67 & 39183.45 & 0 & 10605.75 & 0 & 4681.4 \\
\hline 7477.28 & 267646 & 18 & 327453.32 & 93831.6 & 0 & 20981.13 & 15141.51 & 3399.27 \\
\hline 7477.35 & 387579 & 17 & 268154 & 74651.57 & 0 & 23339.51 & 21604.68 & 26514.72 \\
\hline 7477.41 & 507582 & 15 & 73201.65 & 23620.27 & 0 & 9570.4 & 0 & 12291.94 \\
\hline
\end{tabular}




\begin{tabular}{|c|c|c|c|c|c|c|c|c|}
\hline True Depth & XRF tCOUNTS & XRF Live Time & $\mathrm{Si}(\mathrm{ppm})$ & $\mathrm{Al}(\mathrm{ppm})$ & $\mathrm{P}(\mathrm{ppm})$ & $\mathrm{S}(\mathrm{ppm})$ & $\mathrm{K}(\mathrm{ppm})$ & $\mathrm{Ca}(\mathrm{ppm})$ \\
\hline 7477.48 & 407802 & 16 & 157316.25 & 46714.27 & 0 & 20982.93 & 7794.66 & 20234.17 \\
\hline 7477.55 & 414729 & 16 & 170552.72 & 52035.83 & 0 & 14475.1 & 6378.16 & 6434.63 \\
\hline 7477.61 & 328666 & 17 & 313138.72 & 86876.02 & 0 & 14109.2 & 30715.86 & 0 \\
\hline 7477.68 & 343761 & 17 & 241103.44 & 69874.03 & 0 & 12705.36 & 23092.4 & 2258.33 \\
\hline 7477.74 & 321716 & 17 & 304497.06 & 85102.04 & 0 & 15244.8 & 29887.6 & 1266.2 \\
\hline 7477.81 & 315071 & 17 & 291839.41 & 86416.81 & 0 & 12340.85 & 31758.54 & 1551.6 \\
\hline 7477.87 & 328417 & 17 & 218644.45 & 66934.58 & 0 & 9463.2 & 24854.51 & 3430.87 \\
\hline 7477.94 & 361982 & 17 & 178490.99 & 54932.23 & 0 & 9466.36 & 20752.33 & 4551.93 \\
\hline 7478.01 & 326084 & 17 & 324184.01 & 89042.95 & 0 & 17603.14 & 31746.79 & 2828.97 \\
\hline 7478.07 & 343654 & 17 & 293256.47 & 84317.98 & 0 & 22837.44 & 26801.88 & 3618.66 \\
\hline 7478.14 & 369581 & 17 & 205040 & 62946.18 & 0 & 19358.36 & 19763.9 & 7387.56 \\
\hline 7478.20 & 359931 & 17 & 174073.38 & 53048.96 & 0 & 15215.91 & 16504.28 & 8219.64 \\
\hline 7478.27 & 311324 & 17 & 296352.17 & 84419.41 & 0 & 14603.94 & 29082.11 & 1553.82 \\
\hline 7478.33 & 435948 & 16 & 96776.49 & 30103.02 & 0 & 8456.33 & 0 & 5985.38 \\
\hline 7478.40 & 318878 & 17 & 268558.43 & 77849.76 & 0 & 14355.08 & 29736.27 & 1594.95 \\
\hline 7478.46 & 307283 & 17 & 291392.61 & 85594.26 & 0 & 13721.98 & 32155.42 & 1230.42 \\
\hline 7478.53 & 411832 & 16 & 123642.85 & 38648.71 & 0 & 5942.18 & 8885.75 & 4191.3 \\
\hline 7478.60 & 444659 & 16 & 84169.97 & 26324.37 & 0 & 3763.88 & 0 & 2462.66 \\
\hline 7478.66 & 593893 & 14 & 39057.72 & 11375.52 & 0 & 8274.38 & 0 & 5417.2 \\
\hline 7478.73 & 416514 & 16 & 146943.27 & 42683.91 & 0 & 20351.28 & 0 & 18652.44 \\
\hline 7478.79 & 329400 & 17 & 305088.52 & 83383.76 & 0 & 19158.49 & 28460.09 & 19212.43 \\
\hline 7478.86 & 350933 & 17 & 271628.65 & 75384.65 & 0 & 20491.79 & 22091.33 & 30914.07 \\
\hline 7478.92 & 376795 & 17 & 41798.25 & 10439.18 & 0 & 4127.74 & 0 & 4091.26 \\
\hline 7478.99 & 170630 & 19 & 73265.2 & 20625.48 & 0 & 4846.87 & 9169.03 & 4279.92 \\
\hline 7479.06 & 291019 & 18 & 44020.18 & 10359.26 & 0 & 6080.9 & 0 & 71544.4 \\
\hline 7479.12 & 479066 & 16 & 10034.58 & 1380.85 & 0 & 2635.78 & 0 & 1588.14 \\
\hline 7479.19 & 506923 & 15 & 13823.77 & 2825.89 & 0 & 2857.66 & 0 & 3948.18 \\
\hline 7479.25 & 326543 & 17 & 257551.15 & 76808.45 & 0 & 14130.3 & 20191.29 & 5125.83 \\
\hline 7479.32 & 333204 & 17 & 266872.05 & 74170.71 & 0 & 14288.36 & 25115.85 & 6892.79 \\
\hline 7479.38 & 454988 & 16 & 75104.06 & 21910.62 & 0 & 6043.94 & 0 & 7698.32 \\
\hline 7479.45 & 318367 & 17 & 268647.09 & 74229.07 & 0 & 14317.23 & 25135.15 & 4823.09 \\
\hline 7479.51 & 342110 & 17 & 165874.73 & 47673 & 0 & 8912.28 & 14799.38 & 3374.41 \\
\hline 7479.58 & 311033 & 17 & 282712.51 & 75368.51 & 0 & 18537.43 & 24253.8 & 6405.32 \\
\hline
\end{tabular}




\begin{tabular}{|c|c|c|c|c|c|c|c|c|}
\hline True Depth & XRF tCOUNTS & XRF Live Time & $\mathrm{Si}(\mathrm{ppm})$ & $\mathrm{Al}(\mathrm{ppm})$ & $\mathrm{P}(\mathrm{ppm})$ & $\mathrm{S}(\mathrm{ppm})$ & $\mathrm{K}(\mathrm{ppm})$ & $\mathrm{Ca}(\mathrm{ppm})$ \\
\hline 7479.65 & 381084 & 17 & 124538.34 & 36674.84 & 0 & 6559.65 & 5700.23 & 4133.33 \\
\hline 7479.71 & 299892 & 17 & 289711.38 & 77270.11 & 0 & 13043.03 & 26355.26 & 1942.83 \\
\hline 7479.78 & 433611 & 16 & 83944.16 & 23396.28 & 0 & 4947.52 & 0 & 4963.17 \\
\hline 7479.84 & 482518 & 16 & 75690.25 & 22503.37 & 0 & 5065.44 & 0 & 3438.3 \\
\hline 7479.91 & 298103 & 17 & 255946.41 & 71781.84 & 0 & 10863.38 & 20599.19 & 1662.35 \\
\hline 7479.97 & 317591 & 17 & 175662.76 & 49035.42 & 0 & 7247.08 & 16801.89 & 4192.06 \\
\hline 7480.04 & 351449 & 17 & 111046.84 & 28972.4 & 0 & 5545.14 & 6418.99 & 4537.99 \\
\hline 7480.10 & 342546 & 17 & 140572.91 & 38888.66 & 0 & 5589.25 & 9379.62 & 3402.93 \\
\hline 7480.17 & 290309 & 18 & 141575.5 & 41225.72 & 0 & 5495.42 & 10396.23 & 3516.24 \\
\hline 7480.24 & 446032 & 16 & 46342.37 & 14066.78 & 0 & 2466.29 & 0 & 1880.48 \\
\hline 7480.30 & 374354 & 17 & 157804.41 & 44453.9 & 0 & 6058.54 & 10035.88 & 4093.77 \\
\hline 7480.37 & 261238 & 18 & 236063.9 & 63288.61 & 0 & 11790.04 & 22824.02 & 5002.84 \\
\hline 7480.43 & 496983 & 15 & 45421.25 & 11652.23 & 0 & 3420.16 & 0 & 2495.21 \\
\hline 7480.50 & 343589 & 17 & 286705.54 & 79485.64 & 0 & 16098.1 & 22153.66 & 3130.1 \\
\hline 7480.56 & 413609 & 16 & 76723.47 & 19999.52 & 0 & 4723.63 & 0 & 3663.07 \\
\hline 7480.63 & 301714 & 17 & 172038.92 & 47378.29 & 0 & 11969.46 & 18477.47 & 7066.57 \\
\hline 7480.70 & 259652 & 18 & 231494.64 & 60126.85 & 0 & 13694.97 & 26123.02 & 9834.81 \\
\hline 7480.76 & 329053 & 17 & 259393.11 & 70603.16 & 0 & 13601.65 & 23524.16 & 20643.23 \\
\hline 7480.83 & 314678 & 17 & 263283.87 & 71861.76 & 0 & 13083.9 & 20818.83 & 19359.33 \\
\hline 7480.89 & 493359 & 15 & 110490.79 & 28747.31 & 0 & 4583.84 & 0 & 102854.7 \\
\hline 7480.96 & 578549 & 14 & 63477.81 & 12650.66 & 0 & 2465.81 & 0 & 231581.21 \\
\hline 7481.02 & 521460 & 15 & 109867.99 & 23495.49 & 0 & 4875.67 & 0 & 267188.24 \\
\hline 7481.09 & 503426 & 15 & 50903.88 & 11362.78 & 0 & 2543 & 0 & 222685.85 \\
\hline 7481.15 & 481464 & 16 & 57651.76 & 9423.65 & 0 & 2804.92 & 0 & 276099.49 \\
\hline 7481.22 & 389612 & 17 & 50122.75 & 11057.41 & 0 & 2895.17 & 0 & 268671.98 \\
\hline 7481.29 & 568582 & 15 & 145844.86 & 36419.93 & 0 & 5138.91 & 0 & 279659.71 \\
\hline 7481.35 & 623398 & 14 & 43365.15 & 9747.58 & 0 & 2220.33 & 0 & 145333.95 \\
\hline 7481.42 & 523356 & 15 & 66541.02 & 18146.68 & 0 & 2347.27 & 0 & 15226.85 \\
\hline 7481.48 & 448908 & 16 & 80377.43 & 22399.35 & 0 & 2712.88 & 0 & 45222.11 \\
\hline 7481.55 & 451291 & 16 & 70885.65 & 17402.23 & 0 & 3000.17 & 0 & 53094.9 \\
\hline 7481.61 & 476819 & 16 & 133125.48 & 31489.49 & 0 & 4464.86 & 0 & 186744.29 \\
\hline 7481.68 & 467367 & 16 & 190708.66 & 49841.93 & 0 & 3694.85 & 0 & 183284.51 \\
\hline 7481.75 & 564298 & 15 & 149172.23 & 39243.14 & 0 & 4610.82 & 0 & 266849.41 \\
\hline
\end{tabular}




\begin{tabular}{|c|c|c|c|c|c|c|c|c|}
\hline True Depth & XRF tCOUNTS & XRF Live Time & $\mathrm{Si}(\mathrm{ppm})$ & $\mathrm{Al}(\mathrm{ppm})$ & $\mathrm{P}(\mathrm{ppm})$ & $\mathrm{S}(\mathrm{ppm})$ & $\mathrm{K}(\mathrm{ppm})$ & $\mathrm{Ca}(\mathrm{ppm})$ \\
\hline 7481.81 & 505681 & 15 & 110080.62 & 28807.8 & 0 & 1344.25 & 0 & 169597.38 \\
\hline 7481.88 & 497049 & 15 & 42524.87 & 9031.71 & 0 & 1199.49 & 0 & 133475.14 \\
\hline 7481.94 & 229416 & 18 & 260007.53 & 68592.03 & 0 & 8588.76 & 0 & 81961.23 \\
\hline 7482.01 & 423804 & 16 & 262786.58 & 65482.29 & 0 & 5959.52 & 7581.92 & 119676.14 \\
\hline 7482.07 & 480056 & 16 & 212542.66 & 53418.52 & 0 & 11500.27 & 0 & 151533.5 \\
\hline 7482.14 & 577072 & 14 & 67302.89 & 18547.86 & 0 & 5244.93 & 0 & 71683.88 \\
\hline 7482.20 & 516406 & 15 & 106868.2 & 29957.6 & 0 & 6201.81 & 0 & 106625.36 \\
\hline 7482.27 & 437063 & 16 & 154862.68 & 43553.14 & 0 & 9727.77 & 0 & 54017.24 \\
\hline 7482.34 & 615663 & 14 & 30005.62 & 6773.18 & 0 & 5105.74 & 0 & 47689.39 \\
\hline 7482.40 & 611492 & 14 & 42235.99 & 9059.19 & 0 & 6162.37 & 0 & 85911.43 \\
\hline 7482.47 & 548999 & 15 & 19365.33 & 3506.82 & 0 & 3194.49 & 0 & 13006.1 \\
\hline 7482.53 & 399464 & 17 & 53285.08 & 13239.19 & 0 & 4781.14 & 0 & 26157.09 \\
\hline 7482.60 & 359592 & 17 & 54389.91 & 13819.3 & 0 & 45515.16 & 0 & 8326.66 \\
\hline 7482.66 & 247992 & 18 & 140406.77 & 38066.01 & 0 & 8300.35 & 0 & 12126.95 \\
\hline 7482.73 & 471978 & 16 & 100168.38 & 27986.84 & 0 & 8038.65 & 0 & 11055.77 \\
\hline 7482.80 & 580204 & 14 & 45734.25 & 10493.59 & 0 & 5092.55 & 0 & 9996.78 \\
\hline 7482.86 & 584576 & 14 & 54928.11 & 13678.75 & 0 & 6033.33 & 0 & 49541.2 \\
\hline 7482.93 & 380759 & 17 & 32724.74 & 6828.01 & 0 & 4752.57 & 0 & 4177.05 \\
\hline 7482.99 & 297675 & 18 & 65226.58 & 15967.87 & 0 & 4851.97 & 0 & 10535.5 \\
\hline 7483.06 & 406598 & 16 & 26572.26 & 5286.26 & 0 & 4303.49 & 0 & 6005.26 \\
\hline 7483.12 & 424829 & 16 & 38253.42 & 9213.96 & 0 & 3872.89 & 0 & 3516.25 \\
\hline 7483.19 & 361005 & 17 & 94397.04 & 25758.37 & 0 & 6543.7 & 0 & 6333.88 \\
\hline 7483.25 & 135056 & 19 & 31751.58 & 9497.24 & 0 & 2799.84 & 0 & 4423.58 \\
\hline 7483.32 & 368287 & 17 & 169530.9 & 46102.09 & 0 & 9813.67 & 11827.98 & 6243.84 \\
\hline 7483.39 & 465214 & 16 & 84527.73 & 23199.62 & 0 & 5149.47 & 0 & 10261.1 \\
\hline 7483.45 & 342591 & 17 & 181474.01 & 46067.91 & 0 & 9976.44 & 9399.28 & 2695.44 \\
\hline 7483.52 & 423066 & 16 & 71041.93 & 18091.32 & 0 & 4628.56 & 0 & 3327.71 \\
\hline 7483.58 & 490563 & 16 & 44795.8 & 9306.96 & 0 & 3529.67 & 0 & 2410.28 \\
\hline 7483.65 & 581831 & 14 & 40920.05 & 9670.92 & 0 & 3896.05 & 0 & 3155.12 \\
\hline 7483.71 & 356576 & 17 & 274450.88 & 69754.87 & 0 & 14910.87 & 12482.16 & 24397.21 \\
\hline 7483.78 & 346654 & 17 & 273929.24 & 70662.05 & 0 & 14448.11 & 16738.03 & 12082.96 \\
\hline 7483.85 & 319942 & 17 & 316527.75 & 81761.05 & 0 & 15162.39 & 19829.45 & 7091.72 \\
\hline 7483.91 & 349587 & 17 & 122097.69 & 33270.2 & 0 & 5527.95 & 0 & 5322.19 \\
\hline
\end{tabular}




\begin{tabular}{|c|c|c|c|c|c|c|c|c|}
\hline True Depth & XRF tCOUNTS & XRF Live Time & $\mathrm{Si}(\mathrm{ppm})$ & $\mathrm{Al}(\mathrm{ppm})$ & $\mathrm{P}(\mathrm{ppm})$ & $\mathrm{S}(\mathrm{ppm})$ & $\mathrm{K}(\mathrm{ppm})$ & $\mathrm{Ca}(\mathrm{ppm})$ \\
\hline 7483.98 & 503290 & 15 & 77213.65 & 21923.28 & 0 & 4037.01 & 0 & 3877 \\
\hline 7484.04 & 464733 & 16 & 81590.4 & 21110.23 & 0 & 5681.9 & 0 & 8445.81 \\
\hline 7484.11 & 576015 & 15 & 38171.55 & 10069.2 & 0 & 3329.34 & 0 & 2351.36 \\
\hline 7484.17 & 443222 & 16 & 114485.23 & 30453.99 & 0 & 6820.68 & 0 & 3444.29 \\
\hline 7484.24 & 437904 & 16 & 98803.72 & 25642.5 & 0 & 7025.83 & 0 & 6118.46 \\
\hline 7484.30 & 357665 & 17 & 200957.14 & 54461.88 & 0 & 8181.92 & 8052.23 & 2460.55 \\
\hline 7484.37 & 545269 & 15 & 53722.99 & 16025.05 & 0 & 4403.39 & 0 & 3408.37 \\
\hline 7484.44 & 539014 & 15 & 53822.89 & 13732 & 0 & 4113.8 & 0 & 2828.63 \\
\hline 7484.50 & 359395 & 17 & 250455.82 & 65046.12 & 0 & 9684.3 & 13303.85 & 2394.82 \\
\hline 7484.57 & 396423 & 17 & 157078.66 & 43045.53 & 0 & 5744.99 & 0 & 2279.39 \\
\hline 7484.63 & 637431 & 14 & 16568.39 & 3269.81 & 0 & 3651.53 & 0 & 2069.28 \\
\hline 7484.70 & 450927 & 16 & 86798.16 & 22812.71 & 0 & 4333.47 & 0 & 2543.42 \\
\hline 7484.76 & 383836 & 17 & 137141.43 & 39053.21 & 0 & 6186.01 & 8711.11 & 2863.4 \\
\hline 7484.83 & 641679 & 14 & 19161.36 & 4580.23 & 0 & 2794.92 & 0 & 1015.57 \\
\hline 7484.90 & 449190 & 16 & 94129.34 & 26303.1 & 0 & 5130.15 & 0 & 3320.41 \\
\hline 7484.96 & 374078 & 17 & 165928.32 & 45904.3 & 0 & 6974.79 & 0 & 1811.86 \\
\hline 7485.03 & 389090 & 17 & 178052.29 & 47970.9 & 0 & 8120.7 & 5815.54 & 2733.32 \\
\hline 7485.09 & 582643 & 14 & 48076.02 & 13283.56 & 0 & 3494.18 & 0 & 2443.43 \\
\hline 7485.16 & 593849 & 14 & 36516.04 & 10580.68 & 0 & 3459.28 & 0 & 2355.76 \\
\hline 7485.22 & 408582 & 16 & 74325.56 & 24098.56 & 0 & 4156.86 & 0 & 2880.05 \\
\hline 7485.29 & 218676 & 18 & 410244.18 & 99163.23 & 0 & 17590.71 & 0 & 3000.79 \\
\hline 7485.35 & 479333 & 16 & 87673.05 & 22453.71 & 0 & 4655.91 & 0 & 3972.22 \\
\hline 7485.42 & 490072 & 16 & 53566.82 & 14081.46 & 0 & 3035.13 & 0 & 2230.51 \\
\hline 7485.49 & 451040 & 16 & 57760.62 & 14116.28 & 0 & 3026 & 0 & 3431.07 \\
\hline 7485.55 & 430982 & 16 & 63700.77 & 14837.1 & 0 & 3718.94 & 0 & 4888.05 \\
\hline 7485.62 & 439163 & 16 & 60182.51 & 15010.58 & 0 & 3100.3 & 0 & 4276.53 \\
\hline 7485.68 & 253660 & 18 & 180730.8 & 50412.14 & 0 & 6638.66 & 0 & 5462.03 \\
\hline 7485.75 & 315172 & 17 & 232412.16 & 60510.8 & 0 & 7497.88 & 18131.32 & 2691.68 \\
\hline 7485.81 & 309864 & 17 & 133842.22 & 33164.77 & 0 & 4851.62 & 7096.04 & 2611.54 \\
\hline 7485.88 & 156723 & 19 & 138821.65 & 31346.61 & 0 & 7083.33 & 0 & 5002.49 \\
\hline 7485.94 & 273306 & 18 & 85908.43 & 22055.87 & 0 & 3542.42 & 0 & 3523.69 \\
\hline 7486.01 & 305161 & 17 & 124825.41 & 30573.54 & 0 & 5773.24 & 7802.07 & 5793.69 \\
\hline 7486.08 & 235415 & 18 & 201336.74 & 48926.92 & 0 & 9007.67 & 17372.37 & 5844.55 \\
\hline
\end{tabular}




\begin{tabular}{|c|c|c|c|c|c|c|c|c|}
\hline True Depth & XRF tCOUNTS & XRF Live Time & $\mathrm{Si}(\mathrm{ppm})$ & $\mathrm{Al}(\mathrm{ppm})$ & $\mathrm{P}(\mathrm{ppm})$ & $\mathrm{S}(\mathrm{ppm})$ & $\mathrm{K}(\mathrm{ppm})$ & $\mathrm{Ca}(\mathrm{ppm})$ \\
\hline 7486.14 & 250709 & 18 & 203526.29 & 50822.2 & 0 & 9292.38 & 19860.84 & 5194.85 \\
\hline 7486.21 & 262482 & 18 & 124035.97 & 29101.75 & 0 & 6454.25 & 11678.62 & 7031.76 \\
\hline 7486.27 & 282354 & 18 & 83712.25 & 19628.13 & 0 & 3944.71 & 0 & 7148.86 \\
\hline 7486.34 & 247885 & 18 & 137146.34 & 33708.16 & 0 & 5448.94 & 7287.51 & 3442.71 \\
\hline 7486.40 & 351206 & 17 & 55748.39 & 12980.97 & 0 & 3469.05 & 0 & 6548.22 \\
\hline 7486.47 & 304232 & 17 & 65725.48 & 17286.89 & 0 & 3215.96 & 0 & 8472.37 \\
\hline 7486.54 & 358918 & 17 & 142424.61 & 34625.96 & 0 & 5484.19 & 4577.44 & 9048.13 \\
\hline 7486.60 & 340067 & 17 & 186197.33 & 45457.04 & 0 & 6026.86 & 8214.29 & 7791.46 \\
\hline 7486.67 & 306578 & 17 & 209839.56 & 50630.77 & 0 & 8429.73 & 14193.89 & 10440.2 \\
\hline 7486.73 & 286391 & 18 & 103429.14 & 25690.12 & 0 & 4195.58 & 0 & 9964.16 \\
\hline 7486.80 & 382084 & 17 & 189573.15 & 49629.16 & 0 & 5765.5 & 6385.35 & 9624.08 \\
\hline 7486.86 & 499716 & 15 & 73610.77 & 18992.62 & 0 & 5187.48 & 0 & 9350.37 \\
\hline 7486.93 & 338441 & 17 & 291759.19 & 73928.98 & 0 & 9855.36 & 13735.15 & 9878.54 \\
\hline 7486.99 & 430970 & 16 & 120005.7 & 30567.55 & 0 & 7824.66 & 0 & 11006.36 \\
\hline 7487.06 & 497310 & 15 & 66144.37 & 16099.29 & 0 & 4967.51 & 0 & 16372.39 \\
\hline 7487.13 & 349334 & 17 & 274289.95 & 71084.64 & 0 & 10320.13 & 15793.91 & 5462.68 \\
\hline 7487.19 & 382872 & 17 & 181589.62 & 47007.33 & 0 & 8382.83 & 4531.9 & 7322.77 \\
\hline 7487.26 & 131478 & 19 & 14402.86 & 9686.1 & 0 & 2485.53 & 0 & 2609.26 \\
\hline 7487.32 & 114705 & 19 & 186467.23 & 50280.77 & 0 & 10629.93 & 0 & 7766.41 \\
\hline 7487.39 & 325665 & 17 & 301769.51 & 74902.45 & 0 & 12065.19 & 19789.37 & 3174.1 \\
\hline 7487.45 & 350651 & 17 & 184353.64 & 47338.77 & 0 & 7249.88 & 12377.6 & 4413.74 \\
\hline 7487.52 & 368508 & 17 & 132826.39 & 36037.87 & 0 & 5319.54 & 0 & 4406.13 \\
\hline 7487.59 & 328290 & 17 & 264581.42 & 69073.1 & 0 & 13098.9 & 17628.88 & 7484.4 \\
\hline 7487.65 & 349189 & 17 & 265945.54 & 68307.84 & 0 & 14171.58 & 19338.8 & 6049.61 \\
\hline 7487.72 & 378959 & 17 & 197470.71 & 52488.98 & 0 & 9718.2 & 8282.38 & 4984.22 \\
\hline 7487.78 & 413485 & 16 & 152971.36 & 42066.49 & 0 & 8326.68 & 0 & 4078.76 \\
\hline 7487.85 & 603048 & 14 & 31387.94 & 8073.95 & 0 & 4833.5 & 0 & 3864.8 \\
\hline 7487.91 & 530833 & 15 & 67148.48 & 18794.2 & 0 & 5557.83 & 0 & 5067.35 \\
\hline 7487.98 & 405975 & 16 & 138775.01 & 35066.52 & 0 & 7279.66 & 0 & 12298.61 \\
\hline 7488.04 & 475231 & 16 & 90232.73 & 25070.9 & 0 & 6232.79 & 0 & 17500.06 \\
\hline 7488.11 & 401935 & 16 & 148973.84 & 40216.04 & 0 & 6093.79 & 5104.33 & 5454.16 \\
\hline 7488.18 & 435699 & 16 & 87787.45 & 23600.3 & 0 & 6793.23 & 0 & 9267.23 \\
\hline 7488.24 & 351501 & 17 & 222127.05 & 55758.98 & 0 & 9565.51 & 12819 & 15458.57 \\
\hline
\end{tabular}




\begin{tabular}{|c|c|c|c|c|c|c|c|c|}
\hline True Depth & XRF tCOUNTS & XRF Live Time & $\mathrm{Si}(\mathrm{ppm})$ & $\mathrm{Al}(\mathrm{ppm})$ & $\mathrm{P}(\mathrm{ppm})$ & $\mathrm{S}(\mathrm{ppm})$ & $\mathrm{K}(\mathrm{ppm})$ & $\mathrm{Ca}(\mathrm{ppm})$ \\
\hline 7488.31 & 323964 & 17 & 239299.27 & 59997.16 & 0 & 9420.5 & 17952.58 & 6966.25 \\
\hline 7488.37 & 329337 & 17 & 231681.69 & 64226.18 & 0 & 8176.88 & 16333.73 & 8977.36 \\
\hline 7488.44 & 388282 & 17 & 297874.65 & 78109.76 & 0 & 16801.83 & 11538.92 & 36615.79 \\
\hline 7488.50 & 323990 & 17 & 354844.26 & 86559.41 & 0 & 13596.53 & 19146.64 & 6031.71 \\
\hline 7488.57 & 356970 & 17 & 252124.22 & 64932.79 & 0 & 15082.98 & 15308.21 & 6749.25 \\
\hline 7488.64 & 371681 & 17 & 187229.91 & 46739.48 & 0 & 9630.33 & 7656.36 & 8452.65 \\
\hline 7488.70 & 385995 & 17 & 178601.2 & 47417.71 & 0 & 13176.2 & 8129.88 & 9614.68 \\
\hline 7488.77 & 415457 & 16 & 140161.57 & 39078.07 & 0 & 9323.94 & 0 & 10359.56 \\
\hline 7488.83 & 426065 & 16 & 110205.15 & 30011.18 & 0 & 6727.64 & 0 & 8117.24 \\
\hline 7488.90 & 339711 & 17 & 235324.06 & 62431.32 & 0 & 9501.08 & 15783.18 & 5055.97 \\
\hline 7488.96 & 556944 & 15 & 57565.83 & 15935.96 & 0 & 4603.94 & 0 & 4114.57 \\
\hline 7489.03 & 348149 & 17 & 294715.99 & 75252.69 & 0 & 10304.57 & 18050.76 & 3613.58 \\
\hline 7489.09 & 425609 & 16 & 138678.78 & 38134.87 & 0 & 8004.96 & 0 & 7141.98 \\
\hline 7489.16 & 436013 & 16 & 109003.82 & 30988.91 & 0 & 5865.83 & 0 & 7247.64 \\
\hline 7489.23 & 337776 & 17 & 283191.71 & 75118.89 & 0 & 12034.12 & 21410.31 & 8788.97 \\
\hline 7489.29 & 389172 & 17 & 163667.45 & 43585.54 & 0 & 8595.67 & 9725.79 & 6527.07 \\
\hline 7489.36 & 300026 & 17 & 101236.09 & 30254.6 & 0 & 5101.14 & 0 & 5160.02 \\
\hline 7489.42 & 512200 & 15 & 69009.31 & 18949.15 & 0 & 5198.59 & 0 & 5118.91 \\
\hline 7489.49 & 347259 & 17 & 202711.98 & 54742.65 & 0 & 8071.33 & 13317.23 & 10651.06 \\
\hline 7489.55 & 324538 & 17 & 233962.9 & 59689.63 & 0 & 10753.67 & 19314.77 & 13064.62 \\
\hline 7489.62 & 391032 & 17 & 89673.3 & 24432.23 & 0 & 4644.54 & 0 & 10767.11 \\
\hline 7489.69 & 298330 & 17 & 252293.52 & 65031.27 & 0 & 7420.3 & 24206.95 & 10311.38 \\
\hline 7489.75 & 438999 & 16 & 125641.6 & 35655.8 & 0 & 5252.26 & 0 & 9344.21 \\
\hline 7489.82 & 340431 & 17 & 274241.97 & 75503.56 & 0 & 10233.55 & 18875.86 & 8518.18 \\
\hline 7489.88 & 515576 & 15 & 69985.83 & 20713.3 & 0 & 4863.96 & 0 & 4941.29 \\
\hline 7489.95 & 388707 & 17 & 148815.4 & 42372.91 & 0 & 8595.59 & 9035.12 & 7145.68 \\
\hline 7490.01 & 427378 & 16 & 99238.21 & 30178.34 & 0 & 5624.48 & 0 & 5455.54 \\
\hline 7490.08 & 488306 & 16 & 71170.38 & 20266.4 & 0 & 5997.52 & 0 & 5695.13 \\
\hline 7490.14 & 402650 & 16 & 125781.49 & 35268.17 & 0 & 8529.4 & 0 & 7145.32 \\
\hline 7490.21 & 353030 & 17 & 261106.67 & 69093.46 & 0 & 11831.75 & 16705.75 & 4977.85 \\
\hline 7490.28 & 374535 & 17 & 153388.36 & 44628.88 & 0 & 8013.14 & 0 & 4637.65 \\
\hline 7490.34 & 359582 & 17 & 194900.25 & 52415.57 & 0 & 12273.77 & 12092.17 & 7132.29 \\
\hline 7490.41 & 370559 & 17 & 207377.65 & 54810.42 & 0 & 10302.13 & 11966.93 & 6993.37 \\
\hline
\end{tabular}




\begin{tabular}{|c|c|c|c|c|c|c|c|c|}
\hline True Depth & XRF tCOUNTS & XRF Live Time & $\mathrm{Si}(\mathrm{ppm})$ & $\mathrm{Al}(\mathrm{ppm})$ & $\mathrm{P}(\mathrm{ppm})$ & $\mathrm{S}(\mathrm{ppm})$ & $\mathrm{K}(\mathrm{ppm})$ & $\mathrm{Ca}(\mathrm{ppm})$ \\
\hline 7490.47 & 363920 & 17 & 203076.13 & 54310.32 & 0 & 9272.99 & 9365.69 & 6563.64 \\
\hline 7490.54 & 452348 & 16 & 92496.74 & 25526.03 & 0 & 4475.4 & 0 & 8813.68 \\
\hline 7490.60 & 358677 & 17 & 253451.46 & 65958.44 & 0 & 10905.11 & 16918.6 & 16869.06 \\
\hline 7490.67 & 477986 & 16 & 85391.83 & 23022.8 & 0 & 5489.81 & 0 & 19402.36 \\
\hline 7490.73 & 361669 & 17 & 238732.54 & 62496.26 & 0 & 10125.17 & 18376.55 & 31141.68 \\
\hline 7490.80 & 342714 & 17 & 228161.13 & 61755.32 & 0 & 8156.18 & 20215.25 & 26489.76 \\
\hline 7490.87 & 519208 & 15 & 63704.83 & 18389.46 & 0 & 7139.05 & 0 & 11235.37 \\
\hline 7490.93 & 386605 & 17 & 183643.33 & 50481.61 & 0 & 7745.97 & 8076.04 & 8582.01 \\
\hline 7491.00 & 373050 & 17 & 196134.45 & 52255.99 & 0 & 12825.54 & 13856.51 & 9942.32 \\
\hline 7491.06 & 428089 & 16 & 78720.85 & 19629.98 & 0 & 5453.82 & 0 & 8696.94 \\
\hline 7491.13 & 454881 & 16 & 87048.04 & 23025.28 & 0 & 8731.56 & 0 & 17949.39 \\
\hline 7491.19 & 636220 & 14 & 19007.37 & 4543.82 & 0 & 3847.95 & 0 & 2870.91 \\
\hline 7491.26 & 335936 & 17 & 278626.28 & 72562.34 & 0 & 10678.46 & 19488.93 & 8808.52 \\
\hline 7491.33 & 554117 & 15 & 20898.78 & 4697.8 & 0 & 3267.71 & 0 & 1808.63 \\
\hline 7491.39 & 123129 & 19 & 379216.69 & 100490.56 & 0 & 22595.79 & 0 & 15179.93 \\
\hline 7491.46 & 348801 & 17 & 268098.46 & 74541.56 & 0 & 11234.19 & 21147.22 & 14261.07 \\
\hline 7491.52 & 345934 & 17 & 230416.98 & 60532.76 & 0 & 11716.26 & 22137.68 & 9293.67 \\
\hline 7491.59 & 319984 & 17 & 305473.22 & 81174.94 & 0 & 15169.68 & 24872.44 & 7845.81 \\
\hline 7491.65 & 339560 & 17 & 262426.99 & 70322.69 & 0 & 15818.39 & 22117.12 & 5654.5 \\
\hline 7491.72 & 324756 & 17 & 314627.11 & 82131.18 & 0 & 17907.61 & 22606.55 & 7347.66 \\
\hline 7491.78 & 393860 & 17 & 179968.13 & 50378.67 & 0 & 15469.25 & 11404.46 & 17489.93 \\
\hline 7491.85 & 394296 & 17 & 136141.4 & 38125.38 & 0 & 9122.2 & 0 & 13778.57 \\
\hline 7491.92 & 384253 & 17 & 304687.28 & 79155.66 & 0 & 24612.86 & 17036.15 & 42491.01 \\
\hline 7491.98 & 357318 & 17 & 304668.51 & 79032.56 & 0 & 25981.4 & 19801.48 & 24134.56 \\
\hline 7492.05 & 347838 & 17 & 303358.81 & 79215.99 & 0 & 24006.76 & 15380.41 & 27200.56 \\
\hline 7492.11 & 374265 & 17 & 169296.06 & 50115.12 & 0 & 9595.87 & 9839.45 & 7337.41 \\
\hline 7492.18 & 547265 & 15 & 38875.91 & 10959.17 & 0 & 4130.04 & 0 & 5827.85 \\
\hline 7492.24 & 340798 & 17 & 281439.44 & 72856.87 & 0 & 19215.35 & 19813.63 & 23592.63 \\
\hline 7492.31 & 454157 & 16 & 109971.21 & 32465.1 & 0 & 6925.41 & 0 & 9133.17 \\
\hline 7492.38 & 343090 & 17 & 354607.01 & 92818.36 & 0 & 22181.04 & 26684.26 & 7555.3 \\
\hline 7492.44 & 409464 & 16 & 157993.82 & 44722.65 & 0 & 8532.56 & 0 & 8956.39 \\
\hline 7492.51 & 362429 & 17 & 196124.22 & 53525.78 & 0 & 9472.55 & 15133.27 & 9267.74 \\
\hline 7492.57 & 321355 & 17 & 272490.22 & 74102.7 & 0 & 11405.6 & 23566.26 & 5316.37 \\
\hline
\end{tabular}




\begin{tabular}{|c|c|c|c|c|c|c|c|c|}
\hline True Depth & XRF tCOUNTS & XRF Live Time & $\mathrm{Si}(\mathrm{ppm})$ & $\mathrm{Al}(\mathrm{ppm})$ & $\mathrm{P}(\mathrm{ppm})$ & $\mathrm{S}(\mathrm{ppm})$ & $\mathrm{K}(\mathrm{ppm})$ & $\mathrm{Ca}(\mathrm{ppm})$ \\
\hline 7492.64 & 283655 & 18 & 200653.76 & 57195.41 & 0 & 8511.61 & 13212.26 & 4965.26 \\
\hline 7492.70 & 390581 & 17 & 186616.39 & 51829.01 & 0 & 9304.85 & 8433.36 & 6073.29 \\
\hline 7492.77 & 341350 & 17 & 264427.16 & 72540.31 & 0 & 14419.47 & 17166.49 & 10577.86 \\
\hline 7492.83 & 408057 & 16 & 129976.38 & 36718.04 & 0 & 5884.49 & 5483.98 & 5575.75 \\
\hline 7492.90 & 401508 & 16 & 130339.28 & 36620.99 & 0 & 6805.29 & 4692.84 & 6936.54 \\
\hline 7492.97 & 409134 & 16 & 142793.74 & 41091.97 & 0 & 6643.39 & 0 & 7479.26 \\
\hline 7493.03 & 364567 & 17 & 215926.3 & 59328.83 & 0 & 10226.57 & 20155.87 & 5590.46 \\
\hline 7493.10 & 327284 & 17 & 268463.92 & 68838.91 & 0 & 14426.56 & 22835.3 & 4092.78 \\
\hline 7493.16 & 358162 & 17 & 161566.22 & 44777.62 & 0 & 8490.39 & 10450.17 & 5497.77 \\
\hline 7493.23 & 373714 & 17 & 151606.22 & 43754.96 & 0 & 7696.04 & 11109.13 & 6122.44 \\
\hline 7493.29 & 379753 & 17 & 191839.39 & 54305.02 & 0 & 8213.77 & 12947.55 & 5516.93 \\
\hline 7493.36 & 293319 & 18 & 307750.63 & 87177.89 & 0 & 14209.44 & 22664.42 & 6512.27 \\
\hline 7493.43 & 328626 & 17 & 319219.83 & 84983.88 & 0 & 15409.91 & 20361.17 & 6085.44 \\
\hline 7493.49 & 516793 & 15 & 67716.43 & 19890.04 & 0 & 4075.75 & 0 & 2910.9 \\
\hline 7493.56 & 369950 & 17 & 172484.53 & 47411.01 & 0 & 8817.47 & 11436.3 & 5982.08 \\
\hline 7493.62 & 470030 & 16 & 74148.08 & 21006.12 & 0 & 4706.97 & 0 & 3591.69 \\
\hline 7493.69 & 391902 & 17 & 149220.11 & 42034.55 & 0 & 6070.54 & 7576.47 & 3380.73 \\
\hline 7493.75 & 628296 & 14 & 15202.08 & 3701.83 & 0 & 2250.5 & 0 & 660.85 \\
\hline 7493.82 & 304990 & 17 & 309235.15 & 80953.7 & 0 & 13352.31 & 26046 & 5368.5 \\
\hline 7493.88 & 322825 & 17 & 242181.75 & 64660.04 & 0 & 10896.95 & 21888.58 & 4093.21 \\
\hline 7493.95 & 318635 & 17 & 228761.97 & 61516.04 & 0 & 13423.58 & 13651.46 & 2434.57 \\
\hline 7494.02 & 365216 & 17 & 294123.03 & 72147.33 & 0 & 17087.21 & 14057.81 & 26293.83 \\
\hline 7494.08 & 461730 & 16 & 89400.51 & 22687.29 & 0 & 6752.63 & 0 & 23903.38 \\
\hline 7494.15 & 556272 & 15 & 37435.06 & 9941.89 & 0 & 3174.27 & 0 & 5240.18 \\
\hline 7494.21 & 348260 & 17 & 180475.93 & 47897.55 & 0 & 9107.73 & 12257.52 & 5570.96 \\
\hline 7494.28 & 437236 & 16 & 100681.33 & 26705.76 & 0 & 5073.58 & 0 & 6438.78 \\
\hline 7494.34 & 372797 & 17 & 168700.04 & 44677.78 & 0 & 9320.89 & 9944.16 & 3876.68 \\
\hline 7494.41 & 484103 & 16 & 65319.95 & 16904.39 & 0 & 4492.12 & 0 & 3845.89 \\
\hline 7494.48 & 378818 & 17 & 203182.99 & 53736.64 & 0 & 8159.4 & 7567.44 & 15130.81 \\
\hline 7494.54 & 537885 & 15 & 59003.35 & 14857.39 & 0 & 2812.49 & 0 & 2820.26 \\
\hline 7494.61 & 570226 & 15 & 32265.51 & 8438.68 & 0 & 2320.49 & 0 & 2142.96 \\
\hline 7494.67 & 371013 & 17 & 139325.29 & 36029.67 & 0 & 5518.34 & 5626.81 & 10092.13 \\
\hline 7494.74 & 446246 & 16 & 126098.94 & 32240.58 & 0 & 5732.46 & 0 & 39388.28 \\
\hline
\end{tabular}




\begin{tabular}{|c|c|c|c|c|c|c|c|c|}
\hline True Depth & XRF tCOUNTS & XRF Live Time & $\mathrm{Si}(\mathrm{ppm})$ & $\mathrm{Al}(\mathrm{ppm})$ & $\mathrm{P}(\mathrm{ppm})$ & $\mathrm{S}(\mathrm{ppm})$ & $\mathrm{K}(\mathrm{ppm})$ & $\mathrm{Ca}(\mathrm{ppm})$ \\
\hline 7494.80 & 494945 & 15 & 59137.97 & 17112.91 & 0 & 4221.5 & 0 & 6554.17 \\
\hline 7494.87 & 525859 & 15 & 47419.8 & 12737.73 & 0 & 4262.98 & 0 & 7504.18 \\
\hline 7494.93 & 386860 & 17 & 211670.83 & 55165.42 & 0 & 8533.33 & 7139.85 & 72198.74 \\
\hline 7495.00 & 488006 & 16 & 82478.25 & 24178.11 & 0 & 3954.45 & 0 & 4083.49 \\
\hline 7495.07 & 359862 & 17 & 102458.19 & 26204.28 & 0 & 4235.57 & 0 & 10471.2 \\
\hline 7495.13 & 339477 & 17 & 84824.94 & 20791.34 & 0 & 3913.69 & 0 & 6680.84 \\
\hline 7495.20 & 337820 & 17 & 91332.24 & 23077.92 & 0 & 4750.78 & 0 & 11962.59 \\
\hline 7495.26 & 266316 & 18 & 237144.99 & 54882.9 & 0 & 11439.3 & 20830.81 & 4800.14 \\
\hline 7495.33 & 376398 & 17 & 36753.04 & 8463.41 & 0 & 3179.27 & 0 & 2703.4 \\
\hline 7495.39 & 201604 & 18 & 186335.56 & 46187.08 & 0 & 8661.53 & 0 & 5945.74 \\
\hline 7495.46 & 334957 & 17 & 337242.46 & 78591.83 & 0 & 18362.66 & 17392.93 & 7215.23 \\
\hline 7495.52 & 377333 & 17 & 166076.53 & 42416.32 & 0 & 10343.32 & 0 & 9057.8 \\
\hline 7495.59 & 362418 & 17 & 215299.02 & 56615.2 & 0 & 8599.67 & 8343.8 & 10946.55 \\
\hline 7495.66 & 503191 & 15 & 77718.94 & 19884.31 & 0 & 5675.4 & 0 & 7118.37 \\
\hline 7495.72 & 402568 & 16 & 159436.43 & 40123.33 & 0 & 6774.39 & 0 & 33141.46 \\
\hline 7495.79 & 301073 & 17 & 142682.41 & 37543.51 & 0 & 6581.64 & 0 & 18545.18 \\
\hline 7495.85 & 371351 & 17 & 124336.85 & 30412.35 & 0 & 6259.24 & 0 & 11159.74 \\
\hline 7495.92 & 351529 & 17 & 85759.91 & 20943.45 & 0 & 4688.28 & 0 & 11405.34 \\
\hline 7495.98 & 307813 & 17 & 101061.42 & 22627.86 & 0 & 4793.06 & 0 & 10348.74 \\
\hline 7496.05 & 318582 & 17 & 117372.64 & 23903.47 & 0 & 5641.3 & 0 & 19967.6 \\
\hline 7496.12 & 322890 & 17 & 87128.37 & 18890.76 & 0 & 5636.56 & 0 & 9144.99 \\
\hline 7496.18 & 374280 & 17 & 112543.58 & 26853.19 & 0 & 5749.46 & 0 & 5461.81 \\
\hline 7496.25 & 335368 & 17 & 122697.71 & 25920.44 & 0 & 7190.57 & 0 & 8065.39 \\
\hline 7496.31 & 289049 & 18 & 27128.58 & 2960.27 & 0 & 3554.04 & 0 & 9605.83 \\
\hline 7496.38 & 238933 & 18 & 36321.42 & 6939.64 & 0 & 3828.54 & 0 & 8815.89 \\
\hline 7496.44 & 364362 & 17 & 60460.83 & 11938.51 & 0 & 4233.31 & 0 & 7127.67 \\
\hline 7496.51 & 484035 & 16 & 66159.76 & 15247.62 & 0 & 7252.61 & 0 & 9879.14 \\
\hline 7496.57 & 300452 & 17 & 239640.98 & 50992.45 & 0 & 12786.13 & 0 & 21627.55 \\
\hline 7496.64 & 570112 & 15 & 42356.47 & 8138.74 & 0 & 4330.04 & 0 & 40394.63 \\
\hline 7496.71 & 538732 & 15 & 38032.21 & 7420.76 & 0 & 3856.88 & 0 & 15537.65 \\
\hline 7496.77 & 414260 & 16 & 94294.89 & 19480.72 & 0 & 6498.9 & 0 & 28789.46 \\
\hline 7496.84 & 366151 & 17 & 171275.72 & 39568.2 & 0 & 10525.37 & 0 & 9597.97 \\
\hline 7496.90 & 371678 & 17 & 154537.83 & 34332.67 & 0 & 10969.45 & 0 & 11257.72 \\
\hline
\end{tabular}




\begin{tabular}{|c|c|c|c|c|c|c|c|c|}
\hline True Depth & XRF tCOUNTS & XRF Live Time & $\mathrm{Si}(\mathrm{ppm})$ & $\mathrm{Al}(\mathrm{ppm})$ & $\mathrm{P}(\mathrm{ppm})$ & $\mathrm{S}(\mathrm{ppm})$ & $\mathrm{K}(\mathrm{ppm})$ & $\mathrm{Ca}(\mathrm{ppm})$ \\
\hline 7496.97 & 277682 & 18 & 193756.66 & 44432.61 & 0 & 8610.66 & 5579.81 & 6200.15 \\
\hline 7497.03 & 331903 & 17 & 144258.38 & 32366.3 & 0 & 10665.26 & 0 & 16494.34 \\
\hline 7497.10 & 379155 & 17 & 190190.19 & 46089.62 & 0 & 10347.65 & 0 & 16619.92 \\
\hline 7497.17 & 341778 & 17 & 219060.3 & 52646.51 & 0 & 10206.07 & 0 & 8327.25 \\
\hline 7497.23 & 432164 & 16 & 46251.79 & 10772.48 & 0 & 4415.59 & 0 & 4362.62 \\
\hline 7497.30 & 294263 & 18 & 90723.1 & 23113.29 & 0 & 5756.84 & 0 & 18899.2 \\
\hline 7497.36 & 502317 & 15 & 81087.36 & 21157.53 & 0 & 7502.39 & 0 & 7239.25 \\
\hline 7497.43 & 290450 & 18 & 331004.74 & 85134.55 & 0 & 20800.97 & 0 & 8972.65 \\
\hline 7497.49 & 363740 & 17 & 241063.64 & 59930.27 & 0 & 15520.08 & 11706.43 & 9098.67 \\
\hline 7497.56 & 380096 & 17 & 151253.17 & 38741.6 & 0 & 11062.81 & 4606.83 & 20578.12 \\
\hline 7497.62 & 319265 & 17 & 222357.84 & 52304 & 0 & 12535.92 & 13563.99 & 12572.42 \\
\hline 7497.69 & 337624 & 17 & 245023.9 & 56477.22 & 0 & 16779.54 & 10993.39 & 10714.33 \\
\hline 7497.76 & 425361 & 16 & 99425.35 & 23105.25 & 0 & 9789.79 & 0 & 13579.74 \\
\hline 7497.82 & 313708 & 17 & 277830.48 & 66191.86 & 0 & 17393.24 & 14368.07 & 11662.38 \\
\hline 7497.89 & 341077 & 17 & 108998.04 & 28344.2 & 0 & 6680.38 & 0 & 13518.1 \\
\hline 7497.95 & 356978 & 17 & 323059.41 & 80662.41 & 0 & 22530.55 & 12347.5 & 16396.79 \\
\hline 7498.02 & 364276 & 17 & 323866.2 & 76734.26 & 0 & 21331.19 & 9324.89 & 20600.78 \\
\hline 7498.08 & 335433 & 17 & 95048.06 & 21573.78 & 0 & 6845.09 & 0 & 13379.05 \\
\hline 7498.15 & 367878 & 17 & 251387.68 & 61689.81 & 0 & 24387.69 & 6933.87 & 22961.57 \\
\hline 7498.22 & 336141 & 17 & 250515.79 & 61385.89 & 0 & 18495.35 & 8040.56 & 11176.8 \\
\hline 7498.28 & 307433 & 17 & 173402.41 & 40019.92 & 0 & 17624 & 7154.76 & 19409.64 \\
\hline 7498.35 & 284042 & 18 & 171533.13 & 38890.39 & 0 & 12762.56 & 8727.15 & 18342.09 \\
\hline 7498.41 & 301965 & 17 & 193046.77 & 43352.44 & 0 & 15515.54 & 7825.48 & 17725.23 \\
\hline 7498.48 & 475615 & 16 & 89066.09 & 24181.1 & 0 & 8198.71 & 0 & 15169.15 \\
\hline 7498.54 & 410355 & 16 & 184296.42 & 44843.8 & 0 & 15424.25 & 0 & 25383.69 \\
\hline 7498.61 & 416153 & 16 & 170066.85 & 40025.96 & 0 & 16109.15 & 0 & 21957.41 \\
\hline 7498.67 & 374559 & 17 & 109223.35 & 28918.43 & 0 & 8782.62 & 0 & 15442.14 \\
\hline 7498.74 & 360183 & 17 & 299127.83 & 70265.47 & 0 & 25674.81 & 4915.08 & 20561.41 \\
\hline 7498.81 & 404745 & 16 & 155263.73 & 36414.43 & 0 & 11087.33 & 0 & 11912.41 \\
\hline 7498.87 & 365862 & 17 & 257006.21 & 55649.05 & 0 & 28550.06 & 0 & 16728.66 \\
\hline 7498.94 & 384972 & 17 & 227299.19 & 50947.76 & 0 & 19128.65 & 0 & 32249.04 \\
\hline 7499.00 & 359842 & 17 & 279237.95 & 57341.78 & 0 & 21809.39 & 0 & 46011.85 \\
\hline 7499.07 & 354186 & 17 & 298616.97 & 65105.38 & 0 & 19972.99 & 0 & 33412.12 \\
\hline
\end{tabular}




\begin{tabular}{|c|c|c|c|c|c|c|c|c|}
\hline True Depth & XRF tCOUNTS & XRF Live Time & $\mathrm{Si}(\mathrm{ppm})$ & $\mathrm{Al}(\mathrm{ppm})$ & $\mathrm{P}(\mathrm{ppm})$ & $\mathrm{S}(\mathrm{ppm})$ & $\mathrm{K}(\mathrm{ppm})$ & $\mathrm{Ca}(\mathrm{ppm})$ \\
\hline 7499.13 & 368072 & 17 & 282727.71 & 60788.19 & 0 & 19550.48 & 0 & 38682.14 \\
\hline 7499.20 & 337184 & 17 & 333905.9 & 70898.1 & 0 & 23085.53 & 0 & 22513.61 \\
\hline 7499.27 & 338476 & 17 & 309206.08 & 66381.63 & 0 & 23424.16 & 5186.74 & 19850.11 \\
\hline 7499.33 & 356098 & 17 & 233962.48 & 52052.79 & 0 & 20036.41 & 0 & 20324.32 \\
\hline 7499.40 & 359853 & 17 & 263011.8 & 54556.98 & 0 & 23035.14 & 0 & 23708.55 \\
\hline 7499.46 & 336992 & 17 & 334670.07 & 72729.27 & 0 & 22121.15 & 6867.81 & 12277.77 \\
\hline 7499.53 & 345115 & 17 & 249144.7 & 54629.26 & 0 & 23096.71 & 0 & 10946.53 \\
\hline 7499.59 & 392368 & 17 & 188607.82 & 40818.14 & 0 & 18446.84 & 0 & 19425.38 \\
\hline 7499.66 & 408116 & 16 & 140039.48 & 29418.98 & 0 & 13377.66 & 0 & 24449.49 \\
\hline 7499.72 & 377406 & 17 & 152333.68 & 34482.4 & 0 & 10074.2 & 0 & 17285.5 \\
\hline 7499.79 & 363327 & 17 & 265534.16 & 55132.09 & 0 & 15274.36 & 0 & 87676.83 \\
\hline 7499.86 & 393502 & 17 & 234388.58 & 49800.59 & 0 & 12177.53 & 0 & 104301.95 \\
\hline 7499.92 & 444709 & 16 & 183931.89 & 39974.72 & 0 & 13670.43 & 0 & 101489.66 \\
\hline 7499.99 & 436492 & 16 & 148619.28 & 32139.52 & 0 & 15771.14 & 0 & 23446.28 \\
\hline 7500.05 & 622815 & 14 & 25458.93 & 5468.6 & 0 & 4734.42 & 0 & 5054.18 \\
\hline 7500.12 & 368021 & 17 & 248800.47 & 53145.24 & 0 & 20091.56 & 0 & 15356.43 \\
\hline 7500.18 & 340507 & 17 & 338421.65 & 68235.27 & 0 & 29184.88 & 0 & 14599.92 \\
\hline 7500.25 & 335206 & 17 & 243092.61 & 51726.25 & 0 & 25321.76 & 0 & 11490.43 \\
\hline 7500.31 & 299299 & 17 & 99785.19 & 18268.1 & 0 & 8106.84 & 0 & 36926.82 \\
\hline 7500.38 & 354709 & 17 & 135307.64 & 31713.54 & 0 & 11850.04 & 0 & 49574.45 \\
\hline 7500.45 & 413137 & 16 & 81321.05 & 17691.17 & 0 & 8165.95 & 0 & 86681.86 \\
\hline 7500.51 & 360790 & 17 & 77003.18 & 15685.84 & 0 & 8832.71 & 0 & 47278.3 \\
\hline 7500.58 & 247711 & 18 & 85174.85 & 16274.75 & 0 & 8990.94 & 0 & 19550.39 \\
\hline 7500.64 & 301489 & 17 & 51801.59 & 8007.15 & 0 & 6368.01 & 0 & 12636.76 \\
\hline 7500.71 & 302536 & 17 & 88374.71 & 15480.27 & 0 & 6892.12 & 0 & 11520.12 \\
\hline 7500.77 & 337508 & 17 & 91961.25 & 16481.95 & 0 & 6157.57 & 0 & 12174.16 \\
\hline 7500.84 & 292986 & 18 & 208196.58 & 37930.41 & 0 & 10811.27 & 0 & 11421.34 \\
\hline 7500.91 & 333375 & 17 & 91522.76 & 16405.66 & 0 & 5550.29 & 0 & 10089.1 \\
\hline 7500.97 & 288562 & 18 & 157011.43 & 29309.07 & 0 & 9784.08 & 0 & 11703.1 \\
\hline 7501.04 & 349020 & 17 & 104828.01 & 18126.04 & 0 & 9748.23 & 0 & 12682.16 \\
\hline 7501.10 & 424956 & 16 & 104380.61 & 20050.94 & 0 & 10223.67 & 0 & 11004.4 \\
\hline 7501.17 & 426880 & 16 & 100636.42 & 21716.27 & 0 & 9560.05 & 0 & 9736.14 \\
\hline 7501.23 & 474101 & 16 & 140618.26 & 24734.49 & 0 & 19706.9 & 0 & 17123.42 \\
\hline
\end{tabular}




\begin{tabular}{|c|c|c|c|c|c|c|c|c|}
\hline True Depth & XRF tCOUNTS & XRF Live Time & $\mathrm{Si}(\mathrm{ppm})$ & $\mathrm{Al}(\mathrm{ppm})$ & $\mathrm{P}(\mathrm{ppm})$ & $\mathrm{S}(\mathrm{ppm})$ & $\mathrm{K}(\mathrm{ppm})$ & $\mathrm{Ca}(\mathrm{ppm})$ \\
\hline 7501.30 & 321113 & 17 & 67618.71 & 12408.94 & 0 & 4094.37 & 0 & 7662.44 \\
\hline 7501.36 & 393828 & 17 & 72050.34 & 10911.1 & 0 & 17775.54 & 0 & 10628.9 \\
\hline 7501.43 & 189049 & 18 & 27175.23 & 4544.07 & 0 & 4654.77 & 0 & 4245.29 \\
\hline 7501.50 & 332354 & 17 & 337669.36 & 64043.65 & 0 & 20582.88 & 0 & 12265.23 \\
\hline 7501.56 & 497699 & 15 & 75218.13 & 14456.7 & 0 & 36005.42 & 0 & 5947.1 \\
\hline 7501.63 & 317592 & 17 & 90103.24 & 16837.79 & 0 & 4199.76 & 0 & 7339.13 \\
\hline 7501.69 & 308756 & 17 & 205525.32 & 38349.83 & 0 & 9098.98 & 0 & 10725.21 \\
\hline 7501.76 & 332552 & 17 & 368368.51 & 63818.82 & 0 & 19843.94 & 0 & 10912.56 \\
\hline 7501.82 & 384625 & 17 & 183729.81 & 31352.06 & 0 & 10852.39 & 0 & 13547.51 \\
\hline 7501.89 & 442580 & 16 & 89990.88 & 14189.31 & 0 & 5660.19 & 0 & 14577.93 \\
\hline 7501.96 & 407355 & 16 & 109875.03 & 19842.74 & 0 & 7767.15 & 0 & 18038.37 \\
\hline 7502.02 & 333427 & 17 & 227050.47 & 40085.79 & 0 & 12141.51 & 0 & 13596.49 \\
\hline 7502.09 & 374359 & 17 & 210556.72 & 34096.96 & 0 & 19591.13 & 0 & 16984.5 \\
\hline 7502.15 & 369409 & 17 & 328092.41 & 51774.81 & 0 & 51960.5 & 0 & 22974.17 \\
\hline 7502.22 & 177311 & 19 & 171288.36 & 19697.22 & 0 & 9015.84 & 0 & 13025.93 \\
\hline 7502.28 & 177221 & 19 & 166806.14 & 24024.77 & 0 & 8672.94 & 0 & 11647 \\
\hline 7502.35 & 229716 & 18 & 238363.33 & 34006.18 & 0 & 14023.89 & 0 & 15210.55 \\
\hline 7502.41 & 246996 & 18 & 301821.86 & 50579.47 & 0 & 21066 & 0 & 11251.77 \\
\hline 7502.48 & 284276 & 18 & 161664.85 & 25352.92 & 0 & 10967.09 & 0 & 14915.96 \\
\hline 7502.55 & 291006 & 18 & 122073.16 & 19635.08 & 0 & 6860.95 & 0 & 9972.81 \\
\hline 7502.61 & 237225 & 18 & 246640.86 & 36810.45 & 0 & 15353.73 & 0 & 14893.3 \\
\hline 7502.68 & 231419 & 18 & 279944.91 & 40308.65 & 0 & 15760.38 & 0 & 17516.41 \\
\hline 7502.74 & 349863 & 17 & 300879.34 & 47468.31 & 0 & 11761.07 & 0 & 11438.9 \\
\hline 7502.81 & 538836 & 15 & 75265.25 & 12361.48 & 0 & 3090.24 & 0 & 4723.11 \\
\hline 7502.87 & 309250 & 17 & 362281.14 & 51768.96 & 0 & 18534.99 & 0 & 13211.34 \\
\hline 7502.94 & 336984 & 17 & 320751.08 & 48648 & 0 & 15739.84 & 0 & 11750.67 \\
\hline 7503.01 & 341176 & 17 & 131982.49 & 20109.69 & 0 & 9398.25 & 0 & 11641.14 \\
\hline 7503.07 & 337410 & 17 & 127454.59 & 19610.67 & 0 & 7950.99 & 0 & 11411.74 \\
\hline 7503.14 & 316510 & 17 & 384168.05 & 58573.04 & 0 & 17769.25 & 0 & 13676.23 \\
\hline 7503.20 & 356830 & 17 & 217283.03 & 32602.76 & 0 & 11273.92 & 0 & 10744.92 \\
\hline 7503.27 & 318474 & 17 & 300035.17 & 42440.7 & 0 & 19154.02 & 0 & 13439.06 \\
\hline 7503.33 & 339858 & 17 & 200935.14 & 29052.36 & 0 & 14593.9 & 0 & 15462.4 \\
\hline 7503.40 & 278199 & 18 & 267233.94 & 38244.89 & 0 & 15720.04 & 0 & 13855.25 \\
\hline
\end{tabular}




\begin{tabular}{|c|c|c|c|c|c|c|c|c|}
\hline True Depth & XRF tCOUNTS & XRF Live Time & $\mathrm{Si}(\mathrm{ppm})$ & $\mathrm{Al}(\mathrm{ppm})$ & $\mathrm{P}(\mathrm{ppm})$ & $\mathrm{S}(\mathrm{ppm})$ & $\mathrm{K}(\mathrm{ppm})$ & $\mathrm{Ca}(\mathrm{ppm})$ \\
\hline 7503.46 & 311687 & 17 & 285715.09 & 43223.69 & 0 & 16911.89 & 0 & 21796.55 \\
\hline 7503.53 & 272733 & 18 & 378923.44 & 59939.02 & 0 & 17958.2 & 0 & 23881.22 \\
\hline 7503.60 & 334159 & 17 & 311906.05 & 46037.29 & 0 & 16500.9 & 0 & 21988.09 \\
\hline 7503.66 & 404928 & 16 & 153239.95 & 25084.36 & 0 & 11602.56 & 0 & 16572.39 \\
\hline 7503.73 & 306747 & 17 & 356367.82 & 56106.03 & 0 & 18957 & 0 & 13607.6 \\
\hline 7503.79 & 355707 & 17 & 197541.74 & 32880.84 & 0 & 11354.82 & 0 & 15625.12 \\
\hline 7503.86 & 353570 & 17 & 218008.33 & 32698.54 & 0 & 11815.43 & 0 & 16207.69 \\
\hline 7503.92 & 489676 & 16 & 80695.63 & 12105.77 & 0 & 6475.53 & 0 & 13591.35 \\
\hline 7503.99 & 320528 & 17 & 385826.4 & 58050.36 & 0 & 18481.53 & 0 & 15151.03 \\
\hline 7504.06 & 341094 & 17 & 334764.58 & 52002.83 & 0 & 17365.86 & 0 & 19278.24 \\
\hline 7504.12 & 491206 & 16 & 82680.98 & 13613.47 & 0 & 6349.24 & 0 & 11114.3 \\
\hline 7504.19 & 345119 & 17 & 360523.01 & 53168.98 & 0 & 22151.92 & 0 & 20658.23 \\
\hline 7504.25 & 334955 & 17 & 398564.61 & 58623.02 & 0 & 26307.87 & 0 & 21629.11 \\
\hline 7504.32 & 343566 & 17 & 249645.78 & 36991.28 & 0 & 15917.94 & 0 & 23018.74 \\
\hline 7504.38 & 321735 & 17 & 331460.37 & 49230.74 & 0 & 21167.55 & 0 & 26975.87 \\
\hline 7504.45 & 335250 & 17 & 268814.41 & 41755.73 & 0 & 14008.23 & 0 & 22986.73 \\
\hline 7504.51 & 316697 & 17 & 358214.4 & 51727.21 & 0 & 18838.82 & 0 & 25026.5 \\
\hline 7504.58 & 307540 & 17 & 351222.79 & 53442.55 & 0 & 16723.39 & 0 & 25323.82 \\
\hline 7504.65 & 316281 & 17 & 347824.81 & 49143.34 & 0 & 18396.32 & 0 & 33124.76 \\
\hline 7504.71 & 305602 & 17 & 364486.78 & 59927.34 & 0 & 19134.7 & 0 & 30655.98 \\
\hline 7504.78 & 320161 & 17 & 360896.97 & 55530.81 & 0 & 18483.42 & 0 & 22209.49 \\
\hline 7504.84 & 319535 & 17 & 357828.16 & 55872.78 & 0 & 19201.09 & 0 & 21335.3 \\
\hline 7504.91 & 325366 & 17 & 363051.23 & 59166.48 & 0 & 21176.26 & 0 & 24194.65 \\
\hline 7504.97 & 317230 & 17 & 349769.27 & 57926.8 & 0 & 19549.5 & 0 & 21290.91 \\
\hline 7505.04 & 456916 & 16 & 82323.27 & 12025.65 & 0 & 6736.7 & 0 & 22147.98 \\
\hline 7505.10 & 300669 & 17 & 295977.25 & 46250.27 & 0 & 15927.2 & 0 & 32632.72 \\
\hline 7505.17 & 343844 & 17 & 241711.86 & 41211.27 & 0 & 11917.05 & 0 & 23258.64 \\
\hline 7505.24 & 486753 & 16 & 95134.04 & 16343.52 & 0 & 6996.45 & 0 & 22176.04 \\
\hline 7505.30 & 342936 & 17 & 367406.61 & 58604.15 & 0 & 20785.91 & 0 & 36051.56 \\
\hline 7505.37 & 382501 & 17 & 174792.23 & 30886.94 & 0 & 11161.93 & 0 & 16737.95 \\
\hline 7505.43 & 384702 & 17 & 166982.77 & 28796.64 & 0 & 12081.66 & 0 & 19771.39 \\
\hline 7505.50 & 366361 & 17 & 174734.69 & 30419.96 & 0 & 8913.26 & 0 & 17960.67 \\
\hline 7505.56 & 163885 & 19 & 376941.86 & 64681.61 & 0 & 21706.94 & 0 & 22306.28 \\
\hline
\end{tabular}




\begin{tabular}{|c|c|c|c|c|c|c|c|c|}
\hline True Depth & XRF tCOUNTS & XRF Live Time & $\mathrm{Si}(\mathrm{ppm})$ & $\mathrm{Al}(\mathrm{ppm})$ & $\mathrm{P}(\mathrm{ppm})$ & $\mathrm{S}(\mathrm{ppm})$ & $\mathrm{K}(\mathrm{ppm})$ & $\mathrm{Ca}(\mathrm{ppm})$ \\
\hline 7505.63 & 306873 & 17 & 325233.86 & 52825.09 & 0 & 21575.11 & 0 & 27694.55 \\
\hline 7505.70 & 282074 & 18 & 280419.83 & 45938.01 & 0 & 19861.88 & 0 & 17571.61 \\
\hline 7505.76 & 272395 & 18 & 280358.24 & 43372.11 & 0 & 17130.51 & 0 & 16410.1 \\
\hline 7505.83 & 286107 & 18 & 309006.57 & 50319.6 & 0 & 25508.96 & 0 & 11695.52 \\
\hline 7505.89 & 350296 & 17 & 247730.78 & 43456.04 & 0 & 24548.1 & 0 & 30618.07 \\
\hline 7505.96 & 362185 & 17 & 139051.81 & 30246.52 & 0 & 11548.18 & 0 & 18011.36 \\
\hline 7506.02 & 365072 & 17 & 317189.14 & 65106.24 & 0 & 33626.24 & 0 & 24736.4 \\
\hline 7506.09 & 389669 & 17 & 159965.51 & 31861.09 & 0 & 15265.76 & 0 & 17235.11 \\
\hline 7506.15 & 387526 & 17 & 197860.09 & 40072.12 & 0 & 18504.53 & 0 & 13683.61 \\
\hline 7506.22 & 346442 & 17 & 284738.86 & 53630.65 & 0 & 26604.53 & 0 & 10807.4 \\
\hline 7506.29 & 373374 & 17 & 288510.85 & 54867.86 & 0 & 36015.61 & 0 & 20985.02 \\
\hline 7506.35 & 344524 & 17 & 296112.69 & 56682.77 & 0 & 35016.16 & 0 & 17400.75 \\
\hline 7506.42 & 348530 & 17 & 383410 & 70768.65 & 0 & 36402.88 & 0 & 4523.09 \\
\hline 7506.48 & 339254 & 17 & 392880.33 & 68911.45 & 0 & 31933.17 & 0 & 8909.64 \\
\hline 7506.55 & 318728 & 17 & 367220.3 & 63588.05 & 0 & 20065.14 & 0 & 12576.48 \\
\hline 7506.61 & 322791 & 17 & 234674.29 & 41323.55 & 0 & 13336.89 & 0 & 14826.37 \\
\hline 7506.68 & 326105 & 17 & 378525.13 & 65002.52 & 0 & 20536.42 & 0 & 13687.67 \\
\hline 7506.75 & 374651 & 17 & 184548.74 & 35660.58 & 0 & 11296.08 & 0 & 14269.93 \\
\hline 7506.81 & 364981 & 17 & 200106.68 & 35743.22 & 0 & 13942.03 & 0 & 17019.17 \\
\hline 7506.88 & 350276 & 17 & 315975.32 & 54647.57 & 0 & 20908.1 & 0 & 20585.13 \\
\hline 7506.94 & 313753 & 17 & 361933.39 & 61395.24 & 0 & 18346.62 & 0 & 18079.99 \\
\hline 7507.01 & 357484 & 17 & 244745.92 & 45584.93 & 0 & 17255.45 & 0 & 19360.05 \\
\hline 7507.07 & 328953 & 17 & 341016.61 & 58718.54 & 0 & 27742.48 & 0 & 22670.68 \\
\hline 7507.14 & 410679 & 16 & 151679.43 & 27696.06 & 0 & 8257.04 & 0 & 11217.12 \\
\hline 7507.20 & 372087 & 17 & 271991.11 & 47704.54 & 0 & 19708.62 & 0 & 19682 \\
\hline 7507.27 & 322518 & 17 & 354502.11 & 57366.78 & 0 & 22086.61 & 0 & 22932.75 \\
\hline 7507.34 & 312620 & 17 & 358444.47 & 58890.47 & 0 & 21297.29 & 0 & 17515.33 \\
\hline 7507.40 & 307728 & 17 & 345988.35 & 55055.61 & 0 & 24802.68 & 0 & 18913.53 \\
\hline 7507.47 & 307607 & 17 & 243197.61 & 42144.01 & 0 & 14693.58 & 0 & 14154.83 \\
\hline 7507.53 & 305349 & 17 & 258379.03 & 46426.05 & 0 & 16715.13 & 0 & 11994.18 \\
\hline 7507.60 & 321783 & 17 & 363417.73 & 65182.18 & 0 & 19662.22 & 0 & 11712.48 \\
\hline 7507.66 & 297194 & 17 & 326858.74 & 55895.26 & 0 & 16962.79 & 0 & 11577.54 \\
\hline 7507.73 & 263582 & 18 & 333957.1 & 54422.6 & 0 & 20987.2 & 0 & 7067.78 \\
\hline
\end{tabular}




\begin{tabular}{|c|c|c|c|c|c|c|c|c|}
\hline True Depth & XRF tCOUNTS & XRF Live Time & $\mathrm{Si}(\mathrm{ppm})$ & $\mathrm{Al}(\mathrm{ppm})$ & $\mathrm{P}(\mathrm{ppm})$ & $\mathrm{S}(\mathrm{ppm})$ & $\mathrm{K}(\mathrm{ppm})$ & $\mathrm{Ca}(\mathrm{ppm})$ \\
\hline 7507.80 & 339400 & 17 & 302785.84 & 49401.3 & 0 & 26189.4 & 0 & 6640.35 \\
\hline 7507.86 & 317655 & 17 & 343955.54 & 55966.65 & 0 & 30455.96 & 0 & 13115.61 \\
\hline 7507.93 & 297460 & 17 & 318295.43 & 56706.19 & 0 & 24626.23 & 0 & 15518.58 \\
\hline 7507.99 & 298580 & 17 & 299167.53 & 55416.75 & 0 & 23946.07 & 0 & 21365.15 \\
\hline 7508.06 & 342116 & 17 & 344995.15 & 65722.44 & 0 & 25789.66 & 0 & 22782.07 \\
\hline 7508.12 & 436330 & 16 & 124865.13 & 23713.23 & 0 & 8581.91 & 0 & 12277.52 \\
\hline 7508.19 & 295250 & 17 & 170890.73 & 25819.82 & 0 & 10577.11 & 0 & 10613.82 \\
\hline 7508.25 & 253731 & 18 & 271861.38 & 42078.15 & 0 & 17947.66 & 0 & 12617.51 \\
\hline 7508.32 & 357660 & 17 & 96598.1 & 15265.57 & 0 & 13173.42 & 0 & 13339.29 \\
\hline 7508.39 & 272435 & 18 & 339484.63 & 49751.21 & 0 & 23465.38 & 0 & 9687.9 \\
\hline 7508.45 & 279583 & 18 & 276688.47 & 42201.5 & 0 & 21002.28 & 0 & 10695.71 \\
\hline 7508.52 & 260883 & 18 & 335058.87 & 48281.79 & 0 & 22132.24 & 0 & 6845.41 \\
\hline 7508.58 & 278852 & 18 & 277060.99 & 51131.71 & 0 & 17305.06 & 0 & 8884.61 \\
\hline 7508.65 & 295724 & 18 & 348637.71 & 59477.27 & 0 & 20434.73 & 0 & 14854.5 \\
\hline 7508.71 & 272786 & 18 & 323576.96 & 52273.26 & 0 & 19366.1 & 0 & 13905.34 \\
\hline 7508.78 & 286857 & 18 & 163024.12 & 27168.02 & 0 & 11045.24 & 0 & 18869.42 \\
\hline 7508.85 & 256449 & 18 & 224487.36 & 35771.9 & 0 & 15568.02 & 0 & 21988.67 \\
\hline 7508.91 & 219121 & 18 & 269637.76 & 43596.84 & 0 & 13554.96 & 0 & 12194.63 \\
\hline 7508.98 & 262868 & 18 & 193219.74 & 28060.27 & 0 & 11044.17 & 0 & 8464.81 \\
\hline 7509.04 & 277572 & 18 & 157274.58 & 23540.03 & 0 & 8886.17 & 0 & 6654.34 \\
\hline 7509.11 & 242842 & 18 & 183022.08 & 25986.69 & 0 & 10777.8 & 0 & 9267.33 \\
\hline 7509.17 & 255715 & 18 & 207498.96 & 29103.1 & 0 & 11467.66 & 0 & 17137.84 \\
\hline 7509.24 & 518077 & 15 & 90349.57 & 14976.96 & 0 & 3866.95 & 0 & 3990.87 \\
\hline 7509.30 & 408820 & 16 & 102172.6 & 19119.55 & 0 & 5188.17 & 0 & 17473.44 \\
\hline 7509.37 & 451192 & 16 & 83470.27 & 13810.17 & 0 & 5038.04 & 0 & 19824.31 \\
\hline 7509.44 & 355042 & 17 & 146879.57 & 26166.68 & 0 & 7865.15 & 0 & 25118.9 \\
\hline 7509.50 & 356984 & 17 & 167236.86 & 26924.13 & 0 & 9993.76 & 0 & 23170.71 \\
\hline 7509.57 & 296667 & 17 & 196409.48 & 25646.86 & 0 & 12093.78 & 0 & 72115.08 \\
\hline 7509.63 & 365018 & 17 & 264216.98 & 51362.16 & 0 & 14604.03 & 0 & 40499.35 \\
\hline 7509.70 & 343595 & 17 & 327799.77 & 56183.09 & 0 & 16229.21 & 0 & 56849.76 \\
\hline 7509.76 & 367696 & 17 & 277616.43 & 47176.82 & 0 & 14518.1 & 0 & 65930.67 \\
\hline 7509.83 & 332760 & 17 & 308683.52 & 50488.1 & 0 & 16208.19 & 0 & 57498.12 \\
\hline 7509.90 & 314925 & 17 & 253204.73 & 42832.43 & 0 & 14057.16 & 0 & 24675.8 \\
\hline
\end{tabular}




\begin{tabular}{|c|c|c|c|c|c|c|c|c|}
\hline True Depth & XRF tCOUNTS & XRF Live Time & $\mathrm{Si}(\mathrm{ppm})$ & $\mathrm{Al}(\mathrm{ppm})$ & $\mathrm{P}(\mathrm{ppm})$ & $\mathrm{S}(\mathrm{ppm})$ & $\mathrm{K}(\mathrm{ppm})$ & $\mathrm{Ca}(\mathrm{ppm})$ \\
\hline 7509.96 & 315346 & 17 & 329786.81 & 52405.98 & 0 & 18934.46 & 0 & 37571.13 \\
\hline 7510.03 & 520290 & 15 & 82749.37 & 14441.69 & 0 & 4801.77 & 0 & 13026.55 \\
\hline 7510.09 & 312576 & 17 & 319078.6 & 52996.64 & 0 & 19657.7 & 0 & 34241.4 \\
\hline 7510.16 & 475082 & 16 & 154487.29 & 18020.53 & 0 & 26078.66 & 0 & 83133.42 \\
\hline 7510.22 & 340061 & 17 & 300477.66 & 52613.8 & 0 & 19778.79 & 0 & 24804.2 \\
\hline 7510.29 & 372939 & 17 & 147872.62 & 24578.13 & 0 & 8164.91 & 0 & 10991.53 \\
\hline 7510.35 & 430592 & 16 & 54276.46 & 7398.29 & 0 & 4943.77 & 0 & 3796.9 \\
\hline 7510.42 & 262171 & 18 & 338996.76 & 48209.03 & 0 & 13528.06 & 0 & 11814.86 \\
\hline 7510.49 & 404077 & 16 & 255569.6 & 25241.48 & 0 & 40413.71 & 0 & 102282.84 \\
\hline 7510.55 & 313809 & 17 & 386469.05 & 58798.44 & 0 & 16041.97 & 0 & 18534.19 \\
\hline 7510.62 & 317062 & 17 & 315221.46 & 55178.51 & 0 & 11158.7 & 0 & 23034.69 \\
\hline 7510.68 & 239591 & 18 & 309804.81 & 47836.53 & 0 & 12659.44 & 0 & 12426.44 \\
\hline 7510.75 & 292471 & 18 & 361423.23 & 54193.56 & 0 & 17130.9 & 0 & 13995.84 \\
\hline 7510.81 & 256301 & 18 & 278583 & 42714.47 & 0 & 14773.93 & 0 & 14546.61 \\
\hline 7510.88 & 258577 & 18 & 339162.17 & 63461.75 & 0 & 15392.48 & 0 & 12217.75 \\
\hline 7510.94 & 325959 & 17 & 346402.05 & 61569.26 & 0 & 22671.63 & 0 & 15518.21 \\
\hline 7511.01 & 324308 & 17 & 258755.27 & 43566.04 & 0 & 14527.45 & 0 & 10758.13 \\
\hline 7511.08 & 373820 & 17 & 181092.32 & 31897.13 & 0 & 14833.47 & 0 & 9655.08 \\
\hline 7511.14 & 307453 & 17 & 365487.4 & 57102.55 & 0 & 19438.66 & 0 & 10746.58 \\
\hline 7511.21 & 331281 & 17 & 303306.89 & 50271.8 & 0 & 20793.75 & 0 & 7521.54 \\
\hline 7511.27 & 412980 & 16 & 128720.65 & 20565.47 & 0 & 11467.3 & 0 & 7281.6 \\
\hline 7511.34 & 296448 & 17 & 354831.29 & 56167.72 & 0 & 23199.72 & 0 & 9369.76 \\
\hline 7511.40 & 332728 & 17 & 253699.44 & 44621.57 & 0 & 18029.17 & 0 & 15764.66 \\
\hline 7511.47 & 382513 & 17 & 169638.39 & 30465.47 & 0 & 14930.16 & 0 & 13688.8 \\
\hline 7511.54 & 482714 & 16 & 84644.41 & 16830.05 & 0 & 5381.73 & 0 & 7643.05 \\
\hline 7511.60 & 158570 & 19 & 417019.9 & 76569.52 & 0 & 33570.89 & 0 & 12559.73 \\
\hline 7511.67 & 331817 & 17 & 181833.08 & 31040.57 & 0 & 18429.4 & 0 & 6875.72 \\
\hline 7511.73 & 271067 & 18 & 322613.92 & 49856.52 & 0 & 15854.19 & 0 & 7217 \\
\hline 7511.80 & 322076 & 17 & 180694.13 & 29067.78 & 0 & 13566.67 & 0 & 7746.19 \\
\hline 7511.86 & 387508 & 17 & 115954.31 & 19328.51 & 0 & 11490.29 & 0 & 7954.5 \\
\hline 7511.93 & 289106 & 18 & 131594.32 & 18773.83 & 0 & 10412.97 & 0 & 14751.56 \\
\hline 7511.99 & 295801 & 17 & 327864.91 & 59990.48 & 0 & 20024.22 & 0 & 10107.45 \\
\hline 7512.06 & 303836 & 17 & 325426.36 & 54964.13 & 0 & 20027.83 & 0 & 18744.95 \\
\hline
\end{tabular}




\begin{tabular}{|c|c|c|c|c|c|c|c|c|}
\hline True Depth & XRF tCOUNTS & XRF Live Time & $\mathrm{Si}(\mathrm{ppm})$ & $\mathrm{Al}(\mathrm{ppm})$ & $\mathrm{P}(\mathrm{ppm})$ & $\mathrm{S}(\mathrm{ppm})$ & $\mathrm{K}(\mathrm{ppm})$ & $\mathrm{Ca}(\mathrm{ppm})$ \\
\hline 7512.13 & 298387 & 17 & 306625.48 & 52310.82 & 0 & 22153.52 & 0 & 20102.71 \\
\hline 7512.19 & 290741 & 17 & 308409.9 & 53018.61 & 0 & 23858.56 & 0 & 18404.4 \\
\hline 7512.26 & 207264 & 18 & 256986.82 & 41858.55 & 0 & 15395.72 & 0 & 16406.87 \\
\hline 7512.32 & 418214 & 16 & 60392.35 & 9254.09 & 0 & 3736.24 & 0 & 2053.64 \\
\hline 7512.39 & 270668 & 18 & 320153.28 & 49370.23 & 0 & 23610.12 & 0 & 9641.65 \\
\hline 7512.45 & 340366 & 17 & 318796.39 & 51252.59 & 0 & 46896.99 & 0 & 11808.48 \\
\hline 7512.52 & 301788 & 17 & 324465.57 & 46527.37 & 0 & 27664.49 & 0 & 14612.51 \\
\hline 7512.59 & 394971 & 16 & 159925.07 & 26298.97 & 0 & 11237.01 & 0 & 9428.56 \\
\hline 7512.65 & 293022 & 17 & 343690.59 & 55746.65 & 0 & 20592.1 & 0 & 12484.44 \\
\hline 7512.72 & 472865 & 16 & 97611.95 & 18074.57 & 0 & 4276.43 & 0 & 5850.89 \\
\hline 7512.78 & 455678 & 16 & 94949.1 & 17125.89 & 0 & 4940.78 & 0 & 8761.09 \\
\hline 7512.85 & 321357 & 17 & 342716.72 & 56179.24 & 0 & 20902.21 & 0 & 20224.86 \\
\hline 7512.91 & 308282 & 17 & 292798.4 & 49606.64 & 0 & 18101.55 & 0 & 14253.93 \\
\hline 7512.98 & 376690 & 17 & 165092.25 & 27853.09 & 0 & 11008.69 & 0 & 8225.97 \\
\hline 7513.04 & 304927 & 17 & 243485.25 & 42343.39 & 0 & 9969.1 & 0 & 7915.04 \\
\hline 7513.11 & 373966 & 17 & 136373.39 & 23111.55 & 0 & 8207.97 & 0 & 6154.09 \\
\hline 7513.18 & 268383 & 18 & 310212.45 & 46030.52 & 0 & 23295.87 & 0 & 5780.56 \\
\hline 7513.24 & 407062 & 16 & 150784.22 & 25246.2 & 0 & 7759.75 & 0 & 4353.36 \\
\hline 7513.31 & 473128 & 16 & 93239.86 & 16135.51 & 0 & 5209.14 & 0 & 3235.66 \\
\hline 7513.37 & 313501 & 17 & 370473.78 & 63673.76 & 0 & 20685.97 & 0 & 8246.68 \\
\hline 7513.44 & 308212 & 17 & 314999.56 & 53281.42 & 0 & 17264.99 & 0 & 13207.49 \\
\hline 7513.50 & 341287 & 17 & 213807.47 & 37134.36 & 0 & 12972.41 & 0 & 11975.62 \\
\hline 7513.57 & 339182 & 17 & 352790.96 & 61043.88 & 0 & 22958.03 & 0 & 21794.48 \\
\hline 7513.64 & 253899 & 18 & 363832.66 & 66666.5 & 0 & 21366.17 & 0 & 18098.16 \\
\hline 7513.70 & 347644 & 17 & 235355.1 & 42949.91 & 0 & 14005.16 & 0 & 26699.15 \\
\hline 7513.77 & 295351 & 17 & 331394.51 & 54396.01 & 0 & 18838.32 & 0 & 13053.49 \\
\hline 7513.83 & 302580 & 17 & 352538.92 & 54698.19 & 0 & 23014.71 & 0 & 10582.23 \\
\hline 7513.90 & 332202 & 17 & 319955.04 & 53748.31 & 0 & 30698.71 & 0 & 9580.83 \\
\hline 7513.96 & 279779 & 18 & 331277.28 & 53285.24 & 0 & 19859.02 & 0 & 9964.48 \\
\hline 7514.03 & 347684 & 17 & 264678.63 & 47995.38 & 0 & 13409.12 & 0 & 16383.13 \\
\hline 7514.09 & 350248 & 17 & 255192.09 & 42732.73 & 0 & 22832.24 & 0 & 10921.97 \\
\hline 7514.16 & 467273 & 16 & 77127.83 & 12391.55 & 0 & 4618.62 & 0 & 7081.03 \\
\hline 7514.23 & 303695 & 17 & 367681.89 & 59282.18 & 0 & 17834.76 & 0 & 11647.32 \\
\hline
\end{tabular}




\begin{tabular}{|c|c|c|c|c|c|c|c|c|}
\hline True Depth & XRF tCOUNTS & XRF Live Time & $\mathrm{Si}(\mathrm{ppm})$ & $\mathrm{Al}(\mathrm{ppm})$ & $\mathrm{P}(\mathrm{ppm})$ & $\mathrm{S}(\mathrm{ppm})$ & $\mathrm{K}(\mathrm{ppm})$ & $\mathrm{Ca}(\mathrm{ppm})$ \\
\hline 7514.29 & 377717 & 17 & 372503.59 & 60016.32 & 0 & 45240.84 & 0 & 16264.07 \\
\hline 7514.36 & 358937 & 17 & 262291.57 & 43849.05 & 0 & 16411.83 & 0 & 28103.36 \\
\hline 7514.42 & 408519 & 16 & 114981.59 & 21777.1 & 0 & 5941.9 & 0 & 14104.43 \\
\hline 7514.49 & 552620 & 15 & 41291.66 & 7131.37 & 0 & 2372.43 & 0 & 4457.97 \\
\hline 7514.55 & 319540 & 17 & 279831.81 & 46079.22 & 0 & 16145.16 & 0 & 19180.48 \\
\hline 7514.62 & 314216 & 17 & 337434.73 & 52160.67 & 0 & 20438.73 & 0 & 29171.05 \\
\hline 7514.69 & 303286 & 17 & 337670.25 & 52190.58 & 0 & 13386.47 & 0 & 22130.95 \\
\hline 7514.75 & 309340 & 17 & 359192.41 & 52713.25 & 0 & 14737.17 & 0 & 26637.15 \\
\hline 7514.82 & 344517 & 17 & 362284.8 & 56216.69 & 0 & 17175.25 & 0 & 49945.82 \\
\hline 7514.88 & 370704 & 17 & 307230.28 & 48227.93 & 3581.42 & 15627.47 & 0 & 92925.4 \\
\hline 7514.95 & 599599 & 14 & 33372.32 & 4721.63 & 0 & 3518.17 & 0 & 10056.24 \\
\hline 7515.01 & 394837 & 17 & 89172.94 & 14257.8 & 0 & 5022.85 & 0 & 21559.02 \\
\hline 7515.08 & 311998 & 17 & 104151.69 & 15073.58 & 0 & 4906.47 & 0 & 27353 \\
\hline 7515.14 & 356446 & 17 & 57327.36 & 8712.48 & 0 & 4880.49 & 0 & 19006.96 \\
\hline 7515.21 & 636904 & 14 & 23271.08 & 2864.03 & 0 & 2771.43 & 0 & 9430.91 \\
\hline 7515.28 & 643500 & 14 & 17781.47 & 2410.88 & 0 & 3587 & 0 & 8967.52 \\
\hline 7515.34 & 664732 & 13 & 18176.39 & 1891.94 & 0 & 2722.59 & 0 & 1732.81 \\
\hline 7515.41 & 626438 & 14 & 13958.39 & 1202.27 & 0 & 3427 & 0 & 5878.22 \\
\hline 7515.47 & 431113 & 16 & 55917.68 & 8960.24 & 0 & 4970.03 & 0 & 13299.44 \\
\hline 7515.54 & 619384 & 14 & 30947.04 & 5269.3 & 0 & 3712.69 & 0 & 10177.18 \\
\hline 7515.60 & 645312 & 14 & 28692.3 & 4653.2 & 0 & 3221.11 & 0 & 13399.89 \\
\hline 7515.67 & 114084 & 19 & 105658.48 & 24686.77 & 0 & 11610.16 & 0 & 87165.39 \\
\hline 7515.73 & 489214 & 16 & 85232.39 & 14538.98 & 0 & 5986.22 & 0 & 68627.89 \\
\hline 7515.80 & 324001 & 17 & 261033.73 & 50701.03 & 0 & 16415.68 & 0 & 22076.35 \\
\hline 7515.87 & 468890 & 16 & 59323.19 & 11352.2 & 0 & 4843.69 & 0 & 25121.74 \\
\hline 7515.93 & 582443 & 14 & 36341.85 & 6102.92 & 0 & 3550.77 & 0 & 16048.13 \\
\hline 7516.00 & 497286 & 15 & 73781.08 & 17045.97 & 0 & 4905.3 & 0 & 13847.89 \\
\hline 7516.06 & 426813 & 16 & 106276.92 & 22028.21 & 0 & 6911.05 & 0 & 16112.64 \\
\hline 7516.13 & 348626 & 17 & 184811.31 & 41938.75 & 0 & 9539.98 & 0 & 15808.78 \\
\hline 7516.19 & 549494 & 15 & 52745.1 & 10534.98 & 0 & 4113.88 & 0 & 4669.26 \\
\hline 7516.26 & 548876 & 15 & 59614.08 & 11662.91 & 0 & 12000.84 & 0 & 4702.99 \\
\hline 7516.33 & 372035 & 17 & 184877.37 & 40976.58 & 0 & 10818.98 & 0 & 11072.19 \\
\hline 7516.39 & 527741 & 15 & 48765.79 & 9595.24 & 0 & 2923.05 & 0 & 2038.13 \\
\hline
\end{tabular}




\begin{tabular}{|c|c|c|c|c|c|c|c|c|}
\hline True Depth & XRF tCOUNTS & XRF Live Time & $\mathrm{Si}(\mathrm{ppm})$ & $\mathrm{Al}(\mathrm{ppm})$ & $\mathrm{P}(\mathrm{ppm})$ & $\mathrm{S}(\mathrm{ppm})$ & $\mathrm{K}(\mathrm{ppm})$ & $\mathrm{Ca}(\mathrm{ppm})$ \\
\hline 7516.46 & 422332 & 16 & 77672.53 & 17505.15 & 0 & 4891.27 & 0 & 8136.62 \\
\hline 7516.52 & 400342 & 16 & 204969.26 & 45913.82 & 562.73 & 18645.56 & 0 & 18670.22 \\
\hline 7516.59 & 497255 & 15 & 71274.26 & 15336.03 & 0 & 7206.85 & 0 & 10584.45 \\
\hline 7516.65 & 365720 & 17 & 127543.15 & 26643.92 & 0 & 13510.7 & 0 & 12201.92 \\
\hline 7516.72 & 370791 & 17 & 113614.49 & 22859.99 & 0 & 10763.78 & 0 & 12225.72 \\
\hline 7516.78 & 301737 & 17 & 178264.33 & 42562.41 & 0 & 17648.86 & 0 & 17499.13 \\
\hline 7516.85 & 302406 & 17 & 186255.09 & 43530.82 & 0 & 18070.02 & 0 & 21105.76 \\
\hline 7516.92 & 561168 & 15 & 54307.58 & 13235.79 & 0 & 6836.25 & 0 & 9763.51 \\
\hline 7516.98 & 536661 & 15 & 121005.04 & 19673.66 & 0 & 10475.72 & 0 & 218381.75 \\
\hline 7517.05 & 462345 & 16 & 129229.02 & 26372.4 & 0 & 8652.72 & 0 & 138456.96 \\
\hline 7517.11 & 556827 & 15 & 96535.94 & 17392.89 & 0 & 12836.39 & 0 & 265719.46 \\
\hline 7517.18 & 580098 & 14 & 74686.13 & 8042.42 & 0 & 13940.25 & 0 & 308454.22 \\
\hline 7517.24 & 561972 & 15 & 122375.24 & 24541.21 & 0 & 17308.7 & 0 & 263699.3 \\
\hline 7517.31 & 522081 & 15 & 46761.2 & 11903.16 & 0 & 6066.06 & 0 & 40854.55 \\
\hline 7517.38 & 374299 & 17 & 265251.5 & 64685.61 & 0 & 26223.28 & 6793.54 & 65199.37 \\
\hline 7517.44 & 329585 & 17 & 309510.15 & 70577.99 & 0 & 24371.25 & 9911.39 & 20318.64 \\
\hline 7517.51 & 311347 & 17 & 200012.26 & 48472.89 & 0 & 15454.83 & 5324.35 & 12433.34 \\
\hline 7517.57 & 360611 & 17 & 234026.57 & 56638.7 & 0 & 18285.35 & 8246.33 & 17433.11 \\
\hline 7517.64 & 322004 & 17 & 287668.53 & 67462.56 & 0 & 21184.28 & 12740.34 & 25716.79 \\
\hline 7517.70 & 315120 & 17 & 138377.6 & 37624.69 & 0 & 10351.64 & 0 & 22971.27 \\
\hline 7517.77 & 278812 & 18 & 270242.62 & 61265.17 & 0 & 15171.37 & 5796.56 & 42176.16 \\
\hline 7517.83 & 326000 & 17 & 227342.9 & 45960.95 & 0 & 11006.2 & 0 & 62641.58 \\
\hline 7517.90 & 474119 & 16 & 71762.46 & 16505.7 & 0 & 5433.73 & 0 & 30978.95 \\
\hline 7517.97 & 549272 & 15 & 44806.73 & 9295.91 & 0 & 3967.73 & 0 & 15931.77 \\
\hline 7518.03 & 379103 & 17 & 227014.86 & 53768.14 & 0 & 12622.08 & 9735.66 & 59007.44 \\
\hline 7518.10 & 616642 & 14 & 38857.12 & 2796.25 & 0 & 1745.34 & 0 & 324542.39 \\
\hline 7518.16 & 635620 & 14 & 66971.74 & 3783.64 & 0 & 2036.99 & 0 & 406155.05 \\
\hline 7518.23 & 639114 & 14 & 119446.22 & 6606.42 & 0 & 3318.97 & 0 & 385283.84 \\
\hline 7518.29 & 616558 & 14 & 55629.58 & 4409.14 & 0 & 1856.64 & 0 & 228495.88 \\
\hline 7518.36 & 545921 & 15 & 204408.69 & 12149.08 & 0 & 13544.05 & 0 & 306141.86 \\
\hline 7518.43 & 620536 & 14 & 32715.53 & 1683.28 & 0 & 10775.23 & 0 & 387641.01 \\
\hline 7518.49 & 632466 & 14 & 37942.3 & 4891.59 & 0 & 5046.39 & 0 & 420756.26 \\
\hline 7518.56 & 637098 & 14 & 48900.58 & 6632.61 & 0 & 1745.01 & 0 & 418985.55 \\
\hline
\end{tabular}




\begin{tabular}{|c|c|c|c|c|c|c|c|c|}
\hline True Depth & XRF tCOUNTS & XRF Live Time & $\mathrm{Si}(\mathrm{ppm})$ & $\mathrm{Al}$ (ppm) & $\mathrm{P}(\mathrm{ppm})$ & $\mathrm{S}(\mathrm{ppm})$ & $\mathrm{K}(\mathrm{ppm})$ & $\mathrm{Ca}(\mathrm{ppm})$ \\
\hline 7518.62 & 302806 & 17 & 43459.43 & 8629.83 & 0 & 2643.59 & 0 & 423196.97 \\
\hline 7518.69 & 542088 & 15 & 21214.34 & 1929.21 & 0 & 1299.41 & 0 & 364325.04 \\
\hline 7518.75 & 517061 & 15 & 38335.16 & 9764.14 & 0 & 1082.18 & 0 & 351258.1 \\
\hline 7518.82 & 513099 & 15 & 27703.35 & 0 & 0 & 1156.5 & 0 & 389515.49 \\
\hline 7518.88 & 451361 & 16 & 20295.89 & 0 & 0 & 1121.78 & 0 & 349290.98 \\
\hline 7518.95 & 520598 & 15 & 41661.43 & 2780.77 & 0 & 1793.48 & 0 & 383895.46 \\
\hline 7519.02 & 531156 & 15 & 30573.71 & 1951.33 & 0 & 1398.88 & 0 & 384377.68 \\
\hline 7519.08 & 574427 & 15 & 20300.29 & 0 & 0 & 1413.85 & 0 & 331134.47 \\
\hline 7519.15 & 555803 & 15 & 57127.69 & 4221.03 & 0 & 2274.83 & 0 & 372902.6 \\
\hline 7519.21 & 469131 & 16 & 31810.98 & 0 & 0 & 1455.98 & 0 & 331284.78 \\
\hline 7519.28 & 620432 & 14 & 29795.31 & 1910.36 & 0 & 1719.24 & 0 & 345820.28 \\
\hline 7519.34 & 620402 & 14 & 45180.39 & 4278.86 & 0 & 1958.3 & 0 & 384842.1 \\
\hline 7519.41 & 627859 & 14 & 72429.99 & 7888.51 & 0 & 2410.07 & 0 & 382472.98 \\
\hline 7519.48 & 659809 & 13 & 36809.29 & 2484.09 & 0 & 1934.99 & 0 & 214753.13 \\
\hline 7519.54 & 594526 & 14 & 58657.62 & 4192.03 & 0 & 2294.99 & 0 & 301761.46 \\
\hline 7519.61 & 492364 & 15 & 30135.34 & 4946.08 & 0 & 2485.24 & 0 & 92427.52 \\
\hline 7519.67 & 404871 & 16 & 201116.12 & 55447.52 & 0 & 12561.81 & 10776.81 & 83386.39 \\
\hline 7519.74 & 209358 & 18 & 319679.64 & 76623.72 & 0 & 17617.88 & 0 & 70236.02 \\
\hline 7519.80 & 476854 & 16 & 114712.24 & 26007.64 & 0 & 17201.49 & 0 & 38371.48 \\
\hline 7519.87 & 452488 & 16 & 95090.48 & 21714.79 & 0 & 4993.46 & 0 & 46913.29 \\
\hline 7519.93 & 321586 & 17 & 186331.11 & 44558.29 & 0 & 7918.7 & 0 & 50709.85 \\
\hline 7520.00 & 459834 & 16 & 240785.71 & 47906.26 & 0 & 48996.44 & 0 & 128171.91 \\
\hline 7520.07 & 444424 & 16 & 212786.13 & 38363.49 & 0 & 8506.57 & 0 & 163690.39 \\
\hline 7520.13 & 361345 & 17 & 261138.58 & 54087.23 & 0 & 13016.81 & 0 & 90022.48 \\
\hline 7520.20 & 417863 & 16 & 234046.97 & 49786.46 & 0 & 10153.32 & 0 & 157271.01 \\
\hline 7520.26 & 565307 & 15 & 146995.64 & 23088.92 & 0 & 6221.57 & 0 & 300848.51 \\
\hline 7520.33 & 548158 & 15 & 107987.5 & 12774.45 & 0 & 3531.38 & 0 & 281304.88 \\
\hline 7520.39 & 530922 & 15 & 117865.04 & 15426.51 & 0 & 4059.08 & 0 & 275923.74 \\
\hline 7520.46 & 459453 & 16 & 173331.22 & 32270.65 & 0 & 7697.64 & 0 & 204629.64 \\
\hline 7520.52 & 361309 & 17 & 207230.74 & 37667.28 & 0 & 8951.61 & 0 & 88481.87 \\
\hline 7520.59 & 312423 & 17 & 199687.11 & 36026.37 & 0 & 10206.08 & 0 & 25557.32 \\
\hline 7520.66 & 346560 & 17 & 181986.58 & 35636.97 & 0 & 8203.6 & 0 & 14419.99 \\
\hline 7520.72 & 307116 & 17 & 336908.57 & 61601.45 & 0 & 14226.8 & 0 & 21569.71 \\
\hline
\end{tabular}




\begin{tabular}{|c|c|c|c|c|c|c|c|c|}
\hline True Depth & XRF tCOUNTS & XRF Live Time & $\mathrm{Si}(\mathrm{ppm})$ & $\mathrm{Al}(\mathrm{ppm})$ & $\mathrm{P}(\mathrm{ppm})$ & $\mathrm{S}(\mathrm{ppm})$ & $\mathrm{K}(\mathrm{ppm})$ & $\mathrm{Ca}(\mathrm{ppm})$ \\
\hline 7520.79 & 365211 & 17 & 191082.87 & 43499.01 & 0 & 6582 & 0 & 24103.93 \\
\hline 7520.85 & 345491 & 17 & 295473.22 & 64682.78 & 0 & 15065.94 & 6324.03 & 46357.54 \\
\hline 7520.92 & 298323 & 17 & 306379.59 & 69281.2 & 0 & 13436.69 & 9269.71 & 14951.73 \\
\hline 7520.98 & 411741 & 16 & 83711.77 & 19898.35 & 0 & 3707.24 & 0 & 4828.59 \\
\hline 7521.05 & 307956 & 17 & 327948.1 & 70201.99 & 0 & 19891.47 & 7388.4 & 10437.98 \\
\hline 7521.12 & 304664 & 17 & 263208.17 & 69157.13 & 0 & 18229.52 & 17976.42 & 8720.77 \\
\hline 7521.18 & 389002 & 17 & 204402.22 & 50499.47 & 0 & 24675.79 & 6069.63 & 15604.95 \\
\hline 7521.25 & 366340 & 17 & 168559.59 & 42791.3 & 0 & 10517.04 & 6858.1 & 10805.48 \\
\hline 7521.31 & 294533 & 18 & 282154.6 & 68489.98 & 0 & 15487.2 & 16514.49 & 10813.27 \\
\hline 7521.38 & 276033 & 18 & 286137.24 & 72511.97 & 0 & 15963.65 & 14540.38 & 7804.29 \\
\hline 7521.44 & 304849 & 17 & 320756.11 & 75806.05 & 0 & 16295.69 & 15867.64 & 6461.81 \\
\hline 7521.51 & 475936 & 16 & 67323.44 & 16943.67 & 0 & 5137.19 & 0 & 7604.3 \\
\hline 7521.57 & 380439 & 17 & 135690.62 & 33796.35 & 0 & 10943.69 & 0 & 15291.98 \\
\hline 7521.64 & 327209 & 17 & 176599.34 & 44621.65 & 0 & 11162.3 & 10662.02 & 17581.12 \\
\hline 7521.71 & 235365 & 18 & 256186.67 & 63280.24 & 0 & 12605.94 & 12792.64 & 13492.85 \\
\hline 7521.77 & 288730 & 18 & 257226.44 & 69139.57 & 0 & 15471.72 & 4924.92 & 16026.54 \\
\hline 7521.84 & 333205 & 17 & 255859.21 & 68370.77 & 0 & 9802.73 & 14306 & 13436.06 \\
\hline 7521.90 & 327307 & 17 & 304579.31 & 72904.55 & 0 & 21454.4 & 13846.1 & 15039.98 \\
\hline 7521.97 & 383437 & 17 & 150674.36 & 37574.28 & 0 & 11053.37 & 0 & 13507.56 \\
\hline 7522.03 & 319970 & 17 & 232134.36 & 56406.03 & 0 & 13596.24 & 14753.6 & 16394.35 \\
\hline 7522.10 & 464196 & 16 & 74754.88 & 18402.75 & 0 & 18217.97 & 0 & 11785.02 \\
\hline 7522.17 & 337002 & 17 & 243381.34 & 63296.7 & 0 & 10260.97 & 15018.4 & 19464.7 \\
\hline 7522.23 & 365727 & 17 & 185928.91 & 50066.11 & 0 & 9451.19 & 5334.97 & 23205.74 \\
\hline 7522.30 & 445261 & 16 & 106145.94 & 26626.37 & 0 & 8777.25 & 0 & 22266.66 \\
\hline 7522.36 & 407197 & 16 & 137552.79 & 37902.42 & 0 & 11027.38 & 7820.07 & 27545.11 \\
\hline 7522.43 & 328262 & 17 & 217280.33 & 54396.01 & 0 & 13091.83 & 11451.92 & 22521.12 \\
\hline 7522.49 & 322874 & 17 & 230412.75 & 58483.38 & 0 & 13999.43 & 12425.59 & 15647.06 \\
\hline 7522.56 & 496493 & 15 & 51666.38 & 14580.37 & 0 & 7404.7 & 0 & 5154.07 \\
\hline 7522.62 & 538087 & 15 & 42942.91 & 11747.63 & 0 & 4351.8 & 0 & 5222.6 \\
\hline 7522.69 & 325489 & 17 & 300391.55 & 69640.55 & 0 & 18285.13 & 15254.22 & 24186.92 \\
\hline 7522.76 & 411183 & 16 & 92052.3 & 22388.89 & 0 & 6769.63 & 0 & 23287.82 \\
\hline 7522.82 & 327299 & 17 & 185948.03 & 43872.27 & 0 & 10539.75 & 0 & 20283.19 \\
\hline 7522.89 & 309108 & 17 & 279893.82 & 61194.2 & 0 & 17635.17 & 13249.64 & 29742.95 \\
\hline
\end{tabular}




\begin{tabular}{|c|c|c|c|c|c|c|c|c|}
\hline True Depth & XRF tCOUNTS & XRF Live Time & $\mathrm{Si}(\mathrm{ppm})$ & $\mathrm{Al}(\mathrm{ppm})$ & $\mathrm{P}(\mathrm{ppm})$ & $\mathrm{S}(\mathrm{ppm})$ & $\mathrm{K}(\mathrm{ppm})$ & $\mathrm{Ca}(\mathrm{ppm})$ \\
\hline 7522.95 & 389833 & 17 & 113357.83 & 29461.98 & 0 & 7934.32 & 0 & 26971.05 \\
\hline 7523.02 & 329726 & 17 & 250560.08 & 63890.73 & 0 & 14801.2 & 11402.69 & 53425.53 \\
\hline 7523.08 & 411237 & 16 & 171324.16 & 43747.61 & 0 & 12287.22 & 0 & 49311.76 \\
\hline 7523.15 & 595153 & 14 & 114251.99 & 17752.01 & 0 & 3351.59 & 0 & 328214.31 \\
\hline 7523.22 & 491609 & 16 & 69775.67 & 21725.01 & 0 & 4172.24 & 0 & 59340.11 \\
\hline 7523.28 & 421604 & 16 & 214855.79 & 50853.09 & 0 & 11163.47 & 0 & 137526.11 \\
\hline 7523.35 & 340200 & 17 & 250931.24 & 65894.43 & 0 & 9473.04 & 8433.89 & 85471.82 \\
\hline 7523.41 & 473099 & 16 & 92663.34 & 23892 & 0 & 4890.14 & 0 & 64942.49 \\
\hline 7523.48 & 432365 & 16 & 123477.9 & 31726.77 & 0 & 7013.39 & 0 & 51597.14 \\
\hline 7523.54 & 331793 & 17 & 272765.29 & 70941.62 & 0 & 11464.46 & 16568.93 & 48108.7 \\
\hline 7523.61 & 450373 & 16 & 65909.24 & 17433.55 & 0 & 4193.17 & 0 & 26053.18 \\
\hline 7523.67 & 384240 & 17 & 78759.89 & 20960.55 & 0 & 4887.07 & 0 & 20729.91 \\
\hline 7523.74 & 449453 & 16 & 98383.38 & 26534.75 & 0 & 4098.4 & 0 & 31745.98 \\
\hline 7523.81 & 98472 & 19 & 95138.85 & 33686.1 & 0 & 3116.69 & 0 & 28363.04 \\
\hline 7523.87 & 382782 & 17 & 148690.38 & 40491.7 & 0 & 2296.77 & 4555.05 & 38512.39 \\
\hline 7523.94 & 284936 & 18 & 183939.06 & 46978.6 & 0 & 8229.85 & 9135.19 & 42226.61 \\
\hline 7524.00 & 349772 & 17 & 284749.14 & 66643.46 & 0 & 8726.39 & 11845.14 & 67199.41 \\
\hline 7524.07 & 420483 & 16 & 117202.8 & 27338.05 & 0 & 6501.05 & 0 & 57040.41 \\
\hline 7524.13 & 374084 & 17 & 248823.22 & 58111.03 & 0 & 9445.06 & 10215.02 & 97534.97 \\
\hline 7524.20 & 317854 & 17 & 256977.11 & 66070.96 & 0 & 6830.62 & 17841.33 & 50516.52 \\
\hline 7524.27 & 416171 & 16 & 104172.63 & 29969.82 & 0 & 2346.72 & 0 & 33128.7 \\
\hline 7524.33 & 467490 & 16 & 61923.19 & 15633.68 & 0 & 1197.95 & 0 & 23863.2 \\
\hline 7524.40 & 415087 & 16 & 170233.96 & 39605.46 & 0 & 3448.86 & 0 & 170786.96 \\
\hline 7524.46 & 628671 & 14 & 93308.26 & 13460.77 & 0 & 2587.9 & 0 & 340335.77 \\
\hline 7524.53 & 541271 & 15 & 111919.76 & 18880.52 & 0 & 2919.15 & 0 & 257179.37 \\
\hline 7524.59 & 551232 & 15 & 47408.14 & 8798.63 & 0 & 51721.76 & 0 & 273719.46 \\
\hline 7524.66 & 600176 & 14 & 27177.85 & 3481.24 & 0 & 5401.28 & 0 & 402075.89 \\
\hline 7524.72 & 576497 & 14 & 33750.92 & 5321.16 & 0 & 14890.66 & 0 & 373694.31 \\
\hline 7524.79 & 642939 & 14 & 34769.97 & 7474.43 & 0 & 1895.22 & 0 & 415333.94 \\
\hline 7524.86 & 638326 & 14 & 40259.88 & 6504.64 & 0 & 1461.95 & 0 & 409829.32 \\
\hline 7524.92 & 638049 & 14 & 52992.24 & 6445.65 & 0 & 1791.77 & 0 & 405743.53 \\
\hline 7524.99 & 512080 & 15 & 166241.24 & 30384.03 & 0 & 3154.76 & 0 & 235418.24 \\
\hline 7525.05 & 463900 & 16 & 166527.68 & 29474.23 & 0 & 4039.56 & 0 & 205965.92 \\
\hline
\end{tabular}




\begin{tabular}{|c|c|c|c|c|c|c|c|c|}
\hline True Depth & XRF tCOUNTS & XRF Live Time & $\mathrm{Si}(\mathrm{ppm})$ & $\mathrm{Al}(\mathrm{ppm})$ & $\mathrm{P}(\mathrm{ppm})$ & $\mathrm{S}(\mathrm{ppm})$ & $\mathrm{K}(\mathrm{ppm})$ & $\mathrm{Ca}(\mathrm{ppm})$ \\
\hline 7525.12 & 428051 & 16 & 221448.17 & 51795.22 & 0 & 7015.54 & 0 & 119247.76 \\
\hline 7525.18 & 616466 & 14 & 42879.49 & 6938.39 & 0 & 1486.98 & 0 & 90211.88 \\
\hline 7525.25 & 470522 & 16 & 83491.45 & 19474.77 & 0 & 1509.07 & 0 & 41709.04 \\
\hline 7525.31 & 482479 & 16 & 74248.96 & 19084.82 & 0 & 2322.16 & 0 & 41401.27 \\
\hline 7525.38 & 396154 & 17 & 94096.5 & 23665.45 & 0 & 1942.84 & 0 & 44593.81 \\
\hline 7525.45 & 363356 & 17 & 234624.62 & 54906.01 & 0 & 5392.98 & 6847.64 & 82203.98 \\
\hline 7525.51 & 471889 & 16 & 82104.7 & 18992.27 & 0 & 1484.87 & 0 & 77408.57 \\
\hline 7525.58 & 495175 & 15 & 201349.92 & 36537.74 & 0 & 3400.2 & 0 & 207525.9 \\
\hline 7525.64 & 485819 & 16 & 89663.25 & 21166.08 & 0 & 3089.69 & 0 & 144294.04 \\
\hline 7525.71 & 368672 & 17 & 222291.73 & 52022.27 & 0 & 6616.27 & 5743.98 & 71959.14 \\
\hline 7525.77 & 336431 & 17 & 280612.25 & 65741.69 & 0 & 3547.7 & 12630.53 & 74815.3 \\
\hline 7525.84 & 372477 & 17 & 60204.39 & 14086.74 & 0 & 2632.38 & 0 & 17529.41 \\
\hline 7525.91 & 310349 & 17 & 256805.61 & 61620.59 & 0 & 5325.47 & 16823.11 & 61467.19 \\
\hline 7525.97 & 365225 & 17 & 155771.86 & 39068.17 & 0 & 2969.11 & 5354.17 & 48348.57 \\
\hline 7526.04 & 424053 & 16 & 85201.34 & 23414.71 & 0 & 1674.25 & 0 & 35335.26 \\
\hline 7526.10 & 355027 & 17 & 153967.62 & 36901.4 & 0 & 4766.3 & 0 & 59214.2 \\
\hline 7526.17 & 399735 & 16 & 222557.39 & 42322.73 & 0 & 5335.74 & 0 & 138683.54 \\
\hline 7526.23 & 413206 & 16 & 221234.82 & 41030.32 & 0 & 7797.4 & 0 & 132913.25 \\
\hline 7526.30 & 631716 & 14 & 16813.51 & 2340.95 & 0 & 2330.63 & 0 & 23324.14 \\
\hline 7526.36 & 598639 & 14 & 49154.05 & 9450.74 & 0 & 2952.23 & 0 & 132457.66 \\
\hline 7526.43 & 385067 & 17 & 186027.24 & 47482.01 & 0 & 13210.16 & 5553.32 & 56555.63 \\
\hline 7526.50 & 341917 & 17 & 250011.02 & 67771.67 & 0 & 11476.84 & 17257.73 & 65003.75 \\
\hline 7526.56 & 553589 & 15 & 44095.03 & 7280.03 & 524.96 & 3156.61 & 0 & 76201.33 \\
\hline 7526.63 & 408389 & 16 & 124727.44 & 28882.75 & 0 & 15569.7 & 0 & 50504.25 \\
\hline 7526.69 & 350194 & 17 & 241524.13 & 52373.86 & 0 & 32271.97 & 8946.08 & 43352.67 \\
\hline 7526.76 & 510337 & 15 & 51332.31 & 12527.01 & 0 & 6933.88 & 0 & 10025.08 \\
\hline 7526.82 & 374521 & 17 & 117820.65 & 26097.39 & 0 & 12614.8 & 0 & 15871.31 \\
\hline 7526.89 & 307837 & 17 & 174072.56 & 35893.09 & 0 & 9723.39 & 0 & 17458.37 \\
\hline 7526.96 & 332339 & 17 & 88474.23 & 19240.49 & 0 & 5745.87 & 0 & 12034.06 \\
\hline 7527.02 & 413486 & 16 & 77213.03 & 12572.97 & 0 & 6210.63 & 0 & 13973.15 \\
\hline 7527.09 & 370225 & 17 & 83325.67 & 13057.8 & 0 & 6231.43 & 0 & 13265.22 \\
\hline 7527.15 & 351607 & 17 & 54376.25 & 8389.62 & 0 & 4492.1 & 0 & 11192.25 \\
\hline 7527.22 & 481290 & 16 & 82139.72 & 12366.63 & 0 & 4563.98 & 0 & 18074.85 \\
\hline
\end{tabular}




\begin{tabular}{|c|c|c|c|c|c|c|c|c|}
\hline True Depth & XRF tCOUNTS & XRF Live Time & $\mathrm{Si}(\mathrm{ppm})$ & $\mathrm{Al}(\mathrm{ppm})$ & $\mathrm{P}(\mathrm{ppm})$ & $\mathrm{S}(\mathrm{ppm})$ & $\mathrm{K}(\mathrm{ppm})$ & $\mathrm{Ca}(\mathrm{ppm})$ \\
\hline 7527.28 & 487293 & 16 & 95596.88 & 15950.16 & 0 & 6998.38 & 0 & 19139.21 \\
\hline 7527.35 & 306173 & 17 & 297973.14 & 48448.92 & 0 & 21931.41 & 0 & 30380.51 \\
\hline 7527.41 & 331972 & 17 & 347001.87 & 54337.18 & 0 & 24648.85 & 0 & 27400.58 \\
\hline 7527.48 & 370060 & 17 & 196916.59 & 32973.87 & 0 & 16949.69 & 0 & 30859.15 \\
\hline 7527.55 & 336765 & 17 & 247618.56 & 38161.92 & 0 & 16605.69 & 0 & 41244.5 \\
\hline 7527.61 & 357131 & 17 & 290553.23 & 49024.73 & 0 & 20393.86 & 0 & 37335.42 \\
\hline 7527.68 & 361058 & 17 & 256897.14 & 42456.92 & 0 & 15906.76 & 0 & 32008.27 \\
\hline 7527.74 & 349396 & 17 & 247593.14 & 37116.21 & 0 & 17508.34 & 0 & 23013.39 \\
\hline 7527.81 & 269628 & 18 & 234862.16 & 39362.43 & 0 & 18372.46 & 0 & 21310.04 \\
\hline 7527.87 & 338202 & 17 & 238918.16 & 35724.69 & 0 & 12317.24 & 0 & 41410.57 \\
\hline 7527.94 & 297455 & 17 & 334445.76 & 54698.49 & 0 & 18414.99 & 0 & 23149.44 \\
\hline 7528.01 & 315283 & 17 & 335844.54 & 52989.75 & 0 & 18755.39 & 0 & 24407.75 \\
\hline 7528.07 & 360924 & 17 & 214401.45 & 38865.96 & 0 & 15983.69 & 0 & 22670.97 \\
\hline 7528.14 & 349713 & 17 & 261882.89 & 45698.02 & 0 & 16092.13 & 0 & 32353.84 \\
\hline 7528.20 & 367220 & 17 & 252584.28 & 44908.4 & 0 & 15478.53 & 0 & 36909.05 \\
\hline 7528.27 & 363545 & 17 & 332023.83 & 66192.99 & 0 & 20485.28 & 0 & 53405.8 \\
\hline 7528.33 & 361699 & 17 & 317432.14 & 59712.38 & 0 & 22198.69 & 0 & 32676.83 \\
\hline 7528.40 & 469072 & 16 & 129355.2 & 19843.66 & 0 & 6006.37 & 0 & 80795.97 \\
\hline 7528.46 & 417557 & 16 & 283034.72 & 42028.74 & 0 & 11667.39 & 0 & 149909.46 \\
\hline 7528.53 & 377792 & 17 & 205491.03 & 36782.07 & 0 & 10633.67 & 0 & 62714.91 \\
\hline 7528.60 & 409774 & 16 & 233030.7 & 37843.8 & 0 & 13476.09 & 0 & 128272.53 \\
\hline 7528.66 & 350216 & 17 & 191224.33 & 39040.85 & 0 & 9726.82 & 0 & 70551.03 \\
\hline 7528.73 & 452587 & 16 & 128557.25 & 23840.72 & 0 & 9020.73 & 0 & 60306.1 \\
\hline 7528.79 & 362403 & 17 & 252649.71 & 49412.1 & 0 & 15686.78 & 0 & 49489.6 \\
\hline 7528.86 & 398286 & 16 & 247672.13 & 53181.64 & 0 & 54027.01 & 0 & 38642.88 \\
\hline 7528.92 & 320270 & 17 & 320056.78 & 63635.96 & 0 & 18159.91 & 0 & 36521.28 \\
\hline 7528.99 & 392416 & 17 & 134806.04 & 26944.19 & 0 & 8064.04 & 0 & 26905.62 \\
\hline 7529.06 & 329409 & 17 & 284939.34 & 59439.31 & 0 & 29526.06 & 0 & 11041.03 \\
\hline 7529.12 & 280240 & 18 & 278789.34 & 60046.09 & 0 & 31527.42 & 0 & 15261.39 \\
\hline 7529.19 & 494519 & 15 & 86825.66 & 18817.97 & 0 & 9443 & 0 & 12177.5 \\
\hline 7529.25 & 348542 & 17 & 316228.5 & 66949.66 & 0 & 31176.27 & 6396.07 & 20624.75 \\
\hline 7529.32 & 295551 & 17 & 176978.15 & 37732.21 & 0 & 14434.28 & 5014.05 & 16518.69 \\
\hline 7529.38 & 320087 & 17 & 87945.45 & 16658.87 & 0 & 7200.16 & 0 & 19620.26 \\
\hline
\end{tabular}




\begin{tabular}{|c|c|c|c|c|c|c|c|c|}
\hline True Depth & XRF tCOUNTS & XRF Live Time & $\mathrm{Si}(\mathrm{ppm})$ & $\mathrm{Al}(\mathrm{ppm})$ & $\mathrm{P}(\mathrm{ppm})$ & $\mathrm{S}(\mathrm{ppm})$ & $\mathrm{K}(\mathrm{ppm})$ & $\mathrm{Ca}(\mathrm{ppm})$ \\
\hline 7529.45 & 415794 & 16 & 90808.98 & 19617.46 & 0 & 8083.52 & 0 & 23091.17 \\
\hline 7529.51 & 530647 & 15 & 30552.1 & 5360.12 & 0 & 3624.64 & 0 & 7336.48 \\
\hline 7529.58 & 459789 & 16 & 63184.42 & 12152.92 & 0 & 5176.3 & 0 & 15161.28 \\
\hline 7529.65 & 443352 & 16 & 37352.18 & 5245.45 & 0 & 5771.7 & 0 & 11499.78 \\
\hline 7529.71 & 333208 & 17 & 83174.25 & 15181.78 & 0 & 6189.94 & 0 & 22383.19 \\
\hline 7529.78 & 289556 & 18 & 58422.94 & 11263.53 & 0 & 6390.78 & 0 & 20148.95 \\
\hline 7529.84 & 256343 & 18 & 285045.43 & 65782.88 & 0 & 33731.61 & 0 & 33765.45 \\
\hline 7529.91 & 440944 & 16 & 58698.05 & 12183.32 & 0 & 5790.03 & 0 & 17306.32 \\
\hline 7529.97 & 368822 & 17 & 53915.23 & 11184.93 & 0 & 6564.8 & 0 & 14713.32 \\
\hline 7530.04 & 262001 & 18 & 302998.66 & 61984.83 & 0 & 19002.21 & 0 & 16644.87 \\
\hline 7530.10 & 376478 & 17 & 267780.68 & 57103.27 & 0 & 21692.21 & 0 & 20434.81 \\
\hline 7530.17 & 339122 & 17 & 238896.45 & 52488.76 & 0 & 14812.35 & 0 & 15376.53 \\
\hline 7530.24 & 338100 & 17 & 99542.44 & 20704.58 & 0 & 8374.66 & 0 & 11593.99 \\
\hline 7530.30 & 367693 & 17 & 158730.82 & 27847.95 & 0 & 39611.01 & 0 & 10870.05 \\
\hline 7530.37 & 550849 & 15 & 37901.85 & 7391.34 & 0 & 6551.91 & 0 & 5021.88 \\
\hline 7530.43 & 355231 & 17 & 223318.51 & 43525.94 & 0 & 11690.68 & 0 & 29426.02 \\
\hline 7530.50 & 399852 & 16 & 246093.53 & 35588.76 & 0 & 11747.56 & 0 & 132977.73 \\
\hline 7530.56 & 399351 & 16 & 133831.11 & 25894.5 & 0 & 7791.79 & 0 & 21835.36 \\
\hline 7530.63 & 443799 & 16 & 118659.8 & 23092.16 & 0 & 6651.64 & 0 & 18490.23 \\
\hline 7530.70 & 436174 & 16 & 92378.96 & 19567.18 & 0 & 5653.26 & 0 & 15859.45 \\
\hline 7530.76 & 453535 & 16 & 132523.43 & 8108 & 0 & 81158.96 & 0 & 1662.8 \\
\hline 7530.83 & 454042 & 16 & 59162.55 & 11679.41 & 0 & 4683.09 & 0 & 6124.87 \\
\hline 7530.89 & 453830 & 16 & 218361.27 & 9352.86 & 0 & 110308.37 & 0 & 0 \\
\hline 7530.96 & 339725 & 17 & 311713.76 & 63367.61 & 0 & 20502.31 & 5202.51 & 17649.31 \\
\hline 7531.02 & 633272 & 14 & 9302.38 & 989.59 & 0 & 6602.97 & 0 & 2304.79 \\
\hline 7531.09 & 364493 & 17 & 78049.54 & 12780.44 & 0 & 4629.23 & 0 & 13497.22 \\
\hline 7531.15 & 263056 & 18 & 236848.52 & 41066.68 & 0 & 12198.05 & 0 & 30784.57 \\
\hline 7531.22 & 329758 & 17 & 193667.94 & 36326.25 & 0 & 9329.22 & 0 & 41047.45 \\
\hline 7531.29 & 436727 & 16 & 98498.34 & 19474.61 & 0 & 6380.15 & 0 & 28768.93 \\
\hline 7531.35 & 436543 & 16 & 195381.5 & 39089.74 & 0 & 60411.8 & 8891.84 & 33118.69 \\
\hline 7531.42 & 485534 & 16 & 65073.12 & 12540.31 & 0 & 4298.04 & 0 & 12901 \\
\hline 7531.48 & 352685 & 17 & 94667 & 17426.89 & 0 & 12883.54 & 0 & 13716.33 \\
\hline 7531.55 & 364774 & 17 & 43714.11 & 6798.13 & 0 & 7677.09 & 0 & 18933.05 \\
\hline
\end{tabular}




\begin{tabular}{|c|c|c|c|c|c|c|c|c|}
\hline True Depth & XRF tCOUNTS & XRF Live Time & $\mathrm{Si}(\mathrm{ppm})$ & $\mathrm{Al}$ (ppm) & $\mathrm{P}(\mathrm{ppm})$ & $\mathrm{S}(\mathrm{ppm})$ & $\mathrm{K}(\mathrm{ppm})$ & $\mathrm{Ca}(\mathrm{ppm})$ \\
\hline 7531.61 & 362992 & 17 & 76643.42 & 14000.49 & 0 & 5808.34 & 0 & 13250.46 \\
\hline 7531.68 & 467359 & 16 & 58458.25 & 10183.09 & 0 & 3517.44 & 0 & 7560.24 \\
\hline 7531.75 & 304817 & 17 & 122981.95 & 20517.43 & 0 & 5276.32 & 0 & 26652.34 \\
\hline 7531.81 & 430795 & 16 & 38685.45 & 5444.67 & 0 & 3451.37 & 0 & 9517.97 \\
\hline 7531.88 & 254710 & 18 & 86952.75 & 22432.73 & 0 & 7758.03 & 0 & 85102.63 \\
\hline 7531.94 & 512970 & 15 & 54550.65 & 10292.32 & 0 & 3252.1 & 0 & 11283.83 \\
\hline 7532.01 & 306823 & 17 & 159646.77 & 27654.56 & 0 & 7406.45 & 0 & 35982.12 \\
\hline 7532.07 & 298107 & 18 & 187869.86 & 30726.69 & 0 & 12803.61 & 0 & 62318.12 \\
\hline 7532.14 & 317668 & 17 & 119101.23 & 20957.33 & 0 & 5091.35 & 0 & 117583.89 \\
\hline 7532.20 & 372300 & 17 & 68353.56 & 8929.27 & 0 & 12334.15 & 0 & 157224.89 \\
\hline 7532.27 & 457665 & 16 & 188550.08 & 32643.04 & 0 & 7470.09 & 0 & 207471.13 \\
\hline 7532.34 & 464291 & 16 & 156311.1 & 22606 & 0 & 20227.28 & 0 & 191919.18 \\
\hline 7532.40 & 519335 & 15 & 121365.34 & 15631.8 & 0 & 4900.23 & 0 & 223333.54 \\
\hline 7532.47 & 447598 & 16 & 189516.18 & 27426.78 & 0 & 7109.86 & 0 & 187493.1 \\
\hline 7532.53 & 394404 & 17 & 85122.82 & 15220.72 & 0 & 6148.81 & 0 & 54998 \\
\hline 7532.60 & 422578 & 16 & 181084.42 & 35897.01 & 0 & 8587.7 & 0 & 75990.96 \\
\hline 7532.66 & 384918 & 17 & 248744.62 & 52070.21 & 0 & 12647.75 & 0 & 65429.54 \\
\hline 7532.73 & 507656 & 15 & 65974.5 & 12393.88 & 0 & 3048.37 & 0 & 17818.26 \\
\hline 7532.80 & 352194 & 17 & 278942.28 & 53966.48 & 0 & 13423.16 & 0 & 59143.87 \\
\hline 7532.86 & 552054 & 15 & 82625.75 & 15436.59 & 0 & 136295.41 & 0 & 8267.58 \\
\hline 7532.93 & 378869 & 17 & 239066.37 & 46758.12 & 0 & 11301.48 & 0 & 47707.67 \\
\hline 7532.99 & 516066 & 15 & 57715.78 & 11455.12 & 0 & 3888.58 & 0 & 18369.72 \\
\hline 7533.06 & 419392 & 16 & 119229.96 & 23969.76 & 0 & 5968.97 & 0 & 47452.97 \\
\hline 7533.12 & 449683 & 16 & 90690.74 & 18479.54 & 0 & 5371.91 & 0 & 44107.31 \\
\hline 7533.19 & 383749 & 17 & 179352.57 & 37863.67 & 0 & 8953.24 & 0 & 62507.01 \\
\hline 7533.25 & 465276 & 16 & 125502.21 & 26946.94 & 0 & 8349.84 & 0 & 61141.49 \\
\hline 7533.32 & 412397 & 16 & 250856.97 & 49634.25 & 0 & 16432.32 & 0 & 94284.64 \\
\hline 7533.39 & 588372 & 14 & 28015.35 & 4718.39 & 0 & 4040.92 & 0 & 11761.58 \\
\hline 7533.45 & 381325 & 17 & 259682.71 & 48661.01 & 0 & 14387.86 & 0 & 93445.27 \\
\hline 7533.52 & 393983 & 17 & 177340.57 & 33144.03 & 0 & 9678.99 & 0 & 76883.35 \\
\hline 7533.58 & 508638 & 15 & 54079.03 & 10505.73 & 0 & 4214.4 & 0 & 27276.98 \\
\hline 7533.65 & 372589 & 17 & 250377.72 & 50277.51 & 0 & 14674.93 & 0 & 66298.89 \\
\hline 7533.71 & 501844 & 15 & 88932.21 & 16973.1 & 0 & 5772.2 & 0 & 69082.99 \\
\hline
\end{tabular}




\begin{tabular}{|c|c|c|c|c|c|c|c|c|}
\hline True Depth & XRF tCOUNTS & XRF Live Time & $\mathrm{Si}(\mathrm{ppm})$ & $\mathrm{Al}$ (ppm) & $\mathrm{P}(\mathrm{ppm})$ & $\mathrm{S}(\mathrm{ppm})$ & $\mathrm{K}(\mathrm{ppm})$ & $\mathrm{Ca}(\mathrm{ppm})$ \\
\hline 7533.78 & 450388 & 16 & 142250.57 & 27342.22 & 0 & 7043.6 & 0 & 69781.88 \\
\hline 7533.85 & 371307 & 17 & 330078.57 & 62579.35 & 0 & 18698.92 & 0 & 73880.35 \\
\hline 7533.91 & 304571 & 17 & 126098.56 & 24969.22 & 0 & 8330.77 & 0 & 40219.58 \\
\hline 7533.98 & 351580 & 17 & 310823.05 & 59730.65 & 0 & 19182.36 & 0 & 59242.54 \\
\hline 7534.04 & 420587 & 16 & 122625.6 & 23399.4 & 0 & 9139.74 & 0 & 61223.97 \\
\hline 7534.11 & 389112 & 17 & 247309.84 & 48343.37 & 0 & 15180.62 & 0 & 129932.98 \\
\hline 7534.17 & 453300 & 16 & 228320.05 & 38396.88 & 0 & 12060.83 & 0 & 184458.54 \\
\hline 7534.24 & 506902 & 15 & 186422.15 & 28919.57 & 0 & 9211.19 & 0 & 230336.74 \\
\hline 7534.30 & 479480 & 16 & 178274.15 & 28882.73 & 0 & 9388.5 & 0 & 207236.39 \\
\hline 7534.37 & 463943 & 16 & 129008.15 & 22415.81 & 0 & 6266.95 & 0 & 144512.04 \\
\hline 7534.44 & 405999 & 16 & 263410.04 & 50421.9 & 0 & 13710.2 & 0 & 134492.61 \\
\hline 7534.50 & 361622 & 17 & 170187.6 & 32688.4 & 0 & 9663.71 & 0 & 105915.19 \\
\hline 7534.57 & 400168 & 16 & 187296.04 & 37458.13 & 0 & 9407.06 & 0 & 88494.36 \\
\hline 7534.63 & 381511 & 17 & 254079.08 & 50339.27 & 0 & 13217.31 & 0 & 96683.27 \\
\hline 7534.70 & 363123 & 17 & 197234.52 & 38416.71 & 0 & 11594.43 & 0 & 69718.98 \\
\hline 7534.76 & 351280 & 17 & 295270.27 & 60295.84 & 0 & 17033.95 & 0 & 83247.63 \\
\hline 7534.83 & 366819 & 17 & 206943.46 & 42865.24 & 0 & 12064.26 & 0 & 70359.03 \\
\hline 7534.90 & 523090 & 15 & 66411 & 13855.26 & 0 & 4718.58 & 0 & 40112.47 \\
\hline 7534.96 & 416528 & 16 & 95595.2 & 19402.92 & 0 & 5311.91 & 0 & 24218.45 \\
\hline 7535.03 & 391302 & 17 & 92717.63 & 19233.48 & 0 & 6158.68 & 0 & 21588.91 \\
\hline 7535.09 & 332813 & 17 & 126667.83 & 22888.28 & 0 & 7750.94 & 0 & 18736.06 \\
\hline 7535.16 & 259650 & 18 & 259297.44 & 52947.98 & 0 & 18683.52 & 0 & 24085.94 \\
\hline 7535.22 & 342677 & 17 & 280547.74 & 53844.44 & 0 & 17689.36 & 0 & 24463.14 \\
\hline 7535.29 & 324701 & 17 & 294449.05 & 57431.13 & 0 & 18895.75 & 0 & 29937.9 \\
\hline 7535.35 & 291522 & 18 & 313578.08 & 57123.41 & 0 & 21476.84 & 0 & 18453.62 \\
\hline 7535.42 & 438341 & 16 & 123027.28 & 25170.66 & 0 & 7859.86 & 0 & 9697.11 \\
\hline 7535.49 & 431299 & 16 & 74299.39 & 13580.74 & 0 & 5787.91 & 0 & 9565.48 \\
\hline 7535.55 & 335596 & 17 & 340085.36 & 74554.05 & 0 & 23436.38 & 10552.07 & 9275.39 \\
\hline 7535.62 & 295975 & 17 & 334377.35 & 65154.92 & 0 & 19969.54 & 7241.85 & 9743.74 \\
\hline 7535.68 & 374276 & 17 & 172683.83 & 35591.47 & 0 & 9113.72 & 0 & 7973.52 \\
\hline 7535.75 & 376888 & 17 & 120013.49 & 22215.34 & 0 & 8025.03 & 0 & 10744.52 \\
\hline 7535.81 & 601458 & 14 & 18308.2 & 2132.11 & 0 & 2749.21 & 0 & 3103.53 \\
\hline 7535.88 & 221840 & 18 & 137483.58 & 21942.48 & 0 & 8874.62 & 0 & 6470.56 \\
\hline
\end{tabular}




\begin{tabular}{|c|c|c|c|c|c|c|c|c|}
\hline True Depth & XRF tCOUNTS & XRF Live Time & $\mathrm{Si}(\mathrm{ppm})$ & $\mathrm{Al}(\mathrm{ppm})$ & $\mathrm{P}(\mathrm{ppm})$ & $\mathrm{S}(\mathrm{ppm})$ & $\mathrm{K}(\mathrm{ppm})$ & $\mathrm{Ca}(\mathrm{ppm})$ \\
\hline 7535.94 & 297170 & 18 & 21072.06 & 3164.32 & 0 & 1881.88 & 0 & 1149.72 \\
\hline 7536.01 & 434922 & 16 & 71235.99 & 14080.5 & 0 & 8367.96 & 0 & 19423.94 \\
\hline 7536.08 & 512604 & 15 & 73484.23 & 15255.53 & 0 & 7630 & 0 & 7897.24 \\
\hline 7536.14 & 323625 & 17 & 241621.72 & 48384.83 & 0 & 21168.78 & 0 & 10399.68 \\
\hline 7536.21 & 253259 & 18 & 140875.53 & 27500.41 & 0 & 9984.61 & 0 & 7775.37 \\
\hline 7536.27 & 303368 & 17 & 140858.03 & 27153.4 & 0 & 7245.28 & 0 & 5719.66 \\
\hline 7536.34 & 309048 & 17 & 164191.35 & 32073.32 & 0 & 7697.59 & 0 & 6326.64 \\
\hline 7536.40 & 318970 & 17 & 259330.1 & 52190.24 & 0 & 12244.4 & 0 & 8674.21 \\
\hline 7536.47 & 329687 & 17 & 307430.26 & 55829.28 & 0 & 18277.96 & 0 & 11081.62 \\
\hline 7536.54 & 445441 & 16 & 96845.67 & 18806.33 & 0 & 6555.03 & 0 & 8444.97 \\
\hline 7536.60 & 595190 & 14 & 17648.73 & 1672.94 & 0 & 2771.39 & 0 & 13561.79 \\
\hline 7536.67 & 511303 & 15 & 49981.53 & 8443 & 0 & 5886.53 & 0 & 5066.72 \\
\hline 7536.73 & 370496 & 17 & 144715.13 & 25432.32 & 0 & 12922.63 & 0 & 17741.63 \\
\hline 7536.80 & 300456 & 17 & 321905.6 & 53531.1 & 0 & 19803.44 & 0 & 32444.51 \\
\hline 7536.86 & 335831 & 17 & 158246.95 & 28881.64 & 0 & 9969.03 & 0 & 27760.95 \\
\hline 7536.93 & 431698 & 16 & 137440.5 & 23706.62 & 0 & 6880.11 & 0 & 21717.13 \\
\hline 7536.99 & 336816 & 17 & 303317.2 & 53006.05 & 0 & 15374.94 & 0 & 25628.64 \\
\hline 7537.06 & 321527 & 17 & 271383.54 & 50100.55 & 0 & 16808.73 & 0 & 19470.9 \\
\hline 7537.13 & 398077 & 17 & 62912.54 & 11439.57 & 0 & 4603.18 & 0 & 7476.74 \\
\hline 7537.19 & 308552 & 17 & 341706.28 & 64806.33 & 0 & 21647.63 & 0 & 19467 \\
\hline 7537.26 & 352906 & 17 & 167222.37 & 29714.87 & 0 & 9783.59 & 0 & 15140.95 \\
\hline 7537.32 & 313851 & 17 & 214537.59 & 39208.26 & 0 & 11494.21 & 0 & 13526.35 \\
\hline 7537.39 & 360646 & 17 & 76952.71 & 14001.77 & 0 & 5422.81 & 0 & 13058.94 \\
\hline 7537.45 & 361836 & 17 & 307393.71 & 51509.29 & 0 & 33813.8 & 0 & 27469.52 \\
\hline 7537.52 & 465048 & 16 & 85644.02 & 14458.92 & 0 & 7237.78 & 0 & 20043.87 \\
\hline 7537.59 & 339631 & 17 & 313274.48 & 57551.05 & 0 & 21583.29 & 0 & 31356.54 \\
\hline 7537.65 & 345738 & 17 & 235938.55 & 44625.29 & 0 & 19410.4 & 0 & 27555.98 \\
\hline 7537.72 & 471118 & 16 & 86041.95 & 15433.73 & 0 & 9048.22 & 0 & 21501.72 \\
\hline 7537.78 & 375908 & 17 & 233157.11 & 42720.57 & 0 & 19998.97 & 0 & 24433.59 \\
\hline 7537.85 & 344122 & 17 & 208609.03 & 39744.29 & 0 & 16619.23 & 0 & 28033.33 \\
\hline 7537.91 & 310216 & 17 & 320529.54 & 60187.51 & 0 & 27876.26 & 0 & 31301.82 \\
\hline 7537.98 & 179536 & 19 & 252105.46 & 52571.76 & 0 & 16632.64 & 0 & 36124.01 \\
\hline 7538.04 & 347000 & 17 & 335125.35 & 62850.01 & 0 & 23266.48 & 0 & 21222.22 \\
\hline
\end{tabular}




\begin{tabular}{|c|c|c|c|c|c|c|c|c|}
\hline True Depth & XRF tCOUNTS & XRF Live Time & $\mathrm{Si}(\mathrm{ppm})$ & $\mathrm{Al}(\mathrm{ppm})$ & $\mathrm{P}(\mathrm{ppm})$ & $\mathrm{S}(\mathrm{ppm})$ & $\mathrm{K}(\mathrm{ppm})$ & $\mathrm{Ca}(\mathrm{ppm})$ \\
\hline 7538.11 & 422081 & 16 & 108187.03 & 20827.81 & 0 & 8011.19 & 0 & 16059.09 \\
\hline 7538.18 & 348365 & 17 & 202459.86 & 40431.56 & 0 & 14877.99 & 0 & 20192.59 \\
\hline 7538.24 & 310488 & 17 & 247182.42 & 49373.92 & 0 & 22745.43 & 0 & 24843.55 \\
\hline 7538.31 & 328845 & 17 & 314951.38 & 66984.24 & 0 & 43961.54 & 0 & 21873.31 \\
\hline 7538.37 & 428090 & 16 & 171796.55 & 35927.93 & 0 & 15810.68 & 0 & 16867.16 \\
\hline 7538.44 & 365350 & 17 & 301553.05 & 62211.08 & 0 & 25057.53 & 0 & 25934.32 \\
\hline 7538.50 & 355659 & 17 & 288582.16 & 56577.66 & 0 & 27371.98 & 0 & 34936.17 \\
\hline 7538.57 & 440424 & 16 & 96057.82 & 20647.83 & 0 & 11467.4 & 0 & 17959.79 \\
\hline 7538.64 & 340797 & 17 & 216971.87 & 41117.13 & 0 & 18720.82 & 0 & 18017.5 \\
\hline 7538.70 & 334267 & 17 & 321915.75 & 62231.23 & 0 & 25438.54 & 0 & 20377.66 \\
\hline 7538.77 & 390751 & 17 & 157458.06 & 33744.89 & 0 & 19124.46 & 0 & 16356.52 \\
\hline 7538.83 & 386713 & 17 & 140525.74 & 29204.73 & 0 & 14890.48 & 0 & 21703.58 \\
\hline 7538.90 & 357471 & 17 & 230313.69 & 48848.76 & 0 & 17789.14 & 0 & 15208.12 \\
\hline 7538.96 & 379035 & 17 & 212234.17 & 48305.39 & 0 & 20269.46 & 0 & 15875.08 \\
\hline 7539.03 & 410788 & 16 & 91411.23 & 18728.18 & 0 & 11172.87 & 0 & 12621.99 \\
\hline 7539.09 & 420478 & 16 & 114537.95 & 24964.13 & 0 & 12607.73 & 0 & 10503.77 \\
\hline 7539.16 & 332743 & 17 & 259075.15 & 55164.46 & 0 & 25314.01 & 6057.89 & 12660.5 \\
\hline 7539.23 & 338174 & 17 & 236153.53 & 47683.34 & 0 & 41948.54 & 0 & 12845.63 \\
\hline 7539.29 & 424909 & 16 & 140718.29 & 29995.08 & 0 & 14335.05 & 0 & 22809.19 \\
\hline 7539.36 & 332403 & 17 & 303209.71 & 59645.06 & 0 & 27847.15 & 0 & 13110.29 \\
\hline 7539.42 & 416316 & 16 & 69185.32 & 13893.34 & 0 & 8311.93 & 0 & 9601.58 \\
\hline 7539.49 & 390478 & 17 & 105919.69 & 22884.7 & 0 & 8668.94 & 0 & 19142.94 \\
\hline 7539.55 & 323170 & 17 & 56365.56 & 9869.95 & 0 & 4958.54 & 0 & 12625.25 \\
\hline 7539.62 & 475293 & 16 & 71960.41 & 15435.07 & 0 & 4896.85 & 0 & 11544.57 \\
\hline 7539.69 & 384045 & 17 & 220792.36 & 46511.62 & 0 & 13075.18 & 0 & 15669.3 \\
\hline 7539.75 & 416779 & 16 & 129436.17 & 26066.73 & 0 & 6895.32 & 0 & 9040.35 \\
\hline 7539.82 & 360306 & 17 & 145617.38 & 29674.64 & 0 & 7986.55 & 0 & 9667.7 \\
\hline 7539.88 & 346952 & 17 & 371278.42 & 70481.2 & 0 & 25243.42 & 0 & 24531.86 \\
\hline 7539.95 & 175871 & 19 & 224043.11 & 45572.42 & 0 & 11455.68 & 0 & 13781.02 \\
\hline 7540.01 & 533369 & 15 & 78184.29 & 15452.97 & 0 & 5690.2 & 0 & 12149.63 \\
\hline 7540.08 & 350719 & 17 & 333817.01 & 61713.98 & 0 & 25731.43 & 0 & 20955.67 \\
\hline 7540.14 & 519779 & 15 & 69563.59 & 13843.78 & 0 & 4960.89 & 0 & 4635.37 \\
\hline 7540.21 & 419986 & 16 & 104511.31 & 19125.34 & 0 & 8894.12 & 0 & 7045.41 \\
\hline
\end{tabular}




\begin{tabular}{|c|c|c|c|c|c|c|c|c|}
\hline True Depth & XRF tCOUNTS & XRF Live Time & $\mathrm{Si}(\mathrm{ppm})$ & $\mathrm{Al}(\mathrm{ppm})$ & $\mathrm{P}(\mathrm{ppm})$ & $\mathrm{S}(\mathrm{ppm})$ & $\mathrm{K}(\mathrm{ppm})$ & $\mathrm{Ca}(\mathrm{ppm})$ \\
\hline 7540.28 & 447332 & 16 & 76457.42 & 13860.37 & 0 & 5411.36 & 0 & 9853.68 \\
\hline 7540.34 & 358579 & 17 & 342224.36 & 64526.48 & 0 & 24833.96 & 0 & 41532.06 \\
\hline 7540.41 & 409113 & 16 & 180324.91 & 37505.06 & 0 & 12654.36 & 0 & 28681.73 \\
\hline 7540.47 & 586321 & 14 & 49365.74 & 8816.81 & 0 & 8828.58 & 0 & 14445.07 \\
\hline 7540.54 & 665217 & 13 & 16135.42 & 1810.09 & 0 & 1701.59 & 0 & 833.78 \\
\hline 7540.60 & 277124 & 18 & 17571.27 & 3206.32 & 0 & 4236.24 & 0 & 195169.61 \\
\hline 7540.67 & 383519 & 17 & 34547.05 & 5946.82 & 0 & 4066.95 & 0 & 32929.32 \\
\hline 7540.73 & 410896 & 16 & 12374.75 & 4179.28 & 0 & 3225.04 & 0 & 9499.59 \\
\hline 7540.80 & 610446 & 14 & 40409.44 & 7823.08 & 0 & 4003.7 & 0 & 8254.62 \\
\hline 7540.87 & 313227 & 17 & 84132.22 & 17223.06 & 0 & 8180.39 & 0 & 25146.85 \\
\hline 7540.93 & 603410 & 14 & 52943.83 & 10220.4 & 0 & 12329.95 & 0 & 16711.49 \\
\hline 7541.00 & 266998 & 18 & 108391.17 & 19471.62 & 0 & 15624.6 & 0 & 41287.95 \\
\hline 7541.06 & 272582 & 18 & 89780.02 & 15304.03 & 0 & 14384.72 & 0 & 28041.53 \\
\hline 7541.13 & 284771 & 18 & 208522.89 & 39512.77 & 0 & 30435.98 & 0 & 28552.76 \\
\hline 7541.19 & 411888 & 16 & 87708.63 & 18624.41 & 0 & 15149.91 & 0 & 22475.11 \\
\hline 7541.26 & 284665 & 18 & 141734.6 & 28109.54 & 0 & 19405.32 & 0 & 33482.33 \\
\hline 7541.33 & 392343 & 17 & 80324.64 & 13936.83 & 0 & 13939.92 & 0 & 27340.56 \\
\hline 7541.39 & 288663 & 18 & 164892.5 & 32063.58 & 0 & 25936.13 & 0 & 19199.19 \\
\hline 7541.46 & 307316 & 17 & 64931.86 & 11872.73 & 0 & 14039.59 & 0 & 15312.33 \\
\hline 7541.52 & 309772 & 17 & 46410.61 & 6927.98 & 0 & 13904.08 & 0 & 16873.52 \\
\hline 7541.59 & 302030 & 17 & 38179.28 & 5204.94 & 0 & 13952.54 & 0 & 11968.44 \\
\hline 7541.65 & 307072 & 17 & 134562.76 & 20870.11 & 0 & 29910.47 & 0 & 34379.47 \\
\hline 7541.72 & 284227 & 18 & 80124.67 & 14830.57 & 0 & 18352.98 & 0 & 22480.37 \\
\hline 7541.78 & 265464 & 18 & 46440.26 & 6660.99 & 0 & 14146.01 & 0 & 16917.96 \\
\hline 7541.85 & 365919 & 17 & 103643.45 & 18562.35 & 0 & 16704.38 & 0 & 41198.1 \\
\hline 7541.92 & 439763 & 16 & 182984.94 & 36275.29 & 0 & 28004.48 & 0 & 40610.02 \\
\hline 7541.98 & 360428 & 17 & 132171.35 & 28100.81 & 0 & 26003.25 & 0 & 20806.37 \\
\hline 7542.05 & 349909 & 17 & 209767.37 & 35872.2 & 0 & 41995.64 & 0 & 49435.97 \\
\hline 7542.11 & 333450 & 17 & 180446.85 & 26467.39 & 0 & 35539.9 & 0 & 38385.34 \\
\hline 7542.18 & 332229 & 17 & 104495.32 & 15948.43 & 0 & 19799.28 & 0 & 43955.93 \\
\hline 7542.24 & 320211 & 17 & 230420.35 & 39563.73 & 0 & 30056.07 & 0 & 63715.87 \\
\hline 7542.31 & 388161 & 17 & 81484.47 & 14264.43 & 0 & 13791.59 & 0 & 58505.02 \\
\hline 7542.38 & 341012 & 17 & 145091.98 & 16182.28 & 0 & 15646.01 & 0 & 96917.35 \\
\hline
\end{tabular}




\begin{tabular}{|c|c|c|c|c|c|c|c|c|}
\hline True Depth & XRF tCOUNTS & XRF Live Time & $\mathrm{Si}(\mathrm{ppm})$ & $\mathrm{Al}(\mathrm{ppm})$ & $\mathrm{P}(\mathrm{ppm})$ & $\mathrm{S}(\mathrm{ppm})$ & $\mathrm{K}(\mathrm{ppm})$ & $\mathrm{Ca}(\mathrm{ppm})$ \\
\hline 7542.44 & 377708 & 17 & 151145.72 & 16711.99 & 0 & 11748.99 & 0 & 165889.67 \\
\hline 7542.51 & 562030 & 15 & 58723.03 & 0 & 0 & 2723.56 & 0 & 283390.87 \\
\hline 7542.57 & 627316 & 14 & 20305.23 & 0 & 0 & 1025.62 & 0 & 418964.07 \\
\hline 7542.64 & 690021 & 13 & 13257.81 & 1629.21 & 0 & 1091.65 & 0 & 463720.97 \\
\hline 7542.70 & 682211 & 13 & 21472.15 & 0 & 0 & 1729.45 & 0 & 464964.68 \\
\hline 7542.77 & 701062 & 13 & 25710.01 & 4576.86 & 0 & 2117.57 & 0 & 463263.01 \\
\hline 7542.83 & 642887 & 14 & 17037.54 & 1995.88 & 0 & 1510.73 & 0 & 449659.59 \\
\hline 7542.90 & 678580 & 13 & 37458.44 & 1988.44 & 0 & 2358.61 & 0 & 443663.04 \\
\hline 7542.97 & 696120 & 13 & 15610.6 & 1909.83 & 0 & 2510.68 & 0 & 315482.06 \\
\hline 7543.03 & 703070 & 13 & 15743.12 & 3355.2 & 0 & 1724.56 & 0 & 316134.86 \\
\hline 7543.10 & 610544 & 14 & 126576.92 & 4156.62 & 0 & 7469.83 & 0 & 369035.89 \\
\hline 7543.16 & 506214 & 15 & 248221.94 & 7825.8 & 0 & 9028.5 & 0 & 264016.56 \\
\hline 7543.23 & 349425 & 17 & 239890.94 & 6552.71 & 0 & 6512.07 & 0 & 181257.17 \\
\hline 7543.29 & 464204 & 16 & 65103.64 & 4705.2 & 0 & 5970.69 & 0 & 149787.38 \\
\hline 7543.36 & 419979 & 16 & 115470.46 & 21969.33 & 0 & 19301.96 & 0 & 86279.35 \\
\hline 7543.43 & 376387 & 17 & 209745.19 & 38933.25 & 0 & 26772.55 & 0 & 39529.94 \\
\hline 7543.49 & 338436 & 17 & 201327.35 & 30477.16 & 0 & 30285.35 & 0 & 39430.06 \\
\hline 7543.56 & 323357 & 17 & 214797.6 & 34638.68 & 0 & 31721.7 & 0 & 51440.08 \\
\hline 7543.62 & 337011 & 17 & 206741.38 & 36154.86 & 0 & 27585.57 & 0 & 53196.58 \\
\hline 7543.69 & 341500 & 17 & 279044.58 & 41267.02 & 0 & 37578.08 & 0 & 34365.38 \\
\hline 7543.75 & 467016 & 16 & 114947.76 & 18568.54 & 0 & 78864.61 & 0 & 25645.57 \\
\hline 7543.82 & 302062 & 17 & 226670.15 & 37096.93 & 0 & 35883.66 & 0 & 31532.03 \\
\hline 7543.88 & 360487 & 17 & 209172.44 & 37751.2 & 0 & 26280.15 & 0 & 58387.02 \\
\hline 7543.95 & 445080 & 16 & 158243.66 & 18461.32 & 0 & 16247.45 & 0 & 155693.03 \\
\hline 7544.02 & 319105 & 17 & 285201.24 & 46906.94 & 0 & 41667.83 & 0 & 93104.43 \\
\hline 7544.08 & 384806 & 17 & 290761.65 & 49888.39 & 0 & 48857.08 & 0 & 29755.62 \\
\hline 7544.15 & 342837 & 17 & 262130.31 & 44773.4 & 0 & 36840.37 & 0 & 48389.02 \\
\hline 7544.21 & 417313 & 16 & 173974.89 & 24148.42 & 0 & 18231.14 & 0 & 189836.2 \\
\hline 7544.28 & 483777 & 16 & 140823.85 & 25731.61 & 0 & 20515.13 & 0 & 126664.09 \\
\hline 7544.34 & 423108 & 16 & 242407.07 & 42609.64 & 0 & 38455.37 & 0 & 116086.52 \\
\hline 7544.41 & 396142 & 17 & 243030.75 & 42263.63 & 0 & 44626.23 & 0 & 52994.38 \\
\hline 7544.48 & 383653 & 17 & 135288.19 & 25782.8 & 0 & 16965.17 & 0 & 25834.78 \\
\hline 7544.54 & 453067 & 16 & 156482.21 & 25996.52 & 0 & 52434.07 & 0 & 30553.43 \\
\hline
\end{tabular}




\begin{tabular}{|c|c|c|c|c|c|c|c|c|}
\hline True Depth & XRF tCOUNTS & XRF Live Time & $\mathrm{Si}(\mathrm{ppm})$ & $\mathrm{Al}(\mathrm{ppm})$ & $\mathrm{P}(\mathrm{ppm})$ & $\mathrm{S}(\mathrm{ppm})$ & $\mathrm{K}(\mathrm{ppm})$ & $\mathrm{Ca}(\mathrm{ppm})$ \\
\hline 7544.61 & 359974 & 17 & 277971.66 & 38532.58 & 0 & 25436.56 & 0 & 71925.63 \\
\hline 7544.67 & 375621 & 17 & 253145.5 & 37271.34 & 0 & 43887.84 & 0 & 35280.12 \\
\hline 7544.74 & 376409 & 17 & 299240.1 & 44353.6 & 0 & 36991.93 & 0 & 51227.34 \\
\hline 7544.80 & 394440 & 17 & 289117.99 & 43664.32 & 0 & 40040.84 & 0 & 58714.07 \\
\hline 7544.87 & 399479 & 16 & 187734.83 & 32735.98 & 0 & 30015.56 & 0 & 58767.95 \\
\hline 7544.93 & 379347 & 17 & 164494.45 & 28107.81 & 0 & 23092.93 & 0 & 58465.83 \\
\hline 7545.00 & 395249 & 17 & 125950.74 & 15360.18 & 0 & 19164.76 & 0 & 90572.66 \\
\hline 7545.07 & 393200 & 17 & 230927.91 & 39625.81 & 0 & 33505.35 & 0 & 52183.05 \\
\hline 7545.13 & 335749 & 17 & 172348 & 25024.9 & 0 & 27450.63 & 0 & 28054.36 \\
\hline 7545.20 & 307639 & 17 & 278703.24 & 35229.75 & 0 & 32989.12 & 0 & 33526.06 \\
\hline 7545.26 & 282291 & 18 & 297729.84 & 33476.1 & 0 & 29943.88 & 0 & 44436.91 \\
\hline 7545.33 & 391510 & 17 & 181847.26 & 21228.26 & 0 & 22220.23 & 0 & 46692.51 \\
\hline 7545.39 & 480716 & 16 & 87116.75 & 12053.45 & 0 & 22446.04 & 0 & 28589.52 \\
\hline 7545.46 & 375430 & 17 & 235784.48 & 34730.5 & 0 & 61187.34 & 0 & 36659.57 \\
\hline 7545.52 & 336817 & 17 & 241960.5 & 33645.85 & 0 & 33131.24 & 0 & 41576.05 \\
\hline 7545.59 & 424141 & 16 & 93689.79 & 12706.38 & 0 & 18180.21 & 0 & 29957.64 \\
\hline 7545.66 & 435006 & 16 & 130164.87 & 15546.1 & 0 & 12592.78 & 0 & 46223.08 \\
\hline 7545.72 & 392903 & 17 & 108403.73 & 14984.9 & 0 & 10963.29 & 0 & 29114.6 \\
\hline 7545.79 & 405717 & 16 & 344345.78 & 46365.05 & 0 & 49286.99 & 0 & 50422.48 \\
\hline 7545.85 & 427772 & 16 & 193361.28 & 28344.17 & 0 & 23664.27 & 0 & 39799.49 \\
\hline 7545.92 & 602420 & 14 & 29684.76 & 4264.5 & 0 & 7730.92 & 0 & 9576.52 \\
\hline 7545.98 & 346451 & 17 & 305847.22 & 45833.86 & 0 & 26578.21 & 0 & 45724.44 \\
\hline 7546.05 & 142576 & 19 & 31246.07 & 6377.52 & 0 & 3657.39 & 0 & 11283.23 \\
\hline 7546.12 & 346017 & 17 & 231110.99 & 38714.33 & 0 & 35384.66 & 0 & 42220.94 \\
\hline 7546.18 & 361851 & 17 & 313163.09 & 50108.96 & 0 & 35514.03 & 0 & 36050.67 \\
\hline 7546.25 & 362520 & 17 & 309499.81 & 49130.02 & 0 & 42861.49 & 0 & 26514.2 \\
\hline 7546.31 & 365462 & 17 & 267987.23 & 40061.99 & 0 & 43783.44 & 0 & 27655.38 \\
\hline 7546.38 & 376612 & 17 & 287908.84 & 40788.06 & 0 & 41930.41 & 0 & 48406.79 \\
\hline 7546.44 & 391287 & 17 & 274866.68 & 39261.41 & 0 & 32235.76 & 0 & 85086.11 \\
\hline 7546.51 & 487723 & 16 & 103737.71 & 9485.77 & 0 & 10693.8 & 0 & 140947.51 \\
\hline 7546.57 & 519133 & 15 & 158518.23 & 16137.68 & 0 & 38057.38 & 0 & 183301.8 \\
\hline 7546.64 & 378586 & 17 & 164464.28 & 24188.04 & 0 & 38784.4 & 0 & 126688.97 \\
\hline 7546.71 & 545124 & 15 & 72846.71 & 12738.92 & 0 & 41215.07 & 0 & 19993.16 \\
\hline
\end{tabular}




\begin{tabular}{|c|c|c|c|c|c|c|c|c|}
\hline True Depth & XRF tCOUNTS & XRF Live Time & $\mathrm{Si}(\mathrm{ppm})$ & $\mathrm{Al}(\mathrm{ppm})$ & $\mathrm{P}(\mathrm{ppm})$ & $\mathrm{S}(\mathrm{ppm})$ & $\mathrm{K}(\mathrm{ppm})$ & $\mathrm{Ca}(\mathrm{ppm})$ \\
\hline 7546.77 & 354766 & 17 & 280852.47 & 45399.6 & 0 & 42827.93 & 0 & 40802.75 \\
\hline 7546.84 & 336188 & 17 & 294201.48 & 46207.23 & 0 & 49305.74 & 0 & 35774.99 \\
\hline 7546.90 & 372301 & 17 & 336174.45 & 46087.03 & 0 & 44203.37 & 0 & 27201.58 \\
\hline 7546.97 & 354393 & 17 & 320090.84 & 41319.27 & 0 & 41183.06 & 0 & 34419.93 \\
\hline 7547.03 & 446132 & 16 & 289557.78 & 44897.7 & 0 & 73566.79 & 0 & 29005.22 \\
\hline 7547.10 & 358018 & 17 & 324671.86 & 41883.79 & 0 & 46921.91 & 0 & 22720.88 \\
\hline 7547.17 & 327083 & 17 & 243547.19 & 31254.81 & 0 & 31129.96 & 0 & 56388.43 \\
\hline 7547.23 & 348609 & 17 & 229146.41 & 33263.72 & 0 & 27388.25 & 0 & 71011.68 \\
\hline 7547.30 & 464646 & 16 & 101988.75 & 14171.72 & 0 & 15511.43 & 0 & 127541.16 \\
\hline 7547.36 & 354266 & 17 & 186448.34 & 23146.12 & 0 & 24909.28 & 0 & 94752.57 \\
\hline 7547.43 & 438651 & 16 & 54447.64 & 6625.77 & 0 & 10026.53 & 0 & 51110.47 \\
\hline 7547.49 & 426688 & 16 & 235164.66 & 32461.52 & 0 & 32162.34 & 0 & 85784.16 \\
\hline 7547.56 & 337922 & 17 & 229397.91 & 32957.8 & 0 & 28670.9 & 0 & 75701.16 \\
\hline 7547.62 & 367860 & 17 & 190185.96 & 24807.85 & 0 & 21330.78 & 0 & 89845.42 \\
\hline 7547.69 & 368096 & 17 & 73349.21 & 9918.3 & 0 & 13524.54 & 0 & 29418.85 \\
\hline 7547.76 & 335700 & 17 & 82594.33 & 11455.38 & 0 & 10940.08 & 0 & 55150.66 \\
\hline 7547.82 & 216191 & 18 & 41720.57 & 11484.36 & 0 & 8592.78 & 0 & 20083.35 \\
\hline 7547.89 & 206146 & 18 & 102212.81 & 17206.98 & 0 & 15593.73 & 0 & 18253.14 \\
\hline 7547.95 & 252465 & 18 & 60537.6 & 6283.05 & 0 & 12907.66 & 0 & 32826.95 \\
\hline 7548.02 & 309434 & 17 & 339886.67 & 50175.98 & 0 & 45692.94 & 0 & 25029.51 \\
\hline 7548.08 & 379521 & 17 & 288446.62 & 44883.16 & 0 & 49642.08 & 0 & 47409.81 \\
\hline 7548.15 & 323139 & 17 & 272580.18 & 33579.2 & 0 & 28795.02 & 0 & 54730.99 \\
\hline 7548.22 & 378285 & 17 & 197109.28 & 21914.54 & 0 & 44561.74 & 0 & 63679.31 \\
\hline 7548.28 & 386686 & 17 & 332024.15 & 43482.1 & 0 & 34098.48 & 0 & 61231.17 \\
\hline 7548.35 & 373841 & 17 & 223543 & 27395.62 & 0 & 21417.54 & 0 & 42263.82 \\
\hline 7548.41 & 388108 & 17 & 298838.92 & 43817.22 & 0 & 35806.9 & 0 & 61643.81 \\
\hline 7548.48 & 343644 & 17 & 315630.39 & 39849.93 & 0 & 39638.73 & 0 & 24297.1 \\
\hline 7548.54 & 388029 & 17 & 195340.88 & 24124.19 & 0 & 24219.62 & 0 & 17337.24 \\
\hline 7548.61 & 361954 & 17 & 327976.53 & 42597.34 & 0 & 39146.36 & 0 & 38640.61 \\
\hline 7548.67 & 316792 & 17 & 257900.02 & 27570.51 & 0 & 25197.44 & 0 & 16984.08 \\
\hline 7548.74 & 340851 & 17 & 234051.31 & 25180.12 & 0 & 23368.52 & 0 & 16981.34 \\
\hline 7548.81 & 323392 & 17 & 271989.34 & 27228.14 & 0 & 22353.58 & 0 & 17352.36 \\
\hline 7548.87 & 294573 & 17 & 275654.43 & 33998.4 & 0 & 22801.39 & 0 & 16205.77 \\
\hline
\end{tabular}




\begin{tabular}{|c|c|c|c|c|c|c|c|c|}
\hline True Depth & XRF tCOUNTS & XRF Live Time & $\mathrm{Si}(\mathrm{ppm})$ & $\mathrm{Al}(\mathrm{ppm})$ & $\mathrm{P}(\mathrm{ppm})$ & $\mathrm{S}(\mathrm{ppm})$ & $\mathrm{K}(\mathrm{ppm})$ & $\mathrm{Ca}(\mathrm{ppm})$ \\
\hline 7548.94 & 325716 & 17 & 368638.9 & 35775.93 & 0 & 22868.22 & 0 & 31586.76 \\
\hline 7549.00 & 343257 & 17 & 383635.37 & 44288.77 & 0 & 31341.38 & 0 & 13712.19 \\
\hline 7549.07 & 411155 & 16 & 315038.27 & 47337.78 & 0 & 35848.23 & 0 & 20266.43 \\
\hline 7549.13 & 346720 & 17 & 340001.74 & 42592.8 & 0 & 38140.57 & 0 & 16067.7 \\
\hline 7549.20 & 320219 & 17 & 326642.54 & 42981.33 & 0 & 35070.9 & 0 & 15294.99 \\
\hline 7549.27 & 331519 & 17 & 353407.31 & 43749.04 & 0 & 33083.39 & 0 & 18936.58 \\
\hline 7549.33 & 322074 & 17 & 358146.01 & 47576.5 & 0 & 31174.13 & 0 & 11604.33 \\
\hline 7549.40 & 340123 & 17 & 327715.58 & 41763.67 & 0 & 39864.22 & 0 & 14043.75 \\
\hline 7549.46 & 332217 & 17 & 337524.75 & 34392.48 & 0 & 36672.41 & 0 & 12926.52 \\
\hline 7549.53 & 337687 & 17 & 275030.94 & 25446.89 & 0 & 23006.37 & 0 & 8466.37 \\
\hline 7549.59 & 315617 & 17 & 335437.1 & 29023.65 & 0 & 26310.09 & 0 & 11415.6 \\
\hline 7549.66 & 341552 & 17 & 246463.72 & 18581.1 & 0 & 18515.59 & 0 & 16699.45 \\
\hline 7549.72 & 332828 & 17 & 353490.64 & 43439.8 & 0 & 37047.18 & 0 & 16251.16 \\
\hline 7549.79 & 348902 & 17 & 204065.69 & 20679.66 & 0 & 18106.55 & 0 & 17199.03 \\
\hline 7549.86 & 240287 & 18 & 393157.94 & 37543.41 & 0 & 28953.07 & 0 & 11671.5 \\
\hline 7549.92 & 398178 & 17 & 163213.46 & 17161.99 & 0 & 23386.74 & 0 & 13439.75 \\
\hline 7549.99 & 361801 & 17 & 247249.7 & 21385 & 0 & 19165.59 & 0 & 33931.39 \\
\hline 7550.05 & 334540 & 17 & 327924.46 & 35935.66 & 0 & 24100.68 & 0 & 52596.82 \\
\hline 7550.12 & 352080 & 17 & 200754.57 & 18768.65 & 0 & 11923.17 & 0 & 144842.83 \\
\hline 7550.18 & 308908 & 17 & 237220.48 & 31847.62 & 0 & 36530.49 & 0 & 66480.13 \\
\hline 7550.25 & 345583 & 17 & 305660.14 & 34898.83 & 0 & 37153.16 & 0 & 46048.84 \\
\hline 7550.31 & 326988 & 17 & 302631.24 & 38599.41 & 0 & 24092.04 & 0 & 35871.46 \\
\hline 7550.38 & 285002 & 18 & 305345.51 & 38137.6 & 0 & 24436.43 & 0 & 32703.64 \\
\hline 7550.45 & 410633 & 16 & 188294.77 & 24205.05 & 0 & 19549.38 & 0 & 44136.42 \\
\hline 7550.51 & 357005 & 17 & 355963.03 & 42409.74 & 0 & 27701.41 & 0 & 56496.19 \\
\hline 7550.58 & 331350 & 17 & 254876.85 & 25439.15 & 0 & 13476.57 & 0 & 54479.23 \\
\hline 7550.64 & 307575 & 17 & 266452.47 & 24827.63 & 0 & 23016.86 & 0 & 29494.93 \\
\hline 7550.71 & 298388 & 17 & 133828.63 & 8156.42 & 0 & 10422.44 & 0 & 32315.84 \\
\hline 7550.77 & 288072 & 18 & 184991.99 & 15280.76 & 0 & 16452.18 & 0 & 38305.24 \\
\hline 7550.84 & 297542 & 17 & 292669.23 & 26049.36 & 0 & 21295.33 & 0 & 24082.23 \\
\hline 7550.91 & 356169 & 17 & 207001.5 & 23405.92 & 0 & 17969.68 & 0 & 20699.24 \\
\hline 7550.97 & 439871 & 16 & 95721.27 & 9656.14 & 0 & 14780.55 & 0 & 20867.85 \\
\hline 7551.04 & 284262 & 18 & 336316.34 & 30915.15 & 0 & 16119.69 & 0 & 40195.09 \\
\hline
\end{tabular}




\begin{tabular}{|c|c|c|c|c|c|c|c|c|}
\hline True Depth & XRF tCOUNTS & XRF Live Time & $\mathrm{Si}(\mathrm{ppm})$ & $\mathrm{Al}(\mathrm{ppm})$ & $\mathrm{P}(\mathrm{ppm})$ & $\mathrm{S}(\mathrm{ppm})$ & $\mathrm{K}(\mathrm{ppm})$ & $\mathrm{Ca}(\mathrm{ppm})$ \\
\hline 7551.10 & 387980 & 17 & 229608.87 & 21032.71 & 0 & 15271.3 & 0 & 46686.61 \\
\hline 7551.17 & 390662 & 17 & 155543.31 & 17541.12 & 0 & 15241.15 & 0 & 26770.79 \\
\hline 7551.23 & 384290 & 17 & 191365 & 25336.94 & 0 & 20034.18 & 0 & 20393.89 \\
\hline 7551.30 & 343324 & 17 & 157488.21 & 13750.15 & $\overline{0}$ & 9709.31 & $\overline{0}$ & 16721.73 \\
\hline 7551.36 & 400252 & 16 & 198761.91 & 18533.37 & 0 & 22306.96 & 0 & 8227.68 \\
\hline 7551.43 & 294144 & 18 & 214137.84 & 12937.11 & 0 & 12121.68 & 0 & 13375.52 \\
\hline 7551.50 & 281646 & 18 & 135391.97 & 8411.23 & 0 & 8353.26 & 0 & 15176.47 \\
\hline 7551.56 & 207154 & 18 & 244853.85 & 24677.48 & 0 & 13453.82 & 0 & 13400.38 \\
\hline 7551.63 & 373687 & 17 & 306797.25 & 33102.87 & 0 & 33076.21 & 0 & 35707.3 \\
\hline 7551.69 & 522992 & 15 & 84412.52 & 9503.23 & 0 & 12852.69 & 0 & 23196.28 \\
\hline 7551.76 & 466215 & 16 & 74994.82 & 7720.46 & 0 & 10275.11 & 0 & 22966.99 \\
\hline 7551.82 & 305425 & 17 & 173033.68 & 16081.62 & 0 & 30990.3 & 0 & 22108.78 \\
\hline 7551.89 & 326390 & 17 & 139477.05 & 11135.24 & 0 & 11634.98 & 0 & 17910.95 \\
\hline 7551.96 & 382728 & 17 & 199591.02 & 28619.16 & 0 & 16256.27 & 0 & 18772.4 \\
\hline 7552.02 & 331394 & 17 & 219534.28 & 30538.02 & 0 & 18408.06 & 0 & 49756.77 \\
\hline 7552.09 & 280862 & 18 & 162622.38 & 25701.2 & 0 & 10749.51 & 0 & 27044.29 \\
\hline 7552.15 & 540508 & 15 & 89044.76 & 9529.89 & 0 & 2873.55 & 0 & 347831.99 \\
\hline 7552.22 & 654116 & 14 & 53779.7 & 4241.74 & 0 & 1938.97 & 0 & 423088.1 \\
\hline 7552.28 & 651836 & 14 & 32763.14 & 2255.22 & 0 & 1520.4 & 0 & 408968.13 \\
\hline 7552.35 & 590349 & 14 & 29540.96 & 3184.85 & 0 & 2316.09 & 0 & 397989.64 \\
\hline 7552.41 & 630531 & 14 & 25153.68 & 3559.98 & 0 & 1682.22 & 0 & 409727.39 \\
\hline 7552.48 & 659914 & 13 & 19836.47 & 0 & 0 & 1391.63 & 0 & 398584.03 \\
\hline 7552.55 & 612197 & 14 & 21662.88 & 1622.17 & 0 & 1174.36 & 0 & 431628.73 \\
\hline 7552.61 & 623372 & 14 & 11961.41 & 1020.32 & 0 & 1021.6 & 0 & 213258.8 \\
\hline 7552.68 & 641246 & 14 & 14459.27 & 1809.2 & 0 & 1103.67 & 0 & 329570.13 \\
\hline 7552.74 & 648064 & 14 & 21001.5 & 2756.61 & 0 & 1571.35 & 0 & 394034.93 \\
\hline 7552.81 & 518325 & 15 & 14112.63 & 1589.24 & 0 & 1304.73 & 0 & 322842.52 \\
\hline 7552.87 & 698043 & 13 & 27650.84 & 0 & 0 & 701.76 & 0 & 448758.61 \\
\hline 7552.94 & 680298 & 13 & 22925.95 & 0 & 0 & 712.17 & 0 & 444062.15 \\
\hline 7553.01 & 666638 & 13 & 19849.06 & 0 & 0 & 981.37 & 0 & 448606.15 \\
\hline 7553.07 & 660865 & 13 & 21874.04 & 4280.37 & 0 & 786.18 & 0 & 443405.52 \\
\hline 7553.14 & 661870 & 13 & 18294.69 & 0 & 0 & 1147.77 & 0 & 450463.26 \\
\hline 7553.20 & 619399 & 14 & 38499.81 & 5590.28 & 0 & 7008.67 & 0 & 396773.13 \\
\hline
\end{tabular}




\begin{tabular}{|c|c|c|c|c|c|c|c|c|}
\hline True Depth & XRF tCOUNTS & XRF Live Time & $\mathrm{Si}(\mathrm{ppm})$ & $\mathrm{Al}(\mathrm{ppm})$ & $\mathrm{P}(\mathrm{ppm})$ & $\mathrm{S}(\mathrm{ppm})$ & $\mathrm{K}(\mathrm{ppm})$ & $\mathrm{Ca}(\mathrm{ppm})$ \\
\hline 7553.27 & 549064 & 15 & 43322.28 & 0 & 0 & 2843.58 & 0 & 321335.39 \\
\hline 7553.33 & 630129 & 14 & 63212.29 & 2109.02 & 0 & 4430.71 & 0 & 401657.22 \\
\hline 7553.40 & 702584 & 13 & 13189.21 & 0 & 0 & 4098.94 & 0 & 53778.75 \\
\hline 7553.46 & 428979 & 16 & 56116.03 & 7905.06 & 0 & 6754.03 & 0 & 29104.18 \\
\hline 7553.53 & 309440 & 17 & 171772.1 & 26923.45 & 0 & 17130.93 & 0 & 41723.89 \\
\hline 7553.60 & 531825 & 15 & 30746.73 & 2232.53 & 0 & 4707.56 & 0 & 4478.29 \\
\hline 7553.66 & 568001 & 15 & 28201.57 & 6359.95 & 0 & 3183.31 & 0 & 1460 \\
\hline 7553.73 & 472000 & 16 & 67332.75 & 10021.5 & 0 & 7404.37 & 0 & 14089.99 \\
\hline 7553.79 & 559909 & 15 & 38061.38 & 8893.42 & 0 & 2452.79 & 0 & 34433.16 \\
\hline 7553.86 & 373558 & 17 & 16653.62 & 4117.52 & 0 & 934.26 & 0 & 0 \\
\hline 7553.92 & 488440 & 16 & 23516.61 & 9366.62 & 0 & 1273.68 & 0 & 0 \\
\hline 7553.99 & 474909 & 16 & 83680.36 & 10063.61 & 0 & 2739.49 & 0 & 10480.94 \\
\hline 7554.06 & 103833 & 19 & 93195.86 & 7636.31 & 0 & 5017.01 & 0 & 30871.34 \\
\hline 7554.12 & 234252 & 18 & 221244.11 & 38124.07 & 0 & 13480.36 & 0 & 22474.69 \\
\hline 7554.19 & 308455 & 17 & 300354.36 & 36027.26 & 0 & 19716.16 & 0 & 29278.77 \\
\hline 7554.25 & 300956 & 17 & 322171.58 & 30704.99 & 0 & 20898.96 & 0 & 48213.99 \\
\hline 7554.32 & 349789 & 17 & 146176.93 & 17921.64 & 0 & 13351.59 & 0 & 19982.11 \\
\hline 7554.38 & 334600 & 17 & 158716.69 & 15500.73 & 0 & 7804.15 & 0 & 10180.49 \\
\hline 7554.45 & 416475 & 16 & 130601.13 & 17144.09 & 0 & 8229.16 & 0 & 7266.77 \\
\hline 7554.51 & 431868 & 16 & 145913.57 & 21970.92 & 0 & 14027.85 & 0 & 17022.37 \\
\hline 7554.58 & 442751 & 16 & 120183.66 & 17426.24 & 0 & 16173.6 & 0 & 16528.61 \\
\hline 7554.65 & 439788 & 16 & 100334.11 & 13442.48 & 0 & 6199.74 & 0 & 19748.19 \\
\hline 7554.71 & 428671 & 16 & 86978.21 & 14183.75 & 0 & 5310.66 & 0 & 12475.76 \\
\hline 7554.78 & 467874 & 16 & 31371.38 & 11763.1 & 0 & 2756.97 & 0 & 0 \\
\hline 7554.84 & 209670 & 18 & 78916.7 & 13588.76 & 0 & 2239.47 & 0 & 5985.24 \\
\hline 7554.91 & 297736 & 17 & 347320.81 & 37991.85 & 0 & 15370.28 & 0 & 8536.29 \\
\hline 7554.97 & 324677 & 17 & 341065.64 & 47266.2 & 0 & 23963.34 & 0 & 30228.39 \\
\hline 7555.04 & 311056 & 17 & 301260.1 & 26684.62 & 0 & 10365.4 & 0 & 53106.88 \\
\hline 7555.10 & 346152 & 17 & 175855.83 & 23422.07 & 0 & 11117.5 & 0 & 30011.62 \\
\hline 7555.17 & 449410 & 16 & 72417.94 & 10948.22 & 0 & 5306.77 & 0 & 84233.08 \\
\hline 7555.24 & 396367 & 17 & 211650.53 & 32755.22 & 0 & 12690.22 & 0 & 82603.35 \\
\hline 7555.30 & 637873 & 14 & 21677.3 & 2011.09 & 0 & 8315.18 & 0 & 215857.25 \\
\hline 7555.37 & 652761 & 14 & 15214.28 & 1819.75 & 0 & 2455.07 & 0 & 114909.9 \\
\hline
\end{tabular}




\begin{tabular}{|c|c|c|c|c|c|c|c|c|}
\hline True Depth & XRF tCOUNTS & XRF Live Time & $\mathrm{Si}(\mathrm{ppm})$ & $\mathrm{Al}(\mathrm{ppm})$ & $\mathrm{P}(\mathrm{ppm})$ & $\mathrm{S}(\mathrm{ppm})$ & $\mathrm{K}(\mathrm{ppm})$ & $\mathrm{Ca}(\mathrm{ppm})$ \\
\hline 7555.43 & 648299 & 14 & 35130.84 & 1148.63 & 0 & 2094.02 & 0 & 140313.2 \\
\hline 7555.50 & 641791 & 14 & 74544.57 & 3392.59 & 693.67 & 1328 & 0 & 356094.51 \\
\hline 7555.56 & 515587 & 15 & 94711.4 & 2130.12 & 0 & 7400.82 & 0 & 177461.23 \\
\hline 7555.63 & 580364 & 14 & 42519.43 & 9598.33 & 0 & 6977.28 & 0 & 20771.01 \\
\hline 7555.70 & 654100 & 14 & 18862.35 & 0 & 0 & 2888.82 & 0 & 152916.46 \\
\hline 7555.76 & 523233 & 15 & 61527.68 & 8795.54 & 0 & 6677.76 & 0 & 94977.5 \\
\hline 7555.83 & 633206 & 14 & 21267.63 & 3292.75 & 0 & 4835.43 & 0 & 15971.04 \\
\hline 7555.89 & 578099 & 14 & 44209.95 & 11847.53 & 0 & 3953.18 & 0 & 20489.62 \\
\hline 7555.96 & 604713 & 14 & 152063.6 & 8970.89 & 0 & 7465.72 & 0 & 377658.05 \\
\hline 7556.02 & 574704 & 15 & 108505.7 & 2129.73 & 0 & 1506.25 & 0 & 353114.05 \\
\hline 7556.09 & 559671 & 15 & 56463.78 & 1771.7 & 0 & 1637.1 & 0 & 308346.84 \\
\hline 7556.15 & 243095 & 18 & 266949.56 & 39783.72 & 0 & 11299.02 & 0 & 78373.27 \\
\hline 7556.22 & 481452 & 16 & 151691.3 & 9536.25 & 0 & 1649.29 & 0 & 264677.38 \\
\hline 7556.29 & 181733 & 19 & 56070.65 & 21472.65 & 0 & 3281.23 & 0 & 5768.25 \\
\hline 7556.35 & 248799 & 18 & 69841.18 & 27714.05 & 0 & 3578.11 & 17633.93 & 19951.53 \\
\hline 7556.42 & 219315 & 18 & 273521.69 & 80044.34 & 0 & 13570.91 & 47439.7 & 13462.81 \\
\hline 7556.48 & 281531 & 18 & 120699.24 & 9771.49 & 0 & 1979.03 & 0 & 16929.35 \\
\hline 7556.55 & 352126 & 17 & 230796.08 & 23949.53 & 0 & 11427.17 & 0 & 51832.1 \\
\hline 7556.61 & 334439 & 17 & 341267.55 & 34019.56 & 0 & 13812.65 & 0 & 62749.37 \\
\hline 7556.68 & 332439 & 17 & 318284.05 & 27452.37 & 0 & 12082.2 & 0 & 58601.58 \\
\hline 7556.75 & 344621 & 17 & 227133.04 & 18086.92 & 0 & 8447.46 & 0 & 63158.79 \\
\hline 7556.81 & 367617 & 17 & 248534.89 & 25088.23 & 0 & 12069.65 & 0 & 98627.52 \\
\hline 7556.88 & 379264 & 17 & 280195.15 & 21011.76 & 0 & 8924.17 & 0 & 123099.06 \\
\hline 7556.94 & 430301 & 16 & 235129.87 & 8232.17 & 0 & 23235.57 & 0 & 144193.2 \\
\hline 7557.01 & 378212 & 17 & 197863.16 & 24426.07 & 0 & 13448.69 & 0 & 87742.11 \\
\hline 7557.07 & 398513 & 17 & 311244.24 & 30242.82 & 0 & 21035.24 & 0 & 86237.59 \\
\hline 7557.14 & 612431 & 14 & 55730.98 & 3193.67 & 0 & 7076.39 & 0 & 180727.68 \\
\hline 7557.20 & 413155 & 16 & 158932.01 & 15961.8 & 0 & 9006.16 & 0 & 65216.83 \\
\hline 7557.27 & 433351 & 16 & 131958.15 & 35224.38 & 0 & 12578.42 & 3924.18 & 77576.97 \\
\hline 7557.34 & 348719 & 17 & 257246.57 & 37599.48 & 0 & 13876.51 & 0 & 73029.11 \\
\hline 7557.40 & 415157 & 16 & 241635.8 & 46746.28 & 0 & 21309.95 & 0 & 114917.1 \\
\hline 7557.47 & 481344 & 16 & 26077.78 & 2577.98 & 0 & 3197.54 & 0 & 14016.36 \\
\hline 7557.53 & 462535 & 16 & 112996.12 & 1509.96 & 0 & 9739.67 & 0 & 14578.95 \\
\hline
\end{tabular}




\begin{tabular}{|r|r|r|r|r|r|r|r|r|}
\hline \multicolumn{1}{|c|}{ True Depth } & XRF tCOUNTS & XRF Live Time & \multicolumn{1}{c|}{$\mathrm{Si}(\mathrm{ppm})$} & $\mathrm{Al}(\mathrm{ppm})$ & $\mathrm{P}(\mathrm{ppm})$ & $\mathrm{S}(\mathrm{ppm})$ & $\mathrm{K}(\mathrm{ppm})$ & $\mathrm{Ca}(\mathrm{ppm})$ \\
\hline 7557.60 & 450726 & 16 & 103507.1 & 866.78 & 0 & 1452.81 & 0 & 67392.78 \\
\hline 7557.66 & 481631 & 16 & 83060.85 & 1866.81 & 0 & 1724.32 & 0 & 36476.89 \\
\hline 7557.73 & 498842 & 15 & 90938.13 & 1676.85 & 0 & 2341.48 & 0 & 20318.51 \\
\hline 7557.80 & 606336 & 14 & 10057.84 & 0 & 0 & 916.08 & 0 & 54826.8 \\
\hline 7557.86 & 587553 & 14 & 59975.57 & 0 & 0 & 457.87 & 0 & 354369.34 \\
\hline 7557.93 & 648111 & 14 & 39856.02 & 1592.08 & 0 & 474.09 & 0 & 411790.19 \\
\hline 7557.99 & 664767 & 13 & 44206.42 & 0 & 0 & 377.34 & 0 & 419904.38 \\
\hline 7558.06 & 623808 & 14 & 91911.43 & 0 & 0 & 446.61 & 0 & 403167.79 \\
\hline 7558.12 & 573901 & 15 & 7703.4 & 0 & 0 & 1542 & 0 & 69185.24 \\
\hline
\end{tabular}

